DEPARTMENT OF THE INTERIOR UNITED STATES GEOLOGICAL SURVEY GEORGE OTIS SMITH, DIRECTOR

BULLETIN 419

\title{
ANALYSES OF ROCKS AND MINERALS
}

FROM THE

$\circ$

\section{LABORATORY OF THE UNITED STATES GEOLOGICAL SURVEY}

\section{To 1908}

\author{
TABULATED BY \\ F. W. CLARKE, Chief Chemist
}

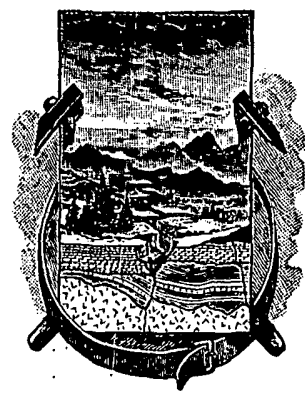

W ASHINGTON

GOVERNMENT PRINTING OFFICE 



\section{CONTENTS.}

Page.

Introduction $\ldots \ldots \ldots \ldots \ldots \ldots \ldots \ldots \ldots \ldots \ldots \ldots \ldots \ldots \ldots \ldots \ldots \ldots, 1$

The average composition of rocks................................ 4

Analyses of igneous and crystalline rocks........................... 13

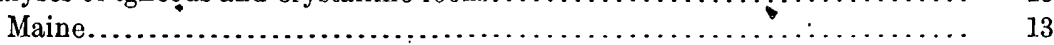

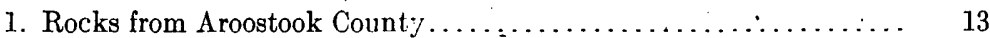

2. Miscellaneous rocks.................................. 14

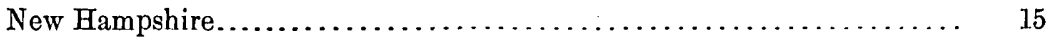

Vermont.................................................... 15

1. Rocks of Mount Ascutney .............................. 15

2. Miscellaneous rocks.............................. $\quad 17$

Massachusetts.......................................... ${ }_{18}$

1. Magnesian rocks.......................................... 18

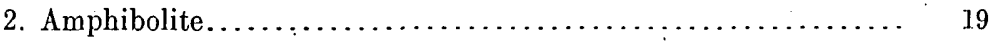

3. Miscellaneous rocks................................. 21

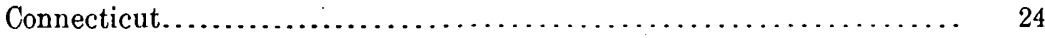

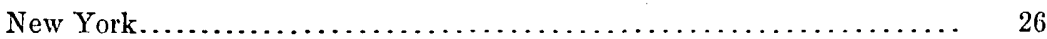

1. Rocks of the Adirondack region......................... 26

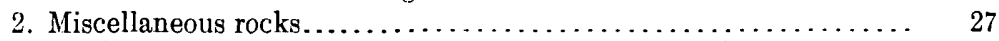

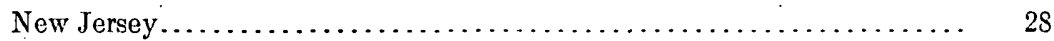

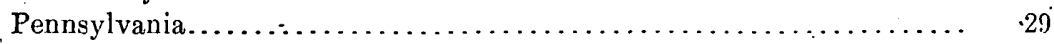

Maryland and the District of Columbia........................ 30

1. Peridotite and pyroxenite............................ 30

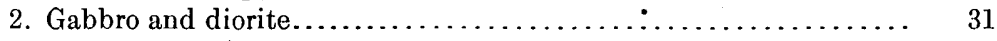

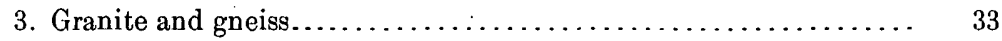

4. Miscellaneous rocks................................ 34

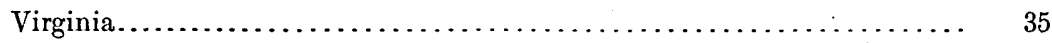

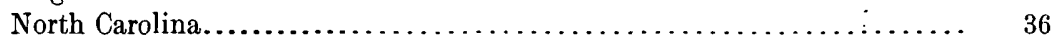

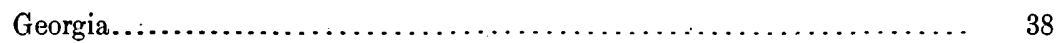

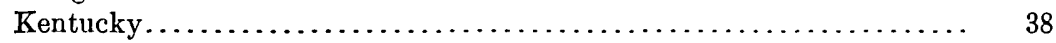

1. Elliott County dike................................... 38

2. Crittenden County dike................................. 39

Tennessee................................................ ${ }_{40}$

Missouri.................................................... 40

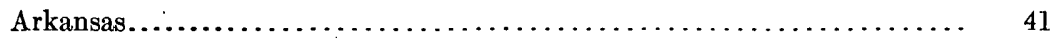

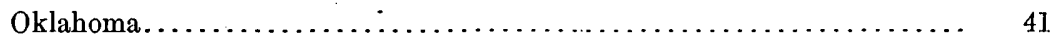

Texas............................................... 41.

Lake Superior region...................................... 45

1. Marquette region, Michigan......................... 45

2. Crystal Falls district, Michigan ....................... 46

3. Keweenaw Point, Michigan......................... 48

4. Menominee River................................. 48

Sturgeon Falls gabbro................................. 48

Lower Quinnesec Falls............................ $\quad 49$

Upper Quinnesec Falls.............................. $\quad 49$

Schist from the Aragon iron mine...................... 50 
Analyses of igneous and crystalline rocks-Continued.

Lake Superior region-Continued.

5. Penokee-Gogebic region. ...............

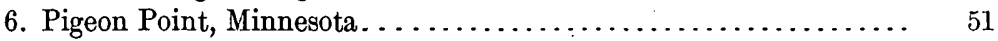

7. Mesabi district, Minnesota ......................... 54

8. Miscellaneous rocks from Minnesota . . . . . . . . . . . . . . . 55

South Dakota........................................ 57

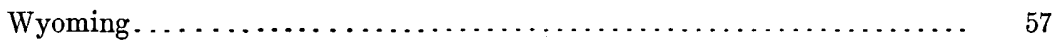

Yellowstone National Park and the Absaroka Range.................. $\quad 60$

1. Electric Peak ...................................... $\quad 60$

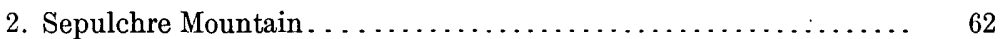

3. Absaroka Range..................................... 63

Crandall Basin.................................. 63

Sunlight intrusives. . . . . . . . . . . 65

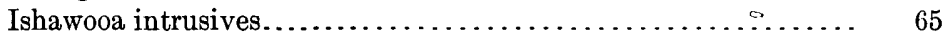

Dikes in breccia.................................. $\quad 66$

4. Absarokite-shoshonite-banakite series.................... 67

5. Miscellaneous rocks.............................. 70

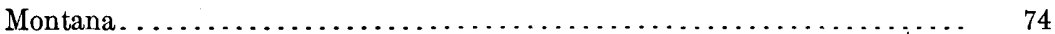

1. Madison and Gallatin valleys.......................... 74

2. Butte district.................................... 77

3. Boulder batholith.................................. $\quad 79$

4. Crazy Mountains................................... 80

5. Little Belt Mountains. . . . . . . . . . . . . . . . . . . . 83

6. Castle Mountain district........................... $\quad 86$

7. Highwood Mountains............................... 87

8. Little Rocky Mountains............................. $\quad 90$

9. Bearpaw Mountains................................ 90

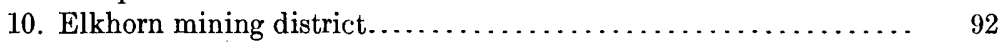

11. Bitterroot Range.................................... 93

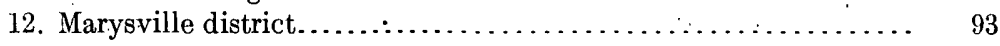

13. Livingston quadrangle........................... 94

14. Philipsburg quadrangle................................ 95

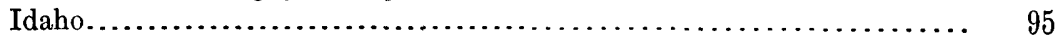

Colorado ................................................. 98

1. Denver Basin.......................................... 98

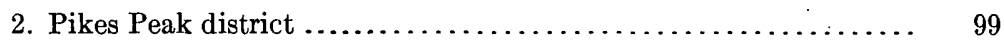

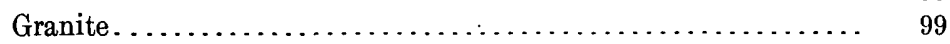

Rocks of Cripple Creek district........................ 100

Miscellaneous rocks.............................. 103

3. Silver Cliff and Rosita. . . . . . . . . . . . . . . . . . . . . . . . . . . 104

4. Buffalo Peaks. . . . . . . . . . . . . . . . . . . . . . . . . . . . . . 108

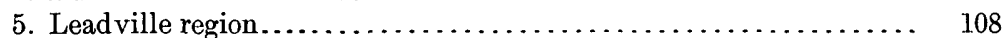

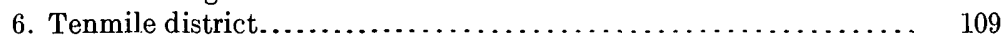

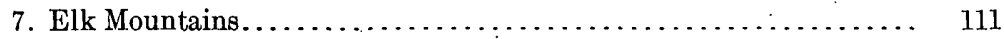

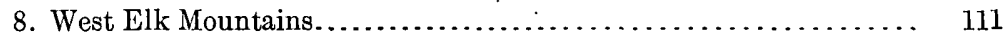

9. La Plata Mountains. ............................ 112

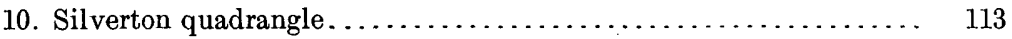

11. Ouray quadrangle.................................. 114

12. Telluride quadrangle................................. 115

13. Miscellaneous rocks................................ 115

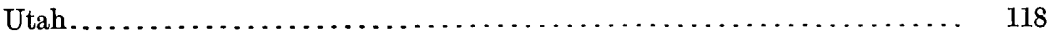

1. Tintic district.................................. 118

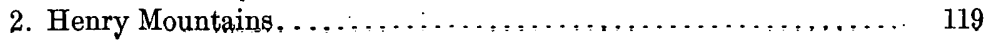


Analyses of igneous and crystalline rocks-Continued.

Utah-Continued.

3. Bingham district.............................. 120

4. La Sal Mountains. .............................. 120

5. Park City district............................. 121

6. Miscellaneous rocks............................. 122

New Mexico...................................... 122

1. Basalts from the Rio Grande Canyon.................... 123

2. Mount Taylor region. . . . . . . . . . . . . . . . . . . . 123

3. Colfax County ................................ 123

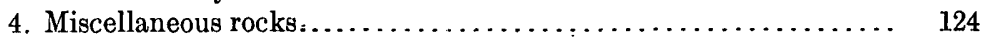

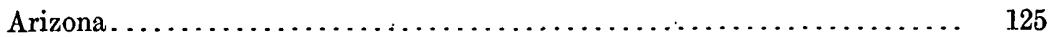

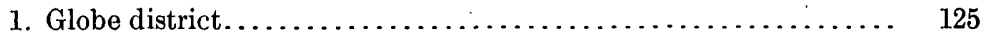

2. Bradshaw Mountains quadrangle.................. 126

3. Morenci district. ................................ 127

4. Miscellaneous rocks........................... 127

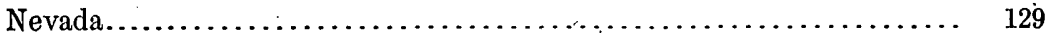

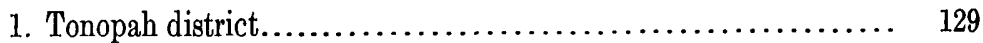

2. Goldfield district.............................. 130

3. Bullfrog district.............................. 132

4. Miscellaneous rocks......................... 132

California . . . ................................... 135

1. Mount Shasta................................ 135

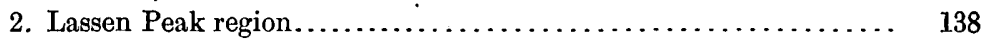

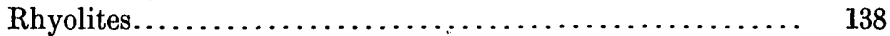

Dacites and andesites. . . . . . . . . . . . . . . . . . 139

Basalts.................................. 142

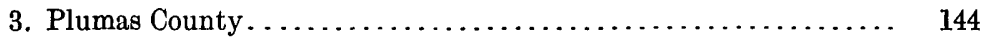

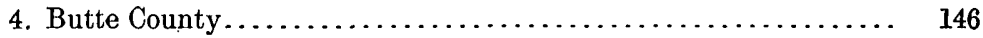

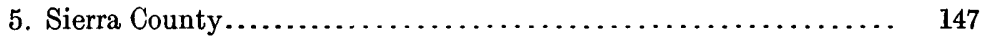

6. Nevada City and Grass Valley....................... 149

7. Placer County .................................... 150

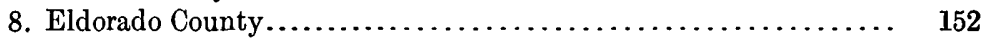

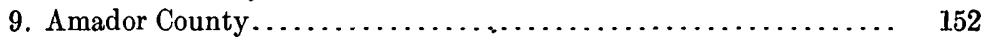

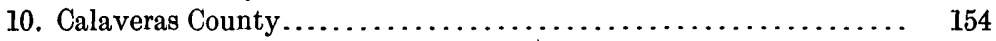

11. Tuolumne County................................ 154

12. Mariposa County............................... 157

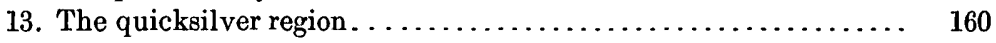

14. Mount Diablo.................................. 161

15. Miscellaneous rocks.............................. 163

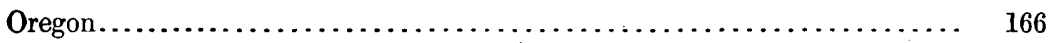

1. Basalt, Mount Thielsen........................... 166

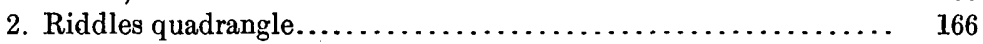

3. Crater Lake..................................... 168

4. Port Orford quadrangle........................... 169

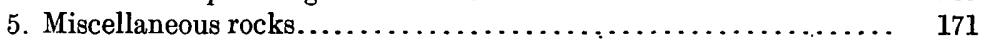

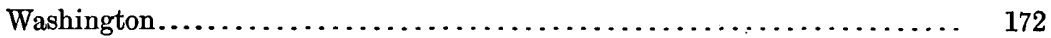

1. Mount Stuart quadrangle........................... 172

2. Snoqualmie quadrangle $\ldots \ldots \ldots \ldots \ldots \ldots \ldots \ldots \ldots \ldots \ldots \ldots \ldots \ldots$

3. Miscellaneous rocks............................... 174

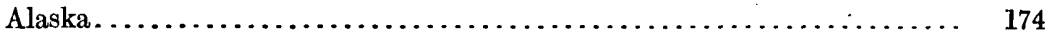

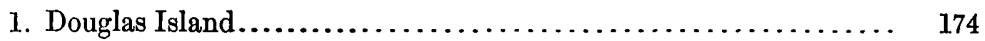

2. Copper River Basin............................ 175

3. Miscellaneous rocks............................ 176 
Analyses of igneous and crystalline rocks-Continued. Page.

British Columbia..................................... 178

Hawaiian Islands. ...................................... 178

Guatemala............................................ 179

West Indies.............................................. 179

Brazil.............................................. 180

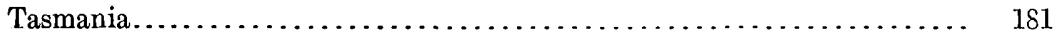

Analyses of sandstones, cherts, and sinters....................... 102

Sandstones from Eastern States............................... 182

Sandstones from Michigan, Wisconsin, and Iowa................... 182

Sandstones from Ohio..................................... 183

Sandstones from Kentucky, Mississippi, Missouri, and Arkansas........ 183

Sandstones from Kansas.................................... 183

Sandstones from Colorado, Utah, and Arizona................... 184

Sandstones from California.................................. 185

1. Mount Diablo...................................... 185

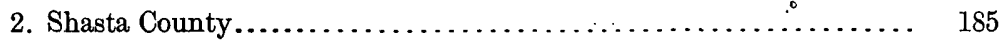

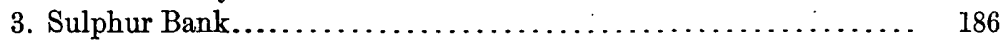

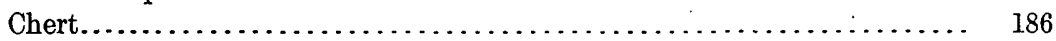

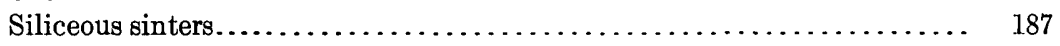

Analyses of carbonate rocks.................................... 189

Maine and Vermont.......................................... 189

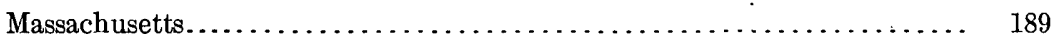

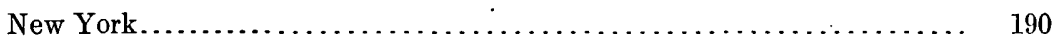

Pennsylvania............................................ 190

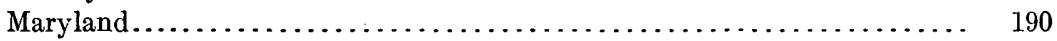

Virginia and West Virginia................................... 191

North Carolina and South Carolina............................ 191

Georgia.............................................. 192

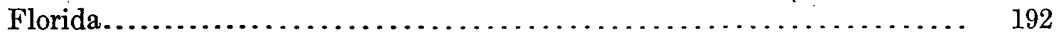

1. Coral and shell rocks.............................. 192

2. Miscellaneous rocks................................... 193

Kentucky ......................................... 194

Tennessee, Alabama, and Louisiana........................ 194

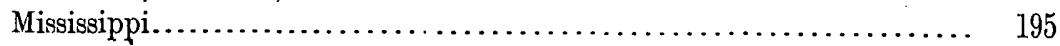

Texas....................................................... 195

Ohio.................................................. 195

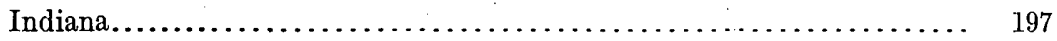

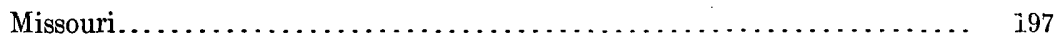

Kansas.................................................... 197

Michigan, Wisconsin, Minnesota, and Canada.................... 198

South Dakota and Wyoming............................ 200

Yellowstone National Park ................................ 201

Montana................................................. 202

Idaho .................................................... 202

Colorado............................................... 203

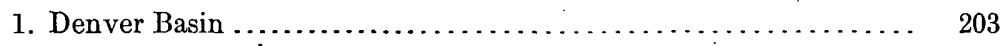

2. Leadville district................................ 203

3. Fairplay, Park County................................... 204

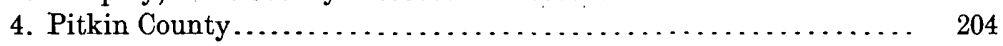

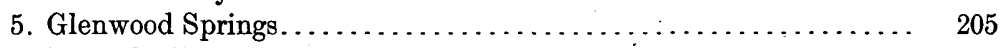

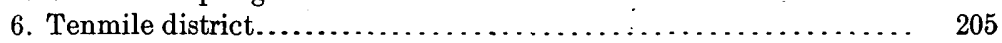

7. Rico district........................................ 206

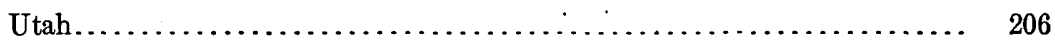


Analyses of carbonate rocks-Continued. Page.

- Arizona................................................. 208

Nevada and California.................................... 208

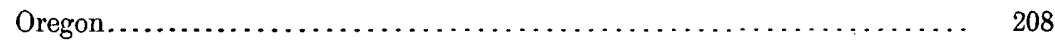

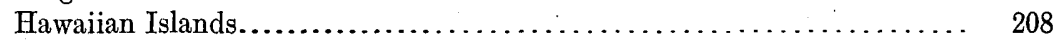

Analyses of slates and shales................................ 211

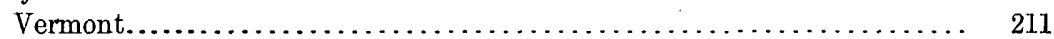

New York.............................................. 212

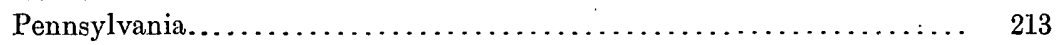

Kentucky, Georgia, and Alabama......................... 214

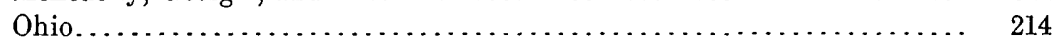

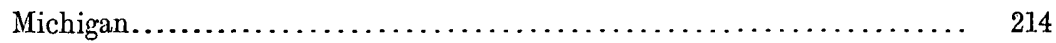

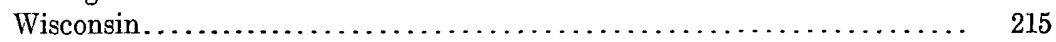

Minnesota................................................. 215

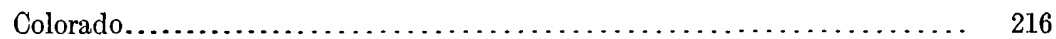

New Mexico.......................................... 217

Arizona..................................................... 217

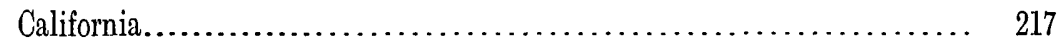

Analyses of clays, soils, etc................................... 219

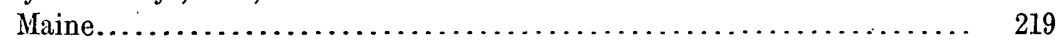

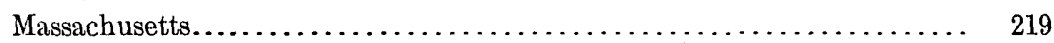

Connecticut....................................................... 220

New York and Pennsylvania................................. 220

Maryland .................................................. 221

Virginia and North Carolina................................. 222

South Carolina and Georgia.................................... 222

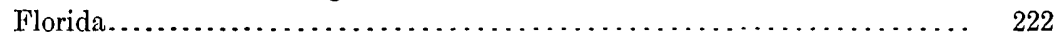

Alabama and Mississippi...................................... 223

Kentucky and Tennessee..................................... 224

Illinois, Iowa, and Minnesota............................ 224

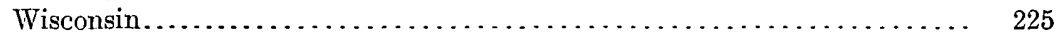

Missouri and Arkansas....................................... 225

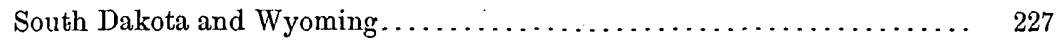

Colorado................................................. 227

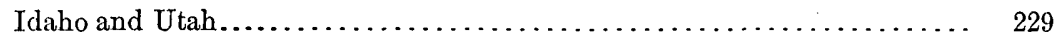

New Mexico and Arizona.................................... 229

Nevada............................................ 230

California, Washington, and Hawaiian Islands................. 230

Oceanic clays........................................... 231

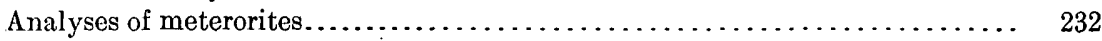

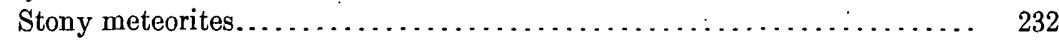

1. Rockwood, Tennessee............................ 232

2. Hamblen County, Tennessee....................... 232

3. Allegan, Michigan.............................. 233

4. Winnebago County, Illinois..................... 233

5. Taney County, Missouri.......................... 234

6. Washington County, Kansas........................ 234

7. Kiowa County, Kansas.............................. 235

8. Travis County, Texas............................ 235

9. Bluff, Fayette County, Texas...................... 236

10. San Bernardino County, California.................... 237

11. Beaver Creek, British Columbia...................... 237

12. Llano del Inca, Chile.......................... 238

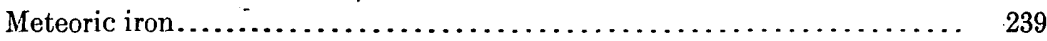


Anaiyses of minerals

I. Native elements............................... 241

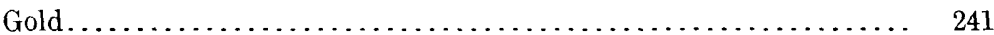

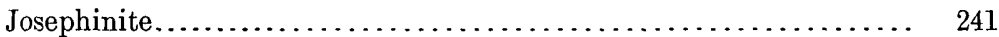

II. Sulphides and arsenides............................ 242

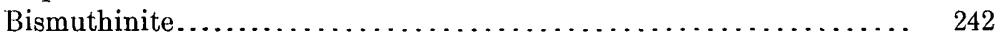

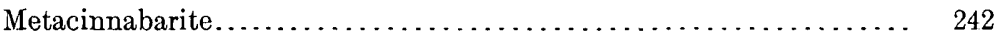

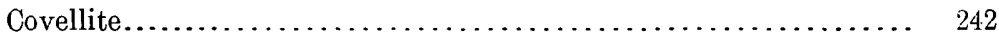

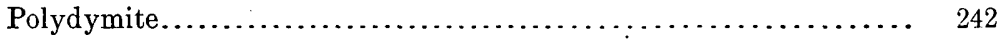

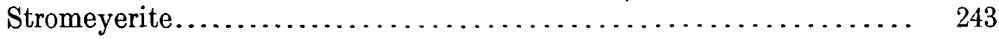

Sulphide of silver, copper, and zinc ................... 243

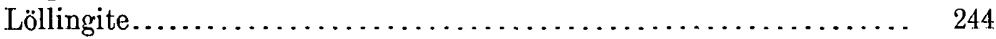

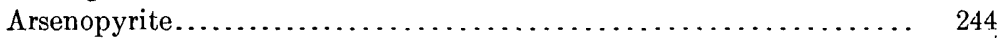

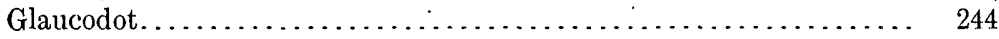

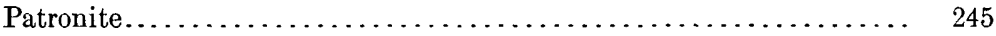

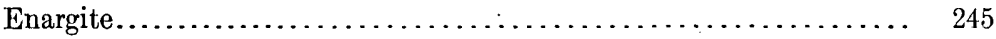

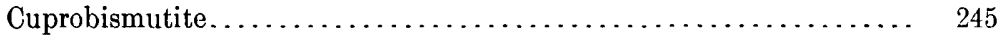

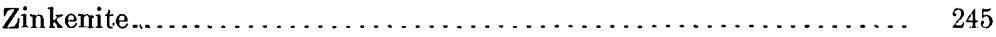

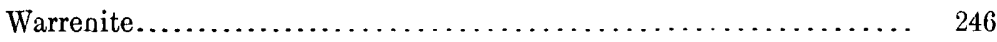

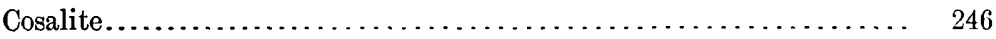

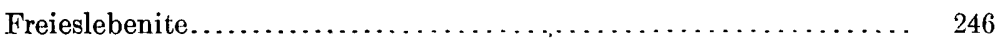

Bournonite. . . . . . . . . . . . . . . . . . . . . . . . . . 246

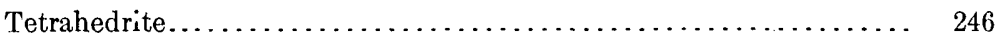

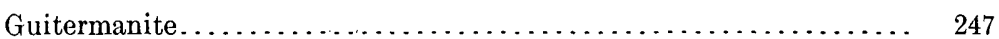

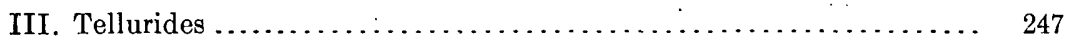

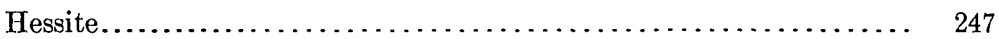

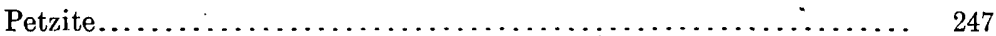

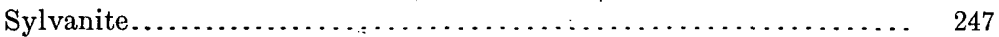

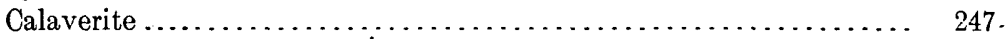

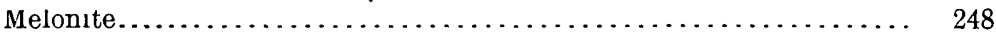

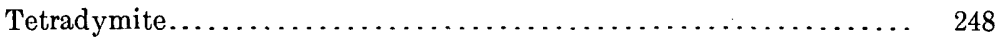

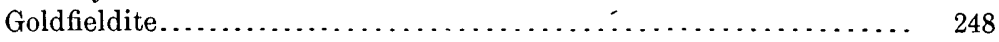

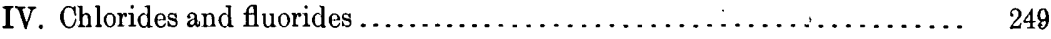

Halite.................................... 249

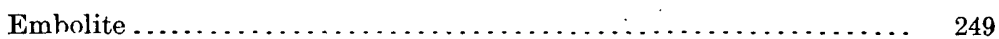

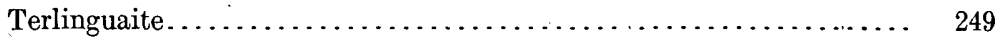

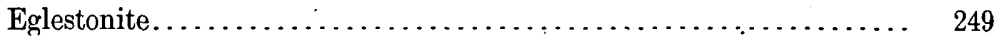

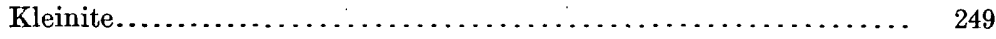

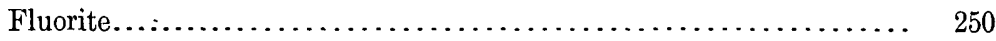

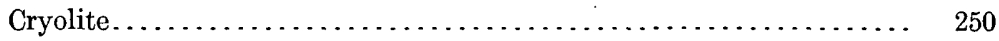

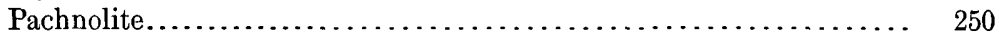

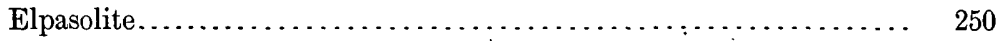

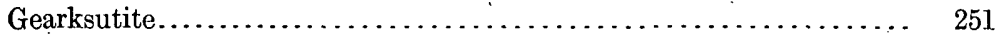

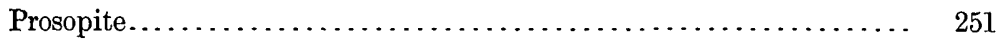

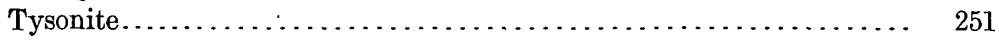

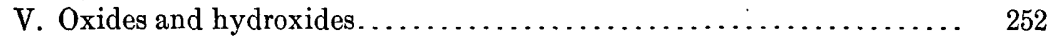

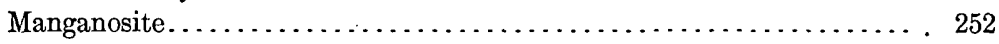

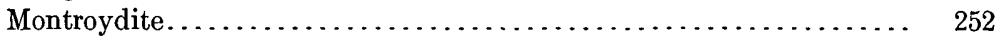

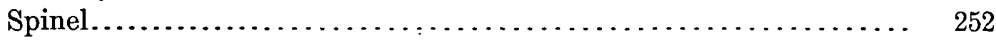

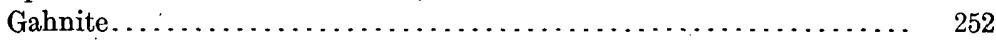

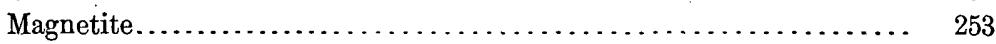

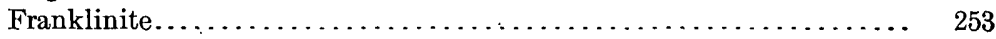

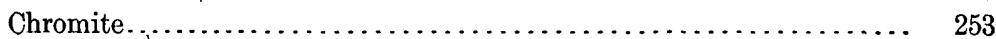


Analyses of minerals-Continued.

V. Oxides and hydroxides-Continued.

Rutile............................................ 253

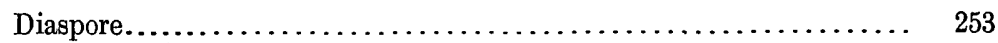

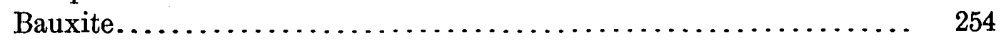

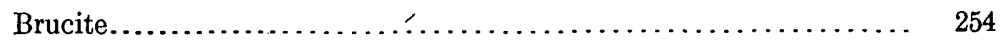

Psilomelane........................................... 254

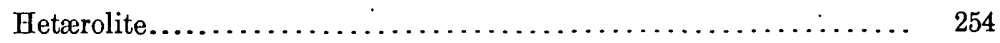

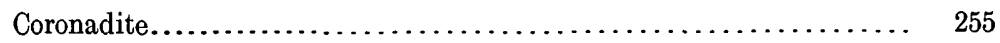

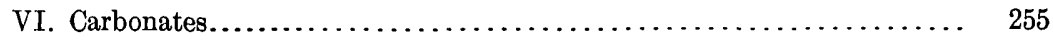

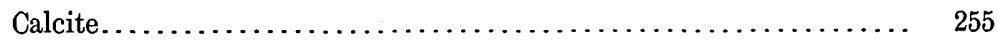

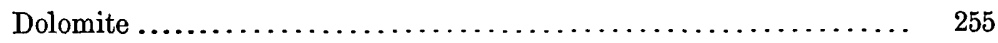

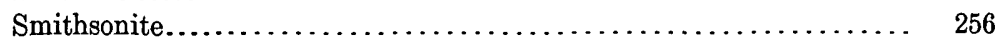

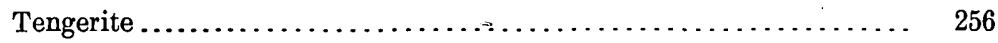

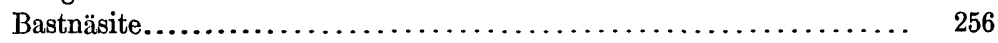

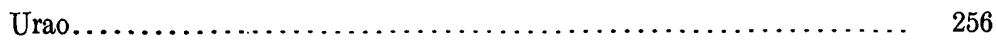

VII. Silicates............................................. 257

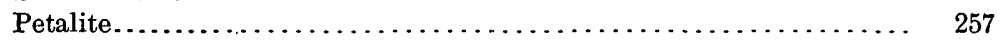

Orthoclase and microcline............................. 257

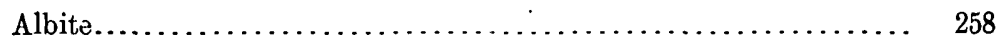

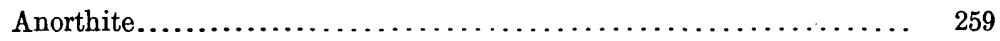

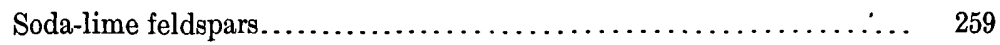

Leucite........................................ 260

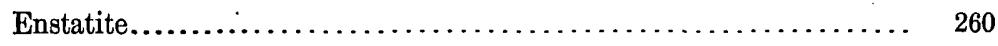

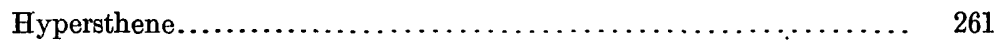

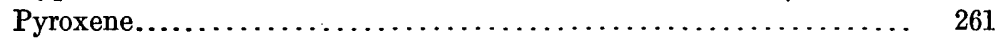

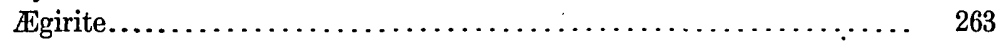

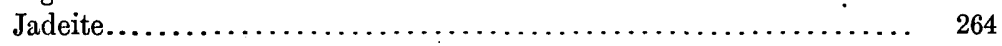

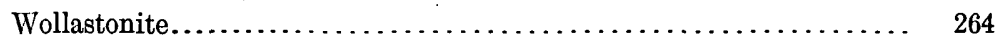

Pectolite............................................. 264

Amphibole........................................ 264

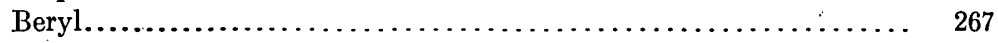

Nephelite......................................... 267

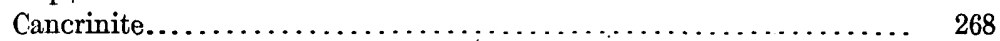

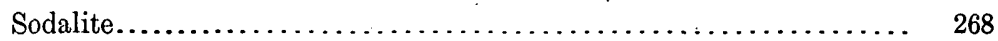

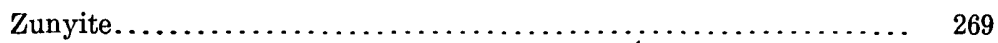

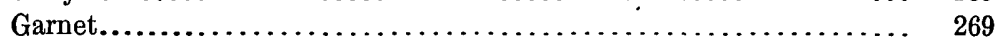

Chrysolite............................................ $\quad 270$

Willemite............................................ $\quad 270$

Friedelite............................................ 271

Mizzonite............................................. 271

Vesuvianite.......................................... 271

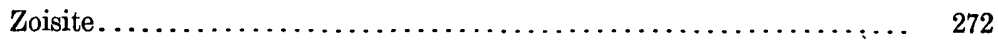

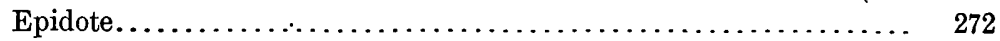

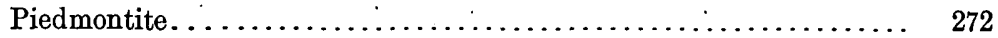

Allanite............................................. 273

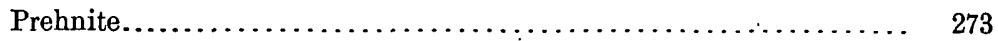

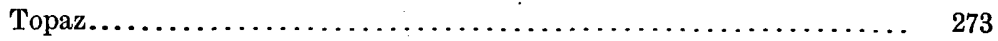

Sillimanite......................................... 274

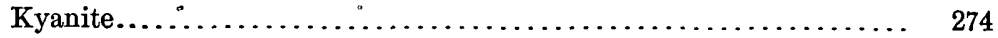

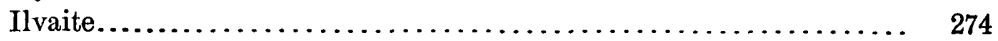

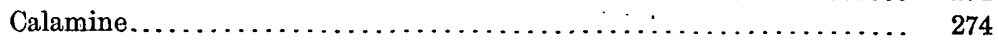

Lawsonite........................................... 274 
Analyses of minerals-Continued. Page.

VII. Silicates-Continued.

Staurolite ........................................ $\quad 275$

Gadolinite...................................... 275

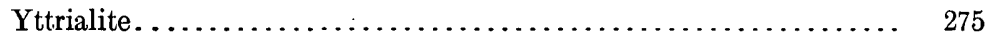

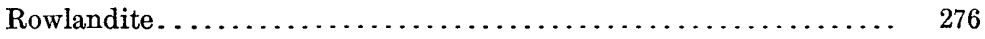

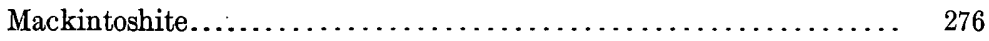

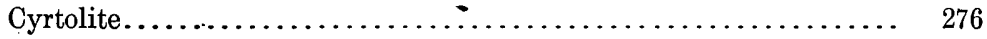

Danburite.......................................... 277

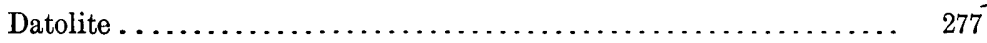

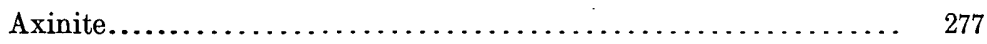

Dumortierite.................................... 277

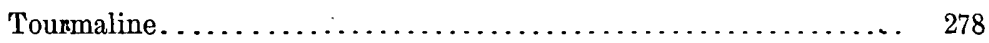

Lithia tourmaline........................... 278

Magnesian and iron tourmaline..................... 279

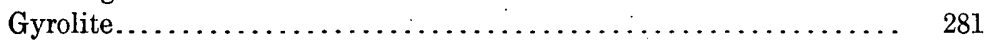

Apophyllite......................................... 281

Ptilolite............................................. 282

Heulandite. . . . . . . . . . . . . . . . . . . . . . . . . . . $\quad 282$

Stilbite......................................... $\quad 282$

Laumontite........................................ 283

Chabazite.......................................... 283

Levynite........................................... 283

Analcite............................................ 283

Natrolite............................................ 284

Scolecite........................................... 284

Mesolite............................................ 285

Thomsonite.......................................... 285

Hydronephelite. . . . . . . . . . . . . . . . . . . . . . . . . . . 285

Muscovite......................................... 285

Lepidolite...................................... 287

Cookeite......................................... . 288

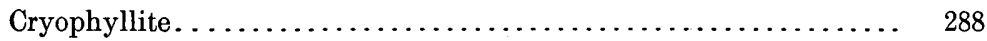

Biotite............................................... 288

Phlogopite....................................... 289

Lepidomelane ....................................... 290

Roscoelite..................................... $\quad 290$

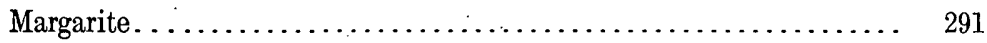

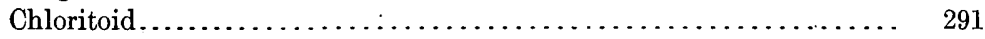

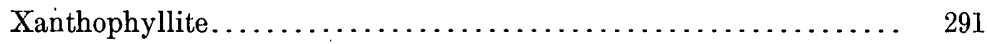

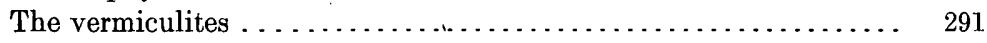

Clinochlore.................................... $\quad 293$

Prochlorite........................................ $\quad 294$

Serpentine...................................... 294

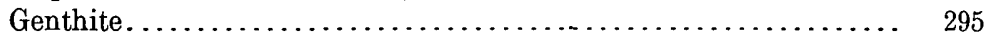

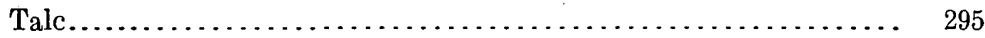

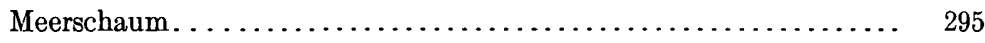

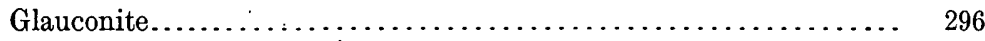

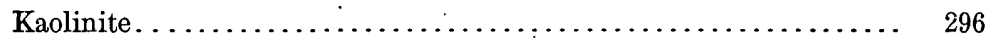

Halloysite . . . . . . . . . . . . . . . . . . . . . . . . . . . . 296

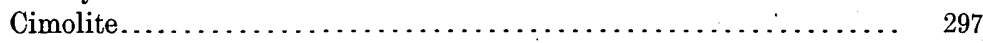

Pyrophyllite........................................ $\quad 297$

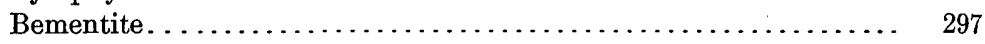

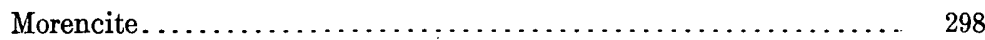

Bole.......................................... 298 
Analyses of minerals-Continued. Page.

VIII. Titanates and titano-silicates......................... 298

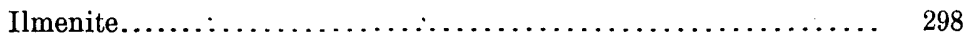

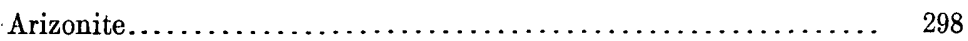

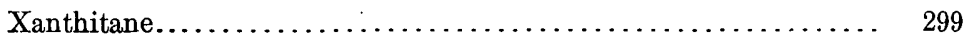

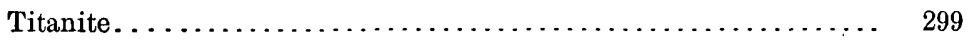

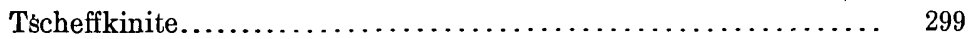

Astrophyllite.................................... 299

IX. Columbo-tantalates .............................. 300

Samarskite. . . . . . . . . . . . . . . . . . . . . . . . . 300

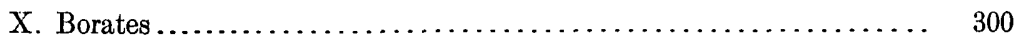

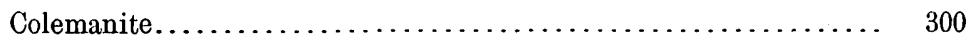

Ulexite. . . . . . . . . .

Ludwigite. . . . . . . . . . . .

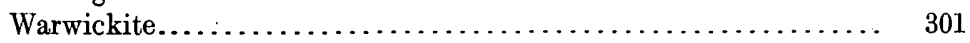

XI. Nitrates..................................... 301

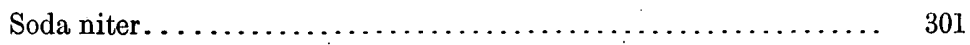

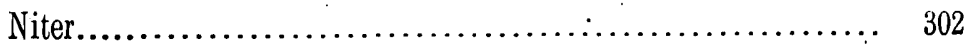

XII. Phosphates.................................... 302

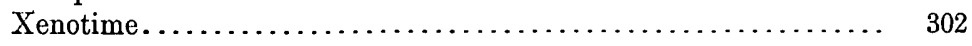

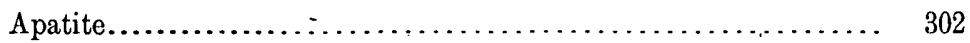

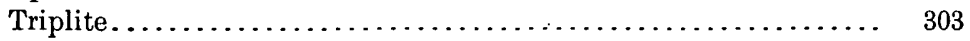

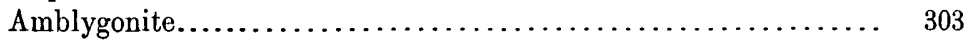

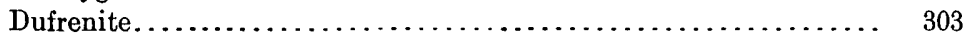

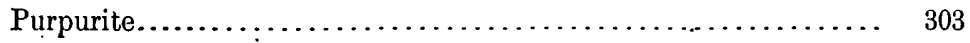

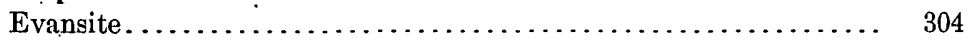

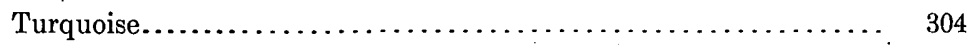

XIII. Vanadates ...................................... 304

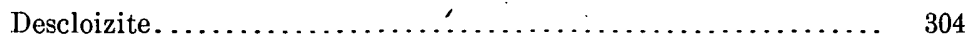

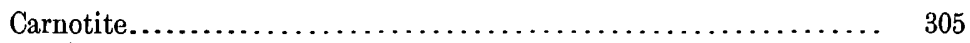

XIV. Arsenates and antimonites........................ 305

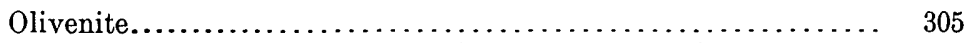

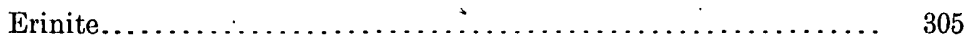

Clinoclasite................................... 306

Conichalcite.................................... 306

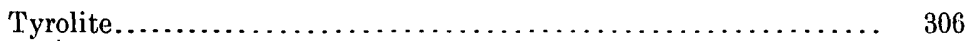

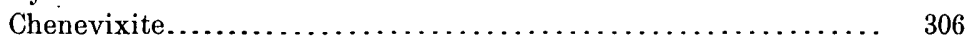

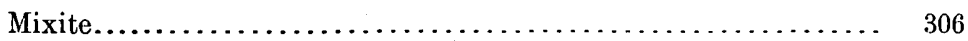

Scorodite.............................. 307

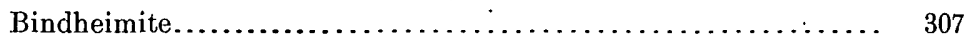

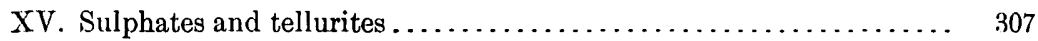

Anhydrite...................................... 307

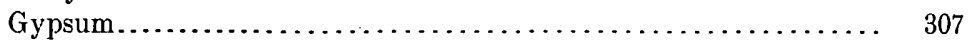

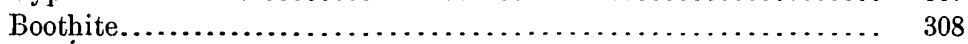

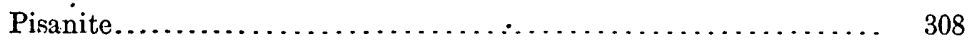

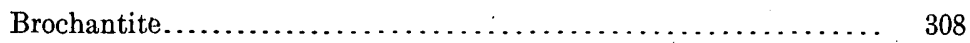

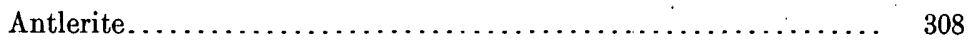

Picrallumogene.............................. 309

Halotrichite................................... 309

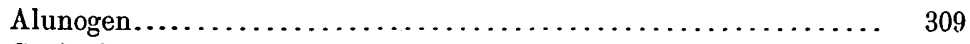

Copiapite.................................. 310

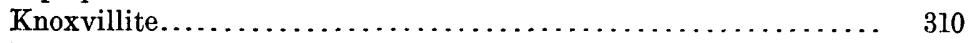

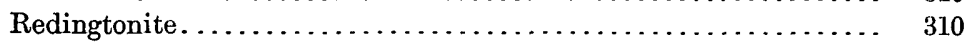

Alunite...................................... 310

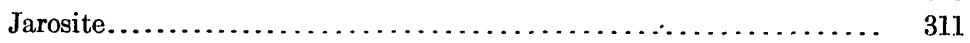


Analyses of minerals-Continued.

Page.

XV. Sulphates and tellurites-Continued.

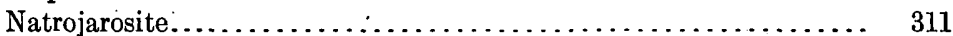

Plumbojarosite...................................... 311

Emmonsite.......................................... 311

XVI. Molybdates, tungstates, and uranates.................... 312

Powellite........................................ 312

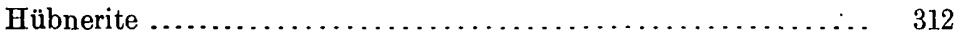

Molybdic ocher.................................. 313

Uraninite.......................................... 313

XVII. Carbonaceous minerals.............................. 315

Quisqueite................................... $\quad 315$

Index............................................... 317 


\section{ANALYSES OF ROCKS AND MINERALS FROM THE LABORATORY OF THE UNITED. STATES GEOLOGICAL SURVEY, 1880-1908.}

By F. W. Clarke.

\section{INTRODUCTION.}

The present Geological Survey of the United States was organized in 1879. In 1880, in connection with the Colorado work, a chemical laboratory was established at Denver in charge of Dr. W. F. Hillebrand, with whom were associated Mr. Antony Guyard and, later, Mr. L. G. Eakins. In 1882 Dr. W. H. Melville was placed in charge of a second laboratory at San Francisco, and in the autumn of 1883 the central laboratory was started in Washington, with myself as chief chemist. In November, 1885, Doctor Hillebrand was transferred to Washington; early in 1888 he was followed by Mr. Eakins, and the Denver laboratory was discontinued. In the spring of 1890 Doctor Melville also was transferred to Washington, and since then the geochemical work of the Survey has been concentrated at headquarters. The special laboratories of the water-resources and technologic branches of the Survey are not included in this statement. Their work is not represented in this bulletin.

Up to January 1, 1909, over 6,000 analyses have been made in the laboratory at Washington. These represent rocks, minerals, ores, waters, sediments, coals, metals, and so on through all the range of substances with which geology has to do. There were also some hundreds of analyses made in the laboratories at Denver and San Francisco. A fair amount of research work upon mineralogical and analytical problems has also been done. In all of this work the following chemists have been employed: E. T. Allen, Charles Catlett, T. M. Chatard, F. W. Clarke, L. G. Eakins, F. A. Gooch, Antony Guyard, W. F. Hillebrand, W. H. Melville, H. C. McNeil, Chase Palmer, R. B. Riggs, W. T. Schaller, E. A. Schneider, George Steiger, H. N. Stokes, E. C. Sullivan, William Valentine, R. C. Wells, and J. E. Whitfield. As many as eight of these have been at work simultaneously; at 
present only five are connected with the Survey. Other officers of the Survey have been occupied more or less with chemical questions, but the men named in this list were connected directly with the laboratory. Some work for the chemical division has also been done by chemists not regularly on the rolls of the Survey, but their analyses, with the exception of a single group to be noted later (see pp. 86-89), do not fall within the scope of this paper.

Quite naturally, on account of the activity of the petrographers, a dominant feature of the laboratory work has been the analysis of rocks. These have been studied in great numbers and in the most thorough way. The results have appeared in widely scattered publications, official reports, monographs, bulletins, American and foreign journals, and so on. The object of this bulletin is to bring together this valuable material, together with such bibliographic and petrographic data as seem. to be necessary, in order to identify the specimens and to facilitate chemical discussion. Analyses of minerals have also been made in considerable numbers, and they are collected in the final section of this book. In some instances such analyses were made with direct reference to petrographic studies, and therefore are cited in connection with the rocks to which they belong. Meteorites, of which twenty-nine have been analyzed, are brought into the work on account of their petrographic relations, and the groups of clays and soils have been admitted because of the bearing of these substances on the study of slates and shales. The actual number of analyses given in the bulletin is as follows:

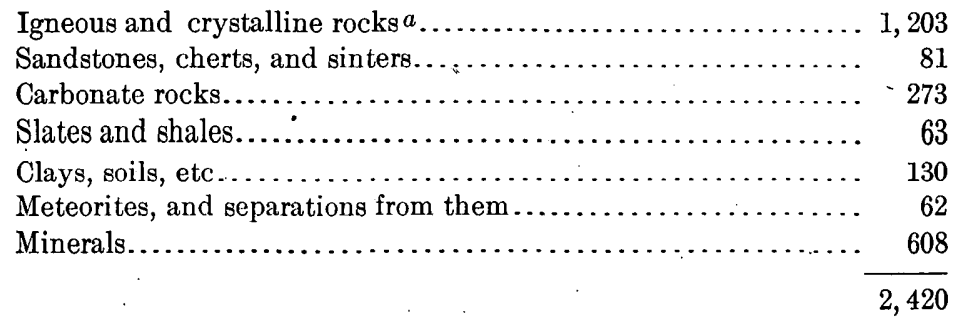

It may be observed that the classification thus indicated has not been rigorously followed. In a few instances the study of a sedimentary rock has been so related to that of its igneous neighbors that the analyses are best tabulated together; but these exceptional cases are few, and all are properly noted. The heading "Igneous and crystalline rocks" has been used in the broadest and most liberal way, and doubtless many of the analyses given under it might properly be otherwise classified. In such cases of uncertainty, convenience has furnished the rule to follow.

Within each division of the analyses the classification chosen has been geographic. The petrographic grouping of the rocks would 
doubtless be best were petrographers agreed on it; but their differences are many, and the chemist will do well to avoid them. The geographic method, moreover, has some advantages of its own. It facilitates the study of areas, it simplifies the bibliographic references, and it brings together, in great measure, the work of each petrographer for whom analyses have been made. Thus, most of Diller's work has been in California, most of Cross's in Colorado, and most of Iddings's in the Yellowstone National Park, and in each case the analyses are massed, and their discussion is practically uniform in character. As regards nomenclature, each rock has received the designation given it by its describer and no liberties have been taken. This plan may cause some lack of uniformity; but no other procedure seemed to be practicable. Whenever it was possible, however, I have inserted in italics the new names proposed by Cross, Iddings, Pirsson, and Washington in their classification of the igneous rocks. In most cases these names were taken from Washington's great compilation of analyses published by the Survey in 1903. ${ }^{a}$ This addition, it is believed, will assist petrographers in their study of the material thus brought together.

It will be noticed by anyone who uses this bulletin that the analyses vary as regards completeness. Among the sedimentary rocks especially partial analyses are common, but in the igneous group thoroughness is more general. In the early days of the chemical division many analyses were made along the older lines, just as they are still made in many laboratories to-day-that is, only the main constituents, those having direct petrographic significance, were determined. In such analyses the minor ingredients, like titanium, phosphorus, barium, strontium, chlorine, etc., were ignored; and, although the results were satisfactory in some respects, they left much to be desired. Latterly greater completeness has been sought for; the work done has been much fuller, and the data obtained can be discussed with much higher approaches to accuracy. The old form of "complete analysis" is to be discouraged. It leads too often to erroneous conclusions, and only the best modern methods of work and of statement should be tolerated. The fuller analyses, moreover, have brought some interesting points to light; titanium now appears to be one of the more abundant elements, and barium and strontium are found to be almost universally diffused in igneous rocks in quite perceptible quantities.

On general principles the analysis of a rock and its petrographic description should be two parts of the same investigation, matching each other completely. In practice, however, this rule does not always hold, and the departures from it are in two opposite directions. 
For example, an analysis of the older type says nothing of titanium and phosphorus, while the microscope reveals the presence of sphene and apatite. In this case the petrographer has been more thorough than the chemist. On the other hand, a full and perfect analysis may be given, accompanied by a petrographic description of the most general kind, in which only the main mineral constituents of the rock are noted. Here the analysis has been incompletely used and the petrographic discussion is defective. It is hoped that the publication of this material may lead to a clearer recognition of the mutuality which should exist between the chemical and the microscopic researches, and so bring, in the future, both lines of investigation more into harmony. Hitherto the chemist and the petrographer have worked too much apart and each has too often misunderstood the purpose of the other. If the study of the thin section could always precede the analysis, the petrographic problems could be stated more clearly and the chemical evidence might be rendered much more pertinent and satisfactory.

During the preparation of this- bulletin much assistance was rendered by the petrographers and geologists connected with the Survey, especially with reference to analyses hitherto unpublished. In each case credit has been given for the data thus added. Twenty-eight analyses of rocks from Montana, executed by or under the direction of Prof. L. V. Pirsson, of Yale University, and made in connection with regular Survey work, are included in the tabulations. With this exception all of the analyses given were made in the Survey laboratories. To those executed in the laboratory at Washington "record numbers" are attached, which serve to identify them on the record books of the division of chemistry. Of the abbreviations used for bibliographic reference only five need explanation, and they refer to the official publications of the Survey. "Ann." for Annual Report, "Mon." for Monograph, "Bull." for Bulletin, "P. P." for Professional Paper, and "Folio," are the five in question. The others relate to well-known journals and are familiar to all geologists. The letters P. R. C., following the description of a rock, refer to the petrographic reference collection of the Survey and are followed by the number assigned to the rock in that series.

\section{THE AVERAGE COMPOSITION OF ROCKS. 。}

In a paper published some years ago, ${ }^{a}$ on the relative abundance of the chemical elements, I computed the average composition of the primitive crust of the earth from 880 analyses of eruptive and crystal- 
line rocks. Of these analyses only 207 were from the laboratories of the Survey, while 673 were collected from various other American and foreign sources. A large proportion of them were incomplete, regarded from a modern point of view, and yet the results obtained were fairly conclusive.

In Bulletin 148 a similar estimate was given, based upon 680 complete analyses found in the Survey records, plus some hundreds of determinations of silica, lime, and alkalies. Again, in Bulletin 168, a third estimate was presented, representing 830 complete analyses and some partial determinations, all made in the Survey laboratories. In 1899 Harker $^{a}$ published a computation covering 397 analyses of British rocks, and Washington ${ }^{b}$ has also worked out the average of 1,811 analyses given in his compilation.

For the more important constituents of igneous rocks the five estimates mentioned above agree remarkably well, and yet they are not thoroughly comparable. The 397 analyses discussed by Harker were in most cases incomplete, at least when considered from a modern standpoint. In only 34 of them was titanium taken into account, and in only 55 is phosphorus mentioned. These omissions affect the percentages of other things and lessen the value of the computation very materially. Excluding manganese the five averages may be tabulated as follows. Minor constituents will be considered later:

\begin{tabular}{|c|c|c|c|c|c|}
\hline & \multicolumn{3}{|c|}{ Clarke. } & \multirow{2}{*}{ Harker. } & \multirow{2}{*}{$\begin{array}{l}\text { Washing- } \\
\text { ton. }\end{array}$} \\
\hline & First. & Socond. & Third. & & \\
\hline \multirow[t]{2}{*}{ 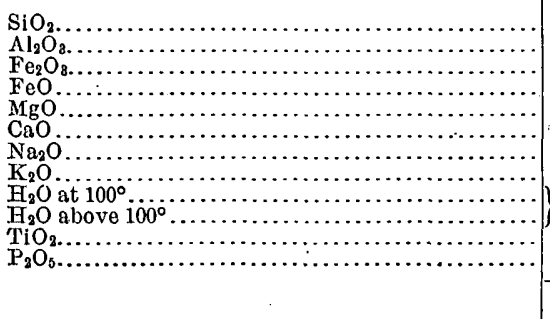 } & $\begin{array}{r}58.59 \\
15.04 \\
3.94 \\
3.48 \\
4.49 \\
5.29 \\
3.20 \\
2.90 \\
1.96 \\
.55 \\
.22\end{array}$ & \begin{tabular}{r}
59.77 \\
15.38 \\
2.65 \\
3.44 \\
4.40 \\
4.81 \\
3.61 \\
.2 .83 \\
\hdashline 1.51 \\
. .53 \\
. .21
\end{tabular} & \begin{tabular}{r}
59.71 \\
15.41 \\
2.63 \\
3.52 \\
4.36 \\
4.90 \\
3.55 \\
2.80 \\
\hdashline 1.7. \\
1.52 \\
.60
\end{tabular} & $\begin{array}{r}58.75 \\
15.64 \\
5.34 \\
2.40 \\
4.09 \\
4.98 \\
3.25 \\
2.74 \\
2.23 \\
.12 \\
.02\end{array}$ & $\begin{array}{r}58.239 \\
15.796 \\
3.334 \\
3.874 \\
3.843 \\
5.221 \\
3.912 \\
3.161 \\
.363 \\
1.428 \\
1.039 \\
.0373\end{array}$ \\
\hline & 99.66 & 99.14 & 99.22 & 99.56 & 100.583 \\
\hline
\end{tabular}

An examination of the foregoing table will show several discrepancies, and one of them is in the variable treatment given to water. In two columns hygroscopic water does not appear; in two others total water is given; in Washington's estimate a discrimination has been made. If we reject the figures for water and recalculate the remain- 
ing constituents to 100 per cent, the comparison of estimates will assume the following form:

\begin{tabular}{|c|c|c|c|c|c|}
\hline & \multicolumn{3}{|c|}{ Clarke. } & \multirow{2}{*}{ Tarker. } & \multirow{2}{*}{$\begin{array}{c}\text { Washing- } \\
\text { ton. }\end{array}$} \\
\hline & First. & Second. & Third. & & \\
\hline 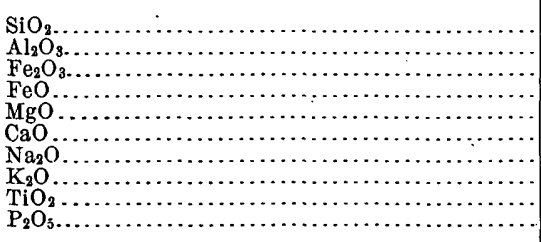 & $\begin{array}{r}59.97 \\
15.39 \\
4.03 \\
3.56 \\
4.60 \\
5.41 \\
3.28 \\
2.97 \\
.56 \\
.23\end{array}$ & 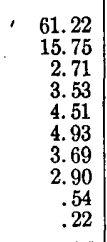 & $\begin{array}{r}61.12 \\
15.77 \\
2.69 \\
3.60 \\
4.46 \\
5.02 \\
3.63 \\
2.87 \\
.61 \\
.23\end{array}$ & $\begin{array}{r}60.36 \\
16.07 \\
5.48 \\
2.48 \\
4.20 \\
5.12 \\
3.34 \\
2.83 \\
.12 \\
.02\end{array}$ & $\begin{array}{r}58.96 \\
15.99 \\
3.37 \\
3.93 \\
3.89 \\
5.28 \\
3.96 \\
3.20 \\
1.05\end{array}$ \\
\hline -. & 100.00 & 100.00 & 100.00 & 100.00 & 100.00 \\
\hline
\end{tabular}

Between Washington's computation and mine there is one fundamental difference-a difference of method. In my own calculations analyses were averaged together as if each one was complete-an assumption which is not justifiable. In some cases minor constituents were not reported; in other cases they were determined with great care. The average of analyses varying in this way will obviously give too low a result for the rarer substances. Washington's policy was to consider the rock-forming elements separately, averaging each according to the actual number of determinations made, and thus to secure a truer group of estimates. For example, his average represents 1,811 determinations of $\mathrm{Al}_{2} \mathrm{O}_{3}$ and $\mathrm{SiO}_{2}, 1,625$ of $\mathrm{Fe}_{2} \mathrm{O}_{3}$ and $\mathrm{FeO}$, 1,139 of $\mathrm{TiO}_{2}, 955$ of $\mathrm{P}_{2} \mathrm{O}_{5}$, and only 731 of $\mathrm{MnO}$. The data thus utilized, however, coming from many laboratories and representing various methods of analysis, are evidently of very unequal value; and this consideration counts for something against the result finally obtained. I have therefore thought it desirable to repeat the computation, applying Washington's method to the determinations made in the laboratories of the Survey alone, and using all of the analyses, partial or complete, for my purpose. Every determination-made upon an igneous rock has been thus utilized. When an element was definitely reported as absent, it has counted as zero in the averaging. To the statement of a "trace" I have arbitrarily assigned the value of 0.01 per cent. In this way I have obtained the following averages, which represent all of the data accumulated by the chemists of the Survey down to January 1, 1908:

Silica.-Average of 1,530 determinations, 60.97 per cent.

Alumina.-Average of 1,042 determinations, 15.29 per cent. In 93 additional determinations, $\mathrm{TiO}_{2}$ and $\mathrm{P}_{2} \mathrm{O}_{5}$ were not separated; and these, therefore, are not included in the computation.

Ferric oxide.-1,093 determinations. Average, 2.63 per cent. 
Ferrous oxide. $-1,093$ determinations. Average, 3.40 per cent. In 38 analyses the oxides of iron were not separately determined, and in them the figure for iron is excluded from the calculation.

Magnesia.-1,163 determinations. Average, 3.96 per cent.

Lime.-1,384 determinations. Average, 4.89 per cent.

Soda.-1,442 determinations. Average, 3.47 per cent.

Potash.-1,434 determinations. Average, 3.00 per cent.

Water.--In 288 analyses, total water was determined; average, 1.86 per cent. In 752 analyses, water lost below $100^{\circ}-110^{\circ}$ was estimated; average, 0.48 per cent. In 816 analyses, water retained above $100^{\circ}-110^{\circ}$ gave an average of 1.51 per cent. Combining these data, the probable percentages are, $\mathrm{H}_{2} \mathrm{O}-, 0.47$, and $\mathrm{H}_{2} \mathrm{O}+$, 1.48 per cent.

Titanic oxide.-989 determinations. Average, 0.74 per cent.

Zirconia.-281 determinations. Average, 0.026 per cent. These determinations were not made so generally as to give the average any great importance.

Phosphoric oxide.-995 determinations. Average, 0.26 per cent.

Baryta.-710 determinations. Average, 0.11 per cent.

Strontia. -596 determinations. Average, 0.04 per cent.

Manganese oxide. $-1,011$ determinations. Average, 0.10 per cent.

Carbon dioxide.-599 determinations. Average, 0.50 per cent. This is doubtless too high, for in many rocks carbon dioxide was not looked for. In 142 of the analyses in which it was reported the percentage was zero.

Lithia.-Commonly reported in traces, but often absent. Reckoning a "trace" as 0.01 , the average of 571 analyses is 0.011 per cent. This is probably not far from a true estimate.

Nickel oxide.-273 determinations. Average, 0.026 per cent, which is probably too high.

Chromic oxide. -262 determinations. Average, 0.05 per cent. Probably too high.

Vanadium trioxide.-Only 83 determinations are reported; in average, 0.024 per cent. Determinations too few to give this value any weight. The figure, however, is not without interest. $a$

Chlorine.-Determined or proved to be absent in 258 cases. Average, 0.065 per cent. Probably too high.

Fluorine.-Only determined or proved to be absent in 90 analyses. Average, 0.11 per cent, which is undoubtedly too high. An inferior limit, however, may be fixed with reference to $\mathrm{P}_{2} \mathrm{O}_{5}$. If we assume that to represent apatite, the equivalent amount of fluorine should be 0.023 per cent. The true average must be slightly higher.

Sulphur.-This element is reported in three forms-as $\mathrm{S}$, as $\mathrm{SO}_{3}$, and as $\mathrm{FeS}_{2}$. Reported as $\mathrm{S}, 253$ analyses give an average 0.051 per cent. $\mathrm{As} \mathrm{SO}_{3}, 321$ analyses give 0.11 per cent, equivalent to 0.044 per cent of $S$. In 143 analyses 0.68 per cent $\mathrm{FeS}_{2}$ was found, equivalent to 0.36 of sulphur and 0.32 of $\mathrm{Fe}$. The latter must be taken into account in estimating total iron. General average for sulphur, 0.109 per cent.

a See Hillebrand, W. F., Bull. U. S. Geol. Survey No. 167, 1900, on the vanadium found in 57 rocks. 
Now, omitting the minor constituents and considering only those which appear in Washington's calculation, his average and mine may be compared as follows:

\begin{tabular}{|c|c|c|c|c|}
\hline & \multicolumn{2}{|c|}{ As given. } & \multicolumn{2}{|c|}{$\begin{array}{l}\text { Reduced to } 100 \text { per } \\
\text { cent. }\end{array}$} \\
\hline & Clarke. & $\begin{array}{l}\text { Washing- } \\
\text { ton. }\end{array}$ & Clarke. & $\begin{array}{l}\text { Washing- } \\
\text { ton. }\end{array}$ \\
\hline \multirow[t]{2}{*}{ 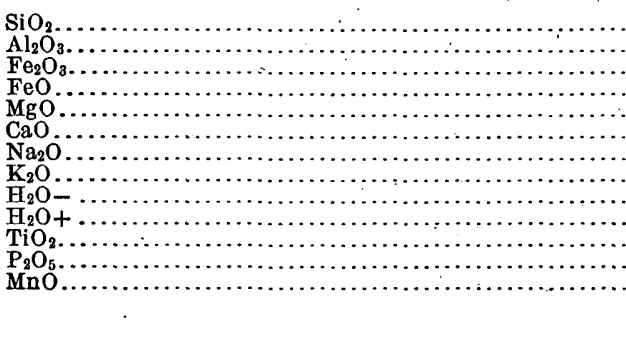 } & $\begin{array}{r}60.97 \\
15.29 \\
2.63 \\
3.40 \\
3.96 \\
4.89 \\
3.47 \\
3.00 \\
.47 \\
1.48 \\
.74 \\
.26 \\
.10\end{array}$ & $\begin{array}{r}58.239 \\
15.796 \\
3.334 \\
3.874 \\
3.843 \\
5.221 \\
3.912 \\
3.161 \\
.363 \\
1.428 \\
1.039 \\
.373 \\
.219\end{array}$ & $\begin{array}{r}60.57 \\
15.18 \\
2.61 \\
3.38 \\
3.93 \\
4.86 \\
3.45 \\
2.98 \\
.47 \\
1.47 \\
.74 \\
.26 \\
.10\end{array}$ & $\begin{array}{r}57.78 \\
15.67 \\
3.31 \\
3.84 \\
3.81 \\
5.18 \\
3.88 \\
3.13 \\
.36 \\
1.42 \\
1.03 \\
.37 \\
.22\end{array}$ \\
\hline & 100.66 & 100.802 & 100.00 & 100.00 \\
\hline
\end{tabular}

One reason for the difference between my estimate and that of Washington is not far to seek. The two columns of reduced averages are not strictly comparable, for one contains. many partial analyses, and the other relates only to those which are nominally complete. For the interpretation of a femic ${ }^{a}$ rock, which is mineralogically complex, a full analysis is necessary. For the simpler salic ${ }^{a}$ rocks, determinations of silica, lime, and alkalies are usually sufficient for petrographic purposes. The partial analyses, therefore, represent mainly salic varieties, and their inclusion in the average tends to raise the percentage of silica and to lower that of magnesia or iron. But the salic rocks are undoubtedly more abundant than those of the other class, and so I am inclined to regard the higher figure for silica as more probable than the lower. If, however, we include all the minor constituents of rocks as given in my computation, the final result assumes the form given on the following page.

\footnotetext{
a The terms "salic" and "femic," proposed by Cross, Iddings, Pirsson, and Washington, are here used instead of the conventional "acid" and "basic." The latter terms have precise chemical meaning, and their use in another sense by petrographers is inexact and misleading. The terms persilicic, mediosilicic, and subsilicic are even better.
} 


\begin{tabular}{|c|c|c|c|c|}
\hline & Found. & $\begin{array}{c}\text { Reduced } \\
\text { to } 100 .\end{array}$ & In elementary form. & \\
\hline \multirow[t]{2}{*}{$\begin{array}{l}\mathrm{S}_{\mathrm{O}} \mathrm{O}_{2} \\
\mathrm{Al}_{2} \mathrm{O}_{3} \\
\mathrm{Fe}_{2} \mathrm{O}_{3} \\
\mathrm{Fe} \\
\mathrm{FeO} \\
\mathrm{MgO} \\
\mathrm{Mg} \\
\mathrm{CaO}\end{array}$} & $\begin{array}{l}60.97 \\
15.29 \\
2.63+ \\
3.40+ \\
3.96 \\
4.89 \\
3.47 \\
3.00 \\
.17 \\
1.48 \\
.74 \\
.03- \\
.50- \\
.26 \\
.11 \\
.06 \\
.02+ \\
.11 \\
.04 \\
.10 \\
.03- \\
.05- \\
.02 \\
.01\end{array}$ & $\begin{array}{l}59.99 \\
1.5 .04 \\
2.59+ \\
3.34+ \\
3.89 \\
4.81 \\
3.41 \\
2.95 \\
.47 \\
1.45 \\
.73 \\
.03- \\
.49- \\
.26 \\
.11 \\
.06 \\
.02+ \\
.11 \\
.04 \\
.10 \\
.03- \\
.05- \\
.02 \\
.01\end{array}$ & \multirow[t]{2}{*}{ 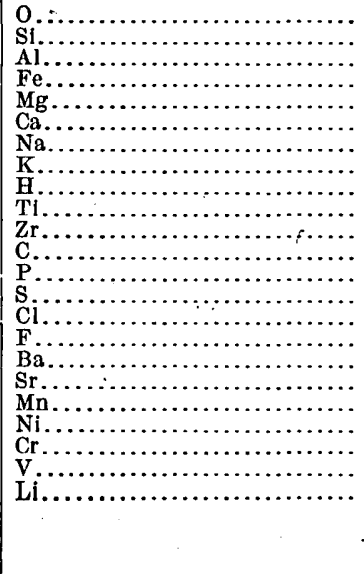 } & $\begin{array}{c}47.08 \\
28.30 \\
7.99 \\
4.47 \\
2.36 \\
3.45 \\
2.54 \\
2.46 \\
.16 \\
.44 \\
.026- \\
.13 \\
.11 \\
.11 \\
.06 \\
.02+ \\
.09 \\
.034 \\
.089 \\
.023- \\
.034- \\
.014- \\
.01\end{array}$ \\
\hline & 101.64 & 100.00 & & 100.000 \\
\hline
\end{tabular}

In the elementary column the iron reported in iron pyrites is included, but hygroscopic water is thrown out. The elements not included in the calculation represent minor corrections, to be applied whenever the necessity for doing so may arise. For estimates of their probable amounts Vogt's papers may be consulted. ${ }^{a}$ The percentages assigned to $\mathrm{C}, \mathrm{Zr}, \mathrm{Cl}, \mathrm{F}, \mathrm{Ni}, \mathrm{Cr}$, and $\mathrm{V}$ are-nothing more than very rough approximations.

By a similar statistical process I have tried to ascertain something with regard to the relative abundance of the more important rockforming minerals. Nearly 700 analyses of igneous rocks were studied, and the foregoing table of averages was also taken into account. For apatite, and for the titanium minerals titanite, ilmenite, and rutile the calculation was simple, but the other figures in the following table are approximative only:

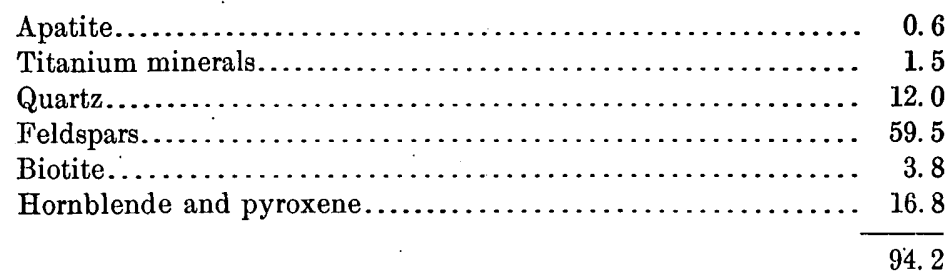

The less frequent minerals make up the remaining 5.8 per cent. The computation, although it is by no means conclusive, is not without some significance. It is interesting to note that the average 
igneous rock has very nearly metasilicate ratios and is close to an andesite in composition. Its place in the new classification of rocks has been fully discussed by Washington in the memoir already mentioned.

For computing the average composition of the sedimentary rocks the existing analyses of individual samples are inadequate. They are too few and too incomplete to yield any conclusions of value. Attempts have been made to partly use the data, as, for example, by Joly ${ }^{a}$ and it seems probable, therefore, that better material will not be without interest or scientific value.

-About fourteen years ago, at the request of Mr. G. K. Gilbert, a series of composite analyses of sedimentary rocks was made in this laboratory. Many samples were mixed into one uniform sample, from which, by a single analysis, an average composition was determined. The material was selected and the samples were prepared by Mr. Gilbert, assisted by Mr. G. W. Stose, and the analyses were made by Dr. H: N. Stokes. The data obtained may be tabulated as follows:

A. Composite analysis of 27 Mesozoic and Cenozoic shales. Each individual shale was taken in amount roughly proportional to the mass of the formation which it represented.

B. Composite analysis of 51 Paleozoic shales, weighted as in the former case.

C. General average of $\mathrm{A}$ and $\mathrm{B}$, giving them, respectively, weights as 3 to 5 . This average represents 78 rocks.

D. Composite analysis of 253 sandstones, about 1 gram of each being taken in preparing the average sample.

E. Composite analysis of 371 sandstones used for building purposes. Equal weights taken.

F. Composite analysis of 345 limestones, equal weights being taken.

G. Composite analysis of 498 limestones used for building purposes. Equal weights taken.

\begin{tabular}{|c|c|c|c|c|c|c|c|}
\hline & A. & B. & C. & D. & E. & F. & G. \\
\hline 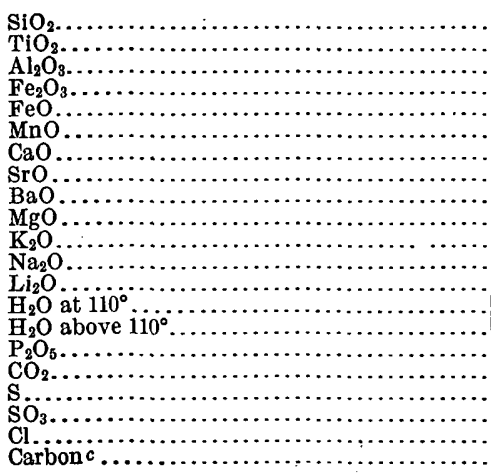 & $\begin{array}{r}55.43 \\
.46 \\
13.84 \\
4.00 \\
1.74 \\
\text { trace } \\
5.96 \\
\text { none } \\
.06 \\
2.67 \\
2.67 \\
1.80 \\
\text { trace } \\
2.11 \\
3.45 \\
.20 \\
4.62 \\
\ldots . .78 \\
.78 \\
.69\end{array}$ & $\begin{array}{r}60.15 \\
.76 \\
16.45 \\
4.04 \\
2.90 \\
\text { trace } \\
1.41 \\
\text { none } \\
.04 \\
2.32 \\
3.60 \\
1.01 \\
\text { trace } \\
.89 \\
3.82 \\
.15 \\
1.46 \\
\ldots . .2 . \\
.58 \\
\ldots . . .3 \\
.88\end{array}$ & $\begin{array}{r}58.38 \\
.65 \\
15.47 \\
4.03 \\
2.46 \\
\text { trace } \\
3.12 \\
\text { none } \\
.05 \\
2.45 \\
3.25 \\
1.31 \\
\text { trace } \\
1.34 \\
3.68 \\
.17 \\
2.64 \\
\ldots \ldots . . . \\
.65 \\
\ldots . . . . \\
.81\end{array}$ & $\begin{array}{r}78.66 \\
.25 \\
4.78 \\
1.08 \\
.30 \\
\text { trace } \\
5.52 \\
\text { trace } \\
.05 \\
1.17 \\
1.32 \\
.45 \\
\text { trace } \\
.31 \\
\text { b } 1.33 \\
.08 \\
5.04 \\
\ldots \ldots . . . \\
.07 \\
\text { trace } \\
\ldots \ldots . .\end{array}$ & $\begin{array}{r}84.86 \\
.41 \\
5.96 \\
1.39 \\
.84 \\
\text { trace } \\
1.05 \\
\text { none } \\
.01 \\
.52 \\
1.16 \\
.76 \\
\text { trace } \\
.27 \\
\text { b } 1.47 \\
.06 \\
1.01 \\
\therefore \ldots . . . \\
.09 \\
\text { trace }\end{array}$ & $\begin{array}{r}5.19 \\
.06 \\
.81 \\
.54 \\
\text { undet. } \\
.05 \\
42.61 \\
\text { none } \\
\text { none } \\
7.90 \\
.33 \\
.05 \\
\text { trace } \\
.21 \\
\text { b.56 } \\
.04 \\
41.58 \\
.09 \\
.05 \\
.02 \\
\ldots \ldots . .\end{array}$ & $\begin{array}{r}14.09 \\
.08 \\
1.75 \\
.77 \\
\text { undet. } \\
.03 \\
40.60 \\
\text { none } \\
\text { none } \\
4.49 \\
.58 \\
.62 \\
\text { trace } \\
.30 \\
\text { b. } 88 \\
.42 \\
35.58 \\
.07 \\
.07 \\
.01 \\
\ldots \ldots\end{array}$ \\
\hline & 100.48 & 100.46 & 100.46 & 100.41 & 99.86 & 100.09 & 100.34 \\
\hline
\end{tabular}

a An estimate of the geological age of the earth: Sci. Trans. Royal Dublin Soc., vol. 7, 1899, p. 23. $b$ Includes organic matter.

$c$ Of organic origin. 
These analyses may be used for a variety of purposes. For example, they can help in tracing the change from an average igneous rock to an average sediment. They suggest something as to the characteristic features which distinguish a good building stone from other limestones and sandstones. They are applicable to the discussion of a variety of large theoretical problems like that chosen by Professor Joly. These considerations alone justify their publication here. 



\title{
ANALYSES OF IGNEOUS AND CRYSTALLINE ROCKS
}

\author{
MAINE.
}

1. RQCKS FROM AROOSTOOK COUNTY.

Described by H. E. Gregory in Bull. 165. Analyses by W. F. Hillebrand, record No. 1795.

A. Quartz trachyte (bostonite), Quoggy Joe Mountain, Presque Isle Township. Liparose. Contains quartz, orthoclase, albite, and magnetite, with siderite, kaolin, and chlorite.

B. Teschenite, Mapleton Township. Akerose. In dikes cutting shales. Contains andesine, augite, biotite, apatite, analcite, and magnetite.

C. Andesite, Edmunds Hill, Chapman Township. Tonalose. Contains labradorite, orthoclase, pyroxene, apatite, and magnetite.

D. Calciferous sandstone, New Sweden Township. Contains calcite, alkali feldspar, quartz, magnetite, muscovite, and siderite. Included here because studied as one of the group.

\begin{tabular}{|c|c|c|c|c|}
\hline & A. & $\mathrm{B}$. & C. & D. \\
\hline 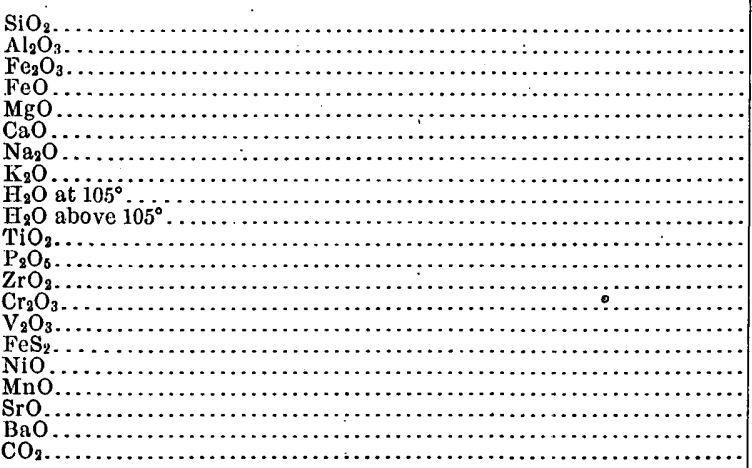 & $\begin{array}{r}72.77 \\
12.15 \\
.44 \\
3.06 \\
.22 \\
.07 \\
3.38 \\
4.67 \\
.17 \\
.55 \\
.20 \\
\text { trace } \\
.04 \\
\text { none } \\
(?) \\
.12 \\
\text { none } \\
.16 \\
\text { none } \\
.03 \\
2.06\end{array}$ & $\begin{array}{r}46.77 \\
14.91 \\
7.80 \\
4.90 \\
2.94 \\
6.30 \\
4.97 \\
2.37 \\
.92 \\
4.28 \\
2.31 \\
.98 \\
\text { none } \\
\text { none } \\
.02 \\
.07 \\
\text { trace } \\
.29 \\
.03 \\
.04 \\
\text { trace? }\end{array}$ & $\begin{array}{r}61.40 \\
16.59 \\
2.13 \\
3.05 \\
2.73 \\
6.17 \\
3.83 \\
1.34 \\
.82 \\
.88 \\
.79 \\
.20 \\
\text { none } \\
\text { trace } \\
.02 \\
\text { none } \\
\text { trace } \\
.13 \\
\text { trace? } \\
.02 \\
\text { none }\end{array}$ & $\begin{array}{r}54.23 \\
7.38 \\
.54 \\
1.37 \\
3.29 \\
14.56 \\
1.65 \\
1.74 \\
.25 \\
1.22 \\
.28 \\
.07 \\
(?) \\
? ? \\
? ? \\
\text { ?? } \\
\text { none } \\
\text { undet. } \\
\text { none } \\
\text { none } \\
\text { 13. } 48\end{array}$ \\
\hline · & 100.09 & 99.90 & 100.10 & 100.06 \\
\hline
\end{tabular}

Traces of lithia present in all. $\mathrm{F}$ and $\mathrm{Cl}$ not looked for.

E. Diabase (basaltic glass), Mars Hill. Ornose. Not resolvable into minerals.

F. Rhyolite, Haystack Mountain. Liparose. Contains quartz, albite, and orthoclase, with sphene and accessory chlorite and kaolin.

G. Volcanic tuff, southeast base of Castle Hill. Contains fragments of trachyte, andesite, devitrified glass, and lapilli.

H. Diabase, Aroostook Falls, near Maine boundary line. Beerbachose. Dike. Contains plagioclase, pyroxene, pyrite, apatite, chlorite, and a little calcite. 


\begin{tabular}{|c|c|c|c|c|}
\hline & E. & F. & G. & H. \\
\hline 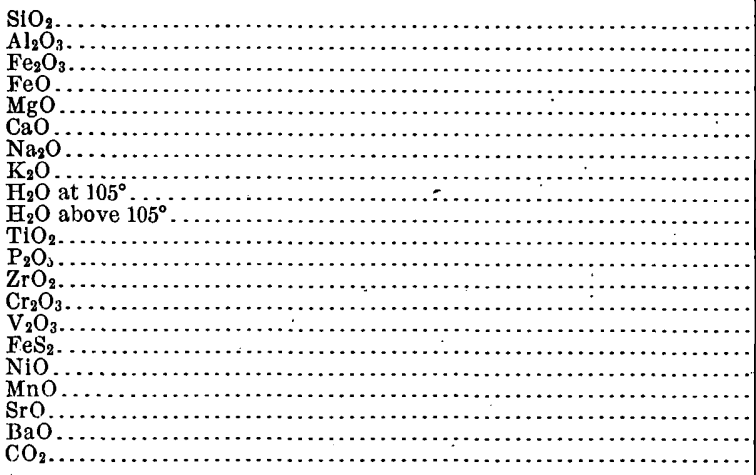 & $\begin{array}{r}42.25 \\
16.87 \\
5.24 \\
10.72 \\
6.91 \\
3.33 \\
3.96 \\
.77 \\
.43 \\
5.58 \\
2.93 \\
.34 \\
\text { none } \\
.03 \\
.07 \\
\text { trace } \\
.01 \\
.40 \\
\text { none } \\
\text { trace? } \\
\text { none }\end{array}$ & $\begin{array}{c}75.98 \\
12.34 \\
.85 \\
.93 \\
.15 \\
.13 \\
4.02 \\
4.44 \\
.24 \\
.64 \\
: 17 \\
.03 \\
.03 \\
(?) \\
(?) \\
\text { none } \\
\text { none } \\
\text { trace? } \\
\text { trace? } \\
.07 \\
\text { none }\end{array}$ & $\begin{array}{r}31.42 \\
11.57 \\
2.37 \\
7.48 \\
5.32 \\
16.71 \\
2.26 \\
.74 \\
.76 \\
4.17 \\
2.30 \\
.46 \\
\text { none } \\
\text { trace } \\
.06 \\
.16 \\
\text { trace } \\
.38 \\
\text { none } \\
.64 \\
13.13\end{array}$ & $\begin{array}{r}49.64 \\
15.07 \\
1.66 \\
8.82 \\
5.43 \\
7.23 \\
4.19 \\
.89 \\
.45 \\
2.81 \\
2.32 \\
.29 \\
\text { none } \\
\text { trace } \\
.04 \\
.79 \\
\text { trace } \\
.25 \\
.05 \\
.02 \\
.32\end{array}$ \\
\hline & 99.84 & 100.02 & 99.93 & 100. 27 \\
\hline
\end{tabular}

Traces of lithia present in all. Cl and F not looked for.

\section{MiscellaNEOUS ROCKS.}

A. Elæolite syenite, var. litchfieldite, from Litchfield. Nordmarkose. Described by Bayley in Bull. 150, p. 201; and in Bull. Geol. Soc. America, vol. 3, p. 231. Contains elæolite, two feldspars, and lepidomelane, with sodalite, cancrinite, and zircon as accessories. Analysis by L. G. Eakins, record No. 1298. P. R. C. 77 and $949 . a$

B. Syenite porphyry, Appleton, Knox County. Prowersose. Described by E. S. Bastin in Jour. Geology, vol. 14, p. 173. Contains potash feldspar, albite, biotite, hornblende, zircon, titanite, apatite, iron ores, and a little quartz. Analysis by G. Steiger, record No. 2232.

C. Pyrrhotitic peridotite, East Union. Lermondose. Described by Bastin in Jour. Geology, vol. 16, p. 124. Contains pyrrhotite, with some pyrite and chalcopyrite, olivine, plagioclase, hornblende, and magnetite. Also small amounts of biotite and spinel, with secondary serpentine, amphibole, chlorite, and calcite. Analysis by W. F. Hillebrand, record No. 2301.

D, E. Two pegmatites (graphic-granite), Topsham. Collected by Bastin. Essentially quartz and orthoclase. Analyses by Steiger, records Nos. 2345, 2357.

\begin{tabular}{|c|c|c|c|c|c|}
\hline • & A. & B. & C. & D. & E. \\
\hline 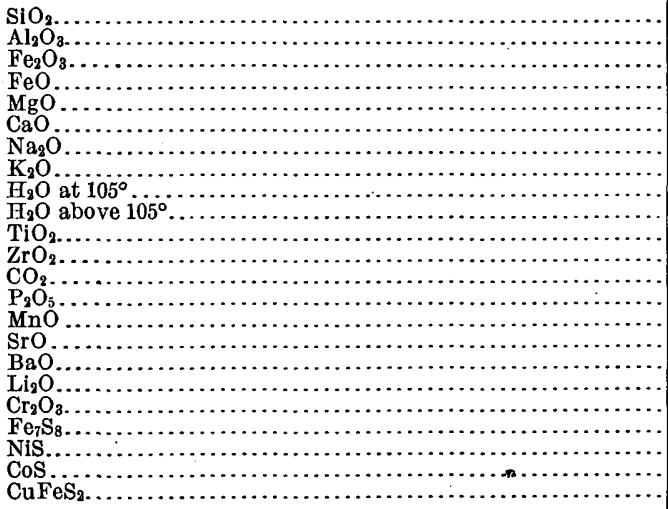 & 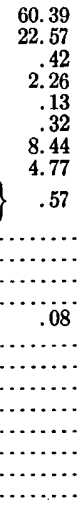 & $\begin{array}{r}52.26 \\
10.63 \\
2.47 \\
5.45 \\
9.32 \\
5.62 \\
1.60 \\
5.99 \\
.98 \\
1.97 \\
1.92 \\
.08 \\
.75 \\
.98 \\
.12 \\
\ldots \ldots . . \\
\ldots \ldots . . \\
\ldots \ldots . \\
\ldots \ldots . . \\
\ldots \ldots \ldots \\
\ldots \ldots \ldots \\
\ldots \ldots \ldots \\
\ldots \ldots \ldots\end{array}$ & $\begin{array}{c}28.04 \\
3.51 \\
? \\
14.95 \\
21.97 \\
1.78 \\
.28 \\
.08 \\
1.48 \\
2.54 \\
.20 \\
(?) \\
1.01 \\
.04 \\
.24 \\
\text { trace? } \\
(?) \\
\text { none } \\
\text { trace } \\
21.53 \\
.94 \\
.03 \\
1.03\end{array}$ & 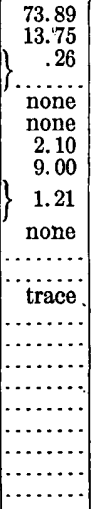 & 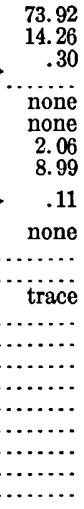 \\
\hline & 99.95 & 100.14 & 99.65 & 100.21 & 99.64 \\
\hline
\end{tabular}

a For analyses of feldspar, elæolite, hydronephelite, lepidomelane, sodalite, and cancrinite from this rock, see the closing section of this volume. 
NEW HAMPSHIRE.

A. Elæolite syenite, Red Hill, Moultonboro. Umptekose. Described by Bayley in Bull. Geol. Soc. America, vol. 3, p. 231. Contains elæolite, augite, hornblende, biotite, sodalite, albite, and orthoclase, with accessory apatite, sphene, magnetite, and an occasional zircon. Fibrous decomposition products are also present. P. R. C. 203.

B. Mixed albite and orthoclase from A.

C. Nepheline (elæolite) from A. Analysis by W. F. Hillebrand, record No. 1321. The mixed nepheline and feldspar were treated with dilute hydrochloric acid, and the residue was extracted with sodium carbonate solution. C represents the soluble part and $B$ the insoluble.

D. Camptonite, Campton Falls. Analysis by L. G. Eakins, record No. 1298. Described by J. P. Iddings in Bull. 150, p. 239. Contains hornblende, plagioclase, orthoclase, augite, iron ore, biotite, apatite, pyrite, and a mineral which appears to be analcite. Also variable calcite, serpentine, and chlorite. P. R. C. 92.

E. Quartz porphyry, Pemigewasset. Toscanose. Analysis by Eakins, No. 1298.

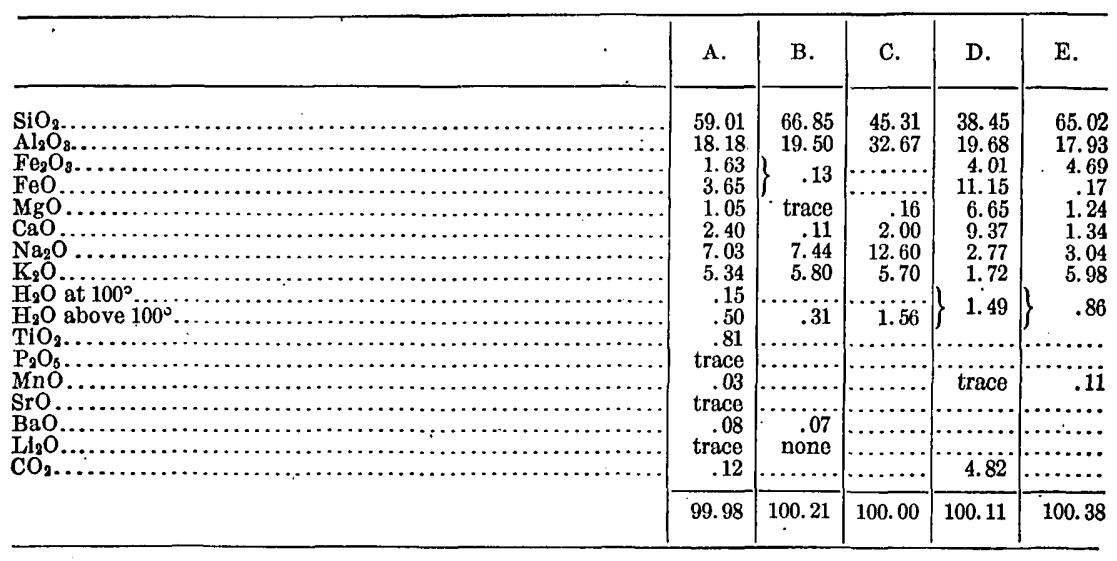

VERMONT.

\section{ROCKS OF MOUNT ASCUTNEY.}

Described by R. A. Daly in Bull. 209. Analyses by W. F. Hillebrand, record Nos. 1621, 1657.

A. Biotite granite. Liparose. Contains quartz, orthoclase, plagioclase (microperthite), biotite, magnetite, sphene, apatite, and zircon. P. R. C. 1340.

B. Basic segregation in biotite granite. Akerose. Contains biotite, hornblende, quartz, plagioclase', microperthite, much sphene and apatite, some magnetite and zircon. P. R. C. 1338.

C. Another sample of B, but containing more hornblende. Akerose. P. R. C. 1339.

D. Nordmarkite. Phlegrose. Contains orthoclase, plagioclase, quartz, hornblende, magnetite, apatite, and zircon, with very little biotite, titanite, diopside, and allanite. P. R. C. 1341.

E. Basic segregation in nordmarkite. Akerose. Contains hornblende, augite, microperthite, orthoclase, plagioclase, quartz, magnetite, zircon, and apatite. P. R.C 1342.

F. Hornblende paisanite. Liparose. Dike. Contains orthoclase; quartz, hornblende, magnetite, apatite, and zircon. P. R. C. 1347. 


\begin{tabular}{|c|c|c|c|c|c|c|}
\hline ' & A. & B. & C. & D. & E. & F. \\
\hline $\begin{array}{l}\mathrm{S}_{\mathrm{O}} \mathrm{A}_{2} \\
\mathrm{Al}_{2} \mathrm{O}_{3} \\
\mathrm{Fe}_{2} \mathrm{O}_{8} \\
\mathrm{FeO}\end{array}$ & $\begin{array}{r}71.90 \\
14.12 \\
1.20 \\
.86 \\
.33 \\
1.13 \\
4.52 \\
4.81 \\
.18 \\
.42 \\
.35 \\
.11 \\
.04 \\
.05 \\
\text { none } \\
.04 \\
.21 \\
.06 \\
.02 \\
\text { trace }\end{array}$ & $\begin{array}{r}56.01 \\
a 15.19 \\
2.34 \\
4.89 \\
4.67 \\
4.85 \\
5.66 \\
2.16 \\
.36 \\
.90 \\
1.13 \\
.53 \\
\ldots . .70 \\
.40 \\
.03 \\
\text { trace? } \\
\text { undet. } \\
\text { undet. } \\
\text { undet. } \\
.09\end{array}$ & $\begin{array}{r}59.27 \\
15.76 \\
2.07 \\
3.57 \\
3.04 \\
3.69 \\
5.63 \\
3.33 \\
.23 \\
.74 \\
1.12 \\
.42 \\
.04 \\
.37 \\
\text { trace } \\
\text { trace? } \\
.30 \\
.42 \\
.03 \\
.07\end{array}$ & $\begin{array}{c}65.43 \\
16.11 \\
1.15 \\
2.85 \\
.40 \\
1.49 \\
5.00 \\
5.97 \\
.19 \\
.39 \\
.50 \\
.13 \\
.11 \\
.23 \\
(?) \\
.03 \\
\text { trace? } \\
.08 \\
.05 \\
.07\end{array}$ & $\begin{array}{r}56.51 \\
16.59 \\
1.35 \\
6.59 \\
2.52 \\
4.96 \\
5.15 \\
3.05 \\
.21 \\
.71 \\
1.20 \\
.41 \\
.04 \\
.24 \\
\text { trace? } \\
.03 \\
.33 \\
.24 \\
.07 \\
.06\end{array}$ & $\begin{array}{r}73.03 \\
13.43 \\
.40 \\
1.49 \\
.14 \\
.79 \\
4.91 \\
4.54 \\
.18 \\
.35 \\
.30 \\
.06 \\
.06 \\
.15 \\
.(?) \\
\text { trace? } \\
\text { trace? } \\
.08 \\
.03 \\
.09\end{array}$ \\
\hline \multirow[t]{2}{*}{ 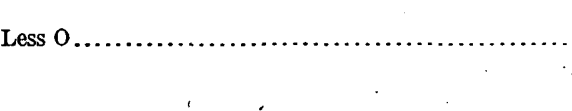 } & $\begin{array}{r}100.35 \\
.03\end{array}$ & $\begin{array}{r}99.21 \\
\ldots \ldots\end{array}$ & $\begin{array}{r}100.10 \\
.19\end{array}$ & $\begin{array}{r}100.18 \\
.04\end{array}$ & $\begin{array}{r}100.26 \\
.11\end{array}$ & $\begin{array}{r}100.03 \\
.04\end{array}$ \\
\hline & 100.32 & & 99.91 & 100.14 & 100.15 & 99.99 \\
\hline
\end{tabular}

a Including $\mathrm{ZrO}_{2}$.

G. Nordmarkite. Phlegrose. Contains orthoclase, plagioclase, microperthite, hornblende, quartz, augite, magnetite, biotite, apatite, and zircon. P. R. C. 1343.

H. Hornblende paisanite. Liparose. Composition like D, but with more quartz. P. R. C. 1348.

I. Basic segregation in H. Akerose. Contains hornblende, green and brown augite, biotite, quartz, microperthite, magnetite, little apatite, and zircon. P. R. C. 1349.

J. Diorite. Andose. Contains hornblende, augite, biotite, plagioclase, titaniferous magnetite, sphene, zircon, and quartz. P. R. C. 1344.

K. Windsorite. Toscanose. Contains plagioclase, orthoclase, quartz, biotite, magnetite, ilmenite, and very small amounts of diopside, apatite, and zircon. $P$. R. C. 1345 .

\begin{tabular}{|c|c|c|c|c|c|}
\hline 0 & G. & H. & I. & J. & $\mathrm{K}$. \\
\hline 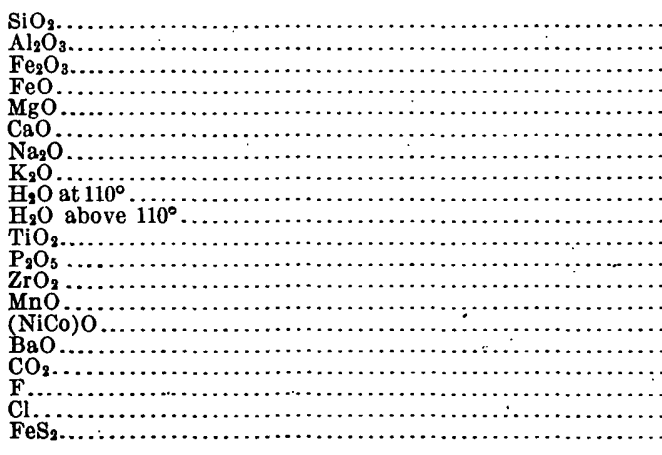 & $\begin{array}{r}64.88 \\
16.24 \\
1.37 \\
2.70 \\
.89 \\
1.92 \\
5.00 \\
5.61 \\
.19 \\
.46 \\
.69 \\
.13 \\
.13 \\
.14 \\
\text { none } \\
.06 \\
\text { none } \\
.08 \\
.04 \\
\text { none }\end{array}$ & $\begin{array}{r}73.69 \\
12.46 \\
1.21 \\
1.75 \\
.17 \\
.36 \\
4.47 \\
4.92 \\
.14 \\
.24 \\
.28 \\
.04 \\
.14 \\
.15 \\
\text { none } \\
\text { none } \\
\text { trace } \\
.05 \\
.02 \\
\text { none }\end{array}$ & $\begin{array}{r}56.53 \\
16.47 \\
1.58 \\
5.40 \\
2.67 \\
4.90 \\
5.59 \\
3.80 \\
.23 \\
.60 \\
.1 .40 \\
.27 \\
.03 \\
.20 \\
\text { trace } \\
\text { trace } \\
.05 \\
.19 \\
.07 \\
\text { trace }\end{array}$ & $\begin{array}{r}52.12 \\
16.35 \\
3.68 \\
6.02 \\
4.14 \\
7.25 \\
3.65 \\
2.34 \\
.25 \\
.88 \\
2.10 \\
.89 \\
.02 \\
.17 \\
\text { trace } \\
.04 \\
.07 \\
.03 \\
.09 \\
.24\end{array}$ & $\begin{array}{r}64.62 \\
16.46 \\
1.82 \\
2.14 \\
1.10 \\
2.39 \\
4.57 \\
5.21 \\
.13 \\
.39 \\
.81 \\
.21 \\
.03 \\
.12 \\
\text { none } \\
.03 \\
.11 \\
\text { undet. } \\
.05 \\
.19\end{array}$ \\
\hline Less $0 .$. & $\begin{array}{r}100.53 \\
.04\end{array}$ & $\begin{array}{r}100.09 \\
.02\end{array}$ & $\begin{array}{r}99.98 \\
.09\end{array}$ & $\begin{array}{r}100.33 \\
.03\end{array}$ & $\begin{array}{r}100.38 \\
.01\end{array}$ \\
\hline 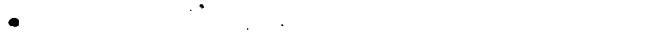 & 100.49 & 100.07 & 99.89 & 100.30 & 100.37 \\
\hline
\end{tabular}


L. Basic segregation in diorite. Akerose. Contains hornblende, augite, biotite, plagioclase, magnetite, apatite, zircon, and a little quartz. . P. R. C. 1346.

M. Diabase. Auvergnose. Contains plagioclase, augite, and magnetite. P. R. C. 1351 .

N. Camptonite. Camptonose. Contains brown hornblende, plagioclase, a little augite, olivine, magnetite, and apatite. P. R. C. 1350.

O. Phyllite. Contains quartz, sericite, graphite?, magnetite, pyrite, rare orthoclase, plagioclase, sphene, and rutile. A quartz-sericite schist. P. R. C. 1352.

P. Cordierite-microperthite hornfels. Akerose. Contains-biotite, quartz, red garnet, corundum, magnetite, iolite, microperthite, and rarely pleonaste.

Q. Cordierite hornfels. Contains quartz, biotite, pleonaste, corundum, iolite, magnetite, plagioclase, red garnet, and epidote?.

\begin{tabular}{|c|c|c|c|c|c|c|}
\hline & L. & M. & N. & 0 & P. & Q. \\
\hline 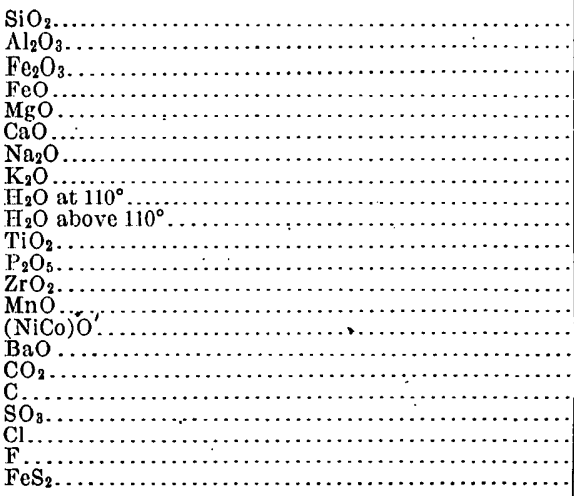 & $\begin{array}{r}55.28 \\
17.23 \\
1.54 \\
6.23 \\
2.69 \\
5.60 \\
5.42 \\
2.12 \\
.20 \\
.71 \\
1.64 \\
.73 \\
\text { trace } \\
.24 \\
(?) \\
.06 \\
.04 \\
\ldots \ldots . . \\
\text { none } \\
.07 \\
.28 \\
.07\end{array}$ & $\begin{array}{r}49.63 \\
14.40 \\
2.85 \\
8.06 \\
7.25 \\
9.28 \\
2.47 \\
.70 \\
.27 \\
1.47 \\
1.68 \\
.25 \\
\text { trace? } \\
.17 \\
.04 \\
\text { trace? } \\
1.36 \\
\ldots . . . \\
\text { none } \\
.07 \\
\text { trace } \\
.22\end{array}$ & $\begin{array}{r}48.22 \\
14.27 \\
2.46 \\
9.00 \\
6.24 \\
8.45 \\
2.90 \\
1.93 \\
.28 \\
1.66 \\
2.79 \\
.64 \\
.03 \\
.20 \\
.03 \\
.04 \\
.15 \\
\ldots \ldots \\
\text { none } \\
.10 \\
.05 \\
.36\end{array}$ & $\begin{array}{r}90.91 \\
4.18 \\
.22 \\
1.27 \\
.37 \\
.22 \\
.77 \\
.58 \\
.06 \\
.74 \\
.28 \\
.05 \\
.02 \\
\text { trace } \\
\text { none } \\
\text { trace } \\
.18 \\
.10 \\
\text { none } \\
\ldots \ldots . . \\
\text { trace } \\
.11\end{array}$ & $\begin{array}{r}58.35 \\
21.30 \\
.03 \\
6.41 \\
2.10 \\
.85 \\
1.60 \\
5.63 \\
.31 \\
.86 \\
.87 \\
.18 \\
\text { none } \\
.13 \\
.03 \\
.05 \\
\ldots . . . . \\
.40 \\
\text { none } \\
.03 \\
\text { (?) } \\
.58\end{array}$ & $\begin{array}{r}45.30 \\
30.51 \\
.24 \\
8.80 \\
3.11 \\
.90 \\
1.65 \\
4.84 \\
.26 \\
1.05 \\
1.48 \\
.12 \\
\text { none } \\
.20 \\
.02 \\
.03 \\
\ldots .27 \\
.17 \\
.04 \\
.04 \\
.04 \\
1.07\end{array}$ \\
\hline \multirow[t]{2}{*}{ 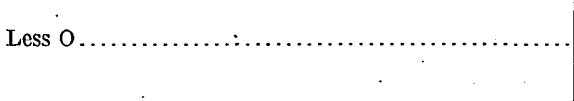 } & $\begin{array}{c}100.15 \\
. .13\end{array}$ & $\begin{array}{r}100.17 \\
.02\end{array}$ & $\begin{array}{r}99.80 \\
.04\end{array}$ & $\begin{array}{l}100.06 \\
\ldots \ldots\end{array}$ & & $\begin{array}{r}99.87 \\
\ldots\end{array}$ \\
\hline & 100.02 & 100.15 & 99.76 & & & \\
\hline
\end{tabular}

In these rocks the sulphur is all reckoned as pyrite, although pyrrhotite also is probably present. The carbonic acid represents either dolomite or siderite; not calcite. Traces of lithia and strontia occur in nearly all. Samples $H, L, O$, and $Q$ contain traces of copper. In $\mathrm{N}$ there is $0.03 \mathrm{~V}_{2} \mathrm{O}_{3}$, a supplementary determination by Hillebrand.

\section{MiSCELLANEOUS ROCKS.}

A. Amphibolite, Guilford." Described by Emerson in Mon. XXIX. Contains hornblende needles, with albite and rutile. Analysis by L. G. Eakins, record No. 1326. P. R. C. 1615.

B. Granitoid gneiss, north of Lincoln's.

C. The same, west slope of Little Peco.

D. Chloritic granite. Yellowstonose. East Clarendon section.

E. Hornblende granite. Hessose. East Clarendon.

Rocks B, C, D, and E collected by C. L. Whittle, but not described. Analyses by H. N. Stokes, record No. 1396. 


\begin{tabular}{|c|c|c|c|c|c|}
\hline & A. & B. & C. & D. & E. \\
\hline 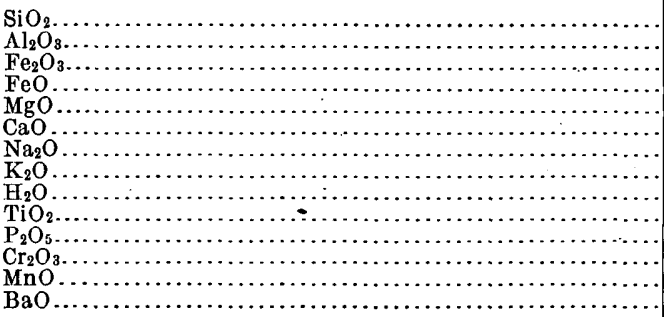 & $\begin{array}{r}49.16 \\
16.43 \\
3.92 \\
7.19 \\
8.19 \\
9.21 \\
3.70 \\
.41 \\
.45 \\
1.03 \\
.16 \\
\operatorname{trace} \\
.23 \\
.02\end{array}$ & $\begin{array}{r}71.02 \\
15.00 \\
1.12 \\
1.81 \\
.69 \\
.31 \\
2.48 \\
5.79 \\
1.14 \\
.35 \\
.13 \\
\text { trace } \\
\text { trace }\end{array}$ & $\begin{array}{r}69.97 \\
14.90 \\
2.16 \\
.96 \\
.37 \\
.45 \\
2.85 \\
6.54 \\
.66 \\
.44 \\
.12 \\
\ldots . . . \\
\text { trace } \\
.09\end{array}$ & $\begin{array}{r}67.33 \\
16.20 \\
1.40 \\
2.73 \\
1.31 \\
2.81 \\
3.15 \\
2.14 \\
1.84 \\
.80 \\
.16 \\
\ldots . . . \\
\text { trace } \\
.05\end{array}$ & $\begin{array}{r}52.60 \\
18.45 \\
2.47 \\
6.11 \\
4.22 \\
7.55 \\
3.24 \\
1.12 \\
2.53 \\
1.11 \\
.20 \\
\ldots \ldots .23 \\
\ldots \ldots . .\end{array}$ \\
\hline & 100.10 & 99.84 & 99.51 & 99.92 & 99.83 \\
\hline
\end{tabular}

\section{MASSACHUSETTS.}

\section{MAGNESIAN ROCKS.}

A. Cortlandtite, Belchertown. Belcherose. Described by B. K. Emerson in Mon. XXIX. Contains hornblende, pyroxene, biotite, olivine, and magnetite. Analysis by L. G. Eakins, record No. 1326 . P. R. C. 1616.

B. Wehrlite, New Braintree. Cookose. Description furnished by Emerson. Contains diallage, enstatite, augite, anorthite, biotite, apatite, chromite, magnetite, and pyrrhotite. Analysis by L. G. Eakins, record No. 1327. P. R. C. 1617.

C. Black, serpentinized boltonite, Stow. Collected by Emerson. Analysis by W. F. Hillebrand, record No. 1555. P. R. C. 1618.

\begin{tabular}{|c|c|c|c|}
\hline - & A. & B. & C. \\
\hline 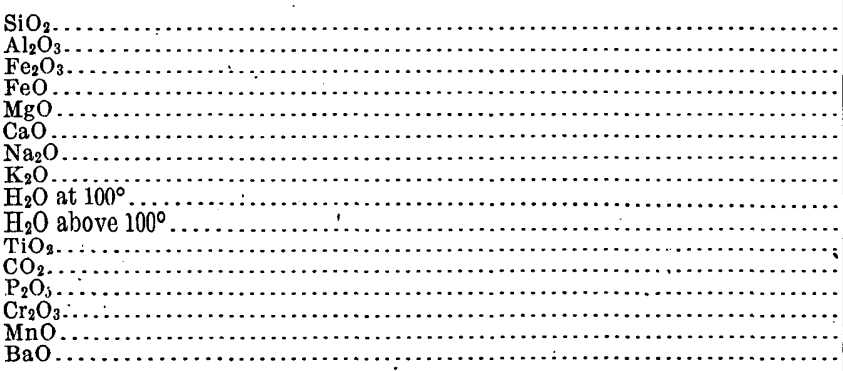 & $\begin{array}{r}48.63 \\
5.32 \\
2.91 \\
3.90 \\
21.79 \\
13.04 \\
.34 \\
.23 \\
2.81 \\
.47 \\
\operatorname{trace} \\
.21 \\
.36 \\
.12 \\
\text { trace }\end{array}$ & $\begin{array}{r}50.64 \\
7.93 \\
1.41 \\
14.82 \\
18.58 \\
3.41 \\
.96 \\
.21 \\
.87 \\
.82 \\
\ldots . . . \\
.27 \\
.05 \\
.16 \\
\ldots . . .\end{array}$ & $\begin{array}{r}36.92 \\
.10 \\
1.19 \\
.87 \\
43.99 \\
.59 \\
. . .05 \\
.05 \\
.72 \\
14.70 \\
\text { none } \\
.90 \\
\text { trace } \\
\text { none } \\
\text { trace } \\
\text { none }\end{array}$ \\
\hline & 100.13 & 100.13 & 100.03 \\
\hline
\end{tabular}

The following serpentinous rocks are described by Emerson in Mon. XXIX:

D. Serpentine, derived from salite, Osburn's soapstone quarry, Blandford. P. R. C. 1619 .

E. Dark-green, oily serpentine, center of large Middlefield bed. P. R. C. 1620.

F. Enstatite, slightly altered, from Granville. For comparison with G. P. R. C. 1621.

G. Serpentine, derived from enstatite, Granville. P. R. C. 1622.

Analyses D, E, and F by W. F. Hillebrand, record No. 1555. Analysis G by George Steiger, No. 1536. 


\begin{tabular}{|c|c|c|c|c|}
\hline & D. & E. & F. & G. \\
\hline 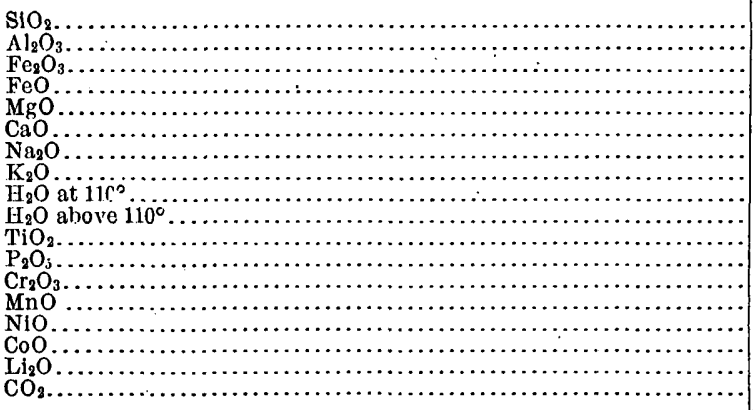 & \begin{tabular}{r}
40.77 \\
1.16 \\
3.56 \\
1.47 \\
39.37 \\
none \\
.14 \\
.10 \\
.49 \\
12.48 \\
none \\
trace \\
.28 \\
.09 \\
.17 \\
\hdashline trace \\
none
\end{tabular} & $\begin{array}{r}38.62 \\
.35 \\
3.44 \\
3.99 \\
40.61 \\
.40 \\
.10 \\
.08 \\
.36 \\
10.91 \\
\text { none } \\
\text { trace } \\
.39 \\
.10 \\
.21 \\
\ldots . . . \\
\text { trace } \\
.52\end{array}$ & $\begin{array}{r}54.04 \\
.52 \\
1.51 \\
3.90 \\
34.40 \\
\text { none } \\
.08 \\
.70 \\
3.07 \\
\text { none } \\
\text { none } \\
.14 \\
.11 \\
.23 \\
\ldots \ldots \ldots \\
\ldots \ldots . . \\
1.32\end{array}$ & $\begin{array}{r}37.82 \\
.61 \\
7.92 \\
1.15 \\
37.94 \\
\text { none } \\
\text { trace } \\
.75 \\
12.50 \\
\text { trace } \\
\text { trace } \\
.19 \\
\ldots . .85 \\
.45 \\
.05 \\
\ldots \ldots . .\end{array}$ \\
\hline & 100.08 & 100.08 & 100.02 & 99.38 \\
\hline
\end{tabular}

H. Rich, dark-green serpentine, Rowe. P. R. C. 1623.

I. Black serpentine, containing marmolite, Atwater's quarry, Russell. P. R. C. 1624.

J. Blackish-green serpentine, containing much chromite. From "The Crater," North Blandford. P. R. C. 1625.

K. Gray, splintery serpentine, Chester. P. R. C. 1626.

Analyses by Steiger, record No. 1536 .

\begin{tabular}{|c|c|c|c|c|}
\hline & H. & I. & J. & $\mathbf{K}$. \\
\hline 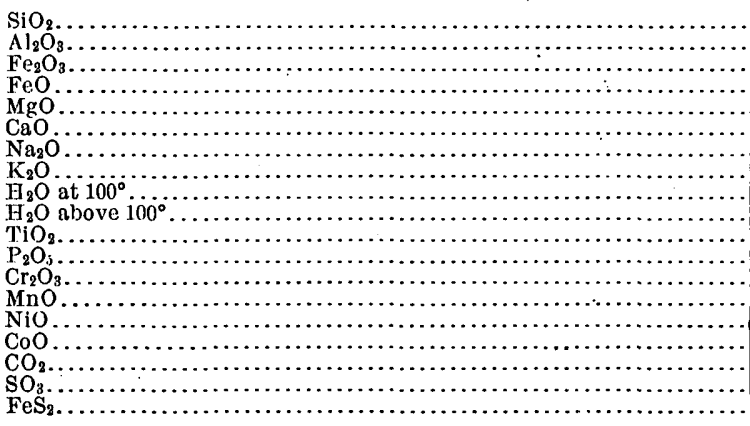 & $\begin{array}{r}40.42 \\
1.86 \\
2.75 \\
4.27 \\
35.95 \\
.66 \\
.16 \\
.21 \\
10.51 \\
\text { none } \\
\text { trace } \\
.28 \\
\text { trace } \\
.53 \\
\text { trace } \\
1.44 \\
\text { trace } \\
.43\end{array}$ & $\begin{array}{r}36.94 \\
.50 \\
6.04 \\
1.94 \\
38.35 \\
\text { none } \\
\text { none } \\
.71 \\
12.07 \\
\text { trace } \\
\text { trace } \\
.33 \\
\text { trace } \\
.40 \\
\text { none } \\
1.85 \\
.20 \\
\ldots . . .\end{array}$ & \begin{tabular}{|r}
39.14 \\
1.18 \\
4.46 \\
3.14 \\
41.45 \\
none \\
none \\
\\
9.34 \\
none \\
.02 \\
.33 \\
none \\
.47 \\
trace \\
none \\
none \\
$\ldots . . .$.
\end{tabular} & $\begin{array}{r}33.87 \\
.77 \\
2.81 \\
4.25 \\
38.57 \\
\text { none } \\
\text { none } \\
.38 \\
7.00 \\
\text { none } \\
\text { trace } \\
.38 \\
.04 \\
.33 \\
10.82 \\
.20 \\
\ldots . .\end{array}$ \\
\hline & 99.47 & 99.33 & 100.01 & 99.42 \\
\hline
\end{tabular}

\section{AMPHIBOLITE.}

Rocks $\mathrm{A}$ to $\mathrm{G}$ are described by Emerson in Mon. XXIX.

A. Amphibolite, Bernardston. A black, heavy, massive hornblende rock. Analysis by L. G. Eakins, record No. 1327. P. R. C. 1627.

B. Porphyritic amphibolite, Heath. Analysis by Eakins, record No. 1325. P. R. C. 1628 .

C. Amphibolite, New Salem. Analysis by Eakins, record No. 1325 . P. R. C. 1629.

D. Amphibolite, Whitmans Ferry, Sunderland. Thin, shaly, aphanitic. Analysis by Eakins, record No. 1325 . P. C. R. 1630. 


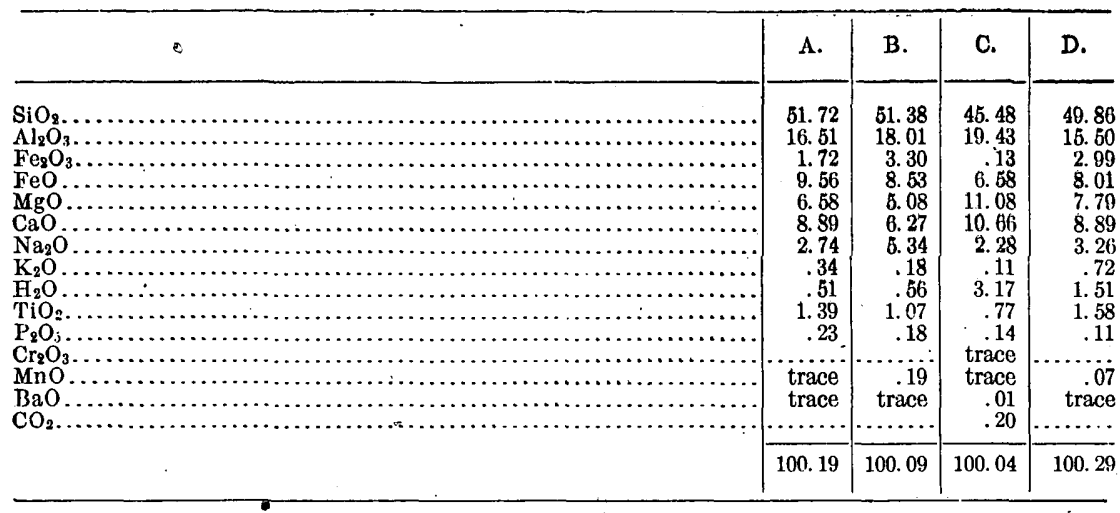

E. Amphibolite, South Leverett. Deep green, ligniform. Analysis by L. G. Eakins, record No. 1327. P. R. C. 1631.

F. Amphibolite, Goshen. Derived from Conway limestone. Analysis by Eakins, record No. 1414. P. R. C. 1632.

G. Black, fissile amphibólite, Worthington. Nearly pure, matted hornblende. Titanite and sometimes zircon present. Analysis by Eakins, record No. 1326. P. R. C. 1633.

H. Black, fissile, porphyritic amphibolite, Warwick. Analysis by Eakins, record No. 1414. Collected by Emerson, but not described in Mon. XXIX. P. R. C. 1634.

\begin{tabular}{|c|c|c|c|c|}
\hline . & $\mathrm{E}$ & F. & G. & H. \\
\hline 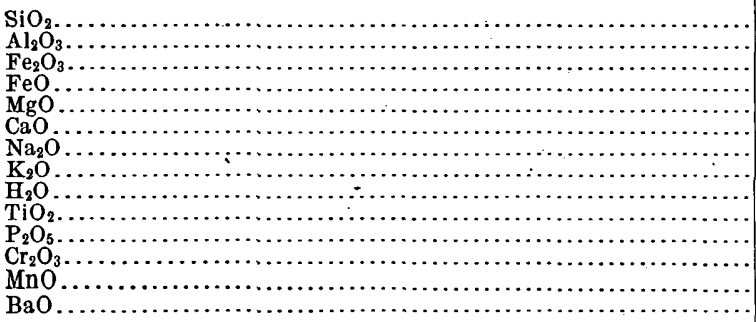 & $\begin{array}{r}47.56 \\
16.13 \\
1.80 \\
9.39 \\
9.21 \\
6.67 \\
2.52 \\
1.58 \\
3.51 \\
1.24 \\
.21 \\
\text { trace } \\
.08 \\
\text { trace }\end{array}$ & $\begin{array}{r}55.64 \\
16.27 \\
1.22 \\
7.20 \\
5.58 \\
9.23 \\
.91 \\
.19 \\
3.11 \\
.50 \\
.23 \\
.28\end{array}$ & $\begin{array}{r}48.53 \\
16.35 \\
2.03 \\
10.52 \\
9.71 \\
9.83 \\
1.36 \\
.32 \\
.79 \\
.51 \\
.07 \\
\cdots .17 \\
\text { trace }\end{array}$ & $\begin{array}{r}50.65 \\
13.03 \\
.27 \\
12.67 \\
16.96 \\
1.73 \\
1.37 \\
.04 \\
2.96 \\
.50 \\
\text { trace } \\
\text { trace } \\
.15\end{array}$ \\
\hline & 99.90 & 100.36 & 100.19 & 100.33 \\
\hline
\end{tabular}

The following amphibolites, from Palmer Center, were also collected by Emerson:

I. Amphibolite dike. P. R. C. 1635.

J. Hornblende, separated from I.

K. Feldspar, separated from I.

L. Amphibolite bed. P. R. C. 1636.

M. Hornblende, separated from $L$.

N. Feldspar, separated from $L$.

Analyses I to N by W. F. Hillebrand, record No. 1895. 


\begin{tabular}{|c|c|c|c|c|c|c|}
\hline & I. & J. & $\mathbf{K}$. & L. & M. & N. \\
\hline $\begin{array}{l}\mathrm{PLO}_{2} \\
\mathrm{Al}_{2} \mathrm{O}_{2} \ldots \\
\mathrm{Fe}_{2} \mathrm{O}_{3} \\
\mathrm{FeO}\end{array}$ & $\begin{array}{r}49.57 \\
14.23 \\
3.95 \\
8.01 \\
6.14 \\
10.19 \\
3.06 \\
.95 \\
.14 \\
1.33 \\
2.03 \\
\text { trace } \\
.21 \\
.02 \\
.04 \\
\text { trace } \\
\text { trace } \\
\text { trace? } \\
\text { trace } \\
\text { trace }\end{array}$ & $\begin{array}{r}43.11 \\
11.10 \\
4.97 \\
13.04 \\
9.35 \\
11.76 \\
1.18 \\
1.27 \\
.16 \\
1.92 \\
1.32 \\
.10 \\
\text { trace } \\
\text { trace } \\
\text { none } \\
\text { none } \\
\text { (?) }\end{array}$ & \begin{tabular}{r}
62.91 \\
23.37 \\
$\ldots \ldots \ldots .$. \\
\hdashline$\ldots . . .$. \\
5.83 \\
7.78 \\
.20 \\
.42
\end{tabular} & \begin{tabular}{|}
51.25 \\
16.53 \\
1.81 \\
7.67 \\
5.87 \\
9.32 \\
3.35 \\
.78 \\
.19 \\
1.26 \\
1.84 \\
$\cdots .31$ \\
$(?)$ \\
undet. \\
trace \\
trace \\
(?) \\
(?) \\
trace?
\end{tabular} & $\begin{array}{r}44.09 \\
10.68 \\
2.72 \\
12.96 \\
10.75 \\
11.58 \\
1.19 \\
.88 \\
.21 \\
1.91 \\
1.73 \\
\ldots .10\end{array}$ & 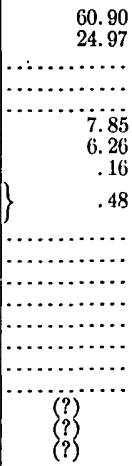 \\
\hline Specific gravity.. & & $\begin{array}{r}99.78 \\
3.220,21.5^{\circ}\end{array}$ & $\begin{array}{r}100.51 \\
2.667,24^{\circ}\end{array}$ & $\begin{array}{c}100.46 \\
\cdots \cdots \ldots . .\end{array}$ & $\begin{array}{r}99.12 \\
3.217,29^{\circ}\end{array}$ & $\begin{array}{r}100.62 \\
2.677,22^{\circ}\end{array}$ \\
\hline
\end{tabular}

\section{MISCELLANEOUS ROCKS.}

A. Keratophyre, Marblehead Neck. Liparose. Described by Sears, in Bull. Mus. Comp. Zool., vol. 16, No. 9, p. 170. Contains crystals of feldspar, with a decomposed base, irregular patches of quartz, some scales of biotite and grains of magnetite, and also some limonite and earthy matter. The feldspar is anorthoclase. P. R. C. $149 \hat{1}$. 1176.

B. Feldspar, separated from A. Analyses A and B by T. M. Chatard, record No.

C. Highly metamorphosed feldspathic conglomerate, graduating into arkose gneiss, electric railroad cut, Marlboro. Analysis by George Steiger, record No. 1536. P. R. C. 1637.

D. Egirite tinguaite, Southboro. Miaskose. Analyeis by H. N. Stokes, record No. 1653. Contains traces of chlorine and fluorine; 63.2 per cent of the rock is decomposable by hydrochloric acid. P. R. C. 1639.

Rocks $\mathrm{C}$ and $\mathrm{D}$ were collected by B. K. Emerson, but have not been fully described.

\begin{tabular}{|c|c|c|c|c|}
\hline & A. & B. & C. & D. \\
\hline 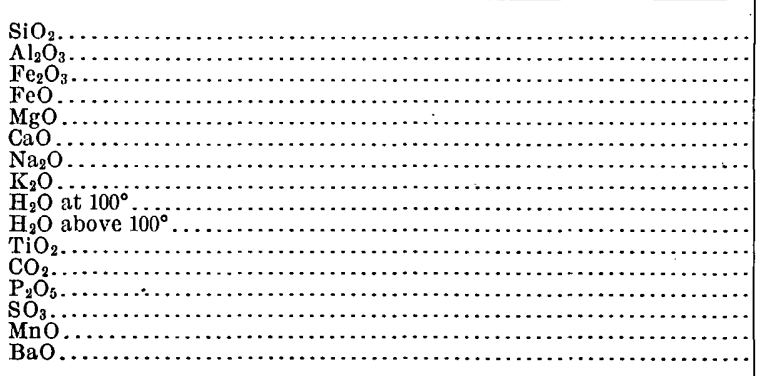 & $\begin{array}{r}70.23 \\
15.00 \\
1.99 \\
\text { undet. } \\
.38 \\
.33 \\
4.98 \\
4.99 \\
.91 \\
1.28 \\
(?) .03 \\
\cdots \\
.06 \\
.24 \\
. .24 \\
. . .\end{array}$ & $\begin{array}{r}65.66 \\
20.05 \\
\text { trace } \\
\text { trace } \\
.18 \\
.67 \\
6.56 \\
6.98 \\
.04 \\
.37 \\
\text { undet. } \\
\text { undet. } \\
\cdots \\
\ldots . .13 \\
\ldots . . . .\end{array}$ & $\begin{array}{r}75.35 \\
13.03 \\
.62 \\
.94 \\
.21 \\
1.33 \\
2.44 \\
5.14 \\
.15 \\
.73 \\
.21 \\
.03 \\
.08 . \\
.03 \\
\text { none } \\
.07\end{array}$ & $\begin{array}{r}54.22 \\
20.20 \\
2.35 \\
1.02 \\
.29 \\
.70 \\
9.44 \\
4.85 \\
.42 \\
5.57 \\
.38 \\
\text { trace } \\
.11 \\
\text { none } \\
.19 \\
\text { trace }\end{array}$ \\
\hline & 100.42 & 100.64 & 100.36 & 99.74 \\
\hline
\end{tabular}

E. Massive, coarse, altered diabase, Leverett. Ornose. Contains saussuritic feldspar with black hornblende. Analysis by Eakins, record No. 1325. P. R. C. 1640.

F. Tonalite, South Leverett. Andose. Dark green, chloritic. Contains reddish feldspar, dark hornblende, and a network of dark-green epidotic quartz veins. Analysis by Eakins, record No. 1326. P. R. C. 1641. 
G. Biotite granite, Moore's quarry, Florence. Lassenose. Very feldspathic. Quartz rare, with fluid inclusions. Feldspar mostly triclinic, orthoclase and microcline present in small quantities. Little muscovite, some rutile. Analysis by Eakins, record No. 1414. P. R. C. 1642:

Rocks E, F, and G are described by Emerson in Mon. XXIX.

H. Holyokeite, east foot of Mount Tom, Northampton. Probably tuolumnose. Described by Emerson in Jour. Geology, vol. 10, p. 508. Contains àlbite, 70 per cent; orthoclase, 9.4 per cent; calcite, 16.4 per cent, with minor amounts of ilmenite, dolomite, chalcopyrite, pyrite, and apatite. Analysis by W. F. Hillebrand, record No. 1924. P. R. C. 1643.

\begin{tabular}{|c|c|c|c|c|}
\hline . & E. & F. & G. & H. \\
\hline 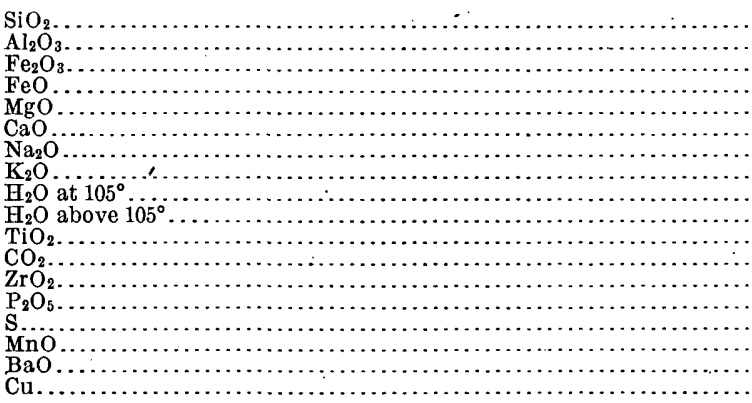 & $\begin{array}{r}51.56 \\
14.82 \\
4.30 \\
7.21 \\
7.36 \\
7.09 \\
4.21 \\
.17 \\
1.47 \\
1.97 \\
\ldots \ldots \\
\ldots .09 \\
\text { trace } \\
\text { trace } \\
\ldots \ldots\end{array}$ & \begin{tabular}{|r|}
55.51 \\
16.51 \\
1.68 \\
4.57 \\
6.73 \\
6.73 \\
3.19 \\
2.46 \\
1.53 \\
.91 \\
$\ldots \ldots$. \\
$\ldots \ldots .17$ \\
$\ldots \ldots .11$ \\
.02 \\
$\ldots \ldots .$.
\end{tabular} & $\begin{array}{r}73.27 \\
15.51 \\
.33 \\
1.14 \\
.15 \\
2.74 \\
4.79 \\
1.66 \\
.68 \\
.10 \\
\ldots \ldots \ldots \\
\ldots \ldots \ldots \\
\text { trace } \\
\ldots \ldots \ldots . \\
\text { trace } \\
\ldots \ldots \ldots . \\
\ldots \ldots . . .\end{array}$ & $\begin{array}{r}53.83 \\
16.36 \\
\ldots . . . \\
.89 \\
.13 \\
9.81 \\
7.89 \\
1.58 \\
.15 \\
.36 \\
.86 \\
7.47 \\
.02 \\
.11 \\
.17 \\
\text { undet. } \\
\text { (?) } \\
.14\end{array}$ \\
\hline . & 100.25 & 100.12 & 100.37 & 99.77 \\
\hline
\end{tabular}

Analyses I to $\mathrm{P}$ are of rocks collected by B. K. Emerson, and as yet, with two exceptions, undescribed.

I. Gneissoid granite, north well of Flint's quarry, Monson. Amadorose. Analysis by W. F. Hillebrand, record No. 1924. P. R. C. 1645.

J. Gneissoid granite, Frawley's quarry, Erving. Tehamose-toscanose. Analysis by George Steiger, record No. 1941. P. R. C. 1648.

$\mathrm{K}$. Gneissoid granite, Alderman's quarry, Becket. Toscanose-lassenose: Analysis by Steiger, No. 1944. P. R. C. 1649.

L. Gneissoid biotite granite, Wood's'quarry, Pelham. Toscanose. Analysis by Steiger, No. 1960. P. R. C. 1650.

\begin{tabular}{|c|c|c|c|c|}
\hline & I. & $\mathbf{J}$ & $\mathbf{K}$. & L. \\
\hline 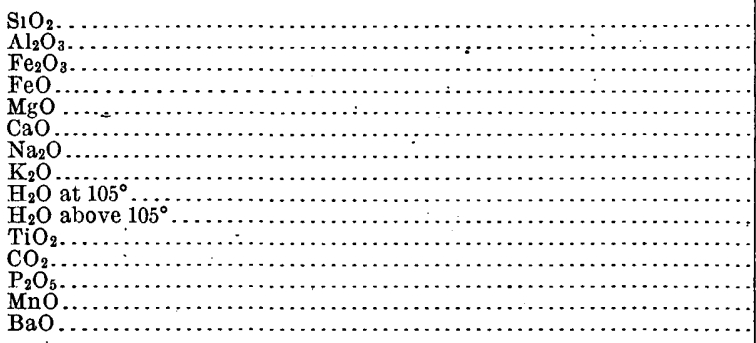 & $\begin{array}{r}65.02 \\
18.37 \\
1.21 \\
2.06 \\
1.49 \\
6.20 \\
3.96 \\
.64 \\
.09 \\
.42 \\
.33 \\
\text { none } \\
.14 \\
.09 \\
\text { trace }\end{array}$ & $\begin{array}{r}74.15 \\
13.35 \\
1.26 \\
.53 \\
.23 \\
1.92 \\
2.84 \\
4.58 \\
.13 \\
.50 \\
.12 \\
\text { none } \\
.06 \\
\ldots \ldots . . .\end{array}$ & $\begin{array}{r}70.62 \\
15.31 \\
1.06 \\
.43 \\
.29 \\
1.30 \\
4.55 \\
4.01 \\
.16 \\
.72 \\
.29 \\
.88 \\
.07 \\
\ldots \ldots . . \\
\ldots . . .\end{array}$ & $\begin{array}{r}72.45 \\
13.32 \\
1.93 \\
.63 \\
.44 \\
1.81 \\
3.55 \\
3.86 \\
.59 \\
1.51 \\
.27 \\
\text { none } \\
.06 \\
\ldots \ldots . . \\
\ldots \ldots . .\end{array}$ \\
\hline & 100.02 & 99.67 & 99.69 & 100.42 \\
\hline
\end{tabular}

M. Aplite (alsbachite), Fallon Hill, Enfield.- Kallerudose. Garnetiferous biotite granite dike. P. R. C. 1651.

N. Another sample, same locality as M. Lassenose-alsbachose, P. R. C. 1652. 
Analyses M, N, by George Steiger, record No. 1975.

O. Quartz diabase, west of Ashley reservoir, Holyoke. Vaalose. Contains calcite and radiated quartz. P. R. C. 1653.

$P$. Palagonite, from blebs in $\mathrm{O}$.

Analyses O, P, by Steiger, No. 2039; rocks described by Emerson in Bull. Geol. Soc. America, vol. 16, p. 91.

\begin{tabular}{|c|c|c|c|c|}
\hline$\cdot$ & M. & N. & 0. & P. \\
\hline 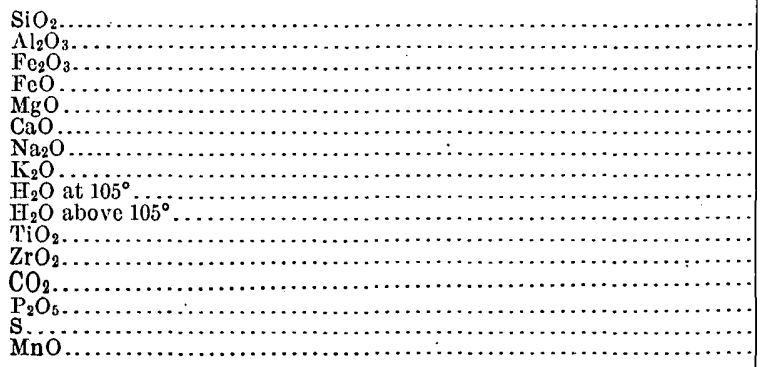 & $\begin{array}{r}77.00 \\
13.60 \\
.41 \\
\ldots \ldots \\
\text { none } \\
.70 \\
5.78 \\
1.50 \\
.23 \\
.48 \\
.07 \\
\ldots \ldots \ldots \\
\text { trace } \\
\ldots \ldots \ldots\end{array}$ & $\begin{array}{r}73.09 \\
13.42 \\
1.44 \\
1.13 \\
.35 \\
1.89 \\
4.52 \\
1.59 \\
.47 \\
1.41 \\
.26 \\
. . . \\
.05 \\
. \\
.10\end{array}$ & $\begin{array}{r}53.52 \\
9.70 \\
8.06 \\
9.45 \\
2.52 \\
5.64 \\
2.24 \\
1.50 \\
1.67 \\
2.16 \\
1.98 \\
.03 \\
1.02 \\
.36 \\
.10 \\
.26\end{array}$ & $\begin{array}{r}40.35 \\
5.11 \\
24.99 \\
3.55 \\
5.48 \\
1.32 \\
.18 \\
1.44 \\
8.51 \\
8.51 \\
.20 \\
\ldots \ldots \\
\text { none } \\
\text { none } \\
.22\end{array}$ \\
\hline & 99.77 & 99.72 & 100.21 & 99.86 \\
\hline
\end{tabular}

Q. Granite gneiss, Hoosac Mountain. Toscanose. Contains quartz, microcline, albite, muscovite, biotite, magnetite, titanite, epidote, apatite, and zircon. Analysis by E. T. Allen, record No. 2064. P. R. C. 1718.

R. Albite schist, Hoosac Mountain. Varingose. Same minerals as in $\mathrm{Q}$, with garnet and pyrite. Same analyst and number. P. R. C. 1719.

Rocks $Q$ and $R$ collected by J. E. Wolff, but not yet described. The following feldspars, all albite, separated from schists of the same region, are described by Wolff is Mon. XXIII, pp. 60 and 187. Analyses by R. B. Riggs, record Nos. 507 and 567.

S. From feldspathic schist, central shaft of the Hoosac tunnel. P. R. C. 129.

T. U. From the porphyritic mica schist of Greylock Mountain.

\begin{tabular}{|c|c|c|c|c|c|}
\hline & Q. & $\mathrm{R}$. & S. & 'T. & U. \\
\hline 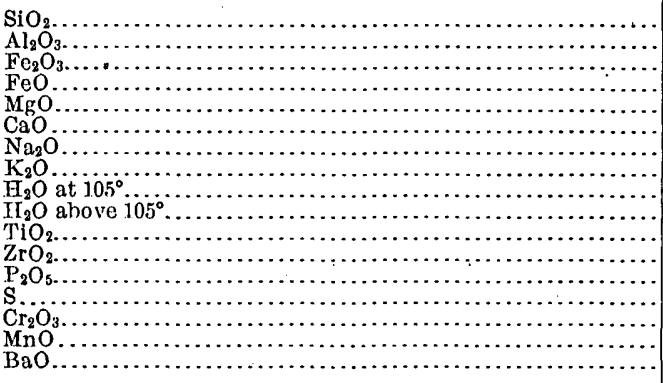 & $\begin{array}{r}67.12 \\
14.97 \\
2.61 \\
2.19 \\
.54 \\
1.69 \\
3.92 \\
5.15 \\
.19 \\
1.13 \\
.37 \\
.03 \\
.14 \\
\text { none } \\
\text { trace } \\
.02 \\
.19\end{array}$ & $\begin{array}{r}70.95 \\
9.99 \\
3.08 \\
6.10 \\
1.08 \\
.38 \\
1.69 \\
3.74 \\
.40 \\
1.82 \\
.63 \\
.04 \\
.23 \\
.05 \\
\text { trace } \\
\text { trace } \\
.04\end{array}$ & 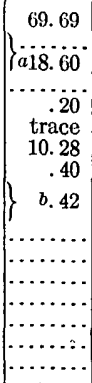 & 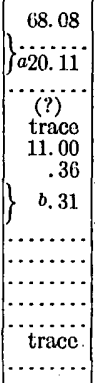 & 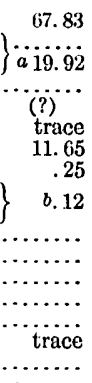 \\
\hline . & 100.26 & 100.22 & 99.59 & 99.86 & 99.77 \\
\hline
\end{tabular}

$a \mathrm{Fe}_{2} \mathrm{O}_{3}$ less than 0.5 per cent.

$b$ L.oss on ignition.

V. Pyroxene-titanite aplite, South Peru. Analysis by W. T. Schaller, record No. 2143.

W. Diorite, railroad cut west of Ironstone. Analysis by E. C. Sullivan.

$X$. Pre-Cambrian quartzite gneiss, Northbridge. Analysis by Sullivan.

Rocks V, W, X, collected by B. K. Emerson, but no description furnished. 


\begin{tabular}{|c|c|c|c|}
\hline & V. & $\mathrm{W}$ & X. \\
\hline 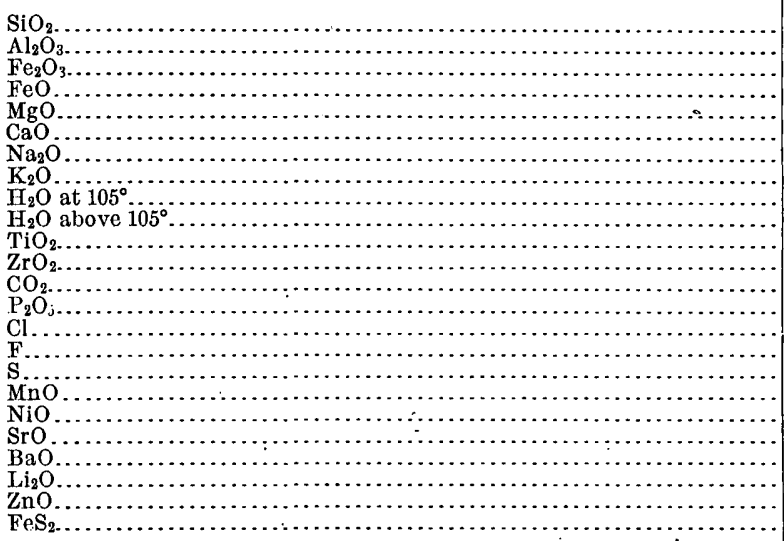 & $\begin{array}{r}60.44 \\
16.26 \\
.07 \\
3.52 \\
1.75 \\
7.86 \\
7.13 \\
.44 \\
.29 \\
.38 \\
1.33 \\
.06 \\
\text { none } \\
.58 \\
\text { undet. } \\
\text { undet. } \\
\text { none } \\
.06 \\
\text { trace } \\
\text { trace? } \\
\text { trace? } \\
\text { none } \\
\ldots \ldots . . . \\
.10\end{array}$ & $\begin{array}{r}47.18 \\
16.90 \\
5.21 \\
7.22 \\
3.71 \\
9.69 \\
2.76 \\
.68 \\
.92 \\
1.66 \\
2.94 \\
\text { trace } \\
.63 \\
.38 \\
\text { trace } \\
\text { trace? } \\
.22 \\
.14 \\
\text { trace } \\
\text { trace } \\
.01 \\
\ldots \ldots . . \\
.03\end{array}$ & $\begin{array}{r}75.05 \\
12.83 \\
1.11 \\
.53 \\
.31 \\
.85 \\
3.02 \\
5.14 \\
.72 \\
.62 \\
.20 \\
.02 \\
.07 \\
.04 \\
\text { trace } \\
\text { trace? } \\
.01 \\
.03 \\
\ldots \ldots . . \\
\text { trace } \\
.03 \\
\text { trace } \\
\ldots \ldots \ldots . . \\
\ldots \ldots \ldots\end{array}$ \\
\hline & 100.27 & 100.28 & 100.58 \\
\hline
\end{tabular}

\section{CONNECTICUT.}

A. Olivine basalt, main flow, Pine Hill, South Britain. Auvergnose. Contains plagioclase, pink augite, olivine, and magnetite. The rock is quite fresh. P. R. C. 1456.

B. Olivine basalt, anterior flow, South Britain. Contains plagioclase, uralitized augite, olivine, and magnetite. Rock much altered, and containing numerous amygdules of calcite and prehnite stained by iron. P. R. C. 1457.

Rocks A and B are described by W. H. Hobbs in 21st Ann. Rept., pt. 3, p. 60. Analyses by W. F. Hillebrand, record No. 1842.

C. Basic pitchstone (tachylyte), from the so-called "ash bed" northeast of Meriden. SR. 1-2 of auvergnase. Described by Emerson in Bull. Geol. Soc. America, vol. 8, p. 77. Analysis by H. N. Stokes, record No. 1641. P. R. C. 1654.

\begin{tabular}{|c|c|c|c|}
\hline . & A. & B. & C. \\
\hline 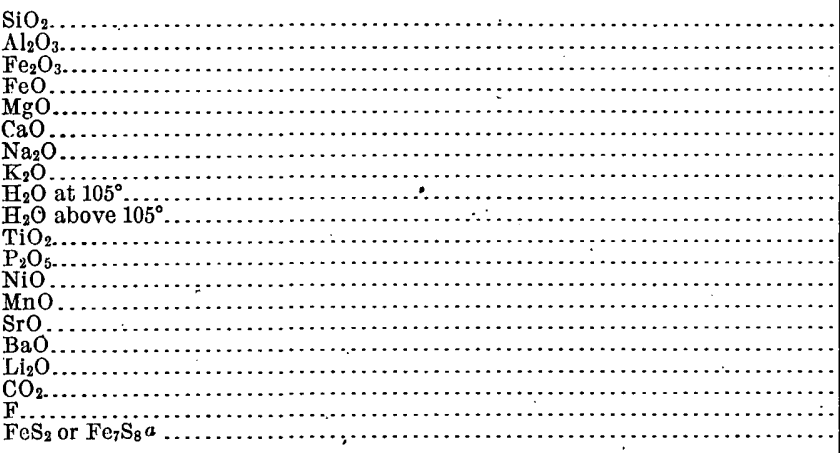 & $\begin{array}{r}52.40 \\
13.55 \\
2.73 \\
9.79 \\
5.53 \\
10.01 \\
2.32 \\
.40 \\
.62 \\
1.05 \\
1.08 \\
.12 \\
\text { trace } \\
.26 \\
\text { none } \\
\text { trace? } \\
\text { none } \\
\ldots . . . . \\
\ldots . . . . \\
.13\end{array}$ & $\begin{array}{r}47.52 \\
13.91 \\
7.06 \\
3.76 \\
6.84 \\
5.71 \\
3.06 \\
.77 \\
1.75 \\
4.55 \\
1.19 \\
.15 \\
\text { trace } \\
.18 \\
\text { none } \\
\text { trace } \\
\text { trace } \\
3.68 \\
\ldots . . . \\
\ldots . . . .\end{array}$ & $\begin{array}{r}46.86 \\
13.96 \\
.5 .23 \\
4.67 \\
7.69 \\
9.42 \\
1.85 \\
2.02 \\
1.29 \\
3.43 \\
1.13 \\
.15 \\
\ldots . . . \\
\text { trace } \\
\text { trace } \\
.03 \\
\text { trace } \\
2.19 \\
\text { trace }\end{array}$ \\
\hline & 99.99 & 100.13 & 99.92 \\
\hline
\end{tabular}


The following rocks, D to H, are from Prospect Hill, west of Litchfield. Collected by W. H. Hobbs, who furnishes the petrographic data:

D. Mica-hornblende gabbro. Bandose. Contains plagioclase, hypersthene, biotite, subordinate green hornblende, and magnetite. P. R. C. 1677.

E. Hornblende diorite. Hessose. Contains plagioclase, green hornblende, subordinate biotite, and magnetite. P. R. C. 1678.

F. Hornblendite. III. 6. 4. 4, 5. Almost entirely green hornblende. Contains also magnetite, a little biotite, and very little plagioclase. P. R. C. 1679.

G. Cortlandtite. IV. $1^{2} \cdot 1^{2}$. 2. Mainly hypersthene, with subordinate olivine, green hornblende, and magnetite. P. R. C. 1680.

H. Hornblende norite. Auvergnose. Contains greenish hornblende and a little more hypersthene. Rich in plagioclase, with accessory biotite and magnetite. P. R. C. 1681 .

Analyses D, G, H, :by W. F. Hillebrand, record No. 2074; E and F by George Steiger, record No 2071.

\begin{tabular}{|c|c|c|c|c|c|}
\hline & D. & E.o & F. & G. & H. \\
\hline 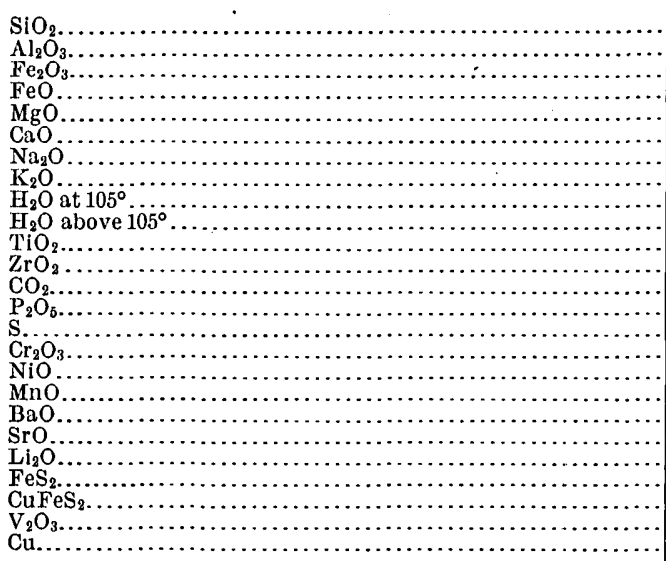 & $\begin{array}{r}50.46 \\
19.65 \\
1.60 \\
\mathbf{5} .15 \\
5.31 \\
9.66 \\
3.15 \\
1.57 \\
.74 \\
1.14 \\
1.18 \\
\text { trace? } \\
\text { none } \\
.18 \\
\text { trace } \\
\ldots \ldots . . \\
\ldots \ldots . . \\
.15 \\
.09 \\
.03 \\
\text { trace? } \\
\ldots \ldots . . . \\
\ldots \ldots \ldots \\
\ldots \ldots \ldots . . \\
\text { trace? }\end{array}$ & $\begin{array}{r}47.97 \\
17.41 \\
2.06 \\
9.09 \\
5.93 \\
9.12 \\
3.08 \\
.85 \\
.30 \\
1.11 \\
2.10 \\
\ldots . . \\
.43 \\
.37 \\
\ldots . . .\end{array}$ & $\begin{array}{r}38.02 \\
14.64 \\
5.69 \\
10.33 \\
10.26 \\
9.11 \\
1.90 \\
1.66 \\
.74 \\
2.35 \\
4.84 \\
\ldots . . . \\
\text { none } \\
.09\end{array}$ & $\begin{array}{r}47.87 \\
6.09 \\
1.40 \\
8.14 \\
16.33 \\
14.49 \\
.87 \\
.55 \\
.26 \\
1.07 \\
1.20 \\
\text { trace? } \\
.75 \\
.07\end{array}$ & $\begin{array}{r}49.28 \\
15.76 \\
1.86 \\
6.94 \\
8.21 \\
10.51 \\
2.58 \\
.76 \\
.47 \\
1.10 \\
.87 \\
\text { trace? } \\
.36 \\
.11 \\
\ldots . .5 \\
.03 \\
.09 \\
.20 \\
\text { trace? } \\
\text { none } \\
\text { none } \\
.99 \\
.13 \\
\text { (?) } \\
\ldots . . .\end{array}$ \\
\hline & 100.12 & 99.92 & 99.75 & 100.16 & 100.25 \\
\hline
\end{tabular}

The following rocks were collected by G. F. Loughlin. Petrographic data supplied by H. E. Gregory. Analyses by G. Steiger.

I. Gabbro, near northeast boundary, between Preston and Griswold. Contains plagioclase, pyroxene, hornblende, epidote, zoisite, chlorite, serpentine, pyrite, ilmenite, apatite, and a little calcite.

J. Quartz diorite, 1 mile east of Preston City post-office. Contains quartz, plagioclase, little orthoclase, hornblende, little biotite, rare muscovite, epidote, zoisite, pyrite, ilmenite, apatite, and zircon.

K. Granite dike, southern slope of Barnes Hill, North Stonington. Contains quartz, orthoclase, oligoclase, biotite, muscovite, titanite slightly altered to leucoxene, epidote, chlorite, ilmenite, pyrite (?), zircon, and apatite. Somewhat kaolinized.

L. Amphibolite dike, east base of Prospect Hill, northwest of Preston City postoffice. Contains plagioclase, hornblende, epidote, ilmenite, pyrite, apatite, and a little kaolin and sericite. 


\begin{tabular}{|c|c|c|c|c|}
\hline & I. & J. & K. & L. \\
\hline 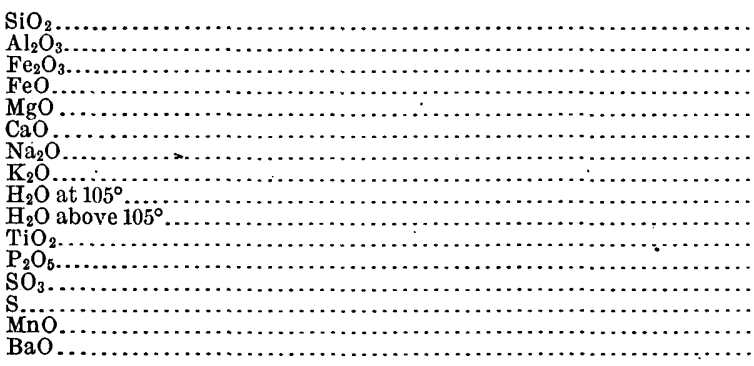 & $\begin{array}{r}49.98 \\
17.65 \\
3.48 \\
4.85 \\
7.78 \\
9.42 \\
2.75 \\
.17 \\
1.06 \\
2.23 \\
.87 \\
\text { trace } \\
\ldots \ldots . . \\
\text { none }\end{array}$ & $\begin{array}{r}49.90 \\
14.64 \\
4.65 \\
7.52 \\
6.06 \\
9.14 \\
2.73 \\
.46 \\
.46 \\
1.98 \\
1.98 \\
.38 \\
\ldots . . . \\
.02 \\
.18 \\
.02\end{array}$ & $\begin{array}{r}73.52 \\
12.86 \\
1.48 \\
1.54 \\
.57 \\
2.08 \\
4.36 \\
1.41 \\
.35 \\
1.21 \\
.51 \\
.07 \\
\text { none } \\
\text { none } \\
.08 \\
.02\end{array}$ & $\begin{array}{r}47.63 \\
15.49 \\
2.60 \\
8.70 \\
8.40 \\
10.04 \\
3.09 \\
.36 \\
.35 \\
1.78 \\
1.93 \\
.11 \\
\ldots \ldots . . \\
\ldots \ldots 22 \\
.2 .\end{array}$ \\
\hline 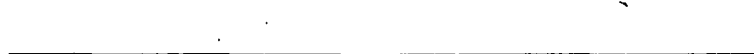 & 100.19 & 100.12 & 100.06 & 100.70 \\
\hline
\end{tabular}

NEW YORK.

\section{ROCKS OF THE ADIRONDACK REGION.}

Collected by J. F. Kemp, who furnishes the petrographic data. A and G, together with the four iron ores, are described by Kemp in 19th Ann., pt. 3, p. 383 . Analyses A and B by George Steiger, record No. 1715; C to K by W. F. Hillebrand, record Nos. 1714 and 1717.

A. Wall rock of iron mine near Lincoln Pond, Elizabethtown. Camptonose. Varies from norite to gabbro. Chief minerals, green augite, hypersthene, brown hornblende, plagioclase, and magnetite. Microperthitic feldspar less common. Garnet varies from absence to abundance.

B. Coarse gabbro, top of Whiteface Mountain. Hessose. A pyroxenic phase of the anorthosite. Contains much labradorite, abundant light-green augite, brown hornblende, shreds of more or less bleached brown biotite, and magnetite.

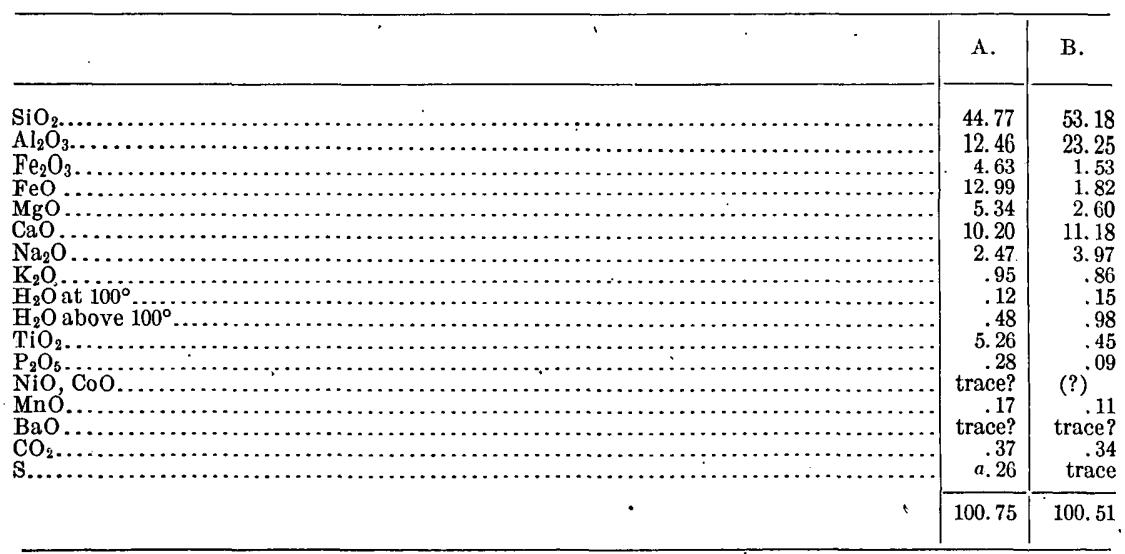

a Mainly present as pyrrhotite.

C. Pyroxenic anorthosite, Ẹlizabethtown. Hessose. Contains largely labradorite, subordinate light-green augite, less brown hornblende, and a little magnetite.

D. Norite, with close affinity to gabbro. Camptonose. Intrusion in C. Contains labradorite, hypersthene, garnets, green augite, brown hornblende, a little brown biotite, magnetite, and apatite.

E. Diabasic norite or gabbro, Elizabethtown. Auvergnose. Contains the same minerals as $\mathrm{D}$. 
F. Gneissoid derivative, by pressure, of E. Camptonose. The same minerals, but with hornblende more abundant.

G. Gabbro, wall rock of Split Rock mine. Hessose. Contains augite, hypersthene, brown hormblende, garnet, plagioclase, magnetite, and possibly spinel. P. R. C. 1471.

\begin{tabular}{|c|c|c|c|c|c|}
\hline & C. & D. & E. & F. & G. \\
\hline 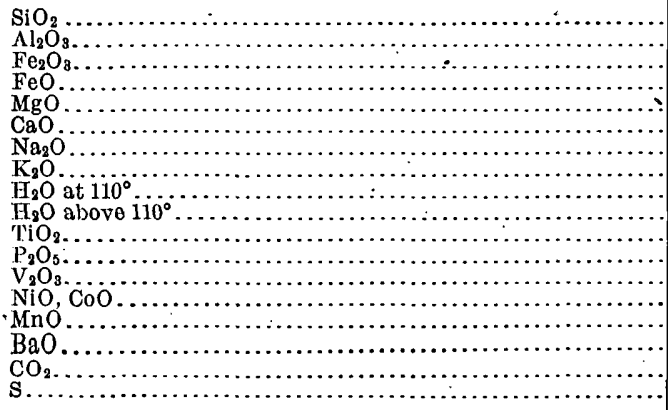 & $\begin{array}{r}56.94 \\
20.82 \\
.83 \\
3.02 \\
2.36 \\
9.41 \\
3.36 \\
1.58 \\
.21 \\
.59 \\
.44 \\
.07 \\
\text { (?) } \\
\text { trace } \\
.11 \\
.05 \\
.45 \\
\text { trace }\end{array}$ & $\begin{array}{r}47.16 \\
14.45 \\
1.61 \\
13.81 \\
5.24 \\
8.13 \\
3.09 \\
1.20 \\
.12 \\
.48 \\
3.37 \\
.57 \\
(?) \\
.02 \\
.24 \\
\operatorname{trace} \\
.35 \\
.14\end{array}$ & $\begin{array}{r}44.97 \\
15.38 \\
2.29 \\
12.39 \\
10.89 \\
7.50 \\
3.02 \\
.56 \\
.10 \\
.65 \\
1.18 \\
.14 \\
.02 \\
.02 \\
.22 \\
\text { trace } \\
.23 \\
.06\end{array}$ & $\begin{array}{r}46.74 \\
16.63 \\
2.17 \\
10.60 \\
6.11 \\
8.66 \\
3.81 \\
.86 \\
.12 \\
.73 \\
2.54 \\
.33 \\
(?) \\
.03 \\
.26 \\
\text { trace } \\
.07 \\
.11\end{array}$ & $\begin{array}{r}47.88 \\
18.90 \\
1.39 \\
10.45 \\
7.10 \\
8.36 \\
2.75 \\
.81 \\
.18 \\
.43 \\
1.20 \\
.20 \\
(?) \\
.02 \\
.16 \\
\text { trace } \\
.12 \\
.07\end{array}$ \\
\hline & 100.24 & 99.98 & 99.64 & 99.77 & 100.02 \\
\hline
\end{tabular}

$\mathrm{ZrO}_{2}, \mathrm{Cl}$, and $\mathrm{F}$ not looked for. $\mathrm{Cr}_{2} \mathrm{O}_{3}, \mathrm{Li}_{2} \mathrm{O}$, and $\mathrm{SrO}$ present in traces. $\mathrm{S}$ is, in part at least, present as pyrrhotite.

The following titaniferous magnetites, from Elizabethtown, are regarded by Kemp as being of magmatic origin, and are therefore included in this tabulation.

H. Near Lincoln Pond.

I. Oak Fill pit.

J. Tunnel Hill.

K. Split Rock mine.

$\mathrm{H}$ and $\mathrm{I}$ are classed by Washington as Adirondackiase, $\mathrm{J}$ and $\mathrm{K}$ as Champlainiase.

\begin{tabular}{|c|c|c|c|c|}
\hline & H. & I. & J. & K. \\
\hline \multirow[t]{2}{*}{ 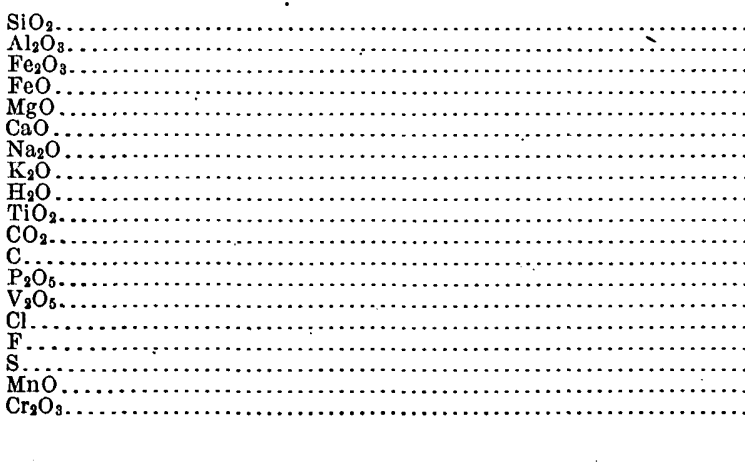 } & $\begin{array}{r}12.42 \\
6.46 \\
30.68 \\
27.92 \\
3.35 \\
3.95 \\
.50 \\
.26 \\
.64 \\
12.31 \\
.32 \\
.05 \\
.82 \\
.04 \\
.12 \\
\text { trace } \\
.04 \\
\text { undet. } \\
\text { none }\end{array}$ & \begin{tabular}{|r}
21.42 \\
7.03 \\
30.34 \\
22.81 \\
6.92 \\
3.59 \\
.53 \\
.41 \\
.95 \\
5.21 \\
trace \\
trace \\
.14 \\
undet. \\
.42 \\
$(?)$ \\
.04 \\
undet. \\
none
\end{tabular} & \begin{tabular}{|r}
13.35 \\
8.78 \\
20.35 \\
28.82 \\
6.63 \\
2.15 \\
undet. \\
undet. \\
1.68 \\
16.45 \\
.17 \\
trace \\
.02 \\
.61 \\
little \\
$(?)$ \\
.09 \\
undet. \\
.55
\end{tabular} & $\begin{array}{r}17.90 \\
10.23 \\
15.85 \\
27.94 \\
6.04 \\
2.86 \\
\text { undet. } \\
\text { undet. } \\
1.33 \\
15.66 \\
\text { trace } \\
\text { none } \\
.04 \\
.55 \\
\text { none } \\
(?) \\
\text { undet. } \\
.51\end{array}$ \\
\hline & 99.88 & 99.81 & 99.65 & 99.15 \\
\hline
\end{tabular}

2. MiSCELLANEOUS ROCKS.

A. Peridotite, from Dewitt, near Syracuse. A rock described by Darton and Kemp, Am. Jour. Sci., 3d ser., vol. 49, p. 456. Contains olivine, partly serpentinized, biotite, and augite, with magnetite, apatite, and perofskite. Possibly a little chromite also. Classed by Kemp as limburgite. P. R. C. 296. 
Analysis by H. N. Stokes, record No. 1491. The $\mathrm{FeO}$ represents the total iron, because the sulphides present, possibly as pyrrhotite, prevent the separate estimation of the two iron oxides.

B. Syenite, Fort Ann, Washington County. Adamellose. Contains orthoclase, microperthite, hornblende, green augite, and a little quartz.

C. Quartz-biotite-garnet gneiss, Fort Ann. Consists essentially of quartz, garnet, biotite, orthoclase, some plagioclase, and zircons.

B and C were collected by J.F. Kemp, who supplies the petrographic data. Analyses by W. F. Hillebrand, record No. 1930.

\begin{tabular}{|c|c|c|c|}
\hline$\cdot$ & A. & B. & C. \\
\hline 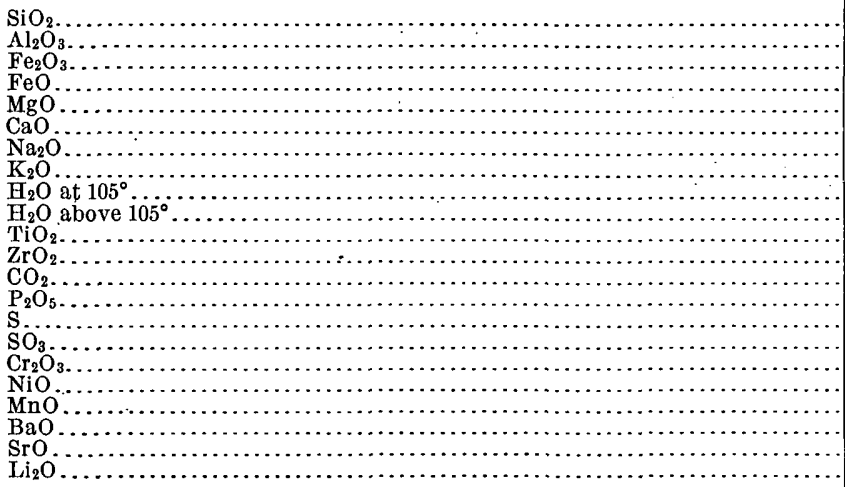 & $\begin{array}{r}36.80 \\
4.16 \\
\ldots . . . \\
8.33 \\
25.98 \\
8.63 \\
.17 \\
2.48 \\
.51 \\
6.93 \\
1.26 \\
\ldots .95 \\
2.95 \\
.47 \\
.95 \\
.06 \\
.20 \\
.09 \\
.13 \\
.12 \\
\text { trace } \\
\ldots . . .\end{array}$ & $\begin{array}{r}64.47 \\
10.51 \\
1.11 \\
7.37 \\
5.21 \\
3.10 \\
2.21 \\
3.63 \\
.18 \\
.75 \\
.65 \\
\text { none } \\
.58 \\
.25 \\
.12 \\
\ldots . . \\
\text { trace? } \\
.04 \\
.15 \\
.04 \\
\text { trace } \\
\text { none }\end{array}$ & $\begin{array}{r}65.09 \\
16.37 \\
.93 \\
5.64 \\
2.40 \\
2.40 \\
3.31 \\
1.93 \\
.13 \\
.58 \\
.93 \\
.01 \\
.07 \\
.11 \\
.03 \\
\ldots . . \\
\text { trace } \\
\text { trace } \\
.16 \\
.03 \\
\text { trace } \\
\text { trace }\end{array}$ \\
\hline \multirow[t]{2}{*}{ 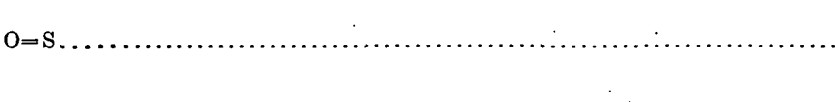 } & $\begin{array}{r}100.22 \\
.47\end{array}$ & $\begin{array}{r}100.37 \\
.06\end{array}$ & $\begin{array}{r}100.12 \\
\ldots \ldots\end{array}$ \\
\hline & 99.75 & 100.31 & \\
\hline
\end{tabular}

NEW JERSEY.

Rocks A, B, and C described by J. P. Iddings in Bull. 150, pp. 254, 209, 236. Analyses by Eakins, record No. 1299.

A. Basalt, Watchung Mountain, Orange. Auvergnose. Contains pyroxene, mostly malacolite, plagioclase, magnetite, and glass, with variable amounts of serpentine or chlorite. The feldspar is partly altered into a mineral which is probably prehnite. P. R. C. 102.

B. Elæolite syenite, Beemersville. Beemerose. Contains nephelite, orthoclase, ægirite, and biotite, with melanite, sphene, apatite, zircon, and magnetite in smaller amounts. Sodalite is probably present also. P. R. C. 78 .

C. Minette, Franklin Furnace. Contains alkali feldspar, biotite, monoclinic pyroxene, magnetite, epidote, calcite, chlorite, apatite, sphene, pyrite, and a little secondary quartz. P. R. C. 91.

D. Nepheline syenite, Brookville. Viezzenose. Collected by N. H. Darton and described by F. L. Ransome in Am. Jour. Sci., 4th ser., vol. 8, p. 417. Contains alkali feldspars, nepheline largely altered to secondary minerals, brown amphibole, biotite, cancrinite, plagioclase, muscovite, ægirine-augite, apauite, titanite, fluorite, and a few specks of magnetite. Also secondary analcite, sericite, and natrolite (?). Analysis by Steiger, record No. 1807. P. R. C. 223. 


\begin{tabular}{|c|c|c|c|c|}
\hline & A. & $B$. & C. & D. \\
\hline 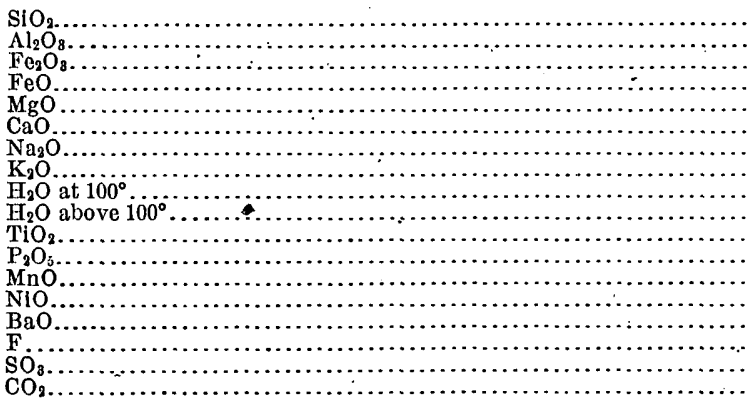 & 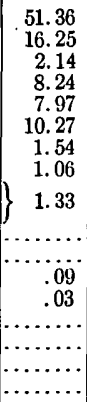 & $\begin{array}{r}53.56 \\
24.43 \\
2.19 \\
1.22 \\
.31 \\
1.24 \\
6.48 \\
.9 .50 \\
.93\end{array}$ & 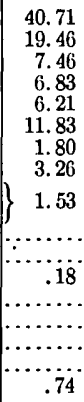 & $\begin{array}{r}54.68 \\
21.63 \\
2.22 \\
2.00 \\
1.25 \\
2.86 \\
7.03 \\
4.58 \\
.27 \\
1.88 \\
.79 \\
.28 \\
\text { trace } \\
\ldots . .0 \\
.05 \\
.22 \\
.07 \\
\text { none }\end{array}$ \\
\hline \multirow[t]{2}{*}{ 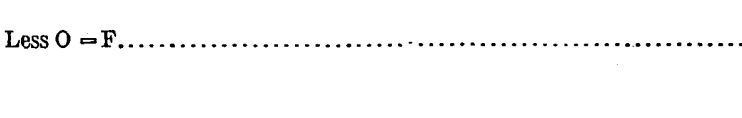 } & 100.28 & $\begin{array}{r}99.96 \\
\cdots\end{array}$ & 100.01 & $\begin{array}{r}99.81 \\
.09\end{array}$ \\
\hline & & & & 99.72 \\
\hline
\end{tabular}

Rocks E to J collected by W. S. Bayley, who describes E, F, G in Folio 167. Analyses by W. T. Schaller, record Nos. 2252, 2255.

E. Pochuck gneiss, Greenwood Lake quadrangle. Contains oligoclase, orthoclase, diopside, hornblende, hypersthene, biotite, magnetite, and quartz.

F. Byram gneiss, 1 mile west of Hibernia. Contains potash feldspars (microperthite), but less hornblendic and pyroxenic minerals than the Pochuck gneiss.

G. Losee gneiss, northeast of Berkshire Valley, Lake Hopatcong quadrangle. Contains plagioclase, quartz, diopside, hypersthene, biotite, apatite, magnetite, zircon, and titanite.

H. Augitic Losee granite, Lake Hopatcong quadrangle.

I. Intermediate gneiss, Oxford tunnel, Hackettstown quadrangle.

J. Micaceous Oxford gneiss, Oxford tunnel.

K. Quartz-orthoclase-augite rock, near Marion Station, Jersey City. Collected by J. Volney Lewis. Analysis by Schaller, record No. 2261.

\begin{tabular}{|c|c|c|c|c|c|c|c|}
\hline & E. & ${ }_{0}^{\mathbf{F}}$ & G. & H. & I. & J. & K. \\
\hline 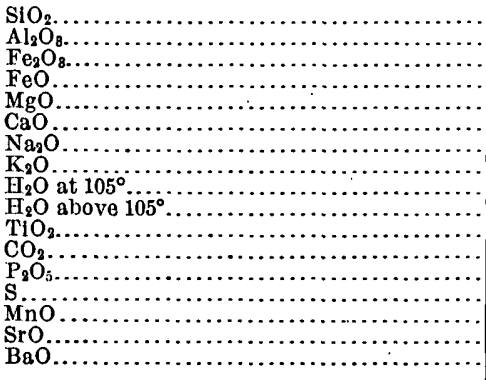 & $\begin{array}{r}43.98 \\
12.01 \\
6.60 \\
12.20 \\
5.46 \\
11.99 \\
2.93 \\
1.10 \\
.29 \\
1.04 \\
2.25 \\
.18 \\
.28 \\
.05 \\
. .05 \\
\cdots \ldots . .\end{array}$ & $\begin{array}{r}77.07 \\
12.61 \\
.71 \\
.73 \\
\text { trace } \\
.87 \\
3.43 \\
4.06 \\
.23 \\
.62 \\
.12 \\
\text { trace } \\
\text { trace? }\end{array}$ & $\begin{array}{r}77.53 \\
13.60 \\
.23 \\
.16 \\
\text { trace } \\
.73 \\
6.65 \\
1.20 \\
.15 \\
.18 \\
.16 \\
\operatorname{trace} \\
.03 \\
\ldots \ldots . . \\
\text { trace? } \\
\ldots \ldots . . . \\
\ldots \ldots\end{array}$ & $\begin{array}{r}52.97 \\
23.27 \\
1.31 \\
3.31 \\
2.20 \\
10.08 \\
4.09 \\
.62 \\
.53 \\
.69 \\
.38 \\
.26 \\
.22 \\
\ldots .19 \\
.19 \\
\ldots \ldots . . \\
\ldots \ldots . .\end{array}$ & $\begin{array}{r}61.54 \\
17.98 \\
3.11 \\
3.21 \\
.32 \\
2.29 \\
5.85 \\
4.77 \\
.09 \\
.78 \\
\ldots .42 \\
.18 \\
\ldots .08 \\
. .0 . \\
\ldots . . . \\
. .2\end{array}$ & $\begin{array}{r}58.75 \\
17.16 \\
5.18 \\
3.94 \\
.91 \\
.62 \\
5.72 \\
5.40 \\
.35 \\
.73 \\
.65 \\
.13 \\
.20 \\
\ldots .10 \\
.10 \\
\ldots \ldots . . . \\
\ldots \ldots . .\end{array}$ & $\begin{array}{r}74.97 \\
10.39 \\
1.56 \\
1.47 \\
1.18 \\
1.83 \\
3.68 \\
2.34 \\
.59 \\
.92 \\
.70 \\
.11 \\
.14 \\
\text { trace? } \\
.07 \\
\text { trace? } \\
.02\end{array}$ \\
\hline & 100.36 & 99.54 & 100.62 & 100.12 & 100.62 & 99.84 & 99.97 \\
\hline
\end{tabular}

\section{PENNSYLVANIA.}

A: Aporhyolite, Monterey, Franklin County. Kallerudose. Described by Florence Bascom in Bull. 150, p. 343. Contains alkali feldspar, quartz, sericite, epidote, titaniferous magnetite, leucoxene, and rarely sphene. Analysis by H. N. Stokes, record No. 1479. P. R. C. 136. 
B. Quartz porphyry, same locality. Alaskose. Analyzed for G. H. Williams, but never described. Analysis by L. G. Eakins, record No. 1350.

C. Baltimore gneiss. Yellowstonose. Contains quartz, orthoclase, microcline, oligoclase, biotite, hornblende, magnetite, and minor accessories. P. R. C. 1701 to 1705 .

D. Mica gneiss. III. 3. 2. 3. Consists chiefly of quartz and muscovite, with feldspars and minor accessories. P. R. C. 1707 to 1710.

E. Hornblende gneiss. Auvergnose: Contains largely green hornblende and colorless anthophyllite, with plagioclase, magnetite, and scanty .secondary quartz. P. R. C. 1710 to 1711 .

F. Hypersthene gabbro. Hessose. Contains quartz, orthoclase, labradorite, diallage, hypersthene, magnetite, apatite, and minor accessories. P. R. C. 1712 to 1714.

Rocks $\mathrm{C}$ to $\mathrm{F}$ are average samples collected by F. Bascom within the Philadelphia quadrangle. Analyses by W. F. Hillebrand, record No. 1872. The mica gneiss is described in the Cecil County volume of the Maryland Geological Survey.

G. Sericite schist, Pine Grove Furnace, South Mountain. Collected by G. W. Stose. Analysis by W. T. Schaller, record No. 2302.

\begin{tabular}{|c|c|c|c|c|c|c|c|}
\hline & A. & B. & C. & D. & E. & F. & G. \\
\hline 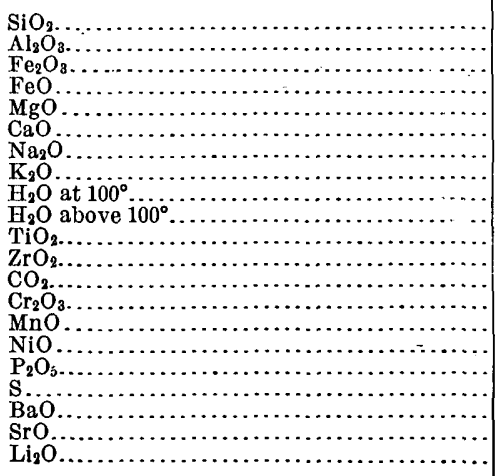 & \begin{tabular}{r}
76.34 \\
11.60 \\
2.41 \\
.30 \\
.06 \\
.55 \\
5.50 \\
2.75 \\
.10 \\
.39 \\
.26 \\
\hdashline$\ldots \ldots$ \\
\hdashline trace \\
\hdashline$\ldots \ldots$ \\
trace \\
\hdashline$\ldots \ldots$ \\
$\ldots \ldots . .$. \\
.09
\end{tabular} & 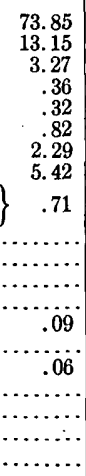 & $\begin{array}{r}70.21 \\
13.95 \\
1.05 \\
3.08 \\
1.26 \\
3.10 \\
3.27 \\
2.69 \\
.19 \\
.48 \\
.52 \\
\text { trace } \\
.11\end{array}$ & $\begin{array}{r}66.13 \\
15.11 \\
2.52 \\
3.19 \\
2.42 \\
1.87 \\
2.71 \\
2.86 \\
.24 \\
1.55 \\
.82 \\
(?) \\
\text { none } \\
.20 \\
\text { trace } \\
.22 \\
.03 \\
\text { trace } \\
\text { trace } \\
\text { none }\end{array}$ & $\begin{array}{r}48.68 \\
14.39 \\
4.00 \\
10.09 \\
6.32 \\
9.23 \\
2.31^{-} \\
.47 \\
.46 \\
2.03 \\
1.69 \\
(?) \\
\text { none } \\
.22 \\
\text { trace } \\
.29 \\
\text { trace } \\
\text { trace } \\
\text { none } \\
\text { trace? }\end{array}$ & $\begin{array}{r}54.03 \\
16.71 \\
1.37 \\
7.70 \\
5.66 \\
8.84 \\
2.99 \\
.67 \\
.14 \\
.53 \\
.84 \\
(?) \\
.40 \\
\text { trace } \\
.13 \\
\text { trace } \\
.13 \\
.09 \\
\text { trace } \\
\text { trace } \\
\text { trace? }\end{array}$ & $\begin{array}{r}73.45 \\
13.77 \\
2.76 \\
\ldots . . .22 \\
1.22 \\
\text { none } \\
.18 \\
4.47 \\
a 3.08 \\
.21 \\
\ldots \ldots . . . \\
\ldots \ldots . .\end{array}$ \\
\hline & 100.35 & 100.34 & 100.30 & 99.87 & 100.18 & 100.23 & 99.17 \\
\hline
\end{tabular}

a Loss on ignition.

\section{MARYLAND AND THE DISTRICT OF COLUMBIA.}

\section{PERIDOTITE AND PYROXENITE.}

Rocks A to I, inclusive, described by G. H. Williams in Am. Geologist, vol. 6, p. 35.

A. Porphyritic lherzolite, Johnny Cake road, Baltimore County. SR. 1 of baltimoriase. Contains olivine, bronzite, and diallage, the olivine partly serpentinized. Analysis by T. M. Chatard, record No. 1094.

B. Websterite, Johnny Cake road.'Baltimorose. Consists entirely of hypersthene and diallage. P. R. C. 110, and 1740 .

C, D. Alterations of B. Maricose. B, C, and D dried at 104. P. R. C. 1741.

E. Smaragdite rock, altered pyroxenite, Dogwood road, Baltimore County. Cecilose.

Änalyses B, C, D, and E by J. E. Whitfield, record Nos. 975, 976. C, D, and E are from the laboratory record and do not appear in the published paper. 


\begin{tabular}{|c|c|c|c|c|c|}
\hline & A. & B. & C. & D. & F. \\
\hline 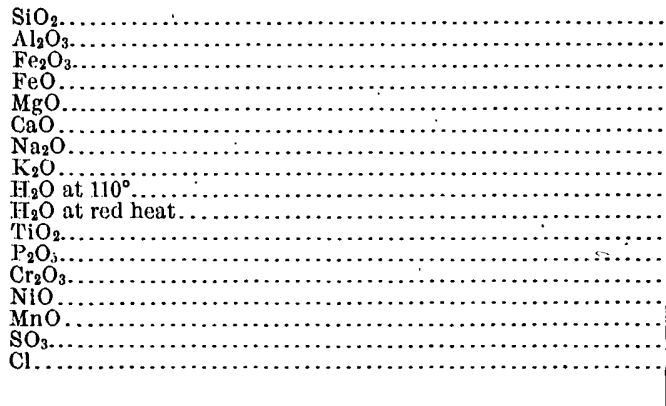 & $\begin{array}{r}43.87 \\
1.64 \\
8.94 \\
2.60 \\
27.32 \\
6.29 \\
.50 \\
1.08 \\
7.64 \\
.12 \\
\operatorname{trace} \\
.44 \\
\operatorname{trace} \\
.19 \\
\cdots \ldots . . \\
\cdots \ldots . . . \\
\end{array}$ & $\begin{array}{r}50.80 \\
3.40 \\
1.39 \\
8.11 \\
22.77 \\
12.31 \\
\text { trace } \\
\\
.52 \\
\text { none } \\
\text { trace } \\
.32 \\
\cdots .17 \\
\text { trace } \\
.24 \\
\end{array}$ & $\begin{array}{r}50.10 \\
2.00 \\
2.38 \\
8.68 \\
26.85 \\
5.06 \\
\text { none } \\
\\
4.16 \\
\text { none } \\
\text { none } \\
.36 \\
\ldots . . . \\
.29 \\
\text { trace } \\
.26 \\
\end{array}$ & $\begin{array}{r}51.94 \\
2.53 \\
2.88 \\
9.38 \\
25.97 \\
3.60 \\
\text { none } \\
\\
2.82 \\
\text { none } \\
\text { none } \\
.60 \\
\ldots \ldots . . \\
\text { trace } \\
.19 \\
.16\end{array}$ & $\begin{array}{r}53.22 \\
3.14 \\
\ldots . . . \\
7.95 \\
20.09 \\
14.44 \\
\text { trace } \\
.98 \\
\text { none } \\
\ldots .23 \\
. .23 \\
.11 \\
\text { trace } \\
.26\end{array}$ \\
\hline
\end{tabular}

F, G. Two samples of websterite, Hebbville, 6 miles west of Baltimore. Websterose. P. R. C. 1742.

H. Bronzite from G. P. R. C. 1743 .

I. Diopside from G. P. R. C. 1744.

The rock consists entirely of bronzite and diopside. Analyses by T. M. Chatard, record Nos. 1094, 1123.

J. Websterite, Oakwood, Cecil County. Cecilose. Composed of hypersthene and diallage. Analysis by W. F. Hillebrand, record No. 1755. Described by A. G. Leonard in Am. Geologist, vol. 28, p. 135.

K. Cortlandtite, Ilchester, Howard County. Cortlandtose. Analysis by W. F. Hillebrand, record No. 1422. Published by Williams in 15th Ann., p. 674. The rock consists of olivine, pyroxene, and large hornblende crystals, the latter considerably altered to talc. P. R. C. 1745 .

\begin{tabular}{|c|c|c|c|c|c|c|}
\hline & $\mathrm{F}$. & G. & $\mathrm{H}$ & I. & J. & K. \\
\hline 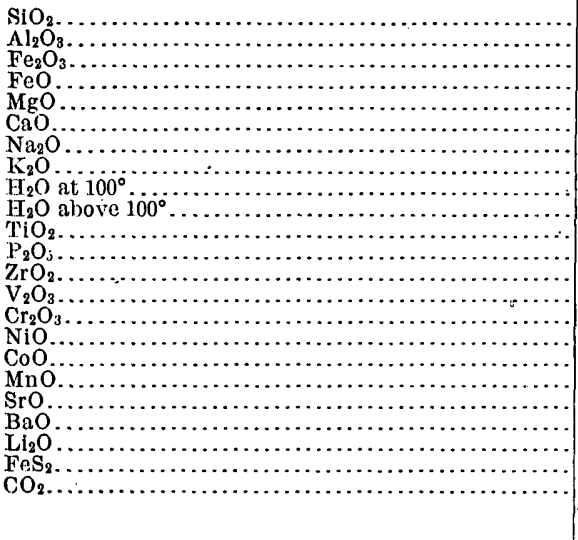 & 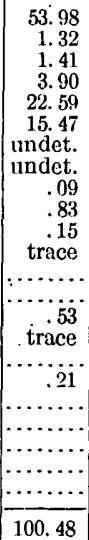 & $\left\{\begin{array}{r}52.55 \\
2.71 \\
1.27 \\
4.90 \\
20.39 \\
16.52 \\
.27 \\
1.09 \\
.14 \\
\text { trace } \\
\ldots \ldots . . \\
\ldots \ldots . . . \\
. \ldots .4 \\
\ldots \ldots . . . \\
. \ldots 24 \\
\ldots \ldots . .\end{array}\right.$ & $\left\{\begin{array}{r}54.53 \\
1.93 \\
1.70 \\
8.92 \\
29.51 \\
2.25 \\
\ldots \ldots . . \\
1.14 \\
\text { undet. } \\
\text { trace } \\
\ldots \ldots . . . \\
\ldots .30 \\
\ldots . . . .\end{array}\right.$ & 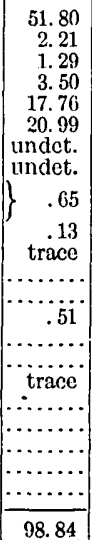 & $\begin{array}{r}53.21 \\
1.94 \\
1.44 \\
7.92 \\
20.78 \\
13.12 \\
.11 \\
.07 \\
.14 \\
.87 \\
.26 \\
\text { trace } \\
\text { trace } \\
.03 \\
.20 \\
.03 \\
.22 \\
\text { none } \\
\text { none } \\
\text { trace } \\
.03 \\
.10\end{array}$ & $\begin{array}{r}39.20 \\
4.60 \\
-3.45 \\
6.15 \\
31.65 \\
3.23 \\
.42 \\
.14 \\
\ldots .50 \\
9.38 \\
.52 \\
\text { trace } \\
\ldots \ldots . . \\
\ldots . .41 \\
.30\end{array}$ \\
\hline
\end{tabular}

\section{GABBRO AND DIORITE.}

Rocks A to E, inclusive, described by G. H. Williams, but not in detail, in 15th Ann., pp. 673,674 . Some of the rocks, with other analyses, are discussed by him in Bull. 28. Analyses by W. F. Hillebrand, record No. 1422.

A. Olivine gabbro, Orange Grove, Baltimore County. IV. $1^{2} \cdot 1^{2} .2$. Containg plagioclase, diallage, hypersthene, fresh olivine, magnetite, and apatite, and sometimes hornblende. P. R. C. 1746. 
B. Hypersthene gabbro, Wetheredville, Baltimore County. Kedabekose. Rich in hypersthene and diallage, with plagioclase, magnetite, and apatite. P. R. C. 1747.

C. Gabbro diorite, Ilchester, Howard County. Hessose. A coarse anorthite-hornblende rock, probably an altered gabbro. P. R. C. 1748 .

D. Biotite diorite, Triadelphia, Montgomery County. Bandose. P. R. C. 1749.

E. Biotite diorite, Georgetown, D. C. Bandose. P. R. C. 1750.

To these may be added an analysis made for Williams by L. G. Eakins, record No. 1350, as follows:

F. Hornblende diorite, Rock Creek tunnel shaft, Washington, D. C. III. 4. 4. 4, 5. Relations to $\mathrm{E}$ not stated.

\begin{tabular}{|c|c|c|c|c|c|c|}
\hline & A. & B. & C. & D. & E. & F. \\
\hline 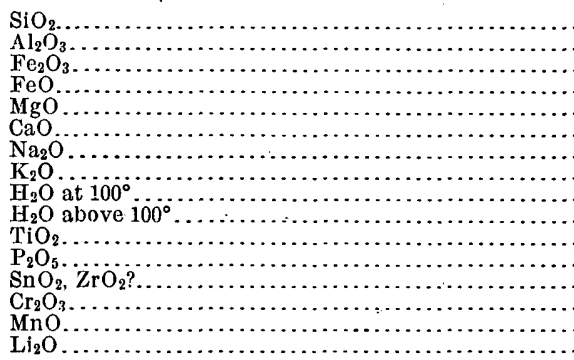 & $\begin{array}{r}48.91 \\
8.81 \\
1.04 \\
9.52 \\
15.19 \\
14.69 \\
.64 \\
.10 \\
.07 \\
.52 \\
.37 \\
\text { trace } \\
.15 \\
.16 \\
\text { trace }\end{array}$ & $\begin{array}{r}44.76 \\
18.82 \\
2.19 \\
4.73 \\
11.32 \\
14.58 \\
.89 \\
.11 \\
.17 \\
2.36 \\
.13 \\
\text { none } \\
.08 \\
.15 \\
\text { trace }\end{array}$ & $\begin{array}{r}43.42 \\
22.37 \\
.81 \\
9.25 \\
5.75 \\
13.34 \\
1.24 \\
1.13 \\
.09 \\
1.54 \\
1.25 \\
.10\end{array}$ & $\begin{array}{r}55.97 \\
15.60 \\
1.21 \\
6.28 \\
6.83 \\
7.31 \\
2.23 \\
1.25 \\
.18 \\
1.85 \\
1.11 \\
.16 \\
.04 \\
.08 \\
\text { trace }\end{array}$ & $\begin{array}{r}56.41 \\
15.19 \\
1.60 \\
6.24 \\
7.18 \\
6.77 \\
2.21 \\
1.34 \\
.08 \\
2.00 \\
.69 \\
.05 \\
.14 \\
.05 \\
.11 \\
\text { trace }\end{array}$ & $\begin{array}{r}56.18 \\
14.76 \\
2.12 \\
6.98 \\
8.11 \\
7.97 \\
1.62 \\
.80 \\
1.37 \\
\ldots \ldots . . \\
.08 \\
\ldots \ldots . . \\
\ldots .17 \\
\ldots \ldots . .\end{array}$ \\
\hline & 100.17 & 100.29 & 100.35 & 100.10 & 100.06 & 100.16 \\
\hline
\end{tabular}

The following rocks, from Cecil County, are described by A. G. Leonard in Am. Geologist, vol. 28, p. 135.

G. Quartz-mica-hornblende diorite, near the foundry on Stone Run. Bandose. Contains hornblende; biotite, quartz, plagioclase, and a little orthoclase, 'with accessory zircon, apatite, titanite, and magnetite, and secondary chlorite and epidote.

H. Like G, near Porter's Bridge on Octoraro Creek. Bandose.

I. Hornblende diorite poor in quartz, three-fourths' mile northwest of Rising Sun. Corsose.

J. Norite, 1 mile west of Oak Grove schoolhouse. Kedabekose. Contains hypersthene, bytownite, a little diallage, apatite, magnetite, and secondary hornblende.

Analyses by Hillebrand, record No. 1755 .

\begin{tabular}{|c|c|c|c|c|}
\hline & G. & H. & I. & J. \\
\hline 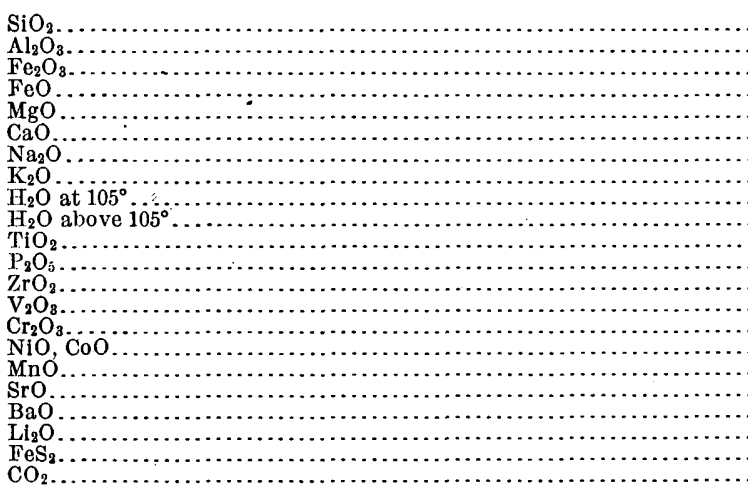 & $\begin{array}{r}58.57 \\
16.10 \\
2.89 \\
6.12 \\
2.33 \\
7.39 \\
2.11 \\
1.01 \\
.21 \\
1.27 \\
1.41 \\
.37 \\
.09 \\
.02 \\
\text { none } \\
\text { none } \\
.18 \\
\text { trace } \\
\text { trace } \\
\text { trace } \\
\text { trace } \\
\text { none }\end{array}$ & $\begin{array}{r}55.16 \\
17.51 \\
2.62 \\
5.83 \\
4.35 \\
8.50 \\
1.83 \\
1.08 \\
.18 \\
2.01 \\
.64 \\
.21 \\
.02 \\
.04 \\
\text { trace } \\
.01 \\
.15 \\
\text { trace } \\
\text { trace } \\
\text { trace } \\
.03 \\
\text { none }\end{array}$ & $\begin{array}{r}44.04 \\
20.01 \\
4.22 \\
8.61 \\
5.01 \\
11.68 \\
1.24 \\
.15 \\
.11 \\
1.90 \\
2.24 \\
.52 \\
.10 \\
.05 \\
\text { none } \\
.01 \\
.28 \\
\text { none } \\
\text { none } \\
\text { trace } \\
.25 \\
\text { none }\end{array}$ & $\begin{array}{r}48.02 \\
20.01 \\
1.13 \\
7.29 \\
10.05 \\
11.42 \\
.51 \\
.05 \\
.10 \\
.57 \\
.23 \\
\text { trace } \\
\text { none } \\
.02 \\
.03 \\
.01 \\
.18 \\
\text { none } \\
\text { none } \\
\text { trace } \\
.11 \\
.25\end{array}$ \\
\hline 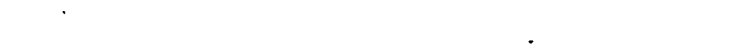 & 100.07 & 100.17 & 100.42 & 99.98 \\
\hline
\end{tabular}




\section{GRANITE AND GNEISS.}

For descriptions see Williams, 15th Ann., p. 657, and Keyes, idem, p. 685. The Rowlandsville granite is described by Grimsley in Jour. Cincinnati Soc. Nat. Hist., vol. 17, p. 78 .

A. White granite, Brookville, Montgomery County. Liparose. (Williams.) P. R. C., 1751.

B. Binary granite, Guilford, Howard County. Toscanose. Contains quartz, orthoclase, a little plagioclase, muscovite, and biotite, with occasional zircon and apatite. (Keyes.)

C. Biotite granite, Woodstock." Toscanose. Light colored, fine grained. Quartz, feldspar, and biotite, with accessory allanite and epidote. (Keyes.)

D. Biotite granite, Rowlandsville, Cecil County. Tonalose. Dark colored. Contains plagioclase, orthoclase, quartz, epidote, biotite, sphene, magnetite, and apatite, with a little secondary muscovite. The percentages of the several minerals are computed by Grimsley from the analysis.

Analyses by W. F. Hillebrand, record Nos. 1220, 1422, 1455. In B and C manganese was present, barium and strontium were not tested for, and the alumina contains possible titanium and phosphoric acid.

\begin{tabular}{|c|c|c|c|c|}
\hline & $\Lambda$. & B. & C. & D. . \\
\hline 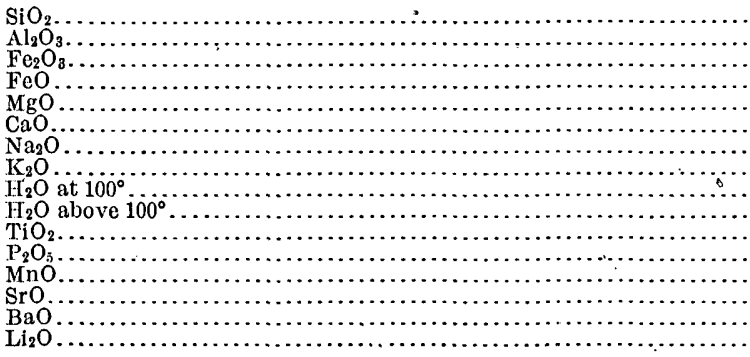 & \begin{tabular}{r}
74.87 \\
14.27 \\
trace \\
.51 \\
.16 \\
.48 \\
3.06 \\
5.36 \\
.26 \\
.66 \\
.05 \\
.21 \\
trace \\
$\ldots \ldots .$. \\
\hdashline$\ldots$ \\
trace
\end{tabular} & $\begin{array}{r}72.57 \\
15.11 \\
.59 \\
1.02 \\
.30 \\
1.65 \\
3.92 \\
4.33 \\
.47 \\
\text { undet. } \\
\text { undet. } \\
\text { undet. } \\
\text { undet. } \\
\text { undet. } \\
\text { trace }\end{array}$ & $\begin{array}{r}71.79 \\
15.00 \\
.77 \\
1.12 \\
.51 \\
2.50 \\
3.09 \\
4.75 \\
.64 \\
\text { undet. } \\
\text { undet. } \\
\text { undet. } \\
\text { undet. } \\
\text { undet. } \\
\text { trace }\end{array}$ & $\begin{array}{r}66.68 \\
14.93 \\
1.58 \\
3.32 \\
2.19 \\
4.89 \\
2.65 \\
2.05 \\
.16 \\
1.09 \\
.50 \\
.10 \\
.10 \\
\text { trace } \\
.08 \\
\text { trace }\end{array}$ \\
\hline & 99.89 & 99.96 & 100.17 & 100.32 \\
\hline
\end{tabular}

E. Biotite granite, Dorseys Run, Howard County. Yellowstonose. Typical, dark colored. Quartz, feldspar, and biotite, with accessory allanite and epidote. (Keyes.) P. R. C., 1752.

F. Same locality, light-colored dikes. Toscanose.

G. Inclusions in E. SR. 2 of tonalase. Derived from gneiss.

H. The typical gneiss of the Dorseys Run area. Perhaps of sedimentary origin.

Descriptions by Keyes. Analyses by Hillebrand. The remarks appertaining to $\mathrm{B}$ and $\mathrm{C}$ apply here also. Record No. 1220.

\begin{tabular}{|c|c|c|c|c|}
\hline & E. & F. & G. & H. \\
\hline 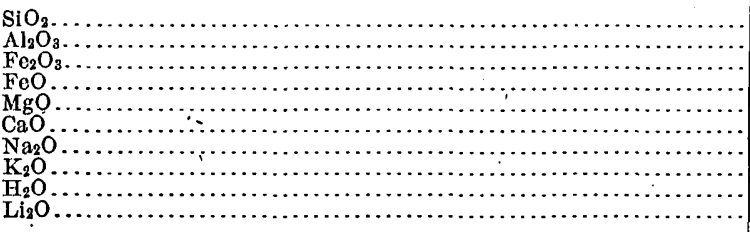 & $\begin{array}{r}62.91 \\
19.13 \\
.98 \\
3.20 \\
1.69 \\
4.28 \\
3.94 \\
3.38 \\
.63 \\
\text { trace }\end{array}$ & $\begin{array}{r}70.45 \\
15.98 \\
.75 \\
1.84 \\
.77 \\
2.60 \\
3.83 \\
3.59 \\
.45 \\
\text { trace }\end{array}$ & $\begin{array}{r}57.33 \\
15.31 \\
3.39 \\
8.19 \\
4.36 \\
3.95 \\
1.22 \\
4.57 \\
1.80 \\
\text { trace }\end{array}$ & $\begin{array}{r}48.92 \\
16.57 \\
4.21 \\
9.18 \\
5.98 \\
9.69 \\
2.47 \\
1.56 \\
1.68 \\
\text { trace }\end{array}$ \\
\hline & 100.14 & 100.26 & 100.12 & 100.26 \\
\hline
\end{tabular}


I. Biotite granite, Sykesville. Tehamose. Contains quartz, feldspar, and biotite, with accessory magnetite, zircon, and apatite.

J. Inclusion in I, derived from limestone. Yellowish central portion. Consists of epidote and quartz, with a little chlorite.

$\mathrm{K}$. Same inclusion, fine-grained dark outer zone. Quartz, garnet, and epidote, with a little magnetite. Feldspar and muscovite in some portions. Descriptions by Keyes. Analyses by Hillebrand, record No."1220, with the same limitations as in the Guilford, Woodstock, and Dorseys Run granites.

\begin{tabular}{|c|c|c|c|}
\hline & I. & J. & K. \\
\hline 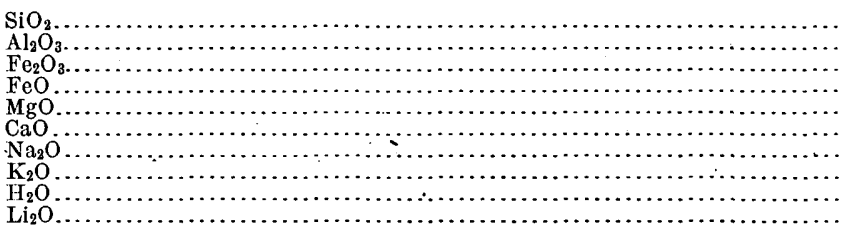 & $\begin{array}{r}71.45 \\
14.36 \\
2.07 \\
2.78 \\
1.17 \\
1.58 \\
1.95 \\
3.28 \\
1.30 \\
\text { trace }\end{array}$ & $\begin{array}{r}67.02 \\
13.77 \\
4.64 \\
1.02 \\
.65 \\
11.09 \\
.66 \\
.09 \\
1.16 \\
\text { trace }\end{array}$ & $\begin{array}{r}47.35 \\
29.76 \\
2.94 \\
3.15 \\
1.60 \\
2.20 \\
2.84 \\
6.83 \\
3.15 \\
\text { trace }\end{array}$ \\
\hline$\cdot$ & 99.94 & 100.10 & 99.82 \\
\hline
\end{tabular}

L. Typical gneiss of Washington, D. C., from quarry of Potomac Stone Company, 1 mile below Chain Bridge. A "basic" granite.

M. Fine-grained, fissile chloritic gneiss, from northwest of Cabin John Bridge. Also a "basic" granite. P. R. C. 1753.

N. Fine-grained, hard gneiss, from the second lock at the Great Falls of the Potomac. Alsbachose. Probably of sedimentary origin.

Described by Williams. Analyses by Hillebrand, record No. 1459.

\begin{tabular}{|c|c|c|c|}
\hline - & L. & M. & N. \\
\hline 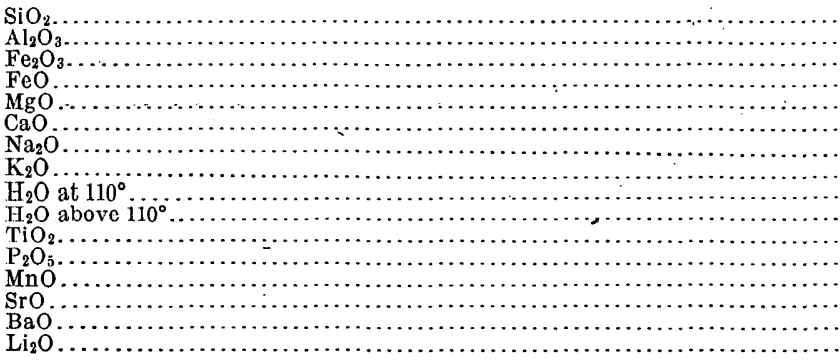 & $\begin{array}{r}67.22 \\
15.34 \\
2.78 \\
3.41 \\
1.65 \\
1.36 \\
2.00 \\
3.26 \\
.29 \\
1.68 \\
.84 \\
.14 \\
.13 \\
\operatorname{trace} \\
.04 \\
\operatorname{trace}\end{array}$ & $\begin{array}{r}63.43 \\
16.69 \\
3.36 \\
3.87 \\
2.33 \\
.80 \\
2.38 \\
3.22 \\
.23 \\
2.67 \\
.91 \\
.11 \\
.09 \\
\text { trace } \\
.03 \\
\text { trace }\end{array}$ & $\begin{array}{r}78.28 \\
9.96 \\
1.85 \\
1.78 \\
.95 \\
1.68 \\
2.73 \\
1.35 \\
.12 \\
.83 \\
.70 \\
.11 \\
.08 \\
\text { trace } \\
.02 \\
\text { trace }\end{array}$ \\
\hline$\cdot$ & 100.14 & 100.12 & 100.44 \\
\hline
\end{tabular}

\section{MISCELLANEOUS ROCKS.}

A. Typical diabase, Rocky Ridge. Auvergnose. Analyzed for J. S. Diller, but not described. Analysis by E. A. Schneider, record No. 1370.

B. Ottrelite-phyllite rock, Liberty, Frederick County. Analyzed for G. H. Williams, but not described. P. R. C. 1754 .

C. Chloritoid separated from B. Analyses B and C by L. G. Eakins, record No. 1349.

D. Quartz schist, Shoemaker quarry, near Stevenson Station, Green Spring Valley. Described by Bayley in Bull. 150, p. 302. Contains quartz, muscovite, occasional tourmalines, microcline, zircon, and iron stains. Analysis by Schneider, No. 1370. P. R. C. 119.

E. Mica separated from D. Analysis by Schneider, No. 1377. 


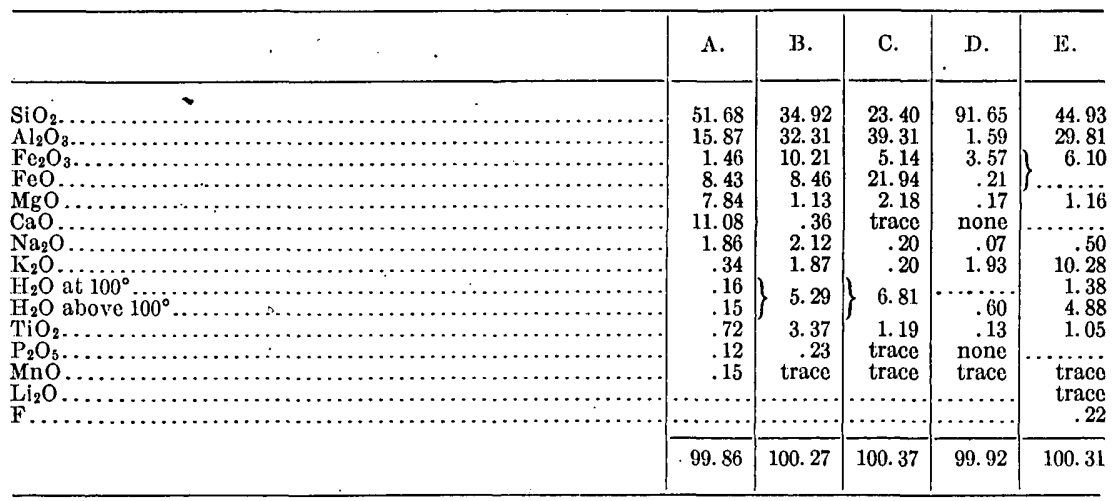

F. Sericite schist, Ladiesburg. Described by Bayley in Bull. 150, p. 317. Contains quartz, sericite, chlorite, a mineral thought to be kaolin, zircon, iron oxide, and rutile. Analysis by George Steiger, record No. 1600. P. R. C. 126.

G. Another sample of F. Analysis by Schneider, No. 1370.

H. Metarhyolite, south of Port Deposit. Dike. Vulcanose. Described by F. Bascom, in Maryland Geological Survey, Cecil County volume, p. 136. Contains quartz, orthoclase, plagioclase, biotite, and hornblende, with accessory magnetite, apatite, titanite, garnet, pyrrhotite, and muscovite, and secondary chlorite and epidote. Analysis by W. F. Hillebrand, record No. 1928, P. R. C. 1706.

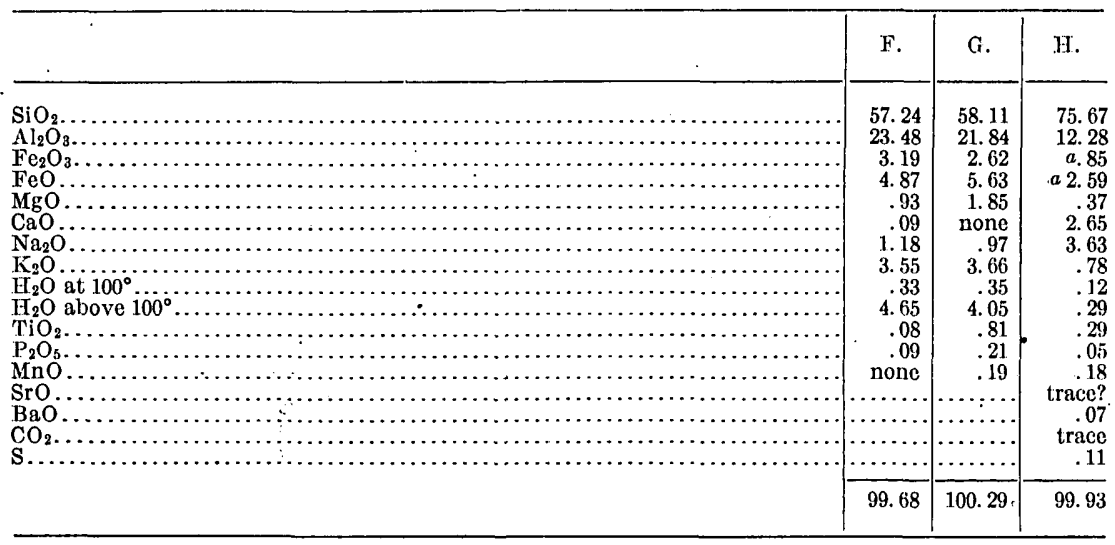

$a$ Uncertain because sulphides are present.

\section{VIRGINIA.}

A. Andesite, $3 \frac{1}{2}$ miles east of Front Royal. SR. 4 of orendase. Described by Keith in 14th Ann., p. 305. Intermediate between diabase and quartz porphyry. Contains plagioclase, quartz, magnetite, ilmenite, and a little epidote. Analysis by George Steiger, record No. 1450.

B. Porphyritic felsite or felsophyre, forks of Straight Creek, 3 miles east-northeast of Monterey. Toscanose. Described by Darton and Keith in Am. Jour. Sci., 4th ser., vol. 6, p. 305 . Contains phenocrysts of biotite, orthoclase, and plagioclase, the mica and feldspar being about equal in amount. In the groundmass are feldspar, quartz, magnetite, or ilmenite, and a little secondary chlorite with less muscovite. No glass. Analysis by Hillebrand; record No. 1665. P. R. C. 342. 


\begin{tabular}{|c|c|c|}
\hline - & A. & B. \\
\hline 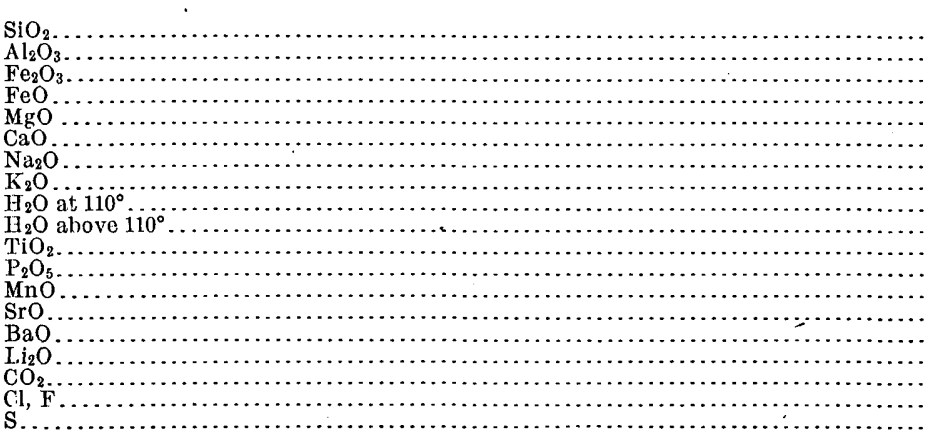 & \begin{tabular}{r}
51.08 \\
11.37 \\
11.17 \\
5.64 \\
3.96 \\
5.20 \\
.5 .54 \\
1.50 \\
1.31 \\
.19 \\
2.67 \\
.39 \\
.22 \\
$\ldots \ldots .$. \\
$\ldots \ldots . .$. \\
\hdashline$\ldots . .$. \\
$\ldots \ldots .$. \\
$\ldots \ldots .$.
\end{tabular} & $\begin{array}{r}69.56 \\
15.52 \\
1.67 \\
1.19 \\
.41 \\
1.20 \\
4.46 \\
4.68 \\
.34 \\
.67 \\
.31 \\
.08 \\
.07 \\
\text { trace } \\
.10 \\
\text { trace } \\
\text { none } \\
\text { (?) } \\
\text { trace }\end{array}$ \\
\hline & 100.24 & 100.26 \\
\hline
\end{tabular}

\section{NORTH CAROLINA.}

Rocks A to F collected by Arthur Keith, who furnishes the petrographic data. Analyses B, C, D, and E by W. F. Hillebrand, record No. 1707. Analyses A and F by H. N. Stokes, record No. 1710.

A. Porphyritic rhyolite, 2 miles west of Barmers Elk, Watauga County. Adamellose. Contains orthoclase and plagioclase, with less quartz, epidote, chlorite, and pyrite.

B. Quartz porphyry, $2 \frac{1}{2}$ miles northwest of Blowing Rock, Watauga County. Magdeburgose. Contains quartz and orthoclase, with subordinate sericite, chlorite, and biotite.

C. Diorite, east end of Hump Mountain, Mitchell County. Auvergnose. Contains plagioclase, orthoclase, and hornblende, with less quartz, biotite, magnetite, and garnet.

D. Garnetiferous diabase, $1 \frac{3}{4}$ miles southeast of Cranberry. III. 4.4.4, 5. Contains plagioclase and hornblende, with less garnet, biotite, and magnetite.

\begin{tabular}{|c|c|c|c|c|}
\hline - & A. & B. & C. & D. \\
\hline 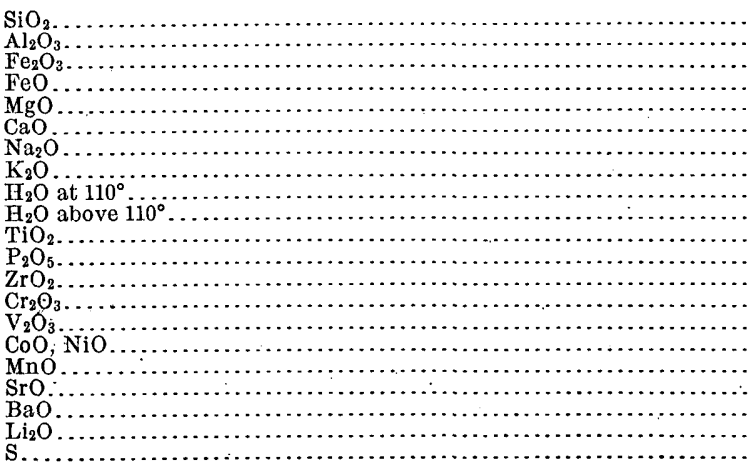 & $\begin{array}{r}62.35 \\
.13 .24 \\
3.52 \\
6.33 \\
.85 \\
3.34 \\
2.79 \\
3.95 \\
.11 \\
1.21 \\
1.18 \\
.57 \\
\ldots \ldots+. \\
\text { none } \\
\ldots \ldots . . \\
\text { none } \\
.08 \\
\text { trace } \\
\text { trace } \\
\ldots . . . .\end{array}$ & $\begin{array}{r}79.75 \\
10.47 \\
.64 \\
.92 \\
.13 \\
.15 \\
1.36 \\
6.01 \\
.08 \\
.60 \\
.15 \\
\text { trace } \\
.05 \\
\ldots \ldots . \\
\ldots \ldots . . \\
\text { none } \\
\text { trace } \\
\text { trace } \\
.06 \\
\text { trace } \\
\text { none }\end{array}$ & $\begin{array}{r}46.91 \\
15.85 \\
2.86 \\
9.95 \\
7.01 \\
9.62 \\
2.65 \\
.69 \\
.24 \\
1.62 \\
2.03 \\
.26 \\
\text { none } \\
.01 \\
.03 \\
.03 \\
.22 \\
\text { trace? } \\
\text { trace? } \\
\text { trace } \\
\text { none }\end{array}$ & $\begin{array}{r}52.11 \\
13.70 \\
1.22 \\
9.86 \\
8.08 \\
12.16 \\
1.31 \\
.16 \\
.06 \\
.53 \\
.32 \\
.05 \\
\text { none } \\
\ldots \ldots \\
\ldots . . . \\
.03 \\
.20 \\
\text { none } \\
\text { none } \\
\text { trace } \\
\text { trace }\end{array}$ \\
\hline & 99.68 & 100. 37 & 99.98 & 99.79 \\
\hline
\end{tabular}


E. Epidote-chlorite schist, one-fourth mile northeast of Montezuma, Mitchell County. Contains epidote and feldspar, with less chlorite, hornblende, and magnetite.

F. Metamorphosed amygdaloid, 3 miles southeast of Boone, Watauga County. Contains orthoclase and plagioclase, with less sericite, chlorite, and magnetite.

G. Pyroxenite, var. websterite, from Webster. Websterose. Described by Williams, Am. Geologist, vol. 6, p. 35. Consists of diopside and bronzite. Analysis by E. A. Schneider, record No. 1096. Material dried at $105^{\circ}$. P: R. C. 388.

H. Spherulitic rhyolite, Sam Christian gold mine, Montgomery County. Alaskose. Described by Diller, Am. Jour. Sci., 4th ser., vol. 7, p. 337. The rock of the supposed fossil Palzotrochis. Contains feldspar and quartz, with a little biotite and sericite. Analysis by Hillebrand, record No. 1796.

\begin{tabular}{|c|c|c|c|c|}
\hline & $\mathrm{E}$. & F. & G. & H. \\
\hline 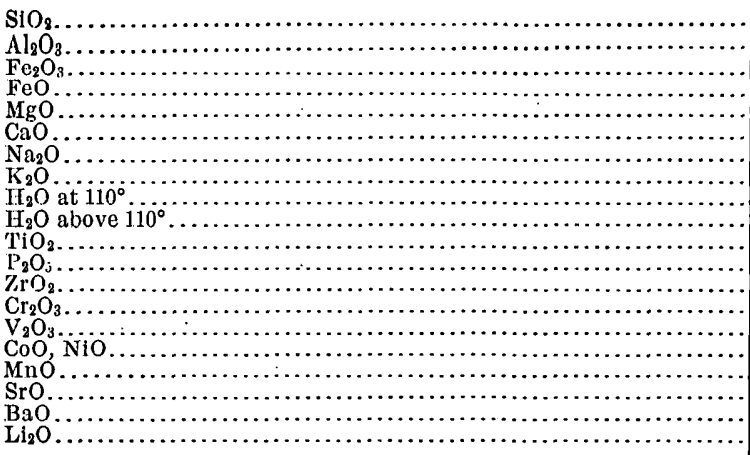 & $\begin{array}{r}47.85 \\
16.51 \\
4.16 \\
7.43 \\
6.24 \\
7.00 \\
3.20 \\
.82 \\
.21 \\
4.00 \\
2.28 \\
.35 \\
.03 \\
.01 \\
.05 \\
.03 \\
.24 \\
\text { trace? } \\
\text { trace? } \\
\text { trace }\end{array}$ & $\begin{array}{r}43.62 \\
17.30 \\
14.13 \\
6.83 \\
2.34 \\
1.63 \\
3.45 \\
3.03 \\
.30 \\
2.93 \\
2.75 \\
1.34 \\
\ldots \ldots . . \\
\text { none } \\
\ldots \ldots . . . \\
\text { none } \\
\text { trace } \\
\text { trace } \\
.09 \\
\text { trace }\end{array}$ & $\begin{array}{r}55.14 \\
.66 \\
3.48 \\
4.73 \\
26.66 \\
8.39 \\
.30 \\
\ldots \ldots . . \\
.38 \\
\text { trace } \\
1.23 \\
\ldots .25 \\
\ldots \ldots . . \\
.11 \\
.03 \\
\ldots \ldots \ldots . \\
\ldots \ldots . . . \\
\ldots \ldots . .\end{array}$ & 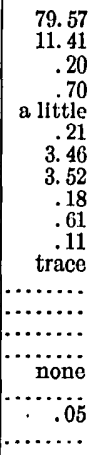 \\
\hline & 100.41 & 99.74 & 100.36 & 100.02 \\
\hline
\end{tabular}

The following rocks from Corundum Hill were collected, analyzed, and described by T. M. Chatard, Bull. 42 , p. 45.

I. Altered gneiss. Made up of micaceous scales, with grains of quartz and some earthy matter. Record No. 238.

J. Dunite. Dunose. Olivine rock containing a little chromite. Record No. 256. K, L. Yellow, claylike alterations of dunite. Record Nos. 254, 255.

Other analyses of altered rocks are given in the paper, and also analyses of associated minerals.

\begin{tabular}{|c|c|c|c|c|}
\hline & I. & J. & $\mathbf{K}$. & L. \\
\hline 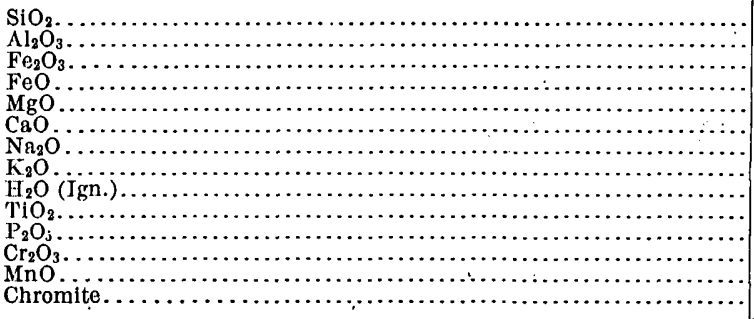 & $\begin{array}{r}64.27 \\
16.75 \\
6.08 \\
.89 \\
1.74 \\
.25 \\
.89 \\
3.09 \\
4.97 \\
1.32 \\
.05 \\
.07 \\
\ldots\end{array}$ & $\begin{array}{r}40.11 \\
.88 \\
1.20 \\
6.09 \\
48.58 \\
\ldots \ldots . . \\
\ldots \ldots . . . \\
\cdots 2.74 \\
\ldots \ldots . . \\
\ldots .18 \\
\ldots \ldots \\
.56\end{array}$ & 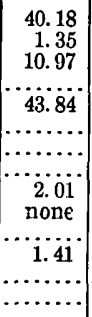 & 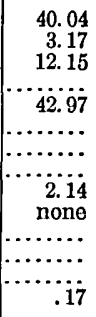 \\
\hline$\cdot$ & 100.37 & 100.34 & 99.76 & 100.64 \\
\hline
\end{tabular}




\section{GEORGIA.}

Rocks collected by A. H. Brooks, who supplies the petrographic data. Analyses by H. N. Stokes. Record No. 1727.

A. Meta-quartz diorite, 2 miles southwest of Sweden, Gordon County. . Gordonose. Contains plagioclase, near labradorite, green hornblende, sometimes diallage, much vitreous quartz, and accessory magnetite, ilmenite, and orthoclase. Also secondary epidote, zoisite, uralite, chlorite, garnet, calcite, and leucoxene: P. R. C. 1530.

B. Augite-microcline granite, 1 mile east of Rowland, Bartow County. Toscanose. Contains microcline, some plagioclase, abundant pyroxene partly altered into chiefly uralite and chlorite, some biotite with frequent inclusions of rutile, much blue vitreous quartz, apatite, zircon, and magnetite. P. R. C. 1531.

C. Quartz gabbro, 2 miles southeast of Walleska, Cherokee County. Harzose. Closely related to B. Contains essentially plagioclase, near labradorite, and augite. Accessory magnetite, ilmenite, apatite, and zoisite. Orthoclase is sparingly present. Quartz occurs in vitreous masses. P. R. C. 1532.

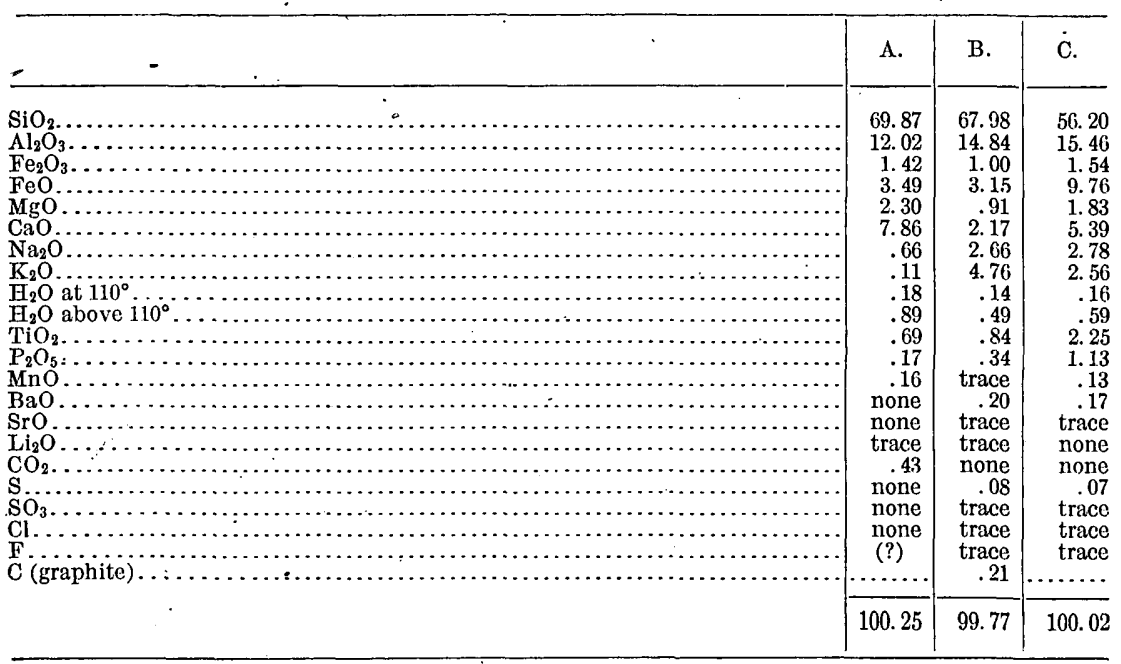

KENTUCKY.

1. ELLIOTT COUNTY DIKE.

Described by Diller in Bull. 38. Also in Am. Jour. Sci., 3d ser., vol. 32, p. 125.

A peridotite, var. kimberlite, consisting largely of olivine, sometimes altered to serpentine. Pyrope, ilmenite, a few scales of biotite, a little enstatite, and a trace of apatite are present as primary minerals. Secondary minerals are serpentine, dolomite, magnetite, and octahedrite.

$\Lambda$ granitic rock occurs with the peridotite. It consists chiefly of feldspar, orthoclase, and plagioclase, with a considerable amount of quartz and ilmenite, and traces of hornblende, sphene, and apatite.

Analyses by T. M. Chatard, record Nos. 272, 273, 282, 305, 351, 352, 353, 354, and 358.
A. Granite. Monzonose.
B. Peridotite (kimberlite). P. R. C. 799.
C. Olivine from peridotite.
D. Garnet from peridotite.
E. Ilmenite from peridotite. 


\begin{tabular}{|c|c|c|c|c|c|}
\hline . & $\Lambda$. & B. & C. & D. & E. \\
\hline 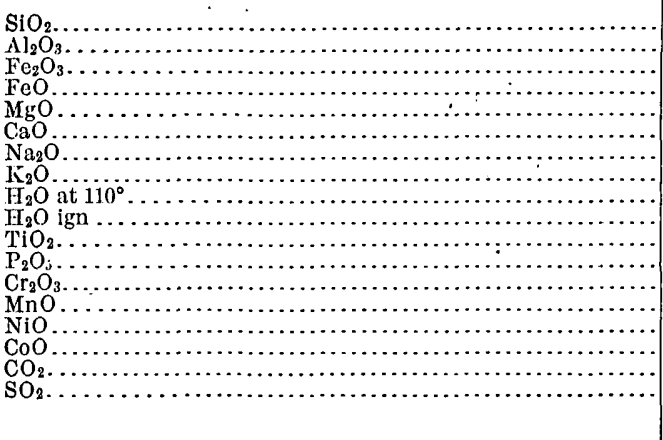 & 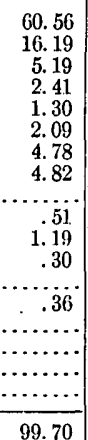 & $\begin{array}{r}29.81 \\
2.01 \\
5.16 \\
4.35 \\
32.41 \\
7.69 \\
.11 \\
.20 \\
8.92 \\
2.20 \\
.35 \\
.43 \\
.23 \\
.05 \\
6.66 \\
.28 \\
100.86\end{array}$ & $\begin{array}{r}40.05 \\
.30 \\
2.36 \\
7.14 \\
46.68 \\
1.16 \\
.08 \\
.21 \\
.14 \\
.66 \\
.07 \\
.04 \\
.24 \\
.20 \\
\ldots \ldots \\
\text { trace } \\
(?) \\
\ldots \ldots . . \\
\end{array}$ & $\begin{array}{r}41.32 \\
21.21 \\
4.21 \\
7.93 \\
19.32 \\
4.94 \\
.07 \\
\ldots \ldots \ldots \\
.17 \\
.16 \\
. \text { none } \\
.91 \\
.34 \\
\ldots \ldots \ldots \\
\ldots \ldots \ldots . \\
\ldots \ldots \ldots \\
\ldots \ldots \ldots . \\
100.58\end{array}$ & $\left\{\begin{array}{r}0.76 \\
2.84 \\
9.13 \\
: 7.81 \\
\ldots 68 \\
.23 \\
.10 \\
\ldots \ldots \\
.20 \\
49.32 \\
\operatorname{trace} \\
.74 \\
.20 \\
\ldots \ldots . \\
\ldots \ldots \ldots \\
\ldots \ldots \ldots \\
\ldots \ldots . . \\
100.10\end{array}\right.$ \\
\hline
\end{tabular}

The following analyses are of sedimentary rocks adjoining the dike:

F. Calcareous sandstone.

G. Fine-grained fissile sandstone.

H. Indurated shale.

I. Fragment of shale included in the peridotite.

\begin{tabular}{|c|c|c|c|c|}
\hline & F. & G. & H. & I. \\
\hline 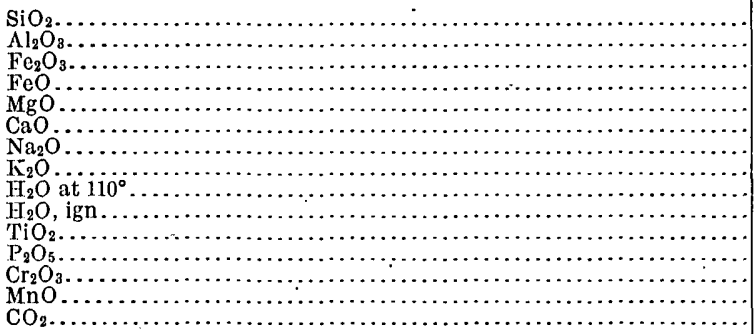 & $\begin{array}{r}60.78 \\
10.54 \\
3.27 \\
1.59 \\
10.15 \\
1.41 \\
2.36 \\
.85 \\
2.32 \\
.03 \\
.09 \\
. .10 \\
6.29\end{array}$ & $\begin{array}{r}60.25 \\
20.18 \\
1.53 \\
3.42 \\
3.52 \\
.51 \\
.39 \\
3.17 \\
1.94 \\
5.17 \\
.23 \\
.10 \\
\ldots . . . \\
.10 \\
\ldots \ldots . . .\end{array}$ & \begin{tabular}{r}
41.32 \\
20.71 \\
2.59 \\
5.46 \\
1.91 \\
9.91 \\
7.19 \\
.88 \\
\hdashline .78 \\
8.78 \\
.48 \\
.08 \\
$\operatorname{trac\theta }$ \\
.17 \\
.55
\end{tabular} & $\begin{array}{r}35.53 \\
18.23 \\
2.46 \\
4.81 \\
2.01 \\
21.17 \\
2.53 \\
1.08 \\
1.40 \\
9.00 \\
.95 \\
.08 \\
\ldots .13 \\
.13 \\
.88\end{array}$ \\
\hline & 99.78 & 100.51 & 100.03 & 100.26 \\
\hline
\end{tabular}

H was dried five hours at $110^{\circ}$ previous to analysis.

\section{CRITTENDEN COUNTY DIKE.}

A mica peridotite, described by Diller in Am. Jour. Sci., 3d ser., vol. 44, p. 286. Contains biotite, serpentine, and perofskite, with less apatite, muscovite, magnetite, calcite, chlorite, and some other secondary products. P. R. C. 800.

A. The rock described by Diller. ' $S R$.' 1 of casseliase. Analysis by W. F. Hillebrand, record No. 1241.

B. An analysis of probably the same rock, from a shaft 40 feet deep at Marion. Collected by J. R. Procter, analyzed by L. G. Eakins, record No. 965.

\begin{tabular}{|c|c|c|}
\hline . & A. & $B$. \\
\hline 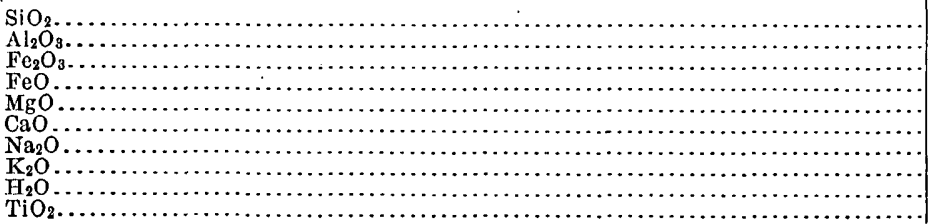 & $\begin{array}{r}33.84 \\
5.88 \\
7.04 \\
5.16 \\
22.96 \\
9.46 \\
.33 \\
2.04 \\
7.50 \\
3.78\end{array}$ & $\begin{array}{r}34.50 \\
14.37 \\
2.85 \\
4.46 \\
21.81 \\
11.43 \\
.51 \\
1.50 \\
7.14\end{array}$ \\
\hline
\end{tabular}




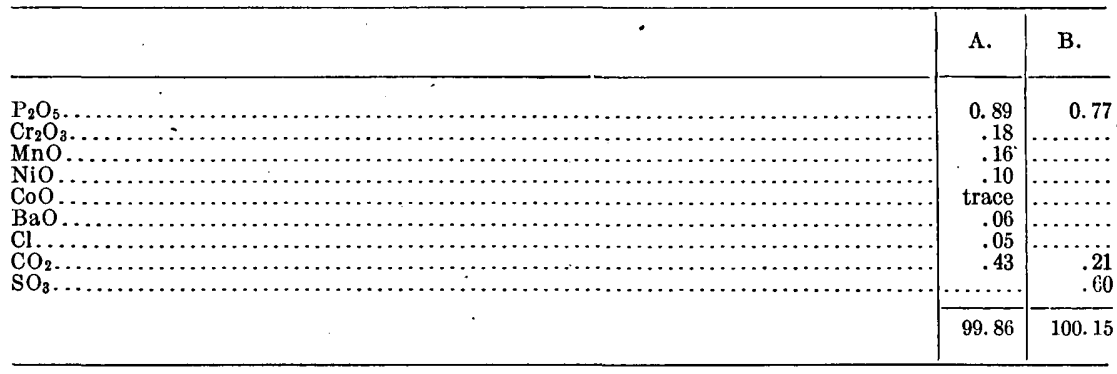

\section{TENNESSEE.}

Gabbro, 2 miles south of Limestone Cove, Unicoi County. Camptonose. Collected by Arthur Keith, who furnishes the petrographic data. Analysis by W. F. Hillebrand, record No. 1707. Contains hypersthene, plagioclase, and magnetite.

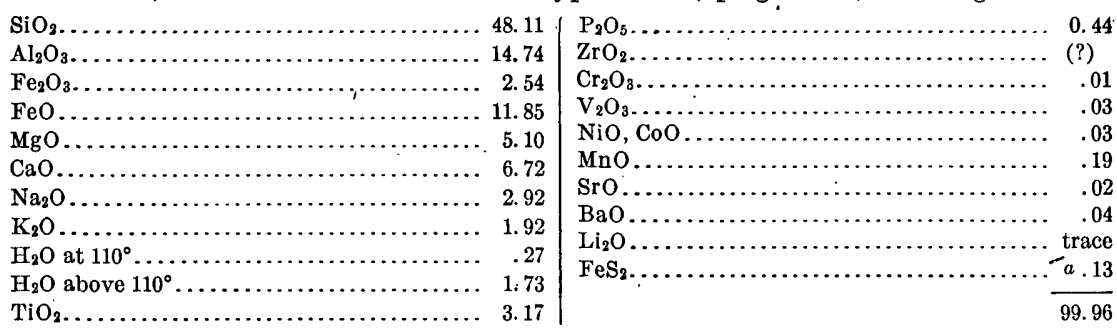

\section{MISSOURI.}

Rocks A to D, granite and porphyry, 6 miles east of Ironton. Described by Haworth in Missouri Geol. Survey, vol. 8, Annual Report, pp. 140, 180, and 213. Analyses by W. H. Melville, record No. 1206.

A, B. Granite. A, P. R. C. 1027 ; B, P. R. C. 1028 .

C, D. Porphyry. C, P. R. C. 1029 ; D, P. R. C. 1030.

Rocks composed principally of orthoclase and quartz, with some microcline, plagioclase, and biotite, and minor accessory minerals. A is toscanose; $\mathrm{B}, \mathrm{C}$, and $\mathrm{D}$ are liparose.

E. Devonite, Mount Devon. Hessose. Collected and described by A. Johannsen. Contains phenocrysts of a plagioclase rich in potassium. The groundmass consists of feldspar and augite, with titaniferous magnetite and chlorite. Analysis by W. F. Hillebrand, record No. 2350.

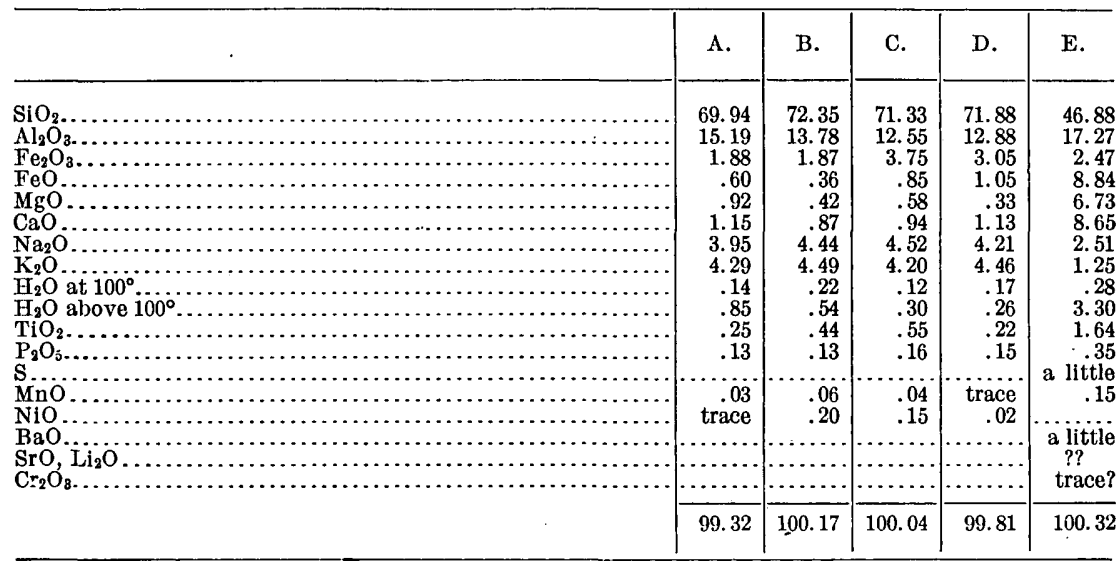

a Equivalent to $0.07 \mathrm{~S}$. Assumed to be pyrite, no pyrrhotite being present. 


\section{ARKANSAS.}

Ouachitite, dike near Maple Spring, 4 miles southwest of Hot Springs. SR. 2 of etindase. Described by Kemp, in Ann. Rept. Geol. Survey Arkansas, 1890, vol. 2, p. 399. A dike rock of the monchiquite group. Contains abundant and conspicuous augite and biotite, magnetite, and minor accessory minerals in a groundmass considered by Kemp to be glass. Calcite and other secondary products are also present. According to Pirsson, Jour. Geology, vol. 4, p. 679, the so-called "glass" in the monchiquites is really analcite. Analysis by L. G. Eakins, record No. 1023. P. R. C. 395.

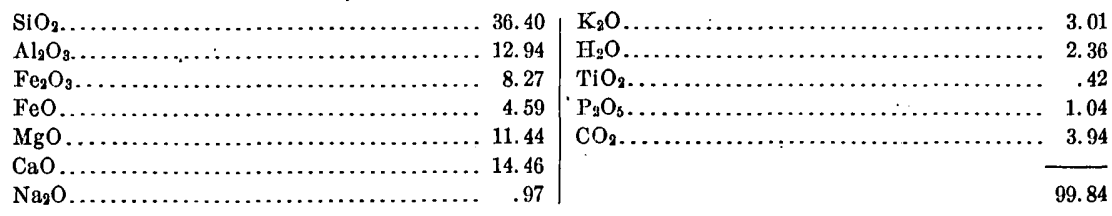

\section{OKLAHOMA.}

Granite, west of Mount Sheridan, Wichita Mountains, collected by.J. P. Iddings. Analysis by G. Steiger, record No. 2335.

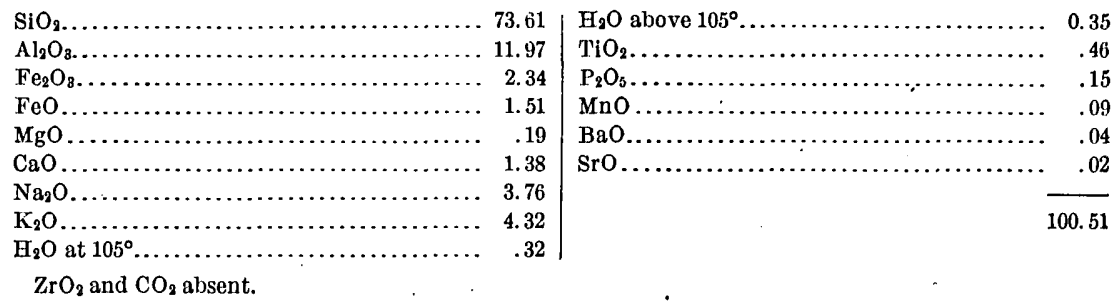

\section{TEXAS.}

A. Quartz pantellerite, Vieja Mountains, San Carlos, Presidio County. Liparose. Description furnished by E. C. E. Lord. Contains anorthoclase, augite, and grains of quartz in a groundmass of æegirine-augite, a brown hornblende which is probably barkevikite, orthoclase, and quartz. Magnetite and apatite are present as accessory minerals. Analysis by George Steiger, record No. 1581.

The following rocks, analyses $\mathrm{B}$ to $\mathrm{M}$, from Uvalde County were collected by $\mathrm{T}$. Wayland Vaughan. Petrographic data furnished by Whitman Cross. Analyses by W. F. Hillebrand, record No. 1681.

B. Plagioclase basalt, Pinto Mountain, Brackett quadrangle. Limburgose. Contains olivine, augite, plagioclase (labradorite), biotite, a very little alkali feldspar (?), magnetite, and apatite. Sp. gr., 3.118, 20 . P. R. C. 1067.

C. Basanite, Mount Inge, Uvalde quadrangle. Lujavrose. Cōntains sanidine, nephelite, hornblende, augite, ægirine-augite, olivine, magnetite, apatite, and a trace of pyrite. Sp. gr., $2.770,20^{\circ}$. P. R. C. 1069.

D. Rock of basaltic habit, allied to C, 1 mile northeast of Big Mountain, Uvalde quadrangle. Essexose. Contains alkali feldspar, augite, magnetite, and variable amounts of olivine, nephelite, ægirite, biotite, and zeolitic minerals. Sp. gr., 2.742, $23^{\circ}$. P. R. C. 1068. 


\begin{tabular}{|c|c|c|c|c|}
\hline i & A. & B. & C. & D. \\
\hline 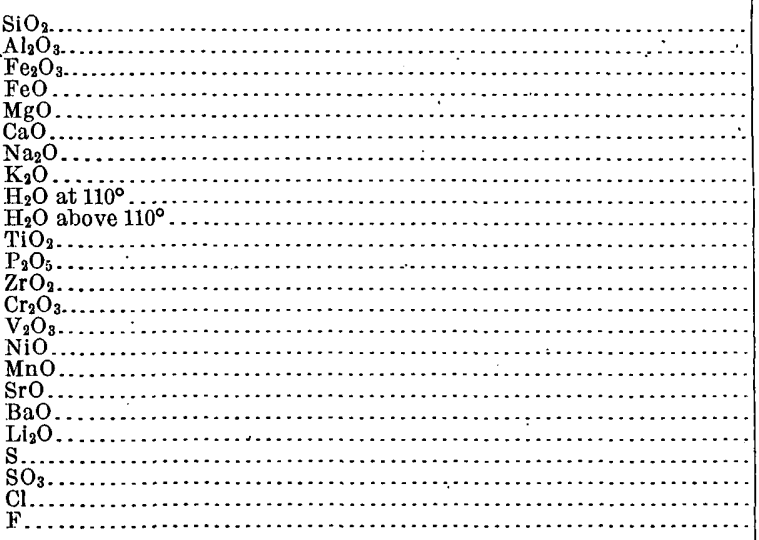 & $\begin{array}{r}68.71 \\
13.45 \\
5.31 \\
.75 \\
.19 \\
.96 \\
4.63 \\
5.51 \\
.13 \\
.36 \\
.21 \\
.04 \\
\ldots \ldots . \\
\ldots \ldots . . \\
\ldots \ldots . . . \\
\text { none } \\
\text { none } \\
\text { none } \\
\ldots \ldots . . . \\
.05 \\
\ldots \ldots . . \\
\ldots \ldots . . .\end{array}$ & $\begin{array}{r}45.11 \\
12.44 \\
2.67 \\
9.36 \\
11.56 \\
10.61 \\
3.05 \\
1.01 \\
.16 \\
.78 \\
2.34 \\
.51 \\
(?) \\
\ldots \ldots \\
.04 \\
.04 \\
.22 \\
\text { trace } \\
\text { trace } \\
\text { none } \\
.01 \\
\ldots \ldots .11 \\
\text { undet. }\end{array}$ & $\begin{array}{r}48.13 \\
18.44 \\
3.41 \\
4.30 \\
3.06 \\
5.89 \\
8.00 \\
3.80 \\
.18 \\
1.59 \\
1.74 \\
.49 \\
.05 \\
\text { none } \\
\ldots . . \\
.02 \\
.19 \\
.10 \\
.10 \\
\text { trace } \\
.09 \\
\ldots . . . \\
.29 \\
.06\end{array}$ & $\begin{array}{r}48.23 \\
17.43 \\
2.77 \\
5.92 \\
2.99 \\
6.38 \\
6.87 \\
2.78 \\
.54 \\
2.84 \\
2.00 \\
.69 \\
.04 \\
\text { none } \\
.04 \\
\text { trace } \\
.18 \\
.08 \\
.08 \\
\text { trace } \\
.08\end{array}$ \\
\hline Less $0 \ldots \ldots \ldots \ldots \ldots \ldots$ & $\begin{array}{c}100.44 \\
\ldots . . .\end{array}$ & $\begin{array}{r}100.02 \\
.02\end{array}$ & $\begin{array}{r}99.93 \\
.09\end{array}$ & \\
\hline & & 100.00 & 99.84 & \\
\hline
\end{tabular}

$a$ Including $\mathrm{Cr}_{2} \mathrm{O}_{3}$.

E. Phonolite, hill between Black and Big mountains, Uvalde quadrangle. Laurdalose. Contains sanidine, nephelite, and ægirite, and very little brown hornblende, augite, and magnetite. Sp. gr., 2.559, 19.5 . P. R. C. 1070.

$\mathrm{F}$. The portion of $\mathrm{E}$ soluble in 1:40 dilute nitric acid.

G. Nepheline basalt, Tom Nunns Hill, Uvalde quadrangle. Uvaldose. Contains olivine, augite, nephelite, magnetite, and apatite. Sp. gr., 3.148, 19². P. R. C. 1065.

$\mathrm{H}$. The portion of $\mathrm{G}$ soluble in 1:40 dilute nitric acid.

\begin{tabular}{|c|c|c|c|c|}
\hline & E. & F. & G. & H. \\
\hline 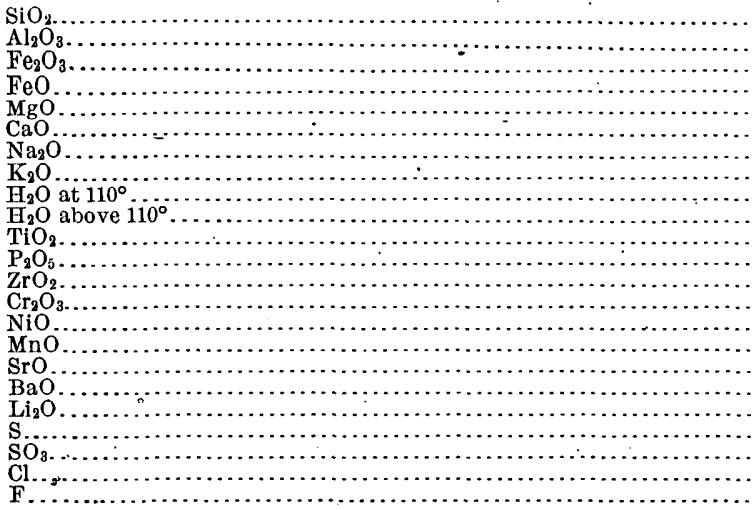 & $\begin{array}{r}54.42 \\
20.76 \\
2.64 \\
1.33 \\
.22 \\
1.34 \\
10.41 \\
4.89 \\
.22 \\
2.50 \\
.40 \\
.11 \\
.15 \\
\text { none } \\
\text { none } \\
.15 \\
\text { trace } \\
.04 \\
\text { trace } \\
.01 \\
\ldots . .23 \\
\text { none }\end{array}$ & 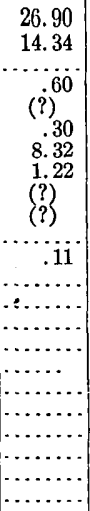 & $\begin{array}{r}40.32 \\
a 9.46 \\
4.75 \\
7.48 \\
18.12 \\
10.55 \\
2.62 \\
1.10 \\
.57 \\
1.25 \\
2.66 \\
.68 \\
\text { none } \\
\cdots . .0 \\
.06 \\
.25 \\
.03 \\
.06 \\
\text { trace } \\
.01 \\
.03 \\
.05 \\
.04\end{array}$ & 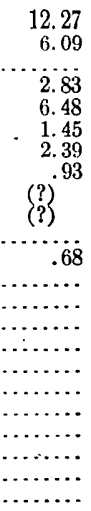 \\
\hline \multirow[t]{2}{*}{ Less 0 . } & $\begin{array}{r}99.82 \\
.05\end{array}$ & 51.79 & $\begin{array}{r}100.09 \\
.03\end{array}$ & 33.12 \\
\hline & 99.77 & & 100.06 & \\
\hline
\end{tabular}


I. Nepheline basalt, Black Mountain, Uvalde quadrangle: Uvaldose. Contains olivine, augite, nephelite, magnetite, and apatite. Sp. gr., $3.200,21.5^{\circ}$. P. R. C. 1066.

$\mathrm{J}$. The portion of I soluble in 1:40 dilute nitric acid.

K. Augite from I. Violet in color, very pure.

L. Nepheline-melilite basalt, from about 3 miles southwest of Uvalde. Casselose. Contains nephelite, melilite, olivine, augite, magnetite, ảnd apatite. Sp. gr., 3.150, 20.5 . P. R. C. 1064.

M. The portion of $\mathrm{L}$ soluble in 1:40 dilute nitric acid.

\begin{tabular}{|c|c|c|c|c|c|}
\hline & I. & J. & K. & I. & M. \\
\hline 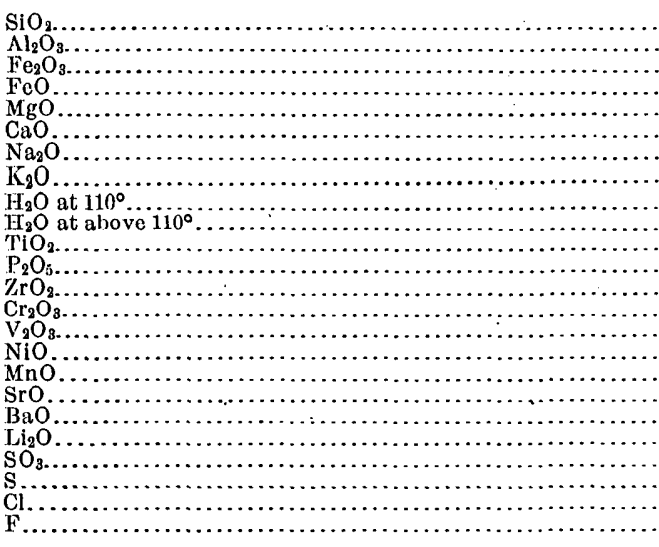 & $\begin{array}{r}39.92 \\
8.60 \\
4.40 \\
8.00 \\
20.17 \\
10.68 \\
1.91 \\
1.03 \\
.43 \\
1.45 \\
2.70 \\
.51 \\
\text { none } \\
.14 \\
.04 \\
.06 \\
.24 \\
.04 \\
.06 \\
\text { trace } \\
\ldots \ldots \\
\text { trace } \\
\text { trace } \\
.07\end{array}$ & 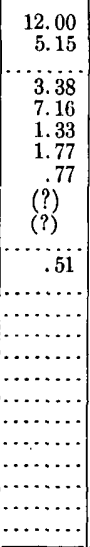 & \begin{tabular}{r}
45.23 \\
7.73 \\
2.95 \\
4.07. \\
12.25 \\
23.37 \\
.47 \\
.12 \\
.37 \\
4.28 \\
none \\
$\ldots \ldots . .$. \\
\hdashline$\ldots . .$. \\
\hdashline. .65 \\
.05 \\
none \\
none \\
trace
\end{tabular} & $\begin{array}{r}37.96 \\
10.14 \\
3.69 \\
7.59 \\
14.69 \\
16.28 \\
2.18 \\
.69 \\
.39 \\
1.82 \\
2.93 \\
1.13 \\
\text { none } \\
.08 \\
.05 \\
.04 \\
.22 \\
.05 \\
.06 \\
\text { trace } \\
.03 \\
.04 \\
\text { trace } \\
.07\end{array}$ & 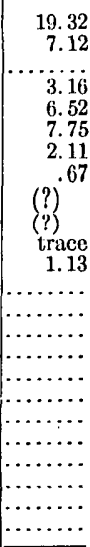 \\
\hline \multirow[t]{2}{*}{ Less $0 .}$. & $\begin{array}{r}100.45 \\
.03\end{array}$ & 32.07 & 100.96 & $\begin{array}{r}100.13 \\
.03\end{array}$ & $\begin{array}{r}47.78 \\
\ldots . . .\end{array}$ \\
\hline & 100.42 & $\cdots \cdots \cdot$ & $\cdots \cdots$ & 100.10 & \\
\hline
\end{tabular}

Rocks $\mathrm{N}$ to $\mathrm{U}$ collected by $\mathrm{R}$. T. Hill in the trans-Pecos region. Descriptions supplied by Whitman Cross. Analyses by W. F. Hillebrand, record No. 1901.

N. Rhyolite, summit of Chisos Mountain, Big Bend of the Rio Grande. Liparose near alaskose. Pink porphyry. Rich in alkali feldspars and quartz, with very little riebeckite and barkevikite (?). .Sp. gr., 2.602, $15.5^{\circ}$.

O. Rhyolite, near Shafter, Shafter quadrangle. Liparose near alaskose. Phenocrysts of sanidine and quartz. Groundmass of alkali fieldspars, quartz, riebeckite (?), and ægirite. Spherulitic bands traverse the rock. Sp. gr., 2.61.7, 1.5.5०:

P. Rhyolite, north summit of Chisos Mountain. Liparose. Consists chiefly of alkali feldspars and quartz, with riebeckite and a little magnetite. Sp. gr., 2.611, $15.5^{\circ}$.

Q. Rhyolite, west of Paisano Mountain, Alpine quadrangle. Liparose. Contains alkali feldspars, quartz, arfvedsonite, and ægirite. Sp. gr., $2.635,15.5^{\circ}$. 


\begin{tabular}{|c|c|c|c|c|}
\hline & N. & O. & $\mathrm{P}$. & Q. \\
\hline 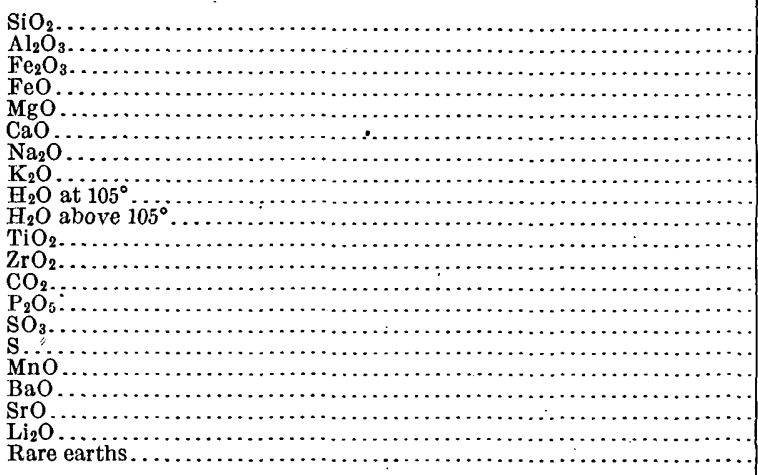 & $\begin{array}{r}76.30 \\
11.53 \\
1.83 \\
.76 \\
.03 \\
.16 \\
4.01 \\
4.70 \\
.19 \\
.34 \\
.16 \\
.11 \\
\text { trace } \\
\text { none } \\
\ldots . . . . \\
\text { trace } \\
\text { trace } \\
\text { none } \\
\text { none } \\
\text { trace } \\
.02\end{array}$ & $\begin{array}{r}75.12 \\
10.94 \\
2.88 \\
. .86 \\
.07 \\
.20 \\
4.46 \\
4.54 \\
.18 \\
.19 \\
.20 \\
.13 \\
.04 \\
\text { none } \\
\text { none } \\
.05 \\
.08 \\
\text { none } \\
\text { none } \\
\text { trace } \\
.04\end{array}$ & $\begin{array}{r}74.85 \\
12.83 \\
1.40 \\
.37 \\
.04 \\
.48 \\
4.24 \\
5.12 \\
.24 \\
.30 \\
.15 \\
.09 \\
\text { trace? } \\
\text { trace } \\
\ldots . . . \\
\text { trace } \\
\text { trace } \\
\text { none } \\
\text { none } \\
\text { trace } \\
.07\end{array}$ & $\begin{array}{r}72.86 \\
11.74 \\
2.71 \\
1.66 \\
.06 \\
.24 \\
4.63 \\
4.92 \\
.51 \\
.40 \\
.20 \\
.28 \\
\text { trace } \\
\text { trace } \\
\ldots . . . \\
\text { trace } \\
.07 \\
\text { none } \\
\text { none } \\
\text { trace } \\
.05\end{array}$ \\
\hline & 100.14 & 99.98 & 100.18 & 100.33 \\
\hline
\end{tabular}

$\mathrm{Cl}$ and $\mathrm{F}$ not looked for.

R. Grorudite, about 2 miles north of the summit of Chisos Mountain. Grorudosepantellerose. Contains alkali feldspars and subordinate quartz, with riebeckite and ægirite in irregular interstitial patches. Sp. gr., 2.648, $15.5^{\circ}$.

S. Pulaskite, Santiago Mountain. Nordmarkose. Consists of alkali feldspars, with subordinate ægirite, ægirite-augite, riebeckite, magnetite, and rare lovenite. Sp. gr., $2.581,25.5^{\circ}$.

T. Syenite porphyry, Iron Mountain, near Marathon. Nordmarkose. Contains many oligoclase-albite phenocrysts in a groundmass of alkali feldspar, with very little quartz. Titanite, apatite, and magnetite are present in small amounts. Sp. gr., $2.577,15.5^{\circ}$.

U. Essexite, Big Hill Canýon, Rio Grande. Essexose. Contains predominant orthoclase, soda-rich plagioclase, and nephelite, with considerable augite, olivine, and magnetite, and small amounts of biotite and apatite. Sp. gr., 2.686, 25.5 ${ }^{\circ}$.

V. Syenite porphyry, Hueco Tanks, El Paso County. Phlegrose near nordmarkose. Collected and described by G. B. Richardson. Contains orthoclase, albite, oligoclase, biotite, augite, quartz, ilmenite, magnetite, and apatite. Analysis by G. Steiger, record No. 2162 .

\begin{tabular}{|c|c|c|c|c|c|}
\hline . & $\mathrm{R}$. & S. & 'T. & U. & V. \\
\hline 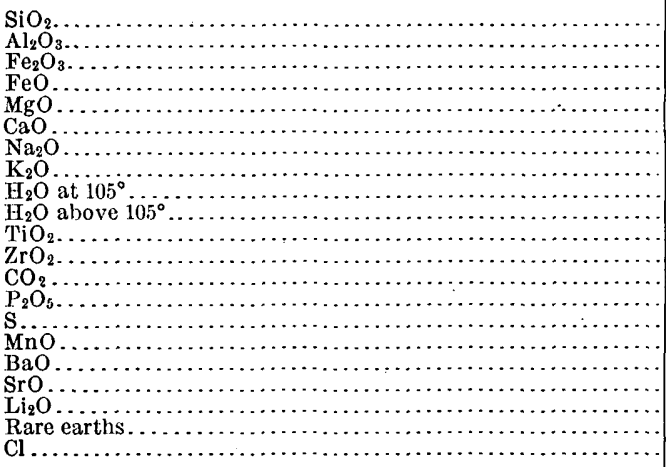 & $\begin{array}{r}68.25 \\
13.60 \\
3.66 \\
1.43 \\
.02 \\
.54 \\
6.52 \\
4.73 \\
.16 \\
.32 \\
.26 \\
.25 \\
\text { trace } \\
.02 \\
\text { trace } \\
.04 \\
\text { trace } \\
\text { trace } \\
\text { trace } \\
.08 \\
\cdots \ldots\end{array}$ & $\begin{array}{r}62.46 \\
17.10 \\
2.49 \\
2.65 \\
.28 \\
1.27 \\
6.84 \\
5.44 \\
.15 \\
.49 \\
.38 \\
.10 \\
\text { trace? } \\
.11 \\
\text { none } \\
.18 \\
\text { none } \\
\text { none } \\
\text { trace } \\
.03\end{array}$ & $\begin{array}{r}65.47 \\
17.93 \\
2.15 \\
.43 \\
.06 \\
1.10 \\
6.21 \\
5.21 \\
.19 \\
.41 \\
.29 \\
.07 \\
\text { trace? } \\
.19 \\
\text { trace } \\
\text { trace } \\
.16 \\
\text { trace } \\
\text { none } \\
.05 \\
\ldots . . .\end{array}$ & $\begin{array}{r}53.34 \\
17.92 \\
2.27 \\
5.51 \\
1.40 \\
4.19 \\
6.41 \\
4.32 \\
.58 \\
2.08 \\
1.60 \\
\text { trace } \\
\text { trace? } \\
.44 \\
.03 \\
.17 \\
.09 \\
.06 \\
\text { trace } \\
.05 \\
\ldots . . .\end{array}$ & 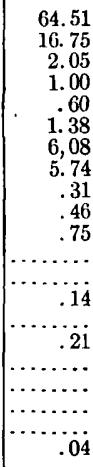 \\
\hline & 99.88 & 99.97 & 99.92 & 100.46 & 100.02 \\
\hline
\end{tabular}




\section{LAKE SUPERIOR REGION.}

\section{MARQUETTE REGION, MICHIGAN.}

Rocks mostly described by Van Hise and Bayley. When not otherwise specified the descriptions have been published in Mon. XXVIII, and partly in 15th Ann., p. 485 .

A. Peridotite, near Opin Lake, E. $\frac{1}{4}$ sec. 27 , T. 48 N., R. 27 W. Marquettose. Contains diallage, olivine, magnetite, and plagioclase. The diallage is partly chloritized, and the olivine is partly serpentinized. Analysis by W. F. Hillebrand, record No. 1452 . P. R. C. 996.

-B. Altered greenstone, Marquette district. Camptonose. Analysis by George Steiger, record No. 1586, hitherto unpublished. P. R. C. 988.

C. Grünerite-magnetite schist, sec. 11, T. 47 N., R. 27 W. Mainly impure grünerite, with magnetite and quartz. Analysis by W. H. Melville, record No. 1403. P. R. C. 984.

D. Like C, from sec. 18, T. 47 N., R. 28 W. Analysis by Melville, record No. 1403. P. R. C. 980.

E. Like C and D, from sec. 20 , T. 46 N., R. 30 W. Analysis by H. N. Stokes, record No. 1546. C, D, and $\mathrm{E}$ are similar schists, and alike in mineralogical composition. P. R. C. 981 .

\begin{tabular}{|c|c|c|c|c|c|}
\hline & A. & B. & C. & D. & E. \\
\hline 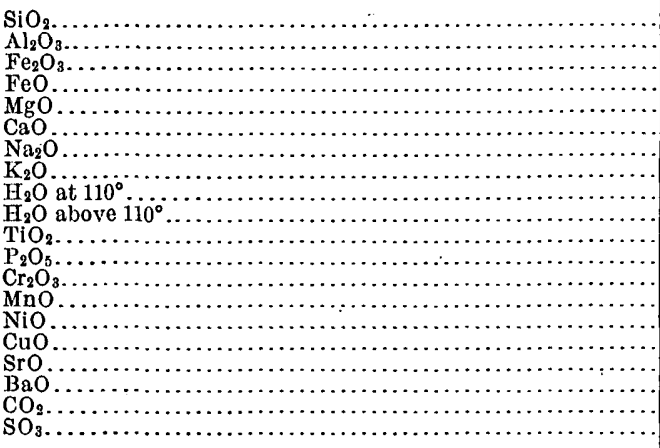 & $\begin{array}{r}39.37 \\
4.47 \\
4.96 \\
9.13 \\
26.53 \\
3.70 \\
.50 \\
.26 \\
.87 \\
7.08 \\
.66 \\
.17 \\
.68 \\
.12 \\
.21 \\
. . \cdots \\
\text { trace } \\
\text { trace } \\
1.23 \\
\ldots \ldots\end{array}$ & $\begin{array}{r}48.85 \\
15.83 \\
2.50 \\
10.79 \\
5.82 \\
6.20 \\
2.79 \\
1.31 \\
.27 \\
3.77 \\
1.28 \\
.22 \\
\ldots . . \\
.11\end{array}$ & $\begin{array}{r}46.94 \\
.66 \\
4.51 \\
33.72 \\
6.64 \\
3.22 \\
.16 \\
\ldots \ldots . . \\
.67 \\
\text { none } \\
.07 \\
\ldots \ldots .31 \\
. \ldots \ldots \\
\ldots \ldots . . \\
\ldots \ldots . . \\
\ldots \ldots .79 \\
\ldots \ldots . .\end{array}$ & 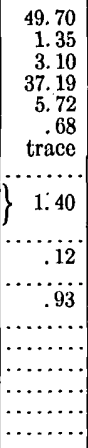 & $\begin{array}{r}46.25 \\
.92 \\
30.62 \\
16.92 \\
2.13 \\
1.69 \\
\text { none } \\
\text { none } \\
\ldots . . . \\
.42 \\
\text { none } \\
.07 \\
\ldots \ldots .01 \\
\text { none } \\
\text { trace } \\
\text { none } \\
\text { none } \\
\text { none } \\
\ldots . . .\end{array}$ \\
\hline$\cdot$ & 99.94 & 99.89 & 99.69 & 100.19 & 100.03 \\
\hline
\end{tabular}

F. Green schist, sec. 34, T. 48 N., R. 27 W., near center of section. Contains plagioclase, chlorite, and quartz. Analysis by George Steiger, record No. 1447. P. R. C. 986.

G. Sericite schist associated with the preceding. Mainly sericite and quartz. Analysis by Steiger, No. 1447. P. R. C. 985 .

H. Dark-gray, foliated schist, southeast corner of sec. 30, T. 47 N., R. 30 W. Contains quartz, plagioclase, orthoclase, and biotite. Analysis by Steiger, No. 1525. P. R. C. 991.

I. Schistose gneiss, NW. $\frac{1}{4}$ sec. 35 , T. 47 N., R. 26 W. Contains quartz, kaolin, sericite, plagioclase, chlorite, magnetite, and apatite. Analysis by Steiger, No. 1525.

J. Novaculite from Marquette. Described by Williams in Bull. 62. Contains quartz and sericite. Analysis by W. F. Hillebranc, record No. 759. 


\begin{tabular}{|c|c|c|c|c|c|}
\hline & F. & G. & H. & I. & J. \\
\hline 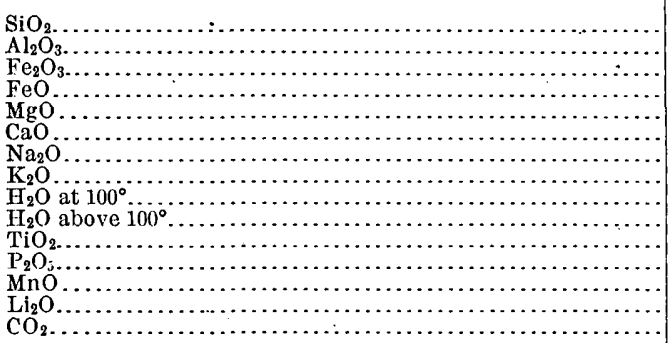 & $\begin{array}{r}61.35 \\
16.45 \\
.94 \\
4.20 \\
3.12 \\
3.46 \\
5.24 \\
1.05 \\
.10 \\
-2.51 \\
.26 \\
.18 \\
\ldots \ldots\end{array}$ & $\begin{array}{r}70.76 \\
14.83 \\
1.46 \\
3.09 \\
1.99 \\
.36 \\
.47 \\
3.50 \\
.09 \\
2.70 \\
.33 \\
.26 \\
\ldots . . . \\
\ldots . . .\end{array}$ & $\begin{array}{r}63.50 \\
17.89 \\
1.12 \\
5.38 \\
1.22 \\
2.34 \\
2.55 \\
2.43 \\
.22 \\
2.04 \\
.62 \\
.19 \\
\text { trace }\end{array}$ & $\begin{array}{r}82.38 \\
11.32 \\
.97 \\
.26 \\
.17 \\
.22 \\
.59 \\
1.04 \\
.18 \\
2.33 \\
.14 \\
.09 \\
\text { none. } \\
\cdots \\
\ldots \ldots . .\end{array}$ & $\begin{array}{r}76.99 \\
13.92 \\
.45 \\
.77 \\
1.12 \\
.32 \\
.56 \\
3.65 \\
2.35 \\
\text { trace } \\
\text { trace } \\
\text { trace } \\
\ldots . . . .\end{array}$ \\
\hline & 100.84 & $\begin{array}{c}99.84 \\
.\end{array}$ & 99.50 & .99 .69 & 100.1 .3 \\
\hline
\end{tabular}

\section{CRYSTAL FALLS DISTRICT, MICHIGAN.}

Rocks described by J. Morgan Clements and H. L. Smyth, in Mon. XXXVI. A, B, C, and D by Smyth, p. 274. Analyses by H. N. Stokes, record No. 1721.

A: Granite, sec. 2, T. 41 N., R. 30 W. Magdeburgose. Contains quartz, orthoclase, microcline, plagioclase, biotite, muscovite, magnetite, hematite, titanite, and occasional apatite.

B. Gneiss, sec. 35, T. 42 N., R. 29 W. Same minerals as in A.

C. Mica schist, sec. 35, T. 42 N., R. 29 W. Contains biotite, quartz, some microcline, and magnetite.

D. Amphibolite, sec. 32 , T. 42 N., R. 28 W. Contains hornblende, plagioclase, biotite, and quartz, with a little rutile and magnetite:

\begin{tabular}{|c|c|c|c|c|}
\hline , & A. & B. & C. & D. \\
\hline 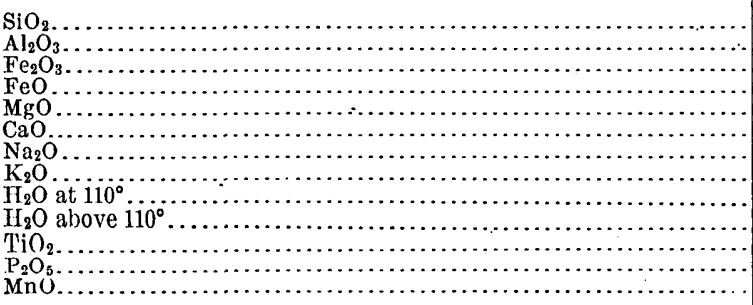 & $\begin{array}{r}76.10 \\
12.95 \\
.65 \\
.09 \\
.14 \\
.12 \\
2.36 \\
6.50 \\
.17 \\
.48 \\
.07 \\
.02 \\
\text { trace }\end{array}$ & $\begin{array}{r}74.37 \\
13.34 \\
.92 \\
.21 \\
.27 \\
.50 \\
2.50 \\
6.70 \\
.12 \\
.44 \\
.07 \\
.01 \\
\text { trace }\end{array}$ & $\begin{array}{r}64.71 \\
16.43 \\
1.83 \\
3.84 \\
2.97 \\
.08 \\
.11 \\
5.63 \\
.31 \\
2.79 \\
.72 \\
.02 \\
\text { trace }\end{array}$ & $\begin{array}{r}50.36 \\
13.26 \\
6.30 \\
9.34 \\
5.55 \\
7.85 \\
2.11 \\
1.14 \\
.16 \\
1.55 \\
1.77 \\
.20 \\
\text { trace }\end{array}$ \\
\hline & 99.65 & 99.45 & 99.44 & 99.59 \\
\hline
\end{tabular}

$\mathrm{Ba}, \mathrm{Sr}, \mathrm{Cl}, \mathrm{F}, \mathrm{S}, \mathrm{SO}_{3}$ not looked for.

The following rocks, with one exception, are described by Clements in his portion of Mon. XXXVI:

E. Mica diorite, sec. 28, T. 42 N., R. 32 W., southeast of Crystal Falls. Shoshonose. Contains plagioclase (andesine), orthoclase, quartz, biotite, hornblende, and titaniferous magnetite. Analysis by Stokes, record No. 1721.

F. Hornblende gabbro, sec. 29, T. 42 N., R. 31 W., west bank Michigamme River, east of Crystal Falls. Hessose. Contains labradorite, hornblende, and iron oxide, with subordinate pyroxene, biotite, and orthoclase. Analysis by George Steiger, record No. 1712.

G. Bronzite norite, same locality as F. Auvergnose. Contains bronzite, hornblende, and labradorite. Analysis by Steiger, record No. 1712.

H. Wehrlite, sec. 29 , T. 42 N., R. 31 W., on Michigamme River, east of Crystal Falls. Rossweinose. Contains hornblende, pyroxene, olivine, biotite, and iron oxide. Analysis by Stokes, record No. 1721. 
I. Picrite porphyry, sec. 27, T. 44 N., R. 32 W., northwest of Mansfield. Principal minerals serpentine, amphibole, chlorite, ilmenite, all but the last being secondary. Analysis by Stokes, record No. 1721.

\begin{tabular}{|c|c|c|c|c|c|}
\hline . & E. & F. & G. & H. & I. \\
\hline 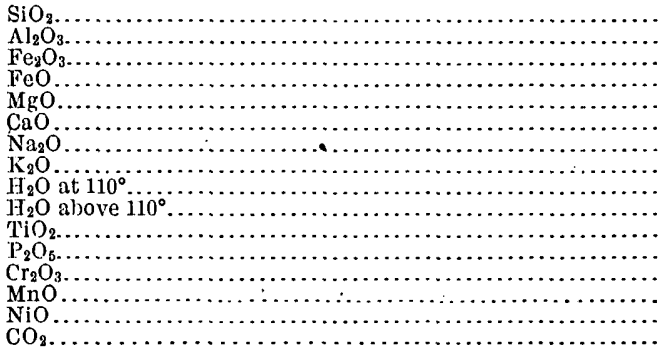 & $\begin{array}{r}58.51 \\
16.32 \\
2.11 \\
4.43 \\
3.73 \\
3.92 \\
0.11 \\
4.08 \\
.23 \\
2.00 \\
.72 \\
.30 \\
\text { trace } \\
\text { none }\end{array}$ & $\begin{array}{r}49.80 \\
19.96 \\
6.32 \\
.49 \\
7.05 \\
11.33 \\
2.22 \\
.61 \\
.13 \\
1.71 \\
.79 \\
.07 \\
\ldots \ldots . . \\
\ldots \ldots . . \\
\ldots . .75\end{array}$ & $\begin{array}{r}48.23 \\
18.26 \\
1.26 \\
6.10 \\
10.84 \\
9.39 \\
1.34 \\
.73 \\
.26 \\
2.00 \\
1.00 \\
.07 \\
\ldots \ldots . \\
\ldots \ldots . \\
.43\end{array}$ & $\begin{array}{r}44.99 \\
5.91 \\
3.42 \\
8.30 \\
21.02 \\
9.79 \\
.91 \\
.74 \\
.63 \\
3.19 \\
.97 \\
.05 \\
.25 \\
\text { trace } \\
\ldots \ldots . . \\
\text { trace? }\end{array}$ & $\begin{array}{r}37.36 \\
4.76 \\
6.61 \\
6.12 \\
31.11 \\
1.19 \\
\text { trace } \\
\text { trace } \\
.65 \\
10.37 \\
.79 \\
.00 \\
.62 \\
\text { trace } \\
.04 \\
\text { none }\end{array}$ \\
\hline & 99.46 & 100.63 & 99.91 & 99.17 & 99.68 \\
\hline
\end{tabular}

$\mathrm{Ba}, \mathrm{Sr}, \mathrm{Cl}, \mathrm{F}, \mathrm{S}, \mathrm{SO}_{3}$ not looked for.

J. Pre-Cambrian nonporphyritic metabasalt, from the Hemlock formation. Beerbachose. Contains plagioclase, light-green hornblende, epidote-zoisite, chlorite, calcite, muscovite, apatite, sphene, quartz, pyrite, and magnetite.

K. Porphyritic metabasalt, Hemlock formation. Same minerals as in $J$, with feldspar phenocrysts.

L. Porphyritic metabasalt, like K. SR. 5 of monzonase.

M: Metadolerite, large dike in T, 43 N., R. 31 W., east of Mansfield. Auvergnose. Petrographic data furnished by C. R. Van Hise. Contains uralite, labradorite, and ilmenite.

Analyses J, K, and L by H. N. Stokes, record No. 1617 .

Analysis M by George Steiger, record No. 1814.

\begin{tabular}{|c|c|c|c|c|}
\hline & J. & K. & I. & M. \\
\hline 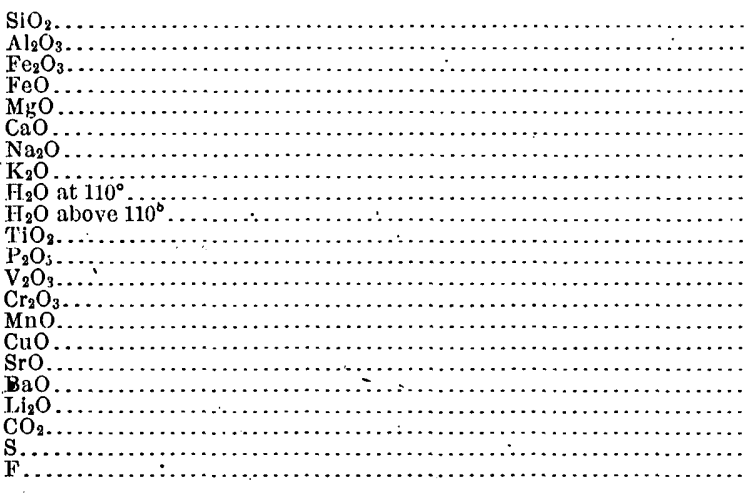 & $\begin{array}{r}46.47 \\
16.28 \\
3.15 \\
8.96 \\
6.56 \\
7.90 \\
3.64 \\
.21 \\
.28 \\
3.89 \\
1.28 \\
.13 \\
\cdots \\
.01 \\
.09 \\
\text { trace } \\
\text { none } \\
\text { none } \\
\text { trace } \\
1.26 \\
\text { none } \\
\text { trace }\end{array}$ & $\begin{array}{r}47.20 \\
15.36 \\
3.06 \\
8.87 \\
4.20 \\
5.05 \\
4.72 \\
1.40 \\
.16 \\
3.04 \\
3.30 \\
.36 \\
\ldots \ldots . . \\
\text { none } \\
.20 \\
\text { trace } \\
\text { trace } \\
\text { trace } \\
\text { trace } \\
3.34 \\
\text { trace } \\
\text { trace }\end{array}$ & $\begin{array}{r}52.59 \\
15.89 \\
6.12 \\
3.96 \\
5.04 \\
5.55 \\
5.79 \\
.67 \\
.16 \\
2.16 \\
1.36 \\
.15 \\
.04 \\
\text { trace } \\
.25 \\
\text { trace } \\
\text { none } \\
\text { trace } \\
\text { none } \\
\text { none } \\
\text { none } \\
\text { trace }\end{array}$ & 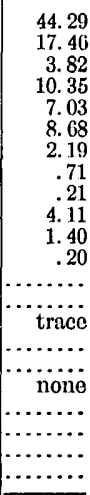 \\
\hline & 100.11 & $\cdot 100.26$ & 99.73 & 100.45 \\
\hline
\end{tabular}

N. Adinole, sec. 8, T. 43 N., R. 31 W., near Mansfield. Contains actinolite, albite, quartz, some chlorite, and epidote. Analysis by George Steiger, record No. 1709.

O. Spilosite. Contains quartz, feldspar, chlorite, epidote, and a little biotite. 
P. Spilosite. Contains quartz, feldspar, actinolite, and epidote.

Analyses $\mathrm{O}$ and $\mathrm{P}$ by $\mathrm{H}$. N. Stokes, record No. 1617. These three rocks are contact derivatives of the Mansfield clay slate, q. .v.

\begin{tabular}{|c|c|c|c|}
\hline . & N. & O. & $P$. \\
\hline 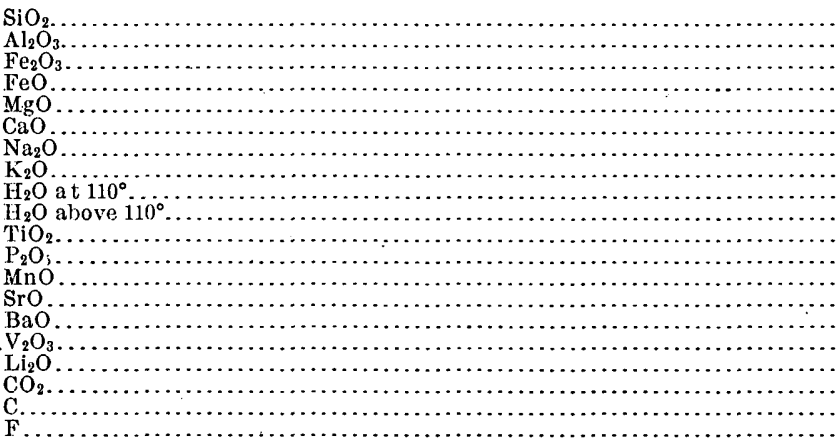 & $\begin{array}{r}74.16 \\
11.85 \\
.82 \\
1.66 \\
2.10 \\
2.10 \\
6.57 \\
.15 \\
.05 \\
.52 \\
.37 \\
.08 \\
.06 \\
\text { none } \\
\text { none } \\
\ldots \ldots . . \\
\ldots \ldots . . . \\
.09 \\
.18 \\
\ldots . . . .\end{array}$ & \begin{tabular}{r}
52.51 \\
19.00 \\
3.31 \\
7.19 \\
3.29 \\
1.55 \\
6.72 \\
.70 \\
.34 \\
3.26 \\
1.70 \\
.15 \\
trace \\
trace \\
trace \\
\hdashline$\ldots . .$. \\
trace \\
none \\
$\ldots \ldots . .$. \\
trace
\end{tabular} & $\begin{array}{r}57.77 \\
19.33 \\
1.29 \\
3.37 \\
4.35 \\
1.71 \\
8.22 \\
.22 \\
.18 \\
2.34 \\
.92 \\
.04 \\
\text { trace } \\
\text { trace } \\
\text { none } \\
.02 \\
\text { none } \\
\text { none } \\
\text {..... } \\
\text { none }\end{array}$ \\
\hline & 100.76 & 99.72 & 99.76 \\
\hline
\end{tabular}

3. KEWEENAW POINT, MICHIGAN. I

Analyses made by G. Steiger for A. N. Winchell, who has published them in Jour. Geology, vol. 16, p. 772, but without detailed description of the rocks.

A. Olivine diabase, Greenstone Cliff. Auvergnose.

B. "Ashbed" diabase, bed 65, Eagle River section. Camptonose.

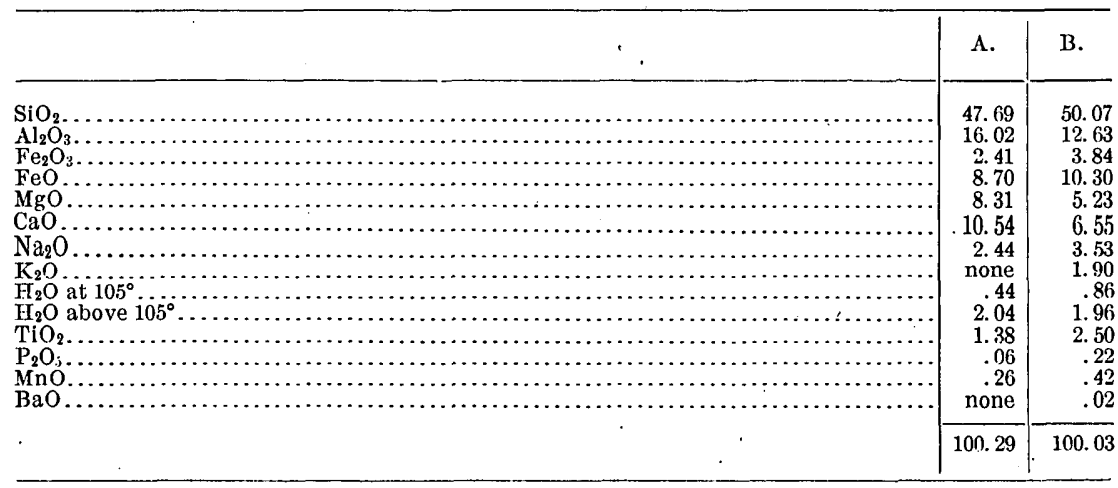

$\mathrm{ZrO}_{2}, \mathrm{CO}_{2}, \mathrm{~S}, \mathrm{SO}_{3}$, and $\mathrm{SrO}$ absent.

\section{MENOMINEE RIVER.}

STURGEON FALLS GABBRO.

From Sturgeon Falls, Menominee River, sec. 27, T. 39 N., R. 29 W., Michigan. Described by Williams, Bull. 62, p. 67.

A. Saussurite gabbro. Auvergnose. Contains plagioclase, almost wholly altered to saussurite, diallage, hornblende, and ilmenite, with quartz, calcite, and chlorite as alteration products. P. R. C. 1755.

B. The same, altered and somewhat schistose. Feldspar much altered into calcite, with secondary quartz and sericite; pyroxene and hornblende changed to chlorite. Leucoxene common. 
C. Light-gray, silvery schist, derived from gabbro. Contains chlorite, calcite, and a little quartz, with remnants of feldspar and some leucoxene. Analyses by R. B. Riggs, record Nos. 389, 390, 391. Material dried at $105^{\circ}$. $\mathrm{TiO}_{2}$ undetermined.

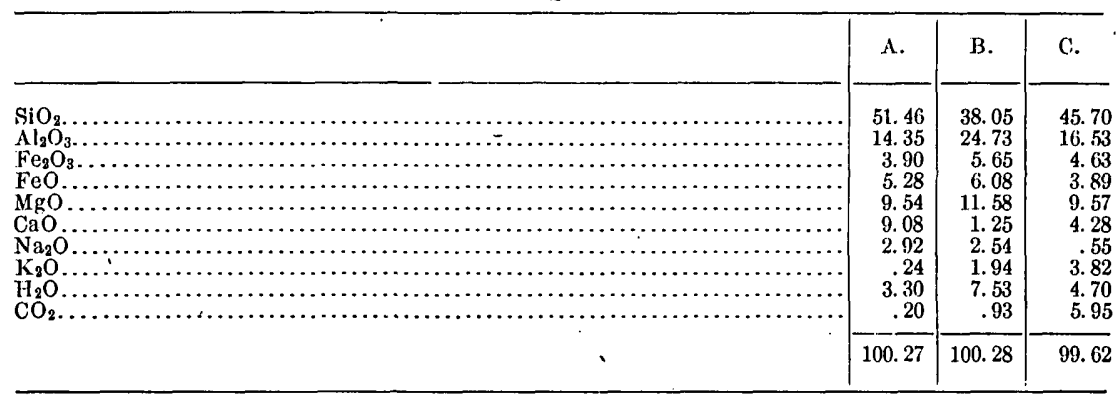

LOWER QUINNESEC FALLS, MENOMINEE RIVER.

Described oy Williams, Bull. 62, pp. 89, 91.

A. Gabbro-diorite, shore below falls, Wisconsin side. Auvergnose. Contains saussurite, hornblende, and ilmenite. Some calcite in the saussurite. Hornblende partly altered to chlorite, and ilmenite to leucoxene.

B. The same, schistose form. Contains less saussurite. Hornblende and ilmenite completely altered into chlorite and leucoxene. Calcite present, and also porphyritic feldspar.

C. Silvery schist, adjoining B, and derived from gabbro. Resembles B in general, without the feldspar crystals. Calcite and sericite are present, and rutile in place of leucoxene.

D. Dark massive greenstone. Contains hornblende, chlorite, epidote, quartz, leucoxene, with some ilmenite and traces of original feldspar.

E. Dark schistose greenstone, forming a band in D. Chlorite entirely replaces hornblende, and rutile replaces leucoxene. Some feldspar, quartz, and calcite.

Analyses by R. B. Riggs, record Nos. $384,385,386,387,388$. Material dried at $105^{\circ}$.

\begin{tabular}{|c|c|c|c|c|c|}
\hline & A. & B. & C. & $\mathrm{J}$. & E. \\
\hline \multirow[t]{2}{*}{ 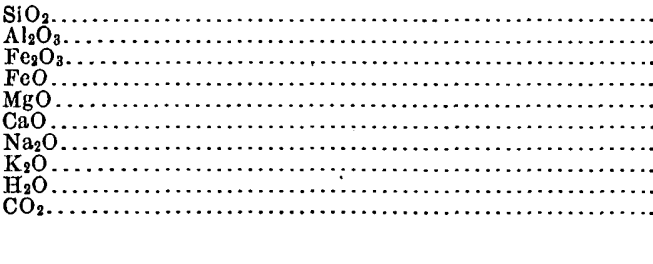 } & $\begin{array}{r}47.96 \\
16.85 \\
4.33 \\
4.17 \\
9.15 \\
13.25 \\
1.25 \\
.30 \\
2.89 \\
.08\end{array}$ & $\begin{array}{r}49.19 \\
18.71 \\
5.03 \\
4.04 \\
7.98 \\
5.92 \\
1.44 \\
.77 \\
5.05 \\
1.82\end{array}$ & $\begin{array}{r}46.21 \\
18.38 \\
3.30 \\
3.90 \\
7.03 \\
6.28 \\
2.14 \\
.35 \\
3.82 \\
8.32\end{array}$ & $\begin{array}{r}43.80 \\
16.08 \\
9.47 \\
10.50 \\
6.54 \\
7.81 \\
1.96 \\
.34 \\
3.99 \\
.08\end{array}$ & $\begin{array}{r}44.49 \\
16.37 \\
5.07 \\
5.50 \\
7.50 \\
7.94 \\
2.59 \\
.56 \\
4.99 \\
5.38\end{array}$ \\
\hline & 100.23 & 99.95 & 99.73 & 100.57 & 100.39 \\
\hline
\end{tabular}

UPPER QUINNESEC FALLS, MENOMINEE RIVER.

Described by Williams, Bull. 62, pp. 104, 113, 114, 121.

A. Light greenstone. Auvergnose. Contains hornblende, feldspar much altered to saussurite, ilmenite with leucoxene border, and some secondary quartz.

B. Mica diorite porphyry. I. 5. 3. 4. Mainly andesitic feldspar and biotite, with apatite, zircon, sphene, and some calcite.

C. Biotite gneiss, Michigan side. Contains biotite, soda orthoclase, and quartz. Sphene common, zircon and apatite present. 
D. Schistose quartz porphyry. Toscanose. Contains quartz, feldspar, sericite, some chlorite, anatase, and tourmaline, with zircon and apatite sparingly. A, B, and $\mathrm{D}$ are from the Wisconsin side of the river.

Analyses by R. B. Riggs, record Nos. 392, 393, 394, 395. Material dried at $105^{\circ}$.

\begin{tabular}{|c|c|c|c|c|}
\hline . & $\Lambda$. & B. & C. & D. \\
\hline 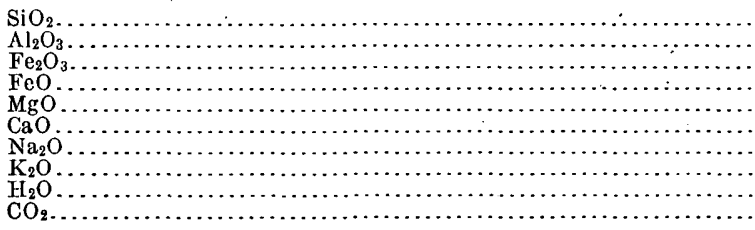 & $\begin{array}{r}48.35 \\
15.40 \\
4.04 \\
4.63 \\
11.61 \\
10.38 \\
1.87 \\
.35 \\
3.60 \\
.08\end{array}$ & $\begin{array}{r}54.83 \\
25.49 \\
1.61 \\
1.65 \\
1.96 \\
6.08 \\
5.69 \\
1.87 \\
1.18 \\
.18\end{array}$ & $\begin{array}{r}67.77 \\
16.61 \\
2.06 \\
1.96 \\
1.26 \\
1.87 \\
4.35 \\
2.35 \\
1.69 \\
.19\end{array}$ & $\begin{array}{r}66.69 \\
16.69 \\
2.06 \\
.93 \\
1.15 \\
1.40 \\
2.46 \\
5.23 \\
1.70 \\
1.42\end{array}$ \\
\hline . & 100.31 & 100.54 & 100.11 & 99.73 \\
\hline
\end{tabular}

SCHIST FROM THE ARAGON IRON MINE.

Described by Bayley in Mon. XLVI, p. 122. Consists of quartz and micaceous minerals, either talc or kaolin and serpentine. Some limonite is present. Analy'isis by George Steiger, record No. 1835.

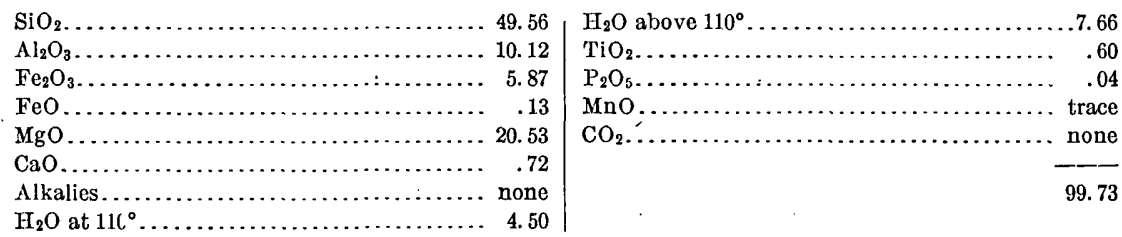

\section{PENOKEE-GOGEBIC REGION.}

Rocks mostly described by Van Hise in Mon. XIX. Analyses A to G by T. M. Chatard, record Nos. 991, 992, 993, 994, 995.

A. Diabase, near southeast corner of sec. 13, T. 47 N., R. 46 W., Michigan. Auvergnose. Contains plagioclase, augite, magnetite, apatite, and olivine, with some ilmenite and leucoxene. P. R. C. 1001.

B. Same dike as A, partly altered. The pyroxene is altered to amphibole, and the latter partly to biotite. Biotite has also been derived from feldspar. P. R. C. 1000 .

C. Feldspar separated from A.

D. Altered diabase, Aurora mine, NE. $\frac{1}{4}$ SW. $\frac{1}{4}$ sec. 23, T. 47 N., R. 47 W., Michigan. P. R. C. 1002.

\begin{tabular}{|c|c|c|c|c|}
\hline & A. & B. & C. & D. \\
\hline 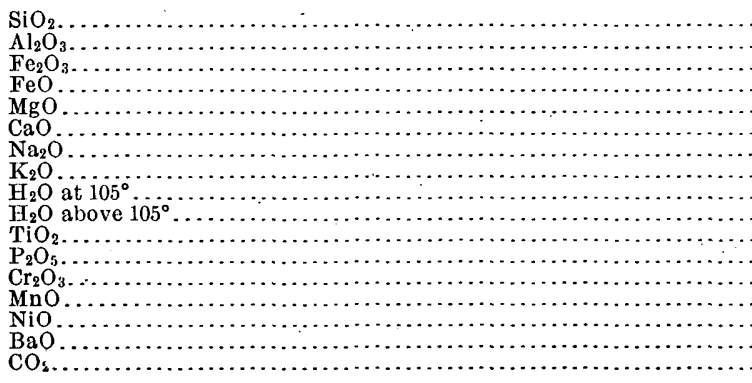 & $\begin{array}{r}47.90 \\
15.60 \\
3.69 \\
8.41 \\
8.11 \\
9.99 \\
2.05 \\
.23 \\
.15 \\
2.34 \\
.82 \\
.13 \\
\text { trace } \\
.17 \\
.10 \\
.05 \\
.38\end{array}$ & $\begin{array}{r}46.85 \\
22.62 \\
5.12 \\
1.58 \\
2.01 \\
1.25 \\
.80 \\
2.66 \\
3.12 \\
8.25 \\
1.12 \\
.16 \\
2.54 \\
.08 \\
\because .10 \\
1.89\end{array}$ & \begin{tabular}{r}
51.18 \\
27.00 \\
3.19 \\
undet. \\
1.92 \\
11.70 \\
3.48 \\
.41 \\
1.19 \\
$\ldots \ldots . .$. \\
\hdashline$\ldots . .$. \\
\hdashline .17 \\
$\ldots \ldots . .$. \\
\hdashline$\ldots . .$. \\
$\ldots . .$.
\end{tabular} & $\begin{array}{r}41.60 \\
37.20 \\
3.21 \\
.30 \\
.02 \\
.23 \\
.07 \\
\ldots . .2 \\
.29 \\
13.54 \\
3.79 \\
.14 \\
.08 \\
.0 . \\
\text { trace } \\
.38\end{array}$ \\
\hline • & 100.12 & 100.15 & 100.24 & 100.85 \\
\hline
\end{tabular}


E. Feldepàr separated from gabbro, sec. 14, 'T. 44 N., R. 4 W., Wisconsin.

F, G. Feldspars separated from olivine diabase, NE. $\frac{1}{4}$ sec. 13, T. 45 N., R. 1 W., Wisconsin. P. R. C. 1003.

\begin{tabular}{|c|c|c|c|}
\hline & E. & F. & G. \\
\hline \multirow[t]{2}{*}{ 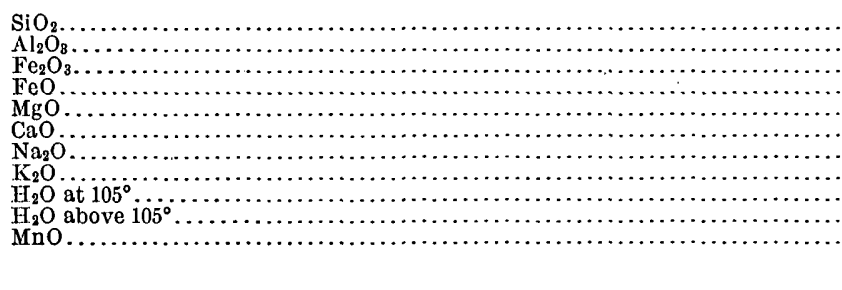 } & $\begin{array}{r}51.99 \\
29.32 \\
1.23 \\
.63 \\
12.60 \\
2.91 \\
.28 \\
.03 \\
.54 \\
\text { trace }\end{array}$ & $\begin{array}{r}61.65 \\
19.91 \\
2.28 \\
\text { undet. } \\
.61 \\
4.12 \\
4.74 \\
5.72 \\
.95 \\
\text { trace }\end{array}$ & $\begin{array}{r}56.15 \\
26.05 \\
1.98 \\
\text { undet. } \\
.54 \\
8.70 \\
4.79 \\
1.56 \\
.13 \\
.64 \\
.13\end{array}$ \\
\hline & 99.53 & 99.98 & 100.67 \\
\hline
\end{tabular}

The following analyses, by W. F. Hillebrand, of separations from the gabbro of Ashland County, Wis:, were made for the late R. D. Irving, but were never published by him:

H. Magnetite, record No. 687. Partial analysis.

I. Feldspar, record No. 688 .

J. Diallage, record No..686.

For convenience I also include here, although it is decidedly out of classification-

K. Graywacke, Hurley, Wis. Described by Bayley in Bull. 150, p. 84 . Analysis by H. N. Stokes, record No. 1314. Contains quartz, feldspars, iron oxides, and probably kaolin. In the cement are chlorite, quartz, magnetite, pyrite, rutile, occasionally biotite, and either muscovite or kaolin. P. R. C. 20 .

\begin{tabular}{|c|c|c|c|c|}
\hline . & II. & I. & J. & $\mathbf{K}$ \\
\hline 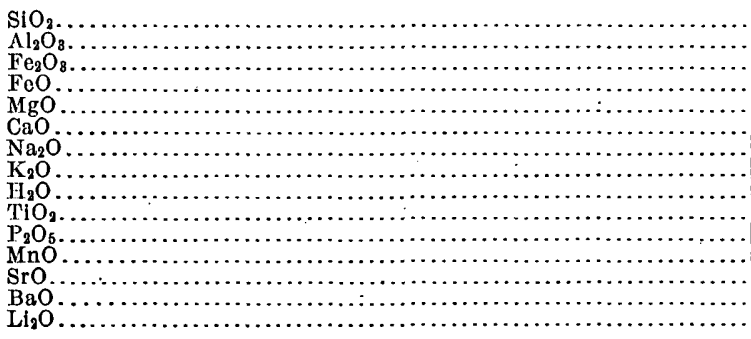 & 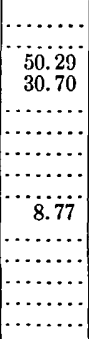 & $\begin{array}{r}53.30 \\
29.03 \\
.55 \\
.23 \\
.13 \\
11.40 \\
4.87 \\
.40 \\
.23 \\
\text { trace } \\
\text { trace } \\
\text { none } \\
\text { trace } \\
\text { trace } \\
\text { none }\end{array}$ & $\begin{array}{r}49.80 \\
2.86 \\
2.48 \\
10.82 \\
15.33 \\
16.50 \\
.51 \\
.12 \\
.33 \\
1.29 \\
\text { trace } \\
.37 \\
\text { none } \\
\text { none } \\
\text { trace? }\end{array}$ & 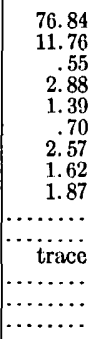 \\
\hline & 89.46 & 100.14 & 100.41 & 100.18 \\
\hline
\end{tabular}

6. PIGEON POINT, MINNESOTA.

Described by Bayley in Bull. 109. Also partly in Am. Jour. Sci., 3d ser., vol. 37, p. 54. A and B are also described in Bull. 150, p. 274 .

A. Olivine diabase. Hessose. Contains labradorite, olivine, pyroxene, apatite, and magnetite. Analysis by W. F. Hillebrand, record No. 496. P. R. C. 107.

B. Diallage separated from diabase. Partial analysis by R. B. Riggs, record No. 607 .

C. Rock lying between the diabase and the adjacent red porphyry. Adamellose. Contains red feldspar, quartz, hornblende, chlorite, magnetite, apatite, and rutile. Analysis by W. F. Hillebrand, record No. 500. P. R. C. 1018. 


\begin{tabular}{|c|c|c|c|}
\hline . & A. & B. & c. \\
\hline 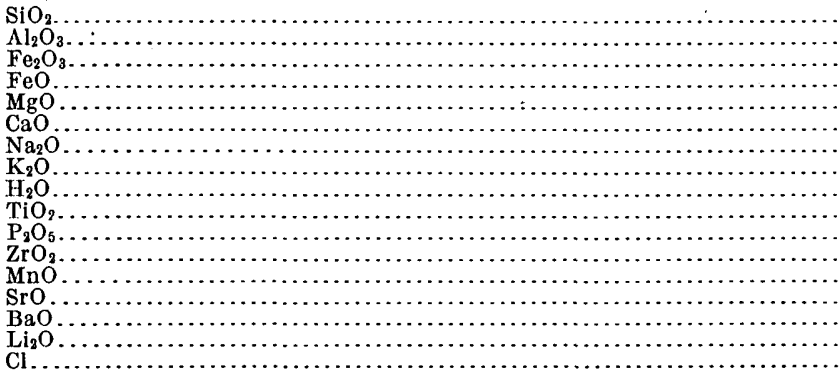 & $\begin{array}{r}49.88 \\
18.55 \\
2.06 \\
8.37 \\
5.77 \\
9.72 \\
2.59 \\
.68 \\
1.04 \\
1.19 \\
.16 \\
\text { none } \\
.09 \\
\text { trace } \\
.02 \\
\text { none } \\
\text { trace }\end{array}$ & 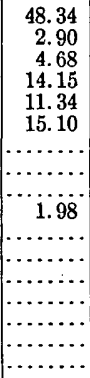 & $\begin{array}{r}57.98 \\
13.58 \\
3.11 \\
8.68 \\
2.87 \\
2.01 \\
3.56 \\
3.44 \\
2.47 \\
1.75 \\
.29 \\
\ldots .13 \\
.13 \\
\text { trace } \\
.04 \\
\text { trace } \\
\text { trace }\end{array}$ \\
\hline & 100.12 & 98.49 & 99.91 \\
\hline
\end{tabular}

D. Red soda granite. Liparose. Contains feldspar, quartz, chlorite, some muscovite, rutile, leucoxene, hematite, and apatite, with sometimes secondary calcite. Granular variety. Analysis by W. F. Hillebrand, record No. 495 . Dried at $110^{\circ}$.

E. Same as D, porphyritic variety. Toscanose. Called by Bayley a quartz keratophyre. Analysis by Hillebrand, record No. 497.

F. Similar red porphyry, Little Brick Island, Lake Superior, near Pigeon Point. Liparose. Consists chiefly of feldspar and quartz, with augite occasionally present. Analysis by L.: G. Eakins, record No. 1059.

G. Red feldspar, anorthoclase, separated from D. Contains hematite inclusions. Analysis by J. E. Whitfield, record No. 521. Dried at $104^{\circ}$.

\begin{tabular}{|c|c|c|c|c|}
\hline 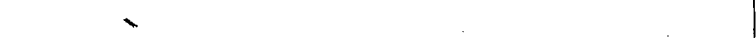 & D. & E. & F. & G. \\
\hline 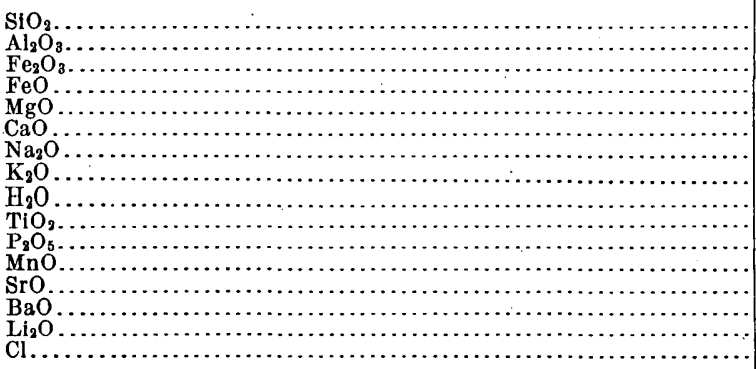 & $\begin{array}{r}72.42 \\
13.04 \\
.68 \\
2.49 \\
.58 \\
.66 \\
3.44 \\
4.97 \\
1.21 \\
.40 \\
.20 \\
.09 \\
\text { trace? } \\
.15 \\
\text { trace? } \\
\text { trace }\end{array}$ & $\begin{array}{r}74.00 \\
12.04 \\
.78 \\
2.61 \\
.42 \\
.85 \\
3.47 \\
4.33 \\
.86 \\
.34 \\
.06 \\
.05 \\
\text { trace } \\
.12 \\
\text { trace? } \\
\text { trace }\end{array}$ & 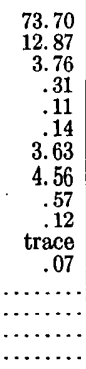 & 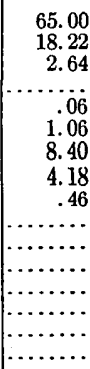 \\
\hline & 100.33 & 99.93 & 99.84 & -100.02 \\
\hline
\end{tabular}

H. Dark vitreous quartzite. Contains quartz, a little red feldspar, some chlorite, some green mica, a few scales of biotite, and grains of magnetite. P. R. C. 1007.

I. Lighter interbedded quartzite. Feldspar in it much altered. P. R. C. 1004.

J. Epidotic quartzite. P. R. C. 1023.

K. Unaltered quartzite. P. R. C. 1006.

Analyses by R. B. Riggs, record Nos. $569,571,572,575$. Material dried at $105^{\circ}$. 


\begin{tabular}{|c|c|c|c|c|}
\hline & . $\mathrm{H}$. & I. & J. & K. \\
\hline 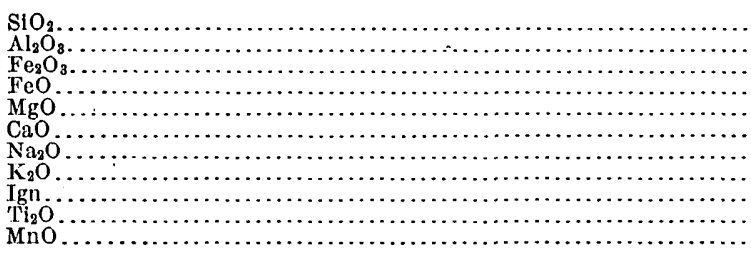 & $\begin{array}{r}74.22 \\
10.61 \\
7.45 \\
.85 \\
1.48 \\
.56 \\
2.12 \\
1.08 \\
1.79 \\
.16 \\
\text { none }\end{array}$ & $\begin{array}{r}73.65 \\
11.08 \\
7.24 \\
.77 \\
1.52 \\
.40 \\
1.67 \\
1.65 \\
1.88 \\
\text { trace? } \\
\text { trace }\end{array}$ & $\begin{array}{r}73.14 \\
12.60 \\
7.57 \\
1.31 \\
1.67 \\
.43 \\
1.78 \\
1.00 \\
.83 \\
.04 \\
\text { trace }\end{array}$ & $\begin{array}{r}73.64 \\
11.25 \\
6.24 \\
1.04 \\
1.57 \\
.36 \\
3.04 \\
1.42 \\
1.98 \\
\text { trace } \\
\text { none }\end{array}$ \\
\hline & 100.32 & 99.86 & 100.37 & 100.54 \\
\hline
\end{tabular}

$\mathrm{Ba}$ and $\mathrm{Sr}$ not looked for.

L. Altered quartzite. Contains more fibrous chlorite, some sericite, a little kaolin and biotite, grains of magnetite, earthy matter, and sometimes a little calcite. Analysis by R. B. Riggs, record No. 570. P. R. C. 1009.

M. Mottled quartzite. Analysis by Riggs, record No. 573. P. R. C. 1021.

N. Purplish slate, containing many small crystals of red feldspar and scales of mica. Analyses by J. E. Whitfield, record No. 520. P. R. C. 1024.

. O. Very slightly altered slate. Analysis by Riggs, record No. 576. P. R. C. 1011. Material for analysis dried at $104^{\circ}-105^{\circ}$.

\begin{tabular}{|c|c|c|c|c|}
\hline & I. & M. & N. & 0. \\
\hline 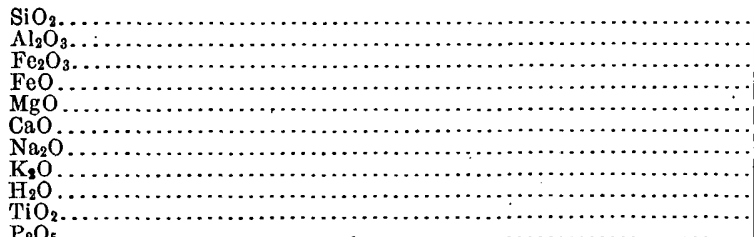 & $\begin{array}{r}71.00 \\
12.88 \\
6.69 \\
.65 \\
1.68 \\
.21 \\
1.43 \\
2.95 \\
2.03 \\
.44\end{array}$ & $\begin{array}{r}72.25 \\
10.73 \\
8.01 \\
.38 \\
1.85 \\
.42 \\
2.03 \\
2.56 \\
2.05 \\
\text { trace }\end{array}$ & $\begin{array}{r}63.82 \\
14.65 \\
3.16 \\
5.12 \\
2.08 \\
.70 \\
1.95 \\
2.81 \\
2.62 \\
2.66\end{array}$ & $\begin{array}{r}59.71 \\
18.32 \\
8.11 \\
.85 \\
3.54 \\
1.05 \\
1.93 \\
3.43 \\
3.24 \\
\text { trace }\end{array}$ \\
\hline 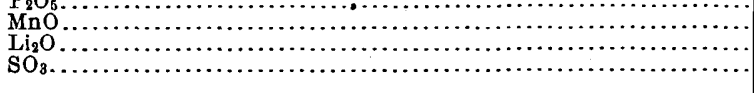 & 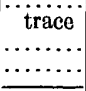 & $\begin{array}{l}\text { trace } \\
. . . . . .\end{array}$ & $\begin{array}{r}.15 \\
\text { none } \\
\text { none } \\
.33\end{array}$ & $\begin{array}{c}\text { none } \\
\cdots \ldots \ldots \\
\cdots \cdots\end{array}$ \\
\hline & 99.96 & 100.28 & 100.09 & 100.18 \\
\hline
\end{tabular}

$\mathrm{Ba}$ and $\mathrm{Sr}$ not looked for.

P. Red granitic rock resembling $\mathrm{D}$, but with more dark spots, found in contact with the purplish slate, N. Liparose. Consists mainly of red feldspar, quartz, and chlorite. Analysis by J. E. Whitfield, record No. 519. - Dried at 104². P. R. C. 1014.

Q. Groundmass of red mottled quartzite. Analysis by W. F. Hillebrand, record No. 499. P. R. C. 1022.

R. Green mottlings from Q. Mostly quartz and sericite. Analysis by Fillebrand, record No. 498.

S. Another sample of the green mottlings, selected with especial care. Analysis by L. G. Eakins, record No. 1058.

T. Brilliantly red vitreous quartzite. Analysis by R. B. Riggs, record No. 574. Dried at $105^{\circ}$. P. R. C. 1008.

15619-Bull. $419-10-5$ 


\begin{tabular}{|c|c|c|c|c|c|}
\hline & P. & Q. & $\mathrm{R}$ & S. & $\mathrm{T}$ \\
\hline 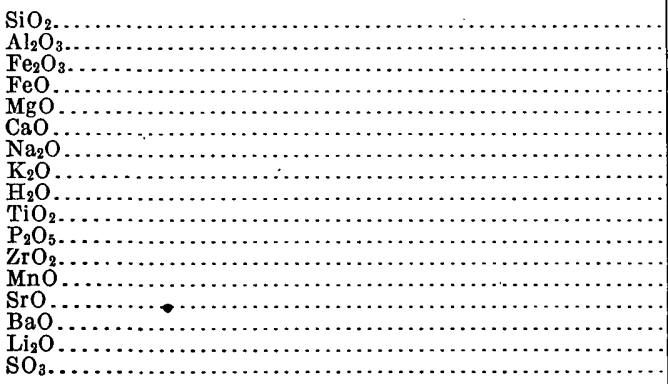 & $\begin{array}{r}68.36 \\
13.76 \\
2.65 \\
2.75 \\
.68 \\
.70 \\
3.56 \\
4.48 \\
.98 \\
1.57 \\
.33 \\
\ldots . . . \\
\text { trace } \\
\text { undet. } \\
\text { undet. } \\
\text { none } \\
.66\end{array}$ & $\begin{array}{r}76.57 \\
9.21 \\
1.67 \\
3.94 \\
1.51 \\
.73 \\
3.07 \\
1.02 \\
1.89 \\
.42 \\
\text { trace } \\
\text { none } \\
.05 \\
\text { trace? } \\
\text { none } \\
\text { trace } \\
\ldots . . . . .\end{array}$ & $\begin{array}{c}77.70 \\
7.67 \\
3.55 \\
3.29 \\
1.83 \\
.26 \\
1.96 \\
1.04 \\
a 2.36 \\
.30 \\
\text { none } \\
\ldots \ldots . . \\
\text { (?) } \\
\text { none } \\
\text { trace } \\
\ldots . . . .\end{array}$ & 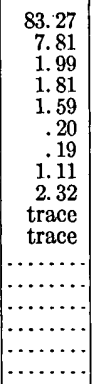 & $\begin{array}{r}83.69 \\
7.50 \\
1.81 \\
.38 \\
.35 \\
.39 \\
2.46 \\
2.61 \\
.72 \\
\text { trace? } \\
\ldots \ldots . . \\
\ldots \ldots . . \\
\text { trace } \\
\text { undet. } \\
\text { undet. } \\
\ldots \ldots . . . \\
\ldots . . .\end{array}$ \\
\hline r & 100.48 & 100.08 & 100.00 & 100.29 & 99.91 \\
\hline
\end{tabular}

a Determined by difference.

\section{MESABI DISTRICT, MINNESOTA.}

The following rocks, described by Leith in Mon. XLIII, do not fall legitimately within this section of the present bulletin. There seems, however, to be no other convenient place for them, and so the niceties of,classification have been ignored for reasons of expediency.

A. Greenalite rock, test pit, Cincinnati mine. Contains green and brown transparent granules, with opaque brown to black ones, in a matrix of chert. P. R. C. 1668 .

B. Greenalite rock; same locality as A. Contains greenish-yellow granules, slightly altered to amphibole in a matrix of amphibole with subordinate chert. P. R. C. 1669.

C. Greenalite rock, near W. quarter post, sec. 35, T. 59 N., R. 15 W. Resembles A. P. R. C. 1670 .

D. Greenalite rock, near SE. corner of sec. 22 , T. 59 N., R. 15 W. Contains yellowish-brown, transparent, and dark-brown to black and opaque granules in a matrix which is mainly amphibole, possibly actinolite. All four rocks contain oxides of iron, either limonite or magnetite. P. R. C. 1671.

Analyses by George Steiger, record Nos. 1931 and 1992. In three of the rocks the portions soluble and insoluble in hydrochloric acid were analyzed separately.

\begin{tabular}{|c|c|c|c|c|c|c|c|}
\hline & \multicolumn{2}{|c|}{ A. } & \multicolumn{2}{|c|}{ B. } & \multicolumn{2}{|c|}{ C. } & \multirow{2}{*}{ D. } \\
\hline & Insoluble. & Soluble. & Insoluble. & Soluble. & Insoluble. & Soluble. & \\
\hline 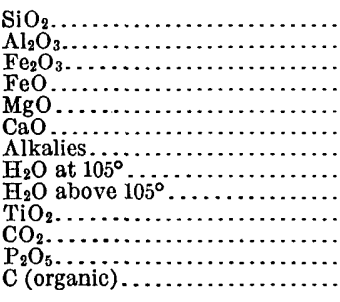 & $\begin{array}{r}36.50 \\
.76\end{array}$ & $\begin{array}{r}19.30 \\
.61 \\
13.83 \\
17.57 \\
3.22 \\
\text { none } \\
\text { none } \\
2.38 \\
5.74 \\
\text { none } \\
\text { none } \\
\text { none }\end{array}$ & 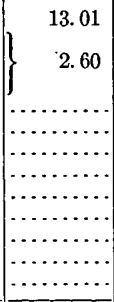 & $\begin{array}{r}33.11 \\
.56 \\
6.44 \\
30.93 \\
5.35 \\
\text { none } \\
\text { none } \\
1.34 \\
6.13 \\
\text { none } \\
\text { none } \\
\text { none } \\
\ldots \ldots . .\end{array}$ & 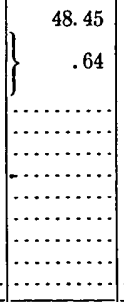 & $\begin{array}{r}13.45 \\
.37 \\
15.00 \\
10.28 \\
2.33 \\
.28 \\
\text { none } \\
2.50 \\
4.17 \\
\text { none } \\
2.04 \\
\text { none } \\
\end{array}$ & $\begin{array}{r}50.96 \\
1.09 \\
5.01 \\
30.37 \\
5.26 \\
.04 \\
\text { none } \\
.75 \\
6.41 \\
\text { none } \\
\text { none } \\
\text { none } \\
.21 \\
\end{array}$ \\
\hline \multirow[t]{2}{*}{ Insoluble.. } & $\begin{array}{r}37.26 \\
\cdots \cdots \\
\end{array}$ & $\begin{array}{r}62.65 \\
37.26 \\
\end{array}$ & 15.61 & $\begin{array}{l}83.86 \\
15.61 \\
\end{array}$ & 49.09 & $\begin{array}{l}50.42 \\
49.09 \\
\end{array}$ & $\begin{array}{r}100.10 \\
\cdots \cdots \\
\end{array}$ \\
\hline & & 99.91 & & 99.47 & & 99.51 & $\therefore \ldots \ldots$, \\
\hline
\end{tabular}

E. Ferruginous chert, sec. 28, T. 58 N., R. 17 W. P. R. C. 1672.

F. Ferruginous chert, horizon of ore deposits, Oliver mine. P. R. C. 1673.

G. Amphibolitic chert, Old Chicago mine. P. R. C. 1674. 
H. Amphibolitic chert, one-half mile southwest of Virginia. P. R. C. 1675.

I. Amphibolitic chert, Donora mine. P. R. C. 1676.

$\mathrm{G}, \mathrm{H}$, and $\mathrm{I}$ are largely amphibole in a matrix of chert. Analyses by George Steiger, record Nos. 1931, 1948.

\begin{tabular}{|c|c|c|c|c|c|}
\hline & E. & $\mathrm{F}$. & G. & H. & I. \\
\hline 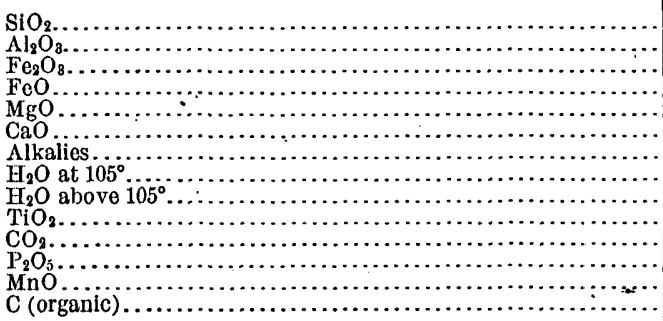 & $\begin{array}{r}63.92 \\
\text { none } \\
31.13 \\
3.13 \\
.49 \\
\text { none } \\
\text { trace } \\
.48 \\
1.12 \\
\text { trace } \\
\text { none } \\
.05 \\
.10 \\
\ldots . . .\end{array}$ & $\begin{array}{r}32.56 \\
\text { none } \\
66.02 \\
.30 \\
\text { none } \\
.18 \\
\text { none } \\
.32 \\
.90 \\
.16 \\
\text { none } \\
.12 \\
.14 \\
\ldots \ldots\end{array}$ & $\begin{array}{r}50.36 \\
.64 \\
6.46 \\
32.91 \\
3.94 \\
.23 \\
\text { none } \\
.27 \\
4.64 \\
\text { none } \\
\text { none } \\
\text { none } \\
\text { none } \\
.18\end{array}$ & $\begin{array}{r}83.82 \\
.39 \\
4.46 \\
8.77 \\
\text { nono } \\
.60 \\
\text { none } \\
.13 \\
1.37 \\
\text { none } \\
.72 \\
.02 \\
\text { none } \\
\text {....... }\end{array}$ & $\begin{array}{r}44.10 \\
1.05 \\
10.80 \\
28.73 \\
2.43 \\
.33 \\
\text { none } \\
.51 \\
2.47 \\
\text { none } \\
9.71 \\
.04 \\
\text { none } \\
. . . .\end{array}$ \\
\hline & 100.42 & 100.70 & 99.63 & 100.28 & 100.17 \\
\hline
\end{tabular}

8. MISCELLANEOUS ROCKS FROM MINNESOTA.

A. Granulitic hypersthene gabbro, from SIE. $\frac{1}{4}$ sec. 20 , T. 65 N., R. 4 W. Cookose. Described by Bayley, Jour. Geology, vol. 3, p. 1. Contains hypersthene, biotite, diallage, magnetite, and plagioclase. Analysis by H. N. Stokes, record No. 1267.

B. Hypersthene from A. Analysis by E. A. Schneider, record No. 1358.

C. Granulitic diallage gabbro, SE. $\frac{1}{4}$ NW. $\frac{1}{4}$ sec. 26 , T. 64 N., R. $8 \mathrm{~W}$ Auvergnose. Described by Bayley, Jour. Geology, vol. 3, p. 1. Contains diallage, hypersthene, magnetite, and plagioclase. Analysis by W. H. Melville, record No. 1403.

D. Olivine gabbro, west side of Birch Lake, SE. 4 sec. 19, T. 63 N., R. 4 W. Auvergnose. Described by Bailey, Jour. Geology, vol. 1, p. 688. Contains a large proportion of diallage and olivine. Analysis by Stokes, No. 1267.

E. Olivine from D. Analysis by W. F. Hillebrand, record No. 1308.

\begin{tabular}{|c|c|c|c|c|c|}
\hline & A. & B. & C. & D. & E. \\
\hline 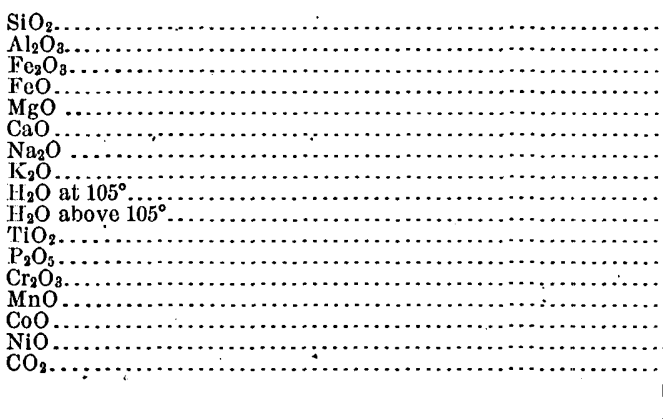 & $\begin{array}{r}46.96 \\
14.13 \\
.76 \\
14.95 \\
15.97 \\
2.32 \\
.35 \\
1.68 \\
.07 \\
1.26 \\
.62 \\
.03 \\
\text { trace } \\
.93 \\
\cdots . .6 \\
. .06 \\
\end{array}$ & 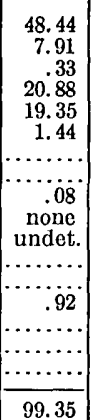 & $\begin{array}{r}49.56 \\
17.81 \\
2.76 \\
9.48 \\
5.93 \\
9.70 \\
2.87 \\
\ldots \ldots . \\
.50 \\
.48 \\
.67 \\
\ldots . \ldots \\
.06 \\
\ldots \ldots \ldots \\
\text { trace }\end{array}$ & $\begin{array}{r}45.66 \\
16.44 \\
.66 \\
13.90 \\
11.57 \\
7.23 \\
2.13 \\
.41 \\
.07 \\
.83 \\
.92 \\
\text { trace } \\
\text { trace } \\
\ldots . .76 \\
\ldots .16 \\
\ldots \ldots . . \\
100.03\end{array}$ & $\begin{array}{r}35.58 \\
.92 \\
33.91 \\
\ldots \ldots .86 \\
26.86 \\
.90 \\
\ldots \ldots . . \\
\cdots \\
.11 \\
.20 \\
1.22 \\
\cdots \ldots . . \\
\text { trace } \\
.35 \\
.20 \\
\ldots \ldots \ldots \\
100.25\end{array}$ \\
\hline
\end{tabular}

F. Average gabbro, south quarter post, sec. 35 , T. 61 N., R. 12 W. Hessose. Described by Bayley in Jour. Geology, vol. 1, p. 688 . Contains plagioclase, olivine, pyroxene, and magnetite. Analysis by H. N. Stokes, record No. 1267.

G. Feldspar, from preceding gabbro. Analysis by W. T. Hillebrand, record No. 1308.

H. Feldspar, from gabbro east side of North Fowl Lake. Analysis by Hillebrand, No. 1308. 
I. Feldspar, from gabbro, NW. $\frac{1}{4}$ SE. $\frac{1}{4}$ sec. 23, T. 62 N., R. 10 W.

J. Feldspar, from gabbro, center of sec. 25, T. 64 N., R. 8 W.

K. Feldspar, from gabbro, Duluth and Iron Range Railroad.

Analyses I, J, and K by Hillebrand, record. No. 1115.

$\mathrm{H}, \mathrm{I}, \mathrm{J}$, and $\mathrm{K}$ analyzed for W. S. Bayley.

\begin{tabular}{|c|c|c|c|c|c|c|}
\hline & F. & G. & H. & I. & J. & $\mathrm{K}$. \\
\hline 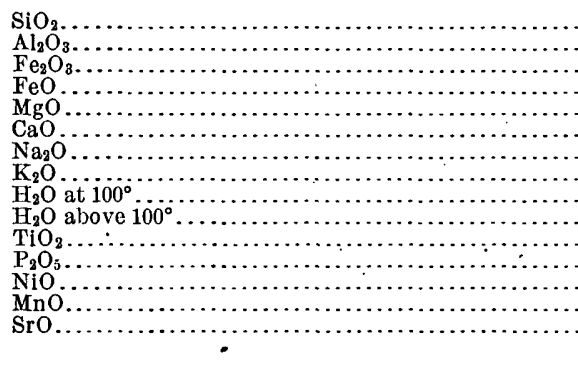 & $\begin{array}{r}46.45 \\
21.30 \\
.81 \\
9.57 \\
7.90 \\
9.83 \\
2.14 \\
.34 \\
.14 \\
1.02 \\
1.19 \\
.02 \\
.04 \\
\text { trace } \\
\cdots \cdots\end{array}$ & $\begin{array}{r}51.89 \\
29.68 \\
.32 \\
.37 \\
.38 \\
12.62 \\
3.87 \\
.00 \\
.07 \\
.39 \\
\cdots \\
\cdots \\
\cdots \\
\cdots \\
\cdots \\
\end{array}$ & $\begin{array}{r}62.71 \\
19.20 \\
1.08 \\
.93 \\
.81 \\
.44 \\
2.96 \\
10.41 \\
.23 \\
.92 \\
\text { trace } \\
\cdots \\
\text { trace } \\
\text { trace }\end{array}$ & 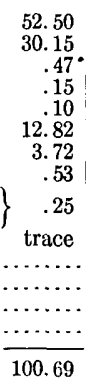 & \begin{tabular}{r}
52.61 \\
29.80 \\
.57 \\
.23 \\
.20 \\
12.25 \\
3.80 \\
.53 \\
.29 \\
trace \\
\hdashline$\ldots .$. \\
\hdashline trace \\
\hdashline$\ldots .$. \\
\end{tabular} & $\begin{array}{r}53.45 \\
29.77 \\
.33 \\
.15 \\
.11 \\
11.33 \\
4.33 \\
.68 \\
.23 \\
\text { trace } \\
\ldots . . .\end{array}$ \\
\hline
\end{tabular}

.L. Garnetiferous gabbro, Granite Falls. Bandose. Described by W. S. Bayley in Bull. 150, p. 282 . Contains plagioclase, augite, garnet, magnetite, a little hornblende, some quartz grains, and apatite. Analysis by H. N. Stokes, record No. 1296. Sp. gr., 3.105. P. R. C. 109.

M. Gabbro-diorite, Minnesota Falls. Hessose. Described by Bayley in Bull. 150, p. 369. Essentially plagioclase and hornblende, with some kaolin, augite, and biotite. Analysis by Stokes, No. 1296. Sp. gr., 2.935. P. R. C. 144.

N. Quartz norite gneiss, Odessa. Described by Bayley in Bull. 150, p. 358. Contains quartz, plagioclase, pyroxene, biotite, garnet, magnetite, and sulphide of iron. Analysis by Stokes, No. 1296. Sp. gr., 2.770. P. R. C. 140.

O. Mica schist, bed of Cross River, near Gunflint Lake. Description furnished by C. R. Van Hise. Contains biotite, quartz, feldspar (?), and pyrites. Analysis by T. M. Chatard, record No. 896.

P. Actinolite-magnetite schist, SE. $\frac{1}{4}$ SE. $\frac{1}{4} \mathrm{sec} .34$, T. 61 N., R. 12 W. Described by Bayley in Am. Jour. Sci., 3d ser., vol. 46, p. 178. Mainly actinolite and magnetite. Analysis by W. H. Melville, record No. 1403.

Q. Magnetite rock near the preceding, also described with $\mathrm{P}$ by Bayley. Analysis by Melville, No. 1403 .

\begin{tabular}{|c|c|c|c|c|c|c|}
\hline . & L. & M. & $N$. & O. & P. & Q. \\
\hline 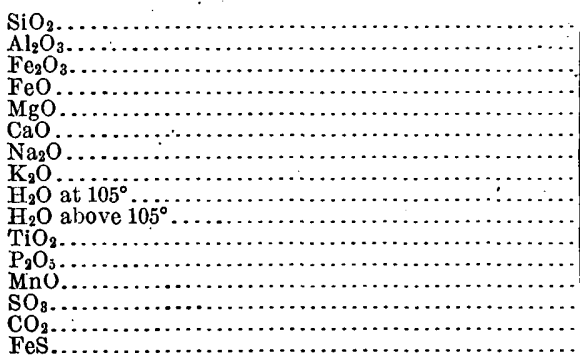 & $\begin{array}{r}52.31 \\
18.35 \\
5.90 \\
11.06 \\
1.00 \\
7.33 \\
2.90 \\
.49 \\
.35\end{array}$ & 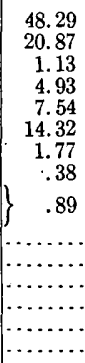 & 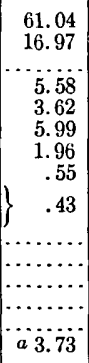 & $\begin{array}{r}64.77 \\
14.45 \\
1.84 \\
4.54 \\
2.34 \\
2.33 \\
1.37 \\
5.03 \\
.07 \\
1.92 \\
.60 \\
.20 \\
.11 \\
.60 \\
.41 \\
\ldots . .\end{array}$ & $\begin{array}{r}12.35 \\
.10 \\
58.68 \\
21.34 \\
4.08 \\
1.91 \\
\text { trace } \\
\ldots \ldots . . \\
.19 \\
.12 \\
.25 \\
1.22 \\
\ldots \ldots \ldots \\
\ldots \ldots \ldots . \\
\ldots \ldots \ldots\end{array}$ & $\begin{array}{r}1.16 \\
1.81 \\
69.08 \\
27.10 \\
.25 \\
.53 \\
\ldots \ldots . . \\
\ldots \ldots . . \\
\ldots \ldots . . \\
\text { none } \\
.06 \\
.33 \\
\ldots \ldots . . \\
\ldots \ldots . . \\
\ldots \ldots . .\end{array}$ \\
\hline$\cdot$ & 99.69 & 100.12 & 99.87 & 100.58 & 100.24 & 100.32 \\
\hline
\end{tabular}

a Probably pyrrhotite. 


\section{SOUTH DAKOTA.}

A. Phonolite, Black Hills. Miaskose. Described by Whitman Cross in Bull. 150, p. 191. Contains sanidine, nephelite, ægirite, nosean, and sodalite, with accessory sphene, apatite, and zircon, and possibly some rare zirconates or titanates. Also, sparingly, secondary zeolites and calcite. No magnetite, but minute ferritic flakes are disseminated through the rock. Analysis by W. F. Hillebrand, record No. 1618. P. R. C. 73.

B. An earlier analysis of A, by H. N. Stokes, record No. 1314. This was made along the ordinary lines as regards "completeness," as requested by the collector, and without regard to minor accessory constituents. It serves well to illustrate the difference between the two modes of treatment, in comparison with the fuller analysis.

C. Tourmaline-biotite schist, north of Harney Peak, Black Hills. Described by Bayley in Bull. 150, p. 327. Contains quartz, biotite, tourmaline, a little garnet and rarely muscovite, iron ore, and apatite. Analysis by Stokes, record No. 1296. P. R. C. 130.

D. Volcanic ash, 3 miles west of Argyle, Custer County. Amiatose. Collected by N. H. Darton. Analysis by George Steiger, record No. 1875. P. R. C. 599.

\begin{tabular}{|c|c|c|c|c|}
\hline & A. & B. & C. & D. \\
\hline 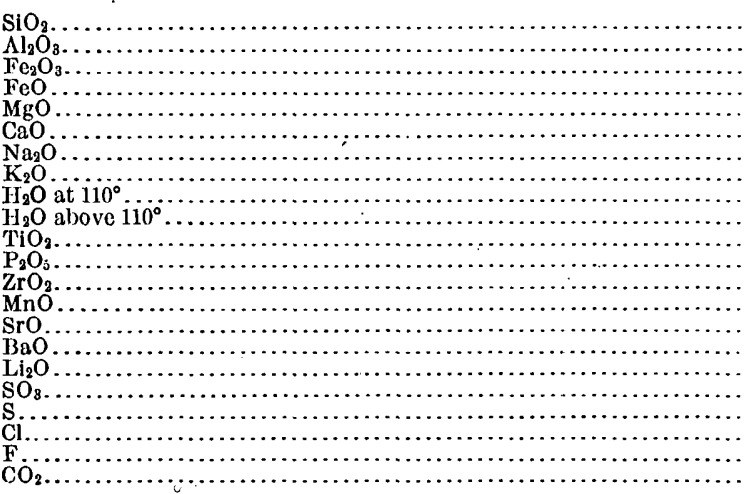 & $\begin{array}{r}57.86 \\
20.26 \\
2.35 \\
.39 \\
.04 \\
.89 \\
9.47 \\
5.19 \\
.21 \\
2.40 \\
.22 \\
.03 \\
.15 \\
.21 \\
.04 \\
.09 \\
\text { trace } \\
.06 \\
.03 \\
.08 \\
\text { (?) } \\
\text { none }\end{array}$ & 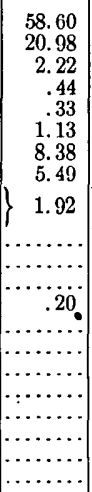 & 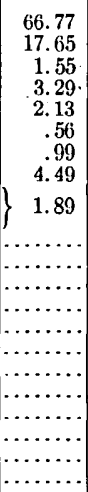 & 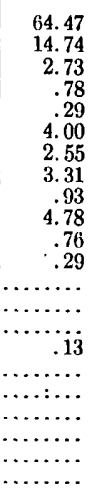 \\
\hline & 99.97 & 99.69 & 99.32 & 99.76 \\
\hline
\end{tabular}

\section{WYOMING.}

Rocks A to J, inclusive, studied by Whitman Cross. The Leucite Hills series is described in Am. Jour. Sci., 4th ser., vol. 4, p. 115. Analysis J is by L. G. Eakins, record No. 1078. The others are by W. F. Hillebrand, one made in the Denver laboratory, and record Nos. 343, 355, 362, 368, 403, 463, 1668, and 1682.

A. Wyomingite, Boars Tusk, Leucite Hills. Wyomingose. Contains phlogopite, leucite, diopside, and apatite. Sp. gr., $2.779,13.5^{\circ}$. P. R. C. 566.

B. Wyomingite. Fifteenmile Spring, Leucite Hills. Orendose. Composition like A. Sp. gr., $2.627,30^{\circ}$. P. R. C. 567 .

C. Portion of $\mathrm{B}$ soluble in 2.5 per cent nitric acid.

D. Phlogopite from wyomingite.

E. Madupite, Pilot Butte, Leucite Hills. Madupose. Contains predominating diopside and phlogopite, with perofskite and magnetite, in a glassy base which has approximately the composition of leucite. Sp. gr., 2.857, $22^{\circ}$. P. R. C. 576. 


\begin{tabular}{|c|c|c|c|c|c|}
\hline . & A. & B. & C. & D. & E. \\
\hline 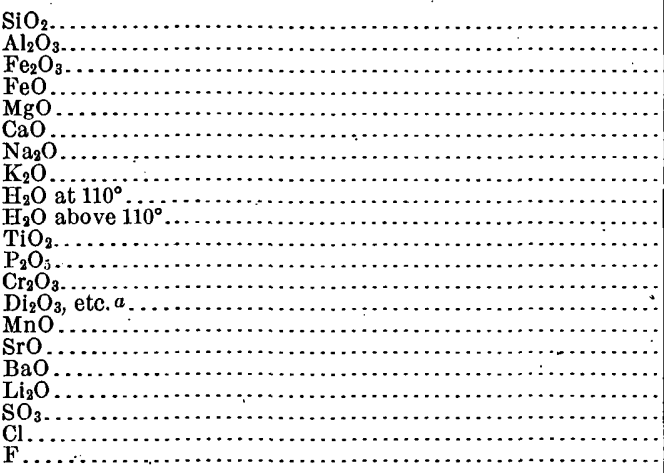 & $\begin{array}{r}50.23 \\
11.22 \\
3.34 \\
1.84 \\
7.09 \\
5.99 \\
1.37 \\
9.81 \\
.93 \\
1.72 \\
2.27 \\
1.89 \\
.10 \\
.03 \\
.05 \\
.24 \\
1.23 \\
\text { trace } \\
.74 \\
.03 \\
.50\end{array}$ & $\begin{array}{r}53.70 \\
11.16 \\
3.10 \\
1.21 \\
6.44 \\
3.46 \\
1.67 \\
11.16 \\
.80 \\
2.61 \\
1.92 \\
1.75 \\
.04 \\
\text { none } \\
.04 \\
.19 \\
.62 \\
\text { trace } \\
.06 \\
.03 \\
.44\end{array}$ & $\begin{array}{c}6.08 \\
.91 \\
\ldots . . . \\
.50 \\
1.51 \\
2.13 \\
.28 \\
1.21 \\
(?) \\
(?) \\
.21 \\
1.54 \\
\ldots \ldots \\
\ldots \ldots \\
\ldots .10 \\
.14 \\
\ldots \ldots . \\
.06 \\
.03 \\
\ldots \ldots .\end{array}$ & \begin{tabular}{r}
42.56 \\
12.18 \\
2.73 \\
.90 \\
22.40 \\
.20 \\
.44 \\
10.70 \\
\hdashline 2.35 \\
2.09 \\
$\ldots .06$ \\
.73 \\
$\ldots \ldots$ \\
$\ldots \ldots$. \\
trace \\
1.00 \\
trace \\
$\ldots \ldots$ \\
$\ldots \ldots$ \\
$\ldots 2.46$
\end{tabular} & $\begin{array}{r}42.65 \\
9.14 \\
5.13 \\
1.07 \\
10.89 \\
12.36 \\
.90 \\
7.99 \\
2.04 \\
2.18 \\
.1 .64 \\
1.52 \\
.07 \\
.11 \\
.12 \\
.33 \\
.89 \\
\text { trace } \\
.58 \\
.03 \\
.47\end{array}$ \\
\hline \multirow[t]{2}{*}{ 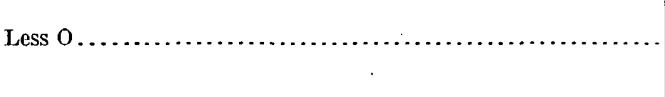 } & $\begin{array}{r}100.62 \\
.22\end{array}$ & $\begin{array}{r}100.40 \\
.19\end{array}$ & $\begin{array}{c}14.70 \\
\ldots \ldots\end{array}$ & $\begin{array}{r}100.80 \\
1.03\end{array}$ & $\begin{array}{r}100.11 \\
.20\end{array}$ \\
\hline & 100.40 & 100.21 & & 99.77 & 99.91 \\
\hline
\end{tabular}

a Probably contains other rare earths.

F. Orendite, Fifteenmile Spring, Leucite Hills. Orendose. Contains predominating leucite and sanidine, with phlogopite, a little biotite, diopside, and amphibole, and accessory apatite and rutile. Sp. gr., 2.686, 23. $5^{\circ}$. P. R. C. 72 and 572 .

G. Orendite, North Table Butte, Leucite Hills. Orendose. Composition like F. Sp. gr., 2.699, $19^{\circ}$. P. R. C. 570 .

H. Groundmass of F. Sp. gr., 2.615, $19^{\circ}$. Not cited in published paper.

I. Diopside separated from mixed wyomingite and madupite. Sp. gr., $3.290,20^{\circ}$. Analyzed in the Denver laboratory. $\mathrm{P}_{2} \mathrm{O}_{5}$ included with $\mathrm{TiO}_{2}$.

J. Dacite (?), Garfield Peak. Phenocrysts of plagioclase and hornblende, with microscopic sphene and apatite in a groundmass containing plagioclase, orthoclase, quartz, and magnetite. Sp. gr., 2.576, 26.2 ${ }^{\circ}$. P. R. C. 159.

\begin{tabular}{|c|c|c|c|c|c|}
\hline & F. & G. & $\mathrm{H}$. & I & J. \\
\hline $\mathrm{SiO}_{2} \ldots \ldots \ldots \ldots \ldots$ & 54.08 & 54.17 & 58.13 & 50.86 & 67.78 \\
\hline $\mathrm{Al}_{2} \mathrm{O}_{3}$ & 9.47 & 10.16 & $\begin{array}{l}28.13 \\
11.72\end{array}$ & & 16.67 \\
\hline $\mathrm{Fe}_{2} \mathrm{O}_{3} \ldots$ & 3.19 & 3.34 & 3.01 & 1.19 & 1.99 \\
\hline $\mathrm{FeO} \ldots .$. & 1.03 & .65 & 1.01 & 1.82 & .51 \\
\hline $\mathrm{MgO}$. & 6.74 & 6.62 & 5.79 & 17.42 & .71 \\
\hline $\mathrm{CaO}$. & 3.55 & 4.19 & 2.24 & 23.32 & 2.67 \\
\hline $\mathrm{Na}_{2} \mathrm{O}$. & 1. 39 & $\begin{array}{l}1.121 \\
1.21\end{array}$ & 1. 36 & .76 & 4.91 \\
\hline $\mathrm{K}_{2} \mathrm{O} \ldots \ldots \ldots$ & 11.76 & 11.91 & 12.58 & .42 & 3.43 \\
\hline $\mathrm{H}_{2} \mathrm{O}$ at $110^{\circ} \ldots$ & .79 & .52 & .99 & & 1.44 \\
\hline $\mathrm{H}_{2} \mathrm{O}$ above $110^{\circ} \ldots$. & 2.71 & 1.01 & 1. 16 & .31 & 1.44 \\
\hline $\mathrm{TiO}_{2} \ldots \ldots \ldots \ldots$ & 2.08 & 2.67 & 1. 48 & 3.03 & \\
\hline $\mathrm{P}_{2} \mathrm{O}_{5} \ldots$ & 1. 36 & -1.59 & & ........ & .19 \\
\hline $\mathrm{ZrO}_{2}$ & undet. & .22 & undet. & & $\cdots$ \\
\hline $\begin{array}{ll}\mathrm{N}_{2} \mathrm{O}_{3} \\
\mathrm{Cr}_{2} \mathrm{O}_{3}\end{array}$ & .02 & .05 & .......... & & $\cdots$ \\
\hline $\mathrm{NiO}$. & & trace & trose & 03 & trace \\
\hline $\begin{array}{l}\mathrm{MnO} \text {. } \\
\mathrm{SrO} .\end{array}$ & .05 & $\begin{array}{r}.00 \\
.18\end{array}$ & trace & & trace \\
\hline $\mathrm{BaO}$. & .67 & .59 & .29 & & …?... \\
\hline $\mathrm{Li}_{2} \mathrm{O} \ldots$ & trace & trace & trace & & $\cdots \cdots$ \\
\hline 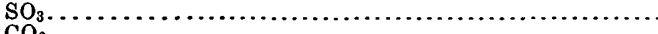 & .29 & .16 & .13 & & \\
\hline $\begin{array}{ll}\mathrm{Cl} . \\
\mathrm{Cl}\end{array}$ & .04 & .06 & trace & & \\
\hline & .49 & .36 & trace & & \\
\hline \multirow{3}{*}{ Less } & 99.98 & 100.21 & 100.31 & 99.16 & 100.30 \\
\hline & & & & & \\
\hline & 99.77 & 100.04 & & & \\
\hline
\end{tabular}


The four following rocks from Encampment quadrangle are described by A. C. Spencer in P. P. 25. Analyses by E. T. Allen, record No. 2048.

K. Norite, near head of Cow Creek, about three-fourths of a mile from Bridger Peak. Auvergnose. Contains chiefly hypersthene and labradorite, the latter slightly altered to sericite. Biotite and magnetite are present, with a few grains of diallage and a little hornblende.

L. Diorite derived from norite, altered form of $\mathrm{K}$. The pyroxene is completely changed to uralite and the feldspar is largely decomposed.

M. Diorite derived from norite, 2 miles west of the Verdi mine. Contains uralite and labradorite, with a little biotite and magnetite.

N. Diorite, probably derived from gabbro, near head of Big Sandstone Creek. Contains much zoisite derived from the feldspars.

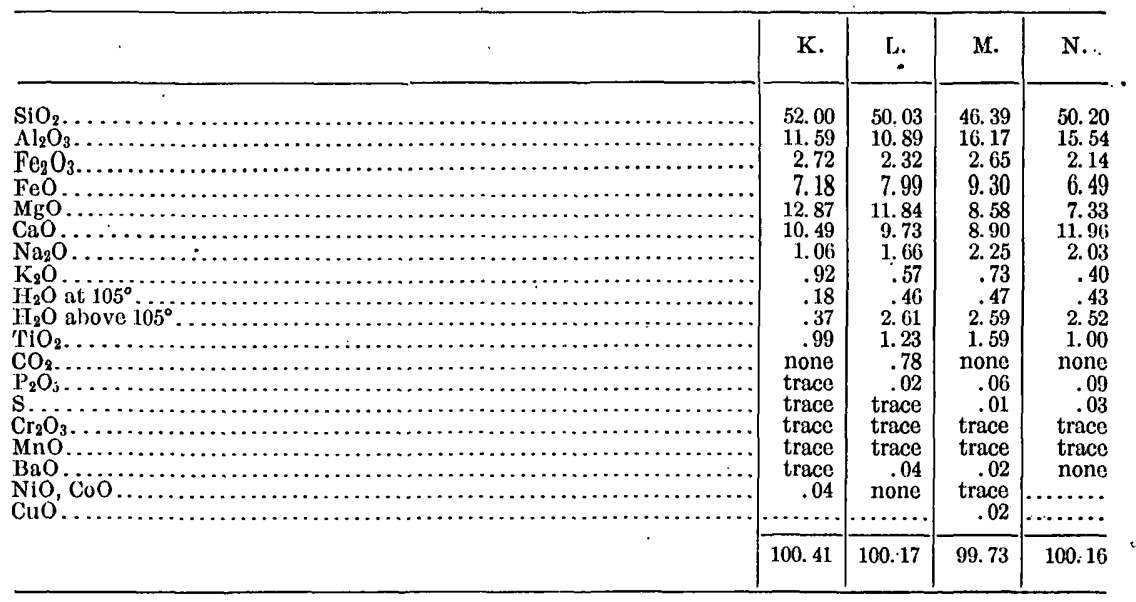

The following rocks; from Sundance quadrangle, are described by W. S. T. Smith in Folio 127. Analyses by George Steiger, record No. 1999.

O. Trachytoid phonolite. Pulaskose. Contains phenocrysts of orthoclase, ægirineaugite, a little augite, nepheline (?), magnetite, garnet, titanite, and apatite. Groundmass mainly feldspar, probably orthoclase, and other minerals as above, with possibly some species of the sodalite group. P. R. C. 1756.

P. Phonolite. Essexose. Contains phenocrysts of feldspar, apparently orthoclase and anorthoclase, ægirine-augite or little augite, garnet, probably nosean, titanite, magnetite, and apatite. In the groundmass, feldspar (orthoclase ?), ægirine-augite, and possibly sodalite. P. R. C. 1757 .

Q. Trachytoid phonolite. Akerose-laurvikose. Contains phenocrysts of feldspar (orthoclase and plagioclase ?), ægirine-augite, magnetite, titanite, and apatite. In the groundmass, feldspar, magnetite, ægirine-augite, and apatite. P. R. C. 1758.

R. Augite vogesite. Kentallenose. Contains phenocrysts of augite, magnetite, apatite, biotite, orthoclase, and plagioclase. Same minerals in the groundmass, with with perhaps some species of the nepheline-sodalite group. 


\begin{tabular}{|c|c|c|c|c|}
\hline . & O. & $\mathbf{P}$. & Q. & R. \\
\hline 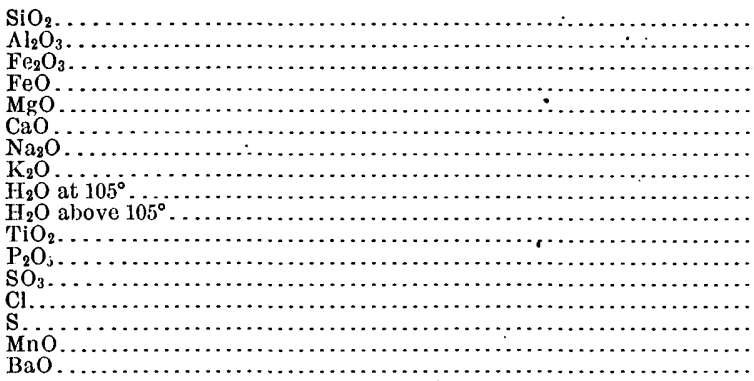 & $\begin{array}{r}55.14 \\
18.98 \\
2.60 \\
1.62 \\
.32 \\
3.96 \\
5.38 \\
6.64 \\
.63 \\
3.70 \\
.50 \\
.17 \\
.10 \\
\text { trace } \\
.03 \\
\text { trace } \\
\ldots . . .\end{array}$ & $\begin{array}{r}57.46 \\
18.41 \\
2.40 \\
1.28 \\
\text { none } \\
4.02 \\
9.23 \\
4.93 \\
.45 \\
1.12 \\
.42 \\
.11 \\
.50 \\
.23 \\
.05 \\
.01 \\
\text { none }\end{array}$ & $\begin{array}{r}58.08 \\
18.38 \\
3.02 \\
1.42 \\
.96 \\
3.85 \\
6.22 \\
5.11 \\
.37 \\
1.55 \\
.58 \\
.21 \\
.07 \\
\text { trace } \\
\text { none } \\
.10 \\
\text { none }\end{array}$ & $\begin{array}{r}42.95 \\
12.44 \\
10.16 \\
5.18 \\
5.82 \\
13.11 \\
2.10 \\
2.29 \\
.91 \\
1.98 \\
1.34 \\
1.37 \\
.15 \\
.07 \\
\text { none } \\
.29 \\
\text { none }\end{array}$ \\
\hline \multirow{2}{*}{ 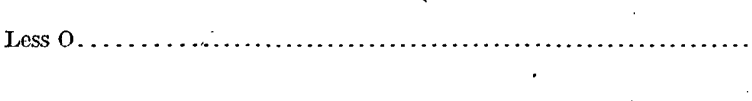 } & $\begin{array}{r}99.77 \\
.01\end{array}$ & $\begin{array}{r}100.72 \\
.08\end{array}$ & $\begin{array}{r}99.92 \\
\ldots \ldots\end{array}$ & $\begin{array}{r}100.16 \\
.02\end{array}$ \\
\hline & 99.76 & 100.64 & & 100. 14 \\
\hline
\end{tabular}

No $\mathrm{CO}_{2}$ in any of these rocks.

YELLOWSTONE NATIONAL PARK AND THE ABSAROKA RANGE.

1. ELECTRIC PEAK.

Rocks described by Iddings in 12th Ann., p. 577. Also in Bull. Philos. Soc. Washington, vol. 11, p. 206. For analyses $\mathrm{O}, \mathrm{P}$, see Mon. XXXII, pt. 2.

A. Pyroxene-mica diorite. Tonalose. Contains augite, hypersthene, plagioclase, quartz, biotite, hornblende, magnetite, and apatite. Analysis by J. E. Whitfield, record No. 830. P. R. C. 94.

B. Pyroxene-mica diorite. Andose. Same minerals as under A. Analysis by Whitfield, No. 921.

C. Pyroxene-mica diorite. Tonalose. Like A and B. Analysis by W. H. Melville, record No. 1231.

D. Pyroxene porphyry. Andose. Contains augite, hypersthene, biotite, magnetite, plagioclase, and quartz. Analysis by Whitfield, No. 830 .

E. Quartz-pyroxene-mica diorite. Tonalose. Contains biotite, hornblende, augite, hypersthene, magnetite, plagioclase, orthoclase, and quartz. Analysis by Melville, No. 1231.

\begin{tabular}{|c|c|c|c|c|c|}
\hline & A. & B. & C. & D. & E. \\
\hline 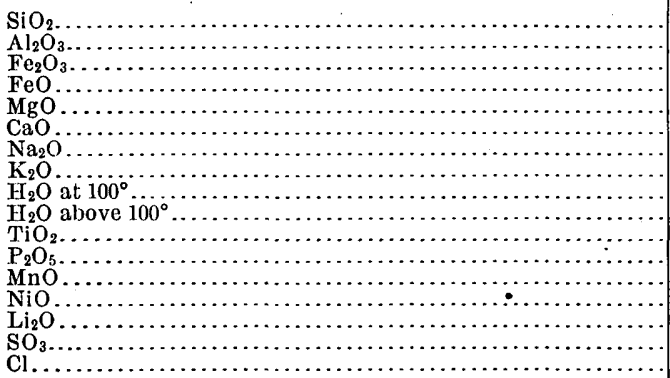 & $\begin{array}{r}56.28 \\
14.23 \\
4.69 \\
4.05 \\
6.37 \\
7.94 \\
2.98 \\
1.23 \\
.93 \\
.84 \\
.40 \\
.16 \\
.01 \\
\text { trace } \\
.17\end{array}$ & $\begin{array}{r}58.05 \\
18.00 \\
2.49 \\
4.56 \\
3.55 \\
6.17 \\
3.64 \\
2.18 \\
.86 \\
1.05 \\
.17 \\
\text { none } \\
\ldots \ldots \\
\text { none } \\
.07 \\
\text { trace }\end{array}$ & $\begin{array}{r}61.22 \\
16.14 \\
3.01 \\
2.58 \\
4.21 \\
5.46 \\
4.48 \\
1.87 \\
.04 \\
.40 \\
.61 \\
.25 \\
\text { trace } \\
.09 \\
\ldots \ldots . . \\
\ldots \ldots . . . \\
\ldots . . .\end{array}$ & $\begin{array}{r}57.38 \\
16.86 \\
2.49 \\
5.17 \\
5.51 \\
7.32 \\
3.33 \\
1.45 \\
.42 \\
\text { trace } \\
\text { trace } \\
\text { trace } \\
\cdots . .39 \\
.39 \\
.21 \\
.17\end{array}$ & $\begin{array}{r}64.07 \\
15.82 \\
3.40 \\
1.44 \\
3.39 \\
4.43 \\
4.06 \\
2.27 \\
.10 \\
.42 \\
.45 \\
.18 \\
\text { trace } \\
.05 \\
\ldots \ldots . . \\
\ldots \ldots . . . \\
\ldots . . .\end{array}$ \\
\hline 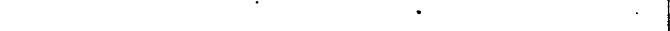 & 100.28 & 100.79 & 100.36 & 100.70 & 100.08 \\
\hline
\end{tabular}


F. Quartz-mica diorite. Tonalose. Contains biotite, hornblende, augite, hypersthene, plagioclase, orthoclase, and quartz. Analysis by Whitfield, No. 921 .

G. Quartz-mica diorite. Yellowstonose. Contains biotite, hornblende, plagioclase, pyroxene, quartz, and orthoclase. Analysis by Whitfield, No. 921.

H, I, J. Quartz-mica diorites. Yellowstonose. Contain hornblende, biotite, plagioclase, orthoclase, and quartz. Analyses by Whitfield, Nos. 921 and 830 .

\begin{tabular}{|c|c|c|c|c|c|}
\hline & F. & G. & H. & I. & J. \\
\hline $\mathrm{SLO}^{\mathrm{Al}_{2} \mathrm{O}_{2}} \mathrm{~F}_{\mathrm{F}} \mathrm{F}_{2} \mathrm{O}_{2}$ & $\begin{array}{r}65.11 \\
16.21 \\
1.06 \\
3.19 \\
2.57 \\
3.97 \\
4.00 \\
2.51 \\
.94 \\
.71 \\
.02 \\
\text { none } \\
.04 \\
\text { trace } \\
\text { none } \\
\end{array}$ & $\begin{array}{r}65.60 \\
17.61 \\
.95 \\
2.76 \\
1.49 \\
3.72 \\
4.36 \\
2.36 \\
.59 \\
.75 \\
.16 \\
\text { none } \\
\text { trace } \\
\text { none } \\
\end{array}$ & $\begin{array}{r}64.85 \\
16.57 \\
2.10 \\
2.15 \\
2.14 \\
4.01 \\
3.71 \\
3.10 \\
.35 \\
.91 \\
.14 \\
\text { none } \\
\text { none } \\
\text { trace } \\
\text { none } \\
\end{array}$ & $\begin{array}{r}66.05 \\
16.96 \\
2.59 \\
1.38 \\
2.08 \\
3.37 \\
4.20 \\
2.53 \\
.69 \\
.34 \\
\text { trace } \\
\text { none } \\
\text { none } \\
.03 \\
\text { trace } \\
\end{array}$ & $\begin{array}{r}67.54 \\
17.02 \\
2.97 \\
.34 \\
.13 \\
3.36 \\
4.62 \\
2.28 \\
.55 \\
.80 \\
\text { trace } \\
\text { traco } \\
.03 \\
.26 \\
.15 \\
100.05 \\
\end{array}$ \\
\hline
\end{tabular}

K. Quartz-mica diorite porphyry. Yellowstonose. Contains biotite, hornblende, plagioclase, orthoclase, and quartz. Analysis by Whitfield, No. 830 .

L. Quartz-mica diorite porphyry. Lassenose. Contains quartz, biotite, plagioclase, alkali feldspar, and hornblende. Analysis by Whitfield, No. 830 .

M. Hornblende porphyry. Tonalose. Intrusive sheet. Contains hornblende, plagioclase, magnetite, and quartz. Analysis by Whitfield, No. 429.

N. Hornblende-mica porphyry. Yellowstonose. Intrusive sheet. Contains hornblende, plagioclase, biotite, magnetite, and quartz. Analysis by Whitfield, No. 420 . The Indian Creek laccolith.

O. Augite andesite porphyry. Monzonose. Intrusive sheet, upper portion. Contains malacolite, plagioclase, probably orthoclase, magnetite, and little biotite, with secondary chlorite or serpentine and actinolite. Analysis by Whitfield, No. 830 .

P. Same sheet as O, lower portion. Kentallenose. Analysis by W. F. Hillebrand, record No. 1571.

\begin{tabular}{|c|c|c|c|c|c|c|}
\hline . & K. & L. & M. & N. & 0. & P. \\
\hline 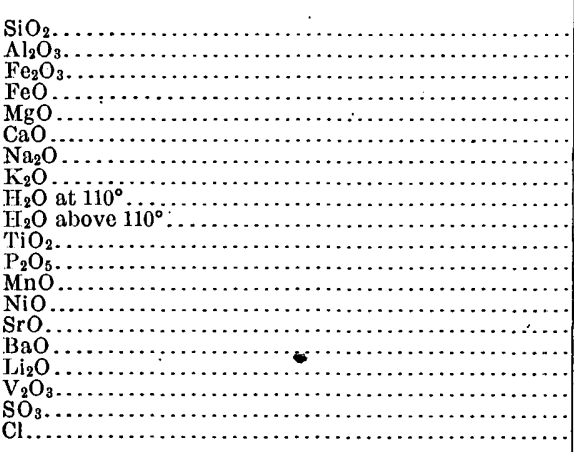 & $\begin{array}{r}65.97 \\
16.53 \\
2.59 \\
1.72 \\
2.11 \\
3.37 \\
3.41 \\
2.67 \\
1.23 \\
\text { trace } \\
\text { none } \\
\ldots \ldots \\
\ldots \ldots \\
\ldots .09 \\
. . . \\
.13 \\
.09\end{array}$ & $\begin{array}{r}69.24 \\
15.30 \\
1.72 \\
.69 \\
.95 \\
2.98 \\
4.46 \\
2.52 \\
1.30 \\
.65 \\
\text { trace } \\
\text { trace } \\
\ldots \ldots . \\
\ldots \ldots . . \\
\ldots \ldots . . \\
\text { none } \\
\ldots \ldots . . . \\
\text { trace }\end{array}$ & $\begin{array}{r}58.49 \\
16.70 \\
3.85 \\
2.37 \\
3.12 \\
5.90 \\
3.47 \\
1.59 \\
2.44 \\
1.71 \\
\text { trace } \\
.24 \\
\ldots \ldots . \\
\ldots \ldots \ldots \\
\ldots \ldots . . . \\
. \ldots .01 \\
\ldots .63 \\
\ldots \ldots . .\end{array}$ & 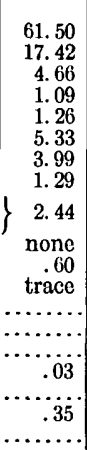 & $\begin{array}{r}52.10 \\
16.34 \\
3.84 \\
6.82 \\
4.33 \\
4.73 \\
4.02 \\
4.20 \\
1.74 \\
.79 \\
.68 \\
\operatorname{trace} \\
\ldots \ldots . \\
\ldots \ldots . . \\
\ldots \ldots . . \\
.13 \\
\ldots \ldots . . \\
.22 \\
.24\end{array}$ & $\begin{array}{r}50.59 \\
11.49 \\
1.83 \\
7.64 \\
11.27 \\
8.79 \\
2.27 \\
2.33 \\
.21 \\
1.76 \\
.80 \\
.48 \\
.17 \\
.06 \\
.03 \\
.10 \\
\text { trace } \\
.04 \\
\text { none } \\
\text { trace }\end{array}$ \\
\hline \multirow[t]{2}{*}{$\mathrm{O}=\mathrm{Cl}$} & $\begin{array}{r}100.33 \\
.02\end{array}$ & $\begin{array}{c}100.08 \\
\ldots \ldots \ldots\end{array}$ & $\begin{array}{c}100.52 \\
\ldots \ldots \ldots\end{array}$ & $\begin{array}{c}99.96 \\
\ldots \ldots\end{array}$ & $\begin{array}{r}100.18 \\
.05\end{array}$ & $\begin{array}{r}99.86 \\
\ldots \ldots \ldots\end{array}$ \\
\hline & 100.31 & & & $\ldots$ & 100.13 & \\
\hline
\end{tabular}




\section{SEPULCHRE MOUNTAIN.}

Rocks described by Iddings, 12th Ann., p. 633. Also in Bull. Philos. Soc. Washington, vol. 11, p. 210, and in Mon. XXXII, pt. 2.

A. Pyroxene andesite. Tonalose. Essentially composed of augite, hypersthene, and plagioclase. Analysis by J. E. Whitfield, record No. 923.

B. Pyroxene andesite. Andose. Composition like A. Analysis by Whitfield, No. 923 .

C. Hornblende andesite. Andose. Essentially plagioclase and hornblende. Analysis by Whitfield, No. 922 .

D. Hornblende-pyroxene andesite. Tonalose. Contains augite, hypersthene, plagioclase, and hornblende. Analysis by T. M. Chatard, record No. 712.

E. Hornblende-pyroxene andesite. Tonalose. Minerals as in D, with magnetite also. Analysis by Chatard, No. 712.

\begin{tabular}{|c|c|c|c|c|c|}
\hline & A. & B. & C. & D. & E. \\
\hline 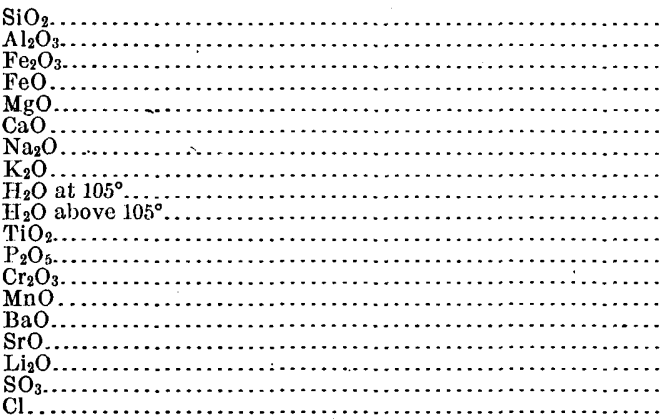 & $\begin{array}{r}55.83 \\
17.11 \\
4.07 \\
3.75 \\
5.05 \\
7.40 \\
2.94 \\
1.71 \\
1.28 \\
1.05 \\
.21 \\
\ldots \ldots \\
\text { none } \\
\cdots \cdots \\
\text { none } \\
\text { trace } \\
\text { none }\end{array}$ & $\begin{array}{r}57.17 \\
17.25 \\
2.48 \\
4.31 \\
4.83 \\
6.61 \\
3.44 \\
2.03 \\
1.20 \\
1.03 \\
.05 \\
\ldots \ldots . . \\
\text { none } \\
\ldots \ldots . . \\
\ldots \ldots . . . \\
\text { trace } \\
\text { trace } \\
\text { trace }\end{array}$ & \begin{tabular}{r}
55.92 \\
17.70 \\
3.16 \\
4.48 \\
4.34 \\
5.90 \\
4.08 \\
2.24 \\
1.42 \\
.94 \\
.18 \\
\hdashline$\ldots .$. \\
trace \\
\hdashline$\ldots .$. \\
\hdashline$\ldots . .$. \\
trace \\
none
\end{tabular} & $\begin{array}{r}56.61 \\
13.62 \\
5.89 \\
2.60 \\
5.48 \\
6.61 \\
3.13 \\
2.71 \\
1.20 \\
1.07 \\
.79 \\
.06 \\
.05 \\
.35 \\
.14 \\
\text { trace } \\
\ldots . . . \\
\text { (?) }\end{array}$ & $\begin{array}{r}60.30 \\
16.31 \\
4.35 \\
1.41 \\
2.39 \\
5.62 \\
3.99 \\
2.36 \\
.64 \\
1.86 \\
.76 \\
.20 \\
\text { trace? } \\
.13 \\
.15 \\
\text { trace } \\
\ldots . . .10 \\
. .10 \\
\ldots . . . .\end{array}$ \\
\hline & 100.40 & 100.40 & 100.45 & 100.31 & 100.57 \\
\hline
\end{tabular}

F. Hornblende-mica andesite. Yellowstonose. Contains plagioclase, hornblende, biotite, and magnetite. Analysis by Whitfield, No.'924.

G. Hornblende-mica andesite. Dacose. Contains plagioclase, hornblende, and biotite. Analysis by Chatard, No. 712 .

H. Dacite. Yellowstonose. Contains plagioclase, hornblende, biotite, and quartz.. Analysis by Whitfield, No. 830 .

I. Dacite. Lassenose. Minerals as in H. Analysis by L. G. Eakins, record No. 1135.

J. Andesite breccia. Lassenose. Not in paper cited. See Mon. XXXII, pt. 2. Analysis by Chatard, No. 712. Reported by Iddings as hornblende-mica andesite, containing hornblende, plagioclase, quartz, biotite, and a little magnetite. 


\begin{tabular}{|c|c|c|c|c|c|}
\hline & F. & G. & F. & I. & $\mathbf{J}$ \\
\hline 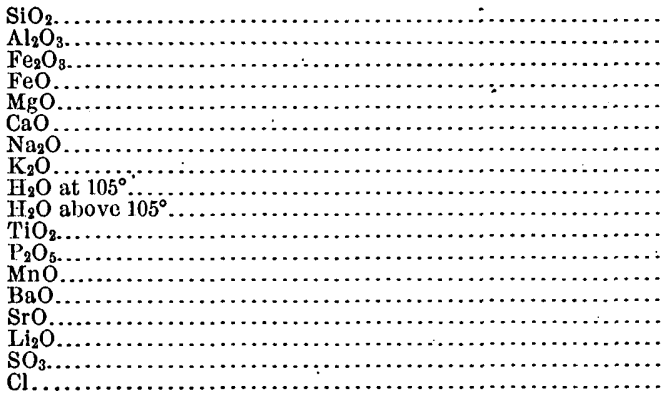 & $\begin{array}{r}64.27 \\
17.84 \\
3.36 \\
1.29 \\
2.00 \\
3.42 \\
3.84 \\
2.48 \\
1.32 \\
.32 \\
.16 \\
\text { none } \\
\cdots \\
\text { trace } \\
\text { none }\end{array}$ & $\begin{array}{r}65.50 \\
14.94 \\
1.72 \\
2.27 \\
2.97 \\
2.33 \\
5.46 \\
2.76 \\
.24 \\
1.13 \\
.45 \\
.09 \\
.20 \\
.13 \\
\text { trace? } \\
\ldots \ldots . . . \\
.06 \\
\ldots \ldots . .\end{array}$ & $\begin{array}{r}65.66 \\
15.61 \\
2.10 \\
2.07 \\
2.46 \\
3.64 \\
3.65 \\
2.03 \\
1.07 \\
1.37 \\
\text { trace } \\
\text { none } \\
\ldots \ldots . \\
\ldots . . . \\
.36 \\
.13 \\
.12\end{array}$ & $\begin{array}{r}67.49 \\
16.18 \\
1.30 \\
1.22 \\
1.34 \\
2.68 \\
4.37 \\
2.40 \\
2.69 \\
.13 \\
.13 \\
.08 \\
\ldots \ldots . . \\
\ldots \ldots \ldots . \\
\ldots \ldots \ldots . \\
\ldots \ldots \ldots .\end{array}$ & $\begin{array}{r}67.95 \\
14.98 \\
2.33 \\
.95 \\
1.42 \\
3.98 \\
4.39 \\
2.86 \\
.37 \\
.61 \\
.45 \\
.07 \\
.09 \\
.23 \\
\text { trace? } \\
\ldots \ldots . . . \\
.11 \\
\ldots \ldots . .\end{array}$ \\
\hline & 100.33 & 100.25 & 100.27 & 100.01 & 100.79 \\
\hline
\end{tabular}

\section{AQBSAROKA RANGE.}

CRANDALL BASIN.

Rocks described by Iddings in Mon. XXXII, pt. 2. The analyses also appeared in Bull. Philos. Soc. Washington, vol. 12, p. 204.

A. Gabbro porphyry, Hurricane Ridge. Camptonose. Contains augite, plagioclase, hypersthene, biotite, magnetite, and a little olivine. Analysis by I.. G. Eakins, record No. 1089.

B. Basalt flow, north side of Timber Creek. Andose. Rich in olivine, augite, and magnetite: Analysis by Eakins, No. 1087.

C. Basalt dike, ridge south of Hurricane Ridge, Shoshonose. Iike B. Contains also labradorite and a little orthoclase. Analysis by Eakins, No. 1087.

D. Mica gabbro porphyry, Hurricane Ridge. Camptonose. Contains plagioclase, augite, hypersthene, biotite, and magnetite. Analysis by Eakins, No. 1089.

E. Mica gabbro, Hurricane. Ridge. Andose. Like D, with some orthoclase and a little quartz and olivine. Analysis by Eakins, No. 1089.

F. Basalt-glass brecicia, ridge south of Indian Peak. Andose. A glass showing crystals of olivine, augite, plagioclase, and magnetite. Analysis by Eakins, No. 1087.

\begin{tabular}{|c|c|c|c|c|c|c|}
\hline . & A. & B. & C. & D. & IE. & F. \\
\hline \multirow[t]{2}{*}{ 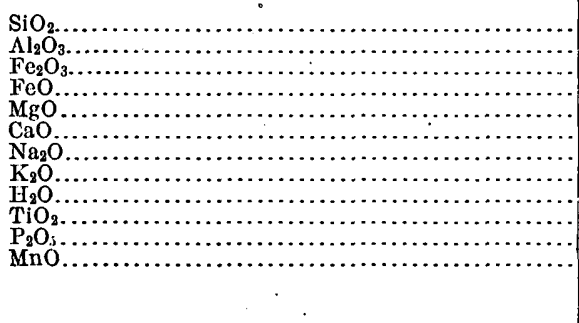 } & $\begin{array}{r}51.81 \\
15.24 \\
3.66 \\
4.86 \\
8.89 \\
9.06 \\
2.83 \\
2.08 \\
.67 \\
.77 \\
.18 \\
.08\end{array}$ & $\begin{array}{r}52.09 \\
17.84 \\
4.27 \\
.4 .56 \\
5.33 \\
8.03 \\
3.39 \\
1.98 \\
1.77 \\
.39 \\
.27 \\
.14\end{array}$ & $\begin{array}{r}52.11 \\
16.58 \\
3.66 \\
4.99 \\
6.87 \\
6.43 \\
3.25 \\
3.20 \\
1.99 \\
.53 \\
.63 \\
.23\end{array}$ & $\begin{array}{r}53.56 \\
16.07 \\
3.21 \\
5.29 \\
7.23 \\
8.77 \\
3.06 \\
1.94 \\
.19 \\
.68 \\
.18 \\
.11\end{array}$ & $\begin{array}{r}53.71 \\
18.00 \\
3.99 \\
4.05 \\
5.19 \\
6.88 \\
3.50 \\
3.10 \\
.55 \\
.74 \\
.38 \\
.24\end{array}$ & $\begin{array}{r}53.89 \\
18.81 \\
4.92 \\
2.81 \\
3.29 \\
5.42 \\
3.65 \\
2.98 \\
2.99 \\
.49 \\
.52 \\
.17\end{array}$ \\
\hline & 100.13 & 100.06 & 100.47 & 100.29 & 100.33 & 99.94 \\
\hline
\end{tabular}


G. Orthoclase gabbro-diorite, rich in mica; Hurricane Ridge. Andose. Contains orthoclase, plagioclase, quartz, biotite, augite, hypersthene, magnetite, and hornblende. Analysis by Eakins, No. 1089.

H. Another sample, like G. Andose. Same analyst and record number.

I. Dioritic facies of gabbro, Hurricane Ridge. Andose. Very feldspathic. Same analyst and number.

J. Monzonite, Hurricane Ridge. Andose. Contains orthoclase, plagioclase, biotite, augite, hypersthene, and magnetite. Analysis by W. H. Melville, record No: 1233.

K. Augite andesite porphyry. Andose. Intrusive sheet, Hurricane Ridge. Analysis by Eakins, No. 1088. Contains plagioclase, augite, hypersthene, magnetite, biotite, quartz, and microscopic orthoclase.

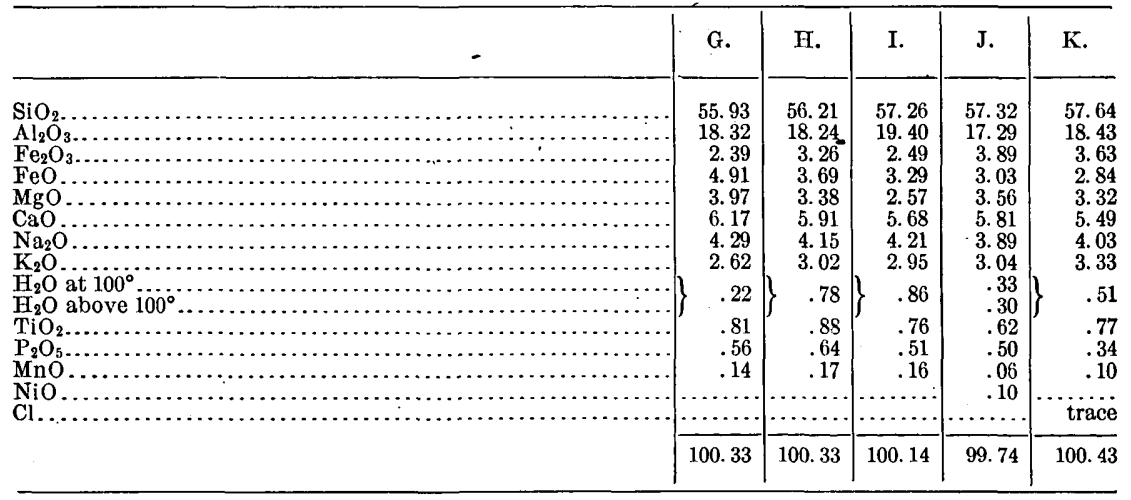

L. Hornblende-mica andesite porphyry dike, ridge south of Hurricane Ridge. Tonalose. Contains plagioclase, hornblende, biotite, augite, hypersthene, and magnetite, with a little chlorite or serpentine. Analysis by Eakins, No. 1087.

M. Quartz diorite porphyry, Hurricane Ridge. Yellowstonose. Contains plagioclase, orthoclase, quartz, augite, hypersthene, magnetite, and a little biotite. Analysis by Melville, No. 1234.

N. Quartz-mica diorite, Hurricane Ridge. Adamellose. Contains andesine, orthoclase, quartz, biotite, hornblende, magnetite, and a little pyroxene. Analysis by Melville, No. 1234.

0. Quartz-mica diorite porphyry, Hurricane Ridge. Toscanose. Contains andesine, orthoclase, quartz, and biotite. Analysis by Melville, No. 1234.

P. Aplite dike, Hurricane Ridge. Toscanose. Contains quartz, orthoclase, oligoclase, biotite, magnetite, some chlorite, and a little hornblende. Analysis by Eakins, No. 1088

\begin{tabular}{|c|c|c|c|c|c|}
\hline & L. & M. & N. & 0. & P. \\
\hline 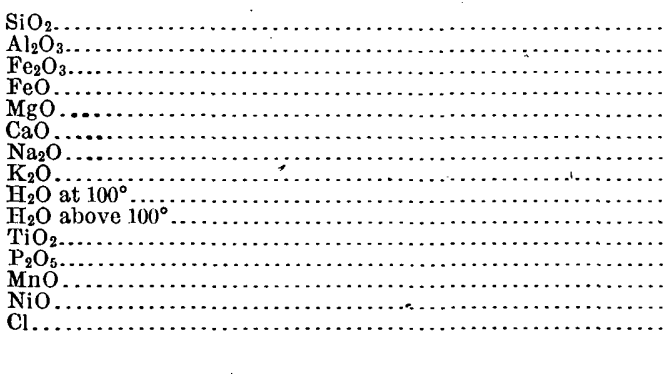 & $\begin{array}{r}61.16 \\
16.17 \\
2.89 \\
2.18 \\
3.89 \\
4.26 \\
3.87 \\
3.20 \\
2.09 \\
.23 \\
.13 \\
\text { trace } \\
\cdots \cdots \cdots \\
\cdots \cdots\end{array}$ & \begin{tabular}{r|}
63.42 \\
17.16 \\
3.09 \\
1.50 \\
1.64 \\
4.65 \\
4.51 \\
3.04 \\
.16 \\
.28 \\
.35 \\
.26 \\
.04 \\
.19 \\
$\cdots$ \\
100.29 \\
\end{tabular} & $\begin{array}{r}63.97 \\
15.78 \\
2.35 \\
1.87 \\
2.84 \\
3.71 \\
4.36 \\
4.01 \\
.09 \\
.49 \\
.48 \\
.40 \\
.05 \\
\text { trace } \\
\end{array}$ & $\begin{array}{r}64.40 \\
15.77 \\
2.47 \\
1.15 \\
2.12 \\
3.54 \\
4.10 \\
3.81 \\
.31 \\
1.93 \\
.40 \\
.16 \\
.04 \\
.17 \\
\ldots \ldots . . \\
100.27\end{array}$ & $\begin{array}{r}71.62 \\
14.99 \\
1.27 \\
1.01 \\
.74 \\
1.33 \\
3.62 \\
4.81 \\
.41 \\
.08 \\
\text { trace } \\
.17 \\
\ldots \ldots . \\
\text { trace }\end{array}$ \\
\hline
\end{tabular}




\section{SUNLIGHT INTRUSIVES.}

Descriptions supplied by Arnold Hague and T. A. Jaggar, jr. Analyses $\Lambda, B$, and C by W. F. Hillebrand, record No. 1801; D by H. N. Stokes, No. 1804.

A. Quartz syenite, Copper Creek Basin. Laurvikose. Contains biotite, hornblende, epidote, orthoclase, augite, titanite, magnetite; and apatite. Hornblende very pale in color.

B. Syenite porphyry, Sulphur Creek Basin. Lassenose. Contains oligoclase and biotite, in a groundmass of quartz and feldspar.

C. Augite syenite porphyry, Copper Creek Basin. Laurvikose. Contains augite, biotite, orthoclase, a little hornblende, and quartz.

D. Gabbro, southwest of Beams Hill, Sunlight Valley. Andose. Contains plagioclase, pyroxene, magnetite, apatite, and a little biotite.

\begin{tabular}{|c|c|c|c|c|}
\hline . & A. & $B$. & C. & D. \\
\hline 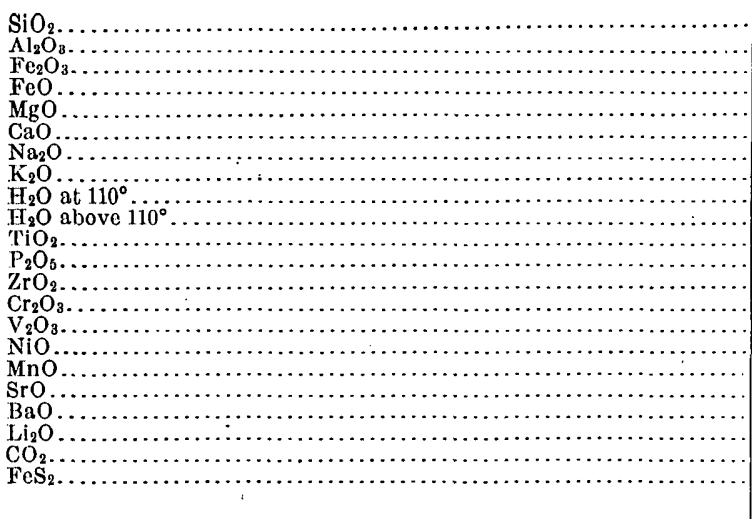 & $\begin{array}{r}63.07 \\
17.47 \\
2.09 \\
1.38 \\
1.44 \\
2.27 \\
5.77 \\
4.59 \\
.25 \\
.43 \\
.38 \\
.18 \\
\text { trace } \\
\text { trace } \\
\text { trace } \\
\text { none } \\
.03 \\
.15 \\
.32 \\
\text { trace? } \\
\text { none } \\
.02\end{array}$ & $\begin{array}{r}66.64 \\
16.22 \\
1.84 \\
1.06 \\
1.25 \\
2.41 \\
5.11 \\
3.86 \\
.52 \\
.55 \\
.29 \\
.16 \\
.01 \\
\text { trace } \\
.01 \\
\text { none } \\
\text { trace } \\
.14 \\
.27 \\
\text { none } \\
\text { none } \\
\text { trace? }\end{array}$ & $\begin{array}{r}64.40 \\
16.90 \\
1.86 \\
1.37 \\
1.13 \\
2.60 \\
5.79 \\
4.56 \\
.16 \\
.39 \\
.23 \\
.21 \\
.02 \\
(?) \\
(?) \\
\text { none } \\
.07 \\
.14 \\
.27 \\
\text { trace } \\
\text { none } \\
\text { trace? }\end{array}$ & $\begin{array}{r}53.57 \\
17.78 \\
3.19 \\
4.93 \\
4.36 \\
6.22 \\
4.04 \\
3.04 \\
.27 \\
.80 \\
.89 \\
.44 \\
\ldots \ldots \ldots \\
\ldots \ldots \ldots \\
\ldots \ldots . . \\
\text { none } \\
: 07 \\
.13 \\
.21 \\
\text { trace } \\
\text { none } \\
\ldots \ldots . . \\
. . .\end{array}$ \\
\hline & 99.84 & 100.34 & 100.10 & 99.94 \\
\hline
\end{tabular}

\section{ISHÄWOOA INTRUSIVES.}

Descriptions supplied by Arnold Hague and T..A. Jaggar, jr. Analyses A and B by H. N. Stokes, record No. 1804; C and D by W. F. Hillebrand, No. 1765.

A. Granite porphyry, base of Crater Mountain. Yellowstonose. Contains oligoclase, orthoclase, biotite,-and quartz.

B. Diorite porphyry, Cabin Creek. Tonalose. Contains plagioclase, orthoclase, quartz, and abundant hornblende. This rock is intermediate between granite porphyry and diorite porphyry, but nearer to the latter.

C. Diorite, base of Needle Mountain. Yellowstonose. Contains plagioclase, quartz, biotite, with subordinate hornblende and orthoclase. Structure granitic.

D. Diabase, ềntrance to Shoshone Canyon. Andose. Contains plagioclase, augite, and chlorite. 


\begin{tabular}{|c|c|c|c|c|}
\hline & A. & B. & C. & D. \\
\hline 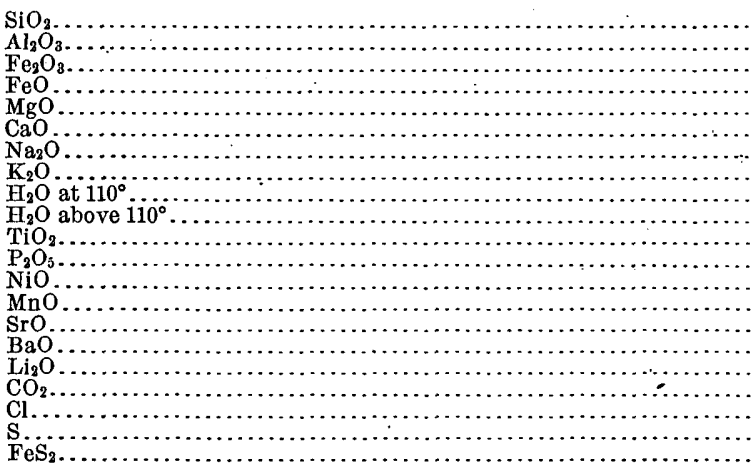 & $\begin{array}{r}64.23 \\
16.34 \\
1.07 \\
1.58 \\
2.47 \\
3.07 \\
3.49 \\
2.59 \\
.47 \\
1.76 \\
.50 \\
.18 \\
\text { none } \\
\text { trace } \\
.06 \\
.19 \\
\text { trace } \\
.30\end{array}$ & $\begin{array}{r}60.00 \\
16.37 \\
2.28 \\
2.46 \\
3.81 \\
4.96 \\
3.73 \\
2.70 \\
.61 \\
1.42 \\
.59 \\
.35 \\
\text { none } \\
.05 \\
.11 \\
.26 \\
\text { trace } \\
.17 \\
\ldots . . . \\
\ldots \ldots . . \\
\ldots . . .\end{array}$ & $\begin{array}{c}63.76 \\
16.01 \\
2.22 \\
1.96 \\
2.43 \\
4.55 \\
3.98 \\
2.84 \\
.28 \\
.57 \\
.52 \\
.25 \\
\text { none } \\
.09 \\
.09 \\
.17 \\
\text { trace } \\
.23 \\
\text { trace } \\
\text { (?) } \\
\ldots . . . .\end{array}$ & $\begin{array}{r}52.18 \\
18.19 \\
3.31 \\
4.36 \\
4.69 \\
6.51 \\
4.58 \\
1.88 \\
.75 \\
2.00 \\
.99 \\
.29 \\
\text { trace } \\
.14 \\
.06 \\
.11 \\
\text { trace } \\
\text { none } \\
\text { trace } \\
\text { none } \\
\ldots . . . .\end{array}$ \\
\hline & 99.91 & 99.87 & 99.95 & 100.04 \\
\hline
\end{tabular}

DIKES IN BRECCIA.

Descriptions supplied by Arnold Hague and T. A. Jaggar, jr. Analyses A, B, D, by H. N. Stokes, record No. 1804; C, E, F, G, H, by W. F. Hillebrand, Nos. 1765 and 1801 .

A. Hornblende-pyroxene andesite, Eagle Creek. Camptonose. Contains plagioclase, hornblende, pyroxene, quartz, and apatite.

B. Leucite absarokite, Sunlight Valley. Lamarose. Contains phenocrysts of olivine and augite, with secondary alteration of the olivine to serpentine. The groundmass contains magnetite, augite, plagioclase, and orthoclase. Leucite abundant.

C. Gabbro porphyry, Deer Creek. Shoshonose. Contains plagioclase, pyroxene, olivine, ilmenite, and apatite.

D. Hornblende-augite andesite, Wind River Plateau. Tonalose. Contains phenocrysts of augite, hypersthene, and hornblende, plagioclase, and fine magnetite grains.

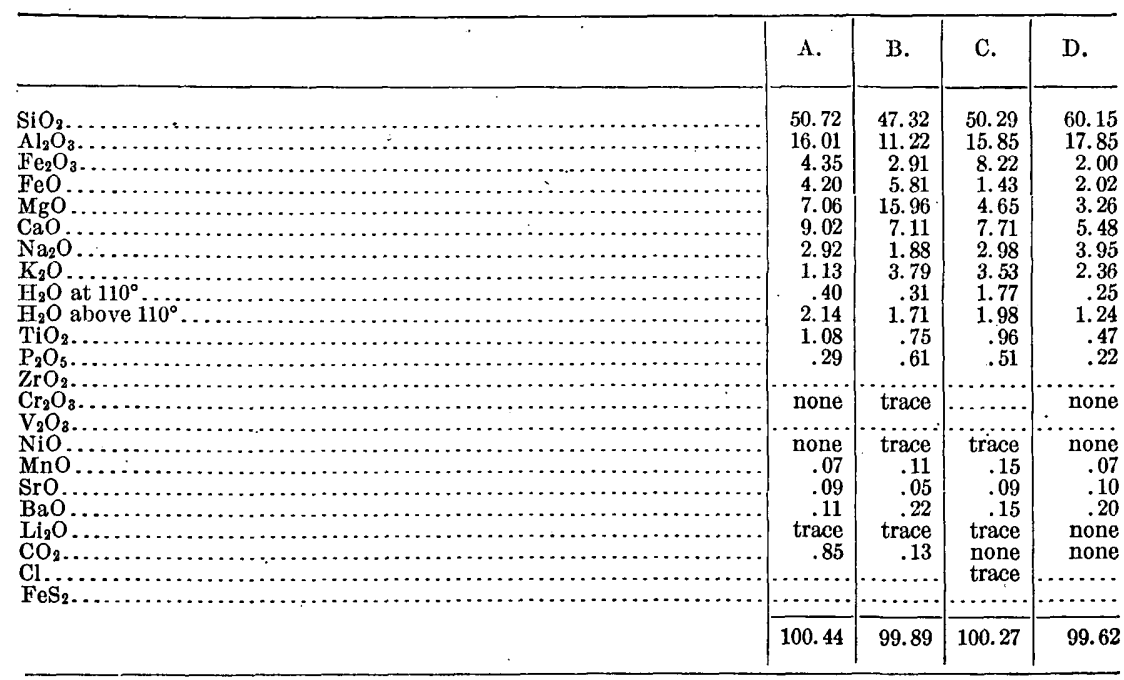


E. Augite andesite, Dike Mountain. Shoshonose. Contains augite, plagioclase, serpentinized olivine, magnetite, and apatite.

F. Trachyte andesite, Dike Mountain. Akerose. Contains plagioclase, orthoclase, chlorite, apatite, and magnetite. Very little augite.

G. Biotite trachyte, Dike Mountain. Nordmarkose. Contains plagioclase, orthoclase, biotite, magnetite, and chlorite.

H. Biotite trachyte, Dike Mountain. Pulaskose. Contains orthoclase, plagioclase, biotite, and magnetite.

\begin{tabular}{|c|c|c|c|c|}
\hline - & $\mathrm{E}$. & F. & G. & $\mathrm{H}_{\mathrm{t}}$ \\
\hline 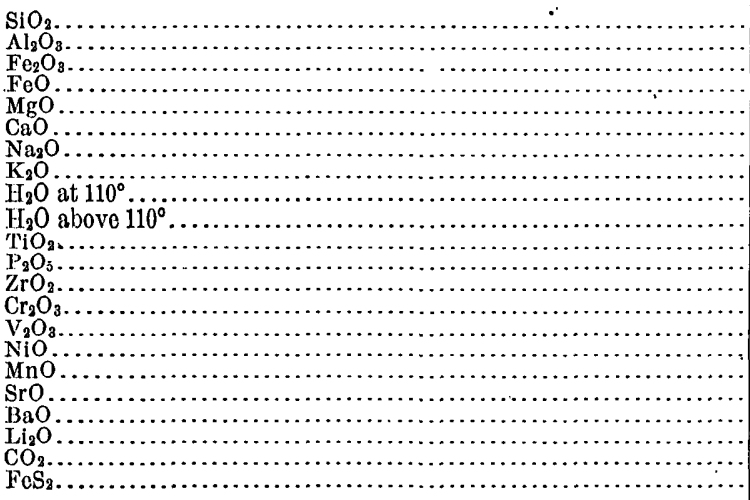 & $\begin{array}{r}51.17 \\
16.14 \\
4.11 \\
4.48 \\
4.82 \\
7.72 \\
2.99 \\
3.54 \\
.63 \\
2.24 \\
1.01 \\
.48 \\
\text { none } \\
\text { trace } \\
.04 \\
.01 \\
.21 \\
.10 \\
.20 \\
\text { trace } \\
\text { none } \\
.05\end{array}$ & $\begin{array}{r}52.47 \\
18.23 \\
3.31 \\
3.85 \\
2.85 \\
4.56 \\
4.83 \\
3.81 \\
.68 \\
2.03 \\
.97 \\
.64 \\
.02 \\
\text { trace } \\
.03 \\
\text { trace } \\
.15 \\
.11 \\
.23 \\
\text { trace } \\
1.01 \\
.04\end{array}$ & $\begin{array}{r}63.24 \\
17.98 \\
2.67 \\
.85 \\
.63 \\
.93 \\
6.27 \\
5.47 \\
.37 \\
.80 \\
.38 \\
.22 \\
\text { trace } \\
\text { none } \\
.01 \\
\text { none } \\
.04 \\
.03 \\
.25 \\
\text { trace } \\
\text { none } \\
\text {.trace }\end{array}$ & $\begin{array}{r}57.73 \\
18.93 \\
1.97 \\
1.92 \\
.91 \\
2.78 \\
5.52 \\
6.11 \\
.22 \\
2.93 \\
.33 \\
.25 \\
\text { trace } \\
\text { traco } \\
.01 \\
\text { trace? } \\
.06 \\
.09 \\
.16 \\
\text { traco } \\
.26 \\
.02\end{array}$ \\
\hline & 99.94 & 99.82 & 100.14 & 100.20 \\
\hline
\end{tabular}

\section{ABSAROKITE-SHOSHONITE-BANAKITE SERIES.}

Rocks from the Yellowstone Park and the Absaroka Range, described in Mon. XXXII, pt. 2, and also by Iddings in Jour. Geology, vol. 3, pp. 938, 943, 947. The rock A, from Ishawooa Canyon, is also described by Hague in Am. Jour. Sci., 3d ser., vol. 38, p. 46 .

A. Leucite absarokite, Ishawooa Canyon, Wyoming. Camptonose. Bowlder. Contains olivine and augite in a groundmass of orthoclase and leucite. Accessory minerals, magnetite, apatite, and a few flakes of brown mica. Analysis by J. E. Whitfield, record No. 1057 . Material dried at $104^{\circ}$.

B. Absarokite dike, head of Lamar River. Lamarose. Contains olivine and augite in a groundmass of orthoclase and plagioclase, with accessory magnetite, biotite, and ilmenite.

C. Absarokite dike, south of Clark Fork. Absarokose. Contains augite, quartz, biotite, magnetite, orthoclase, plagioclase, and sometimes analcite.

D. Absarokite lava flow, head of Raven Creek. Kentallenose. . Contains olivine, augite, orthoclase, labradorite, magnetite, apatite, and a little serpentine.

E. Absarokite dike, divide east of Cache Creek. Absarokose. Contains orthoclase, plagioclase, augite, biotite, magnetite, serpentine, and occasional quartz.

Analyses B, C, D, and E by L. G. Eakins, record Nos. 1086, 1365. 


\begin{tabular}{|c|c|c|c|c|c|}
\hline . & A. & B. & C. & D. & 'E. \\
\hline 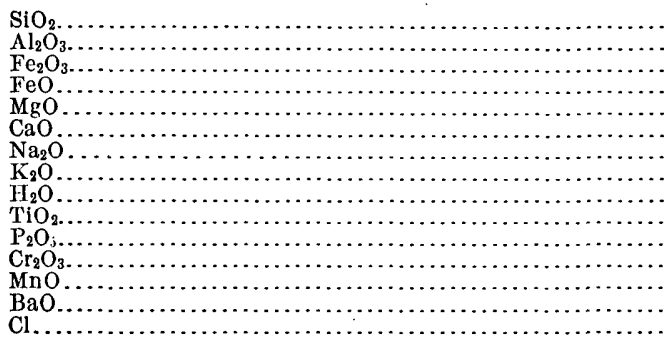 & $\begin{array}{r}47.28 \\
11.56 \\
3.52 \\
5.71 \\
13.17 \\
9.20 \\
2.73 \\
2.17 \\
2.96 \\
.88 \\
.59 \\
\ldots . .13\end{array}$ & $\begin{array}{r}48.95 \\
12.98 \\
3.63 \\
4.68 \\
11.73 \\
7.66 \\
2.31 \\
3.96 \\
3.16 \\
.49 \\
.67 \\
\ldots \ldots . . \\
.13 \\
\ldots \ldots . . . \\
\ldots \ldots . . .\end{array}$ & $\begin{array}{r}48.36 \\
12.42 \\
5.25 \\
2.48 \\
9.36 \\
8.65 \\
1.46 \\
3.97 \\
5.54 \\
1.18 \\
.84 \\
\text { trace } \\
.13 \\
.29\end{array}$ & $\begin{array}{r}51.76 \\
12.36 \\
4.88 \\
4.60 \\
9.57 \\
7.14 \\
1.99 \\
3.83 \\
3.05 \\
.47 \\
.56 \\
\ldots .11 \\
\ldots \ldots . . . \\
\ldots . . . .\end{array}$ & $\begin{array}{r}49.71 \\
13.30 \\
4.41 \\
3.37 \\
7.96 \\
8.03 \\
1.49 \\
4.81 \\
4.07 \\
1.57 \\
.66 \\
\text { trace } \\
.17 \\
.46 \\
\ldots . . .\end{array}$ \\
\hline \multirow[t]{2}{*}{ 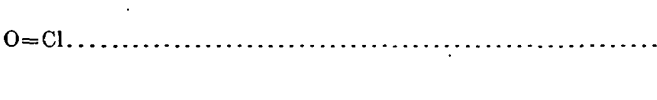 } & $\begin{array}{r}100.08 \\
.04\end{array}$ & $\begin{array}{c}100.35 \\
\ldots \ldots\end{array}$ & 99.93 & $\begin{array}{c}100.32 \\
\cdots\end{array}$ & $\begin{array}{r}100.01 \\
\ldots \ldots\end{array}$ \\
\hline & 100.04 & & & & \\
\hline
\end{tabular}

F. Shoshonite lava sheet, Lamar River, south of Bison Peak. Shoshonose. Contains plagioclase, orthoclase, augite, olivine, magnetite, and serpentine, with amygdules of zeolite and calcite. Analysis by Eakins, No. 1086.

G. Shoshonite lava sheet, southeast fork of Beaverdam Creek. Shoshonose. Contains plagioclase, orthoclase, augite, and serpentinized olivine.

H. Leucite (?) shoshonite lava sheet, mountain east of Pyramid Peak. Shoshonose. Contains feldspars, olivine, serpentine, augite, magnetite, brown mica, and impure leucite (?).

I. Olivine-free shoshonite dike, northeast of Indian Peak. Shoshonose. Contains augite, plagioclase, biotite, and magnetite.

J. Shoshonite lava sheet, Two Ocean Pass. Shoshonose. Contains orthoclase, serpentinized olivine, magnetite, augite, chlorite, biotite, apatite, labradorite. Dried at $104^{\circ}$ 906.

Analyses G, H, I by Eakins, Nos. 1371, 1375, 1379. Analysis J by Whitfield, No.

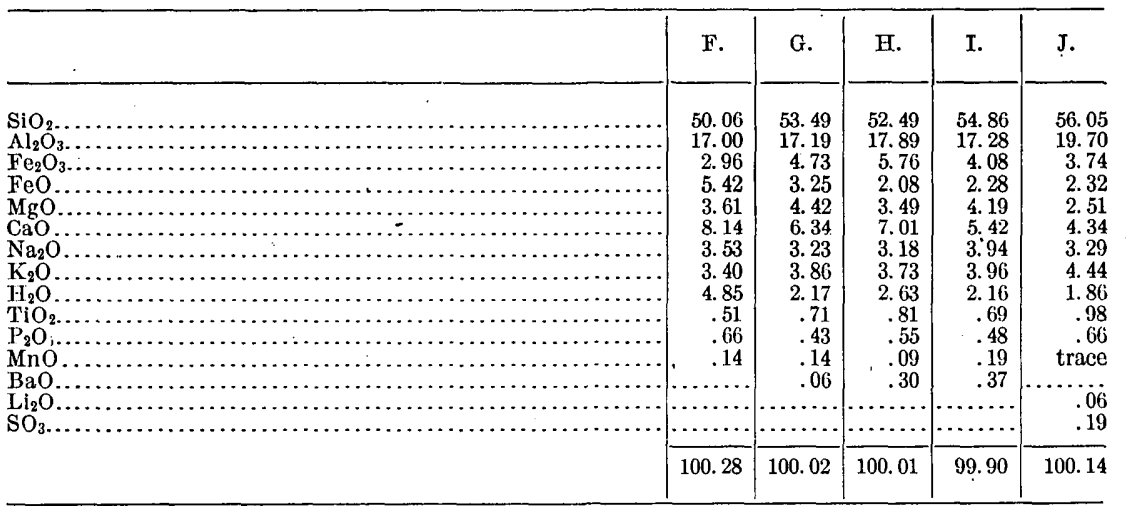

K. Banakite dike, head of Lamar River. Monzonose. Contains augite, serpentinized olivine, orthoclase, plagioclase, biotite, magnetite, ilmenite, apatite, and analcite. Adjoins rock B. Analysis by Eakins, No. 1375.

L. Banakite dike, Hoodoo Mountain. Monzonose. Like K, but with amygdaloidal zeolites. Analysis by Eakins, No. 1371.

M. Banakite dike, Ishawooa Canyon, Wyoming. Monzonose. Like $\mathrm{K}$ and $\mathrm{L}$, but more feldspathic. Contains a little serpentine, probably from olivine; also possibly analcite or sodalite. Analysis by Eakins, No. 1086. 
N. Banakite dike, near head of Stinkingwater River. Monzonose. Like M, but with more serpentine. Analysis by W. H. Melville, record No. 1232.

\begin{tabular}{|c|c|c|c|c|}
\hline & K. & L. & M. & N. \\
\hline 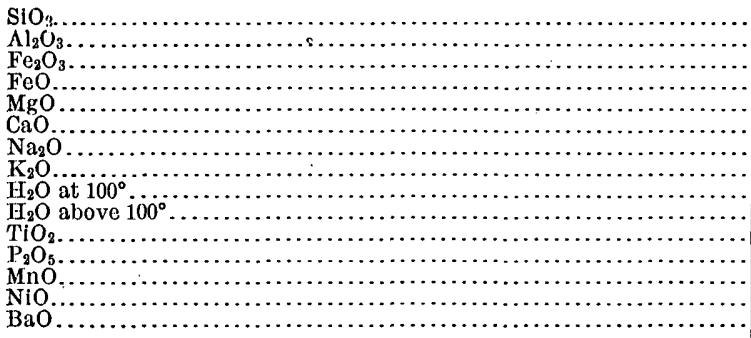 & $\begin{array}{r}51.82 \\
16.75 \\
4.56 \\
3.36 \\
4.03 \\
4.94 \\
3.91 \\
5.02 \\
3.97 \\
.71 \\
.52 \\
.23 \\
.26\end{array}$ & $\begin{array}{r}52.63 \\
16.87 \\
4.52 \\
3.11 \\
3.69 \\
4.77 \\
3.86 \\
5.17 \\
3.65 \\
.81 \\
.63 \\
.10 \\
\ldots . . . \\
.29\end{array}$ & $\begin{array}{r}51.46 \\
18.32 \\
4.61 \\
2.71 \\
2.91 \\
6.03 \\
4.11 \\
4.48 \\
3.89 \\
.83 \\
.86 \\
.17 \\
\ldots . . .\end{array}$ & $\begin{array}{r}52.33 \\
18.70 \\
4.95 \\
1.83 \\
2.69 \\
4.71 \\
4.51 \\
5.45 \\
.74 \\
2.71 \\
.71 \\
.81 \\
.03 \\
.14 \\
\ldots . .\end{array}$ \\
\hline & 100.08 & 100.10 & 100.38 & 100. 31 \\
\hline
\end{tabular}

O. Leucite banakite lava sheet, southeast fork of Beaverdam Creek. Shoshonose. Overlies rock G. Contains olivine, augite, leucite, feldspars, magnetite, apatite, and a little brown mica. Analysis by Eakins, No. 1378.

P. Earlier analysis of O, another sample, by J. E. Whitfield, record No. 907.

Q. Quartz banakite dike, near head of Stinkingwater River. Monzonose. Contains plagioclase, orthoclase, quartz, biotite, magnetite, augite, and a little calcite. Analysis by Melville, No. 1232.

R. Quartz banakite dike, near Q. Pulaskose. Contains plagioclase, orthoclase, quartz, biotite, magnetite, augite, chlorite, and serpentine. Analysis by Melville, No. 1232 .

\begin{tabular}{|c|c|c|c|c|}
\hline$\cdot$ & 0 & P. & Q. & $\mathrm{R}$. \\
\hline 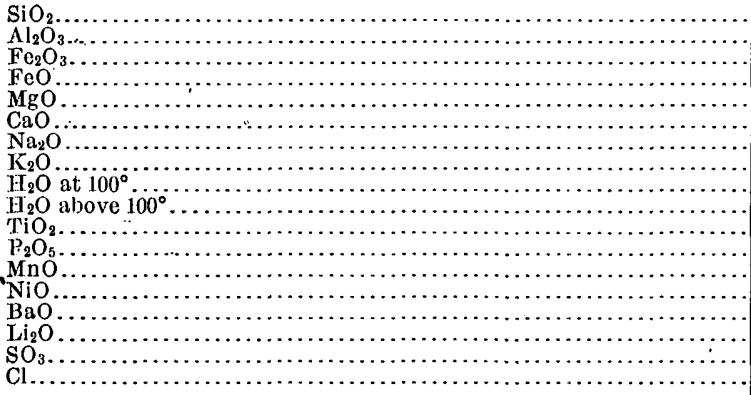 & $\begin{array}{r}52.93 \\
19.67 \\
3.07 \\
3.50 \\
2.88 \\
4.69 \\
4.20 \\
4.75 \\
2.73 \\
.72 \\
.59 \\
.15 \\
\ldots . . . \\
21 \\
\ldots \ldots\end{array}$ & $\begin{array}{r}51.56 \\
21.00 \\
5.17 \\
2.76 \\
2.52 \\
4.83 \\
4.37 \\
4.13 \\
2.27 \\
: 65 \\
69 \\
\operatorname{trace} \\
\cdots \ldots \\
. \cdots \\
.21 \\
\operatorname{trace}\end{array}$ & $\begin{array}{r}57.29 \\
18.45 \\
4.38 \\
1.20 \\
2.08 \\
3.57 \\
4.43 \\
5.43 \\
.17 \\
2.01 \\
.72 \\
.46 \\
\text { trace } \\
.12 \\
\ldots \ldots . . \\
\ldots \ldots \ldots \\
\ldots \ldots . . \\
\ldots \ldots . .\end{array}$ & $\begin{array}{r}6089 \\
17.14 \\
3.32 \\
.95 \\
1.16 \\
3.58 \\
4.54 \\
5.71 \\
.39 \\
1.22 \\
.49 \\
.27 \\
.09 \\
.19 \\
\ldots \ldots . . \\
\ldots \ldots . . \\
\ldots \ldots . . \\
\ldots \ldots .\end{array}$ \\
\hline & 100.09 & 100.29 & 10031 & 99.94 \\
\hline
\end{tabular}

S. Absarokite, Two Ocean Pass. Kentallenose. Contains augite, olivine, orthoclase, magnetite, serpentine, little biotite, apatite, and an isotropic substance, probably glass. Analysis by J. E. Whitfield, record No. 906.

T. Shoshonite, Beaverdam Creek. Shoshonose. Analysis by Whitfield, No. 907. Probably the rock already represented by analysis $\mathrm{G}$.

U. Shoshonite, northeast spur of Sepulchre Mountain. Shoshonose. Contains augite and serpentinized olivine, in a groundmass of plagioclase, orthoclase, augite, magnetite, and apatite. Analysis by Whitfield, No. 908.

V. Shoshonite, Baldy Mountain, Bear Gulch, Montana. Shoshonose. Contains hypersthene, augite, olivine, plagioclase, orthoclase, and magnetite. Analysis by Whitfield, No. 909.

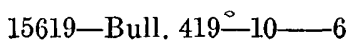




\begin{tabular}{|c|c|c|c|c|}
\hline . & S. & $\mathbf{T}$ & U. - & V. \\
\hline 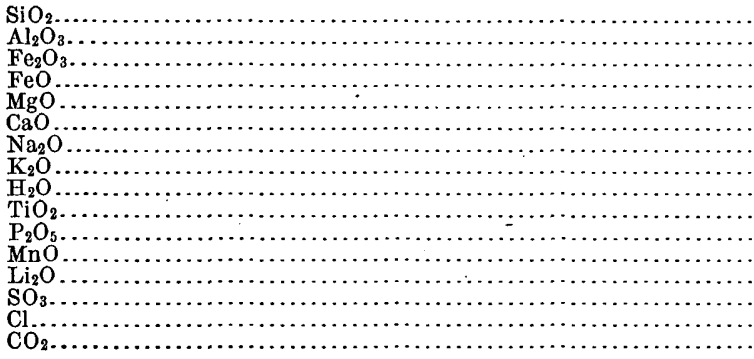 & $\begin{array}{r}51.68 \\
14.07 \\
4.71 \\
4.57 \\
7.72 \\
6.65 \\
2.45 \\
4.16 \\
2.09 \\
1.08 \\
.72 \\
\text { trace } \\
\text { trace } \\
.13 \\
\cdots \cdots \\
\cdots \cdots\end{array}$ & $\begin{array}{r}52.86 \\
17.51 \\
5.18 \\
3.31 \\
4.18 \\
6.51 \\
3.22 \\
3.41 \\
1.76 \\
1.04 \\
.53 \\
\text { trace } \\
.04 \\
.22 \\
.16 \\
\ldots \ldots . .\end{array}$ & $\begin{array}{r}51.75 \\
17.48 \\
6.42 \\
1.46 \\
4.05 \\
8.20 \\
3.33 \\
3.72 \\
2.26 \\
.86 \\
.67 \\
\text { trace } \\
\text { trace } \\
.17 \\
\text { trace } \\
\ldots \ldots . .\end{array}$ & $\begin{array}{r}54.97 \\
18.38 \\
3.06 \\
4.22 \\
2.38 \\
5.43 \\
3.45 \\
3.37 \\
.82 \\
.97 \\
-.42 \\
\text { trace } \\
.03 \\
.03 \\
\text { trace } \\
2.92\end{array}$ \\
\hline & 100.03 & 99.93 & 100.37 & 100.45 \\
\hline
\end{tabular}

5. MISCELLANEOUS ROCKS.

First group: Collected and investigated by Arnold Hague and J. P. Iddings.

A. Black obsidian, Obsidian Cliff. Liparose. Described by Iddings, 7th Ann. Contains microlites of augite and magnetite, with traces of quartz and feldspar. Analysis by J. E. Whitfield, record No. 224.

B. Red obsidian, Obsidian Cliff. Alaskose. Described by Iddings, 7th Ann. Like A, with ferric oxide replacing magnetite. Analysis by Whitfield, No. 223.

C. Obsidian, east of Willow Park. Lassenose. Black and opaque. Described by Iddings, Bull. Philos. Soc. Washington, vol. 12, p. 204. Analysis by Whitfield, No. 222.

D. Lithoidite, Obsidian Cliff. Lassenose. Described by Iddings, Bull. 150, p. 153. Contains quartz and sanidine, with a little magnetite and augite. Analysis by Whitfield, No. 425 . P. R. C. 62 .

E. Rhyolite, Upper Geyser Basin. Liparose. See Iddings, Bull. Philos. Soc. Washington, vol. 12, p. 204. Analysis by F. A. Gooch, record No. 114.

F. Rhyolite, Tower Creek. Toscanose. See Iddings, Bull. Philos. Soc. Washington, vol. 12, p. 204. Analysis by Gooch, No. 115.

\begin{tabular}{|c|c|c|c|c|c|c|}
\hline & A. & B. & C. & D. & E. & F. \\
\hline 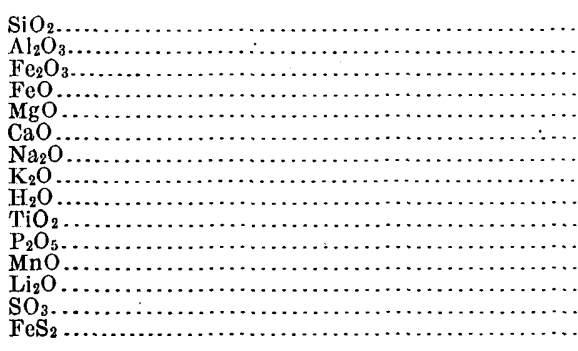 & $\begin{array}{r}74.70 \\
13.72 \\
1.01 \\
.62 \\
.14 \\
.78 \\
3.90 \\
4.02 \\
.62 \\
\text { none } \\
\text { none } \\
\text { trace } \\
\cdots \cdots \\
.40\end{array}$ & $\begin{array}{r}75.52 \\
14.11 \\
1.74 \\
.08 \\
.10 \\
.78 \\
3.92 \\
3.63 \\
.39 \\
\text { none } \\
\text { none } \\
\ldots . . .\end{array}$ & $\begin{array}{r}72.59 \\
13.47 \\
1.58 \\
1.32 \\
1.05 \\
2.12 \\
4.63 \\
2.52 \\
.18 \\
.52 \\
\ldots \ldots \\
\text { none } \\
\ldots \ldots \\
.26\end{array}$ & $\begin{array}{r}75.50 \\
13.25 \\
1.02 \\
.91 \\
.07 \\
.90 \\
4.76 \\
2.85 \\
.41 \\
\text { none } \\
\text { none } \\
\text { none } \\
.06 \\
.32 \\
\ldots \ldots \ldots \\
. . .\end{array}$ & $\begin{array}{r}70.92 \\
13.24 \\
3.54 \\
.66 \\
.23 \\
1.42 \\
4.28 \\
4.25 \\
.57 \\
.16 \\
. .18 \\
.14 \\
\text { none } \\
\ldots . . .\end{array}$ & $\begin{array}{r}71.85 \\
13.17 \\
0.17 \\
1.34 \\
.63 \\
2.25 \\
4.06 \\
3.89 \\
.43 \\
.43 \\
.14 \\
.12 \\
\ldots \ldots . . \\
\ldots . . .\end{array}$ \\
\hline & 99.91 & 100.38 & 100.24 & 100.05 & 100.59 & 100.48 \\
\hline
\end{tabular}

G. Rhyolite, "Great Paint Pots." Liparose. Analysis by Gooch, No. 113.

H. Rhyolite, "Elephants Back." Porphyritic obsidian. -Tehamose. Analysis by Whitfield, No. 423 . Reported by Iddings as containing quartz, sanidine, and a little augite and magnetite, in a glassy, microlitic groundmass.

I. Rhyolite, Mount Sheridan. Tehamose. Composition reported by Iddings as quartz and sanidine, with a little magnetite and augite. Analysis by Whitfield, No. 426 , 
J. Sanidine quartz rhyolite, Sheridan volcano. Analysis by G. Steiger, record No. 21.54 .

K. Rhyolite, Madison Plateau. Like I. Near alaskose. Analysis by Whitfield, No. 427.

L. Trachytic rhyolite, Elk Creek. Lassenose. Analysis by Whitfield, No. 428. Reported by Iddings as containing sanidine, labradorite, and little biotite, in a groundmass of quartz and alkali feldspar.

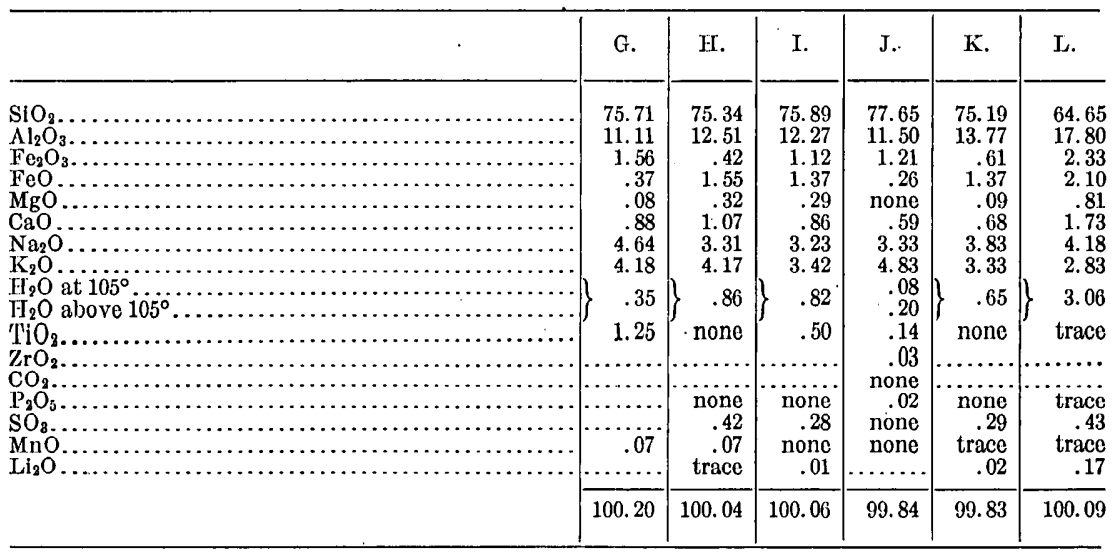

M. Trachytic rhyolite tuff, Two Ocean Pass. Analysis by Whitfield, No. 906. Reported by Iddings as containing sanidine, labradorite, biotite, magnetite, and augite, in a somewhat altered, glassy groundmass. Also contains fragments of andesite.

N. Glassy trachyte, approaching rhyolite, Sunset Peak, Bear Gulch. Toscanose: Contains phenocrysts of sanidine, plagioclase, and biotite. See Iddings, Bull. Philos. Soc. Washington, vol. 12, p. 205; also Mon. XXXII, pt. 2. Analysis by L. G. Eakins, record No. 1378.

O. Altered rhyolite, "Iron Pot," Lower Basin. Collected by W. H. Weed. Analysis by Whitfield, No. 546 .

P. Dacite porphyry, Echo Peak. Near alaskose.

Q. Same as $\mathrm{O}$, Mount Holmes. Alsbachose. Analyses $\mathrm{O}$ and $\mathrm{P}$ by Whitfield, Nos. 421, 422. Reported by Iddings as containing plagioclase, probably oligoclase, quartz, little biotite, and magnetite.

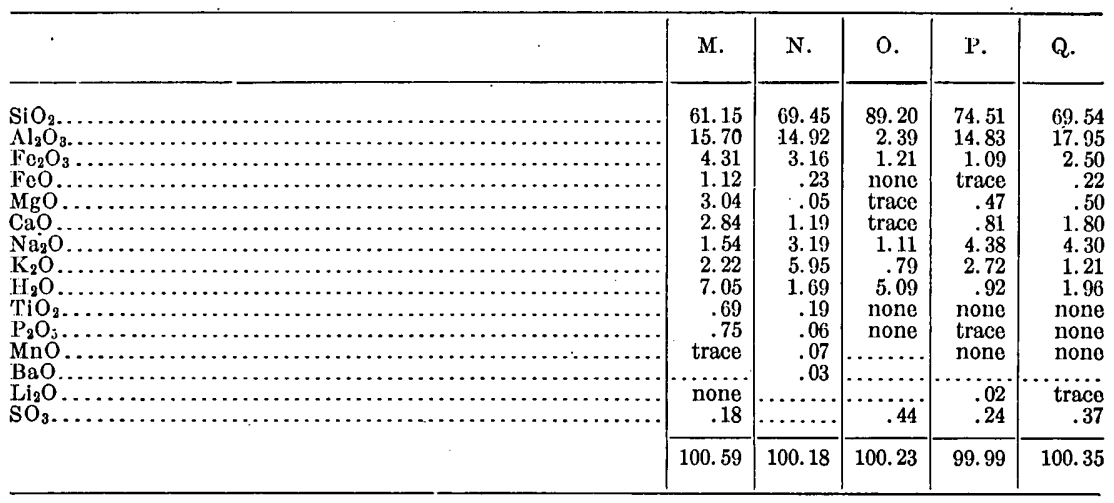

R. Mica dacite porphyry, Bunsen Peak. Lassenose. Analysis by Whitfield, No. 419. Reported by Iddings as containing oligoclase-andesine, quartz, ${ }^{\circ}$ biotite little magnetite, apatite; and zircon, 
S. Mica dacite porphyry, Birch Hills. Lassenose. Like Q in composition. Analysis by Whitfield, No. 718.

T. Hornblende-mica andesite porphyry, Fan Creek. Yellowstonose. Analysis by Whitfield, No. 713. Reported by Iddings as containing andesine-oligoclase, hornblende, and biotite in a groundmass of feldspar (probably oligoclase and orthoclase), with a little magnetite, biotite, and hornblende.

U. Hornblende-mica andesite porphyry, Gray Peak. Lassenose. Analysis by Whitfield, No. 715. Composition similar to that of S.

\begin{tabular}{|c|c|c|c|c|}
\hline . & R. & S. & $\mathrm{T}$. & U. \\
\hline 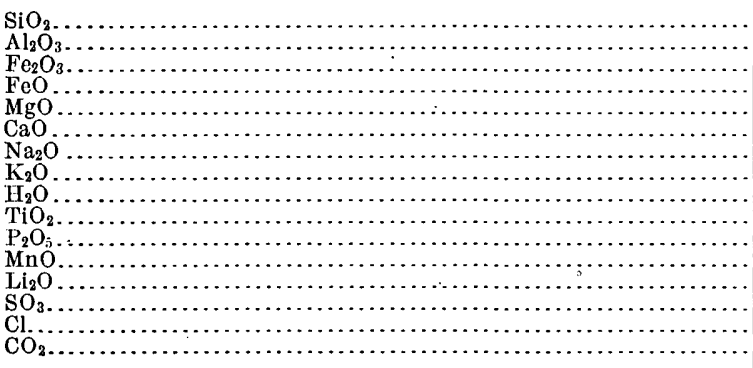 & $\begin{array}{r}70.52 \\
15.85 \\
2.28 \\
.36 \\
.09 \\
2.59 \\
3.93 \\
3.43 \\
.35 \\
\operatorname{trace} \\
.17 \\
.09 \\
\text { trace } \\
.29 \\
\ldots \ldots \ldots\end{array}$ & $\begin{array}{r}70.24 \\
17.36 \\
1.38 \\
.79 \\
.53 \\
2.74 \\
3.69 \\
2.65 \\
.71 \\
\text { trace } \\
\text { trace } \\
\text { none } \\
\text { none } \\
\text { trace } \\
\text { none } \\
\text { none }\end{array}$ & $\begin{array}{r}65.63 \\
17.00 \\
2.55 \\
1.19 \\
2.03 \\
3.48 \\
4.42 \\
1.64 \\
2.00 \\
\text { trace } \\
.07 \\
\text { none } \\
.04 \\
\text { trace } \\
\text { trace } \\
.27\end{array}$ & $\begin{array}{r}65.64 \\
17.29 \\
3.07 \\
1.29 \\
1.78 \\
1.98 \\
5.77 \\
2.44 \\
1.03 \\
\text { none } \\
.23 \\
\text { trace } \\
.04 \\
\text { trace } \\
\text { trace } \\
.17\end{array}$ \\
\hline 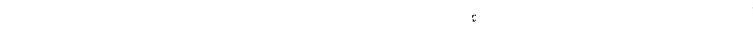 & 99.95 & 100.09 & 100.32 & 100.73 \\
\hline
\end{tabular}

V. Hornblende-mica andesite, Crescent Hill. Yellowstonose: Analysis by Whitfield, No: 432 Reported by Iddings as containing oligoclase-andesine, biotite, and subordinate decomposed hornblende, in a groundmass of feldspar and quartz, with a little magnetite and biotite.

W. Hornblende andesite, Tower Creek. Dacose. Analysis by Gooch, No. 117. Reported by Iddings as containing plagioclase, hornblende, and a little augite.

X. Pyroxene andesite, Agate Creek. Tonalose. Analysis by Whitfield, No. 432. Reported by Iddings as containing augite, hypersthene, labradorite, and magnetite, in a glassy microlitic groundmass.

Y. Rhyolitic perlite. Toscanose. Described by Iddings, Bull. 150, p. 1.53. Analysis by H. N. Stokes, No. 1314. From a bluff opposite the Midway Geyser Basin. Reported by Iddings as containing quartz, sanidine, plagioclase, and rarely augite and magnetite, with microscopic zircon and apatite, in a glassy groundmass. P. R. C. 61 .

\begin{tabular}{|c|c|c|c|c|}
\hline & V. & $W_{:}$ & $\mathrm{X}$. & $\mathbf{Y}$ \\
\hline 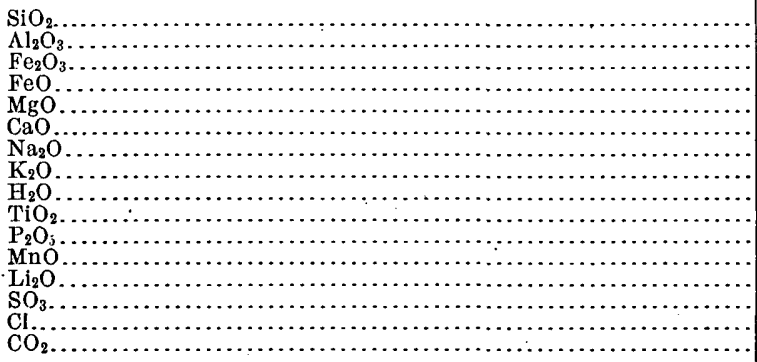 & $\begin{array}{r}64.61 \\
18.62 \\
2.78 \\
.95 \\
.85 \\
4.20 \\
4.37 \\
2.36 \\
.93 \\
\text { none } \\
.30 \\
\text { trace } \\
.01 \\
\text { trace } \\
\text { trace } \\
.25\end{array}$ & $\begin{array}{r}61.56 \\
14.73 \\
4.47 \\
1.23 \\
3.57 \\
4.87 \\
5.10 \\
2.24 \\
1.42 \\
.87 \\
.04 \\
.34 \\
\ldots \ldots \ldots . \\
\ldots \ldots \ldots . . \\
\ldots \ldots \ldots . .\end{array}$ & $\begin{array}{r}61.45 \\
15.07 \\
4.46 \\
1.18 \\
3.02 \\
5.37 \\
4.00 \\
1.22 \\
1.23 \\
2.80 \\
\text { trace } \\
\text { none } \\
.05 \\
.29 \\
\ldots \ldots . . . \\
\ldots \ldots . . .\end{array}$ & $\begin{array}{r}73.84 \\
12.47 \\
.32 \\
.90 \\
.25 \\
1.08 \\
2.88 \\
5.38 \\
2.76 \\
\ldots \ldots . . \\
\ldots \ldots . . \\
\text { trace } \\
\ldots \ldots . . . \\
\ldots \ldots . . . \\
\ldots \ldots . . .\end{array}$ \\
\hline . & 100.23 & 100.44 & 100.14 & 99.88 \\
\hline
\end{tabular}


Second group: Collected and investigated by Arnold Hague and J. P. Iddings.

A. Pyroxene andesite, west of Dunraven Peak. Andose. Analysis by F. A. Gooch, record No. 116. Reported by Iddings as containing labradorite, augite, hypersthene, and magnetite, in a microlitic groundmass.

B. Basalt, southwest of Dunraven Peak. Camptonose. Analysis by Gooch, No. 118. Reported by Iddings as containing augite, olivine, labradorite-bytownite, and magnetite, in a globulitic glassy groundmass.

C. Basalt, north spur of Prospect Peak. Auvergnose. Analysis by J. E. Whitfield, record No. 431. Reported by Iddings as containing labradorite, augite, olivine, and magnetite. Little glass in groundmass.

D. Basalt, Yellowstone Canyon. Andose. Analysis by Whitfield, No. 430. Reported by Iddings as containing labradorite-bytownite, augite, olivine, magnetite, and a little brown glass.

E. Basalt, Stinkingwater Canyon. Andose. See Iddings, Bull. Philos. Soc. Washington, vol. 12; p. 205. Analysis by W. H. Mélville, record No. 1232.

\begin{tabular}{|c|c|c|c|c|c|}
\hline · & A. & B. & C. & D. & E. \\
\hline 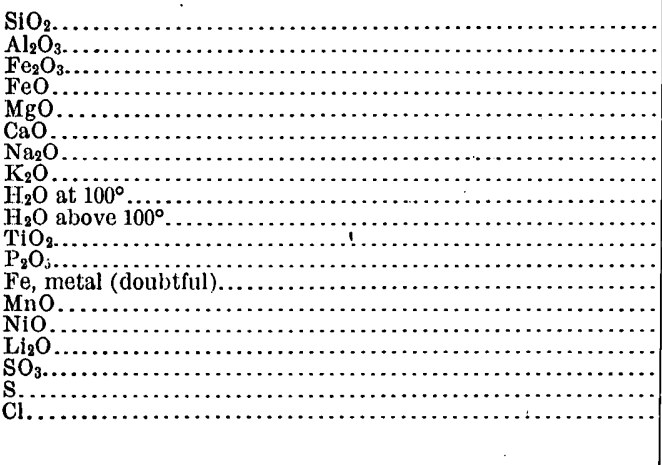 & $\begin{array}{r}56.47 \\
15.33 \\
2.54 \\
4.53 \\
5.08 \\
6.93 \\
3.81 \\
1.66 \\
1.65 \\
.99 \\
.54 \\
\ldots .18\end{array}$ & 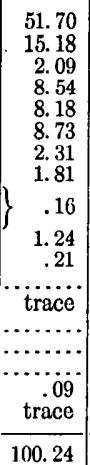 & $\begin{array}{r}47.17 \\
17.85 \\
7.42 \\
1.18 \\
6.54 \\
10.12 \\
2.94 \\
.56 \\
.65 \\
2.13 \\
.20 \\
3.26 \\
\text { none } \\
\cdots \\
.02 \\
.51 \\
\cdots \cdots \cdots \\
100.55 \\
\ldots \ldots . . \\
10.5 \\
\end{array}$ & $\begin{array}{r}51.70 \\
17.90 \\
-7.24 \\
1.00 \\
2.77 \\
6.94 \\
4.17 \\
1.62 \\
1.15 \\
3.17 \\
.41 \\
1.81 \\
\text { trace } \\
\cdots \ldots . . \\
.03 \\
.32 \\
\ldots \ldots . . . \\
\ldots \ldots . . . \\
100.23\end{array}$ & 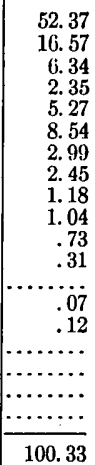 \\
\hline
\end{tabular}

F. Camptonite (?), dike in Stinkingwater Canyon. Andose. See Iddings, Bull. Philos. Soc. Washington, vol. 12, p. 205. Analysis by Melville, No. 1232.

G. Kersantite, Bighorn Pass. Kentallenose. Described by Iddings in Mon. XXXII, pt. 2. Contains hornblende, plagioclase, orthoclase, quartz, augite, biotite, magnetite, chlorite, calcite, and apatite. Augite and hornblende partly decomposed. Analysis by Whitfield, No. 714 .

H. Basalt, dike, north spur of Mount Washburn. Andose. Analysis by Whitfield, No. 717. Reported by Iddings as approaching pyroxene andesite in composition. Contains labradorite, augite, serpentinized olivine, and magnetite, in a groundmass of globulitic and microlitic brown glass. 


\begin{tabular}{|c|c|c|c|}
\hline 1 & F. & G. & H. \\
\hline 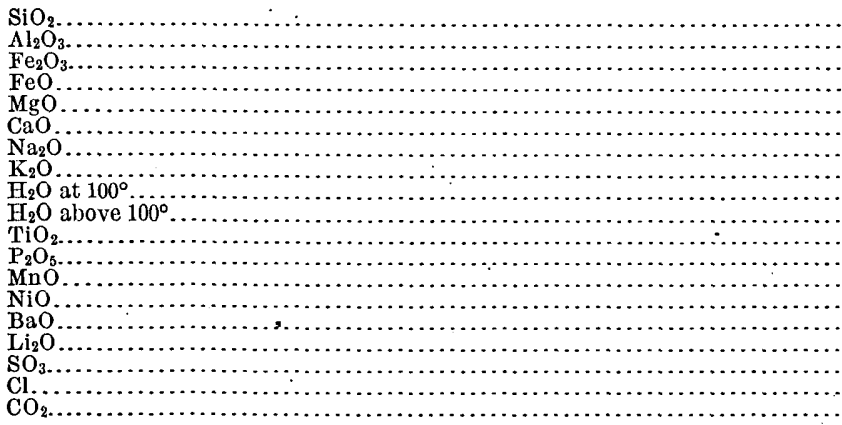 & 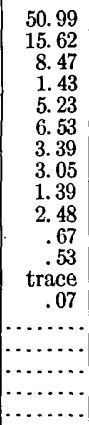 & $\begin{array}{r}48.73 \\
11.92 \\
4.79 \\
4.56 \\
5.93 \\
9.24 \\
2.62 \\
2.47 \\
1.52 \\
1.34 \\
.32 \\
.36 \\
\ldots \ldots \ldots c e \\
\text { trace } \\
\text { trace } \\
.34 \\
.11 \\
5.80\end{array}$ & $\begin{array}{r}53.75 \\
20.75 \\
4.50 \\
3.53 \\
3.76 \\
7.18 \\
4.16 \\
1.37 \\
1.55 \\
\text { none } \\
.15 \\
\text { trace } \\
\ldots \ldots . . \\
\cdots \\
\text { trace } \\
\text { trace } \\
\text { none } \\
\text { none }\end{array}$ \\
\hline & 99.85 & 100.05 & $100^{\circ} .70$ \\
\hline
\end{tabular}

\section{MONTANA.}

\section{MADISON AND GALLATIN VALLEYS.}

Rocks collected by A. C. Peale and G. P. Merrill. Described by Merrill in Proc. U. S. Nat. Mus., vol. 17, p. 637. See also Bull. 110, p. 47.

A. Basalt (?), east side of Bozeman Creek, $2 \frac{1}{2}$ miles southeast of Bozeman. Kentallenose. Contains olivine and augite, chloritized and serpentinized. The colorless ground.nass contains apparently two feldspars and a pyroxene, with grains of iron oxide. Analysis by T. M. Chatard, record No. 517. Sp. gr., 2.86. P. R. C. 971.

B. Pyroxene from A. Analysis by L. G. Eakins, record No. 1046.

C. Portion of $A$ soluble in hydrochloric acid: Contains olivine, iron oxides, and decomposition products. Analysis by Eakins, record No. 817.

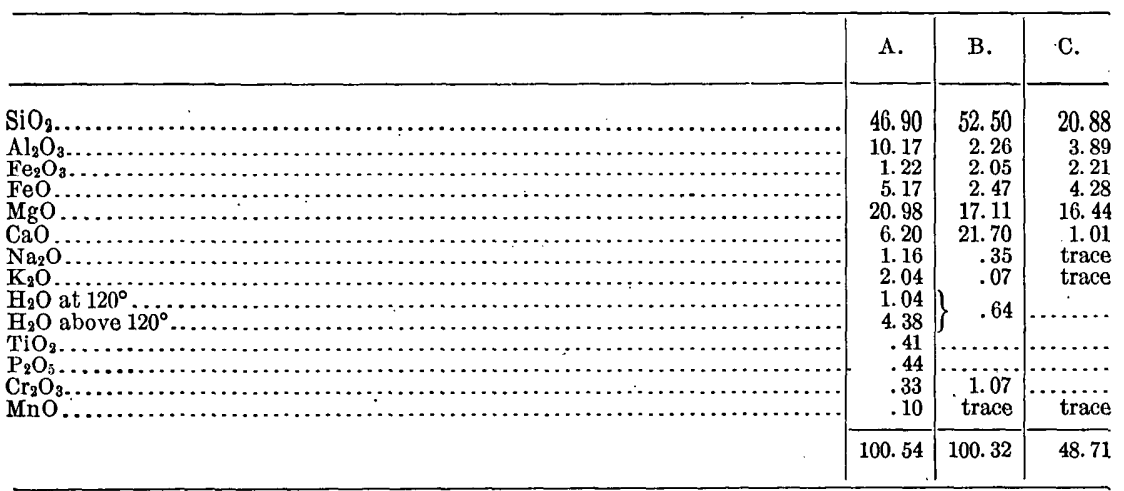

D. Highly altered porphyrite (?), hills 1 mile north of East Gallatin River, near camp No. 6. Rock contains hornblende paramorphs after augite in a devitrified base, with amygdules of calcite, chloritic and ferruginous matter derived from porphyritic augite and olivine. The base is also filled with needles, which may be mica. Analysis by Eakins, record No. 820. P. R. C. 968.

E. Lamprophyre, Cottonwood Creek. Monzonose. Contains porphyritic augite and olivine in an indeterminate groundmass carrying augite, iron oxides, and mica. Analysiis by Chatard, record No. 516. P. R. C. 979. 
F. Augite porphyry, Cottonwood Creek. Shoshonose. Contains feldspars, augite, and brown mica, with iron oxides, apatite, glass, and secondary calcite and chlorite. Carries porphyritic plagioclase and augite. No unaltered olivine visible. Sp. gr., 2.785. Analysis by Eakins, record No. 819. P. R. C. 965.

\begin{tabular}{|c|c|c|c|}
\hline & D. & E. & F. \\
\hline 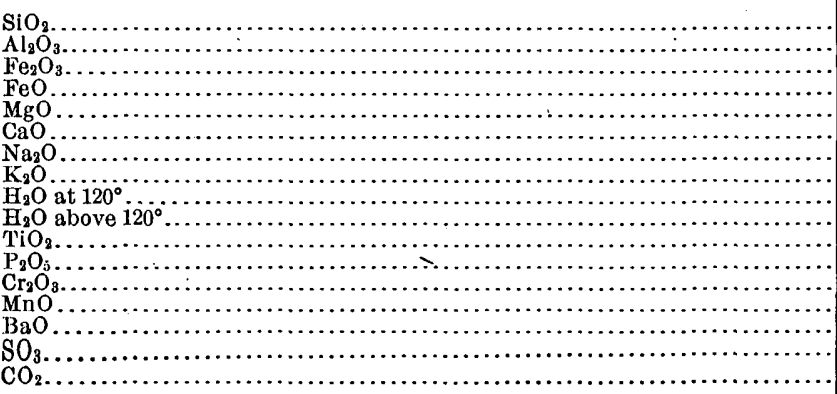 & $\begin{array}{r}49.47 \\
12.15 \\
1.93 \\
4.07 \\
10.86 \\
9.30 \\
2.08 \\
2.42 \\
4.14 \\
.21 \\
.37 \\
\operatorname{trace} \\
.10 \\
.03 \\
\ldots \ldots . . . \\
3.31\end{array}$ & $\begin{array}{r}51.65 \\
13.89 \\
2.70 \\
4.80 \\
11.56 \\
4.07 \\
2.99 \\
4.15 \\
1.30 \\
1.89 \\
.55 \\
.21 \\
.08 \\
.15 \\
.19 \\
.19 \\
\ldots \ldots\end{array}$ & $\begin{array}{r}52.33 \\
15.09 \\
4.31 \\
4.03 \\
6.73 \\
7.06 \\
3.14 \\
3.76 \\
2.68 \\
.14 \\
1.02 \\
\ldots .09 \\
.0 .07 \\
\ldots \ldots \ldots\end{array}$ \\
\hline & 100.44 & 100.37 & 100.45 \\
\hline
\end{tabular}

G. Basalt (?), Bear Creek. Lamarose. Resembles A, but with a more crystalline groundmass. Contains plagioclase, possibly sanidine, augite, olivine, and iron oxides. Analysis by Chatard, record No. 1154. P. R. C. 967.

H. Lamprophyre, between South Boulder and Antelope creeks. Kentallenose. Contains porphyritic augite and olivine in a feldspathic groundmass, with apatite, augite, grains of iron oxide, and shreds of brown mica. Sp. gr., 2.96. Analysis by Eakins, record No. 1266 . P. R. C. 966.

I. Lamprophyre, hills east of South Boulder Creek. Near auvergnose. Nodules from a decomposed mass. Shows sanidine, plagioclase, brown mica, and altered olivine.

\begin{tabular}{|c|c|c|c|}
\hline - & G. & H. & I. \\
\hline 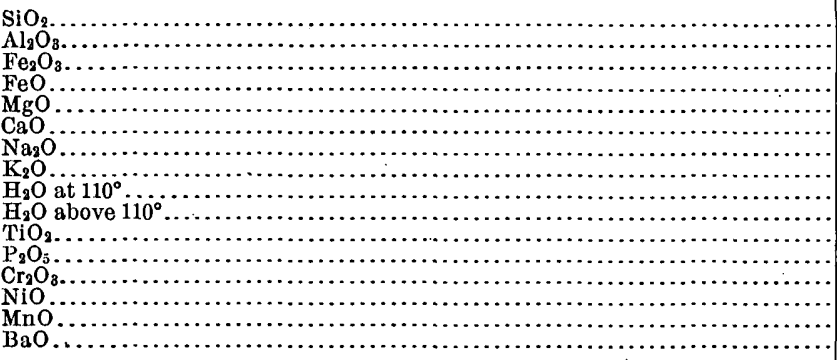 & $\begin{array}{r}49.13 \\
9.05 \\
3.57 \\
5.05 \\
17.21 \\
5.68 \\
2.01 \\
2.24 \\
.84 \\
3.50 \\
.42 \\
.38 \\
.39 \\
\operatorname{trace} \\
.15 \\
.05\end{array}$ & $\begin{array}{r}50.82 \\
11.44 \\
.25 \\
8.94 \\
14.01 \\
8.14 \\
1.79 \\
3.45 \\
.58 \\
.59 \\
.20 \\
.03 \\
\text { trace } \\
.19 \\
.06\end{array}$ & $\begin{array}{r}50.03 \\
14.08 \\
2.92 \\
6.11 \\
10.73 \\
7.46 \\
1.46 \\
2.64 \\
3.70 \\
.61 \\
.42 \\
\text { trace } \\
\text { trace } \\
.08 \\
.04\end{array}$ \\
\hline & 99.67 & 100.49 & 100.28 \\
\hline
\end{tabular}

J. Hornblende picrite, North Meadow Creek. Wehrlose. Contains hornblende, abundant fresh olivine, grains of pleonaste and iron oxides, and occasionally hypersthene. Sp. gr., 3.35. P. R. C. 973.

K. Pyroxenite, divide between Meadow and Granite creeks. Cookose. Contains hornblende and hypersthene, with grains of iron oxide. P. R. C. 972. 
L. Hypersthene andesite, northwest of Red Bluff. Tonalose. Contains plagioclase and pyroxene, with an amorphous glassy base, and sometimes olivine altered to chloritic matter.

M. Peridotite, var. wehrlite, hills 3 miles northwest of Red Bluff. Wehrlose. Contains olivine, diallage, brown mica, rarely plagioclase, and secondary iron oxides. Sp. gr., 3.37. Analyses J, K, L, and M by Eakins, record No. 1266. P. R. C. 975 and 976 .

\begin{tabular}{|c|c|c|c|c|}
\hline & J. & K. & L. & M. \\
\hline 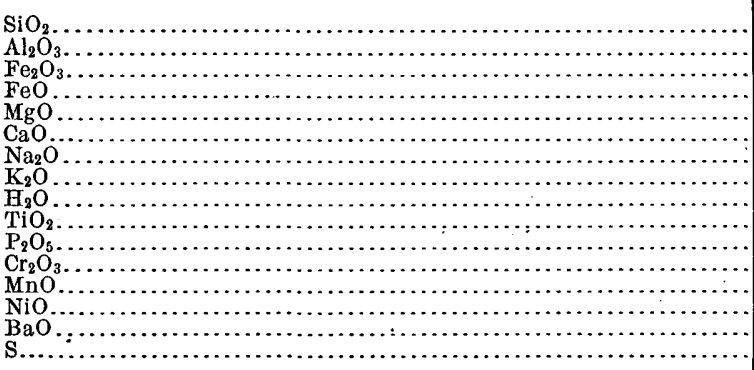 & $\begin{array}{r}46.13 \\
4.69 \\
.73 \\
16.87 \\
25.17 \\
4.41 \\
.08 \\
\operatorname{trace} \\
1.38 \\
.73 \\
.07 \\
.04 \\
\operatorname{trace} \\
.09 \\
\operatorname{trace} \\
.24\end{array}$ & $\begin{array}{r}51.83 \\
7.98 \\
1.48 \\
8.28 \\
24.10 \\
5.26 \\
.35 \\
.06 \\
.29 \\
.29 \\
.09 \\
.31 \\
\text { trace } \\
.11 \\
\ldots \ldots . . . \\
\ldots . . . . .\end{array}$ & $\begin{array}{r}59.48 \\
16.37 \\
3.21 \\
3.17 \\
3.29 \\
4.88 \\
3.30 \\
2.81 \\
2.01 \\
.93 \\
.41 \\
.03 \\
.19 \\
\text { trace } \\
.13 \\
\ldots \ldots . . .\end{array}$ & $\begin{array}{r}48.95 \\
5.69 \\
1.20 \\
12.11 \\
23.49 \\
5.33 \\
1.58 \\
.79 \\
.18 \\
.81 \\
.12 \\
.05 \\
.08 \\
.16 \\
\text { trace } \\
\ldots . . . .\end{array}$ \\
\hline \multirow[t]{2}{*}{ Less 0.} & $\begin{array}{r}100.63 \\
.12\end{array}$ & $\begin{array}{c}100.43 \\
\ldots \ldots\end{array}$ & $\begin{array}{c}100.21 \\
\ldots \ldots\end{array}$ & $\begin{array}{r}100.54 \\
\ldots \ldots\end{array}$ \\
\hline & 100.51 & & & \\
\hline
\end{tabular}

The following rocks, at first supposed to be Pliocene sandstones, were also described by Merrill, Am. Jour. Sci., 3d ser., vol. 32, p. 119. All consist of pumiceous volcanic glass. C, from Idaho, is included here for convenience. . Analyses by J. E. Whitfield, record No. 382.

A. Little Sage Creek.

B. Devils Pathway.

C. Marsh Creek Valley, Idaho.

Tron and alumina weighed together. The iron is mostly in the ferrous form.

\begin{tabular}{|c|c|c|c|}
\hline & A. & B. & C. \\
\hline \multirow[t]{2}{*}{ 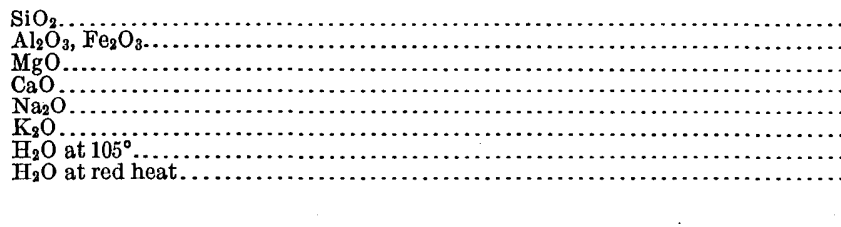 } & $\begin{array}{r}65.56 \\
18.24 \\
.72 \\
2.58 \\
2.08 \\
3.94 \\
1.12 \\
6.50\end{array}$ & $\begin{array}{r}65.76 \\
17.18 \\
\text { trace } \\
2.30 \\
2.22 \\
3.14 \\
3.46 \\
5.60\end{array}$ & $\begin{array}{r}68.92 \\
16.22 \\
\text { trace } \\
1.62 \\
1.56 \\
4.00 \\
1.60 \\
6.00\end{array}$ \\
\hline & 100.74 & 99.66 & 99.92 \\
\hline
\end{tabular}

The following examples of volcanic dust or sand, from the Gallatin Valley, were collected by A. C. Peale. Analyses A, B, C, and D by F. W. Clarke, record No. 379. Analysis E by H. N. Stokes, record No. 1314.

A. Dry Creek Valley, above the mouth of Pass Creek.

B, C. Near Bozeman.

D. Near Fort Ellis.

E. Essentially rhyolitic glass, described by Iddings in Bull. 150, p. 146. Gallatin Valley. P. R: C. 58. 


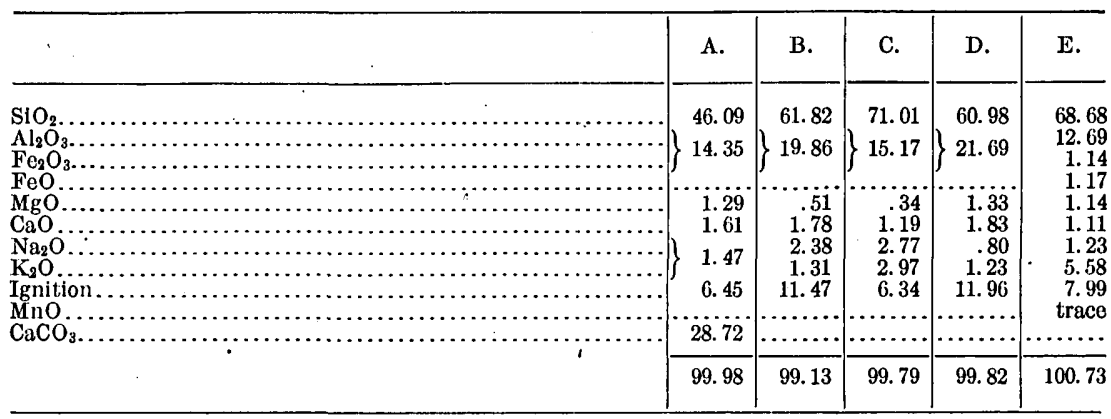

\section{BUTTE DISTRICT.}

Rocks received from W. H. Weed and G. W. Tower. Some of the granite rocks are described by Weed in Jour. Geology, vol. 7, p. 737.

A. Butte granite. Amiatose. A quartz monzonite, variety banatite, from Walkerville Station. Contains quartz, andesine, orthoclase, hornblende, and biotite, with a little titanite, apatite, and magnetite.

B. Biotite from $A$.

C. Amphibole from $A$.

Analyses A, B, C, by H. N. Stokes, record Nos. 1686, 1808.

D. Butte granite, Gagnon mine, Butte. Amiatose.

E. Butte granite, Atlantic mine, Butte. Harzose.

F. Butte granite, Alice mine, Butte. Harzose. Analyses D, E, and F, by W. F. Hillebrand, record No. 1692. Mineralogically the rocks are like A and D.

\begin{tabular}{|c|c|c|c|c|c|c|}
\hline & A. & B. & C. & D. & E. & F. \\
\hline 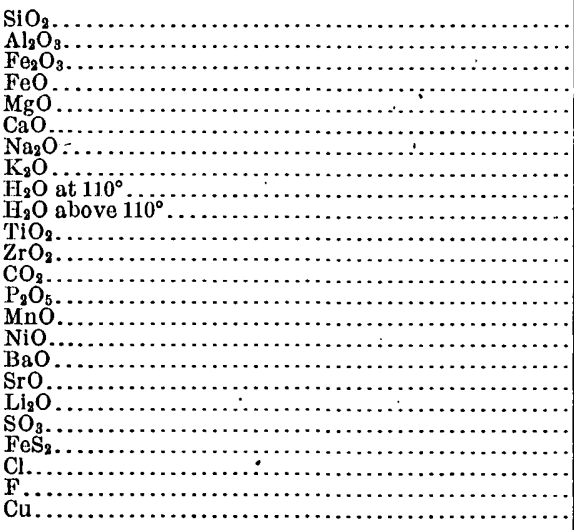 & $\begin{array}{r}63.88 \\
15.84 \\
2.11 \\
2.59 \\
2.13 \\
3.97 \\
2.81 \\
4.23 \\
.22 \\
.66 \\
.65 \\
\ldots \ldots . . \\
\ldots .21 \\
.07 \\
\ldots \ldots .09 \\
.09 \\
\text { trace } \\
.34 \\
\ldots \ldots . . \\
\text { trace }\end{array}$ & $\begin{array}{r}35.79 \\
13.70 \\
5.22 \\
13.72 \\
12.13 \\
.05 \\
.15 \\
9.09 \\
1.21 \\
3.64 \\
3.51 \\
\ldots \ldots \\
.10 \\
.19 \\
. .13 \\
\text { none } \\
\text { trace } \\
\ldots \ldots . . . \\
.20 \\
.76\end{array}$ & $\begin{array}{r}45.73 \\
6.77 \\
4.94 \\
10.39 \\
12.32 \\
11.25 \\
.77 \\
1.22 \\
.49 \\
2.29 \\
1.43 \\
\ldots \ldots . . \\
\ldots \ldots .35 \\
. .54 \\
. .54 \\
\text { none } \\
\text { none } \\
\text { trace }\end{array}$ & $\begin{array}{c}64.05 \\
15.38 \\
2.20 \\
2.74 \\
2.08 \\
4.30 \\
2.74 \\
4.00 \\
.27 \\
.83 \\
.60 \\
(?) \\
.35 \\
.21 \\
.11 \\
\text { trace? } \\
.08 \\
.04 \\
\text { trace } \\
\ldots . . . \\
.07\end{array}$ & $\begin{array}{c}64.34 \\
15.72 \\
1.62 \\
2.94 \\
2.17 \\
4.24 \\
2.76 \\
4.04 \\
.25 \\
.76 \\
.53 \\
.02 \\
.03 \\
.14 \\
.12 \\
\text { trace? } \\
.06 \\
.03 \\
\text { trace } \\
. . .2 . \\
.03\end{array}$ & $\begin{array}{r}63.87 \\
15.39 \\
1.93 \\
3.08 \\
2.23 \\
4.30 \\
2.76 \\
4.18 \\
.19 \\
.69 \\
.65 \\
.03 \\
.15 \\
.17 \\
.11 \\
\text { trace } \\
.07 \\
.04 \\
\text { trace } \\
\ldots . . . \\
.07 \\
\ldots . . .\end{array}$ \\
\hline \multirow[t]{2}{*}{ 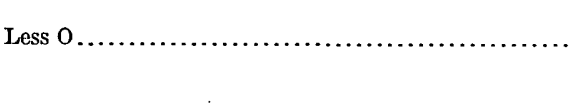 } & $\begin{array}{r}99.82 \\
\ldots \ldots \ldots\end{array}$ & $\begin{array}{r}99.59 \\
.37\end{array}$ & $\begin{array}{r}98.77 \\
.12\end{array}$ & $\begin{array}{c}100.055 \\
\ldots \ldots \ldots\end{array}$ & $\begin{array}{c}99.805 \\
\ldots \ldots \ldots\end{array}$ & $\begin{array}{r}99.915 \\
\ldots \ldots \ldots\end{array}$ \\
\hline & & 99.22 & 98.65 & & & \\
\hline
\end{tabular}

The six following analyses represent alterations of quartz monzonite, the so-called Butte granite:

G. Weathered Butte granite. 
H. Altered Butte granite. Decomposed near quartz-pyrite veins. Shows opaline silica, with sericite derived from feldspar. Hornblende gone; mica recognizable only as sericite masses having the form of biotite.

Analyses $\mathrm{G}$ and $\mathrm{H}$ by Stokes, record Nos. 1792, 1787.

I. Altered Butte granite, 300-foot level, Colusa mine. Derived from A. Contains quartz, altered orthoclase and plagioclase, and sericite.

J. Altered Butte granite, wall rock, 1,300-foot level, Parrot mine. Derived from A. Contains quartz, sericite, pyrite, bornite, etc.

K. Altered Butte granite, enargite vein, 1,000-foot level, Leonard mine. Contains quartz, kaolin, pyrite, etc.

I. Altered Butte granite, veinlets in Original mine. Contains quartz, sericite, partly altered feldspars, calcite, zinc blende, etc.

Analyses I, J, by E. T. Allen, record No. 1964; K, L, by W. F. Hillebrand, record No. 1971.

\begin{tabular}{|c|c|c|c|c|c|c|}
\hline & G. & H. & I. & J. & $\mathbf{K}$. & I.. \\
\hline ' & 65.14 & 64.81 & 56.80 & 0209 & 6690 & \\
\hline (1) & 65. 14 & 64. 81 & 56.80 & 62. 09 & 60. 90 & 54.30 \\
\hline 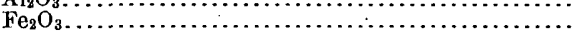 & $\begin{array}{r}15.05 \\
2.37\end{array}$ & $\begin{array}{r}19.44 \\
1.82\end{array}$ & $\begin{array}{r}21.02 \\
3.06\end{array}$ & $\begin{array}{r}10.49 \\
8.52\end{array}$ & $\begin{array}{l}15.85 \\
(?)\end{array}$ & $\begin{array}{r}13.63 \\
1.89\end{array}$ \\
\hline 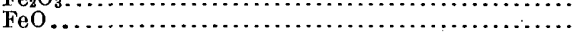 & 2.13 & $\begin{array}{r}1.04 \\
.16\end{array}$ & $\begin{array}{r}0.00 \\
.90\end{array}$ & $\{\ldots .0$ & $(?)$ & $\begin{array}{l}1.09 \\
2.22\end{array}$ \\
\hline 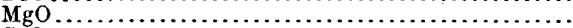 & 1.85 & .19 & 1. 21 & .42 & trace & 2.13 \\
\hline 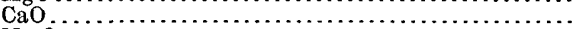 & 3. 62 & .18 & .35 & .20 & .05 & 7.36 \\
\hline$\ldots \ldots \ldots \ldots \ldots \ldots \ldots \ldots \ldots \ldots \ldots$ & 2.63 & .21 & .50 & .37 & .08 & .16 \\
\hline $\mathrm{K}_{2} \mathrm{O} \ldots \ldots$ & 4. 29 & 5. 30 & 4. 78 & 4. 34 & .03 & 4. 41 \\
\hline 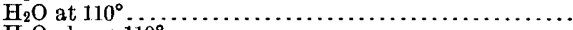 & .37 & 1. 41 & 2.88 & 1. 20 & .87 & 2.19 \\
\hline 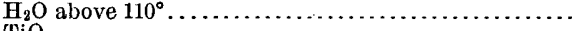 & .75 & 5. 25 & 7.68 & 3.01 & 3.88 & 4. 09 \\
\hline 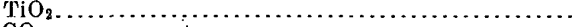 & .59 & .73 & $\because 60$ & .51 & .68 & .6 \\
\hline 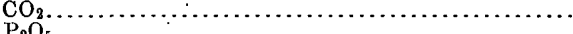 & none & none & none & none & none & 5. \\
\hline 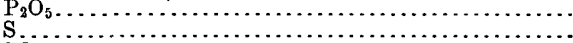 & .16 & .10 & $\begin{array}{l}.05 \\
.17\end{array}$ & .13 & $\cdots$ & \\
\hline 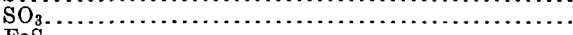 & .05 & .31 & none & none & none & non \\
\hline 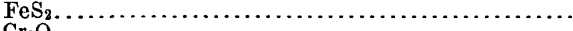 & $\ldots \ldots$ & ..... & & $\ldots \ldots \ldots$ & $\ldots \ldots \ldots$ & \\
\hline 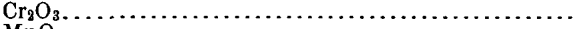 & & & trace & none & $\ldots$. & $\cdots$ \\
\hline 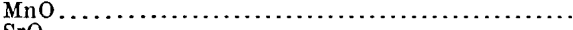 & trace & trace & trace & trace? & none & \\
\hline 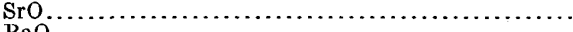 & trace & trace & $\ldots \ldots$ & $\ldots \ldots$. & ........ & \\
\hline 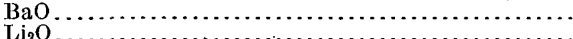 & .10 & .10 & .05 & trace & $\ldots \ldots$. & trac \\
\hline $\begin{array}{l}\mathrm{Li}{ }_{2} \mathrm{O} \\
\mathrm{ZnO}\end{array}$ & trace & trace & $\cdots \cdots \cdots$ & …...... & $\cdots \cdots \cdots$ & $\cdots$ \\
\hline 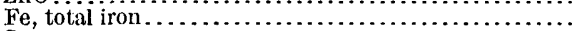 & $\cdots$ & & & & 4. 37 & \\
\hline \multirow[t]{2}{*}{ 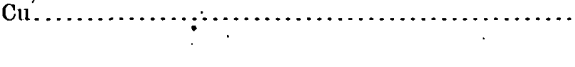 } & & & .10 & .25 & present & \\
\hline & 99.68 & 100.01 & 100. 15 & 102.00 & 98.15 & 100.01 \\
\hline \multirow[t]{2}{*}{ Less 0.} & $\ldots \ldots \ldots$ & & .06 & 2.05 & $\cdots$ & \\
\hline & & & 100.09 & 99.95 & & \\
\hline
\end{tabular}

M. Bluebird granite, Nettie mine. Alaskose. An aplite. Contains orthoclase and quartz, with a little plagioclase and a sprinkling of biotite. Analysis by $\mathrm{H} . \mathrm{N}$. Stokes, record No. 1686.

N. Same rock and locality as M. Analysis by W. F. Hillebrand, record No. 1692.

O. Pitchstone, or rhyolite-dacite obsidian, Butte. Lassenose. Phenocrysts of andesine, quartz, and orthoclase in a glassy base.

P. Rhyolite, Hyde Park dike. Tehamose. Contains sanidine, quartz, plagioclase, and biotite in a groundmass of quartz and feldspar. Analyses $0, \mathrm{P}$, by Stokes, record No. 1686.

Q. Quartz porphyry, Modoc mine. Toscanose. Contains quartz, orthoclase, and plagioclase in a groundmass of quartz and feldspar. A few small biotites are present. Feldspar partly altered to sericite. Analysis by Hillebrand, record No. 1692.

R. Altered rhyolite porphyry, 1,800-foot level, Anaconda mine. Contains quartz phenocrysts, with sericite, pyrite, etc. Analysis by Hillebrand, record No. 1971, partial only. 


\begin{tabular}{|c|c|c|c|c|c|c|}
\hline . & M. & N. & 0. & P. & Q. & $\mathrm{R}$. \\
\hline 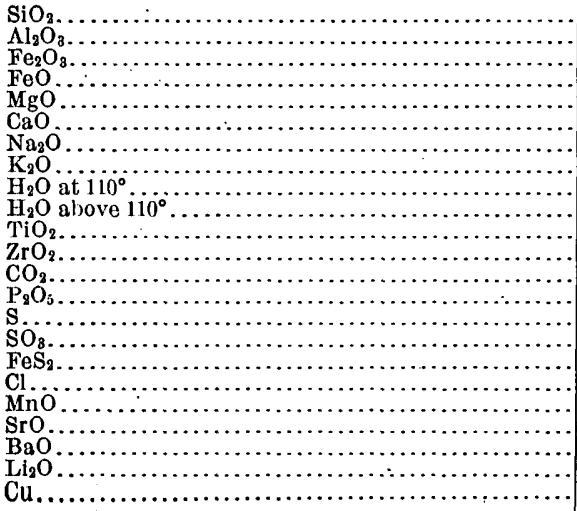 & \begin{tabular}{r}
76.87 \\
12.52 \\
.67 \\
none \\
.09 \\
.49 \\
2.47 \\
5.78 \\
.25 \\
.52 \\
.11 \\
$\ldots . .6$ \\
\hdashline. .6 \\
.05 \\
trace
\end{tabular} & 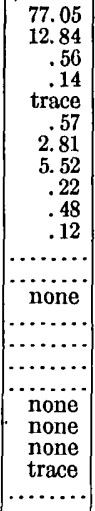 & $\begin{array}{r}67.55 \\
15.68 \\
.98 \\
1.02 \\
1.11 \\
2.51 \\
4.15 \\
2.86 \\
.38 \\
2.76 \\
.34 \\
\text { none } \\
\text { none } \\
.12 \\
\ldots \ldots . . \\
\text { none } \\
. \cdots .05 \\
\text { trace } \\
.03 \\
.11 \\
\text { none }\end{array}$ & $\begin{array}{r}74.34 \\
12.97 \\
.75 \\
.54 \\
.86 \\
.85 \\
2.49 \\
4.72 \\
1.03 \\
.11 \\
.18 \\
.05 \\
\text { none } \\
.07 \\
\ldots \ldots . . \\
.03 \\
\ldots \ldots . . \\
\text { none } \\
\text { trace } \\
\text { trace } \\
.07 \\
\text { trace }\end{array}$ & \begin{tabular}{|r|}
69.95 \\
15.14 \\
.38 \\
.83 \\
.56 \\
1.45 \\
2.70 \\
6.36 \\
.40 \\
.91 \\
.24 \\
.02 \\
.37 \\
.10 \\
$\ldots .10$ \\
$a .39$ \\
undet. \\
.08 \\
.02 \\
.13 \\
trace \\
.03 \\
\end{tabular} & 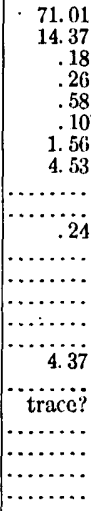 \\
\hline & 99.82 & 100.31 & 99.65 & 100.06 & 100.06 & 97.20 \\
\hline
\end{tabular}

$n$ Other sulphides than pyrite are present.

\section{BOULDER BATHOLITH.}

Rocks collected by W. H. Weed, who supplies the petrographic data.

A. Quartz monzonite, or "Butte granite," from the Frohner mine, 10 miles south of Helena. Harzose. Contains quartz, andesine, orthoclase, hornblende, and biotite, with minor accessories.

B. Granite, Boulder type, near Boulder. Near adamellite. Toscanose. Contains quartz, feldspar, black mica, and dark-green hornblende. Has an unusual amount of plagioclase.

C. Diorite, Red Mountain. Andose. Contains pale-green to colorless hornblende, plagioclase varying from labradorite to albite, some orthoclase, and a little quartz. Biotite, apatite, and magnetite also occur.

D. Diorite, Red Mountain. Kentallenose. Contact of granite with limestones. Consists mainly of green hornblende and "basic" plagioclase. Little quartz present.

E. Quartz monzonite, Rèd Rock Creek. Harzose. Contains hornblende, augite, biotite, plagioclase, and orthoclase, with a little magnetite and apatite.

Analyses by H. N. Stokes, record Nos. 1686, 1787.

\begin{tabular}{|c|c|c|c|c|c|}
\hline & A. & B. & C. & D. & E. \\
\hline 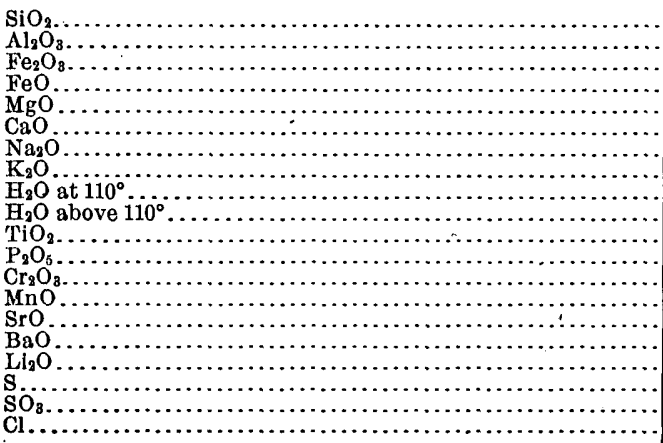 & $\begin{array}{r}64.17 \\
15.25 \\
2.16 \\
2.98 \\
2.60 \\
4.24 \\
2.62 \\
4.34 \\
.16 \\
.65 \\
.67 \\
.16 \\
.04 \\
\text { trace } \\
.07 \\
\text { trace } \\
\cdots .07 \\
\text { trace }\end{array}$ & $\begin{array}{r}67.12 \\
15.00 \\
1.62 \\
2.23 \\
1.74 \\
3.43 \\
2.76 \\
4.52 \\
.09 \\
.58 \\
.48 \\
.15 \\
\text { none } \\
.06 \\
.03 \\
.07 \\
\text { trace } \\
\ldots \ldots . . . \\
\text { trace } \\
\ldots . . . .\end{array}$ & $\begin{array}{r}56.41 \\
17.62 \\
1.24 \\
3.55 \\
3.97 \\
8.66 \\
3.25 \\
2.61 \\
.14 \\
.76 \\
.68 \\
.49 \\
\ldots . .6 \\
.08 \\
.08 \\
.09 \\
\text { none } \\
\text { none } \\
\text { none } \\
.07\end{array}$ & $\begin{array}{r}49.22 \\
12.02 \\
2.77 \\
8.80 \\
9.29 \\
10.56 \\
1.90 \\
1.70 \\
.27 \\
1.63 \\
.95 \\
.43 \\
\ldots . . \\
\text { trace } \\
.03 \\
.03 \\
\text { trace } \\
.05 \\
.04 \\
.08\end{array}$ & $\begin{array}{r}61.64 \\
15.63 \\
3.39 \\
2.69 \\
2.82 \\
4.90 \\
2.64 \\
3.72 \\
.28 \\
.91 \\
.71 \\
.21 \\
\text { trace } \\
.04 \\
.04 \\
.08 \\
\text { trace } \\
\ldots . . . \\
\text { none } \\
\text {...... }\end{array}$ \\
\hline & 100.18 & 99.88 & 99.70 & 99.77 & 99.70 \\
\hline
\end{tabular}


F. Rhyolite, top of Red Mountain, Rimini. Liparose. Quartz and feldspar phenocrysts in a groundmass of the same minerals.

G. Andesite porphyry, Hiawatha Creek, above basin. Amiatose. Contains plagioclase, orthoclase, and augite.

H. Andesite porphyry, Zosel mining district, near Daylight. Near bandose. Somewhat altered. Contains augite, andesine, and olivine in a groundmass of plagioclase, augite, and iron oxide. Ferromagnesian minerals altered to serpentine. Analyses by H. N. Stokes, record Nos. 1686, 1787.

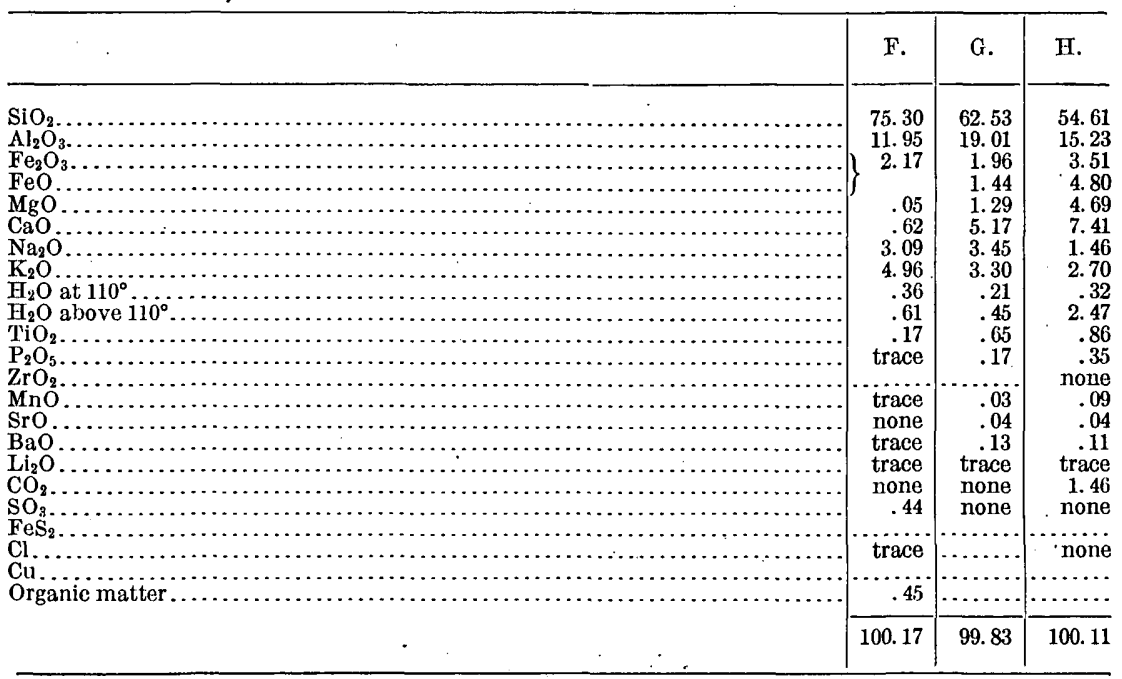

\section{CRAZY MOUNTAINS.}

The greater number of the rocks in this group were received from J. E. Wolff, who has supplied the petrographic data. The few exceptions are properly indicated. The three tinguaites and the elæolite syenite were described by Wolff and Tarr in Bull. Mus. Comp. Zool., vol. 16, No. 12, 1893, in a paper upon "Acmite-Trachyte from the Crazy Mountains." The renaming of these rocks is due to Wolff. The analyses, except when otherwise stated, are by W. F. Hillebrand, record No. 1438.

A. Granitite, Big Timber Creek. Toscanose. Small dikes in main diorite stock. Contains quartz, orthoclase, plagioclase (oligoclase), and biotite. P. R. C. 1720.

B. Hornblende granitite, main stock, Big Timber Creek. Dacose. Contains biotite, hornblende, quartz, orthoclase, and plagioclase (oligoclase). P. R. C. 1721.

C. Granite porphyry, intrusive sheet, north part of the mountains. Lassenose. Contains orthoclase, albite, quartz, biotite, and green hornblende in a groundmass of quartz and untwinned feldspar. P. R. C. 1722.

D. Porphyrite, intrusive sheet, Sweet Grass Creek. Lassenose. Contains hornblende and andesine in a groundmass of plagioclase, orthoclase, and quartz, with accessory magnetite. P. R. C. 1723.

E. Porphyrite, dike in contact zone, Sweet Grass Creek. Toscanose. Contains brown hornblende, biotite, and labradorite in a groundmass of plagioclase, biotite, and hornblende, with a little quartz and orthoclase. P. R. C. 1724.

F. Porphyrite, intrusive sheet, middle peak of Three Peaks. Akerose. Contains andesine, hornblende, augite, and biotite in a groundmass of plagioclase, orthoclase, and quartz. P. R. C. 1725. 


\begin{tabular}{|c|c|c|c|c|c|c|}
\hline & A. & B. & C. & D. & E. & F. \\
\hline 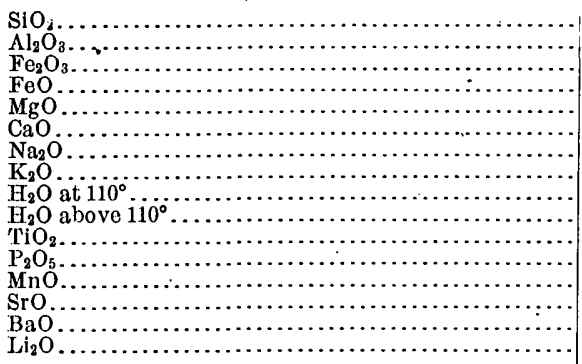 & $\begin{array}{r}74.37 \\
13.12 \\
.73 \\
.87 \\
.35 \\
1.26 \\
2.57 \\
6.09 \\
.05 \\
.25 \\
.29 \\
.06 \\
\text { trace } \\
\text { trace } \\
.10 \\
\text { trace }\end{array}$ & $\begin{array}{r}64.47 \\
15.45 \\
2.25 \\
2.25 \\
2.68 \\
3.63 \\
4.54 \\
3.19 \\
.05 \\
.63 \\
.75 \\
.22 \\
.06 \\
.04 \\
.23 \\
\text { trace? }\end{array}$ & $\begin{array}{r}69.93 \\
14.95 \\
1.78 \\
.55 \\
.60 \\
1.46 \\
5.30 \\
3.99 \\
.12 \\
.32 \\
.33 \\
.33 \\
\text { trace } \\
.06 \\
.29 \\
\operatorname{traco}\end{array}$ & $\begin{array}{r}66.28 \\
16.21 \\
.80 \\
2.06 \\
1.57 \\
3.53 \\
4.36 \\
3.20 \\
.12 \\
.78 \\
.50 \\
.20 \\
\text { trace } \\
.05 \\
.34 \\
\text { trace? }\end{array}$ & $\begin{array}{r}64.49 \\
17.25 \\
.86 \\
2.42 \\
1.24 \\
3.79 \\
4.19 \\
4.15 \\
.06 \\
.54 \\
.51 \\
.23 \\
\text { trace } \\
.08 \\
.30 \\
\text { trace }\end{array}$ & $\begin{array}{r}61.08 \\
16.62 \\
2.87 \\
2.56 \\
1.65 \\
3.66 \\
4.75 \\
3.90 \\
.44 \\
.97 \\
.73 \\
.63 \\
\text { trace } \\
.08 \\
.32 \\
\text { trace }\end{array}$ \\
\hline & 100.11 & 100.44 & 100.01 & 100.00 & 100.11 & 100.26 \\
\hline
\end{tabular}

G. Porphyrite, intrusive sheet; north of Shields River basin. Akerose. Contains plagioclase, hornblende, and biotite, in a groundmass of plagioclase, a little orthoclase, augite, hornblender and magnetite. P. R. C. 1726.

H. Porphyrite, intrusive sheet, northern part of the mountains. Akerose. Contains brown hornblende, green augite, and plagioclase, in a groundmass of plagioclase, augite, and magnetite, with accessory apatite. P. R. C. 1727.

I. Syenite, ridge north of Shields River basin. Akerose. Contains hornblende, green augite, and anorthoclase, with accessory sphene, apatite, and magnetite. P. R. C. 1728 .

J. Diabase-porphyrite (?), dike south of Shields River basin. I. 5. 3. 4. Contains labradorite and decomposed augite in a groundmass of plagioclase, epidote, and chlorite. P. R. C. 1729.

K. Diorite-porphyrite, Big Timber Creek. Andose. Contains labradorite, augite, hornblende, biotite, orthoclase, quartz, magnetite, and apatite. P. R. C. 1730.

L. Quartz diorite, main stock on Sweet Grass Creek. Andose. Contains hornblende, biotite, augite, labradorite, orthoclase, and quartz, with accessory apatite, magnetite, olivine, and hypersthene. P. R. C. 1731.

\begin{tabular}{|c|c|c|c|c|c|c|}
\hline & G. & H. & I. & J. & K. & I. \\
\hline 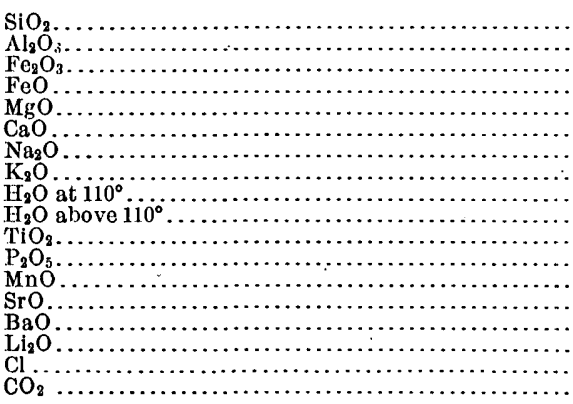 & $\begin{array}{r}56.75 \\
16.40 \\
4.78 \\
3.10 \\
3.22 \\
5.34 \\
4.19 \\
3.36 \\
.40 \\
.82 \\
.86 \\
. .52 \\
.17 \\
.10 \\
.33 \\
\text { trace? }\end{array}$ & $\begin{array}{r}54.69 \\
16.53 \\
4.54 \\
2.83 \\
2.99 \\
5.34 \\
5.19 \\
3.93 \\
.32 \\
1.05 \\
.91 \\
.73 \\
.07 \\
.06 \\
.37 \\
\text { trace? } \\
\text { trace } \\
.83\end{array}$ & $\begin{array}{r}58.28 \\
17.89 \\
3.20 \\
1.73 \\
1.51 \\
3.69 \\
5.89 \\
5.34 \\
.17 \\
.98 \\
.64 \\
.26 \\
.06 \\
.05 \\
. .36 \\
\text { trace } \\
\ldots . . .\end{array}$ & $\begin{array}{r}58.28 \\
19.37 \\
1.35 \\
2.98 \\
1.30 \\
4.78 \\
4.40 \\
3.75 \\
.44 \\
1.78 \\
.96 \\
.35 \\
.07 \\
.09 \\
.25 \\
\text { trace? } \\
.33\end{array}$ & $\begin{array}{r}54.56 \\
17.58 \\
4.30 \\
4.98 \\
2.86 \\
6.00 \\
4.43 \\
2.70 \\
.02 \\
.38 \\
1.34 \\
.60 \\
.06 \\
.08 \\
.27 \\
\text { trace }\end{array}$ & $\begin{array}{r}53.48 \\
19.35 \\
2.37 \\
4.90 \\
3.67 \\
7.55 \\
4.07 \\
1.41 \\
.16 \\
.80 \\
1.07 \\
.62 \\
.06 \\
.11 \\
.19 \\
\text { trace? } \\
.08\end{array}$ \\
\hline & 100.34 & 100.38 & 100.05 & 100.48 & 100.16 & 99.89 \\
\hline
\end{tabular}

M. Diorite, head of Rock Creek. Shoshonose. Contains biotite, labradorite, and augite, in a groundmass of plagioclase, orthoclase, and quartz, with accessory magnetite, apatite, and hornblende. P. R. C. 1732.

N. Diorite, main stock, Big Timber Creek. 'Andose. Contains biotite, augite, labradorite, quartz, orthoclase, apatite, and magnetite. P. R. C. 1733. 
O. Olivine gabbro, Big Timber Creek. IV. $2^{2} \cdot 1^{2} .2$. Contains labradorite (?); brown hornblende, augite, olivine, and magnetite. P. R. C. 1734.

P. Rock from Musselshell River, north of Crazy Mountains. Monchiquose. Received from J. S. Diller, but undescribed. Regarded by W. H. Weed as monchiquite. Analysis by L. G. Eakins, record No. 1021.

Q. Hornstone, metamorphosed shale, contact zone, Sweet Grass Creek. An aggregate of augite, quartz, triclinic feldspar, and biotite. P. R. C. 17.35.

\begin{tabular}{|c|c|c|c|c|c|}
\hline & M. & N. & $\mathrm{O}$ & P. & Q. \\
\hline 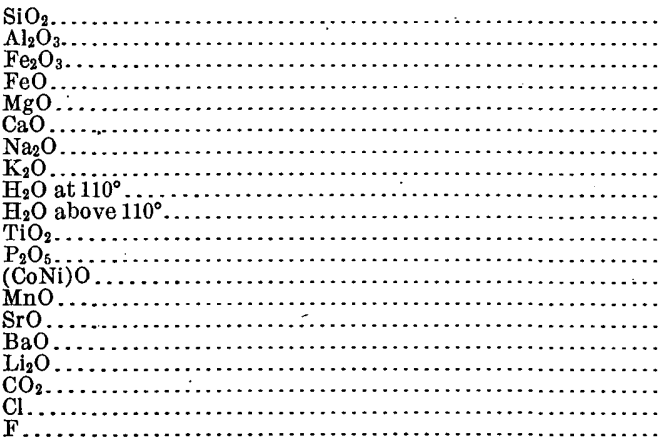 & $\begin{array}{r}57.97 \\
15.65 \\
.73 \\
2.80 \\
4.96 \\
10.93 \\
3.03 \\
3.16 \\
.22 \\
.38 \\
.60 \\
.15 \\
\text { trace } \\
.02 \\
.09 \\
\text { trace } \\
\text { trace } \\
\text { trace }\end{array}$ & $\begin{array}{r}50.73 \\
19.99 \\
3.20 \\
4.66 \\
3.48 \\
8.55 \\
4.03 \\
1.89 \\
.11 \\
.66 \\
1.59 \\
.81 \\
\ldots .05 \\
.05 \\
.11 \\
.27 \\
\text { trace } \\
\ldots \ldots . . \\
\ldots \ldots . . .\end{array}$ & $\begin{array}{r}40.42 \\
9.98 \\
9.83 \\
10.67 \\
11.56 \\
10.78 \\
1.26 \\
.60 \\
.45 \\
1.17 \\
2.51 \\
.63 \\
.02 \\
.25 \\
.02 \\
.05 \\
\text { trace }\end{array}$ & $\begin{array}{r}44.66 \\
12.12 \\
5.81 \\
3.20 \\
8.77 \\
8.14 \\
4.47 \\
2.75 \\
4.33 \\
1.02 \\
2.02 \\
\cdots .21 \\
\cdots \cdots \\
\cdots \cdots \\
\cdots \cdots \\
\cdots \\
\cdots \\
\cdots\end{array}$ & $\begin{array}{r}57.31 \\
14.24 \\
1.00 \\
3.24 \\
4.60 \\
11.31 \\
2.64 \\
4.55 \\
.25 \\
.24 \\
.52 \\
.18 \\
\ldots . .08 \\
.08 \\
\text { trace? } \\
.19 \\
\text { trace? } \\
.17\end{array}$ \\
\hline & 100.69 & 100.13 & 100.20 & 99.69 & 100.52 \\
\hline
\end{tabular}

R. Elæolite syenite, Peaked Butte, northeast side of the mountains. Umptekose. Described by Wolff and Tarr, loc. cit. Contains anorthoclase, augite, occasionally sodalite, ægirine, apatite, magnetite, and some interstitial nephelite. Analysis by W. H. Melville, record No. 1291. P. R. C. 1736.

Ra. Anorthoclase from R. Analysis by W. F. Hillebrand, record No. 1297.

S. Tinguaite, var. sölvsbergite, intrusive sheet north of Shields River. P. R. C. 1737.

T. Tinguaite, var. sölvsbergite, dike 'north part of mountains. Nordmarkose. P. R. C. 1738.

U. Tinguaite; var. sölvsbergite, dike at head of Sixteenmile Creek. Nordmarkose. P. R. C. 1739.

$\mathrm{S}, \mathrm{T}$, and $\mathrm{U}$ are the rocks described by Wolff and Tarr in their paper upon "Acmite Trachyte." Mineral composition the same as under R. Analyses by W. H. Melville, record No. 1291.

\begin{tabular}{|c|c|c|c|c|c|}
\hline & R. & Ra. & S. & $\mathrm{T}$. & U. \\
\hline 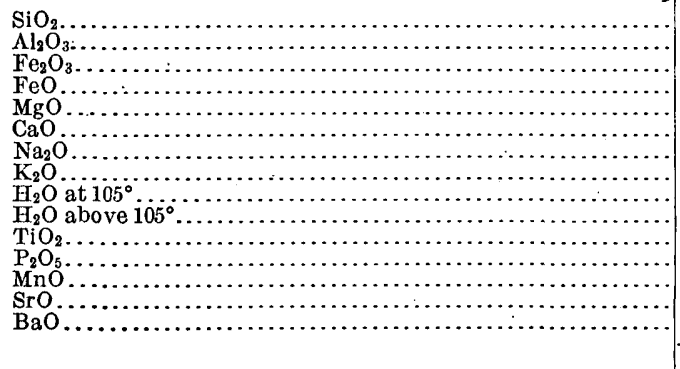 & $\begin{array}{r}59.66 \\
16.97 \\
3.18 \\
1.15 \\
. .80 \\
2.32 \\
8.38 \\
4.17 \\
.07 \\
2.53 \\
\text { trace } \\
.14 \\
.19 \\
\cdots \cdots \\
\end{array}$ & 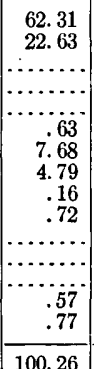 & $\begin{array}{r}58.70 \\
19.26 \\
3.37 \\
.58 \\
1.76 \\
8.45 \\
4.53 \\
2.07 \\
2.57 \\
\text { trace } \\
.10 \\
.10 \\
\cdots \ldots . . . \\
\end{array}$ & $\begin{array}{r}62.17 \\
18.58 \\
2.15 \\
1.05 \\
1.53 \\
7.57 \\
7.56 \\
3.88 \\
1.07 \\
\text { trace } \\
\text { trace } \\
\ldots \ldots \ldots . \\
\cdots \cdots \cdots . . \\
\end{array}$ & $\begin{array}{r}64.33 \\
17.52 \\
3.06 \\
.94 \\
.34 \\
.56 \\
7.30 \\
4.28 \\
.04 \\
.95 \\
\text { trace } \\
\text { trace } \\
.35 \\
\ldots \ldots \ldots . . \\
9.67 \\
99.67\end{array}$ \\
\hline & 99.56 & 100.26 & 100.00 & 99.50 & 99.67 \\
\hline
\end{tabular}


V. Theralite, Gordons Butte. Malignose. Contains green augite, ægirine, biotite, olivine, nephelite, sodalite, and a feldspar, partly sanidine, containing $\mathrm{K}, \mathrm{Na}, \mathrm{Ba}, \mathrm{Sr}$, and $\mathrm{Ca}$; also accessory apatite, magnetite, and sphene.

W. Theralite, Gordons Butte; another sample. Kamerunose. Analysis by E. A. Schneider, record No. 1281. P. R. C. 75 .

$\mathrm{X}$. Theralite, north of Alabaugh Creek. Essexose. Described by Wolff for the Educational Series (Bull. 150). Contains augite, ægirine, biotite, olivine, magnetite, apatite, nephelite partly zeolitized, a mineral of the sodalite group, sanidine, and analcite. Analysis by Schneider, No. 1281. P. R. C. 76.

Rocks V, W, and X are described by Wolff in Bull. 150, pp. 197, 199.

Y. Altered theralite, head of Shields River, west of Loco Mountain. Received from W. H. Weed. Analysis by H. N. Stokes, record No. 1547.

Z. Hornblende picrite, Conical Peak.' Auvergnose. Data supplied by J. P. Iddings. Contains hornblende, plagioclase, hypersthene, augite, olivine, very little primary quartz, and probably some magnetite and apatite. Analysis by L. G. Eakins, record No. 1379.

\begin{tabular}{|c|c|c|c|c|c|}
\hline - & V. & W. & $\mathrm{X}$. & $Y$. & $Z$. \\
\hline 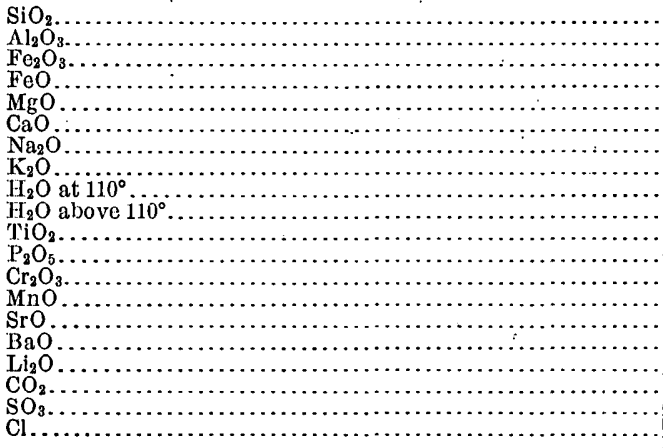 & $\begin{array}{r}44.65 \\
13.87 \\
6.06 \\
2.94 \\
5.15 \\
9.57 \\
5.67 \\
4.49 \\
.96 \\
2.10 \\
.95 \\
1.50 \\
\ldots .17 \\
.37 \\
.76 \\
\text { trace } \\
.11 \\
.61 \\
\text { trace }\end{array}$ & 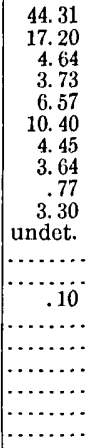 & $\begin{array}{r}47.67 \\
18.22 \\
3.65 \\
3.85 \\
6.35 \\
8.03 \\
4.93 \\
3.82 \\
.38 \\
2.97 \\
\text { undet. } \\
\ldots \ldots . . \\
\ldots \ldots . . . \\
.28 \\
\ldots \ldots . . . \\
\ldots \ldots . . . \\
\ldots \ldots . . . \\
\ldots \ldots . . .\end{array}$ & $\begin{array}{r}48.90 \\
14.70 \\
4.14 \\
3.68 \\
3.95 \\
8.26 \\
5.22 \\
.56 \\
.52 \\
2.44 \\
.95 \\
.79 \\
\ldots . . \\
.03 \\
.13 \\
.31 \\
\text { trace } \\
5.42 \\
.04\end{array}$ & $\begin{array}{r}45.71 \\
10.80 \\
4.43 \\
9.35 \\
13.75 \\
10.48 \\
1.58 \\
.85 \\
.97 \\
1.83 \\
.11 \\
.10 \\
.17 \\
\ldots \ldots . \\
\text { trace } \\
\ldots \ldots \ldots \\
\ldots \ldots \ldots \\
\ldots \ldots \ldots . . \\
\ldots \ldots \ldots\end{array}$ \\
\hline & 99.93 & 99.11 & 100.15 & 100.04 & 100.13 \\
\hline
\end{tabular}

\section{LITTLE BELT MOUNTAINS.}

Rocks collected by W. H. Weed and L. V. Pirsson. Described in a paper on the Neihart and Barker mining districts in Pt. III of the 20th Ann. Analyses by W. F. Hillebrand, record No. 1476, and H. N. Stokes, record No. 1547.

A. Rhyolite porphyry, Yogo Peak, sheet at head of Belt and Running Wolf creeks. Toscanose. Phenocrysts of orthoclase and quartz in a groundmass of quartz and alkali feldspar, with a little white mica and some kaolin. Chlorite, limonite, and calcite are also present, pseudomorphous after biotite and perhaps hornblende. Total amount of secondary minerals very small. Analysis by Hillebrand. P. R. C. 1474.

B. Granite porphyry, Wolf Butte. Toscanose. Phenocrysts of quartz, orthoclase, plagioclase, and biotite in a groundmass of quartz and alkali feldspar. A little apatite and iron ore, with secondary calcite, limonite, chlorite, and white mica. Analysis by Stokes.' P. R. C. 1475.

C. Granite porphyry, top of Barker Mountain. Toscanose. Phenocrysts of orthoclase, oligoclase, biotite, green homblende, sphene, and iron ore, in a groundmass of quartz and alkali feldspar. Also a little apatite and some secondary chlorite and limonite. Analysis by Stokes, P. R, C. 1476. 
D. Syenite, Wright and Edwards mine, Hughesville, near Barker. Toscanose. Very fresh rock. Contains magnetite, ilmenite, hormblende, anorthoclase, albite, subordinate quartz, a little chlorite, calcite, and limonite, and white mica in traces. Analysis by Stokes. P. R. C. 1477.

E. Syenite, Yogo Peak. Monzonose. Described in Am. Jour. Sci.; 3d ser., vol. 50, p. 471. Contains apatite, sphene, iron ore, pyroxene, hornblende, biotite, orthoclase, oligoclase, and quartz, with traces of chlorite and limonite and a little kaolin. Analysis by Hillebrand. P. R. C. 1478.

F. Granite porphyry, dike at head of Sheep Creek. Toscanose. Phenocrysts of orthoclase, some plagioclase, and green hornblende, in a groundmass of alkali feldspar, with some quartz. Also contains a little apatite and iron ore, with some secondary calcite and kaolin. Analysis by Hillebrand. P. R. C. 1479.

\begin{tabular}{|c|c|c|c|c|c|c|}
\hline & A. & B. & C. & D. & E. & F. \\
\hline 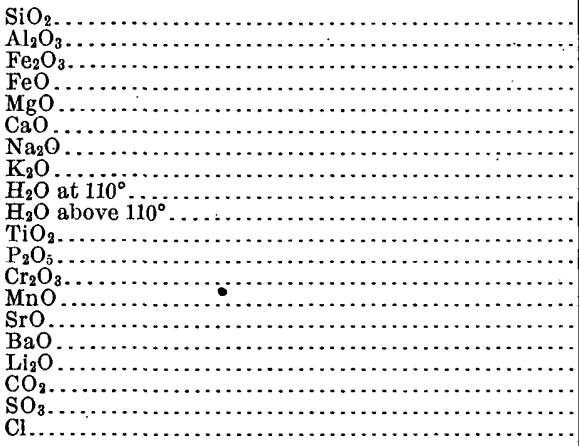 & $\begin{array}{r}73.12 \\
14.27 \\
.51 \\
.26 \\
.24 \\
1.10 \\
3.43 \\
4.90 \\
.68 \\
.73 \\
.08 \\
.03 \\
\text { none } \\
.06 \\
\text { trace } \\
\text { trace } \\
\text { trace } \\
.77 \\
\cdots \ldots . . \\
\cdots \ldots . .\end{array}$ & $\begin{array}{r}69.68 \\
14.97 \\
.79 \\
.34 \\
.66 \\
2.10 \\
3.38 \\
4.40 \\
1.09 \\
.92 \\
.28 \\
.17 \\
\ldots . . . \\
\text { trace } \\
.06 \\
.14 \\
\text { trace } \\
.88 \\
\text { trace } \\
\text { trace }\end{array}$ & $\begin{array}{r}68.60 \\
16.13 \\
2.22 \\
.44 \\
.72 \\
1.36 \\
4.37 \\
4.89 \\
.20 \\
.58 \\
.32 \\
.18 \\
\ldots \ldots \\
\text { trace } \\
.09 \\
.27 \\
\text { trace } \\
\ldots \ldots . . \\
\text { trace } \\
\text { trace }\end{array}$ & $\begin{array}{r}64.64 \\
16.27 \\
2.42 \\
1.58 \\
1.27 \\
2.65 \\
4.39 \\
4.98 \\
.09 \\
.27 \\
.51 \\
.37 \\
\ldots \ldots \\
\text { trace } \\
.08 \\
.18 \\
\ldots . .2 \\
.37 \\
\text { trace } \\
.05\end{array}$ & $\begin{array}{r}61.65 \\
15.07 \\
2.03 \\
2.25 \\
3.67 \\
4.61 \\
.4 .35 \\
4.50 \\
.26 \\
.41 \\
.56 \\
.33 \\
\operatorname{trace} \\
.09 \\
.10 \\
.27 \\
\operatorname{trace} \\
\ldots \ldots \ldots \\
\ldots \ldots \ldots \\
\ldots \ldots \ldots\end{array}$ & $\begin{array}{r}66.29 \\
15.09 \\
1.37 \\
1.17 \\
2.39 \\
2.38 \\
3.96 \\
4.91 \\
.39 \\
.60 \\
.27 \\
.15 \\
\text { none } \\
.06 \\
.07 \\
.30 \\
\text { trace } \\
.45 \\
\ldots \ldots . . \\
\ldots \ldots\end{array}$ \\
\hline . & 100.18 & $\dot{99} .86$ & 100.37 & 100.12 & 100.15 & 99.85 \\
\hline
\end{tabular}

G. Syenite porphyry, intrusive sheet, between Yogo Peak and Big Baldy Mountain. Lassenose. Abundant phenocrysts of hornblende and orthoclase, with less biotite and plagioclase, in a groundmass of alkali feldspar with accessory quartz. Also contains iron ore and apatite, with secondary calcite, chlorite, sericite, and kaolin. Analysis by Hillebiand. P. R. C. 1480.

H. Granite porphyry, north end of Thunder Mountain. Toscanose. Phenocrysts of orthoclase, oligoclase, hornblende, and biotite, in a groundmass of alkali feldspar and very abundant quartz. Also contains a little sphene, iron ore, and apatite, very little secondary chlorite and limonite, and a trace of kaolin. Analysis by Stokes. P. R. C. 1481.

I. Granite porphyry, top of Big Baldy Mountain. Toscanose. Phenocrysts of orthoclase, plagioclase, biotite, iron ore, brown hornblende, and sphene, in a groundmass of quartz and alkali feldspar. Also contains a little apatite, with traces of chlorite, limonite, and kaolin. Analysis by Hillebrand. P. R. C. 1482.

J. Quartz monzonite, talus slope on west side of Bear Park. Dacose. Phenocrysts of biotite, hornblende, plagioclase, and orthoclase, in a groundmass of quartz and alkali feldspar. Also contains a little magnetite, chlorite, white mica, and apatite. Analysis by Stokes. - P. R. C. 1483.

K. Diorite porphyry, Steamboat Mountain. Adamellose. Phenocrysts of orthoclase, plagioclase, hornblende, and biotite, with a little iron ore and apatite, in a groundmass of orthoclase, plagioclase, and quartz. Analysis by Stokes. P. R. C. 1484.

L. Diorite, Carpenter Creek, near Neihart. Andose. Contains green hornblende, biotite, and plagioclase, with some apatite, iron ore, calcite, kaolin, and muscovite, and a very little quartz and orthoclase. Analysis by Hillebrand. P. R. C. 1485. 


\begin{tabular}{|c|c|c|c|c|c|c|}
\hline & G. & H. & I. &. $\mathrm{J}$ & K. & L. \\
\hline \multirow[t]{2}{*}{ 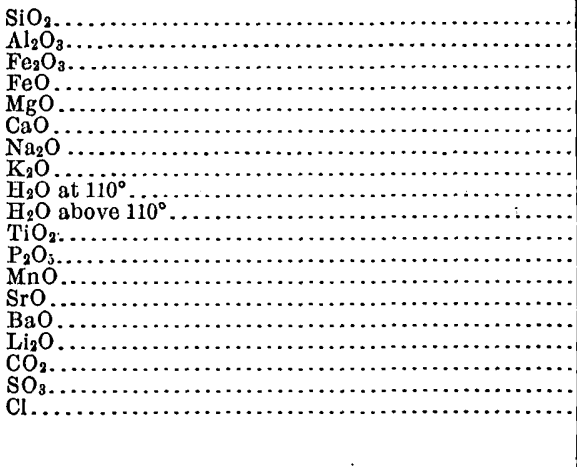 } & $\begin{array}{r}62.58 \\
16.42 \\
2.46 \\
1.96 \\
1.84 \\
2.47 \\
4.57 \\
3.91 \\
.38 \\
1.40 \\
.40 \\
.33 \\
.08 \\
.10 \\
.41 \\
\operatorname{trace} \\
.77 \\
\ldots \ldots \ldots \\
\ldots \ldots . . .\end{array}$ & $\begin{array}{r}67.44 \\
15.78 \\
1.58 \\
.85 \\
1.43 \\
2.38 \\
4.11 \\
4.87 \\
.32 \\
.70 \\
.32 \\
.21 \\
\text { trace } \\
.09 \\
.24 \\
\text { trace } \\
\ldots \ldots . . \\
\text { trace } \\
\text { trace }\end{array}$ & \begin{tabular}{|r|}
67.04 \\
15.25 \\
1.69 \\
1.13 \\
1.75 \\
2.17 \\
4.09 \\
5.10 \\
.56 \\
.51 \\
.20 \\
.21 \\
.05 \\
.03 \\
.33 \\
trace? \\
$\ldots \ldots .$. \\
$\ldots \ldots .$. \\
$\ldots \ldots .$. \\
$\ldots \ldots$.
\end{tabular} & $\begin{array}{r}65.95 \\
15.44 \\
2.02 \\
1.60 \\
2.65 \\
3.07 \\
4.25 \\
3.87 \\
.26 \\
.85 \\
.39 \\
.25 \\
\text { trace } \\
.10 \\
.35 \\
\ldots \ldots \\
\ldots \ldots . . \\
.02 . \\
.04\end{array}$ & $\begin{array}{r}62.18 \\
15.77 \\
1.83 \\
2.44 \\
3.55 \\
4.13 \\
3.92 \\
3.91 \\
.30 \\
.70 \\
.55 \\
.32 \\
\text { trace } \\
.16 \\
.43 \\
\text { trace } \\
\ldots . . . . \\
\text { trace } \\
.04\end{array}$ & $\begin{array}{r}55.13 \\
20.27 \\
1.52 \\
4.29 \\
1.80 \\
7.05 \\
4.31 \\
2.84 \\
.14 \\
.95 \\
.74 \\
.40 \\
.13 \\
.06 \\
.11 \\
\text { trace } \\
.26 \\
\ldots \ldots . . \\
\ldots \ldots . .\end{array}$ \\
\hline & 100.08 & 100.32 & 100.11 & 100.11 & 100.23 & 100.00 \\
\hline
\end{tabular}

'M. Minette, intrusive sheet, head of Sheep Creek. . Monzonose. Chiefly biotite, augite, and orthoclase, with accessory apatite, plagioclase, quartz, and iron ore, and some secondary calcite, chlorite, and kaolin. Analysis by Hillebrand. P. R. C. 1486.

N. Monzonite, Yogo Peak. Monzonose. Described in Am. Jour. Sci., 3d ser., vol. 50 , p. 473 , and 4 th ser., vol. 1, p. 356. Contains apatite, sphene, iron ore, pyroxene, hornblende, biotite, orthoclase, and oligoclase, and a little secondary kaolin. Analysị by Hillebrand. P. R. C. 1487.

O. Monchiquite, dike on Big Baldy Mountain. Monchiquose. Contains much pyroxene, a few serpentinized olivines, iron ore, and apatite in a colorless base of analcite. Analysis by Hillebrand. P. R. C. 1488.

P. Monchiquite, dike on Bandbox Mountain. Near wyomingose. Contains olivine, augite, biotite, analcite, and apatite, with traces of serpentine and chlorite. Analysis by Stokes. P. R. C. 1489.

Q. Shonkinite, Yogo Peak. Shonkinose. Described in Am. Jour. Sci., 3d ser., vol. 50, p. 474. Chiefly augite and orthoclase, with a considerable amount of accessory biotite, iron ore, and andesine, less apatite and olivine, and a trace of kaolin. Analysis by Hillebrand. P. R. C. 1490.

\begin{tabular}{|c|c|c|c|c|c|}
\hline - & M. & N. & 0 & P. & Q. \\
\hline 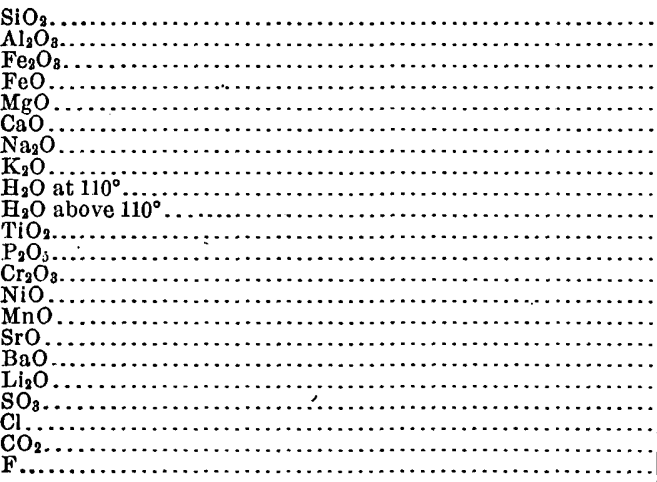 & $\begin{array}{r}52.26 \\
13.96 \\
2.76 \\
4.45 \\
8.21 \\
7.06 \\
2.80 \\
3.87 \\
1.53 \\
1.34 \\
.58 \\
.52 \\
\operatorname{trace} \\
\ldots .14 \\
.05 \\
.23 \\
\operatorname{trace} \\
\ldots \ldots \\
\ldots . . . \\
. . .\end{array}$ & $\begin{array}{r}54.42 \\
14.28 \\
3.32 \\
4.13 \\
6.12 \\
7.72 \\
3.44 \\
4.22 \\
.22 \\
.38 \\
.80 \\
.59 \\
\text { trace } \\
\ldots . .10 \\
.10 \\
.13 \\
.32 \\
\text { trace } \\
\ldots \ldots \ldots \\
\ldots \ldots . . . \\
\ldots \ldots . . \\
\ldots \ldots . .\end{array}$ & $\begin{array}{r}48.35 \\
13.27 \\
4.38 \\
3.23 \\
8.36 \\
9.94 \\
3.35 \\
3.01 \\
.90 \\
2.89 \\
.52 \\
.40 \\
\text { trace } \\
.04 \\
.19 \\
09 \\
.54 \\
\text { trace } \\
\ldots \ldots . \\
\ldots . . . \\
.30 \\
25\end{array}$ & $\begin{array}{r}48.39 \\
11.6 .1 \\
4.09 \\
3.57 \\
12.55 \\
7.64 \\
4.14 \\
3.24 \\
.28 \\
2.56 \\
.73 \\
.45 \\
.07 \\
\ldots \ldots . . \\
\text { trace } \\
.15 \\
.32 \\
\text { trace } \\
.08 \\
\text { trace } \\
\ldots \ldots . . . \\
\ldots \ldots . . .\end{array}$ & $\begin{array}{r}48.98 \\
12.29 \\
2.88 \\
5.77 \\
9.19 \\
9.65 \\
2.22 \\
4.96 \\
.26 \\
.56 \\
1.44 \\
.98 \\
\text { trace } \\
\ldots . . . \\
.08 \\
.08 \\
.43 \\
\text { trace } \\
\ldots \ldots . \\
\ldots \ldots . . \\
\ldots \ldots . . \\
.22\end{array}$ \\
\hline 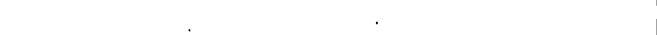 & 100.25 & 100.19 & 100.01 & 99.90 & 99.99 \\
\hline
\end{tabular}




\section{CASTLE MOUNTAIN DISTRICT.}

Rocks described by Weed and Pirsson in Bull. 139. Analyses made by Pirsson in the laboratory of the Sheffield Scientific School.

A. Rhyolite, between Fourmile and Fivemile creeks, near Smith River. Liparose. Contains quartz, soda orthoclase, tourmaline, and a little iron ore and zircon. P. R. C. 560 .

B. Quartz-tourmaline porphyry, upper Fourmile Creek. Liparose. Contains quartz, orthoclase, plagioclase, tourmaline, fluorite, and a little white mica, apatite, and zircon. P. R. C. 558.

C. Granite, Elk Peak. Liparose. Contains quartz, orthoclase, oligoclase, biotite, hornblende, iron ore, apatite, sphene, and zircon. The last three minerals are rare. P. R. C. 551 .

D. Quartz porphyry, sheet or ridge between Fourmile and Checkerboard creeks. Liparose. Contains quartz, plagioclase, an untwinned feldspar and biotite, with occasional grains of iron ore and crystals of apatite and zircon. P. R. C. 557.

E. Rhyolite-pitchstone, forks of Checkerboard Creek. Kallerudose. Essentially glass, inclosing grains of iron ore. P. R. C. 561.

F. Aplitic granite, dike between Blackhawk and Robinson. Liparose. Contains quartz and unstriated feldspar, some plagioclase, and occasional biotite. P. R. C. 553 .

G. Quartz porphyry, Musselshell Canyon. Liparose. Contains quartz, biotite, plagioclase, apatite, iron ore, and zircon. P. R. C. 556.

\begin{tabular}{|c|c|c|c|c|c|c|c|}
\hline . & A. & B. & C. & D. & E. & F. & G. \\
\hline 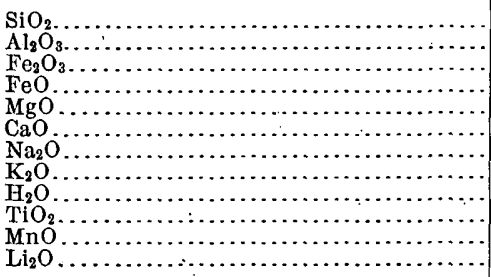 & $\begin{array}{r}74.90 \\
13.64 \\
.66 \\
.50 \\
\text { trace } \\
.61 \\
4.22 \\
4.64 \\
.33 \\
.15 \\
\text { trace } \\
\text { trace }\end{array}$ & $\begin{array}{r}74.82 \\
13.80 \\
.37 \\
.30 \\
.10 \\
.17 \\
4.33 \\
4.81 \\
.83 \\
.25 \\
\text { trace } \\
\text { trace }\end{array}$ & $\begin{array}{r}72.48 \\
13.14 \\
1.66 \\
1.02 \\
.15 \\
1.04 \\
4.22 \\
4.88 \\
.42 \\
.32 \\
\text { trace } \\
\text { trace }\end{array}$ & $\begin{array}{r}72.38 \\
14.71 \\
1.09 \\
.82 \\
.70 \\
.67 \\
4.28 \\
4.15 \\
.92 \\
.10 \\
\text { trace } \\
\text { trace }\end{array}$ & $\begin{array}{r}72.56 \\
12.33 \\
.80 \\
.82 \\
\text { trace } \\
\text { trace } \\
5.36 \\
3.08 \\
4.59 \\
.20 \\
\text { trace }\end{array}$ & $\begin{array}{r}72.88 \\
12.90 \\
.74 \\
1.05 \\
.75 \\
.81 \\
3.72 \\
5.03 \\
1.22 \\
.45 \\
.05 \\
\ldots . . .\end{array}$ & $\begin{array}{r}71.67 \\
15.82 \\
1.18 \\
.35 \\
. .13 \\
.25 \\
4.46 \\
4.45 \\
1.21 \\
.10 \\
\text { trace } \\
\ldots . . .\end{array}$ \\
\hline Sp. gr $\ldots \ldots, \ldots \ldots \ldots \ldots \ldots \ldots \ldots \ldots \ldots \ldots \ldots \ldots \ldots \ldots$ & $\begin{array}{r}99.65 \\
2.61\end{array}$ & $\begin{array}{r}99.78 \\
2.59\end{array}$ & $\begin{array}{r}99.33 \\
2.62\end{array}$ & $\begin{array}{r}99.82 \\
2.61\end{array}$ & $\begin{array}{r}99.74 \\
2.37\end{array}$ & $\begin{array}{r}99.60 \\
2.64\end{array}$ & $\begin{array}{r}99.62 \\
2.60\end{array}$ \\
\hline
\end{tabular}

H. Feldspar porphyry, dike below Castle. Lassenose: Contains orthoclase, less plagioclase, hornblende, biotite partly altered to chlorite, sometimes allanite, and occasional iron ore, apatite, and zircon. P. R. C. 555.

I. Rhyolitic tuff, near forks of Checkerboard Creek. Contains fragments of shale, quartz, plagioclase, sanidine, and hornblende, in a brownish material carrying pumiceous glass. Much decomposed, with formation of kaolin. P. R. C. 562.

J. Syenitic mass included in granite, head of Cottonwood Creek. Akerose. Contains orthoclase, plagioclase, quartz, hornblende, biotite, apatite, and iron ore. A hornblende-mica syenite. P. R. C. 554.

K. Diorite, between Blackhawk and Robinson. Andose. Contains plagioclase, orthoclase, quartz, biotite, diallage, hypersthene, iron ore, apatite, and zircon. P. R.C 559 .

L. Basalt, Volcano Butte. Kilauose. Contains labradorite, augite, olivine, a little serpentine, occasional quartz grains, small patches of glass, iron ore, chiefly ilmenite, apatite, a trace of calcite, and a mineral which may be nephelite or analcite. P.R. C. 565. 
M. Augite vogesite, dike on west side of upper Fourmile Creek. Kentallenose. Contains augite, hornblende, iron ore, a little plagioclase, orthoclase, calcite, and some decomposition products. P. R. C. 563.

$N$. Monchiquite-like dike rock, west side of upper Willow Creek. Ourose. Contains augite, olivine, biotite, ilmenite, and a colorless base which appears to be partially zeolitized glass. Also a little serpentine, chlorite, and calcite. P. R. C. 564.

\begin{tabular}{|c|c|c|c|c|c|c|c|}
\hline & H. & I. & J. & K. & L. & M. & N. \\
\hline 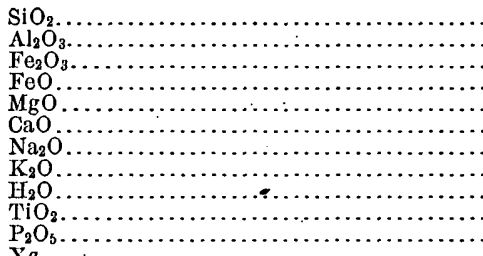 & $\begin{array}{r}65.87 \\
16.82 \\
1.58 \\
1.23 \\
1.54 \\
2.65 \\
4.72 \\
3.15 \\
1.43 \\
.37 \\
\text { trace }\end{array}$ & $\begin{array}{r}61.21 \\
15.67 \\
4.06 \\
.62 \\
1.58 \\
2.18 \\
1.57 \\
2.75 \\
10.20 \\
.56\end{array}$ & $\begin{array}{r}61.87 \\
17.26 \\
2.35 \\
2.43 \\
1.82 \\
33.23 \\
5.18 \\
3.83 \\
1.07 \\
.87 \\
\text { trace }\end{array}$ & $\begin{array}{r}56.80 \\
18.30 \\
1.64 \\
5.58 \\
3.63 \\
5.31 \\
4.35 \\
3.28 \\
.53 \\
.46 \\
\text { trace }\end{array}$ & $\begin{array}{r}46.52 \\
10.48 \\
4.40 \\
7.79 \\
10.58 \\
9.49 \\
3.12 \\
1.55 \\
1.79 \\
2.98 \\
.83\end{array}$ & $\begin{array}{r}45.15 \\
15.39 \\
2.76 \\
5.64 \\
6.38 \\
8.83 \\
2.67 \\
2.77 \\
2.85 \\
2.80 \\
.56\end{array}$ & $\begin{array}{r}42.46 \\
12.04 \\
3.19 \\
5.34 \\
12.40 \\
12.14 \\
1.21 \\
2.68 \\
4.03 \\
2.47 \\
.84\end{array}$ \\
\hline 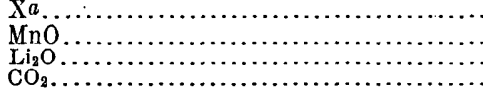 & $\begin{array}{c}\text { } \begin{array}{c}\text { trace } \\
\ldots \ldots \ldots \ldots\end{array} \\
\cdots \ldots \ldots \ldots\end{array}$ & $\begin{array}{r}.10 \\
\text { trace }\end{array}$ & $\begin{array}{r}.03 \\
\text { trace }\end{array}$ & $\underset{\text { trace }}{\text { trace }}$ & $\begin{array}{r}.73 \\
\text { trace } \\
\text { trace }\end{array}$ & $\begin{array}{r}.14 \\
\text { trace } \\
4.27\end{array}$ & $\begin{array}{r}.16 \\
\text { trace } \\
.55\end{array}$ \\
\hline Sp.gr.... & $\begin{array}{r}99.36 \\
2.62\end{array}$ & $\begin{array}{l}100.50 \\
\cdots \cdots \cdots\end{array}$ & $\begin{array}{r}99.94 \\
2.67\end{array}$ & $\begin{array}{r}99.88 \\
2.83\end{array}$ & $\begin{array}{r}100.37 \\
2.99\end{array}$ & $\begin{array}{r}100.21 \\
2.70\end{array}$ & $\begin{array}{r}99.51 \\
2.94\end{array}$ \\
\hline
\end{tabular}

$a$ Unseparated rare earths or metallic acids, such as columbic, tantalic, etc.

7. HIGHWOOD MOUNTAINS.

Rocks collected by W. H. Weed and L. V. Pirsson, and described in Bull. 237. Analyses A to N, inclusive, made in the laboratory of the Sheffield Scientific School, under the direction of Professor Pirsson.

A. Trachy-andesite, head of north fork of Willow Creek. Adamellose. Contains hornblende, biotite, iron ore, apatite, plagioclase, and orthoclase, with a little kaolin and limonite. E. B. Hurlbut and B. Barnes, analysts. P. R. C. 1492.

B. Tinguaite porphyry, dike 1 mile north of divide in Highwood Gap. Highwoodose. Contains abundant phenocrysts of orthoclase and augite in a groundmass of the same minerals; also a little biotite, apatite, and iron ore, and some secondary kaolin. E. B. Hurlbut, analyst. P. R. C. 1493.

C. Sölvsbergite porphyry, dike north end of South Mountain. Pulaskose. Contains phenocrysts of alkali feldspar, ægirine-augite, and melanite, in a groundmass of alkali feldspar. A patite, iron ore, a trace of calcite, and some kaolin are also present. H. W. Foote, analyst. P. R. C. 1494.

D. Gauteite, dike on Aspen Creek. Monzonose. Contains alkali-hornblende and alkali-feldspar phenocrysts, in a groundmass of alkali feldspars; also some apatite and iron ore. Rock stained by limonite. H. W. Foote, analyst. P. R. C. 1495.

E. Syenite porphyry, dike at head of Shonkin Creek. Borolanose. Contains augite, biotite, iron ore, and orthoclase, in a groundmass of alkali feldspar; also apatite and some kaolin. W. M. Bradley, analyst. P. R. C. 1496. 


\begin{tabular}{|c|c|c|c|c|c|}
\hline & A. & B. & C. & D. & E. \\
\hline 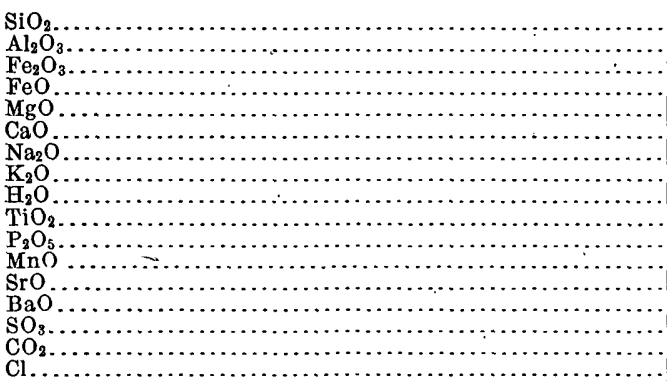 & $\begin{array}{r}59.24 \\
1.84 \\
5.46 \\
1.36 \\
4.79 \\
5.60 \\
3.13 \\
4.22 \\
2.02 \\
.22 \\
.34 \\
\text { trace } \\
\text { none } \\
\text { trace } \\
.08 \\
\text { none } \\
.04\end{array}$ & $\begin{array}{r}58.04 \\
17.24 \\
2.49 \\
1.24 \\
1.79 \\
3.50 \\
3.37 \\
10.06 \\
1.95 \\
.30 \\
.22 \\
\text { trace } \\
\text { undet. } \\
\text { undet. } \\
\text { trace } \\
\ldots .38\end{array}$ & $\begin{array}{r}57.18 \\
18.54 \\
3.65 \\
1.15 \\
.69 \\
2.31 \\
4.48 \\
8.58 \\
2.10 \\
.30 \\
.05 \\
\text { trace } \\
\text { trace } \\
.49 \\
.06 \\
.77\end{array}$ & $\begin{array}{r}55.23 \\
18.31 \\
4.90 \\
2.06 \\
1.85 \\
3.62 \\
4.02 \\
6.43 \\
1.84 \\
.42 \\
.58 \\
\text { trace } \\
\text { trace } \\
.46 \\
.23 \\
\text { none } \\
.32\end{array}$ & $\begin{array}{r}51.94 \\
15.78 \\
4.07 \\
3.71 \\
3.48 \\
6.04 \\
3.44 \\
7.69 \\
2.17 \\
.39 \\
.59 \\
\text { trace } \\
.28 \\
.42 \\
.29 \\
\ldots .08 \\
.08\end{array}$ \\
\hline Less $0 \ldots$ & $\begin{array}{r}100.34 \\
.01\end{array}$ & $\begin{array}{r}100.58 \\
.09\end{array}$ & $\begin{array}{r}100.35 \\
.17\end{array}$ & $\begin{array}{r}100.27 \\
.08\end{array}$ & $\begin{array}{r}99.83 \\
.02\end{array}$ \\
\hline - & 100.33 & 100.49 & 100.18 & 100.19 & 99.81 \\
\hline
\end{tabular}

F. Syenite, top of Palisade Butte. Borolanose. Contains augite, iron ore, apatite, alkali feldspar, a little biotite, some limonite in cracks, much natrolite, and possibly other zeolites. H. W. Foote, analyst. P. R. C. 1497.

G. Leucite shonkinite, head of Davis Creek. Shonkinose. Contains augite, iron ore, olivine, biotite, apatite, alkali feldspar, leucite, and some zeolites, with traces of limonite and serpentine. E. B. Hurlbut, analyst. P. R. C. 1498.

H. Analcite basalt, dike east side of Highwood Gap. Monchiquose. Contains augite, olivine, biotite, iron ore, apatite, and analcite, with some serpentine and a little kaolin. H. W. Foote, analyst. P. R. C. 1499.

I. Leucite basalt, saddle between Highwood and Pinewood peaks. Shonkinose. Contains augite, iron ore, leucite, apatite, altered olivine, chlorite, calcite, and zeolites. H. W. Foote, analyst. P. R. C. 1500.

J. Mica basalt, dike on Arrow Peak. Cascadose. Phenocrysts of augite, olivine, and biotite, with some leucite, in a groundmass of the same minerals. Also analcite, iron ore, and apatite. H. W. Foote, analyst. P. R. C. 1501.

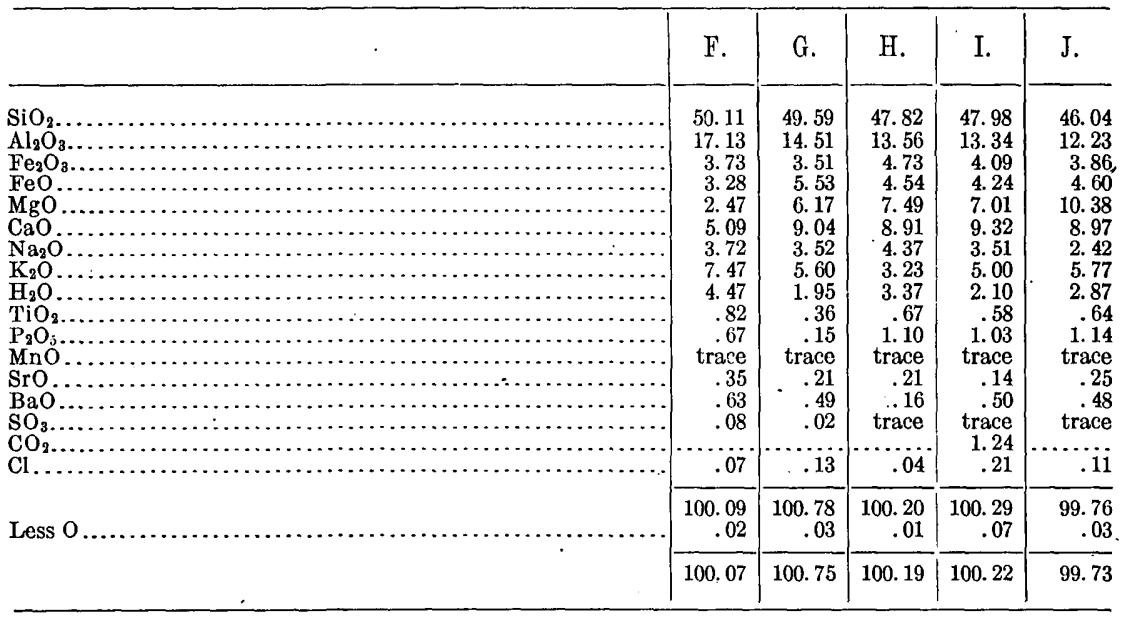

K. Missourite, head of Shonkin Creek. Albanose. Described in Am. Jour. Sci., 4th ser., vol. 2, p. 315 . Contains apatite, iron ore, olivine, biotite, augite, and leucite, 
the two latter being the chief minerals. Some zeolites and analcite are also present. E. B. Hurlbut, analyst. P: R. C. 356.

L. Fergusite, head of Shonkin Creek. Fergusose. Contains augite, olivine, biotite, iron ore, apatite, orthoclase, nephelite, zeolites, and traces of serpentine, limonite, and kaolin. Contains pseudomorphs after leucite. E. B. Hurlbut, analyst. P. R. C. 1502 .

M. Monzonite, Highwood Peak. Shoshonose. Contains augite, biotite, iron ore, apatite, plagioclase, and alkali feldspar. E. B. Hurlbut, analyst. P. R. C. 1503.

N. Syenite, Middle Peak. Borolanose. Contains augite, olivine, biotite, iron ore, apatite, plagioclase, and orthoclase. The two latter, with augite, are the chief minerals. E. B. Hurlbut, analyst. P. R. C. 1504.

\begin{tabular}{|c|c|c|c|c|}
\hline . & K. & L. & M. & $\mathbf{N}:$ \\
\hline 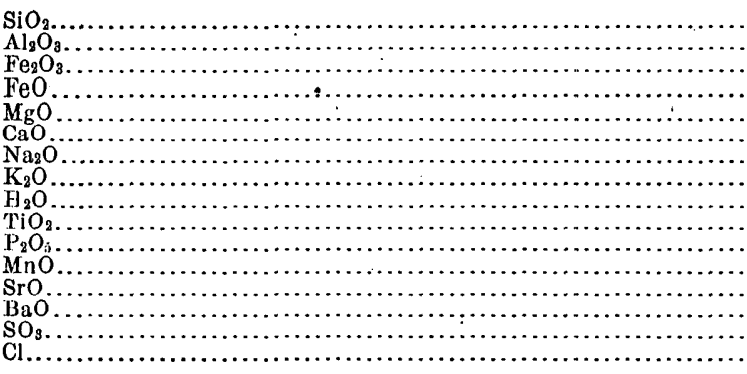 & $\begin{array}{r}46.06 \\
10.01 \\
3.17 \\
5.61 \\
14.74 \\
10.55 \\
1.31 \\
5.14 \\
1.44 \\
.73 \\
.21 \\
\text { trace } \\
.20 \\
.32 \\
.05 \\
.03\end{array}$ & $\begin{array}{r}51.75 \\
14.52 \\
5.08 \\
3.58 \\
4.55 \\
7.04 \\
2.93 \\
7.61 \\
2.25 \\
.23 \\
.18 \\
\text { trace } \\
.07 \\
.30 \\
\text { trace } \\
.05\end{array}$ & $\begin{array}{r}51.00 \\
17.21 \\
2.41 \\
4.23 \\
6.19 \\
9.15 \\
2.88 \\
4.93 \\
.63 \\
.13 \\
.33 \\
\text { trace } \\
.14 \\
.34 \\
.03 \\
\text { trace }\end{array}$ & $\begin{array}{r}52.05 \\
15.02 \\
2.65 \\
5.52 \\
5.39 \\
8.14 \\
3.17 \\
6.10 \\
.35 \\
.47 \\
.21 \\
\text { trace } \\
.28 \\
.42 \\
.02 \\
.24\end{array}$ \\
\hline \multirow[t]{2}{*}{ Less 0 . } & $\begin{array}{r}99.57 \\
.01\end{array}$ & $\begin{array}{r}100.14 \\
.01\end{array}$ & $\begin{array}{r}99.60 \\
\ldots \ldots \ldots\end{array}$ & $\begin{array}{r}100.03 \\
.06 \\
\end{array}$ \\
\hline & 99.56 & 100.13 & & 99.97 \\
\hline
\end{tabular}

O. White syenite of the Shonkin Sag laccolith. Borolanose. Partial analysis, P. R. C. 1505.

P. Shonkinite, Shonkin Sag laccolith. Montanose. Contains augite, olivine, biotite, and orthoclase. P. R. C. 1506.

Rocks O, P, described by Weed and Pirsson in Am. Jour. Sci., 4th ser., vol. 12, p.1. Analyses by W. F. Hillebrand, record No: 1885.

\begin{tabular}{|c|c|c|}
\hline & 0 & P. \\
\hline 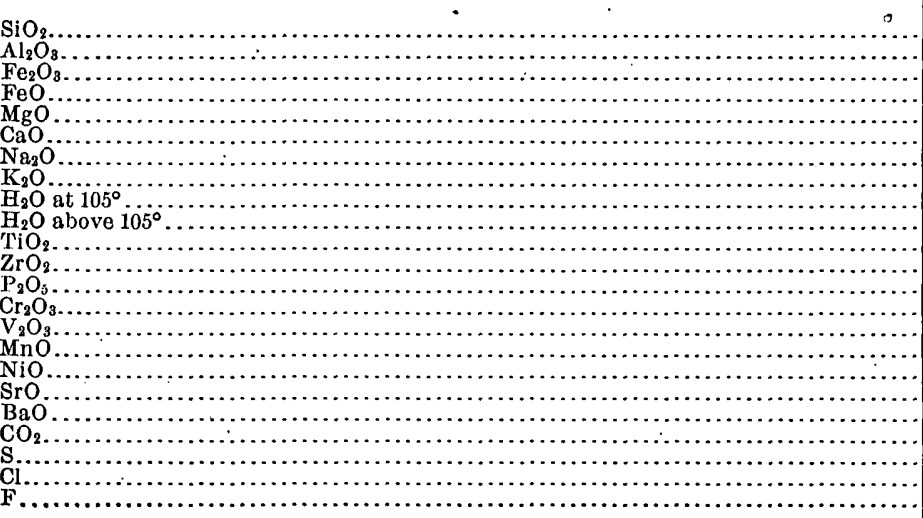 & 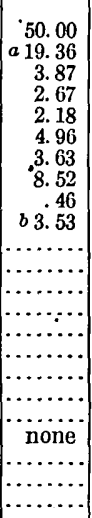 & $\begin{array}{c}47.88 \\
12.10 \\
3.53 \\
4.80 \\
8.64 \\
9.35 \\
2.94 \\
5.61 \\
.70 \\
1.52 \\
.77 \\
.03 \\
1.11 \\
.035 \\
.04 \\
.15 \\
\text { trace } \\
.13 \\
.46 \\
.12 \\
.025 \\
\text { trace } \\
.05\end{array}$ \\
\hline & 99.18 & 99.99 \\
\hline
\end{tabular}


The following rock and separations, from Square Butte, at the east end of the Highwood Mountains, are described by Lindgren in Am. Jour. Sci., 3d ser., vol. 45, p. 286. Analyses by W. H. Melville, record No. 1268.

A. Post-Cretaceous sodalite syenite. Pulaskose. Contains orthoclase, some albite, hornblende, sodalite, analcite, and apatite. Orthoclase predominates. The sodalite amounts to 8 per cent. P. R. C. 184 and 201.

B. Hornblende separated from A. Near barkevikite.

C. Sodalite from A. Sp. gr., 2.265 .

D. Analcite from A. Sp. gx., 2.255.

In addition, the orthoclase gave 3.88 per cent $\mathrm{Na}_{2} \mathrm{O}$ and 11.03 per cent $\mathrm{K}_{2} \mathrm{O}$. A separation of mixed feldspars (sp. gr., 2.56) gave 6.08 per cent $\mathrm{Na}_{2} \mathrm{O}$ and 8.91 per cent $\mathrm{K}_{2} \mathrm{O}$.

\begin{tabular}{|c|c|c|c|c|}
\hline . & A. & B. & C. & D. \\
\hline 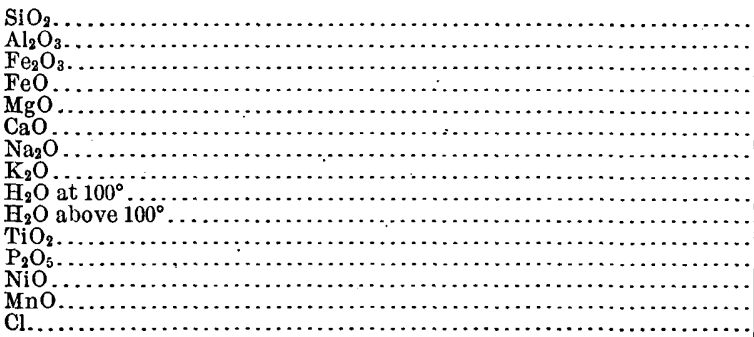 & $\begin{array}{r}56.45 \\
20.08 \\
1.31 \\
4.39 \\
.63 \\
2.14 \\
5.61 \\
7.13 \\
.26 \\
1.51 \\
.29 \\
.13 \\
\text { trace } \\
.09 \\
.43\end{array}$ & \begin{tabular}{r}
38.41 \\
17.65 \\
3.75 \\
21.75 \\
2.54 \\
10.52 \\
2.95 \\
1.95 \\
\hdashline$\ldots$. \\
.24 \\
\hdashline$\ldots .$. \\
trace \\
.15
\end{tabular} & \begin{tabular}{r}
41.56 \\
29.48 \\
$\ldots \ldots . \ldots$ \\
.49 \\
.15 \\
.49 \\
19.21 \\
.91 \\
.45 \\
3.73 \\
\hdashline$\ldots . .$. \\
\hdashline$\ldots . .$. \\
\hdashline$\ldots .79$ \\
$\ldots . .$.
\end{tabular} & $\begin{array}{r}49.54 \\
25.07 \\
\ldots \ldots . . \\
.40 \\
.20 \\
.22 \\
15.32 \\
.89 \\
\text { undet. } \\
\text { undet. } \\
\ldots \ldots . . \\
\ldots \ldots . . . \\
\ldots \ldots . . .\end{array}$ \\
\hline \multirow[t]{2}{*}{ Less $0 .}$. & $\begin{array}{r}100.45 \\
.10\end{array}$ & $\begin{array}{r}99.91 \\
\ldots .\end{array}$ & $\begin{array}{r}101.26 \\
1.08\end{array}$ & $\begin{array}{r}93.31 \\
.38\end{array}$ \\
\hline & 100. 35 & $\ldots \ldots$ & 100.18 & 92.93 \\
\hline
\end{tabular}

8. LITTLE ROCKY MOUNTAINS.

Granite syenite porphyry. Liparose. "Described by Weed and Pirsson, Jour. Geology, vol. 4, p. 399 . Contains orthoclase, quartz, oligoclase, iron oxides, and a little muscovite. Analysis by H. N. Stokes, record No. 1558. P. R. C. 905.

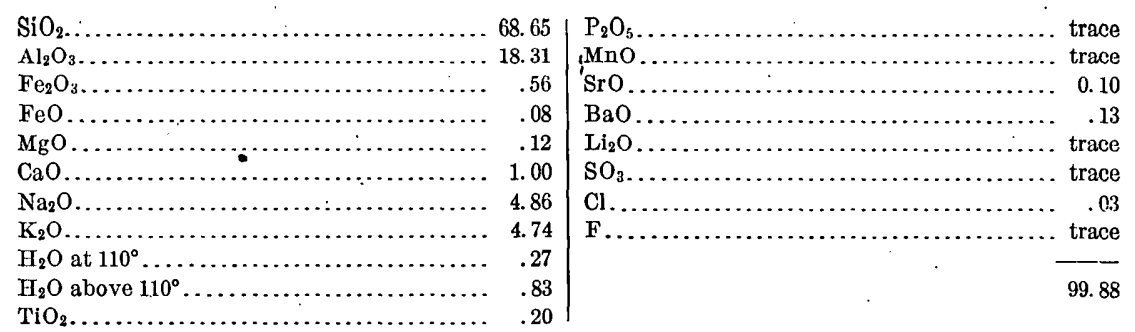

9. BEARPAW MOUNTAINS.

Described by Weed and Pirsson, Am. Jour. Sci., 4th ser., vol. 1, pp. 283 and 351, and vol. 2, pp. 136 and 188. Analyses by H. N. Stokes, record Nos. 1558 and 1572.

A. Quartz syenite porphyry, Gray Butte. Nordmarkose. Contains anorthoclase, microlites of plagioclase, ægirite, augite, quartz, and apatite, with an occasional zircon, and very few biotite leaves. P. R. C. 897.

B. Quartz syenite, Beaver Creek stock. Liparose. Contains orthoclase, albite, quartz, augite, and iron oxides, with very little biotite, hornblende, and sphene. P. R. C. 900 . 
C. Basic syenite or monzonite (yogoite), Beaver Creek. Monzonose. Contains orthoclase, plagioclase, diopside, biotite, iron oxides, and apatite. P. R. C. 902.

\begin{tabular}{|c|c|c|c|}
\hline . & A. & B. & C. \\
\hline 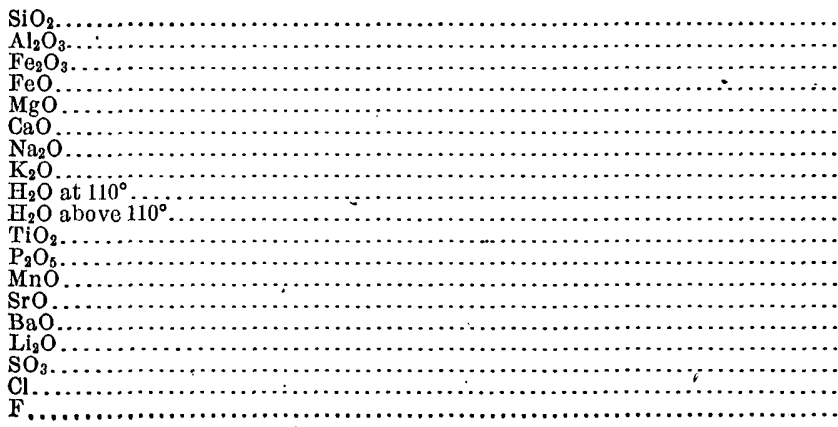 & $\begin{array}{r}66.22 \\
16.22 \\
1.98 \\
.16 \\
.77 \\
1.32 \\
6.49 \\
5.76 \\
.08 \\
.24 \\
.22 \\
.10 \\
\text { trace } \\
.06 \\
.29 \\
\text { trace } \\
.02 \\
.04 \\
\text { trace }\end{array}$ & $\begin{array}{r}68.34 \\
15.32 \\
1.90 \\
.84 \\
.54 \\
.92 \\
5.45 \\
5.62 \\
.15 \\
.30 \\
.21 \\
.13 \\
.07 \\
.04 \\
.08 \\
\text { none } \\
\text { trace } \\
.04 \\
\text { none? }\end{array}$ & $\begin{array}{r}52.81 \\
15.66 \\
3.06 \\
4.76 \\
4.99 \\
7.57 \\
3.60 \\
4.84 \\
.16 \\
.93 \\
.71 \\
.75 \\
\text { trace } \\
.09 \\
.24 \\
\text { trace } \\
\text { trace } \\
.07 \\
\text { trace }\end{array}$ \\
\hline & 99.97 & 99.95 & 100.24 \\
\hline
\end{tabular}

D. Shonkinite, Beaver Creek. SR. $\&$ of uyomingase. Contains anorthoclase, diopside, biotite, iron oxides, and apatite, with very little olivine and probably a trace of nephelite. P. R. C. 901.

E. Leucitite, Bearpaw Mountains. Chotose. An olivine-free leucite basalt. Contains leucite, augite, iron oxides, rarely biotite, and very little glassy base. P. R. C. 903.

F. Tinguaite, dike on Bear Creek. Judithose. Contains orthoclase, nephelite, cancrinite, augite, ægirite, apatite, a little sodalite, and a doubtful fibrous hornblende. P. R. C. 899.

G. Pseudoleucite-sodalite tinguaite. Beaver Creek. Janeirose. Contains orthoclase, nephelite, sodalite, nosean, ægirite, diopside, and fluorite. P. R. C. 904.

\begin{tabular}{|c|c|c|c|c|}
\hline & D.. & E. & F. & G. \\
\hline 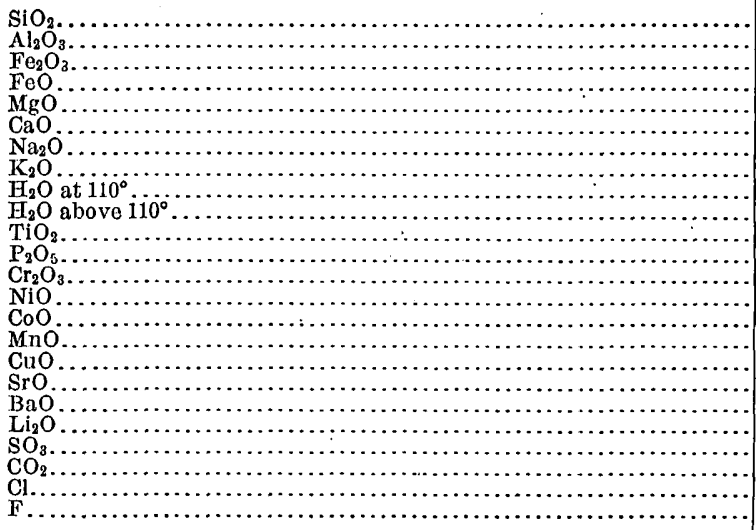 & $\begin{array}{r}50.00 \\
9.87 \\
3.46 \\
5.01 \\
11.92 \\
8.31 \\
2.41 \\
5.02 \\
.17 \\
1.16 \\
.73 \\
.81 \\
.11 \\
.07 \\
\ldots . . . \\
\text { trace } \\
\ldots . . . \\
.07 \\
.32 \\
\text { trace } \\
.02 \\
.31 \\
.08 \\
.16\end{array}$ & $\begin{array}{r}46.51 \\
11.86 \\
7.59 \\
4.39 \\
4.73 \\
7.41 \\
2.39 \\
8.71 \\
1.10 \\
2.45 \\
.83 \\
.80 \\
\text { none } \\
.04 \\
\text { trace } \\
.22 \\
\text { trace } \\
.16 \\
.50 \\
\text { trace } \\
.05 \\
\text { none } \\
.04 \\
\text { trace }\end{array}$ & $\begin{array}{r}57.46 \\
15.40 \\
4.87 \\
.87 \\
1.37 \\
2.59 \\
5.48 \\
9.44 \\
.09 \\
.82 \\
.60 \\
.21 \\
\ldots \ldots . . \\
\ldots \ldots . . \\
\text { trace } \\
\ldots .16 \\
.60 \\
\text { trace } \\
.13 \\
.13 \\
.20 \\
\text { trace }\end{array}$ & $\begin{array}{r}51.93 \\
20.29 \\
3.59 \\
1.20 \\
.22 \\
1.65 \\
8.49 \\
9.81 \\
.10 \\
.99 \\
.20 \\
.06 \\
\ldots \ldots \\
\ldots \ldots . . \\
\ldots \ldots \\
\text { trace } \\
\ldots . .07 \\
.07 \\
.09 \\
\text { trace } \\
.67 \\
.25 \\
.70 \\
.27\end{array}$ \\
\hline \multirow{2}{*}{ 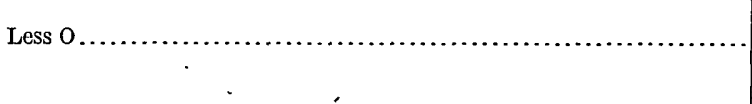 } & $\begin{array}{r}100.01 \\
. \quad .08\end{array}$ & $\begin{array}{r}99.78 \\
.02\end{array}$ & $\begin{array}{r}100.42 \\
.05\end{array}$ & $\begin{array}{r}100.58 \\
.27\end{array}$ \\
\hline & 99.93 & 99.76 & 100.37 & 100.31 \\
\hline
\end{tabular}


10. ELKHORN MINING DISTRICT.

Rocks collected by W. H. Weed. Described in detail by J. S. Barrell in 22d Ann., pt. 2, p. 511. Analyses by H. N. Stokes, record No. 1858.

A. Gabbro, west side of Black Butte. Hessose. Contains labradorite, augite, hornblende, biotite, and magnetite, with a little quartz and zircon. P. R. C. 351.

B. Andesite, Elkhorn Mountain. Harzose. Contains plagioclase, hornblende, biotite, and pyroxene. P. R. C. 413.

C. Hornblende andesite porphyry, southwestern part of the district. Contains plagioclase and hornblende phenocrysts in a groundmass of hornblende. Pyrite, pyrrhotite, and perhaps magnetite are also present. P. R. C. 412.

D. Quartz'monzonite, Elkhorn. Harzose. Contains labradorite, orthoclase, quartz, biotite, hornblende, augite, magnetite, apatite, and titanite. P. R. C. 407.

E. Aplite, north of Black Butte, middle of intrusion. Toscanose-tehamose. P. R.C. 410.

F. Like E, eastern side of intrusion. E and F contain soda-orthoclase and quartz, with a little:plagioclase and occasional crystals of magnetite and biotite. P. R. C. 411.

\begin{tabular}{|c|c|c|c|c|c|c|}
\hline & A. & B. & C. & D. & E. & F. \\
\hline 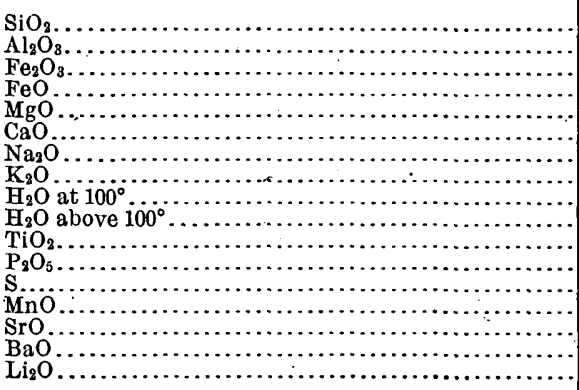 & $\begin{array}{r}47.44 \\
18.21 \\
5.37 \\
6.05 \\
5.53 \\
9.98 \\
2.58 \\
1.17 \\
.13 \\
1.08 \\
1.38 \\
.68 \\
a .03 \\
.06 \\
.08 \\
.08 \\
\text { trace }\end{array}$ & $\begin{array}{r}59.64 \\
16.64 \\
2.33 \\
4.35 \\
2.10 \\
4.59 \\
3.31 \\
4.16 \\
.14 \\
.69 \\
1.08 \\
.49 \\
\text { a.01 } \\
\text { trace } \\
.05 \\
.10 \\
\text { trace }\end{array}$ & $\begin{array}{r}54.50 \\
17.97 \\
8.55 \\
3.37 \\
9.36 \\
2.34 \\
1.62 \\
.14 \\
.96 \\
.94 \\
.31 \\
.63 \\
\text { trace } \\
.05 \\
.06 \\
\text { trace }\end{array}$ & $\begin{array}{r}64.31 \\
15.44 \\
2.43 \\
2.58 \\
2.21 \\
4.22 \\
2.71 \\
4.09 \\
.19 \\
.79 \\
.71 \\
.22 \\
\text { trace } \\
\text { trace } \\
\text { trace } \\
.07 \\
\text { trace }\end{array}$ & $\begin{array}{r}76.26 \\
12.94 \\
.69 \\
.13 \\
.17 \\
1.10 \\
2.73 \\
5.26 \\
.17 \\
.43 \\
.11 \\
.06 \\
a .01 \\
\text { trace } \\
\text { none } \\
. .07 \\
\text { trace }\end{array}$ & $\begin{array}{r}74.61 \\
13.36 \\
.83 \\
.36 \\
.35 \\
1.35 \\
2.49 \\
5.70 \\
.19 \\
.42 \\
.11 \\
.06 \\
\text { trace } \\
\text { trace } \\
\text { trace } \\
.10 \\
\text { trace }\end{array}$ \\
\hline & 99.85 & 99.68 & 100.80 & 99.97 & 100.13 & 99.93 \\
\hline
\end{tabular}

$a$ May be present either as $\mathrm{S}_{\text {or }} \mathrm{SO}_{3}$.

$\mathrm{Cr}_{2} \mathrm{O}_{3}, \mathrm{NiO}, \mathrm{CO}_{2}$ are all absent.

The following rocks were also analyzed for Weed, who supplies the petrographic data:

G. Grossularite hornfels. Contains diopside, grossularite, calcite, and quartz.

H. Lime-enriched gabbro. Contains labradorite, pyroxene, and scapolite. Analyses G, H, by W. T. Schaller, record No. 2158.

I. Grossularite hornstone, a modified marble. Contains calcite, chlorite, grossularite, and augite.

J. Mica diorite, the normal batholith rock. Contains labradorite, soda orthoclase, quartz, biotite, hornblende, augite, magnetite, and apatite. Analyses I, J, by E. C. Sullivan, record No. 2195. In these the $\mathrm{TiO}_{2}$ and $\mathrm{P}_{2} \mathrm{O}_{5}$ were not separated from the alumina. 


\begin{tabular}{|c|c|c|c|c|}
\hline & G. & H. & I. & J. \\
\hline \multirow[t]{2}{*}{ 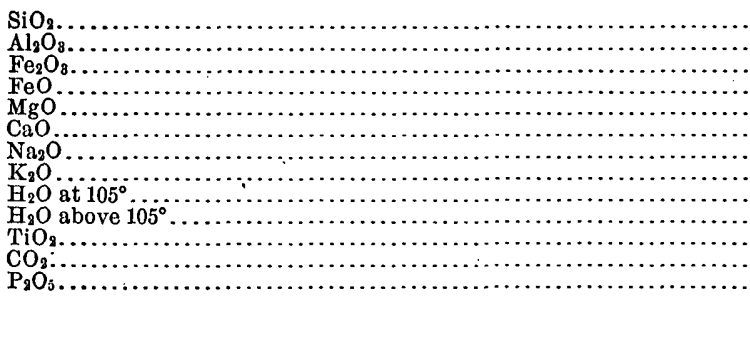 } & $\begin{array}{r}40.31 \\
12.11 \\
8.67 \\
.40 \\
2.65 \\
33.61 \\
.79 \\
.12 \\
.23 \\
.22 \\
.78 \\
\text { none } \\
.69\end{array}$ & $\begin{array}{r}49.42 \\
13.58 \\
3.98 \\
3.59 \\
5.06 \\
18.15 \\
2.22 \\
1.42 \\
.28 \\
.50 \\
.71 \\
\text { undet. } \\
1.38\end{array}$ & $\begin{array}{r}36.91 \\
6.54 \\
19.43 \\
.67 \\
1.07 \\
31.09 \\
\text { trace } \\
.18 \\
.70 \\
1.57 \\
\text { undet. } \\
1.91 \\
\text { undet. }\end{array}$ & $\begin{array}{r}60.84 \\
16.36 \\
2.40 \\
3.23 \\
3.85 \\
4.96 \\
2.90 \\
4.10 \\
.48 \\
1.03 \\
\text { undet. } \\
\text {....... } \\
\text { undet. }\end{array}$ \\
\hline & 100.58 & 100.29 & 100.07 & 100.05 \\
\hline
\end{tabular}

\section{BITTERROOT RANGE.}

Quartz monzonite, near head of Mill Creek. Toscanose. Described by Lindgren in P. P. 27. Analysis by W. F. Hillebrand, record No. 1921. Contains quartz, potash, feldspar, plagioclase, biotite, apatite, titanite, and magnetite. P. R. C. 1519.

\begin{tabular}{|c|c|c|c|}
\hline 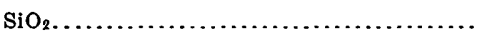 & 72.07 & $\mathrm{H}_{2} \mathrm{O}$ at $105^{\circ} \ldots \ldots \ldots$ & .03 \\
\hline $\mathrm{Al}_{2} \mathrm{O}_{3} \ldots$ & 15. 51 & $\mathrm{H}_{2} \mathrm{O}$ above $105^{\circ} \ldots$ & .30 \\
\hline 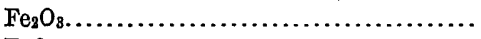 & .31 & 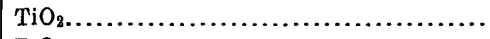 & .16 \\
\hline 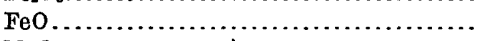 & 1.01 & 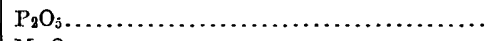 & .11 \\
\hline $\operatorname{MgO} \ldots \ldots \ldots \ldots \ldots \ldots \ldots \ldots \ldots \ldots \ldots \ldots \ldots \ldots \ldots \ldots \ldots \ldots \ldots$ & .35 & Mno................ & trace \\
\hline 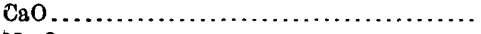 & 1.93 & $\mathrm{CO}_{2} \ldots$ & none \\
\hline 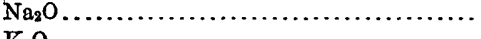 & 4. 02 & & \\
\hline 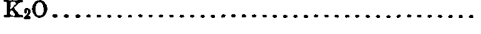 & 4.09 & & 99.89 \\
\hline
\end{tabular}

Other elements not looked for.

\section{MARYSVILLE DISTRICT.}

Rocks described by Joseph Barrell, in P. P. 57. Analyses by George Steigér, record No. 2021.

A. Hornblende microdiorite, near West Belmont mine, east slope of Mount Belmont. Andose-shoshonose. Contains feldspars, 60 per cent; hornblende, 35 per cent; and small amounts of quartz, magnetite, biotite, augite, and apatite.

B. Hornblende porphyrite, north slope of the town of Bald Butte. Andose. Contains feldspar, largely oligoclase, about 75 per cent; and hornblende, 23 per cent; with secondary hornblende, calcite, and chlorite.

C. Quartz diorite, road up Woodchopper Gulch. Yellowstonose. Typical of the Marysville batholith. Contains andesine, 52 per cent; quartz, 31 per cent; orthoclase, 11 per cent; and minor biotite, hornblende, magnetite, titanite, and apatite.

\begin{tabular}{|c|c|c|c|}
\hline$\checkmark$ & A. & B. & C. \\
\hline 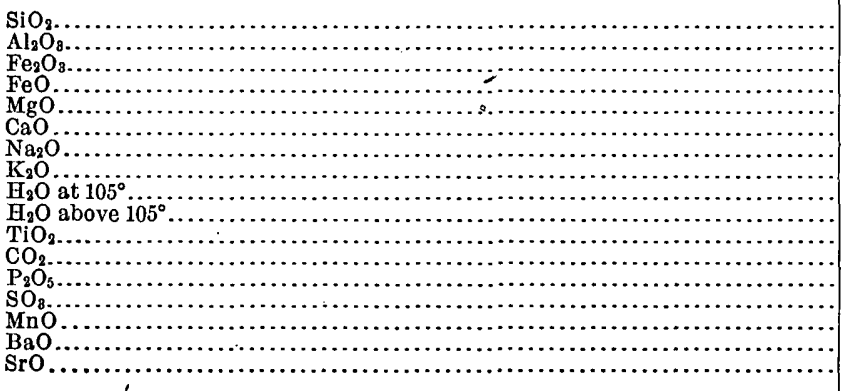 & $\begin{array}{r}52.07 \\
15.99 \\
4.77 \\
5.59 \\
4.54 \\
7.50 \\
2.97 \\
2.79 \\
.34 \\
1.60 \\
1.08 \\
\text { none } \\
\ldots .40 \\
\ldots \ldots . . . \\
\ldots \ldots . . . \\
\ldots . . .\end{array}$ & $\begin{array}{r}56.88 \\
15.61 \\
2.95 \\
2.34 \\
6.35 \\
5.23 \\
3.59 \\
2.39 \\
.67 \\
3.03 \\
.49 \\
\text { none } \\
.13 \\
\ldots \ldots . . . \\
\cdots \ldots . . . \\
\cdots\end{array}$ & $\begin{array}{r}63.55 \\
16.57 \\
2.36 \\
1.98 \\
1.53 \\
4.69 \\
3.78 \\
2.78 \\
.31 \\
1.11 \\
.42 \\
.69 \\
.21 \\
.06 \\
.13 \\
.15 \\
.04\end{array}$ \\
\hline 3 & 99.64 & 99.66 & 100.36 \\
\hline
\end{tabular}




\section{LIVINGSTON QUADRANGLE.}

Rocks analyzed for J. P. Iddings, who furnishes the descriptions. Published by W. H. Emmons, in Jour. Geology, vol. 16, p. 193. Analyses by G. Steiger, record Nos. $2135,2147$.

A. Granodiorite, 11 miles northwest of Haystack Mountain. Adamellose. Contains plagioclase, orthoclase, quartz, hornblende, biotite, pyroxene, and magnetite.

B. Orthoclase gabbro, 1 mile northeast of Haystack Mountain. Shoshonose. Contains piagioclase, orthoclase, quartz, pyroxene, biotite, magnetite, apatite, and very little altered olivine.

C. Pyroxene separated from B.

D. Olivine gabbro, one-half mile northeast of Haystack Mountain. Hessose. Contains olivine, plagioclase, pyroxene, biotite, and magnetite.

\begin{tabular}{|c|c|c|c|c|}
\hline & A. & B. & C. & D. \\
\hline 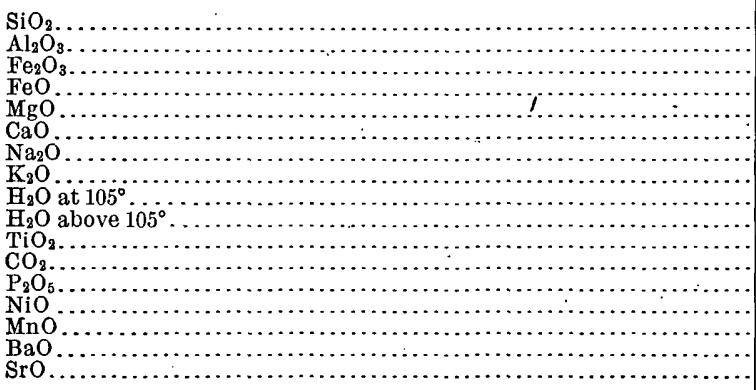 & $\begin{array}{r}65.06 \\
14.71 \\
2.82 \\
1.31 \\
2.48 \\
3.43 \\
3.86 \\
3.48 \\
.31 \\
1.10 \\
.61 \\
\text { none } \\
.18 \\
\text { none } \\
.18 \\
.10 \\
.05\end{array}$ & $\begin{array}{r}54.09 \\
16.00 \\
2.92 \\
5.54 \\
5.19 \\
7.37 \\
3.38 \\
2.67 \\
.20 \\
.77 \\
.99 \\
\text { none } \\
.35 \\
\text { none } \\
.15 \\
.10 \\
.06\end{array}$ & $\begin{array}{r}50.95 \\
2.72 \\
1.70 \\
13.86 \\
15.58 \\
11.39 \\
.31 \\
\ldots \ldots \ldots \\
60 \\
1.20 \\
1.42 \\
\ldots \ldots . . \\
\ldots \ldots \ldots \\
\ldots \ldots . . . \\
.26 \\
\ldots \ldots . . . \\
\ldots . . .\end{array}$ & $\begin{array}{r}47.87 \\
16.34 \\
3.59 \\
7.17 \\
7.80 \\
10.33 \\
2.43 \\
.92 \\
.28 \\
1.25 \\
1.02 \\
.44 \\
.41 \\
.02 \\
.14 \\
.03 \\
\text { undet. }\end{array}$ \\
\hline 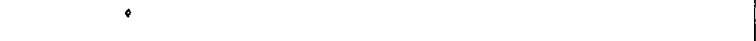 & 99.68 & 99.78 & 99.99 & 100.04 \\
\hline
\end{tabular}

$\mathrm{S}, \mathrm{SO}_{3}$ and $\mathrm{ZrO}_{2}$ absent.

E. Granodiorite porphyry, 1 mile northeast of Haystack Mountain. Hàrzose. Contains plagioclase, subordinate orthoclase, quartz, biotite, pyroxene, magnetite, and little hornblende.

F. Quartz diorite, north of Haystack Mountain. Tonalose. Nearly the same mineral composition as $\mathrm{E}$.

G. Biotite-orthoclase gabbro, north of Haystack Mountain, between Blue and Múd Lakes. Monzonose. Contains plagioclase, orthoclase, a little quartz, pyroxene, biotite, magnetite, and apatite.

H. Mica separated from $G$.

\begin{tabular}{|c|c|c|c|c|}
\hline · & E. & F. & G. & H. \\
\hline 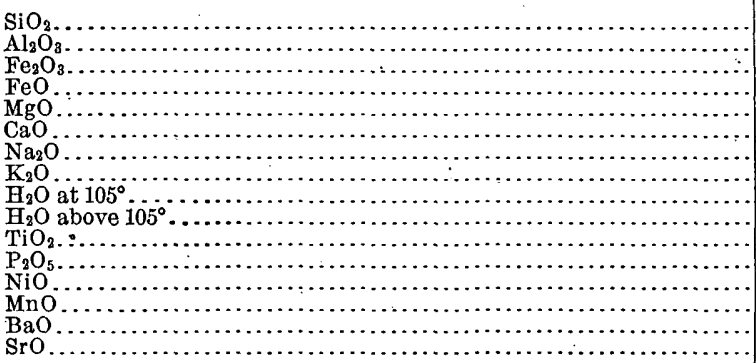 & $\begin{array}{r}64.09 \\
16.20 \\
2.61 \\
2.40 \\
2.06 \\
4.51 \\
3.88 \\
2.51 \\
.22 \\
.44 \\
.49 \\
.24 \\
\text { none } \\
.09 \\
.15 \\
.03\end{array}$ & $\begin{array}{r}57.98 \\
17.01 \\
3.34 \\
3.34 \\
2.74 \\
-7.35 \\
3.92 \\
2.02 \\
.14 \\
.49 \\
.90 \\
.43 \\
\text { trace } \\
.12 \\
.06 \\
.02\end{array}$ & $\begin{array}{r}54.84 \\
16.41 \\
3.63 \\
4.54 \\
4.71 \\
6.64 \\
3.27 \\
2.83 \\
.34 \\
.93 \\
.99 \\
.35 \\
\text { none } \\
\text { none } \\
.12 \\
.05\end{array}$ & 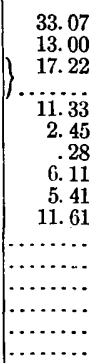 \\
\hline . & 99.92 & 99.86 & 99.65 & 100.48 \\
\hline
\end{tabular}

$\mathrm{CO}_{2}, \mathrm{ZrO}_{2}, \mathrm{~S}$, and $\mathrm{SO}_{3}$ absent. 
14. PHIIIPSBURGG QUADRANGLE.

Rocks collected by F: C. Calkins, who supplies the petrographic data.

A. Quartz monzonite, Royal mine. Contains quartz, plagioclase, orthoclase, and biotite, with accessory muscovite, magnetite, apatite, zircon, and tourmaline.

B. Quartz monzonite, from head of east fork of Rock Creek. Contains quartz, plagioclase, orthoclase, biotite, and a little hornblende, with accessory apatite, zircon, titanite, and magnetite.

C. Quartz monzonite, from a quarry one-half mile southwest of Cable mine. Contains plagioclase, alkali feldspar, quartz, biotite, hornblende, and pyroxene; with accessory magnetite, apatite, and zircon, and secondary chlorite, epidote, calcite, kaolin, muscovite, and titanite. Analyses A, B, C, by G. Steiger, record No. 2367.

D. Pyroxene aplite, about 1 mile northeast of Rumsey Mountain. Contains plagioclase, quartz, orthoclase, pyroxene, titanite, scapolite, apatite, and zircon.

E. Scapolite-pyroxene aplite, from cliff east of Foster Creek, about one-half mile north of the county boundary. Contains scapolite, plagioclase, quartz, alkali feldspar, pyroxene, hornblende, titanite, apatite, and zircon, with secondary calcite, epidote, and sericite. Analyses D, E, by W. T. Hillebrand, record No. 2365.

\begin{tabular}{|c|c|c|c|c|c|}
\hline$\cdot$ & A. & $B$. & C. & D. & E. \\
\hline 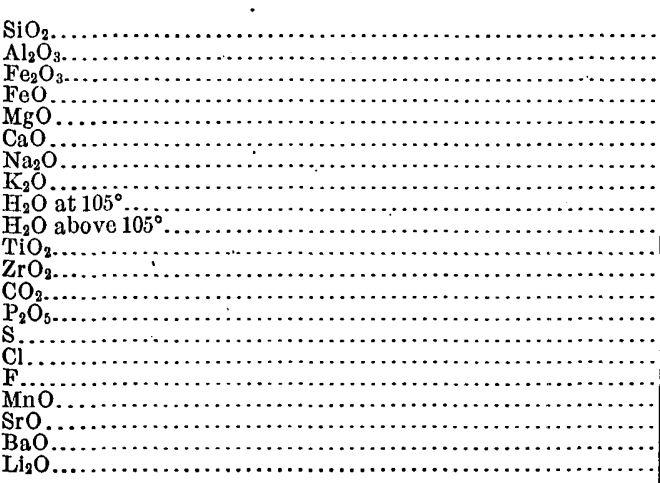 & $\begin{array}{r}68.40 \\
16.34 \\
.17 \\
1.56 \\
.64 \\
3.77 \\
3.39 \\
3.91 \\
.29 \\
.55 \\
.29 \\
\text { none } \\
\text { none } \\
.22 \\
\text { none } \\
\ldots \ldots \ldots \\
\ldots \ldots . . \\
.07 \\
.05 \\
.15 \\
\ldots \ldots . .\end{array}$ & $\begin{array}{r}70.05 \\
15.04 \\
.70 \\
1.32 \\
1.04 \\
2.46 \\
4.03 \\
3.33 \\
.70 \\
1.12 \\
.36 \\
.02 \\
\text { none } \\
.08 \\
\text { none } \\
\ldots \ldots . . \\
\ldots \ldots . . \\
.03 \\
.05 \\
.10 \\
\ldots \ldots .\end{array}$ & \begin{tabular}{|r|}
60.19 \\
17.39 \\
2.04 \\
4.28 \\
2.10 \\
5.69 \\
3.30 \\
2.67 \\
.31 \\
.89 \\
.85 \\
.04 \\
.21 \\
.30 \\
none \\
$\ldots \ldots .$. \\
$\ldots \ldots .$. \\
.11 \\
.02 \\
.08 \\
$\ldots \ldots .$.
\end{tabular} & $\begin{array}{r}68.00 \\
16.33 \\
.26 \\
.70 \\
1.41 \\
5.90 \\
6.20 \\
.38 \\
.06 \\
.25 \\
.43 \\
.02 \\
\text { none } \\
.22 \\
.01 \\
.13 \\
.07 \\
.02 \\
.03 \\
.03 \\
\text { none }\end{array}$ & $\begin{array}{r}57.98 \\
19.32 \\
.44 \\
.83 \\
1.93 \\
8.87 \\
3.62 \\
4.01 \\
.57 \\
.67 \\
.66 \\
.04 \\
.53 \\
.17 \\
.01 \\
.17 \\
.07 \\
.07 \\
.05 \\
.12 \\
\text { trace }\end{array}$ \\
\hline \multirow[t]{2}{*}{ 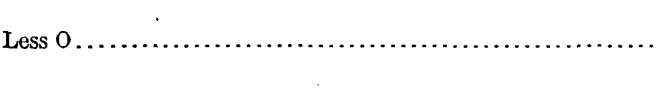 } & \multirow[t]{2}{*}{$\begin{array}{r}99.80 \\
\ldots \ldots\end{array}$} & \multirow[t]{2}{*}{$\begin{array}{c}100.43 \\
\ldots \ldots\end{array}$} & \multirow[t]{2}{*}{$\begin{array}{c}100.47 \\
\ldots \ldots \ldots\end{array}$} & $\begin{array}{r}100.45 \\
.06\end{array}$ & $\begin{array}{r}100.13 \\
.07\end{array}$ \\
\hline & & & & 100. 39 & 100.06 \\
\hline
\end{tabular}

IDAHO.

Rocks A to K, inclusive, are described by Waldemar Lindgren in 20th Ann., pt. 3, p. 75.

A. Quartz monzonite, Idaho-Democrat mine, Hailey. Toscanose. Contains quartz, orthoclase, microcline, oligoclase, biotite, apatite, titanite, and magnetite. Sp. gr., $2.672,27.5^{\circ}$.

B. Same rock and locality as $A$, but in altered condition. Contains quartz, sericite, chlorite, calcite, pyrite, rutile, etc. Sp. gr., $2.472,29^{\circ}$.

C. Diorite, Crosus mine, Hailey. Andose. Contains labradorite, biotite; diallage, hypersthene, hornblende, quartz, titanite, magnetite, orthoclase, and chlorite. Sp. gr., $2,826,28^{\circ}$.

D. Same rock and locality as $\mathrm{C}$, but in altered condition. Contains quartz, sericite, chlorite, calcite, pyrite, arsenopyrite, rutile, etc. Sp. gr., $2.898,28^{\circ}$.

Analyses A to D by W. F. Hillebrand, record No. 1826. The metals which are bracketed with sulphur represent sulphides. Traces of lithia are present in all four. 


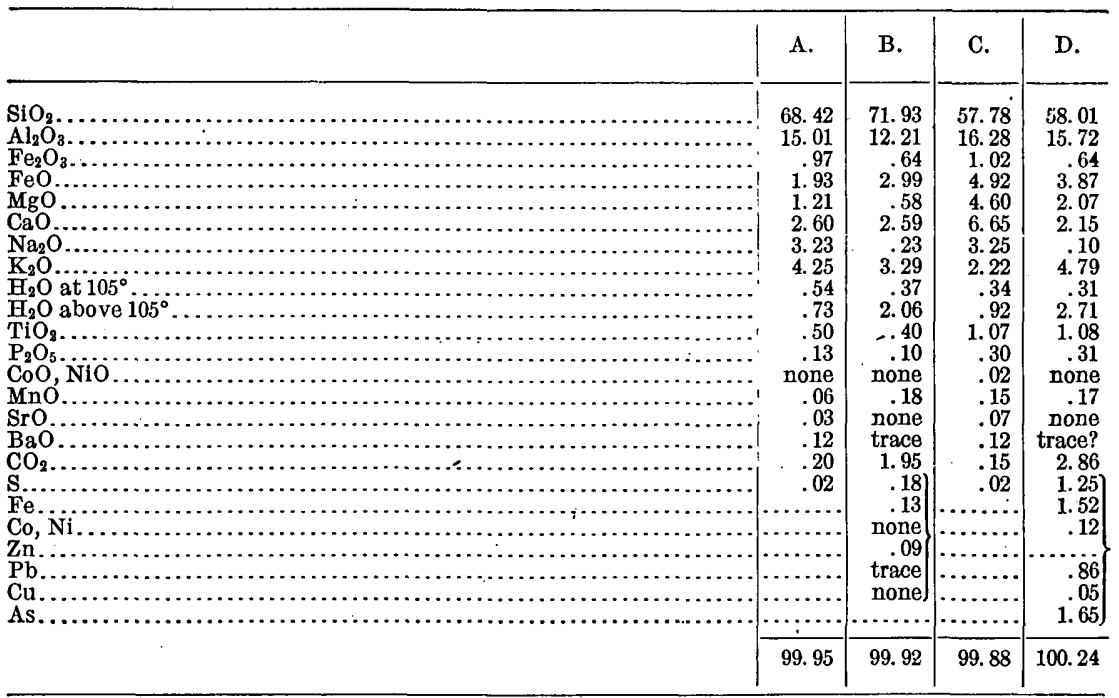

E. Altered rhyolite, De Lamar mine, Silver City. Contains quartz, sericite, pyrite, apatite, and rutile, with some undeterminable magnesian mineral. Analysis by H. N. Stokes, record No. 1731.

F. Altered rhyolite, De Lamar mine.' Contains sericite, quartz, kaolinite, and pyrite. Sp. gr., $2.655,23^{\circ}$.

G. Altered rhyolite, De Lamar mine. Contains quartz, sericite, kaolinite, and pyrite. Sp. gr., $2.576,24^{\circ}$.

H. Diabasic basalt, Trade Dollar mine, Silver City. Camptonose. Contains labradorite, augite, chlorite, magnetite, secondary quartz, etc.

Analyses F, G, and H, by Hillebrand, record No. 1826.

\begin{tabular}{|c|c|c|c|c|}
\hline & E. & F. & G. & H. \\
\hline 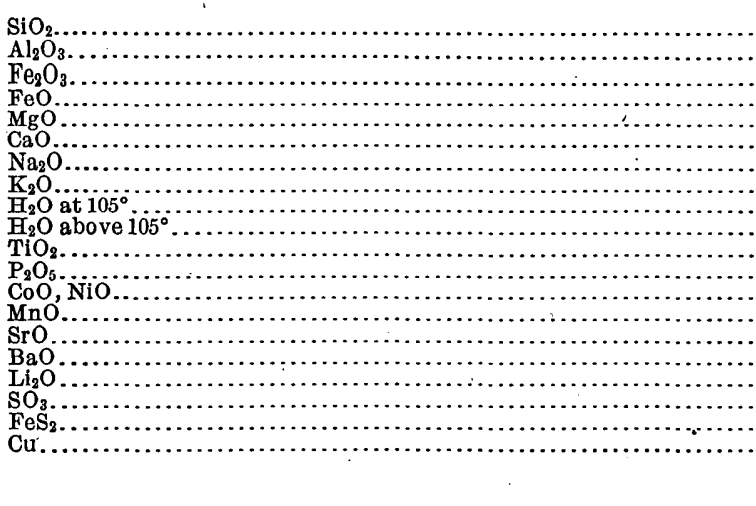 & $\begin{array}{r}66.69 \\
15.40 \\
1.84 \\
\text { undet. } \\
.85 \\
.09 \\
.16 \\
3.50 \\
.83 \\
2.97 \\
2.11 \\
.08 \\
. \text { trace } \\
.09 \\
.09 \\
.11 \\
3.99 \\
\cdots\end{array}$ & $\begin{array}{r}87.37 \\
7.44 \\
.09 \\
.18 \\
.12 \\
.10 \\
.14 \\
1.79 \\
.51 \\
1.39 \\
.09 \\
\text { trace } \\
\text { none } \\
\text { trace? } \\
\text { none } \\
.02 \\
\text { trace }\end{array}$ & $\begin{array}{r}78.59 \\
12.13 \\
\text { none } \\
.09 \\
.41 \\
.16 \\
.10 \\
2.55 \\
.82 \\
2.47 \\
.12 \\
\text { trace } \\
\text { none } \\
\text { trace? } \\
\text { none } \\
.02 \\
\text { trace }\end{array}$ & $\begin{array}{r}48.47 \\
16.07 \\
4.12 \\
7.47 \\
5.96 \\
4.84 \\
2.43 \\
1.41 \\
2.30 \\
4.63 \\
1.51 \\
.44 \\
\text { trace } \\
.23 \\
\text { trace } \\
.03 \\
\text { trace }\end{array}$ \\
\hline
\end{tabular}

Heavy metals not looked for in $\mathrm{E}$.

I. Quartz monzonite, Schafer Butte, Boise County. Lassenose. Contains quartz, orthoclase, oligoclase, biotite, apatite, titanite, and magnetite. Analysis by George Steiger, record No. 1802 . P. R. C. 1520.

J. Granodiorite, Silver Wreath mine, Boise County. Yellowstonose. Contains orthoclase, oligoclase, apatite, biotite, titanite, and magnetite. Described in 18th Ann., pt. 3, p. 642 . Sp. gr., 2.714, $23^{\circ}$. 
K. Same as $J$, but in altered condition. Also described in 18th Ann. Contains sericite, quartz, titanite, apatite, and pyrite, with carbonates of calcium, magnesium, and iron. Sp. gr., $2.774,23^{\circ}$.

Analyses J and K by George Steiger, record No. 1691.

L. Volcanic sand, Nez Perces region. Collected by I. C. Russell.

Analysis by W. F. Hillebrand, record No. 1906.

M. Typical basalt, Cinder Buttes, west side of Snake River plains. Camptonose. Analysis by W. F. Hillebrand, record No. 1950. Analysis published by Russell in Bull. 199, but the mineralogical composition of the rock is not given. Sp. gr., 2.907 at $24^{\circ}$.

\begin{tabular}{|c|c|c|c|c|c|}
\hline & I. & J. & $\mathbf{K}$. & L. & M. \\
\hline 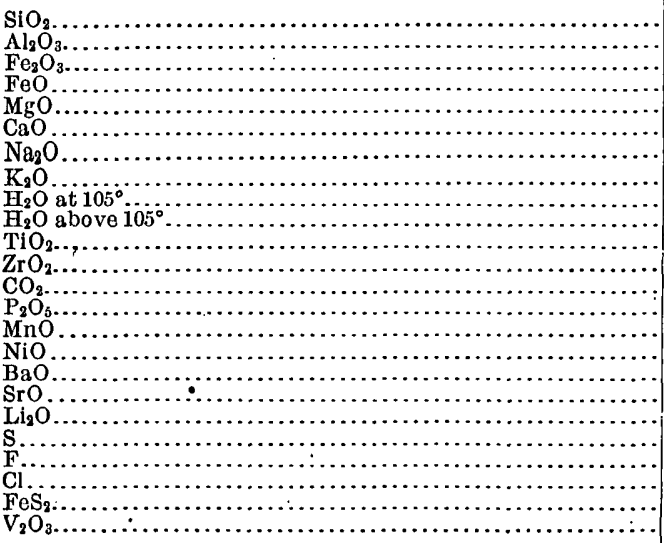 & 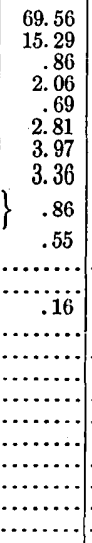 & 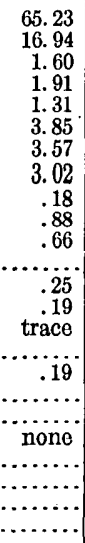 & 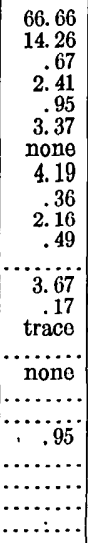 & $\begin{array}{c}68.95 \\
14.33 \\
1.17 \\
1.23 \\
.47 \\
2.13 \\
5.08 \\
2.58 \\
.28 \\
3.63 \\
.42 \\
.03 \\
\ldots \ldots . \\
\text { trace } \\
\ldots \ldots . . \\
\text { trace } \\
\text { trace } \\
\text { trace } \\
\text { ? } \\
\ldots \ldots . . \\
\ldots \ldots \ldots \\
\ldots \ldots \ldots\end{array}$ & $\begin{array}{r}51.14 \\
13.95 \\
2.15 \\
12.97 \\
2.21 \\
6.56 \\
3.59 \\
2.33 \\
.12 \\
.22 \\
2.41 \\
.12 \\
\ldots . .9 \\
1.59 \\
.44 \\
\text { trace } \\
.25 \\
\text { trace } \\
\text { none } \\
\ldots . .0 \\
\text { trace } \\
.15 \\
\text { trace }\end{array}$ \\
\hline \multirow[t]{2}{*}{ 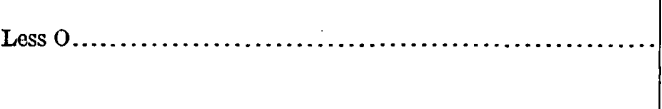 } & $\begin{array}{c}100.17 \\
\ldots\end{array}$ & $\begin{array}{r}99.78 \\
\cdots\end{array}$ & $\begin{array}{r}100.11 \\
.24\end{array}$ & $\begin{array}{r}100.48 \\
\cdots \cdots\end{array}$ & $\begin{array}{r}100.30 \\
\ldots \ldots \\
\end{array}$ \\
\hline & & & 100.07 & & \\
\hline
\end{tabular}

N. Quartz monzonite, near Gem. Pulaskose. Contains plagioclase, .alkali feldspar, hornblende, and $\bullet$ quartz, with a little biotite, pyroxene, titanite, and magnetite.

O. Syenite porphyry, near Bradyville. Monzonose. Contains microcline, plagioclase, hornblende, pyroxene, apatite, magnetite, and titanite, with secondary epidote, sericite, and green amphibole.

Rocks N, O, from the Cœur d'Alene district, are described by F. L. Ransome and F. C. Calkins in P. P. 62 . Analyses by Steiger, record No. 2194.

\begin{tabular}{|c|c|c|}
\hline & N. & 0. \\
\hline 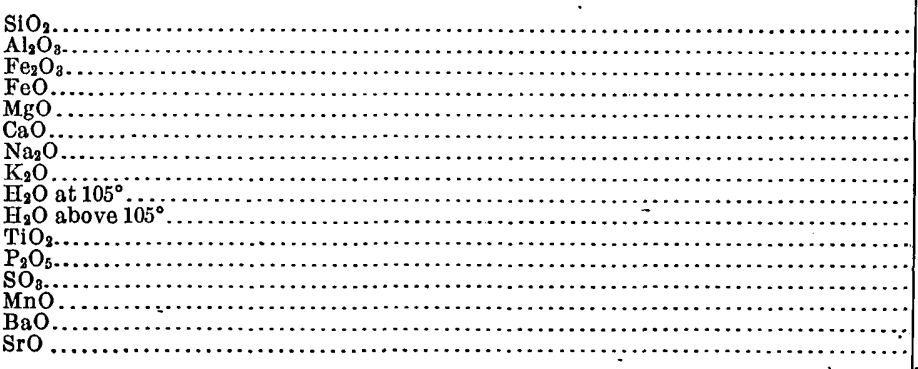 & $\begin{array}{r}61.41 \\
17.99 \\
2.93 \\
1.39 \\
1.20 \\
4.75 \\
4.01 \\
4.59 \\
.11 \\
.68 \\
.53 \\
.19 \\
.05 \\
.16 \\
.11 \\
.14 \\
\end{array}$ & $\begin{array}{r}58.53 \\
16.85 \\
3.49 \\
2.37 \\
1.46 \\
3.93 \\
4.05 \\
7.12 \\
.12 \\
.49 \\
.71 \\
.24 \\
.04 \\
.19 \\
.10 \\
.14\end{array}$ \\
\hline & 100.24 & 99.83 \\
\hline
\end{tabular}

$\mathrm{CO}_{2}, \mathrm{ZrO}_{3}$, and $\mathrm{NiO}$ absent. 


\section{COLORADO.}

\section{DENVER BASIN.}

Rocks described by Cross in Mon. XXVII. Analyses A, B, D, and E by L. G. Eakins, $\mathrm{C}$ by W. F. Hillebrand. All but A were made in the Denver laboratory.

A. Dolerite, dike near Valmont. Shoshonose. Contains augite, plagioclase, olivine, orthoclase, and biotite, with accessory magnetite and apatite. Record No. 1145. P. R. C. 534 .

B. Augite separated from A. P. R. C. 105.

C. Basalt, Table Mountain, lower capping sheet. Shoshonose. Contains plagioclase, orthoclase, augite, magnetite, and apatite, with olivine much serpentinized. Sp. gr., $2.83,22.5^{\circ}$.

D. Basalt, earlier flow, south slope of North Table Mountain. Shoshonose. Contains augite, olivine, plagioclase, probably orthoclase, magnetite, apatite, and a little biotite. P. R. C. 535.

E. Augite-mica syenite, from north fork of Turkey Creek, Jefferson County. Shoshonose. Contains orthoclase, augite, biotite, rhombic pyroxene, hornblende, plagioclase, quartz, apatite, and magnetite. Sp. gr., 2.857, 29.5 ${ }^{\circ}$ P. R. C. 532.

\begin{tabular}{|c|c|c|c|c|c|}
\hline & A. & B. & C. & D. & E. \\
\hline 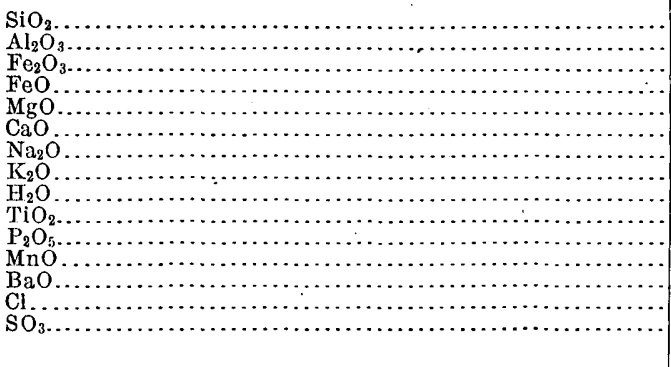 & $\begin{array}{r}48.25 \\
16.73 \\
3.99 \\
6.28 \\
5.77 \\
8.32 \\
3.24 \\
4.08 \\
1.72 \\
.89 \\
.68 \\
\text { trace } \\
.01 \\
.08 \\
.12 \\
\end{array}$ & 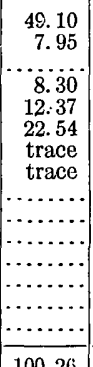 & $\begin{array}{r}52.59 \\
17.91 \\
3.81 \\
5.18 \\
4.11 \\
7.24 \\
2.94 \\
3.83 \\
1.24 \\
.84 \\
.14 \\
\text { trace } \\
\ldots \ldots .05 \\
. \ldots . . . \\
\ldots . .0 \\
\end{array}$ & $\begin{array}{r}49.69 \\
18.06 \\
2.64 \\
6.19 \\
5.73 \\
8.24 \\
2.99 \\
3.90 \\
.91 \\
.85 \\
.81 \\
.13 \\
\ldots .13 \\
. .13 \\
\ldots . . \\
\end{array}$ & $\begin{array}{r}56.90 \\
18.50 \\
.17 \\
4.61 \\
5.10 \\
6.17 \\
2.99 \\
4.14 \\
.51 \\
.19 \\
.79 \\
\text { trace }\end{array}$ \\
\hline
\end{tabular}

The following rocks from the Denver Basin were analyzed by L. G. Eakins in the Denver laboratory, but the analyses do not appear in the monograph. The subjoined data have been supplied by Whitman Cross.

A. Enstatite diabase porphyry, Mount Morrison. Bandose. Contains labradorite` and enstatite in a groundmass of considerable amount, which is colorless and crypto. crystalline, probably feldspathic, and carries magnetite and other indistinct ferritic matter. P. R. C. 533.

B. Augite andesite, Table Mountain. I. 5. 3. 3. Contains plagioclase (andesine), with rare augite and biotite, in a groundmass of plagioclase, augite, magnetite, and minor accessories. This rock contained ptilolite in its vesicular equivalent. Described in Proc. Colorado Sci. Soc., 1886, p. 72.

C. Augite andesite, a pebble from the Denver beds, Table Mountain. Yellowstonose. A few augite and andesite phenocrysts in a groundmass of oligoclase, orthoclase, and quartz (?) grains, with some augite and magnetite. 


\begin{tabular}{|c|c|c|c|}
\hline & $\Lambda$. & B. & C. \\
\hline 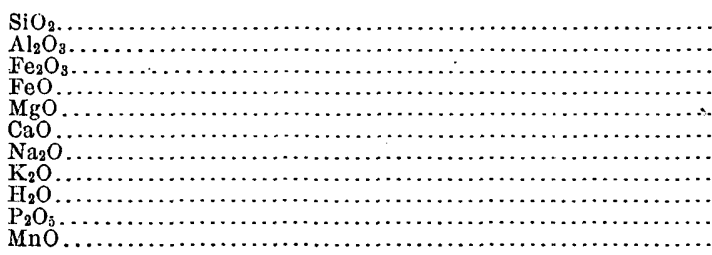 & $\begin{array}{r}56.74 \\
18.80 \\
.15 \\
6.91 \\
5.57 \\
7.34 \\
2.32 \\
.77 \\
1.09 \\
.20 \\
.07\end{array}$ & $\begin{array}{r}59.26 \\
23.63 \\
.30 \\
.57 \\
.31 \\
5.93 \\
4.94 \\
4.78 \\
.74 \\
\hdashline\end{array}$ & $\begin{array}{r}59.29 \\
21.27 \\
3.33 \\
1.04 \\
1.12 \\
5.25 \\
3.39 \\
3.00 \\
1.63 \\
.23 \\
.21\end{array}$ \\
\hline Sp.gr $\ldots \ldots \ldots \ldots, \ldots, \ldots, \ldots, \ldots, \ldots$, & $\begin{array}{r}99: 96 \\
2.876,27^{\circ}\end{array}$ & $\begin{array}{r}100.46 \\
2.625,31^{\circ}\end{array}$ & 2. $596,14.5^{\circ}$ \\
\hline
\end{tabular}

2. PIKES PEAK DISTRICT.

GRANITE.

Rocks A to G described by E. B. Mathews.' For description of A, C, E, and F see Jour. Geology, vol. 8, p. 214. Analyses by W. F. Hillebrand, record No. 1470.

A. Granitite, Sentinel Point, western part of Pikes Peak massif. Alaskose. Contains microcline, microcline-perthite, quartz, biotite, a little oligoclase, and accessory fluorite, apatite, zircon, sphene, magnetite, and állanite. P. R. C. 67 and 600.

B. Granitite, near road between Florissant and the Platte River. Kallerudose. Consists chiefly of microcline in perthitic intergrowth with albite, quartz, and biotite. P. R. C. 606.

C. Porphyritic granitite, south side of Pikes Peak, ridge between Middle and North Beaver creeks. Liparose. Contains microcline, perthite, orthoclase, oligoclase, quartz, biotite, and accessory apatite, fluorite, zircon, and magnetite. P. R. C. 602.

D. Sheared granite, Currant Creek Canyon; north of Twelvemile Park. Magdeburgose. Contains perthitic microcline, quartz, muscovite, and sericitic aggregates replacing plagioclase and a part of the microcline. P. R. C. 604.

E. Granite, Currant Creek Canyon, north of Twelvemile Park. Omeose. Consists chiefly of perthitic microcline, quartz, greenish biotite, muscovite, and plagioclase altered to a sericitic mass. Also flakes of limonite. Accessory minerals rare. P. R. C. 603.

F. Granitite, Middle Beaver Creek, south side of Pikes Peak. Liparose. Contains microcline, orthoclase, perthitic albite, oligoclase, abundant quartz, biotite, and a little accessory magnetite, fluorite, and zircon. P. R. C. 601.

G. Granite gneiss, north of Twin Creek. Kallerudose. Containsmmicrocline, orthoclase, quartz, biotite, abundant fluorite, and a little sphene and apatite. P. R. C. 605 .

\begin{tabular}{|c|c|c|c|c|c|c|c|}
\hline & A: & B. & C. & D. & E. & $\mathrm{F}$ & $\mathrm{G}$. \\
\hline 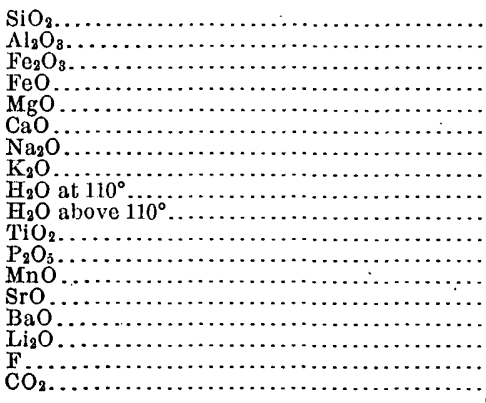 & $\begin{array}{r}77.03 \\
12.00 \\
.76 \\
.86 \\
.04 \\
.80 \\
3.21 \\
4.92 \\
.14 \\
.30 \\
.13 \\
\text { trace } \\
\text { trace } \\
\text { none } \\
\text { trace } \\
\text { trace } \\
.36 \\
\cdots\end{array}$ & $\begin{array}{r}75.92 \\
12.96 \\
.33 \\
1.40 \\
\text { trace } \\
.15 \\
4.60 \\
4.15 \\
.16 \\
.32 \\
.05 \\
\text { trace } \\
.04 \\
\text { none } \\
\text { trace } \\
\text { trace } \\
.12 \\
.03\end{array}$ & $\begin{array}{r}75.17 \\
12.66 \\
.23 \\
1.40 \\
.05 \\
.82 \\
2.88 \\
5.75 \\
.16 \\
.66 \\
.10 \\
.03 \\
\text { trace } \\
\text { trace? } \\
.03 \\
\text { trace } \\
.31 \\
\ldots . . .\end{array}$ & $\begin{array}{r}74.40 \\
14.43 \\
.89 \\
.22 \\
.07 \\
.58 \\
1.76 \\
6.56 \\
.15 \\
.92 \\
.12 \\
.22 \\
\text { trace } \\
\text { none } \\
\text { trace } \\
\text { trace } \\
.04\end{array}$ & $\begin{array}{r}.73 .90 \\
13.65 \\
.28 \\
.42 \\
.14 \\
.23 \\
2.53 \\
7.99 \\
.16 \\
.33 \\
.07 \\
.05 \\
\text { trace } \\
\text { none } \\
\text { trace } \\
\text { trace } \\
\text { none } \\
\text {........ }\end{array}$ & $\begin{array}{r}73.51 \\
13.28 \\
.94 \\
.97 \\
.05 \\
1.11 \\
3.79 \\
5.22 \\
.16 \\
.62 \\
.18 \\
\text { trace } \\
\text { trace } \\
\text { none } \\
\text { trace } \\
\text { trace } \\
.55 \\
\ldots . . . .\end{array}$ & $\begin{array}{r}66.90 \\
14.86 \\
.93 \\
3.41 \\
.31 \\
1.23 \\
5.56 \\
5.02 \\
.16 \\
.31 \\
.43 \\
.12 \\
.15 \\
\text { none } \\
.14 \\
.06 \\
1.00 \\
\ldots . . .\end{array}$ \\
\hline \multirow[t]{2}{*}{ 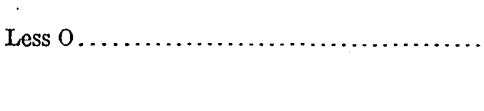 } & $\begin{array}{r}100.55 \\
.15\end{array}$ & $\begin{array}{r}100.23 \\
.05\end{array}$ & $\begin{array}{r}100.25 \\
.13\end{array}$ & $\begin{array}{r}100.36 \\
.02\end{array}$ & $\begin{array}{r}99.75 \\
\ldots \ldots\end{array}$ & $\begin{array}{r}100.38 \\
.22\end{array}$ & $\begin{array}{r}100.59 \\
.42\end{array}$ \\
\hline & 100. 40 & 100.18 & 100.12 & 100.34 & n..... & 100.16 & 100.17 \\
\hline
\end{tabular}


The four following rocks, from the Colorado Springs quadrangle, were collected by G. I. Finlay. Descriptions furnished by Cross. Analyses by G. Steiger, record No. 2236 .

H. Riebeckite granite, Rosemount. Contains quartz and alkali feldspar, with considerable amounts of riebeckite, less barkevikite, and very little zircon, magnetite, and titanite.

I. Aplitic granite, east of St. Peters Dome. Consists chiefly of quartz and alkali feldspar, with small amounts of riebeckite and a mica rich in iron.

J. Micaceous granite, east of Fairview. Rich in lepidomelane and riebeckite, with quartz and alkali feldspar. Accessory constituents rare, fluorite probably among them.

K. Lamprophyre dike in granite, Bear Creek Canyon. Consists of alkali feldspar, plagioclase, and hornblende, with small amounts of biotite, quartz, muscovite, and other minor accessories.

\begin{tabular}{|c|c|c|c|c|}
\hline$"=$ & H. & I. & J. & $\mathbf{K}$. \\
\hline 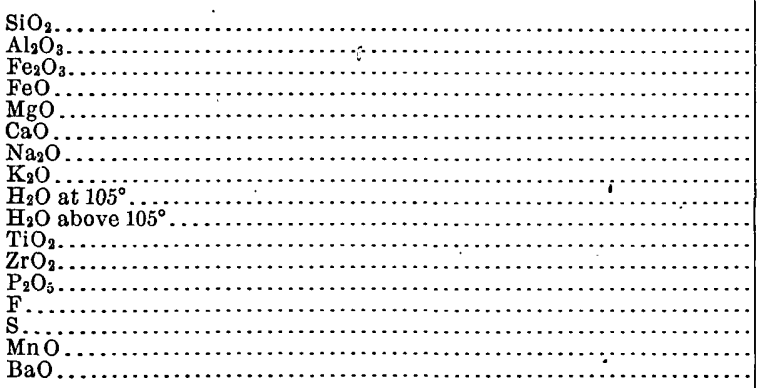 & $\begin{array}{r}73.82 \\
10.59 \\
2.18 \\
2.98 \\
.04 \\
.28 \\
4.20 \\
4.57 \\
.39 \\
.49 \\
.13 \\
\text { none } \\
.02 \\
.06 \\
\text { none } \\
\text { none } \\
\text { none }\end{array}$ & $\begin{array}{r}77.31 \\
12.45 \\
.43 \\
.33 \\
\text { none } \\
.50 \\
4.72 \\
3.84 \\
.42 \\
.40 \\
.06 \\
\text { none } \\
\text { none } \\
.15 \\
\text { none } \\
.01 \\
\text { none }\end{array}$ & $\begin{array}{r}73.22 \\
10.93 \\
3.94 \\
1.20 \\
\text { none } \\
.41 \\
3.63 \\
4.59 \\
.87 \\
.99 \\
.22 \\
\text { none } \\
\text { none } \\
.10 \\
\text { none } \\
.03 \\
\text { none }\end{array}$ & $\begin{array}{r}61.46 \\
14.55 \\
2.30 \\
5.78 \\
.50 \\
2.74 \\
4.71 \\
4.88 \\
.54 \\
.74 \\
1.07 \\
.02 \\
.27 \\
.14 \\
.01 \\
.25 \\
.17\end{array}$ \\
\hline \multirow[t]{2}{*}{ 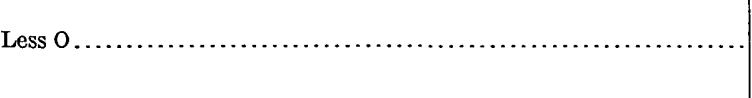 } & $\begin{array}{r}99.75 \\
.02\end{array}$ & $\begin{array}{r}100.62 \\
.06\end{array}$ & $\begin{array}{r}100.13 \\
.04\end{array}$ & $\begin{array}{r}100.13 \\
.06\end{array}$ \\
\hline & 99.73 & 100.56 & 100.09 & 100.07 \\
\hline
\end{tabular}

$\mathrm{CO}_{2}$ and $\mathrm{SO}_{3}$ absent; $\mathrm{SrO}$ not looked for.

ROCKS OF THE CRIPPIE CREEK DISTRICT:

Rocks A to L described by Cross. Descriptions published, except when otherwise stated, in 16th Ann., pt. 2, pp. 38-50. Analyses, with two exceptions, which are properly noted, by W. F. Hillebrand, record Nos. 1448, 1453, and 1524.

A. Phonolite, dike in granite northeast of Big Bull Mountain. Miaskose. Contains anorthoclase, nepheline, sodalite, ægirine-augite, some ægirine, biotite, magnetite, and limonite. Nosite not distinguishable. P. R. C. 616.

B. Phonolite, Mitre Peak. Miaskose. Contains sanidine, nepheline, sodalite, ægirine, nosite, and colorless particles which may be låvenite. P. R. C. 608.

C. Phonolite, hill 1 mile south of Straub Mountain. Miaskose. Contains anorthoclase, sanidine, nepheline, sodalite, nosite, analcite, and ægirine, with sometimes ægirine-augite. P. R. C. 612 .

D. Phonolite, Rhyolite Mountain. Miaskose. Contains nepheline, nosite, analcite, ægirine, and some ægirine-augite, in a feldspathic groundmass. Sp. gr., 2.52, $23^{\circ}$. P. R. C. 618.

E. Phonolite, between Florissant and Manitou. Miaskose. Analysis by L. G. Eakins, made in the Denver laboratory, inserted here for comparison with the Cripple Creek samples. Described by Cross in Proc. Colorado Sci. Soc., vol. 2, p. 167. Contains sanidine, nepheline, hornblende, with accessory pyroxene (?), magnetite, apatite, and sphene. Sp. gr., 2.576, $13^{\circ}$. P. R. C. 607.

F. The portion of $\mathrm{E}$ soluble in hydrochloric acid. This portion amounted to 25.39 per cent, and is recalculated here to 100. Analysis by Eakins. 


\begin{tabular}{|c|c|c|c|c|c|c|}
\hline , & A. & B. & C. & D. & E. & F. \\
\hline 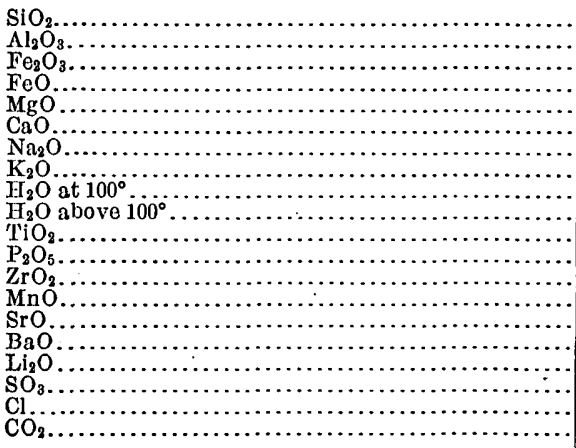 & $\begin{array}{r}59.00 \\
20.07 \\
1.58 \\
.65 \\
.10 \\
1.05 \\
8.34 \\
5.63 \\
.24 \\
2.03 \\
.29 \\
.05 \\
.20 \\
.12 \\
\text { none } \\
\text { trace } \\
\text { trace } \\
.07 \\
.24 \\
.26\end{array}$ & $\begin{array}{r}58.98 \\
20.54 \\
1.65 \\
.48 \\
.11 \\
.67 \\
9.95 \\
5.31 \\
.19 \\
.97 \\
.24 \\
.04 \\
.20 \\
.26 \\
\text { none } \\
\text { none } \\
\text { trace } \\
.20 \\
.28 \\
\ldots . . .\end{array}$ & $\begin{array}{r}58.78 \\
20.03 \\
1.87 \\
.49 \\
.16 \\
.83 \\
9.36 \\
5.50 \\
.31 \\
1.57 \\
.29 \\
.03 \\
.17 \\
.15 \\
\text { none } \\
\text { none } \\
\text { trace } \\
.12 \\
.58\end{array}$ & $\begin{array}{r}58.64 \\
19.62 \\
2.17 \\
.42 \\
.37 \\
1.24 \\
8.39 \\
5.26 \\
.34 \\
2.40 \\
.20 \\
.03 \\
.09 \\
.20 \\
\text { trace } \\
\text { trace } \\
\text { trace } \\
\text { trace? } \\
.14 \\
.23\end{array}$ & 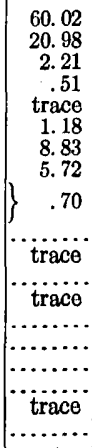 & 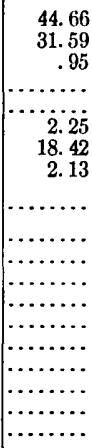 \\
\hline & 99.92 & 100.07 & 100.24 & 99.74 & 100.15 & 100.00 \\
\hline
\end{tabular}

G. Trachytic phonolite, dike on west slope of Bull Cliff. (The latite-phonolite of Graton.) Miaskose. Contains alkali feldspars, nepheline, nosite, sodalite, augite, scanty ægirine, brown hornblende, magnetite, sphene, apatite, and sometimes låvenite. P. R. C 624 .

H. Altered phonolite, Washington shaft, Victor. Contains potash feldspars, with some alteration to muscovite. Crystals of pyrite and fluorite are visible, but nepheline and ægirine have disappeared. P. R. C. 623.

I. Nepheline syenite, near the Longfellow mine. Akerose. Containsalkali feldspars, nepheline, sodalite, augite, some ægirine, hornblende, biotite, sphene, apatite, and magnetite. Sp. gr., 2. $68,23^{\circ}$. P. R. C. 626.

J. Nepheline basalt, Appie Ellen shaft. Much altered. Rich in olivine, augite, and magnetite. Also contains nepheline, feldspars in small amount, and biotite. Sp. gr., $2.99,23^{\circ}$.

K. Altered nepheline basalt, Anna Lee mine.

L. Local facies of a phonolitic mass, Bull Cliff. Akerose. Contains abundant augite, plagioclase, alkali feldspar, magnetite, and a little red-brown biotite, with a colorless isotropic base in the darker spots. P. R. C. 627.

\begin{tabular}{|c|c|c|c|c|c|c|}
\hline & G. & H. & I. & J. & $\mathbf{K}$. & L. \\
\hline 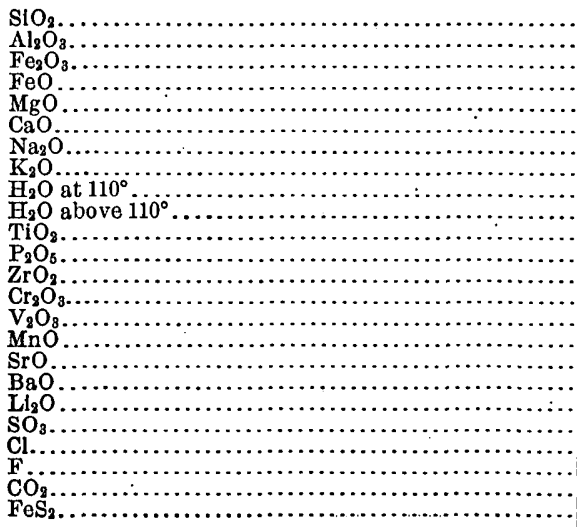 & $\begin{array}{r}59.38 \\
19.47 \\
1.60 \\
1.19 \\
.36 \\
1.96 \\
7.80 \\
5.83 \\
.11 \\
.69 \\
.58 \\
.08 \\
.10 \\
\ldots \ldots \\
\ldots . .2 \\
.15 \\
.03 \\
.13 \\
\text { trace } \\
.37 \\
.22 \\
\ldots . . .\end{array}$ & 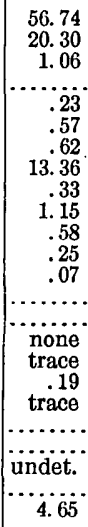 & $\begin{array}{r}54.34 \\
19.21 \\
3.19 \\
2.11 \\
1.28 \\
4.53 \\
6.38 \\
5.14 \\
.14 \\
1.17 \\
1.09 \\
.27 \\
.07 \\
\ldots .02 \\
.02 \\
.08 \\
.16 \\
.24 \\
\text { trace } \\
.07 \\
.28 \\
\text { undet. } \\
\text { none } \\
\ldots \ldots . .\end{array}$ & \begin{tabular}{r}
35.03 \\
9.80 \\
5.55 \\
4.98 \\
9.78 \\
15.09 \\
2.04 \\
2.16 \\
.41 \\
2.05 \\
2.20 \\
1.99 \\
none \\
$\ldots$ \\
$\cdots \ldots .$. \\
\hdashline .06 \\
.17 \\
.14 \\
trace \\
none \\
trace \\
undet. \\
7.83 \\
.38
\end{tabular} & $\begin{array}{r}48.61 \\
20.74 \\
4.29 \\
.22 \\
2.11 \\
.25 \\
.16 \\
.77 \\
12.10 \\
7.07 \\
3.57 \\
.29 \\
\ldots \ldots . . \\
\text { trace } \\
\ldots \ldots \ldots . \\
\text { none } \\
. \text { none } \\
\text { none } \\
(?) . \\
\ldots \ldots . . . \\
\ldots \ldots . . . \\
. .63 \\
\ldots \ldots . . . \\
\ldots \ldots . . .\end{array}$ & $\begin{array}{r}49.84 \\
17.78 \\
5.86 \\
2.62 \\
3.02 \\
7.35 \\
5.20 \\
3.04 \\
.34 \\
2.02 \\
1.43 \\
.76 \\
.03 \\
\ldots . .03 \\
.03 \\
.21 \\
.18 \\
.22 \\
\text { trace } \\
\text { none } \\
\text { trace } \\
\text { undet. } \\
.52 \\
\ldots . . .\end{array}$ \\
\hline & 100.05 & 100.10 & 99.77 & 99.66 & 100.81 & 100.45 \\
\hline
\end{tabular}


The following Cripple Creek rocks, M to Y, are described in P. P. 54, mainly by L. C. Graton.

M. Granite, Ajax mine. Contains microcline, oligoclase, quartz, biotite, and apatite.

N. Altered granite adjacent to M. Contains quartz, potash feldspar, fluorite, and pyrite. Described as an ore. Descriptions of $\mathrm{M}$ and $\mathrm{N}$ furnished by F. L. Ransome.

O. Biotite trachyte, Portland mine. Phlegrose.

P. Syenite, Portland mine. Monzonose. Contains pyroxene, plagioclase, orthoclase, hornblende, apatite, magnetite, and titanite.

Q. Latite-phonolite, Portland mine. Essexose. Contains pyroxene, plagioclase, orthoclase, hornblende, analcite, nosean, sodalite, apatite, magnetite, and titanite.

R. Latite-phonolite, 3,300 feet $10^{\circ}$ east of south from Bull Cliff. Essexose. Contains pyroxene, plagioclase, orthoclase, hómblende, nosean, analcite, apatite, magnetite, titanite, sodalite, and secondary zeolites. Analyses $\mathrm{M}$ to $\mathrm{R}$ by W. F. Hillebrand, record No. 2132. All contain traces of lithia.

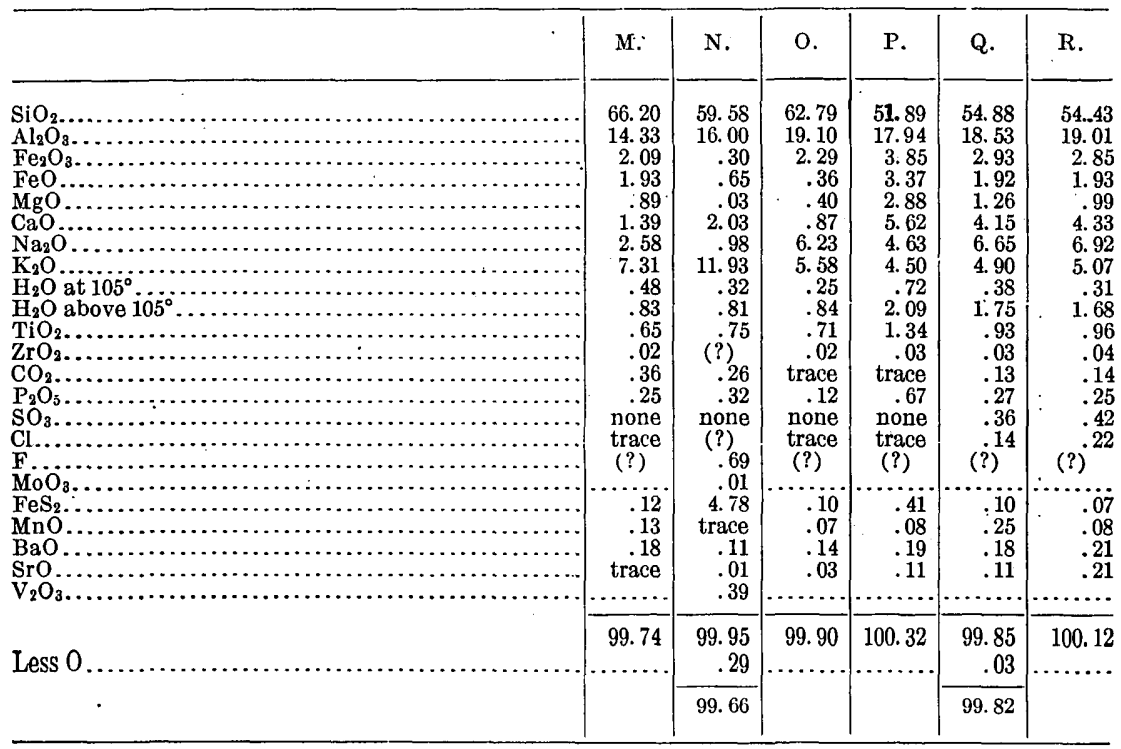

S. Latite-phonolite, Portland mine. Akerose. Contains pyroxene, plagioclase, orthoclase, magnetite, apatite, and very little sodalite.

T. Latite-phonolite, Anaconda mine. Akerose. Contains pyroxene, plagioclase, orthoclase, magnetite, apatite, titanite, hornblende, sodalite, analcite, and a little biotite. Analyses S, T by G. Steiger, record No. 2152.

U. Trachydolerite, Isabella dike. Akerose. Contains plagioclase, pyroxene, olivine, orthoclase, analcite, apatite, magnetite, biotite, and hornblende.

V. Vogesite, Jennie Sample mine. Monzonose. Contains orthoclase, hornblende, pyroxene, biotite, plagioclase, brown mica, iron ores, rare apatite, some analcite, and often olivine.

W. Monchiquite, Block 8 mine. Ourose. Contains pyroxene, olivine, analcite, biotite, apatite, magnetite, and alteration products. Analyses $\mathrm{S}$ to $\mathrm{W}$ by W. T. Schaller, record No. 2171: 
X. Granitic gneiss. Contains albite, orthoclase, quartz, and biotite.

Y. Mica schist. Contains quartz, sillimanite, muscovite, and magnetite. Descriptions of $\mathrm{X}$ and $\mathrm{Y}$ supplied by W. Lindgren. Analyses by Schaller, record No. 2163.

\begin{tabular}{|c|c|c|c|c|c|c|c|}
\hline & S. & T. & U. & V. & W. & X. & Y. \\
\hline \multirow[t]{2}{*}{$\begin{array}{l}\mathrm{SiO}_{2} \\
\mathrm{Al}_{2} \mathrm{O}_{3} \ldots \ldots \\
\mathrm{Fe}_{2} \mathrm{O}_{2} \ldots \ldots \\
\mathrm{FeO} \\
\mathrm{MeO} \\
\mathrm{MgO} \\
\mathrm{Ca} \\
\mathrm{Ca} \\
\mathrm{Na}\end{array}$} & $\begin{array}{r}56.01 \\
17.92 \\
4.22 \\
2.52 \\
2.04 \\
4.80 \\
4.92 \\
4.21 \\
.31 \\
1.10 \\
1.20 \\
.02 \\
\text { none } \\
.55 \\
.02 \\
\text { none } \\
\text { trace } \\
.13 \\
.16 \\
.06 \\
\text { none }\end{array}$ & $\begin{array}{r}58.05 \\
17.66 \\
3.51 \\
1.65 \\
1.55 \\
4.48 \\
5.80 \\
4.06 \\
.35 \\
.87 \\
.91 \\
.02 \\
\text { none } \\
.40 \\
\ldots .0 \\
.04 \\
\text { trace } \\
.13 \\
.19 \\
.08 \\
\text { none }\end{array}$ & \begin{tabular}{|r}
48.76 \\
17.04 \\
5.04 \\
5.52 \\
4.57 \\
8.64 \\
4.27 \\
3.39 \\
.69 \\
1.84 \\
1.34 \\
trace \\
.22 \\
.79 \\
.06 \\
trace \\
.01 \\
.08 \\
.15 \\
.07 \\
trace? \\
trace
\end{tabular} & $\begin{array}{r}47.31 \\
16.21 \\
5.11 \\
2.90 \\
3.08 \\
7.11 \\
3.92 \\
3.73 \\
.87 \\
2.17 \\
1.64 \\
.01 \\
4.98 \\
.90 \\
.06 \\
.05 \\
.05 \\
\text { trace } \\
.17 \\
.02 \\
\text { trace? } \\
\text { trace }\end{array}$ & $\begin{array}{r}44.08 \\
12.80 \\
4.58 \\
3.84 \\
7.22 \\
11.21 \\
2.97 \\
3.31 \\
.77 \\
2.35 \\
1.43 \\
\text { none } \\
4.14 \\
.70 \\
.14 \\
.01 \\
.04 \\
.14 \\
.13 \\
.06 \\
.05 \\
\text { trace }\end{array}$ & 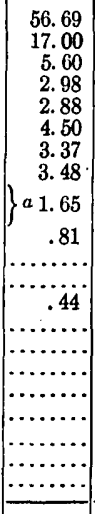 & 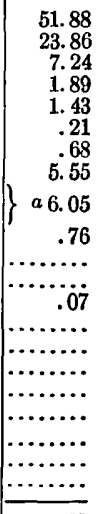 \\
\hline & 100.19 & 99.75 & 100.48 & 100.29 & 99.97 & 99.40 & 99.62 \\
\hline
\end{tabular}

a Loss on ignition.

\section{MISCELLANEOUS ROCKS}

Analyses, elsewhere unpublished, except when otherwise stated, by W. F. Hillebrand, record Nos. 1448, 1453, and 1669. Petrographic data supplied by Whitman Cross. Names marked with a query are provisional designations only.

A. Rhyolite, near Robbins's ranch. Toscanose. Has scattered phenocrysts of alkali feldspar, oligoclase, and biotite in a predominant trachytic groundmass of alkali feldspar, tridymite, particles of residual glass, and ferritic flakes and grains. P. R. C. 1326.

B. Trachyte (?), Wicher Mountain. Toscanose. Shows biotite and a few glassy. feldspars in a groundmass resembling that of A. P. R. C. 1328.

C. Quartz latite, Bare Hills. Pulaskose. Composed mainly of plagioclase and alkali feldspar, with small augites and much ferritic matter. Minute prisms of a yellowish-brown amphibole (?) and scales of tridymite are also visible. P. R. C. 1327.

D. Pyroxene latite, Wicher Mountain. Monzonose. Contains phenocrysts of plagioclase, a few of augite, minute specks of iddingsite, and flakes of limonite. In the groundmass are plagioclase, orthoclase (?), augite, iddingsite, and hypersthene. In the pores tridymite appears. P. R. C. 1325.

E. Plagioclase basalt, mesa east of Mac Gulch. Andose. Contains plagioclase, augite, olivine, iddingsite, magnetite, biotite, and apatite. P. R. C. 1324.

F. Plagioclase basalt, Saddle Mountain. Andose. Phenocrysts of augite and olivine in a groundmass of plagioclase, orthoclase, augite, magnetite, biotite, and apatite. Very fresh. See Cross, Jour. Geology, vol. 5, p. 684. P. R. C. 1323. 


\begin{tabular}{|c|c|c|c|c|c|c|}
\hline & A. & B. & C. & D. & E. & F. \\
\hline 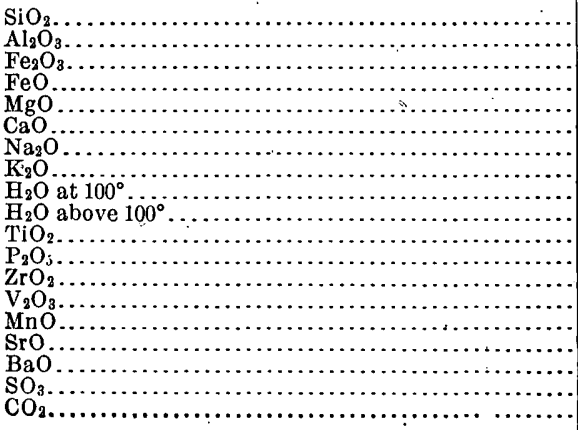 & $\begin{array}{r}69.52 \\
15.44 \\
1.90 \\
.09 \\
.17 \\
1.70 \\
4.54 \\
5.04 \\
.33 \\
.27 \\
.23 \\
.14 \\
.05 \\
\ldots .08 \\
.08 \\
.04 \\
.19 \\
\ldots . . \\
.17\end{array}$ & $\begin{array}{r}66.12 \\
17.21 \\
2.43 \\
\text { trace } \\
.35 \\
2.11 \\
4.70 \\
5.57 \\
.14 \\
.71 \\
.29 \\
.11 \\
.06 \\
\ldots \ldots . . \\
.08 \\
.05 \\
.25 \\
\ldots \ldots . . \\
\ldots \ldots . .\end{array}$ & $\begin{array}{r}62.64 \\
17.82 \\
3.91 \\
.31 \\
.47 \\
3.22 \\
4.47 \\
4.99 \\
.58 \\
.65 \\
.59 \\
.25 \\
.08 \\
\ldots . . \\
.04 \\
.07 \\
.28 \\
\ldots \ldots . \\
\ldots \ldots .\end{array}$ & $\begin{array}{r}57.48 \\
18.02 \\
5.73 \\
.73 \\
1.17 \\
5.03 \\
4.28 \\
4.15 \\
.62 \\
.55 \\
1.00 \\
.66 \\
.04 \\
.02 \\
\text { trace } \\
.12 \\
.20 \\
.16 \\
\ldots . . .\end{array}$ & $\begin{array}{r}52.97 \\
18.31 \\
1.86 \\
6.73 \\
3.04 \\
6.51 \\
3.74 \\
3.35 \\
.44 \\
.31 \\
1.04 \\
.81 \\
.05 \\
\ldots . . . \\
.09 \\
.14 \\
.18 \\
\ldots \ldots . . \\
\ldots \ldots . .\end{array}$ & $\begin{array}{r}48.76 \\
15.89 \\
6.04 \\
4.56 \\
5.98 \\
8.15 \\
3.43 \\
2.93 \\
.40 \\
1.48 \\
1.65 \\
.60 \\
\text { none } \\
\ldots \ldots .0 \\
.13 \\
.06 \\
.17 \\
\ldots \ldots . . . \\
\ldots \ldots . . .\end{array}$ \\
\hline & 99.90 & 100.18 & 100.37 & 99.96 & 99.57 & 100.23 \\
\hline
\end{tabular}

G. Analcite basalt, from the Basin. Monchiquose. Contains phenocrysts of augite, olivine, and analcite. Also magnetite, with subordinate amounts of alkali feldspars, biotite, and apatite. P. R. C. 1322.

H. Portion of the analcite basalt soluble in hydrochloric acid.

I. Augite separated from $\mathrm{G}$.

J. Analcite separated from G. After deduction of 4.22 per cent of substance insoluble in boiling dilute hydrochloric acid, and later removal of liberated silica with weak potash solution. Sixty-two one-hundredths per cent of the water goes off over sulphuric acid. A trace of lithia was found.

The analcite basalt and its fractions are described by Cross in Jour. Geology, vol. 5, p. 684 .

\begin{tabular}{|c|c|c|c|c|}
\hline - & G. & H. & I. & J. \\
\hline 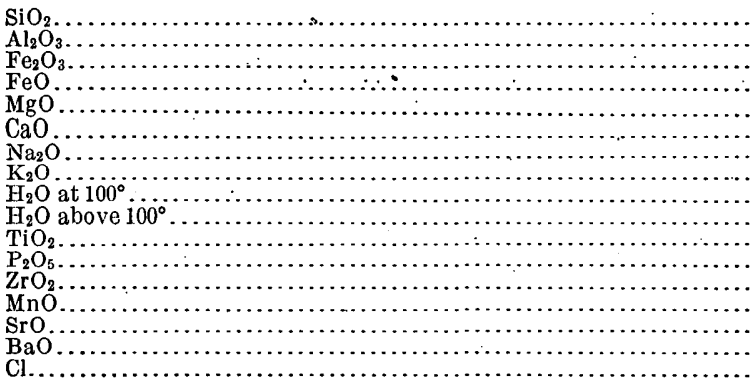 & $\begin{array}{r}45.59 \\
12.98 \\
4.97 \\
4.70 \\
8.36 \\
11.09 \\
4.53 \\
1.04 \\
.51 \\
3.40 \\
1.32 \\
.91 \\
.03 \\
.14 \\
.12 \\
.13 \\
.05\end{array}$ & $\left\{\begin{array}{r}44.44 \\
20.11 \\
7.50 \\
\\
5.81 \\
3.94 \\
8.17 \\
1.13 \\
7.91 \\
\ldots \ldots \ldots \\
\ldots \ldots \ldots \\
\ldots \ldots \\
\text { trace } \\
.16 \\
(?) \\
.10\end{array}\right.$ & \begin{tabular}{|r|}
49.26 \\
6.01 \\
3.31 \\
4.23 \\
12.40 \\
21.79 \\
.79 \\
.41 \\
undet. \\
undet. \\
1.53 \\
$\ldots \ldots .$. \\
$\ldots$ undet. \\
$\ldots .06$ \\
(?) \\
$\ldots \ldots . .$.
\end{tabular} & 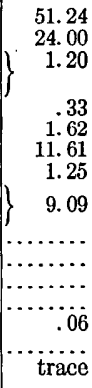 \\
\hline$\cdot$ & 99.87 & 99.27 & 99.79 & 100.40 \\
\hline
\end{tabular}

$a$ Includes $\mathrm{P}_{2} \mathrm{O}_{5}$ and possible $\mathrm{ZrO}_{2}$ and $\mathrm{TiO}_{2}$.

\section{SILVER CLIFF AND ROSITA.}

Rocks described by Cross, mostly in 17th Ann., pt. 2, p. 263 . Also, partly in Proc. Colorado Sci. Soc., vol. 2; p. 228 . Analyses, with one exception, by L. G. Eakins. Those with record numbers attached were made in the Washington laboratory; all others in the laboratory at Denver.

A. Peridotite, Cottonwood Gulch. Custerose. Contains hornblende, biotite, hypersthene, olivine, a little plagioclase, apatite, pyrrhotite, and sillimanite (?). P. R. C. 519. 
B. Augite diorite, Mount Fairview, Rosita Hills. Salemose. Contains augite, biotite, labradorite, and accessory orthoclase, olivine, magnetite, and apatite. Sp. gr., $2.870,32^{\circ}$. Olivine a subordinate consituent. P. R. C. 526.

C. The same as B, but with orthoclase in much larger amount. Akerose. S Sp. gr., 2.768, $34^{\circ}$. Record No. 1091. P. R. C. 529.

D. Trachyte, Game Ridge. Phlegrose. Contains sanidine, plagioclase, a little biotite, magnetite, apatite, and zircon in a groundmass of orthoclase, with a little quartz. Sp. gr., 2.592, $29^{\circ}$. . P. R. C. 70 and 524 .

E. Trachyte, dark-colored dike. Pulaskose. Contains more magnetite than D, but otherwise the two are practically identical. Sp. gr., 2.621, $24^{\circ}$. P. R. C. 589.

\begin{tabular}{|c|c|c|c|c|c|}
\hline & A. & B. & C. & D. & $\mathrm{I}$. \\
\hline 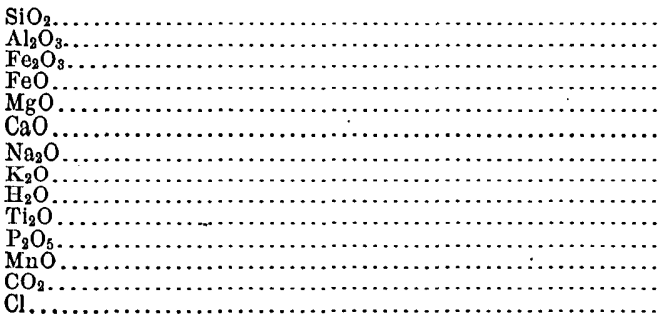 & $\begin{array}{r}46.03 \\
9.27 \\
2.72 \\
9.94 \\
25.04 \\
3.53 \\
1.48 \\
.87 \\
.64 \\
.17 \\
.40 \\
\cdots\end{array}$ & $\begin{array}{r}50.47 \\
18.73 \\
4.19 \\
4.92 \\
3.48 \\
8.82 \\
4.62 \\
3.56 \\
.58 \\
.51 \\
.10 \\
.11 \\
\text { trace } \\
\text { trace }\end{array}$ & $\begin{array}{r}53.80 \\
20.13 \\
3.57 \\
2.63 \\
2.26 \\
5.60 \\
5.20 \\
4.49 \\
.90 \\
.43 \\
.56 \\
.29 \\
\ldots \ldots . . \\
\ldots \ldots . . .\end{array}$ & $\begin{array}{r}66.03 \\
18.49 \\
2.18 \\
.22 \\
.39 \\
.96 \\
5.22 \\
5.86 \\
.85 \\
\ldots . . . \\
.04 \\
\text { trace } \\
\text { trace } \\
\ldots . . .\end{array}$ & $\begin{array}{r}65.41 \\
18.78 \\
.94 \\
.72 \\
.16 \\
1.58 \\
5.91 \\
5.41 \\
1.38 \\
\ldots \ldots . . \\
\text { trace } \\
\text { trace } \\
\ldots \ldots . . . \\
\ldots \ldots . . .\end{array}$ \\
\hline & 100.09 & 100.09 & 99.86 & 100.24 & 100.29 \\
\hline
\end{tabular}

F. Andesite, Pringle Hill. Pulaskose. Contains plagioclase, orthoclase, quartz, biotite, augite, magnetite, and apatite, with sometimes a little hornblende. Sp. gr., 2.651, $17.8^{\circ}$. Record No. 1124 . P. R. C. 528.

G. Pringle andesite, dike. Laurvikose. Like F, but often contains sphene. Sp. gr., 2.690, $28^{\circ}$. P. R. C. 527.

H. Bunker andesite, Lookout Mountain. Akerose. Contains plagioclase, orthoclase, augite, biotite, hornblende, quartz, magnetite, and apatite. Sp. gr., 2.699, $34^{\circ}$. Record No. 1091.

${ }^{\circ}$ I. Altered Bunker andesite, Robinson Plateau. Toscanose: Biotite gone, magnetite altered to limonite, feldspars not much attacked. Record No. 11.24. Sp. gr., $2.580,19.7^{\circ}$.

J. Much-decomposed Bunker andesite, ridge near Knickerbocker Hill. Liparose. Augite, hornblende, and biotite all replaced by decomposition products, plagioclase much muscovitized. Record No. 1124. P. R. C. 592.

\begin{tabular}{|c|c|c|c|c|c|}
\hline V & F. & G. & H. & I. & J. \\
\hline 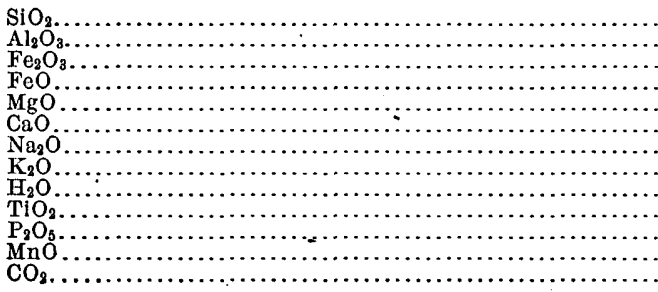 & $\begin{array}{r}58.94 \\
17.19 \\
2.63 \\
1.98 \\
1.52 \\
4.45 \\
4.20 \\
3.90 \\
4.53 \\
.27 \\
.23 \\
.10\end{array}$ & $\begin{array}{r}63.49 \\
18.40 \\
2.44 \\
1.09 \\
.66 \\
2.30 \\
5.70 \\
4.62 \\
1.04 \\
\text { trace } \\
\text { trace } \\
.16 \\
\text { trace }\end{array}$ & $\begin{array}{r}57.01 \\
18.41 \\
3.69 \\
2.36 \\
2.34 \\
4.29 \\
4.95 \\
3.72 \\
2.29 \\
.27 \\
.42 \\
.21\end{array}$ & $\begin{array}{r}63.88 \\
19.96 \\
2.21 \\
.57 \\
.58 \\
2.03 \\
4.19 \\
3.88 \\
2.03 \\
\ldots \ldots \ldots . . \\
\text { trace }\end{array}$ & $\begin{array}{r}67.13 \\
18.41 \\
.45 \\
.07 \\
.44 \\
.55 \\
4.17 \\
5.28 \\
2.98 \\
.30 \\
\text { trace } \\
\text { trace }\end{array}$ \\
\hline & 99.94 & 99.90 & 99.96 & 99.93 & 99.78 \\
\hline
\end{tabular}


K. Dacite, Bald Mountain, near Rosita. Lassenose. Contains plagioclase, biotite, hornblende, sometimes augite, magnetite, and quartz. Sp. gr., 2.574, 24 ${ }^{\circ}$. Record No. 1068. P. R. C. 530.

L. Mica dacite. Lassenose. Contains plagioclase, sanidine, biotite, and quartz. Sp. gr., 2.563, $24^{\circ}$. Record No. 1068.

M. Rhyolite, Pennsylvania Hill. Toscanose. Sp. gr., 2.470, $26^{\circ}$. Record No. 1079. P. R. C. 525.

N. Rhyolite, Round Mountain. Omeose. Contains sanidine, quartz, some glass, and accessory garnet. P. R. C. 521 .

O. Rhyolite, Silver Cliff. Magdeburgose. Sp. gr., 2,560, 15. Record No. 1125. P. R. C. 522 .

\begin{tabular}{|c|c|c|c|c|c|}
\hline & K. & L. & M. & N. & 0 \\
\hline 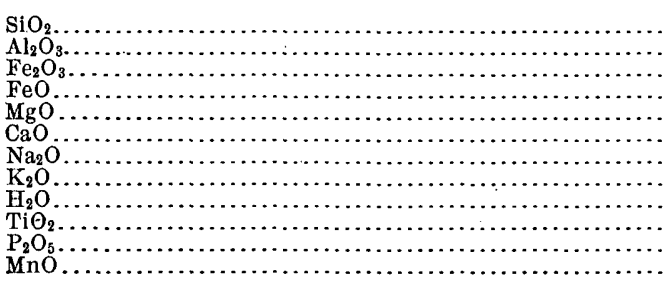 & $\begin{array}{r}66.46 \\
17.91 \\
2.42 \\
.35 \\
.49 \\
2.89 \\
4.79 \\
3.74 \\
1.01 \\
\cdots \\
\text { trace }\end{array}$ & $\begin{array}{r}67.49 \\
17.76 \\
2.54 \\
.08 \\
.35 \\
1.67 \\
5.03 \\
4.40 \\
.52 \\
\ldots \ldots \ldots \\
\text { trace } \\
\text { trace }\end{array}$ & $\begin{array}{r}70.87 \\
15.18 \\
2.18 \\
.12 \\
.60 \\
1.58 \\
3.47 \\
5.04 \\
1.08 \\
\text { trace } \\
\text { trace } \\
\text { trace }\end{array}$ & $\begin{array}{r}75.20 \\
12.96 \\
.37 \\
.27 \\
.12 \\
.29 \\
2.02 \\
8.38 \\
.58 \\
\ldots \ldots \\
\text { trace } \\
.03\end{array}$ & $\begin{array}{r}75.39 \\
13.65 \\
.38 \\
.18 \\
.15 \\
.51 \\
1.84 \\
6.81 \\
1.13 \\
\text { trace } \\
\text { trace } \\
.14\end{array}$ \\
\hline & 100.06 & 99.84 & 100.12 & 100.22 & 100.18 \\
\hline
\end{tabular}

P. Rhyolitic tuff, a lake-bed deposit east of the Blue Mountains. Mainly composed of glassy rhyolite dust.

Q. Devitrified pitchstone, the gangue of ptilolite, 3 miles southeast of Silver Cliff. Riesenose. Shows feldspar, quartz, and barite. Described by Cross and Eakins in Am. Jour. Sci., 3d ser., vol. 44, p. 96. Record No. 1342. P. R. C. 590.

R. Pitchstone, Rosita. Alaskose. Record No. 1033. P. R. C. 531.

S. Pitchstone, Fleetwood tunnel, Silver Cliff. Liparose. P. R. C. 523.

T. Alteration product of S. Gonsists mainly of silica and kaolin. Record No. of $\mathrm{S}$ and $\mathrm{T}$ 1034. P.R. C. 591.

U. Decomposition product of pitchstone, Silver Cliff. Analysis by W. F. Hillebrand.

\begin{tabular}{|c|c|c|c|c|c|c|}
\hline & P. & Q. & R. & S. & T. & U. \\
\hline \multirow{10}{*}{ 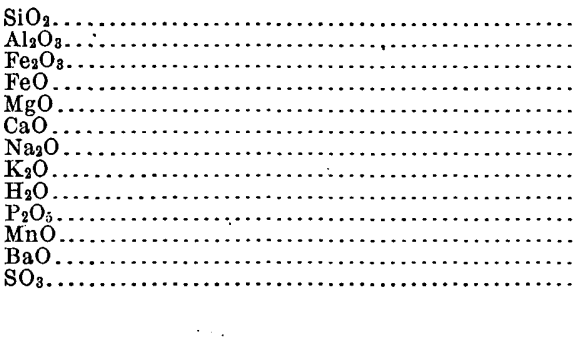 } & $\begin{array}{r}71.02 \\
14.27 \\
1.22\end{array}$ & $\begin{array}{r}65.67 \\
13.48 \\
1.51\end{array}$ & $\begin{array}{r}73.11 \\
13.16 \\
.62\end{array}$ & $\begin{array}{r}71.56 \\
13.10 \\
.66\end{array}$ & $\begin{array}{r}71.71 \\
12.36 \\
1.10\end{array}$ & $\begin{array}{r}84.77 \\
8.46 \\
.52\end{array}$ \\
\hline & & & .23 & & & \\
\hline & trace & . 31 & .19 & .14 & 1.21 & $\begin{array}{r}92 \\
18\end{array}$ \\
\hline & $\begin{array}{l}1.00 \\
2.28\end{array}$ & 1. 52 & 2. 85 & 3.77 & $\begin{array}{r}1.11 \\
.17\end{array}$ & 1.18 \\
\hline & 3. 97 & 2. 42 & 5. 10 & 4. 06 & .36 & \\
\hline & 6.12 & 12. 27 & 4.05 & 5. 52 & 11.97 & 4. 11 \\
\hline & & $\begin{array}{l}\text { trace } \\
\text { trace }\end{array}$ & .14 & .16 & .17 & \\
\hline & & .32 & & & & \\
\hline & & & & & & \\
\hline & 100.26 & 100.19 & 99.99 & 99.99 & 100.16 & 99.96 \\
\hline
\end{tabular}

V. Syenite, Silver Cliff. Monzonose. Contains orthoclase and plagioclase in nearly equal amounts, colored by ferric hydroxide, with amphibole, a little biotite, and secondary epidote, calcite, and chlorite. Sp. gr., 2.689, $20^{\circ}$. P. R. C. 71 and 520 . 
W. Quartz-alunite rock, Democrat Hill. About two-thirds quartz and one-third alunite. 'Record No. 1126 . P. R. C. 596.

X. The same, Mount Robinson. About one-fourth alunite. Record No. 1248. P. C. R. 593 .

Y. Quartz-diaspore rock, Mount Robinson. About 18 per cent diaspore, the rest quartz. Record No. 1167 . P. R. C. $59 \dot{4}$.

Rocks W, X, and Y are also described in Am. Jour. Sci., 3d ser., vol. 41, p. 471. These three rocks are decomposition products of rhyolite.

\begin{tabular}{|c|c|c|c|c|}
\hline & V. & W. & $\mathrm{x}$ & Y. \\
\hline 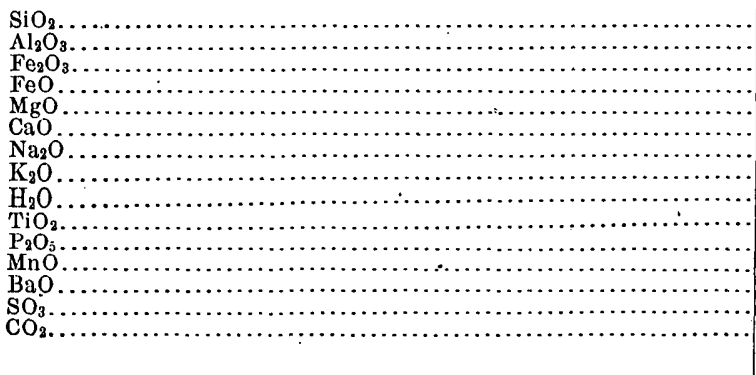 & $\begin{array}{r}59.78 \\
16.86 \\
3.08 \\
3.72 \\
.69 \\
2.96 \\
5.39 \\
5.01 \\
1.58 \\
\ldots \ldots \\
.14 \\
\ldots . . . \\
.75\end{array}$ & $\begin{array}{r}65.94 \\
12.95 \\
.33 \\
.07 \\
.05 \\
.10 \\
1.19 \\
2.32 \\
4.47 \\
\cdots \cdots \cdots \\
\text { trace } \\
\text { trace } \\
12.47 \\
\ldots \ldots \ldots\end{array}$ & 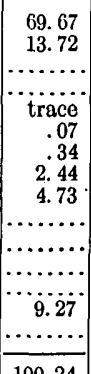 & \begin{tabular}{r}
76.22 \\
19.45 \\
trace \\
$\ldots \ldots .$. \\
\hdashline trace \\
trace \\
trace \\
3.82 \\
.11 \\
.13 \\
\hdashline$\ldots .$. \\
.29 \\
$\ldots \ldots$. \\
\end{tabular} \\
\hline & 99.96 & 99.89 & 100.24 & 100.02 \\
\hline
\end{tabular}

The following analyses, all by L. G. Eakins, do not appear in the published memoir just cited. A and B were made in the Denver laboratory. The petrographic details have been supplied by Whitman Cross.

A. Spherulite in rhyolite, ridge west of Mount Tyndall. Mainly composed of quartz and feldspar needles, with some ferritic coloring due to decomposition of trichites. P. R. C. 1077.

B. Rhyolitic residual glass, same locality as A. P. R. C. 1077.

C. Interspherulitic mass, Rosita. Record No. 1285.

D. Spherulite, Rosita. Record No. 1286.

E, F, G. Spherulites, Silver Cliff. Record Nos. 1285, 1286.

H. Spherulite of rhyolite, Fleetwood tunnel, Silver Cliff. Sp. gr., 2.389, $20^{\circ}$. P. R. C. 1075.

The spherulites, C, D, E, F, and G, are made up of orthoclase needles, with free silica in fibers or grains, or rarely as tridymite.

The "soluble silica" is that which is dissolved by sodium carbonate solution.

\begin{tabular}{|c|c|c|c|c|c|c|c|c|}
\hline . & A. & B. & C. & D. & E. & F. & G. & H. \\
\hline 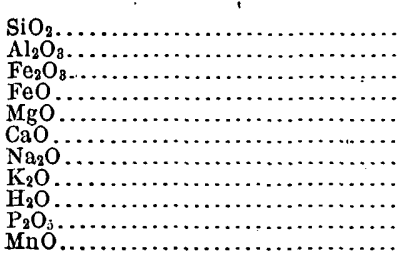 & $\begin{array}{r}71.27 \\
16.02 \\
1.41 \\
.17 \\
\text { trace } \\
.35 \\
5.76 \\
4.08 \\
1.14 \\
\text { trace } \\
\text { trace }\end{array}$ & $\begin{array}{r}85.50 \\
7.42 \\
1.23 \\
.34 \\
.82 \\
.37 \\
.74 \\
2.64 \\
1.22 \\
\text { none } \\
.08\end{array}$ & $\begin{array}{r}74.47 \\
13.87 \\
\ldots \ldots \ldots \\
\cdots \ldots \ldots \\
\text { trace } \\
.51 \\
2.10 \\
7.46 \\
1.88 \\
\cdots \ldots . . \\
\cdots\end{array}$ & $\begin{array}{r}80.61 \\
10.94 \\
\ldots \ldots . . \\
\cdots \ldots . .69 \\
.09 \\
2.90 \\
3.02 \\
2.20\end{array}$ & $\begin{array}{r}83.91 \\
9.54 \\
7 . \ldots . \\
\text { trace } \\
.19 \\
.62 \\
5.06 \\
.69\end{array}$ & $\begin{array}{r}79.21 \\
12.24 \\
\ldots \ldots . . \\
\cdots . .11 \\
.11 \\
.43 \\
2.58 \\
5.26 \\
.66\end{array}$ & $\begin{array}{r}78.77 \\
12.46 \\
\ldots \ldots . . \\
\cdots \cdots \\
.09 \\
.34 \\
2.12 \\
5.84 \\
.70\end{array}$ & $\begin{array}{r}.09 \\
.16 \\
2.21 \\
5.84 \\
.92 \\
\ldots .13\end{array}$ \\
\hline Soluble $\mathrm{SiO}_{2}$. & 100.20 & 100.36 & $\begin{array}{r}100.29 \\
12.72\end{array}$ & $\begin{array}{r}100.02 \\
11.12\end{array}$ & $\begin{array}{r}100.01 \\
1.06\end{array}$ & $\begin{array}{r}100.49 \\
1.27\end{array}$ & $\begin{array}{r}100.32 \\
1.25\end{array}$ & $\begin{array}{r}100.10 \\
\ldots \ldots \ldots\end{array}$ \\
\hline
\end{tabular}




\section{BUFFALO PEAKS.}

Hypersthene andesite, described by Cross in Bull. 1. Contains hypersthene, augite, plagioclase, magnetite, and apatite. Sp. gr., 2.742, $16^{\circ}$. Analyses made by W. F. Hillebrand in the Denver laboratory.

A. Hypersthene andesite. Andose. P. R. C. 86 and 588.

B, C, D. Hypersthene separated from the rock. Sp. gr., of D, $3.307,23^{\circ}$. In B and $\mathrm{C}$ alkalies were not tested for. In $\mathrm{C}$ and $\mathrm{D}$ all the iron is given as $\mathrm{FeO}$.

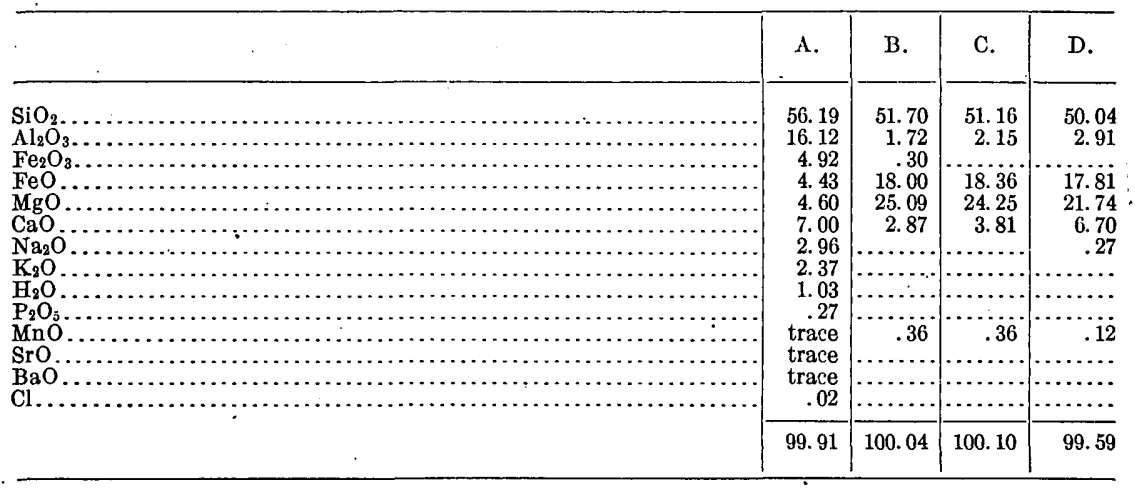

\section{LEADVILLE REGION.}

Rocks described by Cross in Mon. XII, Appendix A. Analyses made in the Denver laboratory.

A. Porphyry, Mount Zion. Toscanose. Contains orthoclase, plagioclase, quartz, biotite, apatite, magnetite, and zircon. Analysis by L. G. Eakins. P. R. C. 504.

B. White or Leadville porphyry. Riesenose. Contains orthoclase, plagioclase, quartz, muscovite, magnetite, apatite, and zircon, with crystals which appear to be rutile and anatase. Sp. gr., 2.680, $16^{\circ}$. Analysis by W. F. Hillebrand. P. R. C. 587.

C. Porphyry, summit of Mount Lincoln. Lassenose. Contains quartz, orthoclase, plagioclase, biotite, apatite, sphene, magnetite, zircon, and allanite. The sample analyzed showed some muscovite, chlorite, and calcite. Sp. gr., 2.670, $16^{\circ}$. Analysis by W. F. Hillebrand. P. R. C. 505.

\begin{tabular}{|c|c|c|c|}
\hline & A. & B. & C. \\
\hline 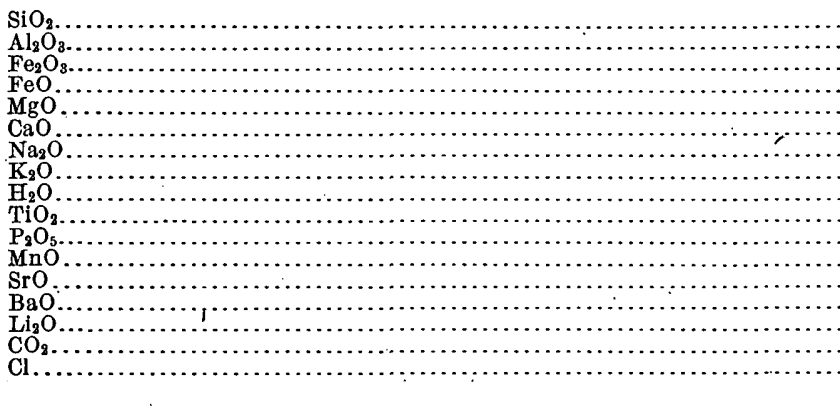 & $\begin{array}{r}73.50 \\
14.87 \\
.95 \\
.42 \\
.29 \\
2.14 \\
3.46 \\
3.56 \\
.90 \\
\quad \text { none } \\
.03 \\
\text { trace } \\
\cdots \cdots \\
\cdots\end{array}$ & \begin{tabular}{r}
70.74 \\
14.68 \\
.69 \\
.58 \\
.28 \\
4.12 \\
2.29 \\
2.59 \\
2.09 \\
$\cdots$ \\
\hdashline .06 \\
trace \\
.03 \\
2.14 \\
trace \\
100.29 \\
\end{tabular} & $\begin{array}{r}66.45 \\
15.84 \\
2.59 \\
1.43 \\
1.21 \\
2.90 \\
3.92 \\
2.89 \\
.84 \\
.10 \\
.36 \\
.09 \\
.07 \\
\ldots \ldots \\
\text { trace } \\
1.35 \\
.05 \\
100.09\end{array}$ \\
\hline
\end{tabular}

D. Gray porphyry, Johnson Gulch, near Leadville. Yellowstonose. Slightly altered. Contains orthoclase, plagioclase, biotite, and quartz, with decomposition products probably derived from original hornblende. Sp. gr., $2.736,16^{\circ}$. Analysis by Hillebrand. 
E. Pink orthoclase crystals from D. Analysis by Hillebrand.

F. Hornblendic porphyrite, lower Buckskin Gulch. Andose. Contains plagioclase, orthoclase, quartz, hornblende, biotite, magnetite, apatite, and zircon, with a little secondary calcite and chlorite. Sp. gr. $2.768,16^{\circ}$. Analysis by Hillebrand. P. R. C. 89 and 506.

G. Biotite porphyrite, dike in gneiss in the North Mosquito amphitheater. Tonalose. Composition like F, but with no hornblende, much biotite, and some pyrite. Sp. gr., 2.740, $16^{\circ}$. Analysis by Hillebrand. P. R. C. 507.

\begin{tabular}{|c|c|c|c|c|}
\hline$=$ & D. & $\mathrm{E}$. & F. & G. \\
\hline 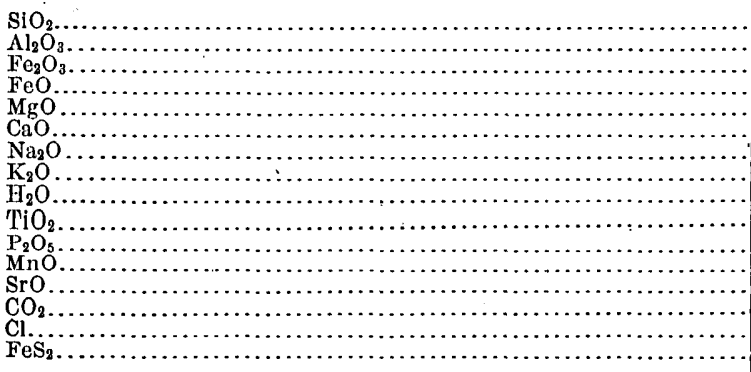 & $\begin{array}{r}68.10 \\
14.97 \\
2.78 \\
1.10 \\
1.10 \\
3.04 \\
3.46 \\
2.93 \\
1.28 \\
.07 \\
.16 \\
.09 \\
.08 \\
.92 \\
.03 \\
\ldots . .\end{array}$ & 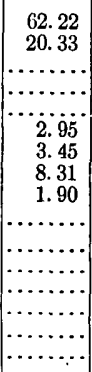 & $\begin{array}{r}56.62 \\
16.74 \\
4.94 \\
3.27 \\
4.08 \\
7.39 \\
3.50 \\
1.97 \\
.92 \\
\ldots \ldots \ldots \\
\text { trace } \\
.15 \\
\text { trace } \\
1.15 \\
\ldots \ldots \ldots \\
\ldots \ldots \ldots\end{array}$ & $\begin{array}{r}64.81 \\
15.73 \\
1.68 \\
2.91 \\
2.82 \\
4.22 \\
3.98 \\
1.43 \\
. .62 \\
.08 \\
.23 \\
.08 \\
\text { trace } \\
1.08 \\
.04 \\
.90\end{array}$ \\
\hline & 100.11 & $99.1 \overline{6}$ & $100.73^{\circ}$ & 100.61 \\
\hline
\end{tabular}

H. Rhyolite (nevadite), from Chalk Mountain. Liparose. Mainly quartz and feldspar, the latter being sanidine and plagioclase. A little biotite, magnetite, apatite, and zircon are present. Analysis by Hillebrand. P. R. C. 64, 512, and 1289.

I. Sanidine from H. Analysis by Hillebrand. P. R. C. 64 .

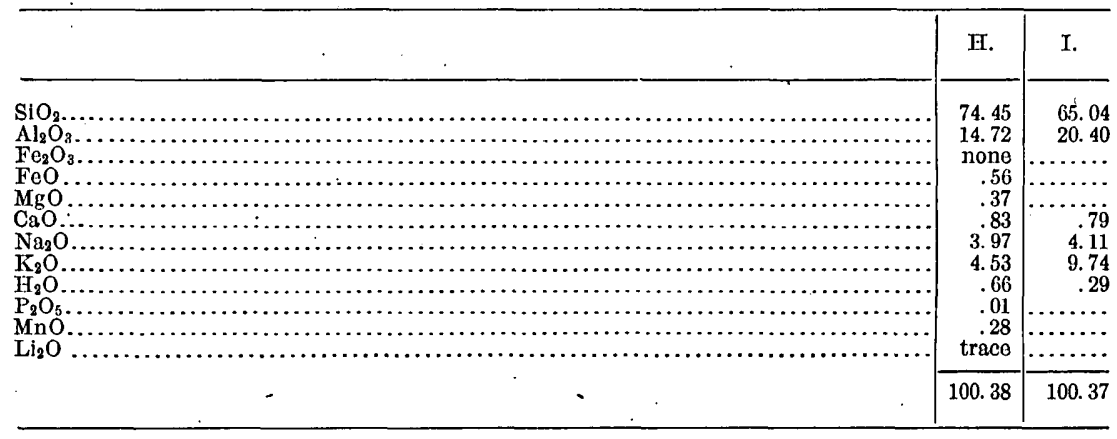

\section{TENMILE DISTRICT.}

Rocks described by Cross in 14th Ann., p. 165. Analyses made in the Denver laboratory-A and C by W. F: Hillebrand, B by L. G. Eakins.

A. Quartz-hornblende-mica porphyrite, Gold Hill. Yellowstonose. Contains plagioclase, hornblende, biotite, and quartz in a groundmass of quartz, orthoclase, and a little plagioclase. P. R. C. 510 .

B. Quartz porphyrite, Sugar Loaf. Toscanose. Contains plagioclase, biotite, and quartz, in a groundmass of quartz and orthoclase. P. R. C. 509.

C. Quartz porphyrite, Chicago Mountain. Lassenose. Contains plagioclase, orthoclase, biotite, and quartz. P. R. C. 508. 


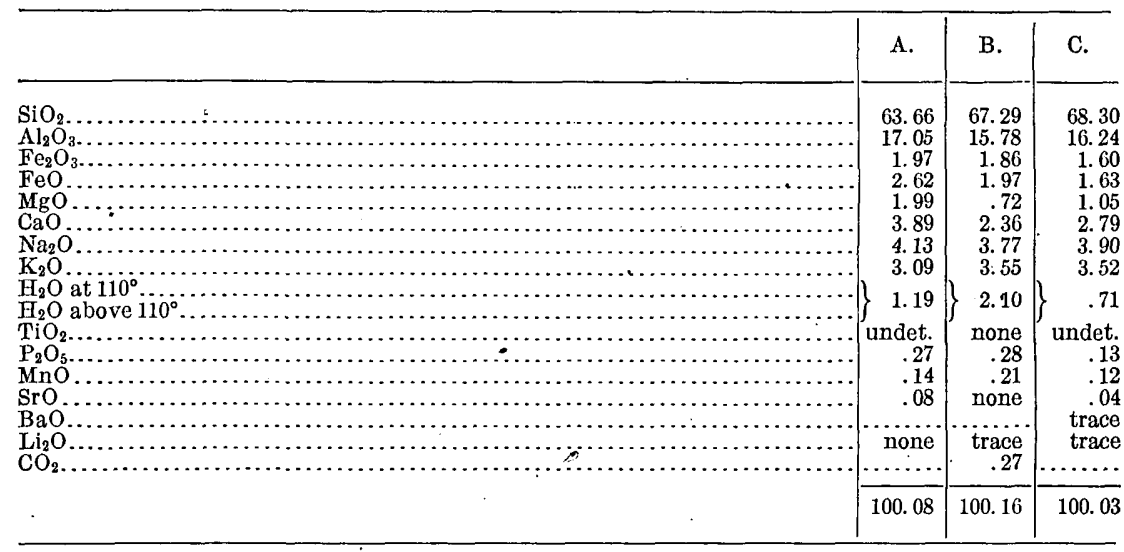

The following, rocks were also analyzed in the Denver laboratory. Petrographic data furnished by Whitman Cross.

A. Granite porphyry, McNulty Gulch. Toscanose. Contains phenocrysts of orthoclase, oligoclase, andesine, quartz, biotite, and altered hornblende, in a groundmass of quartz, orthoclase, and magnetite. Accessory sphene, allanite, apatite, and zircon, and a little secondary chlorite are also present. Analysis by W. F. Hillebrand. P. R. C. 586.

B. Granite porphyry, Jefferson tunnel. Toscañose. Contains orthoclase, oligoclase, quartz, and biotite, in a groundmass of mainly quartz and orthoclase; also accessory magnetite, apatite, zircon, and allanite. Chlorite appears as a decomposition product of biotite, and calcite and magnetite are present in small amounts. Analysis by Hillebrand.

C. Same as B. Toscanose. Analysis by L. G. Eakins. P. R. C. 583.

D. Diorite porphyry, Copper Mountain. Yellowstonose. Contains oligọclase, hornblende, and biotite, in a groundmass of quartz, orthoclase, plagioclase, and magnetite; also accessory zircon, sphene, and apatite, and a little secondary chlorite and epidote. Analysis by Eakins. P. R. C. 585.

E. Diorite porphyry, McNulty type. Lassenose. Contains oligoclase, andesine, hornblende, biotite, and magnetite, in a groundmass of orthoclase, plagioclase, quartz; magnetite, apatite, allanite, and sphene; also secondary chlorite, epidote, and calcite. Analysis by Eakins. P. R. C. 584 .

\begin{tabular}{|c|c|c|c|c|c|}
\hline . & A. & B. & C. & D. & E. \\
\hline 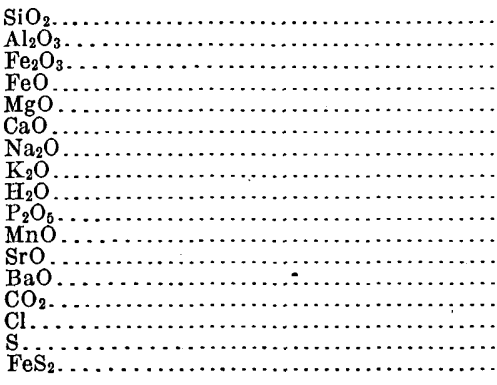 & 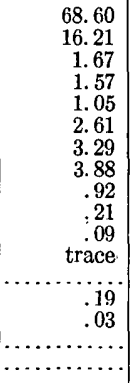 & $\begin{array}{r}65.94 \\
16.00 \\
.60 \\
1.74 \\
1.02 \\
2.87 \\
3.85 \\
4.56 \\
1.13 \\
.23 \\
.14 \\
\operatorname{trace} \\
\ldots \ldots .55 \\
. .03 \\
. .60 \\
.60\end{array}$ & 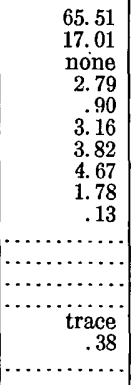 & $\begin{array}{r}67.01 \\
18.03 \\
.66 \\
.72 \\
.84 \\
3.99 \\
4.42 \\
3.53 \\
.91 \\
.10 \\
.09 \\
\ldots . . . \\
.10 \\
\ldots \ldots . . \\
\ldots \ldots . . \\
\ldots \ldots . . \\
\ldots \ldots . . \\
\ldots\end{array}$ & 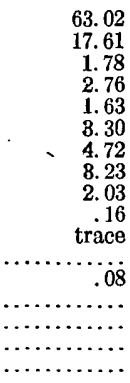 \\
\hline Sp. gr $\ldots . . .$. & $\begin{array}{r}100.32 \\
2.640,27^{\circ}\end{array}$ & $\begin{array}{r}100.26 \\
2.672,21^{\circ}\end{array}$ & $\begin{array}{r}100.15 \\
2.666,26^{\circ}\end{array}$ & $\begin{array}{c}100.40 \\
\ldots . \ldots\end{array}$ & $\begin{array}{r}100.32 \\
2.689,16.5^{\circ}\end{array}$ \\
\hline
\end{tabular}




\section{ELK MOUNTAINS.}

Analyses made by L. G. Eakins in the Denver laboratory. Petrographic data supplied by Whitman Cross.

A. Rhyolite, East Mountain, Crested Butte district. Alsbachose. Phenocrysts of orthoclase, oligoclase, quartz, and biotite, in a microspherulitic and cryptocrystalline groundmass. P. R. C. 514.

B. Rhyolite, Round Mountain, Crested Butte district. Toscanose. Small phenocrysts of quartz. sanidine, biotite, and oligoclase, in a groundmass of quartz and orthoclase. P. R. C. 513.

C. Diorite, Brush Creek, Gunnison County. Tonalose. Contains several varieties of plagioclase, with orthoclase, quartz, hornblende, biotite, augite, sphene, apatite, and magnetite. P. R. C. 93.

\begin{tabular}{|c|c|c|c|}
\hline & . A. & 13. & C. \\
\hline 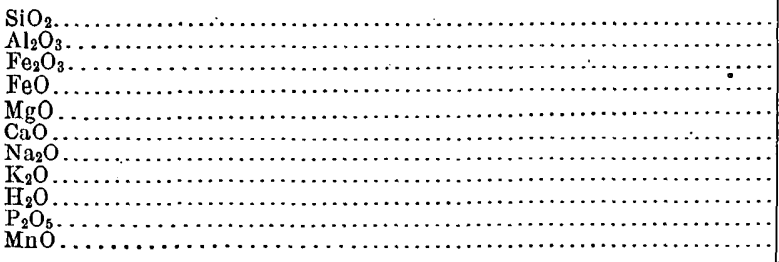 & $\begin{array}{r}74.84 \\
14.05 \\
.17 \\
.31 \\
\text { trace } \\
1.57 \\
3.66 \\
3.14 \\
2.33 \\
\cdots\end{array}$ & $\begin{array}{r}71.56 \\
14.91 \\
1.47 \\
1.04 \\
.08 \\
1.98 \\
3.78 \\
4.94 \\
.44 \\
\text { trace } \\
\cdots\end{array}$ & $\begin{array}{r}62.71 \\
17.06 \\
3.79 \\
2.74 \\
1.78 \\
5.51 \\
3.54 \\
2.96 \\
.24 \\
\text { none } \\
\text { trace }\end{array}$ \\
\hline Sp. gr. . & $\begin{array}{r}100.07 \\
2.38,17^{\circ}\end{array}$ & $\begin{array}{r}100.20 \\
2.59,18^{\circ}\end{array}$ & $\begin{array}{r}100.33 \\
2.791,30^{\circ}\end{array}$ \\
\hline
\end{tabular}

8. WEST ELK MOUNTAINS.

Rocks described by Cross in 14th Ann., p. 165.

A. Hornblende-mica porphyrite, Cliff Creek. Adamellose. Contains plagioclase, hornblende, and biotite, in a groundmass of quartz, feldspar, and mica. Analysis by W. F. Hillebrand, record No. 1429.

B. Porphyrite, Storm Ridge. Yellowstonose. Contains plagioclase, biotite, hypersthene, hornblende, and augite, in a groundmass of quartz and orthoclase. Analysis by L. G. Eakins, record No. 1238 . P. R. C. 517.

C. Porphyrite-diorite, Mount Marcellina. Yellowstonose. Contains plagioclase, hornblende, and a little biotite, in a groundmass of quartzand orthoclase. Analysis by T. M. Chatard, record No. 1238. P. R. C. 516.

D. Quartz porphyrite, Mount Carbon. Amiatose. Contains plagioclase, orthoclase, biotite, hornblende, augite, and quartz. Analysis by Chatard, record No. 1238. P. R. C. 518 .

E. Quartz porphyrite, Crested Butte. Lassenose. Contains plagioclase, orthoclase, hornblende, biotite, quartz, and a little augite. Analysis made by Eakins in the Denver laboratory. P. R. C. 515 .

\begin{tabular}{|c|c|c|c|c|c|}
\hline & A. & B. & C. & $\mathrm{D}$. & E. \\
\hline \multirow[t]{2}{*}{ 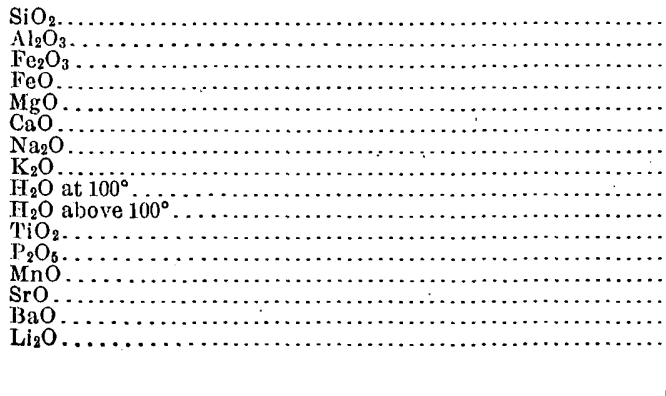 } & $\begin{array}{r}63.05 \\
15.58 \\
2.92 \\
2.11 \\
1.70 \\
4.15 \\
3.77 \\
3.66 \\
.55 \\
1.38 \\
.60 \\
.27 \\
.12 \\
.07 \\
.13 \\
\text { trace }\end{array}$ & $\begin{array}{r}61.42 \\
17.69 \\
4.24 \\
1.74 \\
1.81 \\
5.29 \\
3.14 \\
3.19 \\
.97 \\
.37 \\
.14 \\
.19 \\
\ldots . . . \\
.09 \\
\ldots . . .\end{array}$ & $\begin{array}{r}62.85 \\
16.21 \\
3.08 \\
1.46 \\
1.47 \\
4.72 \\
3.49 \\
3.10 \\
.29 \\
2.03 \\
.41 \\
.48 \\
.15 \\
\ldots .17 \\
.11\end{array}$ & $\begin{array}{r}65.36 \\
15.48 \\
3.09 \\
1.21 \\
1.53 \\
4.14 \\
3.58 \\
3.41 \\
.82 \\
.70 \\
.52 \\
.25 \\
.19 \\
\ldots . . . \\
.08 \\
\ldots \ldots\end{array}$ & $\begin{array}{r}65.71 \\
18.30 \\
1.19 \\
1.53 \\
.98 \\
2.17 \\
5.00 \\
3.95 \\
1.39 \\
\text { undet. } \\
\ldots \ldots .02 \\
\ldots \ldots . . \\
\ldots \ldots . . .\end{array}$ \\
\hline & 100.06 & 100.28 & 99.85 & 100. 36 & 100.24 \\
\hline
\end{tabular}




\section{LA PLATA MOUNTAINS.}

Rocks described by Whitman Cross in Folio 60.

A. Monzonitic facies of diorite mass. Akerose. Contains augite, hornblende, plagioclase, and orthoclase in large amount, with biotite, quartz, sphene, apatite, and magnetite as subordinate constituents. Also secondary chlorite, muscovite, and calcite. Analysis by W. F. Hillebrand, record No. 1640. Sp. gr., 2.79, $21^{\circ}$. Described by Cross in 21st Ann., pt. 2. P. R. C. 1284.

B. Diorite porphyry, Deadwood Gulch. Akerose. Contains phenocrysts of hornblende, plagioclase, occasional quartz, sphene, apatite, and magnetite in a groundmass of orthoclase, plagioclase, and quartz. Also secondary epidote, chlorite, and calcite. Analysis by Hillebrand, record No. 1636. Sp. gr:, 2.677, 24․ A trace of sulphur is present. Described by Cross in 21st Ann., pt. 2. P. R. C. 1283.

C. Augite syenite, between Tirbircio and Schurman gulches. Monzonose. Contains much alkali feldspar, some oligoclase, augite, biotite, and hornblende, with a little titanite, magnetite, and apatite. Analysis by H. N. Stokes, record No. 1764. Sp. gr., 2.704, 25․ P. R. C. 1286.

D. Augitic monzonite, Babcock Peak. Andose. Contains orthoclase and plagioclase in about equal amounts, with augite and hornblende, and a little quartz, titanite, magnetite, and apatite. Analysis by Stokes, record No. 1764. Sp. gr., 2.767, $26^{\circ}$. Described by Cross in 21st Ann., pt. 2. P. R. C. 1285.

E. Porphyritic lamprophyre, allied to camptonite, Snowstorm Peak. Kentallenose. Contains numerous phenocrysts of green hornblende, augite, and plagioclase, in a groundmass of plagioclase, orthoclase, augite, magnetite, and apatite. Some secondary calcite. Analysis by Hillebrand, record No. 1640. Sp. gr., 2.906, $21^{\circ}$. P. R. C. 1287.

F. Basic dike rock, Indian Trail Ridge, La Plata quadrangle. Camptonose. Contains phenocrysts of green hornblende and colorless diopside in a subordinate groundmass of plagioclase, orthoclase (?), augite, magnetite, and apatite. Much secondary calcite and some serpentine. Analysis by W. F. Hillebrand, record No. 1640. Sp. gr., 2.912, $19.5^{\circ}$. P. R. C. 1288.

\begin{tabular}{|c|c|c|c|c|c|c|}
\hline . & A. & B. & C. & D. & E. & F. \\
\hline 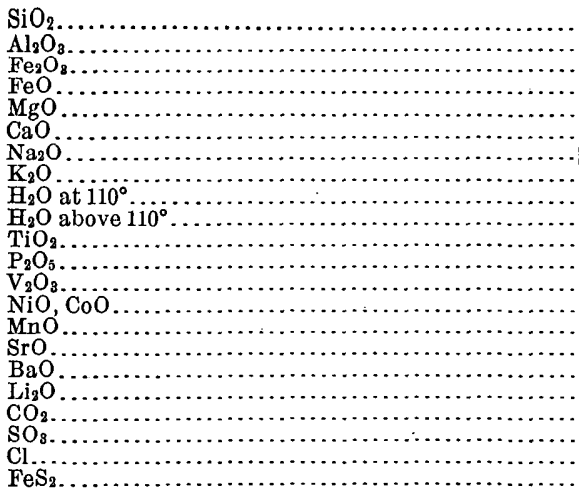 & $\begin{array}{r}55.53 \\
16.78 \\
4.06 \\
3.35 \\
3.00 \\
6.96 \\
4.31 \\
3.57 \\
.09 \\
.55 \\
.95 \\
.47 \\
.02 \\
\operatorname{trace} \\
.16 \\
.11 \\
.13 \\
\operatorname{trace} \\
.09 \\
\cdots \cdots \\
. .2 \\
.04\end{array}$ & $\begin{array}{r}60.44 \\
16.65 \\
2.31 \\
3.09 \\
2.18 \\
4.22 \\
5.18 \\
2.71 \\
.36 \\
1.07 \\
.60 \\
.29 \\
.02 \\
\text { none } \\
.13 \\
.11 \\
.12 \\
\text { trace } \\
.48 \\
\cdots \cdots . . . \\
\cdots \cdots . . .\end{array}$ & $\begin{array}{r}59.79 \\
17.25 \\
3.60 \\
1.59 \\
1.24 \\
3.77 \\
5.04 \\
5.05 \\
.19 \\
.39 \\
.67 \\
.35 \\
\ldots \ldots . . \\
\ldots .20 \\
.11 \\
.14 \\
\text { trace } \\
.72 \\
.04 \\
\text { trace } \\
\ldots \ldots . . .\end{array}$ & \begin{tabular}{r}
57.42 \\
18.48 \\
3.74 \\
2.10 \\
1.71 \\
6.84 \\
4.52 \\
3.71 \\
.08 \\
.28 \\
.86 \\
.36 \\
$\ldots \ldots .$. \\
\hdashline$\ldots .$. \\
.09 \\
.08 \\
.15 \\
trace \\
none \\
none \\
.03
\end{tabular} & $\begin{array}{r}47.25 \\
15.14 \\
5.05 \\
4.95 \\
6.87 \\
9.98 \\
2.39 \\
2.60 \\
.40 \\
2.12 \\
1.22 \\
.25 \\
.05 \\
.02 \\
.17 \\
.05 \\
.08 \\
\text { trace } \\
1.87 \\
\ldots \ldots . .\end{array}$ & $\begin{array}{r}43.98 \\
13.30 \\
3.67 \\
6.92 \\
7.03 \\
10.66 \\
2.15 \\
1.64 \\
.42 \\
1.52 \\
1.18 \\
.32 \\
\ldots . .63 \\
.03 \\
.22 \\
.05 \\
.06 \\
\text { trace } \\
6.46 \\
\ldots . . . \\
\ldots . .\end{array}$ \\
\hline & 100.17 & 99.96 . & 100.14 & 100.45 & 100.46 & 100.15 \\
\hline
\end{tabular}


10. SILVERTON QUंADRANGLE.

Rocks A, B, C, D described by Whitman Cross in Folio 120. Analyses by W. F. Hillebrand, record Nos. 2024, 2042, 2045.

A. Quartz latite, ridge north of Pole Creek. Amiatose near yellowstonose. Contains phenocrysts of andesine, hornblende, and a little biotite, in a predominant groundmass of orthoclase, plagioclase, quartz, and a little augite, biotite, and magnetite. P. R. C. 1353 .

B. Quartz latite, bench south of Greenhalgh Mountain. Toscanose. Contains phenocrysts of oligoclase-andesine and biotite in a groundmass of orthoclase, quartz, and rare accessories. P. R. C. 1354.

C. Pyroxene andesite, ridge west from Edith Mountain. Tonalose. Contains phenocrysts of labradorite, augite, hypersthene, biotite, and magnetite, in a groundmass which is largely glass, with feldspar microlites and ferritic particles. P. R. C. 1355.

D. Pyroxene andesite, Dolly Varden mine, Henson Creek. Harzose. Contains phenocrysts of labradorite, hypersthene, augite, and magnetite, in a groundmass of plagioclase, orthoclase, quartz, augite, magnetite, etc. P. R. C. 1356.

\begin{tabular}{|c|c|c|c|c|}
\hline . & A. & B. & C. & D. \\
\hline 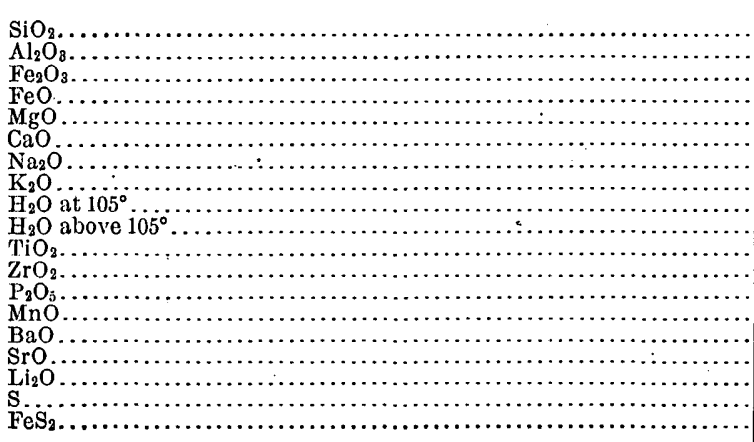 & $\begin{array}{r}62.09 \\
16.77 \\
3.96 \\
.99 \\
1.63 \\
4.26 \\
3.77 \\
3.68 \\
.50 \\
1.32 \\
.73 \\
\text { trace } \\
.25 \\
.14 \\
.10 \\
.05 \\
\text { trace } \\
\text { none } \\
\ldots \ldots\end{array}$ & $\begin{array}{r}64.93 \\
16.79 \\
3.54 \\
.32 \\
.65 \\
2.11 \\
3.33 \\
4.76 \\
1.12 \\
1.65 \\
.53 \\
.03 \\
.17 \\
\text { trace } \\
.15 \\
\text { trace } \\
\text { trace } \\
\text { trace? } \\
\text {........ }\end{array}$ & $\begin{array}{r}58.88 \\
15.93 \\
3.12 \\
2.94 \\
2.30 \\
6.05 \\
3.17 \\
1.86 \\
1.66 \\
2.48 \\
.73 \\
.02 \\
.34 \\
.16 \\
.12 \\
.14 \\
\text { trace } \\
\ldots \ldots . . \\
.07\end{array}$ & $\begin{array}{r}56.03 \\
15.97 \\
4.78 \\
3.00 \\
3.36 \\
6.44 \\
2.85 \\
3.29 \\
1.31 \\
1.08 \\
1.01 \\
\text { trace? } \\
.48 \\
.16 \\
.08 \\
.04 \\
\ldots . . . \\
\text { none }\end{array}$ \\
\hline & 100.24 & 100.08 & 99.97 & 99.88 \\
\hline
\end{tabular}

$\mathrm{CO}_{2}$ absent. $\mathrm{Cl}, \mathrm{F}$, not looked for.

Rocks $\mathrm{E}$ to I described by F. L. Ransome in Bull. 182.

E. Altered andesitic breccia, White Cloud mine. Contains quartz, kaolin, pyrite, rutile, and some undeterminable minerals. Analysis by George Steiger, record No. 1886.

F. Latite, country rock, Polar Star mine, Engineer Mountain. SR. 3 of vaalase. Contains labradorite, orthoclase (?), quartz, diopside, chlorite, calcite, serpentine, kaolin, magnetite, hematite, apatite, rutile, and leucoxene. Mainly feldspar and quartz.

G. Altered latite, same locality as $\mathrm{P}$, wall rock of vein. Contains mainly quartz and kaolin, with diaspore, pyrite, sericite, rutile, and apatite.

H. Monzonitic porphyry, near Yankee Girl mine. Adamellose. Contains albite, quartz, orthoclase, chlorite, calcite, sericite, iron ores, pyrite, and apatite.

I. Altered monzonitic. porphyry, near National Belle mine. Contains quartz, kaolin, pyrite, diaspore, sericite, apatite, and rutile.

Analyses F to I by H. N. Stokes, record No. 1888. 


\begin{tabular}{|c|c|c|c|c|c|}
\hline . & E. & F. & G. & H. & I. \\
\hline 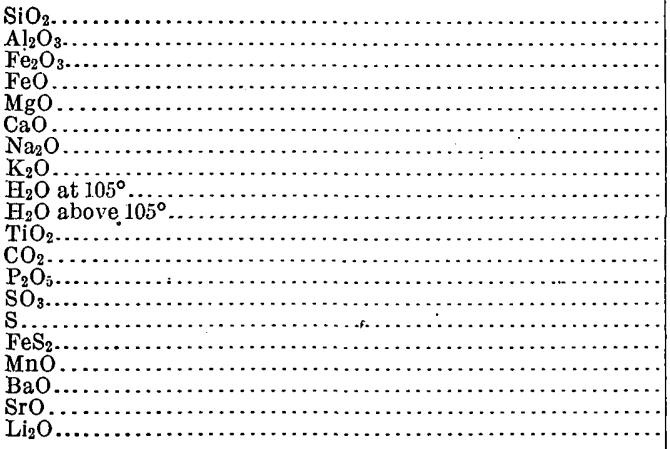 & $\begin{array}{r}85.49 \\
5.49 \\
.23 \\
.25 \\
\text { none } \\
.27 \\
\text { none } \\
\text { none } \\
.46 \\
3.07 \\
.63 \\
\text { none } \\
.07 \\
.46\end{array}$ & $\begin{array}{r}55.61 \\
16.40 \\
5.44 \\
2.37 \\
3.25 \\
5.85 \\
2.61 \\
3.77 \\
.46 \\
1.51 \\
1.10 \\
1.33 \\
.45 \\
\text { trace } \\
\ldots \ldots \\
.09 \\
.03 \\
.05 \\
\text { trace }\end{array}$ & $\begin{array}{r}64.79 \\
18.93 \\
\text { none } \\
\text { none } \\
\text { none } \\
.43 \\
.15 \\
.24 \\
.50 \\
5.39 \\
1.21 \\
\text { none } \\
.51 \\
\ldots \ldots . . \\
\ldots 7.19 \\
7 . . \\
\text { none } \\
.06 \\
\text { trace } \\
\text { trace }\end{array}$ & $\begin{array}{r}58.78 \\
13.52 \\
1.70 \\
2.27 \\
3.29 \\
4.31 \\
3.24 \\
4.06 \\
.25 \\
1.57 \\
.99 \\
3.49 \\
.53\end{array}$ & $\begin{array}{r}73.61 \\
13.97 \\
\text { none } \\
\text { none } \\
\text { none } \\
.31 \\
.04 \\
.06 \\
.58 \\
4.18 \\
.60 \\
\text { none } \\
.33 \\
\ldots \ldots \\
\ldots . . . \\
5.62 \\
\text { trace } \\
.04 \\
\text { trace } \\
\text { trace }\end{array}$ \\
\hline & 99.92 & 100.32 & 99.40 & 99.73 & 99.34 \\
\hline
\end{tabular}

11. OURAY QUADRANGLE.

Rocks described by Ernest Howe in Folio 153. Analyses by G. Steiger, record No. 2200.

A. Quartz-biotite latite, near head of middle fork of Cimarron Creek. Contains. plagioclase, a little orthoclase, biotite, augite, accessory magnetite, and very little doubtful quartz.

B. Quartz-biotite latite, north end of Cow Creek intrusive body, between Wildhorse Creek and the west fork of Cow Creek. Contains plagioclase, very little orthoclase, quartz; biotite, and magnetite.

C. Quartz-pyroxene latite, west side of ridge between the Middle and East Cimarron creeks. Contains andesine, hornblende, pyroxene, biotite, and magnetite.

D. Quartz monzonite porphyry, Porphyry Basin, Middle Cimarron Creek. Contains plagioclase, orthoclase, biotite, hornblende, and magnetite.

\begin{tabular}{|c|c|c|c|c|}
\hline & A. & B. & C. & D. \\
\hline 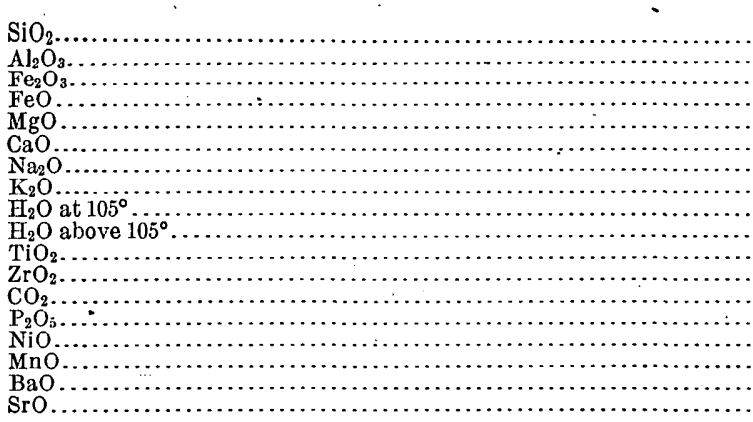 & $\begin{array}{r}59.83 \\
15.86 \\
4.07 \\
2.12 \\
2.73 \\
4.34 \\
3.00 \\
3.55 \\
1.09 \\
2.04 \\
.70 \\
\text { none } \\
.59 \\
.31 \\
\text { none } \\
.06 \\
.08 \\
.02\end{array}$ & $\begin{array}{r}68.81 \\
15.54 \\
1.78 \\
.80 \\
.52 . \\
2.43 \\
4.24 \\
4.07 \\
.50 \\
.78 \\
.28 \\
\operatorname{trace} \\
.48 \\
.13 \\
(?) \\
.12 \\
.13 \\
.04\end{array}$ & $\begin{array}{r}60.69 \\
15.90 \\
4.52 \\
1.72 \\
1.93 \\
5.23 \\
3.55 \\
3.22 \\
.93 \\
.96 \\
.73 \\
\text { trace } \\
.27 \\
.31 \\
\text { none } \\
.13 \\
.10 \\
.03\end{array}$ & $\begin{array}{r}61.36 \\
16.36 \\
3.59 \\
1.45 \\
1.75 \\
3.59 \\
4.04 \\
3.64 \\
1.34 \\
1.56 \\
.51 \\
\text { trace } \\
.64 \\
.36 \\
\text { none } \\
.07 \\
.12 \\
.12\end{array}$ \\
\hline . n & 100.39 & 100.65 & 100.22 & 100.50 \\
\hline
\end{tabular}

$\mathrm{S}$ and $\mathrm{SO}_{3}$ absent. 
12. TELLURIDE QUADRANGLE.

Rocks collected by Whitman Cross. All except A described in Folio 57.

A. Lamprophyre, allied to camptonite, Black Face. Andose. Consists of a fine felt of plagioclase, augite, and brown hornblende microlites, with flakes of biotite, and a cryptocrystalline part, which is probably in large degree orthoclase: Some magnetite and apatite. Analysis by Hillebrand, record No. 1719. Sp. gr., 2.783, 22 ${ }^{\circ}$ P. R. C. 1282 .

B. Quartz monzonite, northeast of San Miguel Peak. Toscanose.' Contains orthoclase and plagioclase in about equal amounts, with abundant quartz and much less augite, hornblende, biotite, magnetite, and apatite. Analysis by H. N. Stokes, record No. 1764. Sp. gr., $2.720,34^{\circ}$. Described by Cross in 21 st Ann., pt. 2. . P. R. C. 1279.

C. Diorite monzonite, Ophir Needles. Tonalose. Contains abundant labradorite, with augite, hypersthene, biotite, orthoclase, magnetite, apatite, and a very little quartz. Analysis by Stokes, record No. 1764. Sp. gr., 2.860, $33^{\circ}$.

D. Gabbro porphyry, pass south of Mount Sneffels. Andose. Contains numerous phenocrysts of labradorite or bytownite, in a groundmass of plagioclase, orthoclase (?), augite, hypersthene, biotite, magnetite, and apatite. Analysis by Stokes, record No. 1764. Sp. gr., $2.949,26.5^{\circ}$. P. R. C. 1280.

E. Vitrophyre, ridge east of Windy Gap. Yellowstonose-lassenose. P. R. C. 1281. 'Analysis by H. N. Stokes.

F. Gabbro, Stony Mountain, Ouray County. Hessose. Analyzed by Eakins in the Denver laboratory. Sp. gr., 2.891, 13.5'. P. R. C. 199.

\begin{tabular}{|c|c|c|c|c|c|c|}
\hline & A.' & B. & C. & D. & E. & F. \\
\hline 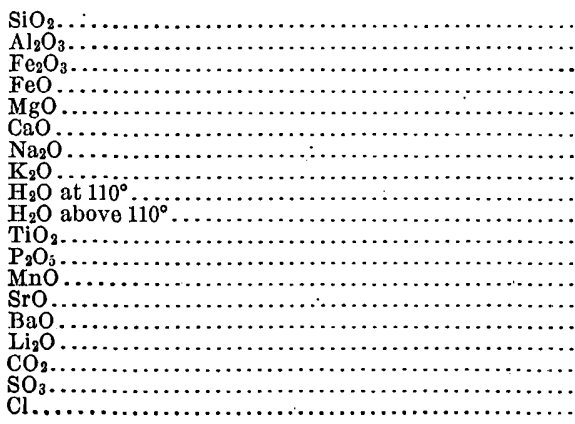 & $\begin{array}{r}55.65 \\
17.04 \\
2.81 \\
5.17 \\
3.42 \\
6.82 \\
3.27 \\
2.29 \\
.46 \\
1.49 \\
.90 \\
.37 \\
.20 \\
.05 \\
.08 \\
\text { trace } \\
\ldots \ldots . . \\
\text { none } \\
\ldots \ldots\end{array}$ & $\begin{array}{r}65.70 \\
15.31 \\
2.54 \\
1.62 \\
1.62 \\
2.56 \\
3.62 \\
4.62 \\
.17 \\
.42 \\
.72 \\
.33 \\
\text { trace } \\
.03 \\
.12 \\
\text { trace } \\
\text { none } \\
.12 \\
.03\end{array}$ & $\begin{array}{r}56.93 \\
17.03 \\
3.67 \\
4.54 \\
3.30 \\
6.51 \\
3.19 \\
2.58 \\
.13 \\
.45 \\
1.03 \\
.44 \\
.10 \\
.06 \\
.08 \\
\text { none } \\
\text { none } \\
\text { none } \\
\text { trace }\end{array}$ & $\begin{array}{r}47.32 \\
16.71 \\
6.92 \\
5.94 \\
5.69 \\
8.51 \\
2.70 \\
2.02 \\
.24 \\
1.04 \\
1.50 \\
.96 \\
.08 \\
.06 \\
.07 \\
\text { trace } \\
\text { none } \\
.19 \\
\text { trace }\end{array}$ & $\begin{array}{r}64.72 \\
14.18 \\
1.58 \\
.40 \\
.50 \\
2.62 \\
3.88 \\
1.82 \\
2.68 \\
6.82 \\
.43 \\
.08 \\
\text { trace } \\
.21 \\
.28 \\
\text { nono } \\
\ldots \ldots . . . \\
\ldots \ldots . . . \\
\ldots \ldots . .\end{array}$ & 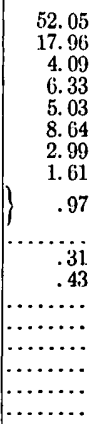 \\
\hline & 100.02 & 99.53 & 100.04 & 99.95 & 100.20 & 100.41 \\
\hline
\end{tabular}

13. MISCELLANEOUS ROCKS.

A. Diabase, east of the Sugar Loaf, Boulder County. Hessose. Description furnished by Whitman Cross. The rock contains labradorite, orthoclase, augite, and magnetite, with small amounts of hornblende, biotite, apatite, and secondary chlorite. Sp. gr., 3.027, $21^{\circ}$. P. R. C. 166.

B. Pyroxene separated from A. Analyses A, B, made by L. G. Eakins in the Denver laboratory.

C. Alkali syenite porphyry, Idaho Springs. Pulaskose. Described by S. H. Ball, in P. P. 63. Contains anorthoclase, orthoclase, ægirine-augite, garnet, titanite, biotite, apatite, magnetite, and often zircon. Analysis by G. Steiger, record No. 2258.

D. Porphyry, Idaho Springs.

E. Biotite latite, Idaho Springs. Rocks D, E, received from J. E. Spurr, but without description. Analyses by W. T. Schaller, récord No. 2263. 
F. Granite, Platte Canyon. Alaskose. Described by E. B. Mathews in Bull. 150, p. 172. Contains microcline, quartz, biotite, oligoclase, and fluorite. Apatite, zircon, magnetite, hematite, limonite, epidote, and rutile (?) are sometimes present. Analysis by $\mathrm{H}$. N. Stokes, record No. 1314.

\begin{tabular}{|c|c|c|c|c|c|c|}
\hline & A. & B. & C. & D. & E. & F. \\
\hline \multirow{16}{*}{$\begin{array}{l}\mathrm{SlO}_{2} \mathrm{Al}_{2} \mathrm{O}_{2} \\
\mathrm{Fe}_{2} \mathrm{O}_{3} \ldots \ldots \ldots \ldots\end{array}$} & & & & & & \\
\hline & 20.99 & & $\begin{array}{l}18.12 \\
18.0\end{array}$ & $\begin{array}{l}0.41 \\
16.23\end{array}$ & $\begin{array}{l}\begin{array}{l}66.444 \\
14.98\end{array}\end{array}$ & \\
\hline & 2. 02 & 2.56 & 2.45 & .85 & 1.57 & \\
\hline & $\begin{array}{l}9.36 \\
4.39\end{array}$ & 14. 40 & $\begin{array}{r}1.25 \\
.28\end{array}$ & $\begin{array}{r}1.14 \\
\times 15\end{array}$ & $\begin{array}{r}.43 \\
.18\end{array}$ & $\begin{array}{r}1.09 \\
.14\end{array}$ \\
\hline & $\begin{array}{l}4.39 \\
8.03\end{array}$ & $\begin{array}{l}13.43 \\
16.0 .0\end{array}$ & $\begin{array}{r}.28 \\
3.89\end{array}$ & $\begin{array}{r}.15 \\
14\end{array}$ & $\begin{array}{r}.18 \\
2.47\end{array}$ & \\
\hline & $\begin{array}{l}\text { 8.03 } \\
\text { 3. }\end{array}$ & & & $\begin{array}{r}.14 \\
3.95\end{array}$ & $\begin{array}{l}\text {. } \\
1.12\end{array}$ & \\
\hline & 1.80 & & 5. 01 & 7.19 & 3. 32 & \\
\hline & 1.18 & & .75 & .67 & $\begin{array}{l}\text { 4. } 60 \\
4.06\end{array}$ & .35 \\
\hline & & $\cdots$ & .55 & .88 & $\begin{array}{r}4.06 \\
.20\end{array}$ & \\
\hline & & & & .11 & & \\
\hline & .15 & & none & $\begin{array}{l}.56 \\
.05\end{array}$ & .67 & \\
\hline & trace & & 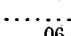 & trace & & \\
\hline & & & & none & trace & \\
\hline & .31 & & .12 & & & \\
\hline & & & & $\begin{array}{l}\text { trace } \\
\text { none }\end{array}$ & none & \\
\hline & 100.22 & 100.16 & 99.84 & 99. 65 & 100.42 & 99.8 \\
\hline
\end{tabular}

G. Rhyolite, east bank of Arkansas River, Nathrop. Liparose. Described by Cross in Proc. Colorado Sci. Soc., vol. 2, p. 69. Contains quartz and sanidine in a groundmass mainly of quartz and alkali feldspar. This rock carries topaz and spessartite in its lithophysæ. Analysis made by L. G. Eakins in the Denver laboratory. Sp. gr., 2,602, $29^{\circ}$. P. R. C. 598.

H. Hornblende porphyrite, Hermano Peak, Sierra EI Late. Lassenose. Described by Cross in 14th Ann., p..165. Contains plagioclase, hornblende, rare quartz, and a little biotite. Analysis by W. F. Hillebrand, record No. 1429.

I. Hornblende porphyrite, Ute Peak, Sierra El Late. Tonalose. Described by Cross in 14th Ann., p. 165. Contains plagioclase, hornblende, and very little augite, in a groundmass of quartz, orthoclase, and plagioclase. Analysis by Hillebrand, No. 1429.

J. Porphyritic augite diorite, Lone Cone, San Miguel Mountains. Tonalose. Described by Cross in 14th Ann., p. 165. Contains plagioclase, augite, hornblende, and biotite, in a groundmass of quartz, orthoclase, and plagioclase. Inclusions of magnetite and apatite in the augite. Analysis by Hillebrand, No. 1429:

\begin{tabular}{|c|c|c|c|c|}
\hline & G. & H. & I. & J. \\
\hline 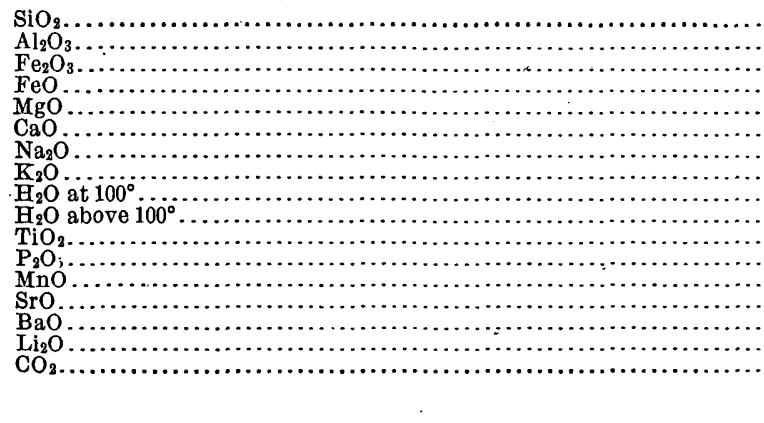 & \begin{tabular}{r}
69.89 \\
17.94 \\
.39 \\
.52 \\
.14 \\
trace \\
4.21 \\
4.38 \\
2.07 \\
\hdashline$\ldots \ldots$ \\
trace \\
.23 \\
trace \\
99.77
\end{tabular} & $\begin{array}{r}62.65 \\
16.68 \\
2.35 \\
2.63 \\
1.43 \\
4.96 \\
4.45 \\
2.75 \\
.27 \\
.66 \\
.42 \\
.28 \\
.16 \\
.11 \\
.13 \\
\text { trace } \\
\ldots \ldots . . \\
99.93\end{array}$ & $\begin{array}{r}59.42 \\
16.79 \\
3.23 \\
3.29 \\
2.24 \\
5.57 \\
4.15 \\
2.82 \\
.27 \\
.79 \\
.68 \\
.35 \\
.13 \\
.07 \\
\text { trace } \\
.44 \\
100.38\end{array}$ & $\begin{array}{r}59.19 \\
18.00 \\
3.07 \\
2.32 \\
1.41 \\
6.55 \\
4.01 \\
2.74 \\
.46 \\
1.06 \\
.58 \\
.29 \\
.19 \\
.13 \\
.18 \\
\text { trace } \\
10 . . . \\
100.18\end{array}$ \\
\hline
\end{tabular}


K. Tinguaite (?), Two Buttes. Laurdalose. Collected by G. K. Gilbert; petrographic data supplied by Whitman Cross. Consists chiefly of pale-green augite, hornblende, apatite, magnetite, and occasional crystals of alkali feldspar, in an obscure, largely isotropic groundmass. Sp. gr., 2.79, 25․ $\quad$ P. R. C. 1393.

L. Pyroxene from F. Sp.gr., $3.43,28^{\circ}$.

M. Portion of $\mathrm{F}$ soluble in 1:40 nitric acid.

N. Syenitic lamprophyre (?), Two Buttes. Prowersose. Collected by Gilbert; described by Cross in Jour. Geol., vol. 14, p. 165. Chief constituents, diopside, alkali feldspar, considerable biotite, magnetite, and olivine. The ferromagnesian minerals predominate. Sp. gr., 2.88, 29 $9^{\circ}$. P. R. C. 1394.

O. Pyroxene from I. Sp. gr., $3.45,25^{\circ}$.

P. Portion of I soluble in 1:40 nitric acid.

Analyses F to K by W. F. Hillebrand, record No. 1604.

\begin{tabular}{|c|c|c|c|c|c|c|}
\hline & K. & L. & M. & N. & 0. & P. \\
\hline 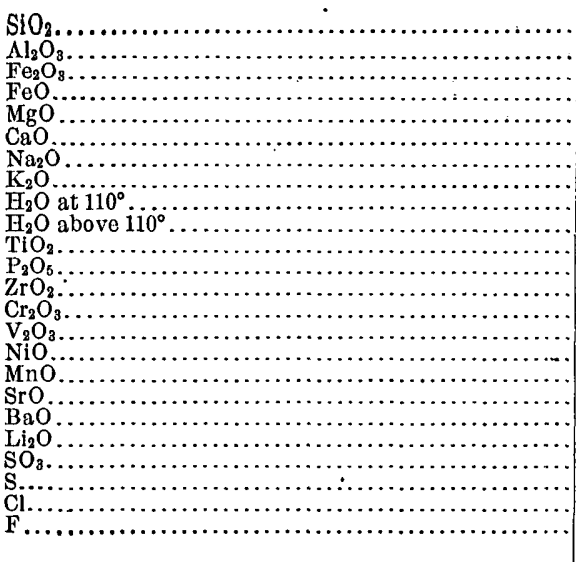 & $\begin{array}{r}47.61 \\
14.26 \\
4.90 \\
4.07 \\
2.62 \\
8.71 \\
6.70 \\
4.08 \\
.26 \\
1.89 \\
1.38 \\
1.38 \\
.18 \\
\text { trace? }\end{array}$ & $\begin{array}{r}47.54 \\
4.14 \\
5.64 \\
6.42 \\
10.05 \\
21.57 \\
1.38 \\
.12 \\
\text { none } \\
\text { undet. } \\
3.00 \\
\ldots \ldots \ldots \\
\text { none } \\
\text { trace? } \\
\ldots \ldots \ldots \\
\text { trace } \\
.36 \\
\text { none } \\
\text { none } \\
\text { trace } \\
\ldots \ldots \ldots . \\
\ldots \ldots \ldots . \\
\ldots \ldots . .\end{array}$ & $\begin{array}{r}13.27 \\
9.40 \\
\ldots \ldots \ldots \\
\text { trace } \\
\text { trace } \\
1.70 \\
5.41 \\
\text { undet. } \\
\text { undet. } \\
\cdots \\
\ldots \ldots .34 \\
\ldots \ldots \ldots\end{array}$ & $\begin{array}{r}50.41 \\
12.27 \\
5.71 \\
3.06 \\
8.69 \\
7.08 \\
.97 \\
7.53 \\
.46 \\
1.80 \\
1.47 \\
.46 \\
\text { none } \\
\text { trace } \\
.03 \\
.04 \\
.15 \\
.06 \\
.23 \\
\text { trace } \\
\text { none } \\
\text { none } \\
\text { trace } \\
\text { trace? }\end{array}$ & 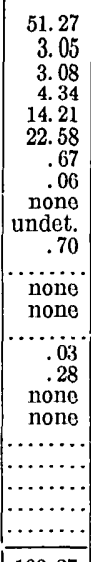 & $\begin{array}{r}1.58 \\
1.00 \\
\text { none } \\
.87 \\
1.22 \\
.68 \\
\text { undet. } \\
\text { undet. } \\
\text { none } \\
\text { undet. } \\
\cdots . . . \\
.46 \\
\cdots \ldots . . . \\
\cdots . . .\end{array}$ \\
\hline & 100.68 & 100.21 & 33.06 & 100.42 & 100.27 & 5.81 \\
\hline
\end{tabular}

Rocks $\mathrm{Q}$ to $\mathrm{V}$ collected by Whitman Cross, who supplies the petrographic data. Hitherto unpublished, except when otherwise stated.

Q. Rhyolitic vitrophyre, near Del Norte, Rio Grande County. Toscanose. Reported by Cross as containing phenocrysts of oligoclase, quartz, biotite, and augite in a dark, fresh, glassy groundmass, the latter being predominant. Analysis made by Eakins in the Denver laboratory. Sp. gr., 2.423, 14 ${ }^{\circ}$. P. R. C. 164.

R. Rhyolite, Summit district, Rio Grande County. Toscanose. Large phenocrysts of sanidine, with smaller ones of oligoclase and biotite, in a groundmass of orthoclase, quartz, oligoclase, biotite, and magnetite. Analysis by Eakins, made in the Denver laboratory. Sp. gr., $2.489,14^{\circ}$.

S. Quartz monzonite, Sultan Mountain, San Juan County. Amiatose. Contains plagioclase, orthoclase, quartz, augite, biotite, magnetite, and apatite. Some chlorite and epidote as alteration products. Analysis by L. G. Eakins in the Denver laboratory. Sp. gr., 2.751, $14^{\circ}$. Described by Cross in 21st Ann., pt. 2. P. R. C. 204.

T. Pyroxene-hornblende andesite, Falls Creek, Lake City quadrangle. Consists chiefly of plagioclase, augite, hypersthene, and hornblende, with little magnetite and apatite, and an abundant glass base. Analysis by G. Steiger, record No. 2244.

U. Decomposed andesite, Slumgullion mud flow, Lake City quadrangle. Analysis by W. T. Schaller, record No. 2368 .

V. Trachyte, Grayrock Peak, Engineer Mountain quadrangle. Analysis by Steiger, record No. 2374.

15619-Bull, 419-10-9 


\begin{tabular}{|c|c|c|c|c|c|c|}
\hline . & Q. & R. & & T. & U. & V. \\
\hline 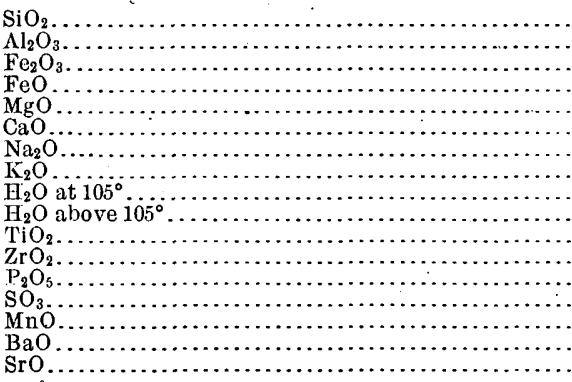 & $\begin{array}{r}68.61 \\
16.43 \\
.73 \\
1.52 \\
.05 \\
1.79 \\
2.82 \\
4.65 \\
3.35\end{array}$ & $\begin{array}{r}68.85 \\
17.01 \\
1.78 \\
.65 \\
\text { trace } \\
1.62 \\
3.44 \\
5.11 \\
1.79 \\
\ldots . . .\end{array}$ & 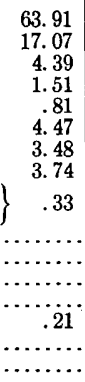 & $\begin{array}{r}63.03 \\
15.86 \\
3.31 \\
1.74 \\
1.69 \\
4.40 \\
3.17 \\
3.75 \\
.43 \\
1.73 \\
.64 \\
\text { none } \\
.24 \\
\text { none } \\
.10 \\
.08 \\
.03\end{array}$ & $\begin{array}{r}56.36 \\
11.51 \\
5.82 \\
1.18 \\
1.42 \\
.44 \\
.54 \\
1.30 \\
8.32 \\
9.80 \\
.87 \\
\text { none } \\
.35 \\
2.50 \\
.08 \\
.04\end{array}$ & $\begin{array}{r}70.73 \\
14.22 \\
1.59 \\
.59 \\
\text { none } \\
.72 \\
4.96 \\
5.57 \\
1.16 \\
.32 \\
.34 \\
.04 \\
.03 \\
\text { none } \\
.11 \\
.01 \\
\ldots . . .\end{array}$ \\
\hline$r$ & 99.95 & 100.25 & 99.92 & 100.20 & 100.53 & 100.39 \\
\hline
\end{tabular}

W. Nepheline tephrite, Elkhead Mountain, Routt County. Undescribed. Sp. gr., $2.888,12.2^{\circ}$.

$\mathrm{X}$. Portion of $\mathrm{N}$ soluble in hydrochloric acid. Recalculated to 100 per cent, 39.95 per cent of the entire rock. Analyses W, X, made by L. G. Eakins in the Denver laboratory.

Y. Olivine basalt, Pilot Knob, Routt County. Collected by H. S. Gale, who furnishes the description. Contains plagioclase, olivine, augite, magnetite, apatite, and possibly other accessory minerals. Analysis by W. F. Hillebrand, record No. 2235.

\begin{tabular}{|c|c|c|c|}
\hline & W. & $\mathrm{X}$. & Y. \\
\hline 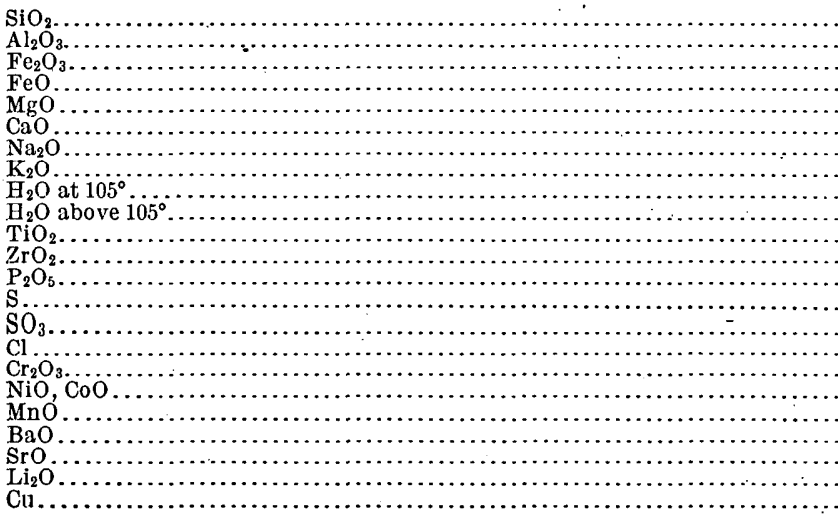 & $\begin{array}{r}46.67 \\
15.90 \\
3.20 \\
7.04 \\
10.17 \\
9.15 \\
3.20 \\
2.54 \\
1.64\end{array}$ & 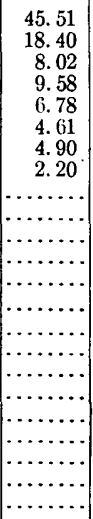 & $\begin{array}{r}48.16 \\
12.85 \\
2.79 \\
7.11 \\
10.45 \\
8.13 \\
3.18 \\
2.79 \\
.45 \\
1.47 \\
1.50 \\
\text { trace } \\
.70 \\
\text { trace } \\
\ldots . . . \\
\text { undet. } \\
.07 \\
.04 \\
.14 \\
.25 \\
.15 \\
\text { trace } \\
\text { trace }\end{array}$ \\
\hline & 100.26 & 100.00 & 100.23 \\
\hline
\end{tabular}

UTAH.

\section{ROCKS FROM THE TINTIC DISTRICT.}

Described by Tower and Smith in 19th Ann., pt. 3, pp. 609 et seq. Analyses by Stokes, record No. 1746.

A. Gray, porphyritic rhyolite,-south of Pinyon Creek. Toscanose. Contains phenocrysts of sanidine, quartz, biotite, plagioclase, and hornblende; the last mineral sparingly. Also tridymite, magnetite, apatite, zircon, and a small amount of glassy residue. P. R. C. 1039.

B. Quartz porphyry, Swansea mine. Toscanose. Phenocrysts of feldspar and quartz, the orthoclase somewhat altered. Microscopic biotite occurs sparingly. Also contains magnetite, apatite, zircon, a little chlorite, and secondary pyrite. P. R. C. 1040. 
C. Andesite, Tintic Mountain. Harzose. Phenocrysts of biotite and feldspar. Contains plagioclase, chiefly labradorite, augite, biotite, hypersthene, magnetite, and apatite, in a dark-gray glass. P. R. C. 1036.

D. Granular monzonite, Iron Duke mine. Harzose. Contains orthoclase, plagioclase, quartz, hornblende, biotite, magnetite, apatite, zircon, and titanite, with a little chlorite and epidote. P. R. C. 1032.

E. Altered monzonite, near Tintic mine. Feldspar and ferromagnesian minerals completely altered. Rutile is present: quartz seems to have been added.

\begin{tabular}{|c|c|c|c|c|c|}
\hline & A. & B. & C. & D. & E. \\
\hline 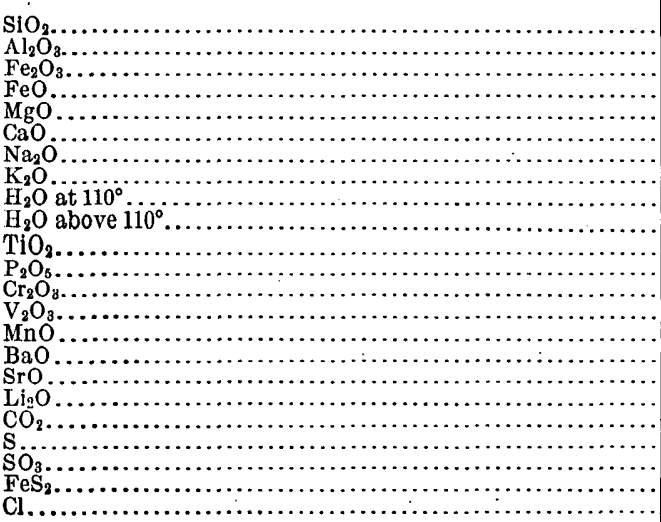 & $\begin{array}{r}69.18 \\
14.36 \\
2.52 \\
.57 \\
.70 \\
1.88 \\
3.58 \\
5.00 \\
.35 \\
.25 \\
.69 \\
.26 \\
\text { trace } \\
.01 \\
.10 \\
.09 \\
\text { trace } \\
\text { trace } \\
\text { none } \\
\text { none } \\
\text { none } \\
\ldots \ldots . . . \\
\text { trace }\end{array}$ & $\left\{\begin{array}{r}71.56 \\
14.27 \\
.89 \\
\ldots . . . \\
.42 \\
1.18 \\
3.00 \\
4.37 \\
.36 \\
.79 \\
.38 \\
.13 \\
\text { trace } \\
.01 \\
\text { trace } \\
.28 \\
\text { trace } \\
\text { none } \\
\text { none } \\
\ldots \ldots . \\
\ldots \ldots .29 \\
2.29 \\
.06\end{array}\right.$ & $\begin{array}{r}60.17 \\
15.77 \\
3.42 \\
2.95 \\
2.52 \\
4.69 \\
2.96 \\
4.16 \\
.25 \\
1.23 \\
.87 \\
.40 \\
\text { none } \\
.01 \\
.11 \\
.14 \\
.09 \\
\text { trace } \\
\text { none } \\
\text { none } \\
\text { none } \\
\ldots . . . . \\
.04\end{array}$ & $\begin{array}{r}59.76 \\
15.77 \\
3.77 \\
3.30 \\
2.16 \\
3.88 \\
3.01 \\
4.40 \\
.31 \\
1.11 \\
.87 \\
.42 \\
\text { none } \\
.02 \\
.12 \\
.09 \\
\text { trace } \\
\text { trace } \\
.78 \\
\text { none } \\
\text { none } \\
\ldots . . . . \\
.04\end{array}$ & $\begin{array}{r}71.14 \\
16.22 \\
.94 \\
.16 \\
1.12 \\
.25 \\
.07 \\
4.96 \\
.49 \\
2.74 \\
.75 \\
.32 \\
\text { none } \\
.02 \\
\text { trace } \\
.05 \\
\text { trace } \\
\text { trace } \\
\text { none } \\
\ldots . . \\
.26 \\
\ldots \ldots . . \\
\text { trace }\end{array}$ \\
\hline$\cdot$ & 99.54 & 99.99 & 99.78 & 99.81 & 99.49 \\
\hline
\end{tabular}

Fluorine was not looked for in the analyses.

\section{ROCKS FROM HENRY MOUNTAINS.}

A. Porphyry. Tonalose. Not hitherto published. Reported by J. S. Diller as containing prominent plagioclase with some augite and hornblende in a crystalline groundmass of quartz and orthoclase. Analysis by R. B. Riggs, record No. 728.

B. Augite porphyrite, dike, north spur of Mount Pennell. Laurvikose. Contains hornblende, augite, and plagioclase in a feldspathic groundmass.

C. Hornblende porphyrite, Mount Hillers. Tonalose. Contains plagioclase, hornblende, quartz, and magnetite.

Analyses B and C by W. F. Hillebrand, record No. 1428. Rocks described by Cross in 14th Ann., p. 165.

\begin{tabular}{|c|c|c|c|}
\hline & A. & R. & C. \\
\hline 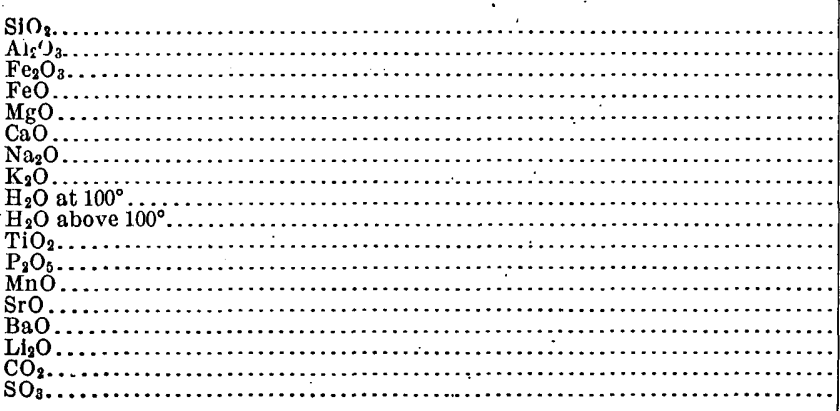 & $\begin{array}{r}63.16 \\
17.21 \\
2.43 \\
2.30 \\
1.27 \\
6.27 \\
4.70 \\
1.84 \\
.69 \\
.21 \\
.12 \\
\text { trace } \\
\text { trace? } \\
.09 \\
\text { trace } \\
\text { trace }\end{array}$ & $\begin{array}{r}60.98 \\
19.09 \\
1.76 \\
1.15 \\
.65 \\
3.67 \\
6.70 \\
3.53 \\
.48 \\
.44 \\
.36 \\
.10 \\
.15 \\
.28 \\
.43 \\
\text { trace } \\
.52 \\
\ldots \ldots\end{array}$ & $\begin{array}{r}62.88 \\
17.13 \\
1.86 \\
2.58 \\
1.48 \\
5.39 \\
4.50 \\
2.25 \\
.16 \\
.42 \\
.51 \\
.26 \\
.16 \\
.12 \\
.16 \\
\text { trace } \\
\ldots \ldots . . \\
\ldots \ldots . .\end{array}$ \\
\hline & 100.29 & 100.29 & 99.86 \\
\hline
\end{tabular}




\section{THE BINGHAM DISTRICT.}

Porphyries collected by J. M. Boutwell, and described in P. P. 38, p. 178. Analyses by E. T. Allen, record No. 1985.

A. From Tribune tunnel, Telegraph mine. Monzonose.

B, C, D. From British tunnel, Last Chance mine. B, SR. 1 of dacase. C. harzose. $\mathrm{D}$, monzonose.

According to Boutwell, $\mathrm{A}$ is the characteristic Bingham porphyry, and is intermediate between diorite porphyry and monzonite. It contains chiefly augite, biotite, and plagioclase, with a few grains of pyrite. B, C, and D are altered forms of this rock.

\begin{tabular}{|c|c|c|c|c|}
\hline & A. & B. & C. & D. \\
\hline 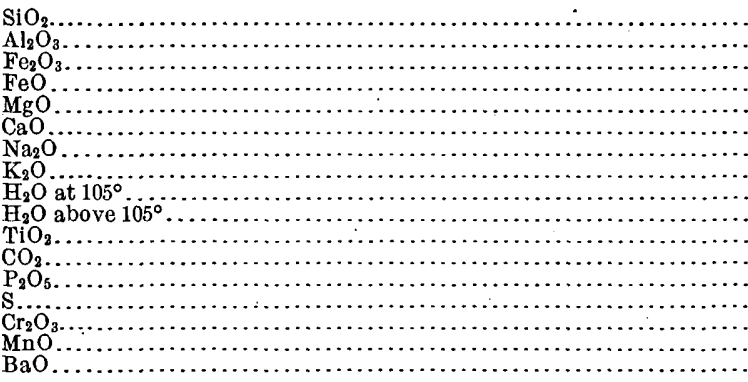 & $\begin{array}{r}57.16 \\
16.69 \\
3.47 \\
2.76 \\
2.47 \\
5.86 \\
3.82 \\
4.49 \\
.83 \\
1.06 \\
.87 \\
\text { none } \\
.41 \\
.02 \\
\text { trace } \\
\text { trace } \\
.30\end{array}$ & $\begin{array}{r}56.78 \\
16.90 \\
6.87 \\
2.34 \\
.03 \\
1.18 \\
.37 \\
7.02 \\
1.32 \\
2.23 \\
.81 \\
.26 \\
.04 \\
5.93 \\
\text { trace } \\
\text { trace } \\
.14\end{array}$ & $\begin{array}{r}56.17 \\
15.94 \\
3.43 \\
1.92 \\
1.60 \\
5.19 \\
2.48 \\
4.91 \\
1.30 \\
2.95 \\
.90 \\
2.01 \\
.20 \\
1.03 \\
\ldots \ldots \\
\text { trace } \\
.18\end{array}$ & $\begin{array}{r}58.64 \\
15.35 \\
3.25 \\
2.54 \\
3.84 \\
5.37 \\
3.60 \\
4.23 \\
.86 \\
1.50 \\
.83 \\
\text { none } \\
.02 \\
.05 \\
\text { trace } \\
\text { trace } \\
.18\end{array}$ \\
\hline \multirow[t]{2}{*}{ Less $O=S . .}$. & $\begin{array}{r}100.21 \\
.01\end{array}$ & $\begin{array}{r}102.22 \\
2.22\end{array}$ & $\begin{array}{r}100.21 \\
.39\end{array}$ & $\begin{array}{r}100.26 \\
.02\end{array}$ \\
\hline & 100.20 & 100.00 & 99.82 & 100.24 \\
\hline
\end{tabular}

4. ROCKS FROM THE LA SAL MOUNTAINS.

Petrographic descriptions by L. M. Prindle. Analyses by W. F. Hillebrand, record No. 2032.

A. Monzonite porphyry, 2 miles west of Mount Peale. Akerose. Contains phenocrysts of plagioclase, partly resorbed hornblende, and pyroxene in a groundmass of partly striated feldspar. It may contain also orthoclase and quartz. P. R. C. 1306.

B. Egirite granite porphyry, about 1.5 miles south of Mount Waas. Omeoseliparose. Contains quartz, feldspar, pyroxene, and iron ore. The pyroxene is probably for the most part ægirite. P. R. C. 1304.

C. Syenite-aplite porphyry resembling grorudite. About 2 miles south of Mount Waas. Liparose. Contains potash and soda-lime feldspars, quartz, pyroxene, titanite, and iron ore. In the groundmass are needles which are probably ægirite. P. R. C. 1301.

D. Syenite porphyry resembling sölvsbergite. About 1 mile northwest of Mount Waas. Phlegrose near nordmarkose. Contains potash and soda-lime feldspars, quartz, pyroxene, and iron ore. Needles of ægirite (?) in the groundmass. P. R. C. 1303.

E. Pulaskite, 1 mile west of Mount Waas. Nordmarkose. Contains potash feldspar, pyroxene, biotite, apatite, titanite, and iron ore. P. R. C. 1305.

- F. Noselite syenite porphyry, dike on northwest shoulder of Mount Waas. Miaskose. Contains feldspar, pyroxene, sodalite or noselite, apatite, titanite, and iron ore. The pyroxene appears to be mostly ægirine-augite and the feldspar mainly potash feldspar. P. R. C. 1302. 


\begin{tabular}{|c|c|c|c|c|c|c|}
\hline , & A. & B. & C. & D. & E. & F. \\
\hline $\begin{array}{l}\mathrm{SiO}_{2} \\
\mathrm{Al}_{2} \mathrm{O}_{3} \\
\mathrm{Fe}_{2} \mathrm{O}_{3}\end{array}$ & $\begin{array}{r}61.21 \\
17.10 \\
2.72 \\
1.88 \\
1.47 \\
4.83 \\
5.66 \\
3.00 \\
.34 \\
.68 \\
.51 \\
.02 \\
\text { none } \\
.24 \\
\text { none } \\
.04 \\
.15 \\
.13 \\
.07 \\
\text { trace? }\end{array}$ & $\begin{array}{r}73.27 \\
13.29 \\
1.16 \\
.13 \\
.07 \\
.21 \\
3.44 \\
7.53 \\
.23 \\
.43 \\
.10 \\
.02 \\
.02 \\
\text { trace } \\
.07 \\
.01 \\
.03 \\
.10 \\
\text { none? } \\
\text { trace? }\end{array}$ & $\begin{array}{r}70.02 \\
14.38 \\
1.17 \\
.13 \\
.61 \\
.66 \\
5.48 \\
5.87 \\
.27 \\
.44 \\
.10 \\
.01 \\
.38 \\
\text { trace } \\
.19 \\
.03 \\
.02 \\
.13 \\
.06 \\
\text { trace }\end{array}$ & $\begin{array}{r}68.96 \\
15.42 \\
1.99 \\
.16 \\
.22 \\
.25 \\
6.59 \\
5.48 \\
.22 \\
.30 \\
.12 \\
.04 \\
.13 \\
\text { trace } \\
\text { none } \\
.01 \\
.07 \\
\text { trace } \\
\text { none } \\
\text { trace }\end{array}$ & $\begin{array}{r}62.64 \\
17.36 \\
2.79 \\
.63 \\
.53 \\
1.70 \\
7.00 \\
4.97 \\
.43 \\
.53 \\
.43 \\
.02 \\
.54 \\
.12 \\
.06 \\
.03 \\
.04 \\
.10 \\
.07 \\
\text { none }\end{array}$ & $\begin{array}{r}58.99 \\
19.01 \\
1.74 \\
.59 \\
.27 \\
2.02 \\
9.11 \\
5.07 \\
.38 \\
1.24 \\
.21 \\
.07 \\
\text { none } \\
.04 \\
.96 \\
.15 \\
.08 \\
.02 \\
.02 \\
\text { trace }\end{array}$ \\
\hline & 100.05 & 100.11 & 99.95 & 99.96 & 99.99 & 99.97 \\
\hline
\end{tabular}

S absent from all.

\section{THE PARK CITY DISTRICT.}

Rocks collected by J. M. Boutwell, who furnishes the petrographic data. Analyses, hitherto unpublished, by W. F. Hillebrand, record No. 21.73.

A. Granite (?), head of Big Cottonwood Canyon. Essential constituents quartz, orthoclase, plagioclase, biotite, hornblende, and augite, with accessory apatite and perhaps sphene.

B. Quartz diorite, Thaynes Canyon. Contains oligoclase, quartz, orthoclase, hornblende, and biotite, with accessory pyrite, apatite, and titanite. A little secondary kaolinite and chlorite.

C. Quartz diorite porphyry, Valeo mine. Contains plagioclase, hornblende, biotite, and quartz, with pyrite and some orthoclase, and secondary calcite, chlorite, and kaolin.

D. Diorite porphyry, Daly West mine. Contains albite, hornblende, quartz, pyrite, and possibly apatite, with secondary chlorite or serpentine, calcite, epidote, kaolin, and muscovite.

E. Diorite, Clayton Peak Amphitheater. Contains plagioclase, orthoclase, hornblende, biotite, and augite, with accessory quartz, apatite, titanite, magnetite, and pyrite.

F. Andesite, Ontario drain tunnel. Contains albite, hornblende, some quartz and magnetite. A green secondary mineral is probably serpentine. 


\begin{tabular}{|c|c|c|c|c|c|c|}
\hline & A. & B. & C. & D. & E. & F. \\
\hline 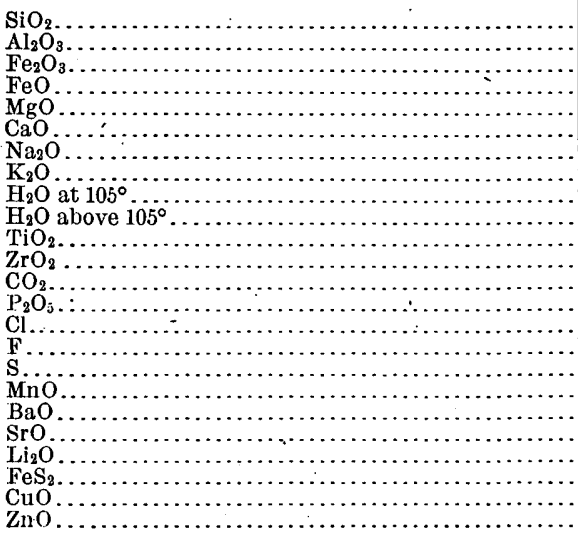 & $\begin{array}{r}65.27 \\
15.75 \\
2.31 \\
.1 .85 \\
1.62 \\
4.09 \\
3.92 \\
3.25 \\
.21 \\
.53 \\
.55 \\
.02 \\
\text { trace } \\
.25 \\
.01 \\
? . \\
\ldots . . . \\
.10 \\
.11 \\
.05 \\
\text { trace } \\
.02 \\
\ldots \ldots . . \\
\ldots \ldots\end{array}$ & $\begin{array}{r}63.46 \\
15.93 \\
2.61 \\
2.31 \\
2.27 \\
4.33 \\
3.66 \\
3.49 \\
.27 \\
.74 \\
.62 \\
.03 \\
\text { trace } \\
.16 \\
.05 \\
\text { trace? } \\
\text { trace } \\
.09 \\
.15 \\
\text { undet. } \\
\text { trace } \\
\ldots \ldots . . \\
\ldots \ldots . . \\
\ldots \ldots . .\end{array}$ & $\begin{array}{r}61.64 \\
14.66 \\
1.95 \\
1.68 \\
2.55 \\
4.65 \\
2.71 \\
3.07 \\
1.04 \\
2.56 \\
.48 \\
.01 \\
2.15 \\
.24 \\
\text { trace } \\
\text { trace } \\
\ldots \ldots . \\
.06 \\
.18 \\
.06 \\
\text { trace } \\
.32 \\
\ldots \ldots\end{array}$ & $\begin{array}{c}59.68 \\
15.61 \\
2.49 \\
2.38 \\
2.52 \\
4.63 \\
3.96 \\
2.96 \\
.51 \\
2.00 \\
.62 \\
.01 \\
2.29 \\
.29 \\
\text { none } \\
? \\
\ldots . . . \\
.08 \\
.15 \\
.07 \\
\text { trace } \\
.02 \\
\ldots \ldots . . \\
\ldots \ldots . .\end{array}$ & $\begin{array}{r}59.35 \\
16.36 \\
2.90 \\
3.36 \\
3.08 \\
5.03 \\
3.73 \\
3.85 \\
.28 \\
.64 \\
.87 \\
.03 \\
\text { trace? } \\
.44 \\
.05 \\
?\end{array}$ & $\begin{array}{r}54.23 \\
17.37 \\
4.00 \\
1.95 \\
3.00 \\
6.67 \\
2.96 \\
2.80 \\
1.60 \\
3.71 \\
.75 \\
.02 \\
.33 \\
.34 \\
\text { trace } \\
? \\
\ldots \ldots .10 \\
.15 \\
.06 \\
\text { trace } \\
.02 \\
\ldots \ldots . . \\
\ldots \ldots . .\end{array}$ \\
\hline . & 99.91 & 100.17 & 100.01 & 100.27 & 100.29 & 100.06 \\
\hline
\end{tabular}

$\mathrm{Cr}_{2} \mathrm{O}_{3}$ and $\mathrm{SO}_{3}$ absent.

\section{MISCELLANEOUS ROCKS.}

A. Rhyolite, Thomas Range. Toscanose. Analysis made by L. G. Eakins in the Denver laboratory. Described by Cross in Proc. Colorado Sci. Soc., vol. 2, p. 69. Contains phenocrysts of quartz and sanidine in a groundmass mainly made up of quartz and alkali feldspar.

B. Rhyolitic glass or pitchstone, edge of Gold Mountain mining district, 8 miles north of west from Marysvale. Liparose. 'Identified by Whitman Cross. Sp. gr., 2.25 at $23.5^{\circ}$. Analysis by W. F. Hillebrand, record No. 1833.

\begin{tabular}{|c|c|c|}
\hline & A. & B. \\
\hline 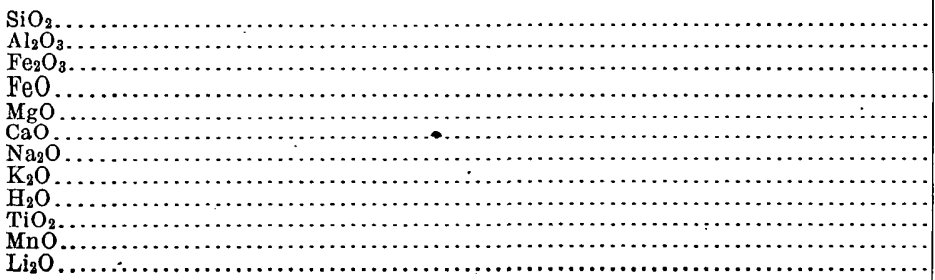 & \begin{tabular}{r|}
74.49 \\
14.51 \\
.57 \\
.32 \\
trace \\
1.03 \\
3.79 \\
4.64 \\
.64 \\
$\ldots \ldots$ \\
trace \\
trace
\end{tabular} & $\begin{array}{r}70.17 \\
11.83 \\
.93 \\
\text { none } \\
.06 \\
.76 \\
3.85 \\
3.74 \\
8.72 \\
.17 \\
.72 \\
7 . .\end{array}$ \\
\hline & 99.99 & 100.23 \\
\hline
\end{tabular}

\section{NEW MEXICO.}

\section{BASALTS FROM RIO GRANDE CANYON.}

Described by Iddings, Bull. 66; also in Am. Jour. Sci., 3d ser., vol. 36, p. 220. Contain plagioclase, augite, olivine, and magnetite. A, B, and C contain quartz also; D is quartzless. Analyses by L. G. Eakins, record Nos. 847, 850. All andose.
A. Light gray, dense. P. R. C. 548.
B. Greenish black, dense.
C. Dark red, vesicular. P. R. C. 549 .
D. Gray, dense. P. R. C. 547. 


\begin{tabular}{|c|c|c|c|c|}
\hline • & A. & B: & $\mathrm{C}$ : & D: \\
\hline 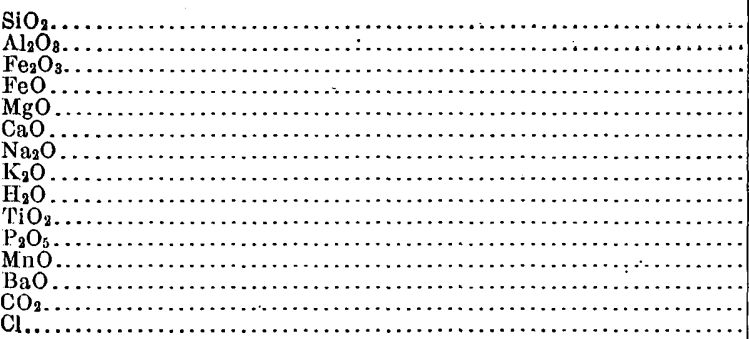 & $\begin{array}{r}62.27 \\
17.68 \\
2.51 \\
5.00 \\
6.05 \\
8.39 \\
4.19 \\
1.58 \\
.82 \\
1.49 \\
.23 \\
.06 \\
\text { trace } \\
\text { trace }\end{array}$ & $\begin{array}{r}52.37 \\
17.01 \\
1.44 \\
5.89 \\
6.86 \\
7.59 \\
3.51 \\
1.59 \\
1.29 \\
1.60 \\
\ldots . .32 \\
.32 \\
.06 \\
.37 \\
\text { trace }\end{array}$ & $\begin{array}{r}51.57 \\
17.72 \\
6.24 \\
1.78 \\
4.91 \\
8.82 \\
3.59 \\
1.99 \\
.64 \\
1.43 \\
\ldots . .75 \\
.45 \\
.16 \\
.58 \\
\ldots . . . .\end{array}$ & $\begin{array}{r}52.38 \\
18.79 \\
2.88 \\
4.90 \\
4.91 \\
7.70 \\
3.99 \\
1.76 \\
.53 \\
1.22 \\
.56 \\
.18 \\
.11 \\
\ldots \ldots . . \\
\ldots . . .\end{array}$ \\
\hline . & 100.27 & 99.90 & 99.88 & 99.91 \\
\hline
\end{tabular}

\section{MOUNT TAYLOR REGION.}

Rocks received from J. S. Diller. Analyses by T. M. Chatard, record Nos. 219, 227, 228, 235, 271, 268, and 269. Petrographic details furnished by Diller.

A. Lava, canyon on east side of San Mateo Mountain. Kallerudose.

B. Andesite, canyon on east side of San Mateo Mountian. Nordmarkose. Contains feldspar and corroded augite, with sometimes olivine, in a groundmass of feldspar and a green ferromagnesian silicate. P. R. C. 1402.

C. Quartz latite, canyon on east side of San Mateo Mountain. Lassenose. Shows prominent plagioclase, with augite and biotite. P. R. C. 1403.

D. Dacite, 6 miles northeast of Grant's. Principally plagioclase and biotite, with less epidote, quartz, sphene, and carbonates. P. R. C. 1401.

E. Basait, 6 miles northeast of Grant's. Auvergnose. Contains chiefly plagioclase, augite, and olivine, with much magnetite. P. R. C. 1400.

F. Augite from E.

G. Feldspar from E. Analysis on three-fourths gram of material.

\begin{tabular}{|c|c|c|c|c|c|c|c|}
\hline - & A. & B. & C. & D. & E. & F. & ' G. \\
\hline 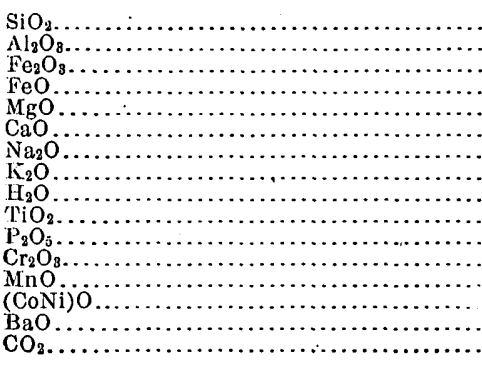 & \begin{tabular}{r}
68.40 \\
17.99 \\
2.66 \\
1.63 \\
.49 \\
.67 \\
4.54 \\
3.54 \\
.52 \\
\hdashline$\ldots .6$ \\
\hdashline .21 \\
.2. \\
.2.
\end{tabular} & $\begin{array}{r}65.51 \\
16.89 \\
1.41 \\
2.52 \\
.39 \\
1.19 \\
6.42 \\
5.02 \\
.16 \\
.92 \\
.07 \\
\ldots .31\end{array}$ & $\begin{array}{r}65.78 \\
17.32 \\
3.68 \\
.46 \\
.47 \\
1.66 \\
5.23 \\
4.64 \\
.14 \\
.27 \\
.13 \\
\ldots . . \\
.32\end{array}$ & $\begin{array}{r}42.80 \\
15.33 \\
\ldots . \ldots . \\
7.44 \\
6.61 \\
7.19 \\
2.71 \\
4.36 \\
1.38 \\
2.67 \\
.73 \\
\ldots . . \\
.30 \\
\ldots . .\end{array}$ & $\begin{array}{r}47.54 \\
16.73 \\
6.69 \\
6.67 \\
6.38 \\
8.74 \\
2.81 \\
1.10 \\
.36 \\
2.76 \\
.51 \\
.19 \\
. .19 \\
.03 \\
(?)\end{array}$ & $\begin{array}{r}47.06 \\
7.77 \\
1.30 \\
8.15 \\
13.52 \\
19.33 \\
.33 \\
.11 \\
.20 \\
1.82 \\
.06 \\
\text { trace } \\
.20 \\
\text { traces } \\
\ldots \ldots . . . \\
\ldots \ldots \ldots . .\end{array}$ & $\begin{array}{r}52.54 \\
31.26 \\
\ldots . . . \\
12.34 \\
3.55 \\
.42 \\
26 \\
\text { undet. } \\
\ldots \ldots . . . \\
\cdots \ldots . . . \\
\text { trace? }\end{array}$ \\
\hline & 100.65 & 100.81 & 100.10 & 101.08 & 100.51 & 99.85 & 100.65 \\
\hline
\end{tabular}

3. COLFAX COUNTY.

Descriptions supplied by Whitman Cross. Analyses by W. F. Hillebrand, record No. 1719 .

A. Phonolite, Pleasant Valley. Miaskose. Contains much nephelite, some ægirite, alkali feldspar, a scanty dust of magnetite, and a few decomposed grains of noselite or sodalite. Sp. gr., $2.619,22^{\circ} ; 40.8$ per cent soluble in dilute $(1: 40)$ nitric acid, of which soluble portion 43.5 per cent is silica. P. R. C. 1334 . 
B. Pyroxene andesite, Sierra Grande. Tonalose. Contains augite, less hypersthene, microliths of plagioclase, apatite, magnetite, and a smoky-brown glassy base. Sp. gr., 2.635, $21^{\circ}$. P. R. C. 1335.

C. Plagioclase basalt, end of San Rafael flow. Camptonose. Contains plagioclase, atgite, olivine, with much iddingsite, magnetite, and apatite. Sp. gr., 2.970, $21.5^{\circ}$. P.,R. C. 1336.

D. Nepheline basanite, Ciruella. Limburgose. Contains augite, olivine, nephelite, plagioclase, magnetite, apatite, and a little biotite. Sp. gr., 3.122, $22^{\circ}$. 0.55 per cent $\mathrm{K}_{2} \mathrm{O}$ and 2.10 per cent $\mathrm{Na}_{2} \mathrm{O}$ soluble in 1:40 nitric acid. P. R. C. 1337.

\begin{tabular}{|c|c|c|c|c|}
\hline & A. & B. & C. & D. \\
\hline 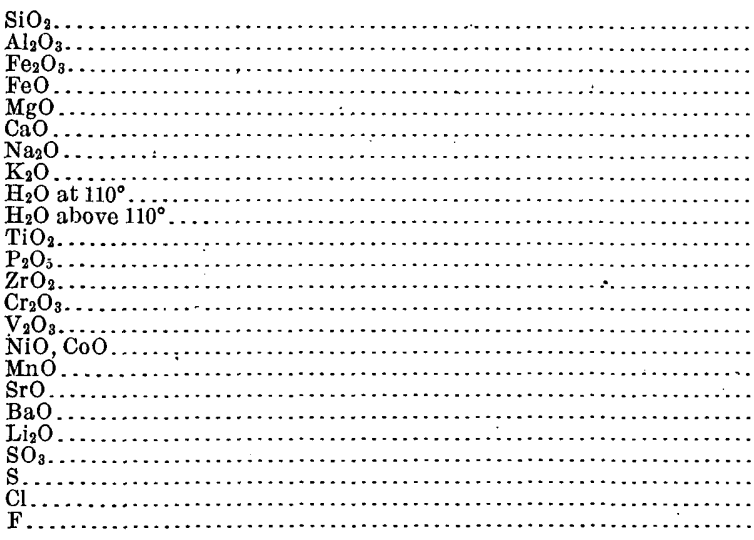 & $\begin{array}{r}56.24 \\
21.43 \\
2.01 \\
.55 \\
.15 \\
1.38 \\
10.53 \\
5.74 \\
.12 \\
.86 \\
.26 \\
.06 \\
.09 \\
\text { none } \\
\ldots . . \\
\text { none } \\
.08 \\
.05 \\
.08 \\
\text { trace } \\
.10 \\
.03 \\
.12 \\
\text { trace }\end{array}$ & $\begin{array}{r}60.16 \\
15.34 \\
3.07 \\
2.18 \\
3.41 \\
5.79 \\
3.88 \\
2.59 \\
.25 \\
1.79 \\
.84 \\
.46 \\
.01 \\
\text { trace? } \\
\ldots \ldots . . \\
\text { trace } \\
.08 \\
.08 \\
.14 \\
\text { trace } \\
.08 \\
\text { trace } \\
\text { undet. } \\
\text { undet. }\end{array}$ & $\begin{array}{r}48.35 \\
15.47 \\
4.80 \\
7.58 \\
8.15 \\
8.81 \\
3.09 \\
.95 \\
.28 \\
.73 \\
1.33 \\
.33 \\
\text { none } \\
\text { trace } \\
\ldots . . . \\
.02 \\
.21 \\
.03 \\
.06 \\
\text { trace } \\
.07 \\
\text { trace } \\
\text { undet. } \\
\text { undet. }\end{array}$ & $\begin{array}{r}42.35 \\
12.29 \\
3.89 \\
7.05 \\
13.09 \\
12.49 \\
2.74 \\
1.04 \\
.32 \\
1.50 \\
1.82 \\
.99 \\
\text { none } \\
.10 \\
.04 \\
.03 \\
.21 \\
.09 \\
.10 \\
\text { trace } \\
.05 \\
\text { trace } \\
\text { undet. } \\
\text { undet. }\end{array}$ \\
\hline & 99.86 & 100.15 & 100.26 & 100. 19 \\
\hline
\end{tabular}

\section{MISCELLANEOUS ROCKS.}

A. ${ }^{\circ}$ Obsidian, Obsidian Hill camp, Tewan Mountains. Liparose. Described by Iddings, 7th Ann., p. 292. A rhyolitic obsidian, containing grains of iron oxide and a few microscopic feldspars. Resembles that from Obsidian Cliff in the Yellowstone National Park. Analysis by L. G. Eakins, record No. 851. Sp. gr., 2.352, 23.5 ${ }^{\circ}$.

B. Trachyte (?), from Los Cerrillos. Described by Diller, Bull. 42, p. 39 . Analysis by F. W. Clarke, record No. 346. Rock composed chiefly of orthoclase, with a considerable amount of biotite, epidote, pyrite, and limonite, and some amorphous substance. It is the matrix or gangue rock of the Los Cerrillos turquois.

C. Gabbro porphyry, Mount McKensie, Los Cerrillos Mountains. Andose. Received from J. F. Kemp. Described by D: W. Johnson, in School of Mines Quart., vol. 25, p. 82. Analysis by George Steiger, record No. 2027. Contains augite, plagioclase, orthoclase, and biotite. P. R. C. 1300.

D. Andesite, Country rock, Bonanza mine, Hillsboro. Shoshonose. Contains plagioclase, orthoclase, augite, and hornblende.

E. Syenite porphyry, Cook's Peak. Dacose. Contains orthoclase, plagioclase, biotite, hornblende, and quartz. Rocks D, E, described by C. H. Gordon. Analyses, as yet unpublished, by G. Steiger, record No. 2238 .

F. Quartz syenite, near Merrimac mine, 3 miles east of Organ City, Organ Mountains. Contains orthoclase, albite, quartz, biotite, augite, titanite, and magnetite. Description furnished by W. Lindgren. Analysis by Steiger, No. 2371. 


\begin{tabular}{|c|c|c|c|c|c|c|}
\hline- & A. & B. & & D. & E. & $\mathrm{F}$. \\
\hline 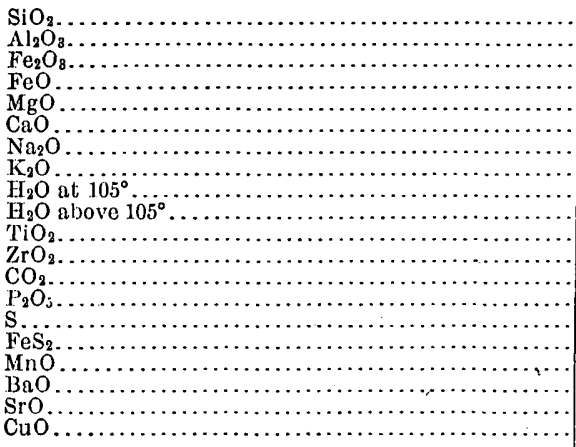 & $\begin{array}{r}76.20 \\
13.17 \\
.34 \\
.73 \\
.19 \\
.42 \\
4.31 \\
4.46 \\
.33 \\
\text { trace } \\
\ldots \ldots \ldots \\
\ldots \ldots \ldots \\
\ldots \ldots \ldots \\
\ldots \ldots \ldots \\
\ldots \ldots . . \\
\ldots \ldots \\
\ldots \ldots\end{array}$ & $\begin{array}{r}56.68 \\
16.62 \\
6.28 \\
.79 \\
.59 \\
1.03 \\
11.18 \\
3.28 \\
.22 \\
\ldots \ldots \\
\ldots \ldots \\
.73 \\
2.21 \\
1.02 \\
\cdots \ldots . . \\
\ldots \ldots \\
\text { trace }\end{array}$ & 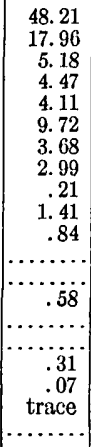 & $\begin{array}{r}54.54 \\
14.66 \\
4.20 \\
2.74 \\
3.21 \\
5.64 \\
3.47 \\
5.28 \\
1.10 \\
1.87 \\
.86 \\
\text { none } \\
2.19 \\
.49 \\
.01 \\
\ldots . . . \\
.29 \\
.07 \\
.05\end{array}$ & $\begin{array}{r}62.95 \\
15.91 \\
3.30 \\
1.37 \\
2.18 \\
4.46 \\
4.05 \\
2.95 \\
.72 \\
1.19 \\
.67 \\
\text { none } \\
\text { none } \\
.18 \\
\ldots \ldots \\
\ldots \ldots . . \\
.08 \\
.03 \\
.03 \\
\ldots \ldots .\end{array}$ & $\begin{array}{r}61.12 \\
15.78 \\
2.69 \\
3.15 \\
1.90 \\
3.95 \\
4.14 \\
4.48 \\
.32 \\
.56 \\
1.30 \\
.04 \\
.22 \\
.45 \\
.05 \\
\ldots \ldots . . \\
.09 \\
.07 \\
.04 \\
\ldots \ldots .\end{array}$ \\
\hline & 100.25 & 100.63 & 99.74 & 100.67 & 100.07 & 100.35 \\
\hline
\end{tabular}

ARIZONA.

\section{GLOBE DISTRICT.}

Rocks described by F. I. Ransome in P. P. 12. Analyses A, F, by W. F. Hillebrand, record No. 1988. The others by E. T. Allen, record Nos. 1952, 1955.

A. Quartz monzonite, Lost Gulch. Toscanose. Contains quartz, plagioclase, microcline, biotite, iron ore, titanite, apatite, and zircon. P. R. C. 1297.

B. Granite porphyry, one-half mile southwest of Hog ranch. Lassenose. Contains orthoclase, quartz, oligoclase, biotite, and iron ore. P. R. C. 1293.

C. Granite porphyry, 2 miles south of Schultze ranch. Lassenose. Contains orthoclase, quartz, oligoclase, and biotite, with some chlorite, epidote, and iron ore derived from biotite. P. R. C. 1292.

D. Granitite, 1 mile west of Schultze ranch. Lassenose. Contains oligoclase, quartz, orthoclase, biotite, muscovite, magnetite, apatite, and zircon. P. R. C. 1291.

\begin{tabular}{|c|c|c|c|c|}
\hline & A. & B. & C. & D. \\
\hline 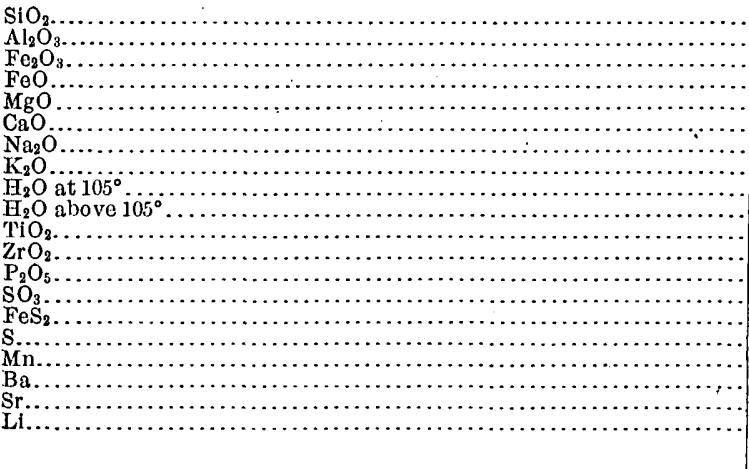 & $\begin{array}{r}68.63 \\
13.68 \\
2.53 \\
1.81 \\
1.10 \\
2.51 \\
2.94 \\
4.04 \\
.70 \\
.87 \\
.69 \\
.01 \\
.24 \\
.11 \\
.15 \\
.15 \\
\text { trace } \\
\text { trace }\end{array}$ & $\begin{array}{r}68.95 \\
15.84 \\
1.14 \\
.56 \\
.24 \\
1.96 \\
4.56 \\
3.69 \\
.86 \\
1.49 \\
.22 \\
.01 \\
.08\end{array}$ & $\begin{array}{r}69.35 \\
15.71 \\
1.18 \\
.43 \\
.36 \\
1.79 \\
4.78 \\
3.63 \\
1.17 \\
.97 \\
.19 \\
\text { trace } \\
.08 \\
\ldots \ldots . \\
\ldots \ldots . . \\
\text { trace } \\
\text { trace } \\
.07 \\
\ldots \ldots . . \\
\ldots \ldots . . \\
\ldots . . .\end{array}$ & $\begin{array}{r}70.95 \\
16.30 \\
1.01 \\
.36 \\
.23 \\
1.85 \\
5.16 \\
3.34 \\
.26 \\
.37 \\
.23 \\
\text { trace } \\
\text { trace } \\
\text { trace } \\
\ldots \ldots . \\
\text { trace } \\
\text { trace } \\
.04 \\
\ldots \ldots . . \\
\ldots \ldots . . \\
10010\end{array}$ \\
\hline & 100.06 & 99.67 & 99.71 & 100.10 \\
\hline
\end{tabular}


E. Biotite dacite, one-fourth mile north of Old Dominion mine. Toscanose. Contains plagioclase, sanidine, quartz, biotite, hornblende, apatite, titanite, zircon, magnetite, and glass. P. R. C. 1299.

F. Quartz-mica diorite, Florence stage road 2 miles south of Pinal Peak. Tonalose. Contains labradorite, quartz, biotite, microcline, muscovite, titanite, apatite, magnetite, and zircon, with secondary chlorite, epidote, sericite, calcite, and a little hornblende. P. R. C. 1295.

G. Quartz-mica diorite, 2 miles south of Hog ranch, Pinal Mountains. SR. 3 of vaalase. Contains labradorite, quartz, biotite, orthoclase, muscovite, magnetite, titanite, apatite, and zircon, with secondary epidote, sericite, and chlorite. P. R. C. 1296.

H. Olivine diabase, 1 mile northwest of Black. Peak. Auvergnose. Contains plagioclase, augite, olivine, biotite, iron ore, apatite, and titanite. Perfectly fresh. P. R. C. 1298.

\begin{tabular}{|c|c|c|c|c|}
\hline & E. & F. & G. . & H. \\
\hline 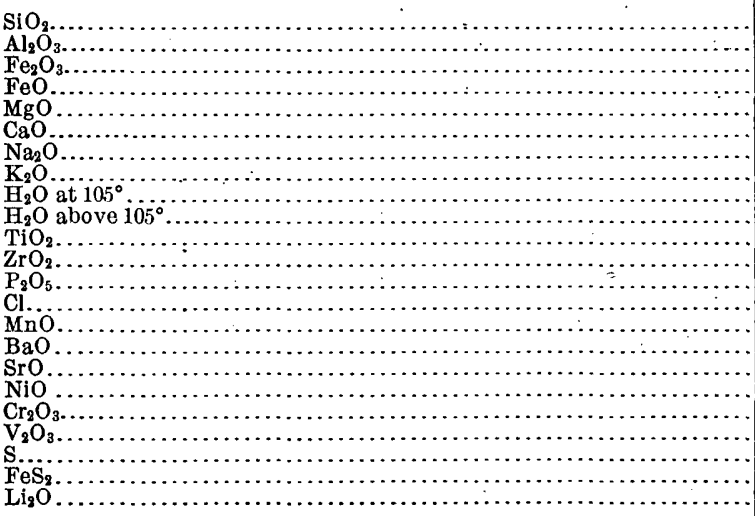 & $\begin{array}{r}68.76 \\
15.48 \\
2.50 \\
.44 \\
.56 \\
2.23 \\
3.89 \\
3.88 \\
.79 \\
.57 \\
.50 \\
.03 \\
.06 \\
.03 \\
.02 \\
.08 \\
\text { none } \\
\text { none } \\
\text { none } \\
\ldots . . . . \\
\text { none } \\
\text { none } \\
\ldots . . . .\end{array}$ & $\begin{array}{r}58.74 \\
16.02 \\
4.16 \\
3.50 \\
2.18 \\
5.12 \\
3.26 \\
2.39 \\
.83 \\
1.60 \\
1.29 \\
.05 \\
.56 \\
\text { undet. } \\
.22 \\
.10 \\
\text { trace } \\
\text { trace? } \\
\ldots \ldots . . \\
\ldots \ldots . . . \\
\ldots \ldots . . . \\
.11 \\
\text { trace }\end{array}$ & $\begin{array}{r}61.99 \\
15.81 \\
3.28 \\
2.69 \\
2.24 \\
4.62 \\
2.73 \\
2.51 \\
.91 \\
1.99 \\
.94 \\
.03 \\
.11 \\
\text { undet. } \\
\text { trace } \\
.06 \\
\text { undet. } \\
\text { undet. } \\
\text { trace } \\
\text {. . ..... } \\
\text { trace } \\
\ldots \ldots . . . \\
\text { undet. }\end{array}$ & $\begin{array}{r}49.00 \\
16.87 \\
2.09 \\
8.50 \\
6.70 \\
10.21 \\
2.57 \\
.66 \\
.72 \\
1.00 \\
1.11 \\
.02 \\
.13 \\
.05 \\
.10 \\
\text { trace } \\
\text { none } \\
\text { none } \\
.02 \\
\text { trace } \\
\text { none } \\
\ldots . . . . \\
\text { undet. }\end{array}$ \\
\hline 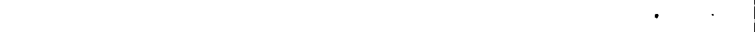 & 99.82 & 100.13 & 99.91 & 99.75 \\
\hline
\end{tabular}

\section{BRADSHAW MOUNTAINS QUADRANGLE.}

Analyses by George Steiger, record No. 1996. Petrographic data furnished by C. Palache. Published by Jaggar and Palache in Folio 126 .

A. Quartz monzonite porphyry, Battle Flat. Tonalose. Contains quartz, orthoclase, oligoclase, green hornblende, and a little apatite and magnetite, with much secondary chlorite and calcite. P. R. C. 1694.

B. Camptonite, Crazy Basin, 2 miles east of Alexandra. Shoshonose. Contains brown hornblende, augite, biotite, feldspar, magnetite, and apatite. The feldspar is apparently about equally orthoclase and andesine, but largely altered to sericite and calcite. P. R. C. 1695 .

C. Basalt, facies of trachydolerite, headwaters of Little Ash Creek. Auvergnose. Contains labradorite, violet augite, olivine, 'magnetite, and abundant apatite, with secondary serpentine. P. R. C. 1696.

D. Trachydolerite, headwaters of Little Ash Creek. Akerose. Contains oligoclase, augite, a little orthoclase and nephelite, ægirite, olivine, and abundant magnetite and apatite. P. R. C. 1697.

E. Zoisite-hornblende diorite, head of Yava Wash. Kedabekase. Contains zoisite, about 47 per cent; actinolite, 17 per cent; quartz, orthoclase, albite, chlorite, kaolin, and magnetite. P. R. C. 1698. 


\begin{tabular}{|c|c|c|c|c|c|}
\hline & A. & B. & C. & D. & E. \\
\hline 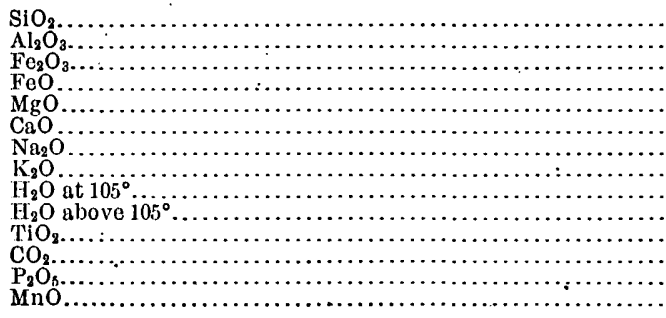 & $\begin{array}{r}60.39 \\
13.94 \\
4.07 \\
2.91 \\
2.39 \\
5.17 \\
2.68 \\
1.88 \\
1.11 \\
2.76 \\
.41 \\
2.10 \\
.07 \\
.08\end{array}$ & $\begin{array}{r}43.68 \\
16.91 \\
5.06 \\
4.01 \\
4.76 \\
8.07 \\
2.37 \\
4.44 \\
1.95 \\
3.39 \\
1.24 \\
3.13 \\
.72 \\
.07\end{array}$ & $\begin{array}{r}46.74 \\
16.96 \\
6.44 \\
4.13 \\
6.18 \\
11.90 \\
3.13 \\
.50 \\
1.24 \\
.89 \\
1.04 \\
.58 \\
.56 \\
.23\end{array}$ & $\begin{array}{r}52.06 \\
15.52 \\
5.49 \\
7.06 \\
2.23 \\
5.46 \\
5.24 \\
2.24 \\
1.00 \\
.59 \\
2.41 \\
\text { none } \\
.32 \\
.12\end{array}$ & $\begin{array}{r}45.73 \\
19.45 \\
5.28 \\
3.18 \\
6.24 \\
13.86 \\
.64 \\
.32 \\
1.57 \\
3.56 \\
.23 \\
.28 \\
\text { trace } \\
\text { none }\end{array}$ \\
\hline & 99.96 & 99.80 & 100.52 & 99.74 & 100.34 \\
\hline
\end{tabular}

\section{MORENCI DISTRICT.}

Rocks collected by Waldemar Lindgren and described in P. P. 43, p. 168. Analyses by W. F. Hillebrand, record No. 1997.

A. Fresh porphyry, Ryerson mine. 100-foot level. Lassenose.

B. Altered porphyry, same locality as A.

C. Altered porphyry, chalcocite zone, Humboldt stopes.

D. Surface alteration of altered porphyry, Copper Mountain.

E. Primary silification of porphyry, Ryerson mine.

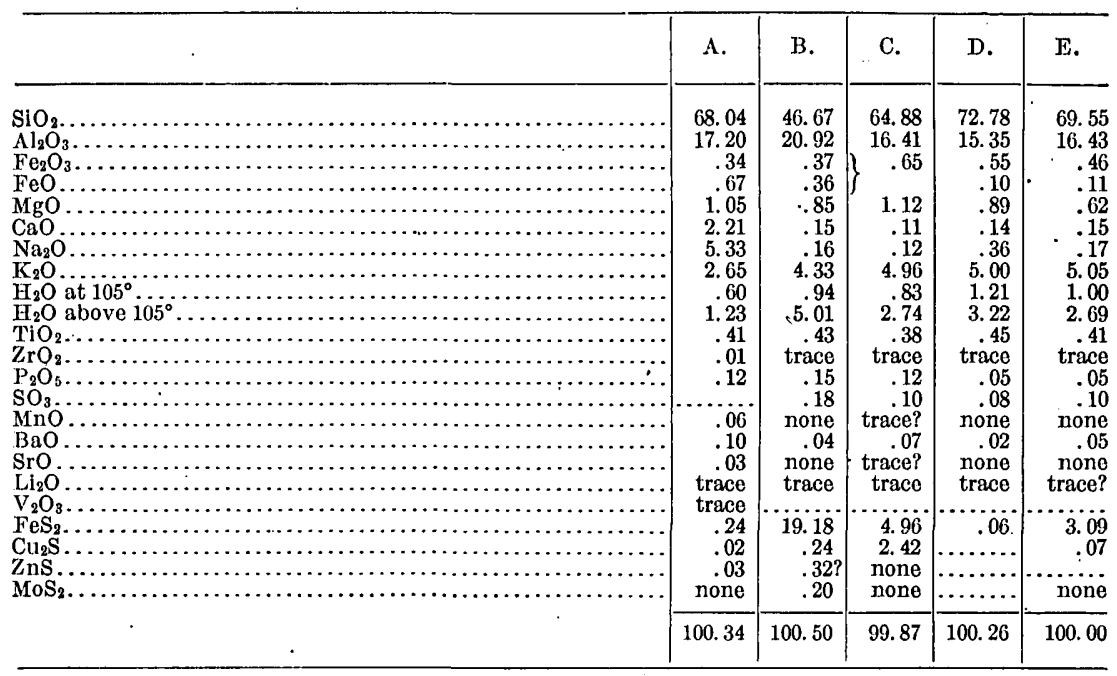

\section{MisCELLANEOUS ROCKS.}

A, B, C, D. Mica basalt; Santa Maria Basin. See Iddings, Bull. Phil. Soc. Washington, vol. 12, p. 212. Not fully described. Analyses by W. F. Hillebrand, record No. 1261.

A. Auvergnose.

$\mathrm{B}, \mathrm{C}, \mathrm{D}$. Monzonose.

E. Hornblende porphyrite, Sierra Carrizo. Dacose. Described by Cross, 14th Ann., p. 165. Contains plagioclase and hornblende, in a groundmass of quartz and orthoclase. Analysis by Hillebrand, No. 1429. 


\begin{tabular}{|c|c|c|c|c|c|}
\hline & A. & B. & C. & D. & E. \\
\hline 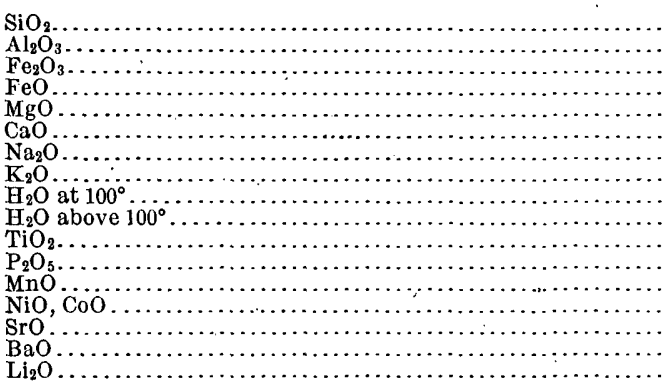 & $\begin{array}{r}49.36 \\
16.35 \\
2.93 \\
8.55 \\
7.06 \\
10.08 \\
2.67 \\
.82 \\
.22 \\
.65 \\
.98 \\
.30 \\
.19 \\
.05 \\
\text { none } \\
.04 \\
\text { none }\end{array}$ & $\begin{array}{r}55.35 \\
12.91 \\
4.67 \\
2.06 \\
6.29 \\
5.77 \\
2.65 \\
4.86 \\
2.67 \\
1.18 \\
.87 \\
.58 \\
.08 \\
.05 \\
\text { trace } \\
.19 \\
\text { trace }\end{array}$ & $\begin{array}{r}57.04 \\
13.66 \\
4.96 \\
1.77 \\
4.43 \\
6.23 \\
3.08 \\
4.95 \\
1.11 \\
1.10 \\
.94 \\
.63 \\
.17 \\
.07 \\
\text { trace } \\
.22 \\
\text { trace? }\end{array}$ & $\begin{array}{r}57.48 \\
14.09 \\
5.21 \\
1.35 \\
3.49 \\
6.05 \\
3.00 \\
4.69 \\
1.20 \\
1.37 \\
.94 \\
.65 \\
.09 \\
.08 \\
\text { trace } \\
.23 \\
\text { none }\end{array}$ & $\begin{array}{r}63.18 \\
16.47 \\
2.36 \\
2.28 \\
1.33 \\
4.77 \\
4.40 \\
2.93 \\
.27 \\
.60 \\
.60 \\
.28 \\
.15 \\
\ldots . .3 \\
.09 \\
.15 \\
\text { trace }\end{array}$ \\
\hline & 100.25 & 99.98 & 100.36 & 99.92 & 99.86 \\
\hline
\end{tabular}

F. Typical hypersthene andesite, San Francisco Mountains. Lassenose. Not described. Analysis by T. M. Chatard, record No. 270.

G. Recent lava, 2 miles south of Mount Trumbull. Camptonose. Not described. Analysis by L. G. Eakins, record No. 1024.

H. Tourmaline-bearing alkali granite, 5 miles northwest of Bisbee. Liparose. Contains microperthitic orthoclase, quartz, oligoclase, biotite, tourmaline, muscovite, apatite, zircon, and iron ore. P. R. C. 1395.

I. Granite porphyry, $3 \frac{1}{4}$ miles north of Waco Junction, Bisbee quadrangle. $S R .1$ of alaskase. Contains quartz, orthoclase, oligoclase, biotite, muscovite, zircon, and iron ore. P. R. C. 1396.

Rocks $\mathrm{H}$ and I are described by F. L. Ransome in P. P. 21. Analyses by George Steiger, record No. 2034.

\begin{tabular}{|c|c|c|c|c|}
\hline . & F. & G. & H. & I. \\
\hline $\begin{array}{l}\mathrm{SiO}_{2} \\
\mathrm{Al}_{2} \mathrm{O}_{3} \\
\mathrm{Fe}_{2} \mathrm{O}_{2} \\
\mathrm{Fe} \\
\mathrm{Mg} \\
\mathrm{MgO} \\
\mathrm{Ca}\end{array}$ & $\begin{array}{r}64.82 \\
18.27 \\
3.48 \\
.56 \\
.85 \\
2.89 \\
5.05 \\
2.67 \\
.20 \\
.56 \\
.23 \\
.20 \\
99.78\end{array}$ & $\begin{array}{r}45.30 \\
14.95 \\
1.98 \\
9.32 \\
8.29 \\
8.87 \\
4.27 \\
1.27 \\
.85 \\
2.66 \\
2.23 \\
\text { trace } \\
99.99\end{array}$ & $\begin{array}{r}75.86 \\
12.17 \\
.85 \\
.36 \\
\text { none } \\
.62 \\
3.60 \\
5.04 \\
.27 \\
.72 \\
.21 \\
\text { trace } \\
\text { none } \\
99.70\end{array}$ & $\begin{array}{r}76.81 \\
10.96 \\
1.18 \\
.08 \\
.14 \\
\text { none } \\
.26 \\
8.50 \\
.48 \\
1.17 \\
.13 \\
\text { trace } \\
\text { none } \\
99.71\end{array}$ \\
\hline
\end{tabular}

The following rocks, from Mohave County; are described by F. C. Schrader in Bull. 397. Analyses by G. Steiger, record No. 2328.

J. Minette, Champion mine, Cerbat district. Shoshonose. Contains andesine, biotite, augite, orthoclase, iron ores, and apatite, with some secondary chlorite.

K. Trachyte, Goldroad mine. Omeose. Contains orthoclase, albite, quartz, iron ores, apatite, and zircon, with secondary serpentine, epidote, and sericite.

L. Trachyte, Goldroad mine. Contains sanidine, biotite, chlorite, epidote, serpentine, and quartz. 


\begin{tabular}{|c|c|c|c|}
\hline & J. & K. & L. \\
\hline 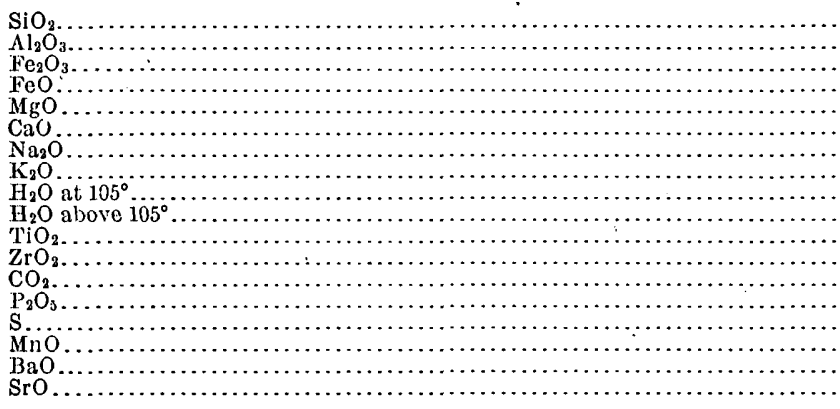 & $\begin{array}{r}49.59 \\
15.30 \\
5.01 \\
4.19 \\
4.50 \\
5.51 \\
2.24 \\
3.80 \\
2.98 \\
3.59 \\
1.50 \\
.05 \\
\text { none } \\
.94 \\
.09 \\
.11 \\
.21 \\
.12\end{array}$ & $\begin{array}{r}66.46 \\
14.14 \\
4.07 \\
.40 \\
.67 \\
.78 \\
1.26 \\
9.26 \\
.78 \\
1.28 \\
.83 \\
.05 \\
\text { none } \\
.25 \\
\text { none } \\
.03 \\
.06 \\
.03\end{array}$ & $\begin{array}{r}58.74 \\
15.09 \\
4.66 \\
.84 \\
2.75 \\
2.68 \\
.25 \\
8.05 \\
2.08 \\
3.09 \\
.98 \\
.02 \\
.61 \\
.40 \\
\text { none } \\
.09 \\
.07 \\
.04\end{array}$ \\
\hline & 99.73 & 100. 35 & 100.44 \\
\hline
\end{tabular}

NEVADA.

1. TONOPAH DISTRICT.

Rocks $A$ to $F$, inclusive, represent hornblende andesite and its various alterations. Collected by J. E. Spurr, and partly published in P. P. 42. Analyses by George Steiger, record No. 2067.

A. Hornblende andesite, California-Tonopah shaft. Pantellerose. The freshest obtainable. Contains andesine, pseudomorphs of chlorite after hornblende, some quartz, pyrite, and apatite.

B. Hornblende andesite, also relatively fresh. No. 2 shaft. Kallerudose near pantellerose. Contains partly altered, striated feldspars, and pseudomorphs of quartz, pyrite, entc., after biotite and hornblende. The rock is partly altered to quartz and muscovite.

C. Kaolinic alteration of the andesite, from a pit in the saddle between Halifax shaft and the Mizpah mine. Entirely altered to quartz, kaolin, and muscovite.

D. The andesite, altered to chlorite and calcite; Mizpah shaft, 675 feet down. Con tains chlorite, calcite, a little quartz, feldspar, sericite, hematite, zircon, and apatite.

E. Hornblende andesite, partly altered to orthoclase (?), Mizpah Hili. Ferromagnesian minerals completely decomposed. Some secondary quartz is present.

F. The andesite altered to quartz and muscovite, Mizpah vein. Little more than quartz and muscovite can be made out.

\begin{tabular}{|c|c|c|c|c|c|c|}
\hline & A. & B. & C. & D. & E. & F. \\
\hline 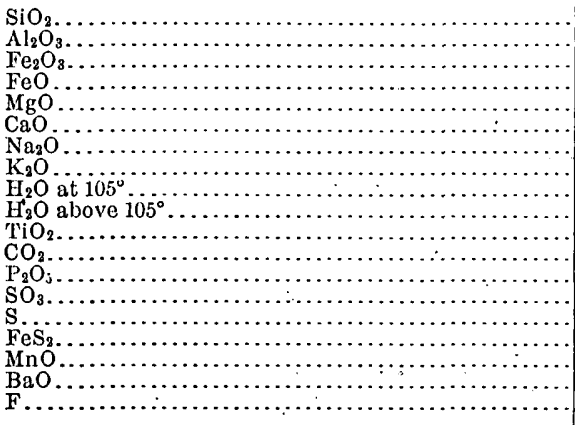 & $\begin{array}{r}58.47 \\
16.85 \\
2.04 \\
3.12 \\
3.84 \\
1.35 \\
4.30 \\
3.14 \\
1.10 \\
3.59 \\
.77 \\
.52 \\
.35 \\
\text { none } \\
\ldots . .2 \\
.49 \\
.26 \\
.11 \\
.12\end{array}$ & $\begin{array}{r}60.45 \\
17.78 \\
5.86 \\
.25 \\
1.55 \\
1.04 \\
3.58 \\
2.11 \\
2.86 \\
2.93 \\
.81 \\
\text { none } \\
.28 \\
\text { none } \\
\ldots \ldots . . . \\
\text { undet. } \\
.06 \\
.07 \\
\text {....... }\end{array}$ & $\begin{array}{r}71.14 \\
15.24 \\
1.77 \\
.26 \\
.16 \\
.09 \\
.24 \\
6.31 \\
.85 \\
2.87 \\
.48 \\
\text { none } \\
.05 \\
.05 \\
.02 \\
\ldots \ldots . . . \\
\text { undet. } \\
.17 \\
\ldots \ldots . . .\end{array}$ & $\begin{array}{r}55.60 \\
16.70 \\
2.23 \\
3.51 \\
2.60 \\
4.27 \\
4.08 \\
3.17 \\
.88 \\
3.06 \\
.72 \\
2.76 \\
.28 \\
\text { none } \\
\text { none } \\
\ldots \ldots . . . \\
\text { undet. } \\
.12 \\
\ldots . . . .\end{array}$ & $\begin{array}{r}73.50 \\
14.13 \\
1.51 \\
.26 \\
.21 \\
.12 \\
.24 \\
5.11 \\
1.07 \\
2.81 \\
.47 \\
\text { none } \\
.09 \\
.17 \\
.03 \\
\ldots \ldots . . \\
\text { undet. } \\
.19 \\
\ldots . . . .\end{array}$ & $\begin{array}{r}72.98 \\
14.66 \\
1.01 \\
.16 \\
.33 \\
.18 \\
\text { none } \\
6.03 \\
.97 \\
2.95 \\
.44 \\
\text { none } \\
.16 \\
\ldots \ldots . . \\
\text {...... } \\
\text { undet. } \\
\text { undet. } \\
\text { undet. } \\
\text {....... }\end{array}$ \\
\hline & 100. 42 & 99.63 & $\begin{array}{l}99.70 \\
\cdots\end{array}$ & 99.98 & 99.91 & 99.87 \\
\hline
\end{tabular}

SrO not looked for. 
Rocks G to M were also collected by Spurr. Analyses L, M by W: F. Hillebrand, record No. 2087; the others by George Steiger, No. 2088.

G. Early andesite, hanging wall of vein, 300-foot level, Mizpah mine. A more advanced stage of quartz-muscovite alteration than $\mathrm{F}$.

H. Extreme stage of alteration of andesite to quartz and muscovite, west drift, Mizpah vein. Quartz, with much muscovite.

I. Augite-biotite andesite, Mizpah extension shaft. Contains phenocrysts of plagioclase and augite, with some alteration to calcite and serpentine.

J. Pyroxene-biotite andesite, completely decomposed. Montana-Tonopah shaft. Feldspars entirely altered to calcite, sericite, and quartz. Biotite and hornblende altered to chlorite, calcite, quartz, sericite, siderite, and pyrite.

K. Biotite dacite, north side of Mount Brougher. Toscanose. Contains plagioclase, possibly orthoclase, biotite, and a glassy groundmass.

L. Biotite andesite, Halifax shaft. Harzose. Contains plagioclase, biotite, augite, and magnetite.

M. Biotite-pyroxene andesite, North Star shaft. Entirely altered. Feldspar altered to calcite. Pyrite, siderite, and rutile are present.

\begin{tabular}{|c|c|c|c|c|c|c|c|}
\hline . & G. & H. & I. & J. & K. & L. & M. \\
\hline 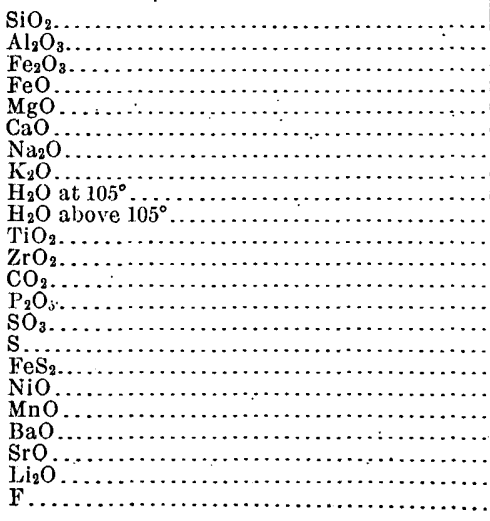 & $\begin{array}{r}76.25 \\
12.84 \\
.54 \\
.33 \\
.56 \\
.16 \\
.12 \\
3.20 \\
2.14 \\
3.17 \\
.37 \\
\ldots \ldots \\
\text { none } \\
.12 \\
\ldots . . . \\
\ldots \ldots . .\end{array}$ & $\begin{array}{r}91.40 \\
4.31 \\
.77 \\
.11 \\
.18 \\
\text { none } \\
.06 \\
1.68 \\
.46 \\
.98 \\
.07 \\
.02 \\
\text { none } \\
.04 \\
\text { none } \\
\text { none } \\
\ldots \ldots \ldots \\
.06 \\
.02 \\
\ldots \ldots \ldots \\
\text { trace }\end{array}$ & $\begin{array}{r}43.00 \\
16.49 \\
2.86 \\
6.31 \\
6.19 \\
5.69 \\
.12 \\
.84 \\
3.00 \\
7.93 \\
.89 \\
\ldots . . . \\
4.19 \\
.36 \\
.08 \\
\ldots \ldots . . \\
2.55 \\
\ldots \ldots . . \\
\ldots \ldots . . \\
.07 \\
\text { none } \\
\ldots \ldots . .\end{array}$ & $\begin{array}{r}57.51 \\
16.55 \\
3.20 \\
2.02 \\
2.30 \\
6.06 \\
2.76 \\
2.81 \\
1.45 \\
2.56 \\
.80 \\
\ldots \ldots . \\
1.91 \\
.30 \\
\text { none } \\
.02 \\
\ldots \ldots \ldots \\
\ldots \ldots . . \\
. \ldots .17 \\
\ldots \ldots . . \\
\ldots \ldots . .\end{array}$ & $\begin{array}{r}71.71 \\
14.00 \\
1.06 \\
.51 \\
.43 \\
2.25 \\
3.21 \\
4.41 \\
.44 \\
1.38 \\
.28 \\
\ldots \ldots . . \\
\text { trace } \\
.07 \\
.54 \\
\text { none } \\
\ldots \ldots . . \\
\ldots \ldots . . \\
\ldots \ldots . . \\
\ldots \ldots . . \\
\ldots \ldots . .\end{array}$ & $\begin{array}{r}56.26 \\
16.18 \\
5.56 \\
1.17 \\
2.78 \\
5.07 \\
3.25 \\
3.43 \\
2.07 \\
2.61 \\
.73 \\
\text { trace? } \\
.62 \\
.32 \\
\text { none } \\
\ldots \ldots . . \\
.03 \\
\text { trace } \\
.21 \\
.12 \\
.06 \\
\text { trace }\end{array}$ & $\begin{array}{r}51.64 \\
1.5 .58 \\
.16 \\
.58 \\
2.79 \\
6.25 \\
.27 \\
2.46 \\
2.56 \\
4.43 \\
.73 \\
\text { trace? } \\
4.24 \\
.31 \\
.03 \\
\ldots . .8 \\
7.89 \\
\text { none } \\
.21 \\
\text { undet. } \\
\text { trace } \\
\text { (?) }\end{array}$ \\
\hline & 99.80 & 100.16 & 100.57 & 100. 42 & 100.29 & 100.47 & 100.13 \\
\hline
\end{tabular}

2. GOLDFIELD DISTRICT.

Rocks collected by F. L. Ransome, who supplies the petrographic data. Analyses by G. Steiger, record Nos. 2249, 2253, 2339.

A. Altered rhyolite, east slope of Vindicator Mountain. Contains quartz, alunite, calcite, and sericite, with a small amount of undeterminable material.

B. Pyroxene-hornblende andesite, first hill northwest of Vindicator Mountain. Tonalose, near yellowstonose. Contains labradorite, augite, orthorhombic pyroxene, hornblende, magnetite, and apatite, in a glassy base.

C. Hornblende-pyroxene andesite, 2 miles northeast of Black Butte. Tonalose. Contains plagioclase, augite, enstatite or bronzite, hornblende, and magnetite, in a glassy groundmass..

D. Hornblende-biotite andesite, 1 mile northeast of Black Butte. Amiatose, near yellowstonose. Contains labradorite, hornblende, and biotite, in a glassy groundmass with specks of magnetite.

E. Pyroxene-hornblende andesite, 1 mile northeast of Columbia Mountain. Tonalose. Contains labradorite; augite, hypersthene, magnetite, apatite, and glass. Traces of secondary calcite and chlorite. 


\begin{tabular}{|c|c|c|c|c|c|}
\hline e & A. & 13. & C. & D. & E. \\
\hline 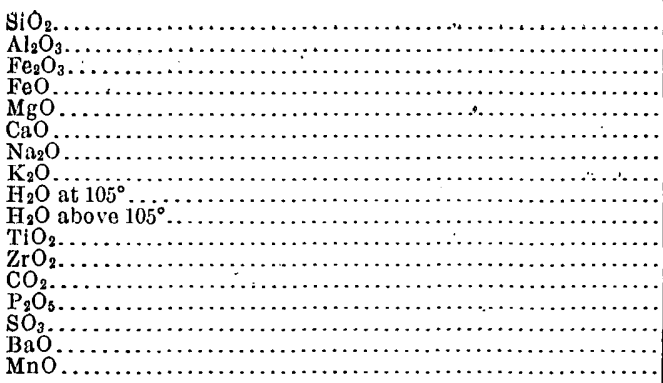 & $\begin{array}{r}75.78 \\
10.65 \\
.68 \\
.16 \\
\text { none } \\
1.67 \\
.22 \\
.96 \\
1.16 \\
3.54 \\
.12 \\
.02 \\
.76 \\
.02 \\
4.73 \\
.02 \\
\text { none }\end{array}$ & $\begin{array}{r}60.41 \\
16.59 \\
3.18 \\
1.70 \\
2.13 \\
5.26 \\
3.94 \\
1.70 \\
1.94 \\
2.64 \\
.62 \\
\ldots . . . \\
\text { none } \\
.31 \\
\ldots . . . \\
\ldots \ldots . . \\
.07\end{array}$ & $\begin{array}{r}58.06 \\
17.48 \\
3.83 \\
1.01 \\
2.60 \\
6.33 \\
3.34 \\
2.61 \\
.97 \\
1.88 \\
.87 \\
\ldots \ldots . . \\
\text { none } \\
.27 \\
\ldots \ldots . . \\
\ldots . . . \\
.09\end{array}$ & $\begin{array}{r}63.16 \\
16.74 \\
3.94 \\
.47 \\
1.12 \\
4.07 \\
3.88 \\
3.71 \\
1.12 \\
.77 \\
.61 \\
\ldots . . . \\
\text { none } \\
.30 \\
\ldots . . . \\
\ldots . . . . \\
.04\end{array}$ & $\begin{array}{r}54.66 \\
17.53 \\
3.18 \\
3.52 \\
3.62 \\
7.33 \\
3.56 \\
2.22 \\
.59 \\
.69 \\
.99 \\
\ldots . .35 \\
1.35 \\
.30 \\
\ldots . . . \\
\ldots . .12\end{array}$ \\
\hline & 100.49 & 100.49 & .100 .19 & 99.93 & 99.66 \\
\hline
\end{tabular}

F. Dacite, near summit of Diamond Peak. Harzose near tonalose. Contains plagioclase, hornblende, augite, biotite, quartz, apatite, magnetite, and glass.

G. Altered dacite, Combination mine. Contains quartz, kaolin, sericite, pyrite and possibly alunite.

H. Biotite dacite vitrophyre, 1 mile southwest of Black Cap Mountain. Amiatose. Contains labradorite, biotite, and quartz in a glassy base.

I. Dacite, one-half mile northeast from summit of Columbia Mountain. Tonalose. Contains the same minerals as $\mathrm{F}$.

J. Mica dolerite, 2 miles east of Black Butte. Hessose. Contains anorthite, augite, biotite, olivine, magnetite, occasional hornblende, scanty apatite, and a little calcite. The olivine is serpentinized.

K. Olivine dolerite, mesa above Rabbit Spring. Hessose. Contains anorthite and olivine in a groundmass of plagioclase, augite, olivine, magnetite, and apatite. A very little hornblende is also present, with a trace of calcite. The olivine is serpentinized.

\begin{tabular}{|c|c|c|c|c|c|c|}
\hline & F. & G. & H. & I. & J. & $\mathbf{K}$ \\
\hline 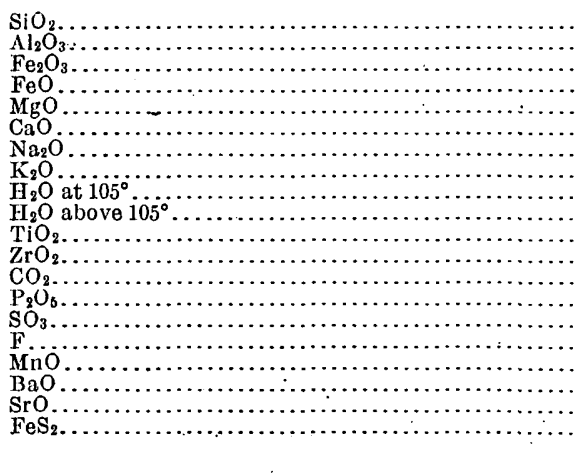 & $\begin{array}{r}61.25 \\
15.92 \\
3.75 \\
1.17 \\
2.28 \\
5.39 \\
3.19 \\
3.23 \\
1.08 \\
1.88 \\
.71 \\
\ldots \ldots \ldots \\
\text { none } \\
.22 \\
\ldots \ldots . . \\
\ldots .08 \\
\ldots \ldots . . . \\
\ldots \ldots . . . \\
100.15\end{array}$ & $\begin{array}{r}60.53 \\
15.32 \\
.20 \\
.14 \\
.06 \\
.41 \\
.84 \\
1.06 \\
1.33 \\
6.60 \\
.80 \\
.01 \\
\text { none } \\
.27 \\
5.97 \\
\text { trace } \\
\text { trace } \\
.06 \\
\text { undet. } \\
7.20 \\
100.80 \\
\end{array}$ & \begin{tabular}{r}
59.99 \\
16.14 \\
4.42 \\
.13 \\
1.51 \\
4.17 \\
3.04 \\
2.82 \\
3.35 \\
4.06 \\
.64 \\
$\ldots \ldots .$. \\
none \\
.24 \\
$\ldots \ldots . .$. \\
\hdashline$\ldots .$. \\
.05 \\
\hdashline$\ldots . .$. \\
\hdashline$\ldots . .$. \\
\hdashline$\ldots . .$. \\
\end{tabular} & $\begin{array}{r}59.95 \\
15.77 \\
3.34 \\
2.34 \\
2.73 \\
5.84 \\
3.07 \\
2.52 \\
.95 \\
2.00 \\
.82 \\
.02 \\
\text { none } \\
.26 \\
\ldots \ldots . . \\
\text { none } \\
.09 \\
.11 \\
.13 \\
\ldots . . . \\
99.94 \\
.13\end{array}$ & 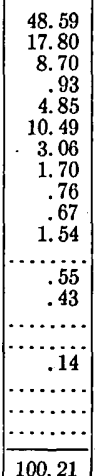 & 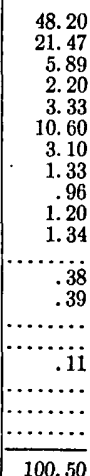 \\
\hline
\end{tabular}




\section{BULLFROG DISTRICT.}

Rocks collected and described by F. L. Ransome. Analyses by G. Steiger, record No. 2272.

A. Porphyritic rhyolite, southeast slope of Bush Peak. Contains quartz, orthoclase, plagioclase, mica, magnetite, and apatite.

B. Rhyolitic glass, west slope of Burton Peak, near summit. Contains phenocrysts of plagioclase, hornblende, and mica.

C. Dacite, east slope of Black Peak. Contains plagioclase, orthoclase, quartz, mica, and magnetite, in a glassy groundmass.

D. Quartz basalt, knob east of summit of Black Peak. Contains plagioclase, quartz, orthoclase, olivine, pyroxene, biotite, and magnetite. Olivine serpentinized.

E. Leucite basalt, road to Indian Springs, $1 \frac{1}{2}$ miles north of Rhyolite. Contains olivine, leucite, augite, apatite, and possibly a little nepheline.

\begin{tabular}{|c|c|c|c|c|c|}
\hline & A. & B. & C. & D. & E. \\
\hline 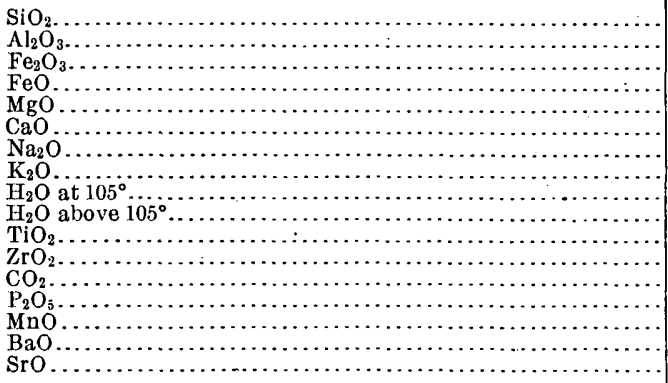 & $\begin{array}{r}77.26 \\
11.54 \\
.85 \\
.13 \\
.20 \\
.58 \\
2.96 \\
4.65 \\
1.03 \\
.96 \\
.18 \\
\ldots . . . \\
\text { none } \\
\text { trace } \\
.03 \\
\text { none } \\
\text { none }\end{array}$ & $\begin{array}{r}71.60 \\
12.44 \\
1.00 \\
.65 \\
.06 \\
1.90 \\
3.30 \\
4.22 \\
.81 \\
3.78 \\
.25 \\
.01 \\
\text { none } \\
.08 \\
.06 \\
.03 \\
.03\end{array}$ & $\begin{array}{r}63.34 \\
15.46 \\
4.14 \\
.39 \\
.66 \\
2.01 \\
3.89 \\
5.31 \\
1.89 \\
1.16 \\
1.53 \\
\ldots \ldots \\
\text { none } \\
.22 \\
.04 \\
.15 \\
.03\end{array}$ & $\begin{array}{r}59.72 \\
14.63 \\
3.40 \\
2.37 \\
2.69 \\
6.55 \\
3.28 \\
3.33 \\
.72 \\
1.38 \\
.95 \\
\ldots .13 \\
1.12 \\
.40 \\
.10 \\
.04 \\
\text { undet. }\end{array}$ & $\begin{array}{r}43.62 \\
12.73 \\
4.89 \\
4.10 \\
9.37 \\
11.62 \\
2.96 \\
1.30 \\
1.91 \\
3.94 \\
1.46 \\
.02 \\
.63 \\
.82 \\
.12 \\
.16 \\
.14\end{array}$ \\
\hline & 100.37 & 100.22 & 100.22 & 100.68 & 99.79 \\
\hline
\end{tabular}

4. MiscellaneOUS ROCKS.

A. Rhyolite, south-southeast of McClellan Peak, Washoe. Mihalose. Contains feldspars, orthoclase predominating over plagioclase, quartz, mica, and hornblende.

B. Dacite, spur northeast of McClellan Peak. Toscanose. More plagioclase than orthoclase, much mica, less hornblende, little quartz.

Rocks $\mathrm{A}$ and $\mathrm{B}$ are described by Hague and Iddings in Bull. 17. Analyses by F. A. Gooch, records Nos. 119, 129. FeO not separately determined.

C. Basalt, summit of Richmond Mountain, Eureka. Hessose. Red, porous. Contains augite, less hypersthene, feldspars, and magnetite, in a glassy base, with accessory olivine and quartz. Analysis by J. E. Whitfield, record No. 424.

D. Andesitic perlite, south of Carbon Ridge, Eureka. Amiatose. Contains plagioclase, hornblende, biotite, quartz, hypersthene, augite, magnetite, apatite, and zircon, with a glassy base. Analysis by W. H. Melville, record No. 1240.

Rocks $\mathrm{C}$ and $\mathrm{D}$ are described by Hague and Iddings in Mon. XX.

E. Dacite, west side of Silver Peak range, south of Emigrant Road. Lassenosetoscanose. Contains phenocrysts of plagioclase, sanidine, possibly quartz, biotite, hornblende, augite, magnetite, and apatite.

F. Spherulitic rhyolite, southeast of Red Mountain, Silver Peak range. Toscanose. Contains sanidine, quartz, biotite, titanite, magnetite, and zircon, in a spherulitic groundmass.

Analyses $\mathrm{E}$ and $\mathrm{F}$ by George Steiger, record No. 1887. Rocks E, F, collected by H. W. Turner. Described in P. P. 55. 


\begin{tabular}{|c|c|c|c|c|c|c|}
\hline & A. & B. & C. & D. & E. & F. \\
\hline 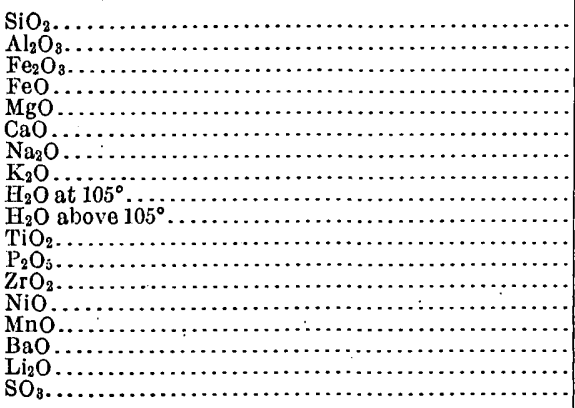 & $\begin{array}{r}73.07 \\
11.78 \\
2.30 \\
.39 \\
2.02 \\
1.19 \\
6.84 \\
a 2.24 \\
\ldots \ldots \ldots\end{array}$ & 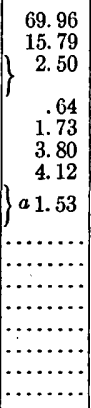 & $\begin{array}{r}50.38 \\
19.83 \\
6.05 \\
2.00 \\
5.36 \\
10.03 \\
2.15 \\
1.76 \\
1.37 \\
\text { none } \\
\text { none } \\
\ldots \ldots . . . \\
\ldots .38 \\
\ldots . . . \\
\text { trace } \\
.83\end{array}$ & \begin{tabular}{|r|}
65.13 \\
15.73 \\
2.24 \\
1.86 \\
1.49 \\
3.62 \\
2.93 \\
3.96 \\
.52 \\
1.91 \\
.58 \\
.23 \\
$\ldots \ldots .$. \\
.07 \\
trace \\
$\ldots \ldots . .$. \\
$\ldots \ldots . .$. \\
$\ldots \ldots . .$.
\end{tabular} & $\begin{array}{r}69.76 \\
14.05 \\
2.05 \\
\text { none } \\
.17 \\
1.73 \\
3.90 \\
3.57 \\
.62 \\
3.65 \\
.19 \\
.07 \\
\ldots \ldots . \\
\ldots .10 \\
.14 \\
\ldots \ldots . . \\
\ldots \ldots . . .\end{array}$ & $\begin{array}{r}72.54 \\
13.32 \\
2.41 \\
.09 \\
.51 \\
1.37 \\
3.40 \\
5.25 \\
.21 \\
.97 \\
.35 \\
.11 \\
.06 \\
\ldots . . . \\
\text { none } \\
.03 \\
\ldots \ldots . . \\
\ldots . . .\end{array}$ \\
\hline & 99.83 & 100.07 & 100.14 & 100.27 & 100.00 & 100.62 \\
\hline
\end{tabular}

a Loss on ignition.

The following rocks, G to X, inclusive, were collected by J. E. Spurr, who furnishes the petrographic data.

G. Olivine basalt, Crater, Silver Peak. Hessose. Analysis by George Steiger, record No. 1883. Contains plagioclase, olivine, and magnetite, in a groundmass of feldspar and olivine.

H. Hornblende-pyroxene-biotite-quartz latite, Coyote Springs, north end of Pahute Range. Harzose. Analysis by W. F. Hillebrand, record No. 1881. Phenocrysts of hornblende, biotite, plagioclase, quartz, and magnetite, in a brown, glassy groundmass.

I. Biotite-quartz monzonite, Cherry Creek, Egan Range. Amiatose. Analysis by H. W. Stokes, record No. 1882. Contains quartz, biotite, and plagioclase.

J. Quartz-muscovite rock, Belmont. Described by Spurr in Amer. Jour. Sci., 4th ser., vol. 10, p. 358. Analysis by Stokes, No. 1882. Contains quartz and muscovite, with some calcite and orthoclase, and a little zircon. P. R. C. 1272.

$\mathrm{K}$. Hornblende-quartz andesite, Masons Butte, Walker River valley. Andose. Described by Spurr, Jour. Geology, vol. 9, p. 593. Analysis by Stokes, record No. 1882. Phenocrysts of feldspar, pale-green hornblende (partly altered to calcite, chlorite, and epidote), and quartz, in a groundmass of feldspar and hornblende, with quartz, pyrite, and siderite.

L., Hornblende-biotite-quartz diorite, Masons Butte. Tonalose. Described by Spurr, as under K. Analysis by Stokes, No. 1882. Contains oligoclase, quartz, hornblende, and biotite.

\begin{tabular}{|c|c|c|c|c|c|c|}
\hline . & G. & H. & I. & J. & I. & L. \\
\hline 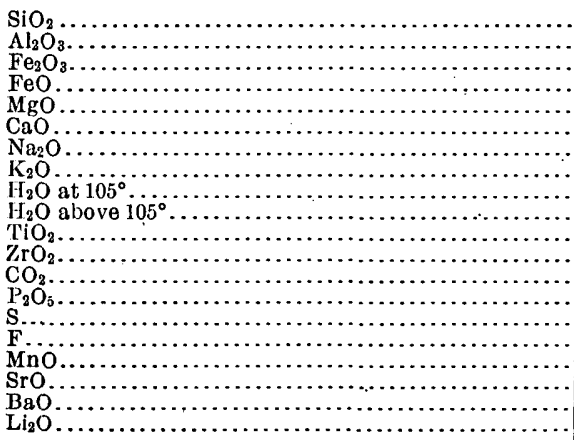 & \begin{tabular}{r|}
47.09 \\
18.40 \\
2.66 \\
5.62 \\
7.06 \\
10.19 \\
2.37 \\
1.34 \\
.66 \\
2.37 \\
1.19 \\
none \\
.54 \\
.03 \\
none \\
undet. \\
.17 \\
$\ldots \ldots$
\end{tabular} & $\begin{array}{r}62.97 \\
15.52 \\
4.03 \\
1.45 \\
2.07 \\
5.31 \\
3.31 \\
3.46 \\
.66 \\
.48 \\
.67 \\
\text { trace } \\
.18 \\
.18 \\
\text { trace } \\
\text { undet. } \\
.10 \\
.02 \\
.09 \\
\text { trace }\end{array}$ & $\begin{array}{r}67.60 \\
15.89 \\
1.77 \\
1.82 \\
.96 \\
3.38 \\
3.39 \\
3.39 \\
.23 \\
.87 \\
.60 \\
\ldots \ldots . . \\
\text { none } \\
.19 \\
\ldots . . . . \\
\ldots . . . \\
\text { trace } \\
\text { trace } \\
\text { undet. }\end{array}$ & $\begin{array}{r}84.15 \\
9.67 \\
.51 \\
.07 \\
.04 \\
.53 \\
2.65 \\
1.57 \\
.21 \\
.74 \\
\text { trace } \\
\ldots \ldots . . \\
\text { none } \\
\text { trace } \\
\ldots . . . \\
.02 \\
\text { trace } \\
\text { trace } \\
\text { undet. }\end{array}$ & $\begin{array}{r}53.37 \\
16.57 \\
3.84 \\
2.45 \\
5.79 \\
6.30 \\
3.40 \\
2.55 \\
.39 \\
2.23 \\
.86 \\
\ldots \ldots 1 \\
1.61 \\
.29 \\
\ldots \ldots . . \\
\ldots \ldots . . . \\
.08 \\
\text { trace } \\
\text { undet. } \\
\ldots . . . .\end{array}$ & $\begin{array}{r}60.25 \\
17.90 \\
3.08 \\
2.44 \\
2.44 \\
5.57 \\
4.29 \\
1.89 \\
.20 \\
1.24 \\
.65\end{array}$ \\
\hline & 99.69 & 100.50 & 100.09 & 100.16 & 99.73 & 100.33 \\
\hline
\end{tabular}


M. Biotite rhyolite, south end of Pine Nut Range. Lassenose. Described by Spurr, Jour. Geology, vol. 9, p. 599. Analysis by Stokes, record No. 1882. Contains quartz, orthoclase, and striated feldspars, in a microcrystalline groundmass.

N. Siliceous granite, south end of Pine Nut Range. Liparose-toscanose. Description by Spurr and analysis by Stokes as under M. Contains quartz, orthoclase, microcline, anorthoclase, albite, biotite, hornblende, and titanite.

O. Tordrillite, Cactus Corral, Ralston Desert. Alaskose-liparose. Analysis by Steiger, record No. 1883. Consists mainly of quartz and orthoclase.

P. Tordrillite, Tybo, Hot Creek Range. SR. 4 of alaskase. Analysis by Steiger, No. 1883. Contains orthoclase and quartz, with secondary sericite.

Q. Siliceous rhyolite, west side of Quinn Canyon Range. Toscanose. Described by Spurr, Jour. Geology, vol. 9, p. 602. Analysis by Steiger, No. 1883.

R. Biotite granite, west side of Quinn Canyon Range. Toscanose. Description by Spurr and analysis by Steiger as under $Q$. Contains essential quartz and orthoclase, with accessory hornblende, titanite, magnetite, and a little striated feldspar.

\begin{tabular}{|c|c|c|c|c|c|c|}
\hline & M. . & N. & O. & P. & Q. & R. \\
\hline $\begin{array}{l}\mathrm{SiO}_{2} \\
\mathrm{Al}_{2} \mathrm{O}_{3} \\
\mathrm{~F}_{2} \mathrm{O}_{3}\end{array}$ & $\begin{array}{r}71.49 \\
15.06 \\
1.51 \\
.88 \\
.35 \\
1.54 \\
4.19 \\
3.39 \\
.16 \\
.88 \\
.20 \\
\text { none } \\
.08 \\
\ldots \ldots . . \\
\text { trace } \\
\text { trace } \\
\text { undet. }\end{array}$ & $\begin{array}{r}75.09 \\
13.51 \\
1.13 \\
.08 \\
.18 \\
.91 \\
3.58 \\
4.71 \\
.17 \\
.25 \\
.22 \\
\text { none } \\
.04 \\
\ldots \ldots . . \\
\ldots \ldots . . \\
\text { trace } \\
\text { trace } \\
\text { undet. }\end{array}$ & $\begin{array}{r}75.70 \\
13.33 \\
.40 \\
.06 \\
.06 \\
.55 \\
3.19 \\
5.39 \\
.31 \\
1.17 \\
.02 \\
\text { none } \\
.07 \\
\ldots . . . \\
.05 \\
\text { trace } \\
\text { undet. } \\
.04\end{array}$ & $\begin{array}{r}77.84 \\
13.20 \\
.80 \\
.25 \\
. .11 \\
.20 \\
4.06 \\
1.55 \\
.37 \\
1.21 \\
\text { none } \\
\text { none } \\
.04 \\
\text { trace } \\
.10 \\
\text { none } \\
\text { none } \\
\text { none }\end{array}$ & $\begin{array}{r}74.67 \\
13.25 \\
1.06 \\
.18 \\
\text { trace } \\
1.26 \\
3.99 \\
4.62 \\
.18 \\
.22 \\
.07 \\
.79 \\
.06 \\
\ldots . . . \\
\text { trace } \\
\text { none } \\
\text { none } \\
\text { none }\end{array}$ & $\begin{array}{r}71.48 \\
13.00 \\
1.25 \\
1.55 \\
.95 \\
2.60 \\
2.60 \\
4.24 \\
.20 \\
1.24 \\
.43 \\
.30 \\
.09 \\
\ldots . . . \\
\text { none } \\
.09 \\
\text { undet. } \\
.09\end{array}$ \\
\hline . & 99.73 & 99.87 & 100:34 & 99.73 & 100.35 & 100.11 \\
\hline
\end{tabular}

S. Biotite-hornblende-quartz latite, Bullionville, near Pioche. Amiatose. Analysis by W. F. Hillebrand, record No. 1881. Contains abundant quartz, plagioclase, biotite, hornblende, and magnetite, in a glassy groundmass.

T. Biotite rhyolite, Meadow Creek Canyon. Liparose-toscanose. Analysis by Hillebrand, No. 1881. Contains quartz, orthoclase, some microcline, and a little biotite, in a glassy groundmass.

U. Tordrillite, Meadow Creek Canyon. Liparose. Analysis by Hillebrand, No. 1881. Contains quartz, orthoclase, and plagioclase, with accessory biotite, in a glassy groundmass.

V. Tordrillite, Sweetwater. 'Toscanose-liparose. Analysis by George Steiger, record No. 1883. Contains orthoclase and a little anorthoclase, in a feldspathic groundmass.

W. Quartz-muscovite rock, Southern Klondike mine. Near SR. 1 of riesenase. Analysis by Hillebrand, No. 2087. Consists mainly of quartz and muscovite.

X. Another sample like W. SR. 1 of dargase. Same analyst, number, and descrip.tion. 


\begin{tabular}{|c|c|c|c|c|c|c|}
\hline . & S. & T. & U. & V. & W. & X. \\
\hline 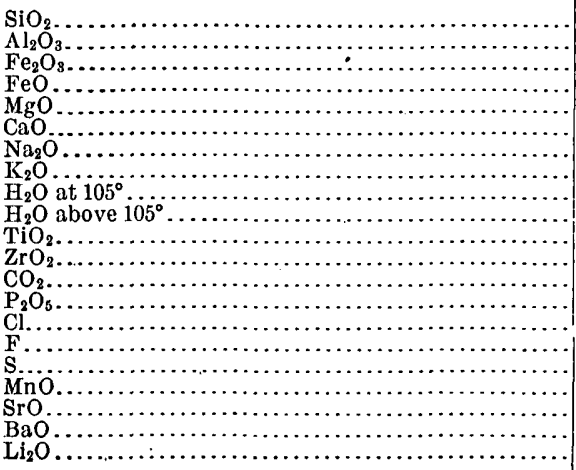 & $\begin{array}{r}63.08 \\
15.74 \\
4.94 \\
.42 \\
1.38 \\
4.10 \\
3.16 \\
3.39 \\
1.70 \\
.98 \\
.71 \\
.02 \\
\text { trace } \\
.17 \\
\cdots \ldots . . \\
\text { trace } \\
.07 \\
.04 \\
.10 \\
\text { trace }\end{array}$ & $\begin{array}{r}72.96 \\
12.32 \\
.76 \\
.03 \\
.35 \\
2.18 \\
3.24 \\
4.88 \\
.80 \\
1.63 \\
.11 \\
\text { trace } \\
.92 \\
.10 \\
\ldots \ldots . \\
\ldots . . . \\
\text { none } \\
\text { trace } \\
\text { trace } \\
\text { trace } \\
\text { trace }\end{array}$ & $\begin{array}{r}74.00 \\
13.48 \\
1.73 \\
.06 \\
.13 \\
.63 \\
4.63 \\
5.12 \\
.11 \\
.15 \\
.16 \\
.04 \\
\text { trace } \\
.06 \\
\ldots . . . \\
\ldots \ldots . . \\
\text { trace } \\
\text { trace } \\
\text { none } \\
.05 \\
\text { trace }\end{array}$ & \begin{tabular}{|r}
74.30 \\
13.29 \\
1.15 \\
.10 \\
.09 \\
.85 \\
3.75 \\
4.83 \\
.91 \\
.50 \\
.20 \\
$\ldots \ldots$ \\
none \\
.07 \\
$\ldots \ldots .$. \\
$\ldots \ldots .$. \\
.03 \\
trace \\
undet. \\
none \\
$\ldots \ldots . .$.
\end{tabular} & \begin{tabular}{|r|}
72.69 \\
$a 13.42$ \\
.97 \\
.32 \\
.26 \\
1.97 \\
.21 \\
3.36 \\
2.36 \\
2.60 \\
undet. \\
$\ldots \ldots .$. \\
undet. \\
trace \\
.25 \\
none \\
present \\
$\ldots \ldots . .$. \\
$\ldots \ldots . .$. \\
$\ldots . . .$.
\end{tabular} & 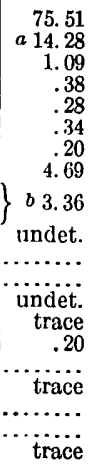 \\
\hline \multirow[t]{2}{*}{ 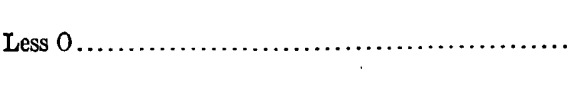 } & $\begin{array}{c}100.00 \\
\ldots \ldots\end{array}$ & $\begin{array}{c}100.28 \\
\ldots \ldots\end{array}$ & $\begin{array}{c}100: 35 \\
\ldots \ldots \ldots\end{array}$ & $\begin{array}{c}100.07 \\
\ldots \ldots\end{array}$ & $\begin{array}{r}99.38 \\
.10\end{array}$ & $\begin{array}{r}100.33 \\
.08\end{array}$ \\
\hline & & & & & 99.28 & 100.25 \\
\hline
\end{tabular}

\section{CALIFORNIA.}

\section{MOUNT SHASTA.}

Description of rocks furnished by J. S. Diller, who also described A, B, and C in Bull. 150, pp. 221, 227.

A. Hornblende andesite, Black Butte, west base of Shasta. Yellowstonose. Contains plagioclase and hornblende in a microlitic groundmass. The latter carries hypersthene, magnetite, and amorphous matter. Analysis by W. H. Melville, record No. $1346 . \quad$ P. R. C. 84.

B. Hypersthene andesite, older flow, west base of Shasta. Yellowstonose. Contains plagioclase, hypersthene, magnetite, and glass. Analysis by Melville, No. 1346. P. R. C. 87.

C. Hypersthene andesite, like B, same locality, later flow. Tonalose. Analysis by Melville, No. 1346. P. R. C. 87.

D. Pyroxene andesite, late flow, eastern side of Shasta. Tonalose. Contains small crystals of plagioclase and hornblende in a dark groundmass. Thin section not examined. Analysis by H. N. Stokes, record No. 1532. P. R. C. 1415.

E. Plagioclase basalt from near McCloud River, south of Mount Shasta. Hessose. A gray cellular rock. Thin section not examined. Analysis by Stokes, No. 1532. P. R. C. 1416.

F. Andesite basalt, Delta, Shasta County. Beerbachose. Much plagioclase and a few hypersthene crystals in a groundmass chiefly of feldspar, pyroxene, magnetite, and trace of olivine. Analysis by Melville, No. 1346. P. R. C. 1417. 


\begin{tabular}{|c|c|c|c|c|c|c|}
\hline & A. & B. & C. & D. & E. & F. \\
\hline 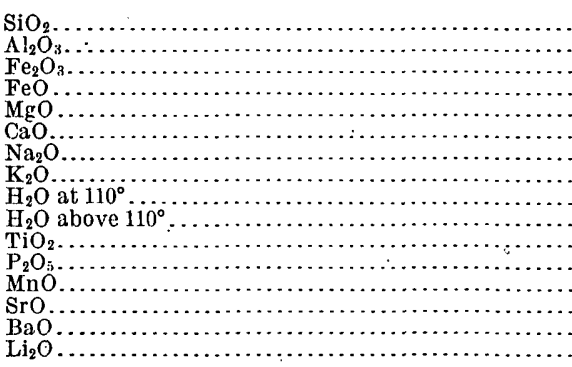 & $\begin{array}{r}64.48 \\
19.28 \\
1.40 \\
1.78 \\
1.64 \\
5.06 \\
4.41 \\
1.12 \\
.06 \\
\text { undet. } \\
\ldots . . . .\end{array}$ & $\begin{array}{r}64.52 \\
18.31 \\
.90 \\
2.51 \\
2.35 \\
5.11 \\
4.64 \\
1.25 \\
\quad .20 \\
\text { undet. } \\
\ldots \ldots . . . \\
\ldots \ldots \ldots . . .\end{array}$ & 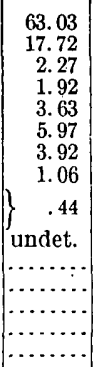 & $\begin{array}{r}61.58 \\
16.96 \\
1.75 \\
2.85 \\
3.67 \\
6.28 \\
3.94 \\
1.28 \\
.24 \\
1.06 \\
.49 \\
.22 \\
\text { trace } \\
\text { trace } \\
.03 \\
\text { trace }\end{array}$ & $\begin{array}{r}47.94 \\
18.90 \\
2.21 \\
8.59 \\
8.21 \\
9.86 \\
2.81 \\
.29 \\
.39 \\
.74 \\
.57 \\
.15 \\
\text { trace } \\
\text { none } \\
\text { none } \\
\text { trace }\end{array}$ & $\begin{array}{r}55.08 \\
18.93 \\
2.02 \\
5.56 \\
5.17 \\
8.40 \\
4.23 \\
.74 \\
.29 \\
\text { trace } \\
\ldots \ldots . . \\
\ldots \ldots . . \\
\ldots \ldots . . . \\
\ldots \ldots . .\end{array}$ \\
\hline 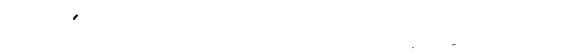 & 99.23 & 99.79 & 99.96 & 100.35 & 100.66 & 100.42 \\
\hline
\end{tabular}

The following rocks are from Shasta County, but not within either the Mount Shasta or the Lassen Peak areas. Collected by J. S. Diller, who furnishes the petrographic data.

A. Dacite porphyry, east fork of Clear Creek, 9 miles above French Gulch. Lassenose. Contains conspicuous phenocrysts of plagioclase, a few of quartz, and smaller - ones of biotite and pyroxene, in a groundmass of quartz and feldspar. Analysis by J. E. Whitfield, record No. 970. P. R. C..1418.

B. Dacite porphyry; Smiths Gulch, 6 miles up Clear Creek from French Gulch. Yellowstonose. Contains phenocrysts of plagioclase, quartz, biotite, and hornblende, in a groundmass chiefly of quartz and feldspar. Analysis by Whitfield, No. 971. Also described by J. P. Iddings in Bull. 150, p. 233 . P. R. C. 90.

C. Diorite, from Ono. Yellowstonose. Not described. Analysis by T. M. Chatard, record. No. 1107.

D. Metarhyolite. The country rock near the Bully Hill mine. Rich in porphyritic quartz.

E. Metabasalt. Country rock, wall of ore body, Bully Hill mine. Porphyritic quartz absent.

Analyses D, E, by E. T. Allen, record No. 1981. The rocks are described by Diller in Bull. 213.

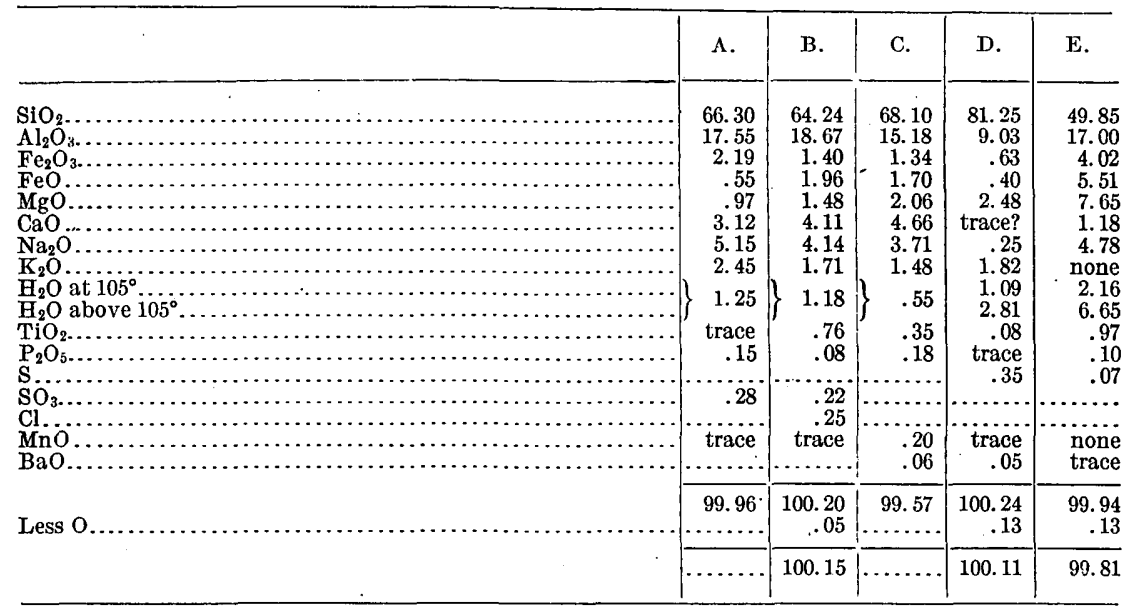

The sixteen following rocks, from mining districts in Shasta County, were collected by B. S. Butler, who furnishes the mineralogical data. All but six of them are technically incomplete. 
A. Granite porphyry, Bully Hill district. Contains quartz, orthoclase, plagioclase, iron ores, apatite, and sphene. Analysis by G. Steiger, record No. 2378.

B. Altered granite porphyry, Bully Hill. Contains quartz, orthoclase, plagioclase, kaolin, and chlorite. Analysis by Chase Palmer, No. 2379.

C. Granite porphyry, near Shasta King mine. Contains quartz, orthoclase, plagioclase, iron ore, apatite, zircon, and sphene. Analysis by Steiger, No. 2378.

D. Altered granite porphyry, near Shasta King mine. Contains quartz, orthoclase, plagioclase, chlorite, and epidote. Analysis by Palmer, No. 2379.

E. Granite porphyry, Afterthought district. Contains quartz, orthoclase, plagioclase, and iron ore. Analysis by Steiger, No. 2378.

F. Granite porphyry, Little Backbone Mountain. Contains quartz, orthoclase, plagioclase, magnetite or ilmenite, apatite, and sphene. Analysis by R. C. Wells, No. 2380 .

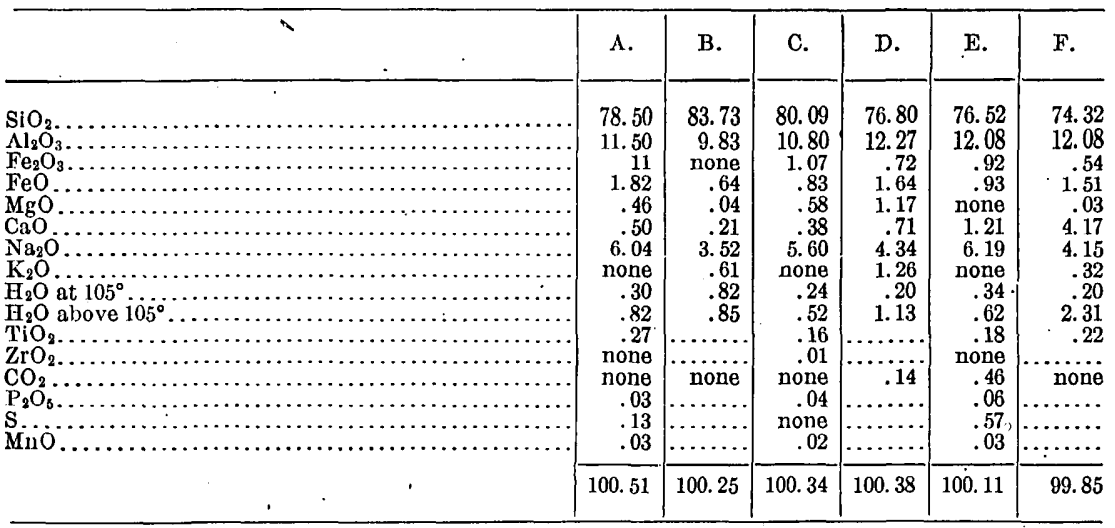

$\mathrm{BaO}, \mathrm{SrO}$, and $\mathrm{SO}_{3}$ absent.

G. Altered granite porphyry, between Mammoth and Summit mines. Contains quartz, orthoclase, plagioclase, chlorite, epidote, calcite, and sericite. Analysis by Wells, No. 2380.

H. Altered granite porphyry, near Iron Mountain mine. Contains quartz, orthoclase, plagioclase, chlorite, and epidote. Analysis by Palmer, No. 2379.

I. Silicified granite porphyry, near Clipper mine. Contains quartz, feldspar, and sericite. Analysis by Palmer, No. 2379.

J. Monzonite, railroad cut near Spring Creek. Contains quartz, orthoclase, plagioclase, hornblende, iron ore, and apatite. Analysis by Steiger, No. 2378.

$\mathrm{K}$. Inclusion in monzonite. Contains quartz, orthoclase, plagioclase, iron ore, chlorite, and epidote. Analysis by W. T. Schaller, No. 2377.

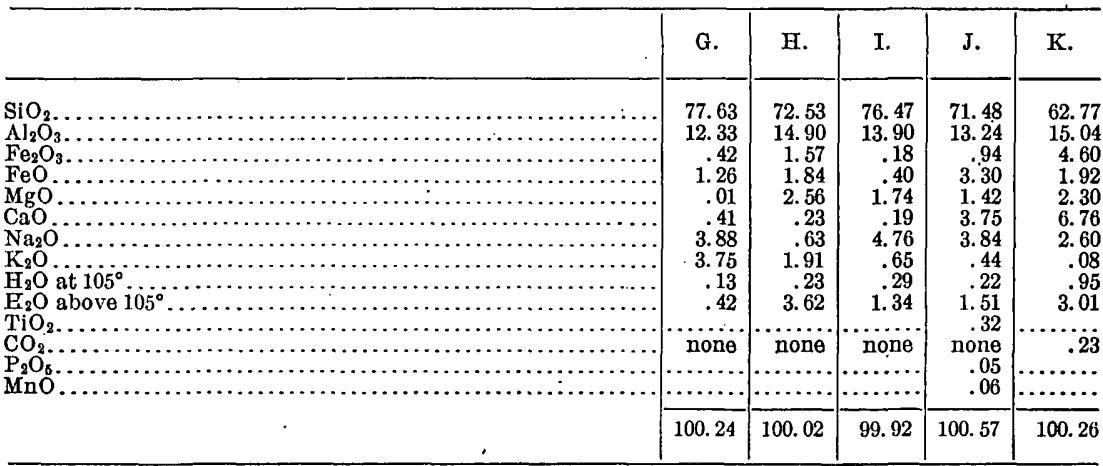


L. Diorite dike, near Minnesota mine. Contains quartz, orthoclase, plagioclase, hornblende, iron ore, chlorite, and epidote. Analysis by Schaller, No. 2377.

M. Diorite porphyry, near Uncle Sam mine. Contains quartz, plagioclase, iron ore, chlorite, and epidote. Analysis by Schaller, No. 2377.

N. Diorite dike, near Keswick. Contains hornblende, plagioclase, orthoclase, and iron ore. Analysis by Wells, No. 2380.

O. Diabase, Butcher Creek. Contains plagioclase, orthoclase, pyroxene, hornblende, and iron ore. Analysis by Wells, No. 2380.

P. Feldspar-epidote rock, dike near Spread Eagle mine. Contains quartz, orthoclase, plagioclase, biotite, epidote, sphene, apatite, and zircon. Analysis by Steiger, No. 2378.

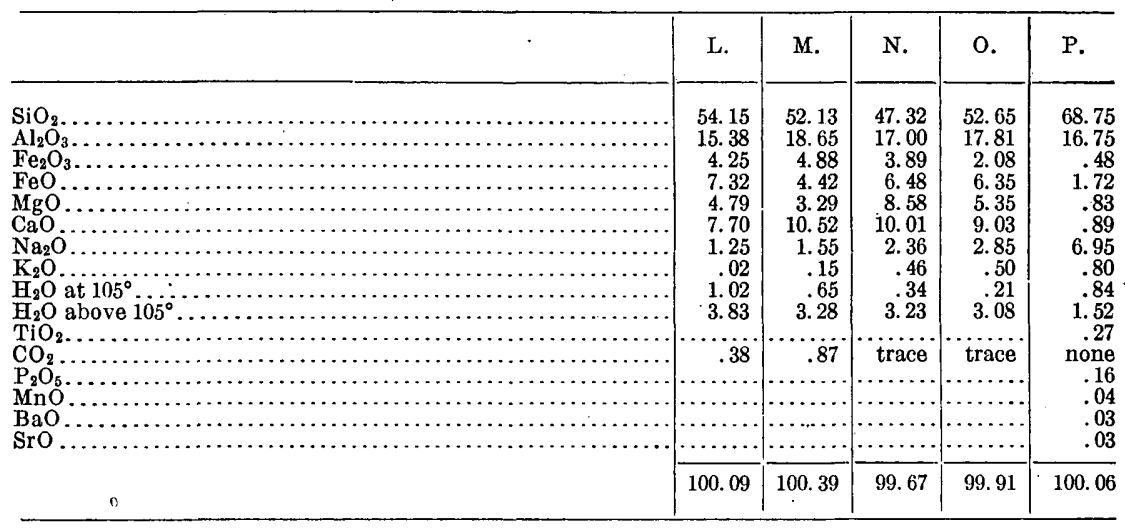

In this group of analyses, $A$ to $\mathrm{P}$, when not otherwise stated, $\mathrm{TiO}_{2}$ and $\mathrm{P}_{2} \mathrm{O}_{5}$ were not separately determined.

\section{LASSEN PEAK REGION.}

Rocks collected by J. S. Diller, who has furnished the petrographic data. Nearly all are from the area covered by the Lassen Peak atlas sheet of the United States Geological Survey. The quartz basalts have been described by Diller in Bull. 79, and partly in Am. Jour. Sci., 3d ser., vol. 33, p. 49. The analyses are so numerous that it seems best to divide them into subordinate groups.

\section{RHYOLITE.}

A. Rhyolite, a short distance northwest of Willow Lake, near the Geyser, Plumas County. Alaskose. A light-gray rock:with occasional phenocrysts of quartz and feldspar in a granular groundmass of the same materials. Analysis by W. F. Hillebrand, record No. 414. P. R. C. 1419.

B. Rhyolite, 2 miles northwest of Deer Creek Meadows, Tehama County. Tehamose. Shows many small crystals of quartz, feldspar, and biotite in a spherulitic groundmass. Analysis by Hillebrand, No. 415. P. R. C. 1420.

C. Rhyolite, Slate Creek, west of Deer Creek Meadows. Tehamose. Composed wholly of spherulites, some of which include crystals of feldspar or biotite. Analysis by Hillebrand, No. 416. P. R. C. 1421.

D. Rhyolite, summit of Mount Stover, Plumas County. Toscanose. Composed almost wholly of spherulites, with a few crystals of feldspar and hornblende. Analysis by Hillebrand, No. 417. P. R. C. 1422.

E. Rhyolite, near Slate Creek, west of Deer Creek Meadows. Toscanose. A pearlite, composed chiefly of little glass balls, with a few spherulites, and crystals of feldspar and biotite. Analysis by Hillebrand, No. 418. 'P. R. C. 1423. 
F. Rhyolite, $1 \frac{1}{2}$ miles northeast of Clipper Mills, on tramway to Rock Creek, Shasta County. Tehamose' A spherulitic rock containing a few microscopic particles of feldspar and hornblende. Analysis by Hillebrand, No. 678 . P. R. C. 1424.

G. Rhyolite tuff, divide between the west fork of Willards Creek and the stage road, in Lassen County. Elevation, 5,800 feet. Composed almost wholly of fine angular particles of clear glass. Analysis by George Steiger, record No. 1427. P. R. C. 1425.

Materials for $\mathrm{A}$ to $\mathrm{F}$ dried at $110^{\circ}$ previous to analysis.

\begin{tabular}{|c|c|c|c|c|c|c|c|}
\hline & A. & B. & C. & D. & E. & F. & G. \\
\hline 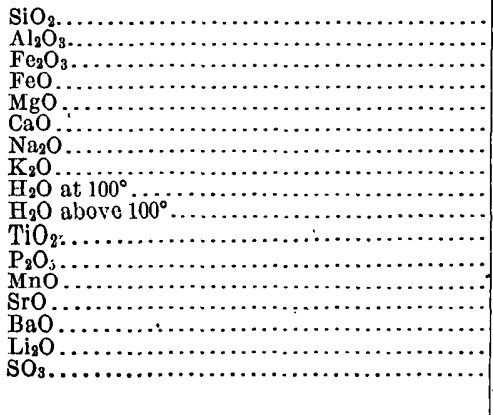 & $\begin{array}{r}74.24 \\
14.50 \\
1.27 \\
.67 \\
.25 \\
.11 \\
3.00 \\
3.66 \\
2.04 \\
.20 \\
.07 \\
.06 \\
\text { trace } \\
.18 \\
\text { none } \\
.03 \\
100.28\end{array}$ & $\begin{array}{r}74.65 \\
14.11 \\
1.08 \\
.29 \\
.20 \\
.80 \\
2.81 \\
4.59 \\
1.40 \\
.21 \\
\text { trace } \\
.11 \\
\text { trace } \\
.08 \\
\text { none } \\
\ldots . . . .\end{array}$ & $\begin{array}{r}73.62 \\
14.24 \\
.93 \\
.67 \\
.33 \\
1.07 \\
3.25 \\
4.28 \\
\ldots . \ldots \\
1.29 \\
.21 \\
.02 \\
.08 \\
\text { trace } \\
.10 \\
\text { none }\end{array}$ & $\begin{array}{r}72.40 \\
14.81 \\
.81 \\
.88 \\
.47 \\
1.94 \\
3.91 \\
3.90 \\
\ldots . .7 \\
.59 \\
.18 \\
.03 \\
.07 \\
.04 \\
.10 \\
\text { trace }\end{array}$ & $\begin{array}{r}73.64 \\
13.44 \\
.60 \\
.74 \\
.26 \\
1.26 \\
3.51 \\
4.50 \\
\cdots .1 . \\
1.99 \\
.11 \\
.06 \\
.06 \\
.02 \\
.11 \\
\text { trace }\end{array}$ & $\begin{array}{r}74.60 \\
13.41 \\
1.28 \\
.30 \\
.26 \\
1.08 \\
3.38 \\
4.50 \\
\cdots . .15 \\
.85 \\
.16 \\
.03 \\
.06 \\
\text { none } \\
.11 \\
\text { trace }\end{array}$ & $\begin{array}{r}70.01 \\
12.61 \\
1.47 \\
.50 \\
.72 \\
1.06 \\
1.94 \\
5.12 \\
2.37 \\
4.68 \\
\ldots \ldots . .04 \\
\text { trace } \\
\ldots \ldots \ldots . . \\
\cdots \ldots \ldots . . \\
\ldots \ldots . . \\
\end{array}$ \\
\hline & 100.28 & 100.33 & 100.08 & 100.13 & 100.30 & 100.02 & 100.52 \\
\hline
\end{tabular}

\section{DACITES AND.ANDESITES.}

A. Gray dacite, Lassen Peak. Lassenose. Contains hornblende, biotite, plagioclase, quartz, scarce pyroxene, magnetite, apatite; and a glassy base. Analysis by T. M. Chatard, record No. 111. P. R. C. 82.

B. Secretion in dacite, Lassen Peak. Composed chiefly of plagioclase and hornblende. Analysis by Chatard, No. 110. P. R. C. 1426.

C. Reddish dacite, Lassen Peak. Essentially like A. Analysis by Chatard, No. 110. P. R. C. 1427.

D. Dacite, near the timber line, west base of Lassen Peak. Lassenose. Small phenocrysts of plagioclase and hornblende, with a few of quartz, in a reddish-gray groundmass containing much amorphous matter. Analysis by W. F. Hillebrand, record No. 668 . P. R. C. 1428.

E. Secretion in D. Hessose. Composed essentially of plagioclase and hornblende. Analysis by Hillebrand, No. 669. P. R. C. 1429.

Rocks A, C, D, and E described by Diller in Bull. 150, p. 217.

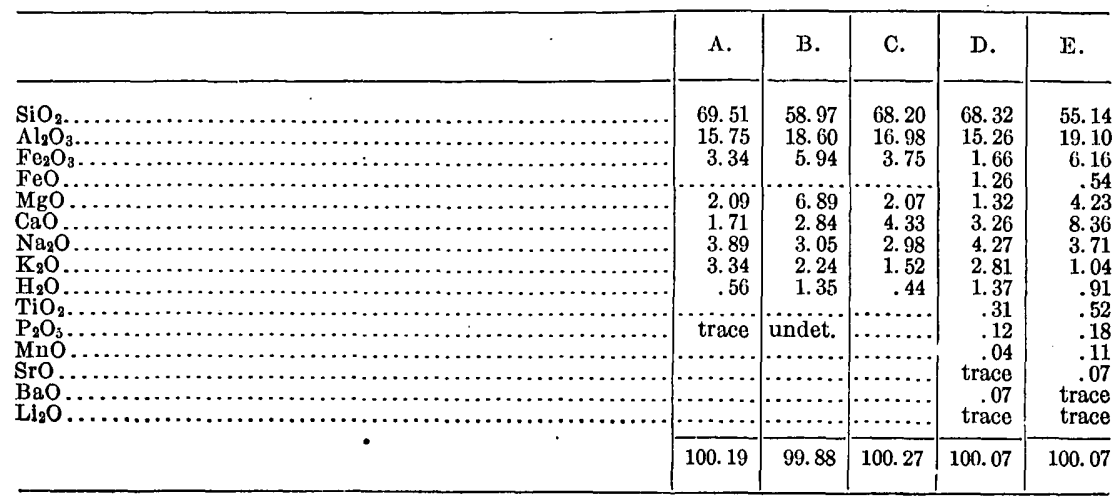


F. Dacite, east end of Chaos, northwest base of Lassen Peak. Lassenose. The youngest dacite of the region. Contains quartz, feldspar, biotite, and hornblende, embedded in a clear pumiceous glass. Analysis by W. F. Hillebrand, record No., 670. Described by Diller in Bull. 150, p. 218. P. R. C. 1430.

G. Secretion in F. Hessose. Consists chiefly of plagioclase and hornblende, with some olivine and clear glass. Analysis by Hillebrand, No. 671. P. R. C. 1431.

H. Dacite (?), west side of old crater rim near the Thumb, at the head of Mill Creek, Shasta County. Yellowstonose. Shows phenocrysts of hornblende, plagioclase, and pyroxene, and apparently of quartz, in a gray, microlitic groundmass. Analysis by Hillebrand, No. 674. P. R. C. 1432.

I. Streaked dacite, falls of south fork of Bear Creek, Shasta County. Lassenose. Contains plagioclase with a little sanidine, hornblende, quartz, magnetite, some pyroxene inclusions, and glass base. Analysis by R. B. Riggs, record No. 524 . P. R. C. 80. Described by Diller in Bull. 150, p. 213.

J. Dacite tuff, Rice's quarry, 6 miles southeast of Paskenta, Tehama County. Clear glass, with fragments of quartz, feldspar, and hornblende. Analysis by George Steiger, record No. 1427. P. R. C. 1433.

J 2. Another sample of J. Analysis by E. T. Allen, record No. 2020. See Diller, Am. Jour. Sci., 4th ser., vol. 15, p. 360. P. R. C. 1434.

\begin{tabular}{|c|c|c|c|c|c|c|}
\hline & F. & G: & H. & I. & J. & J 2. \\
\hline $\begin{array}{l}\mathrm{SiO}_{2} \\
\mathrm{Al}_{2} \mathrm{O}_{3} \\
\mathrm{Fe}_{2} \mathrm{O}_{3}\end{array}$ & $\begin{array}{r}68.72 \\
15.15 \\
1.16 \\
1.76 \\
1.28 \\
3.30 \\
4.26 \\
2.78 \\
.74 \\
.31 \\
.09 \\
.11 \\
.03 \\
.07 \\
. .2 \\
\text { trace }\end{array}$ & $\begin{array}{r}53.35 \\
19.22 \\
3.28 \\
4.48 \\
4.86 \\
9.76 \\
2.89 \\
.99 \\
\ldots .77 \\
.56 \\
\ldots . .70 \\
.15 \\
.03 \\
\text { trace? } \\
\ldots \ldots . . . \\
\text { trace }\end{array}$ & $\begin{array}{r}63.81 \\
17.07 \\
2.11 \\
2.15 \\
2.28 \\
4.97 \\
4.08 \\
1.96 \\
\ldots .03 \\
1.03 \\
.38 \\
\ldots .10 \\
.09 \\
.03 \\
.04 \\
\ldots \ldots . . \\
\text { trace }\end{array}$ & \begin{tabular}{r}
68.10 \\
15.50 \\
3.20 \\
none \\
.10 \\
3.02 \\
4.20 \\
3.13 \\
\hdashline .72 \\
.15 \\
$\ldots \ldots 3$ \\
trace \\
trace \\
.06 \\
$\ldots \ldots .$. \\
none
\end{tabular} & $\begin{array}{r}65.78 \\
14.87 \\
1.27 \\
1.00 \\
1.89 \\
2.41 \\
2.58 \\
2.71 \\
2.87 \\
4.32 \\
\ldots \ldots . . \\
\ldots \ldots . . . \\
.08 \\
\text { trace } \\
\ldots \ldots \ldots . \\
\ldots \ldots . . . \\
\ldots \ldots . . . \\
\ldots \ldots . . .\end{array}$ & $\begin{array}{r}60.23 \\
18.64 \\
3.81 \\
. .88 \\
1.64 \\
6.04 \\
3.87 \\
1.46 \\
.95 \\
1.97 \\
.57 \\
.01 \\
.20 \\
\text { trace } \\
.05 \\
.11 \\
\text { trace } \\
\ldots \ldots . .\end{array}$ \\
\hline & 99.76 & 100.44 & 100.10 & 100.21 & 99.78 & 100.43 \\
\hline
\end{tabular}

K. Pyroxene andesite, west end of Butte Mountain, Plumas County. Hessose. Prominent phenocrysts of pyroxene and minute ones of plagioclase, in a dark groundmass containing much globulitic matter. Analysis by W. F. Hillebrand, record No. 411. P. R. C. 1435.

L. Pyroxene andesite, south base of Burney Butte, Shasta County. Tonalose. Numerous small phenocrysts of plagioclase and a few of pyroxene, in a gray groundmass containing much amorphous matter. Analysis by R. B. Riggs, record No. 684 . P. R. C. 1436.

M. Hornblende andesite, Tuscan Buttes, 7 miles east of Red Bluff. Tonalose. A few small phenocrysts or fragments of hornblende, in a groundmass consisting mainly of plagioclase and gray microlitic matter. Analysis by Hillebrand, No. 412. P. R. C. 1437.

N. Hornblende andesite, near Buntingville, Lassen County. Lassenose. A few phenocrysts of hornblende, in a groundmass consisting mainly of small feldspars. Analysis by T. M. Chatard, record No. 413. P. R. C. 1438.

O. Hornblende andesite, northwest summit, head of Burney Creek, Shasta County. Tonalose. Inconspicuous plagioclase and, rarely, olivine, in a groundmass of plagioclase and pyroxene. Numerous dark spots are due to altered hornblende. Analysis by Riggs, No. 683 . P. R. C. 1439.

Rocks in this group dried at $105^{\circ}$ before analysis. 


\begin{tabular}{|c|c|c|c|c|c|}
\hline-1 & $\mathbf{K}$. & L. & M. & N. & 0 \\
\hline 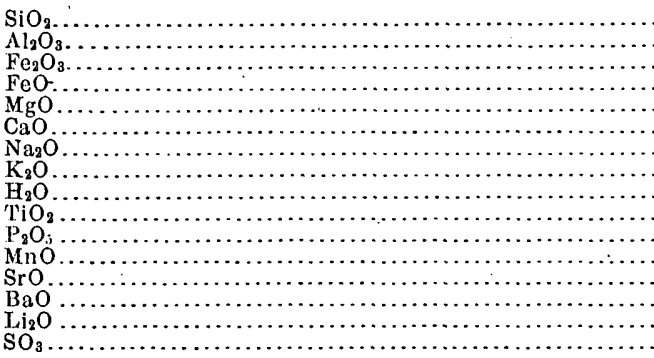 & $\begin{array}{r}55.53 \\
17.63 \\
2.81 \\
3.59 \\
5.85 \\
8.74 \\
3.09 \\
.92 \\
1.24 \\
.56 \\
.21 \\
.08 \\
.06 \\
.02 \\
\text { none } \\
\ldots \ldots\end{array}$ & $\begin{array}{r}62.44 \\
16.39 \\
4.66 \\
1.00 \\
2.65 \\
6.22 \\
3.16 \\
2.25 \\
1.02 \\
.31 \\
.05 \\
\text { trace } \\
\text { trace } \\
.03 \\
\text { trace } \\
\text { trace }\end{array}$ & $\begin{array}{r}60.93 \\
18.56 \\
2.68 \\
2.19 \\
2.37 \\
6.63 \\
3.79 \\
1.33 \\
.90 \\
.61 \\
.18 \\
.10 \\
.12 \\
.02 \\
\text { none } \\
\ldots . . . . .\end{array}$ & $\begin{array}{r}67.89 \\
17.29 \\
2.39 \\
.21 \\
.66 \\
3.01 \\
5.11 \\
1.69 \\
1.34 \\
.21 \\
.12 \\
.12 \\
.04 \\
.03 \\
\ldots \ldots . . \\
\ldots \ldots . .\end{array}$ & $\begin{array}{r}60.04 \\
17.43 \\
5.39 \\
.53 \\
3.51 \\
6.65 \\
4.15 \\
1.24 \\
.90 \\
.49 \\
.04 \\
.08 \\
(?) \\
.04 \\
\text { trace } \\
\text { trace }\end{array}$ \\
\hline & 100.33 & 100.18 & 100.41 & 100.11 & 100. 49 \\
\hline
\end{tabular}

P. Hypersthene andesite, 1 mile west of summit on Bidwell's road, Butte County. Andose. Rich in small phenocrysts of plagioclase and pyroxene, mostly hypersthene, in a groundmass of the same minerals, with magnetite, and probably some amorphous matter. Analysis by W. F. Hillebrand, record No. 410. P. R. C. 1440.

Q. Hypersthene andesite, old crater at head of Mill Creek. Andose. Contains small phenocrysts of plagioclase and hypersthene, in a groundmass of plagioclase, pyroxene, magnetite, etc. Some greenish pseudomorphs suggest former olivine. Analysis by T. M. Chatard, record No. 409. P. R. C. 1441.

R. Hypersthene andesite, 2 miles south of Suppans Mountain, Tehama County. Tonalose. Abundant plagioclase and hypersthene, with traces of hornblende, in a microlitic groundmass. Analysis by Hillebrand, No. 672. P. R. C. 1442.

$\mathrm{S}$. Secretion in R. Bandose. Composed chiefly of plagioclase and hypersthene, with some quartz and amorphous matter. Analysis by Hillebrand, No. 673. P. R. C. 1443.

T. Hypersthene andesite, west base of Suppans Mountain, near Lassen Peak, Tehama County. Tonalose. Contains numerous microscopic crystals of plagioclase and hypersthene, in a microlitic groundmass. Analysis by Hillebrand, No. 676. P. R. C. 1444.

Rocks dried at $100^{\circ}$ to $110^{\circ}$ before analysis.

\begin{tabular}{|c|c|c|c|c|c|}
\hline & $P$. & Q. & R. & s: & 'T. \\
\hline \multirow[t]{2}{*}{ 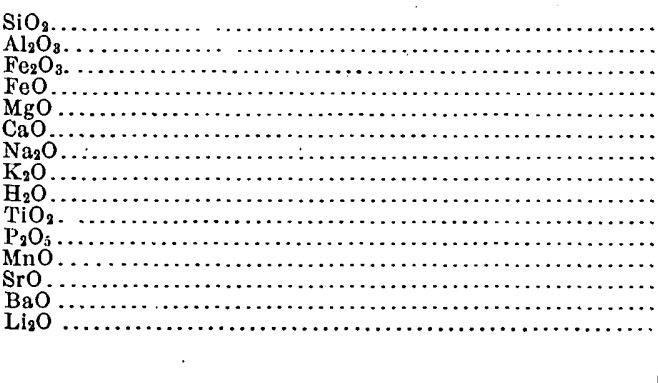 } & $\begin{array}{r}\text { 55. } 20 \\
18.68 \\
3.14 \\
4.42 \\
4.59 \\
8.02 \\
3.66 \\
1.01 \\
.51 \\
.92 \\
.24 \\
.14 \\
.02 \\
.03 \\
\text { none }\end{array}$ & $\begin{array}{r}57.11 \\
17.78 \\
3.54 \\
2.74 \\
3.41 \\
7.21 \\
3.81 \\
1.86 \\
.98 \\
.95 \\
.26 \\
.33 \\
\text { trace? } \\
.03 \\
\ldots . . .\end{array}$ & $\begin{array}{r}63.47 \\
16.75 \\
2.15 \\
2.75 \\
3.04 \\
5.72 \\
3.94 \\
1.62 \\
.55 \\
.37 \\
.13 \\
.09 \\
.04 \\
.04 \\
\text { trace }\end{array}$ & $\begin{array}{r}57.04 \\
19.11 \\
4.37 \\
2.48 \\
3.94 \\
7.34 \\
3.48 \\
1.16 \\
1.09 \\
.47 \\
.08 \\
.12 \\
.02 \\
\text { trace? } \\
\text { trace }\end{array}$ & $\begin{array}{r}58.08 \\
18.37 \\
2.92 \\
3.38 \\
3.35 \\
7.05 \\
3.66 \\
1.33 \\
1.09 \\
.44 \\
.16 \\
.13 \\
.02 \\
.03 \\
\text { trace }\end{array}$ \\
\hline & 100.58 & 100.01 & 100.66 & 100.70 & 100.01 \\
\hline
\end{tabular}


U. Hypersthene andesite, 1 mile southwest of Thumb, head of Bailey Creek, near Lassen Peak. Tonalose. Abundant but inconspicuous plagioclase and hypersthene, in a microlitic groundmass containing many small crystals of plagioclase. Analysis by W. F. Hillebrand, record No. 675. P. R. C. 1445.

V. Hypersthene andesite, west summit of Crater Peak, Shasta County. Yellowstonose. Microphenocrysts of feldspar and hypersthene in a groundmass consisting largely of the same minerals, with some amorphous matter. Analysis by Hillebrand, No. 679 . P. R. C. 1446 .

W. Hypersthene andesite, north slope of Crater Peak. Tonalose. Phenocrysts of plagioclase and hypersthene in a groundmass containing much dark amorphous matter. Analysis by Hillebrand, No. 680. P. R. C. 1447.

$\mathrm{X}$. Secretion in W. Hessose. Composed chiefly of plagioclase, hypersthene, and a globulitic base. Analysis by Hillebrand, No. 681. P. R. C. 1448.

Y. Andesitic tuff, Stillwater Creek, 8 miles northeast of Redding. Contains plagioclase, hornblende, rare hypersthene, magnetite, and glass. Fragments of andesite are inclosed. Analysis by W. H. Melville, record No. 1346. Described by Diller in Bull. 150, p. 211 . P. R. C. 79.

Rocks dried at $100^{\circ}$ to $110^{\circ}$ before analysis.

\begin{tabular}{|c|c|c|c|c|c|}
\hline & U. & v. & w. & $\mathrm{x}$ & $\mathrm{x}$. \\
\hline 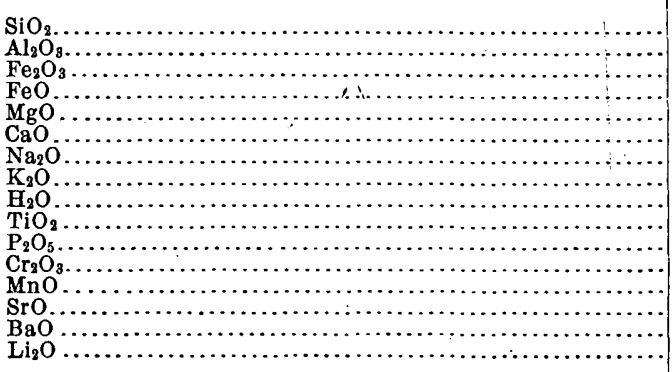 & $\begin{array}{r}59.84 \\
16.81 \\
1.88 \\
3.60 \\
3.85 \\
6.30 \\
3.63 \\
2.13 \\
1.04 \\
.57 \\
.19 \\
\operatorname{trace} \\
.14 \\
.02 \\
.07 \\
\text { trace }\end{array}$ & $\begin{array}{r}68.12 \\
16.24 \\
1.26 \\
2.08 \\
1.35 \\
3.80 \\
3.89 \\
2.54 \\
.40 \\
.25 \\
.14 \\
\text { none } \\
.10 \\
.02 \\
.09 \\
\text { trace }\end{array}$ & $\begin{array}{r}61.17 \\
17.74 \\
1.78 \\
3.51 \\
2.76 \\
5.90 \\
3.79 \\
1.71 \\
.83 \\
.45 \\
.14 \\
\text { none } \\
.12 \\
.04 \\
.06 \\
\text { trace }\end{array}$ & $\begin{array}{r}53.85 \\
18.53 \\
1.96 \\
5.30 \\
5.88 \\
9.66 \\
2.98 \\
.74 \\
.45 \\
.50 \\
.05 \\
\text { trace } \\
.12 \\
.04 \\
.03 \\
\text { trace }\end{array}$ & $\begin{array}{r}69.51 \\
15.61 \\
.56 \\
1.27 \\
.61 \\
2.80 \\
3.43 \\
2.81 \\
3.63 \\
\text { trace }\end{array}$ \\
\hline & 100.07 & 100.28 & 100.00 & 100.09 & 100.23 \\
\hline
\end{tabular}

\section{BASALTS.}

The quartz basalts are described by Diller in Bull. 79. That from Mitylene was analyzed for comparison with the Cinder Cone series.

A. Quartz basalt, Cinder Cone, 10 miles northeast of Lassen Peak. Andose. Contains plagioclase, pyroxene (mostly hypersthene), olivine, quartz, and much unindividualized base; the latter about 25 per cent. Magnetite is also present; augite occurs sparingly. Analysis by W. F. Hillebrand, record No. 407. P. R. C. 101. Also described in Bull. 150, p. 252.

- B. Volcanic bomb from quartz basalt, Cinder Cone. Andose. Analysis by Hillebrand, No. 665 . P. R. C. 1449.

C. Lapilli from quartz basalt, Cinder Cone. Andose. Analysis by Hillebrand, No. 667. P. R. C. 96 . Also described in Bull. 150, p. 249 and 791.

D. Volcanic sand, one-half mile northeast of Cinder Cone. Andose. Analysis by Hillebrand, No. 663. P. R. C. 792.

E. White pumiceous inclosure from quartz basalt, Cinder Cone. Alsbachose. Analysis by Hillebrand, No. 664. Mainly glass. P. R. C. 793.

Rocks dried at $100^{\circ}$ to $110^{\circ}$ before analysis. 


\begin{tabular}{|c|c|c|c|c|c|}
\hline & A. & B. & C. & D. & E. \\
\hline 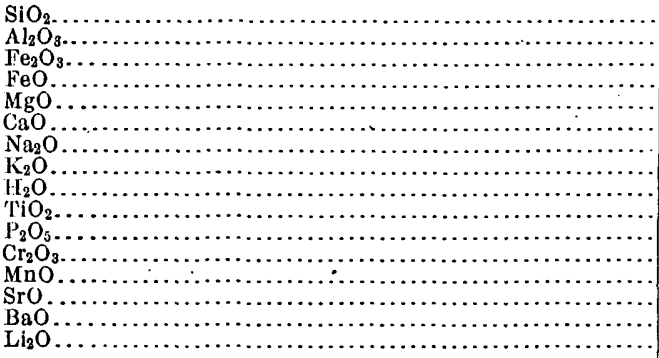 & $\begin{array}{r}57.25 \\
16.45 \\
1.67 \\
4.72 \\
6.74 \\
7.65 \\
3.00 \\
1.57 \\
.40 \\
.60 \\
.20 \\
\text { trace } \\
.03 \\
\text { none }\end{array}$ & $\begin{array}{r}56.70 \\
15.75 \\
1.29 \\
5.32 \\
7.16 \\
7.67 \\
3.36 \\
1.56 \\
.30 \\
.65 \\
.20 \\
\text { trace } \\
.19 \\
\text { trace } \\
.03 \\
\text { trace }\end{array}$ & $\begin{array}{r}56.53 \\
17.50 \\
1.35 \\
5.03 \\
5.94 \\
8.07 \\
3.51 \\
1.55 \\
.27 \\
.54 \\
.15 \\
\text { trace } \\
.12 \\
\text { trace? } \\
\text { trace } \\
\text { trace }\end{array}$ & $\begin{array}{c}55.93 \\
17.34 \\
1.50 \\
5.20 \\
7.29 \\
8.04 \\
3.32 \\
1.35 \\
.26 \\
\text { undet. } \\
\text { undet. } \\
\text { [undet. } \\
\text { unde } \\
\text { (?) } \\
\text { (?) } \\
\text { (?) }\end{array}$ & $\begin{array}{r}79.49 \\
11.60 \\
.33 \\
.49 \\
.09 \\
1.64 \\
4.04 \\
1.52 \\
.68 \\
\text { undet. } \\
\text { undet. } \\
\text {... } \\
\text { none } \\
\text { (?) } \\
\text { ?? } \\
\text { ? }\end{array}$ \\
\hline . & 100.38 & 100.18 & 100.56 & 100.23 & 99.88 \\
\hline
\end{tabular}

F. Quartz basalt, one-half mile south of Cinder Cone, on border of lava field. Camptonose. Analysis by Hillebrand, No. 666. P. R. C. 794.

G. Quartz basalt, west end of Lake Bidwell, on border of Cinder Cone lava field. Andose. Contains a few grains of quartz, much olivine and plagioclase, less pyroxene, and a globulitic base. Analysis by Hillebrand, No. 661. P. R. C. 795.

H. Quartz basalt, Silver Lake, near Lassen Peak. Andose. Contains occasional grains of quartz, much feldspar and olivine, less pyroxene, and a brownish base. Analysis by Hillebrand, No. 662. P. R. C. 790.

I. Quartz basalt, resting on dacite, near west base of Lassen Peak. Bandose. Analysis by Hillebrand, No. 677. P. R. C. 796.

J. Quartz basalt, island of Mitylene, coast of Asia Minor. Tonalose. Analysis for comparison with the Cinder Cone series, by T. M. Chatard, record No. 845.

Rocks $\mathrm{F}$ to I dried at $100^{\circ}$ to $110^{\circ}$ before analysis.

\begin{tabular}{|c|c|c|c|c|c|}
\hline & F. & G. & H. & I. & J. \\
\hline 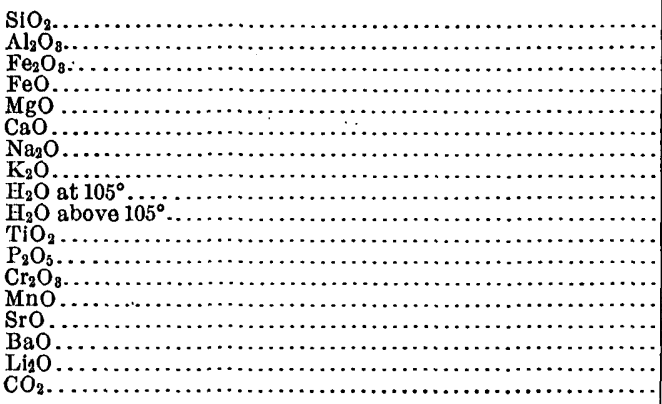 & \begin{tabular}{r}
54.56 \\
16.04 \\
.95 \\
6.07 \\
8.71 \\
8.89 \\
3.05 \\
1.18 \\
\hdashline .28 \\
.53 \\
.18 \\
trace \\
.17 \\
trace \\
.03 \\
trace
\end{tabular} & $\begin{array}{r}56.18 \\
16.59 \\
1.51 \\
5.51 \\
7.26 \\
7.64 \\
3.58 \\
1.47 \\
\cdots \ldots .92 \\
\text { undet. } \\
\text { undet. } \\
\text { undet. } \\
\text { u? } \\
\text { (?) } \\
\text { (?) }\end{array}$ & $\begin{array}{r}57.59 \\
16.49 \\
1.22 \\
4.89 \\
7.72 \\
7.40 \\
3.62 \\
.99 \\
\ldots .86 \\
\text { undet. } \\
\text { undet. } \\
\text { undet. } \\
\text { (?) } \\
\text { (?) } \\
\text { (?) } \\
\ldots . . . .\end{array}$ & $\begin{array}{r}56.51 \\
18.10 \\
4.26 \\
2.68 \\
4.52 \\
8.15 \\
3.23 \\
1.15\end{array}$ & $\begin{array}{r}56.58 \\
14.88 \\
2.31 \\
3.04 \\
3.76 \\
8.69 \\
3.36 \\
2.18 \\
.69 \\
1.43 \\
.77 \\
.15 \\
\text { trace? } \\
.16 \\
\ldots \ldots . . .7 \\
.07 \\
\ldots . .32\end{array}$ \\
\hline$\cdot$ & 100.64 & 100.16 & 100.78 & 100.10 & 100.39 \\
\hline
\end{tabular}

K. Recent basalt, Pit River. Rich in feldspar and augite, poor in olivine. Partial analysis by F. W. Clarke, record No. 109.

L. Basalt, 1 mile southeast of Paynes Creek, on the road from Red Bluff to Lassen Peak. Auvergnose. A normal basalt, rather rich in olivine. Analysis by T. M. Chatard, record No. 405. P. R. C. 1242.

M. Basalt, summit of Inskip Crater, 25 miles east of Red Bluff. Auvergnose. Mainly feldspar and augite, with a few phenocrysts of olivine. Analysis by Hillebrand and Chatard, record No. 406. P. R. C. 1450. 
N. Basalt from the cone at south base of Burney Butte, Shasta County. Andose. Composed of plagioclase and augite, with some olivine and a globulitic base. Analysis by R. B. Riggs, record No. 685 . P. R. C. 1451.

O. Basalt, near east end of rim of Crater Peak, Shasta County. Hessose. Contains plagioclase and pyroxene, some of the latter being hypersthene with a trace of olivine. Analysis by R. B. Riggs, record No. 682. P. R. C. 1452.

P. Hornblende basalt, Kosk Creek near its mouth, by the great bend of Pit River, Shasta County. Auvergnose. Contains abundant phenocrysts of hornblende, with a few of plagioclase, pyroxene, and olivine, in a groundmass of plagioclase, augite, and magnetite. The hornblendes are deeply corroded, and some have disappeared, leaving groups of magnetite grains to mark their former presence. Analysis by L. G. Eakins, record No. 1022. Described by Diller in Am. Geologist, vol. 19, p. 253 . P. R. C. 1453.

Rocks dried at $105^{\circ}$ to $110^{\circ}$ before analysis, except in the case of the rock marked L.

\begin{tabular}{|c|c|c|c|c|c|c|}
\hline . & $\mathbf{K}$. & L. & M. & N. & 0 & P. \\
\hline 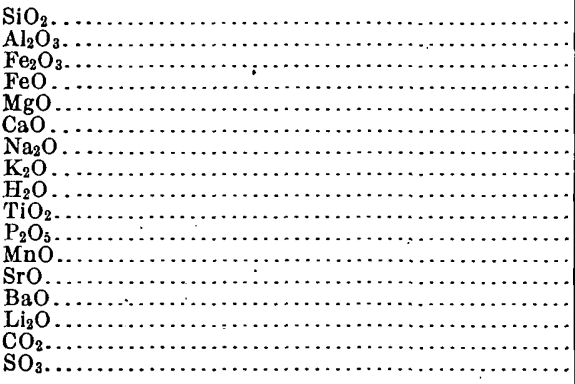 & $\begin{array}{r}51.92 \\
19.76 \\
11.21 \\
3.38 \\
9.30 \\
2.16 \\
.60 \\
1.54 \\
\cdots \\
\cdots \\
\cdots \\
\cdots \\
\cdots \\
\cdots \\
\cdots \\
\cdots\end{array}$ & \begin{tabular}{r}
47.93 \\
18.51 \\
2.07 \\
7.25 \\
9.03 \\
11.14 \\
2.28 \\
.24 \\
.76 \\
.73 \\
.11 \\
.20 \\
$\ldots \ldots . .$. \\
\hdashline$\ldots . .$. \\
\hdashline none \\
$\ldots \ldots . .$.
\end{tabular} & $\begin{array}{r}50.89 \\
16.76 \\
3.86 \\
4.69 \\
8.49 \\
11.72 \\
2.61 \\
.32 \\
.41 \\
.79 \\
.09 \\
.13 \\
\ldots \ldots . . \\
\text { trace } \\
\hdashline \ldots . . \\
\hdashline \ldots . . . \\
\hdashline \ldots . .\end{array}$ & $\begin{array}{r}52.63 \\
17.62 \\
6.49 \\
3.10 \\
5.64 \\
8.62 \\
3.38 \\
1.73 \\
.79 \\
.07 \\
.47 \\
\text { trace } \\
\text { trace } \\
.04 \\
\text { trace } \\
\ldots \ldots . . . \\
\text { trace }\end{array}$ & \begin{tabular}{r}
52.95 \\
18.25 \\
4.36 \\
4.19 \\
4.93 \\
8.73 \\
3.57 \\
.77 \\
1.47 \\
.66 \\
trace \\
.12 \\
trace \\
.01 \\
.$(?)$ \\
\hdashline... \\
trace
\end{tabular} & $\begin{array}{r}44.77 \\
17.82 \\
5.05 \\
6.95 \\
8.22 \\
10.36 \\
2.13 \\
.92 \\
2.64 \\
.53 \\
.72 \\
\text { trace } \\
\ldots \ldots . . . \\
\hdashline \ldots . . \\
\hdashline \ldots . . \\
\hdashline \ldots . . \\
\hdashline \ldots . .\end{array}$ \\
\hline & 99.87 & 100.25 & 100.76 & 100.58 & 100.01 & 100.11 \\
\hline
\end{tabular}

3. Plumas County.

Other rocks from this county are described under the heading of the Lassen Peak area. The following rocks, with two exceptions, were collected by $\mathrm{H}$. W. Turner, who supplies the descriptions:

A. Granite, dike in serpentine, south slope of Grizzly Hill. Near alaskose. Described by Turner in Am. Geologist, vol. 17, p. 375. Contains quartz, albite, and muscovite. Analysis by H. N. Stokes, record No. 1562 . P. R. C. 757.

B. Metarhyolite, near Tower Rock, Grizzly Mountains. Toscanose. Described by Turner in 14th Ann., p. 441. Contains porphyritic quartz, feldspar, and pyrite, in a fine groundmass. Analysis by W. F. Hillebrand, record No. 1273 . P. R. C. 741.

C. Dacite, near Greenville. Yukonose. Collected by Diller, who finds phenocrysts of quartz in a groundmass chiefly of quartz and feldspar. Analysis by Hillebrand, record No. 1458. P. R. C. 1454.

D. Granodiorite, southwest base of Mount Ingalls. Yellowstonose. Description supplied by Turner. Contains plagioclase, quartz, orthoclase, brown mica, green hornblende, iron oxide, and a little apatite, sphene, and epidote. Analysis by Hillebrand, record No. 1456: P. R. C. 727.

E. Granodiorite, Spanish Peak. Tonalose. Description supplied by Turner. Contains plagioclase, quartz, orthoclase, biotite, hornblende, iron ore, and apatite; also abundant secondary epidote and chlorite. Analysis by Stokes, record No. 1562 . P. R. C. 756. 


\begin{tabular}{|c|c|c|c|c|c|}
\hline . & A. & B. & C. & D. & E. \\
\hline 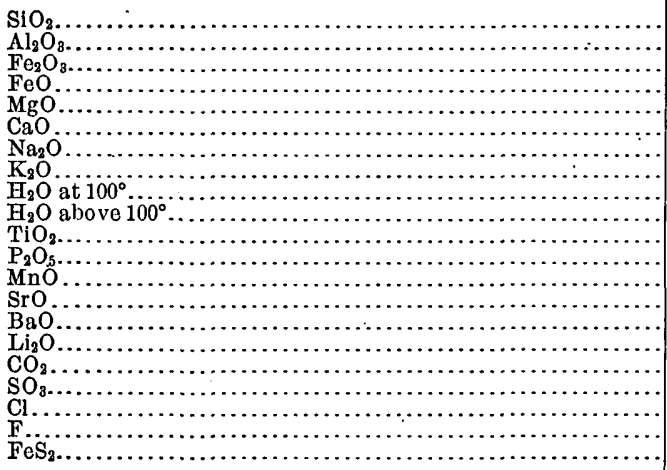 & $\begin{array}{r}76.00 \\
14.88 \\
.65 \\
.10 \\
.06 \\
.19 \\
3.52 \\
2.77 \\
.20 \\
1.42 \\
.04 \\
.11 \\
\operatorname{trace} \\
\ldots \ldots . \\
\text { trace } \\
\ldots \ldots \\
\text { trace } \\
\text { trace } \\
\text { trace } \\
\ldots \ldots\end{array}$ & $\begin{array}{r}73.25 \\
13.25 \\
\ldots . . .7 \\
1.74 \\
.28 \\
2.23 \\
2.69 \\
3.79 \\
.07 \\
1.03 \\
\text { trace } \\
\text { trace } \\
\text { trace } \\
\text { trace? } \\
\text { trace } \\
\text { trace } \\
1.05 \\
\ldots \ldots . . \\
\ldots \ldots . . \\
\ldots \ldots . . . \\
.58\end{array}$ & $\begin{array}{r}72.77 \\
13.00 \\
1.28 \\
2.65 \\
.67 \\
2.47 \\
4.95 \\
.34 \\
.07 \\
1.16 \\
.22 \\
.04 \\
.08 \\
\text { trace } \\
\text { trace } \\
\text { trace } \\
.47 \\
\ldots \ldots . . \\
\ldots \ldots \ldots \\
\ldots \ldots . .\end{array}$ & $\begin{array}{r}67.33 \\
15.93 \\
1.90 \\
1.59 \\
1.63 \\
4.09 \\
3.76 \\
2.46 \\
.19 \\
.66 \\
.36 \\
.11 \\
.09 \\
\operatorname{trace} \\
.08 \\
\operatorname{trace} \\
\ldots \ldots . . . \\
\ldots \ldots . . . \\
\ldots \ldots . . . \\
\ldots \ldots . . .\end{array}$ & $\begin{array}{r}59.68 \\
17.09 \\
2.85 \\
2.75 \\
3.54 \\
6.62 \\
3.87 \\
1.31 \\
.15 \\
1.00 \\
.65 \\
.25 \\
\text { trace } \\
\text { trace } \\
.04 \\
\text { trace } \\
.20 \\
\text { trace } \\
.03\end{array}$ \\
\hline & 99.94 & 99.96 & 100.17 & 100.18 & 100.03 \\
\hline
\end{tabular}

F. Rhyolite, $3 \frac{1}{2}$ miles southwest of Grizzly Peak. Toscanose. Description furnished by Turner. Contains sanidine, with less quartz and biotite, in a glassy groundmass. Analysis by Hillebrand, record No. 1461. P. R. C. 776.

G. Hornblende andesite, 4 miles from Pilot Peak. Tonalose. Described by Turner in 14th Ann., p. 441. Contains plagioclase and hornblende in a groundmass carrying grains of magnetite. Analysis by Hillebrand, record No. 1432. P. R. C. 716.

H. Hornblende-pyroxene andesite, southwest base of Mount Ingalls. Tonalose. Description supplied by Turner. ' Contains plagioclase, rhombic pyroxene, augite, brown hornblende, and magnetite, with much glass in the groundmass. Analysis by Hillebrand, record No. 1456. P. R. C. 728.

I. Hypersthene andesite, Franklin Hill. Hessose. Description supplied by Turner. Contains plagioclase, rhombic pyroxene, augite, and magnetite. Probably no glass. Analysis by Hillebrand, record No. 1548. P. R. C. 754.

\begin{tabular}{|c|c|c|c|c|}
\hline & F. & G. & H. & I. \\
\hline 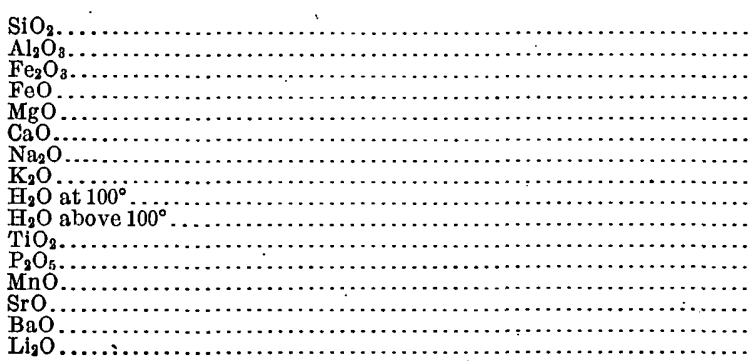 & $\begin{array}{r}71.39 \\
14.13 \\
.63 \\
.37 \\
.08 \\
1.01 \\
2.89 \\
5.69 \\
.42 \\
3.32 \\
.17 \\
.03 \\
\text { trace } \\
\text { trace } \\
.09 \\
\text { trace }\end{array}$ & $\begin{array}{r}60.20 \\
17.21 \\
3.12 \\
2.69 \\
3.18 \\
6.04 \\
3.35 \\
1.44 \\
1.12 \\
1.18 \\
.57 \\
.17 \\
.12 \\
\text { trace } \\
.11 \\
\text { trace }\end{array}$ & $\begin{array}{r}58.47 \\
18.80 \\
3.34 \\
2.64 \\
2.69 \\
6.60 \\
3.58 \\
2.01 \\
.14 \\
.92 \\
.51 \\
.22 \\
.13 \\
.05 \\
.09 \\
\text { trace }\end{array}$ & $\begin{array}{r}56.88 \\
18.25 \\
2.35 \\
4.45 \\
4.07 \\
7.53 \\
3.29 \\
1.42 \\
.24 \\
.50 \\
.45 \\
.30 \\
.18 \\
.04 \\
.11 \\
\text { trace }\end{array}$ \\
\hline . & 100. 22 & 100.50 & 100. 19 & 100.06 \\
\hline
\end{tabular}


J. Dolerite, Mount Ingalls. Hessose. Described by Turner in 14th Ann., p. 441. Contains plagioclase, augite, hypersthene, magnetite, and a few olivines. Analysis by W. F. Hillebrand, record No. 1273 . P. R. C. 739.

K. Dolerite, Mount Ingalls. Hessose. Also in 14th Ann., p. 441. Like J, but with scarcely any olivine. Analysis by Hillebrand, record No. 1432. P. R. C. 740.

L. Basalt, 4 miles southeast of Mount Ingalls. Andose. Also in 14th Ann., p. 441. Contains plagioclase, olivine, augite, and magnetite. Analysis by Hillebrand, record No. 1273.

M. Olivine basalt, $1 \frac{1}{2}$ miles from Franklin Hill. Hessose. Contains plagioclase, augite, partly altered olivine, magnetite, and probably some glass. Description supplied by Turner. Analysis by George Steiger, record No. 1596. P. R. C. 755.

N. Serpentine, Greenville. Described by Diller in Bull. 150, p. 372 . Besides serpentine, the rock contains some magnetite and less chromite, with remnants of the pyroxene from which the serpentine was in great part derived. Analysis by W. H. Melville, record No. 1346. P. R. C. 145.

\begin{tabular}{|c|c|c|c|c|c|}
\hline & J. & K. & L. & M. & N. \\
\hline 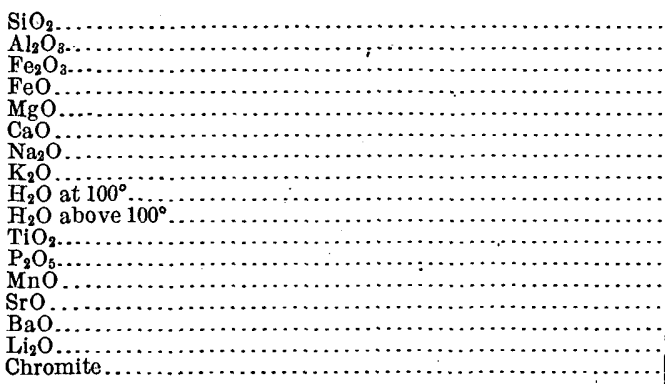 & $\begin{array}{r}53.91 \\
17.95 \\
2.21 \\
4.80 \\
5.52 \\
10.40 . \\
2.90 \\
1.34 \\
.20 \\
.20 \\
.52 \\
.21 \\
.10 \\
\text { trace } \\
.05 \\
\text { trace } \\
\ldots \ldots . .\end{array}$ & $\begin{array}{r}52.81 \\
16.60 \\
2.66 \\
6.13 \\
6.12 \\
10.14 \\
2.79 \\
1.05 \\
.38 \\
.54 \\
.84 \\
.23 \\
\text { undet. } \\
\text { trace } \\
.03 \\
\text { trace } \\
\ldots \ldots . . .\end{array}$ & $\begin{array}{r}50.56 \\
14.71 \\
3.54 \\
8.90 \\
4.07 \\
7.58 \\
2.94 \\
2.10 \\
1.06 \\
1.12 \\
1.71 \\
1.14 \\
.13 \\
\text { trace? } \\
.25 \\
\text { trace? } \\
\ldots . . . . .\end{array}$ & $\begin{array}{r}51.21 \\
17.59 \\
4.71 \\
4.42 \\
7.12 \\
10.36 \\
2.49 \\
.91 \\
.58 \\
1.07 \\
.31 \\
.09 \\
\text { trace } \\
\ldots \ldots . . \\
\text { none } \\
\text { none } \\
\ldots . . . . .\end{array}$ & 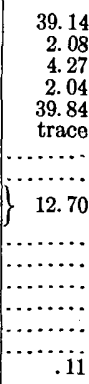 \\
\hline & 100.31 & 100.'32 & 99.81 & 100.86 & 100.18 \\
\hline
\end{tabular}

4. BUTंTE COUNTY.

Rocks from this county are also to be found under the heading of the Lassen Peak region. The following rocks were collected by $\mathrm{H}$. W. Turner, to whom the petrographic data are due. Analyses, with two exceptions, by W. F. Hillebrand, record Nos. 1432, 1456, 1461, and 1548. Analysis G is by H. N. Stokes, record No. 1562.

A. Granodiorite, north side of south fork of Feather River, opposite Enterprise. Lassenose. Described in 14th Ann., p. 441. Contains plagioclase, potash feldspar, quartz, hornblende, brown mica, and accessory minerals. The ferromagnesian minerals are largely altered to chlorite. P. R. C. 720 .

B. Granodiorite, 2 miles east of Bangor. Tonalose. Composition like A. The mica is largely altered to chlorite. See 14th Ann., p. 441. P. R. C. 717.

C. Diorite, South Honcut Creek. Beerbachose. Description supplied by Turner. Contains feldspar, probably all plagioclase, brown hornblende, and a little chlorite. P. R. C. 775 .

D. Quartz diorite, 4.6 miles south of Table Mountain, on ridge between Butte and Plumas counties. Camptonose. Described in 17th Ann., pt. 1, p. 521. Contains hornblende, feldspar, quartz, rutile, and a little secondary chlorite and epidote. P. R. C. 758.

E. Amphibole, separated from E. Analysis by William Valentine, record No. 1723 , $\mathrm{Cr}_{2} \mathrm{O}_{3}$ determination by Hillebrand. 


\begin{tabular}{|c|c|c|c|c|c|}
\hline & A. & B. & C. & D. & E. \\
\hline 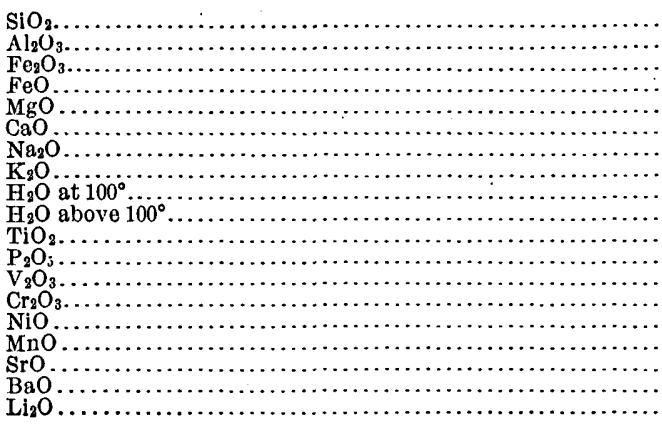 & $\begin{array}{r}70.36 \\
15.47 \\
.98 \\
1.17 \\
.87 \\
3.18 \\
4.91 \\
1.71 \\
.06 \\
1.00 \\
.20 \\
.11 \\
\ldots \ldots \\
\ldots \ldots \\
\text { trace } \\
\text { trace } \\
.06 \\
\text { trace }\end{array}$ & $\begin{array}{r}63.43 \\
14.20 \\
1.54 \\
4.56 \\
2.35 \\
5.51 \\
3.49 \\
2.19 \\
.15 \\
1.50 \\
.73 \\
.11 \\
\ldots \ldots . . \\
\ldots \ldots . . \\
\ldots \ldots 3 \\
\text { trace } \\
.06 \\
\text { none }\end{array}$ & $\begin{array}{r}57.87 \\
16.30 \\
1.71 \\
3.86 \\
5.50 \\
5.53 \\
5.01 \\
.75 \\
.26 \\
2.40 \\
.53 \\
.27 \\
\ldots \ldots \\
\ldots \ldots . \\
\ldots \ldots . \\
.08 \\
\text { trace } \\
.05 \\
\text { trace }\end{array}$ & $\begin{array}{r}54.64 \\
12.06 \\
1.81 \\
5.03 \\
11.86 \\
7.74 \\
2.35 \\
1.01 \\
.12 \\
2.44 \\
.61 \\
.08 \\
.03 \\
\ldots . . . \\
.05 \\
.13 \\
\text { trace } \\
.05 \\
\text { trace }\end{array}$ & 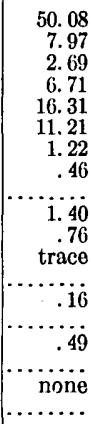 \\
\hline & 100.08 & 99.85 & 100. 12 & 100.01 & 100.46 \\
\hline
\end{tabular}

F. Meta-andesite tuff. Described in 14th Ann., p. 441. Contains plagioclase, augite, epidote, chlorite, and secondary hornblende. P. R. C. 71.9.

G. Uralite diorite, 1 mile southeast of Forbestown. Ornose. Described in 17th Ann., pt. 1, p. 521. Contains plagioclase, hornblende, and magnetite. P. R. C. 751.

H. Basalt, Oroville, Table Mountain. Camptonose. Described in 14th Ann., p. 441. Contains plagioclase, olivine, augite, and magnetite. P. R. C. 718.

I. Altered peridotite, 5 miles northeast of Strawberry Valley. Largely serpentine, with olivine, hornblende, magnetite, and calcite or dolomite. P. R. C. 742.

\begin{tabular}{|c|c|c|c|c|}
\hline - & F. & G. & H. & I. \\
\hline 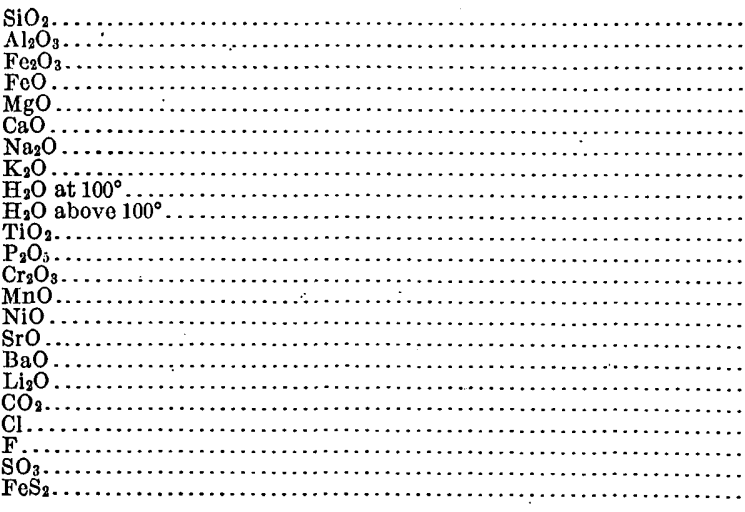 & $\begin{array}{r}54.66 \\
15.85 \\
1.82 \\
5.12 \\
5.64 \\
8.75 \\
3.46 \\
.47 \\
.25 \\
2.48 \\
.67 \\
.15 \\
\ldots .18 \\
.18 \\
\text { trace? } \\
\text { trace } \\
.04 \\
\text { none } \\
.39 \\
\ldots \ldots . . \\
\ldots \ldots . . \\
\ldots \ldots . . \\
.09\end{array}$ & $\begin{array}{r}51.07 \\
14.93 \\
6.44 \\
5.98 \\
4.84 \\
7.89 \\
5.04 \\
.16 \\
.24 \\
1.73 \\
1.65 \\
.19 \\
\ldots \ldots \ldots \\
.22 \\
\ldots \ldots . . \\
\ldots \ldots . . \\
\ldots \ldots . . . \\
\ldots \ldots . . \\
\ldots \ldots \ldots \\
\text { trace } \\
\text { trace } \\
\text { trace }\end{array}$ & $\begin{array}{r}50.66 \\
13.97 \\
2.55 \\
10.20 \\
4.45 \\
8.08 \\
3.32 \\
1.95 \\
.27 \\
.43 \\
2.39 \\
1.01 \\
\ldots \ldots . . \\
.29 \\
\text { trace } \\
\text { trace } \\
.22 \\
\text { none } \\
\ldots \ldots \ldots \\
.02 \\
\ldots \ldots \ldots . \\
\ldots \ldots . . . \\
\ldots \ldots . . .\end{array}$ & $\begin{array}{r}44.81 \\
a 1.88 \\
1.98 \\
4.52 \\
30.91 \\
6.58 \\
.15 \\
.15 \\
6.88 \\
\ldots .02 \\
.29 \\
.13 \\
.09 \\
\text { none } \\
\text { none } \\
\ldots \ldots . . \\
1.79 \\
\ldots \ldots . . \\
\ldots \ldots . . \\
\ldots \ldots . . \\
\ldots \ldots . .\end{array}$ \\
\hline & 100.02 & 100.38 & 99.81 & 100.18 \\
\hline
\end{tabular}

$a$ Includes possible $\mathrm{TiO}_{2}$.

5. SIERRA COUNTY.

Rocks collected and described by H. W. Turner. See paper in 17th Ann., pt. 1, p. 521. Additional details supplied by Turner are given here. Analyses A to $\mathrm{H}$ by W. F. Hillebrand, record Nos. 1456 and 1548. Analysis I by H. N. Stokes, record No. 1514. 
A: Granulite (aplite), Yuba Gap, road east of Sierra Buttes. Toscanose. Contains orthoclase, microcline, quartz, plagioclase, some shreds of greenish mica, and a little iron ore, chlorite, and apatite. P. R. C. 730.

B. Granulite (aplite), dike east of Milton. Toscanose. Contains orthoclase, quartz, plagioclase, a little microcline, brown mica, and iron ore. P. R. C. 734.

C. Biotite-quartz monzonite, Indian Valley. Lassenose. Contains plagioclase, orthoclase, quartz, brown mica, apatite, and iron ore. P. R. C. 737.

D. Quartz diorite porphyry, dike in Indian Valley granite. Yellowstonose. Contains plagioclase, hornblende, biotite, and quartz. P. R. C. 738.

E. Quartz-mica diorite, large area east of Milton. Harzose. Contains plagioclase, a turbid feldspar which is apparently not orthoclase, quartz, green hornblende, brown mica, iron ore, and apatite. P. R. C. 732.

\begin{tabular}{|c|c|c|c|c|c|}
\hline & A. & B. & C. & D. & E. \\
\hline 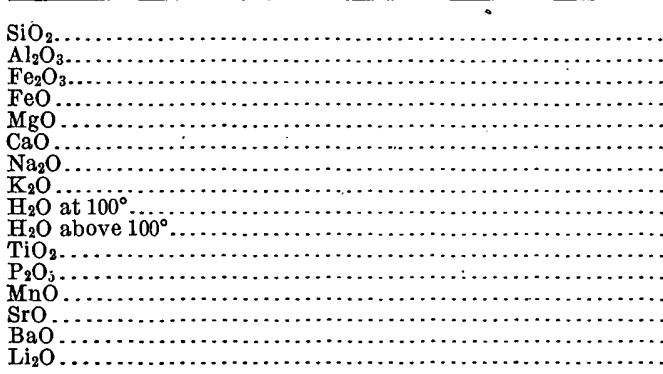 & $\begin{array}{r}76.03 \\
13.39 \\
.48 \\
.31 \\
.05 \\
1.28 \\
2.98 \\
5.18 \\
.15 \\
.34 \\
.07 \\
.03 \\
\text { trace } \\
\text { trace } \\
.04 \\
\text { none }\end{array}$ & $\begin{array}{r}75.97 \\
13.07 \\
.61 \\
.39 \\
.14 \\
1.49 \\
2.51 \\
5.62 \\
.14 \\
.24 \\
.09 \\
\text { trace } \\
\text { trace } \\
.03 \\
.14 \\
\text { trace }\end{array}$ & $\begin{array}{r}68.65 \\
16.34 \\
.93 \\
1.48 \\
1.29 \\
3.07 \\
4.85 \\
1.85 \\
.24 \\
.62 \\
.28 \\
.15 \\
.08 \\
.07 \\
.09 \\
\text { trace }\end{array}$ & $\begin{array}{r}66.65 \\
17.61 \\
.93 \\
1.67 \\
1.26 \\
4.44 \\
4.59 \\
1.70 \\
.03 \\
.41 \\
.33 \\
.18 \\
.07 \\
\ldots . .12 \\
.12 \\
\text { trace }\end{array}$ & $\begin{array}{r}57.26 \\
16.51 \\
3.27 \\
5.19 \\
3.41 \\
6.69 \\
2.65 \\
2.93 \\
.20 \\
.95 \\
.53 \\
.30 \\
.18 \\
.06 \\
.0 \\
\text { trace }\end{array}$ \\
\hline . & 100.33 & 100.44 & 99.99 & 99.99 & 100.23 \\
\hline
\end{tabular}

F. Diabase porphyry, dike east of Milton. Camptonose. Contains labradorite and other plagioclase, augite, and hornblende, the last mineral being perhaps secondary. P. R. C. 733.

G. Hypersthene andesite, point northeast of Goodyears Bar. Yellowstonose. Contains plagioclase and rhombic pyroxene, a little augite; and scales which seem to represent former biotite, now replaced by magnetite. P. R. C. 731.

H. Hornblende-pyroxene andesite, dike southeast of Poker Flat. Tonalose. Contains plagioclase, augite, hornblende, magnetite, some glass, and occasional quartz. P. R. C. 736.

I. Quartz-bearing andesite, northwest of Downieville.' Tonalose. Contains plagioclase, augite, enstatite, magnetite, occasional quartz, and probably glass. P. R. C. 753 .

\begin{tabular}{|c|c|c|c|c|}
\hline$=$ & $\mathbf{F}$. & G. & H. & $\mathrm{x}$. \\
\hline 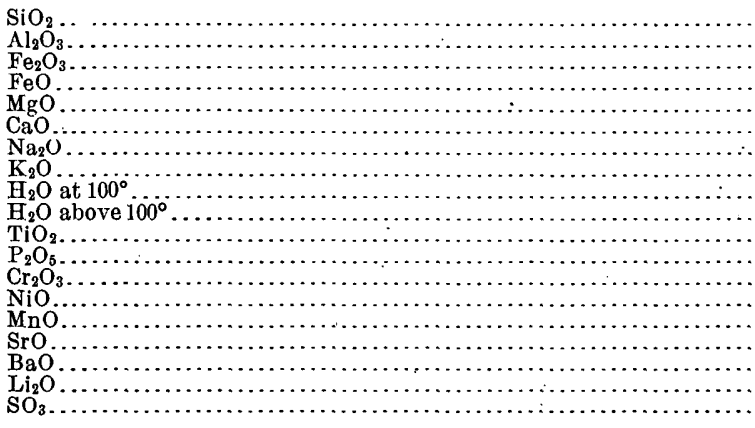 & $\begin{array}{r}51.27 \\
12.14 \\
2.51 \\
6.71 \\
10.88 \\
10.32 \\
2.00 \\
1.63 \\
.17 \\
1.16 \\
.60 \\
.21 \\
\ldots .04 \\
.21 \\
\text { trace? } \\
.07 \\
\text { trace } \\
\ldots \ldots\end{array}$ & $\begin{array}{r}66.94 \\
16.49 \\
1.41 \\
1.87 \\
1.98 \\
4.77 \\
3.88 \\
1.65 \\
.35 \\
.22 \\
.30 \\
.12 \\
\ldots \ldots . \\
\ldots .13 \\
.05 \\
.05 \\
.07 \\
\text { trace } \\
\ldots \ldots . \\
\ldots . .\end{array}$ & $\begin{array}{r}59.34 \\
17.61 \\
3.63 \\
2.28 \\
3.50 \\
6.45 \\
3.40 \\
1.94 \\
.64 \\
.74 \\
.32 \\
.25 \\
\ldots \ldots . \\
\ldots . .2 \\
.12 \\
.04 \\
.11 \\
\text { trace } \\
\ldots . . . \\
. . .\end{array}$ & $\begin{array}{r}60.02 \\
16.07 \\
2.17 \\
3.46 \\
4.57 \\
7.01 \\
3.55 \\
1.59 \\
.24 \\
.45 \\
.42 \\
.17 \\
\text { trace } \\
\ldots . . \\
.10 \\
\text { trace } \\
.08 \\
\text { none } \\
.06\end{array}$ \\
\hline & 99.92 & 100. 23 & 100.37 & 99.96 \\
\hline
\end{tabular}




\section{NEVADA CITY AND GRASS VALLEY.}

Rocks of a mining district in Nevada County, described by Lindgren in 17th Ann., pt. 2 , p. 1.

A. Granodiorite, 1 mile southeast of Nevada City. Yellowstonose. Contains hornblende, biotite, quartz, plagioclase, orthoclase, magnetite, apatite, sphene, and pyrite. Analysis by W. F. Hillebrand, record No. 1478. P. R. C. 1521.

B. Granodiorite, Kate Hayes Hill, Grass Valley. Harzose. Contains plagioclase, orthoclase, quartz, hornblende, pyrite, magnetite, apatite, sphene, and zircon. Analysis by Hillebrand, No. 1478.

C. Hornblende porphyrite, Nevada City. Tonalose. Contains feldspar, hornblende, quartz, epidote, sericite, and biotite. Analysis by H. N. Stokes, record No. 1531.

D. Quartz porphyrite, New Ophir claim, Grass Valley. Tonalose. Contains plagioclase, quartz, uralite, epidote, and augite, and hornblende altered into chlorite. Analysis by Stokes, No. 1531.

E. Diabase, near Maryland mine, Grass Valley. Ornose. Contains feldspar, augite, hornblende, ilmenite, pyrrhotite, pyrite, and some chlorite. Analysis by Stokes, No. 1522.

F. Diabase, Grass Valley. Bandose. Contains feldspar, pyroxene, hornblende, ilmenite, pyrrhotite, pyrite, and chlorite, and probably a little quartz. Analysis by Stokes, No. 1522.

\begin{tabular}{|c|c|c|c|c|c|c|}
\hline . & A. & B. & C. & D. & E. & F. \\
\hline 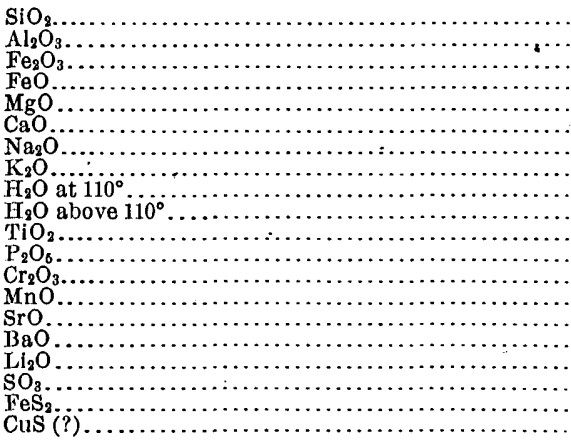 & $\begin{array}{r}66.65 \\
16.15 \\
1.52 \\
2.36 \\
1.74 \\
4.53 \\
3.40 \\
2.65 \\
.18 \\
.72 \\
.38 \\
.10 \\
.10 \\
\text { trace } \\
.07 \\
\text { trace } \\
\ldots \ldots 2 \\
.02\end{array}$ & $\begin{array}{r}63.85 \\
15.84 \\
1.91 \\
2.75 \\
2.07 \\
4.76 \\
3.29 \\
3.08 \\
.28 \\
1.65 \\
.58 \\
.13 \\
\ldots . .0 \\
.07 \\
\text { trace } \\
.06 \\
\text { trace } \\
\ldots \ldots . . \\
.04\end{array}$ & $\begin{array}{r}62.09 \\
16.69 \\
1.45 \\
3.76 \\
1.93 \\
6.08 \\
3.36 \\
1.84 \\
.19 \\
1.47 \\
.32 \\
.39 \\
\ldots \ldots . . \\
\text { trace } \\
\ldots \ldots . . \\
. \ldots \\
\ldots .10 \\
\ldots . . .\end{array}$ & 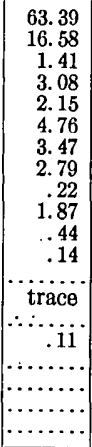 & $\begin{array}{r}51.01 \\
.11 .89 \\
1.57 \\
6.08 \\
8.87 \\
10.36 \\
4.17 \\
.15 \\
.24 \\
2.09 \\
.98 \\
.17 \\
.04 \\
\text { trace } \\
\ldots . . . \\
\text { none } \\
\ldots \ldots . . \\
\ldots \ldots .73 \\
1.73 \\
\text { trace }\end{array}$ & $\begin{array}{r}53.19 \\
17.12 \\
4.35 \\
5.16 \\
3.98 \\
9.39 \\
2.79 \\
.28 \\
.17 \\
1.21 \\
1.34 \\
.13 \\
\text { none } \\
\text { trace } \\
\ldots . . . \\
\text { trace }\end{array}$ \\
\hline & 100.57 & 100.36 & 99.77 & 100.41 & 99.35 & 100.05 \\
\hline
\end{tabular}

G. Wall rock, Federal Loan mine. A siliceous argillite, of sedimentary origin. Contains quartz, feldspar, biotite, pyrrhotite, and a little calcite. Analysis by Hillebrand, No. 1478 .

H. Altered wall rock, Providence mine. Derived from granodiorite. Analysis by Hillebrand, No. 1478.

I. Altered wall rock, Providence mine, back vein. Derived from granodiorite and schist. Analysis by Hillebrand, No. 1478.

J. Altered wall rock, North Star mine. Derived from uralite diabase. Contains quartz, sericite, calcite, pyrite, and sphene. Analysis by Hillebrand, No. 1478.

K. Altered country rock, Idaho mine. Derived from serpentine. Analysis by Hillebrand, No. 1478.

15619-Bull, 419-10-11 


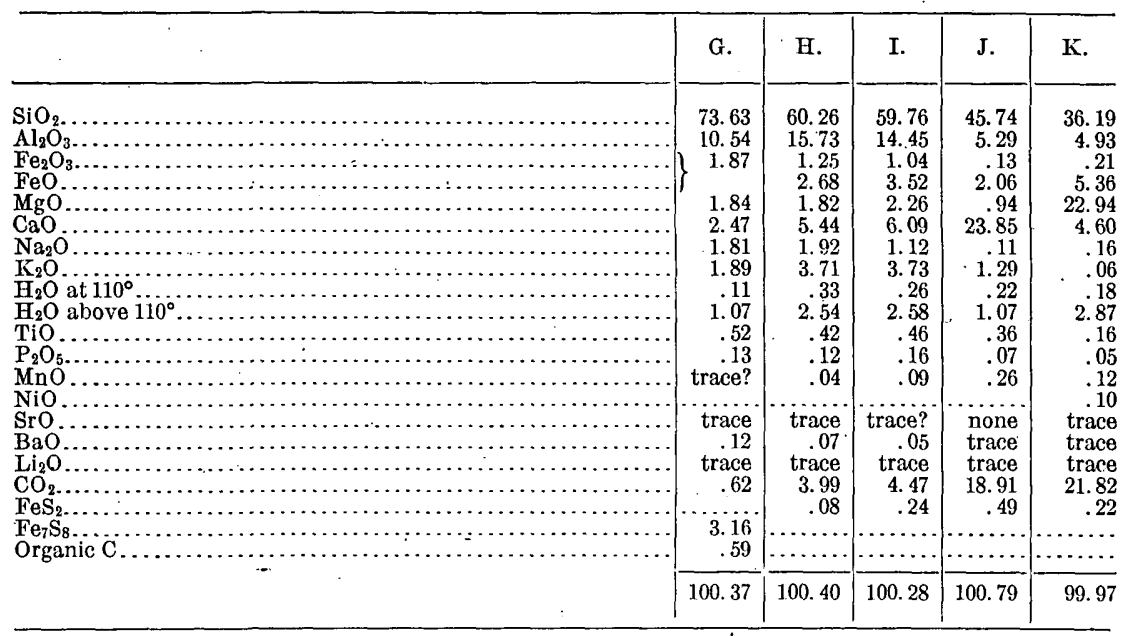

L. Bleached country rock, next to vein, Osborne Hill mine. Derived from sandstone. Anallysis by George Steiger, record No. 1541.

M. Altered wall rock, Empire mine. Derived from granodiorite. Analysis by Steiger, No. 1541 . Sp. gr., 2.782, $20^{\circ}$.

N. Altered wall rock, Ebaugh tunnel. Derived from granodiorite. Mainly quartz and sericite, with pyrite, apatite, sphene, and carbonates. Analysis by Steiger, No. 1541. Sp. gr., $2.747,20^{\circ}$.

o. Altered wall rock, Federal Loan mine. Derived from siliceous argillite. Analysis by Steiger, No. 1541.

\begin{tabular}{|c|c|c|c|c|}
\hline & L. & M. & N.' & O. \\
\hline 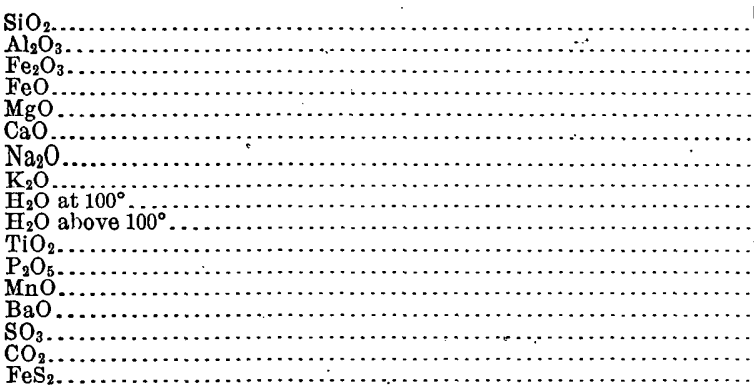 & $\begin{array}{r}71.97 \\
15.75 \\
.77 \\
.45 \\
.80 \\
.80 \\
.33 \\
4.88 \\
.30 \\
2.16 \\
.88 \\
.15 \\
\text { none } \\
\text { trace } \\
\text { trace } \\
.38 \\
.56\end{array}$ & $\begin{array}{r}58.43 \\
17.40 \\
.77 \\
2.19 \\
1.50 \\
5.25 \\
1.76 \\
4.03 \\
.30 \\
2.61 \\
\text { none } \\
.13 \\
\text { none } \\
\text { none } \\
\text { none } \\
4.04 \\
1.59\end{array}$ & $\begin{array}{r}56.25 \\
17.65 \\
.76 \\
2.64 \\
1.69 \\
4.46 \\
.30 \\
6.01 \\
.30 \\
2.36 \\
.25 \\
.21 \\
\text { none } \\
.03 \\
\text { none } \\
4.82 \\
2.87\end{array}$ & $\begin{array}{r}34.91 \\
15.55 \\
.17 \\
4.96 \\
4.58 \\
11.10 \\
.19 \\
4.28 \\
.30 \\
1.86 \\
1.65 \\
.82 \\
\text { none } \\
\text { none } \\
\text { none } \\
15.57 \\
4.20\end{array}$ \\
\hline & 100.18 & 100.00 & 100.60 & 100.14 \\
\hline
\end{tabular}

\section{PLACER COUNTY.}

First, a series of rocks from the Ophir mining district, described by Lindgren in 14th Ann., p. 249. Analyses by W. F. Hillebrand, record Nos. 1419, 1433, 1434.

A. Granodiorite, quarries at Lincoln, 8 miles west of Ophir. Tonalose. Contains feldspars, quartz, biotite, and hornblende. P. R: C. 1526.

B. Pyritiferous amphibolite, Conrad tunnel. Partly altered. Contains pyrite, hornblende, magnetite, feldspars, quartz, epidote, chlorite, a few scales of mica, rutile, and carbonates. Sp. gr., $2.901,23^{\circ}$.

C. Dike rock, near camptonite, Casey's tunnel, Flat Ledge, Duncan Hill. Placerose. Contains hornblende, feldspars, pyrite, and apatite, with secondary epidote and quartz. 
D. Altered wall rock, Mina Rica vein. Sp. gr., 2.979, $20^{\circ}$.

E. Altered wall rock, Plantz vein. These rocks, D and E, contain quartz, muscovite, a little chlorite, pyrite, and sphene, with carbonates of calcium, magnesium, and iron.

\begin{tabular}{|c|c|c|c|c|c|}
\hline ' & A. & B. & C. & D. & E. \\
\hline 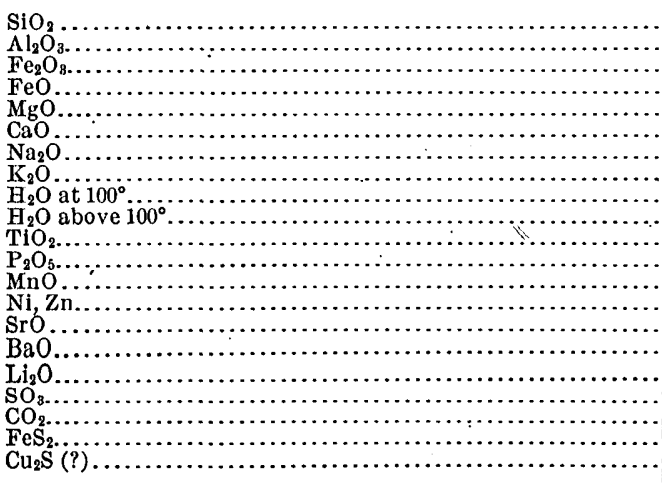 & $\begin{array}{r}65.54 \\
16.52 \\
1.40 \\
2.49 \\
2.52 \\
4.88 \\
4.09 \\
1.95 \\
.12 \\
.59 \\
.39 \\
.18 \\
.06 \\
\ldots \ldots . . \\
\text { trace } \\
\text { trace } \\
\text { trace } \\
\ldots . . . . \\
\ldots . . . . \\
\ldots . . .\end{array}$ & $\begin{array}{r}45.56 \\
14.15 \\
1.20 \\
9.83 \\
6.76 \\
2.30 \\
1.57 \\
1.18 \\
.23 \\
4.84 \\
1.11 \\
.14 \\
.25 \\
\text { traces } \\
\text { trace } \\
\text { trace } \\
\text { trace } \\
.03 \\
3.04 \\
7.86 \\
.10\end{array}$ & $\begin{array}{r}60.09 \\
16.43 \\
2.28 \\
3.01 \\
4.37 \\
5.76 \\
4.52 \\
.70 \\
.20 \\
1.16 \\
.63 \\
.12 \\
.12 \\
\ldots \ldots \\
\text { trace } \\
\text { trace } \\
\text { none } \\
\text { trace } \\
.07 \\
.34\end{array}$ & $\begin{array}{r}37.01 \\
12.99 \\
.43 \\
3.57 \\
5.49 \\
9.78 \\
.13 \\
4.02 \\
.13 \\
1.92 \\
.85 \\
.06 \\
. .24 \\
\text { traces } \\
\text { trace } \\
\text { trace } \\
\text { trace } \\
.04 \\
15.04 \\
7.99\end{array}$ & $\begin{array}{r}46.13 \\
15.82 \\
.89 \\
2.27 \\
2.13 \\
10.68 \\
.17 \\
5.30 \\
.12 \\
2.42 \\
.67 \\
.10 \\
.09 \\
\text { traces } \\
\text { trace } \\
\text { trace } \\
\text { trace } \\
.04 \\
11.24 \\
1.61\end{array}$ \\
\hline . & 100.73 & 100.15 & 99.80 & 99.69 & 99.68 \\
\hline
\end{tabular}

Second, rocks from other localities in Placer County. Studied also by Lindgren, who furnishes the petrographic data. Analysis A by W. H. Melville, record No. 1346; B, C, D, and E by W. F. Hillebrand, record No. 1419.

A. Granite, Rocklin. Lassenose. A normal granite, containing quartz, orthoclase, plagioclase, biotite, muscovite, magnetite, apatite, and zircon, with some secondary chlorite and epidote derived from the biotite. Described by Lindgren in Bull. 150, p. 170 . P. R. C. 66.

B. Granodiorite, Donner Pass. Tonalose. Contains plagioclase, orthoclase, quartz, hornblende, biotite, and sphene. P. R. C. 1525.

C. Gabbro, 2 miles south of Emigrant Gap, on road to Onion Valley. Andose. Contains biotite, hypersthene, diallage, plagioclase, and orthoclase. P. R. C. 1523.

D. Gabbro, same locality as C. Vaalose. Contains hypersthene, diallage, plagioclase, and orthoclase. P. R. C. 1524.

E. Quartz diorite, southeast spur of English Mountain. Placerose. Contains "basic" plagioclase, augite, and quartz. 'P. R. C. 1522.

\begin{tabular}{|c|c|c|c|c|c|}
\hline$=$ & A. & B. & C. & D. & E. \\
\hline 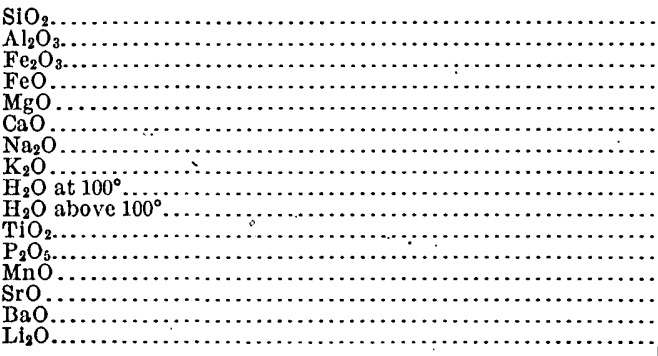 & 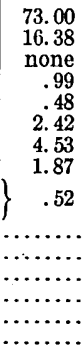 & $\begin{array}{r}59.48 \\
17.25 \\
2.15 \\
4.06 \\
2.67 \\
6.50 \\
3.53 \\
2.27 \\
.09 \\
.71 \\
.93 \\
.33 \\
.11 \\
\text { trace } \\
.09 \\
\text { trace }\end{array}$ & $\begin{array}{r}55.40 \\
15.32 \\
2.70 \\
5.49 \\
5.75 \\
9.90 \\
2.89 \\
1.52 \\
.03 \\
.38 \\
.60 \\
.22 \\
.11 \\
\text { none } \\
.07 \\
\text { trace }\end{array}$ & $\begin{array}{r}55.87 \\
13.52 \\
2.70 \\
5.89 \\
6.51 \\
8.87 \\
2.42 \\
1.72 \\
.09 \\
1.56 \\
.56 \\
.25 \\
.10 \\
\text { none } \\
.02 \\
\text { trace }\end{array}$ & $\begin{array}{r}64.67 \\
16.62 \\
.51 \\
.76 \\
2.26 \\
9.50 \\
4.10 \\
.34 \\
.08 \\
.37 \\
.51 \\
.12 \\
\text { trace } \\
\text { trace } \\
.02 \\
\text { trace }\end{array}$ \\
\hline & 100.19 & 100.17 & 100.38 & 100.08 & 99.86 \\
\hline
\end{tabular}




\section{ELDORADO COUNTY.}

A. Granitite, Placerville canal, one-third mile north of Ditch Camp No. 7. Alaskose. Collected by W. Lindgren, who reports it as containing biotite, orthoclase, plagioclase, and quartz. Analysis by George Steiger, record No. 1591. P. R. C. 1527.

B. Granodiorite, 2 miles south of Silver Lake Hotel. Amiatose. Collected by Lindgren, who reports it as containing hornblende, biotite, plagioclase, and quartz. Analysis by Steiger, No. 1591. Analyses A and B are published by Lindgren in Am. Jour. Sci., 4th ser., vol. 3, p. 306 . P. R. C. 1528.

C. Porphyrite, 1 mile southwest of Latrobe. Dacose. Published by Turner in 17th Ann., pt. 1, p. 521. Contains abundant plagioclase, less augite, calcite or dolomite, iron disulphide, a little chlorite, and secondary greenish mica. Analysis by W. F. Hillebrand, record No. 1432 . P. R. C. 721.

\begin{tabular}{|c|c|c|c|}
\hline 4 & A. & B. & C. \\
\hline 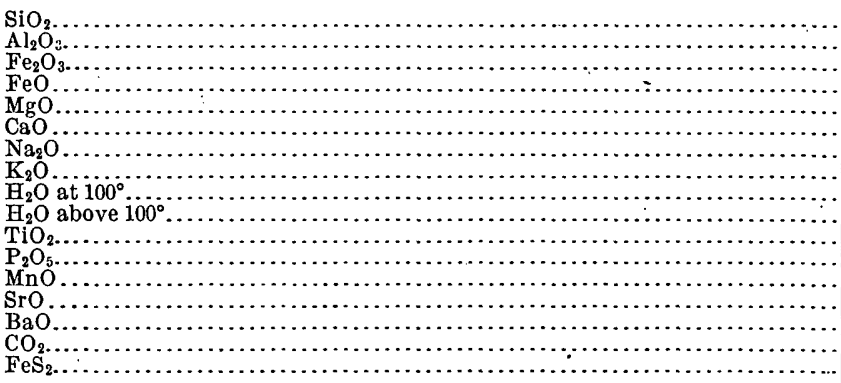 & $\begin{array}{r}77.68 \\
11.81 \\
.72 \\
.51 \\
.18 \\
.72 \\
2.96 \\
5.00 \\
.04 \\
.27 \\
.14 \\
.10 \\
\text { trace } \\
\cdots \ldots . \\
\cdots \ldots .\end{array}$ & \begin{tabular}{r}
67.45 \\
15.51 \\
1.76 \\
2.21 \\
1.10 \\
3.60 \\
3.47 \\
3.66 \\
.14 \\
.63 \\
.58 \\
.12 \\
$\ldots \ldots . .$. \\
\hdashline$\ldots . .$. \\
\hdashline$\ldots . .$. \\
$\ldots \ldots .$. \\
$\ldots . .$.
\end{tabular} & $\begin{array}{r}68.58 \\
13.04 \\
.26 \\
3.40 \\
1.01 \\
3.22 \\
4.94 \\
1.90 \\
.16 \\
1.00 \\
.57 \\
.20 \\
.15 \\
\text { trace } \\
.10 \\
1.31 \\
.15\end{array}$ \\
\hline & 100.13 & 100.23 & 99.99 \\
\hline
\end{tabular}

9. AMADOR COUNTY.

Rocks collected by H. W. Turner, and analyses published in 14th Ann., p. 441; and 17th Ann., pt. 1, p. 521. Additional data supplied by Turner. Analyses by W: F. Hillebrand, record Nos. 1432, 1456, and 1597.

A. Rhyolite, south point of Buena Vista Peak. Magdeburgose. Contains sanidine, quartz, and biotite in a glassy groundmass. P. R. C. 729 .

B. Quartz monzonite, north fork of Mokelumne River. Amiatose. Contains plagioclase, microcline, quartz, abundant biotite, iron ore, sphene, apatite, and perhaps rutile. P. R. C. 770 .

C. Quartz monzonite, north fork of the Mokelumne River. Toscanose. Like B. P. R. C. 765 .

D. Quartz porphyrite schist, $2 \frac{1}{2}$ miles southeast of Buena Vista Peak. Tehamose. Contains porphyritic quartz and hornblende, also calcite and other carbonates. See 14th Ann. P. R. C. 723.

E. Quartz diorite gneiss, north fork of Mokelumne River. Tonalose. Contains plagioclase, hornblende, quartz, brown mica, accessory biotite, and iron oxide. P. R. C. 764.

F. Diorite porphyry, north fork of Mokelumne River. Andose. Contains plagioclase, brown hornblende, epidote, and a little sulphide of iron and chlorite. P. R. C. 769 . 


\begin{tabular}{|c|c|c|c|c|c|c|}
\hline & A. & B. & C. & D. & E. & F. \\
\hline 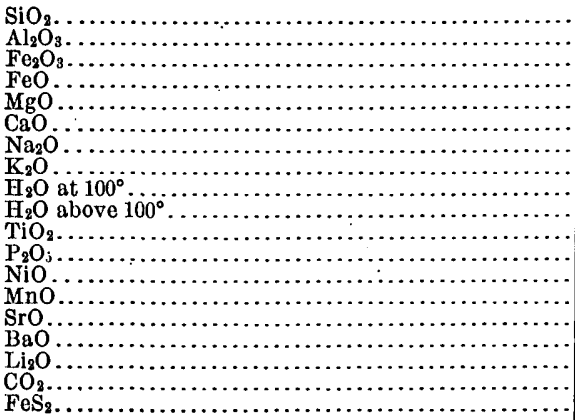 & $\begin{array}{r}73.23 \\
12.73 \\
.99 \\
.16 \\
.22 \\
.61 \\
1.91 \\
5.17 \\
.53 \\
4.51 \\
.09 \\
.02 \\
\ldots \ldots \\
\text { trace } \\
\text { none } \\
.02 \\
\text { trace } \\
\ldots \ldots \ldots \\
\ldots \ldots\end{array}$ & $\begin{array}{r}70.75 \\
15.13 \\
.98 \\
1.43 \\
.73 \\
3.09 \\
3.05 \\
3.62 \\
.10 \\
.51 \\
.42 \\
.10 \\
\text { none } \\
\text { trace } \\
.04 \\
.12 \\
\text { trace } \\
\text { none } \\
.06\end{array}$ & $\begin{array}{c}70.43 \\
15.51 \\
.96 \\
1.28 \\
.37 \\
2.76 \\
2.75 \\
5.14 \\
.08 \\
.40 \\
.24 \\
.11 \\
(?) \\
\text { trace } \\
.05 \\
.20 \\
\text { trace } \\
\text { none } \\
\text { trace }\end{array}$ & $\begin{array}{r}70.29 \\
11.83 \\
1.30 \\
2.08 \\
1.24 \\
2.30 \\
2.68 \\
3.05 \\
.10 \\
1.35 \\
.29 \\
.07 \\
\ldots . . \\
.12 \\
\text { trace? } \\
.07 \\
\text { none } \\
3.25 \\
\ldots . . .\end{array}$ & $\begin{array}{r}57.41 \\
17.71 \\
2.16 \\
5.01 \\
3.38 \\
6.73 \\
3.12 \\
1.82 \\
.20 \\
1.14 \\
1.04 \\
.24 \\
.02 \\
.15 \\
.04 \\
.09 \\
\text { trace } \\
\text { none } \\
\text { none }\end{array}$ & $\begin{array}{r}55.18 \\
17.35 \\
2.77 \\
3.90 \\
4.80 \\
7.98 \\
3.42 \\
1.42 \\
.16 \\
1.52 \\
.83 \\
.20 \\
.03 \\
.15 \\
.06 \\
.04 \\
\text { trace } \\
\text { none } \\
.28\end{array}$ \\
\hline & 100.19 & 100.13 & 100.28 & 100.02 & 100.26 & 100.09 \\
\hline
\end{tabular}

G. Diorite, north fork of Mokelumne River. Amadorose. Contains quartz, feldspar, biotite, sphene, epidote, and secondary chlorite. P. R. C. 771.

H. Diorite, north fork of Mokelumne River. Andose. Contains plagioclase, quartz, hornblende, biotite, apatite, iron ore, epidote, and chlorite. P. R. C. 772.

I. Plagioclase gneiss, north fork of Mokelumne River. Hessose-andose. Contains plagioclase, hornblende, biotite, and apatite. P. R. C. 768 .

J. Plagioclase gneiss, north fork of Mokelumne River. Bandose. Contains plagioclase, hornblende, brown mica, apatite, epidote, and grains of iron ore. P. R. C. 7.67.

\begin{tabular}{|c|c|c|c|c|}
\hline & G. & H. & I. & J. \\
\hline 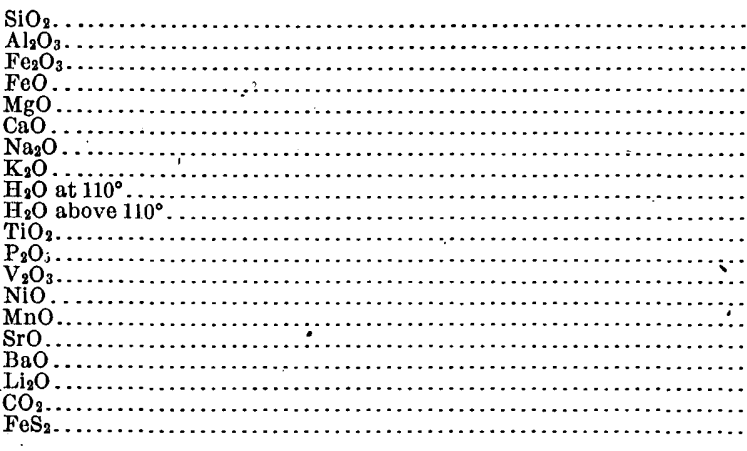 & $\begin{array}{r}69.66 \\
17.57 \\
.21 \\
1.04 \\
.58 \\
4.54 \\
4.91 \\
.71 \\
.05 \\
.50 \\
.21 \\
.03 \\
\text { none } \\
\text { trace } \\
.05 \\
.03 \\
\text { none } \\
\text { none } \\
\text { trace? }\end{array}$ & $\begin{array}{r}55.86 \\
19.30 \\
.91 \\
4.78 \\
2.94 \\
7.31 \\
3.52 \\
1.52 \\
.19 \\
1.23 \\
1.20 \\
.38\end{array}$ & $\begin{array}{r}52.21 \\
18.79 \\
2.71 \\
5.30 \\
5.11 \\
8.01 \\
3.31 \\
1.60 \\
.12 \\
1.35 \\
1.16 \\
.36 \\
\ldots \ldots \\
\text { trace } \\
.06 \\
.08 \\
\text { trace } \\
\text { none } \\
.06\end{array}$ & $\begin{array}{r}46.63 \\
19.47 \\
3.26 \\
6.63 \\
5.37 \\
9.15 \\
3.19 \\
1.55 \\
.10 \\
1.61 \\
1.82 \\
.66 \\
.02 \\
.02 \\
.21 \\
.06 \\
.14 \\
\text { trace } \\
\text { none } \\
.19\end{array}$ \\
\hline & 100.09 & 99.86 & 100.23 & 100.08 \\
\hline
\end{tabular}

K. Wollastonite gneiss, north fork of Mokelumne River. Mainly wollastonite, but garnet, quartz, and sphene are also present. 'P. R. C. 766.

L. Melaphyre tuff, altered basalt, west of Jackson. Contains augite and plagioclase, with secondary quartz, chlorite, and chrysotile. Originally glassy in part, but devitrified. See 14th Ann. P. R. C. 722.

M. Reddish-brown mica separated from pyroxenic gneiss, north fork of Mokelumne River, about 1 kilometer above mouth of Bear River. Described by Turner in Am. Jour. Sci., 4th ser., vol. 7, p. 294. Analysis by William Valentine, record No. 1736. 


\begin{tabular}{|c|c|c|c|}
\hline - & K. & L. & M. \\
\hline 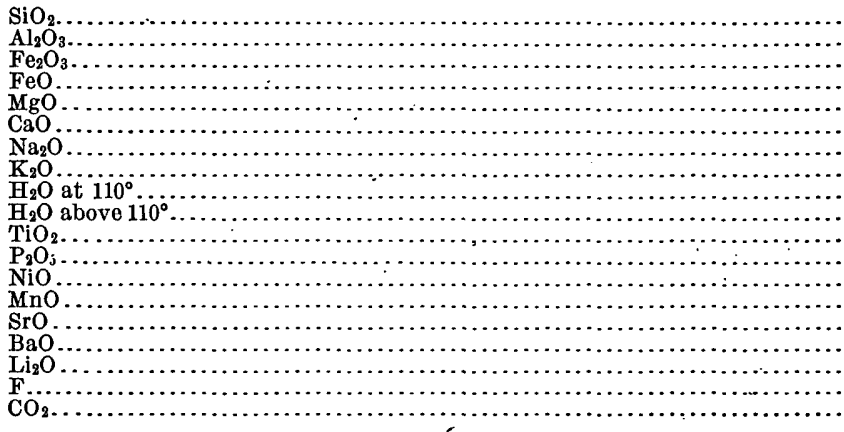 & $\begin{array}{r}50.67 \\
6.37 \\
.31 \\
.50 \\
.58 \\
40.34 \\
.14 \\
.22 \\
.08 \\
.31 \\
.20 \\
\text { none } \\
\text { none } \\
\text { trace } \\
\text { none } \\
\text { none } \\
\text { none } \\
. . . . . \\
.52\end{array}$ & $\begin{array}{r}49: 24 \\
14.79 \\
1.36 \\
8.00 \\
6.89 \\
10.74 \\
2.76 \\
.88 \\
.20 \\
2.97 \\
.96 \\
.17 \\
\ldots . .18 \\
.18 \\
\text { trace } \\
.04 \\
\text { trace } \\
\ldots \ldots . . \\
.90\end{array}$ & $\begin{array}{r}36.62 \\
14.37 \\
4.04 \\
17.09 \\
9.68 \\
1.48 \\
.45 \\
8.20 \\
.90 \\
3.26 \\
3.03 \\
\text { none } \\
\ldots \ldots . . \\
40 \\
\text { trace } \\
.33 \\
\text { trace } \\
.10 \\
\ldots \ldots . .\end{array}$ \\
\hline \multirow[t]{2}{*}{ Less 0 . } & $\begin{array}{r}100.24 \\
\ldots \ldots\end{array}$ & $\begin{array}{r}100.08 \\
\ldots \ldots\end{array}$ & $\begin{array}{r}99.95 \\
.04\end{array}$ \\
\hline & & & 99.91 \\
\hline
\end{tabular}

10. Calaveras county.

Rocks collected by H. W. Turner, and described in 14th Ann., p. 441. Additional data supplied by Turner relative tó analysis B. Ánalyses by W. F. Hillebrand, record No. 1432.

A. Metadacite, $1 \frac{3}{4}$ miles southeast of Milton. Vulcanose. Contains quartz, feldspar, and hornblende. P. R. C. 777.

B. Metadacite, $1 \frac{1}{2}$ miles northeast of Milton. Lassenose. Contains feldspar, quartz, epidote, chlorite, and iron ore, in a groundmass made up probably of feldspar and quartz. P. R. C. 752 .

C. Meta-andesite, $1 \frac{1}{2}$ miles northward from Jenny Lind. Placerose. Contains quartz, plagioclase, epidote, and chlorite derived from augite.

\begin{tabular}{|c|c|c|c|}
\hline & A. & B. & C. , \\
\hline 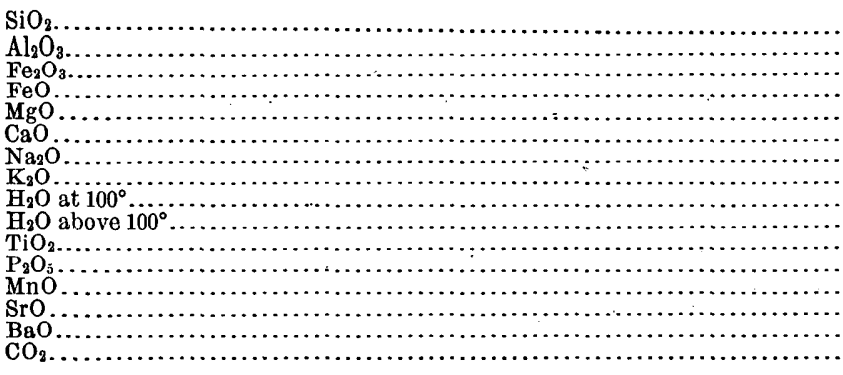 & $\begin{array}{r}72.24 \\
13.84 \\
1.45 \\
1.86 \\
1.10 \\
3.40 \\
4.43 \\
.39 \\
.17 \\
.69 \\
.41 \\
.10 \\
.12 \\
\text { trace } \\
.08 \\
\cdots \\
\cdots\end{array}$ & $\begin{array}{r}71.19 \\
13.81 \\
1.45 \\
1.68 \\
.74 \\
2.87 \\
4.24 \\
1.82 \\
.15 \\
.92 \\
.35 \\
.08 \\
.07 \\
\text { trace } \\
.16 \\
.82\end{array}$ & $\begin{array}{r}61.37 \\
15.41 \\
3.15 \\
3.89 \\
3.48 \\
4.42 \\
3.76 \\
.34 \\
.29 \\
2.70 \\
.60 \\
.08 \\
.47 \\
\text { trace } \\
.08 \\
\ldots . . .\end{array}$ \\
\hline & 100.28 & 100.35 & 100.04 \\
\hline
\end{tabular}

\section{TUOLUMNE COUNTY.}

Rocks collected by H. W. Turner, and partly described in his papers in 14th and 17th Ann. The latites were named and described by Ransome in Bull. 89. Some additional data have been furnished by Turner. 
A. Soda syenite porphyry, dike east of Moccasin Creek. Tuolumnose. Consists mainly of albite, with a greenish mineral which is probably ægirite. Analysis by H. N. Stokes, record No. 1563 . P. R. C. 773.

B. Augite syenite, dike on Turnback Creek, about 1 mile north of Carter post-office. Highwoodose. Contains orthoclase and augite, with less plagioclase and quartz. Analysis by Stokes, No. 1642 . P. R. C. 789.

C. Diorite, dike $1 \frac{1}{2}$ miles southeasterly from Sonora. Tonalose. Contains feldspar largely altered to hornblende. A few black grains are probably iron ore. Analysis by W. F. Hillebrand, record No. 1548 . P. R. C. 759.

D. Quartz-pyroxene diorite, large area east of Sonora. Harzose. Contains plagioclase, quartz, biotite, augite, rhombic pyroxene, and a trace of iron ore. Analysis by Hillebrand, No. 1548 . P. R. C. 760.

E. Diorite, dike about $1 \frac{1}{2}$ miles sourtheasterly from Sonora. Camptonose. Contains altered plagioclase and hornblende, with epidote, chlorite, and iron disulphide as secondary products. Analysis by Hillebrand, No. 1548. P. R. C. 761.

\begin{tabular}{|c|c|c|c|c|c|}
\hline & A. & B. & C. & D. & E. \\
\hline 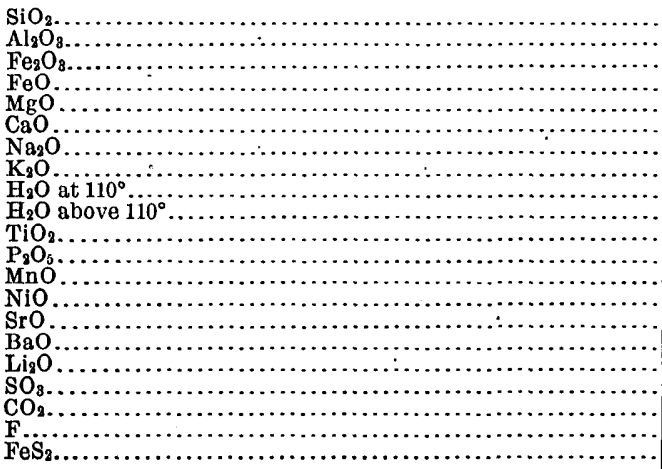 & $\begin{array}{r}67.53 \\
18.57 \\
1.13 \\
.08 \\
.24 \\
.55 \\
11.50 \\
.10 \\
.15 \\
.31 \\
.07 \\
.11 \\
\operatorname{trace} \\
\ldots \ldots . . . \\
\text { trace } \\
\ldots \ldots . . . \\
\text { trace } \\
\ldots \ldots . . . \\
\text { trace }\end{array}$ & $\begin{array}{r}61.28 \\
14.71 \\
1.21 \\
2.85 \\
1.69 \\
5.61 \\
2.99 \\
7.70 \\
.28 \\
.43 \\
.41 \\
.16 \\
\operatorname{trace} \\
\ldots \ldots . . \\
.04 \\
.72 \\
\ldots \ldots . . \\
.08 \\
\ldots \ldots \ldots . \\
\ldots \ldots \ldots . . \\
\ldots \ldots . .\end{array}$ & $\begin{array}{r}58.05 \\
15.46 \\
1.69 \\
5.09 \\
4.84 \\
6.94 \\
2.86 \\
2.14 \\
\vdots .10 \\
2.02 \\
. .72 \\
.16 \\
.14 \\
\text { none } \\
\text { trace } \\
.07 \\
\text { trace } \\
\text {....... } \\
\text { none } \\
\ldots . . . . \\
\text { none }\end{array}$ & $\begin{array}{r}57.80 \\
16.43 \\
1.62 \\
6.51 \\
4.14 \\
7.21 \\
2.35 \\
2.29 \\
.11 \\
.38 \\
.70 \\
.19 \\
.18 \\
.03 \\
\text { trace? } \\
.09 \\
\text { trace } \\
\text {...... } \\
\text { none } \\
\text {...... } \\
\text { none }\end{array}$ & $\begin{array}{r}53.46 \\
14.81 \\
2.60 \\
5.15 \\
7.27 \\
8.44 \\
2.64 \\
1.30 \\
.12 \\
2.13 \\
.70 \\
.16 \\
.18 \\
.05 \\
\text { trace } \\
.05 \\
\text { trace } \\
\ldots . . . .4 \\
.44 \\
\ldots . . .8 \\
.26\end{array}$ \\
\hline , - & 100.34 & 100.16 & 100.28 & 100.03 & 99.76 \\
\hline
\end{tabular}

F. Biotite-augite latite, 4 miles southwest of Clover Meadow. Toscanose. Called "trachyte-andesite tuff" in first edition of this bulletin. Contains plagioclase, biotite, augite, magnetite, apatite, and glass. Analysis by W. F. Hillebrand, record No. 1597. P. R. C. 762.

G. Augite latite, Dardanelle flow, near Clover Meadow. Monzonose. Contains plagioclase, in part labradorite, augite, iron ore, some olivine, apatite, and brown glass. The potassium is probably in the glass, as no potash mineral was observed. Analysis by H. N. Stokes, record No. 1645 . P. R. C. 785.

H. Augite latite, Table Mountain. Shoshonose. Called "basalt" in first edition. Contains labradorite, olivine, augite, and magnetite. Analysis by Hillebrand, record No. 1273. P. R. C. 724 .

I. Augite latite, Table Mountain, near Clover Meadow. Shoshonose. Contains labradorite, augite, olivine, magnetite, apatite, and glass. Analysis by George Steiger, record No. 1697. P. R. C. 763. 


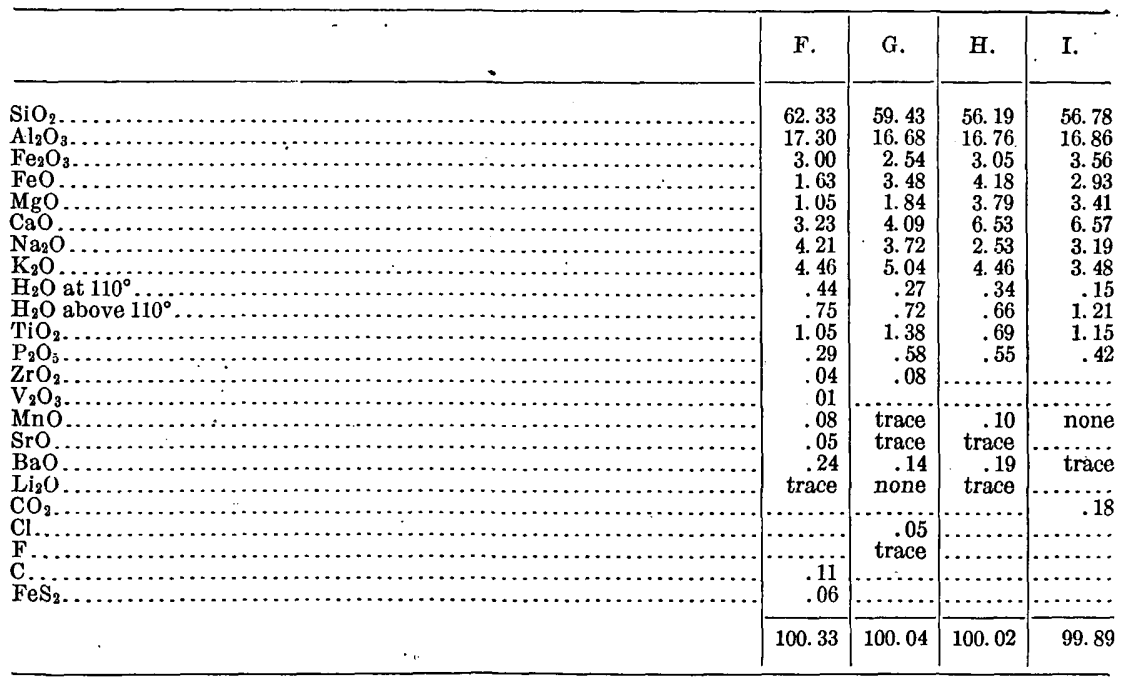

J. Amphibole gabbro, Beaver Creek, Big Trees quadrangle. Hessose. ' Contains labradorite and amphibole, with a little pyrite and pyrrhotite. Analysis by H. N. Stokes, record Nó. 1752.

K. Amphibole separated from J. Analysis by William Valentine, record No. 1733. L. Olivine gabbro, just east of south end of Phoenix reservoir. Corsose. Contains plagioclase, a few grains of alkali feldspar, augite, rhombic pyroxene, amphibole, olivine, magnetite, and iron sulphide, with a little secondary chlorite and epidote. Analysis by H. N. Stokes, record No. 1750.

For description of J and K see Turner, Am. Jour. Sci., 4th ser., vol. 7, p. 294.

\begin{tabular}{|c|c|c|c|}
\hline . & J. & K. & L. \\
\hline 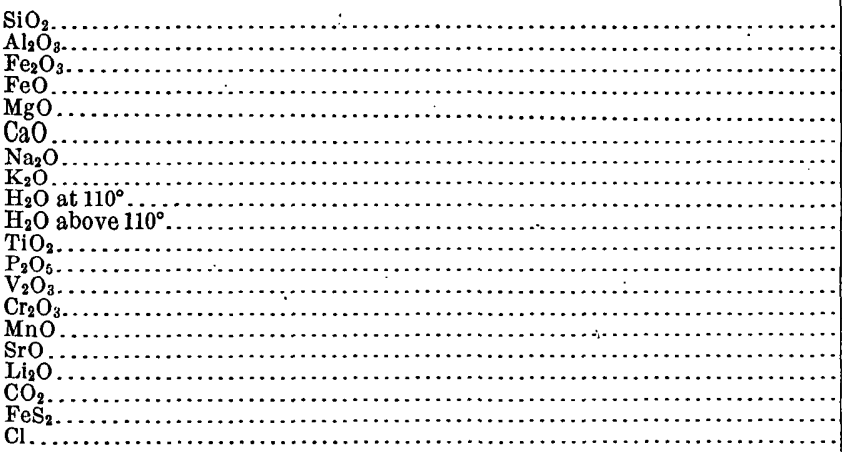 & $\begin{array}{r}47.27 \\
20.82 \\
1.85 \\
4.26 \\
6.44 \\
13.02 \\
2.75 \\
.22 \\
.08 \\
1.27 \\
.92 \\
.74 \\
.02 \\
\text { trace } \\
\text { trace } \\
\text { trace } \\
\text { none } \\
\ldots . . . . \\
\text {. } 20 \\
\text { trace }\end{array}$ & $\begin{array}{r}46.08 \\
10.52 \\
2.81 \\
8.30 \\
14.40 \\
12.64 \\
1.62 \\
.34 \\
.17 \\
1.97 \\
.77 \\
.18 \\
.04 \\
\ldots . . . \\
.15 \\
\ldots \ldots . . . \\
\text { none } \\
\ldots \ldots . . . \\
\ldots \ldots . . . \\
\ldots . . .\end{array}$ & $\begin{array}{r}43.41 \\
23.15 \\
3.72 \\
4.39 \\
7.65 \\
14.27 \\
.82 \\
.22 \\
.18 \\
1.53 \\
.39 \\
.02 \\
\ldots . . \\
\text { none } \\
.08 \\
\ldots . . . \\
\text { trace } \\
.10 \\
.14 \\
\text { trace }\end{array}$ \\
\hline 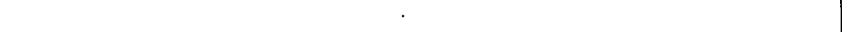 & 99.86 & 99.99 & 100.07 \\
\hline
\end{tabular}




\section{MARIPOSA COUNTY.}

Rocks collected by H. W. Turner, and partly described in his papers in 14th and 17th Ann. Additional data supplied by Turner.

A. Soda granulite or aplite, about 4 miles west of Mariposa. Mariposose. See 17th Ann., pt. 1, p. 721. Contains plagioclase (albite?) and micropegmatite, with less epidote, quartz, sphene, and apatite. Analyses by W. F. Hillebrand, record No. 1461. P. R. C. 748.

B. Micropegmatite, Agua Fria Creek. Lassenose. See 17th Ann., pt. 1, p. 691. Contains quartz, plagioclase, brown mica, epidote, and a little iron ore. Analysis by Hillebrand, No. 1461. P. R. C. 746.

C. Soda granite porphyry, Merced River, below the mouth of the north fork. Lassenose. Published in 17th Ann. Contains feldspar, largely albite, hornblende, muscovite, abundant epidote, apatite, and a little iron ore. Analysis by George Steiger, record No. 1573. P. R. C. 774.

D. Granite porphyry, about one-fourth of a mile north of Lake Tenaya, Yosemite National Park. Toscanose. See 14th and 17th Ann. Contains orthoclase, quartz, plagioclase, and biotite, with a little iron ore and sphene. Analysis by Hillebrand, record No. 1432. P. R. C. 726 .

E. Granite, west of Lake Tenaya, Yosemite National Park. Lassenose. See 14th and 17th Ann. Contains quartz, orthoclase, plagioclase, and biotite, with some hornblende, iron ore, sphene, and apatite. Analysis by Hillebrand, No. 1432. P. R. C. 725 .

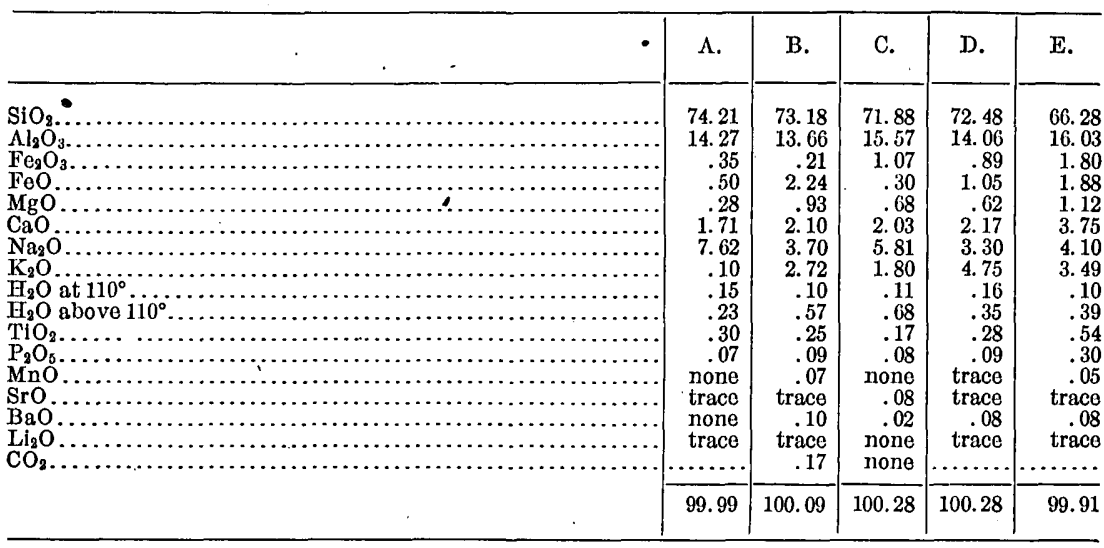

F. Amphibole-biotite granite, Nevada Falls trail, Yosemite Valley. Toscanose. Contains alkali feldspar, plagioclase, quartz, amphibole, biotite, magnetite, and apatite.

G. Biotite granite, base of El Capitan, Yosemite Valley. Toscanose. Contains alkali feldspar, plagioclase, quartz, biotite, titanite, apatite, and iron oxides.

H. Brown mica separated from $G$.

Analyses F, G, and H by William Valentine, record Nos. 1732, 1733.

Samples $G$ and $H$ are described by Turner in Am. Jour. Sci., 4th ser., vol. 7, p. 294. 


\begin{tabular}{|c|c|c|c|}
\hline & F. & G. & H. \\
\hline 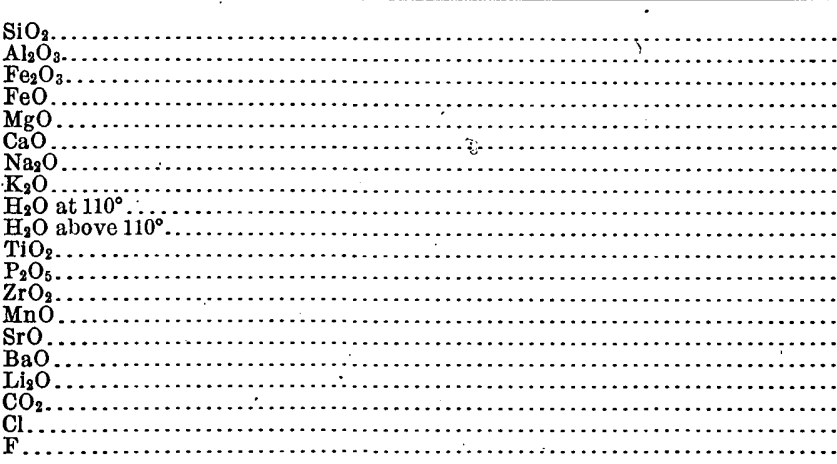 & $\begin{array}{r}66.83 \\
15.24 \\
2.73 \\
1.66 \\
1.63 \\
3.59 \\
3.10 \\
4.46 \\
\text { none } \\
.56 \\
.54 \\
.18 \\
.04 \\
.10 \\
.03 \\
.11 \\
\text { trace } \\
\text { trace } \\
.02 \\
\ldots . . . .\end{array}$ & $\begin{array}{r}71.08 \\
15.90 \\
.62 \\
1.31 \\
.54 \\
2.60 \\
3.54 \\
4.08 \\
\text { none } \\
.30 \\
.22 \\
.10 \\
.08 \\
.15 \\
.02 \\
.04 \\
\text { trace } \\
\text { trace } \\
.02 \\
\ldots . . .\end{array}$ & $\begin{array}{r}35.64 \\
18.62 \\
5.54 \\
14.60 \\
9.72 \\
.90 \\
.38 \\
9.22 \\
.48 \\
2.54 \\
1.12 \\
.20 \\
\ldots .79 \\
\ldots \ldots . \\
\text { trace } \\
\text { trace } \\
\ldots \ldots . . \\
\ldots . . . \\
.26\end{array}$ \\
\hline Less $0 \ldots \ldots \ldots \ldots \ldots \ldots$ & $\begin{array}{c}100.82 \\
\ldots \ldots\end{array}$ & $\begin{array}{c}100.60 \\
\cdots\end{array}$ & $\begin{array}{r}100.01 \\
.11\end{array}$ \\
\hline 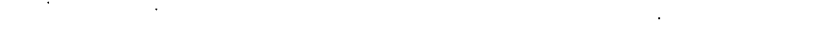 & & & 99.90 \\
\hline
\end{tabular}

I. Amphibole picrite, near Sequoia post-office, Yosemite National Park. Uvaldose. Collected by Turner, but not described. Analysis by George Steiger, record No. 1884.

J. Amphibole-pyroxene rock, perknite, 3 miles northeast of Coulterville. $S R$. 2 of sec. 2 of minnesotase. Described by Turner in Jour. Geology, vol. 9, p. 508. Analysis by George Steiger, record No. 1860. Contains pyroxene and amphibole, with a little quartz and pyrrhotite.

K. Amphibole separated from quartz monzonite, Tioga road, southeast of Mount Hoffman. Sp. gr., $3.203,2.15^{\circ}$.

L. Mica separated from the same rock as I. Sp. gr., $3.05,21^{\circ}$.

Analyses K and L by W. F. Hillebrand, record No. 1774.

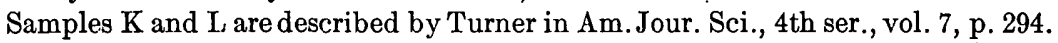

\begin{tabular}{|c|c|c|c|c|}
\hline & I. & J. & $\mathbf{K}$ & L. \\
\hline 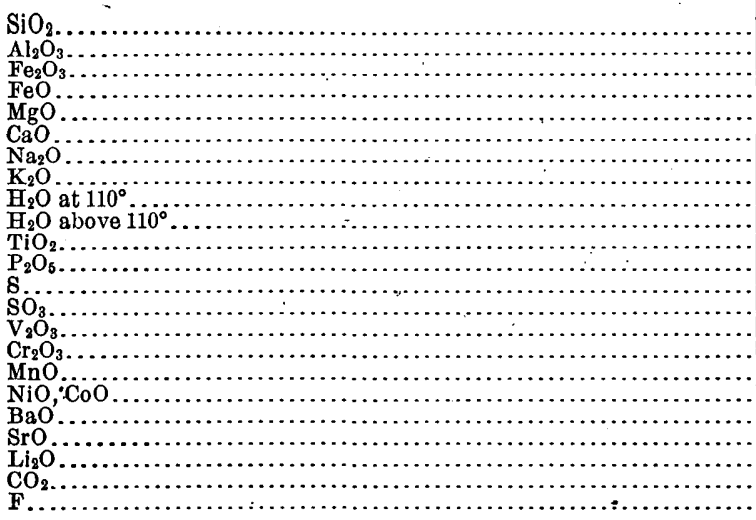 & $\begin{array}{r}43.17 \\
11.42 \\
4.97 \\
6.36 \\
16.97 \\
11.62 \\
1.11 \\
.10 \\
.22 \\
2.51 \\
1.23 \\
.04 \\
.06 \\
\ldots \ldots . . .\end{array}$ & 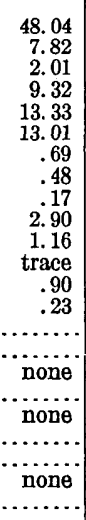 & 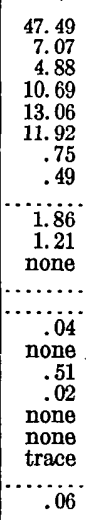 & \begin{tabular}{r}
35.75 \\
14.70 \\
4.65 \\
14.08 \\
12.37 \\
.17 \\
.32 \\
9.19 \\
1.03 \\
3.64 \\
3.16 \\
.03 \\
$\ldots \ldots$. \\
\hdashline .05 \\
trace \\
.45 \\
.02 \\
.12 \\
$(?)$ \\
$\ldots \ldots .$. \\
$\ldots . .17$
\end{tabular} \\
\hline 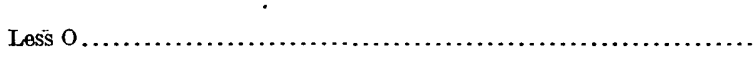 & $\begin{array}{r}100.63 \\
.03\end{array}$ & $\begin{array}{r}100.06 \\
.45\end{array}$ & $\begin{array}{r}100.05 \\
.02\end{array}$ & $\begin{array}{r}99.90 \\
.07\end{array}$ \\
\hline & 100.60 & 99.61 & 100.03 & 99.83 \\
\hline
\end{tabular}


M. Quartz-mica diorite, Chowchilla River. Tonalose. See 17th Ann., pt. I, p. 691. Contains plagioclase, quartz, a little orthoclase (?), brown mica, hornblende, rather abundant apatite, a little iron ore; one zircon-like crystal was noted. Analysis by W. F. Hillebrand, record No. 1461. P. R. C. 745.

N. Quartz-mica diorite, Yaqui Creek. Tonalose. For the Educational Series of Rocks. Contains plagioclase, quartz, biotite, hornblende, a little pyroxene, iron ore and apatite. Analysis by George Steiger, record No. 1643. Described by Turner in Bull. 150, p. 339.

O. Diabase, dike $1 \frac{1}{2}$ miles northeast of Hornitos. Auvergnose. See 17th Ann., pt. 1 , p. 694 . Contains plagioclase, partly labradorite, augite, brown hornblende, and iron ore. Analysis by Hillebrand, No. 1461. P. R. C. 750.

P. Igneous rock, near Cathay Hill. Rossweinose. See 17th Ann., pt. 1, p. 694. Contains two minerals unidentified; neither is olivine. Analysis by Hillebrand, No. 1461. P. R. C. 749.

\begin{tabular}{|c|c|c|c|c|}
\hline.. & M. & N. & O. & P. \\
\hline 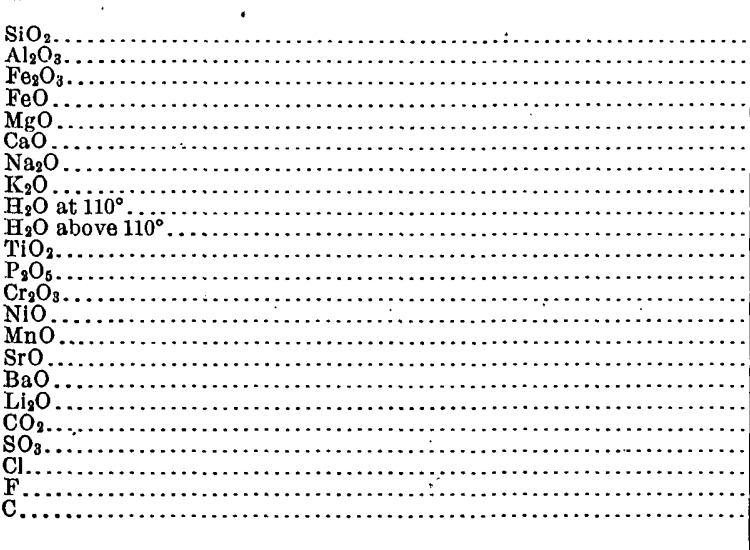 & 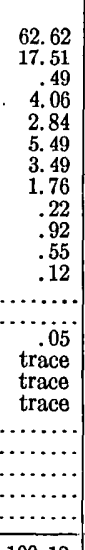 & \begin{tabular}{r}
58.09 \\
17.46 \\
1.12 \\
5.08 \\
4.06 \\
6.24 \\
2.94 \\
2.02 \\
.29 \\
1.45 \\
.95 \\
.17 \\
$\ldots \ldots$. \\
\hdashline$\ldots .$. \\
none \\
.04 \\
.07 \\
none \\
.21 \\
.05 \\
.02 \\
trace \\
.11
\end{tabular} & 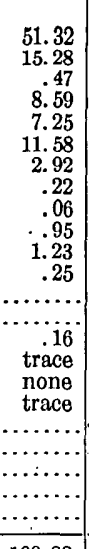 & $\begin{array}{r}47.75 \\
10.56 \\
.74 \\
8.34 \\
19.09 \\
9.62 \\
1.32 \\
.12 \\
.05 \\
2.06 \\
.37 \\
.03 \\
.24 \\
.07 \\
.10 \\
\text { trace } \\
\text { none } \\
\text { trace } \\
\ldots \ldots . . . \\
\ldots \ldots . . \\
\ldots \ldots . . \\
\ldots \ldots . . . \\
\ldots \ldots . \\
\end{array}$ \\
\hline & 100.12 & 100.37 & 100.28 & 100.46 \\
\hline
\end{tabular}

Q. Feldspathic mica schist, Chowchilla River. See 17th Ann., pt. 1, p: 691. Contains quartz, feldspar, biotite, muscovite, apatite, and specular iron. Analysis by W. F. Hillebrand, record No. 1461. P. R. C. 744.

R. Andalusite hornfels, Yaqui Gulch. Principally quartz, andalusite, brown and white mica, black graphite-like grains, a little iron ore, and probably feldspar. Analysis by George Steiger, record No. 1643.. Described by Turner in Bull. 150, p. 342.

S. Andalusite schist, Chowchilla River. See 17th Ann., pt. 1, p. 691. Contains quartz, biotite, andalusite, sericite, a little muscovite, probably graphite, iron ore, a few garnets, and apparently chlorite. Analysis by Hillebrand, No. 1461. P. R. C. 743 .

T. Chiastolite schist, Yaqui Gulch. Contains chiastolite, sillimanite, brown mica, sericite (?), probably graphite, and clear grains which appear to be quartz and feldspar. Analysis by Steiger, No. 1643. Described by Turner in Bull. 150, p.342. P.R.C. 135.

U. Hornfels, Agua Fria Creek. See 17th Ann., pt. 1, p. 691. Contains quartz, brown mica, iron ore, and plagioclase. Analysis by Hillebrand, No. 1461. P. R. C. 747. 


\begin{tabular}{|c|c|c|c|c|c|}
\hline & Q. & R. & S. & T. & U. \\
\hline 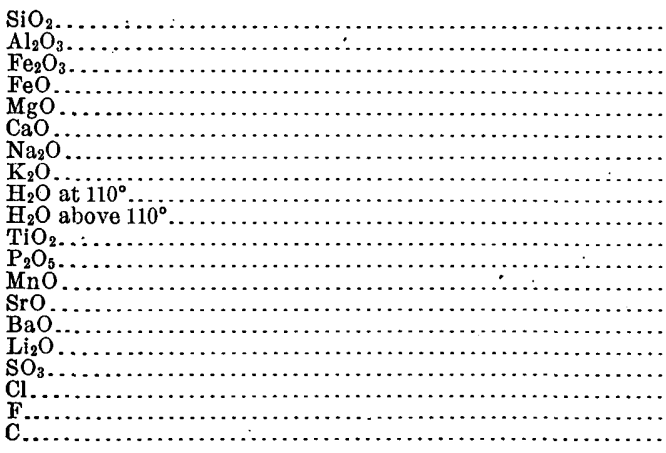 & $\begin{array}{r}70.40 \\
14.70 \\
.65 \\
2.57 \\
1.47 \\
1.63 \\
3.17 \\
3.46 \\
.19 \\
.91 \\
.51 \\
.05 \\
.08 \\
\operatorname{trace} \\
.09 \\
\operatorname{trace} \\
\ldots \ldots \\
\cdots \ldots \\
\cdots \\
\ldots .15\end{array}$ & $\begin{array}{r}65.10 \\
17.77 \\
1.95 \\
3.29 \\
1.43 \\
1.38 \\
2.25 \\
2.45 \\
.47 \\
2.49 \\
.72 \\
.14 \\
\text { none } \\
\text { none } \\
\text { none } \\
\text { none } \\
.03 \\
\text { trace } \\
.12 \\
1.21\end{array}$ & $\begin{array}{r}64.28 \\
17.28 \\
1.10 \\
5.34 \\
2.57 \\
1.19 \\
.91 \\
2.93 \\
.20 \\
2.72 \\
.65 \\
.27 \\
.09 \\
\text { trace } \\
.10 \\
\text { trace } \\
\ldots \ldots . \\
\ldots \ldots . \\
\ldots \ldots . . \\
.43\end{array}$ & $\begin{array}{r}62.15 \\
19.34 \\
4.23 \\
2.25 \\
1.88 \\
1.50 \\
1.60 \\
3.07 \\
.19 \\
1.79 \\
.80 \\
.15 \\
\text { trace } \\
\text { none } \\
.04 \\
\text { none } \\
.13 \\
\text { none } \\
.22 \\
1.12\end{array}$ & $\begin{array}{r}68.27 \\
14.03 \\
.46 \\
4.68 \\
2.23 \\
3.89 \\
2.29 \\
3.35 \\
.08 \\
.98 \\
.57 \\
.21 \\
.04 \\
\text { trace } \\
.08 \\
\text { trace } \\
\ldots \ldots . . \\
\ldots \ldots \ldots \\
\ldots \ldots . . . \\
\ldots \ldots . .\end{array}$ \\
\hline \multirow[t]{2}{*}{ Less 0 . } & $\begin{array}{c}100.03 \\
\ldots \ldots\end{array}$ & $\begin{array}{r}100.80 \\
. .06\end{array}$ & $\begin{array}{c}100.06 \\
\ldots \ldots\end{array}$ & $\begin{array}{r}100.46 \\
.10\end{array}$ & $\begin{array}{r}100.16 \\
\ldots \ldots \ldots\end{array}$ \\
\hline & & 100.74 & $\therefore \ldots \ldots$ & 100.36 & $\ldots$ \\
\hline
\end{tabular}

13. THE QUICKSILVER, REGION.

Rocks described by Becker in Mon. XIII. Analyses made by W. H. Melville in the San Francisco laboratory. With one exception (the serpentine from New Idria) all the rocks are from the districts north of San Francisco.

A. Pseudodiabase, near Mount St. Helena. Ornose. Contains augite, hornblende, oligoclase, albite, zoisite, ilmenite, leucoxene, and a little chlorite. P.' R. C. 1459.

B. Pseudodiabase, Sulphur Bank. Ornose. Contains oligoclase, a little quartz, pyroxene, hornblende, ilmenite, sphene, serpentine, and chlorite. P. R. C. 1460.

C. Pseudodiorite, Knoxville. Rossweinose. Mainly actinolite, with a little white mica, chlorite, serpentine, sphene, rutile, and zircon. P. R. C. 1461.

D. Glaucophane schist, Sulphur Bank. Mainly glaucophane and zoisite. Quartz, albite, muscovite, and sphene are also present. P. R. C. 1462.

\begin{tabular}{|c|c|c|c|c|}
\hline . & A. & B. & C. & D. \\
\hline $\begin{array}{l}\mathrm{SiO}_{2} \\
\mathrm{Al}_{2} \mathrm{O}_{3} \\
\mathrm{Fe}_{2} \mathrm{O}_{2} \\
\mathrm{FeO}\end{array}$ & $\begin{array}{r}49.08 \\
14.68 \\
1.95 \\
9.63 \\
6.69 \\
10.09 \\
4.60 \\
.20 \\
.27 \\
1.18 \\
1.72 \\
.23 \\
.15 \\
. . .\end{array}$ & $\begin{array}{r}51.28 \\
15.05 \\
2.42 \\
8.01 \\
6.07 \\
7.08 \\
4.43 \\
.12 \\
.39 \\
2.96 \\
1.33 \\
.13 \\
\ldots . .2 \\
.25 \\
.10\end{array}$ & $\begin{array}{r}50.44 \\
8.18 \\
1.06 \\
6.29 \\
17.63 \\
11.55 \\
2.98 \\
.50 \\
.07 \\
.92 \\
\ldots \ldots . . \\
\ldots . .78 \\
.21 \\
. \ldots .\end{array}$ & $\begin{array}{r}49.68 \\
13.60 \\
1.86 \\
8.61 \\
6.26 \\
10.97 \\
3.09 \\
.12 \\
\ldots \ldots .84 \\
3.84 \\
1.31 \\
.21 \\
\ldots . . .04 \\
. .04\end{array}$ \\
\hline & 100.47 & 99.62 & 100.31 & 99.59 \\
\hline
\end{tabular}

E. Andesitic obsidian, Clear Lake. Liparose. Shows grains of plagioclase, augite, and hypersthene. Sp. gr., 2.391. P. R. C. 1463.

F. Andesite (asperite), Clear Lake. Yellowstonose. Contains pyroxene, plagioclase, magnetite, and sometimes biotite: Pyroxene mostly rhombic. Sp. gr., 2.664. P. R. C. 1464.

G. Obsidian, south of Borax Lake. Varingose. Sp. gr., 2,390. P. R. C. 1465.

H. Basalt, south of Burns Valley. Andose. Rich in olivine, with a microlitic groundmass of plagioclase and augite. Sp. gr., 2.380. P. R. C. 1466.

I. Ordinary basalt, Knoxville. P. R. C. 1467. 


\begin{tabular}{|c|c|c|c|c|c|}
\hline$\cdot$ & $\mathrm{E}$ & $F$ & $\mathrm{G}$. & H. & I. \\
\hline 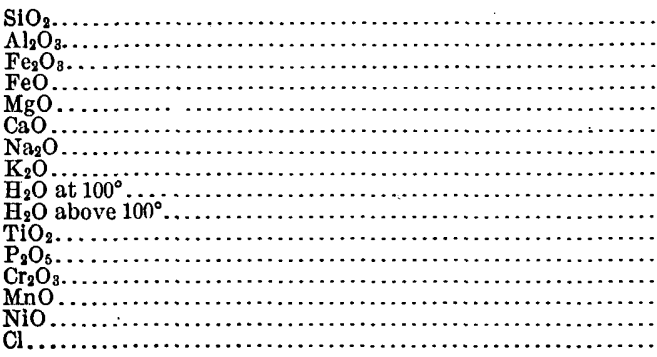 & $\begin{array}{r}74.01 \\
12.95 \\
1.42 \\
.48 \\
.99 \\
5.34 \\
4.65 \\
\ldots .29 \\
.24 \\
.01 \\
\ldots \ldots \\
\text { trace } \\
. \ldots .07\end{array}$ & $\begin{array}{r}65.43 \\
17.10 \\
2.39 \\
1.19 \\
1.48 \\
3.88 \\
3.66 \\
2.83 \\
.20 \\
.36 \\
.83 \\
\text { trace } \\
\ldots \ldots . . \\
.70 \\
.20 \\
\ldots \ldots . .\end{array}$ & $\begin{array}{r}75.40 \\
7.72 \\
1.41 \\
\ldots . \ldots \\
1.26 \\
1.55 \\
8.09 \\
4.52 \\
.43 \\
\ldots \ldots . . . \\
\ldots \ldots .12 \\
\ldots . .12 \\
.12\end{array}$ & $\begin{array}{r}57.37 \\
15.66 \\
2.06 \\
4.46 \\
8.84 \\
4.94 \\
3.05 \\
1.51 \\
.61 \\
.12 \\
.60 \\
.02 \\
\ldots . .27 \\
.27 \\
.41 \\
\ldots . . .\end{array}$ & $\left\{\begin{array}{r}51.66 \\
11.22 \\
7.62 \\
13.61 \\
7.72 \\
5.98 \\
.89 \\
1.06 \\
\text { trace } \\
\ldots \ldots .25 \\
. .12 \\
\ldots \ldots . . .\end{array}\right.$ \\
\hline & 100.45 & 100.25 & 100.62 & 99.92 & 100.13 \\
\hline
\end{tabular}

J. Light-green marmolitic serpentine, New Idria. P. R. C. 1468.

K. Black serpentine, Sulphur Bank. P. R. C. 1469.

L. Light-green serpentine, Sulphur Bank. P. R. C. 1470.

\begin{tabular}{|c|c|c|c|}
\hline & J. & $\mathrm{K}$. & L. \\
\hline \multirow[t]{2}{*}{ 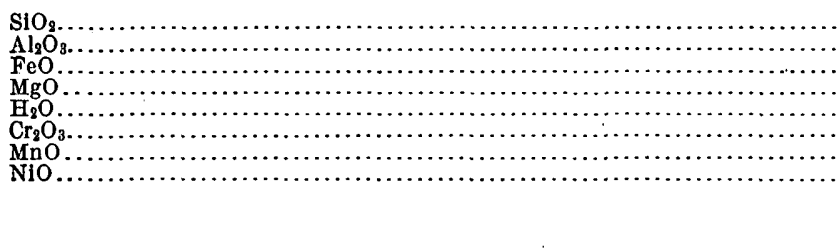 } & $\begin{array}{r}41.54 \\
2.48 \\
1.37 \\
40.42 \\
14.18 \\
\cdots \cdots . . . \\
\cdots \cdots \\
.04\end{array}$ & $\begin{array}{r}39.64 \\
1.30 \\
7.76 \\
37.13 \\
13.81 \\
.29 \\
.12 \\
.33\end{array}$ & $\begin{array}{r}41.86 \\
.69 \\
4.15 \\
38.63 \\
14.16 \\
.24 \\
.20 \\
\text { trace }\end{array}$ \\
\hline & 100.03 & 100. 38 & 99.93 \\
\hline
\end{tabular}

14. MOUNT DIABLO.

Described by Turner and Melville in Bull. Geol. Soc. America, vol. 2, pp. 383-414. Analyses by W. H. Melville. Those with record numbers were made in the Washington laboratory; the others were made in the laboratory at San Francisco.

A. Diabase, Mitchell Canyon. Auvergnose. Composed of augite, plagioclase, and ilmenite, with uralite and chlorite secondary.

B. The same rock, partly altered and partly uralitic. Ornose. Called "diabasediorite" by Turner.

C. Pyroxenite, near Bagley Creek. Cecilose. Composed of bronzite and diallage. Equivalent to the websterite of North Carolina. P. R. C. 735.

D. Glaucophane schist, Pine Canyon. Contains numerous cinnamon garnets.

$\mathrm{A}, \mathrm{B}$, and $\mathrm{C}$ have the record No. 1247 .

\begin{tabular}{|c|c|c|c|c|}
\hline & A. & B. & C. & $\mathrm{D}$ \\
\hline 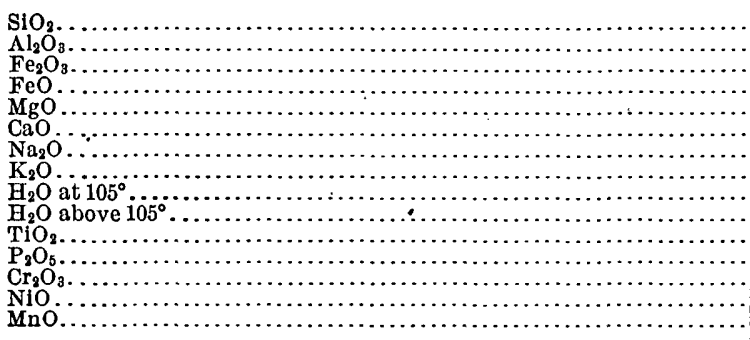 & $\begin{array}{r}52.06 \\
11.34 \\
2.11 \\
7.74 \\
9.26 \\
8.05 \\
1.74 \\
.73 \\
.59 \\
2.90 \\
.47 \\
.13 \\
\cdots \\
\text { trace }\end{array}$ & $\begin{array}{r}51.58 \\
14.99 \\
2.04 \\
8.36 \\
6.51 \\
.59 \\
.08 \\
.31 \\
.34 \\
2.67 \\
1.05 \\
.24 \\
\cdots \\
\text { trace }\end{array}$ & \begin{tabular}{r}
53.25 \\
2.80 \\
.69 \\
5.93 \\
19.91 \\
16.22 \\
.19 \\
trace \\
.05 \\
.24 \\
\hdashline$\ldots .$. \\
\hdashline .54 \\
.07 \\
.09
\end{tabular} & $\begin{array}{r}47.84 \\
16.88 \\
4.99 \\
5.56 \\
7.89 \\
11.15 \\
3.20 \\
.46 \\
.17 \\
1.81 \\
\ldots . .14 \\
.14 \\
\ldots . . . \\
.56\end{array}$ \\
\hline & 100.12 & 99.76 & 99.98 & 100.65 \\
\hline
\end{tabular}


E. Crystalline gabbro, Bagley Creek. Kedabekose. Contains plagioclase and diallage. Record No. 1166.

F. Shaly gabbro. Friable, containing carbonates and sulphates. Somewhat resembles serpentine.

G. Shaly.gabbro, like F, much resembling a true serpentine.

According to Turner, $\mathrm{F}$ and $\mathrm{G}$ are merely weathered layers of the gabbro.

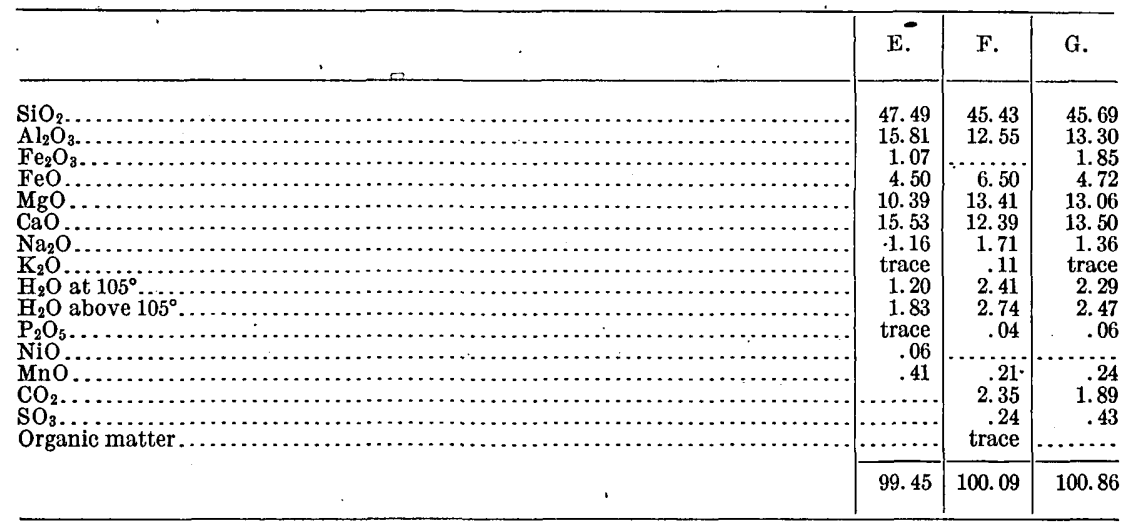

The following analyses are of serpentines derived from a peridotite-pyroxenite dike: H. Almost black. Possibly derived from adjacent shale.

I. Almost black. Derived from pyróxenite.

J. Bastite. Derived from pyroxenite. Record No. 1166.

K. Friable serpentine.

\begin{tabular}{|c|c|c|c|c|}
\hline - & H. & I. & J. & K. \\
\hline 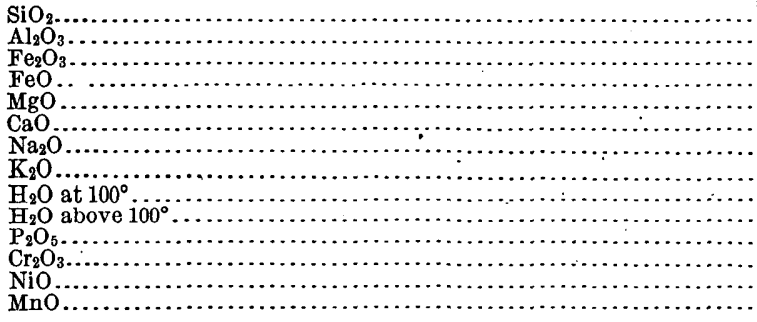 & $\begin{array}{r}38.53 \\
14.55 \\
2.65 \\
4.01 \\
21.79 \\
3.13 \\
.07 \\
.88 \\
4.51 \\
9.56 \\
\text { trace } \\
\text { trace } \\
\text { trace } \\
.32\end{array}$ & $\begin{array}{r}40.50 \\
.78 \\
4.01 \\
2.04 \\
37.43 \\
.39 \\
.28 \\
.16 \\
2.81 \\
10.94 \\
\text { trace } \\
.41 \\
.11 \\
.13\end{array}$ & $\begin{array}{r}36.57 \\
.95 \\
7.29 \\
.37 \\
40.27 \\
.14 \\
.31 \\
\operatorname{trace} \\
.94 \\
12.43 \\
.33 \\
.31 \\
.10\end{array}$ & $\begin{array}{r}36.96 \\
.39 \\
5.00 \\
2.34 \\
33.84 \\
3.81 \\
.34 \\
.14 \\
2.16 \\
14.02 \\
.02 \\
.78 \\
\text { trace } \\
.09\end{array}$ \\
\hline & 100.00 & 99.99 & 100.01 & 99.89 \\
\hline
\end{tabular}

Accidental organic matter was deducted from analyses $H$ and I, with subsequent recalculation of the data to 100 per cent.

L. Serpentine.

M. Talclike yellowish-green serpentine.

N. Weathered serpentine.

Q. Olive colored, probably serpentine.

Samples H, I, and J are from near Bagley Creek; K, L, M, and N from near Arroyo del Cerro; $\mathrm{O}$ from Ferguson ravine. $\mathrm{M}$ and $\mathrm{O}$ carry considerable chromite. 


\begin{tabular}{|c|c|c|c|c|}
\hline : & L. & M. & N. & 0. \\
\hline 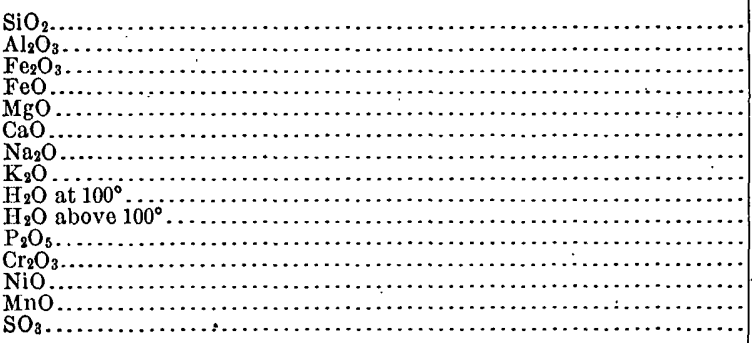 & $\begin{array}{r}34.84 \\
\ldots .42 \\
6.08 \\
1.85 \\
30.74 \\
7.02 \\
.42 \\
.07 \\
1.67 \\
15.72 \\
.04 \\
.68 \\
\text { trace } \\
.01 \\
\ldots . . .\end{array}$ & $\begin{array}{r}32.27 \\
11.45 \\
\text { trace } \\
5.05 \\
33.30 \\
.41 \\
\text { trace } \\
\text { trace } \\
.44 \\
12.40 \\
\text { trace } \\
5.19 \\
.19 \\
\text { trace } \\
\ldots \ldots . .\end{array}$ & 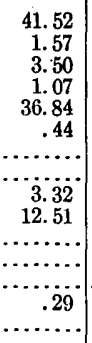 & $\begin{array}{r}30.98 \\
1.04 \\
4.88 \\
2.01 \\
38.44 \\
.22 \\
.40 \\
.16 \\
.39 \\
20.43 \\
\text { trace } \\
.34 \\
\ldots . .42 \\
.44 \\
.44\end{array}$ \\
\hline$\cdot$ & 99.56 & 100.70 & 101.06 & 100.15 \\
\hline
\end{tabular}

15. MISCELLANEOUS ROCKS.

A. Rhyolitic obsidian, Medicine Lake, Modoc County. Toscanose. Collected by J. S. Diller, who reports it to be a banded obsidian, containing a few minute feldspar crystals. The banding is due to a multitude of trichites. Analysis by L. G. Eakins, record No. 1072.

B. Rhyolite, Hyampom, south fork of Trinity River, Trinity County. Tehamose. Almost wholly made up of particles of clear glass. Collected and described by Diller. Analysis by George Steiger, record No. 1427. P. R. C. 1455.

C. Tuff from Redding Creek basin, Trinity County. Collected by J. S. Diller. Analysis by E. T. Allen, record No. 2020.

D. Diabase porphyrite, one-half mile west of Browns Valley, Yuba County. Auvergnose. Collected and described by W. Lindgren. Contains augite, plagioclase, magnetite, chlorite, and epidote. Analysis by W. F. Hillebrand, record No. 1419.

E. Amphibolitic schist, 1 mile northeast of Browns Valley, Yuba County. Collected and described by W. Lindgren. From metamorphosis of C. Contains chiefly green hornblende and feldspar. Analysis by Hillebrand, No. 1419.

\begin{tabular}{|c|c|c|c|c|c|}
\hline . & A. & B. & C. & D. & E. \\
\hline 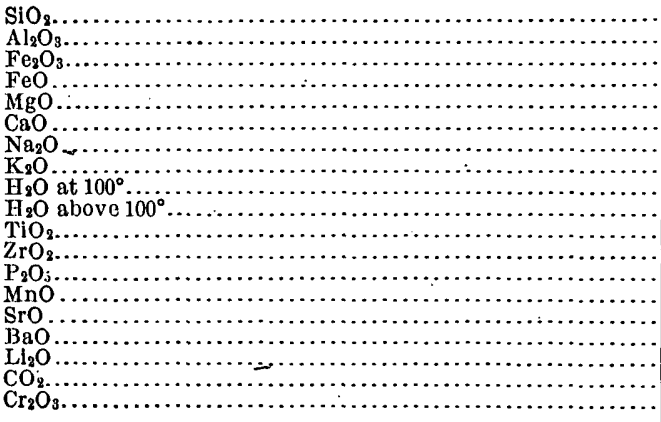 & 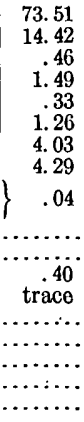 & 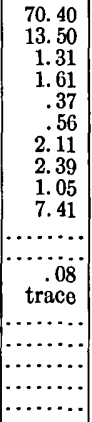 & $\begin{array}{r}60.23 \\
18.64 \\
3.81 \\
.88 \\
1.64 \\
6.04 \\
3.87 \\
1.46 \\
.95 \\
1.97 \\
.57 \\
.01 \\
.20 \\
\text { trace } \\
.05 \\
.11 \\
\ldots . . . \\
\text { none } \\
\text { trace }\end{array}$ & $\begin{array}{r}48.26 \\
14.83 \\
3.27 \\
5.97 \\
8.77 \\
11.38 \\
1.57 \\
1.13 \\
.10 \\
3.37 \\
.51 \\
. .25 \\
.15 \\
\text { trace } \\
.05 \\
\text { trace } \\
1.24 \\
\ldots . . .\end{array}$ & $\begin{array}{r}54.13 \\
14.53 \\
1.50 \\
5.25 \\
10.93 \\
4.91 \\
3.53 \\
.32 \\
.20 \\
4.01 \\
.46 \\
. .09 \\
.15 \\
\text { trace } \\
.02 \\
\text { none } \\
\ldots \ldots . . . \\
\ldots \ldots . .\end{array}$ \\
\hline & 100.23 & 100.79 & 100.43 & 100.85 & 100.03 \\
\hline
\end{tabular}

F. Basalt, base of a lava flow, east of the head of the San Joaquin River, Madera County. Andose. Description supplied by Turner. Hitherto unpublished. Contains pyroxene, partly augite, plagioclase, olivine, and iron ores. Analysis by W. F. Hillebrand, record No. 1767. 
G. Olivine basalt, west peak of the Dardanelles, Alpine County. Hessose. Described by Ransome in Bull. 89. Contains olivine, largely altered to iddingsite, plagioclase, serpentine, and augite. Analysis by George Steiger, record No. 1697.

H. Mica separated from quartz monzonite, near Bloods station, Alpine County. Described by Turner in Am. Jour. Sci., 4th ser., vol. 7, p. 294. Analysis by William Valentine, record No. 1736.

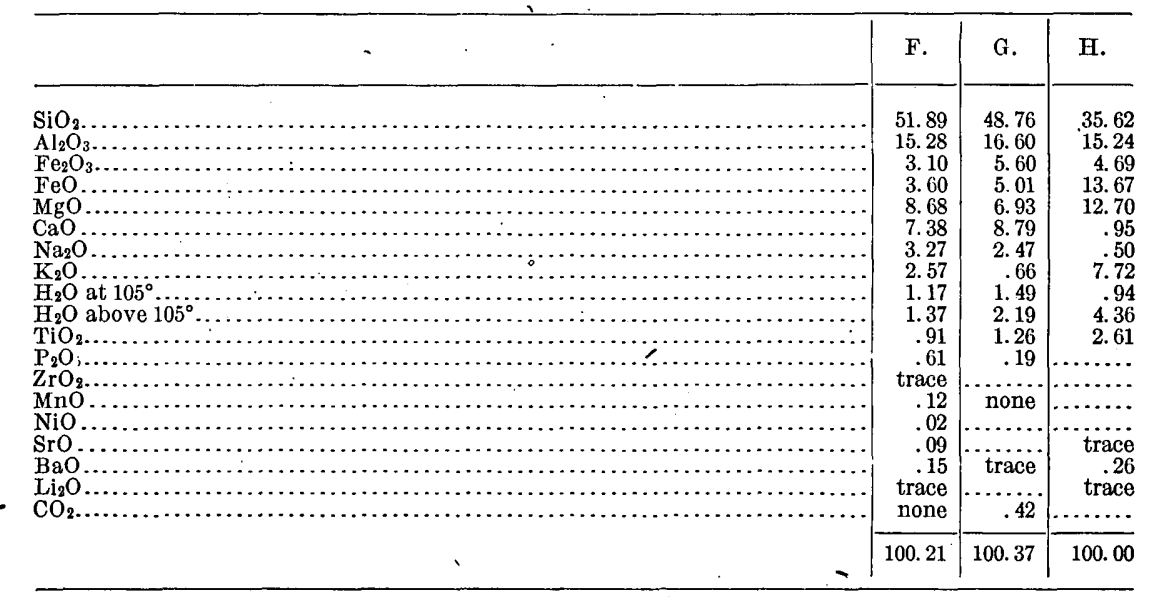

I. Typical diabase, 1 mile north of Bella Vista ranch houses, San Mateo County. Andose. Contains plagioclase, augite, olivine, apatite, ilmenite, and magnetite, with secondary serpentine, chlorite, iron ores, calcite, analcite, and natrolite. P. R. C. 1473 .

J. Diabase, basaltic facies, Mendigo Hill, San Mateo County. 'Andose. Contains plagioclase, augite, olivine, ilmenite, and magnetite, with secondary calcite, serpentine, chlorite, iddingsite, iron oxides, analcite, and natrolite. P. R. C. 1603.

Rocks I and J are described by Haehl and Arnold in Proc. Am. Philos. Soc., vol. 43, p. 16. Analyses by E. T. Allen, record No. 1958.

K. Bronzite-olivine aleutite, south end of Panamint Range. Andose. Contains plagioclase, bronzite, and olivine in a glassy to microcrystalline groundmass carrying much magnetite.

L. Hornblende-quartz diorite, Fremont Peak, Mohave Desert. Contains hornblende, plagioclase, subordinate quartz, and apatite, with secondary muscovite and epidote.

M. Alaskite, Fremont Peak. Toscanose-tehamose. Essential minerals, quartz and orthoclase.

Rocks K, L, M collected and described by J. E. Spurr. Hitherto unpublished. Analyses by W. F. Hillebrand, record No. 1881. 


\begin{tabular}{|c|c|c|c|c|c|}
\hline & I. & J. & . K. & L. & M. \\
\hline 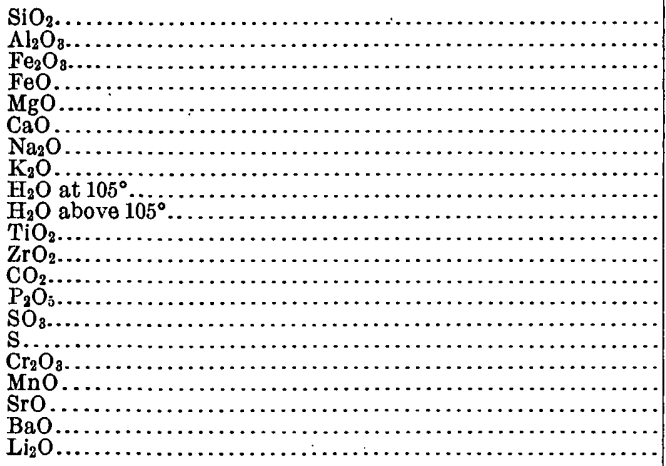 & \begin{tabular}{r|}
50.12 \\
18.52 \\
2.47 \\
4.11 \\
2.68 \\
8.99 \\
5.22 \\
1.46 \\
1.64 \\
3.09 \\
1.33 \\
$\ldots \ldots$ \\
.$\ldots$. \\
.08 \\
$\ldots \ldots$ \\
trace \\
trace \\
$\ldots \ldots .02$ \\
.$\ldots$.
\end{tabular} & $\begin{array}{r}49.60 \\
16.56 \\
4.28 \\
4.44 \\
5.38 \\
9.22 \\
3.31 \\
1.25 \\
1.44 \\
2.58 \\
1.86 \\
\ldots \ldots \\
\ldots \ldots . \\
.30 \\
.17 \\
\ldots \ldots . . \\
.03 \\
.08 \\
\ldots \ldots . . \\
.05 \\
\ldots \ldots . .\end{array}$ & $\begin{array}{r}53.98 \\
17.86 \\
4.61 \\
2.27 \\
3.30 \\
7.55 \\
4.19 \\
2.59 \\
.56 \\
.50 \\
1.10 \\
\text { trace? } \\
.21 \\
.61 \\
.17 \\
.01 \\
\ldots .13 \\
.13 \\
.14 \\
.28 \\
\text { trace }\end{array}$ & $\begin{array}{r}52.55 \\
17.61 \\
4.24 \\
4.98 \\
4.17 \\
8.02 \\
3.41 \\
1.49 \\
.25 \\
2.13 \\
.86 \\
\text { trace? } \\
\text { none } \\
.36 \\
\ldots . . . \\
.02 \\
\ldots . . . \\
.23 \\
.02 \\
.04 \\
\text { none }\end{array}$ & $\begin{array}{r}76.26 \\
13.43 \\
.11 \\
.06 \\
.04 \\
1.06 \\
2.95 \\
5.44 \\
.19 \\
.51 \\
.07 \\
\text { trace } \\
\text { none } \\
\text { trace } \\
\ldots \ldots . \\
\text { trace } \\
\ldots . . \\
\text { none } \\
.03 \\
.10 \\
\text { trace }\end{array}$ \\
\hline & 99.91 & 100.55 & 100.06 & 100.38 & 100.25 \\
\hline
\end{tabular}

N. Scoriaceous rhyolite, Mono Craters, south of Mono Lake. Liparose. Described by Russell in 8th Ann., pt. 1, p. 380. Analysis by T. M. Chatard, record No. 36.

O. Obsidian, Mono Lake. Liparose. Analysis by W. H. Melville, record No. 1346. Described by Lindgren in Bull. 150, p. 149. P. R. C.60.

P. Pumice, Mono Lake. Toscanose. Analysis by Melville, No. 1346. Described by Lindgren in Bull. 150, p. 148 . P. R. C. 59.

Q. Volcanic ash, east shore of Owens Lake. Collected and analyzed by Chatard, record No. 783 .

\begin{tabular}{|c|c|c|c|c|}
\hline & N. & 0. & P. & Q. \\
\hline 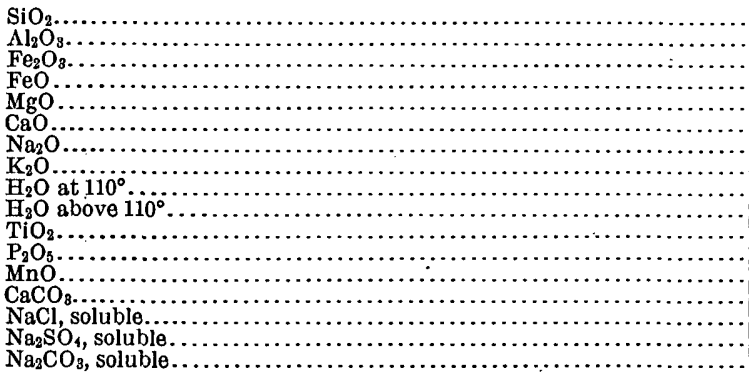 & 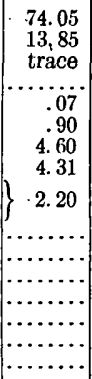 & 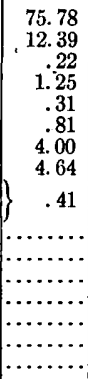 & 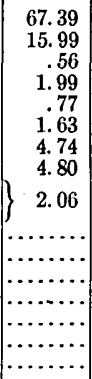 & $\begin{array}{r}55.81 \\
10.07 \\
3.43 \\
.67 \\
2.22 \\
1.05 \\
.75 \\
2.98 \\
.65 \\
2.50 \\
.80 \\
.27 \\
.23 \\
14.44 \\
1.45 \\
.80 \\
2.09\end{array}$ \\
\hline & 99.98 & 99.81 & 99.93 & 100.21 \\
\hline
\end{tabular}

R. Soda syenite, from near Coalinga, Fresno County. Description supplied by Ralph Arnold. Contains plagioclase, hornblende, biotite, apatite, zircon, and iron ore, with secondary calcite, analcite, ægirite, and white mica. Analysis by W. F. Hillebrand, record No. 2366.

S. Quartz-augite rock, Hiriart Hill, Pala, San Diego County. Mainly quartz and augite. Collected and analyzed by W. T. Schaller, record No. 2309.

T. Matrix of dumortierite, near Dehesa, San Diego County. Description and analysis, No. 2120, by Schaller. Consists chiefly of .quartz, with andalusite or sillimanite. For details see Bull. 262, p. 96.

15619-Bull. 419-10-12 


\begin{tabular}{|c|c|c|c|}
\hline . & $\mathrm{R}$ & S. & $\mathbf{T}$ \\
\hline 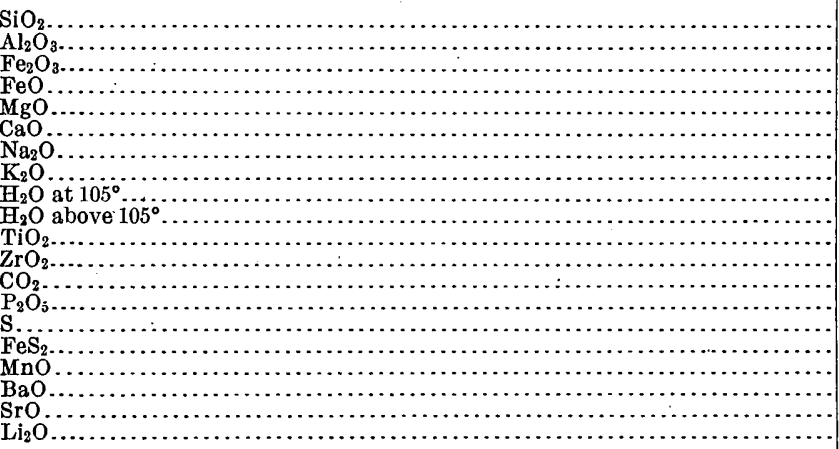 & $\begin{array}{r}60.00 \\
16.88 \\
1.83 \\
3.02 \\
1.40 \\
3.16 \\
9.31 \\
.94 \\
.43 \\
1.53 \\
.42 \\
.03 \\
.59 \\
.14 \\
\text { trace } \\
\ldots . . \\
.12 \\
.06 \\
\therefore .02 \\
\text { trace }\end{array}$ & $\begin{array}{r}57.29 \\
12.07 \\
.89 \\
5.70 \\
4.02 \\
17.77 \\
.39 \\
\text { trace } \\
.37 \\
.31 \\
.70 \\
\ldots \ldots . . \\
.04 \\
\ldots \ldots . . \\
\ldots .58 \\
\ldots \ldots . . \\
\ldots \ldots . . \\
\ldots . . .\end{array}$ & 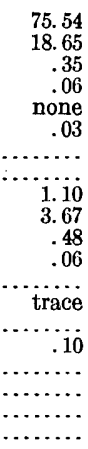 \\
\hline & 99.88 & 100.13 & 100.04 \\
\hline
\end{tabular}

OREGON.

\section{BASALT, MOUNT THIELSÈN.}

Partly described by Diller in Am. Jour. Sci., 3d ser., vol. 28, p. 257. A hypersthene basalt containing hypersthene, olivine, feldspar, and magnetite. In the printed paper only the analysis of the groundmass and the fulgurite formed in it are given.

A. Hypersthene basalt.

B. Pyroxene.

C, D. Feldspars.

E. Groundmass.

F. A fulgurite, or lightning tube.

Analyses A and F by F. W. Clarke, record Nos. 108, 105; B, C, D, and E' by T. M. Chatard, record Nos. 135, 133, 134, 128. These analyses. were made early in the history of the laboratory and are by no means complete.

\begin{tabular}{|c|c|c|c|c|c|c|}
\hline . & A. & B. & C. & D. & E. & F. \\
\hline 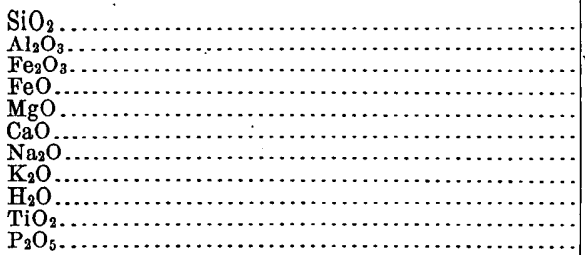 & $\begin{array}{r}55.68 \\
18.93 \\
8.73 \\
4.86 \\
7.99 \\
2.12 \\
.48 \\
.60 \\
\end{array}$ & $\begin{array}{r}53.31 \\
5.99 \\
13.43 \\
21.69 \\
3.69 \\
\ldots \\
\cdots\end{array}$ & $\begin{array}{r}55.48 \\
26.91 \\
2.32 \\
\\
2.27 \\
8.11 \\
3.14 \\
.72 \\
.66 \\
.39 \\
.\end{array}$ & $\begin{array}{r}51.95 \\
28.84 \\
2.24 \\
\\
1.34 \\
11.42 \\
3.22 \\
.59 \\
.40 \\
\operatorname{trace} \\
\end{array}$ & $\begin{array}{r}55.85 \\
2.95 \\
4.59 \\
3.08 \\
8.41 \\
2.16 \\
2.67 \\
.52 \\
\text { trace }\end{array}$ & 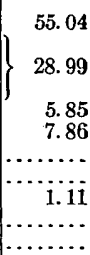 \\
\hline & 99. 39 & 98.11 & 100.00 & 100.00 & 100.23 & 98.85 \\
\hline
\end{tabular}

Iron oxides not separated. Analyses B, C, D, and F made on very small quantities of material. C and $\mathrm{D}$ were analyzed by the hydrofluoric-acid method, and the silica was determined by difference.

\section{RIDDLES QUADRANGLE.}

A. Peridotite, the matrix of the silicate nickel ores. Described by Diller and Clarke in Bull. 60, p. 21. The rock, which may be classed as saxonite, consists essentially of olivine and enstatite, with a little chromite and magnetite. Olivine predominates, and the enstatite forms less than one-third of the mass. Quartz, serpentine, and genthite are present as alteration products. 
B. Olivine separated from A. Analyses A, B, by F. W. Clarke, record Nos. 811, 814.

C. Dacite porphyry, sec. 5 , T. 30 S., R. 6 W.

D. Granodiorite, sec. 26, T. 30 S., R. $3 \cdot W$.

E. Intermediate rock, between greenstone and granodiorite, Evans Creek, near mouth of Sykes Creek.

F. Augite andesite, south bank Umpqua River, $\frac{3}{8}$ mile west of Days Creek.

Rocks C to F collected by G. F. Kay. Analyses by G. Steiger, record No. 2354.

\begin{tabular}{|c|c|c|c|c|c|c|}
\hline & A. & B. & C. & D. & E. & F. \\
\hline 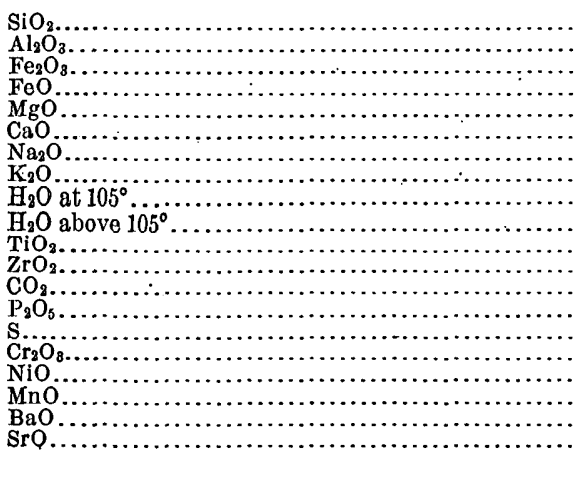 & 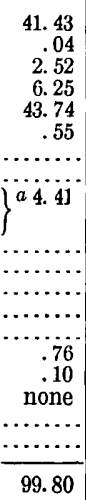 & 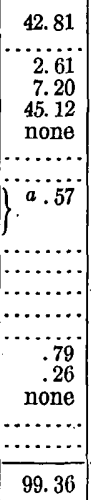 & $\begin{array}{r}70.65 \\
15.57 \\
.57 \\
1.26 \\
.48 \\
3.28 \\
4.91 \\
1.77 \\
.14 \\
.86 \\
.21 \\
.01 \\
\operatorname{trace} \\
.07 \\
.07 \\
\cdots \cdots . . \\
.06 \\
.06 \\
\text { trace }\end{array}$ & $\begin{array}{r}58.25 \\
20.52 \\
.68 \\
3.88 \\
2.03 \\
7.88 \\
4.25 \\
.50 \\
.24 \\
1.10 \\
.57 \\
.01 \\
\text { none } \\
.16 \\
\text { none } \\
\ldots . . . \\
\cdots . .10 \\
.10 \\
\text { none } \\
\text { none }\end{array}$ & $\begin{array}{r}57.06 \\
8.50 \\
1.11 \\
5.40 \\
11.19 \\
12.04 \\
1.39 \\
.95 \\
.18 \\
1.25 \\
.52 \\
\text { none } \\
\text { none } \\
.05 \\
\text { none } \\
\ldots \ldots . . . \\
\cdots \ldots . .7 \\
\text { trace } \\
\text { none }\end{array}$ & $\begin{array}{r}50.01 \\
15.25 \\
2.72 \\
5.35 \\
9.35 \\
10.44 \\
1.50 \\
.60 \\
2.61 \\
1.35 \\
.68 \\
\text { none } \\
\text { none } \\
.03 \\
\text { none } \\
\ldots . . .\end{array}$ \\
\hline
\end{tabular}

a Loss on ignition.

The four following rocks were also collected by Kay. Analyses by Steiger, record No. 2349:

G. Diabasic greenstone, Sec. 2, T. 30 S., R. $6 \mathrm{~W}$.

H. Basaltic greenstone, Sec. 23, T. 31 S., R. 6 W.

I. Gabbroic greenstone, Sec. 2, T. 34 S., R. 6 W.

J. Dioritic greenstone, Sec. 29 , T. 34 S., R. 6 W.

\begin{tabular}{|c|c|c|c|c|}
\hline ' & G.' & H. & I. & J. \\
\hline 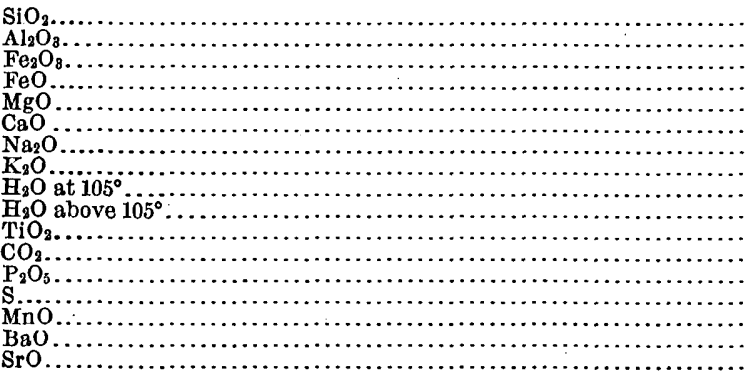 & $\begin{array}{r}50.90 \\
16.71 \\
.40 \\
8.50 \\
5.14 \\
9.74 \\
3.50 \\
.60 \\
.03 \\
2.12 \\
1.80 \\
.51 \\
.07 \\
\text { none } \\
.13 \\
.02 \\
\text { trace }\end{array}$ & $\begin{array}{r}52.58 \\
15.58 \\
2.07 \\
6.68 \\
5.75 \\
10.37 \\
1.79 \\
.82 \\
.22 \\
3.13 \\
.89 \\
.22 \\
.09 \\
\text { none } \\
.15 \\
.03 \\
\text { trace }\end{array}$ & $\begin{array}{r}45.86 \\
15.52 \\
1.84 \\
3.22 \\
11.71 \\
15.57 \\
.86 \\
.12 \\
1.38 \\
3.70 \\
.22 \\
\text { none } \\
\text { none } \\
.06 \\
.07 \\
\text { none } \\
\text { none }\end{array}$ & $\begin{array}{r}46.36 \\
16.88 \\
2.23 \\
6.29 \\
8.15 \\
15.66 \\
1.17 \\
.10 \\
.21 \\
1.48 \\
i .29 \\
\text { none } \\
\text { trace } \\
.01 \\
.10 \\
\text { none } \\
\text { none }\end{array}$ \\
\hline & 100.17 & 100.37 & 100.13 & 99.93 \\
\hline
\end{tabular}

$\mathrm{ZrO}_{3}$ absent. 


\section{CRATER LAKE.}

Rocks collected by J. S. Diller. All except the last one in the series are described by H. B. Patton in P. P. 3. Analyses A to N, inclusive; by H: N. Stokes, record Nos. 1671, 1953.

A. Vitrophyric rhyolite, south edge of Llao Rock flow. . Lassenose. Contains plagioclase, hypersthene, hornblende, and apatite in a glassy. groundmass crowded with augite microlites. P. R. C. 1314.

B. Streaked rhyolite, near "Wine Glass" Grotto Cove. Lassenose. Contains plagioclase, hypersthene, hornblende, and magnetite, with black glass. A few small inclusions of basalt and hypersthene andesite. P. R. C. 1316.

C. Rhyolite, small dike immediately below Llao Rock. Lassenose. Contains plagioclase, hornblende, hypersthene, and magnetite, in a glassy groundmass crowded with microlites of feldspar and augite. P. R. C. 1317.

D. Rhyolite, water's edge, head of Cleetwood Cove. Lassenose. Contains plagioclase, hypersthene, brown hornblende, and magnetite,.in a feldspathic groundmass of trachytic type. P. R. C. 1315.

E. Hypersthene-augite andesite, large dike transsecting the northwestern portion of the crater rim. Tonalose. Contains plagioclase, hypersthene, augite, and magnetite in a groundmass having a moderate amount of glass. P. R. C. 1313.

F. Hypersthene-augite andesite, west edge of Wizard Island. Tonalose. Same minerals as E. P. R. C. 1309.

\begin{tabular}{|c|c|c|c|c|c|c|}
\hline & A. & B. & C. & D. & E. & F: \\
\hline 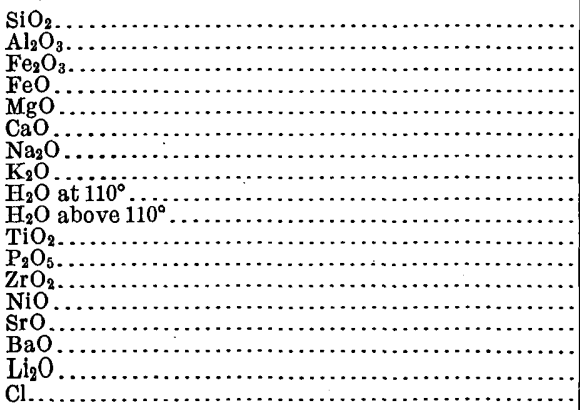 & $\begin{array}{r}70.77 \\
14.83 \\
1.35 \\
1.25 \\
.64 \\
2.12 \\
5.07 \\
2.68 \\
.07 \\
.33 \\
.38 \\
.13 \\
.05 \\
\text { none } \\
.02 \\
.08 \\
\text { trace } \\
.11\end{array}$ & $\begin{array}{r}68.17 \\
15.60 \\
2.31 \\
.94 \\
1.02 \\
2.76 \\
5.15 \\
2.46 \\
.09 \\
.45 \\
.54 \\
.13 \\
\text { none } \\
\text { none } \\
.03 \\
.06 \\
\text { trace } \\
\text { trace }\end{array}$ & $\begin{array}{r}71.78 \\
14.53 \\
1.28 \\
1.02 \\
.48 \\
1.59 \\
5.08 \\
2.84 \\
.06 \\
.22 \\
.41 \\
.10 \\
.04 \\
\text { none } \\
.03 \\
.08 \\
\text { trace } \\
\text { trace }\end{array}$ & $\begin{array}{r}70.10 \\
15.18 \\
1.78 \\
1.09 \\
.74 \\
2.27 \\
5.15 \\
2.58 \\
.10 \\
.19 \\
.48 \\
.13 \\
.04 \\
\text { none } \\
.03 \\
.08 \\
\text { trace } \\
.03\end{array}$ & $\begin{array}{r}60.09 \\
17.85 \\
2.03 \\
3.45 \\
3.50 \\
6.28 \\
4.17 \\
1.31 \\
.12 \\
\therefore 26 \\
.054 \\
.23 \\
\text { none } \\
.05 \\
.05 \\
.05 \\
\text { trace } \\
\text { trace }\end{array}$ & $\begin{array}{r}59.39 \\
18.45 \\
1.79 \\
3.90 \\
3.13 \\
6.29 \\
4.29 \\
1.29 \\
.10 \\
.42 \\
.41 \\
.22 \\
\text { none } \\
\text { none } \\
.04 \\
.05 \\
\text { trace } \\
\text { trace }\end{array}$ \\
\hline ' & 99.88 & 99.71 & 99.63 & 99. 97 & 99.98 & 99.77 \\
\hline
\end{tabular}

Traces of manganese in all. Fluorine not sought for. No $\mathrm{CO}_{2}, \mathrm{~S}, \mathrm{SO}_{3}$, or $\mathrm{Cr}_{3} \mathrm{O}_{3}$ in any.

G. Hypersthene-augite andesite, crater rim, just south of "The Watchman:" "Tonalose. Same minerals as F. P. R. C. 1310.

H. Hypersthene-augite andesite, Palisades, under Round Top, northeast portion of the rim. 'Tonalose. Contains plagioclase, hypersthene, augite, and magnetite. P. R. C. 1312 .

I. Hypersthene-augite andesite, lake level, undér Llao Rock. Tonalose. Same minerals as H. P. R. C. 1311.

J. Basalt, base of Red Cone. Andose. Contains plagioclase, augite, olivine, and magnetite, with some glass base. P. R. C. 1321 . 


\begin{tabular}{|c|c|c|c|c|}
\hline 0 & G. & H. & I. & J. \\
\hline 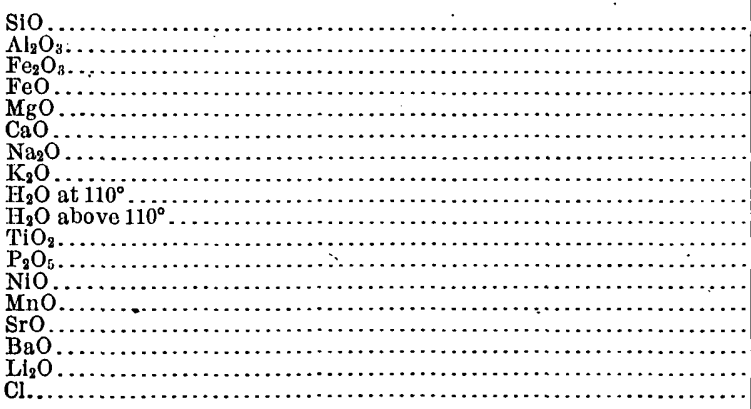 & $\begin{array}{r}60.98 \\
17.82 \\
1.83 \\
3.33 \\
2.76 \\
5.73 \\
4.26 \\
1.43 \\
.13 \\
.45 \\
.71 \\
.17 \\
\text { none } \\
\text { trace } \\
.05 \\
.06 \\
\text { none } \\
\text { trace }\end{array}$ & $\begin{array}{r}62.09 \\
17.03 \\
2.38 \\
2.69 \\
3.08 \\
5.65 \\
4.10 \\
1.67 \\
.04 \\
.13 \\
.65 \\
.19 \\
\text { none } \\
\text { trace } \\
.07 \\
.07 \\
\text { none } \\
\text { trace? }\end{array}$ & $\begin{array}{r}58.41 \\
17.85 \\
2.67 \\
3.29 \\
3.61 \\
6.81 \\
3.77 \\
1.23 \\
.34 \\
.86 \\
.69 \\
.24 \\
\text { none } \\
\text { trace } \\
.05 \\
.05 \\
\text { trace } \\
\text { trace }\end{array}$ & $\begin{array}{r}52.99 \\
16.71 \\
3.80 \\
3.55 \\
6.95 \\
8.49 \\
3.56 \\
1.29 \\
.18 \\
.59 \\
1.18 \\
.42 \\
.02 \\
\text { trace } \\
.12 \\
.07 \\
\text { none } \\
\text { trace }\end{array}$ \\
\hline & 99.71 & 99.84 & 99.87 & 99.92 \\
\hline
\end{tabular}

K. Hypersthene basalt, Anna Creek. Andose-beerbachose. Contains plagioclase, augite, hypersthene, olivine, and magnetite. P. R. C. 1320.

L. Hypersthene basalt of andesitic type, north of Desert Cove. Tonalose. Contains plagioclase, hypersthene, augite, and olivine.

M. Dark secretion from among dacitic ejectamenta, summit of Llao Rock. Tonalose-andose. Contains plagioclase, hornblende, hypersthene, and augite, with a little olivine and apatite, in a dark-brown glassy groundmass. P. R. C. 1318.

N. Light-colored secretion from among dacitic ejectamenta, southern rim of crater, between Sand and Anna creeks. Lassenose. Contains plagioclase, hypersthene, augite, hornblende, biotite, and quartz. P. R. C. 1319.

O. Basalt, 1 mile east of the summit of the Cascade Range, on the road from Fort. Klamath to Crater Lake. Beerbachose. Described by J. S. Diller as a typical basalt, carrying a considerable amount of hypersthene. Analysis by W. F. Hillebrand, record No. 408.

\begin{tabular}{|c|c|c|c|c|c|}
\hline ब & K. & L. & M. & N. & O. \\
\hline 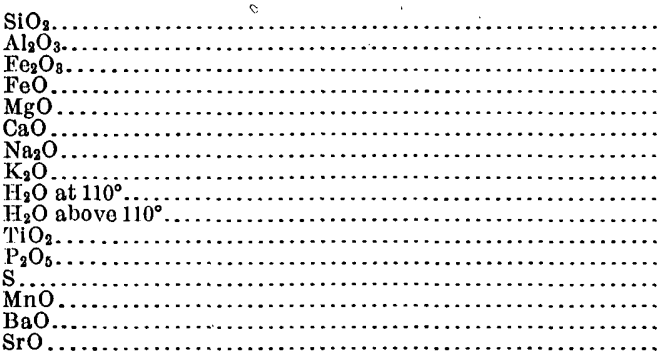 & $\begin{array}{r}56.95 \\
18.84 \\
2.06 \\
4.28 \\
4.37 \\
7.45 \\
3.89 \\
.82 \\
.19 \\
.31 \\
.79 \\
.19 \\
\operatorname{trace} \\
\operatorname{trace} \\
.04 \\
\operatorname{trace}\end{array}$ & $\begin{array}{r}58.65 \\
18.35 \\
1.59 \\
4.21 \\
3.49 \\
6.95 \\
3.70 \\
1.32 \\
.20 \\
.70 \\
.81 \\
.17 \\
\text { none } \\
\text { trace } \\
.06 \\
\text { trace }\end{array}$ & $\begin{array}{r}56.85 \\
18.31 \\
2.88 \\
3.15 \\
3.92 \\
7.20 \\
3.89 \\
1.23 \\
.16 \\
.95 \\
1.08 \\
.22 \\
\text { none } \\
\text { trace } \\
.04 \\
\text { trace }\end{array}$ & $\begin{array}{r}67.41 \\
15.76 \\
1.88 \\
1.76 \\
1.35 \\
3.36 \\
4.54 \\
2.36 \\
.09 \\
.54 \\
.56 \\
.12 \\
.02 \\
\text { trace } \\
.06 \\
\text { trace }\end{array}$ & $\begin{array}{r}57.47 \\
18.86 \\
2.21 \\
4.08 \\
4.27 \\
7.42 \\
3.85 \\
.73 \\
.22 \\
.75 \\
.24 \\
. .10 \\
.10 \\
.03 \\
.11\end{array}$ \\
\hline & 100.18 & 100.20 & 99.88 & 99.81 & 100.34 \\
\hline
\end{tabular}

$\mathrm{CO}_{2}, \mathrm{Cr}_{2} \mathrm{O}_{3}$, and $\mathrm{Ll}_{2} \mathrm{O}$ were absent from all five.

\section{PORT ORFORD QUADRANGLE:}

Rocks collected by J. S. Diller, and partly described by him in Folio 89. Analysis A by George Steiger, record No. 1892. B, D, E, F, by.H. N. Stokes, record No. 1891. C, G, H, I, J, K, L, M, by W. F. Hillebrand, record No. 1897.

A. Serpentine, from 12 miles north of mouth of Boulder Creek.

B. Serpentine, from Iron Mountain crest. Contains, with serpentine, olivine, pyroxene, sometimes hornblende, and magnetite, with picotite or chromite. 
C. Metagabbro, southeast slope of Panther Mountain. Hessose. Much altered. Contains plagioclase and pale-green fibrous hornblende. Fine scales of mica and groups of epidote are also common.

D. Normal metagabbro, summit of Bald Mountain. Auvergnose. . Contains plagioclase, hornblende, numerous grains of magnetite or ilmenite, and traces apparently of pyroxene.

\begin{tabular}{|c|c|c|c|c|}
\hline$\cdot$ & A. & B. & C. & D. \\
\hline 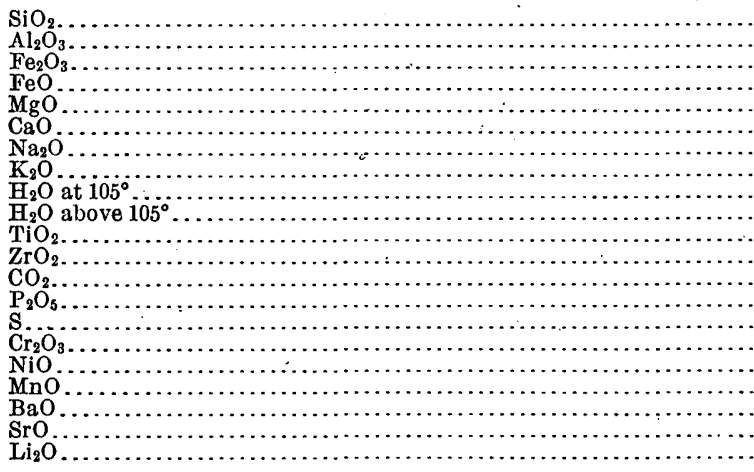 & 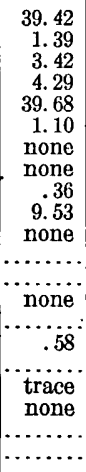 & $\begin{array}{r}38.55 \\
1.32 \\
5.55 \\
2.17 \\
39.06 \\
.85 \\
.10 \\
.05 \\
1.14 \\
10.14 \\
\text { trace } \\
\text { trace } \\
.03 \\
.48 \\
.13 \\
.05 \\
\text { none } \\
\text { none } \\
\text { trace }\end{array}$ & $\begin{array}{r}44.19 \\
20.66 \\
.52 \\
3.26 \\
11.90 \\
10.76 \\
1.35 \\
1.03 \\
.74 \\
5.19 \\
.12 \\
\text { none } \\
.06 \\
\text { trace } \\
\text { trace } \\
.15 \\
.03 \\
.11 \\
.04 \\
.05 \\
(?)\end{array}$ & $\begin{array}{r}50.14 \\
15.26 \\
1.19 \\
8.75 \\
7.21 \\
9.34 \\
2.76 \\
.95 \\
.23 \\
2.22 \\
1.42 \\
\ldots \ldots \\
\text { none } \\
.24 \\
.04 \\
\text { trace }\end{array}$ \\
\hline & 99.77 & 100.13 & 100.16 & 99.78 \\
\hline
\end{tabular}

E. Gabbro, Brush Creek, $1 \frac{1}{2}$ miles southwest of Bald Mountain. Yellowstonose. Rich in quartz and feldspar, with subordinate biotite and hornblende. Contains some chlorite.

F. Gabbro, west of Brush Creek, near summit of Mussel Creek divide. SR. 5 of kilauase. Chiefly feldspar and pyroxene, the latter partly changed to hornblende. A little quartz is present.

G. Gabbro, left bank of Rogue River, 2 miles below the mouth of Illinois River. $S R .5$ of monzonase. . Consists mainly of plagioclase and hornblende.

H. Basalt, Cedar Creek, $1 \frac{1}{2}$ miles northeast of Ophir. Auvergnose. Consists mainly of hornblende and feldspar. Grains of pyroxene are present, and a black dust which appears to be magnetite.

I. Basalt, near fork of West Bend trail, $2 \frac{1}{4}$ miles south of Johnson Creek. Beerbachose. Contains plagioclase and pyroxene, with secondary chlorite and hornblende.

\begin{tabular}{|c|c|c|c|c|c|}
\hline & E. & F. & G. . & H. & I. \\
\hline 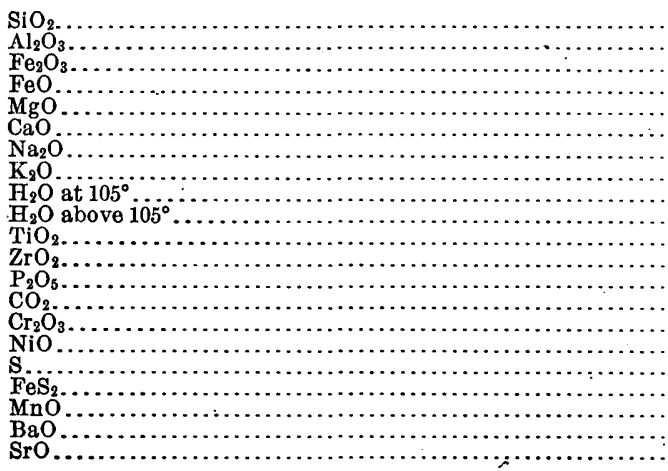 & $\begin{array}{r}60.88 \\
17.71 \\
2.92 \\
2.17 \\
2.21 \\
4.32 \\
4.17 \\
2.68 \\
.54 \\
1.47 \\
.41 \\
.16 \\
\text { none } \\
\text { none } \\
\text { trace } \\
\text { trace } \\
.06 \\
\text { trace }\end{array}$ & $\begin{array}{r}56.45 \\
13.81 \\
1.73 \\
3.95 \\
8.67 \\
6.69 \\
5.03 \\
.46 \\
.67 \\
2.02 \\
.31 \\
\ldots . . . \\
.02 \\
\text { none } \\
\text { trace } \\
\ldots . . . \\
\text { trace }\end{array}$ & $\begin{array}{r}57.43 \\
17.69 \\
1.59 \\
3.48 \\
2.73 \\
5.72 \\
7.19 \\
.58 \\
.48 \\
1.81 \\
.66 \\
\text { none } \\
.17 \\
.10 \\
\ldots \ldots . \\
\ldots \ldots . . \\
.02 \\
\ldots . .17 \\
.17 \\
\text { none } \\
.02\end{array}$ & $\begin{array}{r}50.56 \\
14.49 \\
1.78 \\
10.20 \\
5.90 \\
10.13 \\
2.91 \\
.38 \\
.20 \\
1.50 \\
1.67 \\
\text { none } \\
\text { trace } \\
(?) \\
\cdots \ldots . . \\
\text { trace } \\
\cdots \ldots . . \\
.28 \\
.25 \\
\text { trace } \\
\text { none }\end{array}$ & $\begin{array}{r}52.12 \\
15.21 \\
1.83 \\
8.95 \\
6.01 \\
3.75 \\
4.83 \\
.48 \\
.90 \\
3.74 \\
1.38 \\
\text { none } \\
.14 \\
.09 \\
\text { none } \\
.03 \\
\text { trace } \\
\ldots . . . \\
.19 \\
\text { trace } \\
\text { none }\end{array}$ \\
\hline & 99.70 & 99.83 & 99.84 & 100.25 & 99.65 \\
\hline
\end{tabular}


J. Basalt, Sawtooth Rock. I. 5. 3. 5. Largely feldspar and pyroxene, with some quartz.

K. Dacite porphyry, 6 miles west of Big Bend of Rogue River. Kallerudose. Contains plagioclase and quartz, with some orthoclase, and scattered patches of chlorite and hornblende.

L. Dacite porphyry, head of Boulder Creek. Yellowstonose. Contains abundant quartz, altered feldspar, grains of epidote, hornblende, and chlorite.

M. Dacite porphyry, south slope of Bald Mountain. Alsbachose-lassenose.

\begin{tabular}{|c|c|c|c|c|}
\hline & J. & K. & L. & M. \\
\hline 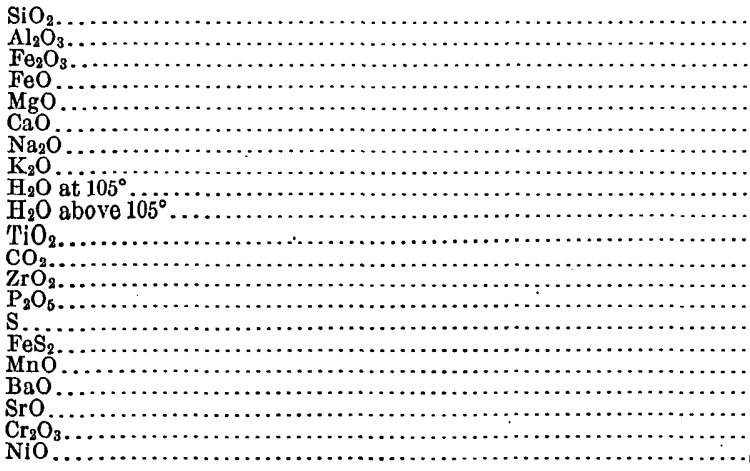 & $\begin{array}{r}53.06 \\
12.83 \\
1.20 \\
5.10 \\
7.50 \\
13.71 \\
3.56 \\
.05 \\
.16 \\
2.16 \\
.42 \\
.25 \\
\text { trace? } \\
\text { trace } \\
\text { trace } \\
\ldots \ldots . . \\
.16 \\
\text { none } \\
\text { none } \\
.06 \\
\text { trace }\end{array}$ & $\begin{array}{r}71.45 \\
14.53 \\
.49 \\
.94 \\
.30 \\
2.01 \\
7.15 \\
2.55 \\
.15 \\
.38 \\
.16 \\
.08 \\
\text { trace } \\
.09 \\
\text { trace } \\
\ldots \ldots . . \\
\text { trace } \\
.03 \\
\text { none } \\
\ldots \ldots . . . \\
\ldots . . . .\end{array}$ & $\begin{array}{r}70.33 \\
15.74 \\
1.43 \\
.83 \\
.53 \\
3.38 \\
4.33 \\
1.87 \\
.20 \\
1.16 \\
.27 \\
\text { trace } \\
\text { none } \\
.06 \\
\text { trace } \\
\ldots \ldots . . \\
\text { trace } \\
\text { trace } \\
\ldots \ldots . . \\
\ldots \ldots . .\end{array}$ & $\begin{array}{r}75.32 \\
13.17 \\
.27 \\
.98 \\
.42 \\
1.48 \\
4.77 \\
2.14 \\
.18 \\
.73 \\
.16 \\
.03 \\
\text { none } \\
.04 \\
\ldots \ldots . . \\
.09 \\
\text { trace } \\
.23 \\
.02 \\
\ldots \ldots . . \\
\ldots \ldots . .\end{array}$ \\
\hline & 100.22 & 100.31 & 100.22 & 100.03 \\
\hline
\end{tabular}

5. MISCELLANEOUS ROCKS.

A. Normal granodiorite from near lake at base of Bald Mountain, northwest of Sumpter. Yellowstonose. Described by Lindgren in 22d Ann., Part II, p. 587. Contains quartz, hornblende, andesine, orthoclase, biotite, and magnetite. Slight alterations to epidote and chlorite are sometimes noticeable. Analysis by W. F. Hillebrand, record No. 1896. P. R. C. 1529.

B. Rock from Wilbur, Douglas County. A tuff partly of igneous, partly of organic, origin. The igneous matter contains a few grains of feldspar and augite, with particles of a rock like diabase. The organic remains are partly calcareous and partly siliceous. Description supplied by J. S. Diller. Analysis by H. N. Stokes, record No. 1737.

C. Basaltic tuff, Columbia River, 25 miles east of Portland. Described by Diller in Bull. 260, p. 343. Analysis by G. Steiger, record No. 2165.

\begin{tabular}{|c|c|c|c|}
\hline - & A. & B. & C. \\
\hline 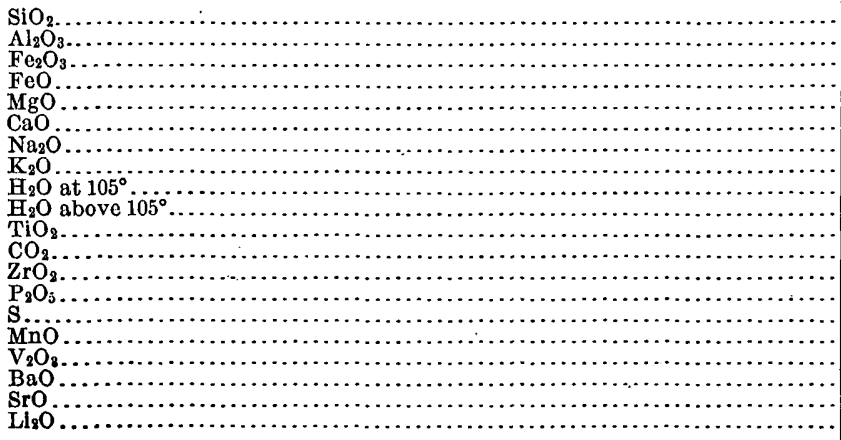 & $\begin{array}{r}71.23 \\
14.61 \\
.93 \\
1.66 \\
1.01 \\
3.29 \\
4.00 \\
1.92 \\
.17 \\
.55 \\
.34 \\
\ldots . .02 \\
.02 \\
.14 \\
\text { trace } \\
.08 \\
\ldots . . . \\
.08 \\
.02 \\
\text { trace }\end{array}$ & 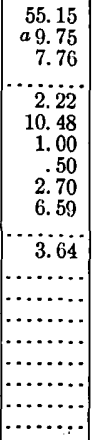 & $\begin{array}{r}40.89 \\
10.41 \\
15.00 \\
.07 \\
3.76 \\
5.18 \\
.47 \\
.53 \\
9.14 \\
10.32 \\
3.37 \\
\text { none } \\
\ldots \ldots . . \\
.52 \\
.03 \\
.90 \\
.01 \\
\ldots \ldots . . \\
\ldots \ldots . . \\
\ldots \ldots . .\end{array}$ \\
\hline & 100.05 & 99.79 & 100.60 \\
\hline
\end{tabular}




\section{WASHINGTON.}

\section{MOUNT STUART QUADRANGLE.}

Rocks described by George Otis Smith, in Folio 106. Analyses A, B, C, F, and G by H. N. Stokes, record No. 1836; D, E, H, and I by.W. F. Hillebrand, record No. 1831; J by George Steiger, record No. 2046.

A. Granodiorite, south slope of Moun't Stuart. Tonalose. Contains plagioclase, orthoclase, hornblende, biotite, quartz, and magnetite. P. R. C. 1407.

B. Granodiorite, ridge between Hardscrabble and Cascade creeks. Tonalose. Contains plagioclase, orthoclase, biotite, hornblende, quartz, magnetite, and apatite. P. R. C. 1412.

C. Granodiorite porphyry, dike 2 miles west of Mount Stuart. Tonalose. Contains plagioclase, biotite, hornblende, orthoclase, and quartz. P. R. C. 1414.

D. Serpentine; Three Brothers. Derived from saxonite. Contains serpentine, bastite, magnetite, and pyrite: P. R. C. 1408.

E. Metamorphic rock, head of Beverly Creek. "Nickel Ledge." Believed to be derived from an inclusion of limestone in the peridotite. P. R. C. 1410.

\begin{tabular}{|c|c|c|c|c|c|}
\hline & A. & B. & C. & D. & E. \\
\hline 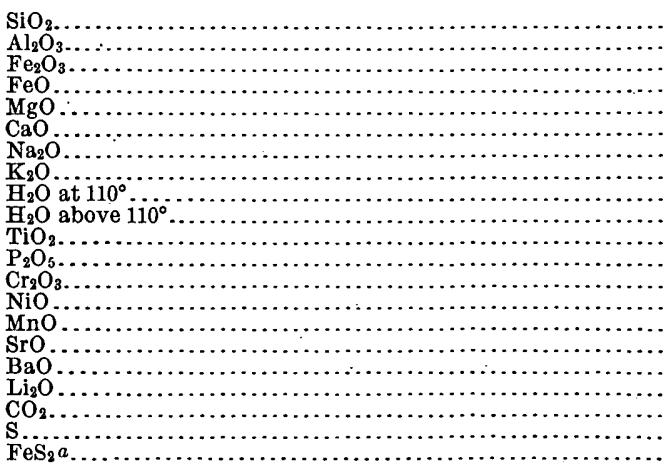 & $\begin{array}{r}64.04 \\
15.58 \\
1.26 \\
3.22 \\
3.23 \\
4.51 \\
4.01 \\
2.22 \\
.19 \\
1.17 \\
.69 \\
.16 \\
\text { none } \\
\text { none } \\
\text { trace } \\
\text { trace } \\
\text { trace } \\
\text { none } \\
\text { trace }\end{array}$ & $\begin{array}{r}63.37 \\
15.90 \\
1.41 \\
3.18 \\
3.33 \\
4.63 \\
4.05 \\
2.10 \\
.18 \\
1.16 \\
.69 \\
.17 \\
\text { none } \\
\text { none } \\
\text { trace } \\
\text { none } \\
\text { troc } \\
\text { trace } \\
\text { none } \\
\text { trace }\end{array}$ & $\begin{array}{r}63.78 \\
16.39 \\
1.12 \\
2.76 \\
3.27 \\
4.07 \\
3.84 \\
2.03 \\
.22 \\
1.82 \\
.44 \\
.11 \\
\text { none } \\
\text { none } \\
.05 \\
\text { trace } \\
.08 \\
\text { trace } \\
\text { none } \\
\text { trace }\end{array}$ & $\begin{array}{r}39.00 \\
1.75 \\
5.16 \\
1.71 \\
38.00 \\
\text { trace } \\
\} \quad .10 \\
1.31 \\
12.43 \\
\text { trace } \\
\text { trace } \\
.47 \\
.10 \\
.15 \\
\text { none } \\
\text { none } \\
\text { none } \\
\text { none } \\
\ldots . . . \\
.03\end{array}$ & $\begin{array}{r}32.12 \\
.82 \\
2.05 \\
3.50 \\
26.73 \\
1.81 \\
.06 \\
.43 \\
.98 \\
\text { trace } \\
\text { trace } \\
.27 \\
.08 \\
.14 \\
\text { none } \\
\text { none }\end{array}$ \\
\hline & $100: 39$ & 100.23 & 99.98 & 100.21 & 100.03 \\
\hline
\end{tabular}

a Actual condition of sulphur not known.

F. Gabbro, east of Beverly Creek. Hessose. Contains diallage and "basic" plagioclase, with pyrite and serpentine as alteration products. P. R. C. 1411.

G. Gabbro, Camas Land. Hessose: Containsaugite, olivine, plagioclase, magnetite, and apatite. P. R. C. 1413.

H. Diabase, dike on ridge west of Turnpike Creek. Tonalose. Contains plagioclase, augite, olivine, magnetite, and apatite. P. R. C. 1406.

I. Basalt, middle fork of Teanaway River. Vaalose. Contains augite, plagioclase, magnetite, and apatite, with a glassy base. P. R. C. 1409

J'. Yakima basalt, Clealum Ridge. Andose. Contains plagioclase, augite, olivine, magnetite, apatite, and glass. P. R. C. 1405. 


\begin{tabular}{|c|c|c|c|c|c|}
\hline & F. & G. & H. & I. & J. \\
\hline 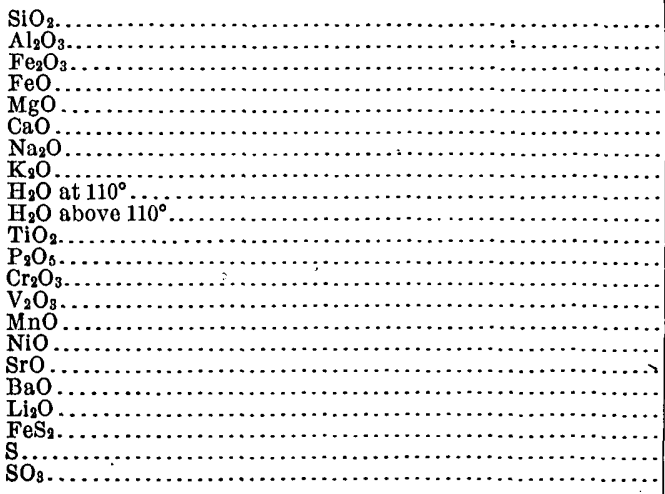 & $\begin{array}{r}48.58 \\
20.23 \\
1.26 \\
3.02 \\
7.59 \\
14.01 \\
2.25 \\
.19 \\
.28 \\
2.68 \\
.09 \\
\text { trace } \\
\text { trace } \\
\text { trace } \\
\text { none } \\
\text { none } \\
\text { none } \\
\text { none } \\
\ldots \ldots . . \\
. . .\end{array}$ & $\begin{array}{r}51.98 \\
15.99 \\
3.10 \\
5.88 \\
5.09 \\
9.68 \\
2.71 \\
.81 \\
.48 \\
2.08 \\
1.71 \\
.31 \\
\text { none } \\
\ldots . . . \\
. .10 \\
\text { none } \\
\text { none } \\
.03 \\
\text { trace } \\
\ldots . . . \\
.01 \\
\ldots . . .\end{array}$ & $\begin{array}{r}57.21 \\
12.99 \\
3.28 \\
10.18 \\
1.59 \\
5.97 \\
3.07 \\
1.61 \\
.68 \\
1.03 \\
1.72 \\
.44 \\
\text { none } \\
\text { none } \\
.24 \\
\text { trace } \\
\text { trace } \\
.06 \\
\text { trace } \\
.13 \\
\ldots \ldots . . \\
\ldots \ldots . .\end{array}$ & $\begin{array}{r}53.35 \\
12.90 \\
2.64 \\
11.28 \\
2.68 \\
6.96 \\
2.83 \\
1.40 \\
.91 \\
1.76 \\
2.44 \\
.45 \\
\text { none } \\
.04 \\
.25 \\
\text { trace } \\
\text { trace } \\
.05 \\
\text { trace } \\
.13 \\
\ldots \ldots . . \\
\ldots . . .\end{array}$ & $\begin{array}{r}54.50 \\
14.43 \\
2.17 \\
8.80 \\
4.24 \\
8.01 \\
3.05 \\
1.29 \\
.29 \\
1.09 \\
1.69 \\
.21 \\
\ldots \ldots . . \\
\ldots \ldots \\
\ldots .10 \\
\text { nowe } \\
.09 \\
.06 \\
\ldots \ldots . \\
\ldots \ldots . . \\
\ldots \ldots .11\end{array}$ \\
\hline & 100.25 & 99.96 & 100.20 & 100.07 & 100.13 \\
\hline
\end{tabular}

2. SNOQUALMIE QUADRANGLE.

Rocks described by Smith and Calkins in Folio 139. Analyses by G. Steiger, record No. 2145.

A. Rhyolite, near Easton. Liparose. Contains albite, quartz, magnetite, zircon, and apatite, with a little secondary kaolin, sericite, and limonite.

B. Hypersthene andesite, Naches Valley. Tonalose. Contains plagioclase, hypersthene, augite, magnetite, and apatite.

C. Granodiorite, head of Gold Creek. Tonalose. Contains plagioclase, orthoclase, quartz, hornblende, biotite, apatite, zircon, magnetite, and titanite.

D. Basalt, 2 miles south-southeast of Naches Pass. Auvergnose. Contains plagioclase, olivine, augite, magnetite, apatite, and very little glass.

\begin{tabular}{|c|c|c|c|c|}
\hline & A. & B. & C. & D. \\
\hline \multirow[t]{2}{*}{ 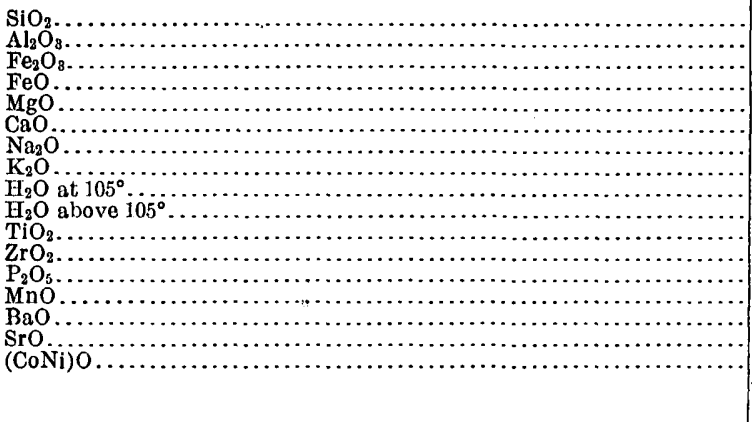 } & $\begin{array}{r}76.17 \\
12.32 \\
1.47 \\
.20 \\
\text { none } \\
.17 \\
3.94 \\
4.27 \\
.23 \\
.79 \\
.20 \\
.03 \\
.04 \\
\text { none } \\
.10 \\
\ldots \ldots . . \\
\text { none }\end{array}$ & $\begin{array}{r}62.77 \\
14.96 \\
1.62 \\
4.36 \\
1.48 \\
3.90 \\
4.31 \\
2.13 \\
.51 \\
2.49 \\
.79 \\
.03 \\
.22 \\
.10 \\
.10 \\
\text { none } \\
\text { none }\end{array}$ & $\begin{array}{r}60.49 \\
17.77 \\
1.98 \\
3.29 \\
2.94 \\
6.20 \\
3.67 \\
1.37 \\
.27 \\
1.13 \\
.69 \\
.02 \\
.08 \\
.09 \\
.04 \\
.02 \\
\text { trace }\end{array}$ & $\begin{array}{r}50.36 \\
15.83 \\
2.29 \\
8.11 \\
7.90 \\
9.25 \\
3.05 \\
.86 \\
.05 \\
.27 \\
1.33 \\
\ldots . .21 \\
.21 \\
.24 \\
\text { trace } \\
\text { trace } \\
\text { none }\end{array}$ \\
\hline & 99.93 & 99.77 & 100.05 & 99.75 \\
\hline
\end{tabular}

$\mathrm{CO}_{2}, \mathrm{~S}$, and $\mathrm{SO}_{8}$ absent. 


\section{MISCELLANEOUS ROCKS.}

A. Minette, Dike, west bank of Columbia River, 1 mile above Northport. $S R$. 2 of vyomingase. Collected by F. L. Ransome, who furnishes the petrographic description. Analysis by W. F. Hillebrand, record No. 1988. Contains orthoclase, possibly some plagioclase, abundant biotite, pyroxene, apatite, and titanite.

B. Brucite-serpentine rock, from quarry of the United States Marble Company, 12 miles north and west of Valley, Stevens County. Resembles ordinary serpentine, but contains also brucite, chlorite, and hydro-magnesite. The brucite amounts to about 60 per cent. Described by Clarke in Am. Jour. Sci., 4th ser., vol. 15, p. 397. Analysis by George Steiger, record No. 2009. P. R. C. 340.

C. Matrix of dumortierite, north fork of Washougal River, Skamania County. Analyzed and described by W. T. Schaller, Bull. 262, p. 105. Contains andalusite, quartz, muscovite, a little dumortierite, and accessory pyrite, magnetite, possibly ilmenite, leucoxene, and apatite. A little $\mathrm{B}_{2} \mathrm{O}_{3}$ present, undetermined.

\begin{tabular}{|c|c|c|c|}
\hline & A. & B. & C. \\
\hline 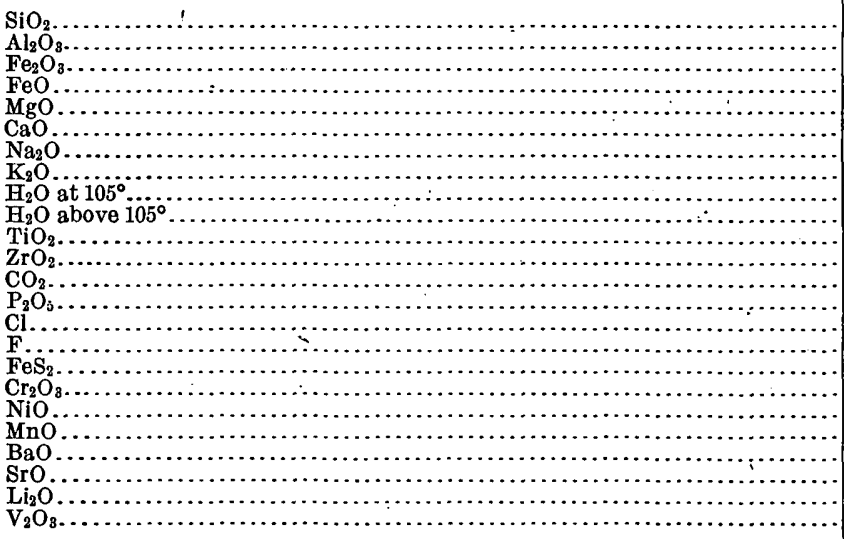 & $\begin{array}{r}41.57 \\
9.75 \\
4.06 \\
4.47 \\
8.65 \\
11.10 \\
1.57 \\
6.10 \\
1.54 \\
2.30 \\
2.36 \\
.02 \\
1.24 \\
4.05 \\
.04 \\
.23 \\
.06 \\
.04 \\
.02 \\
.25 \\
.44 \\
.11 \\
\text { trace } \\
.04\end{array}$ & 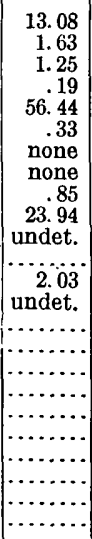 & $\begin{array}{r}57.18 \\
34.10 \\
.54 \\
.28 \\
.10 \\
.63 \\
.39 \\
2.57 \\
.69 \\
2.02 \\
.66 \\
.02 \\
\text { none } \\
.53 \\
\ldots . . \\
\ldots . . \\
.28 \\
\text { none } \\
\text { none } \\
\text { none } \\
.04 \\
\text { trace } \\
\text { none }\end{array}$ \\
\hline \multirow{2}{*}{ 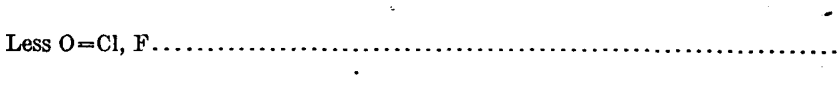 } & $\begin{array}{r}100.01 \\
.11\end{array}$ & $\begin{array}{r}99.74 \\
\ldots \ldots \ldots\end{array}$ & $\begin{array}{r}100.03 \\
\ldots \ldots \ldots\end{array}$ \\
\hline & 99.90 & & \\
\hline
\end{tabular}

ALASKA.

\section{DOUGLAS ISLAND.}

A. Diorite, Treadwell mine, Douglas Island. Tuolumnose. Contains plagioclase, mostly albite, with secondary quartz, calcite, and pyrite, the latter apparently replacing ferromagnesian silicates. Described by G. F. Becker in 18th Ann., pt. 3, p. 7. Analysis by W. F. Hillebrand, record No. 1585.

B. Albite diorite, Treadwell mine. Contains plagioclase, a little microperthite, hornblende, epidote, calcite, and pyrite.

C. Albite diorite, Treadwell mine. Like B, but with sericite, more microperthite, and no hornblende.

D. Altered diorite. Contains calcite, biotite, chlorite, quartz, and pyrrhotite.

E. Amphibolite.

Rocks B to E described by A. C. Spencer, in Bull. 287. Analyses by G. Steiger, record No. 2168. 


\begin{tabular}{|c|c|c|c|c|c|}
\hline & A. & B. & C. & D. & E. \\
\hline 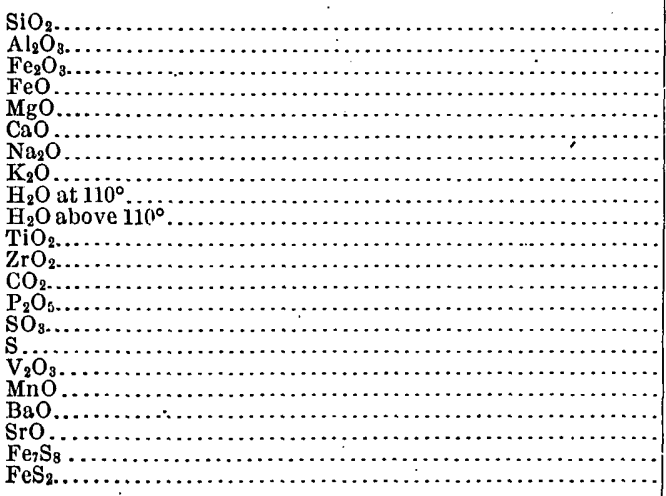 & $\begin{array}{r}63.01 \\
18.47 \\
.06 \\
.32 \\
.06 \\
2.66 \\
10.01 \\
.39 \\
.05 \\
.27 \\
.13 \\
\ldots .01 \\
.06 \\
\ldots \ldots . \\
.01 \\
.06 \\
.02 \\
\text { trace } \\
2.0 \% \\
2.10\end{array}$ & $\begin{array}{r}64.36 \\
18.18 \\
.64 \\
.43 \\
.28 \\
2.56 \\
8.96 \\
.89 \\
.18 \\
.55 \\
.17 \\
.03 \\
1.62 \\
.06 \\
.07 \\
\ldots \ldots \\
\ldots . . . \\
.11 \\
.06 \\
.04 \\
\ldots . .7 \\
.97\end{array}$ & $\begin{array}{r}58.53 \\
17.74 \\
1.58 \\
1.46 \\
1.71 \\
5.08 \\
5.69 \\
3.90 \\
.18 \\
1.18 \\
.81 \\
.06 \\
.90 \\
.27 \\
\text { none } \\
\ldots \ldots . . \\
\ldots . .71 \\
.07 \\
.07 \\
\ldots .05 \\
.96\end{array}$ & $\begin{array}{r}44.69 \\
14.97 \\
.60 \\
7.05 \\
3.92 \\
10.07 \\
2.36 \\
1.76 \\
.36 \\
.20 \\
2.25 \\
.02 \\
8.47 \\
.26 \\
\text { none } \\
\ldots \ldots . . \\
\ldots \ldots . . \\
.1 . \\
.14 \\
.14 \\
\text { undet. } \\
2.25 \\
.27\end{array}$ & $\begin{array}{r}47.76 \\
13.98 \\
1.99 \\
8.72 \\
9.07 \\
12.71 \\
1.65 \\
.20 \\
.22 \\
2.06 \\
1.48 \\
\text { none } \\
\text { none } \\
.12 \\
\text { none } \\
.04 \\
\ldots . .14 \\
.14 \\
\text { trace } \\
\text { none } \\
\ldots . . . . \\
\ldots . . .\end{array}$ \\
\hline & 99.69 & 100.16 & 100.28 & 99.78 & 100.14 \\
\hline
\end{tabular}

\section{COPPER RIVER BASIN.}

Andesites, described in P. P. 41. Analyses A, B, by W. F. Hillebrand, record No. 2075. C, D, by George Steiger, record No. 2080.

A. Dark, vesicular, porphyritic lava, late eruption, Mount Wrangell. Tonalose. Contains labradorite, hypersthene, olivine, and magnetite in a brown glassy base. Collected by W. C. Mendenhall.

B. Gray, porphyritic, well-crystallized older lava, Mount Drum. Tonalose. Contains plagioclase, hornblende, biotite, hypersthene, magnetite, apatite, and zircon in a fine gray hypocrystalline base. Collected by W. C. Mendenhall.

C. Augite andesite, lower north slope of Mount Sanford. Kailerudose. Contains plagioclase, augite, and magnetite in a microcrystalline groundmass of the same minerals. Collected by F. C. Schrader.

D. Hypersthene andesite from near C. Lassenose. Contains plagioclase, hornblende, hypersthene, and magnetite in a glassy groundmass of mainly plagioclase and hypersthene. Collected by F. C. Schrader.

\begin{tabular}{|c|c|c|c|c|}
\hline . & $\Lambda$. & $B$. & C. & D. \\
\hline 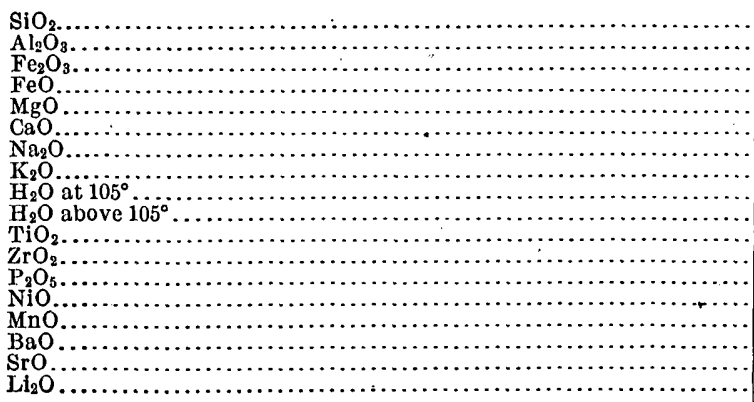 & $\begin{array}{r}61.31 \\
16.70 \\
1.30 \\
4.08 \\
3.44 \\
6.10 \\
4.05 \\
1.58 \\
.22 \\
.36 \\
.73 \\
.01 \\
.18 \\
.02 \\
.14 \\
.05 \\
.02 \\
\text { trace? }\end{array}$ & $\begin{array}{r}62.67 \\
16.62 \\
3.25 \\
1.17 \\
3.08 \\
5.56 \\
4.24 \\
1.67 \\
.23 \\
1.01 \\
.48 \\
.01 \\
.15 \\
.01 \\
.11 \\
.06 \\
.03 \\
\text { trace }\end{array}$ & $\begin{array}{r}70.94 \\
13.96 \\
1.74 \\
1.69 \\
.12 \\
1.13 \\
5.64 \\
4.03 \\
.09 \\
.45 \\
.30 \\
.05 \\
.10 \\
\text { none } \\
.15 \\
.06 \\
\text { trace } \\
\ldots . . . .\end{array}$ & $\begin{array}{r}67.04 \\
16.71 \\
1.46 \\
2.08 \\
1.09 \\
3.26 \\
5.07 \\
1.84 \\
.08 \\
.51 \\
.51 \\
.05 \\
.27 \\
\text { none } \\
.16 \\
\text { trace } \\
.03 \\
. . . .\end{array}$ \\
\hline & 100.29 & 100.35 & 100.45 & 100.16 \\
\hline
\end{tabular}

$\mathrm{CO}_{2}$ and $\mathrm{S}$ absent. 


\section{MISCELLANEOUS ROCKS.}

Rocks A to $G$, inclusive, were collected by G. F. Becker, who furnishes the petrographic data. A, B, C, D, and F are described by Becker in 18th Ann., pt. 3, p. 7. Analyses by Hillebrand, record No. 1585.

. A. Augite-bronzite andesite, Delarof Harbor, Unga Island. Andose. Greenish black. Contains plagioclase, near labradorite, with much smaller proportion of augite and bronzite, in a groundmass of plagioclase, with a little glass and much lightgreen indeterminate material.

B. Augite-brenzite andesite, St. Augustine Volcano, Cook Inlet. Placerose. Purplish gray. Contains labradorite, augite, and bronzite in a groundmass of plagioclase and magnetite.

C. Quartz porphyry, bed of Bear Creek, 4 miles from its mouth, Turnagain Arm, Cook Inlet. Dacose. Resembles D, with more feldspar and less quartz.

D. Quartz porphyry, east of mouth of Indian River, Sitka, Baranof Island. Sitkose. Contains plagioclase, quartz, a little pyroxene, and some carbonaceous matter, with secondary quartz, calcite, and muscovite.

E. Diorite, head of Captains Bay, Unalaska Island. Tonalose. Contains plagioclase, biotite, hornblende, chlorite, magnetite, and sometimes tourmaline.

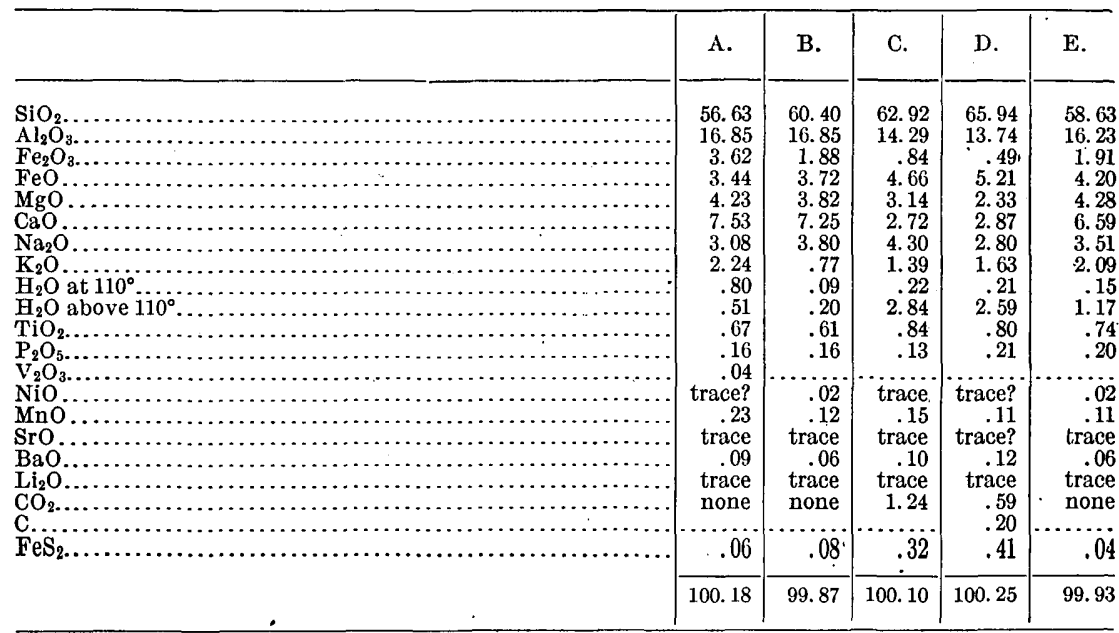

F. Diorite, Karluk Cliffs, Kadiak Island. Bandose. Contains labradorite and hornblende, with subordinate quartz, biotite, and magnetite.

G. Diorite, Lane \& Hayward mine, Silverbow Basin. Shoshonose. Contains plagioclase, biotite, hornblende, and scattering grains of quartz, with secondary chlorite, epidote, and muscovite.

H. Hornblende andesite, Bogoslof Island. Andose. Described by Merrill in Proc.

U. S. Nat. Mus., vol. 8, p. 31. Contains hornblende, augite, $\mathrm{p}^{\mathbf{l}}$ agioclase, tridymite, grains of iron ore, a little apatite, and probably sanidine. P. R. C. 315.

I. Like H, but darker colored. Andose. Contains more hornblende, no glass, and little or no tridymite. Same locality, also described by Merrill. Analyses $\mathrm{H}$ and I by T. M. Chatard, record Nos. 209, 210. P. R. C. 316. 


\begin{tabular}{|c|c|c|c|c|}
\hline & F. & G. & H. & I. \\
\hline 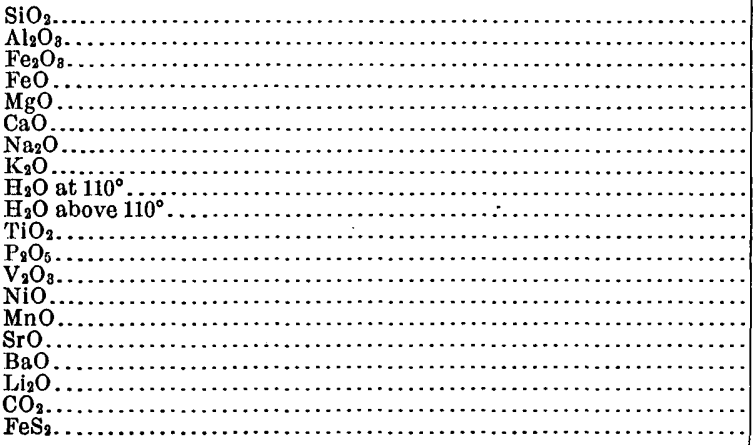 & $\begin{array}{r}61.58 \\
15.89 \\
2.19 \\
5.50 \\
2.69 \\
6.49 \\
3.04 \\
.51 \\
.16 \\
1.26 \\
.63 \\
.12 \\
\text { trace? } \\
.20 \\
\text { trace? } \\
.06 \\
\text { trace } \\
\text { none } \\
.06\end{array}$ & $\begin{array}{r}54.20 \\
15.86 \\
3.32 \\
4.14 \\
3.51 \\
5.32 \\
3.28 \\
3.30 \\
.55 \\
2.40 \\
1.35 \\
.68 \\
\ldots . .2 \\
.02 \\
.19 \\
.04 \\
.41 \\
\text { trace } \\
1.45 \\
.26\end{array}$ & $\begin{array}{r}56.07 \\
19.06 \\
5.39 \\
.92 \\
2.12 \\
7.70 \\
4.52 \\
1.24 \\
.99 \\
1.24 \\
.16 \\
\ldots \ldots . \\
\ldots .23 \\
\ldots \ldots . . \\
\ldots \ldots . . \\
\ldots \ldots . . . \\
\ldots \ldots . . \\
\ldots . . .\end{array}$ & $\begin{array}{r}51.54 \\
20.31 \\
4.64 \\
3.56 \\
3.16 \\
9.55 \\
4.29 \\
2.47 \\
.34 \\
.32 \\
.57 \\
\ldots \ldots . . \\
\ldots \ldots . .32 \\
\ldots \ldots . . \\
\ldots \ldots . . \\
\ldots \ldots . . \\
\ldots \ldots . . \\
\ldots \ldots . . \\
\ldots . .\end{array}$ \\
\hline & 100.38 & 100.28 & 99.64 & 101.07 \\
\hline
\end{tabular}

Rocks $\mathrm{J}$ to R. Collected by J. E. Spurr. Rocks $\mathrm{J}$ to $\mathbf{P}$ are described by him in Am. Geologist, vol. 25, p. 210. Analyses by H.N. Stokes, record Nos. 1809 and 1822.

J. Alaskite, Chilkoot Pass. Liparose. Dike in granite. Contains quartz, orthoclase, and some twinned feldspars, accessory zircon, actinolite, magnetite, and siderite.

K. Alaskite porphyry, Fortymile Creek, near Canyon Creek. Lassenose. Dike. Contains quartz, orthoclase, and some plagioclase, with accessory biotite and epidote.

L. Alaskite, Skwentna River, 12 miles above its mouth. Toscanose. Dike. Contains quartz, orthoclase, and microcline, with no dark minerals.

M. Alaskite, Tordrillo Mountains. Alaskose. Dike. Consists of quartz, orthoclase, and microcline, with no dark minerals. P. R. C. 1275.

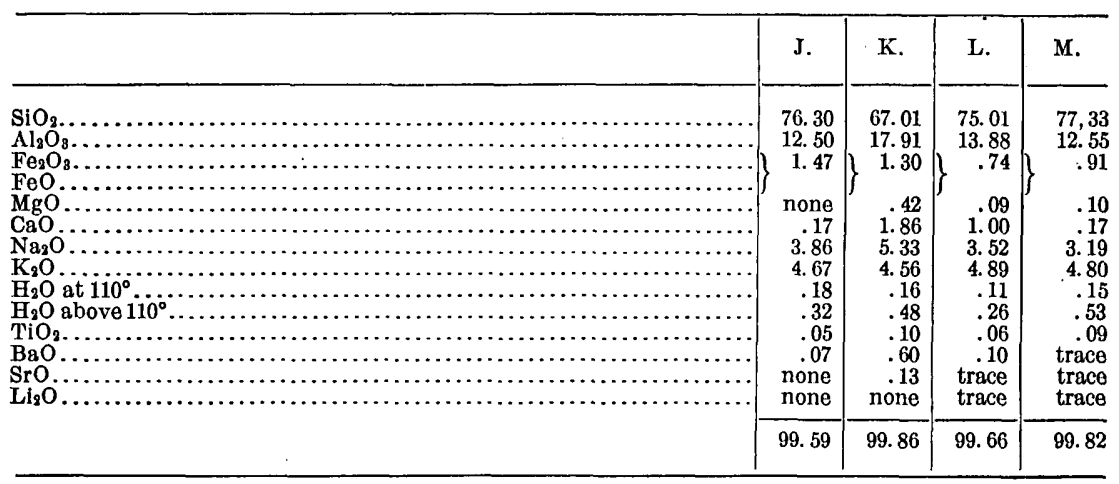

In $\mathrm{J}$ to $\mathrm{M}$ there are traces of $\mathrm{P}_{2} \mathrm{O}_{5}$ but no $\mathrm{CO}_{2}$. Manganese is also present in traces.

N. Augite belugite, Skwentna River, near Hayes River. Hessose. Dike. Contains essential feldspar, intermediate between andesine and labradorite, augite, and some hornblende. Also a considerable amount of pyrite. P. R. C. 1274.

O. Tordrillite, Tordrillo Mountains. Alaskose. Dike. Contains phenocrysts of quartz, orthoclase, anorthoclase, and anorthoclase-albite. Groundmass consists of quartz and orthoclase. No dark minerals except very small quantities of secondary hornblende. P. R. C. 1276.

P. Augite aleutite, near Kalinai Pass, Aleutian Peninsula. Andose. Lava. Chiefly labradorite, tending toward andesine, with a considerable amount of palegreen augite. P. R. C. 1277. 
Q. Plagioclase-scapolite-biotite rock, Skwentna River, 12 to 15 miles above its mouth. Tonalose. Dike. Described by Spurr in Am. Jour. Sci., 4th ser., vol. 10, p. 310. Essential constituents andesine-oligoclase, scapolite (dipyre), and biotite, with accessory apatite and zircon. P. R. C. 1273.

R. Tonalite-aplite or yukonite. Yukonose. Yukon River, above Fort Hamlin. Hitherto undescribed. Consists essentially of quartz and soda-lime feldspars.

\begin{tabular}{|c|c|c|c|c|c|}
\hline . & N. & 0 & P. & Q. & R. \\
\hline 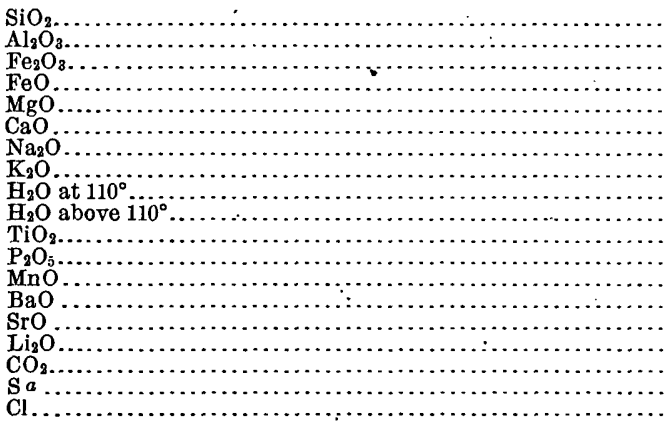 & $\begin{array}{r}50.23 \\
19.46 \\
4.21 \\
4.20 \\
3.59 \\
10.39 \\
3.08 \\
1.32 \\
.16 \\
1.01 \\
1.30 \\
.41 \\
.07 \\
.04 \\
\operatorname{trace} \\
\operatorname{trace} \\
.25 \\
.02 \\
\ldots \ldots\end{array}$ & $\begin{array}{r}75.84 \\
13.38 \\
1.45 \\
.10 \\
.07 \\
3.33 \\
4.73 \\
.18 \\
.71 \\
.09 \\
\text { trace } \\
\text { trace } \\
\text { trace } \\
\text { trace } \\
\text { trace } \\
\text { none } \\
\ldots . . . . \\
\ldots . . . . .\end{array}$ & $\begin{array}{r}56.03 \\
18.31 \\
3.47 \\
4.42 \\
3.64 \\
7.43 \\
3.60 \\
1.18 \\
.12 \\
.31 \\
1.24 \\
.13 \\
.11 \\
\text { trace } \\
\text { trace } \\
\text { trace } \\
\text { none } \\
\text { trace } \\
. . . . . .\end{array}$ & $\begin{array}{r}62.78 \\
17.16 \\
1.96 \\
2.31 \\
2.32 \\
4.84 \\
4.11 \\
2.15 \\
.24 \\
.88 \\
.56 \\
.15 \\
.06 \\
.04 \\
\text { trace } \\
\text { trace } \\
\text { none } \\
.02 \\
\text { trace }\end{array}$ & $\begin{array}{r}74.79 \\
12.59 \\
1.19 \\
\\
.31 \\
3.58 \\
5.10 \\
.21 \\
.09 \\
1.03 \\
.17 \\
\text { trace } \\
\text { trace } \\
\text { none } \\
\text { none } \\
\text { none } \\
.58 \\
\ldots . . . \\
\ldots . . .\end{array}$ \\
\hline & 99.74 & 99.88 & 99.99 & 99.58 & 99.64 \\
\hline
\end{tabular}

$a$ Whether $\mathrm{S}$ or $\mathrm{SO}_{3}$ was not determined.

\section{BRITISH COLUMBIA.}

Peridotite, junction of Eagle Creek and Tulameen River, Yale district. Dunose. Described by J. F. Kemp in Bull. 193. About two-thirds olivine and one-third serpentine, with some magnetite, calcite, and magnesite as accessories. Analysis by W. F. Hillebrand, record No. 1930. P. R. C. 1472.

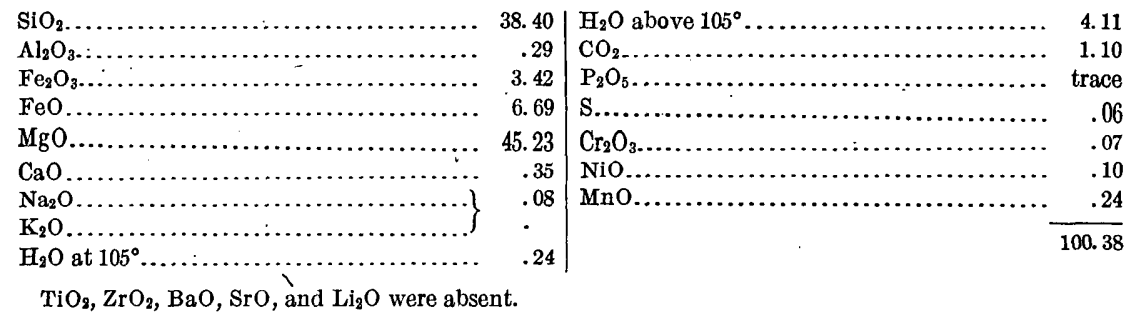

\section{HAWAIIAN ISLANDS.}

Rocks collected by Whitman Cross, who supplies the petrographic data. Analyses by W. F. Hillebrand, record Nos. 2038, 2112. A and B are described by Cross in Jour. Geology, vol. 12, p. 510.

A. Trachyte, from Puu Anahulu, North Kona, Hawaii. Umptekose. Consists chiefly of alkali feldspar, with small amounts of diopside and obscure sodic amphiboles or pyroxenes. Analysis incomplete.

B. Trachyte, from Puuwaaraa, North Kona, Hawaii: Umptekose near nordmarkose. Black glass, containing alkali feldspar microlites and ferritic particles.

C. Basalt, crater of Haleakala, Maui. Essexose. Contains plagioclase, orthoclase, nepheline, augite, much less olivine, magnetite, and apatite.

D. Plagioclase basalt, Makaweli Canyon, Kauai. Auvergnose. Consists essentially of augite, olivine, plagioclase, and magnetite, with a little glassy base. 
E. Melilite-nephelite basalt, Kiláuea Landing, North coast of Kauai. - Uvaldose. Consists essentially of augite, olivine, melilite, nephelite, magnetite, and apatite, with a little analcite. In vesicles, also, an undetermined fibrous zeolite.

F. Portion of $\mathrm{E}$ soluble in 1:40 nitric acid.

\begin{tabular}{|c|c|c|c|c|c|c|}
\hline & A. . & B. & C. & D. & E. & F. \\
\hline 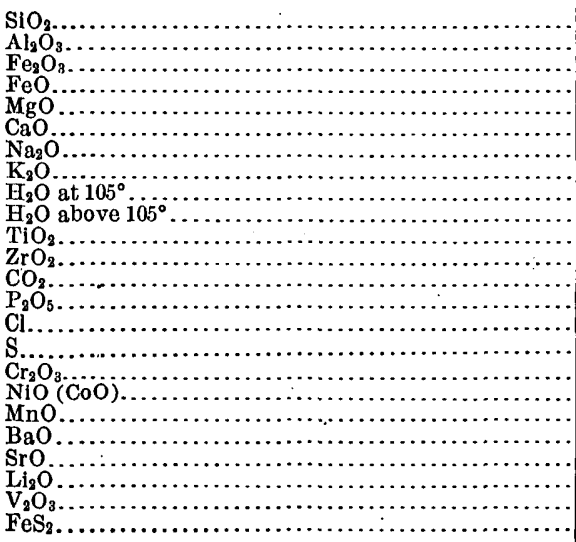 & $\begin{array}{r}.85 \\
6.89 \\
4.82 \\
a 1.60\end{array}$ & $\begin{array}{r}62.19 \\
17.43 \\
1.65 \\
2.64 \\
.40 \\
.86 \\
8.28 \\
5.03 \\
.14 \\
.39 \\
.37 \\
.04 \\
.02 \\
.14 \\
\text { undet. } \\
\text { none } \\
\text { trace } \\
\text { none } \\
.32 \\
.03 \\
\text { none } \\
\text { trace } \\
\text { none } \\
\ldots . . .\end{array}$ & $\begin{array}{c}49.55 \\
17.78 \\
4.65 \\
5.89 \\
2.49 \\
7.01 \\
6.12 \\
2.29 \\
.29 \\
.34 \\
2.09 \\
.01 \\
\text { none } \\
1.10 \\
(?) \\
\ldots . . . \\
\text { none } \\
\text { none } \\
.28 \\
.05 \\
.08 \\
\text { none } \\
.015 \\
.03\end{array}$ & $\begin{array}{r}45.48 \\
11.87 \\
1.98 \\
9.87 \\
13.28 \\
10.97 \\
2.21 \\
.77 \\
.23 \\
.74 \\
1.90 \\
\text { none } \\
\text { none } \\
.25 \\
(?) \\
\ldots . . . \\
.08 \\
.04 \\
.16 \\
.04 \\
\text { trace? } \\
\text { none } \\
.04 \\
.03\end{array}$ & $\begin{array}{r}37.50 \\
9.12 \\
5.59 \\
8.81 \\
13.72 \\
13.85 \\
2.69 \\
.63 \\
1.05 \\
2.35 \\
3.21 \\
.02 \\
.27 \\
.90 \\
.05 \\
\text { trace } \\
.07 \\
.04 \\
.15 \\
.07 \\
.05 \\
\text { trace } \\
.05 \\
\ldots \ldots . .\end{array}$ & $\begin{array}{r}15.84 \\
7.68 \\
1.47 \\
.47 \\
3.39 \\
5.21 \\
2.28 \\
.60 \\
b 1.05 \\
b 2.35 \\
.05 \\
\ldots .27 \\
.90 \\
.03 \\
\ldots \ldots \\
\text { (?) } \\
\text { (?) } \\
\text { in } \mathrm{l}_{2} \mathrm{O}_{3} \\
\text { (?) } \\
\text { in } \mathrm{CaO} \\
\text { (?) } \\
\text { (?) } \\
\text { (?) }\end{array}$ \\
\hline 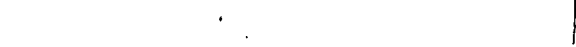 & 99.24 & 99.93 & 100.065 & 99.94 & 100.19 & 41.59 \\
\hline
\end{tabular}

$a$ Loss on ignition.

$b$ Assumed.

\section{GUATEMALA.}

Volcanic dust from the volcano Santa Maria, collected on deck of steamer Luxor, 60 miles distant in San Benito Harbor, October 25, 1902. Yellowstonose. Analysis by E. T. Allen, record No. 2011.

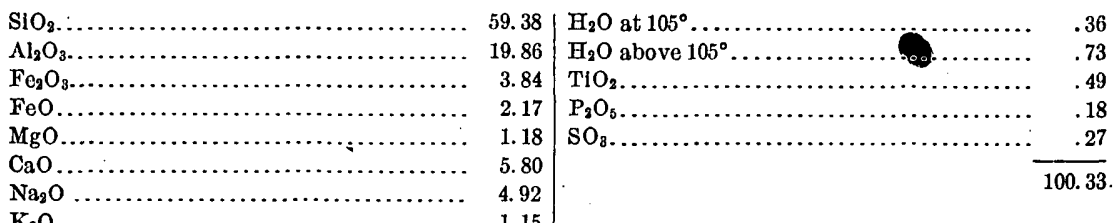

1.15

Traces of $\mathrm{Cr}_{2} \mathrm{O}_{3}, \mathrm{MnO}$, and $\mathrm{BaO}$ are present. $\mathrm{CO}_{2}$ is absent.

\section{WEST INDIES.}

Volcanic ejectamenta from Mont Pelée, Martinique, and La Soufrière, St. Vincent, eruptions of May, 1902: See Diller, Nat. Geog. Mag., vol. 13, p. 285. Also Diller and Steiger, Science, vol. 15, p. 947.

A. Pumice, Mont Pelée, eruption of May 8. Bandose-tonalose. Hypersthene andesite, containing plagioclase, hypersthene, and magnetite in a vesicular, glassy groundmass.

B. Sand, same eruption as A, collected on deck of the steamer Roddam after its arrival at Santa Lucia. Tonalose-bandose.

C. Dust, Mont Pelée, eruption of May 20. Tonalose. Collected on deck of steamer Potomac in harbor at Fort de France. B and C resemble A mineralogically.

D. Volcanic dust from about 1 mile north of Georgetown, St. Vincent. Bandose. Collected by E, O. Hovey and not described in the articles cited above. 
E. Pumice from La Soufrière. Bandose. Collected by R. T. Hill. Hypersthene andesite containing plagioclase, hypersthene, augite, olivine, and magnetite. (Diller.)

F. Dust from La Soufrière. Bandose-placerose. Collected on steamer Coya, at sea, about 275 miles southeast of St. Vincent, May 7, 1902. Contains.feldspars, pyroxenes, magnetite, and possibly other minerals. Contained 0.57 per cent of soluble salts.

Analyses A to D by W. F. Hillebrand, record No. 1991. E and F by George Steiger, record Nos. 1984, 1989.

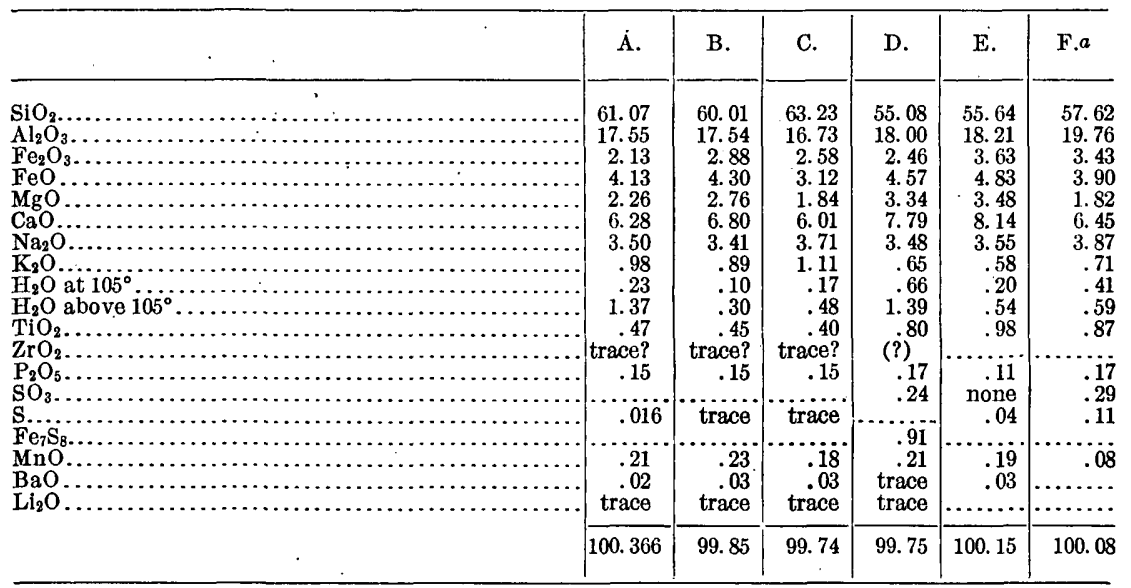

$a$ Soluble in water; $\mathrm{CaO}, 0.20 ; \mathrm{Na}_{2} \mathrm{O}, 0.08 ; \mathrm{SO}_{3}, 0.29$, and a trace of $\mathrm{Cl}$.

$\mathrm{CO}_{2}, \mathrm{Cl}, \mathrm{NiO}$, SrO absent, except a trace of $\mathrm{Cl}$ in $\mathrm{F}$.

\section{BRAZIL.}

A kyanitic schist from Serra do Gigante, near Diamantina, was analyzed at the request of Prof. O. A. Derby, who describes the.rock in Am. Jour. Sci., 4th ser.; vol. 7, p. 343. Analysis by Hillebrand, record No. 1783. Contains kyanite, chlorite, sericite, quartz, and rutile.

A. Bulk analysis of the schist.

B. Portion-soluble in hydrochloric acid.

C. Portion soluble in strong sulphuric acid.

D. Residue insoluble in sulphuric acid.

The bracketed figures are deduced from other columns than the one in which they appear.

\begin{tabular}{|c|c|c|c|c|}
\hline & A. & B. & C. & D.' \\
\hline $\begin{array}{l}\mathrm{SP}_{2} \mathrm{O}_{2} \\
\mathrm{Al}_{2} \mathrm{O}_{2}\end{array}$ & $\begin{array}{r}38.32 \\
28.16 \\
2.24 \\
4.02 \\
12.04 \\
.32 \\
.16 \\
1.11 \\
7.55 \\
7.46 \\
4.93 \\
.47 \\
.09 \\
.04 \\
.16 \\
\text { trace } \\
\text { trace } \\
\text { trace } \\
\text { trace? }\end{array}$ & $\begin{array}{l}10.78 \\
10.42 \\
{[1.78]} \\
{[3.21]} \\
9.34 \\
.34 \\
{[.03]} \\
.26 \\
{[.55]} \\
5.36 \\
.10 \\
.47 \\
\text { trace } \\
\text { (?) } \\
\text { (?) } \\
\text { t?) } \\
\text { trace } \\
\cdots \cdots \cdots\end{array}$ & $\begin{array}{c}14.76 \\
14.77 \\
{[2.24]} \\
{[4.02} \\
{[12.04]} \\
.32 \\
{[.03} \\
.26 \\
{[.55]} \\
6.80 \\
.20 \\
.47 \\
(?) \\
{[.04} \\
{[16]} \\
(?) \\
\text { trace } \\
\cdots \ldots . . \\
\cdots \ldots . .\end{array}$ & 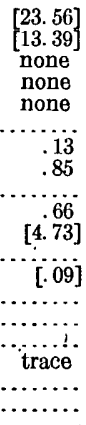 \\
\hline & 100.07 & 42.64 & 56.66 & 43.41 \\
\hline
\end{tabular}


TASMANIA.

Two analyses of so-called "volcanic bombs" or "buttons" were made by W. F. Hillebrand, record No. 2097, at the request of W. H. Twelvetrees, of Launceston, Tasmania. These curious forms of volcanic glass have every appearance of artificiality, but are said to be found under such conditions and in so many localities that the idea of their human origin is precluded. A paper on the subject by R. H. Walcott may be found in Proc. Royal Soc. Victoria, vol. 11, p. 23, 1898.

A. From Upper Weld tindrift. Almerose. Analysis incomplete because of insufficient material. Sp. gr., 2.454 at $18.5^{\circ}$.

B. From Pieman. I. 3. 4. 3. . Sp. gr., 2.428 at $22^{\circ}$.

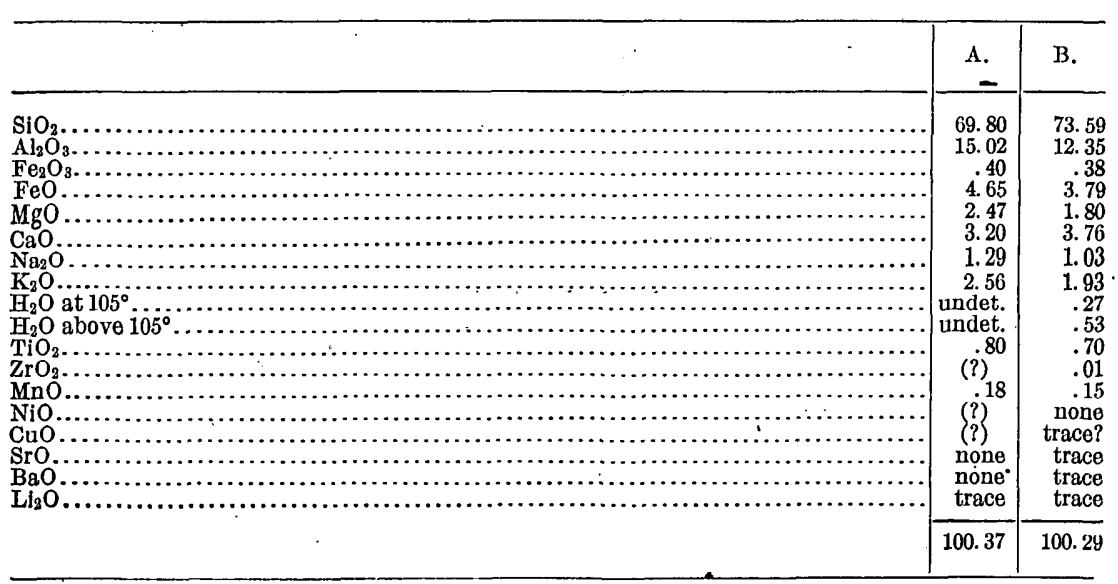

15619-Bull. 419-10-13 


\section{ANALYSES OF SANDSTONES, CHERTS, AND SINTERS.}

\section{SANDSTONES FROM EASTERN STATES.}

A. Calciferous sandstone, New Sweden, Maine. Described by H. E. Gregory in Bull. 165. Analysis by W. F. Hillebrand, record No. 1795. Contains quartz, calcite, feldspar, magnetite, muscovite, and siderite.

B. Brown sandstone, Hummelstown, Pennsylvania. Analysis by E. A. Schneider, record No. 1280. Described by Diller in Bull, 150, p. 77. P. R. C. 14.

C. Triassic sandstone from the Jaittelle quarry, near Hancock, Maryland. Hard, compact, brown. Analysis by F. W. Clarke, record No. 613 .

\begin{tabular}{|c|c|c|c|}
\hline & A. & B. & C. \\
\hline 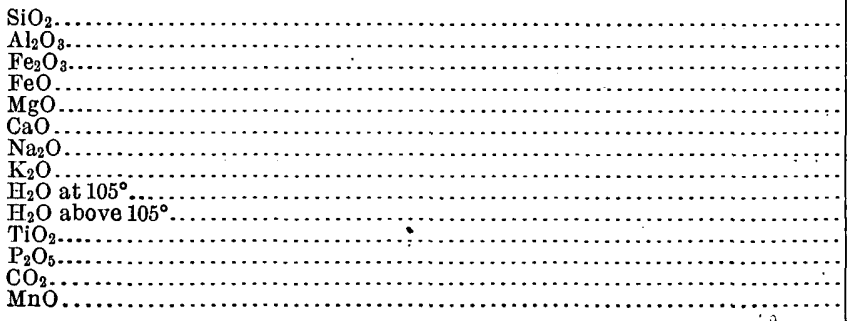 & $\begin{array}{r}54.23 \\
7.38 \\
.54 \\
1.37 \\
3.29 \\
14.56 \\
1.65 \\
1.74 \\
.25 \\
1.22 \\
.28 \\
.07 \\
13.48 \\
\text { undet. }\end{array}$ & $\begin{array}{r}88.13 \\
5.81 \\
1.77 \\
.31 \\
.53 \\
.20 \\
.06 \\
2.63 \\
.23 \\
.26 \\
\ldots \ldots . . \\
\ldots \ldots . . . \\
\ldots \ldots . . . \\
\ldots . . .\end{array}$ & $\begin{array}{r}76.43 \\
17.78 \\
\ldots .92 \\
.92 \\
\text { undet. } \\
\text { undet. } \\
2.79 \\
\ldots \ldots \ldots \\
\ldots \ldots \ldots\end{array}$ \\
\hline Insoluble in $\mathrm{HCl}$ & $\begin{array}{r}100.06 \\
\ldots \ldots\end{array}$ & $\begin{array}{c}99.93 \\
\ldots \ldots\end{array}$ & $\begin{array}{l}98.76 \\
88.68\end{array}$ \\
\hline
\end{tabular}

\section{SANDSTONES FROM MICHIGAN, WISCONSIN, AND IOWA.}

A. Yellow sandstone, Stony Point, Michigan. Analysis by F. W. Clarke; record No. 213.

B. Potsdam sandstone, Ablemans, Sauk County, Wisconsin. Described by Diller in Bull. 150, p. 80. Analysis by Schneider, record No. 1280. P. R. C. 15.

C. From Boscobel, Wisconsin.

D. From Beetown, Wisconsin. Analysis C, D, by G. Steiger, record No. 2227.

E. From Springdale Station, Sioux City, Iowa. Analysis by Steiger, No. 2295.

Rocks C, D, E are St. Peter sandstone, described as "glass sands" by E. F. Burchard in Bull. 285, p. 459, and Bull. 315, p. 377. Partial analyses only.

\begin{tabular}{|c|c|c|c|c|c|}
\hline . & A. & B. & C. & D. & E. \\
\hline 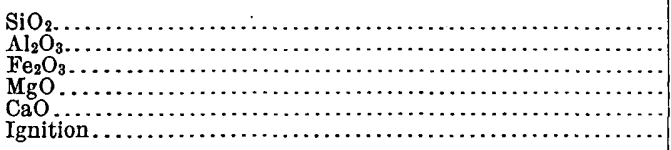 & $\begin{array}{r}84.57 \\
5.90 \\
6.48 \\
.68 \\
1.92\end{array}$ & $\left\{\begin{array}{r}99.42 \\
.31 \\
\cdots \\
\cdots \\
.18\end{array}\right.$ & $\begin{array}{r}99.47 \\
\cdots 07 \\
\cdots \cdots \\
\cdots \cdots \\
\cdots \cdots\end{array}$ & $\begin{array}{r}99.17 \\
.25 \\
.22 \\
\cdots \\
\cdots \\
\cdots\end{array}$ & $\begin{array}{r}96.90 \\
1.22 \\
.28 \\
.05 \\
.14 \\
1.07\end{array}$ \\
\hline Insoluble in $\mathrm{HCl} . \ldots \ldots \ldots \ldots \ldots \ldots \ldots \ldots$ & $\begin{array}{l}99.55 \\
91.87\end{array}$ & $\begin{array}{r}99.91 \\
\ldots \ldots\end{array}$ & $\begin{array}{c}99.54 \\
\ldots \ldots \ldots\end{array}$ & $\begin{array}{c}99.64 \\
\ldots \ldots\end{array}$ & $\begin{array}{r}99.66 \\
\ldots \ldots \ldots\end{array}$ \\
\hline
\end{tabular}




\section{SANDSTONES FROM OHIO.}

A. Blue sandstone from near Cleveland. Analysis by T. M. Chatard, record No. 214.

B. Sandstone from Berea. Analysis by L. G. Eakins, record No. 914.

C, D, E. Three samples of the "Peebles-Henley sandstone," from Portsmouth. Analyses by H. N. Stokes, record No. 1239. Alkalies, etc., undetermined.

\begin{tabular}{|c|c|c|c|c|c|}
\hline & $\Lambda$. & B. & c. & D. & I. \\
\hline 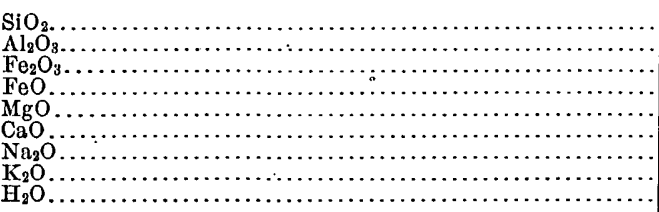 & $\begin{array}{r}91.67 \\
6.92 \\
\text { trace } \\
\cdots \\
.34 \\
.28 \\
.28 \\
1.17\end{array}$ & $\begin{array}{r}92.91 \\
3.78 \\
\text { trace } \\
.91 \\
\text { trace } \\
.31 \\
.34 \\
.61 \\
1.19\end{array}$ & 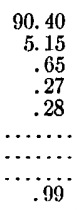 & 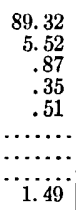 & $\begin{array}{r}87.12 \\
5.96 \\
.85 \\
.85 \\
.77 \\
2.73 \\
2.00\end{array}$ \\
\hline Insoluble in $\mathrm{HCl} . .$. & $\begin{array}{r}100.38 \\
97.50\end{array}$ & $\begin{array}{c}100.05 \\
\cdots \cdots \cdots\end{array}$ & $\begin{array}{l}97.74 \\
98.00\end{array}$ & $\begin{array}{l}98.06 \\
96.90\end{array}$ & $\begin{array}{l}97.51 \\
95.52\end{array}$ \\
\hline
\end{tabular}

\section{SANDSTONES FROM KENTUCKY, MISSISSIPPI, MISSOURI, AND ARKANSAS.}

A. Carboniferous sandstone adjoining the peridotite dike of Elliott County, Kentucky. See Diller, Am. Jour. Sci., 3d ser., vol. 32, p. 125. Analysis by T. M. Chatard, record No. 354 .

B. Fine-grained, fissile sandstone. Same locality and reference as A.

C. "Glass sand," near Iuka, Mississippi. Received from E. C. Eckel. Analysis by G. Steiger, record No. 2268.

D. From Jackson, Missouri.

E. From Versailles, Missouri.

F. From Everton, Arkansas.

Rocks D, E, F are St. Peter sandstones, described by Burchard in Bull. 285, p. 459, and Bull. 315, p. 377. Analyses by Steiger, Nos. 2227, 2295.

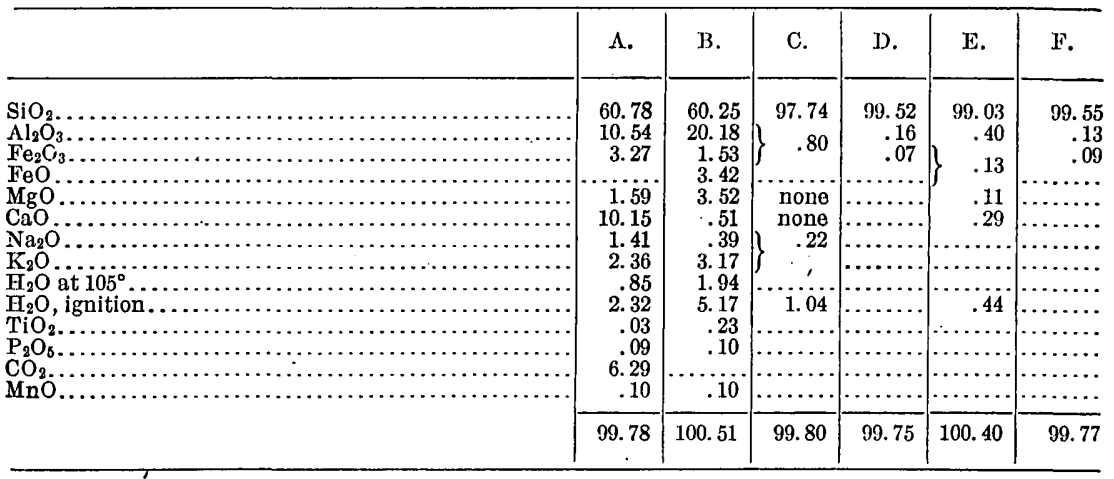

\section{SANDSTONES FROM KANSAS.}

St. Peter sandstones described as "glass sands" by E. F. Burchard in Bull. 285, p. 459, and Bull. 315, p. 377. Analyses by G. Steiger, record Nos. 2222, 2227, 2295. Partial only.

A, B, C, D, from Fredonia.

$\mathrm{E}$, from Neodesha.

F, from Havana. 
G, from Caney.

$\mathrm{H}, \mathrm{I}$, from Niotaze.

J, K, L, from SE. $\frac{1}{4}$ sec. 13, T. 28 S., R. 12 E.

$\mathrm{M}$, from near Fall River station.

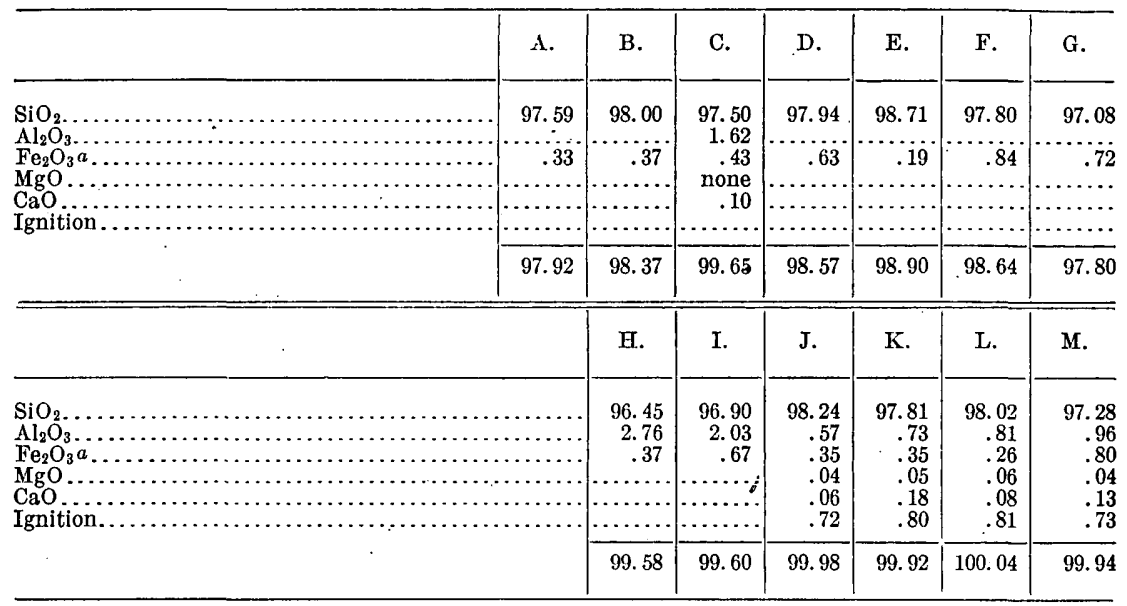

a 'Total Fe.

\section{SANDSTONES FROM COLORADO, UTAH, AND ARIZONA.}

A. Yellow sandstone, Armejo quarry, Colorado. Analysis, partial, by T. M. Chatard, record No. 289.

B. Sandstone, Robinson mine, Summit County, Colorado. Analysis by L. G. Eakins, made in the Denver laboratory, partial.

C. Sandstone from the Logan mine, Rico district, Colorado. Consists mainly of quartz and sericite.

D. Altered sandstone, in contact with C. Same constituents, plus limonite, anglesite, and a hydrous magnesian mineral. Analyses C, D, by W. F. Hillebrand, record No. 1914. Described by Ransome, 22d Ann., pt. 2, p. 287.

E. Banded sandstone, Peoa, Utah. Described by Diller in Bull. 150, p. 80. Analysis by Schneider, record No. 1280 . P. R. C. 16 .

F. Brown sandstone, Flagstaff, Arizona. Analysis by T. M. Chatard, record No. 1144.

\begin{tabular}{|c|c|c|c|c|c|c|}
\hline & A. & B. & C. & D. & E. & F. \\
\hline \multirow{11}{*}{ 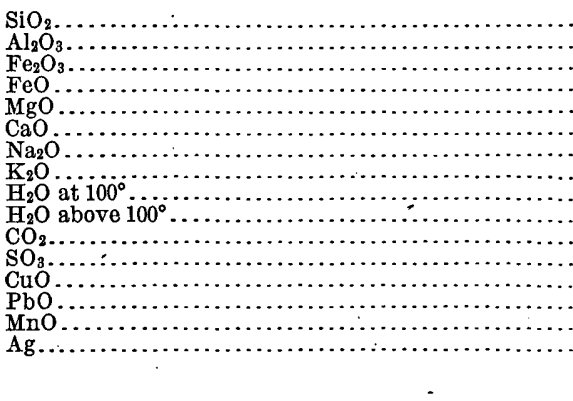 } & $\begin{array}{r}81.27 \\
9.81 \\
1.44\end{array}$ & $\begin{array}{r}56.33 \\
.77 \\
.97\end{array}$ & $\begin{array}{r}83.95 \\
8.92 \\
.48\end{array}$ & $\begin{array}{r}62.88 \\
21.38 \\
1.09\end{array}$ & $\begin{array}{r}96.60 \\
2.02\end{array}$ & $\begin{array}{r}a 79.19 \\
1.30 \\
2.45\end{array}$ \\
\hline & & 7.30 & & 2. 00 & 08 & \\
\hline & & & & & .04 & 7.7 \\
\hline & undet. & undet. & .06 & .07 & $\ldots \ldots . .$. & \\
\hline & undet. & undet. & 3.09 & 6.59 & & \\
\hline & 1.19 & undet. & $b 1.90$ & 5.49 & $\begin{array}{l}.11 \\
.29\end{array}$ & \\
\hline & & $c 19.04$ & none & .53 & & \\
\hline & & & trace & & & \\
\hline & & & tra & $\begin{array}{r}.34 \\
\text { trace }\end{array}$ & & \\
\hline & & & .04 & none & & \\
\hline & 94.57 & 98.42 & 99.66 & 100.77 & 99.14 & 99.9 \\
\hline
\end{tabular}

a Silica and insoluble matter. $\quad b$ Loss on ignition. $\quad c$ Calculated to satisfy bases. 


\section{SANDSTONES FROM CALIFORNIA.}

\section{MOUNT DIABLO.}

Described by Turner and Melville in Bull. Geol. Soc. America, vol. 2, pp. 383-41.4. Analyses made by W. H. Melville in the San Francisco laboratory.

A. Upper Cretaceous, Chico sandstone. Light brown, finely granular, carrying grains of mica and feldspar.

B. Lower Cretaceous, Neocomian sandstone. Hard, granular, greenish. From headwaters of Bagley Creek.

C. Miocene sandstone. Granular, particles light brown and black, friable. From near Wall Point.

D. Chico sandstone. Greenish gray, compact, crystalline.

\begin{tabular}{|c|c|c|c|c|}
\hline & A. & B. & C. & D. \\
\hline 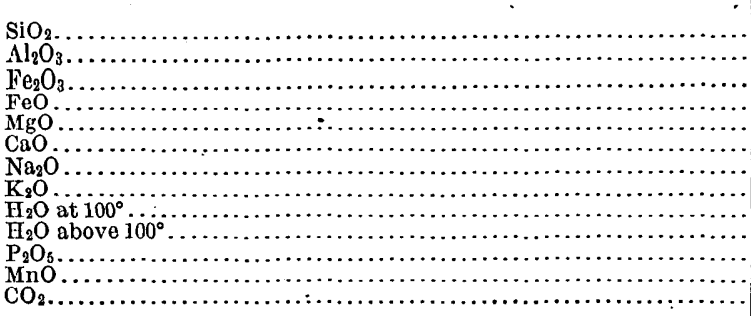 & $\begin{array}{r}73.71 \\
10.40 \\
3.89 \\
1.88 \\
1.62 \\
.96 \\
3.48 \\
.99 \\
1.06 \\
2.60 \\
\text { none } \\
.17 \\
\text { none }\end{array}$ & $\begin{array}{r}56.84 \\
11.37 \\
1.46 \\
4.95 \\
3.10 \\
7.62 \\
3.26 \\
.86 \\
1.45 \\
3.34 \\
.10 \\
.22 \\
5.10 .\end{array}$ & $\begin{array}{r}44.54 \\
12.63 \\
2.50 \\
3.08 \\
5.55 \\
14.65 \\
3.35 \\
1.37 \\
1.43 \\
2.25 \\
.29 \\
7.44 \\
7.76\end{array}$ & $\begin{array}{r}36.93 \\
7.22 \\
1.59 \\
2.95 \\
2.34 \\
29.34 \\
2.94 \\
.64 \\
.57 \\
3.45 \\
.16 \\
.57 \\
a 11.30\end{array}$ \\
\hline . & 100.76 & 99.67 & 99.84 & 100.00 \\
\hline
\end{tabular}

a $\mathrm{CO}_{2}$ determined by difference.

Traces of organic matter are found in all four sandstones, but were not determined.

\section{SHASTA COUNTY.}

Described by J. S. Diller in Bull. Geol. Soc. America, vol. 1, p. 411 . The rock is made up of quartz, feldspar, and biotite, with a calcite cement. Serpentine, sphene, magnetite, and zircon also occur in it, but are less common.

A. From Salt Creek, one-half mile above McNett's.

B. One and one-fourth miles below Ono Bridge, north fork of Cottonwood.

C, D. Three-fourths of a mile below John Allen's, Dry Creek.

E. From John Allen's, Dry Creek.

Analyses A and B by T. M. Chatard, record No. 1106; C, D, and E, by J. E. Whitfield, record Nos. 972, 973.

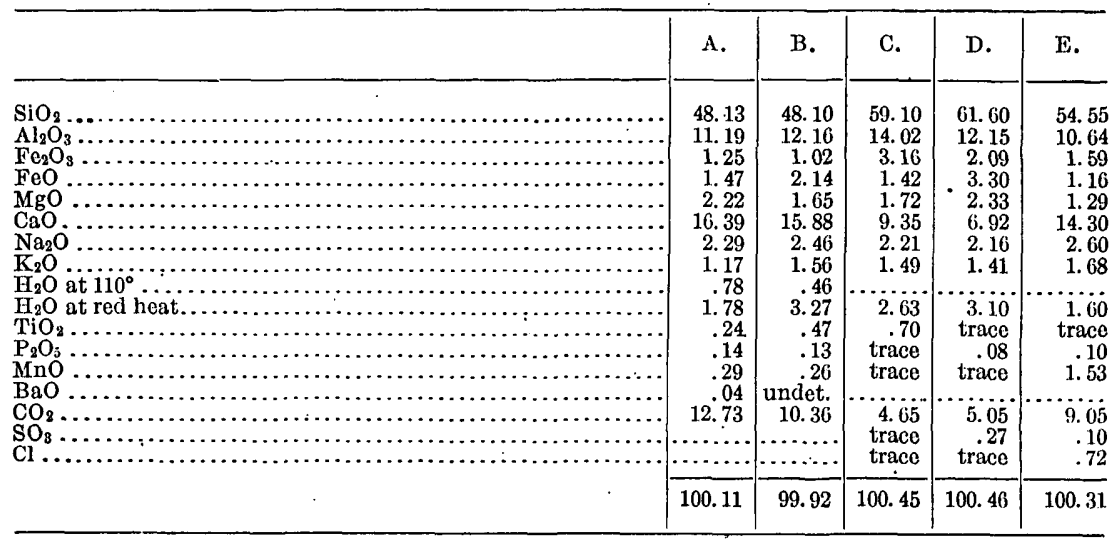


The following bedded sandstones of the same region are also described:

F. From middle fork of Cottonwood, 1 mile above Miller's.

G. Top of cascade, $1 \frac{1}{2}$ miles up Byron Creek from north fork of Cottonwood.

H. Two and one-half miles above John Allen's, Dry Creek.

Analyses $\mathrm{F}$ and $\mathrm{G}$ by Chatard, record No. 1106; H by Whitfield, record No. 974.

\begin{tabular}{|c|c|c|c|}
\hline . & F. & G. & H. \\
\hline 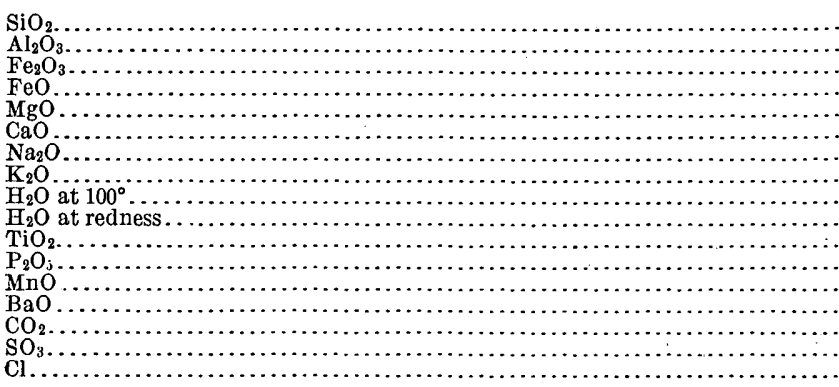 & $\begin{array}{r}55.85 \\
13.20 \\
2.56 \\
4.77 \\
1.90 \\
6.93 \\
2.60 \\
1.89 \\
1.13 \\
2.99 \\
.76 \\
.18 \\
.24 \\
\text { undet. } \\
4.97 \\
\cdots \\
\cdots . . .\end{array}$ & $\begin{array}{r}67.62 \\
13.63 \\
1.25 \\
3.27 \\
2.34 \\
2.80 \\
2.78 \\
1.11 \\
.64 \\
2.83 \\
.48 \\
.08 \\
.15 \\
.03 \\
.72 \\
\ldots . . .\end{array}$ & $\begin{array}{r}60.74 \\
10.25 \\
4.31 \\
6.21 \\
3.69 \\
4.97 \\
1.83 \\
.52 \\
4.36 \\
.86 \\
\text { trace } \\
\text { trace } \\
2.29 \\
.40 \\
\text { trace }\end{array}$ \\
\hline & 99.97 & 99.73 & 100.43 \\
\hline
\end{tabular}

\section{SULPHUR BANK.}

Described by Becker in Mon. XIII, p. 92. Analysis made by Melville in the San Francisco laboratory. An altered sandstone, showing grains of quartz, plagioclase, and orthoclase.

\begin{tabular}{|c|c|c|c|}
\hline 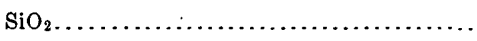 & 68.50 & $\mathrm{H}_{2} \mathrm{O}$ at $100^{\circ} \ldots \ldots \ldots$ & .28 \\
\hline $\mathrm{Al}_{2} \mathrm{O}_{3} \ldots \ldots \ldots \ldots \ldots \ldots \ldots \ldots \ldots \ldots \ldots \ldots \ldots \ldots$ & 12.82 & $\mathrm{H}_{2} \mathrm{O}$ above $100^{\circ} \ldots \ldots \ldots \ldots \ldots \ldots \ldots \ldots \ldots \ldots \ldots \ldots \ldots$ & 2.11 \\
\hline $\mathrm{Fe}_{2} \mathrm{O}_{3} \ldots \ldots \ldots \ldots \ldots \ldots \ldots \ldots \ldots \ldots \ldots \ldots \ldots \ldots$ & 1.29 & $\mathrm{TiO}_{2} \ldots \ldots \ldots \ldots \ldots \ldots \ldots \ldots \ldots \ldots \ldots \ldots \ldots \ldots \ldots$ & .60 \\
\hline 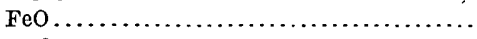 & 3. 37 & $\mathrm{P}_{2} \mathrm{O}_{i} ; \ldots \ldots \ldots \ldots \ldots$ & .16 \\
\hline $\operatorname{MgO} \ldots \ldots \ldots \ldots \ldots \ldots \ldots \ldots \ldots \ldots \ldots \ldots \ldots \ldots \ldots$ & 2.21 & $\mathrm{MnO}_{\ldots} \ldots \ldots \ldots$ & .02 \\
\hline 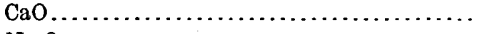 & 1.82 & & \\
\hline 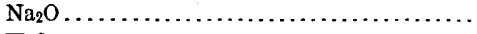 & 6.03 & & 100.47 \\
\hline 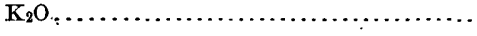 & 1.26 & & \\
\hline
\end{tabular}

\section{CHERT.}

From the lead-zinc region of southwestern Missouri and its extension into Kansas. Collected by W. P. Jenney.

A. Unaltered chert, East Hollow, Belleville, Jasper County, Missouri.

B. Partly altered, same locality.

C. Altered to "cotton rock," same locality.

D. From the Surprise mine, Joplin, Missouri.

E. Blue chert, unaltered, Bonanza shaft, Galena, Kansas.

F. Same locality.

G. Altered, same locality.

H. Jasperite, Joplin, Missouri.

I. Jasperite, Galena, Kansas.

Analyses A to G by E. A. Schneider, record No. 1205; H and I by L. G. Eakins, record No. 1208.

\begin{tabular}{|c|c|c|c|c|c|c|c|c|c|}
\hline 1 & A. & B. & C. & D. & E. & F. & G. & H. & I. \\
\hline 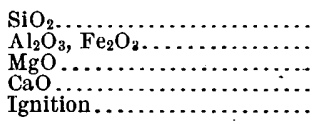 & $\begin{array}{r}98.17 \\
.83 \\
.01 \\
.05 \\
.78\end{array}$ & $\begin{array}{r}98.92 \\
.48 \\
.02 \\
.03 \\
.42\end{array}$ & $\begin{array}{r}98.71 \\
.43 \\
.02 \\
.03 \\
.50\end{array}$ & $\begin{array}{r}99.46 \\
.29 \\
\text { trace } \\
.04 \\
.34\end{array}$ & $\begin{array}{r}99.23 \\
.22 \\
\text { trace } \\
.02 \\
.50\end{array}$ & $\begin{array}{r}98.60 \\
.52 \\
\text { trace } \\
.10 \\
.40\end{array}$ & $\begin{array}{r}99.13 \\
.16 \\
.01 \\
\text { trace } \\
.20\end{array}$ & $\begin{array}{r}95.77 \\
1.84 \\
.24 \\
.54 \\
1.17\end{array}$ & $\begin{array}{r}97.33 \\
1.89 \\
.09 \\
.11 \\
.77\end{array}$ \\
\hline$\because$ & 99.84 & 99.87 & 99.69 & 100.13 & 99.97 & 99.62 & 99.50 & 99.56 & 100.19 \\
\hline
\end{tabular}




\section{SILICEOUS SINTERS.}

From the Yellowstone National Park and similar localities. Analyses A to E by J. E. Whitfield, record Nos. 97, 100, 707, 708, and 861. Discussed by Weed in 9th Ann., p. 619.

A. Dried siliceous jelly from Emerald Spring, Upper Basin.

B. Sinter from Solitary Spring, Upper Basin.

C. Grayish sinter from margin of Splendid Geyser.

D. Compact sinter from Old Faithful Geyser.

E. From Asta Spring, Hillside Group. Sp. gr., 1.7122.

\begin{tabular}{|c|c|c|c|c|c|}
\hline & A. & B. & C. & D. & E. \\
\hline 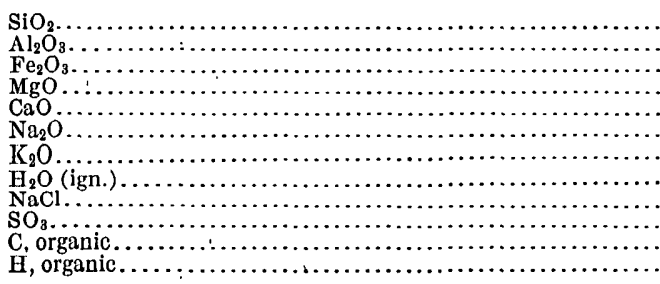 & $\begin{array}{r}93.37 \\
1.16 \\
\text { trace } \\
.05 \\
.29 \\
.11 \\
.02 \\
4.17 \\
.08 \\
.31 \\
.78 \\
.07\end{array}$ & $\begin{array}{r}93.88 \\
1.73 \\
.14 \\
.07 \\
.25 \\
.28 \\
.23 \\
3.37 \\
.18 \\
.20 \\
.2 \ldots\end{array}$ & $\begin{array}{r}81.95 \\
6.49 \\
\text { trace } \\
.15 \\
.50 \\
2.56 \\
.65 \\
7.50 \\
\text { trace } \\
.16\end{array}$ & $\begin{array}{r}89.54 \\
2.12 \\
\text { trace } \\
\text { trace } \\
1.71 \\
1.12 \\
.30 \\
5.13 \\
\text { trace } \\
\text { trace } \\
\cdots \ldots . . . \\
\cdots\end{array}$ & 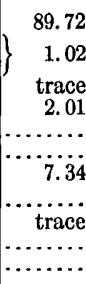 \\
\hline & 100.41 & 100.33 & 100.02 & 99.92 & 100.09 \\
\hline
\end{tabular}

Analyses $\mathrm{F}$ to $\mathrm{O}$, are all of Yellowstone Park deposits. Record Nos. 236, 703, 697, $706,698,709,812,866$, and 1012. Analysis $\mathrm{N}$ by 'T. M. Chatard; all the others by J. E. Whitfield. Samples collected by W. H. Weed.

F. Geyserite incrustation, Spring No. 8, Giant Group, Upper Basin.

G. Incrustation from margin of crater, Surprise Geyser.

H. Deposits from Coral Spring, Norris Basin.

I. Sediment, Vixen Geyser.

J. Deposit from Artemisia Geyser.

K. Opal deposit, Norris Basin.

L. Incrustation, Excelsior Geyser Basin. Bottom layer, compact, opaline.

M. Same as L, top layer, sintery.

$\mathrm{N}$. Incrustation, platform near Union Geyser.

.O. Black coating, the "Minute Man," Norris Basin.

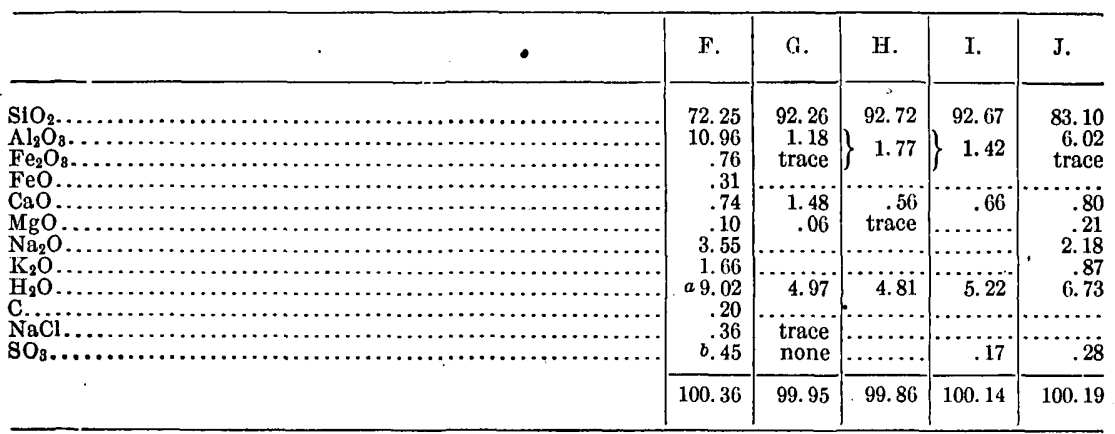




\begin{tabular}{|c|c|c|c|c|c|}
\hline & K. & L. & M. & N. . & o. \\
\hline 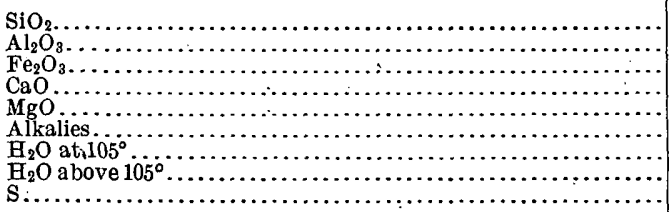 & $\begin{array}{r}93.60 \\
1.06 \\
\text { trace } \\
.50 \\
\text { trace } \\
4.71 \\
\text { trace }\end{array}$ & $\left\{\begin{array}{r}90.85 \\
.83 \\
.46 \\
\text { trace } \\
\ldots \ldots \\
7.90\end{array}\right.$ & $\left\{\begin{array}{r}94.40 \\
.79 \\
\text { none } \\
\text { none } \\
\ldots \\
5.02\end{array}\right.$ & $\begin{array}{r}90.28 \\
2.82 \\
\text { trace } \\
.30 \\
.07 \\
\text { undet. } \\
1.86 \\
3.95 \\
\end{array}$ & $\begin{array}{r}85.08 \\
2.65 \\
1.67 \\
\text { trace } \\
\ldots \ldots \\
a 10.67\end{array}$ \\
\hline & 99.87 & 100.04 & 100.21 & 99.28 & 100.07 \\
\hline
\end{tabular}

$a$ Loss on ignition. Water and organic matter.

The following foreign sinters were analyzed for comparison with the Yellowstone Park specimens:

P, Q, R. Three samples of sinter from Rotorua, New Zealand. Analyses by J. E. Whitfield, record No. 998. Discussed by Weed in 9th Ann., p. 619.

S. Sinter from Mount Morgan gold mine, Queensland. Described by Weed in Am. Jour. Sci., 3d ser., vol. 42, p. 165. Analysis by E. A. Schneider, record No. 1254.

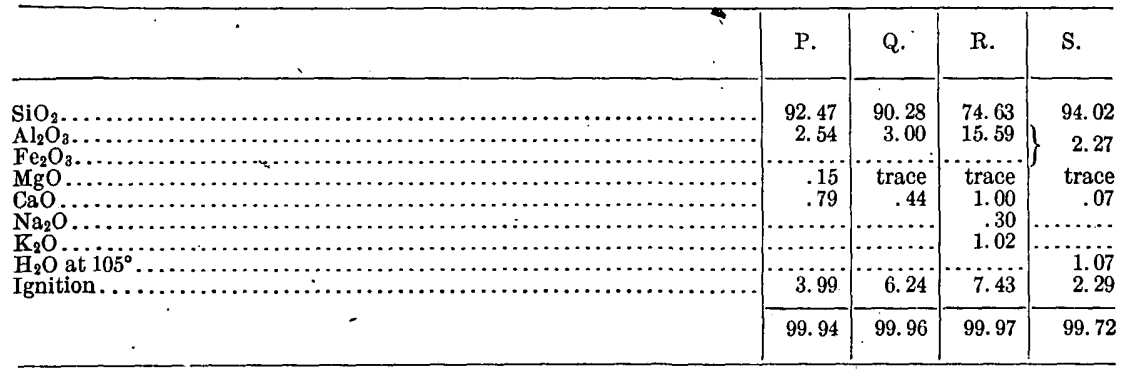




\section{ANALYSES OF CARBONATE ROCKS.}

\section{MAINE AND VERMONT.}

A. Limestone, Islesboro, Maine. Collected by George Otis Smith. Analysis by W. T. Schaller, record No. 2139. Partial only; $\mathrm{CO}_{2}$ calculated to satisfy bases.

B. White marble, Rutland, Vermont. Analysis by L. G. Eakins, record No. 1213.

C. The portion of A insoluble in dilute hydrochloric acid. Same analyst and number.

D. Dolomite, Green Peak quarry, Dorset, Vermont. Analysis by George Steiger, record No. 1938. Described by Dale in Bull. 195.

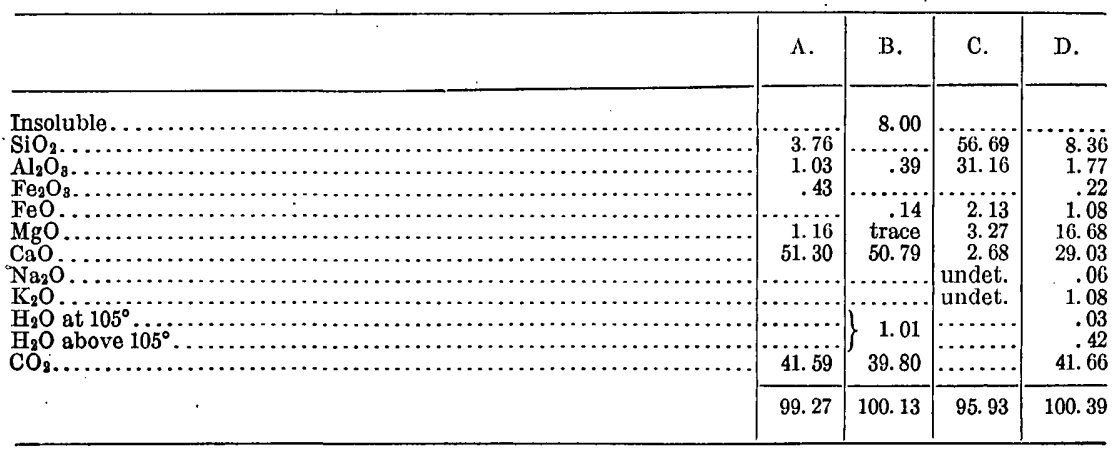

\section{MASSACHUSETTS.}

A. White marble, Lee. Analysis by E. A. Schneider, record No. 1279. Described by Diller in Bull. 150, p.299. P. R. C. 116.

B. Limestone, Lee. Collected by B. K. Emerson. From cut on west side of railroad. Analysis by George Steiger, record No. 1654.

C. Dolomite, Charlemont. Collected by Emerson. Analysis by Eakins, record No. 1343.

D. Dolomite, Webster. Collected by Emerson. Analysis by H. N. Stokes, record No. 1634.

\begin{tabular}{|c|c|c|c|c|}
\hline ' & A. & B. & C. & D. \\
\hline 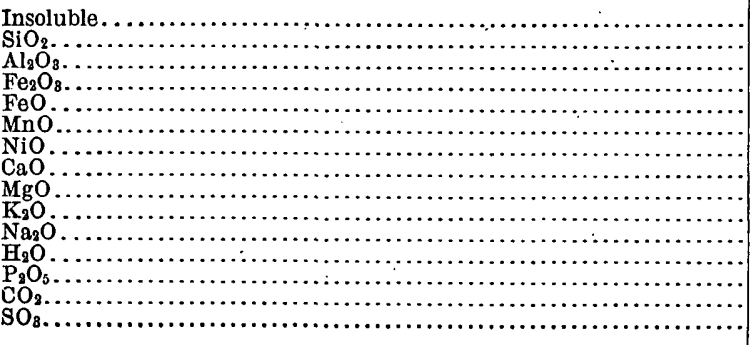 & 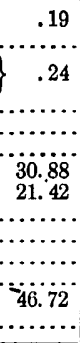 & $\begin{array}{r}.95 \\
.09 \\
\text { none } \\
.10 \\
\ldots \ldots . . . \\
\cdots .74 .75 \\
.56 \\
.15 \\
.02 \\
.08 \\
.03 \\
43.38 \\
.05\end{array}$ & $\begin{array}{r}.67 \\
\text { trace } \\
.08 \\
7.60 \\
1.61 \\
.03 \\
28.63 \\
16.17 \\
\ldots \ldots \ldots . \\
\ldots \ldots \ldots . \\
\ldots .35 .35 \\
\ldots \ldots \ldots .\end{array}$ & $\begin{array}{r}1.01 \\
.17 \\
\text { none } \\
.37 \\
.08 \\
\ldots .30 \\
30.82 \\
21.35 \\
.10 \\
.01 \\
.09 \\
.06 \\
45.84 \\
\ldots . . .\end{array}$ \\
\hline . & 99.45 & 100.16 & 100.14 & 99.90 \\
\hline
\end{tabular}


NEW YORK.

A. Dolomite marble, New York Quarry Company, Tuckahoe, Westchester County. Analysis by W. F. Hillebrand, record Nंo. 746.

B. Hydraulic cement rock, Akron. Analysis by George Steiger, record No. 1655. Described by Diller in Bull. 150.

\begin{tabular}{|c|c|c|}
\hline 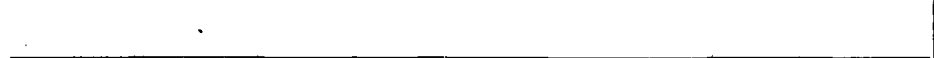 & & B. \\
\hline 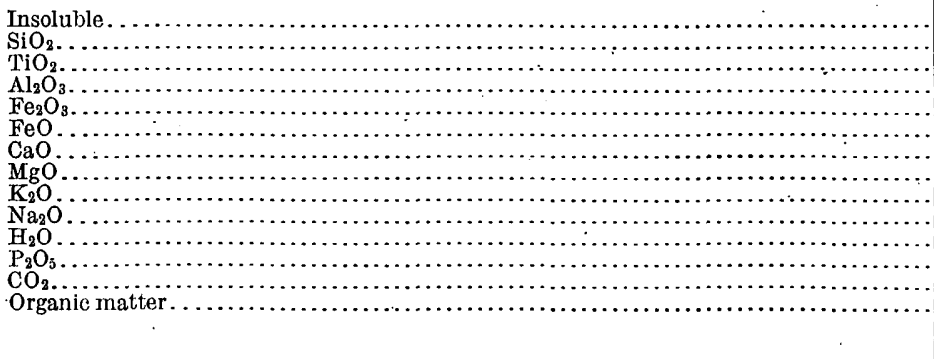 & 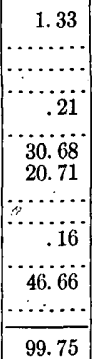 & $\begin{array}{r}9.03 \\
.16 \\
2.25 \\
.85 \\
.52 \\
26.84 \\
18.37 \\
.85 \\
\text { none } \\
.98 \\
.03 \\
40.33 \\
\ldots \ldots . \\
100.21\end{array}$ \\
\hline
\end{tabular}

\section{PENNSYLVANIA.}

A. Compact gray limestone, Greason. Described by:Diller in Bull. 150. Analysis by E. A. Schneider, record No. 1279.

B. Limestone, quarry on south side of Jordan Creek, near.Jordan Bridge, Șouth Whitehall.

C. Quartzose dolomite, same locality as B.

D. Hydraulic limestone, Atlas Cement Company's quarry, Whitehall.

Analyses B, C, D, by W. F. Hillebrand, record No. 2016. Samples collected by T. N. Dale.

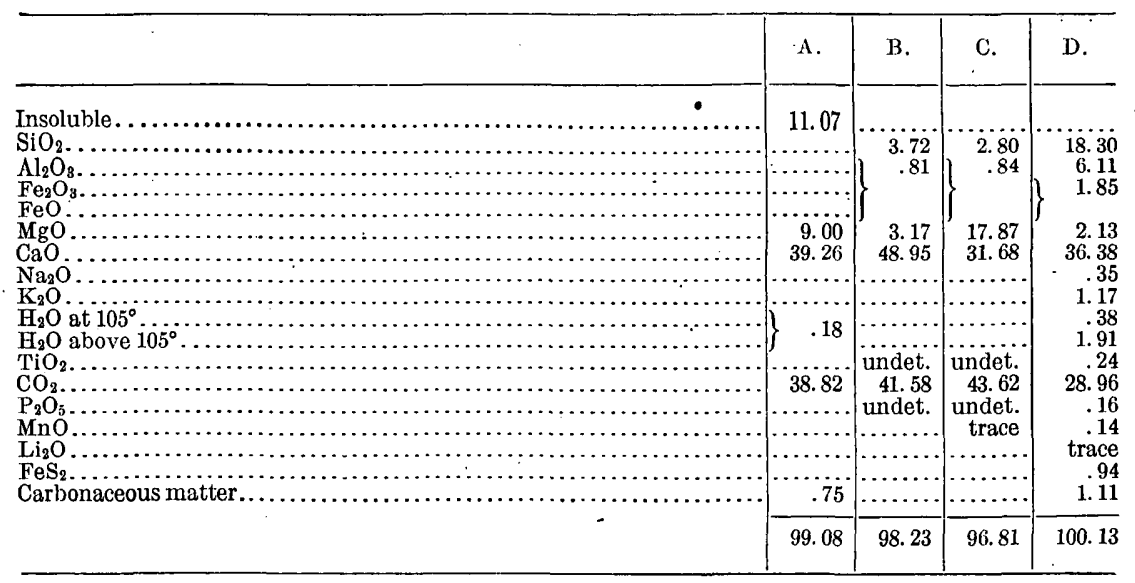

\section{MARYLAND.}

A. Dolomite marble, Cockeysville. Analysis by E. A. Schneider, No. 1279. P. R. C. 117 . 
B. An earlier sample of D. Analysis by J. E. Whitfield, record No. 827. P. R. C. 117. Described by Diller in Bull. 150.

\begin{tabular}{|c|c|c|}
\hline & A. & B. \\
\hline 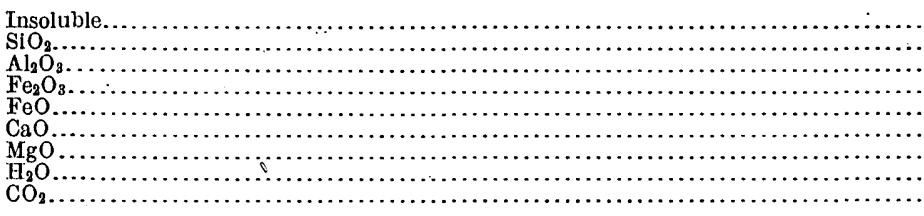 & 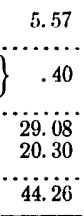 & $\begin{array}{r}.44 \\
1.22 \\
\text { trace } \\
30.73 \\
20.87 \\
1.22 \\
45.85\end{array}$ \\
\hline & 99.61 & 100.33 \\
\hline
\end{tabular}

VIRGINIA AND WEST, VIRGINIA.

A. Limestone, upper ledge, Moundsville Narrows, West Virginia.

B. Same as A, lower ledge. Analyses A and B by T. M. Chatard, record No. 127.

C. Trenton limestone, Lexington, Virginia. Analysis by R. B. Riggs, record No. 365 .

D. Limestone, Staunton, Virginia. Analysis by George Steiger, record No. 1630 .

E. Part of $\mathrm{D}$ insoluble in one-tenth hydrochloric acid.

F. Soluble part of D. Analyses $\mathrm{E}$ and $\mathrm{F}$, also by Steiger, same number. These three analyses are accompanied by analyses of the residual clay, formed by the weathering of the limestone. 'See section on clays.

\begin{tabular}{|c|c|c|c|c|c|c|}
\hline & A. & B. & C. & D. & E. & F. \\
\hline 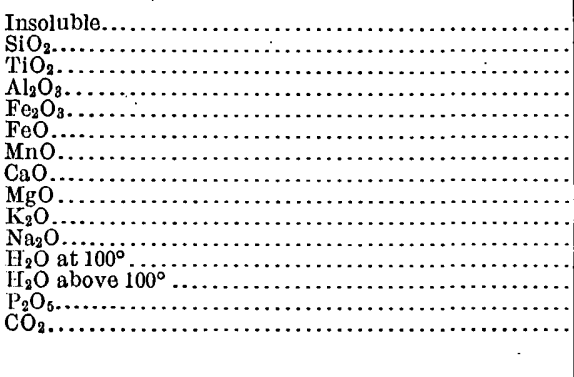 & $\begin{array}{r}10.33 \\
\ldots \ldots \ldots . \\
\ldots \ldots \ldots \\
.90 \\
\ldots \ldots \ldots \\
\text { trace } \\
48.02 \\
1.08 \\
\ldots \ldots \ldots \\
.05 \\
\text { trace } \\
39.18\end{array}$ & $\begin{array}{r}1.53 \\
\ldots . . . . \\
\ldots . . . \\
.96 \\
\text { trace } \\
53.26 \\
.93 \\
\ldots \ldots . . \\
.10 \\
\text { trace } \\
43.16\end{array}$ & 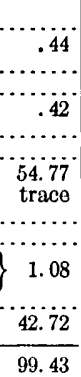 & $\begin{array}{r}7.37 \\
.09 \\
1.92 \\
.29 \\
.63 \\
\text { none } \\
28.39 \\
18.30 \\
1.09 \\
.09 \\
.09 \\
.49 \\
.03 \\
41.85 \\
100.63\end{array}$ & $\begin{array}{r}6.98 \\
.09 \\
1.39 \\
.25 \\
\text { none } \\
\text { none } \\
.04 \\
.15 \\
.91 \\
.04 \\
\text { undet. } \\
.15 \\
\text { none } \\
\text { none } \\
10.00\end{array}$ & $\begin{array}{r}.39 \\
\text { none } \\
.53 \\
.04 \\
.63 \\
\text { none } \\
28.35 \\
18.15 \\
.18 \\
.05 \\
\text { undet. } \\
.34 \\
.03 \\
41.85 \\
90.54\end{array}$ \\
\hline
\end{tabular}

\section{NORTH CAROLINA AND SOUTH CAROLINA.}

A. Marble, from Marshall, Madison County, North Carolina.

B. Marbla, from Bakersville, North Carolina. Analyses A, B, by E. C. Sullivan, record No. 2157 .

C. Marble, from Fletcher, Buncombe County, North Carolina. Analysis (partial) by G. Steiger, No. $2209 . \quad \mathrm{CO}_{2}$ calculated to satisfy bases.

D. Marble, from Caney Fork, Jackson County, North Carolina. Partial analysis by W. T. Schaller, No. $2259 . \quad \mathrm{CO}_{2}$ calculated. 
E. Marl, Charleston, South Carolina. Analysis by W. F. Hillebrand, record No. 2187.

\begin{tabular}{|c|c|c|c|c|c|}
\hline . & A. & B. & C. & D. & E. \\
\hline 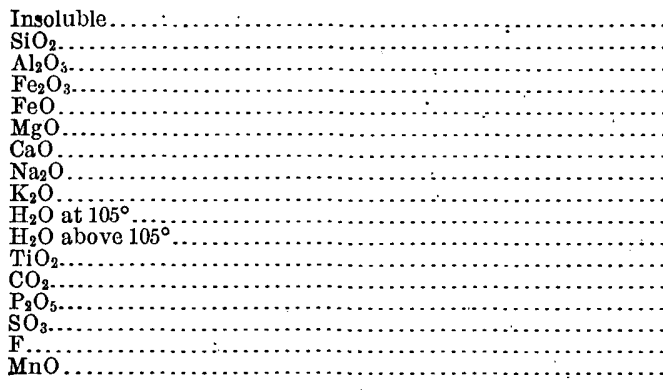 & $\begin{array}{r}13.20 \\
1.82 \\
.42 \\
.82 \\
1.09 \\
47.35 \\
.32 \\
.42 \\
.26 \\
.11 \\
\ldots \ldots \\
34.06 \\
.05 \\
\text { trace } \\
\ldots \ldots . . . \\
.05\end{array}$ & $\left\{\begin{array}{r}.12 \\
\ldots \ldots \\
.11 \\
\ldots \ldots \ldots \\
21.43 \\
30.67 \\
\ldots \ldots \ldots \\
\ldots \ldots \ldots \\
.13 \\
.14 \\
\ldots \ldots .55 \\
\ldots \ldots \ldots\end{array}\right.$ & 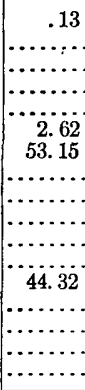 & 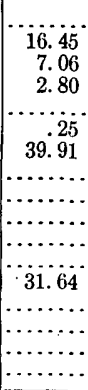 & $\begin{array}{r}10.92 \\
1.47 \\
.75 \\
. .91 \\
45.55 \\
.27 \\
.38 \\
a 3.17 \\
.08 \\
34.42 \\
1.29 \\
.54 \\
.16 \\
\text { trace }\end{array}$ \\
\hline 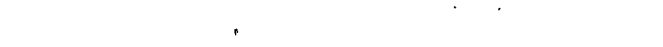 & 99.97 & 100.15 & 100.22 & 98.11 & 99.91 \\
\hline
\end{tabular}

$a$ Includes organic matter.

\section{GEORGIA.}

A. Marble, Happy Valley.

B. "Creole" marble, Happy Valley.

C. Portion of B insoluble in dilute hydrochloric acid.

D. "Cherokee" marble, Happy Valley.

E. Portion of $\mathrm{D}$ insoluble in dilute hydrochloric acid.

Analyses by L. G. Eakins, record Nos. 464, 485.

\begin{tabular}{|c|c|c|c|c|c|}
\hline . & A. & B. & c. & D. & E. \\
\hline \multirow{7}{*}{ 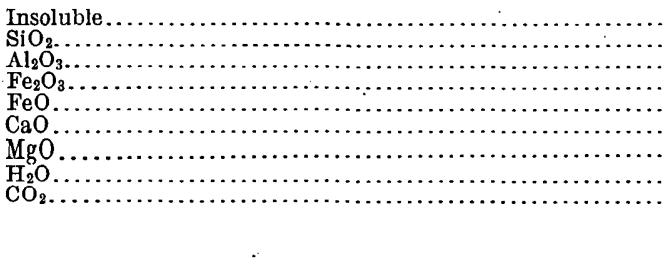 } & 23 & & 582 & 2.01 & \\
\hline & $\begin{array}{r}.20 \\
.91 \\
.22\end{array}$ & .17 & $\begin{array}{r}58.21 \\
7.37\end{array}$ & .15 & $\begin{array}{l}\text { 50. } \\
15.58\end{array}$ \\
\hline & & .05 & .31 & .06 & trace \\
\hline & 52.16 & 53.91 & 12.53 & 53.69 & 14.52 \\
\hline & 2.09 & .83 & 20.42 & & 12.88 \\
\hline & $\begin{array}{r}.45 \\
42.22\end{array}$ & $\begin{array}{r}.13 \\
43.16\end{array}$ & $\ldots \ldots$ & $\begin{array}{r}.17 \\
43.13\end{array}$ & …....... \\
\hline & 100.28 & 99.69 & 98.84 & 100.04 & 98.46 \\
\hline
\end{tabular}

\section{FLORIDA.}

\section{CORAL AND SHELL ROĊKS, COLLECTED BY N. S. SHALER.}

Partial analyses only. Chlorides and sulphates present; alkalies and phosphates not looked for.
A, B, C, D. Coquina gravel, Tortugas.
E. The same, Key West.
F. Near Fort Worth.
G. East side of St. Johns River, near Seville.
H. Corroded surface, Miami Reef.
I. Near Oak Hill.
J. Near Melborne. 
Analyses A to J by F. W. Clarke, record No. 885 .

\begin{tabular}{|c|c|c|c|c|c|c|c|c|c|c|}
\hline & A. & B. & C. & D. & E. & F. & G. & H. & I. & J. \\
\hline $\begin{array}{l}\mathrm{SiO}_{2} \ldots \ldots \ldots \ldots \\
\mathrm{Al}_{2} \mathrm{O}_{3}, \mathrm{Fe}_{2} \mathrm{O}_{3} \ldots \ldots \\
\mathrm{CaO} \ldots \ldots \ldots \ldots \\
\mathrm{MgO} \ldots \ldots \ldots \ldots \ldots \\
\mathrm{CO}_{2} \ldots \ldots \ldots \ldots \ldots \ldots \\
\mathrm{H}_{2} \mathrm{O} \ldots \ldots \ldots \ldots \ldots\end{array}$ & $\begin{array}{r}.19 \\
.19 \\
52.24 \\
1.53 \\
41.46 \\
3.27\end{array}$ & $\begin{array}{r}.22 \\
.47 \\
51.24 \\
2.09 \\
41.07 \\
3.57\end{array}$ & $\begin{array}{r}.32 \\
.56 \\
49.38 \\
1.93 \\
40.39 \\
5.12\end{array}$ & $\begin{array}{r}.21 \\
.76 \\
51.95 \\
1.44 \\
41.53 \\
3.07\end{array}$ & $\begin{array}{r}.25 \\
.56 \\
51.52 \\
2.08 \\
41.58 \\
3.19\end{array}$ & $\begin{array}{r}2.94 \\
.23 \\
51.51 \\
.71 \\
41.59 \\
2.64\end{array}$ & $\begin{array}{r}8.50 \\
.73 \\
47.29 \\
1.51 \\
39.00 \\
3.37\end{array}$ & $\begin{array}{r}2.99 \\
.65 \\
51.22 \\
.06 \\
41.22 \\
2.23\end{array}$ & $\begin{array}{r}5.87 \\
.95 \\
50.34 \\
.37 \\
39.62 \\
3.21\end{array}$ & $\begin{array}{r}17.83 \\
1.18 \\
43.85 \\
.26 \\
34.31 \\
2.53\end{array}$ \\
\hline . & 98.88 & - 98.66 & 97.70 & 98.96 & 99.18 & 99.62 & 100.40 & 98. $37^{\circ}$ & 100.36 & 99.96 \\
\hline
\end{tabular}

K. Coarse shell mass, Senote.

L. Coral rock, Salt Key Bank.

M. Loggerhead Key.

Analyses by L. G. Eakins, record No. 882. . The following analyses, also by Eakins, No. 883 , are included for comparison:

N. Coralline bottom, Barbados.

O. Recent coral (Siderostria), Bermuda.

\begin{tabular}{|c|c|c|c|c|c|}
\hline & K. & I. & M. & N. & o. \\
\hline 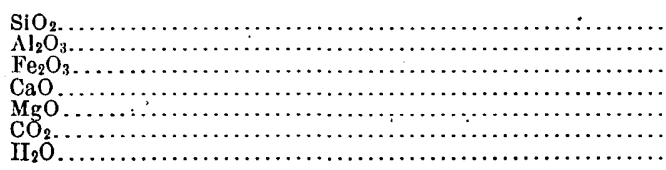 & $\begin{array}{r}.22 \\
.18 \\
54.87 \\
.64 \\
43.89 \\
.11\end{array}$ & $\begin{array}{r}.11 \\
.04 \\
53.54 \\
.71 \\
43.87 \\
1.13\end{array}$ & $\begin{array}{r}.20 \\
.22 \\
53.54 \\
.78 \\
43.71 \\
.81\end{array}$ & $\begin{array}{r}1.17 \\
.31 \\
46.45 \\
5.15 \\
43.40 \\
2.73\end{array}$ & $\begin{array}{r}.23 \\
\text { trace. } \\
55.16 \\
.20 \\
43.74 \\
.54\end{array}$ \\
\hline & 99.91 & 99.40 & 99.26 & 99.21 & 99.87 \\
\hline
\end{tabular}

2. MISCELLANEOUS ROCKS.

A to M, inclusive. Thirteen borings from the artesian well at Key West. Partial analyses by Steiger, record No. 1553. The figures at tops of columns give depths in feet from which samples were taken. For a description of the limestones see E. 0 . Hovey, Bull. Mus. Comp. Zool., vol. 28, p. 63.

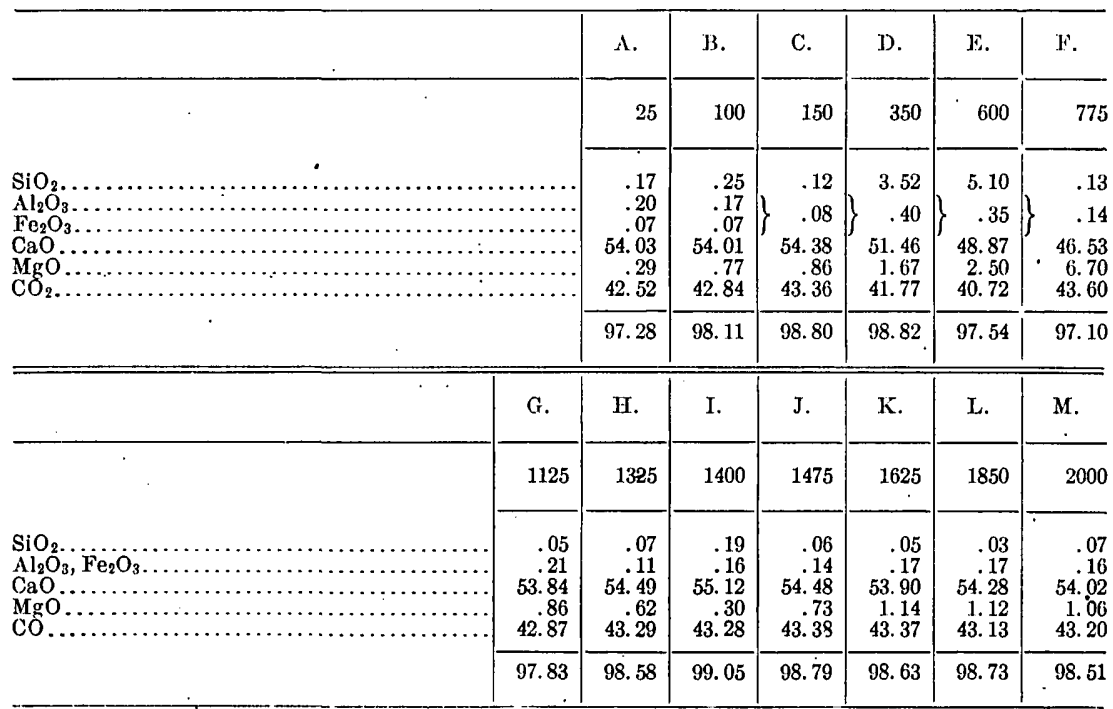

$\mathrm{P}_{2} \mathrm{O}_{5}$ is present. Is included with $\mathrm{Al}_{2} \mathrm{O}_{2}$ and $\mathrm{Fe}_{2} \mathrm{O}_{2}$. 
N. Supposed cement rock, River Junction. Received from D. T. Day. Analysis by George Steiger, record No. 1844.

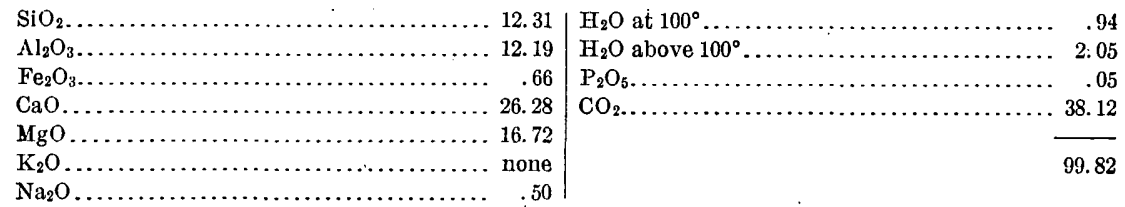

\section{KENTUCKY.}

A. Lithographic stone, 1 mile from Brandenburg, Meade County, on Ohio River.

B. Typical lithographic stone from Solenhofen, Bavaria. Analyzed for comparison with A. Analyses by George Steiger, record No. 1894.

\begin{tabular}{|c|c|c|}
\hline . & A. & B. \\
\hline \multirow[t]{2}{*}{$\left.\begin{array}{l}\mathrm{SiO}_{2} \ldots \ldots . \\
\mathrm{Al}_{2} \mathrm{O}_{3}, \mathrm{Fe}_{2} \mathrm{O}_{3} \\
\mathrm{CaO} \ldots \ldots\end{array}\right\}$ Residue insoluble in $\mathrm{HCl} .\{$} & $\begin{array}{r}3.15 \\
.45 \\
.09\end{array}$ & $\begin{array}{r}1.15 \\
.22 \\
\text { trace }\end{array}$ \\
\hline & 3. 69 & 1. 37 \\
\hline \multirow[t]{2}{*}{ 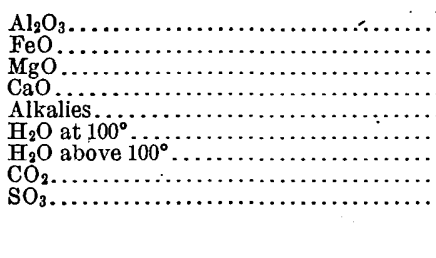 } & $\begin{array}{r}.13 \\
.31 \\
6.75 \\
44.76 \\
.13 \\
.41 \\
.47 \\
43.06 \\
\text { none }\end{array}$ & $\begin{array}{r}.23 \\
.26 \\
.56 \\
53.80 \\
.07 \\
.23 \\
.69 \\
42.69 \\
\text { none }\end{array}$ \\
\hline & 99.71 & 99.90 \\
\hline
\end{tabular}

\section{TENNESSEE, ALABAMA, LOUISIANA.}

A. Limestone, Knoxville, Tennessee. Analysis by L. G. Eakins, record No. 1159.

B. Knox dolomite, Morrisville, Alabama. Described by Russell in Bull. 52, together with a residual clay derived from it. See section on clays. Analysis by W. F. Hillebrand, record No. 797.

C. Limestone from Rayborn's salt lick, Bienville Parish, Louisiana. Analysis by R. B. Riggs, record No. 323 .

D. White marble, streaked with black. From 5 miles west of Winnfield, Louisiana. Analysis by W. F. Hillebrand, record No. 760. In addition to the constituents named in the table, this marble contains traces of barium, strontium, chlorine, and organic matter.

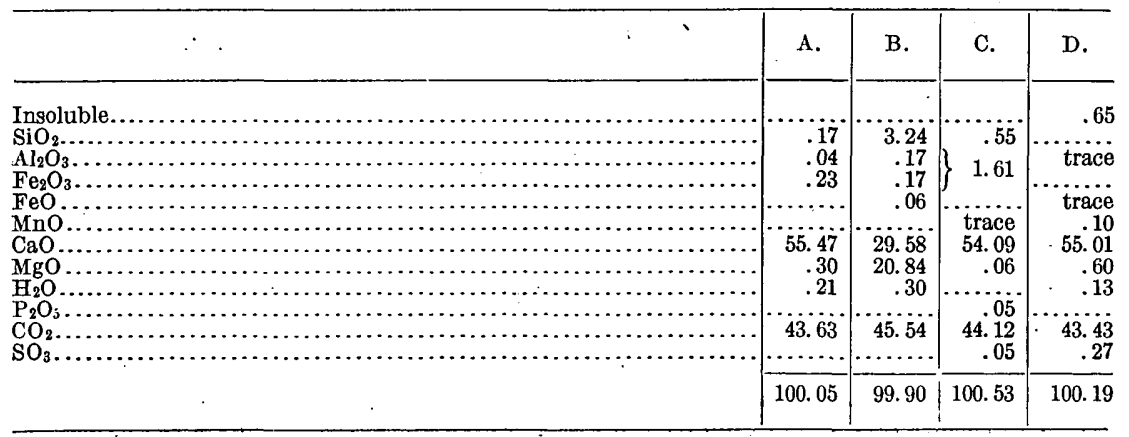




\section{MISSISSIPPI.}

Limestones received from E. C. Eckel. Partial analyses by H. C. McNeil, record No. 2181. $\mathrm{CO}_{2}$ calculated to satisfy bases.
A. From Macon.
B. From Scooba.
C. From Border Creek.
D. From Wahulak Creek.
E. From Prairie Rock.
F. Selma chalk, from north of Macon.
G. From Lime Creek.

\begin{tabular}{|c|c|c|c|c|c|c|c|}
\hline & A. & B. & C. & D. & E. & F. & G. \\
\hline 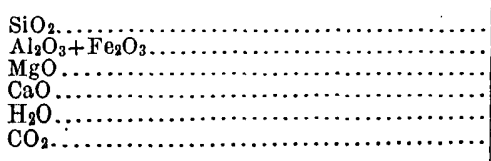 & $\begin{array}{r}9.09 \\
7.47 \\
\text { none } \\
45.38 \\
1.08 \\
35.61\end{array}$ & $\begin{array}{r}16.48 \\
6.97 \\
.33 \\
41.64 \\
.67 \\
33.04\end{array}$ & $\begin{array}{r}10.60 \\
5.90 \\
\text { trace } \\
46.21 \\
.82 \\
36.26\end{array}$ & $\begin{array}{r}20.00 \\
8.92 \\
\text { trace } \\
38.61 \\
1.03 \\
30.30\end{array}$ & $\begin{array}{r}1.13 \\
.68 \\
\text { trace } \\
54.55 \\
42.80 \\
42.81\end{array}$ & $\begin{array}{r}8.52 \\
6.60 \\
\text { none } \\
47.00 \\
1.00 \\
36.88\end{array}$ & $\begin{array}{r}8.06 \\
5.94 \\
\text { none } \\
47.41 \\
1.32 \\
37.20\end{array}$ \\
\hline Insoluble... & $\begin{array}{l}98.63 \\
14.59\end{array}$ & $\begin{array}{l}99.13 \\
20.96\end{array}$ & $\begin{array}{l}99.79 \\
14.21\end{array}$ & $\begin{array}{l}98.86 \\
25.79\end{array}$ & $\begin{array}{r}99.57 \\
1.36\end{array}$ & $\begin{array}{r}100.00 \\
10.00\end{array}$ & $\begin{array}{l}99.93 \\
10.28\end{array}$ \\
\hline
\end{tabular}

TEXAS.

Supposed cement rock, Uvalde quadrangle. Collected by T. Wayland Vaughan. Analysis by W. F. Hillebrand, record No. 1759.

$A$, the rock; $B$, the part insoluble in dilute nitric acid.

\begin{tabular}{|c|c|c|c|}
\hline & A. & & B. \\
\hline \multirow[t]{2}{*}{$\begin{array}{l}\mathrm{SiO}_{2} \ldots \ldots \ldots \\
\mathrm{Fe}_{2} \mathrm{O}_{3}, \mathrm{Al}_{2} \mathrm{O}_{3} \\
\mathrm{CaO} \\
\mathrm{MgO} \\
\mathrm{MgO}\end{array}$} & $\begin{array}{r}.10 \\
.09 \\
40.82 \\
.18 \\
32.41 \\
25.57 \\
.83\end{array}$ & \multirow[t]{2}{*}{ 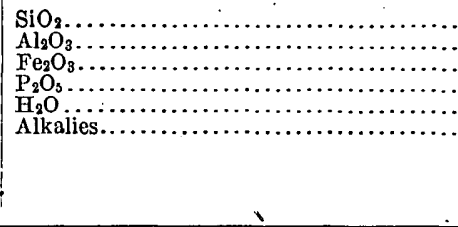 } & $\begin{array}{r}20.80 \\
3.44\end{array}$ \\
\hline & 100.00 & & \\
\hline
\end{tabular}

a By difference.

\section{OHIO.}

A, B, C, D. 'Trenton limestone from New Vienna.

E. Trenton limestone, Arcadia, Hancock County.

F. Air-line Junction, Toledo. This sample contains a great deal of ferrous carbonate.

G. Gas rock, St. Henry's well, Mercer County.

H. Oil rock, Lima.

All Trenton. Partial analyses by F. W. Clarke and R. B. Riggs, record Nos. 729, 730,732 . Iron and alumina are present in soluble form.

\begin{tabular}{|c|c|c|c|c|c|c|c|c|}
\hline & A. & B. & c. & D. & E. & F. & G. & н. \\
\hline \multirow[t]{2}{*}{ 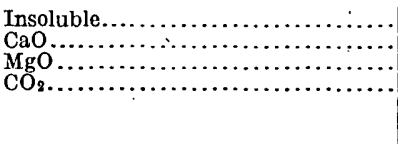 } & $\begin{array}{r}8.47 \\
47.16 \\
1.52 \\
36.20\end{array}$ & $\begin{array}{r}9.93 \\
49.04 \\
37.58 \\
37.64\end{array}$ & $\begin{array}{r}2.12 \\
51.18 \\
3.08 \\
42.04\end{array}$ & $\begin{array}{l}28.43 \\
23.00 \\
12.90 \\
30.82\end{array}$ & $\begin{array}{r}8.56 \\
47.17 \\
2.59 \\
38.54\end{array}$ & $\begin{array}{r}3.52 \\
30.64 \\
18.05 \\
42.82\end{array}$ & $\begin{array}{r}2.27 \\
50.34 \\
2.86 \\
40.96\end{array}$ & $\begin{array}{r}1.64 \\
32.24 \\
17.36 \\
43.92\end{array}$ \\
\hline & 92.35 & 97.19 & 98.42 & 95.15 & 96.86 & 95.03 & 96. 43 & 95.16 \\
\hline
\end{tabular}


The following partial analyses by F. W. Clarke, record No. 738, all relate to Trenton limestones:

A. Well No. 3, Bryan. Gas rock.

B. McElree well, Kenton. Depth, 1,315 feet.

C. Huntsville. Depth, 1,405 feet.

D. Prospect. Depth, 1,650 feet.

E. Findlay street well, Dayton. Depth, 975 feet.

F. Xenia. Depth, 1,075 feet.

G. New Madison. Depth, 1,150 feet.

\begin{tabular}{|c|c|c|c|c|c|c|c|}
\hline & A. & B. & C. & D. & E. & F. & G. \\
\hline \multirow[t]{2}{*}{ 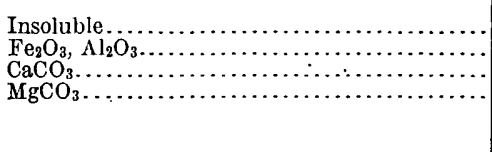 } & $\begin{array}{r}9.22 \\
1.51 \\
49.00 \\
38.59\end{array}$ & $\begin{array}{r}5.26 \\
1.10 \\
84.32 \\
8.43\end{array}$ & $\begin{array}{r}4.41 \\
3.15 \\
57.23 \\
33.16\end{array}$ & $\begin{array}{r}26.12 \\
2.57 \\
66.02 \\
3.77\end{array}$ & $\begin{array}{r}12.34 \\
.58 \\
82.36 \\
1.67\end{array}$ & $\begin{array}{r}9.23 \\
.18 \\
86.54 \\
2.99\end{array}$ & $\begin{array}{r}11.11 \\
3.60 \\
64.91 \\
17.98\end{array}$ \\
\hline & 98.32 & 99.11 & 98.95 & 98.58 & 96.95 & 98.94 & 97.60 \\
\hline
\end{tabular}

In $\mathrm{C}$ and $\mathrm{G}$ there is ferrous carbonate.

Partial analyses by Charles Catlett, record Nos. 754, 755, 756, 757; all of Trenton limestones from the natural-gas belt.

A. London. Depth, 1,594 feet.

B. Air-line Junction, Toledo. Depth, 1,415 feet.

C. Celina. Depth, 1,112 feet.

D. City well No. 2, Upper Sandusky.

E. Sandusky. Depth, 2,260 feet.

F. Gas rock. Pauck well, St. Marys Township, Auglaize County.

G. Gas rock. Bennett well, St. Marys Township. Depth, 1,121 feet.

H. First city well, Carey. Depth, about 1,350 feet.

I. Well No. 2, Fort Recovery. Depth, 1,065 feet.

J. Waggoner well, 6 miles west of Fremont. Gas rock.

K. Loomis and Nyman well, Tiffin. Depth, 1,470 to 1,481 feet.

L. Loomis and Nyman well, Tiffin. Depth, 1,488 to 1,494 feet.

M. Port Clinton. Depth, 1,660 to 1,700 feet.

N. Wauseon. Depth, 2,135 feet.

0 . Napoleon. Depth, 1,830 feet.

P. Kọssuth, Allen County. Oil rock.

Q. Doenze's well, Franklin Township, Mercer County. Depth, 1,107 feet.

\begin{tabular}{|c|c|c|c|c|c|c|c|c|c|}
\hline & A. & B. & C. & D. & E. & F. & G. & H. & I. \\
\hline \multirow[t]{2}{*}{ 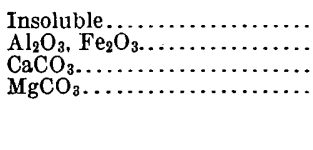 } & $\begin{array}{r}15.90 \\
1.84 \\
77.69 \\
1.89\end{array}$ & $\begin{array}{r}2.88 \\
8.68 \\
54.68 \\
25.73\end{array}$ & $\begin{array}{r}2.95 \\
2.95 \\
68.41 \\
24.18\end{array}$ & $\begin{array}{r}8.18 \\
4.31 \\
64.25 \\
15.93\end{array}$ & $\begin{array}{r}3.65 \\
4.58 \\
54.62 \\
33.67\end{array}$ & $\begin{array}{r}3.18 \\
3.12 \\
52.18 \\
38.42\end{array}$ & $\begin{array}{r}1.66 \\
2.48 \\
56.94 \\
35.55\end{array}$ & $\begin{array}{r}5.72 \\
3.08 \\
80.11 \\
8.09\end{array}$ & $\begin{array}{r}1.89 \\
1.57 \\
87.88 \\
7.43\end{array}$ \\
\hline & 97.32 & 91.97. & 98.49 & 92.67 & 96.52 & 96.90 & 96.63 & 97.00 & 98.77 \\
\hline & & J. & $\mathrm{K}$. & L. & M. & N. & 0. & P. & Q. \\
\hline \multirow{2}{*}{\multicolumn{2}{|c|}{ 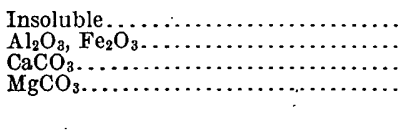 }} & $\begin{array}{r}5.22 \\
6.32 \\
52.93 \\
32.75\end{array}$ & $\begin{array}{r}5.66 \\
4.86 \\
52.89 \\
33.46\end{array}$ & $\begin{array}{r}9.88 \\
1.46 \\
79.39 \\
6.20\end{array}$ & $\begin{array}{r}7.46 \\
4.16 \\
71.96 \\
\cdot 14.34\end{array}$ & $\begin{array}{r}18.24 \\
7.28 \\
42.82 \\
.28 .11\end{array}$ & $\begin{array}{r}2.66 \\
2.14 \\
53.85 \\
37.33\end{array}$ & $\begin{array}{r}1.08 \\
.66 \\
90.72 \\
6.69\end{array}$ & $\begin{array}{r}3.68 \\
8.38 \\
69.53 \\
10.98\end{array}$ \\
\hline & & 97.22 & 96.87 & 96.93 & 97.92 & 96.45 & 95.98 & 99.15 & 92.27 \\
\hline
\end{tabular}


INDIANA.

A. Buff limestone, Hoosier Stone Company, Bedford.

B. Blue limestone, same locality.

Analyses by F. W. Clarke, record Nos. 306, 307.

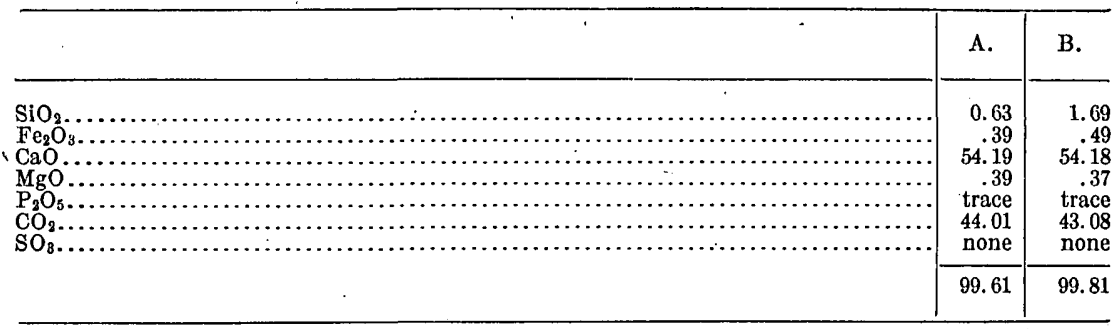

The following Trenton limestones are all from the natural-gas belt:

C. Union City. Depth, 1,160 feet.

D. Bluffton. Depth, 1,062 to 1,067 feet.

E. Muncie. Depth, 920 feet.

F. Greensburg. Depth, 867 feet.

G. Vernon. Depth, 905 feet.

H. Wabash. Depth, about 900 feet.

Analysis C by F. W. Clarke, record No. 738. The remainder by Charles Catlett, Nos. $753,758$.

\begin{tabular}{|c|c|c|c|c|c|c|}
\hline$\cdot$ & C. & D., & E. & F. & G. & H. \\
\hline \multirow[t]{2}{*}{ 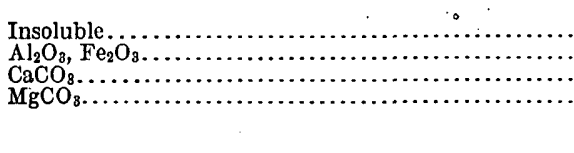 } & $\begin{array}{r}2.14 \\
1.23 \\
83.21 \\
12.48\end{array}$ & $\begin{array}{r}2.37 \\
4.48 \\
53.43 \\
37.47\end{array}$ & $\begin{array}{r}3.30 \\
3.72 \\
51.96 \\
38.11\end{array}$ & $\begin{array}{r}0.87 \\
.55 \\
94.60 \\
.36\end{array}$ & $\begin{array}{r}8.00 \\
.60 \\
85.56 \\
\text { trace }\end{array}$ & $\begin{array}{r}3.52 \\
7.58 \\
53.18 \\
30.53\end{array}$ \\
\hline & 99.06 & 97.75 & 97.09 & 96.38 & 94.16 & 94.81 \\
\hline
\end{tabular}

MISSOURI.

Limestones and dolomites collected by W. P. Jenney. Analyses by L. G. Eakins, record Nos. 1184, 1207. $\mathrm{CO}_{2}$ calculated.

A, B. Cherokee limestone, quarry.near Seneca, Newton County.

C, D. The same, near Grand Falls, Newton County.

E, F. Dolomite, Oswego land, Joplin.

\begin{tabular}{|c|c|c|c|c|c|c|}
\hline & A. & B. & C. & D. & E. & F. \\
\hline \multirow[t]{2}{*}{ 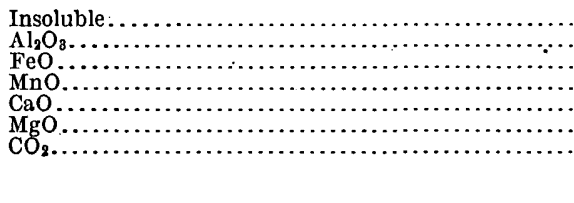 } & $\begin{array}{r}.06 \\
.11 \\
.08 \\
\text { trace } \\
55.29 \\
.23 \\
43.69\end{array}$ & $\begin{array}{r}1.21 \\
.13 \\
.07 \\
\text { trace } \\
54.92 \\
.20 \\
43.31\end{array}$ & $\begin{array}{r}1.01 \\
.08 \\
.05 \\
.03 \\
54.98 \\
.31 \\
43.54\end{array}$ & $\begin{array}{r}1.01 \\
.13 \\
\text { trace } \\
\text { trace } \\
55.11 \\
.32 \\
43.65\end{array}$ & $\left\{\begin{array}{r}29.77 \\
1.32 \\
\cdots \\
21.46 \\
14.79 \\
33.13\end{array}\right.$ & $\begin{array}{r}11.66 \\
1.03 \\
\\
28.72 \\
17.26 \\
41.55\end{array}$ \\
\hline & 100.03 & 99.84 & 100.00 & 100.22 & 100.47 & 100.22 \\
\hline
\end{tabular}

\section{KANSAS.}

A. Limestone, Silverdale. Analysis by Charles Catlett, record No. 967.

B. Cherokee limestone, Short Creek, near Spring River, Cherokee County. Analysis by L. G. Eakins, record No. 1184.

15619-Bull. 419-10 -14 
C. Supposed marl, large surface deposit near Wakeeney, Trego County. Analysis by F. W. Clarke, record No. 212.

D. Limestone, SE. $\frac{1}{4}$ sec. 13, T. 285, R. 12 S. E. Analysis by G. Steiger, record No. 2295.

\begin{tabular}{|c|c|c|c|c|}
\hline . & A. & B. & C. & D. \\
\hline 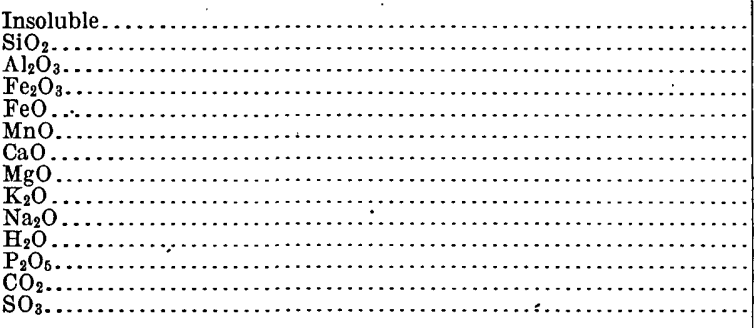 & $\begin{array}{r}5.27 \\
1.07 \\
.71 \\
.32 \\
50.36 \\
.56 \\
.10 \\
.20 \\
.78 \\
.06 \\
40.34 \\
.07\end{array}$ & 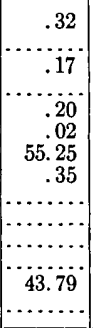 & 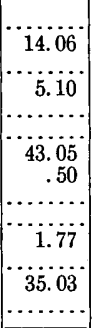 & $\begin{array}{r}2.56 \\
1.55 \\
.50 \\
.47 \\
\ldots . .06 \\
51.98 \\
.06 \\
\ldots \ldots \\
\ldots 1.36 \\
\ldots \\
41.13\end{array}$ \\
\hline$\cdot$ & 99.84 & 100.10 & 99.51 & 99.61 \\
\hline
\end{tabular}

\section{MICHIGAN, WISCONSIN, MINNESOTA, CANADA.}

Most of the rocks considered under this heading were described by Irving and Van Hise in Mon. XIX, pages 131 and 191. A few other analyses, also representing Van Hise's collections, are taken from the laboratory records. The Canadian rocks are from near the boundary line, and relate directly to others gathered upon the Minnesota side.

A. Dolomite, near Sunday Lake, Gogebic district, Michigan. Analysis by W. F. Hillebrand, record No. 767.

B. Dolomite, Penokee region, Wisconsin, NW. $\frac{1}{4} \sec .22$, T. 44 N., R. 5 W. Analysis by Hillebrand, record No. 768 .

C. Limestone, bed of Slate Creek, Huron Bay slate quarries, Michigan. Analysis by T. M. Chatard, record No. 894 . From laboratory records; not in the monograph cited.

D. Limestone, east end of Ogiskemannissi Lake, Minnesota. Analysis by Chatard, record No. 899. Not in monograph.

\begin{tabular}{|c|c|c|c|c|}
\hline & A. & B. & c. & D. \\
\hline $\mathrm{SiO}_{2} \ldots \ldots \ldots$ & 3.07 & .63 & 7.05 & 41. 99 \\
\hline 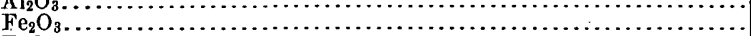 & 09 & & $\begin{array}{r}.48 \\
1.33\end{array}$ & \\
\hline & .86 & .75 & undet. & 4.77 \\
\hline $\begin{array}{l}\mathrm{MnO} . \\
\mathrm{CaO} .\end{array}$ & $\begin{array}{r}.15 \\
29.72\end{array}$ & $\begin{array}{l}.08 \\
30.94\end{array}$ & $\begin{array}{r}.19 \\
50.08\end{array}$ & $\begin{array}{r}.26 \\
16.85\end{array}$ \\
\hline $\mathrm{MgO}$ & 19.95 & 20.68 & .57 & \\
\hline $\begin{array}{l}\mathrm{H}_{2} \mathrm{O} \text { at } 105^{\circ} . \\
\mathrm{H}_{2} \mathrm{O} \text { ign } \ldots . . .\end{array}$ & .30 & .27 & .25 & 1. 02 \\
\hline $\begin{array}{l}\mathrm{P}_{2} \mathrm{O}_{5} \ldots \ldots \ldots \ldots \\
\mathrm{CO}_{2} \ldots \ldots \ldots \ldots\end{array}$ & 45.31 & 46.27 & $\begin{array}{l}.27 \\
39.68\end{array}$ & 24.70 \\
\hline \multirow{3}{*}{ Cla } & trace & trace & .21 & \\
\hline & & & & \\
\hline & 99.45 & 99.65 & 100.11 & 100.08 \\
\hline
\end{tabular}

E. Iron carbonate, SE. $\frac{1}{4}$ sec. 20, T. 47 N., R. 43 W., Michigan.

F. Iron carbonate, south side of Sunday Lake, Michigan.

G. Iron carbonate, Palms mine, Gogebic district, Michigan.

Analyses E, F, and G by W. F. Hillebrand, record Nos. 769, 770, 771.

H. Iron carbonate, Miner \& Wells option, sec. 13, T. 47 N., R. 46 W., Michigan. Analysis by T. M. Chatard, record No. 893. 
I. Iron carbonate, NW. $\frac{1}{4}$ sec. 18, T. 47 N., R. 45 W., Michigan. Analysis by Chatard, No. 895.

\begin{tabular}{|c|c|c|c|c|c|}
\hline & E. & F. & G. & H. & I. \\
\hline 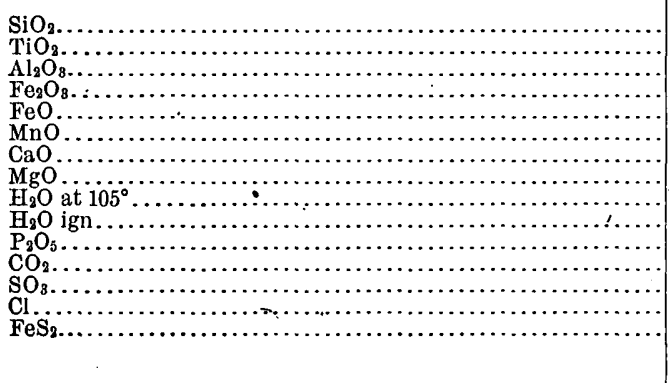 & $\begin{array}{r}3.16 \\
.08 \\
.93 \\
15.18 \\
1.15 \\
26.65 \\
11.01 \\
.54 \\
.06 \\
41.10 \\
\ldots \ldots \\
\text { trace } \\
.34 \\
100.20\end{array}$ & $\begin{array}{r}28.86 \\
.20 \\
1.29 \\
1.01 \\
37.37 \\
.97 \\
.74 \\
3.64 \\
.68 \\
\text { trace } \\
25.21 \\
\cdots \cdots \\
\cdots \cdots \\
\cdots\end{array}$ & $\begin{array}{r}46.47 \\
.10 \\
.70 \\
.86 \\
28.57 \\
.40 \\
.49 \\
2.30 \\
.60 \\
\text { trace } \\
19.24 \\
(?) \\
\ldots \ldots \\
\end{array}$ & 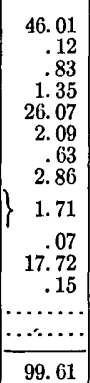 & $\begin{array}{r}36.73 \\
.19 \\
.38 \\
.98 \\
34.81 \\
.52 \\
.48 \\
2.74 \\
.12 \\
1.40 \\
.01 \\
22.44 \\
.16 \\
\ldots \ldots . . \\
\cdots \ldots . . . \\
100.96\end{array}$ \\
\hline
\end{tabular}

J. Iron carbonate, Penokee iron range, NE. $\frac{1}{4}$ sec. 6, T. 45, R. 2 E., Wisconsin. P. R. C. 999.

K, L. Black, slaty, carbonaceous iron carbonates, Animikie formation, Kakabikka Falls, Kaministiquia River, Canada.

M. Iron carbonate, west end of Gunflint Lake, Minnesota.

N. Iron carbonate, north side of Gunflint Lake, Minnesota.

O. Iron carbonate, north side of Gunflint Lake, Canada.

Analyses, J, K, and L by R. B. Riggs, record Nos. 376, 377, 378; M, N, and $\mathrm{O}$ by T. M. Chatard, record Nos. $897,898,900$. Rocks M, N, and $\mathrm{O}$ are described by Clements in Mon. XLV, p. 380.

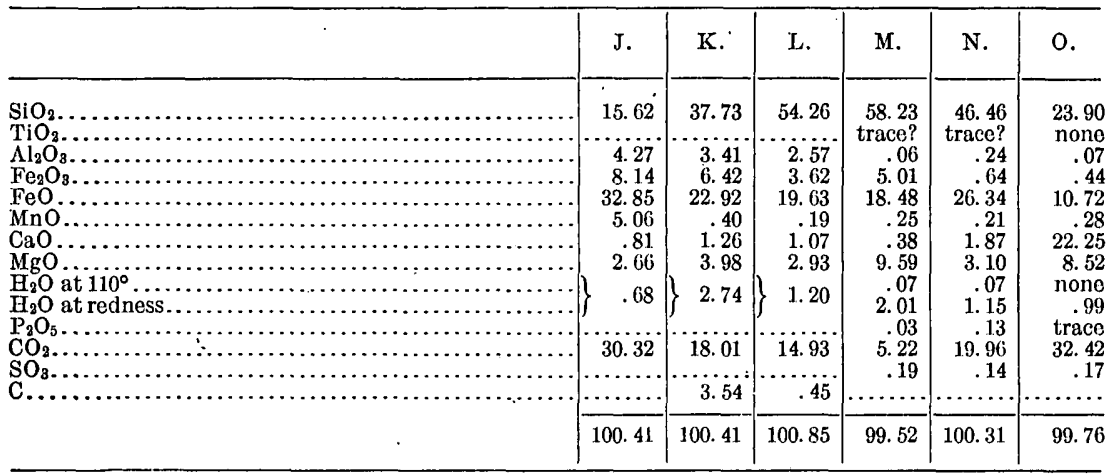

P. Ferrodolomite, Marquette district, Michigan. Analysis by George Steiger, record No. 1473. P. R. C. 994.

Q. Ferrodolomite, Marquette district, Michigan.

$R$. Portion of $\mathrm{Q}$ insoluble in hydrochloric acid.

$\mathrm{S}$. Soluble portion of $\mathrm{Q}$.

Analysis Q, R, and S by George Steiger, record No. 1442. These analyses are not in the monograph cited. 
T. Ferruginous dolomite, Hamburg Hill, Menominee district, Michigan. Described by Bayley in Mon. XLVI, p. 480. Analysis by George Steiger, record No. 2033.

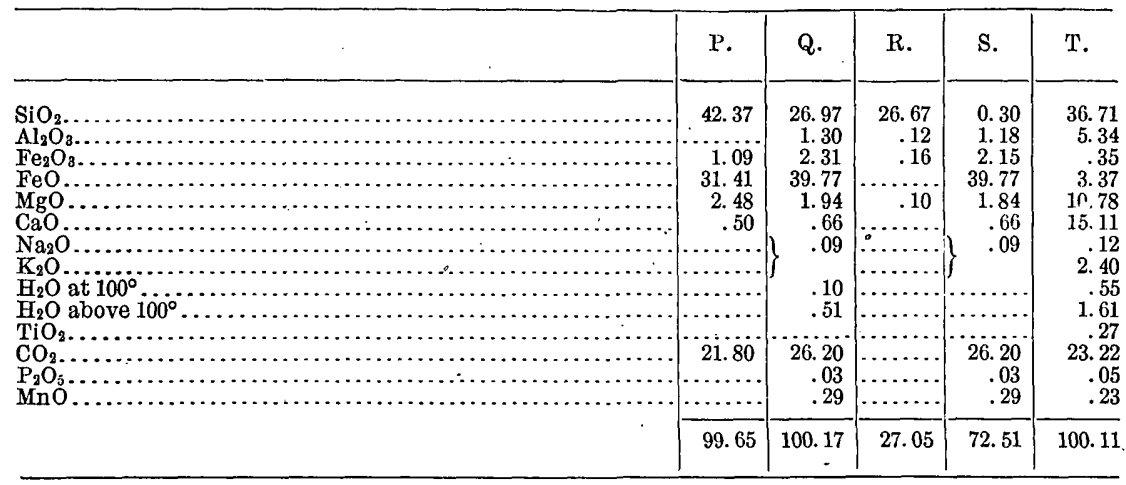

The three following analyses, by E. C. Sullivan, record No. 2160, are of material collected in Michigan by I. C. Russell.

U. Marl, Ore Lake.

V. Dolomite, Maybee, tenth layer from top.

W. Like V, fifth layer from top.

\begin{tabular}{|c|c|c|c|}
\hline & U. & V. & W. \\
\hline 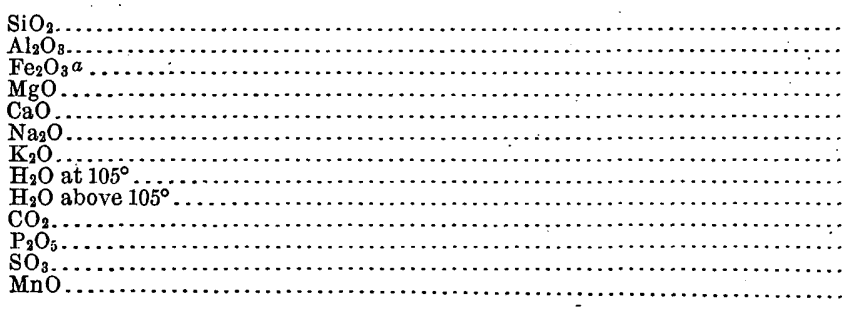 & $\begin{array}{r}0.53 \\
.14 \\
.96 \\
1.10 \\
51.87 \\
.10 \\
.10 \\
.97 \\
1.22 \\
42.30 \\
.06 \\
.14 \\
\text { trace }\end{array}$ & $\begin{array}{r}1.77 \\
.01 \\
.41 \\
20.84 \\
29.65 \\
\cdots \cdots \\
.12 \\
4.48 \\
4.40 \\
\text { trace } \\
.33 \\
\text { trace }\end{array}$ & $\begin{array}{r}1.30 \\
.16 \\
.20 \\
19.79 \\
31.14 \\
\cdots . . . \\
.18 \\
.57 \\
45.18 \\
\text { trace } \\
1.15 \\
\text { trace }\end{array}$ \\
\hline & 99.49 & 100.01 & 99.67 \\
\hline
\end{tabular}

$a$ Total iron.

\section{SOUTH DAKOTA AND WYOMING.}

A. Purple limestone, east of Cascade, Black Hills, South Dakota. Collected by G. B. Richardson. Analysis by George Steiger, record No. 1854 .

B. Dolomite from the Tornado mine, Black Hills. Received from S. F. Emmons. Analysis by W. F. Hillebrand, record No. 1866. 
C. Limestone, Hunt Mountain, Bighorn district, Wyoming. Received from N. H. Darton. Partial analysis by Hillebrand, No. 2250. $\mathrm{CO}_{2}$ calculated to satisfy bases.

\begin{tabular}{|c|c|c|c|}
\hline$\cdot$ & A. & B. & C. \\
\hline 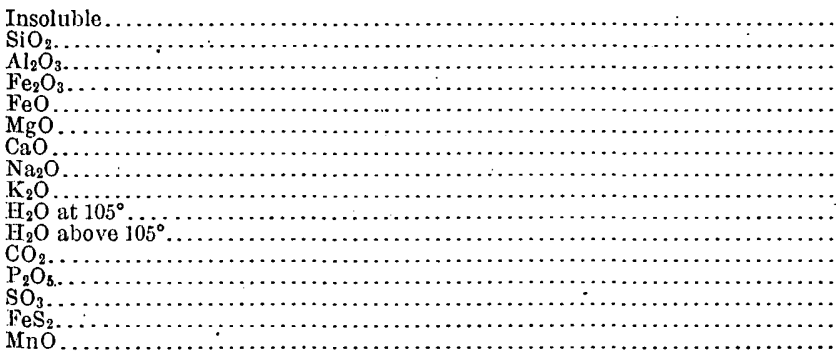 & $\begin{array}{r}1.12 \\
a .36 \\
19.85 \\
31.51 \\
\text { none } \\
\text { none } \\
.07 \\
1.18 \\
45.66 \\
.07 \\
\text { none }\end{array}$ & $\begin{array}{r}5.74 \\
2.02 \\
.64 \\
6.47 \\
14.75 \\
26.81 \\
.07 \\
.53 \\
.34 \\
.67 \\
40.11 \\
.08 \\
.08 \\
.69 \\
.97\end{array}$ & 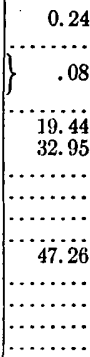 \\
\hline & 99.82 & 99.89 & 99.97 \\
\hline
\end{tabular}

$a$ Includes possible $\mathrm{TiO}_{2}, \mathrm{P}_{2} \mathrm{O}_{5}$, and $\mathrm{Fe}$.

\section{YELLOWSTONE NATIONAL PARK.}

Travertines, tufas, and calcareous sinters. Analyses A to $\mathrm{E}$ reported by W. $\mathrm{H}$. Weed in 9th Ann., p. 619. Travertines.

A. Terrace below the hotel.

B. Cupids Cave.

C. Near Sulphur Spring No. 246, Mammoth Hot Springs.

D. Extinct spring, main terrace, Mammoth Hot Springs.

E. Ridge behind main terrace.

Analysis C by F. A. Gooch, record No. 243; A, B, D, and E by J. E. Whitfield, record Nos. 240, 242, 244, 245.

\begin{tabular}{|c|c|c|c|c|c|}
\hline & A. & B. & C. & D. & E. \\
\hline \multirow{5}{*}{ 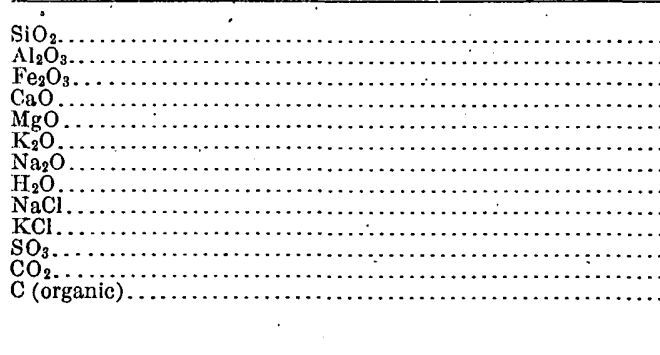 } & $\begin{array}{r}0.08 \\
.15\end{array}$ & $\begin{array}{r}0.15 \\
.49\end{array}$ & $\begin{array}{r}0.01 \\
.05\end{array}$ & $\begin{array}{r}0.06 \\
.14 \\
\quad \ldots \ldots\end{array}$ & $\begin{array}{r}0.26 \\
.11 \\
\ldots .\end{array}$ \\
\hline & $\begin{array}{r}53.83 \\
.90 \\
\ldots \ldots \ldots\end{array}$ & $\begin{array}{r}53.41 \\
.42 \\
.01 \\
.03\end{array}$ & $\begin{array}{r}55.02 \\
.07 \\
.04\end{array}$ & $\begin{array}{r}55.02 \\
.06 \\
\ldots \ldots \ldots\end{array}$ & $\begin{array}{r}54.06 \\
.66 \\
\ldots \ldots .\end{array}$ \\
\hline & $\begin{array}{r}1.43 \\
.02\end{array}$ & $\begin{array}{r}2.44 \\
.13\end{array}$ & $\begin{array}{r}1.61 \\
.12\end{array}$ & $\begin{array}{r}1.06 \\
.20 \\
.08\end{array}$ & $\begin{array}{r}7.19 \\
.26\end{array}$ \\
\hline & $\begin{array}{r}1.72 \\
41.79 \\
.21\end{array}$ & $\begin{array}{r}.55 \\
41.96 \\
.37\end{array}$ & $\begin{array}{r}.49 \\
42.25 \\
.11\end{array}$ & $\begin{array}{r}.70 \\
42.25 \\
.24\end{array}$ & $\begin{array}{l}1.34 \\
42.14 \\
\text { none }\end{array}$ \\
\hline & 100.13 & 99.66 & 99.77 & 99.81 & 100.02 \\
\hline
\end{tabular}

Analyses F, I, J, by F. A. Gooch, record Nos. 93, 246, 257; G, F, K, by J. E. Whitfield, record Nos. 239, 241, 862.

F. Calcareous tufa, Mammoth Hot Springs. 
G. Travertine, from cavity near Pulsating Geyser, Mammoth Hot Springs.

H. Travertine, from bank of Hot River.

I. Travertine, from edge of old cave, highest terrace, Mammoth Hot Springs.

J. Travertine, capping Terrace Mountain, Mammoth Hot.Springs.

K: Calcareous sinter, Hot Lakes. Sp. gr., 2,857.

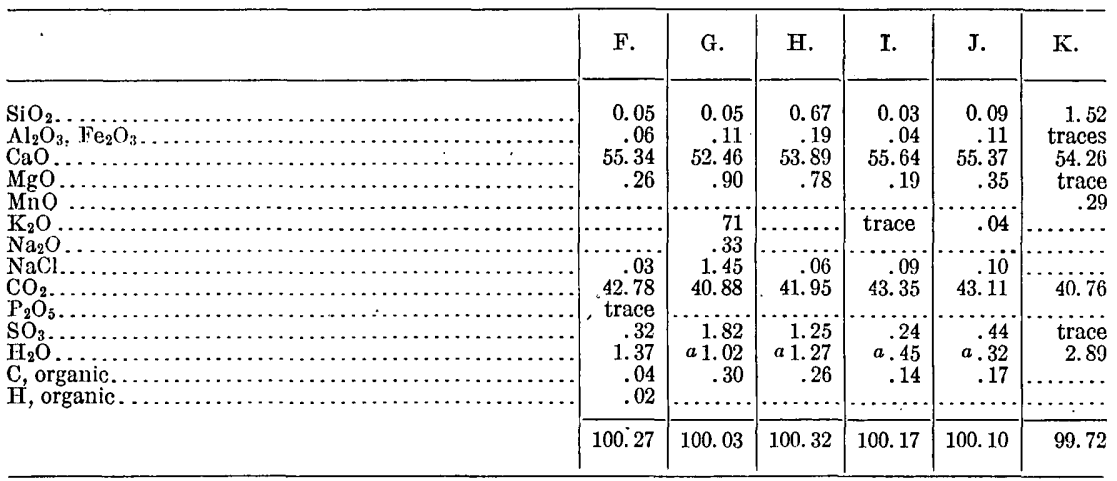

$a$ Includes $\mathrm{H}$ of organic matter.

\section{MONTANA.}

Rocks A to H collected by A. C. Peale. Analyses by Charles Catlett, record Nos: 890,905 .

A. North of East Gallatin River.

B. West of North Boulder River.

C, D. North of East Gallatin River.

E. Base of Carboniferous, west side of Bridger Range.

F. Middle Carboniferous, north of Gallatin River.

G, H. Upper Carboniferous, north of Gallatin River.

Analyses all partial. A, B, C, D, and $\mathrm{H}$ were published by Peale in Bull. 110, pp. 16,28 , and 40 .

\begin{tabular}{|c|c|c|c|c|c|c|c|c|}
\hline & A. & B. & C. & D. & E. & F. & G. & $\mathrm{H}$. \\
\hline 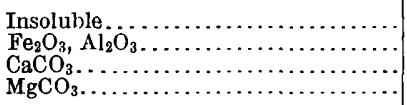 & $\begin{array}{r}.34 \\
.22 \\
54.54 \\
43.63\end{array}$ & $\begin{array}{r}1.78 \\
.40 \\
54.54 \\
42.62\end{array}$ & $\begin{array}{r}23.50 \\
2.50 \\
67.85 \\
6.18\end{array}$ & $\begin{array}{r}35.26 \\
1.92 \\
59.11 \\
1.96\end{array}$ & $\begin{array}{r}9.98 \\
.38 \\
88.50 \\
.95\end{array}$ & $\begin{array}{r}5.99 \\
.58 \\
91.96 \\
1.35\end{array}$ & $\begin{array}{r}50.74 \\
.30 \\
32.28 \\
13.91\end{array}$ & $\begin{array}{r}25.24 \\
5.30 \\
40.21 \\
25.25\end{array}$ \\
\hline . & 99.73 & 99.34 & 100.03 & 98.25 & 99.81 & 99.88 & 97.23 & 96.00 \\
\hline
\end{tabular}

I. Marble, Elkhorn district. Collected by W. H. Weed. Analysis by E. C. Sullivan, record No. 2195 .

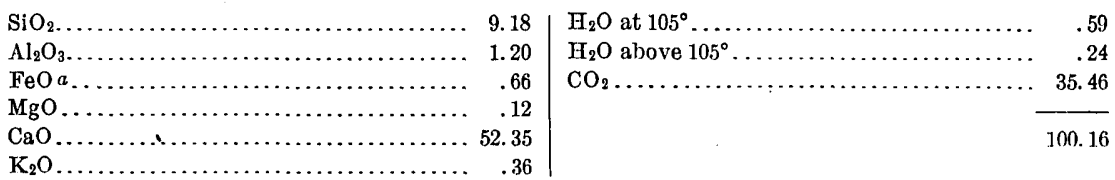

\section{IDAHO.}

A. Marble, Orofino.

B. Limestone, Missouri Creek, Nez Perce County.

Analyses A and B by W. F. Hillebrand, record No. 1905. 
C. Limestone, Snake River canyon. Analysis by George Steiger, record No. 1909.

\begin{tabular}{|c|c|c|c|}
\hline & A. & B. & C. \\
\hline 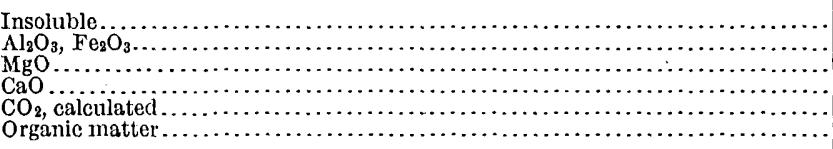 & $\begin{array}{r}.64 \\
.12 \\
51.05 \\
44.06 \\
\text { trace }\end{array}$ & $\begin{array}{r}1.19 \\
.19 \\
.51 \\
54.75 \\
43.50 \\
\text { trace }\end{array}$ & $\begin{array}{r}.36 \\
.10 \\
.10 \\
55.34 \\
43.59 \\
\end{array}$ \\
\hline & 99.85 & 100.14 & 99.49 \\
\hline
\end{tabular}

COLORADO.

1. DENVER BASIN.

Rocks described by Emmons in Mon. XXVII. Analyses made by L. G. Eakins, in the Denver laboratory.

A. Upper Wyoming limestone, Morrison.

B. Niobrara dolomite.

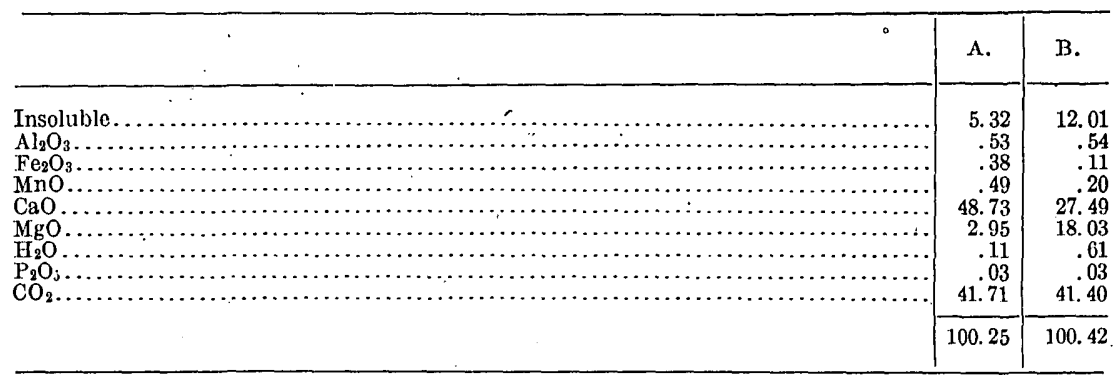

\section{LEADVILLE DISTRICT.}

Rocks described by Emmons in Mon. XII. Analyses A and E by W. F. Hillebrand; B, C, and D by A. Guyard; all made in the Denver laboratory.

A. Upper blue limestone, Silver Wave mine.

B. Upper blue limestone, Dugan quarry.

C. Upper blue limestone, Glass-Pendery mine.

D. Montgomery quarry, near base of blue limestone.

E. White limestone, upper part, Carbonate Hill quarry.

\begin{tabular}{|c|c|c|c|c|c|}
\hline . & A. & B. & C. & D. & E. \\
\hline 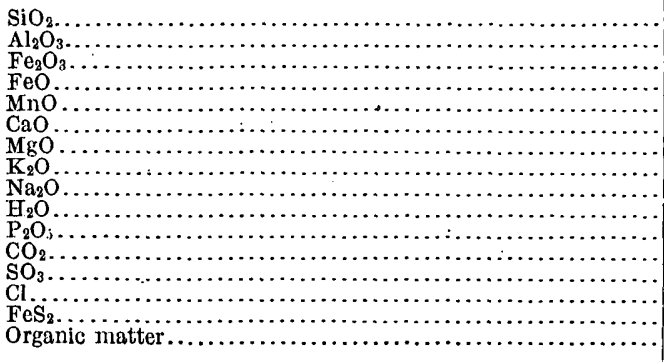 & $\begin{array}{r}.21 \\
.27 \\
.21 \\
.24 \\
\text { trace } \\
30.79 \\
21.14 \\
.03 \\
.06 \\
.22 \\
\text { trace } \\
46.84 \\
\text { trace } \\
.10 \\
\text { trace } \\
.03\end{array}$ & $\begin{array}{r}.70 \\
.17 \\
.11 \\
.38 \\
.05 \\
30.43 \\
20.78 \\
.05 \\
.09 \\
.04 \\
.12 \\
46.93 \\
.13 \\
\text { trace } \\
.03\end{array}$ & $\begin{array}{r}.27 \\
.04 \\
.22 \\
.13 \\
.20 \\
29.97 \\
21.52 \\
.01 \\
.02 \\
.07 \\
.03 \\
47.39 \\
.04 \\
.04 \\
.02\end{array}$ & $\begin{array}{r}7.76 \\
.11 \\
.10 \\
.57 \\
.06 \\
27.26 \\
20.05 \\
.02 \\
.04 \\
.05 \\
.07 \\
43.79 \\
\text { trace } \\
.06 \\
\text { trace } \\
.07\end{array}$ & $\begin{array}{r}11.84 \\
1.66 \\
1.51 \\
.83 \\
\ldots 26.60 \\
17.41 \\
.02 \\
.03 \\
.48 \\
\text { trace } \\
40.01 \\
\ldots .05 \\
. .0 \% \\
\ldots \ldots . .\end{array}$ \\
\hline & 100.14 & 100.02 & 99.93 & 100.01 & 100.44 \\
\hline
\end{tabular}




\section{FAIRPLAY, PARK COUNTY.}

Analyses made by W. F. Hillebrand, in the Denver laboratory.

A. Serpentinous limestone, Buckskin Gulch.

B. Limestone, Fairplay.

C. Limestone, Mount Silverheels.

D. Dolomite-limestone, Mount Silverheels.

Analyses $\mathrm{B}, \mathrm{C}$, and D partial, with $\mathrm{CO}_{2}$ calculated to satisfy bases.

\begin{tabular}{|c|c|c|c|c|}
\hline . & A. & B. & C. & D. \\
\hline 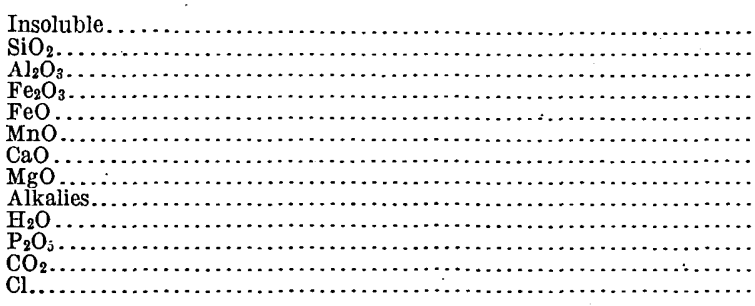 & $\begin{array}{r}17.64 \\
.99 \\
.62 \\
.18 \\
\text { trace } \\
32.23 \\
19.01 \\
.07 \\
3.72 \\
.05 \\
25.33 \\
.08\end{array}$ & $\begin{array}{r}2.37 \\
\ldots \ldots \ldots \\
\ldots \ldots . \ldots \\
\ldots \ldots . \\
.19 \\
53.64 \\
.73 \\
\ldots \ldots \\
.51 \\
\ldots .93 \\
42.93 \\
\ldots \ldots \ldots\end{array}$ & 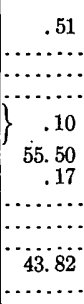 & 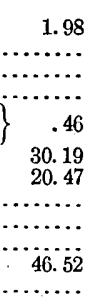 \\
\hline . $\quad$. & 99.92 & 100.37 & 100.10 & 99.62 \\
\hline
\end{tabular}

\section{PITKIN COUNTY.}

Limestones and dolomites collected under the direction of S. F. Emmons. Analyses by George Steiger, record No. 1559. $\mathrm{CO}_{2}$ calculated to satisfy bases. Analyses partial only.

\begin{tabular}{|c|c|c|c|c|c|c|c|}
\hline . & A. & B. & C. & D. & E. & F. & G. \\
\hline 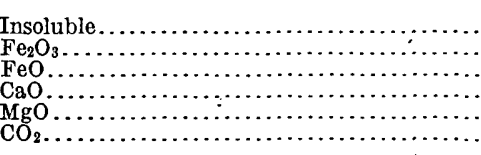 & $\begin{array}{r}.16 \\
.22 \\
.09 \\
30.66 \\
20.94 \\
47.13\end{array}$ & $\begin{array}{r}.80 \\
1.63 \\
.23 \\
31.19 \\
19.69 \\
46.16\end{array}$ & $\begin{array}{r}1.02 \\
2.10 \\
.06 \\
33.74 \\
16.76 \\
44.94\end{array}$ & $\begin{array}{r}13.63 \\
1.88 \\
.64 \\
35.98 \\
8.25 \\
37.35\end{array}$ & $\begin{array}{r}1.42 \\
3.34 \\
.42 \\
31.61 \\
18.06 \\
44.70\end{array}$ & $\begin{array}{r}31.12 \\
.36 \\
.19 \\
37.28 \\
.54 \\
29.88\end{array}$ & $\begin{array}{r}7.78 \\
.88 \\
.22 \\
38.85 \\
9.97 \\
41.47\end{array}$ \\
\hline & 99.20 & 99.70 & 98.62 & 97.73 & 99.55 & 99.37 & 99.17 \\
\hline
\end{tabular}

The following samples from Aspen were analyzed by L. G. Eakins in the Denver laborátory. Partial analyses, $\mathrm{CO}_{2}$ calculated as before.

H. Blue limestone.

I. Limestone.

J. Dolomite.

\begin{tabular}{|c|c|c|c|}
\hline & H. & I. & J. \\
\hline 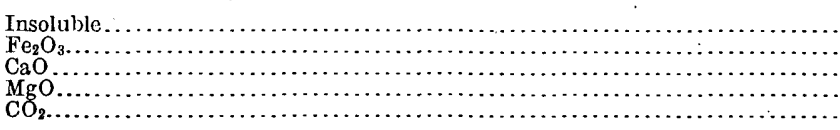 & $\begin{array}{r}.52 \\
.88 \\
31.16 \\
20.64 \\
47.19\end{array}$ & $\begin{array}{r}.33 \\
\operatorname{trace} \\
55.81 \\
.16 \\
44.03\end{array}$ & $\begin{array}{r}.84 \\
30.46 \\
20.90 \\
46.92\end{array}$ \\
\hline . & 100.39 & 100.33 & 100.43 \\
\hline
\end{tabular}




\section{GLENWOOD SPRINGS, GARFIELD COUNTY.}

Limestones and dolomites collected under the direction of S. F. Emmons. Analyses, partial only, by George Steiger, record No. 1559. $\mathrm{CO}_{2}$ calculated to satisfy bases.

\begin{tabular}{|c|c|c|c|c|c|c|c|}
\hline & A. & B. & C. & D. & E. & F. & G. \\
\hline \multirow[t]{2}{*}{ 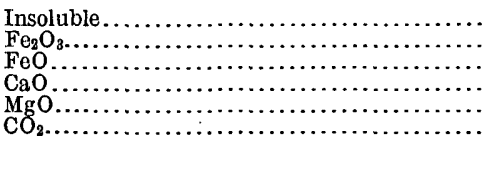 } & $\begin{array}{r}21.45 \\
.97 \\
.23 \\
40.64 \\
.73 \\
32.73\end{array}$ & $\begin{array}{r}47.74 \\
.18 \\
.71 \\
15.87 \\
10.60 \\
24.13\end{array}$ & $\begin{array}{r}6.47 \\
.42 \\
.35 \\
46.65 \\
2.64 \\
39.55\end{array}$ & $\begin{array}{r}3.71 \\
\text { none } \\
.55 \\
47.40 \\
4.49 \\
42.15\end{array}$ & $\begin{array}{r}9.44 \\
.26 \\
.32 \\
39.56 \\
8.56 \\
40.52\end{array}$ & $\begin{array}{r}17.82 \\
.74 \\
.57 \\
26.50 \\
14.86 \\
37.18\end{array}$ & $\begin{array}{r}1.96 \\
.03 \\
.35 \\
32.14 \\
18.72 \\
45.85\end{array}$ \\
\hline & 96.75 & 99.23 & 96.08 & 98.30 & 98.66 & 97.67 & 99.05 \\
\hline & & H. & I. & J. & K. & L. & M. \\
\hline \multirow{2}{*}{\multicolumn{2}{|c|}{ 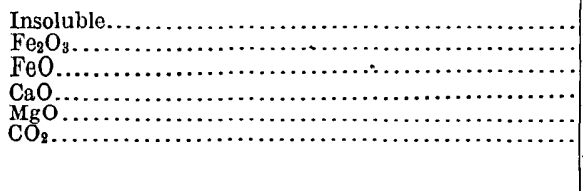 }} & $\begin{array}{r}2.27 \\
.14 \\
53.79 \\
.46 \\
42.76\end{array}$ & $\begin{array}{r}0^{22} \\
\text { trace } \\
55.17 \\
.21 \\
43.58\end{array}$ & $\begin{array}{r}.23 \\
.09 \\
55.49 \\
.24 \\
43.87\end{array}$ & $\begin{array}{l}.06 \\
\text { none } \\
\text { none } \\
55.81 \\
\text { trace } \\
43.85\end{array}$ & $\begin{array}{r}.22 \\
.10 \\
.10 \\
55.45 \\
.24 \\
43.84\end{array}$ & $\begin{array}{r}.11 \\
.03 \\
.07 \\
55.68 \\
\text { trace } \\
43.75\end{array}$ \\
\hline & & 99.42 & 99.18 & 99.92 & 99.72 & 99.95 & 99.64 \\
\hline
\end{tabular}

\section{TENMILE DISTRICT, SUMMIT COUNTY.}

Partial analyses, made in the Denver laboratory, by W. F. Hillebrand. $\mathrm{CO}_{2}$ calculated to satisfy bases. Manganese and iron present as carbonates, but $\mathrm{Fe}_{2} \mathrm{O}_{3}$ and $\mathrm{Al}_{2} \mathrm{O}_{3}$.were not separated from them.
A. A. V. Fletcher shaft, Copper Mountain.
B. Pittston tunnel.
C. Middle Carboniferous, Pearl Hill.
D. Summit quarry.
E. Dolomite, Sheep Mountain.
F. Oolitic limestone, northwest corner of area."
G. Pittston tunnel.

\begin{tabular}{|c|c|c|c|c|c|c|c|}
\hline & A. & B. & C. & D. & E. & F. & G. \\
\hline 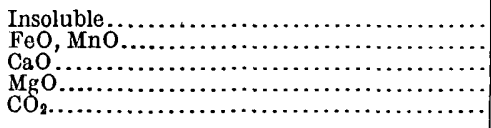 & $\begin{array}{r}2.69 \\
.21 \\
54.23 \\
.21 \\
42.97\end{array}$ & $\begin{array}{r}.62 \\
.25 \\
55.24 \\
.24 \\
43.81\end{array}$ & $\begin{array}{r}10.09 \\
1.19 \\
28.01 \\
18.33 \\
42.63\end{array}$ & $\begin{array}{r}1.75 \\
.32 \\
53.60 \\
1.23 \\
43.65\end{array}$ & $\begin{array}{r}.78 \\
1.50 \\
30.55 \\
20.15 \\
47.04\end{array}$ & $\begin{array}{r}1.37 \\
.20 \\
55.17 \\
.28 \\
43.76\end{array}$ & $\begin{array}{r}7.91 \\
.32 \\
50.83 \\
.70 \\
40.90\end{array}$ \\
\hline e & 100.31 & 100.16 & 100.25 & 100.55 & 100.02 & 100.78 & 100.60 \\
\hline
\end{tabular}

H. Dolomite, Blackbird tunnel, Tucker Mountain.

I. Summit King shaft, Summit City.

J. Middle Carboniferous dolomite, Ptarmigan Hill.

K. Hill north of Sugar Loaf.

L. Quarry on southeast side of Searls Gulch. 
M. Open cut below Sabbath Rest tunnel, Elk Mountain.

N. Triassic, Jacque Mountain.

\begin{tabular}{|c|c|c|c|c|c|c|c|}
\hline & H. & I. & J. & K. & L. & M. & N. \\
\hline \multirow[t]{2}{*}{ 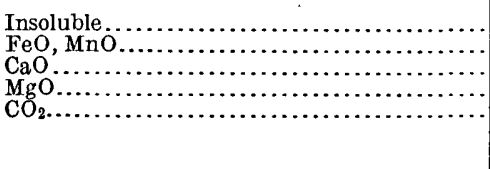 } & $\begin{array}{r}2.68 \\
1.52 \\
31.60 \\
18.27 \\
45.75\end{array}$ & $\begin{array}{r}6.75 \\
3.08 \\
28.05 \\
18.15 \\
43.88\end{array}$ & $\begin{array}{r}.65 \\
1.67 \\
30.90 \\
19.75 \\
47.02\end{array}$ & $\begin{array}{r}4.42 \\
.10 \\
52.97 \\
.40 \\
42.12\end{array}$ & $\begin{array}{r}.36 \\
.17 \\
55.58 \\
.37 \\
44.17\end{array}$ & $\begin{array}{r}.82 \\
.07 \\
55.47 \\
.22 \\
43.86\end{array}$ & $\begin{array}{r}2.04 \\
.15 \\
54.62 \\
.25 \\
43.28\end{array}$ \\
\hline & 99.82 & 99. 91 & 99.99 & 100.01 & 100.65 & 100.44 & 100.34 \\
\hline
\end{tabular}

\section{RICO DISTRICT.}

Limestone described by Ransome in 22d Ann., pt. 2. Analyses by W. F. Hillebrand, record No. 1914.

A. From Forest-Payroll mine.

B. Alteration product of $A$.

C. From Nellie Bly mine.

\begin{tabular}{|c|c|c|c|}
\hline . & A. & B. & C. \\
\hline 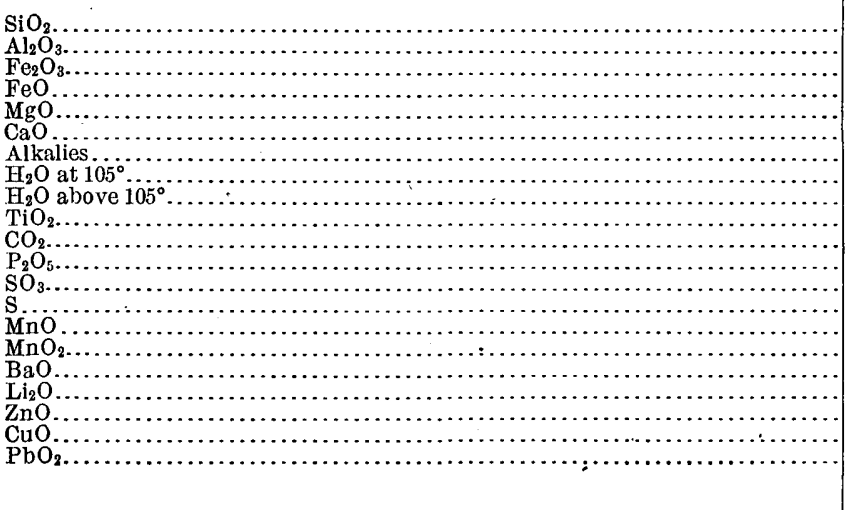 & $\begin{array}{r}23.51 \\
1.56 \\
.30 \\
.56 \\
2.29 \\
39.15 \\
? \\
.51 \\
.66 \\
.06 \\
a 30.86 \\
\text { trace } \\
\ldots \ldots . \\
\ldots \ldots . \\
. .54 \\
\ldots \text { trace } \\
\ldots \ldots . . . \\
\text { trace } \\
\text { trace } \\
\ldots \ldots . . . \\
\end{array}$ & $\begin{array}{r}29.29 \\
12.56 \\
5.36 \\
14.21 \\
1.06 \\
.20 \\
7.33 \\
a 8.88 \\
.27 \\
.17 \\
.14 \\
\text { trace } \\
\ldots \ldots . . \\
\ldots \ldots .27 \\
13.27 \\
\text { trace } \\
\text { trace } \\
1.65 \\
.40 \\
5.21 \\
\end{array}$ & $\begin{array}{r}.80 \\
.04 \\
.07 \\
.10 \\
.25 \\
55.10 \\
\ldots \ldots \ldots \\
\ldots \ldots \ldots . . \\
\ldots \ldots \ldots \\
\text { trace } \\
\text { a } 43.39 \\
\text { undet. } \\
\ldots \ldots . . \\
\text { trace } \\
.13 \\
\ldots \ldots . . \\
\text { none } \\
\ldots \ldots . .09 \\
.09 \\
.03 \\
\ldots . . \\
100.00\end{array}$ \\
\hline
\end{tabular}

a By difference.

UTAH.

A. Marble from the Ontario mine, east end of the 1,000-foot level. Analysis made by L. G. Eakins in the Denver laboratory.

B. Oolitic sand from shore of Great Salt Lake. Analysis by T. M. Chatard, record No. 156.

\begin{tabular}{|c|c|c|}
\hline . & A. & B. \\
\hline 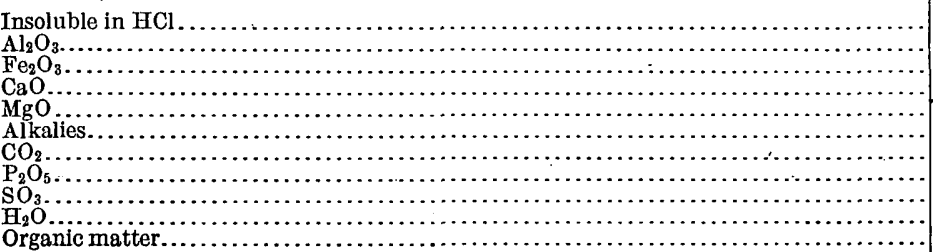 & 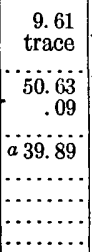 & $\begin{array}{r}4.02 \\
.20 \\
51.33 \\
.72 \\
.63 \\
41.07 \\
\text { trace } \\
.89 \\
.83 \\
.27\end{array}$ \\
\hline 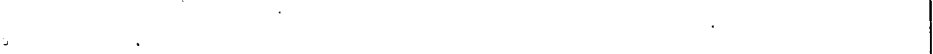 & 100.22 & 99.97 \\
\hline
\end{tabular}


The following samples, $\mathrm{C}$ to $\mathrm{J}$, represent altered limestones from the Bingham district. Received from S. F. Emmons. Partial analyses by W. F. Hillebrand, record No. 1932.

C. White altered limestone, Emma mine, west drift.

D. Same locality as A, gray.

E. White altered limestone, Highland Boy mine, No. 7 tunnel.

F. Same locality as C, gray.

\begin{tabular}{|c|c|c|c|c|}
\hline$\cdot$ & C. & D. & E. & F. \\
\hline 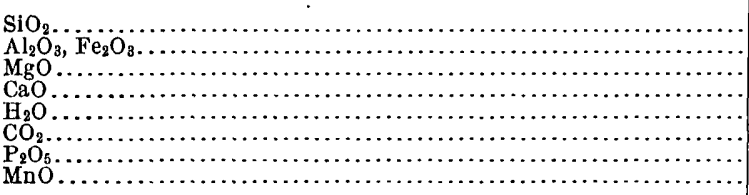 & $\begin{array}{r}50.41 \\
.30 \\
24.57 \\
9.74 \\
11.81 \\
1.61 \\
.15 \\
\text { trace }\end{array}$ & $\begin{array}{r}12.50 \\
1.99 \\
3.66 \\
48.34 \\
4.31 \\
28.06 \\
.56 \\
\ldots . \ldots .\end{array}$ & $\begin{array}{r}43.40 \\
1.31 \\
45.52 \\
.23 \\
8.28 \\
\ldots \ldots \ldots . \\
\ldots \ldots \ldots .\end{array}$ & $\begin{array}{r}4.87 \\
.30 \\
.99 \\
53.50 \\
\ldots . .3 \\
39.32 \\
\ldots \ldots . . \\
\ldots \ldots . .\end{array}$ \\
\hline 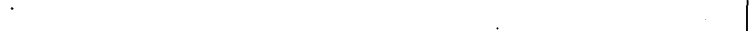 & 98.59 & 99.42 & 98.74 & 98.98 \\
\hline
\end{tabular}

$\mathrm{G}, \mathrm{H}, \mathrm{I}$. Altered limestones from different points on West Mountain.

J. Altered limestone, Bingham Canyon.

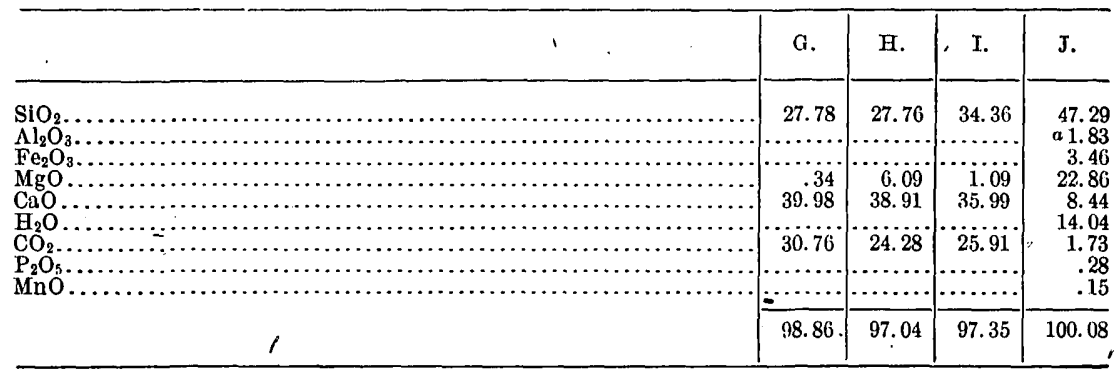

a With a little $\mathrm{TiO}_{2}$.

The following rocks are from the Park City district. Collecterl by J. M. Boutwell. Partial analyses by G. Steiger, record No. 2207.

K. Daly West mine.

L. Scottish Chief mine.

M, N. Silver King mine.

\begin{tabular}{|c|c|c|c|c|}
\hline . & K. & L. & M. & N. \\
\hline 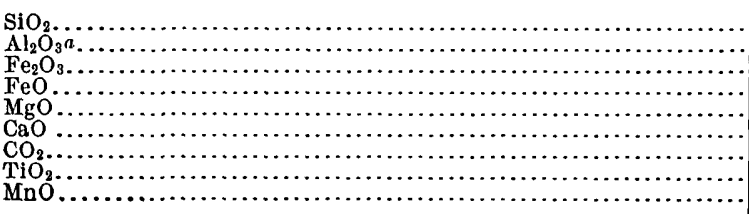 & $\begin{array}{r}3.81 \\
.19 \\
.38 \\
.16 \\
19.54 \\
30.11 \\
44.78 \\
\cdots \\
\cdots\end{array}$ & $\begin{array}{r}1.90 \\
1.70 \\
1.62 \\
1.40 \\
1.04 \\
50.51 \\
39.66 \\
.08 \\
.70\end{array}$ & $\begin{array}{r}1.50 \\
.31 \\
.11 \\
.16 \\
20.41 \\
30.54 \\
46.03 \\
\text { norie } \\
.28\end{array}$ & $\begin{array}{r}4.54 \\
.64 \\
\text { none } \\
.30 \\
19.07 \\
29.69 \\
44.27 \\
.04 \\
.25\end{array}$ \\
\hline & 98.97 & 98.61 & 99.34 & 98.80 \\
\hline
\end{tabular}

a Includes possible $\mathrm{P}_{2} \mathrm{O}_{3}$. 


\section{ARIZONA.}

A. Fresh contact-metamorphosed limestone, Joy mine, Morenci district.

$B$. Primary alteration of $A$, adjoining pyrite vein.

Rocks A and B collected by W. Lindgren, and described in P. P. 43, p. 172. Analyses by W. F. Hillebrand, record No. 1997.

C. Representative "mineralized" limestone, Copper Queen mine, Bisbee.

D. Dark limestone, Copper Queen mine.

E. Alteration product of D. Partial analysis.

Rocks C, D, E, collected by F. L. Ransome. Analyses by W. F. Hillebrand, record No. 2036.

\begin{tabular}{|c|c|c|c|c|c|}
\hline . & A. & B. & C. & D. & E. \\
\hline 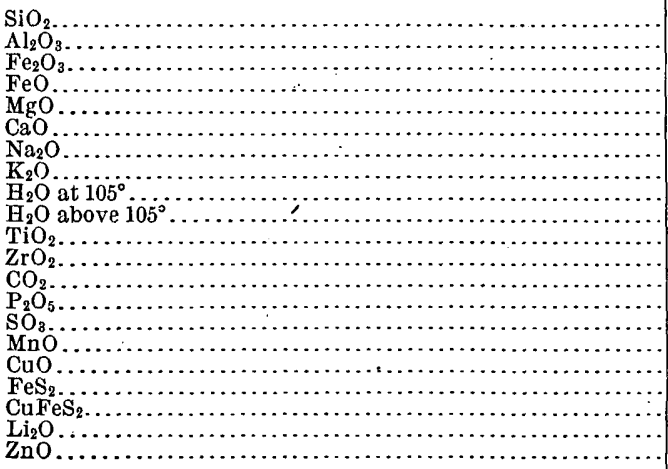 & $\begin{array}{r}17.08 \\
2.34 \\
.76 \\
12.38 \\
32.48 \\
.09 \\
.10 \\
.95 \\
.01 \\
.01 \\
.02 \\
26.85 \\
.16 \\
.13 \\
.26 \\
. .30 \\
1.50 \\
.18 \\
\text { trace } \\
.99\end{array}$ & $\begin{array}{r}42.03 \\
3.16 \\
1.18 \\
.84 \\
20.25 \\
10.41 \\
.06 \\
.12 \\
1.07 \\
4.25 \\
.31 \\
\text { trace } \\
3.58 \\
.42 \\
.75 \\
.18 \\
.83 \\
8.10 \\
1.67 \\
\text { trace } \\
.58\end{array}$ & $\begin{array}{r}28.55 \\
.98 \\
\text { none } \\
.84 \\
13.62 \\
26.20 \\
.14 \\
.06 \\
1.05 \\
3.08 \\
.16 \\
\ldots \ldots .00 \\
19.00 \\
.54 \\
\text { none } \\
.40 \\
\text { trace } \\
5.63 \\
\ldots \ldots . . \\
\text { trace } \\
\ldots \ldots . .\end{array}$ & 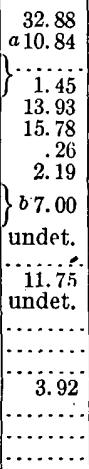 & 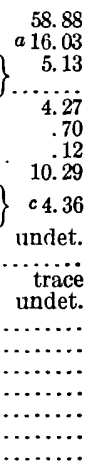 \\
\hline ' & 99.55 & 99.79 & 100.25 & 100.00 & 99.78 \\
\hline
\end{tabular}

$a$ Includes possible $\mathrm{TiO}_{2}$ and $\mathrm{P}_{2} \mathrm{O}_{5} . \quad b$ By difference.

c. Loss on ignition.

\section{NEVADA AND CALIFORNIA.}

A. Crystalline limestone, Eureka, Nevada. Analysis by E. A. Schneider, record No. 1279 :

B. From base of the Hamburg limestone, Eureka district, Nevada.

C. From summit of Hamburg limestone, Eureka.

D. Pogonip limestone (Silurian), Eureka district. Analyses B, C, and D made by W. F. Hillebrand in the Denver laboratory. Described by Hague in Mon. XX, pp. 40,49 . In $\mathrm{D}$ the $\mathrm{CO}_{2}$ was taken by difference.

\begin{tabular}{|c|c|c|c|c|}
\hline C: & A. & B. & C. ' & D. \\
\hline 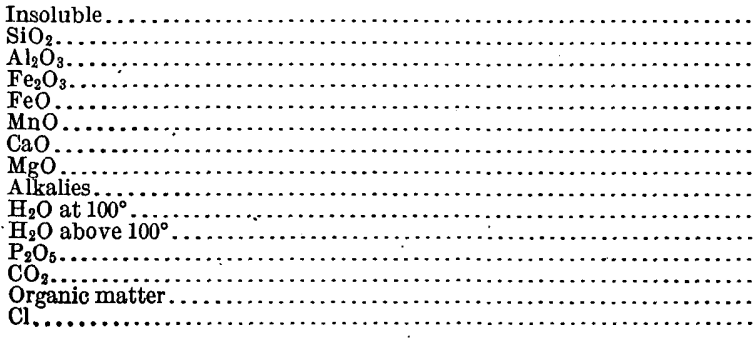 & 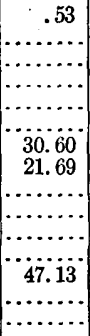 & $\begin{array}{r}24.00 \\
.12 \\
.12 \\
\ldots . \ldots . \\
41.97 \\
.80 \\
\text { traces } \\
.16 \\
.07 \\
32.62 \\
\text { traces } \\
.01\end{array}$ & $\begin{array}{r}3.94 \\
.64 \\
.43 \\
.20 \\
.61 \\
51.96 \\
.52 \\
\text { traces } \\
.37 \\
.50 \\
40.71 \\
.03 \\
.01\end{array}$ & $\begin{array}{r}9.34 \\
.31 \\
.29 \\
\ldots \ldots . . \\
50.01 \\
.54 \\
\text { traces } \\
.13 \\
.24 \\
39.11 \\
\text { traces } \\
.03\end{array}$ \\
\hline . & 99.95 & 99.87 & 99.92 & 100.00 \\
\hline
\end{tabular}


E. Dolomite, 6.3 kilometers west of north from Red Mountain, Silver Peak district, Nevada.

F. Dolomite, 16.3 kilometers northeast of Silver Peak village, Nevada. Analyses $\mathrm{E}$ and $\mathrm{F}$ by George Steiger, record No. 1859.

G. White deposit, White Terrace, west shore of Pyramid Lake, Nevada. Analysis by T. M. Chatard, record No. 34

H. Cretaceous limestone from Mount Diablo, California. Analysis made by W. H. Melville in the San Francisco laboratory and published in Bull. Geol. Soc. America, vol. 2 , p. 409. FeO and alkalies undetermined.

\begin{tabular}{|c|c|c|c|c|}
\hline- & $\mathbf{E}$ & F. & G. & H. \\
\hline 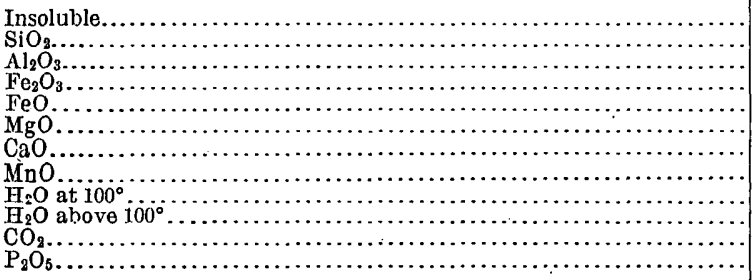 & 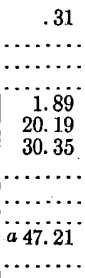 & 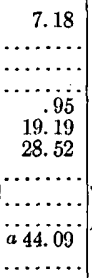 & $\mid \begin{array}{r}\ldots \ldots \\
22.00 \\
5.14 \\
2.04 \\
\ldots \ldots \ldots \\
1.89 \\
37.22 \\
\ldots \ldots \ldots \\
3.32 \\
28.53 \\
\ldots \ldots \ldots\end{array}$ & $\begin{array}{r}21.19 \\
.39 \\
1.52 \\
\ldots .39 \\
1.39 \\
35.61 \\
3.61 \\
.76 \\
2.33 \\
26.84 \\
2.55\end{array}$ \\
\hline$\cdot$ & 99.95 & 99.93 & 100.14 & 96.19 \\
\hline
\end{tabular}

$a$ Calculated to satisfy bases.

\section{OREGON}

Limestones collected in western Oregon by J. S. Diller. Analyses by R. C. Wells, record No. 2381.
A. Ten and one-half miles southwest of Grants Pass.
B. Carter's quarry, $5 \frac{1}{4}$ miles southeast of Gold Hill.
C. Householder's quarry, 5 miles southeast of Gold Hill.
D. Ridge southwest of Gold Hill.
E. Jones's marble quarry, 3 miles southwest of Williams.
F. Applegate River, near mouth of Manzanita Creek.
G. Three miles southeast of Kerby.

\begin{tabular}{|c|c|c|c|c|c|c|c|}
\hline & A. & B. & C. & D. & $\mathrm{E}$. & F. & G. \\
\hline \multirow[t]{2}{*}{ 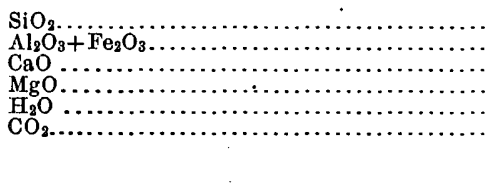 } & $\begin{array}{r}.23 \\
.28 \\
55.28 \\
.03 \\
.50 \\
43.57\end{array}$ & $\begin{array}{r}.37 \\
.20 \\
55.71 \\
.01 \\
.37 \\
43.54\end{array}$ & $\begin{array}{r}.31 \\
.44 \\
55.34 \\
.03 \\
.56 \\
43.23\end{array}$ & $\begin{array}{r}23.86 \\
.32 \\
41.83 \\
\text { trace } \\
32.46 \\
32.57\end{array}$ & $\begin{array}{r}.13 \\
.38 \\
55.55 \\
\text { none } \\
.26 \\
43.63\end{array}$ & $\begin{array}{r}.53 \\
.52 \\
55.05 \\
\text { trace } \\
.50 \\
43.25\end{array}$ & $\begin{array}{r}.06 \\
.62 \\
55.38 \\
\text { trace } \\
.40 \\
43.51\end{array}$ \\
\hline & 99.89 & 100.20 & 99.91 & 99.04 & 99.95 & 99.85 & 99.97 \\
\hline
\end{tabular}

\section{HAWAIIAN ISLANDS.}

Coral and shell rocks, analyzed for N. S. Shaler by L. G. Eakins, record Nos. 886, 887, 889. Analyses only partial.
A. Laie.
B, C. Kohuku Bluff.
D. Kohuku coral flat.
E. Point near coral flat.
F. "Modern chalk," Oahu. 
G. Diamond Head.

H. Under lava, Honululu.

I. Old reef, Waialua.

J. Campbell's ranch, Waianea, Oahu.

K. Wailuku Bay.

L. Reef No. 3, Honolulu.

M. Prison Knoll, Honolulu.

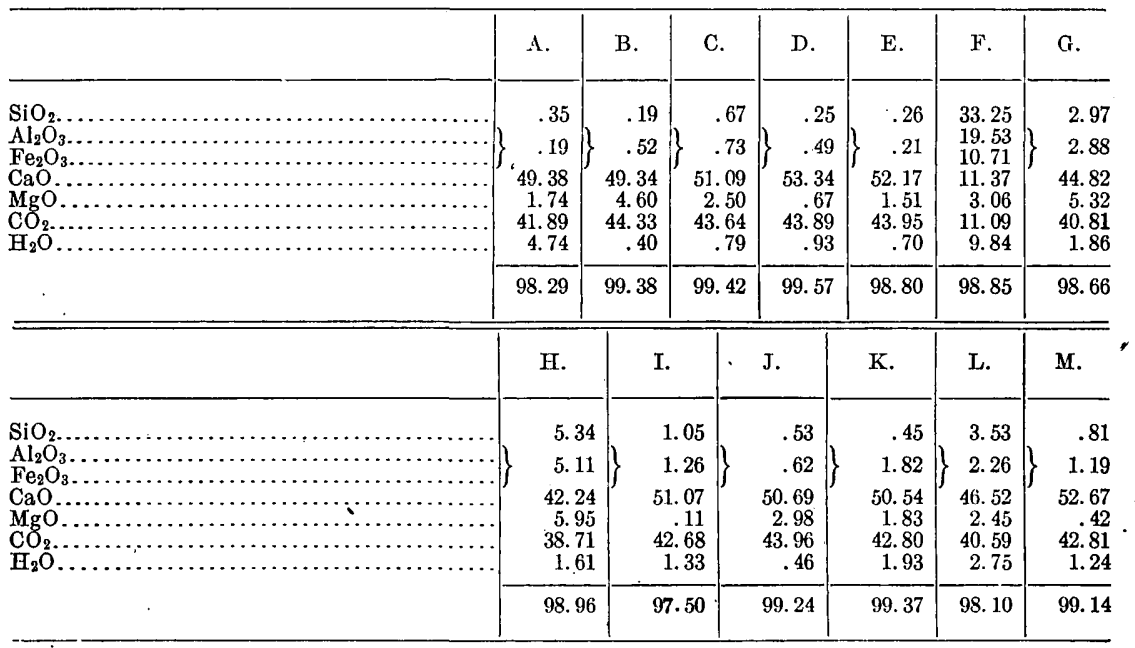




\section{ANALYSES OF SLATES ANP SHALES.}

\section{VERMONT.}

Samples A to I, inclusive, are described by T. Nelson Dale in 19th Ann., pt. 3. Analyses by W. F. Hillebrand, record Nos. 1567, 1656. Roofing slates of Cambrian age.

A. McCarty quarry, South Poultney.

B. Unfading green, Eureka quarry, Poultney.

C. Sea green, Griffith and Nathaniel quarry, South Poultney.

D. Sea green, Rising and Nelson's quarry, Pawlet.

E. Sea green, Brownell quarry, Pawlet.

F. Black, American Black Slate Company, Benson.

\begin{tabular}{|c|c|c|c|c|c|c|}
\hline & A. & B. & C. & D. & E. & $\mathrm{F}$. \\
\hline 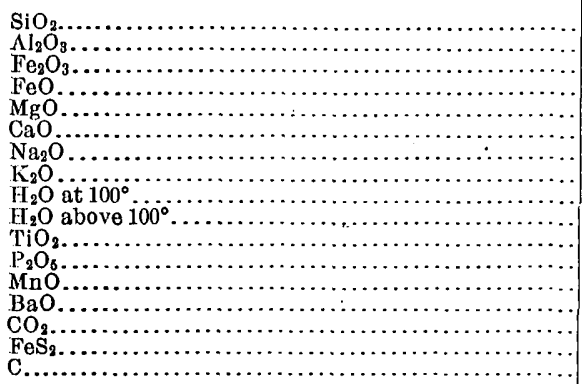 & $\begin{array}{r}61.63 \\
16.33 \\
4.10 \\
2.71 \\
2.92 \\
.50 \\
1.26 \\
5.54 \\
.31 \\
3.24 \\
.68 \\
.16 \\
.09 \\
.06 \\
.41 \\
.04 \\
\text { none }\end{array}$ & $\begin{array}{r}59.27 \\
18.81 \\
1.12 \\
6.58 \\
2.21 \\
.42 \\
1.88 \\
3.75 \\
.32 \\
3.98 \\
.99 \\
.11 \\
.13 \\
.05 \\
.21 \\
.15 \\
\text { none }\end{array}$ & $\begin{array}{r}62.37 \\
15.43 \\
1.34 \\
5.34 \\
3.14 \\
.77 \\
1.14 \\
4.20 \\
.34 \\
3.71 \\
.74 \\
.06 \\
.22 \\
.07 \\
.87 \\
.06 \\
\text { trace }\end{array}$ & $\begin{array}{r}67.76 \\
14.12 \\
.81 \\
4.71 \\
2.38 \\
.63 \\
1.39 \\
3.52 \\
.23 \\
2.98 \\
.71 \\
.07 \\
.10 \\
.04 \\
.40 \\
.22 \\
\text { none }\end{array}$ & $\begin{array}{r}59.84 \\
15.02 \\
1.23 \\
4.73 \\
3.41 \\
2.20 \\
1.12 \\
4.48 \\
.41 \\
3.44 \\
.74 \\
.09 \\
.34 \\
.09 \\
2.98 \\
.05 \\
\text { trace? }\end{array}$ & $\begin{array}{r}59.70 \\
16.98 \\
.52 \\
4.88 \\
3.23 \\
1.27 \\
1.35 \\
3.77 \\
.30 \\
3.82 \\
.79 \\
.16 \\
.16 \\
.08 \\
1.40 \\
1.18 \\
.46\end{array}$ \\
\hline$r$ & 99.98 & 99.98 & 99.80 & 100.07 & 100.17 & 100.05 \\
\hline
\end{tabular}

All six contain traces of lithia, of sulphates, and of nickel or cobalt.

G. Unfading green, Valley Slate Company's quarry, Poultney.

H. Mottled, purple and green, Eureka quarry, Poultney.

I. Purple, I mile south of Hydeville, in Castleton.

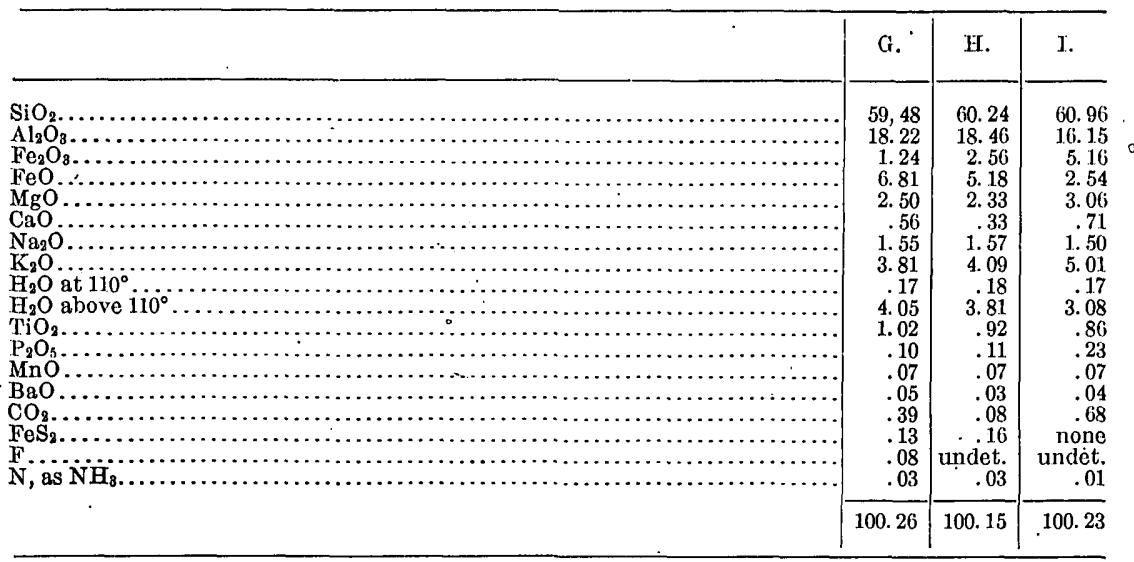

All three contain traces of lithia, nickel, and chlorine, and possibly of zirconia; in G and H traces of strontia, but none in $\mathrm{I}$. 
J. Slate from Guilford, collected for the educational series of rock specimens. Analysis by L. G. Eakins, record No. 1316.

K. Slate from the Lakeshore quarry, Hydeville. Analysis by Eakins, record No. 1159.

\begin{tabular}{|c|c|c|}
\hline . & J. & $\mathbf{K}$ \\
\hline 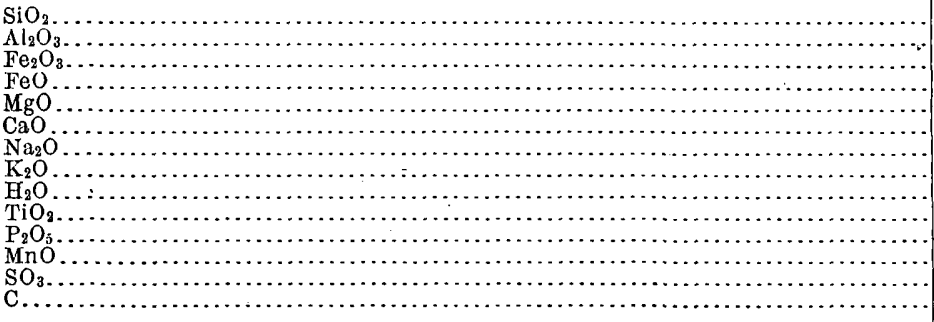 & $\begin{array}{r}60.72 \\
22.59 \\
6.03 \\
2.05 \\
.41 \\
.86 \\
3.69 \\
3.01 \\
. .13 \\
\text { trace } \\
.57\end{array}$ & $\begin{array}{r}58.15 \\
18.93 \\
2.91 \\
5.64 \\
2.70 \\
.60 \\
1.17 \\
3.92 \\
4.56 \\
.93 \\
.12 \\
.07 \\
.16 \\
\ldots . . .\end{array}$ \\
\hline & 100.06 & 99.86 \\
\hline
\end{tabular}

\section{' NEW YORK.}

Roofing slates from Washington County, collected by T. Nelson Dale. Of Cambrian age. Described by Dale in 19th Ann., pt. 3.

A. Red, three-fourths mile south of Hampton Village.

B. Empire Red Slate Company, near Granville.

C. National Red Slate Company, Granville.

D. Green, three-fourths mile northwest of Janesville.

Analyses by W. F. Hillebrand, record No. 1567.

\begin{tabular}{|c|c|c|c|c|}
\hline 4 & A. & B. & C. & D. \\
\hline 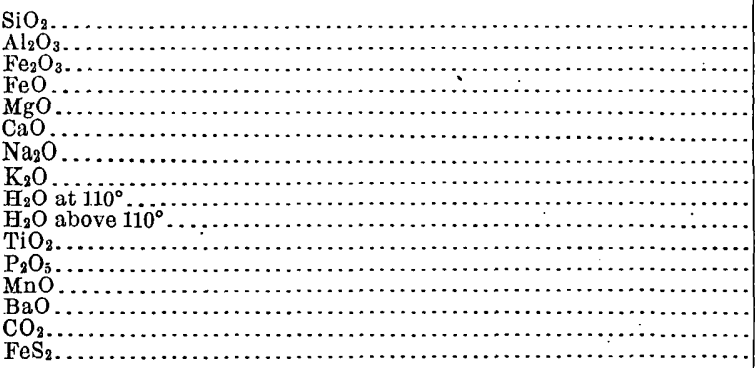 & $\begin{array}{r}67.61 \\
13.20 \\
5.36 \\
1.20 \\
3.20 \\
.11 \\
.67 \\
4.45 \\
.45 \\
2.97 \\
.56 \\
.05 \\
.10 \\
.04 \\
\text { none } \\
.03\end{array}$ & $\begin{array}{r}67.55 \\
12.59 \\
5.61 \\
1.24 \\
3.27 \\
.26 \\
.61 \\
4.13 \\
.40 \\
3.03 \\
.58 \\
.10 \\
.19 \\
.31 \\
.11 \\
.04\end{array}$ & $\begin{array}{r}56.49 \\
11.59 \\
3.48 \\
1.42 \\
6.43 \\
5.11 \\
.52 \\
3.77 \\
.37 \\
2.82 \\
.48 \\
.09 \\
.30 \\
.06 \\
7.42 \\
.03\end{array}$ & $\begin{array}{r}67.89 \\
11.03 \\
1.47 \\
3.81 \\
4.57 \\
1.43 \\
.77 \\
2.82 \\
.36 \\
3.21 \\
.49 \\
.10 \\
.16 \\
.04 \\
1.89 \\
.04\end{array}$ \\
\hline 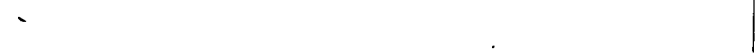 & 100.00 & 100.02 & 100.38 & 100.08 \\
\hline
\end{tabular}

All contain traces of lithia, of sulphates, and of nickel or cobalt. No carbonaceous matter is present. 
E. Red slate from quarry 3 miles north of Raceville

F. Green spot in E.

G. Purple rim of green spot F.

Analyses by W. F. Híllebrand, record No. 1656.

\begin{tabular}{|c|c|c|c|}
\hline & E. & F. & G. \\
\hline \multirow[t]{2}{*}{ 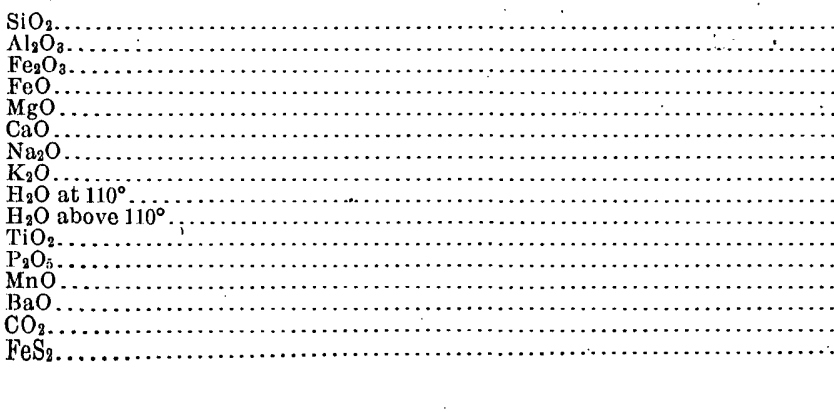 } & $\begin{array}{r}63.88 \\
9.77 \\
3.86 \\
1.44 \\
5.37 \\
3.53 \\
.20 \\
3.45 \\
.27 \\
2.48 \\
.47 \\
.08 \\
.21 \\
.05 \\
5.08 \\
\text { trace }\end{array}$ & $\begin{array}{r}65.44 \\
9.38 \\
1.09 \\
1.06 \\
4.92 \\
4.53 \\
.22 \\
3.57 \\
.25 \\
2.10 \\
.52 \\
.08 \\
.32 \\
.06 \\
6.55 \\
.04\end{array}$ & $\begin{array}{r}64.59 \\
10.23 \\
1.79 \\
1.19 \\
5.12 \\
4.07 \\
.23 \\
3.70 \\
.28 \\
2.29 \\
.51 \\
.08 \\
.26 \\
.05 \\
5.84 \\
\text { trace }\end{array}$ \\
\hline & 100.14 & 100.13 & 100.23 \\
\hline
\end{tabular}

Contain trace of lithia and nickel. Fluorine not determined.

\section{PENNSYLVANIA.}

Slates from Lehigh and Berks counties, collected by T. Nelson Dale. Analyses by W. F. Hillebrand, record No. 2015. Rocks $\Lambda$, B, described in Bull. 275, p. 84 .

A. Black roofing slate, Washington vein, Hazel Dell quarry, north of Slatington. Sp. gr., 2.780, $21^{\circ}$.

B. Black roofing slate, lower Franklin vein, old Franklin quarry, Slatington. Sp. gr., $2.783,21^{\circ}$.

-C. Black roofing slate, partly weathered, $1 \frac{1}{2}$ miles northwest of Waxatawny, Berks County. Dark variety.

D. Like C, light variety.

E. Weathered slate or "shale clay,." one-half mile south of Fogelsville.

F. Like E, one-fourth mile from Guth Station, South Whitehall.

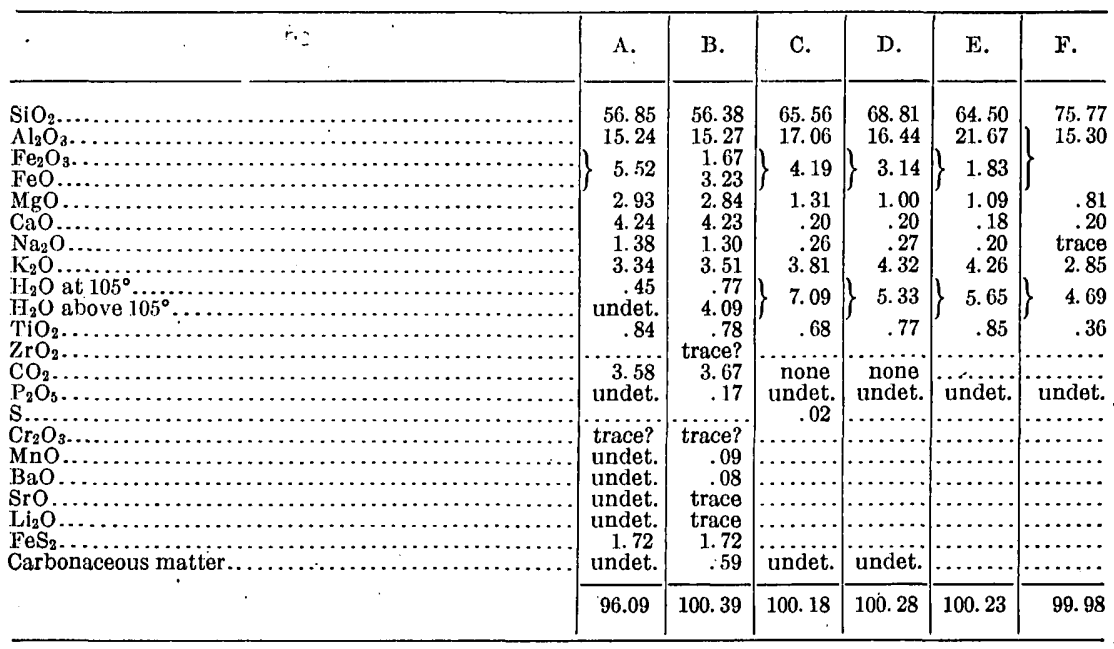

15619-Bull. $419-10-15$ 


\section{KENTUCKY, GEORGIA, ALABAMA.}

A. Indurated Carboniferous shale, in contact with the peridotite dike of Elliott County, Kentucky. Described by Diller in Bull 38. Analysis by T. M. Chatard, record No. 351 .

B. Fragment of shale included in the Elliott County dike. Analysis by Chatard, record No. 353 .

C. Bituminous shale, Dry Gap, Georgia. Analysis by L. G. Eakins, record No. 1316. P. R. C. 22. Described by Diller in Bull. 150, p. 90.

D. Middle Cambrian shale, Coosa Valley, near Blaiue, Cherokee County, Alabama. Analysis by H. N. Stokes, record No. 1549 .

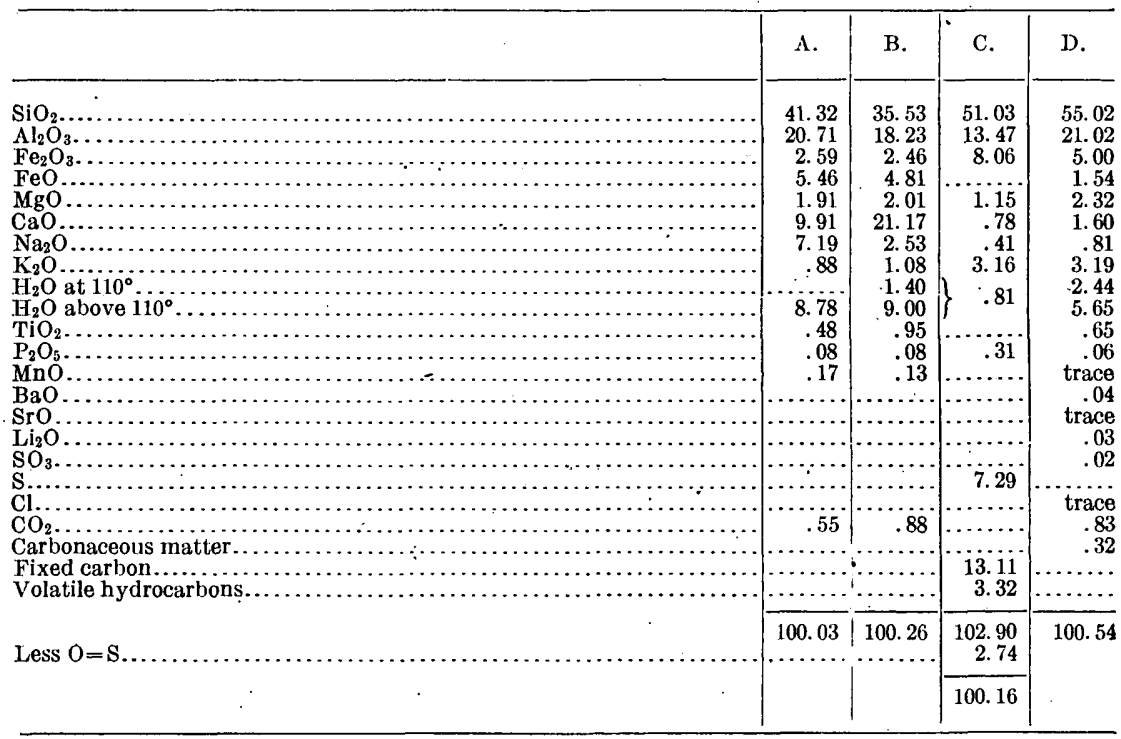

OHIO.

Three samples of Uttica shale from New Vienna. Collected by Edward Orton. Partial analyses by F. W. Clarke and R. B. Riggs, record No. 731.

\begin{tabular}{|c|c|c|c|}
\hline . & A. & B. & C. \\
\hline 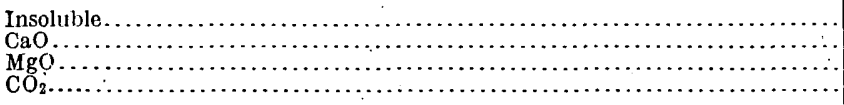 & $\begin{array}{r}60.17 \\
17.11 \\
1.25 \\
15.24\end{array}$ & $\begin{array}{r}29.51 \\
33.43 \\
2.16 \\
27.16\end{array}$ & $\begin{array}{r}25.80 \\
35.27 \\
1.32 \\
27.40\end{array}$ \\
\hline$\therefore$ & 93.77 & 92.26 & 89.79 \\
\hline
\end{tabular}

Iron and alumina are present in the soluble portions of these shales, the solvent being dilute hydrochloric acid.

\section{MICHIGAN.}

A. Clay slate, sec. 17, T. 43 N., R. 31 W., near Mansfield. Contains principally quartz, white mica, actinolite, rutile, hematite, and carbonaceous natter. Described by J. M. Clements in Mon. XXXVI, pp. 59, 61, 210. Analysis by George Steiger, record No. 1709. 
B. Pink slate, from near base of Upper Huronian, Menominee district. Center of sec. 5, T. 39 N., R. 29 W., near Norway mine. Described by Bayley in Mon. XI T'T, p. 298.

C. "Briar slate," Vulcan iron formation, Menominee district. Also described by Bayley, op. cit., p. 330.

Analyses B and C by E.T. Allen, record Nos. 1974, 1994.

\begin{tabular}{|c|c|c|c|}
\hline . & A. & $B$. & C. \\
\hline 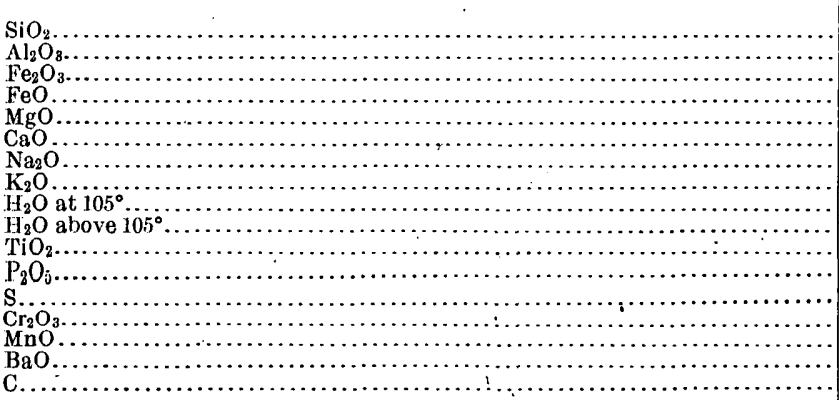 & $\begin{array}{r}60.28 \\
22.61 \\
2.53 \\
.45 \\
1.35 \\
.13 \\
.54 \\
5.73 \\
.60 \\
3.62 \\
.69 \\
.03 \\
\ldots \ldots \\
\ldots \ldots \\
. \\
\text { trace } \\
.04 \\
.97\end{array}$ & $\begin{array}{r}67.04 \\
15.01 \\
3.54 \\
3.18 \\
2.11 \\
.19 \\
.29 \\
4.00 \\
.67 \\
3.73 \\
.69 \\
.03 \\
.02 \\
\text { trace } \\
\text { trace } \\
\text { trace } \\
\ldots . . . .\end{array}$ & $\begin{array}{r}50.15 \\
6.55 \\
33.80 \\
.94 \\
.94 \\
.16 \\
.31 \\
4.38 \\
.81 \\
1.43 \\
.52 \\
.08 \\
\text { trace } \\
\text { trace } \\
\text { none } \\
\text { none } \\
\text {..... }\end{array}$ \\
\hline & 99.57 & 100.50 & 100.07 \\
\hline
\end{tabular}

WISCONSIN.

Slates of the Penokee-Gogebic series, collected by C. R. Van Hise. A and C are described in Mon. XIX, p. 306, as magnetitic clay slates. Analyses by L. G. Eakins; record No. 392.

A. Sec. 6, T. 45 N., R. 2 E.

B. Sec. 1, T. 45 N., R. 1 E.

C. Sec. 4 , T. 44 N., R. 2 W.

\begin{tabular}{|c|c|c|c|}
\hline & $\Lambda$. & B. & C. \\
\hline 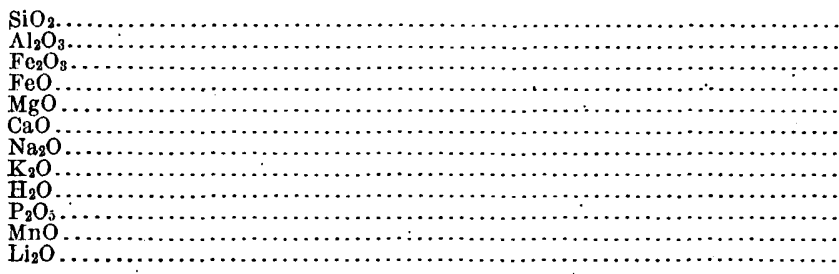 & $\begin{array}{r}53.44 \\
19.62 \\
11.38 \\
5.35 \\
1.58 \\
.42 \\
2.61 \\
1.73 \\
4.07 \\
\text { trace } \\
\text { trace } \\
\text { trace }\end{array}$ & $\begin{array}{r}59.73 \\
22.78 \\
.11 \\
5.98 \\
2.94 \\
.53 \\
1.41 \\
3.48 \\
3.28 \\
.09 \\
.09\end{array}$ & $\begin{array}{r}52.58 \\
20.76 \\
12.17 \\
4.08 \\
1.33 \\
.30 \\
.37 \\
4.87 \\
3.43 \\
.21 \\
\text { trace }\end{array}$ \\
\hline & 100.20 & 100.33 & 100.10 \\
\hline
\end{tabular}

MINNESOT A.

Slates from the Mesabi district, described by C. K. Leith in Mon. XLIII. Analyses by George Steiger, record Nos. 1931, 1992. 
A. Typical "Virginia slate." Average sample. About half chlorite, with quartz and perhaps some feldspar fragments.

B. Siliceous slate, from contact with gabbro, north of Birch Lake.

C. Slaty phase of the iron formation, Moss mine.

\begin{tabular}{|c|c|c|c|}
\hline . & A. & B. & C. \\
\hline 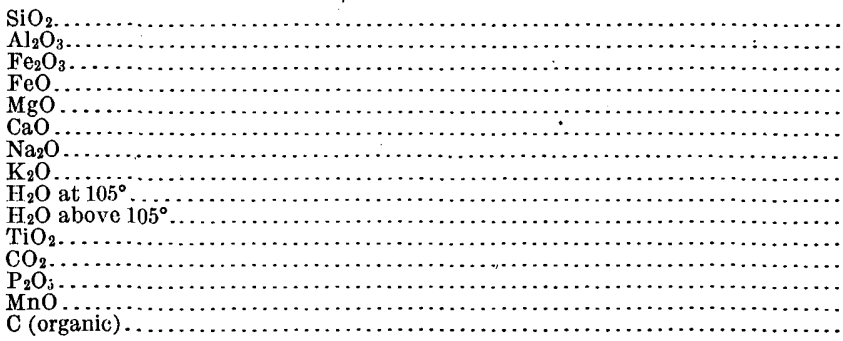 & \begin{tabular}{|r|}
62.26 \\
16.89 \\
1.76 \\
4.55 \\
2.95 \\
.42 \\
2.29 \\
3.02 \\
.70 \\
3.88 \\
.60 \\
none \\
.20 \\
\hdashline$\ldots .$. \\
present
\end{tabular} & $\begin{array}{r}78.95 \\
\text { none } \\
13.89 \\
1.23 \\
.18 \\
.81 \\
\text { none } \\
\text { none } \\
.73 \\
2.21 \\
\text { none } \\
1.59 \\
.04 \\
.11\end{array}$ & $\begin{array}{r}37.11 \\
2.41 \\
17.51 \\
26.13 \\
3.70 \\
.75 \\
.09 \\
.62 \\
.95 \\
2.57 \\
.22 \\
6.16 \\
.09 \\
1.21 \\
.73\end{array}$ \\
\hline , & 99.52 & 99.74 & 100.25 \\
\hline
\end{tabular}

\section{COLORADO.}

Shales from the Pueblo quadrangle, collected by G. K. Gilbert.

A, B. Near Nushbaum Spring.

C. Salt Creek.

D. Head of Rock Creek.

E. Near Rush Creek.

Analyses by George Steiger, record No. 1466.

\begin{tabular}{|c|c|c|c|c|c|}
\hline . & A. & B. & C. & D. & E. \\
\hline 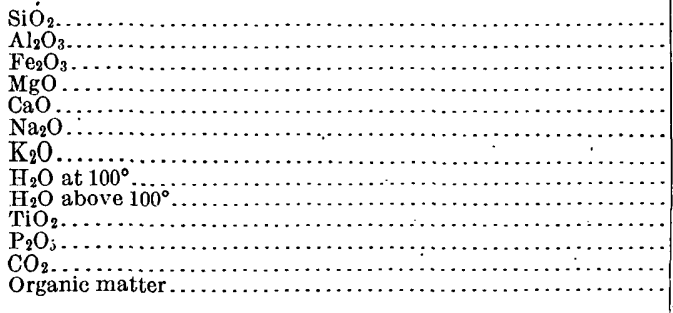 & $\begin{array}{r}60.80 \\
15.63 \\
4.62 \\
2.73 \\
1.63 \\
1.45 \\
2.55 \\
3.19 \\
4.16 \\
.47 \\
.10 \\
2.87\end{array}$ & $\begin{array}{r}51.69 \\
16.50 \\
7.90 \\
2.10 \\
4.41 \\
2.07 \\
2.68 \\
3.02 \\
6.00 \\
.66 \\
.22 \\
3.19 \\
.53\end{array}$ & $\begin{array}{r}60.60 \\
16.42 \\
4.95 \\
1.43 \\
1.61 \\
.92 \\
2.98 \\
3.91 \\
5.72 \\
.35 \\
.31 \\
.84\end{array}$ & $\begin{array}{r}63.60 \\
16.74 \\
4.63 \\
1.19 \\
.68 \\
.29 \\
2.92 \\
2.88 \\
5.99 \\
.66 \\
.16 \\
\ldots .46\end{array}$ & $\begin{array}{r}45.89 \\
13.24 \\
3.88 \\
2.12 \\
12.09 \\
.47 \\
2.31 \\
1.38 \\
4.16 \\
.52 \\
.17 \\
10.38 \\
3.47\end{array}$ \\
\hline . & 100.20 & 100.97 & 100.04 & 100.20 & 100.08 \\
\hline
\end{tabular}

Calcareous shales from Fairplay,.Park County. Partial analyses, by W. F. Hillebrand, made in the Denver laboratory.

\begin{tabular}{|c|c|c|}
\hline & F. & G. \\
\hline \multirow{3}{*}{ 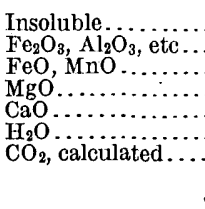 } & $\begin{array}{r}68.72 \\
2.10\end{array}$ & \\
\hline & $\begin{array}{r}5.72 \\
9.06 \\
1.01 \\
13.41\end{array}$ & $\begin{array}{r}2.10 \\
12.55 \\
19.34 \\
.73 \\
30.28\end{array}$ \\
\hline & 100.02 & 100.14 \\
\hline
\end{tabular}


NEW MEXICO.

Shales from Hermosa. Collected by C. H. Gordon. Analyses by G. Steiger, record No. 2239.

\begin{tabular}{|c|c|c|}
\hline 1 & A. & $B$. \\
\hline 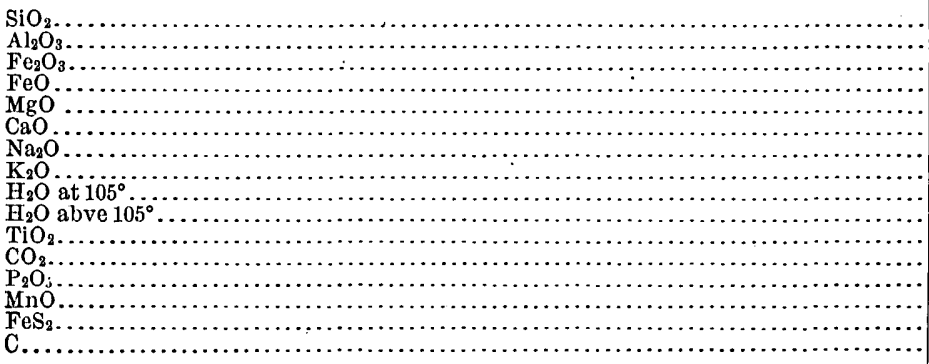 & $\begin{array}{r}34.64 \\
25.58 \\
.35 \\
3.60 \\
17.47 \\
.75 \\
.26 \\
\text { none } \\
3.67 \\
10.38 \\
.83 \\
\text { none } \\
.06 \\
.48 \\
1.70 \\
\ldots \ldots . .\end{array}$ & $\begin{array}{r}49.13 \\
13.92 \\
.77 \\
1.87 \\
5.11 \\
8.73 \\
.20 \\
4.25 \\
1.52 \\
4.69 \\
.66 \\
6.93 \\
.03 \\
.25 \\
.86 \\
2.00\end{array}$ \\
\hline & 99.77 & 100.92 \\
\hline
\end{tabular}

$\mathrm{ZrO}_{2}, \mathrm{SrO}, \mathrm{BaO}$ absent.

ARIZONA.

Shales from the Morenci district, collected by W. Lindgren. Analyses by W. F. Hillebrand, record No. 1997. Described in P. P. 43, p. 130.

A. Fresh, black, Devonian shale, near Longfellow mine.

B. Same locality, adjoining dike. The result of contact metamorphism.

\begin{tabular}{|c|c|c|}
\hline . & A. & B. \\
\hline $\begin{array}{l}\mathrm{SiO}_{2} \\
\mathrm{Al}_{2} \mathrm{O}_{8} \\
\mathrm{Fe}_{2} \mathrm{O}_{1}\end{array}$ & $\begin{array}{r}61.25 \\
15.60 \\
1.35 \\
3.04 \\
4.16 \\
3.40 \\
.44 \\
6.74 \\
.62 \\
2.09 \\
.66 \\
\text { trace? } \\
.08 \\
.07 \\
\text { trace } \\
\text { none } \\
\text { trace } \\
.25 \\
.03 \\
.03\end{array}$ & $\begin{array}{r}60.51 \\
15.81 \\
1.40 \\
2.64 \\
4.25 \\
2.34 \\
1.26 \\
8.27 \\
.59 \\
1.90 \\
.68 \\
\text { trace } \\
.08 \\
.11 \\
.17 \\
.01 \\
\text { trace } \\
.04 \\
\text { trace } \\
.02\end{array}$ \\
\hline 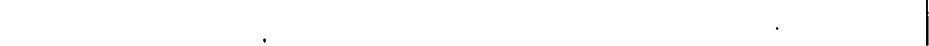 & 99.81 & 100.08 \\
\hline
\end{tabular}

$\mathrm{CO}_{2}$ and $\mathrm{SO}_{2}$ are both absent.

\section{CALIFORNIA.}

Cretaceous shales from Mount Diablo. Described by Turner and Melville, Bull. Geol. Soc. America, vol. 3, pp. 383-414. Analyses by W. H. Melville, made in the laboratory at San Francisco, except F (record No. 1166), which was done in the Washington laboratory.

A. Brownish black, resinous. From Bagley Canyon. 
B. Slate colored, soft, friable, little altered. From near Bagley Creek.

C. Same locality as B, less friable, but considerably altered.

D. Slate colored, friable. From Arroyo del Cerro.

E. Very friable. Same locality as D.

\begin{tabular}{|c|c|c|c|c|c|}
\hline & A. & B. & C. & D. & E: \\
\hline 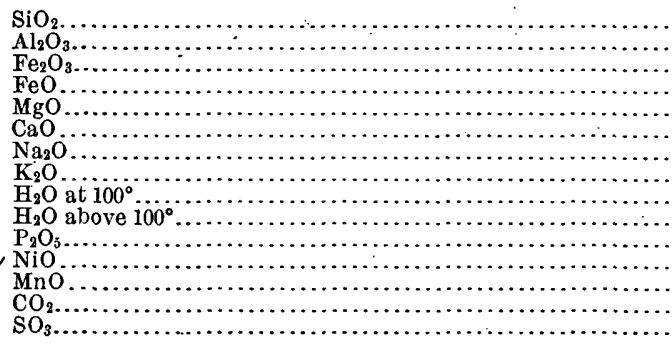 & $\begin{array}{r}56.66 \\
17.64 \\
.49 \\
5.22 \\
3.50 \\
1.67 \\
2.17 \\
2.27 \\
3.01 \\
5.92 \\
.15 \\
.19 \\
. .1 \\
.93\end{array}$ & $\begin{array}{r}53.65 \\
17.64 \\
4.06 \\
3.72 \\
5.15 \\
2.27 \\
2.53 \\
2.22 \\
3.95 \\
4.57 \\
.23 \\
\operatorname{trace} \\
.01 \\
\ldots \ldots \ldots\end{array}$ & $\begin{array}{r}49.14 \\
16.91 \\
4.39 \\
3.82 \\
5.43 \\
3.28 \\
4.67 \\
1.53 \\
3.39 \\
6.97 \\
.24 \\
\text { trace } \\
.22\end{array}$ & $\begin{array}{r}25.05 \\
8.28 \\
.27 \\
2.41 \\
2.61 \\
27.87 \\
\text { udnet. } \\
\text { undet. } \\
1.44 \\
2.86 \\
.08 \\
\text { trace } \\
4.11 \\
24.20 \\
\ldots\end{array}$ & $\begin{array}{r}40.17 \\
12.76 \\
2.10 \\
3.56 \\
15.42 \\
4.24 \\
.57 \\
1.36 \\
9.19 \\
6.73 \\
.08 \\
\text { trace } \\
.16 \\
3.48 \\
\ldots \ldots . .\end{array}$ \\
\hline & 99.82 & 100.00 & 99.99 & 99.18 & 99.82 \\
\hline
\end{tabular}

F. Neocomian shale, altered, light brown, friable. From near Arroyo del Cerro.

G. Calcareous shale, near Arroyo del Cerro. Hard, compact, dark colored. Very much altered.

H. Red shale, metamorphic area at head of Bagley Creek.

I. Silicified shale or phthanite, same locality as $\mathrm{H}$.

J. Clay slate, near the head of Yaqui Gulch, in Mariposa County. Described by Turner in Bull. 150, p. 342. Contains grains of quartz and feldspar, abundant carbonaceous particles, a chloritic substance (?), and a fibrous alteration of sillimanite (?). Analysis by George Steiger, record No. 1643.

\begin{tabular}{|c|c|c|c|c|c|}
\hline . & F. & G. & H. & I. & J. \\
\hline 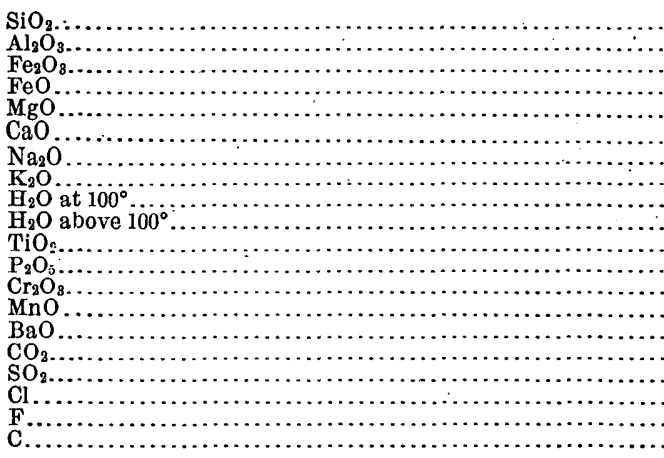 & 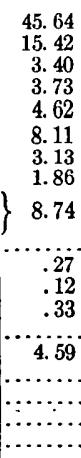 & \begin{tabular}{r}
44.56 \\
3.12 \\
1.27 \\
5.21 \\
3.39 \\
12.70 \\
3.09 \\
.88 \\
1.41 \\
6.24 \\
$\ldots .16$ \\
$\ldots \ldots \ldots$ \\
trace \\
\hdashline 17.62 \\
$\ldots \ldots \ldots$ \\
\hdashline$\ldots \ldots .$. \\
\hdashline$\ldots . .$. \\
$\ldots \ldots . .$.
\end{tabular} & 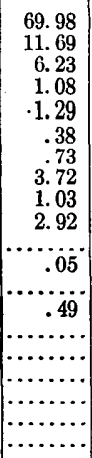 & 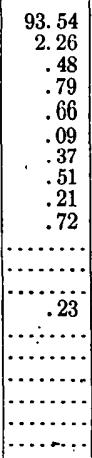 & $\begin{array}{r}60.35 \\
17.62 \\
5.64 \\
2.20 \\
1.04 \\
.45 \\
1.00 \\
3.16 \\
1.02 \\
4.36 \\
.75 \\
.17 \\
\ldots \ldots \\
\text { none } \\
.12 \\
\text { none } \\
.05 \\
.01 \\
\text { trace } \\
1.72\end{array}$ \\
\hline 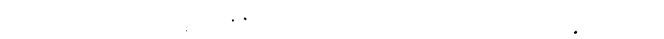 & 99.96 & 99.65 & 99.59 & 99.86 & 99.76 \\
\hline
\end{tabular}




\section{ANALYSES OF CLAYS, SOILS, ETC.}

\section{MAINE.}

Two clays from Thomaston, collected by George Otis Smith. Analyses by W. T. Schaller, record No. 2230.

A. Thomaston brick yard. B. Hayden's Point.

\begin{tabular}{|c|c|c|}
\hline : & A. & B. \\
\hline \multirow[t]{2}{*}{ 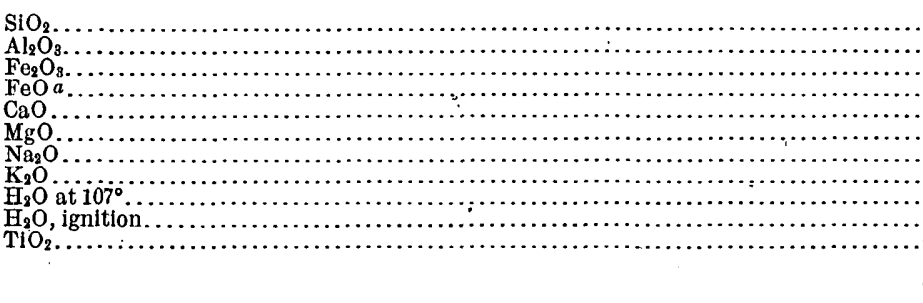 } & $\begin{array}{r}62.80 \\
17.36 \\
4.40 \\
2.00 \\
.88 \\
1.58 \\
1.48 \\
3.05 \\
1.31 \\
4.39 \\
.87\end{array}$ & $\begin{array}{r}62.33 \\
17.70 \\
5.19 \\
1.72 \\
1.00 \\
1.53 \\
2.38 \\
2.41 \\
1.11 \\
3.81 \\
.79\end{array}$ \\
\hline & 100.12 & 99.97 \\
\hline
\end{tabular}

a Uncertain, because of organic matter in the clays. The latter is included under "ignition."

$\mathrm{CO}_{2}$ absent. $\mathrm{P}_{2} \mathrm{O}_{5}$ undetermined.

\section{MASSACHUSETTS.}

Two glacial clays collected by W. C. Alden. Analyses by G. Steiger, record No. 2308.

A. Lancaster. B. Still River.

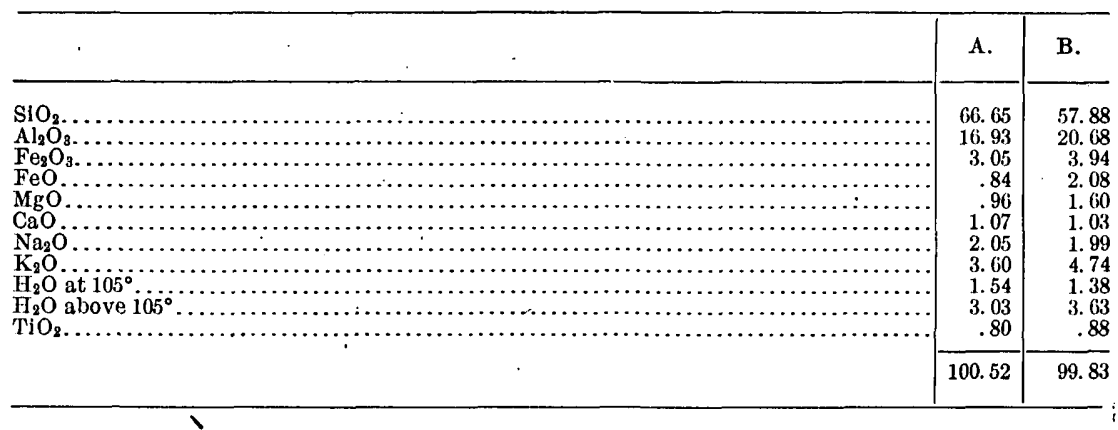

The following clays and soils from Marthas Vineyard were. collected by N. S. Shaler. See 7th Ann., p. 303. Analyses by F. W. Clarke, record Nos. 439, 440, $441,442,443,444,445,446,454$, and 455 . Partial analyses only.

A. Average sample of white clay, east end of Chilmark Cliffs.

B. Average sample of clays, Weyquosque series, Chilmark Cliffs. 
C. Average sample of fine clay and soil, east end of Weyquosque Cliffs.

D. Sandy white clay, south end of Gay Head,Cliffs.

E. Average sample of fine white clay, south end of Gay Head Cliffs.

F. Average sample of clay, north end of Gay Head Cliffs.

G. Average sample of southernmost red clays, Gay Head.

H. Brown clay, south of light-house, Gay Head Cliffs.

I. Average sample of red clay from the greensand, north end of Gay Head.

J. Pyritiferous clay, central part of Gay Head section.

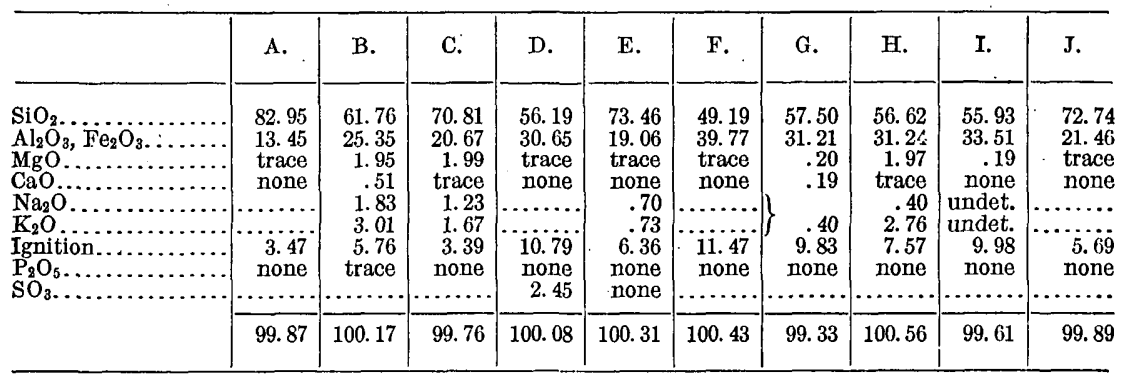

\section{CONNECTICUT.}

Five brick clays, received from H. E. Gregory. Analyses by W. T. Schaller, record No. 2138.

A. East Windsor Hill Brick Company, South .Windsor.

B. Park Brick Company, West Hartford.

C. Berlin Brick Company, Berlin.

D. Tuttle Brick Company, Newfield.

E. I. L. Stiles \& Son, New Haven.

\begin{tabular}{|c|c|c|c|c|c|}
\hline & A. & B. & C. & D. & E. \\
\hline \multirow[t]{2}{*}{ 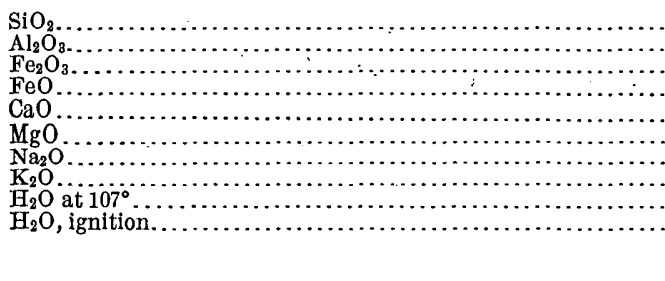 } & $\begin{array}{r}52.73 \\
22.25 \\
4.55 \\
3.14 \\
1.48 \\
3.20 \\
2.22 \\
4.28 \\
1.12 \\
4.91\end{array}$ & $\begin{array}{r}50.33 \\
27.06 \\
2.29 \\
2.62 \\
1.22 \\
3.34 \\
1.78 \\
4.40 \\
1.42 \\
5.24\end{array}$ & $\begin{array}{r}58.02 \\
17.93 \\
4.89 \\
1.24 \\
3.42 \\
1.92 \\
3.33 \\
3.06 \\
.99 \\
5.36\end{array}$ & $\begin{array}{r}55.27 \\
20.52 \\
5.34 \\
1.55 \\
2.21 \\
2.80 \\
2.82 \\
3.43 \\
1.37 \\
5.06\end{array}$ & $\begin{array}{r}56.75 \\
17.54 \\
4.92 \\
.93 \\
4.18 \\
2.34 \\
3.40 \\
3.16 \\
1.24 \\
6.28\end{array}$ \\
\hline & 99.88 & 99.70 & 100.16 & 100.37 & 100.74 \\
\hline
\end{tabular}

$\mathrm{TiO}_{2}, \mathrm{P}_{2} \mathrm{O}_{5}$, etc., not looked for.

\section{NEW YORK, PENNSYLVANIA.}

A. Clay, near Richfield Springs, New York. Partial analysis by Charles Catlett, record No. 946.

B, C. Clays, Northumberland County, Pennsylvania. Analyses by Charles Catlett, record No. 952 . 
D. White clay, derived from sericite schist, South Mountain, Pennsylvania. Collected by G. W. Stose. Analysis by W. T. Schaller, record No. 2302.

\begin{tabular}{|c|c|c|c|c|}
\hline & A. & B. & C. & D. \\
\hline \multirow[t]{2}{*}{ 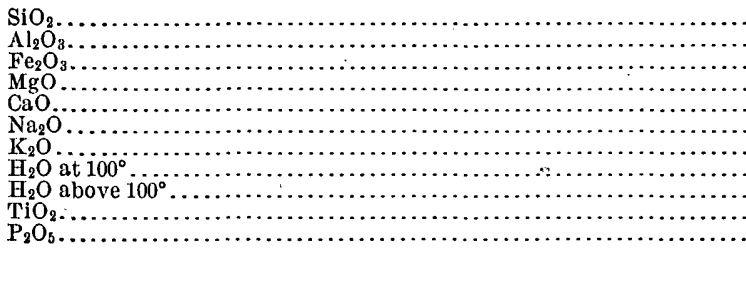 } & $\left\{\begin{array}{r}49.65 \\
23.82 \\
\text { trace } \\
6.48 \\
\text { undet. } \\
\text { undet. } \\
16.18\end{array}\right.$ & $\begin{array}{r}65.97 \\
20.37 \\
2.75 \\
.52 \\
.64 \\
.05 \\
3.32 \\
6.28\end{array}$ & $\begin{array}{r}59.16 \\
18.68 \\
10.32 \\
.67 \\
.52 \\
.11 \\
3.35 \\
6.87 \\
\ldots \ldots \ldots\end{array}$ & $\begin{array}{r}69.61 \\
16.83 \\
.95 \\
1.51 \\
.11 \\
.08 \\
3.41 \\
6.35 \\
.90 \\
.14\end{array}$ \\
\hline & 96.13 & 99.90 & 99.68 & 99.89 \\
\hline
\end{tabular}

E to H. Fire clays from Johnstown quadrangle, Pennsylvania. Collected by W. C. Phalen. Partial analyses by E. C. Sullivan, record No. 2281.

\begin{tabular}{|c|c|c|c|c|}
\hline & E. & F. & G. & $\mathrm{H}$. \\
\hline \multirow[t]{2}{*}{ 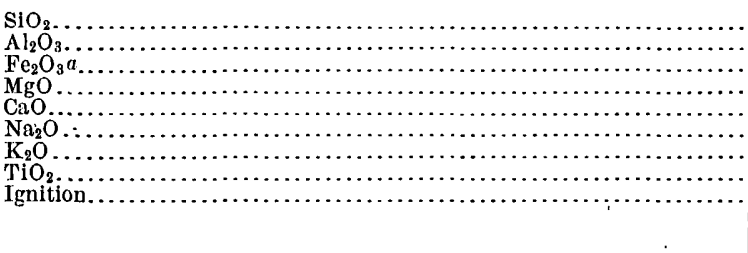 } & $\begin{array}{l}65.9 \\
20.3 \\
1.60 \\
.66 \\
.09 \\
.34 \\
2.98 \\
1.2 \\
6.5\end{array}$ & $\begin{array}{r}66.4 \\
19.8 \\
1.68 \\
.61 \\
.10 \\
.30 \\
3.24 \\
1.0 \\
6.4\end{array}$ & $\begin{array}{r}50.3 \\
21.3 \\
10.4 \\
.61 \\
.39 \\
.18 \\
1.14 \\
.9 \\
12.0\end{array}$ & $\begin{array}{r}53.1 \\
27.8 \\
3.08 \\
.60 \\
.22 \\
.48 \\
3.58 \\
1.2 \\
10.2\end{array}$ \\
\hline & 99.57 & 99.53 & 97.22 & 100.26 \\
\hline
\end{tabular}

a Total iron.

\section{MARYLAND.}

Clays from the Matawan formation, received from W. B. Clark. Analyses by George Steiger, record No. 1684.
A. Below Barnard's wharf, near Betterton, Kent County.
B. Severn River, below Round Bay, Anne Arundel County.
C. Magothy River, near Wilson's wharf, Anne Arundel County.
D. Fort Washington Bluff.

\begin{tabular}{|c|c|c|c|c|}
\hline . & A. & B. & C. & D. \\
\hline \multirow[t]{2}{*}{ 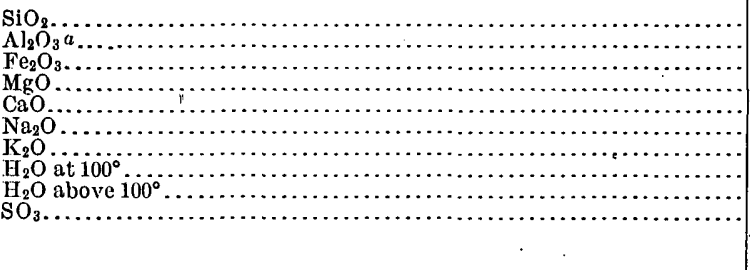 } & $\begin{array}{r}73.47 \\
12.69 \\
4.62 \\
.59 \\
.15 \\
.09 \\
1.55 \\
.89 \\
3.85 \\
.19\end{array}$ & $\begin{array}{r}87.15 \\
6.46 \\
2.15 \\
.27 \\
.10 \\
.14 \\
.90 \\
.32 \\
1.90 \\
\text { none }\end{array}$ & $\begin{array}{r}82.86 \\
6.49 \\
3.54 \\
.52 \\
.29 \\
.16 \\
1.16 \\
.69 \\
2.24 \\
.21\end{array}$ & $\begin{array}{r}73.02 \\
10.00 \\
4.78 \\
.90 \\
.57 \\
.59 \\
1.92 \\
1.09 \\
3.07 \\
1.04\end{array}$ \\
\hline & 98.09 & 99.39 & 98.16 & 96.98 \\
\hline
\end{tabular}

a Titanic and phosphoric oxides not separated.

No carbonates present. Sulphides undetermined. 


\section{VIRGINIA AND NORTH CAROLINA.}

A. Residual clay from decay of Trenton limestone, Lexington, Virginia. Described by Russell in Bull. 52. Analysis by R. B. Riggs, record No. 373. See also analysis of the limestone.

B. Residual clay from limestone, Staunton, Virginia.

C. Portion of $B$ soluble in weak hydrochloric acid.

D. Insoluble portion of B. Analyses B, C, and D by George Steiger, record No. 1630. See also analysis of limestone.

E. Decomposed dolerite, near Wadesboro, North Carolina. Described by Russell in Bull. 52. Analysis by T. M. Chatard, record No. 327.

F. Residual clay from decay of chloritic schist, Cary, 8 miles west of Raleigh, North Carolina. . Analysis by R. B. Riggs, record No. 364. Described by Russell in Bull. 52.

\begin{tabular}{|c|c|c|c|c|c|c|}
\hline - & A. & B. & C. & D. & E. & F. \\
\hline 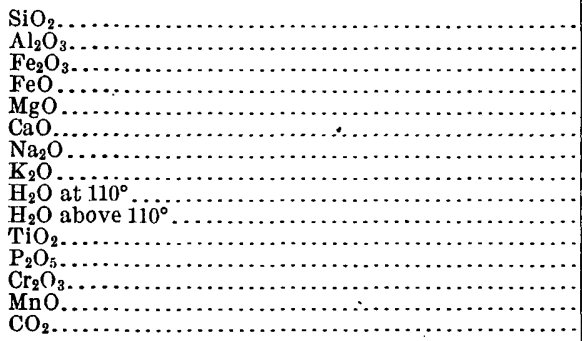 & $\begin{array}{r}43.07 \\
25.07 \\
15.16 \\
\ldots \ldots . . \\
.03 \\
.63 \\
1.20 \\
2.50 \\
12.98\end{array}$ & $\begin{array}{r}55.90 \\
19.92 \\
7.30 \\
.39 \\
1.18 \\
.50 \\
.23 \\
4.79 \\
2.54 \\
6.52 \\
.20 \\
.10 \\
\ldots . . . \\
\text { none } \\
.38\end{array}$ & $\begin{array}{r}3.09 \\
3.96 \\
6.25 \\
.30 \\
.43 \\
.30 \\
.20 \\
.28 \\
2.10 \\
.04 \\
.04 \\
. .0 \\
.38\end{array}$ & $\begin{array}{r}52.81 \\
15.96 \\
1.05 \\
.09 \\
.75 \\
.20 \\
.03 \\
4.51 \\
\hdashline 4.42 \\
.16 \\
.06 \\
\ldots . . . \\
\hdashline \text { none }\end{array}$ & $\begin{array}{r}39.55 \\
28.76 \\
16.80 \\
\ldots . . . \\
.59 \\
37 \\
\text { undet. } \\
\text { undet. } \\
\text { i3. } 26 \\
.64 \\
.10 \\
\text { trace } \\
\text { trace } \\
\ldots . . . .\end{array}$ & 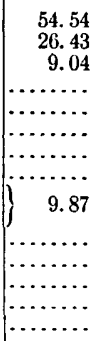 \\
\hline & 100.64 & 99.95 & 17.37 & 80.04 & 100.07 & 99.88 \\
\hline
\end{tabular}

SOUTH CAROLINA AND GEORGIA.

A. Clay from Charleston, South Carolina. Received from E. C. Eckel. Analysis by W. F. Hillebrand, record No. 2187.

B, C, D. Clays from near Augusta, Georgia: Partial analyses by G. Steiger, record No. 1395.

\begin{tabular}{|c|c|c|c|c|}
\hline$\ddots$ & A. & B. & C. & D. \\
\hline 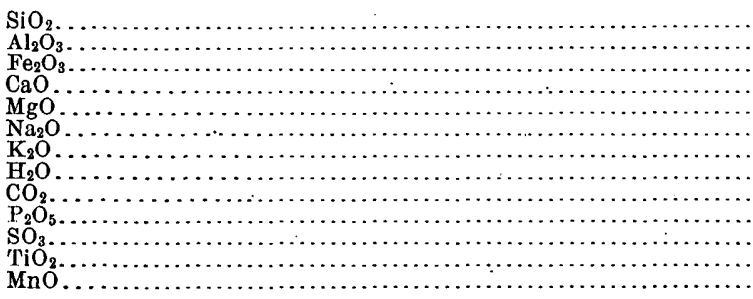 & $\begin{array}{r}50.12 \\
18.04 \\
5.91 \\
6.14 \\
.48 \\
.22 \\
.46 \\
13.91 \\
3.73 \\
.07 \\
.09 \\
.99 \\
(?)^{-09}\end{array}$ & $\begin{array}{r}60.24 \\
26.72 \\
.88 \\
\text { present } \\
\text { undet. } \\
\text { undet. } \\
6.28 \\
\ldots \ldots \\
\cdots \ldots \\
\cdots \ldots\end{array}$ & $\left\{\begin{array}{r}61.36 \\
29.04 \\
.76 \\
\text { present } \\
\text { undet. } \\
\text { undet. } \\
7.46 \\
\ldots \ldots \ldots\end{array}\right.$ & 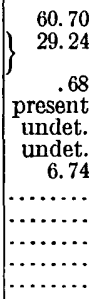 \\
\hline & 100.16 & 94.12 & 98.62 & 97.36 \\
\hline
\end{tabular}

FLORIDA.

A. Hammock clay, Melborne Creek. Collected by N. S. Shaler. Partial analysis by L. G. Eakins, record No. 881 .

B. Clay, Tampa.

C. Clay, Lakeland. 
B and C collected by W. H. Dall. Analyses by L. G. Eakins, record No. 1255, partial.

\begin{tabular}{|c|c|c|c|}
\hline & A. & B. & C. \\
\hline \multirow{4}{*}{ 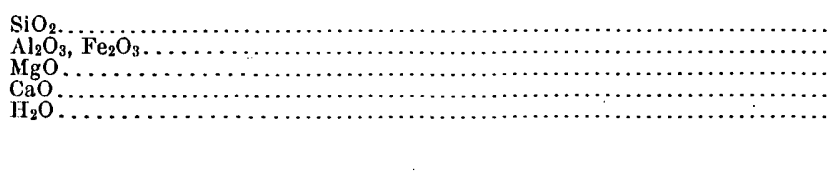 } & $\begin{array}{l}38.04 \\
27.19\end{array}$ & $\begin{array}{l}70.78 \\
11.33\end{array}$ & $\begin{array}{l}80.39 \\
15.03\end{array}$ \\
\hline & $\begin{array}{r}.46 \\
10\end{array}$ & & \\
\hline & $a a_{23.61}^{10.73}$ & $\begin{array}{r}2.18 \\
14.55\end{array}$ & $\begin{array}{l}1.22 \\
4.34\end{array}$ \\
\hline & 100.03 & 98.84 & 100.98 \\
\hline
\end{tabular}

a Includes some $\mathrm{CO}_{2}$.

Clays collected by G. H. Eldridge.

D, E. From the Sandlin place, 2 miles southeast of Marion, Hamilton County.

F. From Richmond's, 6 miles south of Leesburg, Lake County.

$\mathrm{G}, \mathrm{H}$. From Bartow Junction.

Analyses D, E, and F by H. N. Stokes, record No. 1493; G and H by George Steiger, No. 1.545 .

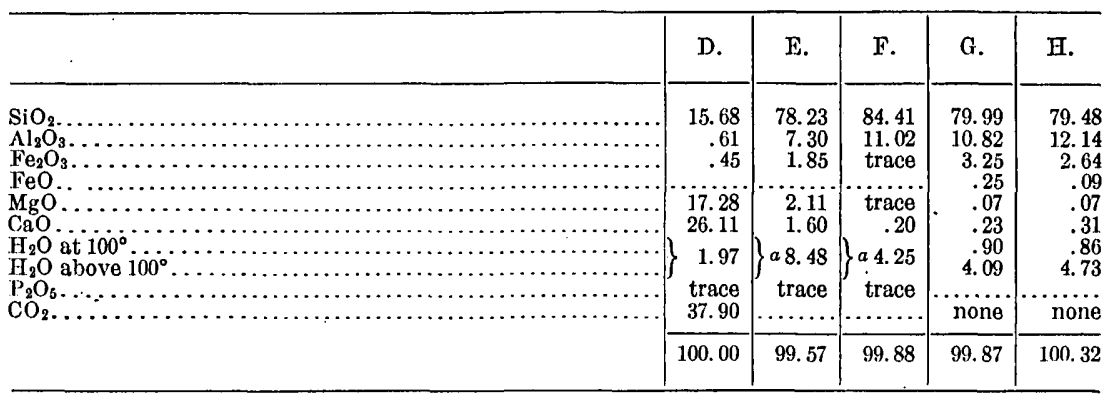

$a$ Includes a little $\mathrm{CO}_{2}$

I. "Filtering clay," Ocala. Received from D. T. Day. Analysis by H. N. Stokes, record No. 1738.

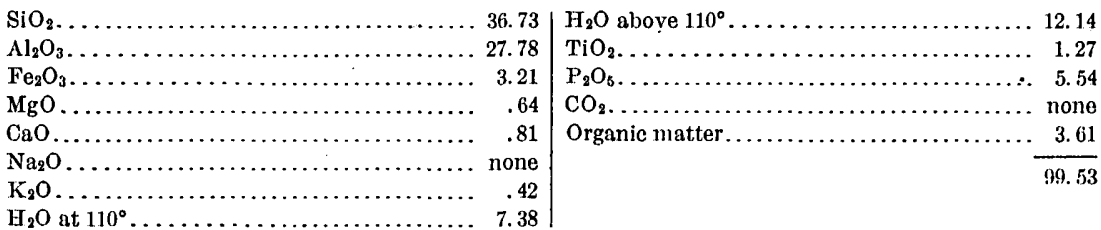

\section{ALABAMA AND MISSISSIPPI.}

A. Kaolin, Greenville, Alabama. Contains about 40 per cent, of kaolin, with fragments of quartz, feldspar, and mica. Analysis by T. M. Chatard, record No. 1148:

B. Residual clay, from decay of Knox dolomite, Morrisville, Alabama. Described by Russell in Bull. 52. Analysis by W. F. Hillebrand, record No. 797. See also analysis of the dolomite.

C. Loess, from Vicksburg, Mississippi. Described by Chamberlin and Salisbury, 6th Ann., p. 282. Analysis by R. B. Riggs, record No. 294. 
D. Stoneware clay, Holly Springs; Mississippi. Analysis by E. C. Sullivan, record No. 2105; Alkalies not separated, calculated as $\mathrm{K}_{2} \mathrm{O}$.

E. Clay, Scooba, Mississippi. Partial analysis by H. C. McNeil, record No. 2181.

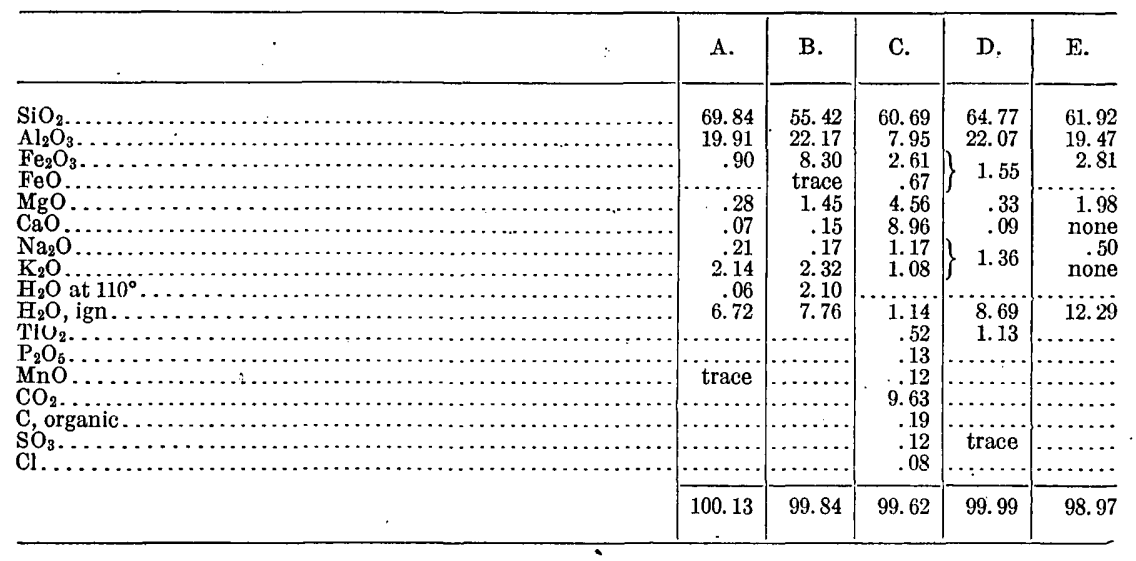

KENTUCKY AND TENNESSEE.

A. Fire clay, Carter County, Kentucky. Analysis by F. W. Clarke, record No. 2073.

B. Black clay, Mandle's pit, Paris, Tennessee.

C. Ball clay, same locality as B.

Analyses B and C by E. C. Sullivan, record No. 2105.

D. Clay, east of Parsons, Tennessee.

E. Clay, Robins \& Henderson pit, Pinson, Tennessee.

Analyses D and E by W. T. Schaller, record No. 2107.

All analyses partial only.

\begin{tabular}{|c|c|c|c|c|c|}
\hline 1 & A. & B. & C. & D. & E. \\
\hline 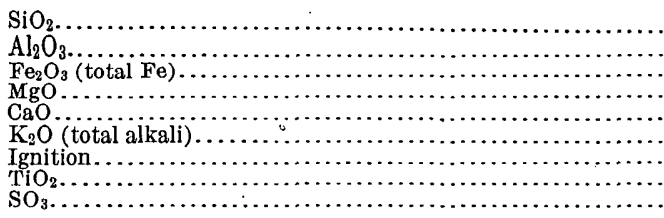 & $\begin{array}{r}42.71 \\
38.88 \\
3.36 \\
\text { none } \\
.13 \\
\text { undet. } \\
15.19 \\
\text { undet. } \\
\text {........ }\end{array}$ & $\begin{array}{r}46.34 \\
30.30 \\
1.19 \\
.34 \\
.24 \\
.78 \\
19.68 \\
1.42 \\
\text { trace }\end{array}$ & $\begin{array}{r}52.31 \\
30.09 \\
1.29 \\
.38 \\
.13 \\
1.72 \\
12.44 \\
1.10 \\
\text { trace }\end{array}$ & $\begin{array}{r}70.76 \\
19.29 \\
1.09 \\
.68 \\
.14 \\
\text { undet. } \\
6.02 \\
\text { undet. } \\
\text { trace }\end{array}$ & $\begin{array}{r}70.56 \\
18.18 \\
1.30 \\
.55 \\
.08 \\
\text { undet. } \\
8.13 \\
\text { undet. } \\
\text { trace }\end{array}$ \\
\hline - & 100.27 & 100.29 & 99.46 & 97.98 & 98.80 \\
\hline
\end{tabular}

\section{ILLINOIS, IOWA, MINNESOTA.}

A, B. Clays from Henry County, Illinois. Analyses by T. M. Chatard, record No. 144.

C. Loess, a stratum overlying residuary clay, 350 feet above the Mississippi River, near Galena, Illinois. Described by Chamberlin and Salisbury, 6th Ann., p. 282. Analysis by R. B. Riggs, record No. 293. Dried at $100^{\circ}$.

D. Loess, 300 feet above the Mississippi, $3 \frac{1}{2}$ miles northwest of Dubuque, Iowa. Described by Chamberlin and Salisbury (loc. cit.), and analyzed by Riggs, No. 292. Dried at $100^{\circ}$. 
E. Tallow clay, lead mine at Lansing, Iowa. Collected by W. P. Jenney. Analysis by H. N. Stokes, record No. 1337. Dried at $100^{\circ}$. Partial analysis.

F. Greenish-gray clay, New Ulm, Minnesota. Analysis by T. M. Chatard, record No. 825 .

\begin{tabular}{|c|c|c|c|c|c|c|}
\hline & A. & B. & C. & D. & E. & F. \\
\hline 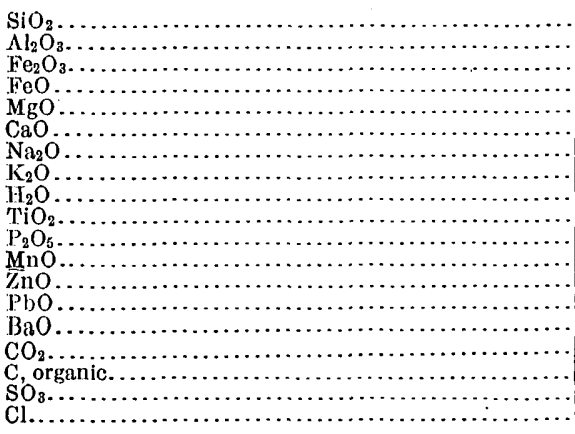 & $\begin{array}{r}46.12 \\
15.24 \\
4.41 \\
\ldots . . . \\
3.63 \\
8.63 \\
1.54 \\
3.79 \\
15.57 \\
.79 \\
.08 \\
.28 \\
\ldots \ldots \ldots \\
\ldots \ldots . . .\end{array}$ & $\begin{array}{r}42.58 \\
12.16 \\
3.90 \\
\ldots . . . \\
4.32 \\
11.33 \\
1.96 \\
3.88 \\
18.64 \\
.64 \\
.10 \\
.09 \\
\ldots \ldots . . \\
\ldots \ldots . .\end{array}$ & $\begin{array}{r}64.61 \\
10.64 \\
2.61 \\
.51 \\
3.69 \\
5.41 \\
1.35 \\
2.06 \\
2.05 \\
.40 \\
.06 \\
.05 \\
\ldots \ldots \\
\ldots . . . \\
6.31 \\
.13 \\
.11 \\
.07\end{array}$ & $\begin{array}{r}72.68 \\
12.03 \\
3.53 \\
.96 \\
1.11 \\
1.59 \\
1.68 \\
2.13 \\
2.50 \\
.72 \\
.23 \\
.06 \\
\ldots \ldots \\
\ldots \ldots . \\
\ldots . . . \\
.39 \\
.09 \\
.51 \\
.01\end{array}$ & 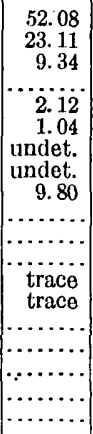 & $\begin{array}{r}61.32 \\
12.27 \\
3.62 \\
4.18 \\
1.76 \\
.99 \\
.42 \\
3.59 \\
10.73 \\
.66 \\
.27 \\
.27 \\
\ldots \ldots . \\
\ldots \ldots .05 \\
\ldots \ldots . . \\
\ldots \ldots . . \\
. \ldots .19 \\
\ldots \ldots .\end{array}$ \\
\hline & 100.08 & 99.60 & 100.06 & 100.22 & 97.49 & 100. 32 \\
\hline
\end{tabular}

WISCONSIN.

Clays, etc., described by Chamberlin and Salisbury in 6th Ann., pp. 250 and 282. Analyses by R. B. Riggs, record Nos. 259, 260, 261, 262, 290, 295 . Dried at $100^{\circ}$.

A. Residuary clay from Dodgeville, $4 \frac{1}{2}$ feet below surface.

B. The same, $8 \frac{1}{2}$ feet below surface.

C. Residuary clay from near Cobb, $4 \frac{1}{2}$ feet below surface.

D. Same as C, $3 \frac{1}{2}$ feet below surface.

E. Red, putty-like clay, containing pebbles, Milwaukee.

F. Red pebble clay, Milwaukee.

\begin{tabular}{|c|c|c|c|c|c|c|}
\hline & A. & B. & C. & D. & E. & F. \\
\hline 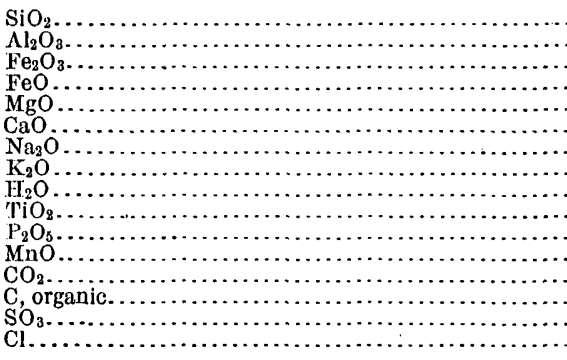 & $\begin{array}{r}71.13 \\
12.50 \\
5.52 \\
.45 \\
.38 \\
.85 \\
2.19 \\
1.61 \\
4.63 \\
.45 \\
.02 \\
.04 \\
.43 \\
.19 \\
.19\end{array}$ & $\begin{array}{r}49.59 \\
18.64 \\
17.19 \\
.27 \\
.73 \\
.93 \\
.80 \\
.93 \\
10.46 \\
.28 \\
.03 \\
.01 \\
.30 \\
\therefore .34\end{array}$ & $\begin{array}{r}49.13 \\
20.08 \\
11.04 \\
.93 \\
1.92 \\
1.22 \\
1.33 \\
1.60 \\
11.72 \\
.13 \\
.04 \\
.06 \\
.39 \\
1.09\end{array}$ & $\begin{array}{r}53.09 \\
21.43 \\
8.53 \\
.86 \\
1.43 \\
.95 \\
1.45 \\
.83 \\
10.79 \\
.16 \\
.03 \\
.03 \\
.29 \\
.22\end{array}$ & $\begin{array}{r}40.22 \\
8.47 \\
2.83 \\
.48 \\
7.80 \\
15.65 \\
.84 \\
2.36 \\
1.95 \\
.35 \\
.05 \\
\text { trace } \\
18.76 \\
.32 \\
.13 \\
.06\end{array}$ & $\begin{array}{r}48.81 \\
7.54 \\
2.53 \\
.65 \\
7.05 \\
11.83 \\
.92 \\
2.60 \\
2.02 \\
.45 \\
.13 \\
.03 \\
15.47 \\
.38 \\
.05 \\
.04\end{array}$ \\
\hline & 100.39 & 100.50 & 100.68 & $100.0 \theta$ & 100.27 & 100.50 \\
\hline
\end{tabular}

\section{MISSOURI AND ARKANSAS.}

A. Typical loess, Kansas City, Missouri. Dried at $100^{\circ}$. Described by Chamberlin and Salisbury, 6th Ann., p. 282. Analysis by R. B. Riggs, record No. 291. 
B, C, D, E. Tallow clays, Joplin, Missouri. Collected by W. P. Jenney. Analyses by T. M. Chatard, record No. 1210 .

F. Tallow clay, Aurora, Missouri. Collected by Jenney. Analysis by Chatard, No. 1210. In analyses B, C, D, E, and $\mathrm{F}$ the percentages of bases relate to the portion soluble in hydrochloric acid. Analyses only partial.

\begin{tabular}{|c|c|c|c|c|c|c|}
\hline & $\Lambda$. & B. & c. & D. & E. & F. \\
\hline Insoluble... & & 40.64 & 43. 07 & 39.34 & 39.62 & 34.04 \\
\hline $\begin{array}{l}\mathrm{SiO}_{2} \ldots \ldots \ldots \\
\mathrm{Al}_{2} \mathrm{O}_{3} \ldots \ldots\end{array}$ & $\begin{array}{l}7.46 \\
12.26\end{array}$ & 5.72 & 7.60 & 6.17 & 6.45 & 10.01 \\
\hline $\mathrm{Fe}_{2} \mathrm{O}_{3} .$. & 3. 25 & 1.30 & 1.12 & 1.16 & 1.53 & 3.62 \\
\hline $\begin{array}{ll}\mathrm{FeO} \\
\mathrm{MgO} . . .\end{array}$ & 1.12 & $.27 \quad \longrightarrow>0>0$ & .32 & .27 & 30 & \\
\hline $\begin{array}{ll}\mathrm{CaO} \\
\mathrm{CaO}\end{array}$ & 1.69 & 1.80 & 1.70 & 2. 13 & 1.77 & 2. 09 \\
\hline $\begin{array}{l}\mathrm{Na}_{2} \mathrm{O} \\
\mathrm{K}_{2} \mathrm{O}\end{array}$ & $\begin{array}{l}1.43 \\
1.83\end{array}$ & & & & & \\
\hline $\mathrm{H}_{2} \mathrm{O} \ldots \ldots \ldots \ldots \ldots \ldots \ldots$ & 2.70 & 17.19 & 16.74 & 17.63 & 16.95 & 16.96 \\
\hline $\begin{array}{l}\mathrm{TiO}_{2-} \\
\mathrm{P}_{2} \mathrm{O}_{5} \cdot\end{array}$ & $\begin{array}{l}.14 \\
.09\end{array}$ & & & & & \\
\hline $\begin{array}{l}\operatorname{MnO} \\
\mathrm{ZnO}\end{array}$ & .02 & 32.46 & 9043 & 34.28 & $33,55,>>>3$ & \\
\hline 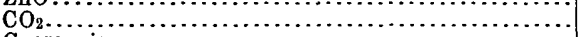 & .49 & & 29.45 & 34.28 & 50.30 & 33. 49 \\
\hline 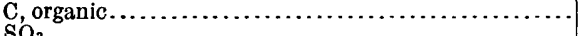 & .12 & $\cdots \cdot$ & ........ & ......... & $\cdots$. & ….. \\
\hline , & .05 & $\cdots \cdots$ & $\cdots \cdot$. & ........ & 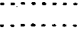 & \\
\hline- & 99.83 & 99.38 & 99.98 & 100.98 & 100.17 & 100.46 \\
\hline
\end{tabular}

The following partial analyses by H. N. Stokes, record No. 1260, all relate to tallow clays collected by W. P. Jenney. The same remarks apply as to B, C, D, E, and F.

G. Cave Springs mine, Jasper County, Missouri.

H. Great Western mine, Granby, Missouri.

I, J, K. Woodcock mine, Granby, Missouri.

L. Coon Hollow, Boone County, Arkansas.

Material dried at $103^{\circ}$.

\begin{tabular}{|c|c|c|c|c|c|c|}
\hline & G. & н. & I. & J. & K. & L. \\
\hline \multirow[t]{2}{*}{ 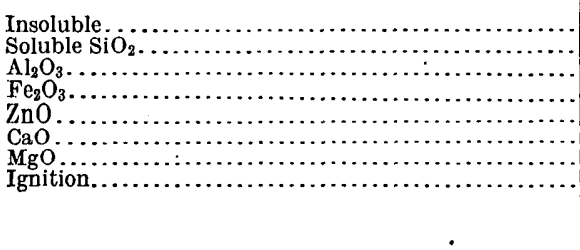 } & $\begin{array}{r}34.89 \\
16.75 \\
7.38 \\
10.34 \\
14.35 \\
1.55 \\
.35 \\
10.37\end{array}$ & $\begin{array}{r}11.25 \\
32.89 \\
10.78 \\
3.89 \\
29.54 \\
2.65 \\
.90 \\
8.22\end{array}$ & $\begin{array}{r}2.41 \\
36.71 \\
8.21 \\
2.75 \\
38.59 \\
2.77 \\
.78 \\
7.99\end{array}$ & $\begin{array}{r}16.17 \\
28.62 \\
8.93 \\
5.98 \\
26.23 \\
2.01 \\
.46 \\
9.19\end{array}$ & $\begin{array}{r}3.85 \\
37.08 \\
6.46 \\
3.49 \\
38.90 \\
2.56 \\
.42 \\
7.52\end{array}$ & $\begin{array}{r}18.18 \\
29.02 \\
6.34 \\
4.40 \\
30.50 \\
1.91 \\
.75 \\
8.36\end{array}$ \\
\hline & 95.98 & 100.12 & 100.21 & 97.59 & 100.28 & 99.46 \\
\hline
\end{tabular}

The following clays, $\mathrm{M}$ to $\mathrm{R}$, from the Hot Springs district, Arkansas, were received through E. C. Eckel. Analyses by G. Steiger, record No. 2221.

\begin{tabular}{|c|c|c|c|c|c|c|}
\hline & M. & N. & 0. & P. & $\mathrm{Q}$. & R. \\
\hline \multirow[t]{2}{*}{ 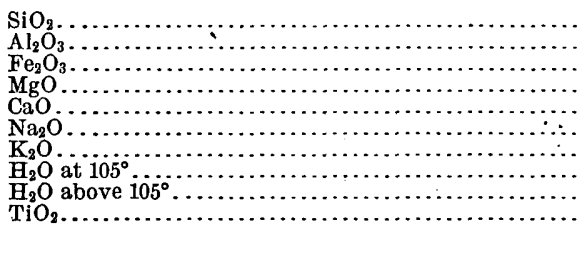 } & $\begin{array}{r}72.06 \\
15.31 \\
1.24 \\
2.26 \\
.17 \\
.15 \\
4.53 \\
-.48 \\
3.50 \\
.95\end{array}$ & $\begin{array}{r}74.55 \\
13.68 \\
1.27 \\
2.03 \\
.20 \\
.10 \\
3.84 \\
.77 \\
3.63 \\
.73\end{array}$ & $\begin{array}{r}73.96 \\
14.54 \\
2.17 \\
.49 \\
.15 \\
.80 \\
2.64 \\
1.36 \\
3.76 \\
.34\end{array}$ & $\begin{array}{r}70.31 \\
17.27 \\
1.85 \\
.91 \\
.23 \\
.26 \\
3.51 \\
.88 \\
4.36 \\
1.00\end{array}$ & $\begin{array}{r}70.29 \\
16.74 \\
2.03 \\
1.50 \\
.10 \\
.08 \\
2.76 \\
1.12 \\
5.00 \\
.64\end{array}$ & $\begin{array}{r}73.07 \\
16.40 \\
1.12 \\
.64 \\
.25 \\
.26 \\
2.75 \\
.36 \\
4.46 \\
1.09\end{array}$ \\
\hline & 100.65 & 100.80 & 100.21 & 100.58 & 100.26 & 100.40 \\
\hline
\end{tabular}




\section{SOUTH DAKOTA AND WYOMING.}

A. Red clay, east of Newcastle, South Dakota.

B. Red clayey sandstone, east of Spearfish, South Dakota. Analyses A and B by George Steiger, record No. 1854.

C. Loess, Cheyenne, Wyoming. Analysis by L. G. Eakins, record No. 1066.

\begin{tabular}{|c|c|c|c|}
\hline . & A. & B. & C. \\
\hline 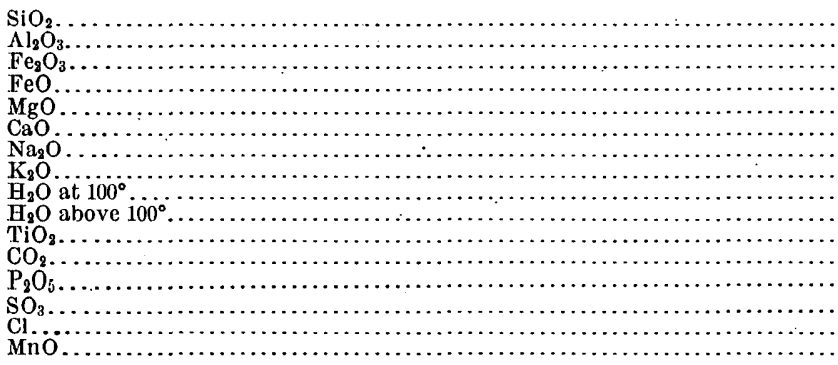 & $\begin{array}{r}56.20 \\
11.50 \\
3.64 \\
.65 \\
4.23 \\
5.83 \\
.98 \\
3.74 \\
1.61 \\
2.84 \\
.77 \\
5.72 \\
.12 \\
2.20 \\
\text { trace } \\
.10\end{array}$ & $\begin{array}{r}58.32 \\
8.59 \\
2.04 \\
.18 \\
3.65 \\
8.45 \\
.72 \\
2.71 \\
.52 \\
1.40 \\
.48 \\
12.08 \\
.05 \\
.43 \\
\text { trace } \\
.07\end{array}$ & $\begin{array}{r}67.10 \\
10.26 \\
2.52 \\
.31 \\
1.24 \\
5.88 \\
1.42 \\
2.68 \\
5.09 \\
\ldots \ldots .67 \\
\ldots \ldots \ldots \\
\ldots \ldots \ldots \\
\ldots \ldots \ldots .11 \\
\ldots \ldots \ldots\end{array}$ \\
\hline & 100.19 & 99.69 & 100.28 \\
\hline
\end{tabular}

COLORADO.

A. Loess, Denver.

B. Loess, Highland.

C. Concretion in loess, Wray.

A, B, and C collected by S. F. Emmons. Analyses by L. G. Eakins, record No. 1066.

D. Clay, Davis ranch, Pueblo quadrangle.

E. Clay, head of Rock Creek, Pueblo quadrangile.

D and E collected by G. K. Gilbert. Analyses by George Steiger, record No. 1457.

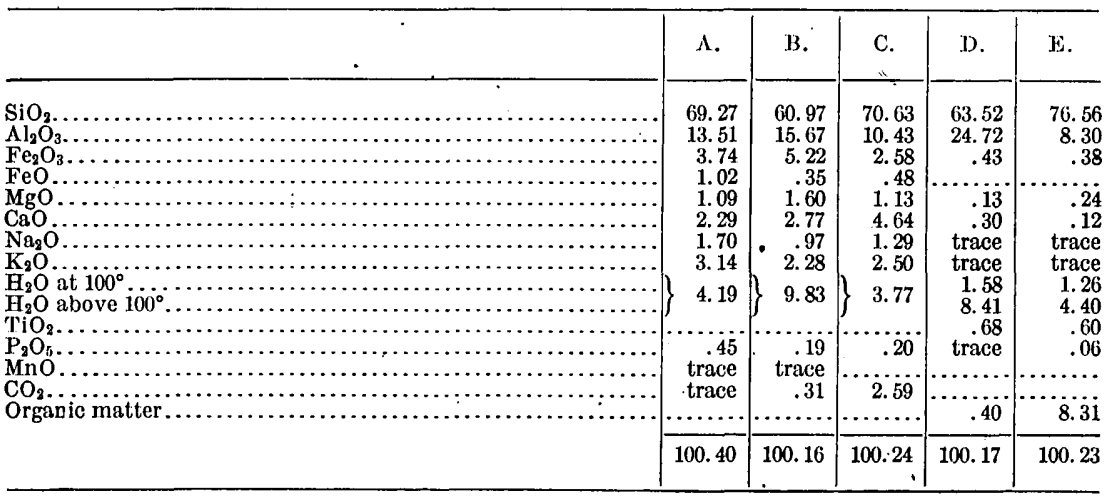


F. From Red Creek canyon, southern part of Colorado Springs quadrangle.

G. From 2 miles southeast of $F$.

H. From near Canyon.

I. Overlying $\mathrm{H}$.

Collected as probable fire clays by G. K. Gilbert. Analyses by George Steiger, record No. 1578. $\mathrm{Fe}_{2} \mathrm{O}_{3}$ represents total iron. $\mathrm{Al}_{2} \mathrm{O}_{3}$ includes $\mathrm{TiO}_{2}$. In $\mathrm{I}$ the ignition includes some $\mathrm{CO}_{2}$, which is absent from the others.

\begin{tabular}{|c|c|c|c|c|}
\hline & F. & G. & $\mathrm{H}$. & I. \\
\hline 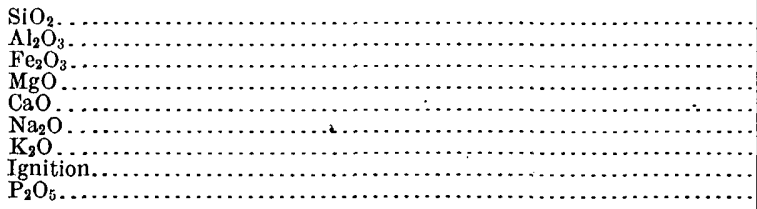 & $\begin{array}{r}85.09 \\
6.98 \\
1.10 \\
.27 \\
.21 \\
\text { none } \\
.13 \\
6.37 \\
.06\end{array}$ & $\begin{array}{r}86.79 \\
8.29 \\
.75 \\
.13 \\
.34 \\
\text { none } \\
.25 \\
3.78 \\
.05\end{array}$ & $\begin{array}{r}57.98 \\
27.51 \\
1.68 \\
.32 \\
.42 \\
.03 \\
.56 \\
11.80 \\
.06\end{array}$ & $\begin{array}{r}69.04 \\
14.51 \\
3.78 \\
.73 \\
1.24 \\
.08 \\
.48 \\
10.50 \\
.07\end{array}$ \\
\hline - & 100.21 & 100.38 & 100.36 & 100.43 \\
\hline
\end{tabular}

$\mathrm{J}$ to R. Supposed fire clays collected in the area of the Apishapa sheet, by G. K. Gilbert. Analyses, partial, by H. N. Stokes, record No. 1503. Titanium present, alkalies undetermined. Analyses made on ignited material, reckoned as 100 . The loss on ignition is separately stated below each analysis.

\begin{tabular}{|c|c|c|c|c|c|c|c|c|c|}
\hline & J. & K. & L. & M. & N. & o. & P. & Q. & $\mathrm{R}$. \\
\hline 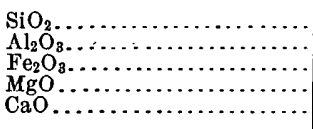 & $\begin{array}{r}86.58 \\
12.72 \\
.45 \\
.11 \\
.11\end{array}$ & $\begin{array}{r}78.07 \\
20.22 \\
.89 \\
.26 \\
\cdots . . .\end{array}$ & $\begin{array}{r}76.96 \\
20.77 \\
1.11 \\
.32 \\
.71\end{array}$ & $\begin{array}{r}61.98 \\
37.51 \\
.45 \\
.09 \\
.19\end{array}$ & $\begin{array}{r}93.11 \\
5.56 \\
1.15 \\
.10 \\
.32\end{array}$ & $\begin{array}{r}85.98 \\
13.67 \\
.41 \\
\cdots .21 \\
.21\end{array}$ & $\begin{array}{r}85.25 \\
11.45 \\
2.24 \\
.21 \\
.26\end{array}$ & $\begin{array}{r}54.93 \\
43.65 \\
.69 \\
.05 \\
.64\end{array}$ & $\begin{array}{r}58.56 \\
39.17 \\
.55 \\
.45 \\
1.08\end{array}$ \\
\hline Ignition.................... & $\begin{array}{r}98.97 \\
4.75\end{array}$ & $\begin{array}{c}99.44^{\circ} \\
7.51\end{array}$ & $\begin{array}{r}99.87 \\
7.98\end{array}$ & $\begin{array}{r}100.22 \\
12.51\end{array}$ & $\begin{array}{r}100.24 \\
4.45\end{array}$ & $\begin{array}{r}100.27 \\
5.07\end{array}$ & $\begin{array}{r}99.41 \\
4.81\end{array}$ & $\begin{array}{l}99.96 \\
16.80\end{array}$ & $\begin{array}{l}99.81 \\
19.58\end{array}$ \\
\hline
\end{tabular}

S. Loesslike alluvium, Golden, Jefferson County.

T. Fire clay, Golden, Jefferson County.

Analyses S and T made by W. F. Hillebrand in the Denver laboratory.

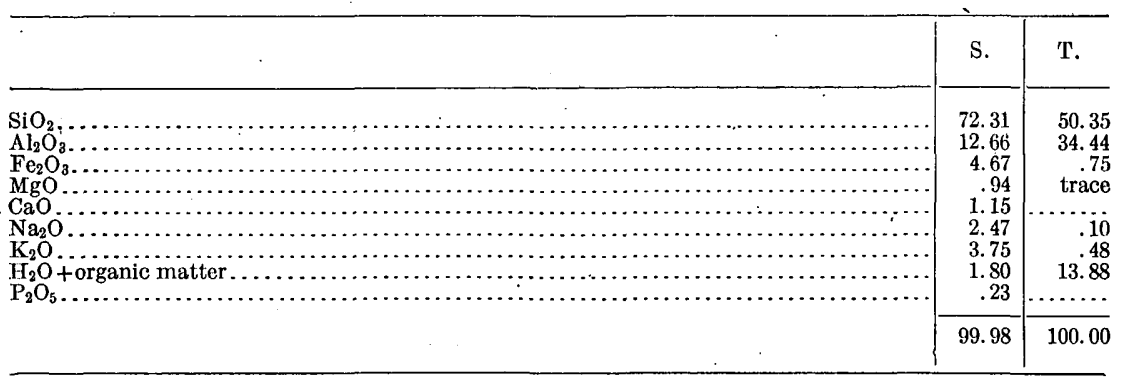




\section{IDAHO AND UTAH.}

A. Adobe soil, Salt Lake City, Utah. Analysis by L. G. Eakins, record No. 996.

B. Lava soil, near Shoshone Falls, Idaho. Described by Russell in Bull. 199. Analysis by W. F. Hillebrand, record No. 1950.

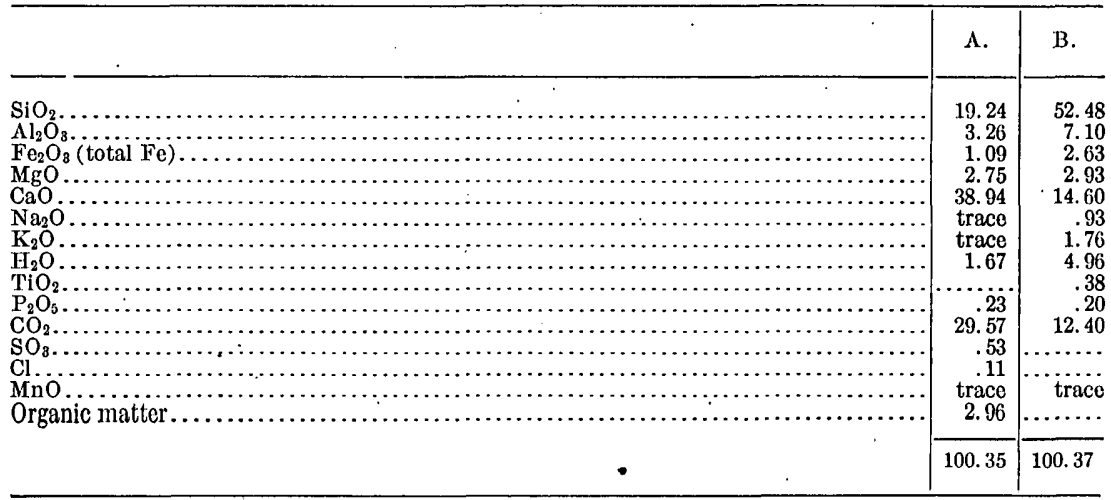

\section{NEW MEXICO AND ARIZONA.}

A. Adobe soil, Santa Fe, New Mexico.

B. Adobe soil, Fort Wingate, New Mexico.

Analyses A and B by L. G. Eakins, record No. 981.

C. Clay, Salt River Valley, Arizona.

D. Clay, about 1 mile from $\mathrm{C}$.

Analyses C, D, by E. T. Allen, record Nos. 1945, 1959.

\begin{tabular}{|c|c|c|c|c|}
\hline . & A. & B. & C. & D. \\
\hline 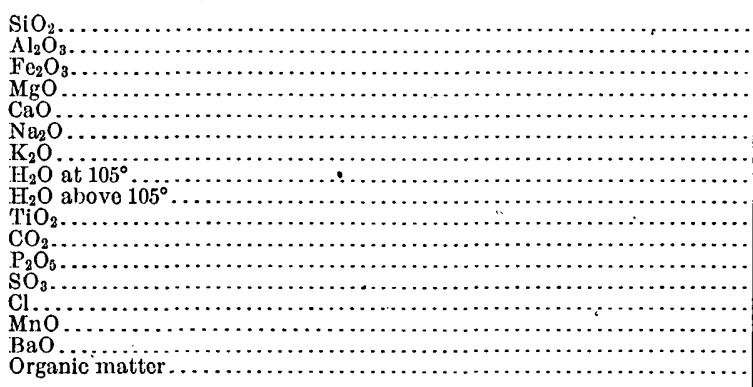 & $\begin{array}{r}66.69 \\
14.16 \\
4.38 \\
1.28 \\
2.49 \\
.67 \\
1.21 \\
4.94 \\
\ldots . .77 \\
.77 \\
.29 \\
.41 \\
.34 \\
.09\end{array}$ & $\begin{array}{r}26.67 \\
.91 \\
.64 \\
.51 \\
36.40 \\
\text { trace } \\
\text { trace } \\
2.26\end{array}$ & 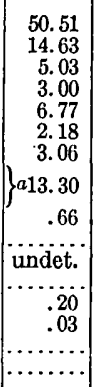 & $\begin{array}{r}50.55 \\
14.89 \\
4.98 \\
2.91 \\
4.82 \\
4.12 \\
3.19 \\
4.75 \\
6.00 \\
.58 \\
2.81 \\
\text { none } \\
\text { trace } \\
.05\end{array}$ \\
\hline 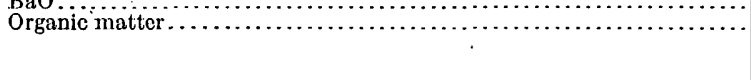 & .99 .72 & 99.97 & 99.37 & 99.65 \\
\hline
\end{tabular}

$a$ Loss on ignition.

15619-Bull. $419-10-16$ 


\section{NEVADA.}

A. Grayish clay from upper Lahontan lake beds, Humboldt River bridge, Mill City.

B. Grayish clay, lower Lahontan beds, same locality. Analyses by T. M. Chatard, record Nos. $32,33$.

C. Adobe soil, Humboldt. Analysis by T. G. Eakins, record No. 981.

\begin{tabular}{|c|c|c|c|}
\hline . & A. & B. & C. \\
\hline 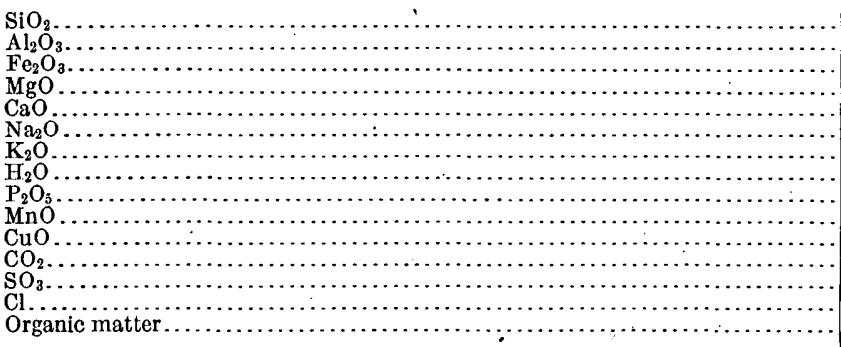 & 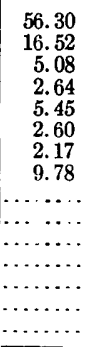 & 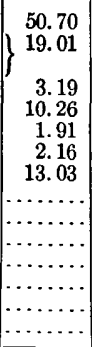 & $\begin{array}{r}44.64 \\
13.19 \\
5.12 \\
2.96 \\
13.91 \\
.59 \\
1.71 \\
3.89 \\
.94 \\
.13 \\
.95 \\
8.55 \\
.64 \\
.14 \\
3.43\end{array}$ \\
\hline & 100.54 & 100.26 & 99.84 \\
\hline
\end{tabular}

CALIFORNIA, WASHINGTON, HAWÄIIAN ISLANDS.

A. Sandy clay, Owens Lake, California.

B. Blue clay, Owens Lake, California. Analyses by T. M. Chatard, record No. 551.

C. Clay from foot of Rickey Hill, Kittle Falls, Stevens County, Washington. Analysis by W. F. Hillebrand, record No. 1428.

D. Typical wheat soil, plateau south of Krupp, Washington.

E. Residuary soil from basalt, Hausen Creek, Kittitas County, Washingtor.. Analy: ses $\mathrm{D}$ and $\mathrm{E}$ by George Steiger, record No. 2028.

F. Lava soil, Diamond Head, Hawaiian Islands. Analysis by L. G. Eakins, record No. 888.

\begin{tabular}{|c|c|c|c|c|c|c|}
\hline & A. & B. & C. & D. & E. & F. \\
\hline 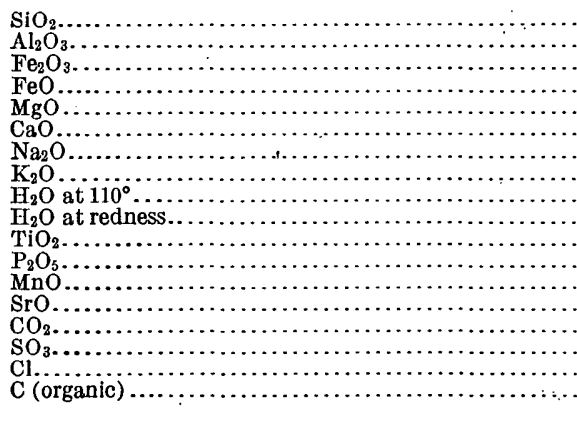 & $\begin{array}{r}53.24 \\
10.84 \\
2.59 \\
.77 \\
5.82 \\
9.18 \\
2.06 \\
2.64 \\
1.41 \\
2.73 \\
.25 \\
.10 \\
.7 . \\
8.75 \\
.08 \\
.05 \\
\cdots \cdots \\
\end{array}$ & $\begin{array}{r}54.92 \\
11.25 \\
2.77 \\
.94 \\
4.91 \\
8.76 \\
2.10 \\
2.77 \\
2.05 \\
2.40 \\
.30 \\
.08 \\
7.24 \\
\text { trace } \\
\text { trace } \\
\end{array}$ & \begin{tabular}{r}
62.74 \\
16.45 \\
2.62 \\
1.91 \\
2.41 \\
3.68 \\
3.05 \\
3.53 \\
2.69 \\
$\ldots \ldots \ldots$ \\
\hdashline$\ldots \ldots$ \\
trace \\
tarce \\
.65 \\
$\ldots \ldots \ldots$ \\
$\ldots \ldots .$.
\end{tabular} & 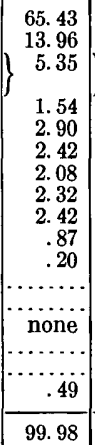 & 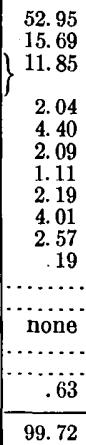 & 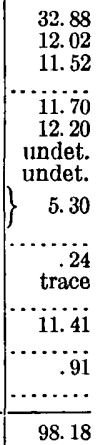 \\
\hline
\end{tabular}




\section{OCEANIC CLAYS.}

Composite analyses of sediments collected by various exploring expeditions. Material contributed by Sir John Murray. The larger number of samples were collected by the Challenger expedition.

A. The red clay. Composite of 51. samples; dredged from the sea bottom in all the great oceans.

B. The portion of A soluble in water. Analyses by G. Steiger, record No. 2300. Additional determinations by Hillebrand revealed the presence in the clay of traces of molybdenum, and E. C. Sullivan also found the following percentages of heavy metals: $\mathrm{CuO}, 0.02 ; \mathrm{PbO}, 0.007 ; \mathrm{ZnO}, 0.004 ; \mathrm{As}_{2} \mathrm{O}_{3}, 0.0007$.

C. Terrigenous clays, dredged at depths from 140 to 2,120 fathoms. Composite of 52 samples.

D. The portion of $\mathrm{C}$ soluble in water. Analyses by Steiger, No. 2323. Analyses $B, D$, represent merely adherent sea salts.

\begin{tabular}{|c|c|c|c|c|}
\hline & A. & B. & C. & J. \\
\hline 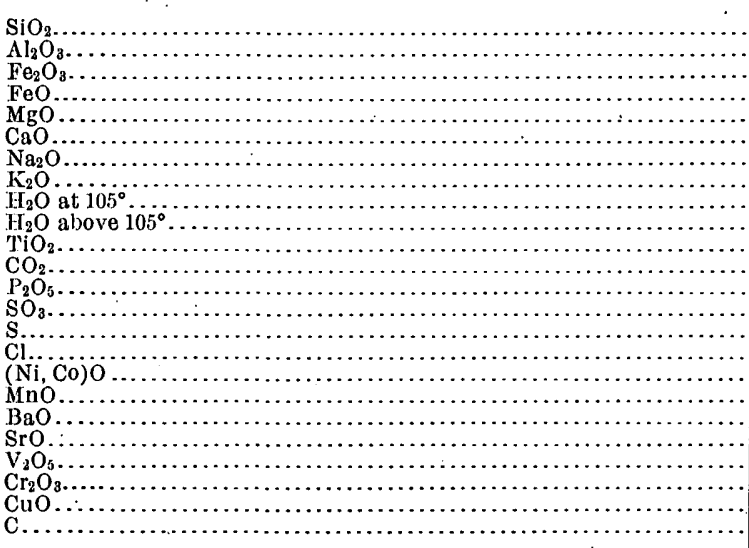 & $\begin{array}{c}45.32 \\
13.26 \\
7.20 \\
.70 \\
3.05 \\
6.82 \\
3.63 \\
2.43 \\
3.28 \\
5.93 \\
.82 \\
3.91 \\
.25 \\
.48 \\
\text { trace? } \\
2.77 \\
.032 \\
.83 \\
.17 \\
.046 \\
.028 \\
.01 \\
.02\end{array}$ & 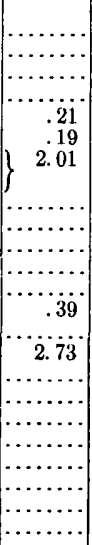 & $\begin{array}{c}46.64 \\
14.08 \\
4.14 \\
1.88 \\
1.95 \\
7.20 \\
2.98 \\
1.84 \\
4.73 \\
5.86 \\
1.04 \\
4.05 \\
.17 \\
.32 \\
.11 \\
2.25 \\
\ldots .10 \\
.10 \\
.05 \\
.025 \\
.028 \\
.044 \\
.016 \\
1.38\end{array}$ & 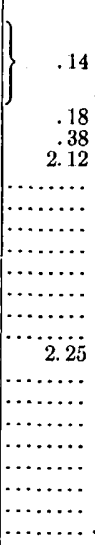 \\
\hline \multirow{2}{*}{ 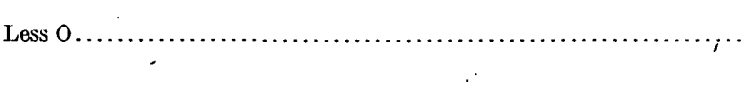 } & $\begin{array}{c}100.986 \\
.62\end{array}$ & $\begin{array}{r}5.53 \\
\ldots \ldots \ldots \\
\end{array}$ & $\begin{array}{c}100.883 \\
.56\end{array}$ & 5.07 \\
\hline & 100.366 & & 100.323 & \\
\hline
\end{tabular}




\section{ANALYSES OF METEORITES.}

\section{STONY METEORITES.}

\section{ROCKWOOD, TENNESSEE.}

From the Crab Orchard Mountains, Cumberland County, about $8 \frac{1}{2}$ miles west of Rockwood. Analyzed by J. E. Whitfield, record No. 735, and described by him in Bull. 60, p. 103.

A. Analysis of the material as a whole.

B. Nickel-iron separated. Forms not over 16 per cent of the mass.

C. Nodule from meteorite.

D. Portion of nodule, 94 per cent, insoluble in hydrochloric acid. May be enstatite.

The stony part of the meteorite appears to be mainly pyroxene and anorthite. $\mathrm{Fe}_{2} \mathrm{O}_{3}$ was not determined, and $\mathrm{FeO}$ represents the total iron oxide.

\begin{tabular}{|c|c|c|c|c|}
\hline & A. & B. & c. & D. \\
\hline 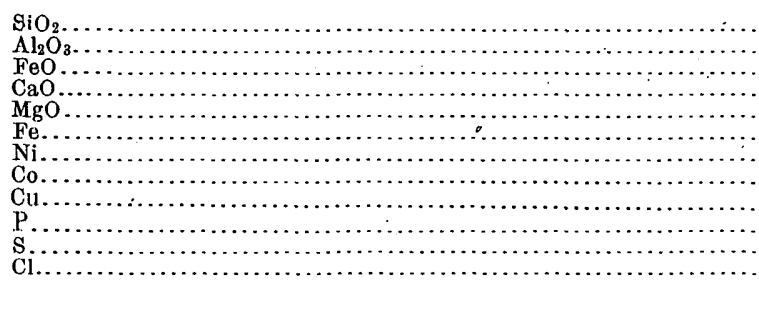 & $\begin{array}{r}41.92 \\
9.27 \\
22.94 \\
9.09 \\
8.76 \\
3.75 \\
1.74 \\
\text { trace } \\
\text { trace } \\
.65 \\
1.58 \\
.18 \\
\end{array}$ & 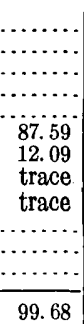 & \begin{tabular}{r}
49.96 \\
4.75 \\
15.97 \\
1.15 \\
28.15 \\
$\ldots \ldots \ldots .$. \\
\hdashline$\ldots \ldots . .$. \\
\hdashline$\ldots \ldots .$. \\
\hdashline$\ldots \ldots .$. \\
\hdashline$\ldots \ldots .$. \\
99.98 \\
\end{tabular} & 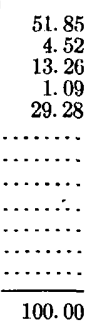 \\
\hline
\end{tabular}

\section{HAMBLEN COUNTY, TENNESSEE.}

Mass of nearly half and half stone and iron found about 6 miles WSW. of Morristown. Analyzed by L. G. Eakins, and described in Bull. 113, p. 61.

A. The nickel-iron.

B. The part of the stony portion soluble in hydrochloric acid; 37.63 per cent, recalculated to 100, with sulphur deducted.

C. Insoluble part of the stony portion; 62.10 per cent, recalculated to 100 .

A petrographic description of this meteorite is given by Merrill in Am. Jour. Sci., 4th ser., vol. 2, p. 149, together with an analysis of the feldspar. He finds it to contain in addition to the nickel-iron, enstatite, diallage, anorthite, olivine or monticellite, oldhamite or secondary gypsum derived from oldhamite, lawrenceite, troilite, and schreibersite. 


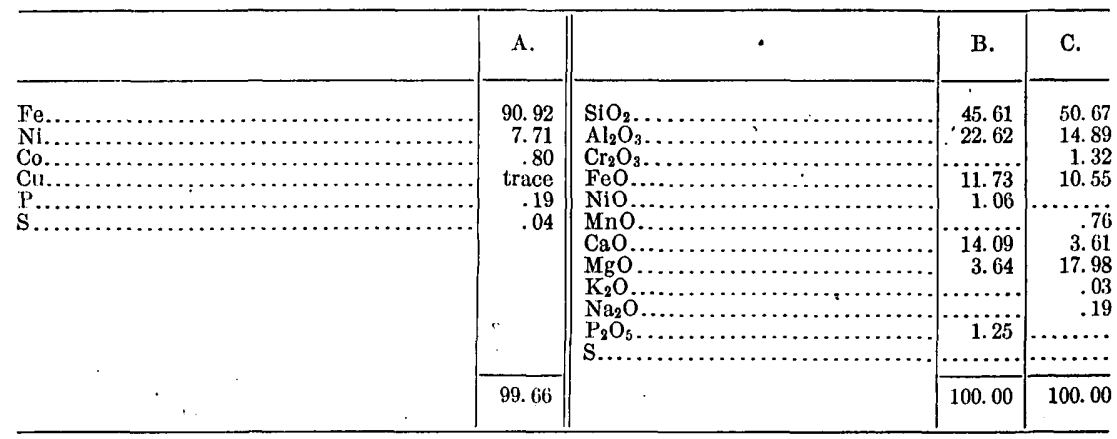

\section{ALLEGAN, MICHIGAN.}

Fell July 10, 1899, on Thomas Hill, Allegan. Analyses by H. N. Stokes, record No. 1856. Described by Merrill and Stokes in Proc. Washington Acad. Sci., vol. 2, p. 41. Sp. gr., 3.905 at $27^{\circ}$, Merrill.

A. Composition of the meteorite as a whole.

B. The metallic portion, analyzed separately.

C. Pàrtial analysis of separated chromite.

D. Composition of the stony portion.

E. Stony material soluble in hydrochloric acid.

F. Stony material insoluble in hydrochloric acid.

The stony matter of the meteorite consisted mainly of olivine and enstatite, and amounted to.76.94 per cent. The metallic portion formed 23.06 per cent. Troilite was present and appears in the analysis of the stony portion.

\begin{tabular}{|c|c|c|c|c|c|c|}
\hline . & A. & B. & C. & D. & E. & $\mathrm{F}$. \\
\hline 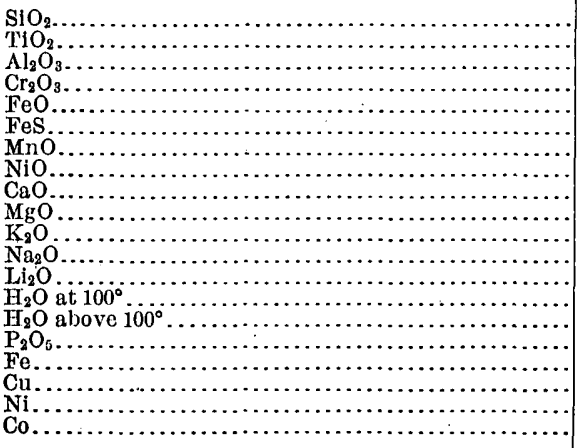 & $\begin{array}{r}34.95 \\
.08 \\
2.55 \\
.53 \\
8.47 \\
5.05 \\
.18 \\
\operatorname{trace} \\
1.73 \\
21.99 \\
.23 \\
.66 \\
\operatorname{trace} \\
.06 \\
.19 \\
.27 \\
21.09 \\
.01 \\
1.81 \\
.15\end{array}$ & 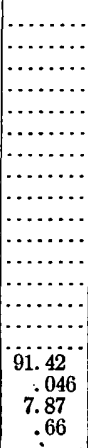 & 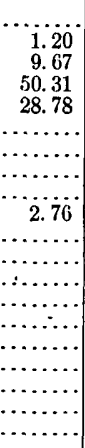 & $\begin{array}{r}45.42 \\
.10 \\
3.31 \\
.69 \\
11.02 \\
6.57 \\
.23 \\
\operatorname{trace} \\
2.24 \\
28.60 \\
.30 \\
.86 \\
\operatorname{trace} \\
.07 \\
.24 \\
.35 \\
\ldots \ldots . . \\
\ldots \ldots \ldots \\
\ldots \ldots \ldots . \\
\ldots \ldots . . .\end{array}$ & 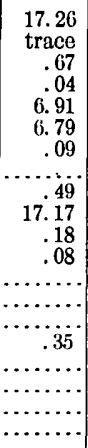 & 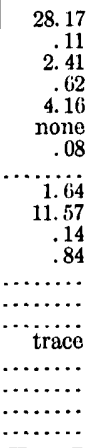 \\
\hline & 100.00 & 99.996 & & 100.00 & 50.03 & 99.74 \\
\hline
\end{tabular}

4. WINNEBAGO COUNTY, IOWA.

Fell May 2, 1890. Sp. gr., 3.804, $28^{\circ} .5$. Analyzed by I. G. Eakins, record No. 1190 , and described in Bull. 78, p. 95. 
Composition of the mass.

Nickel-iron ...................................... 19.40

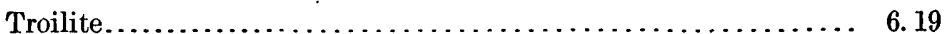

Soluble silicates................................... 36.04

Insoluble silicates................................... 37

100.00

Separate analyses:

A. The nickel iron

B. Silicate soluble in hydrochloric acid, calculated to 100 per cent.

C. Insoluble silicate, recalculated to 100 per cent. The $\mathrm{Cr}_{2} \mathrm{O}_{3}$ probably represents chromite.

\begin{tabular}{|c|c|c|c|c|}
\hline & A. & & B. & c. \\
\hline \multirow[t]{2}{*}{ 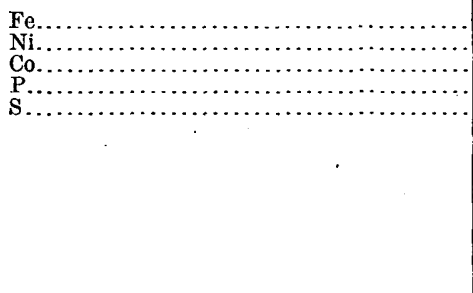 } & $\begin{array}{r}92.65 \\
6.11 \\
.65 \\
\text { trace } \\
\text { trace }\end{array}$ & 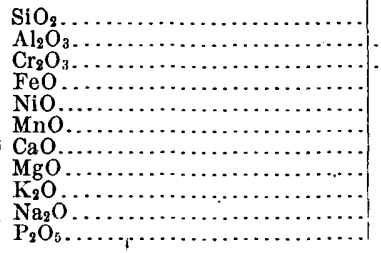 & $\begin{array}{c}39.74 \\
\cdots .32 . \\
18.38 \\
\text { trace } \\
.69 \\
40.77 \\
\text { trace } \\
\text { trace } \\
\text { trace }\end{array}$ & $\begin{array}{r}55.51 \\
5.43 \\
.25 \\
9.45 \\
7.0 . \\
3.00 \\
24.09 \\
.15 \\
2.12 \\
\end{array}$ \\
\hline & 99.41 & & 100.00 & 100.00 \\
\hline
\end{tabular}

\section{TANEY COUNTY, MISSOURI.}

Analysis by J. E. Whitfield, record No. 736. See Bull: 60, p. 106. Sp. gr., 4.484.

A. The separated nickel-iron.

B. The stony portion as a whole.

C. Silicates soluble in hydrochloric acid, recalculated to 100 per cent.

D. Insoluble silicates, recalculated to 100 per cent.

\begin{tabular}{|c|c|c|c|c|c|}
\hline • & A. & & B. & C. & D. \\
\hline \multirow[t]{2}{*}{ 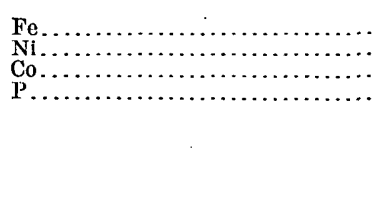 } & $\begin{array}{r}89.41 \\
10.41 \\
.29 \\
.16\end{array}$ & \multirow[t]{2}{*}{ 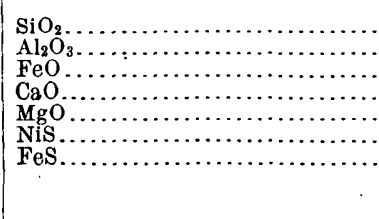 } & $\begin{array}{r}45.88 \\
7.89 \\
19.73 \\
6.02 \\
17.96 \\
1.67 \\
.54\end{array}$ & $\begin{array}{r}26.95 \\
17.69 \\
35.98 \\
15.98 \\
3.40 \\
\cdots \cdots \\
\cdots\end{array}$ & $\begin{array}{r}52.39 \\
7.11 \\
14.68 \\
4.49 \\
21.33 \\
\cdots \cdots\end{array}$ \\
\hline & 100.27 & & 99.69 & 100.00 & 100.00 \\
\hline
\end{tabular}

\section{WASHINGTON COUNTY, KANSAS.}

Fell July 25, 1890. Analyzed by L. G. Eakins, record No. 1227, and described in Bull. 90, p. 45. Sp. gr., 3.49, 21.6

\section{Composition of the mass.}

Nickel-iron

Troilite.

Soluble silicates.

Insoluble silicates. 
Separate analyses.

A. The nickel-iron.

B. Silicates soluble in hydrochloric acid, calculated to 100 per cent.

C. Insoluble silicates, calculated to 100 per cent.

\begin{tabular}{|c|c|c|c|c|}
\hline & A. & & $B$. & C. \\
\hline \multirow{7}{*}{ 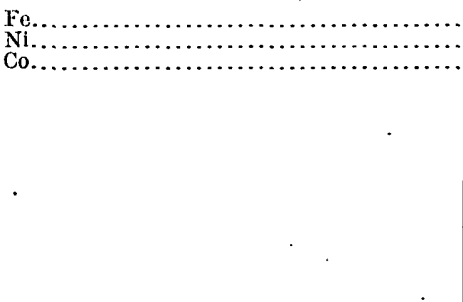 } & 86.76 & \multirow{7}{*}{ 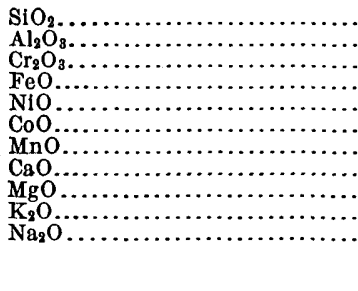 } & 38.50 & 53.80 \\
\hline & $\begin{array}{r}12.18 \\
.83\end{array}$ & & $\ldots \ldots \ldots$ & $\begin{array}{l}4.32 \\
1.41\end{array}$ \\
\hline & & & $\begin{array}{r}23.54 \\
69\end{array}$ & 11.98 \\
\hline & & & $\begin{array}{r}\text { trace } \\
.34\end{array}$ & trace \\
\hline & & & $\begin{array}{r}.12 \\
36.81\end{array}$ & $\begin{array}{r}4.08 \\
22.37\end{array}$ \\
\hline & & & $\begin{array}{l}\cdots \cdots \cdots \\
\cdots \cdots \cdots\end{array}$ & $\begin{array}{r}.27 \\
1.77\end{array}$ \\
\hline & 99.77 & & 100.00 & 100.00 \\
\hline
\end{tabular}

\section{KIOWA COUNTY, KANSAS.}

A pallasite found in Brenham Township. Analyzed by I. G. Eakins, record No. 1.188, and described in Bull. 78, p. 94.

A. The nickei-iron. Sp. gr., $7.93,23.4^{\circ}$.

B. The pure olivine. Sp. gr., $3.376,23.2^{\circ}$.

C. Dark outer zone of olivine, containing troilite.

\begin{tabular}{|c|c|c|c|c|}
\hline & A. & & B. & C. \\
\hline \multirow[t]{3}{*}{ 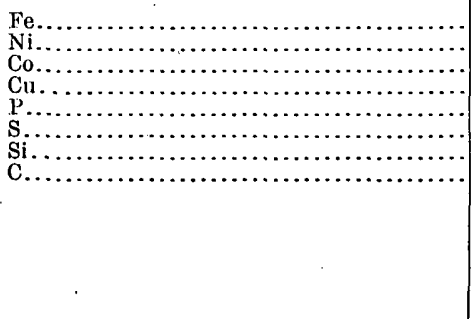 } & \multirow[t]{2}{*}{$\begin{array}{r}88.49 \\
10.35 \\
.57 \\
.03 \\
.14 \\
.08 \\
\text { trace? } \\
\text { trace }\end{array}$} & \multirow{3}{*}{ 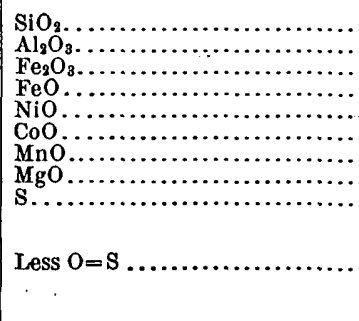 } & $\begin{array}{r}40.70 \\
\text { trace? } \\
\cdot \quad .18 \\
10.79 \\
.02 \\
. .14 \\
48.02 \\
\ldots \ldots .\end{array}$ & $\begin{array}{c}34.14 \\
\cdots \ldots \ldots \\
23.20 \\
\text { trace } \\
.03 \\
.09 \\
40.19 \\
.542\end{array}$ \\
\hline & & & $\begin{array}{r}99.85 \\
\ldots \ldots \ldots\end{array}$ & $\begin{array}{r}103.07 \\
2.71\end{array}$ \\
\hline & 99.66 & & & 100.36 \\
\hline
\end{tabular}

\section{TRAVIS COUNTY, TEXAS.}

Analyzed by L. G. Eakins, record No. 1097, and described in Bull. 78, p. 91 . Sp. gr., $3.543,20^{\circ}$. According to Cross, the stony portion contains olivine and enstatite, with a small amount of a colorless mineral, which is probably feldspar. Chromite is also present.

Approximate composition of the mass.

Nickel-iron.................................... 2.23

Troilite..................................... 5.03

Soluble silicates................................. 39.84

Insoluble silicates................................ 52.42

99.52

A. Total analysis.

B. Nickel-iron. 
C. Silicates soluble in hydrochloric acid, calculated to 100 per cent.

D. Insoluble silicates, calculated to 100 per cent.

\begin{tabular}{|c|c|c|c|c|}
\hline ' & A. & B. & C. & D. \\
\hline 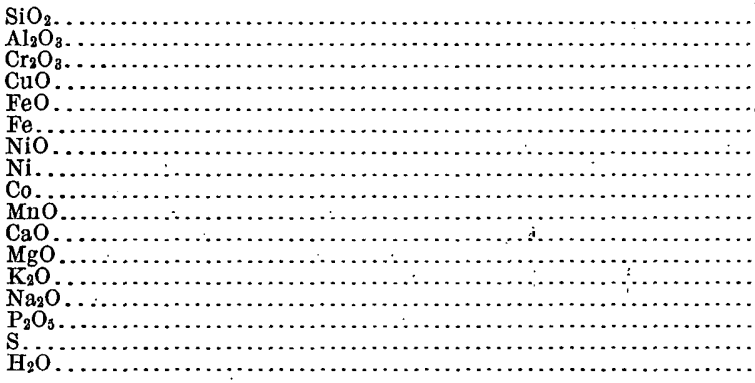 & $\begin{array}{r}44.75 \\
2.72 \\
.52 \\
\operatorname{trace} \\
16.04 \\
1.83 \\
.52 \\
.22 \\
.01 \\
\operatorname{trace} \\
2.23 \\
27.93 \\
.13 \\
1.13 \\
.41 \\
1.83 \\
.84\end{array}$ & 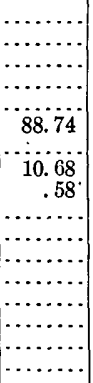 & 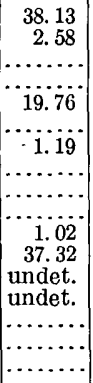 & 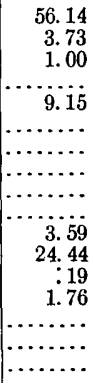 \\
\hline \multirow[t]{2}{*}{ 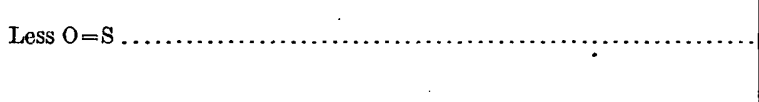 } & $\begin{array}{r}101.11 \\
.92\end{array}$ & $\begin{array}{c}100.00 \\
\ldots \ldots \ldots\end{array}$ & $\begin{array}{c}100.00 \\
\ldots \ldots \ldots\end{array}$ & $\begin{array}{r}100.00 \\
\ldots \ldots\end{array}$ \\
\hline & 100.19 & & & \\
\hline
\end{tabular}

\section{BLUFF, FAYETTE COUNTY, TEXAS.}

Analyzed by J. E. Whitfield, record No. 824, and described in Bull. 60, p. 107. Sp. gr., 3.510. Examined microscopically by G. P. Merrill, who reports, in addition to nickel-iron and pyrrhotite, olivine, enstatite, and what appears to be augite or an allied pyroxene. See Am. Jour. Sci., 3d ser., vol. 36, August, 1888.

A. Total analysis.

B. Nickel-iron (5.67 per cent of total).

C. Part soluble in hydrochloric acid, calculated to 100 per cent (60.62 per cent of total).

D. Insoluble part, calculated to 100 per cent ( 33.3 per cent of total).

\begin{tabular}{|c|c|c|c|c|}
\hline & A. & B. & C. & D. \\
\hline $\begin{array}{l}\mathrm{SiO}_{2} \\
\mathrm{Al}_{2} \mathrm{O}_{3}\end{array}$ & $\begin{array}{r}37.70 \\
2.17 \\
23.82 \\
4.41 \\
1.59 \\
.88 \\
.16 \\
.37 \\
.45 \\
2.20 \\
25.94 \\
.25 \\
1.30\end{array}$ & 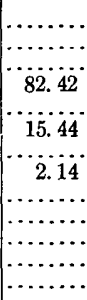 & $\begin{array}{r}33.59 \\
1.34 \\
31.12 \\
2.66 \\
.27 \\
.27 \\
.43 \\
1.00 \\
28.08 \\
.42 \\
2.18\end{array}$ & \begin{tabular}{r}
49.64 \\
4.12 \\
15.56 \\
$\ldots \ldots \ldots$ \\
trace \\
trace \\
\hdashline$\ldots .54$ \\
4.93 \\
25.21 \\
$\ldots \ldots \ldots$.
\end{tabular} \\
\hline Less $\mathrm{O}=\mathrm{S} \ldots \ldots \ldots \ldots \ldots \ldots \ldots \ldots \ldots \ldots \ldots \ldots$ & $\begin{array}{r}101.24 \\
.65\end{array}$ & $\begin{array}{l}100.00 \\
\cdots \cdots \cdots\end{array}$ & $\begin{array}{r}101.09 \\
1.09\end{array}$ & $\begin{array}{r}100.00 \\
\ldots \ldots \ldots\end{array}$ \\
\hline . & 100.59 & & 100.00 & \\
\hline
\end{tabular}

This meteorite also contained a dark vein of specific gravity 3.585 , which carried 2.30 per cent of metallic iron. Analyses, made on less than 0.4 gram of material, gave as follows, recalculated to 100 per cent:

E. Soluble in hydrochloric acid, metal deducted.

F. Insoluble. 


\begin{tabular}{|c|c|c|}
\hline & E. & F. \\
\hline 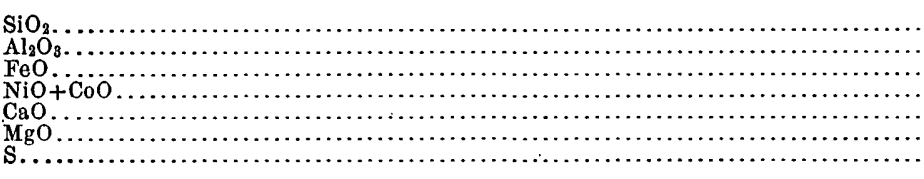 & $\begin{array}{r}27.63 \\
2.41 \\
34.31 \\
3.27 \\
\text { trace } \\
32.12 \\
.52\end{array}$ & $\begin{array}{r}56.52 \\
1.51 \\
12.35 \\
4.09 \\
\text { trace } \\
25.53 \\
7\end{array}$ \\
\hline 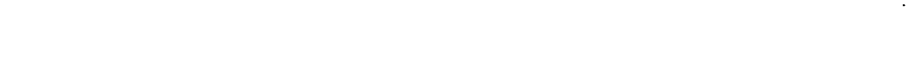 & $\begin{array}{r}100.26 \\
.26\end{array}$ & $\begin{array}{r}100.00 \\
\ldots \ldots\end{array}$ \\
\hline Less $\mathrm{C}=\mathrm{S} \ldots \ldots$ & 100.00 & $\ldots \ldots \ldots$ \\
\hline
\end{tabular}

$\mathrm{E}$ represents 51 per cent and $\mathrm{F} 44$ per cent of the vein.

\section{SAN BERNARDINO COUNTY, CALIFORNIA.}

Found in the San Emigdio Mountains. Analyzed by J. E. Whitfield, record Nos. 804 and 936, and described in Bull. 60, p. 11.4. In fragments, badly altered.

\section{Approximate composition of the mass.}

Nickel-iron

Soluble silicates, etc

Insoluble silicates. 42.23

The soluble part was probably olivine and pyrrhotite, with secondary iron oxide. The insoluble part was enstatite, essentially.

A. The nickel-iron.

B. The enstatite.

\begin{tabular}{|c|c|c|c|}
\hline & A. & & B. \\
\hline \multirow[t]{2}{*}{ 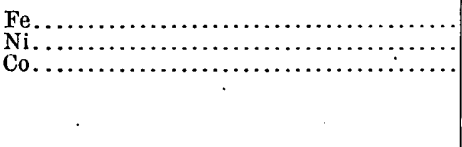 } & $\begin{array}{r}88.25 \\
11.27 \\
.48\end{array}$ & \multirow{2}{*}{ 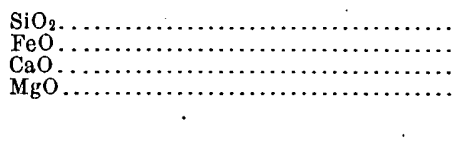 } & $\begin{array}{r}54.42 \\
14.03 \\
2.46 \\
29.11\end{array}$ \\
\hline & 100.00 & & 100.02 \\
\hline
\end{tabular}

\section{BEAVER CREEK, BRITISH COLUMBTA.}

Fell May 26, 1893, near Beaver Creek, West Kootenai district. Déscribed by Howell, Hillebrand, and Merrill in Am. Jour. Sci., 3d ser., vol. 47, p. 430.

\section{Composition of the mass.}

Nickel-iron

Magnetite.

Troilite

Soluble silicates and phosphate.

Insoluble silicates and chromite. 40.43 
According to Merrill, the silicates visible are olivine, enstatite, probably a little plagioclase, and some glassy base.

Analyses by W. F. Hillebrand, record No. 1444.

A. Nickel-iron.

B. Nonmagnetic, stony portion.

C. Portion of B soluble in hydrochloric acid, calculated to 100 per cent.

D. Insoluble portion, calculated to 100 per cent. From $\mathrm{C}$ and D troilite and chromite are excluded. The chromite forms about 0.75 per cent of the stony matter.

\begin{tabular}{|c|c|c|c|c|c|}
\hline & A. & & B. & c. & D. \\
\hline \multirow[t]{2}{*}{$\begin{array}{l}\mathrm{Fe} . \ldots . \\
\mathrm{Ni} . \ldots . \\
\mathrm{Co} \ldots \ldots \\
\mathrm{Cu} . . . \\
\\
\end{array}$} & $\begin{array}{r}90.68 \\
8.80 \\
.49 \\
.03\end{array}$ & \multirow{2}{*}{ 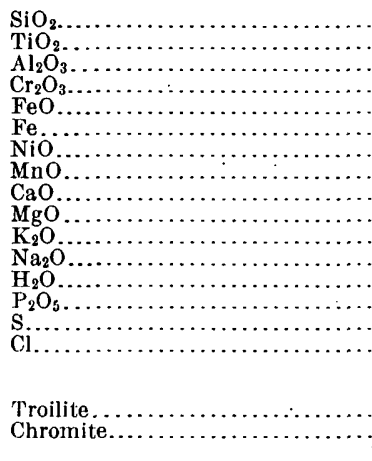 } & $\begin{array}{r}45.87 \\
.09 \\
2.30 \\
.51 \\
12.68 \\
3.87 \\
.07 \\
.26 \\
1.96 \\
28.24 \\
.15 \\
.98 \\
.34 \\
.30 \\
2.21 \\
\text { trace }\end{array}$ & $\begin{array}{r}38.26 \\
.56 \\
19.52 \\
.09 \\
.09 \\
1.03 \\
38.74 \\
.02 \\
.13 \\
.70 \\
.68 \\
\text { trace }\end{array}$ & 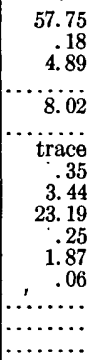 \\
\hline & 100.00 & & $\begin{array}{r}99.83 \\
6.08 \\
.75\end{array}$ & 100.00 & 100.00 \\
\hline
\end{tabular}

12. Llano Del INCA, CHILE.

Analyzed by L. G. Eakins, record No. 1201, and described in Bull. 78, p. 97.

Approximate composition of the mass.

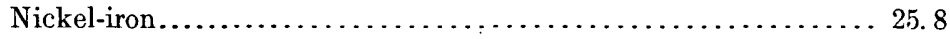

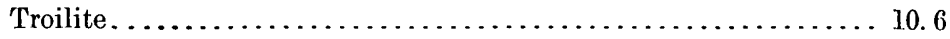

Soluble silicates. . . . . . . . . . . . .

Insoluble silicates. . . . . . . . . . . . . . . . . . . . .

Separate analyses.

A. The nickel-iron.

B. Silicates soluble in hydrochloric acid, calculated to 100 per cent.

C. Insoluble silicates, calculated to 100 per cent.

Is the $\mathrm{P}_{2} \mathrm{O}_{5}$ in $\mathrm{B}$ derived from schreibersite?

\begin{tabular}{|c|c|c|c|c|}
\hline • $\quad \bullet$ & A. & & $B$. & c. \\
\hline \multirow[t]{3}{*}{$\begin{array}{l}\text { Fe... } \\
\text { Ni... } \\
\text { Co... }\end{array}$} & $\begin{array}{r}89.77 \\
9.17 \\
.61\end{array}$ & \multirow{3}{*}{ 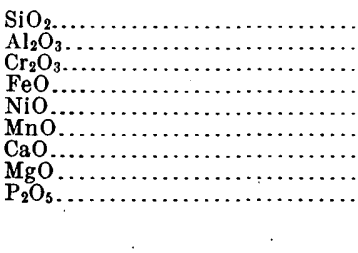 } & $\begin{array}{l}28.08 \\
12.74\end{array}$ & $\begin{array}{r}53.11 \\
2.32 \\
90\end{array}$ \\
\hline & & & $\begin{array}{r}42.52 \\
2.90 \\
.20 \\
9.33 \\
1.98 \\
2.25\end{array}$ & $\begin{array}{r}18.82 \\
\ldots \ldots . . \\
\cdots \\
1.75 \\
23.10 \\
\ldots \ldots . .\end{array}$ \\
\hline & 99.55 & & 100.00 & 100.00 \\
\hline
\end{tabular}




\section{METEORIC IRON.}

A. The Mount Joy meteorite, found near Two Taverns post-office, near Gettysburg, Pennsylvania. Analysis by L. G. Eakins, record No. 1318.

B. From Pulaski County, Virginia. Sp. gr., 7.95, $23^{\circ}$. Analysis by Eakins, No. 1228. Described by Eakins in Bull. 90, p. 45.

C. From Ellenboro, Rutherford County, North Carolina. Described and analyzed by Eakins, Bull. 78, p. 93 . Record No. 1160.

D. From Linnville Mountain, North Carolina. Analyzed by J. E. Whitfield, record No. 822, and described in Bull. 60, p. 107. Sp. gr., 7.778.

E. From Cherokee County, Georgia. Analysis by H. N. Stokes, record No. 1527.

\begin{tabular}{|c|c|c|c|c|c|}
\hline & $\Lambda$. & B. & c. & D. & E. \\
\hline 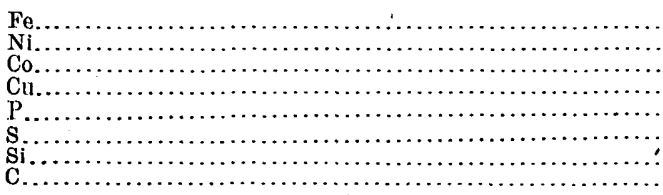 & $\begin{array}{l}93.80 \\
4.81 \\
.51 \\
.005 \\
.19 \\
.01 \\
\cdots \\
\cdots\end{array}$ & $\begin{array}{r}93.59 \\
5.56 \\
.53 \\
\text { trace } \\
.27 \\
.01 \\
\text { trace } \\
\ldots\end{array}$ & $\begin{array}{r}88.05 \\
10.37 \\
.68 \\
.04 \\
.21 \\
.08 \\
.02 \\
.02\end{array}$ & $\begin{array}{r}84.56 \\
14.95 \\
.33 \\
\ldots \ldots . \\
\text { trace } \\
.12 \\
\text { none } \\
\text { trace }\end{array}$ & $\begin{array}{r}91.96 \\
6.70 \\
.50 \\
.03 \\
.11 \\
.01 \\
\text { trace } \\
\text { trace? }\end{array}$ \\
\hline & 99.325 & 99.96 & 99.45 & 99.96 & 99.31 \\
\hline
\end{tabular}

F. From near Holland's store, Chattooga County, Georgia. Analysis by J. E. Whitfield, record No. 765. See Bull. 60, p. 106. Sp. gr., 7.801.

G. From Hamilton County, Texas. Sp. gr., $7.95,27^{\circ}$. Analysis by L. G. Eakins, record No. 1189. See Bull. 78, p. 95.

H. From Mart, McLennan County, Texas. Analysis by H. N. Stokes, record No. 1857. Described by Merrill and Stokes in Proc. Washington Acad., vol. 2, p. 51.

I. From near Scottsville, Allen County, Kentucky. Analysis by Whitfield, record No. 509. See Bull. 55, p. 64.

J. Fell 6 miles east of Cabin Creek, Johnson County, Arkansas, March 27, 1886. Analysis by Whitfield, record No. 505. See Bull. 55, p. 63.

K. From near Grand Rapids, Michigan. Sp. gr., 7.87. Analysis by R. B. Riggs, record No. 296. See Bull. 42, p. 94.

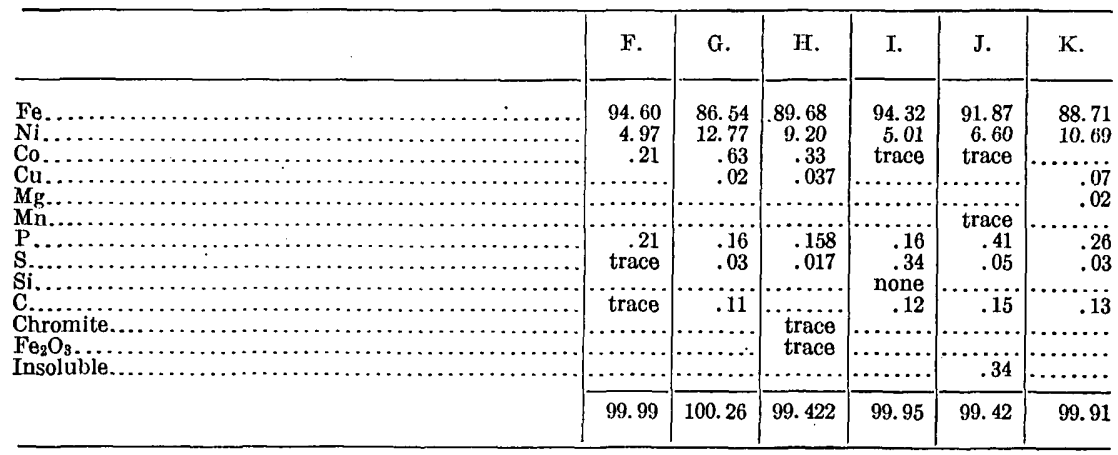

L. The El Capitan iron, from near Bonito, New Mexico. Analysis by H. N. Stokes, record No. 1527.

M. From La Bella Roca, Sierra de San Francisco, Mexico, State of Durango. Analysis by J. E. Whitfield, record No. 1037. Bull. 64, p. 28.

N. Troilite nodule from L, outer part, somewhat altered. 
O. Troilite nodule $\mathrm{N}$, inner part. Analyses $\mathrm{N}$ and $\mathrm{O}$ also by Whitfield, record No. 1037.

P. From Puquios, Chile. Sp. gr., 7.93, 25.2 ${ }^{\circ}$. Analysis by L. G. Eakins, record No. 1.181. See Bull. 78, p. 95 .

Q. The Abert iron, of unknown origin. Analysis by R. B. Riggs, record No. 356. Sp.gr., 7.89. See Bull. 42, p. 94.

\begin{tabular}{|c|c|c|c|c|c|c|}
\hline & L. & M: & N. & 0 & P. & Q. \\
\hline 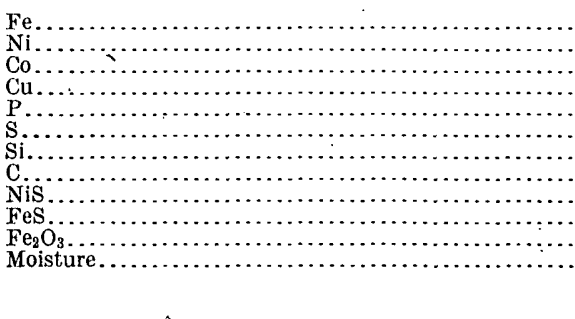 & $\begin{array}{r}90.51 \\
8.40 \\
.60 \\
.05 \\
.24 \\
\text { trace } \\
\cdots . . . . \\
\cdots \\
\cdots \\
\cdots\end{array}$ & 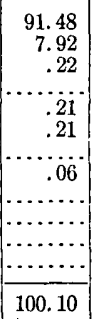 & $\begin{array}{r}2.07 \\
37.51 \\
37.80 \\
19.85 \\
97.23\end{array}$ & 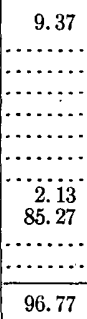 & 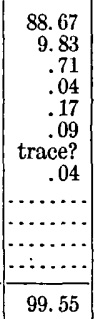 & 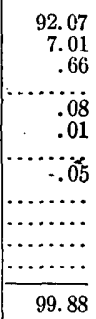 \\
\hline
\end{tabular}

The two following meteoric irons were analyzed by L. G. Eakins in the Denver laboratory.

A. Found near Albuquerque, New Mexico. Described by Eakins in Proc. Colorado Sci. Soc., vol. 2, p. 14.

B. From Wyoming. Partial analysis.

\begin{tabular}{|c|c|c|}
\hline . & A. & B. \\
\hline \multirow[t]{2}{*}{ 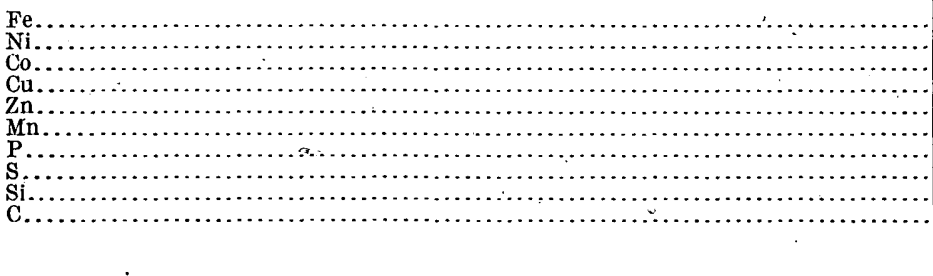 } & $\begin{array}{c}88.76 \\
9.86 \\
.51 \\
.034 \\
.03 \\
\text { trace } \\
.182 \\
.012 \\
.044 \\
\text { undet. }\end{array}$ & 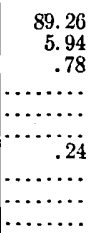 \\
\hline & 99.432 & 96.22 \\
\hline
\end{tabular}




\section{ANALYSES OF MINERALS.}

In the following pages the analyses of over 180 mineral species are given; a considerable number of the species having been originally described as new by chemists connected with the Survey. These species are josephinite, cuprobismutite, warrenite, guitermanite, elpasolite, zunyite, ptilolite, hydronephelite, lucasite, antlerite, knoxvillite, redingtonite, plumbojarosite, emmonsite, powellite, morencite, coronadite, purpurite, goldfieldite, and arizonite. In other cases minerals which were imperfectly described have been more sharply characterized and their true composition made known. . Natrojarosite, for example, is here definitely recognized as a species.

In general the order of Dana's classification has been followed, but with some small variations. Thus the tellurides are put in a group by themselves, the borosilicates are brought together, while the phosphates, vanadates, and arsenates are given as three separate classes. On purely chemical grounds these changes are warranted; on morphological grounds the usual mineralogical classification may be better.

\section{NATIVE ELEMENTS.}

GOLD.

From Persia; exact locality unknown. Analyzed by Charles Catlett.

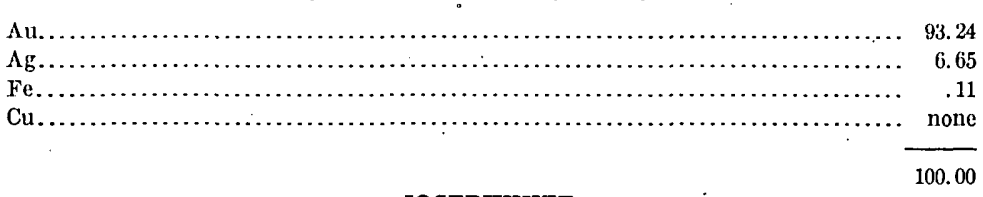

\section{JOSEPHINITE.}

A nickel-iron alloy found in placer gravels in Jackson and Josephine counties, Oregon. Described as a new species by W. H. Melville, Am. Jour. Sci., 3d ser., vol. 43, 1892, p. 509. Waterworn pebbles. Sp. gr., 6.204.

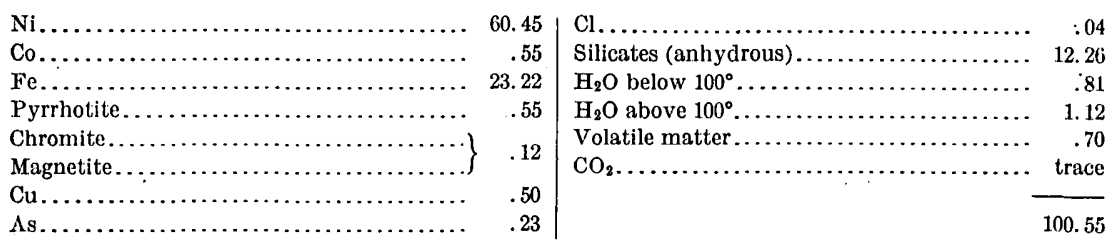

The silicate admixture, including the water, amounts to 13.38 per cent, of which 12.88 per cent, soluble in hydrochloric acid, is serpentine. The insoluble portion may be bronzite. Analyses of the silicates are as follows:

\begin{tabular}{|c|c|c|c|}
\hline ' & Total. & $\begin{array}{l}\text { Insolu- } \\
\text { ble por- } \\
\text { tion. }\end{array}$ & $\begin{array}{l}\text { Soluble } \\
\text { portion. }\end{array}$ \\
\hline \multirow[t]{2}{*}{ 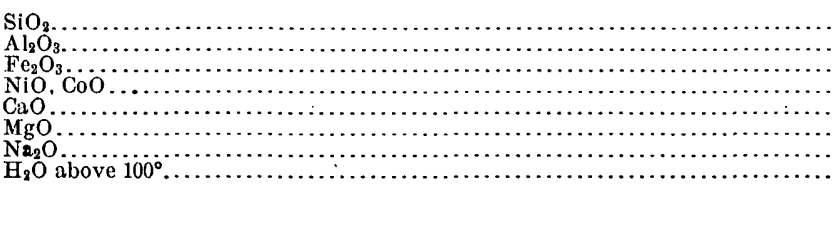 } & $\begin{array}{r}5.14 \\
.33 \\
2.08 \\
.32 \\
1.62 \\
2.69 \\
.08 \\
1.12\end{array}$ & $\begin{array}{r}0.23 \\
.03 \\
.04 \\
\text { trace } \\
.06 \\
.14 \\
\cdots \cdots \\
\cdots\end{array}$ & $\begin{array}{r}4.91 \\
.30 \\
2.04 \\
.32 \\
1.56 \\
2.55 \\
.08 \\
1.12\end{array}$ \\
\hline & 13.38 & .50 & 12.88 \\
\hline
\end{tabular}


For analyses of nickel-iron of meteoric origin, see the preceding section of this bulletin.

\section{SULPHIDES AND ARSENIDES.}

\section{BISMUTHINITE.}

From the Rosario mining district, Sinaloa, Mexico. Analysis by W. H. Melville. Described by him in Bull. 90.

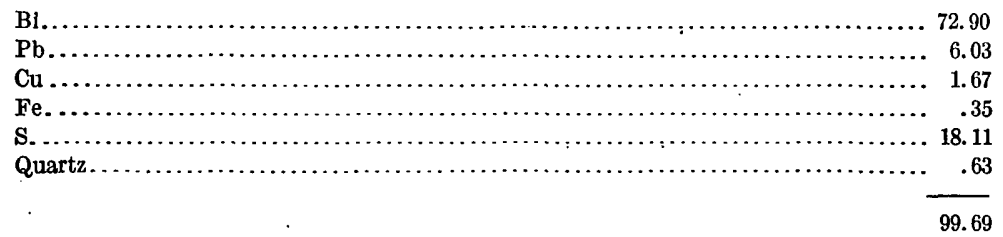

METACINNABARITE.

Crystallized material from New Almaden, California. Analysis by W. H. Melville. Not free from admixtures.

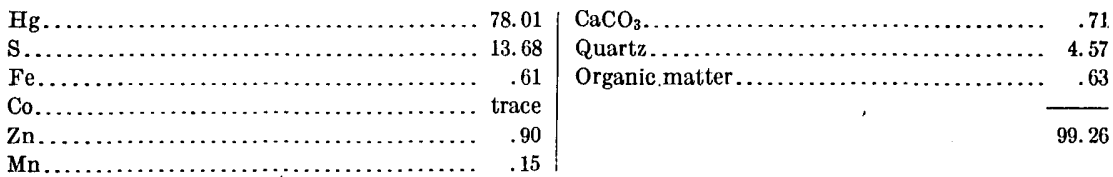

Another specimen of metacinnabarite from Knoxville, California, gave Melville the following figures:

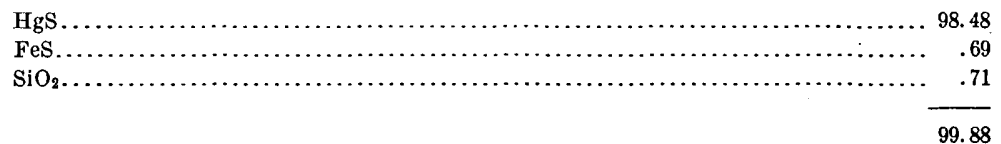

The latter mineral was described by Melville and Lindgren in Bull. 61.

COVELLITE.

From the East Greyrock mine, Butte, Montana. Collected by G. W. Tower. Analysis by W. F. Hillebrand. Color, indigo blue. Massive. Sp. gr., 4.76 at $26^{\circ}$.

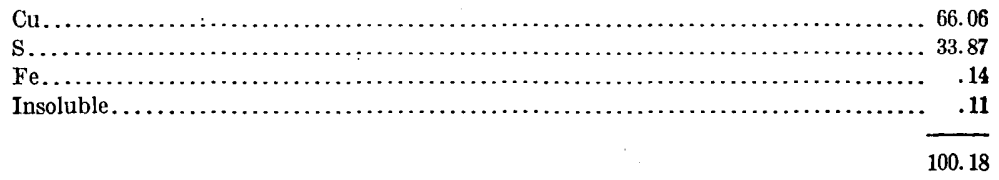

POLYDYMITE.

A massive ore from the mine of the Canadian Copper Company, Sudbury, district of Algoma, Ontario. Specific gravity, 4.541. Analysis by Charles Catlett. Described by Clarke and Catlett in Am. Jour. Sci., 3d ser., vol. 37, 1889, p. 372 . Composition nearly $\mathrm{Ni}_{3} \mathrm{FeS}_{5}$.

A. Actual analysis.

B. Analysis corrected by deduction of quartz and chalcopyrite. 


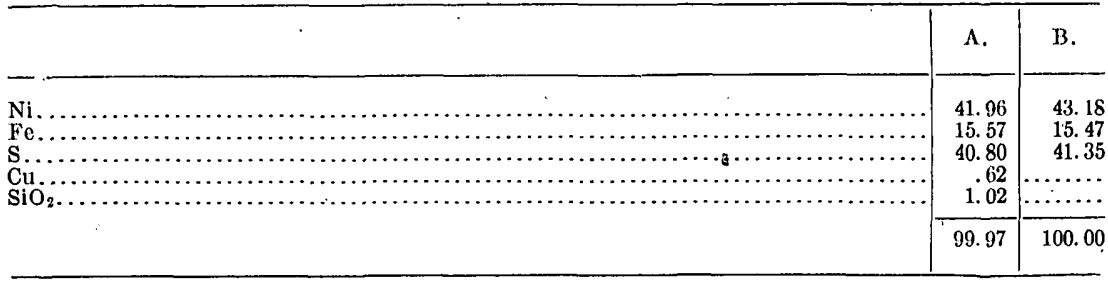

Another nickel-iron sulphide, from the Worthington mine, Sault branch of the Canadian Pacific Railway, 25 miles west of Sudbury, has been analyzed by W. F. Hillebrand. Grayish, with a cast of yellow. Not pyrrhotite. Possibly a mixture of polydymite and pyrite.

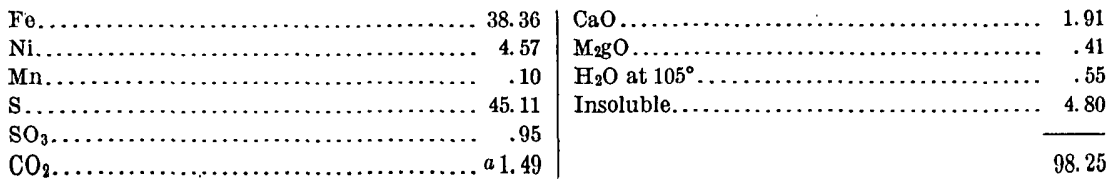

Still another nickel-iron sulphide is described by Hillebrand in Jour. Am. Chem. Soc., vol. 29, p. 1027. It is found in association with the patronite of Minasragra, Peru, and appears to be a new species with the formula $(\mathrm{NiFe}) \mathrm{S}_{2}$. For this the provisional name bravoite is suggested. Analysis by Hillebrand. The deficiency is possibly due to the partial oxidation of the vanadium present. The latter represents unseparated patronite.

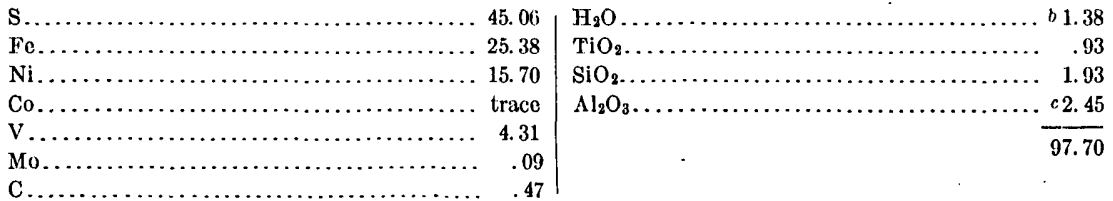

\section{STROMEYERITE.}

From the Silver King mine, Calico, San Bernandino County, California. Sp. gr., 6.28. Analysis by W. H. Melville. Described by Melville and Lindgren in Bull. 61 .

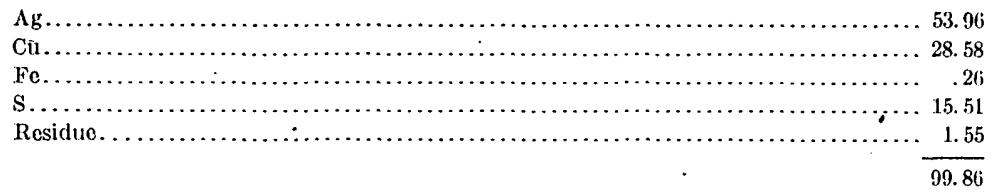

SULPHIDE OF SILVER, COPPER, AND ZINC.

Massive, resembling bornite. Apparently homogeneous, but may be a mixture. Sp. gr., 5.407 at $20^{\circ}$. Analyzed by W. F. Hillebrand and described in Bull. 55 . From the Gagnon mine, Butte, Montana.

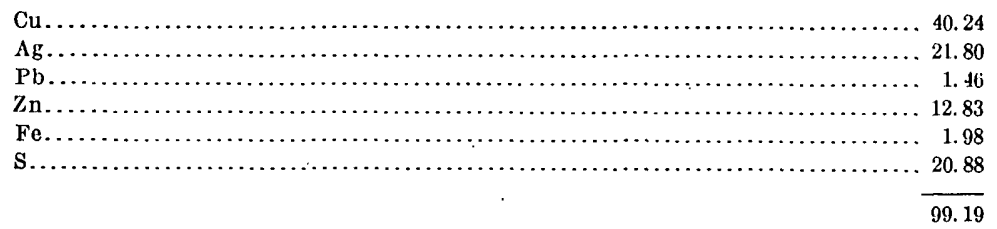

a Calculated to saturate $\mathrm{CaO}$. $\quad$ Partly from $\mathrm{H}$ of carbonaceous matter. c Includes a little $\mathrm{P}_{2} \mathrm{O}_{3}$ 


\section{LÖLLINGITE.}

From Teocalli Mountain, Brush Creek, Gunnison County, Colorado. Sp. gr., 7.400 at $14.5^{\circ}$, corrected for impurity. Analyzed by W. F. Hillebrand and described in Bull. 20 .

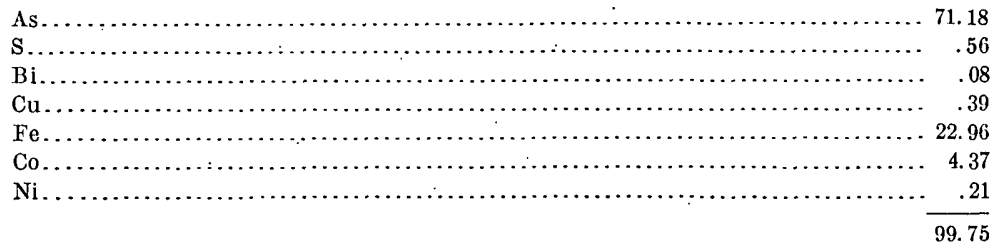

A doubtful arsenide of nickel and cobalt has also been examined by Hillebrand and described in Proc. Colorado Sci. Soc., vol. 3, pt. 1, p. 46. From the Rose mine, Grant County, New Mexico. Sp. gr., 6.644 at $20^{\circ}$. Probably a mixture. Ni: $\mathrm{Co}=3: 1$, approximately.

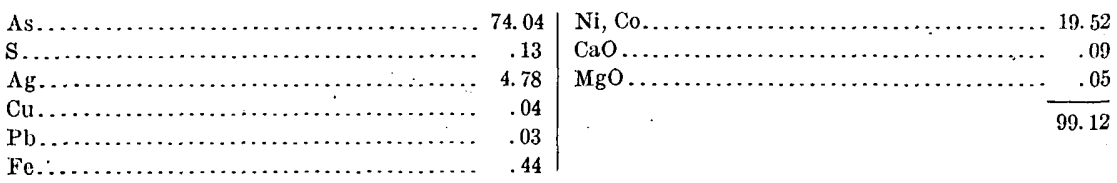

\section{ARSENOPYRITE.}

From Franklin Furnace, New Jersey. Approximate analysis, on only 0.25 gram of material, by E. C. Sullivan. Notable as containing cobalt. The arsenic is probably a trifte too high.

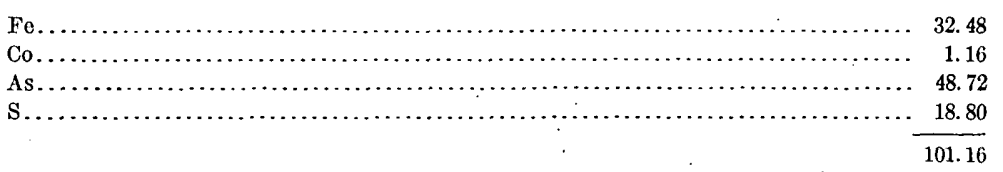

GLAUCODOT.

From the Standard Consolidated gold mine, Sumpter, Oregon. Analyzed by W. T. Schaller, and described in Bull. 262, p. 132.

A. Analysis as made.

B. Analysis recalculated to 100 per cent, after deducting gangue.

\begin{tabular}{|c|c|c|c|}
\hline & . & $\Lambda$. & B. \\
\hline \multirow{2}{*}{\multicolumn{2}{|c|}{ 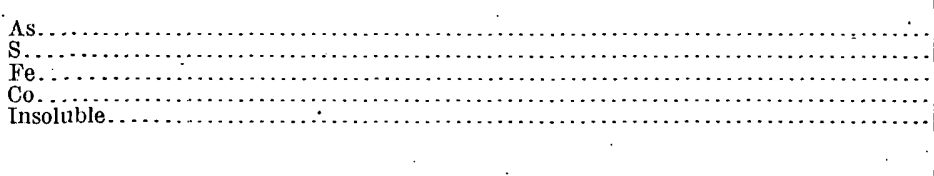 }} & $\begin{array}{r}39.84 \\
18.46 \\
12.45 \\
20.23 \\
9.38\end{array}$ & $\begin{array}{r}43.79 \\
20.29 \\
13.68 \\
22.24 \\
\cdots\end{array}$ \\
\hline & & 100.36 & 100.00 \\
\hline
\end{tabular}




\section{PATRONITE.}

The vanadium sulphide from Minasragra, Peru. Analyzed by W. F. Hillebrand, and described in Jour. Am. Chem. Soc., vol. 29, p. 1019.

\begin{tabular}{|c|c|c|c|}
\hline 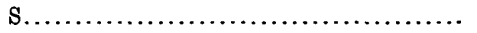 & 58.79 & 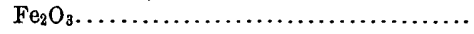 & 0.20 \\
\hline 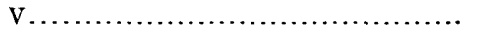 & 19.53 & 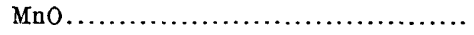 & trace \\
\hline 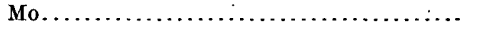 & .18 & 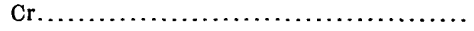 & trace? \\
\hline 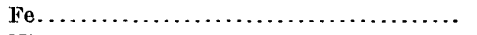 & 2.92 & Alkalies...$\ldots \ldots \ldots \ldots \ldots \ldots \ldots \ldots \ldots$ & $.10 ?$ \\
\hline 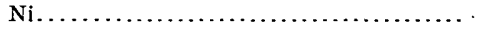 & 1.87 & 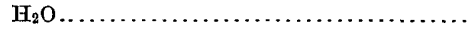 & 1.90 \\
\hline … & 3.47 & O from vanadium sulphate.............. & .38 \\
\hline $\mathrm{SiO}_{2} \ldots \ldots \ldots$ & 6.88 & $\cdot$ & 99.75 \\
\hline$\left[\mathrm{iO}_{2} \ldots \ldots \ldots\right.$ & 1.53 & & \\
\hline $\mathrm{Al}_{2} \mathrm{O}_{3}\left(\mathrm{P}_{2} \mathrm{O}_{5}\right) \ldots \ldots \ldots \ldots$ & 2.00 & & \\
\hline
\end{tabular}

Of the sulphur, 4.5 per cent is free, the remainder is combined. The oxidized constituents represent impurities and gangue.

\section{ENARGITE.}

From the Rarus mine, Butte, Montana. Collected by G. W. Tower. Analysis by W. F. Hillebrand.

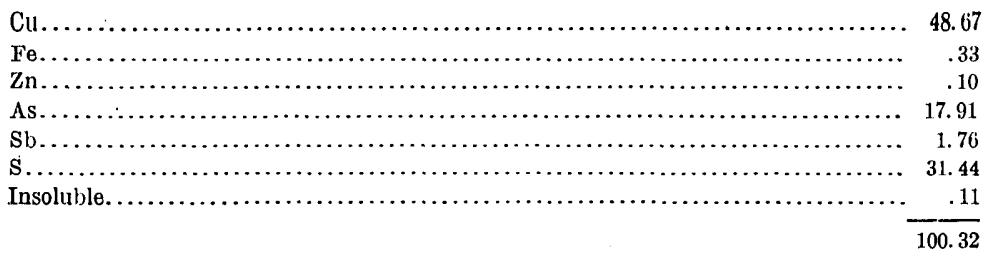

\section{CUPROBISMUTITE.}

New species, discovered by W. F. Hillebrand and described by him in Bull. 20. Named by Dana. Sp. gr., 6.680 at $15^{\circ}$, corrected for impurities. From the Missouri mine, Halls Valley, Park County, Colorado. Analyses, by Hillebrand, of three different samples.

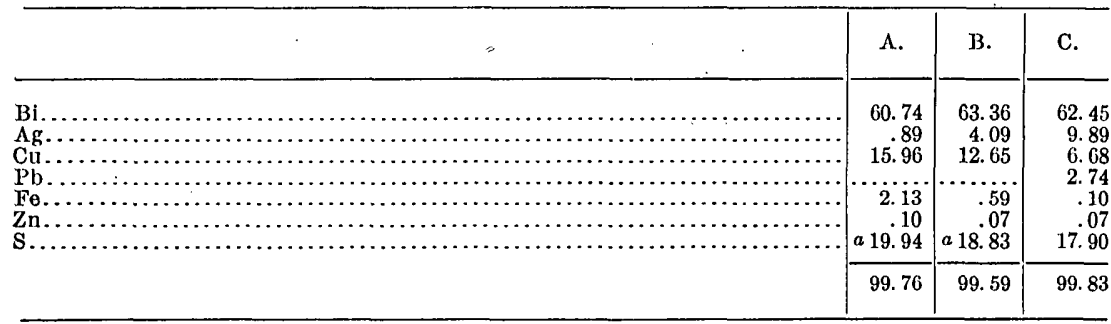

$a$ Calculated.

\section{ZINKENITE.}

From the Brobdignag mine, Red Mountain, San Juan County, Colorado. Sp. gr., 5.21 at $18^{\circ}$. Analyzed by W. F. Hillebrand and described by him in Bull. 20 .

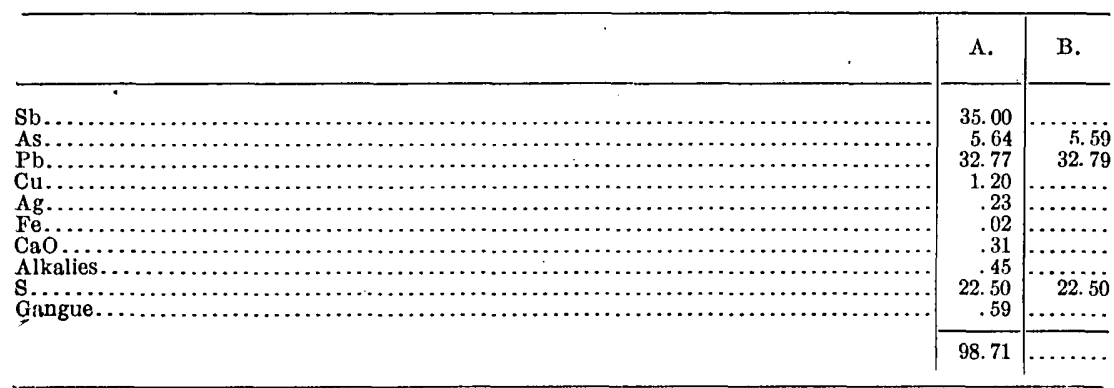




\section{WARRENITE. $a$}

New species, described by L. G. Eakins in Am. Jour. Sci., 3d ser., vol. 36, 1888, p. 450. From the Domingo mine, Gunnison County, Colorado. Occurs in matted, fibrous masses, known locally as "mineral wool." Analysis by Eakins.

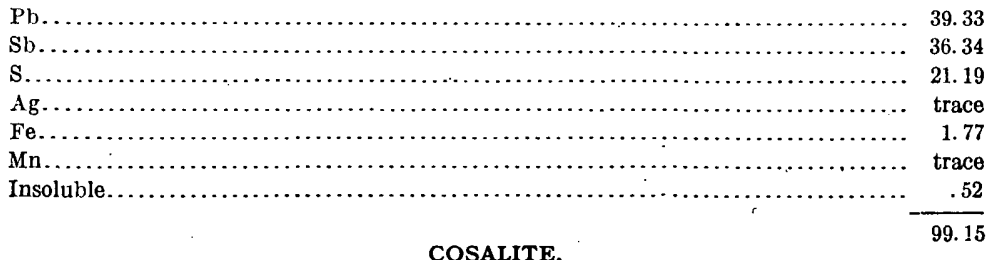

From the Comstock mine, near Parrott City, La Plata County, Colorado. Described by W. F. Hillebrand in Bull. 20.

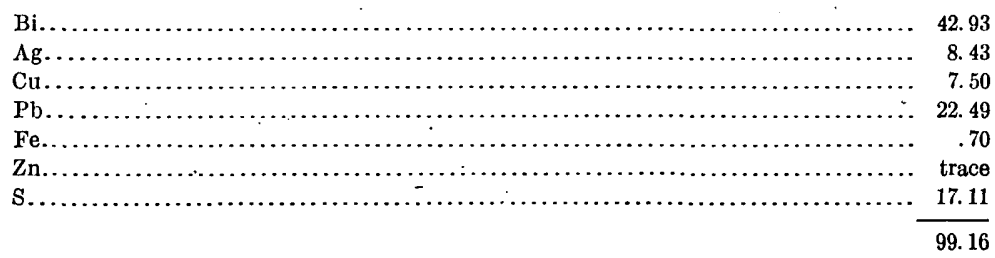

FREIESLEBENITE.

From Augusta Mountain, Gunnison County, Colorado. Known locally as "mineral wool." Remarkable for its freedom from silver. Analyzed by L. G. Eakins and described by him in Am. Jour. Sci., 3d ser., vol. 36, 1888, p. 452.

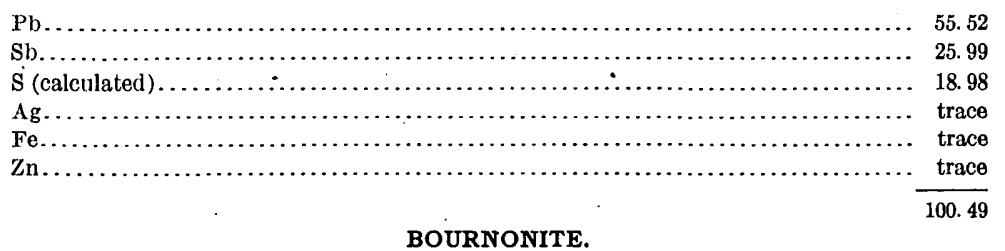

From Boggs mine, Yavapai County, Arizona. Analysis by W. T. Schaller.

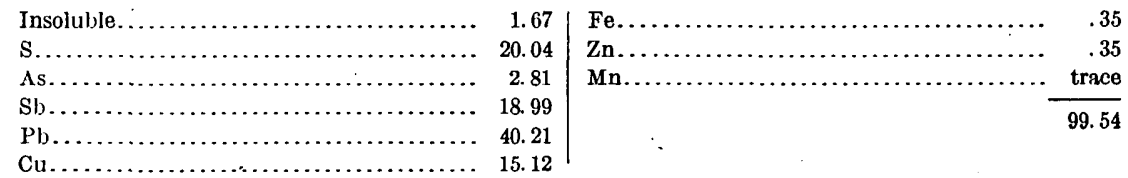

\section{TETRAHEDRITE.}

From Anchor mine, Park City district, Utah. Analysis by G. Steiger.

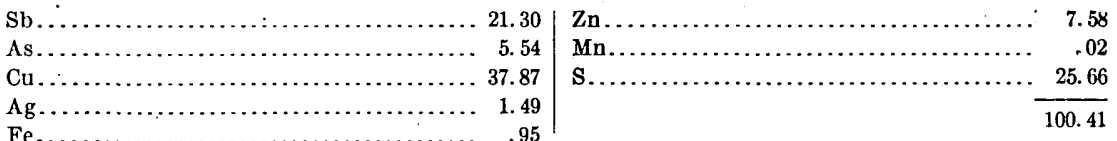

a According to I. J. Spencer, warrenite is identical with jamesonite. In that case it can not be regarded as new. 


\section{GUITERMANITE.}

New species, discovered by W. F. Hillebrand and described by him in Bulletin 20 . From the Zuñi mine, Anvil Mountain, near Silverton, San Juan County, Colorado. Forms the matrix of zunyite. Corrected specific gravity, 5.94 at $17.5^{\circ}$. Analyses by Hillebrand.

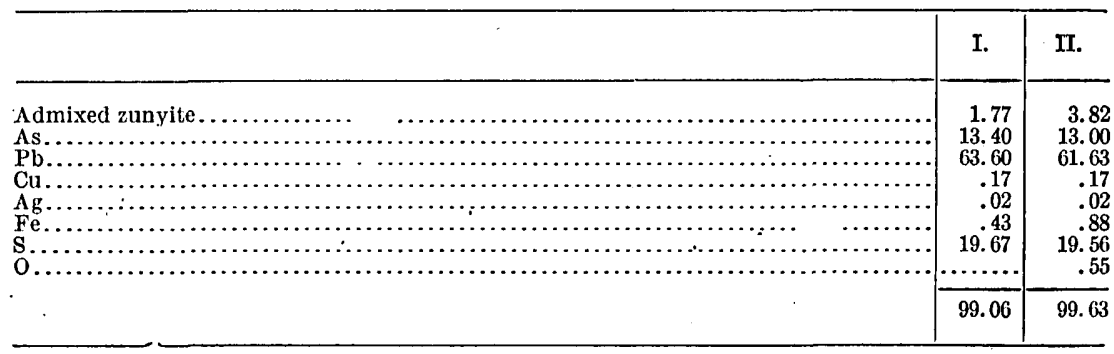

\section{TELLURIDES.}

\section{HESSITE.}

From San Sebastian, Jalisco, Mexico. Sp. gr., 8.24 at $26^{\circ}$. Analysis by W. F. Hillebrand.

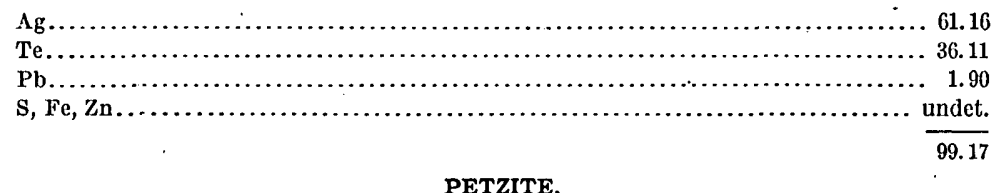

From the Norwegian mine, Calaveras County, California. Collected by F. L. Ransome. Sp. gr., 8.925 at $23^{\circ}$. Analysis by W. F. Hillebrand. Formula, $\mathrm{Au}_{2} \mathrm{Te} .3 \mathrm{Ag}_{2} \mathrm{Te}$.

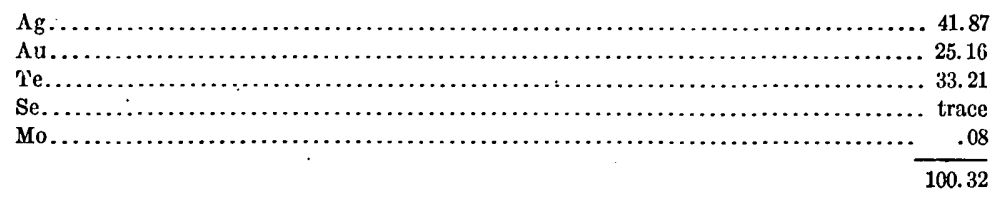

SYLVANITE.

From the Elkton mine, Cripple Creek, Colorado. Analysig by W. F. Hillebrand. Approximate only.

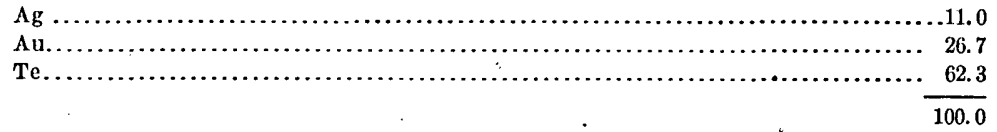

\section{CALAVERITE.}

From Cripple Creek, Colorado. Collected by R. A. F. Penrose, jr. Analyzed by W. F. Hillebrand and described by him in Bull. 167.

A. From the Prince Albert mine. Corrected sp. gr., 9.0 at $24^{\circ}$.

B. Raven mine.

C. C. O. D. mine. 


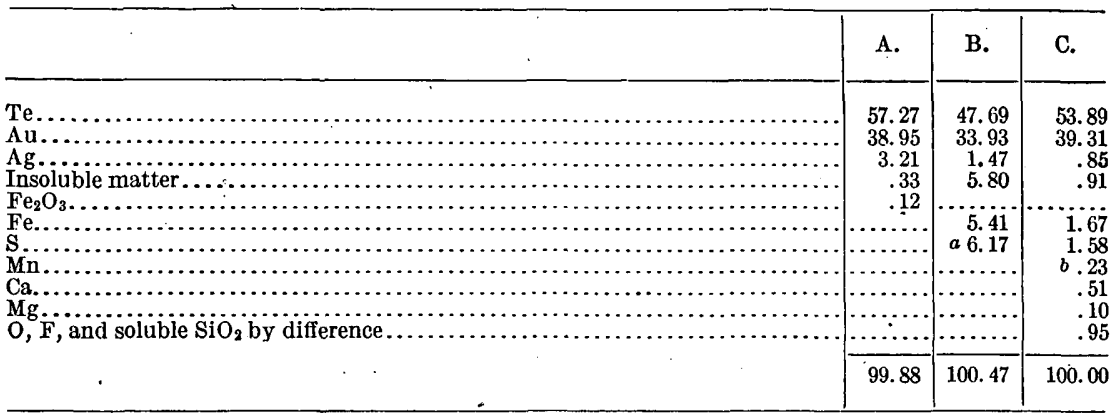

$a$ Calculated from Fe to make $\mathrm{FeS}_{2}$.

$b$ As $\mathrm{MnO}_{2}$ ?

MELONITE.

From the Melones mine, Carson Hill, Calaveras County, California. Collected by F. L. Ransome. Analyzed by W. F. Hillebrand and described by him in Bull. 167. Three samples. Sp. gr. of B, 7.72 at $22.5^{\circ}$, which is probably too high for the pure $\mathrm{NiTe}_{2}$. Sample $\mathrm{C}$ is the purest.

\begin{tabular}{|c|c|c|c|}
\hline & A. & B. & C. \\
\hline 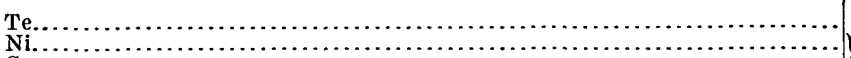 & $\begin{array}{l}75.29 \\
15.71\end{array}$ & $\begin{array}{l}77.72 \\
17.16\end{array}$ & $\begin{array}{l}80.75 \\
18.31\end{array}$ \\
\hline \multirow[t]{2}{*}{ 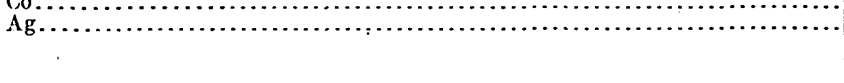 } & 8.44 & 5.09 & .86 \\
\hline & 99.44 & 100.07 & 99.92 \\
\hline
\end{tabular}

TETRADYMITE.

From near Whitehorn, Fremont County, Colorado. Sp. gr., 7.816 at $20^{\circ}$. Analysis by W. F. Hillebrand.

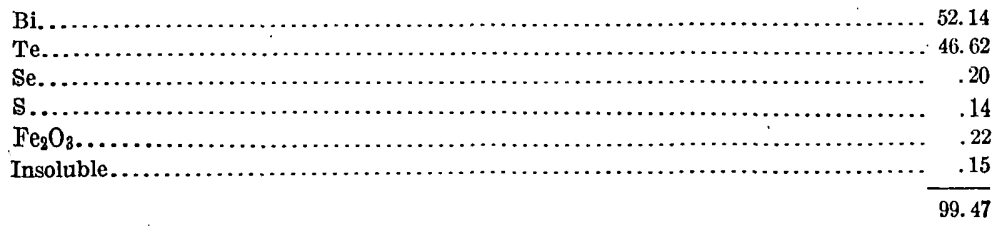

GOLDFIELDITE.

A new mineral, collected by F. L. Ransome, at Goldfield, Nevada, and analyzed by Chase Palmer, has been named goldfieldite. The analysis is only preliminary, for the material was too scanty to admit of the most thorough work. Described by Ransome in P. P. 66, p. 116.

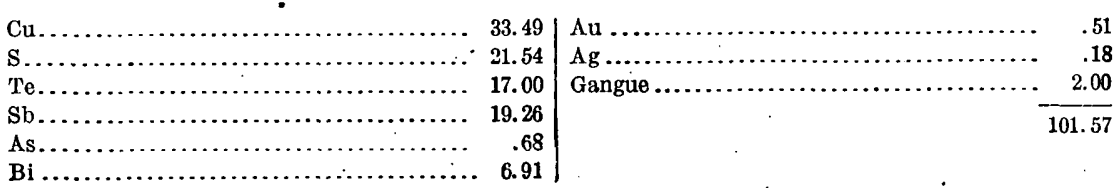


IV. CHLORIDES AND FLUORIDES.

HALITE.

Rock salt from Salton, California. Analysis by E. T. Allen.

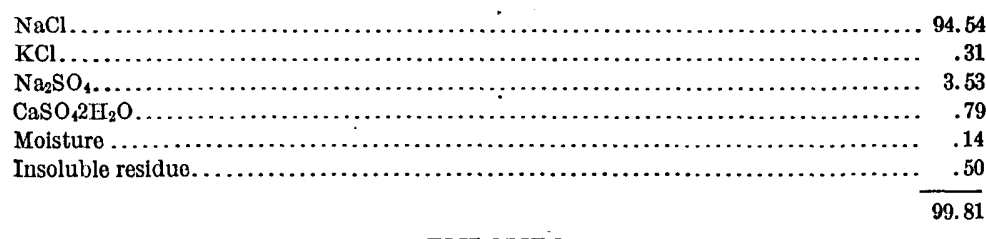

EMBOLITE.

From Broken Hill, Australia. Analysis by L. G. Eakins.

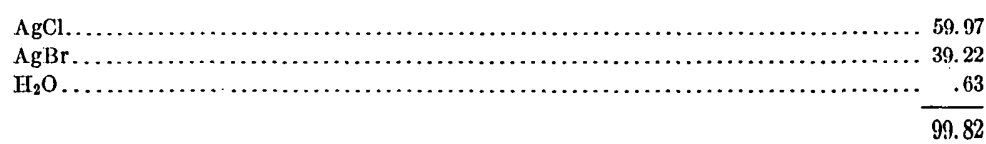

TERLINGUAITE.

From Terlingua, Texas. See Hillebrand and Schaller, Jour. Am. Chem. Soc., vol. 29, p. 11.90. Analysis by Hillebrand, calculated to a gangue-free basis.

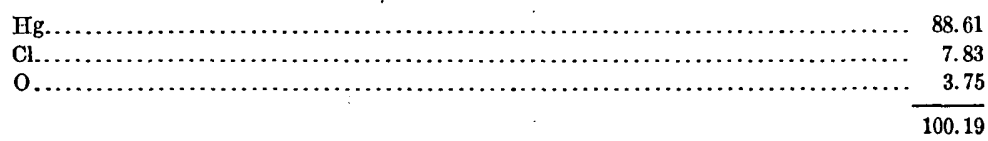

Other determinations gave 88.31 and 88.92 per cent of mercury.

\section{EGLESTONITE.}

From Terlingua, Texas. See Hillebrand and Schaller, Jour. Am. Chem. Soc., vol. 29 , p. 11.92. Three analyses by Hillebrand, on very small amounts of material.

\begin{tabular}{|c|c|c|c|}
\hline & A. & I3. & C. \\
\hline 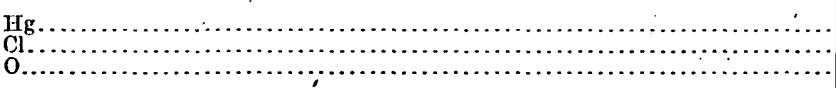 & $\begin{array}{r}88.33 \\
8.32 \\
1.72\end{array}$ & $\begin{array}{r}88.94 \\
8.23 \\
1.84\end{array}$ & $\begin{array}{r}89.73 \\
8.12 \\
1.80\end{array}$ \\
\hline . & 98. 37 & 99.01 & 99.65 \\
\hline
\end{tabular}

Calculated to a gangue-free basis.

\section{KLEINITE.}

From Terlingua, Texas. See Hillebrand and Schaller, Jour. Am. Chem. Soc., vol. 29, p. 1181 . Average of several determinations by Hillebrand, reduced to a ganguefree basis.

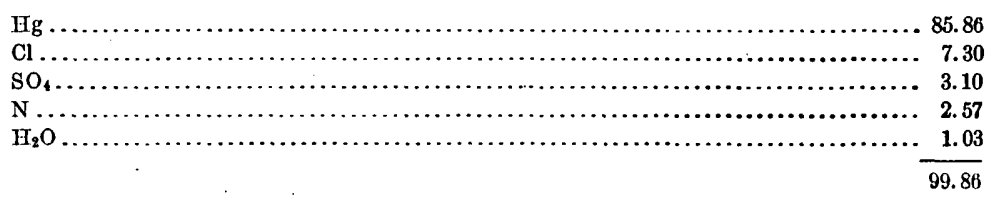




\section{FLUORITE.}

From Franklin Furnace, New Jersey. Partial analysis by G. Steiger, on insufficient material.

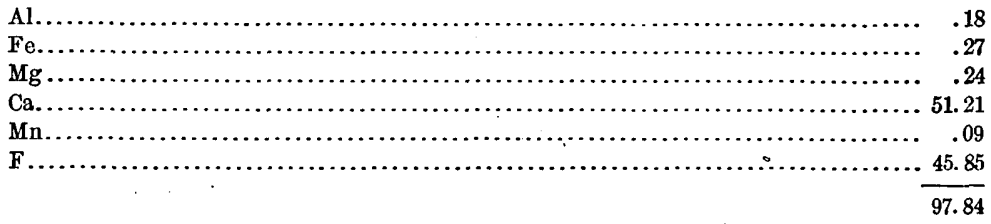

\section{CRYOLITE.}

From St. Peters Dome, Pikes Peak district, Colorado. Described by Cross and Hillebrand in Bull. 20. Massive. Pinkish in color. Sp. gr., 2.972 at $24^{\circ}$. Analysis by W. F. Hillebrand.

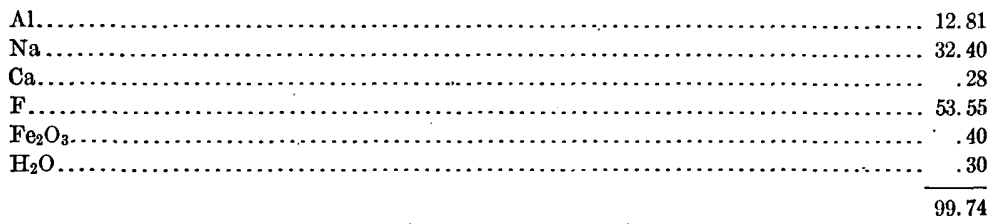

PACHNOLITE.

From St. Peters Dome, Pikes Peak district, Colorado. Described by Cross and Hillebrand in Bull. 20. Analyses by W. F. Hillebrand.

A. Compact bluish variety. Sp. gr., 2.980 at $22^{\circ}$.

B. Crystalline coating.

C. Fresh, transparent, crystalline material.

D. Carefully selected crystals. Sp. gr., 2.965 at $17^{\circ} ; 2.962$ at $22^{\circ}$.

\begin{tabular}{|c|c|c|c|c|}
\hline & A. & B. & c. & D. \\
\hline \multirow{4}{*}{$\begin{array}{l}\mathrm{Al} \\
\mathrm{Ca} \\
\mathrm{Mg} \\
\mathrm{Na} \\
\mathrm{Na} \\
\mathrm{K} \\
\mathrm{H} \\
\mathrm{F}\end{array}$} & $\begin{array}{l}11.94 \\
19.32\end{array}$ & \multirow{3}{*}{$\begin{array}{l}12.93,12.92 \\
15.27,15.17 \\
1.53 \\
10.28 \\
.13 \\
8.64, \quad 8.79\end{array}$} & $\begin{array}{l}12.14 \\
18.06\end{array}$ & $\begin{array}{l}12.27 \\
18.04\end{array}$ \\
\hline & $\begin{array}{r}.13 \\
10.43\end{array}$ & & 10.23 & 10.25 \\
\hline & $\begin{array}{r}7.87,7.95 \\
\ldots \ldots \ldots \ldots . .\end{array}$ & & $\begin{array}{r}8.10, \\
51.33,\end{array}$ & $\begin{array}{r}8.05 \\
a 1.39\end{array}$ \\
\hline & & & 99.86 & 100.00 \\
\hline
\end{tabular}

$a$ By difference.

\section{ELPASOLITE.}

From St. Peters Dome, Pikes Peak district, Colorado. Described as a new species by Cross and Hillebrand in Bull. 20. Incomplete analysis on insufficient material, by W. F. Hillebrand. The fluorine was deduced by calculation on the assumption that the metals are fully combined with it.

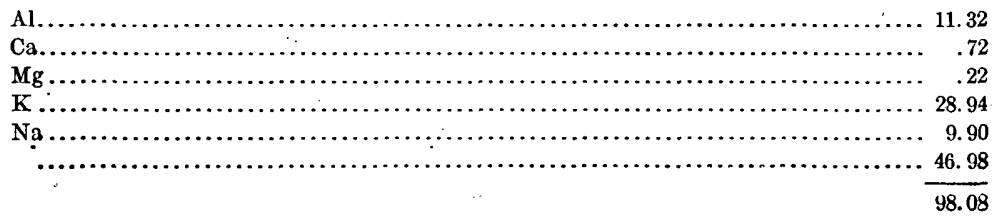




\section{GEARKSUTITE.}

From St. Peters Dome,'Pikes Peak district, Colorado. Described by Cross and.Hillebrand in Bull. 20. Analysis by W. F. Hillebrand.

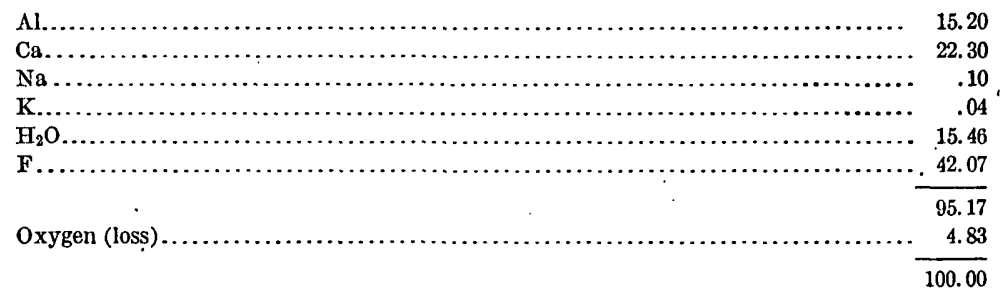

PROSOPITE.

Two samples of prosopite have been analyzed by W. F. Hillebrand, as follows:

A. From St. Peters Dome, Pikes Peak district, Colorado. Described by Cross and Hillebrand in Bull. 20. Sp. gr., 2.880 at $23^{\circ}$. Mean of four analyses.

B. Pale green, massive variety, from the Dugway mining district, Tooele County, Utah. Sp. gr., 2.87 at $21^{\circ}$. Described by Hillebrand in Bull: 167 .

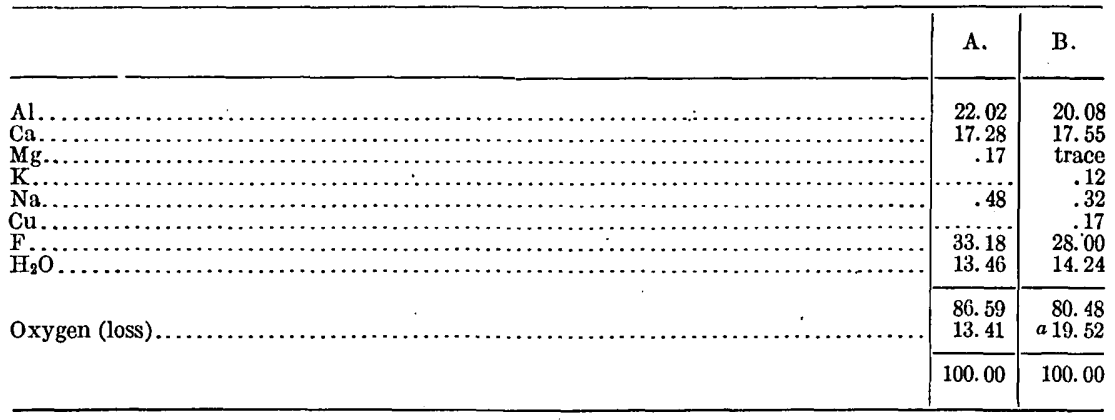

a Including a little quartz; undetermined.

TYSONITE.

From Cheyenne Mountain, near Pikes Peak, Colorado. Analyzed by W. F. Hillebrand and described by him in Bull. 167. Corrected sp. gr., 6.14 at $28^{\circ}$.

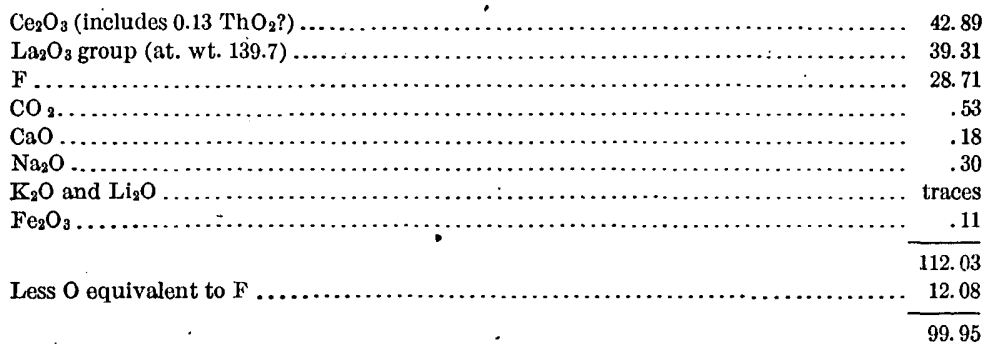




\section{.V. OXIDES AND HYDROXIDES.}

\section{MANGANOSITE.}

From Franklin Furnace, New Jersey. Collected by C. Palache. Analysis by G. Steiger. Sp. gr., 5.364.

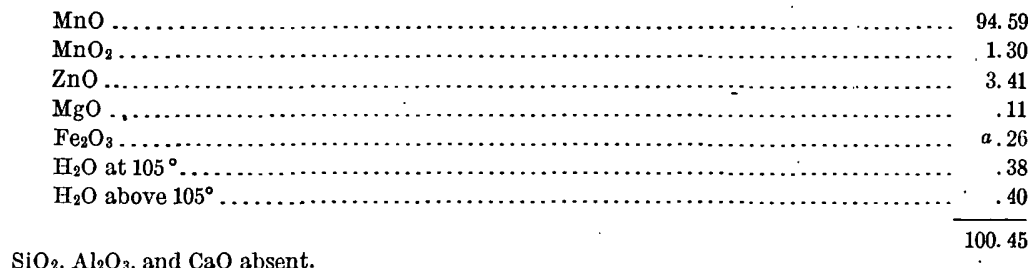

\section{MONTROYDITE.}

From Terlingua, Texas. See Hillebrand and Schaller, Jour. Am. Chem. Soc., vol. 29, p. 1189 . Analysis by W. F. Hillebrand.

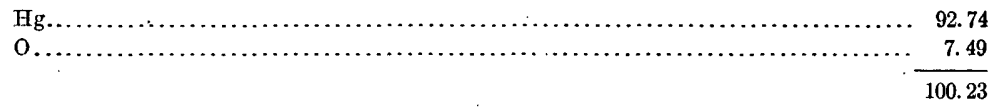

\section{SPINEL.}

Variety pleonaste. Separated from a pyroxenite found between South Meadow and Moore creeks, Madison County, Montana. Rock described by Merrill in Proc. U. S. Nat. Mus., vol. 17, p. 659. Sp. gr. of spinel, $3.89^{\circ}$ at $32^{\circ} .3$. Analysis by L. G. Eakins.

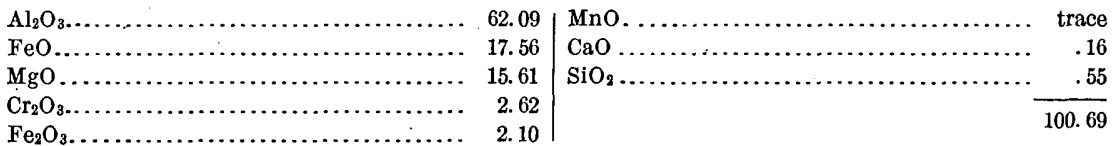

\section{GAHNITE.}

A. From Gilmore's mica mine, Montgomery County, Maryland, about 12 miles north of Washington, District of Columbia. Color, bottle green. Sp. gr., 4.59. Analyzed by T. M. Chatard and described by him in Bull. 9 .

B. Dysluite from Sterling Hill, New Jersey. Collected by C. Palache. Analysis by W. T. Schaller. Sp. gr., 4.56.

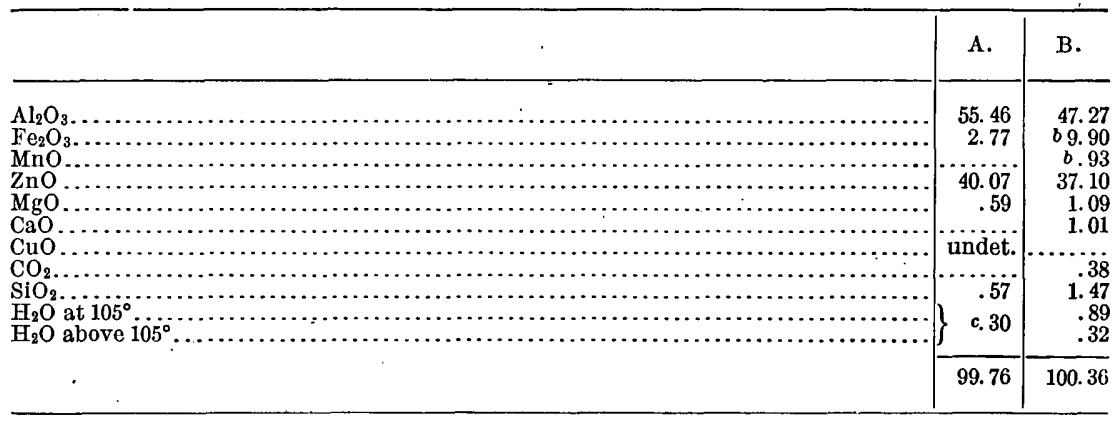

a Total Fe. $\quad$ S State of oxidation uncertain.

c L.oss on ignition. 


\section{MAGNETITE.}

From the Gallatin Range, between Middle and Bozeman creeks, near Bozeman, Montana. Analysis by T. M. Chatard.

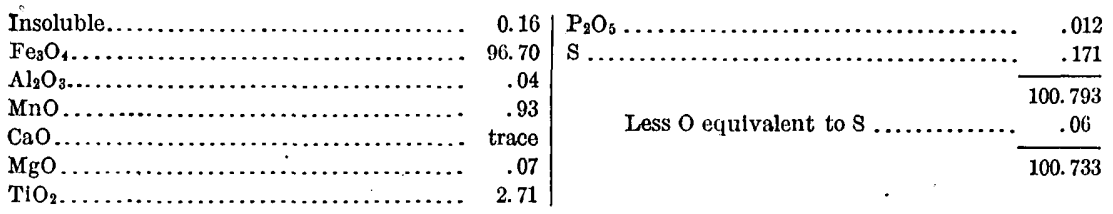

FRANKIINITE.

From Franklin Furnace, New Jersey. Collected by C. Palache. Analysis by W. T. Schaller. Sp. gr., 5.09 .

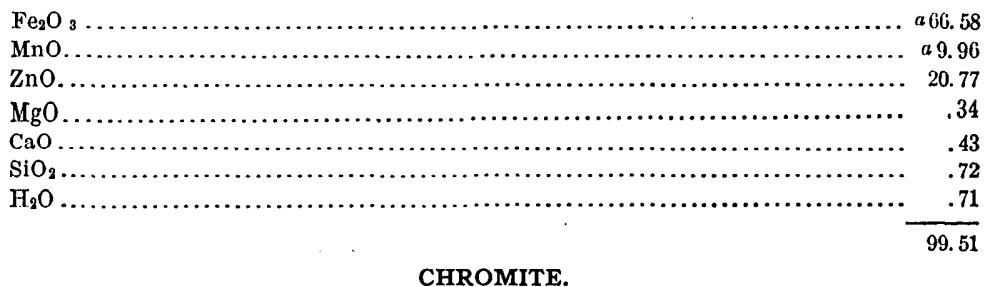

From Corundum Hill, North Carolina. Analysis by T. M. Chatard. $\mathrm{Fe}_{2} \mathrm{O}_{3}$ not separately determined.

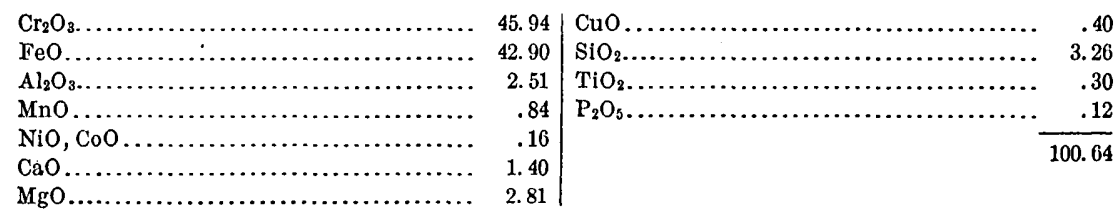

RUTILE.

From near St. Peters Dome, Pikes Peak district, Colorado. Sp. gr., 4.288 at $19^{\circ}$. Analysis by L. G. Eakins.

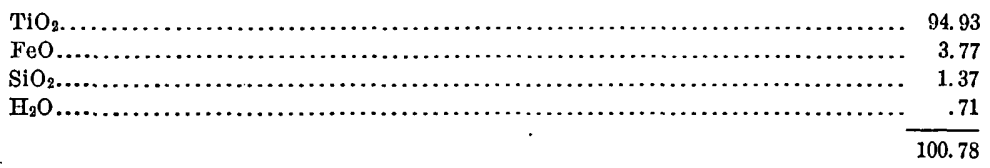

DIASPORE.

From Mount Robinson, Custer County, Colorado. Analysis by L. G. Eakins.

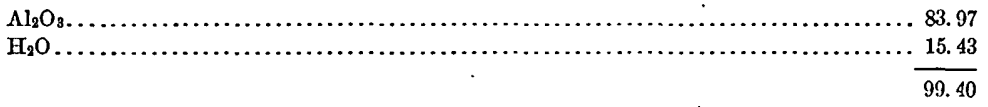




\section{BAUXITE.}

Two samples from Jacksonville, Calhoun County, Alabama. Alkalies, lime, and magnesia not looked for. Analyses by W. F. Hillebrand.

\begin{tabular}{|c|c|c|c|}
\hline & - & Red. & White. \\
\hline \multirow{2}{*}{\multicolumn{2}{|c|}{ 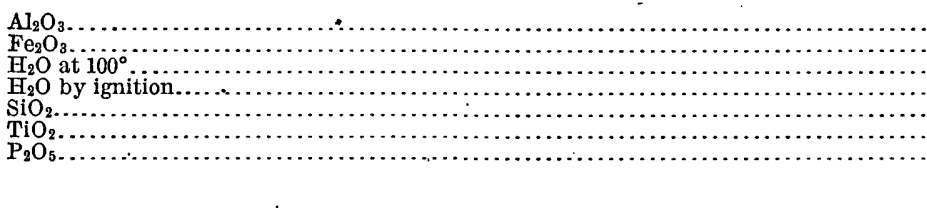 }} & $\begin{array}{r}41.00 \\
25.25 \\
.65 \\
20.43 \\
10.25 \\
2.53 \\
\text { trace }\end{array}$ & $\begin{array}{r}48.92 \\
2.14 \\
.45 \\
23.41 \\
21.08 \\
2.52 \\
\text { trace }\end{array}$ \\
\hline & & 100.11 & 98.52 \\
\hline
\end{tabular}

BRUCITE.

From Texas, Lancaster County, Pennsylvania. Analysis by E. A. Schneider.

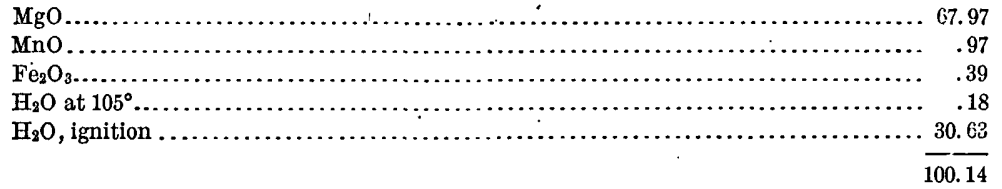

PSILOMELANE.

From a prospect hole on plain south of Round Mountain, Silver Cliff, Colorado. Analysis by W. F. Hillebrand. There were strong spectroscopic reactions for strontia and lithia, but these bases were not estimated. A little insoluble gangue.is included with the silica.

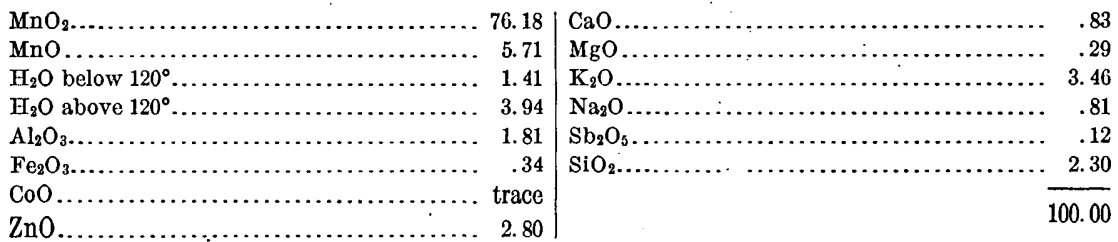

\section{HET EROLITE.}

From Sterling Hill, New Jersey. Collected by C. Palache. Analysis by W. T. Schaller. Sp. gr., 4.85 .

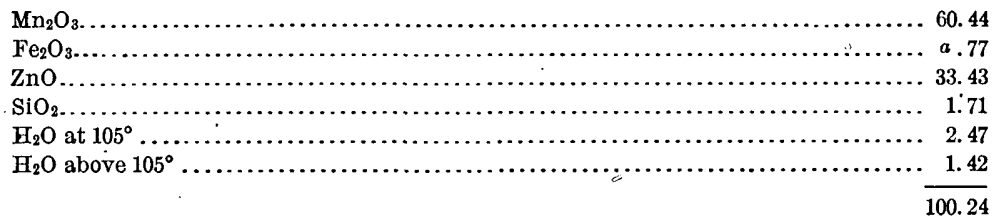

$\mathrm{Al}_{2} \mathrm{O}_{3}, \mathrm{CaO}$, and $\mathrm{MgO}$ absent. 


\section{COŔONADITE.}

New mineral from the Coronado vein, Clifton-Morenci district, Arizona. Described by Lindgren and Hillebrand in Bull. 262, p. 42. Sp. gr., 5.246 at $22^{\circ}$. Analysis by W. F. Hillebrand.

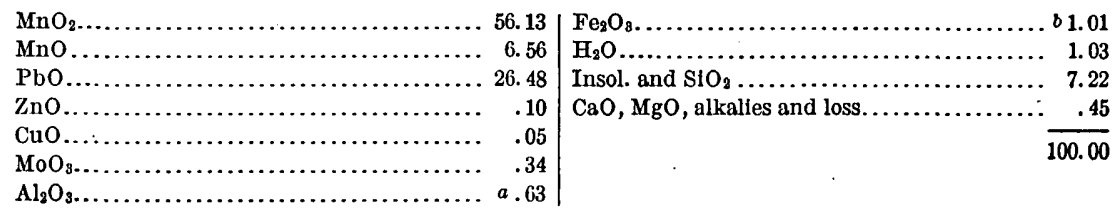

VI. CARBONATES.

\section{CALCITE.}

From the Gibraltar mine, Bullfrog district, Nevada. Analysis by Chase Palmer.

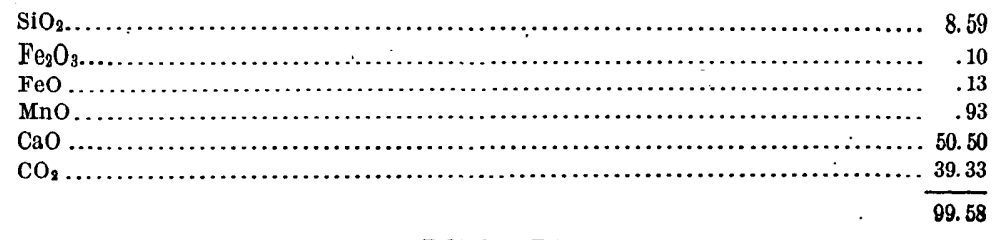

\section{DOLOMITE.}

A. Dolomite marble, New York Quarry Company, Tuckahoe, Westchester County, New York. Analysis by W. F. Hillebrand.

B. Dolomite marble, Cockeysville, Maryland. Analysis by J. E. Whitfield.

C. Same as B. Analysis by E. A. Schneider. Another sample.

D. Dolomite marble, Webster, Massachusetts. Analysis by H. N. Stokes.

E. Pink, crystallized dolomite, Joplin, Missouri. Analysis by E. T: Allen.

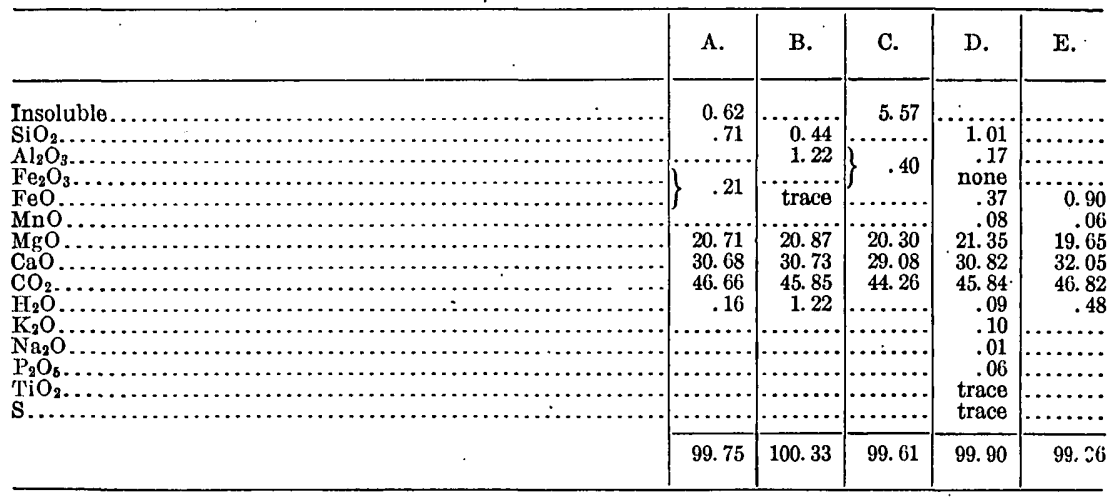




\section{SMITHSONITE.}

From Marion County, Arkansas. Bright yellow variety, known locally as "turkeyfat ore." Analysis by H. N. Stokes.

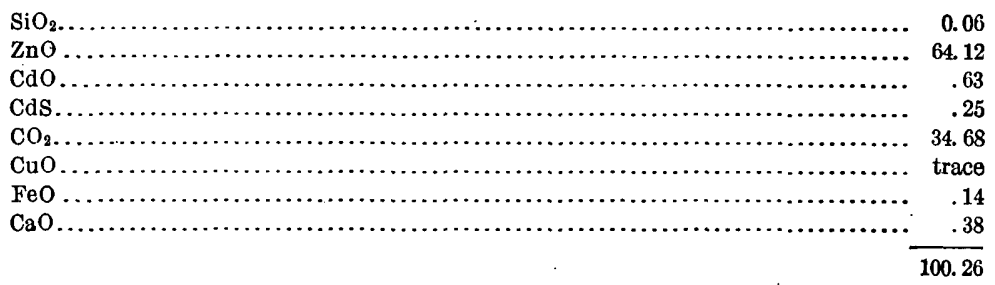

TENGERITE.

From Baringer Hill, Llano County, Texas. Received from W. E. Hidden. Approximate analysis, on insufficient material, by W. F. Hillebrand.

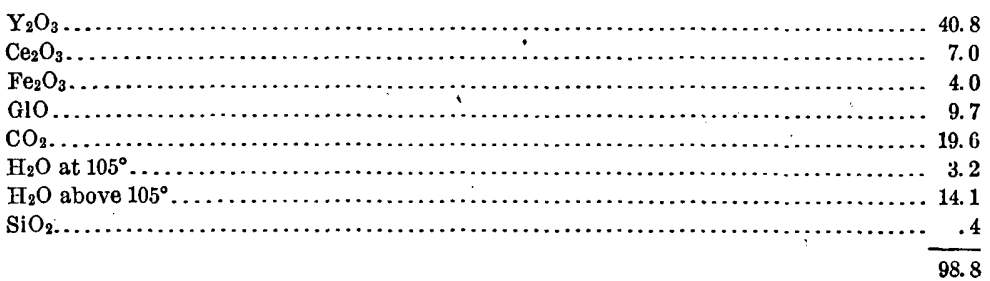

Magnesia, alkalies, etc., undetermined.

\section{BASTNÄSITE.}

Associated with the tysonite of Cheyenne Mountain, near Pikes Peak, Colorado. Sp. gr. 5.12 at $27^{\circ}$. Analyzed by W. F. Hillebrand and described by him in Bull. 167.

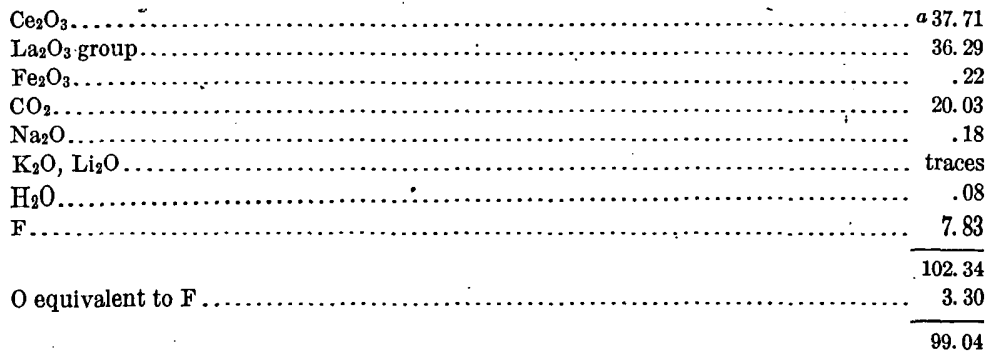

URAO.

Deposited from the waters of Owens Lake, California. Analyses by T. M. Chatard. Described by Chatard in Bull. 60. Five samples analyzed, as follows:

A. The best material. Sp. gr., 2.1473 at $21.7^{\circ}$.

B. Crude urao.

C. Urao deposited upon a grass root.

D. From a small lagoon.

E. From a vat dug on the beach of Owens Lake. 


\begin{tabular}{|c|c|c|c|c|c|}
\hline & A. & B. & C. & D. & E. \\
\hline 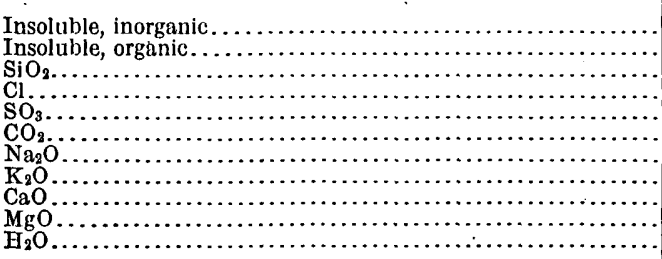 & 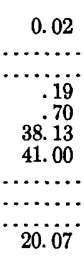 & $\begin{array}{r}0.22 \\
.10 \\
1.57 \\
.79 \\
37.00 \\
41.26 \\
\cdots \\
\cdots \\
\cdots \\
19.62\end{array}$ & \begin{tabular}{r}
2.92 \\
.14 \\
.05 \\
2.73 \\
.76 \\
35.24 \\
40.22 \\
\hdashline$\ldots .$. \\
\hdashline$\ldots \ldots$. \\
18.31
\end{tabular} & $\begin{array}{r}0.40 \\
.12 \\
.09 \\
.21 \\
.63 \\
37.50 \\
40.08 \\
\text { trace } \\
.06 \\
.02 \\
19.94\end{array}$ & $\begin{array}{r}4.10 \\
.27 \\
.04 \\
1.83 \\
.84 \\
35.10 \\
39.36 \\
\ldots \ldots . . \\
\ldots \ldots . \\
18.58\end{array}$ \\
\hline \multirow[t]{2}{*}{ O equivalent to $\mathrm{Cl} . \ldots \ldots \ldots \ldots \ldots \ldots \ldots \ldots \ldots \ldots \ldots \ldots$} & $\begin{array}{r}100.11 \\
.04\end{array}$ & $\begin{array}{r}100.56 \\
.35\end{array}$ & $\begin{array}{r}100.37 \\
.61\end{array}$ & $\begin{array}{r}99.05 \\
.05\end{array}$ & $\begin{array}{r}100.12 \\
.41\end{array}$ \\
\hline & 100.07 & 100.21 & 99. 76 & 99.00. & 99.71 \\
\hline
\end{tabular}

\section{SILICATES.}

\section{PETALITE.}

From Peru, Maine, associated with spodumene. Analysis by F. W. Clarke.

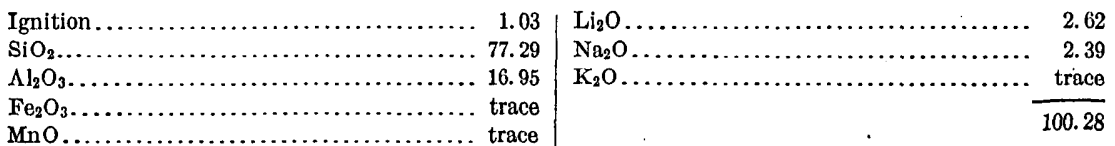

\section{ORTHOCLASE AND MICROCLINE.}

A. Orthoclase from Silver City, Idaho. Occurs as a gangue mineral. Partial analysis by W. F. Hillebrand.

B. Orthoclase from Mitchell County, North Carolina. Large cleavage mass. Analysis by E. T. Allen. See Am. Jour. Sci., ser. 4, vol. 19, p. 119.

C. Feldspar crystal from the nevadite of Chalk Mountain, Summit County, Colorado. Analysis by W. F. Hillebrand.

D. Pink orthoclase crystals, from gray porphyry, Johnson Gulch, near Leadville, Colorado. Described by Cross in Mon. XII, Appendix A. Analysis by W. F. Hillebrand.

E. Feldspar separated from gabbro. East side of North Fowl Lake, Minnesota. Analysis by W. F. Hillebrand.

F. Feldspar from the elæolite syenite of Litchfield, Maine. Described by Bayley in Bull. 150, p. 201. Analysis by W. H. Melville.

\begin{tabular}{|c|c|c|c|c|c|c|}
\hline - & A. & B. & c. & D. & E. & F. \\
\hline $\begin{array}{l}\mathrm{SiO}_{2} \ldots \\
\mathrm{TiO}_{2} \ldots\end{array}$ & 66.28 & 65.49 & 65.04 & 62.22 & $\begin{array}{r}.62 .71 \\
\text { trace }\end{array}$ & 65.14 \\
\hline $\mathrm{Al}_{2} \mathrm{O}_{3}$. & 17.93 & 17.98 & 20.40 & 20.33 & 19.20 & 18.19 \\
\hline $\begin{array}{l}\mathrm{Fe}_{2} \mathrm{O}_{3} \ldots \ldots \\
\mathrm{FeO} \ldots \ldots\end{array}$ & …..... & .36 & $\begin{array}{l}\ldots . . \\
\cdots \cdots \\
\cdots\end{array}$ & ........ & $\begin{array}{r}1.08 \\
.93\end{array}$ & 25 \\
\hline MnO.......... & & & & & trace & \\
\hline $\begin{array}{l}\mathrm{CaO} . . \\
\text { SrO... }\end{array}$ & 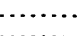 & .42 & .79 & 2.95 & $\stackrel{44}{\operatorname{trace}}$ & .33 \\
\hline $\mathrm{MgO}$. & & none & none & & .81 & 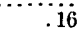 \\
\hline 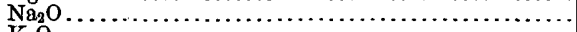 & .25 & 2. 29 & 4:11 & 3. 45 & 2. 96 & 1. 68 \\
\hline 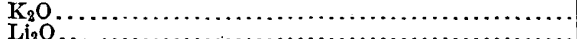 & 15.12 & 12.95 & 9.74 & 8.31 & 10.41 & 14.14 \\
\hline 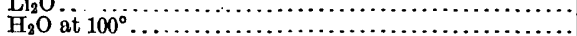 & .... & & & & none & \\
\hline 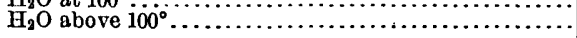 & & .51 & .29 & 1.90 & .28 & .17 \\
\hline . & 99.58 & 100.00 & 100.37 & 99.16 & 99.69 & 100.06 \\
\hline
\end{tabular}


G. Flesh-colored microcline from the pegmatite of Jones Falls, Baltimore, Maryland.

H. Greenish microcline, same locality as G. G and H described by S. L. Powell in Johns Hopkins Univ. Circular, vol. 12, p. 49. Analyses by W. F. Hillebrand.

I. Anorthoclase from the elæolite syenite of Peaked Butte, Crazy Mountains, Montana. See Wolff and Tarr in Bull. Mus. Comp. Zool. Harvard Coll., vol. 16, No. 12; 1893. Analysis by W. F. Hillebrand.

J. Anorthoclase from red soda granite, Pigeon Point, Minnesota. Described by Bayley in Bulletin 109. Analysis by J. E. Whitfield.

K. Anorthoclase from keratophyre, Marblehead Neck, Massachusetts. Described by Sears, Bull. Mus. Comp. Zool. Harvard Coll., vol. 16, No. 9, 1893. Analysis by T. M. Chatard.

\begin{tabular}{|c|c|c|c|c|c|}
\hline & G. & H. & I. & J. & $\mathbf{K}$ \\
\hline \multirow[t]{2}{*}{ 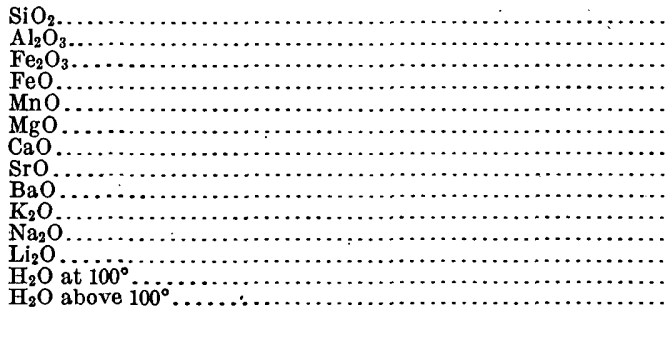 } & $\begin{array}{r}65.06 \\
18.41 \\
\text { trace } \\
\cdots \ldots . . \\
.04 \\
.26 \\
\text { trace } \\
.13 \\
14.30 \\
1.60 \\
\text { trace } \\
.04 \\
.26\end{array}$ & $\begin{array}{r}68.48 \\
16.11 \\
.20 \\
.17 \\
.03 \\
.23 \\
\text { trace } \\
.05 \\
12.90 \\
1.27 \\
\operatorname{trace} \\
.06 \\
.26\end{array}$ & $\begin{array}{r}62.31 \\
22.63 \\
\ldots \ldots \\
\ldots \ldots . .6 \\
.63 \\
.57 \\
.77 \\
4.79 \\
7.68 \\
\ldots .16 \\
.72\end{array}$ & $\begin{array}{r}65.00 \\
18.22 \\
2.64 \\
\ldots \ldots . \\
\ldots \ldots . . \\
.06 \\
1.06 \\
\ldots \ldots \ldots \\
\ldots \ldots .18 \\
8.40 \\
\ldots \ldots \\
.46\end{array}$ & $\begin{array}{r}65.66 \\
20.05 \\
\text { trace } \\
\text { trace } \\
.13 \\
.18 \\
.67 \\
\ldots \ldots . . \\
6.56 \\
6.98 \\
\ldots .04 \\
.04 \\
.37\end{array}$ \\
\hline & 100.10 & 99.85 & 100.26 & 100.02 & 100.64 \\
\hline
\end{tabular}

ALBITE.

A. From feldspathic schist, central shaft of the Hoosac Tunnel, Berkshire County, Massachusetts. Described by Wolff in Mon. XXIII, pp. 60-187. Analysis by R. B. Riggs.

B, C. From the porphyritic mica schist of Greylock Mountain, Massachusetts. Described by Wolff, loc. cit. Analyses by R. B. Riggs.

D. From the elæolite syenite of Litchfield, Maine. Described by Bayley in Bull. 150, p. 201. Sp. gr., 2.622. Analysis by W. H. Melville.

E. From the pegmatite of Jones Falls, Baltimore, Maryland. Described by S. L. Powell in Johns Hopkins Univ. Circular, vol. 12, p. 49. Analysis by W. F. Hillebrand.

F. From Amelia County, Virginia. Analysis by E. T. Allen.

G. From Mitchell County, North Carolina. Analysis by Allen.

\begin{tabular}{|c|c|c|c|c|c|c|c|}
\hline & A. & B. & C. & D. & E. & F. & G. \\
\hline \multirow{7}{*}{ 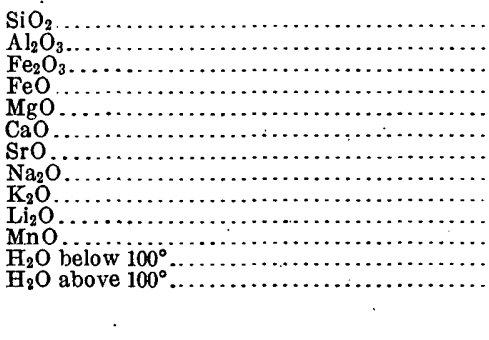 } & $\begin{array}{l}69.69 \\
18.60\end{array}$ & $\begin{array}{l}68.08 \\
20.11\end{array}$ & $\begin{array}{l}67.83 \\
19.92\end{array}$ & $\begin{array}{l}68.28 \\
19.62\end{array}$ & $\begin{array}{l}63.72 \\
22.26\end{array}$ & $\begin{array}{r}68.22 \\
19.06 \\
.15\end{array}$ & $\begin{array}{l}66.03 \\
20.91 \\
.18\end{array}$ \\
\hline & 20 & (?) & & .23 & \multirow{3}{*}{$\begin{array}{r}.06 \\
3.58 \\
\text { trace } \\
8.98 \\
.76 \\
\text { trace }\end{array}$} & & \\
\hline & trace & trace & trace & .31 & & .40 & 2.00 \\
\hline & $\begin{array}{r}10.28 \\
.40\end{array}$ & $\begin{array}{r}11.00 \\
.36\end{array}$ & $\begin{array}{r}11.65 \\
.25\end{array}$ & $\begin{array}{r}10.81 \\
.39\end{array}$ & & $\begin{array}{r}11.47 \\
.20\end{array}$ & $\begin{array}{r}9.97 \\
.70\end{array}$ \\
\hline & & trace & trace & & & & \\
\hline & .42 & & .12 & .09 & $\begin{array}{r}.09 \\
.43\end{array}$ & .69 & .59 \\
\hline & 99.59 & 99.86 & 99.77 & 99.82 & 99.88 & 100.19 & 100.38 \\
\hline
\end{tabular}




\section{ANORTHITE.}

A. From Raymond, Maine. Associated with idocrase, garnet, pyroxene, and scapolite. Analysis by W. H. Melville.

B. From Phippsburg, Maine. Occurrence similar to A. Incomplete analysis by George Steiger.

C. Separated from "hyperite changing to diorite," near Whitaker's ore pit, Wilmington, Delaware. Described by Chester in Bull. 59. Analysis by R. B. Riggs. Sp. gr.; 2.592 to 2.749 .

\begin{tabular}{|c|c|c|c|}
\hline & A. A. & B. & C. \\
\hline 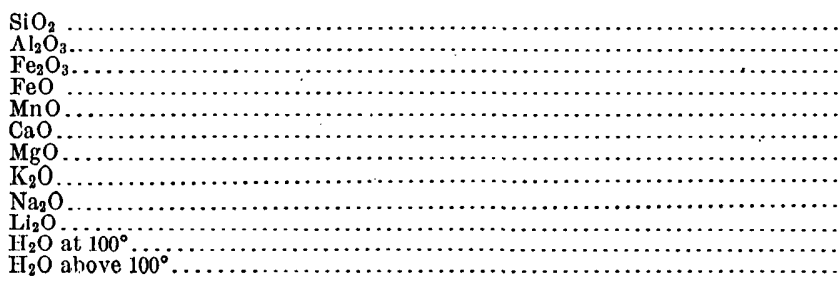 & $\begin{array}{r}43.13 \\
30.95 \\
1.04 \\
\text { trace } \\
\text { trace } \\
19.71 \\
.31 \\
1.29 \\
.69 \\
\text { trace } \\
.22 \\
2.80\end{array}$ & 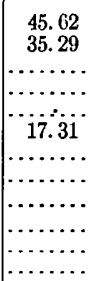 & $\begin{array}{r}44.09 \\
35.41 \\
.51 \\
\ldots \ldots . \\
\ldots . .37 \\
18.47 \\
\text { none } \\
.19 \\
.99 \\
\ldots . \\
.35\end{array}$ \\
\hline & 100.14 & 98.22 & 100.01 \\
\hline
\end{tabular}

SODA-LIME FELDSPARS.

A. Transparent oligoclase, from Bakersville, North Carolina. Analysis by F. W. Clarke.

B. Feldspar separated from porphyrite; Sugar Loaf, northwest of Elk Mountain, Tenmile district, Summit County, Colorado. Analysis by W. F. Hillebrand. Much altered.

C. Feldspar separated from andesite; mesa northwest of Parkdale, Colorado. Analysis by W. F. Hillebrand.

D. Feldspar separated from gabbro; Brandywine Creek, Wilmington, Delaware. Sp. gr., 2.592 to 2.877. Described by Chester in Bull. 59. Analysis by R. B. Riggs.

E. Feldspar from hypersthene gabbro, Wilmington, Delaware. Sp. gr., 2.592 to 2.780. Described by Chester, analysis by Riggs.

F. Feldspar separated from the gabbro of Ashland County, Wisconsin. Analysis by W. F. Hillebrand.

G. Feldspar separated from diabase; near southeast corner of sec. 13, T. 47 N., R. 46 W., Michigan. Described by Van Hise in Mon. XIX. Analysis by T. M. Chatard.

\begin{tabular}{|c|c|c|c|c|c|c|c|}
\hline & A. & B. & C. & D. & E. & F. & G. \\
\hline \multirow{12}{*}{ 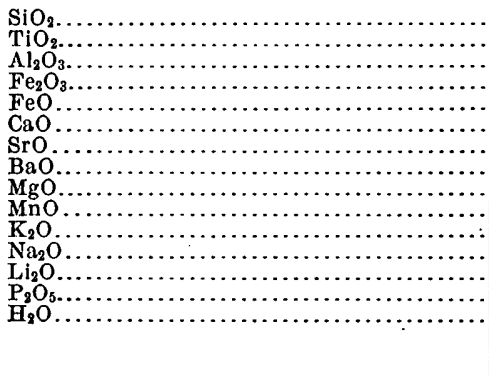 } & 62.92 & 62.96 & 63.02 & 70.37 & 51.44 & \multirow{11}{*}{$\begin{array}{r}53.30 \\
\text { trace } \\
29.03 \\
.55 \\
.23 \\
11.40 \\
\text { trace } \\
\text { trace } \\
.13 \\
\text { none } \\
.40 \\
4.87 \\
\text { none } \\
\text { trace } \\
.23\end{array}$} & 51.18 \\
\hline & 25.32 & 21.51 & 23.05 & 18.36 & 30.05 & & 27.00 \\
\hline & trace & $\cdots, 32$ & & \}$_{\text {undet. }}^{.58}$ & .96 & & $\begin{array}{r}3.19 \\
\text { undet. }\end{array}$ \\
\hline & 4.03 & 4.00 & 3.39 & 5.08 & i3.i9 & & 11.70 \\
\hline & 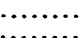 & .13 & trace & & & & $\ldots \ldots \ldots$ \\
\hline & & 30 & trace & .04 & trace & & 1.92 \\
\hline & trace & & & trace & & & .17 \\
\hline & .96 & 1.60 & 3. 92 & .63 & .21 & & \\
\hline & 6.18 & $\begin{array}{l}6.15 \\
\text { trace }\end{array}$ & $\begin{array}{r}6.76 \\
\text { none }\end{array}$ & 4.32 & 4.07 & & $\begin{array}{c}3.48 \\
\ldots \ldots\end{array}$ \\
\hline & & & & & & & \\
\hline & .25 & 2.78 & .26 & .45 & .35 & & 1. 19 \\
\hline & 99.66 & 99.75 & 100.40 & 99.83 & 100.27 & 100.14 & 100.24 \\
\hline
\end{tabular}


H. I. Feldspars separated from olivine diabase; NE. 1 sec. 13; T. 45 N., R. 1 W., Wisconsin. See Van Hise, Mon. XIX. Analyses by.T. M. Chatard.

J. Feldspar from gabbro; southern half of sec. 14, T. 44 N., R. 4 W., Wisconsin. See Van Hise, loc. cit. Analysis by T. M. Chatard.

The following feldspars were separated from Minnesota gabbros for W. S. Bayley and analyzed by W. F. Hillebrand:

K. From average gabbro, south quarter post, sec. 35 , T. 61 N., R. 12 W.

L. From gabbro, NW. $\frac{1}{4}$ SE. $\frac{1}{4}$ sec. 23, T. 62 N., R. 10.

M. From gabbro, center of sec. 25 , T. 64 N., R. 8.

N. From gabbro, Duluth and Iron Range Railroad.

O, P. Two feldspars separated from the amphibolite of Palmer Center, Massachusetts. Analyses by W. F. Hillebrand. Specific gravity of $0,2.667$ at $24^{\circ}$; of $P$, 2.677 at $22^{\circ}$. In $\mathrm{O}$ calculation gives about 7.4 and in $\mathrm{P} 7.6$ per cent of admixed quartz.

\begin{tabular}{|c|c|c|c|c|c|c|c|c|c|}
\hline & H. & I. & J. & K. & L. & M. & N. & o. & P. \\
\hline 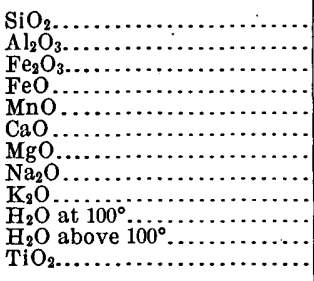 & $\begin{array}{r}61.65 \\
19.91 \\
2.28 \\
\text { undet. } \\
\text { trace } \\
4.12 \\
.61 \\
4.74 \\
5.72 \\
\quad .95\end{array}$ & $\begin{array}{r}56.15 \\
26.05 \\
1.98 \\
\text { undet. } \\
.13 \\
8.70 \\
.54 \\
4.79 \\
1.56 \\
.13 \\
.64\end{array}$ & $\begin{array}{r}51.99 \\
29.32 \\
1.23 \\
\text { trace } \\
12.60 \\
.63 \\
2.91 \\
.28 \\
.03 \\
.54\end{array}$ & $\begin{array}{r}51.89 \\
29.68 \\
.32 \\
.37 \\
12.62 \\
.38 \\
3.87 \\
.50 \\
.07 \\
.39\end{array}$ & $\begin{array}{r}52.50 \\
30.15 \\
.47 \\
.15 \\
\because 12.82 \\
.10 \\
3.72 \\
.53 \\
.25 \\
\text { trace }\end{array}$ & $\begin{array}{r}52.61 \\
29.80 \\
.57 \\
.23 \\
\text { trace } \\
12.25 \\
.20 \\
3.80 \\
.53 \\
.29 \\
.29 \\
\text { trace }\end{array}$ & $\begin{array}{r}53.45 \\
29.77 \\
.33 \\
.15 \\
-11.33 \\
11 . \\
.11 \\
4.33 \\
.68 \\
.23 \\
\text { trace }\end{array}$ & $\begin{array}{r}5.83 \\
7.78 \\
.20 \\
.42 \\
\text { trace }\end{array}$ & $\begin{array}{r}7.85 \\
6.26 \\
.16 \\
.48 \\
\text { trace }\end{array}$ \\
\hline & 99.98 & 100.67 & 99.53 & 100.09 & 100.69 & 100.28 & 100.38 & 100.51 & 100.62 \\
\hline
\end{tabular}

LEUCITE.

From Mount Vesuvius. A fine crystal. Analysis by George Steiger.

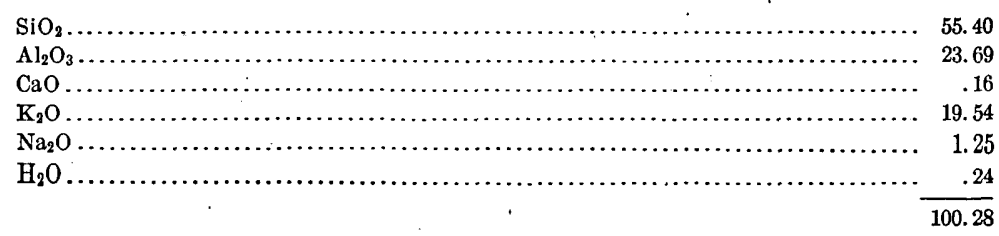

ENSTATITE.

A. Enstatite from Granville, Massachusetts. Slightly altered. Described by Emerson in Mon. XXIX. Analysis by W. F. Hillebrand.

B. Enstatite separated from the San Emigdio meteorite, found in San Bernardino County, California. Analysis by J. E. Whitfield.

C. White, fibrous mineral, near enstatite, from seams in chrysolite rock, Corundum Hill, North Carolina.

D. Altered enstatite, Corundum Hill: Analyses C and D by T. M. Chatard. (See Bull, 42.) Sp. gr., 2.872.

E. Bronzite separated from the websterite of Hebbville, Maryland. Described by Williams in Am. Geologist, vol. 6, p. 35. Analysis by T. M. Chatard. 


\begin{tabular}{|c|c|c|c|c|c|}
\hline . & A. & B. & C. & D. & E. \\
\hline \multirow[t]{2}{*}{ 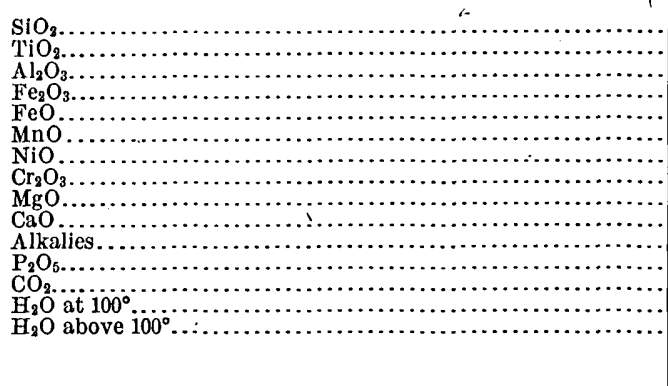 } & $\begin{array}{r}54.04 \\
\text { none } \\
.52 \\
1.51 \\
3.90 \\
.11 \\
.23 \\
.14 \\
34.40 \\
\text { none } \\
.08 \\
\text { none } \\
1.32 \\
.70 \\
3.07\end{array}$ & 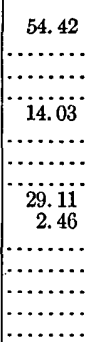 & 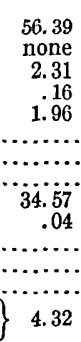 & $\begin{array}{r}56.58 \\
\text { none } \\
1.74 \\
1.89 \\
3.67 \\
.21 \\
\ldots \ldots . .2 \\
30.34 \\
.59 \\
.17 \\
\ldots \ldots . . \\
\ldots \ldots \\
4.55\end{array}$ & $\begin{array}{r}54.53 \\
\text { undet. } \\
1.93 \\
1.70 \\
8.92 \\
.28 \\
\ldots .30 \\
29.51 \\
2.25 \\
\ldots \ldots . \\
\text { trace } \\
\ldots . . \\
1.14\end{array}$ \\
\hline & 100.02 & 100.02 & 99.75 & 99.98 & 100.56 \\
\hline
\end{tabular}

HYPERSTHENE.

A. From the basalt of Mount Thielsen, Oregon. Incomplete analysis by T. M. Chatard.

B. From gabbro, SE. $\frac{1}{4}$ sec. 20, T. 65 N., R. 4 W., Minnesota. Described by Bayley in Jour. Geology, vol. 3, p. 1. Analysis by E. A. Schneider.

C. From the augite andesite of the Tokajer-Berg, Hungary. Analysis by W. F. Hillebrand. Sp. gr., 3.495 at $25^{\circ}$.

D, E, F. Three specimens separated from the hypersthene andesite of the Buffalo Peaks, Colorado. Described by Cross in Bull. 1. Analyses by W. F. Hillebrand. In $\mathrm{D}$ and $\mathrm{E}$ alkalies were disiregarded. In $\mathrm{E}$ and $\mathrm{F}$ all the iron is given as $\mathrm{FeO}$. Sp. gr., of $\mathrm{F}, 3.307$ at $23^{\circ}$.

\begin{tabular}{|c|c|c|c|c|c|c|}
\hline . & A. & B. & C. & D. ${ }^{\circ}$ & E. & F. \\
\hline 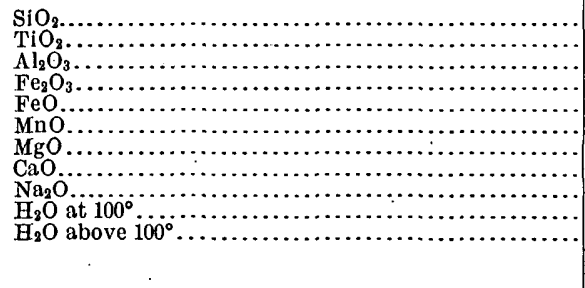 & $\left\{\begin{array}{r}53.31 \\
\cdots \\
5.99 \\
13.43 \\
21.69 \\
3.69 \\
\cdots \ldots \ldots \ldots \\
\cdots \ldots \ldots \\
98.11\end{array}\right.$ & \begin{tabular}{|r}
48.44 \\
undet. \\
7.91 \\
.33 \\
20.88 \\
.92 \\
19.35 \\
1.44 \\
\hdashline .08 \\
none \\
99.35
\end{tabular} & $\begin{array}{r}51.44 \\
.73 \\
.60 \\
2.28 \\
20.77 \\
.88 \\
19.93 \\
3.80 \\
\cdots \\
\cdots \\
\end{array}$ & $\begin{array}{r}51.70 \\
1.72 \\
.30 \\
18.00 \\
.36 \\
25.09 \\
2.87 \\
\cdots \ldots \ldots \\
\cdots \ldots . . \\
\cdots\end{array}$ & $\left\{\begin{array}{r}51.16 \\
2.15 \\
18.36 \\
.36 \\
24.25 \\
3.81 \\
\cdots \\
\cdots \\
\cdots\end{array}\right.$ & $\left\{\begin{array}{r}50.04 \\
\ldots .91 \\
17.81 \\
.12 \\
21.74 \\
6.70 \\
.27 \\
\ldots \ldots . . \\
\cdots \ldots . . \\
99.59\end{array}\right.$ \\
\hline
\end{tabular}

PYROXENE.

A. Diopside separated from the websterite of Hebbville, Maryland. Described by G. H. Williams, Am. Geologist, vol. 6, p. 35. Analysis by T. M. Chatard.

B. Diopside from the leucite rocks of the Leucite Hills, Wyoming. Described by Cross, Am. Jour. Sci., 4th ser., vol. 4, p. 115 . Sp.gr., 3.290 at $20^{\circ}$. Analysis by W. F. Hillebrand.

C. Pyroxene from Moriah, New York. The source of associated serpentine. Analysis by Charles Catlett.

D. Dark-gray pyroxene, Montville, New Jersey. The source of associated serpentine. Analysis by Charles Catlett.

$$
\text { 15619-Bull. 419-10-18 }
$$


E. Diallage from the gabbro of Ashland County, Wisconsin. Analysis by W. F. Hillebrand.

F. Pyroxene from orthoclase gabbro, 1 mile northeast of Haystack Mountain, Livingston quadrangle, Montana. Described by W. H. Emmons, Jour. Geology, vol. 16; p. 193. Analysis by G. Steiger.

\begin{tabular}{|c|c|c|c|c|c|c|}
\hline & A. & B. & C. & D. & E. & F. \\
\hline 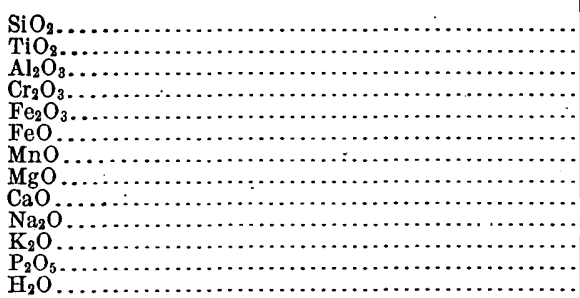 & $\begin{array}{r}51.80 \\
.13 \\
2.21 \\
.51 \\
1.29 \\
3.50 \\
\text { trace } \\
17.76 \\
20.99 \\
\text { undet. } \\
\text { undet. } \\
\text { trace } \\
.65\end{array}$ & $\begin{array}{r}50.86 \\
3.03 \\
\cdots \\
1.19 \\
1.82 \\
.03 \\
17.42 \\
23.32 \\
.76 \\
.42 \\
.31\end{array}$ & 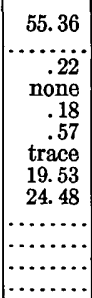 & $\begin{array}{r}51.45 \\
\cdots 2.94 \\
\ldots \ldots .06 \\
1.06 \\
\text { trace } \\
18.43 \\
24.02 \\
\cdots \ldots \ldots \\
\cdots \ldots . . \\
\cdots \ldots .0 \\
1.08\end{array}$ & $\begin{array}{r}49.80 \\
1.29 \\
2.86 \\
\ldots . .78 \\
2.48 \\
10.82 \\
.37 \\
15.33 \\
16.50 \\
.51 \\
.12 \\
\text { trace } \\
.33\end{array}$ & \begin{tabular}{r}
50.95 \\
1.42 \\
2.72 \\
\hdashline 1.70 \\
13.86 \\
.26 \\
15.58 \\
11.39 \\
.31 \\
1.80
\end{tabular} \\
\hline & 98.84 & 99.16 & 100.34 & 99.94 & 100.41 & 99.99 \\
\hline
\end{tabular}

G. Augite from nepheline basalt, 'Black Mountain, Uvalde quadrangle, Texas. Violet colored. Analysis by W. F. Hillebrand.

H. Augite from dolerite dike, near Valmont, Colorado. See Cross, Mon. XXVII. Analysis by L. G. Eakins.

I. Augite from tinguaiite, Two Buttes, Colorado. Sp. gr., 3.43 at $28^{\circ}$. Analysis by W. F. H.illebrand.

J. Pyroxene from syenitic lamprophyre, Two Buttes, Colorado. Sp. gr., 3.45 at $25^{\circ}$. Analysis by W. F. Hillebrand.

K. Augite from granite, north end of Blue Mountains, Silver Cliff, Colorado. Sp. gr., 3.225 at $18^{\circ}$. Analysis by L. G. Eakins.

L. Augite from the Golden King dike, Silver Cliff, Colorado. Sp. gr., 3.281 at $13^{\circ}$. Analysis by L. G. Eakins.

M. Pyroxene from norite, dike east of Sugar Loaf, Boulder County, Colorado. Analysis by L. G. Eakins.

\begin{tabular}{|c|c|c|c|c|c|c|c|}
\hline & G. & H. & I. . & J. & K. & L. & M. \\
\hline 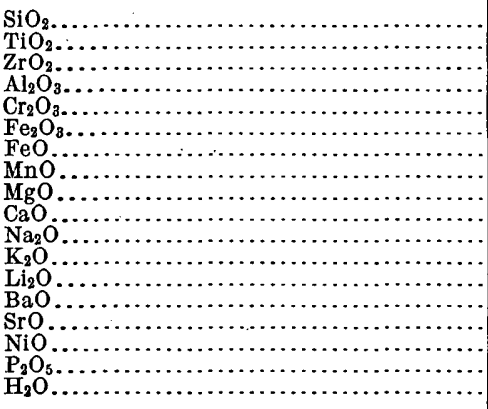 & $\begin{array}{r}45.23 \\
4.28 \\
7.73 \\
2.95 \\
4.07 \\
.07 \\
12.25 \\
23.37 \\
.47 \\
.12 \\
\text { trace } \\
\text { none } \\
\text { none } \\
.05 \\
\text { none } \\
.37\end{array}$ & 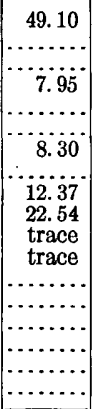 & $\begin{array}{r}47.54 \\
3.00 \\
\text { none } \\
4.14 \\
\text { trace? } \\
5.64 \\
6.42 \\
.36 \\
10.05 \\
21.57 \\
1.38 \\
: 12 \\
\text { trace } \\
\text { none } \\
\text { none } \\
\text { trace } \\
\text { undet. } \\
\text { undet. }\end{array}$ & $\begin{array}{r}51.27 \\
.70 \\
\text { none } \\
3.05 \\
\text { none } \\
3.08 \\
4.34 \\
.28 \\
14.21 \\
22.58 \\
.67 \\
.06 \\
\ldots . . . \\
\text { none } \\
\text { none } \\
.03 \\
\ldots . . .\end{array}$ & 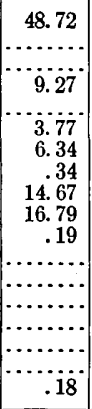 & 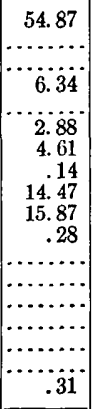 & 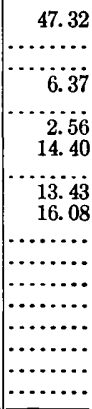 \\
\hline & 100.96 & 100.26 & 100.22 & 100.27 & 100.27 & 99.77 & 100.16 \\
\hline
\end{tabular}

N. Pyroxene from Italian Mountain, Gunnison County, Colorado. Associated with idocrase, scapolite, garnet, epidote, etc. Analysis by L. G. Eakins. Sp. gr., 3.312 at $16.7^{\circ}$. 
O. Augite from basalt, 6 miles northeast of Grants, Mount Taylor region, New Mexico. Analysis by T. M. Chatard.

P. Pyroxene from peridotite, east of Fort Ellis, Montana. Partial analysis by F. W. Clarke.

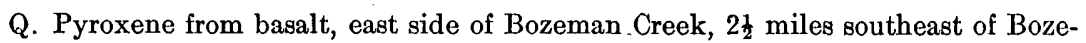
man, Montana. Described by Merrill, Proc. U. S. Nat. Mus., vol. 17, p. 637. Analysis by L. G. Eakins.

\begin{tabular}{|c|c|c|c|c|}
\hline . & N. & O. & P. & Q. \\
\hline 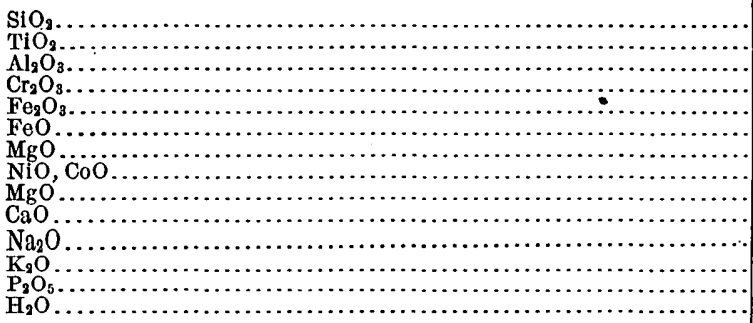 & $\begin{array}{r}47.53 \\
9.88 \\
1.79 \\
\text { trace } \\
\cdots \\
14.43 \\
25.46 \\
\text { trace } \\
\cdots \\
\cdots \\
\cdots\end{array}$ & $\begin{array}{r}47.06 \\
1.82 \\
7.77 \\
\text { trace } \\
1.30 \\
8.15 \\
.20 \\
\text { trace } \\
13.52 \\
19.33 \\
.33 \\
.11 \\
.06 \\
.20\end{array}$ & $\begin{array}{r}51.95 \\
\ldots .21 \\
4.21 \\
\cdots \ldots . . . \\
5.90 \\
\text { undet. } \\
\cdots \\
13.81 \\
23.32 \\
\cdots \ldots . . \\
\cdots \ldots . . . \\
\hdashline \text { undet. }\end{array}$ & $\begin{array}{r}52.50 \\
2.26 \\
1.07 \\
2.05 \\
2.47 \\
\text { trace } \\
\ldots . .11 \\
17.11 \\
21.70 \\
.35 \\
.07 \\
\ldots . . . \\
.64\end{array}$ \\
\hline - & 100.30 & 99.85 & $\cdot 99.19$ & 100.22 \\
\hline
\end{tabular}

R. Jeffersonite from Franklin Furnace, New Jersey. Analyzed by W. F. Hillebrand and described by him in Bull. 167.

S. Jeffersonite, Parker mine, Franklin Furnace, New Jersey. Collected by C. Palache. Analysis by G. Steiger.

T. Manganese pyroxene, Sterling Hill, New Jersey. Collected by C. Palache. Analysis by W. T. Schaller.

\begin{tabular}{|c|c|c|c|}
\hline & $\mathrm{R}$ & S. & T. \\
\hline 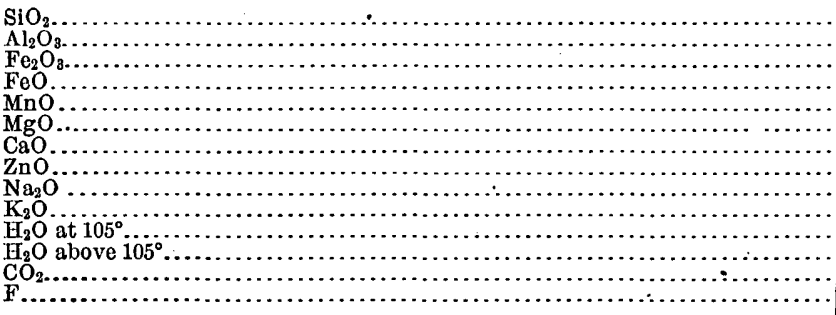 & $\begin{array}{r}51.70 \\
.36 \\
.37 \\
7.43 \\
12.57 \\
23.68 \\
3.31 \\
.12 \\
\operatorname{trace} \\
.65 \\
\ldots \ldots \\
\ldots \ldots\end{array}$ & $\begin{array}{r}49.03 \\
.86 \\
4.22 \\
3.95 \\
7.91 \\
5.81 \\
19.88 \\
7.14 \\
\ldots \ldots \ldots \\
\ldots \ldots . . \\
.60 \\
\ldots .70 \\
\ldots \ldots . . \\
\ldots \ldots .\end{array}$ & $\begin{array}{r}49.80 \\
.26 \\
1.46 \\
1.61 \\
9.69 \\
12.35 \\
21.07 \\
\text { trace } \\
.09 \\
\ldots . .35 \\
1.55 \\
1.31 \\
.43 \\
.31\end{array}$ \\
\hline \multirow[t]{2}{*}{ 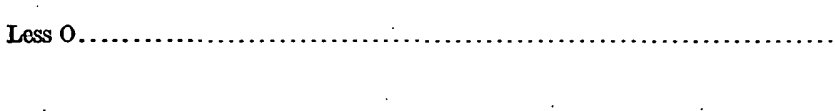 } & $\begin{array}{r}100.19 \\
\ldots \ldots\end{array}$ & 100.10 & $\begin{array}{r}99.93 \\
.13\end{array}$ \\
\hline & & & 99.80 \\
\hline
\end{tabular}

EGIRITE.

From Magnet Cove, Arkansas. Analysis by George Steiger.

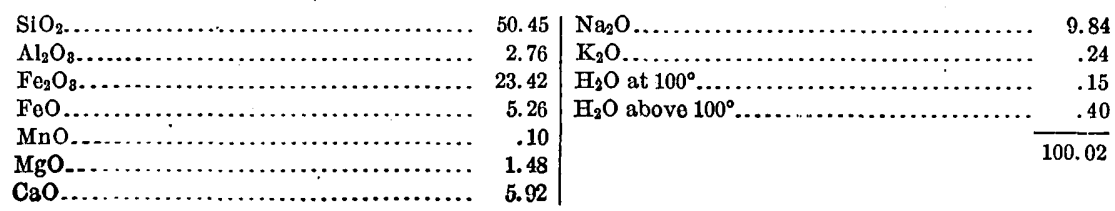


JADEITE.

The following analyses of jadeite, all by $\mathrm{F} . \dot{\mathrm{W}}$. Clarke, were discussed by Clarke and Merrill in the Proceedings of the United States National Museum for 1888. They all represent worked material.

A. Light-colored bead, mottled with emerald green, from State of Oaxaca, Mexico. Sp. gr., 3.007, determined by William Hallock.

B. Carved head, light green, from Zaachita, Oaxaca. Sp. gr., 3.190, Hallock.

C. Fragment from Sardinal, Costa Rica; pale green, translucent. Sp. gr., 3.32, Clarke.

D. Fragment from Culebra, Costa Rica; light green, granular, opaque; quite impure. Sp. gr., 3.27, Clarke.

\begin{tabular}{|c|c|c|c|c|}
\hline . & A. & B. & C. & D. \\
\hline 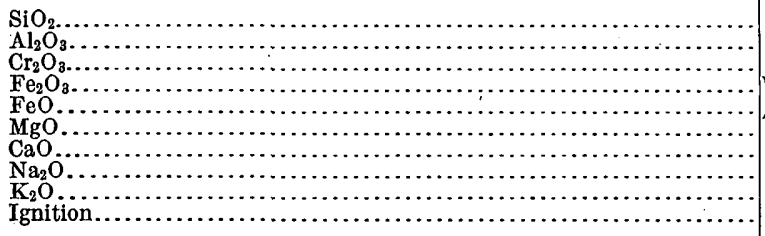 & $\begin{array}{r}58.88 \\
25.93 \\
.12 \\
.24 \\
.36 \\
.40 \\
11.64 \\
.63 \\
1.81\end{array}$ & $\begin{array}{r}58.18 \\
23.53 \\
1.67 \\
1.72 \\
2.35 \\
11.81 \\
.77 \\
.53\end{array}$ & $\begin{array}{r}59.18 \\
22.96 \\
1.87 \\
.67 \\
1.52 \\
12.71 \\
\text { trace } \\
.90\end{array}$ & $\begin{array}{r}58.33 \\
21.63 \\
1.71 \\
.73 \\
3.09 \\
4.92 \\
8.13 \\
.22 \\
.93\end{array}$ \\
\hline & 100.01 & 100.56 & 99.81 & 99.69 \\
\hline
\end{tabular}

WOLLASTONITE.

Compact variety, from Diana, New York. Partial analysis by E. A. Schneider:

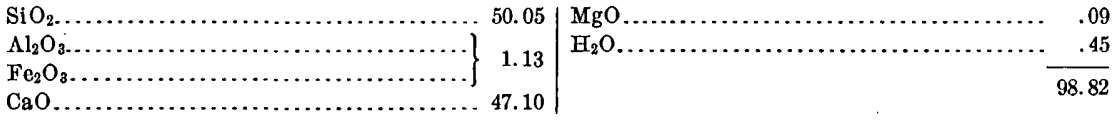

PECTOLITE.

A. Stone hammer, at first supposed to be jade, collected among the Eskimo of Point Barrow, Alaska. Analyzed by F. W. Clarke, and described by him in Bull. 9. Pale apple-green, tough, compact, highly polished. Sp. gr., 2.873 .

B. Radiated pectolite from Bergen Hill, New Jersey. Analyzed by E. A. Schneider.

C. Another sample from Bergen Hill. Analysis by George Steiger.

\begin{tabular}{|c|c|c|c|}
\hline & A. & B. & C. \\
\hline 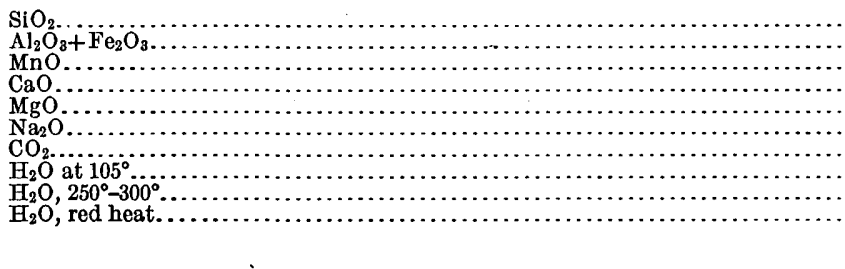 & $\begin{array}{r}53.94 \\
.58 \\
32.21 \\
1.43 \\
8.57 \\
4.09 \\
4.09 \\
\end{array}$ & $\begin{array}{r}53.11 \\
.40 \\
.81 \\
33.88 \\
8.62 \\
.04 \\
.04 \\
.14 \\
2.86 \\
99.86\end{array}$ & $\begin{array}{r}53.34 \\
.33 \\
.45 \\
33.23 \\
9.11 \\
.67 \\
.27 \\
2.70 \\
100.10\end{array}$ \\
\hline
\end{tabular}

AMPHIBOLE.

A. Tremolite, pseudomorphous after sahlite; northeast slope of Canaan Mountain, Connecticut. Analysis by W. F. Hillebrand. 
B. Tremolite, found in the serpentine of Easton, Pennsylvania. Analysis by I. G. Eakins.

C. Actinolite (?), Corundum Hill, North Carolina. Sp. gr., 3.062. Analyzed by T. M. Chatard and described by him in Bull. 42.

D. Nephrite, New Zealand. Fragment from a dark-green bowlder. Analysis by F. W. Clarke.

E. Nephrite, Robenhausen, Lake Pfäffikon, Switzerland. Part of a green, compact, highly polished jade implement. Sp. gr., 3.015;.determined by William Hallock. Analysis by F. W. Clarke.

\begin{tabular}{|c|c|c|c|c|c|}
\hline & A. & B. & C. & D. & E. \\
\hline \multirow{6}{*}{ 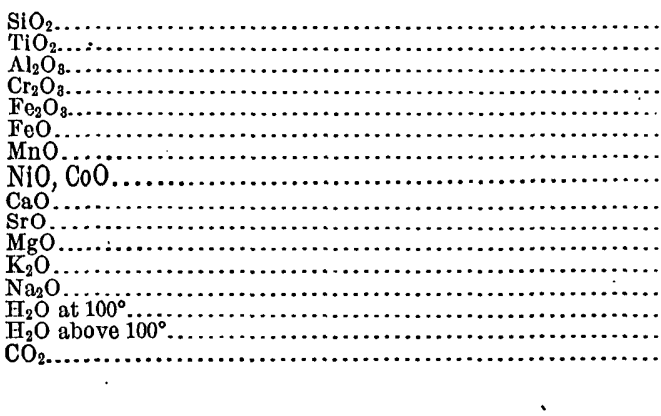 } & $\begin{array}{r}57.97 \\
\text { none } \\
.09\end{array}$ & $\begin{array}{r}58.27 \\
.33\end{array}$ & \multirow{5}{*}{$\begin{array}{r}55.23 \\
\text { none } \\
3.04 \\
.19 \\
1.88 \\
2.51 \\
.26 \\
\text { trace } \\
13.36 \\
22.31 \\
.58 \\
.04 \\
.52 \\
.52\end{array}$} & $\begin{array}{c}56.73 \\
3.22\end{array}$ & \\
\hline & $\begin{array}{r}.11 \\
\text { trace }\end{array}$ & $\begin{array}{l}\text { trace } \\
.08\end{array}$ & & $\begin{array}{r}5.96 \\
\text { trace }\end{array}$ & $\begin{array}{r}6.33 \\
\text { trace }\end{array}$ \\
\hline & \multirow{3}{*}{$\begin{array}{r}15.05 \\
\text { trace } \\
22.45 \\
.12 \\
.20 \\
.03 \\
2.57 \\
1.69\end{array}$} & 11.90 & & 13.24 & 13045 \\
\hline & & $\begin{array}{r}25.93 \\
.42 \\
1.25\end{array}$ & & $\begin{array}{l}19.42 \\
\text { undet. } \\
\text { undet. }\end{array}$ & $\begin{array}{l}\text { 2i.06 } \\
\text { undet. } \\
\text { undet. }\end{array}$ \\
\hline & & 1.22 & & & \\
\hline & 100.46 & 99.40 & 99.92 & 99.40 & 99.84 \\
\hline
\end{tabular}

F. Dark-green nephrite implement, from the Eskimo of Point Barrow, Alaska. Sp. gr., 3.012. Analysis by F. W. Clarke.

G. Nephrite adze, Point Barrow. Nearly black, with grayish-green patches. Sp. gr., 2.922, Hallock. Analysis by F. W. Clarke.

H. Nephrite adze, Cape Prince of Wales, Alaska. Dark green, laminated in two shades, opaque. Sp. gr., 2.989, Hallock. Analysis by F. W. Clarke.

I. Worked nephrite, St. Michael, Alaska. Dull apple green, fairly uniform in tint, semitranslucent at edges. Sp. gr., 3.006, Hallock. Analysis by F. W. Clarke.

J. Jade implement, Diomede Island, Alaska. Dark green, laminated in two shades, opaque. Sp. gr., 3.010, Hallock. Analysis by F. W. Clarke.

\begin{tabular}{|c|c|c|c|c|c|}
\hline & F. & G. & H. & $\mathrm{I}$. & J. \\
\hline \multirow[t]{2}{*}{ 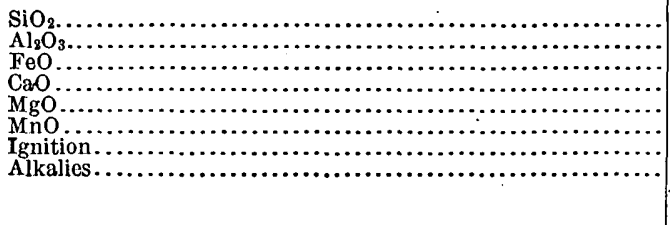 } & $\begin{array}{r}57.01 \\
.42 \\
6.95 \\
12.75 \\
21.36 \\
\cdots 1.41 \\
\text { undet. }\end{array}$ & $\begin{array}{r}57.11 \\
2.57 \\
5.15 \\
11.54 \\
21.38 \\
\text { trace } \\
2.06 \\
\text { undet. }\end{array}$ & $\begin{array}{r}56.01 \\
1.98 \\
6.34 \\
12.54 \\
21.57 \\
\text { trace } \\
1.91 \\
\text { undet. }\end{array}$ & $\begin{array}{r}56.12 \\
7.63 \\
7.45 \\
12.72 \\
20.92 \\
\text { trace } \\
1.42 \\
\text { undet. }\end{array}$ & $\begin{array}{r}56.08 \\
1.01 \\
7.67 \\
13.35 \\
19.96 \\
\text { trace } \\
2.03 \\
\text { undet. }\end{array}$ \\
\hline & 99.90 & 99.81 & 100.35 & 99.26 & 100.10 \\
\hline
\end{tabular}

K, L, M, N. Four samples of nephrite, found in place by Lieutenant Stoney, U. S. Navy, near the Kowak River, Alaska. K, greenish gray, splintery-lamellar. $\mathrm{L}$, like $\mathrm{K}$, but more granular. $\mathrm{M}$, paler, nearly white, closer grained. $\mathrm{N}$, brownish, highly foliated. Analyses by F. W. Clarke. Ferrous iron determinations by R. B. Riggs. O, nephrite from Jordansmühl, Silesia; analysis by George Steiger; typical 
material from an old locality. For details concerning nephrites $\mathrm{D}$ to $\mathrm{N}$, see the memoir by Clarke and Merrill in Proc. U. S. Nat. Mus. for 1888.

\begin{tabular}{|c|c|c|c|c|c|}
\hline & K. & L. & M. & N. & 0. \\
\hline $\mathrm{SiO}_{2} \ldots \ldots \ldots \ldots \ldots \ldots$ & 58.11 & 55.87 & 56.85 & 57.38 & 56.39 \\
\hline $\mathrm{Al}_{2} \mathrm{O}_{3 .}$ & .24 & 2.07 & .88 & .19 & 1.63 \\
\hline $\mathrm{Fe}_{2} \mathrm{FO}_{3}$ & 5.44 & 5.79 & $\begin{array}{r}4.33 \\
.14\end{array}$ & $\begin{array}{l}4.43 \\
1.25\end{array}$ & $\begin{array}{l}1.72 \\
3.70\end{array}$ \\
\hline $\mathrm{MnO}$. & trace & & $\begin{array}{l}1.40 \\
\text { trace }\end{array}$ & $\begin{array}{l}1.20 \\
\text { trace }\end{array}$ & $\begin{array}{l}3.70 \\
.26 \\
13\end{array}$ \\
\hline $\mathrm{CaO}$ & 12.01 & 12.43 & 13.09 & 12.14 & \\
\hline 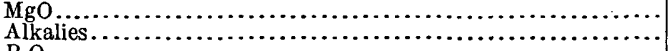 & $\begin{array}{r}21.97 \\
\text { undet. }\end{array}$ & $\begin{array}{l}21.62 \\
\text { undet. }\end{array}$ & $\begin{aligned} 21.56 \\
\text { undet. }\end{aligned}$ & $\begin{array}{r}22.71 \\
\text { undet. }\end{array}$ & $\begin{array}{l}24.63 \\
\text { none }\end{array}$ \\
\hline \multirow{3}{*}{ 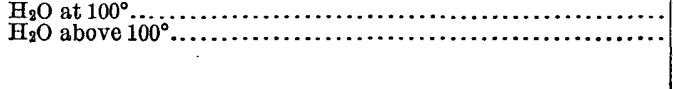 } & & & & & trace \\
\hline & 1.78 & 1.38 & 1.76 & 1.73 & $\begin{array}{r}.65 \\
3.42\end{array}$ \\
\hline & 99.93 & 99.54 & 99.92 & 99.83 & 100.45 \\
\hline
\end{tabular}

P. Brown hornblende, Pierrepont, New York. Analysis by T. M. Chatard.

Q. Hornblende separated from gabbro, east shaft of waterworks extension, Washington, D. C. Analysis by R. B. Riggs.

R. Amphibole from the serpentine of Montville, New Jersey. Analysis by L. G. Eakins.

S. Hornblende separation, gedrite, from south of soapstone quarry, Warwick, Massachusetts. Analysis by E. A. Schneider.

T. Hornblende from amphibolite dike, Palmer Center, Massachusetts. Sp. gr., 3.220 at $31.5^{\circ}$. Analysis by W. F. Hillebrand.

U. Hornblende from amphibolite bed, same locality and analyst. Sp. gr., 3.217 at $29^{\circ}$.

\begin{tabular}{|c|c|c|c|c|c|c|}
\hline . & P. & Q. & R. & S. & T. & U. \\
\hline 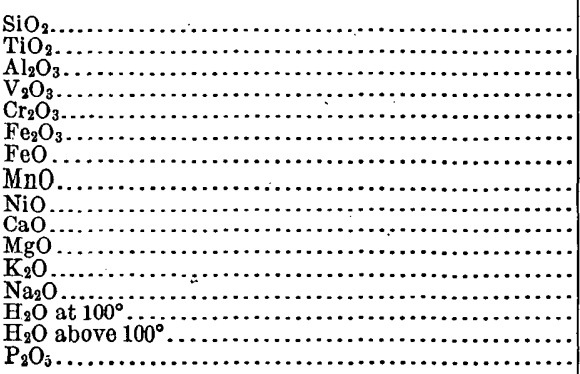 & $\begin{array}{r}56.44 \\
1.77 \\
1.77 \\
. .84 \\
.73 \\
.11 \\
11.83 \\
22.98 \\
.75 \\
2.13 \\
.05 \\
2.41 \\
\text { trace }\end{array}$ & \begin{tabular}{|r|}
52.42 \\
3.15 \\
$\cdots$ \\
3.60 \\
8.36 \\
.11 \\
$\cdots$ \\
14.33 \\
15.85 \\
.12 \\
1.16 \\
1.25 \\
$\cdots \cdots$
\end{tabular} & $\begin{array}{r}43.31 \\
\ldots 17.41 \\
\ldots \ldots . . . \\
.71 \\
.59 \\
.14 \\
\ldots 12.84 \\
19.39 \\
1.36 \\
2.23 \\
1.17 \\
\ldots \ldots\end{array}$ & $\begin{array}{r}47.86 \\
a .63 \\
14.09 \\
\ldots . . . \\
\ldots .33 \\
13.41 \\
.14 \\
\ldots .57 \\
19.89 \\
.06 \\
.93 \\
\text { none } \\
2.46 \\
.05\end{array}$ & $\begin{array}{r}43.11 \\
1.32 \\
11.10 \\
.07 \\
\text { trace } \\
4.97 \\
13.04 \\
.43 \\
\text { trace } \\
11.76 \\
9.35 \\
1.27 \\
1.18 \\
.16 \\
1.92 \\
.10\end{array}$ & $\begin{array}{r}44.09 \\
1.73 \\
10.68 \\
\text { undet. } \\
\text { trace } \\
2.72 \\
12.96 \\
.32 \\
\text { trace } \\
11.58 \\
10.75 \\
.88 \\
1.19 \\
.21 \\
1.91 \\
.10\end{array}$ \\
\hline & 100.15 & 100.35 & 99.15 & 100.42 & 99.78 & 99.12 \\
\hline
\end{tabular}

a Admixed rutile.

V. Hornblende near barkevikite. Separated from the sodalite syenite of Square Butte, Highwood Mountains, Montana. Described by Lindgren in Am. Jour. Sci., 3d ser., vol. 45, p. 286 . Analysis by W. H. Melville.

W. Amphibole separated from quartz diorite, south of Table Mountain, on ridge between Butte and Plumas counties, California. Described by Turner in 17th Ann., pt. 1, p. 521. Analysis by William Valentine. Chromium determination by Hillebrand. 
X. Amphibole separated from amphibole gabbro, Beaver Creek, Big Trees quadrangle, California. Analysis by William Valentine. See Bull. 168, p. 206.

Y. Amphibole separated from quartz monzonite, Tioga road, southeast of Mount Hoffman, Mariposa County, California. Sp. gr., 3.203 at 21. $5^{\circ}$. Analysis by W. F. Hillebrand. See Bull. 168, p. 208.

$\mathrm{Z}$. Amphibole separated from the granite of Butte, Montana. Incomplete analyeis for lack of material. Analysis by H. N. Stokes.

\begin{tabular}{|c|c|c|c|c|c|}
\hline & V. & W. & X. & $\mathbf{Y}$ & $Z$. \\
\hline 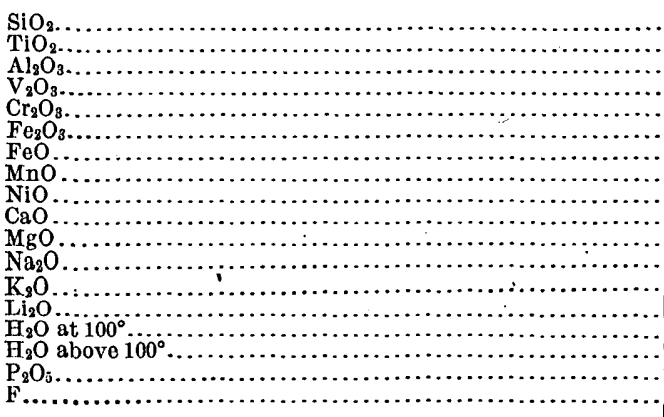 & 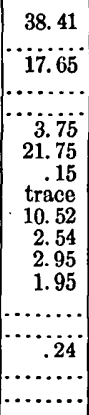 & $\begin{array}{r}50.08 \\
.76 \\
7.97 \\
\ldots . .16 \\
2.69 \\
6.71 \\
.49 \\
\ldots \ldots .21 \\
11.31 \\
1.22 \\
.46 \\
\ldots \ldots . \\
\ldots 1.40 \\
\text { trace } \\
\text { undet. }\end{array}$ & \begin{tabular}{r}
46.08 \\
.77 \\
10.56 \\
$\ldots \ldots .$. \\
\hdashline. .81 \\
8.30 \\
.15 \\
\hdashline$\ldots .$. \\
12.64 \\
14.40 \\
1.62 \\
.34 \\
none \\
.17 \\
1.97 \\
.18 \\
none
\end{tabular} & $\begin{array}{r}47.49 \\
1.21 \\
7.07 \\
.04 \\
\text { none } \\
4.88 \\
10.69 \\
.51 \\
.02 \\
11.92 \\
13.06 \\
.75 \\
.49 \\
\text { trace } \\
\ldots . . .6 \\
1.86 \\
\text { none } \\
.06\end{array}$ & $\begin{array}{r}45.73 \\
1.43 \\
6.77 \\
\ldots \ldots . . \\
\text { none } \\
4.94 \\
10.39 \\
.54 \\
\ldots \ldots . .5 \\
11.25 \\
12.32 \\
.77 \\
1.22 \\
\text { trace } \\
.49 \\
2.29 \\
.35 \\
.28\end{array}$ \\
\hline \multirow[t]{2}{*}{ 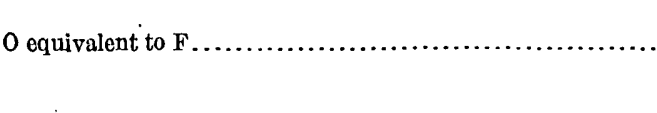 } & $\begin{array}{r}99.91 \\
\cdots\end{array}$ & $\begin{array}{r}99.46 \\
\cdots \ldots . .\end{array}$ & $\begin{array}{r}99.99 \\
\cdots\end{array}$ & $\begin{array}{r}100.05 \\
.02\end{array}$ & $\begin{array}{r}98.77 \\
.12\end{array}$ \\
\hline & & & & 100.03 & 98.65 \\
\hline
\end{tabular}

\section{BERIL.}

A. White beryl from the tin mine at Winslow, Maine. Opaque, milky, associated with mica, fluorite, calcite, tinstone, etc. Sp. gr., 2.707 at $27^{\circ}$. Analysis by W. F. Hillebrand.

B. Green beryl from near Home post-office in eastern Tennessee. Analysis by F. W. Clarke.

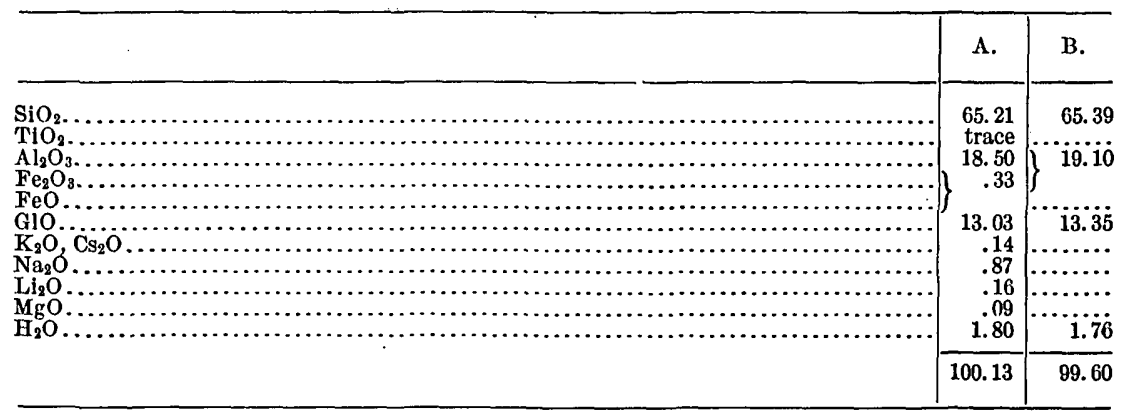

\section{NEPHELITE.}

A. Elæolite from Litchfield, Maine. Dark-gray cleavable masses, of greasy luster. Described by F. W. Clarke in Bull. 42.

B. Another sample from Litchfield. Analysis by George Steiger.

C. Nephelite extracted by solution from the elæolite syenite of Red Hill, New Hampshire. Described by Bayley in Bull. Geol. Soc. America, vol. 3, p. 231. Analysis by W. F. Hillebrand. 


\begin{tabular}{|c|c|c|c|}
\hline , & A. & B. & C. \\
\hline 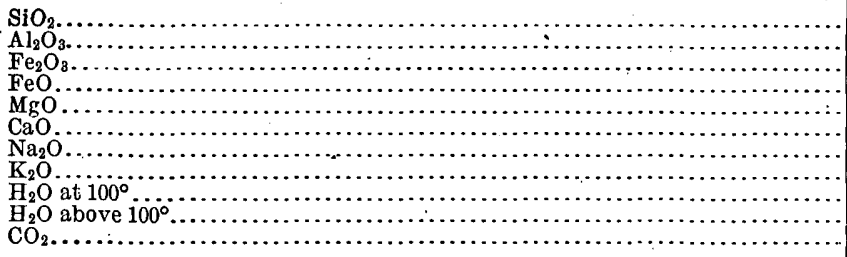 & 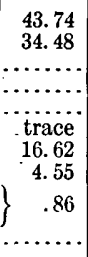 & $\begin{array}{r}45.91 \\
31.14 \\
.34 \\
.23 \\
.33 \\
14.60 \\
5.60 \\
.47 \\
.93 \\
.40\end{array}$ & $\begin{array}{r}45.31 \\
32.67 \\
\ldots \ldots . . \\
2.16 \\
2.00 \\
12.60 \\
5.70 \\
\ldots \ldots \\
1.56\end{array}$ \\
\hline . & 100.25 & 99.95 & 100.00 \\
\hline
\end{tabular}

\section{CANCRINITE.}

All from Litchfield, Maine. See description by Clarke in Bull. 42.

A. Pale yellow, granular.

B. Bright orange-yellow, cleavable, transparent in thin fragments.

C. Dingy pale yellow, otherwise like B.

D. Average yellow sample.

E. Flesh-colored, cleavable mixture of elæolite and cancrinite. Analysis $\mathrm{D}$ by George Steiger; the others by F. W. Clarke. $\mathrm{CO}_{2}$ determinations in $\mathrm{A}, \mathrm{B}, \mathrm{C}, \mathrm{E}$ by R. B. Riggs.

\begin{tabular}{|c|c|c|c|c|c|}
\hline & A. & B. & C. & D. & E. \\
\hline \multirow[t]{2}{*}{ 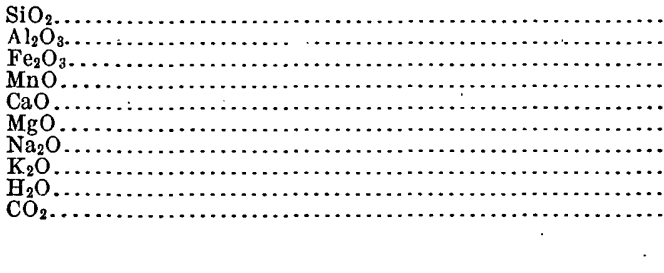 } & $\begin{array}{r}37.22 \\
28.32 \\
\text { trace } \\
\text { trace } \\
4.40 \\
.07 \\
19.43 \\
.18 \\
3.86 \\
6.22\end{array}$ & $\begin{array}{r}36.29 \\
30.12 \\
\text { trace } \\
\text { trace } \\
4.27\end{array}$ & $\begin{array}{r}35.83 \\
29.45 \\
\text { trace } \\
\text { trace } \\
5.12\end{array}$ & $\begin{array}{r}4.72 \\
19.20 \\
.14 \\
4.15 \\
6.11\end{array}$ & $\begin{array}{r}38.93 \\
32.52 \\
\ldots \ldots \\
\text { trace } \\
2.47 \\
\text { none } \\
17.02 \\
3.23 \\
2.83 \\
2.95\end{array}$ \\
\hline & 99.70 & 100.36 & 100.11 & 99.75 & 99.95 \\
\hline
\end{tabular}

SODALITE.

A. Blue sodalite, from Litchfield, Maine. Analysis by F. W. Clarke. See description in Bull. 42.

B. Blue sodalite, from Ice River, near Kicking Horse Pass, British Columbia. Analysis by George Steiger.

C. Sodalite separated from the sodalite syenite of Square Butte, Highwood Mountains, Montana. See Lindgren in Am. Jour. Sci., 3d ser., vol. 45, p. 286. Sp. gr., 2.265. Analysis by W. H. Melville.

\begin{tabular}{|c|c|c|c|}
\hline & $\Lambda$. & B. & C. \\
\hline 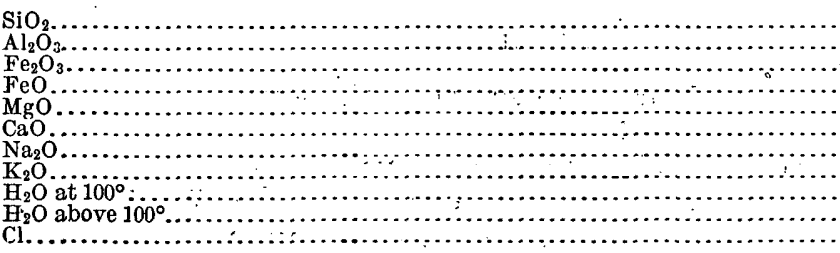 & $\begin{array}{r}37.33 \\
31.87 \\
\ldots \ldots \ldots \\
\ldots \ldots \ldots \\
\cdots \\
24.56 \\
.10 \\
1.07 \\
6.83\end{array}$ & $\begin{array}{r}39.66 \\
30.09 \\
.31 \\
7.18 \\
22.60 \\
1.14 \\
.17 \\
.79 \\
6.12\end{array}$ & $\begin{array}{r}41.56 \\
29.48 \\
. .49 \\
.15 \\
.49 \\
19.21 \\
.91 \\
.45 \\
3.73 \\
4.79\end{array}$ \\
\hline \multirow[t]{2}{*}{ 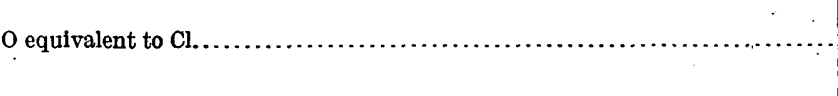 } & $\begin{array}{r}101.76 \\
1.54\end{array}$ & $\begin{array}{r}101.06 \\
1.39\end{array}$ & $\begin{array}{r}101.26 \\
1.08\end{array}$ \\
\hline & 100.22 & 99.67 & 100.18 \\
\hline
\end{tabular}




\section{ZUNYITE.}

From the Zuñi mine, Anvil Mountain, near Silverton, Colorado. Analyzed by W. F. Hillebrand, and described by him as a new species in Bull. 20. Sp. gr., 2.875 at $15^{\circ}$

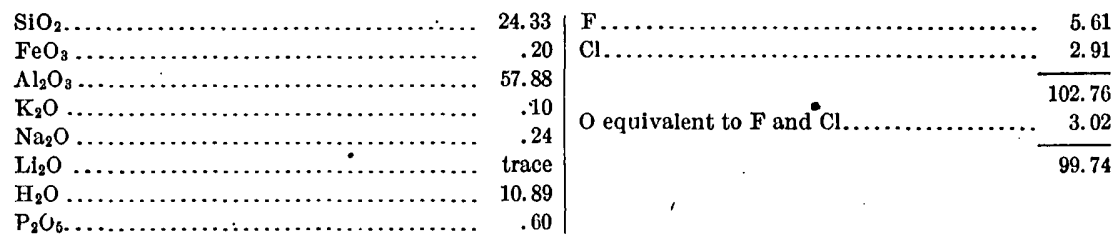

\section{GARNET.}

A. White grossularite; 35 miles east of Selma, Fresno County, California. Analysis by George Steiger. Sp. gr., 3.586.

B. Grossularite. Large waterworn pebble, pale green, very compact. At first, thought to be jade. From Eltoro, 40 miles south of Los Angeles, California. Described by Clarke in Am. Jour. Sci., 3d ser., vol. 50, p. 76. Analysis by Steiger. Sp. gr., 3.485.

C, D, E. Three samples of garnet from Italian Mountain, Gunnison County, Colorado. Sp. gr.: A, 3.72 at $16^{\circ} ; \mathrm{B}, 3.629$ at $23^{\circ} ; \mathrm{C}, 3.721$ at $17.2^{\circ}$. Analyses by L. G. Eakins.

F. Pyrope from the peridotite dike of Elliott County, Kentucky. Described by Diller in Bull. 38. Analysis by T. M. Chatard.

G. Garnets from Hawkes's quarry, Goshen, Massachusetts. Almandite. Analysis by Steiger.

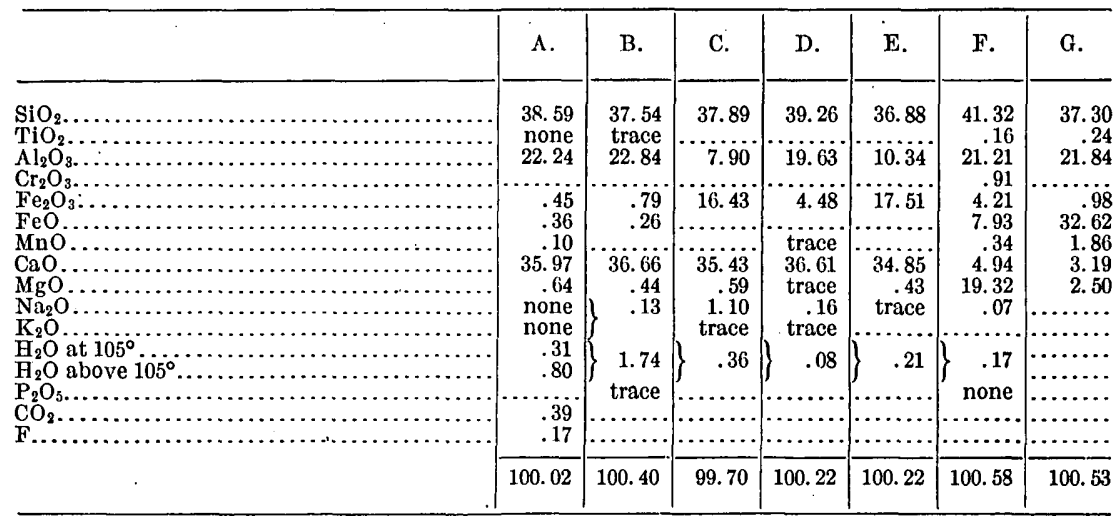

H. Spessartite from Amelia Court House, Amelia County, Virginia. Pale brown, crystalline masses. Analyzed by F. W. Clarke and described in Bull. 60 .

I. Spessartite from Llano County, Texas. Yellow, granular. Analyzed by W. H. Melville and described in Bull. 90. FeO could not be separately determined.

J. Spessartite from, cavities in rhyolite, Nathrop, Colorado. Brilliant crystals. Sp. gr., 4.23 at $18^{\circ}$. Described by Cross in Am. Jour. Sci., 3d ser., vol: 31, p. 432. Analysis by L. G. Eakins.

K. Garnet from the Peacock mining claim, Seven Devils mining district, Idaho. Associated with bornite and powellite. Analyzed by W. H. Melville and described in Bull. 90.

L, M. Two samples andradite from Clifton, Arizona. Analyses by George Steiger.

N. Andradite, from the Jumbo mine, Copper Mountain, Prince of Wales Island, Alaska. Analysis by W. T. Schaller. 


\begin{tabular}{|c|c|c|c|c|c|c|c|}
\hline & H. & I. & J. & $\mathbf{K}$ & L. & M. & N. \\
\hline $\mathrm{SiO}_{2}$ & 35.35 & 35.93 & 35.66 & 38.67 & 36.26 & 42.63 & 35.18 \\
\hline $\mathrm{Al}_{2} \mathrm{O}_{3}$. & 20.41 & $\begin{array}{l}\text { trace } \\
18.08\end{array}$ & 18.55 & 10.08 & $\begin{array}{r}\text { none } \\
.78\end{array}$ & $\begin{array}{r}\text { none } \\
1.53\end{array}$ & 5.15 \\
\hline $\mathrm{Fe}_{2} \mathrm{O}_{3}$. & 2.75 & $\begin{array}{r}4.60 \\
\text { 4. } 60\end{array}$ & $\begin{array}{l}1.00 \\
.32\end{array}$ & 16.00 & 32.43 & 31.41 & 25.05 \\
\hline FeO.. & 1.75 & & . 14.25 & .91 & .32 & .30 & .40 \\
\hline $\mathrm{MnO}$. & 38.70 & 31.77 & 29.48 & & .27 & .43 & \\
\hline $\begin{array}{ll}\mathrm{CaO} \\
\mathrm{BaO} \ldots \ldots . . .\end{array}$ & .94 & $\begin{array}{r}8.48 \\
\text { trace }\end{array}$ & 1.15 & 33. 35 & 29.67 & 23. 37 & 33. 36 \\
\hline $\mathrm{MgO} . . . \ldots \ldots$ & none & .69 & & .77 & none & none & .09 \\
\hline $\mathrm{Na}_{2} \mathrm{O}$. & . & .17 & .21 & $\ldots$. & none & $\ldots=\ldots$ & ..... \\
\hline $\begin{array}{r}\mathrm{K}_{2} \mathrm{O} \\
\mathrm{CuO}\end{array}$ & & & .27 & & none & & \\
\hline \multirow{4}{*}{ 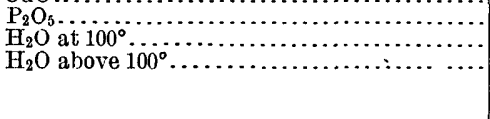 } & & & $\cdots$ & trace & & t & 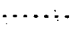 \\
\hline & & $\begin{array}{l}1100 \\
03\end{array}$ & & & $\begin{array}{r}.00 \\
.13\end{array}$ & urace & \\
\hline & .27 & .36 & .44 & .06 & .44 & & .42 \\
\hline & 100.17 & 100.11 & 100.33 & 99.84 & 100.36 & 99.67 & 99.65 \\
\hline
\end{tabular}

\section{CHRYSOLITE.}

A. Transparent green pebbles from near Fort Wingate, New Mexico. Analysis by E. A. Schneider.

B. Transparent olivine from the meteorite of Kiowa County, Kansas. Described in Bull. 78. Sp. gr., 3.376 at $23.2^{\circ}$. Analysis by L. G. Eakins.

C. Olivine from the peridotite dike of Elliott County, Kentucky. Described by Diller in Bull. 38 . Analysis by T. M. Chatard.

D. Olivine from the peridotite at Riddles, Oregon. The rock is the matrix of nickel silicates. Described by Diller and Clarke in Bull. 60. Analysis by F. W. Clarke.

E. Olivine from olivine gabbro, west side of Birch Lake, Minnesota, Described by Bayley, Jour. Geology, vol. 1, p. 688. Analysis by W. F. Hillebrand.

F. Olivine separated from the "hampshirite" of Chester, Massachusetts. Analysis by W. T. Schaller.

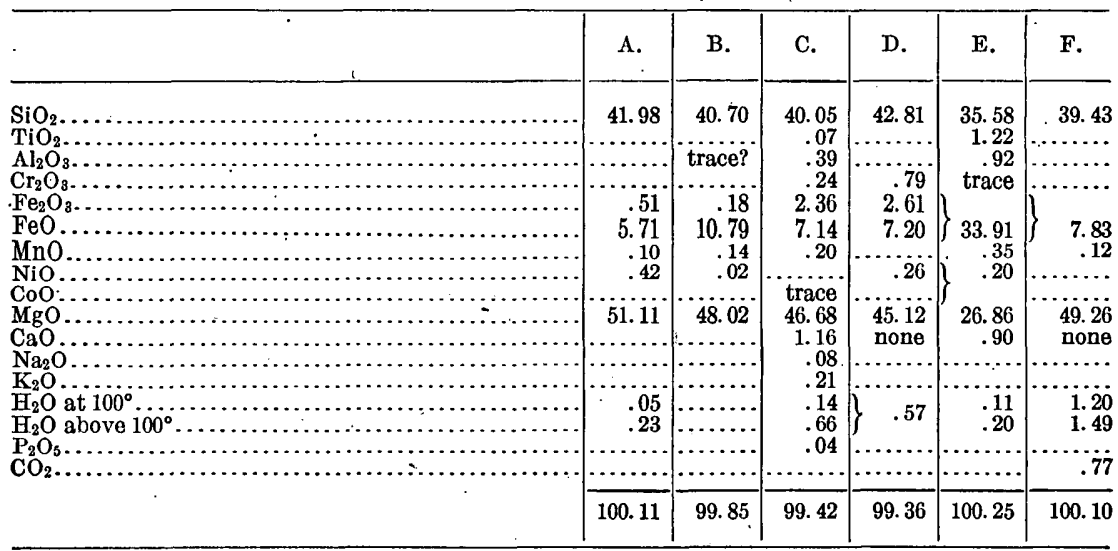

WILLEMITE.

In fine needles, from the Tres Hermanas mine, New Mexico. Collected by W. Lindgren. Analysis by G. Steiger. Mineral not quite pure. Containş admixed carbonates.

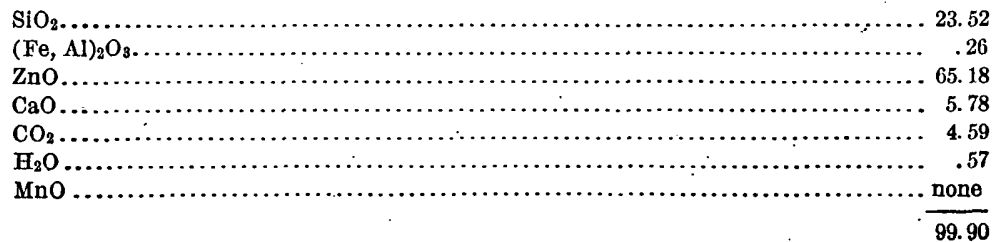




\section{FRIEDELITE.}

From the Taylor mine, Franklin Furnace, New Jersey. Collected by C. Palache. Analysis by W. T. Schaller.

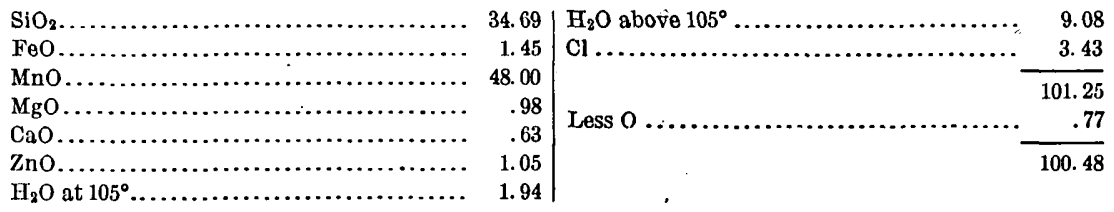

\section{MIZZONITE.}

From a gulch on the side of Italian Mountain, Gunnison County, Colorado. Analysis by L. A. Eakins.

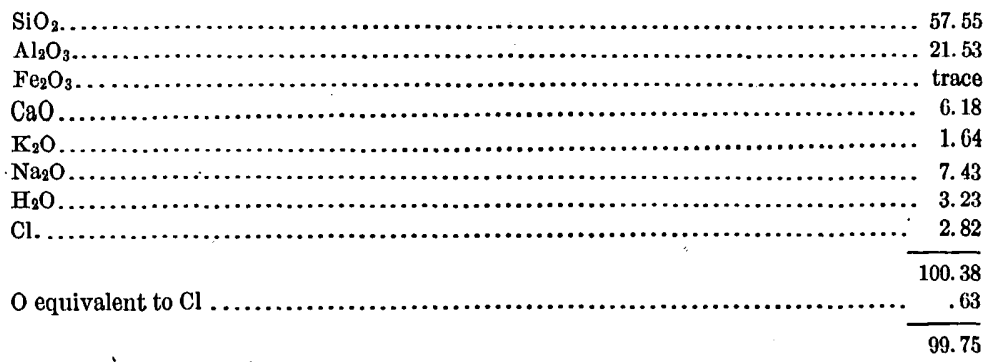

VESUVIANITE.

A. Finely crystallized material from Italian Mountain, Gunnison County, Colorado. Analysis by L. G. Eakins. Sp. gr., 3.394 at $20^{\circ}$.

B. From Nevada, 24 kilometers northeast of Silver Peak. Analysis by George Steiger.

C. Pale green, massive. South Fork of Indian Creek, 12 miles from Happy Camp, Siskiyou County, California. Analysis by Steiger.

D. From 35 miles east of Selma, Fresno County, California. Green, massive, resembling jade. This variety is known as californite. Analysis by Steiger. Sp. gr., 3.359 .

E. From Franklin Furnace, New Jersey. Collected by C. Palache. Analysis by Steiger. Sp. gr., 3.451 .

\begin{tabular}{|c|c|c|c|c|c|}
\hline & A. & B. & C. & D. & E. \\
\hline 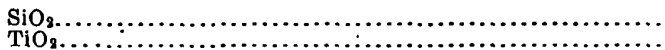 & 37.11 & 36.80 & 35.86 & 36.55 & 36.41 \\
\hline $\mathrm{Al}_{2} \mathrm{O}_{3}$ & 19.30 & $\begin{array}{r}17.50 \\
173\end{array}$ & 18. 35 & 18.89 & 17.35 \\
\hline $\begin{array}{l}\mathrm{Fe}_{2} \mathrm{O}_{3 . .} \\
\mathrm{FeO}\end{array}$ & 3. 31 & $\begin{array}{l}1.56 \\
3.27\end{array}$ & $\begin{array}{r}1.67 \\
.39\end{array}$ & .74 & 1.86 \\
\hline MnO... & & $\begin{array}{r}.21 \\
.48\end{array}$ & .05 & none & 1.75 \\
\hline 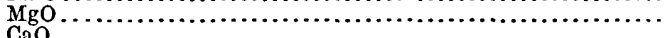 & 3. 89 & 1. 23 & 5. 43 & 2. 33 & 1.38 \\
\hline 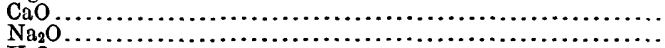 & 36.24 & $\begin{array}{r}35.00 \\
.13\end{array}$ & $\begin{array}{l}33.51 \\
\text { none }\end{array}$ & 35.97 & $\begin{array}{r}33.21 \\
.44\end{array}$ \\
\hline $\mathrm{K}_{2} \mathrm{O} \ldots \mathrm{O}^{\circ}$ & & & none & & .5 \\
\hline $\begin{array}{l}\mathrm{H}_{2}^{\circ} \mathrm{O} \text { at } 100^{\circ} \\
\mathrm{H}_{2} \mathrm{O} \text { above } 100^{\circ} \text {. }\end{array}$ & .06 & .10 & .29 & .58 & .24 \\
\hline $\begin{array}{l}\mathrm{H}_{2} \mathrm{O} \text { above } 100^{\circ} . \\
\mathrm{P}_{2} \mathrm{O}_{5} \ldots \ldots \cdots \cdots \cdots\end{array}$ & & $\begin{array}{r}1.56 \\
.07\end{array}$ & $\begin{array}{r}4.18 \\
.02\end{array}$ & 3.42 & 3.51 \\
\hline $\begin{array}{l}\mathrm{CO} \\
\mathrm{F}\end{array}$ & & .65 & none & .91 & \\
\hline Fno............... & .58 & .88 & none & .13 & $\begin{array}{r}.36 \\
1.74\end{array}$ \\
\hline & & & n......... & ........ & 1. 48 \\
\hline $\mathrm{PbO}$ & & ........ & ........ & $\ldots \ldots \ldots$ & trace \\
\hline \multirow[t]{2}{*}{ Less 0} & $\begin{array}{r}100.49 \\
.24\end{array}$ & $\begin{array}{r}99.92 \\
.36\end{array}$ & 99.85 & 100.26 & $\begin{array}{r}100.23 \\
.15\end{array}$ \\
\hline & 100.25 & 99.56 & & 100.21 & 100.08 \\
\hline
\end{tabular}




\section{ZOISITE.}

A. Zoisite from gabbro, east shaft of waterworks extension, Washington, D. C. Analysis by F. W. Clarke; iron determination by R. B. Riggs.

B. Rose-red zoisite, James's mica mine, Yancey County, North Carolina. Sp. gr., 3.352 at $27^{\circ}$. Analysis by L. G. Eakins.

C. Saussurite from gabbro, Sacramento River road, 37 miles north of Pit River ferry, Shasta County, California. Sp. gr., 3.148. Analysis by.F. W. Clarke. See Bull. 9.

D. Saussurite from the Saas Valley, Switzerland. Pale greenish. Sp. gr., 3.37. Analysis by F. W. Clarke; iron determination by R. B. Riggs.

\begin{tabular}{|c|c|c|c|c|}
\hline & A. & B. & C. & D. \\
\hline 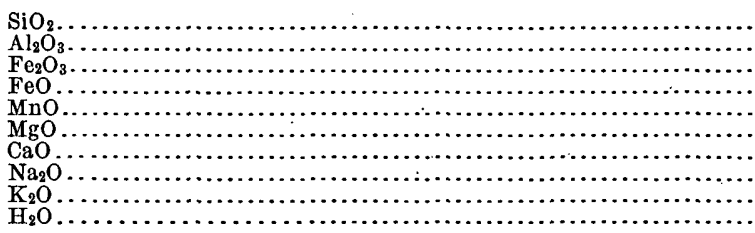 & $\begin{array}{r}45.12 \\
30.53 \\
1.90 \\
17.32 \\
17.34 \\
2.02 \\
1.09 \\
.74\end{array}$ & $\begin{array}{r}38.98 \\
31.02 \\
4.15 \\
.23 \\
23.80 \\
\cdots \\
\cdots \\
2.03\end{array}$ & $\begin{array}{r}42.79 \\
29.43 \\
\ldots \ldots \ldots \\
3.65 \\
\cdots . .70 \\
18.40 \\
18.13 \\
2.51 \\
\cdots 2.42\end{array}$ & $\begin{array}{r}48.29 \\
27.65 \\
\ldots . . . \\
1.45 \\
\ldots . .36 \\
5.36 \\
12.95 \\
3.57 \\
\text { trace } \\
.54\end{array}$ \\
\hline & 99.16 & 100.21 & 100.33 & 99.81 \\
\hline
\end{tabular}

\section{EPIDOTE.}

A. Dark-gray brilliant crystals from Phippsburg, Maine. Analyzed by W. F. Hillebrand and described in Bull. 167.

B, C. Epidote from Italian Mountain, Gunnison County, Colorado. Sp. gr.: B, - 3.448 at $25^{\circ} ;$ C, 3.452 at $17^{\circ}$. Analyses by L. G. Eakins.

D. From Shasta County, California. Analysis by W. T. Schaller.

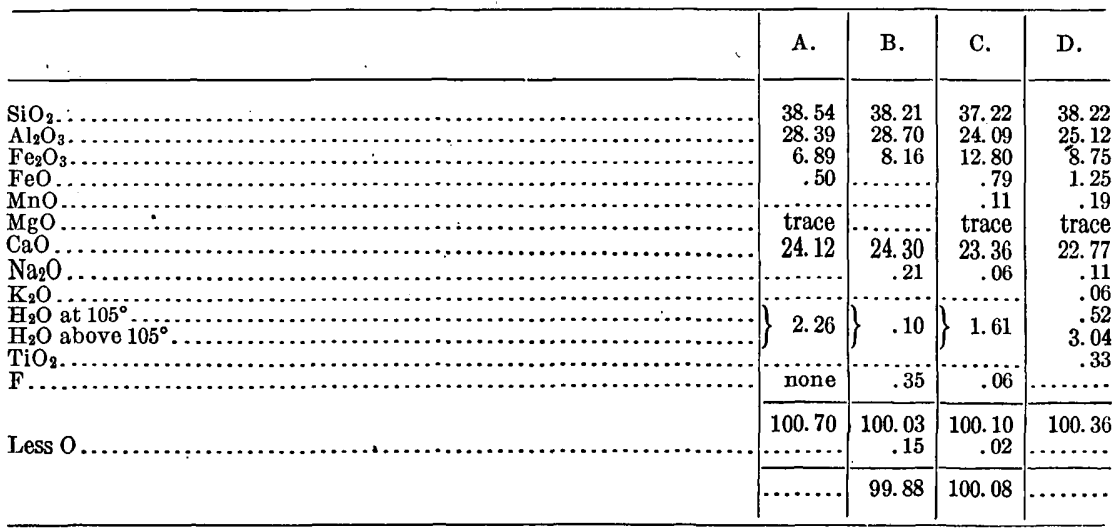

\section{PIEDMONTITE.}

From the rhyolite of Pine Mountain, near Monterey station, Maryland. Contains a little admixed quartz. Analysis by W. F. Hillebrand.

\begin{tabular}{|c|c|c|}
\hline 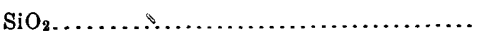 & 47.37 & $\mathrm{MgO} \ldots . .$. \\
\hline 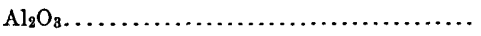 & 18.55 & 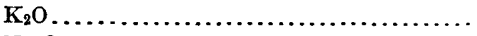 \\
\hline $\mathrm{Ce}_{2} \mathrm{O}_{3} \ldots \ldots \ldots \ldots \ldots \ldots \ldots \ldots \ldots \ldots \ldots \ldots$ & .75 & 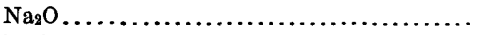 \\
\hline Other rare earths, mol. wt. at $295 . . . \ldots \ldots \ldots$. & 1.28 & $\mathrm{Li}_{2} \mathrm{O} \ldots \ldots \ldots \ldots \ldots \ldots \ldots$ \\
\hline 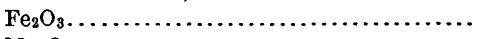 & 4. 02 & $\mathrm{H}_{2} \mathrm{O}$ at $100^{\circ} \ldots \ldots$ \\
\hline 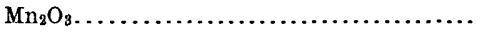 & 6.85 & $\mathrm{H}_{2} \mathrm{O}$ above $100^{\circ} \ldots$ \\
\hline 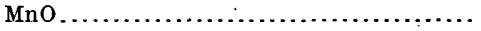 & 1.92 & 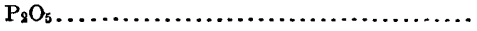 \\
\hline$\ldots \ldots \ldots \ldots \ldots \ldots \ldots \ldots \ldots \ldots \ldots \ldots$ & $\begin{array}{l}.14 \\
.11\end{array}$ & \\
\hline
\end{tabular}




\section{ALlanite.}

From Platte Mountain, Douglas County, Colorado. Sp. gr., 3.52 at $29^{\circ}$. Analysis by L. G. Eakins. See Proc. Colorado Sci. Soc., vol. 2, p. 32.
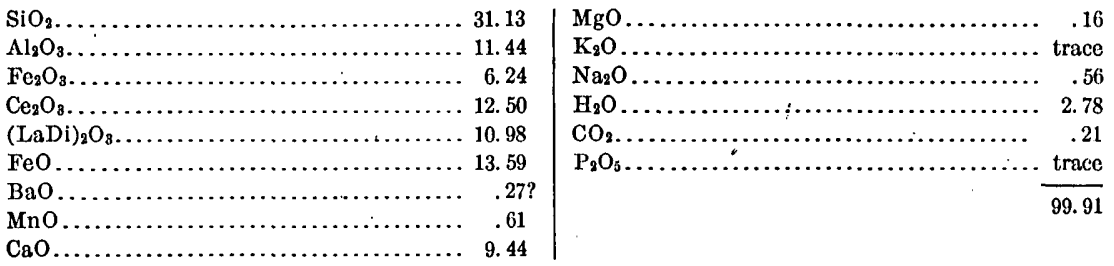

\section{PREHNITE.}

A. From Paterson, New Jersey. Analysis by G. Steiger.

B. From Franklin Furnace, New Jersey. Collected by C. Palache. Analysis by Steiger. Sp. gr., 2.965.

C. From Smiths Mountain, near Oak Grove, San Diego County, California. Sp. gr., 2.895 to 2.909 .

D. Crystals from C. Analyses C, D, by W. T. Schaller.

E. From Fassa, Tyrol. Analysis by E. A. Schneider.

\begin{tabular}{|c|c|c|c|c|c|}
\hline & A. & B. & C. & D. & E. \\
\hline 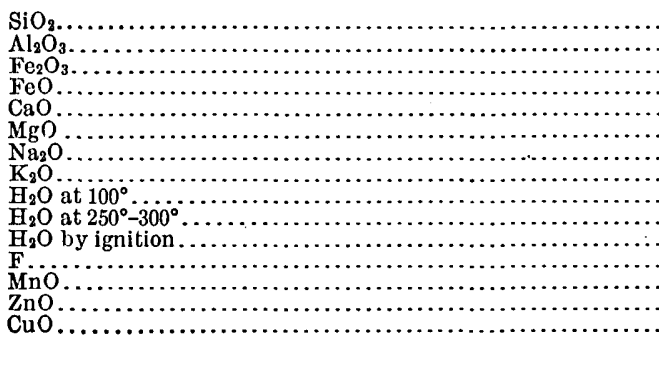 & 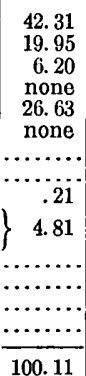 & $\begin{array}{r}43.30 \\
22.70 \\
\quad .34 \\
27.35 \\
\text { none } \\
.93 \\
.18 \\
.24 \\
4.85 \\
. \\
\text { trace } \\
\text { trace? }\end{array}$ & 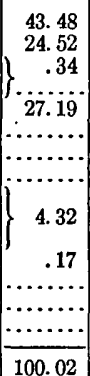 & 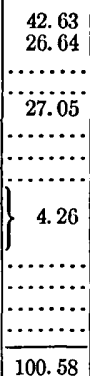 & 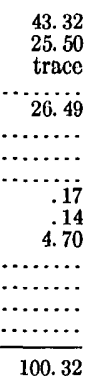 \\
\hline
\end{tabular}

TOPAZ.

A. White, opaque topaz from Stoneham, Maine. Sp. gr:, 3.51 .

B. Alteration product of A, greenish. Sp. gr., 3.42. Analyses A and B by J. E. Whitfield. See discussion by Clarke in Bull. 27.

C. Topaz from Florissart, Colorado. Sp. gr., 3.578 at $22^{\circ}$. Analyzed by W. F. Hillebrand, and described in Bull. 20.

\begin{tabular}{|c|c|c|c|}
\hline . & A. & B. & C. \\
\hline 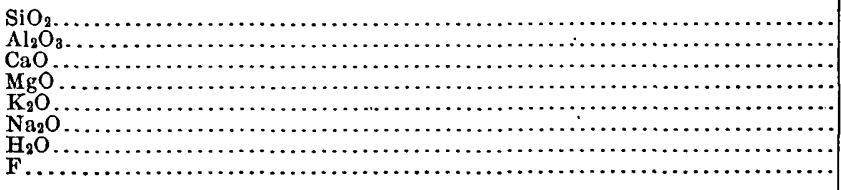 & \begin{tabular}{r}
31.92 \\
57.38 \\
$\ldots \ldots . .$. \\
\hdashline.. .15 \\
1.33 \\
16.99
\end{tabular} & $\begin{array}{r}35.15 \\
53.18 \\
1.32 \\
.17 \\
1.52 \\
1.28 \\
.90 \\
12.88\end{array}$ & 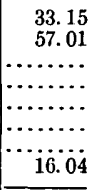 \\
\hline 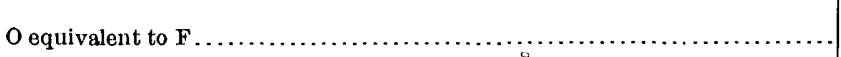 & $\begin{array}{r}107.97 \\
7.16\end{array}$ & $\begin{array}{r}106.40 \\
5.42\end{array}$ & $\begin{array}{r}106.20 \\
6.75\end{array}$ \\
\hline 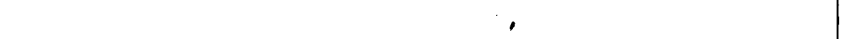 & 100.81 & 100.98 & 99.45 \\
\hline
\end{tabular}


The final alteration of the Stoneham topaz is into muscovite, q. v. The alteration product here given represents the beginning of the process.

\section{SILLIMANITE.}

Fibrolite adze from Brittany. Sp. gr., 3.147, determined by William Hallock. Analysis by F. W. Clarke. See Bull. 60 .

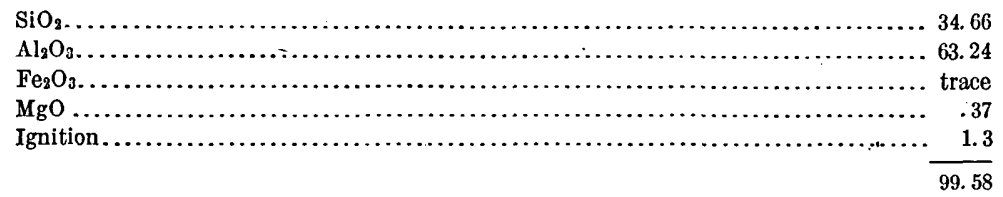

KYANITE.

Pale-green variety, associated with the dumortierite of Clip, Arizona. Analyzed by W. F. Hillebrand. Titanic ox:de is present in appreciable amounts, but was not separated from alumina. The mineral contained a few black, nonmagnetic grains, which may have carried the titanium. Sp. gr., 3.656 at $18.5^{\circ}$.

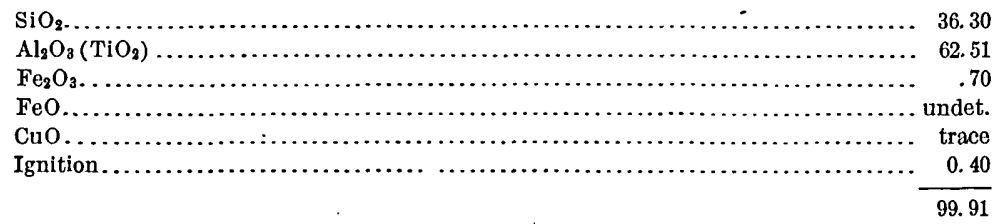

ILVAITE.

From Golconda mine, South Mountain, Owyhee County, Idaho. Analysis by W. F. Hillebrand. Sp. gr., 4.059 at $31^{\circ}$.

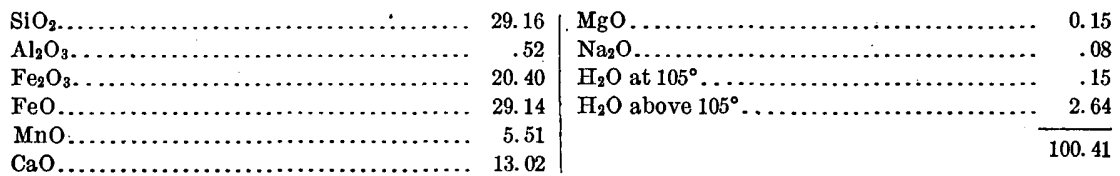

CALAMINE.

White, highly crystalline. From Sterling, New Jersey. Analysis by George Steiger.

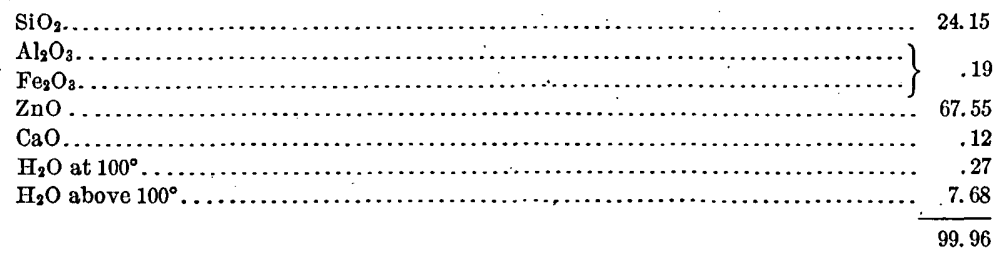

LAWSONITE.

From Tiburon Peninsula, California. Collected by F. L. Ransome. Analysis by W. F. Hillebrand.

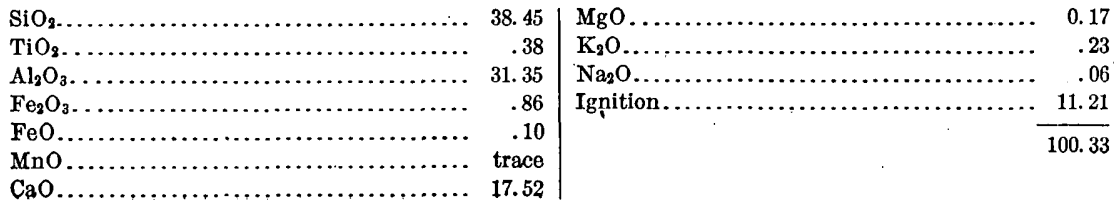


STAUROLITE.

Altered staurolite from Liberty Grove, Cecil County, Maryland. About two-thirds muscovite. Analysis by George Steiger.

\begin{tabular}{|c|c|c|c|}
\hline 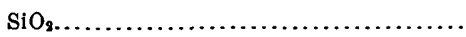 & 50.17 & 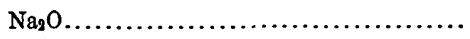 & 0.48 \\
\hline 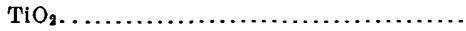 &. .55 & $\mathrm{H}_{2} \mathrm{O}$ at $100^{\circ} \ldots \ldots \ldots \ldots \ldots \ldots \ldots \ldots \ldots \ldots \ldots$ & .42 \\
\hline $\mathrm{Al}_{2} \mathrm{O}_{3} \ldots \ldots \ldots \ldots \ldots \ldots \ldots \ldots \ldots \ldots \ldots \ldots$ & 27.97 & $\mathrm{H}_{2} \mathrm{O}$ above $100^{\circ} \ldots \ldots \ldots \ldots \ldots \ldots \ldots$ & 3.94 \\
\hline 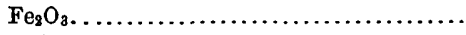 & 6.13 & 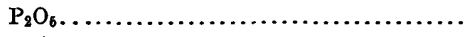 & .06 \\
\hline $\mathrm{FeO} \ldots \ldots \ldots \ldots \ldots \ldots \ldots \ldots \ldots \ldots \ldots \ldots \ldots \ldots$ & 1.18 & & 99.82 \\
\hline 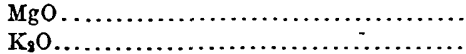 & $\begin{array}{l}1.15 \\
7.77\end{array}$ & & \\
\hline
\end{tabular}

GADOLINITE.

Analyses by L. G. Eakins.,

A. From Llano County, Texas. Sp. gr., 4.239 at $17.4^{\circ}$.

B, C. From Devils Head Mountain, Douglas County, Colorado. Specific gravities: B, 4.56 at $17^{\circ}$; C, 4.59 at $25.5^{\circ}$. Described in Proc. Colorado Sci. Soc., vol. 2, pt. 1, p. 32 .

\begin{tabular}{|c|c|c|c|}
\hline & A. & B. & C. \\
\hline 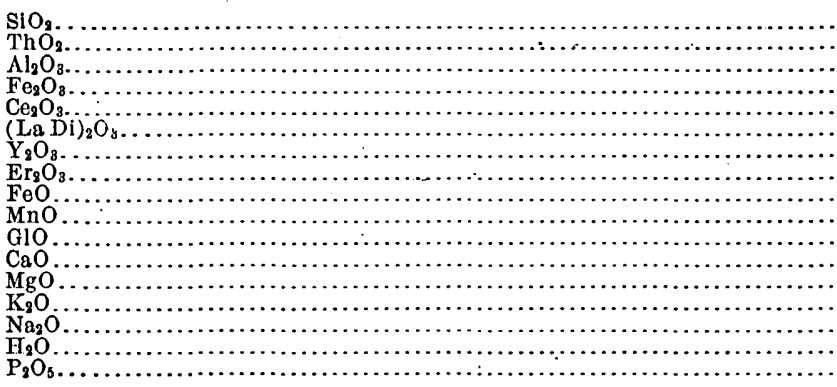 & $\begin{array}{r}23.79 \\
.58 \\
.96 \\
2.62 \\
5.22 \\
a 41.55 \\
12.42 \\
\text { trace } \\
11.33 \\
.74 \\
\text { trace } \\
\text { trace } \\
\text { trace } \\
1.03 \\
.05\end{array}$ & $\begin{array}{r}22.13 \\
.89 \\
2.34 \\
1.13 \\
11.10 \\
21.23 \\
b 9.50 \\
b 12.74 \\
10.43 \\
\ldots \ldots .19 \\
7.19 \\
.34 \\
.14 \\
.18 \\
.28 \\
.86 \\
\ldots . . .\end{array}$ & $\begin{array}{r}21.86 \\
.81 \\
.54 \\
3.59 \\
6.87 \\
19.10 \\
b 12.63 \\
b 15.80 \\
11.36 \\
.11 \\
5.46 \\
.47 \\
.16 \\
.20 \\
.32 \\
.74 \\
\ldots . .\end{array}$ \\
\hline & 100. 29 & 100.48 & 100.02 \\
\hline
\end{tabular}

a Molecular weight, 260.

b Molecular weight Y, Er group: in B, 296; in C, 294.

YTTRIALITE.

From the Baringer mine, Llano County, Texas. Analyzed by W. F. Hillebrand, and discussed in Am. Jour. Sci., 4th ser., vol. 13, p. 145.

\begin{tabular}{|c|c|c|c|}
\hline $\mathrm{O}_{2 . .}$ & 29. 63 & bo. & 0.80 \\
\hline $\mathrm{iO}_{2} \ldots$ & .05 & $\mathrm{CaO}$. & .67 \\
\hline 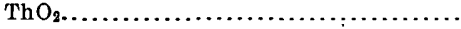 & 10.85 & 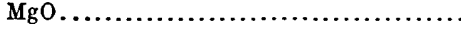 & .16 \\
\hline 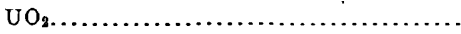 & 1.64 & $\mathrm{H}_{2} \mathrm{O}$ at $105^{\circ} \ldots \ldots \ldots \ldots \ldots \ldots \ldots \ldots \ldots \ldots \ldots \ldots \ldots \ldots$ & .32 \\
\hline ….............. & 3.07 & $\mathrm{H}_{2} \mathrm{O}$ above $105^{\circ} .$. & .04 \\
\hline wt., $335.6 \ldots$. & 5.18 & $\mathrm{CO}_{2} \ldots \ldots \ldots \ldots$ & .11 \\
\hline mol. wt., $265.8 . \ldots \ldots$ & 43. 45 & $\mathrm{P}_{2} \mathrm{O}_{5}$ & $: 12$ \\
\hline & .76 & $\mathrm{~A}, \mathrm{He}, \mathrm{F}$, and alkalies by difference.. & .31 \\
\hline (n) & 1.90 & & \\
\hline 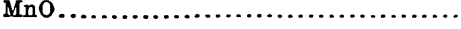 & .88 & & \\
\hline
\end{tabular}




\section{ROWLANDITE.}

From Llano County, Texas. Analysis by W. F. Hillebrand, with discussion in Bull. 113. Sp. gr., 4.513 at $15.5^{\circ}$.

\begin{tabular}{|c|c|c|c|}
\hline $\mathrm{SiO}_{2} \ldots \ldots$ & 26.04 & $\mathrm{MgO} . . .$. & 1.62 \\
\hline $\mathrm{X} a \ldots .$. & .39 & Alkalies.......... & .28 \\
\hline $\mathrm{ThO}_{2} \ldots \ldots \ldots \ldots \ldots \ldots$ & .59 & $\mathrm{H}_{2} \mathrm{O} \ldots \ldots \ldots \ldots \ldots$ & .24 \\
\hline $\mathrm{Ce}_{2} \mathrm{O}_{3} \ldots \ldots \ldots \ldots \ldots \ldots \ldots$ & 5.06 & $\mathrm{CO}_{2} \ldots \ldots \ldots \ldots$ & .34 \\
\hline $\mathrm{La}_{2} \mathrm{O}_{3}$ group, mol. wt., $336.8 \ldots$ & 9.34 & F... & 3.87 \\
\hline $\mathrm{Yt}_{2} \mathrm{O}_{3}$ group, mol. wt., $266.2 \ldots \ldots$ & 47.70 & $\mathrm{P}_{2} \mathrm{O}_{5}$. & trace \\
\hline $\mathrm{Fe}_{2} \mathrm{O}_{3} \ldots \ldots \ldots \ldots \ldots \ldots \ldots$ & .09 & & 101.12 \\
\hline $\begin{array}{l}\mathrm{FeO}, \ldots \ldots \ldots \\
\mathrm{MnO} \ldots \ldots \ldots \ldots \ldots \ldots\end{array}$ & $\begin{array}{r}4.39 \\
.67\end{array}$ & O equivalent & \\
\hline $\mathrm{CaO}$. & .50 & & \\
\hline
\end{tabular}

\section{MACKINTOSHITE.}

From Llano County, Texas. Analyzed by W. F. Hillebrand and discussed in Bull. 113. Sp. gr., 5.43 at $21.4^{\circ}$. Only nine-tenths of a gram available for analysis.

\begin{tabular}{|c|c|c|c|}
\hline 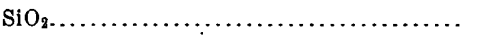 & 13. 90 & $\mathrm{CaO} .$. & 0.59 \\
\hline $\mathrm{UO}_{2} \ldots \ldots$ & 22.40 & MgO. & .10 \\
\hline $\mathrm{ZrO}_{2} ? \ldots \ldots \ldots \ldots \ldots \ldots \ldots \ldots \ldots \ldots \ldots \ldots \ldots \ldots \ldots \ldots$ & .88 & $\mathrm{~K}_{2} \mathrm{O} \ldots \ldots \ldots \ldots$ & .42 \\
\hline 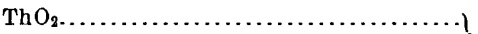 & 45.30 & $(\mathrm{NaLi})_{2} \mathrm{O} \ldots \ldots \ldots \ldots \ldots \ldots \ldots \ldots \ldots \ldots \ldots \ldots \ldots$ & .68 \\
\hline 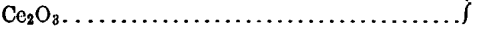 & & $\mathrm{P}_{2} \mathrm{O}_{5} \ldots \ldots \ldots \ldots \ldots \ldots \ldots \ldots \ldots \ldots \ldots \ldots \ldots \ldots \ldots \ldots$ & .67 \\
\hline $\mathrm{La}_{2} \mathrm{O}_{3}$ group $\ldots \ldots \ldots \ldots \ldots \ldots \ldots$ & 1.86 & $\mathrm{H}_{2} \mathrm{O}$ at $100^{\circ} \ldots \ldots \ldots \ldots \ldots \ldots \ldots \ldots$ & .50 \\
\hline 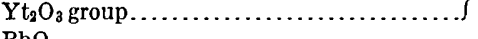 & & $\mathrm{H}_{2} \mathrm{O}$ above $100^{\circ} \ldots \ldots \ldots \ldots \ldots \ldots \ldots \ldots \ldots \ldots$ & 4. 31 \\
\hline 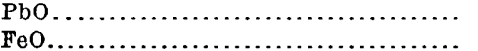 & $\begin{array}{l}3.74 \\
1.15\end{array}$ & & 96.50 \\
\hline
\end{tabular}

\section{- CYRTOLITE.}

A doubtful mineral from Devils Head Mountain, Douglas County, Colorado. Analy ses by W. F. Hillebrand, with description in Proc. Colorado Sci. Soc., vol. 3, pt.1, p. 44. Brown crystalline growths of irregular form. Sp. gr.: A; 3.70; B, 3.60; C, 3.64. The material may be a mixture of cyrtolite or some analogous alteration of zircon with limonite and a phosphate.

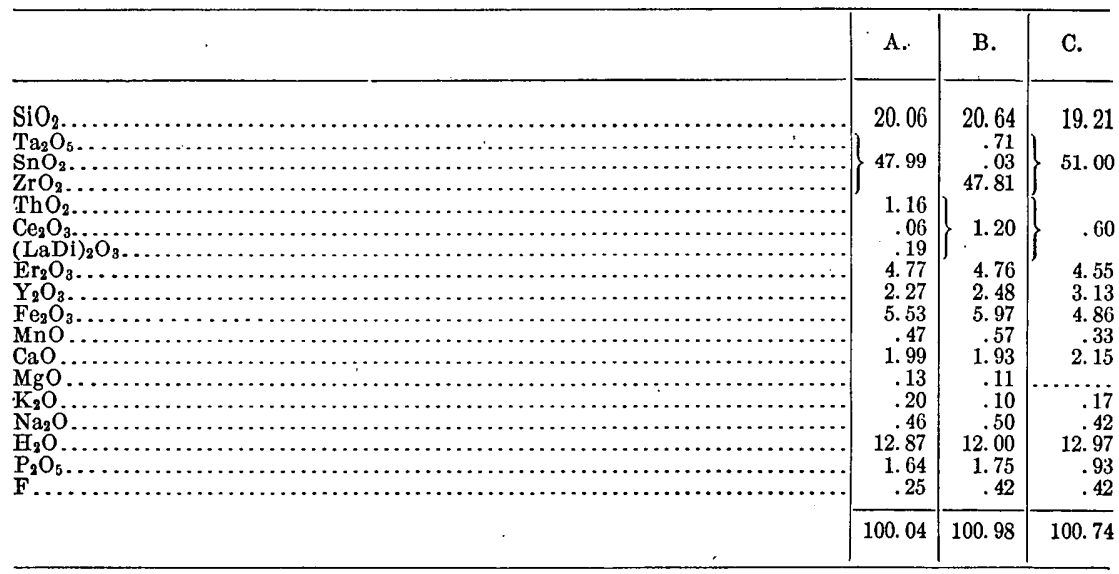

a A mixture of indefinable earths with some uranium and a trace of titanium. 


\section{DANBURITE.}

From Russell, New York. Analysis by J. E. Whitfield. See Bull. 55.

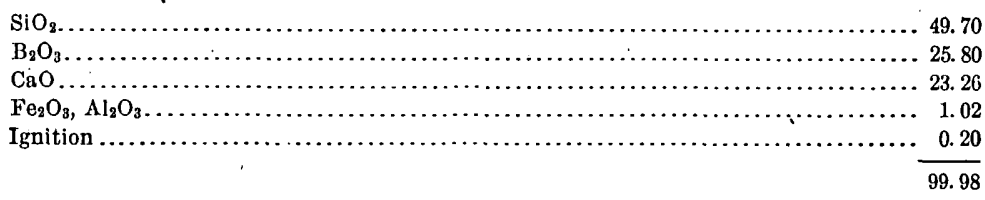

DATOLITE.

From Bergen Hill, New Jersey. Analysis by J. E. Whitfield. See Bull. 55.

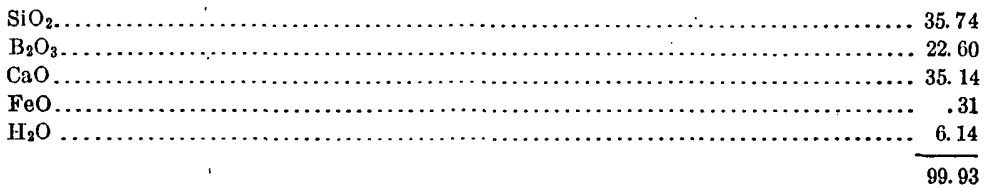

AXINITE.

Analyses A, B, by J. E. Whitfield, with discussion in Bull. 55.

A. Clove brown, from Cornwall, England. - Translucent.

B. Pearl gray, from Bourg d'Oisans, Dauphiny. Transparent:

C. From Moosa Canyon, Bonsall, San Diego County, California.

D. From Consumnes copper mine, Amador County, California.

Analyses C, D, by W. T. Schaller.

\begin{tabular}{|c|c|c|c|c|}
\hline & A. & B. & C. & D. \\
\hline 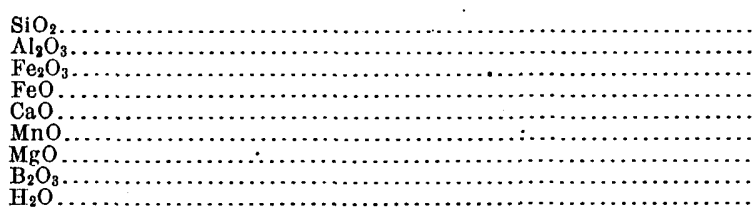 & $\begin{array}{r}42.10 \\
17.40 \\
3.06 \\
5.84 \\
20.53 \\
4.63 \\
.66 \\
4.64 \\
1.80\end{array}$ & $\begin{array}{r}41.53 \\
17.90 \\
3.90 \\
4.02 \\
21.66 \\
3.79 \\
.74 \\
4.62 \\
2.16\end{array}$ & $\begin{array}{r}42.61 \\
17.43 \\
.38 \\
7.53 \\
19.74 \\
4.10 \\
.44 \\
6.04 \\
1.56\end{array}$ & $\begin{array}{r}42.79 \\
16.38 \\
4.22 \\
19.21 \\
8.76 \\
.09 \\
\text { a } 6.70 \\
1.85\end{array}$ \\
\hline & 100.66 & 100.32 & 99.83 & 100.00 \\
\hline
\end{tabular}

a By difference.

DUMORTIERITE.

A. Harlem, New York. Analysis by R. B. Riggs. See Am. Jour. Sci., 3d ser., vol. 34, p. 406 . Contained some tourmaline.

B. Harlem, New York. Analysis by J. E. Whitfield.

C. Near Clip, Yuma County, Arizona. Analysis by J. E. Whitfield..

D. Purified sample from Clip. Analysis by Whitfield. Specific gravity a little over 3.265. See note by Whitfield in Bull. 60, and paper by Diller and Whitfięld in Bull. 64.

E, F. Two samples from Dehesa, San Diego County, Califoruia,

15619-Bull, 419-10-_ 19 
G. North fork of Washougal River, Skamania County, Washington. Analyses E, F, G, by W. T. Schaller, and discussed by him in Bull. 262 .

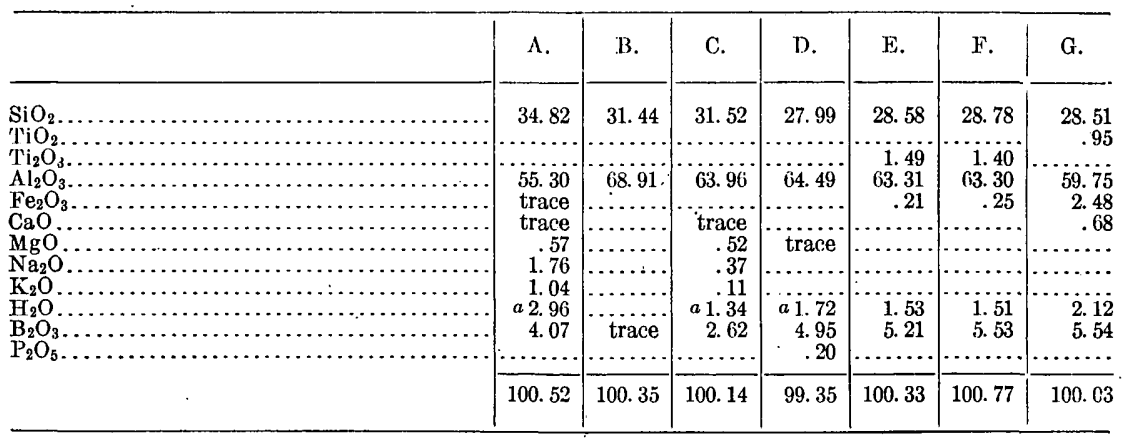

$a$ Loss on ignition.

TOURMALINE.

LITHIA TOURMALINE.

Analyses A to H, inclusive, by R. B: Riggs. 'Discussed by Riggs in Bull. 55 .

A. Pink center of crystals having a green margin, from Calhao, province of Minas Geraes, Brazil. Sp. gr., 3.028.

- B. Pale green, border of A.

C. Olive green, also from Calhao, Brazil.

D. Rose colored, massive, from Black Mountain; Rumford, Maine. Sp. gr., 2.997 .

E. Dark green, massive, same locality as D.

\begin{tabular}{|c|c|c|c|c|c|}
\hline - & $\Lambda$. & B. & C. & D. & E. \\
\hline 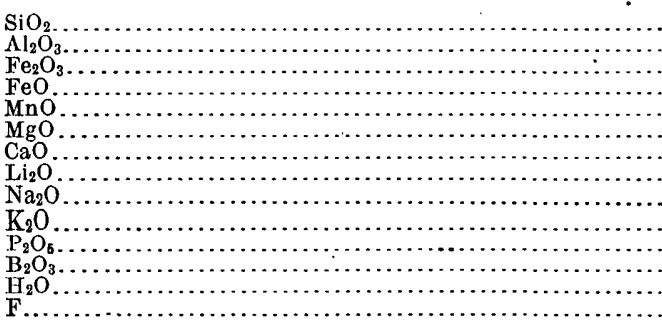 & $\begin{array}{r}37.19 \\
42.43 \\
\text { none } \\
.52 \\
.79 \\
\text { none } \\
.57 \\
1.73 \\
2.24 \\
.23 \\
\text { none } \\
10.06 \\
3.90 \\
\text { trace? }\end{array}$ & $\begin{array}{r}37.39 \\
39.65 \\
.15 \\
2.29 \\
1.47 \\
\text { none } \\
.49 \\
1.71 \\
2.42 \\
.25 \\
\text { trace } \\
10.29 \\
3.63 \\
.32\end{array}$ & $\begin{array}{r}36.91 \\
38.13 \\
.31 \\
3.19 \\
2.22 \\
.04 \\
.38 \\
1.61 \\
2.70 \\
.28 \\
.11 \\
9.87 \\
3.64 \\
.14\end{array}$ & $\begin{array}{r}38.07 \\
42.24 \\
\cdots \\
.26 \\
.35 \\
.07 \\
.56 \\
1.59 \\
2.18 \\
.44 \\
\text { none } \\
9.99 \\
4.26 \\
.28\end{array}$ & $\begin{array}{r}36.53 \\
38.10 \\
\text { none } \\
6.43 \\
.32 \\
\text { none } \\
.34 \\
.95 \\
2.86 \\
.38 \\
\text { trace } \\
10.22 \\
3.52 \\
.16\end{array}$ \\
\hline \multirow[t]{2}{*}{$\mathrm{O}$ equivalent to $\mathrm{F}}$. & $\begin{array}{r}99.66 \\
\ldots . . . . .\end{array}$ & $\begin{array}{r}100.06 \\
.13\end{array}$ & $\begin{array}{r}99.53 \\
.06\end{array}$ & $\begin{array}{r}100.29 \\
.12\end{array}$ & $\begin{array}{r}99.81 \\
.07\end{array}$ \\
\hline & & 99.93 & 99.47 & 100.17 & 99.74 \\
\hline
\end{tabular}

F. Colorless to very pale pinkish or greenish crystals, Auburn, Maine. Sp. gr., 3.07.

G. Light-green crystals, Auburn, Maine.

H. Dark-green, massive tourmaline, Auburn, Maine.

I. Pink, Mesa Grande, San Diego County, California.

J. Pale green, Mesa Grande.

K. Pale-rose color, Island of Elba. Sp. gr., 3.04-3.05. Analyses I, J, K, by W. T. Schaller. Hitherto unpublished. 


\begin{tabular}{|c|c|c|c|c|c|c|}
\hline . & F. & G.. & H. & I. & J. & K. \\
\hline $\begin{array}{l}\mathrm{SiO}_{2} \ldots \\
\mathrm{Al}_{3} \mathrm{O}_{3} \ldots \\
\mathrm{Ti}_{2} \mathrm{O}_{3} \ldots\end{array}$ & $\begin{array}{l}38.14 \\
39.60\end{array}$ & $\begin{array}{l}37.85 \\
37.73\end{array}$ & $\begin{array}{l}36.26 \\
36.68\end{array}$ & $\begin{array}{l}37.57 \\
42.18 \\
\text { trace }\end{array}$ & $\begin{array}{r}36.72 \\
41.27 \\
.06\end{array}$ & $\begin{array}{r}37.89 \\
43.85 \\
.04\end{array}$ \\
\hline $\mathrm{Fe}_{2} \mathrm{O}_{3}$. & .30 & .42 & .15 & & & \\
\hline 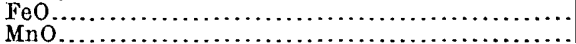 & 1.38 & 3.88 & 7.07 & j .19 & 1.13 & .11 \\
\hline$\ldots \ldots \ldots \ldots$ & 1.38 & .51 & .72 & .24 & 1.48 & .11 \\
\hline $\begin{array}{l}\mathrm{MgO} . \\
\mathrm{CaO} . .\end{array}$ & $\begin{array}{r}\text { trace } \\
.43\end{array}$ & $\begin{array}{l}.04 \\
.49\end{array}$ & $\begin{array}{l}.16 \\
.17\end{array}$ & $\begin{array}{r}\text { none } \\
1.20\end{array}$ & $\begin{array}{r}\text { none } \\
.87\end{array}$ & $\begin{array}{r}\text { none } \\
.07\end{array}$ \\
\hline $\mathrm{L}_{2} \mathrm{O}$. & 1.34 & 1. 34 & 1.05 & 1.92 & 1.76 & 1.66 \\
\hline & 2.36 & 2. 16 & 2.88 & 2.05 & 2.23 & 2. 43 \\
\hline $\mathrm{K}_{2} \mathrm{O} \ldots$ & .27 & .62 & .44 & none & none & none \\
\hline $\begin{array}{l}\mathrm{B}_{2} \mathrm{O}_{3} \\
\mathrm{P}_{2} \mathrm{O}_{5}\end{array}$ & $\begin{array}{l}10.25 \\
\text { trace }\end{array}$ & 10.55 & 9.94 & 10.65 & 10.60 & 10. 28 \\
\hline $\mathrm{H}_{2} \mathrm{O}$ & 4. 16 & 4. 18 & 4. 05 & 3. 38 & 3.33 & 3. 47 \\
\hline & .62 & .62 & .71 & .39 & .31 & .10 \\
\hline \multirow[t]{2}{*}{ Less 0.} & $\begin{array}{r}100.23 \\
.26\end{array}$ & $\begin{array}{l}100.39 \\
.26\end{array}$ & $\begin{array}{l}100.28 \\
.30\end{array}$ & $\begin{array}{l}99.77 \\
.17\end{array}$ & $\begin{array}{r}99.76 \\
.13\end{array}$ & $\begin{array}{r}100.01 \\
.04\end{array}$ \\
\hline & 99.97 & 100.13 & 99.98 & 99.60 & 99.63 & 99.97 \\
\hline
\end{tabular}

The following analyses represent alteration products of lithia tourmaline:

L. From the Rumford rubellite.

M. From the rubellite of Hebron, Maine.

N. Pink tourmaline, Pala, San Diego County, California, partly altered.

$\mathrm{O}$. Tourmaline $\mathrm{N}$, almost completely altered. Analyses' L, M, by Riggs; $\mathrm{N}, \mathrm{O}$, by Schaller.

\begin{tabular}{|c|c|c|c|c|}
\hline : & I.. & M. & N. & 0 \\
\hline 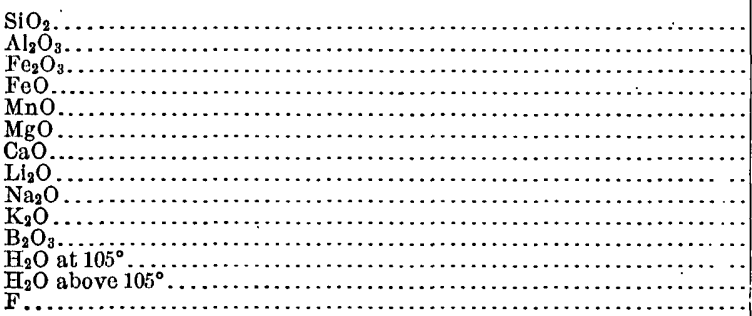 & $\begin{array}{r}53.03 \\
31.67 \\
.51 \\
\text { trace } \\
\text { trace } \\
\text { trace } \\
.26 \\
.54 \\
9.44 \\
\text { trace } \\
4.80 \\
\text { in:ace? }\end{array}$ & $\begin{array}{r}43.90 \\
38.71 \\
.58 \\
.25 \\
.04 \\
.05 \\
.41 \\
\ldots . .05 \\
10.92 \\
\text { trace } \\
4.25 \\
\text { none }\end{array}$ & \begin{tabular}{r}
36.98 \\
43.69 \\
$\ldots \ldots .$. \\
\hdashline$. \ldots .$. \\
trace \\
trace \\
.25 \\
1.28 \\
2.02 \\
2.29 \\
$a 7.66$ \\
1.16 \\
4.67 \\
undet.
\end{tabular} & \begin{tabular}{r}
37.05 \\
44.25 \\
$\ldots \ldots .$. \\
\hdashline... \\
trace \\
.10 \\
.22 \\
1.27 \\
1.06 \\
1.95 \\
$a 2.46$ \\
.83 \\
10.81 \\
undet.
\end{tabular} \\
\hline , & 10.25 & 100.16 & 100.00 & 100.00 \\
\hline
\end{tabular}

a By difference.

MAGNESIAN AND IRON TOURMALIN ES.

Analyses A to L by R. B. Riggs. See Bull. 55 .

A. White to light-brown crystals, Dekalb, St. Lawrence County, New York. Sp. gr., 3.085 .

B. Brown tourmaline, Gouverneur, St. Lawrence County, New York.

C. Cinnamon-brown crystals, Hamburg, New Jersey.

D. Brilliant black crystals, Pierrepont, St. Lawrence County, New York. Sp. gr., 3.08 .

E. Dark-brown crystals, Orford, New Hampshire,

F. Dark-brown crystals, Monroe, Connecticut. 


\begin{tabular}{|c|c|c|c|c|c|c|}
\hline . & . A. & B. & C. & D. & E. & F. \\
\hline 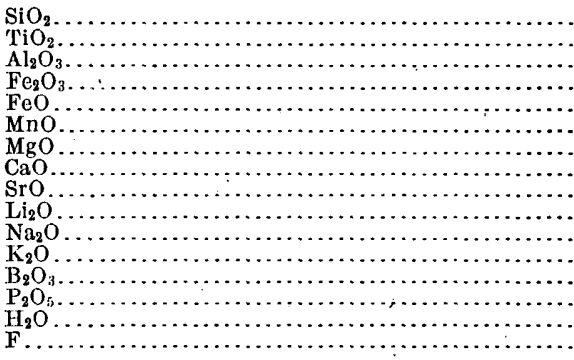 & $\begin{array}{r}36.88 \\
.12 \\
28.87 \\
.52 \\
\text { none } \\
14.53 \\
3.70 \\
\text { trace } \\
\text { trace } \\
1.39 \\
.18 \\
10.58 \\
\text { undet. } \\
3.56 \\
.50\end{array}$ & $\begin{array}{r}37.39 \\
1.19 \\
27.79 \\
.10 \\
.64 \\
\text { none } \\
14.09 \\
2.78 \\
\text { trace } \\
\text { trace } \\
1.72 \\
.16 \\
10.73 \\
\text { none } \\
3.83 \\
\text { trace? }\end{array}$ & $\begin{array}{r}35.25 \\
.65 \\
28.49 \\
\text { none } \\
.86 \\
\text { none } \\
14.58 \\
5.09 \\
\text { trace } \\
\text { trace } \\
.94 \\
.18 \\
10.45 \\
\text { trace } \\
3.10 \\
.78\end{array}$ & $\begin{array}{r}35.61 \\
.55 \\
25.29 \\
.44 \\
8.19 \\
\text { trace } \\
11.07 \\
3.31 \\
\text { none } \\
\text { trace } \\
1.51 \\
.20 \\
10.15 \\
\text { trace } \\
3.34 \\
.27\end{array}$ & $\begin{array}{r}36.66 \\
.23 \\
32.84 \\
\text { none } \\
2.50 \\
\text { trace } \\
10.35 \\
1.35 \\
\text { trace } \\
\text { trace } \\
2.42 \\
.22 \\
10.07 \\
\text { none } \\
3.78 \\
\text { trace? }\end{array}$ & $\begin{array}{r}36.41 \\
1.61 \\
31.27 \\
\text { none } \\
3.80 \\
\text { trace } \\
9.47 \\
.98 \\
\text { trace } \\
\text { none } \\
2.68 \\
.21 \\
9.65 \\
\text { trace } \\
3.79 \\
\text { none }\end{array}$ \\
\hline \multirow[t]{2}{*}{ 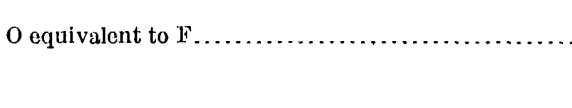 } & $\begin{array}{r}100.83 \\
.21\end{array}$ & $\begin{array}{r}100.42 \\
\ldots \ldots\end{array}$ & $\begin{array}{r}100.37 \\
.33\end{array}$ & $\begin{array}{r}99.93 \\
.11 \\
\end{array}$ & $\begin{array}{r}100.42 \\
\ldots \ldots\end{array}$ & $\begin{array}{r}99.87 \\
\ldots \ldots\end{array}$ \\
\hline & 100.62 & & 100.04 & 99.82 & & \\
\hline
\end{tabular}

G. Massive black tourmaline, Auburn, Maine. Sp. gr., 3.19.

H. Massive black tourmaline, Paris, Maine.

I. Black tourmaline, Calhao, Brazil. Sp. gr., 3.20.

J. Black tourmaline, Haddam, Connecticut.

\begin{tabular}{|c|c|c|c|c|}
\hline & G. & H. & I. & J. \\
\hline 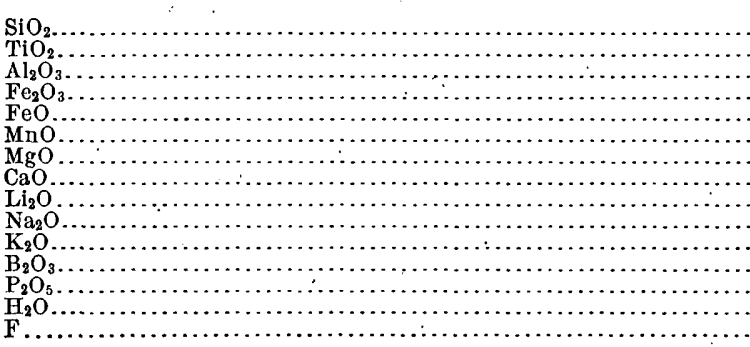 & $\begin{array}{r}34.99 \\
\text { none } \\
33.96 \\
\text { none } \\
14.23 \\
.06 \\
1.01 \\
.15 \\
\text { trace } \\
2.01 \\
.34 \\
9.63 \\
\text { trace } \\
3.62 \\
\text { none }\end{array}$ & $\begin{array}{r}34.44 \\
1.13 \\
12.10 \\
.08 \\
1.81 \\
.24 \\
.07 \\
2.03 \\
.25 \\
9.02 \\
\text { trace } \\
3.69 \\
\text { none }\end{array}$ & $\begin{array}{r}34.63 \\
32.70 \\
.31 \\
13.69 \\
.12 \\
2.13 \\
.33 \\
.08 \\
2.11 \\
.24 \\
9.63 \\
\text { none } \\
3.49 \\
.06\end{array}$ & $\begin{array}{r}34.95 \\
.57 \\
31.11 \\
.50 \\
11.87 \\
.09 \\
4.45 \\
.81 \\
\text { trace } \\
2.22 \\
.24 \\
9.92 \\
\text { trace } \\
3.62 \\
\text { none }\end{array}$ \\
\hline \multirow[t]{2}{*}{$\mathrm{O}$ equivalent to $\mathrm{F} .}$. & $\begin{array}{c}100.00 \\
\cdots \cdots\end{array}$ & 99.89 & $\begin{array}{r}99.52 \\
.02\end{array}$ & $\begin{array}{r}100.35 \\
\ldots\end{array}$ \\
\hline & & & 99.50 & \\
\hline
\end{tabular}

K. Black crystal, Nantic Gulf, Baffin Land. Sp. gr., 3.095.

L. Black crystal, Stony Point, Alexander County, North Carolina. Sp. gr., 3.13.

M. Brown tourmaline from near Colfax, Nevada County, California. Fine, radiating needles. Sp. gr., 3.065. Incomplete analysis by W. H. Melville. See Bull. 90.

N. Chrome tourmaline from near Etchison, Montgomery County, Maryland. Analysis by T. M. Chatard. See Bull. 64 .

O. Black tourmaline, Lost Valley, San Diego County, California.

P. Black tourmaline, Ramona, San Diego County, California.

Analyses O, P by W. T. Schaller. Hitherto unpublished. 


\begin{tabular}{|c|c|c|c|c|c|c|}
\hline & K. & L. & M. & N. & 0 & P. \\
\hline 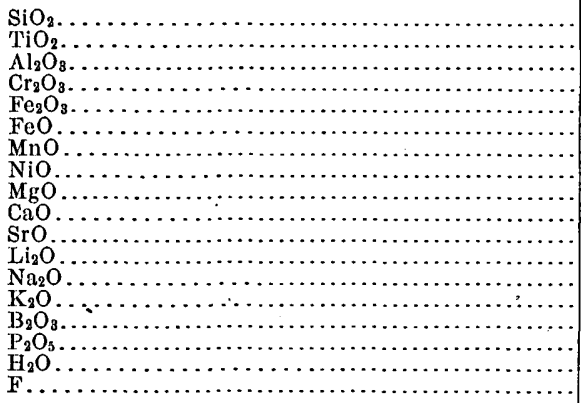 & $\begin{array}{r}35.34 \\
30.49 \\
\text { none } \\
8.22 \\
\text { trace } \\
7.76 \\
2.32 \\
\text { trace } \\
\text { trace } \\
1.76 \\
.15 \\
1045 \\
\text { none } \\
3.60 \\
\text { none }\end{array}$ & $\begin{array}{r}35.56 \\
.55 \\
33.38 \\
\ldots \ldots . . \\
\text { none } \\
8.49 \\
.04 \\
\ldots . .4 \\
5.44 \\
.53 \\
\text { none. } \\
\text { trace } \\
2.16 \\
.24 \\
10.40 \\
(?) \\
3.63 \\
\text { none }\end{array}$ & $\begin{array}{r}36.40 \\
\ldots \ldots \ldots \\
33.64 \\
\ldots \ldots \ldots \\
3.13 \\
\ldots \ldots \ldots \\
\cdots \ldots \ldots .01 \\
10.01 \\
1.51 \\
\ldots \ldots \ldots \\
\ldots 2.49 \\
.1 .2 \\
b 8.74 \\
\cdots \\
3.53 \\
.74\end{array}$ & $\begin{array}{r}36.57 \\
.09 \\
32.58 \\
4.32 \\
.79 \\
\text { trace } \\
.05 \\
9.47 \\
.75 \\
\ldots \ldots . . \\
\text { trace } \\
2.22 \\
.13 \\
8.90 \\
.04 \\
3.74 \\
.06\end{array}$ & $\begin{array}{r}35.96 \\
a .36 \\
33.28 \\
\ldots \ldots . . \\
11.04 \\
.13 \\
\ldots \ldots . . \\
3.48 \\
.42 \\
\ldots \ldots . . \\
\text { none } \\
2.16 \\
\text { none } \\
10.61 \\
\ldots . . . \\
3.31 \\
\text { none }\end{array}$ & $\begin{array}{r}35.21 \\
a .23 \\
36.07 \\
\ldots \ldots . \\
11.11 \\
.98 \\
\ldots \ldots 19 \\
.25 \\
\ldots \ldots . . \\
\text { trace } \\
1.92 \\
\text { none } \\
10.43 \\
\ldots \ldots \\
3.51 \\
\text { none }\end{array}$ \\
\hline \multirow[t]{2}{*}{ O equivalent to $\mathrm{F} \ldots \ldots \ldots \ldots \ldots \ldots \ldots \ldots \ldots \ldots \ldots \ldots \ldots \ldots$} & $\begin{array}{r}100.49 \\
\cdots\end{array}$ & $\begin{array}{c}100.42 \\
\ldots \ldots\end{array}$ & $\begin{array}{r}100.31 \\
.31 \\
\end{array}$ & $\begin{array}{r}99.71 \\
.02\end{array}$ & 100.75 & $\begin{array}{r}99.90 \\
\cdots\end{array}$ \\
\hline & & & 100.00 & 99.69 & & \\
\hline
\end{tabular}

$a \mathrm{Ti}_{2} \mathrm{O}_{3}$.

$b$ By difference.

GYROLITE.

A. Associated with the apophyllite of New Almaden, California. Fibrous. Analyzed by F. W. Clarke and described in Bull. 64.

B. From Fort Point, San Francisco, California. Analyzed' by W. 'T. Schaller, and described in Bull. 262.

\begin{tabular}{|c|c|c|}
\hline & A. & B. \\
\hline 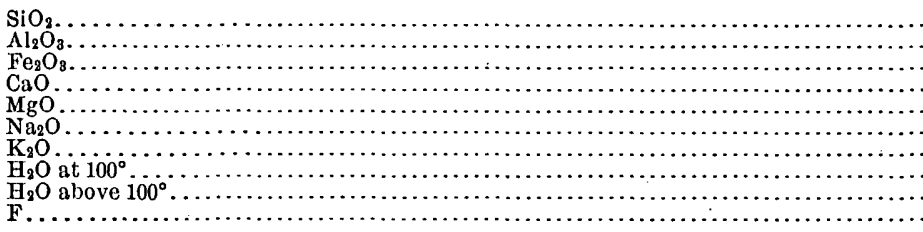 & $\left\{\begin{array}{r}52.54 \\
.71 \\
29.97 \\
\ldots . .27 \\
.27 \\
1.56 \\
14.60 \\
.65\end{array}\right.$ & $\begin{array}{r}53.47 \\
.22 \\
\text { trace } \\
32.00 \\
\text { trace } \\
1.25 \\
\text { trace } \\
5.93 \\
7.28 \\
\text { none }\end{array}$ \\
\hline \multirow[t]{2}{*}{ 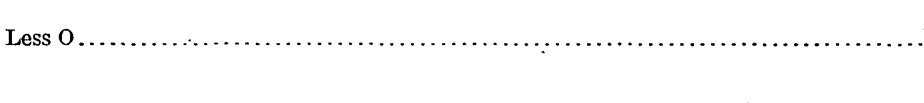 } & $\begin{array}{r}100.30 \\
.27\end{array}$ & \multirow[t]{2}{*}{100.15} \\
\hline & 100.03 & \\
\hline
\end{tabular}

\section{APOPHYLLITE}

From Table Mountain, Golden, Colorado. Described by Cross and Fillebrand in Bull. 20. Analyses by W. F. Hillebrand.

A. Apophyllite.

B. Decomposition product of apophyllite.

\begin{tabular}{|c|c|c|}
\hline & A. & 13. \\
\hline 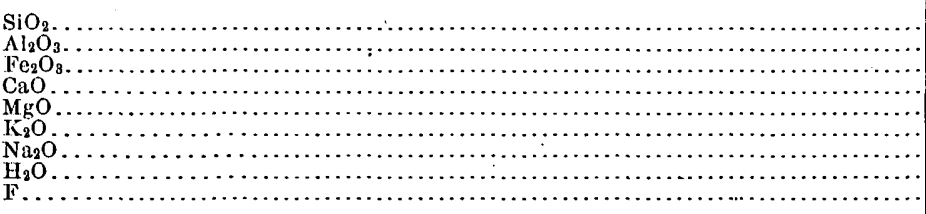 & \begin{tabular}{r}
51.89 \\
1.54 \\
.13 \\
24.51 \\
\hdashline .81 \\
3.81 \\
16.59 \\
1.70
\end{tabular} & $\begin{array}{r}8.96 \\
8.48 \\
1.04 \\
5.47 \\
.53 \\
1.23 \\
\text { a. } 74 \\
14.55 \\
\text { none }\end{array}$ \\
\hline \multirow{2}{*}{ 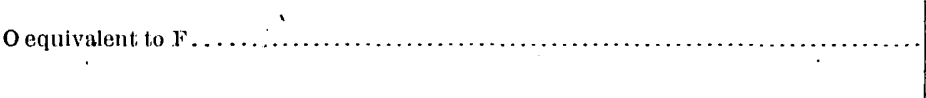 } & $\begin{array}{r}100.69 \\
.72\end{array}$ & \multirow[t]{2}{*}{100.00} \\
\hline & 99.97 & \\
\hline
\end{tabular}


PTILOLITE.

Described as a new species by Cross and Eakins in Am. Jour. Sci., 3d ser., vol. 32, p. 117, and vol. 44, p. 96 . Analyses by L. G. Eakins.

A. From Green Mountain, Jefferson County, Colorado.

B. From 3 miles south of Silver Cliff, Custer County, Colorado.

\begin{tabular}{|c|c|c|}
\hline & A. & B. \\
\hline 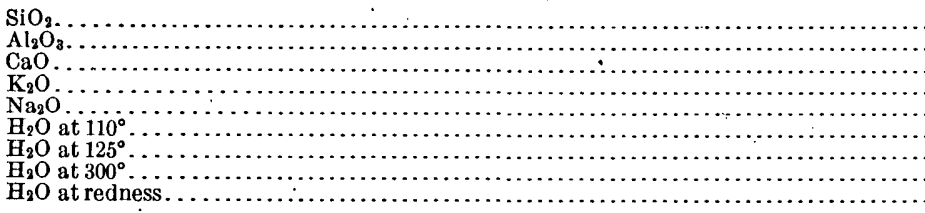 & $\begin{array}{r}70.35 \\
11.90 \\
3.87 \\
2.83 \\
. .77 \\
\\
10.18\end{array}$ & $\begin{array}{r}67.83 \\
11.44 \\
3.30 \\
.64 \\
2.63 \\
3.62 \\
1.31 \\
5.41 \\
3.10\end{array}$ \\
\hline • & 99.90 & 99.28 \\
\hline
\end{tabular}

\section{HEULANDITE.}

A. From Green Mountain, Jefferson County, Colorado. Analysis by L. G. Eakins.

B. From Anthracite Creek, Gunnison County, Colorado. Analysis by L. G. Eakins. Sp. gr., 2.24 at $20.1^{\circ}$.

C. From Berufiörd, Iceland. Analysis by George Steiger.

\begin{tabular}{|c|c|c|c|}
\hline . & A. & B. & c. \\
\hline \multirow{4}{*}{ 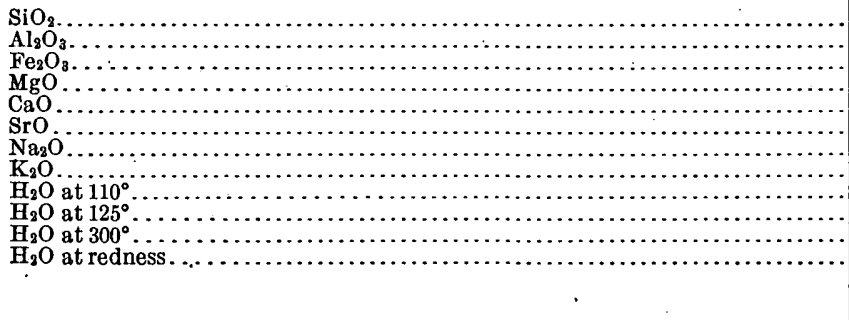 } & $\begin{array}{c}59.17 \\
16.80 \\
\cdots\end{array}$ & $\begin{array}{l}57.38 \\
17.18 \\
\text { trace }\end{array}$ & $\begin{array}{c}57.10 \\
16.82 \\
\ldots \ldots . .\end{array}$ \\
\hline & 7.10 & 8.07 & $\begin{array}{r}.07 \\
6.95\end{array}$ \\
\hline & $\left\{\begin{array}{r}1.37 \\
.34 \\
15.45\end{array}\right.$ & $\begin{array}{r}.82 \\
.40 \\
2.57 \\
1.10 \\
11.70 \\
.90\end{array}$ & $\begin{array}{r}.40 \\
1.25 \\
.42 \\
3.61 \\
13.00\end{array}$ \\
\hline & 100.23 & 100.12 & 99.68 \\
\hline
\end{tabular}

A. From Wassons Bluff, near Parrsboro, Nova Scotia. Analysis by George Steiger.

B. From Italian Mountain, Gunnison County, Colorado. Analysis by L. G. Eakins.

C. From Table Mountain, Golden, Colorado. Described by Cross and Hillebrand in Bull. 20. Analysis by W. F. Hillebrand.

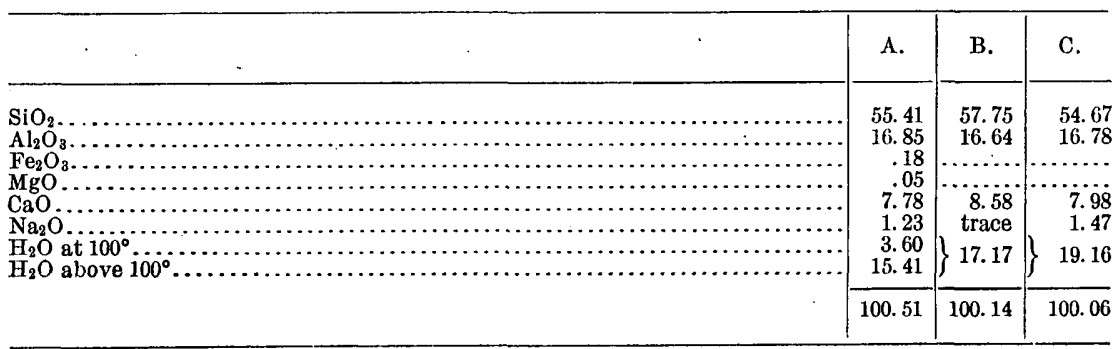




\section{LAUMONTITE:}

From Toble Mountain, Golden, Colorado. Described by Cross and Fillebrand in Bull. 20. Analyses by W. F. Hillebrand.

A. Yellow grains.

B. White crystals.

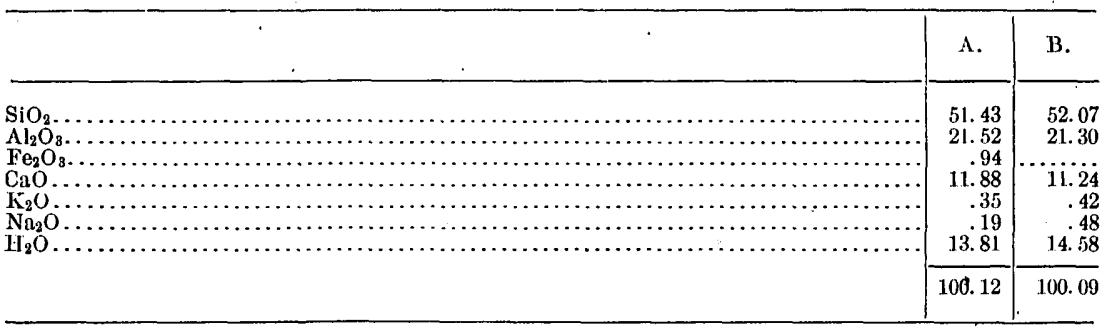

\section{CHABAZITE.}

A. From Wassons Bluff, near Parrsboro, Nova Scotia. Analysis by George Steiger. B, C. From Table Mountain, Golden, Colorado. Described by Cross and Hillebrand in. Bull. 20. Analyses by W. F. Hillebrand.

\begin{tabular}{|c|c|c|c|}
\hline & A. & B. & C. \\
\hline 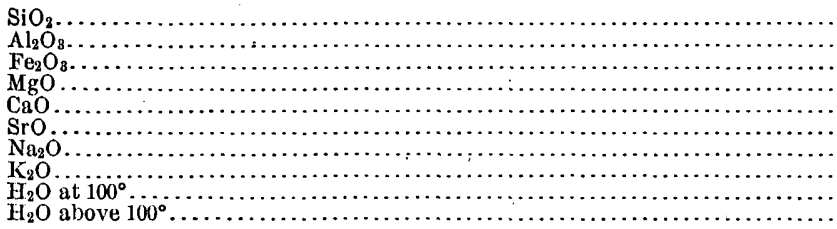 & $\begin{array}{r}50.78 \\
17.18 \\
.40 \\
.04 \\
7.84 \\
\ldots .7 . \\
1.28 \\
.73 \\
5.22 \\
16.63\end{array}$ & $\begin{array}{r}47.86 \\
19.30 \\
.12 \\
\ldots . . .9 \\
9.94 \\
.52 \\
.35 \\
22.07\end{array}$ & $\begin{array}{r}47.18 \\
19.67 \\
\ldots . . . \\
9.74 \\
.43 \\
.51 \\
.37 \\
4.76 \\
17.39\end{array}$ \\
\hline & 100.10 & 100.16 & 100.05 \\
\hline
\end{tabular}

\section{LEVYNITE.}

From Table Mountain, Golden, Colorado. Described by Cross and Hillebrand in Bull. 20. Analyses by W. F. Hillebrand.

A. Crystals of levynite.

B. Associated fibrous variety.

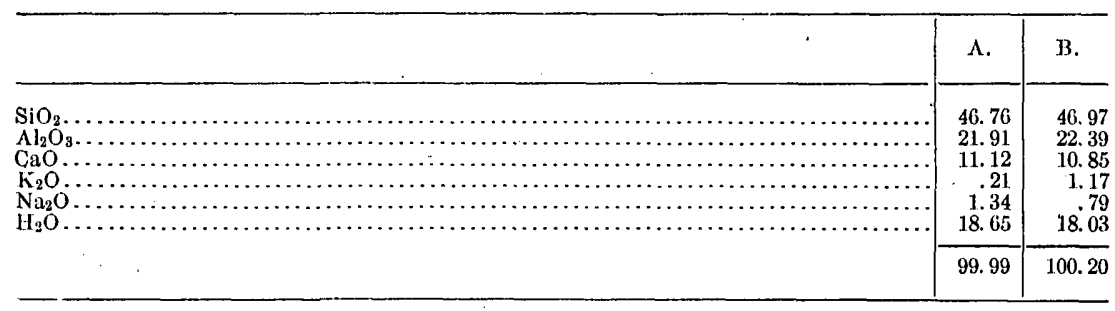

ANALCITE.

A. From Wassons Bluff, near Parrsboro, Nova Scotia. Analysis by George Steiger. See Bull. 207.

B. From Table Mountain, Golden, Colorado. Analysis by George Steiger. See Bull. 207. 
C, D. From Table Mountain. Described by Cross and Hillebrand in Bull. 20. Analyses by W. F. Hillebrand.

\begin{tabular}{|c|c|c|c|c|}
\hline . & A. & B. & C. & D. \\
\hline $\begin{array}{l}\mathrm{SiO}_{2} \\
\mathrm{Al}_{2} \mathrm{O}_{3} \\
\mathrm{Fe}_{2} \mathrm{O}_{8} \ldots \ldots \\
\mathrm{CaO} \\
\mathrm{Na}_{2} \mathrm{O} \\
\mathrm{H}_{2} \mathrm{O} \\
\mathrm{H}_{2} \mathrm{O}\end{array}$ & $\begin{array}{r}57.06 \\
21.48 \\
.13 \\
.16 \\
12.20 \\
.58 \\
8.38\end{array}$ & $\begin{array}{r}55.72 \\
23.06 \\
.17 \\
12.46 \\
.13 \\
8.26\end{array}$ & $\begin{array}{r}55.82 \\
22.42 \\
\cdots \\
13.48 \\
8.38\end{array}$ & $\begin{array}{r}55.80 \\
22.45 \\
\ldots \ldots . \\
\ldots \ldots . . \\
13.45 \\
8.35\end{array}$ \\
\hline . & 99.99 & 99.80 & 100.10 & 100.05 \\
\hline
\end{tabular}

\section{NATROLITE.}

A. From Bergen Hill, New Jersey. Analysis by George Steiger. Şee Bull. 207.

B. From Magnet Cove, Arkansas. Analysis by W. H. Melville. Described in Bull. 90. Sp. gr., 2.261 .

C. From South Table Mountain, Golden, Colorado. Described by Cross and Hillebrand in Bull. 20. Analysis by W. F. Hillebrand.

\begin{tabular}{|c|c|c|c|}
\hline & A. & B. & C. \\
\hline 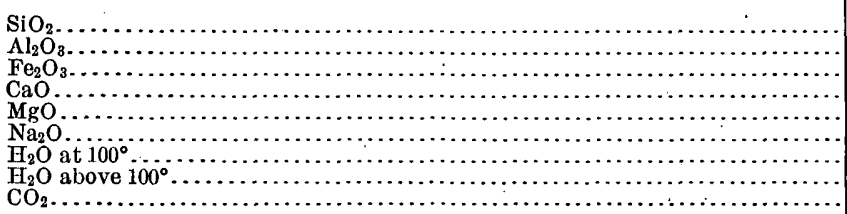 & $\begin{array}{r}46.62 \\
26.04 \\
\text { none } \\
1.48 \\
\text { none } \\
15.67 \\
10.18 \\
10.19\end{array}$ & $\begin{array}{r}47.56 \\
26.82 \\
.23 \\
.13 \\
.09 \\
15.40 \\
.07 \\
9.56 \\
.\end{array}$ & $\begin{array}{r}43.66 \\
24.89 \\
\ldots . \\
4.87 \\
\ldots \quad 14.66 \\
\quad a 8.09 \\
\hdashline \quad \ldots 3.83\end{array}$ \\
\hline & 100.38 & 99.86 & 100.00 \\
\hline
\end{tabular}

$a$ By difference. Too little material for complete analysis. $b$ Calculated to satisfy total lime.

\section{SCOLECITE.}

A. From Whale Cove, Grand Manan, New Brunswick. Analysis by George Steiger. See Bull. 207.

B. From Italian Mountain, Gunnison County, Colorado. Analysis by L. G. Eakins. Sp. gr., 2.247 at $17.2^{\circ}$.

C. From Table Mountain, Golden, Colorado. Described by Cross and Hillebrand in Bull. 20. Analy'sis by W. F. Hillebrand.

\begin{tabular}{|c|c|c|c|}
\hline . & A. & B. & C. \\
\hline 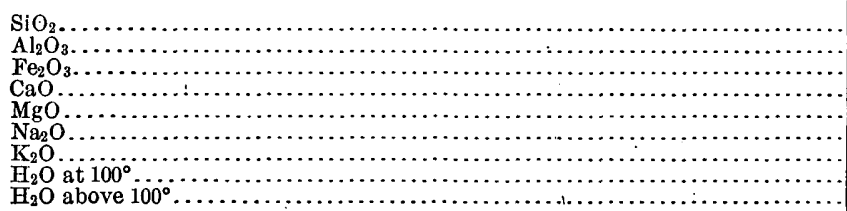 & $\begin{array}{r}45.86 \\
25.78 \\
13.92 \\
\cdots .41 \\
\cdots \\
13.65 \\
1.40\end{array}$ & $\begin{array}{l}45.90 \\
26.51 \\
14.17 \\
\text { trace } \\
\text { trace } \\
\cdots \ldots \\
\cdots \\
13.79\end{array}$ & $\begin{array}{r}46.03 \\
25.28 \\
.27 \\
12.77 \\
\ldots . .25 \\
1.04 \\
.13 \\
a 14.48\end{array}$ \\
\hline & 100.02 & 100.37 & 100.00 \\
\hline
\end{tabular}


MESOLITE.

A. From North Table Mountain, Golden, Colorado. Described by Cross and Hillebrand in Bull. 20. Analysis by W. F. Hillebrand.

B. From Osterö, Faroe Islands. Analysis by G. Steiger.

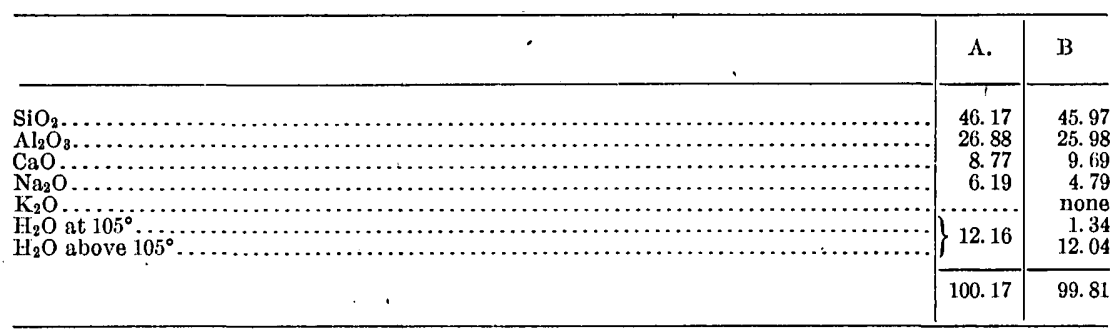

THOMSONITE.

From Table Mountain, Golden, Colorado. Described by Cross and Hillebrand in Bull. 20. Analyse's A, B, C, D, E by W. F. Hillebrand. Analysis F by George Steiger; published in Bull. 207. A represents reddish spherules; F, a mass of fibrous structure.

\begin{tabular}{|c|c|c|c|c|c|c|}
\hline & A. & B. & C. & D. & E. & F. \\
\hline \multirow{5}{*}{ 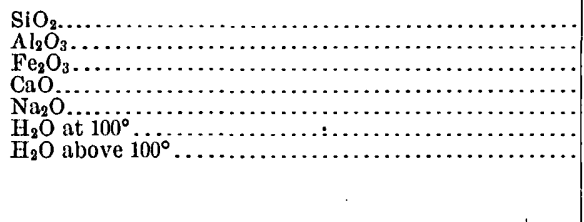 } & $\begin{array}{l}40.52 \\
29.22\end{array}$ & $\begin{array}{l}40.88 \\
29.68\end{array}$ & $\begin{array}{l}40.68 \\
30.12\end{array}$ & $\begin{array}{l}41.21 \\
29.71\end{array}$ & $\begin{array}{l}42.66 \\
29.25\end{array}$ & $\begin{array}{l}41.13 \\
29.58\end{array}$ \\
\hline & 12.43 & i1. 88 & 11.92 & 11.34 & 10.90 & 11.25 \\
\hline & 4.31 & $\begin{array}{r}4.72 \\
\end{array}$ & $\begin{array}{r}4.44 \\
\end{array}$ & $\begin{array}{r}5.62 \\
\end{array}$ & 4.92 & $\begin{array}{l}5.31 \\
1.01\end{array}$ \\
\hline & 12.79 & 12.91 & 12.86 & 12.20 & 12.28 & 12.12 \\
\hline & 100.06 & 100.07 & 100.02 & 100.08 & 100.01 & 100.40 \\
\hline
\end{tabular}

\section{HYDRONEPHELITE.}

From the elæolite syenite of Litchfield, Maine. Described by Clarke as a new species in Bull. 42. Analyses by F. W. Clarke. Sp. gr., 2.263, determined by J. S. Diller.

A, B. Slightly impure. . Two distinct samples.

C. Carefully purified material dried at $100^{\circ}$.

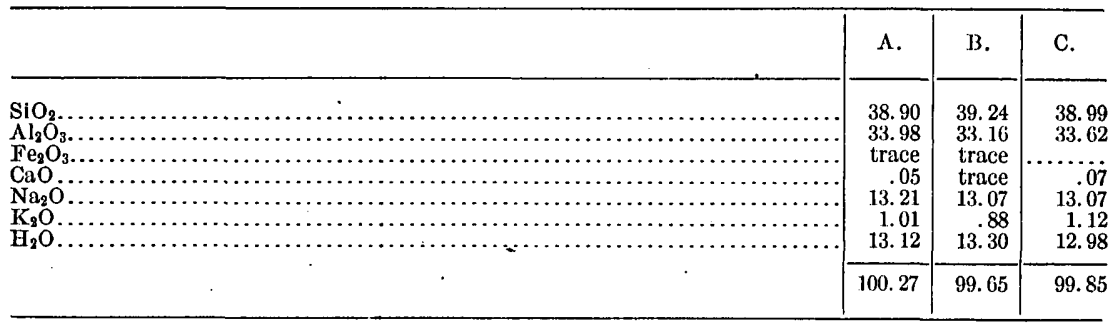

MUSCOVITE.

A. From the Hatch farm, Auburn, Maine. Broad plates, bordered by lepidolite. Analysis by R. B. Riggs.

B. Greenish muscovite, Auburn, Maine. Analysis by E. A. Schneider.

C. Altered muscovite, Mount Mica, Paris, Maine. Occurs as a white enamel on ordinary mica. Analysis by F. W. Clarke.

D. Well-crystallized muscovite from the hiddenite mine, Stony Point, Alexander County, North Carolina. Analysis by F. W. Clarke.

E. White muscovite from Miask, Ural Mountains, Siberia. Analysis by E. A. Schneider. 
F. Mica separated from quartz schist. Shoemaker's quarry, near Stevenson station, Green Spring Valley, Maryland. Analysis by E. A. Schneider.

G. Mica from the Eureka tunnel; St. Peters Dome, near Pikes Peak, Colorado. Analysis by W. F. Hillebrand. See Bull, 20.

H. Pink muscovite, Mesa Grande, San Diego County, California. Analysis by W. T. Schaller.

I. Muscovite associated with the dumortierite of Skamania County, Washington. Analysis by Schaller.

\begin{tabular}{|c|c|c|c|c|c|c|c|c|c|}
\hline & A. & B. & C. & D. & E. & F. & G. & H. & I. \\
\hline $\begin{array}{l}\mathrm{SiO}_{2} \ldots \ldots \\
\mathrm{TiO}_{2} \ldots\end{array}$ & 44.39 & 46.54 & 46.61 & 45.40 & 44.17 & 44.93 & 52.59 & 45.63 & 50.13 \\
\hline $\mathrm{Al}_{2} \mathrm{O}_{3}$ & 35.70 & 34.96 & 35.61 & $\begin{array}{r}1.10 \\
33.66\end{array}$ & 37.35 & $\begin{array}{r}1.00 \\
29.81\end{array}$ & 29.72 & $\begin{array}{l}\text { trace } \\
37.42\end{array}$ & 32.37 \\
\hline $\mathrm{Fe}_{2} \mathrm{O}_{3}$. & 1.09 & 1.59 & & 2.36 & 1.29 & 6.10 & 1.40 & trace & 1.52 \\
\hline 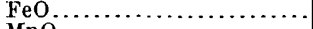 & 1.07 & ........ & & ......... & .20 & & & & \\
\hline $\mathrm{MnO}_{\mathrm{MgO}}$. & trace & $\cdots \cdots$ & trace & $\cdots$ & & trace & in & .06 & \\
\hline $\begin{array}{l}\mathrm{MgO} \\
\mathrm{CaO}\end{array}$ & .10 & & trace & 1.86 & & 1.10 & 2.12 & none & .09 \\
\hline $\mathrm{Li}_{2} \mathrm{O}$. & & & & trace & & trace & & .20 & \\
\hline $\mathrm{Na}_{2} \mathrm{O}$ & 2.41 & .41 & 1.76 & 1.41 & 1.14 & .50 & .50 & 1.43 & \\
\hline $\mathrm{K}_{2} \mathrm{O} \ldots \ldots$ & 9.77 & 10.38 & 8.86 & 8.33 & 10.00 & 10.28 & 8.33 & 9.95 & 9.60 \\
\hline $\mathrm{H}_{2} \mathrm{O}$ at $100^{\circ} \ldots \ldots$ & 5.88 & $\begin{array}{r}.71 \\
4.72\end{array}$ & 6.50 & 5.46 & $\begin{array}{l}1.06 \\
4.67\end{array}$ & $\begin{array}{l}1.38 \\
4.88\end{array}$ & 4.39 & 443 & $\begin{array}{l}7.74 \\
5.08\end{array}$ \\
\hline F.... & .72 & none & & .69 & .90 & .22 & & .77 & \\
\hline \multirow[t]{2}{*}{$\mathrm{O}$ equivalent to $\mathrm{F}$. } & $\begin{array}{r}101.13 \\
.30\end{array}$ & 99.63 & 99.34 & $\begin{array}{r}100.27 \\
.29\end{array}$ & $\begin{array}{r}100.88 \\
.37\end{array}$ & $\begin{array}{r}100.31 \\
.09\end{array}$ & 99.31 & $\begin{array}{r}99.89 \\
.32\end{array}$ & 100.68 \\
\hline & 100.83 & & & 99.98 & 100.51 & 100.22 & & 99.57 & \\
\hline
\end{tabular}

J. Fuchsite. Etchison post-office, Montgomery County, Maryland. Analysis by T. M. Chatard. See Bull. 64.

K. Grayish-green, compact mica from Stoneham, Maine. Structure subfibrous. Analysis by T. M. Chatard.

L. Same locality as I. Specimen broadly foliated, micaceous. Analysis by T. M. Chatard. For analyses I and J see Bull. 9.

M. Stoneham, Maine. From alteration of topaz, the outer portion of an altered crystal. Sp.gr., 2.82. Analysis by J. E. Whitfield. Discussed by Clarke in Bull. 27.

N. Pseudomorph from the Rochelle mine, on Running Water River, Wyoming. Near liebenerite. Sp. gr., 2.831 at $12.5^{\circ}$. Analyzed by W. F. Hillebrand and described in Bull. 20.

O. Mariposite. From vein of the Josephine gold quartz mine, Bear Yalley, Mariposa County, California. Color, green. Sp. gr., 2.817 at $29.5^{\circ}$.

P. White mariposite, same locality as M. Sp. gr., 2.787 at $28.5^{\circ}$. Analyses M and $\mathrm{N}$ by W. F. Hillebrand, and described in Bull. 167.

\begin{tabular}{|c|c|c|c|c|c|c|c|}
\hline & $\mathbf{J}$. & K. & L. & M. & N. & O. & $\mathbf{P}$. \\
\hline 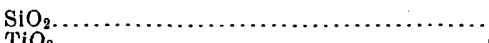 & 42.21 & 45.19 & 45.34 & 44.52 & 45.54 & 55.35 & 56.79 \\
\hline $\begin{array}{l}\mathrm{TiO}_{2} \mathrm{O}_{1} \\
\mathrm{~A}{ }_{2} \mathrm{O}_{3}, \ldots \ldots\end{array}$ & 34.55 & 33.32 & 33.96 & $\cdots .19$ & 37.15 & 25.62 & 25.29 \\
\hline $\mathrm{Cr}_{2} \mathrm{O}_{3}$ & 2.03 & $\ldots . . .$. & ......... & $\ldots . . .$. & .79 & .18 & none \\
\hline 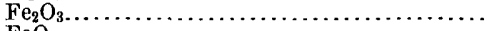 & 1.03 & $\cdots \cdots$ & $\therefore \ldots \ldots$ & $\ldots \ldots \ldots$ & $\ldots \ldots$. & .63 & 1.59 \\
\hline 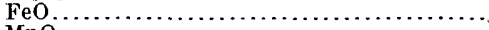 & & 4. 25 & 3.96 & $\ldots \ldots \ldots$ & ........ & .92 & \} \\
\hline 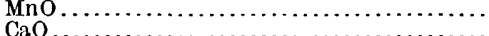 & $\begin{array}{r}\text { trace } \\
47\end{array}$ & $\begin{array}{r}.58 \\
\operatorname{trace}\end{array}$ & $\begin{array}{r}.51 \\
.22\end{array}$ & $\begin{array}{r}.21 \\
30\end{array}$ & & 07 & miz \\
\hline 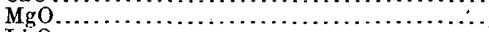 & 3.13 & .36 & .10 & .14 & .38 & 3.25 & 3.29 \\
\hline 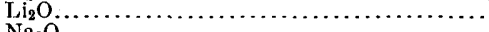 & $\cdots$ & mi & 140 & $\cdots \ddot{p} \ddot{i}$ & & trace & trace \\
\hline 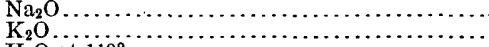 & $\begin{array}{r}.82 \\
9.16\end{array}$ & $\begin{array}{r}1.57 \\
11.06\end{array}$ & $\begin{array}{r}1.49 \\
10.73\end{array}$ & $\begin{array}{l}2.82 \\
2.30\end{array}$ & $\begin{array}{r}.90 \\
10.70\end{array}$ & 9.12 & $\begin{array}{r}.17 \\
8.92\end{array}$ \\
\hline $\begin{array}{l}\mathrm{H}_{2} \mathrm{O} \text { at } 110^{\circ}{ }_{\mathrm{H}_{2}} \mathrm{O} \text { above } 110^{\circ} \ldots \ldots \ldots \ldots \ldots \ldots \ldots \ldots \ldots \ldots \ldots \\
\mathrm{H} \ldots \ldots \ldots \ldots \ldots\end{array}$ & 6.57 & 4.48 & 4.78 & 3.74 & 4.80 & 4.52 & 4. 72 \\
\hline F.............. & $\ldots \ldots \ldots$ & & & .40 & & & \\
\hline \multirow{3}{*}{ o equivalent to $F \ldots \ldots \ldots \ldots \ldots \ldots \ldots \ldots$} & 100.17 & 100.81 & 101.09 & $100.6 \dot{2}$ & 100.26 & 100.13 & 100.84 \\
\hline & $\cdots \ldots \ldots$ & $\cdots \ldots \ldots$ & $\ldots \ldots \ldots$ & & & $\cdots \cdot$ & $\cdots$ \\
\hline & & & & 100.46 & & & \\
\hline
\end{tabular}




\section{LEPIDOLITE.}

Micas A to $\mathrm{G}$ from various localities in Maine. Analyses by R. B. Riggs. Discussion by F. W. Clarke in Bull. 42.

A. From Black Mountain, Rumford. Lilac-purple, granular.

B. From Mount Mica, Paris. Broadly foliated.

C. From Hebron. Purple, coarsely granular.

D. From Auburn. Purple border on plates of muscovite.

E. From Auburn. Purple, coarsely granular.

F. From Norway. White, coarsely granular.

G. From Norway. Brownish, finely granular.

\begin{tabular}{|c|c|c|c|c|c|c|c|}
\hline & A. & B. & C. & D. & E. & F. & G. \\
\hline 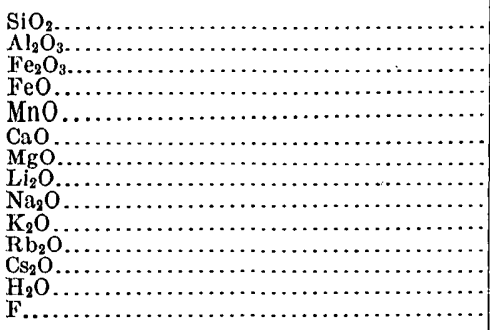 & $\begin{array}{r}51.52 \\
25.96 \\
.31 \\
\text { undet. } \\
.20 \\
.16 \\
.02 \\
4.90 \\
1.06 \\
11.01 \\
7 . . .6 \\
.95 \\
5.80\end{array}$ & $\begin{array}{r}50.92 \\
24.99 \\
.30 \\
.23 \\
\text { trace } \\
\text { trace } \\
\text { trace } \\
4.20 \\
2.11 \\
11.38 \\
\text { trace } \\
\text { trace } \\
1.96 \\
6.29\end{array}$ & $\begin{array}{r}48.80 \\
28.30 \\
.29 \\
.09 \\
.08 \\
.10 \\
.07 \\
4.49 \\
.74 \\
12.21\end{array}$ & $\begin{array}{r}49.62 \\
27.30 \\
.31 \\
.07 \\
.55 \\
\ldots . . . . \\
4.34 \\
2.17 \\
8.03 \\
2.44 \\
.72 \\
1.52 \\
5.45\end{array}$ & $\begin{array}{r}51.11 \\
25.26 \\
.20 \\
.07 \\
.17 \\
.12 \\
.01 \\
4.98 \\
1.43 \\
10.51 \\
1.29 \\
.45 \\
.94 \\
6.57\end{array}$ & $\begin{array}{r}49.52 \\
28.80 \\
. .40 \\
.24 \\
.07 \\
.13 \\
.02 \\
3.87 \\
.13 \\
8.82 \\
3.73 \\
.08 \\
1.72 \\
5.18\end{array}$ & $\begin{array}{r}50.17 \\
25.40 \\
.87 \\
.45 \\
.23 \\
\text { undet. } \\
\text { undet. } \\
4.03 \\
13.40\end{array}$ \\
\hline \multirow[t]{2}{*}{$O$ equivalent to $F$. } & $\begin{array}{r}101.89 \\
2.44\end{array}$ & $\begin{array}{r}102.38 \\
2.64\end{array}$ & $\begin{array}{r}101.86 \\
2.02\end{array}$ & $\begin{array}{r}102.52 \\
2.29\end{array}$ & $\begin{array}{r}103.11 \\
2.76\end{array}$ & $\begin{array}{r}102.71 \\
2.18\end{array}$ & $\begin{array}{r}101.62 \\
2.13\end{array}$ \\
\hline & 99.45 & 99.74 & 99.84 & 100.23 & 100.35 & 100.53 & 99.49 \\
\hline
\end{tabular}

Analyses $\mathrm{H}$ to L, of lepidolites from San Diego County, California, by W.T. Schaller. H. Red purple lepidolite, Pala. Tourmaline Queen mine.

I. Blue purple, same locality as A.

J. Purple lepidolite, Pala.

K. White lepidolite, Pala.

L. Lepidolite border on muscovite, Mesa Grande.

M. Lithia mica, Cassiterite Creek, Cape York, Alaska.

Intermediate between lepidolite and zinnwaldite. Analysis by Schaller.

\begin{tabular}{|c|c|c|c|c|c|c|}
\hline & H. & I. & J. & $\mathbf{K}$. & $\mathrm{L}$. & M. \\
\hline 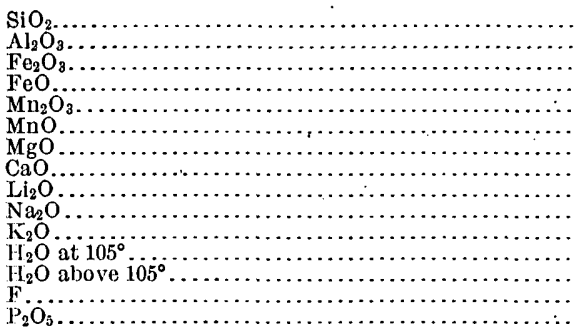 & $\begin{array}{r}51.12 \\
22.70 \\
.80 \\
1.34 \\
1.34 \\
\cdots . . . \\
5.12 \\
2.28 \\
10.60 \\
2.05 \\
6.38 \\
.04\end{array}$ & $\begin{array}{r}50.95 \\
23.97 \\
.82 \\
1.29 \\
1.29 \\
\cdots . . . . \\
4.63 \\
2.39 \\
10.69 \\
1.91 \\
6.11 \\
.04\end{array}$ & $\begin{array}{r}50.34 \\
28.71 \\
.11 \\
\text { trace? } \\
.50 \\
\text { none } \\
\text { trace } \\
2.39 \\
1.59 \\
10.37 \\
3.1 .5 \\
5.02\end{array}$ & $\begin{array}{r}51.25 \\
25.62 \\
.12 \\
\text { none } \\
\ldots .05 \\
\text { none } \\
\text { trace } \\
4.31 \\
1.94 \\
10.65 \\
1.60 \\
7.06\end{array}$ & $\begin{array}{r}50.85 \\
26.78 \\
.60 \\
\ldots \ldots . . \\
\ldots . .67 \\
.07 \\
\text { trace } \\
.10 \\
4.27 \\
1.41 \\
10.30 \\
\ldots .7 \% \\
1.74 \\
6.71\end{array}$ & $\begin{array}{r}46.80 \\
24.50 \\
.50 \\
6.35 \\
1.38 \\
\ldots . .24 \\
.24 \\
3.73 \\
1.73 \\
9.20 \\
.88 \\
8.63\end{array}$ \\
\hline \multirow[t]{2}{*}{ Less 0.} & $\begin{array}{r}102.43 \\
2.69\end{array}$ & $\begin{array}{r}102.80 \\
2.57\end{array}$ & $\begin{array}{r}102.18 \\
2.11\end{array}$ & $\begin{array}{r}102.60 \\
2.97\end{array}$ & $\begin{array}{r}102.83 \\
2.82\end{array}$ & $\begin{array}{r}103.94 \\
3.63 .\end{array}$ \\
\hline & 99.74 & 100.23 & 100.07 & 99.63 & 100.01 & 100.31 \\
\hline
\end{tabular}


COOKEITE.

From Pala, San Diego County, California. Analysis by W. T. Schaller.

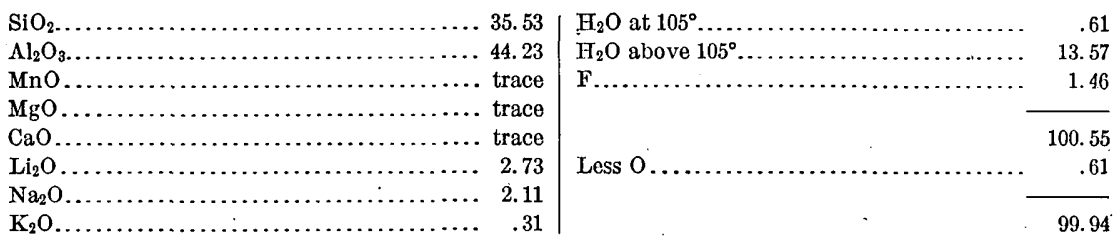

\section{CRYOPHYLLITE.}

From Rockport, Massachusetts. Analyses by R. B. Riggs. Discussed by Clarke in Bull: 42.

A. Brilliant, broadly foliated, blackish-green variety.

B. Paler, dull green, less lustrous, probably somewhat altered.

C. Granular, resembling chlorite.

\begin{tabular}{|c|c|c|c|}
\hline & A. & B. & C. \\
\hline 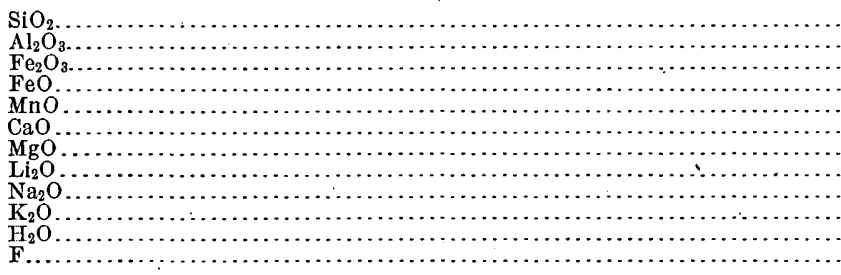 & $\begin{array}{r}51.96 \\
16.89 \\
2.63 \\
6.35 \\
.24 \\
.12 \\
.03 \\
4.93 \\
.92 \\
10.66 \\
1.22 \\
6.78\end{array}$ & $\begin{array}{r}51.46 \\
16.22 \\
2.21 \\
7.66 \\
.06 \\
\text { trace } \\
.17 \\
4.83 \\
.95 \\
10.65 \\
1.06 \\
7.44\end{array}$ & $\begin{array}{r}52.17 \\
16.39 \\
4.11 \\
6.08 \\
.32 \\
\text { trace } \\
\text { trace } \\
5.03 \\
.60 \\
10.54 \\
1.43 \\
7.02\end{array}$ \\
\hline \multirow[t]{2}{*}{$\mathrm{O}$ equivalent to $\mathrm{F}$. } & $\begin{array}{r}102.73 \\
2.86\end{array}$ & $\begin{array}{r}102.71 \\
3.11\end{array}$ & $\begin{array}{r}103.69 \\
2.95\end{array}$ \\
\hline & 99.87 & 99.60 & 100.74 \\
\hline
\end{tabular}

BIOTITE.

A. From Merrow Ledge, Auburn, Maine. Black. Analysis by F. W. Clarke and R. B. Riggs.

B. Bronze mica, Laurel Creek corundum mine, Rabun County, Georgia. Analysis by $\mathrm{E}$. A. Schneider. The loss is due to undetermined volatile matter.

C. Brown mica from the granite of the Yosemite Valley, California. Analysis by William Valentine. Described by Turner, Am. Jour. Sci., 4th ser., vol. 7, p. 294.

D. Mica from quartz monzonite, Tioga road, southeast of Mount Hoffman, Mariposa County, California. Sp.gr., 3.05 at $21^{\circ}$. Analysis by W. F. Hillebrand. Described by Turner, loc. cit.

E. Black mica from quartz monzonite. About 1 kilometer south of Bloods station, Alpine County, California. Analysis by William Valentine. Described by Turner, loc. cit.

F. Reddish-brown mica from pyroxenic gneiss. North Fork of Mokelumne River, above mouth of Bear River, Amador County, California. Analysis by William Valentine. Described by Turner, loc. cit.

G. Biotite from the granite of Butte, Montana. See Weed, Jour. Geology, vol. 7, p. 737. Analysis by H. N. Stokes.

H. Biotite from orthoclase gabbro, between Blue and Mud Lakes, Livingston quadrangle, Montana. See W. H. Emmons, Jour. Geol., vol. 16, p. 193. Analysis by G. Steiger. 


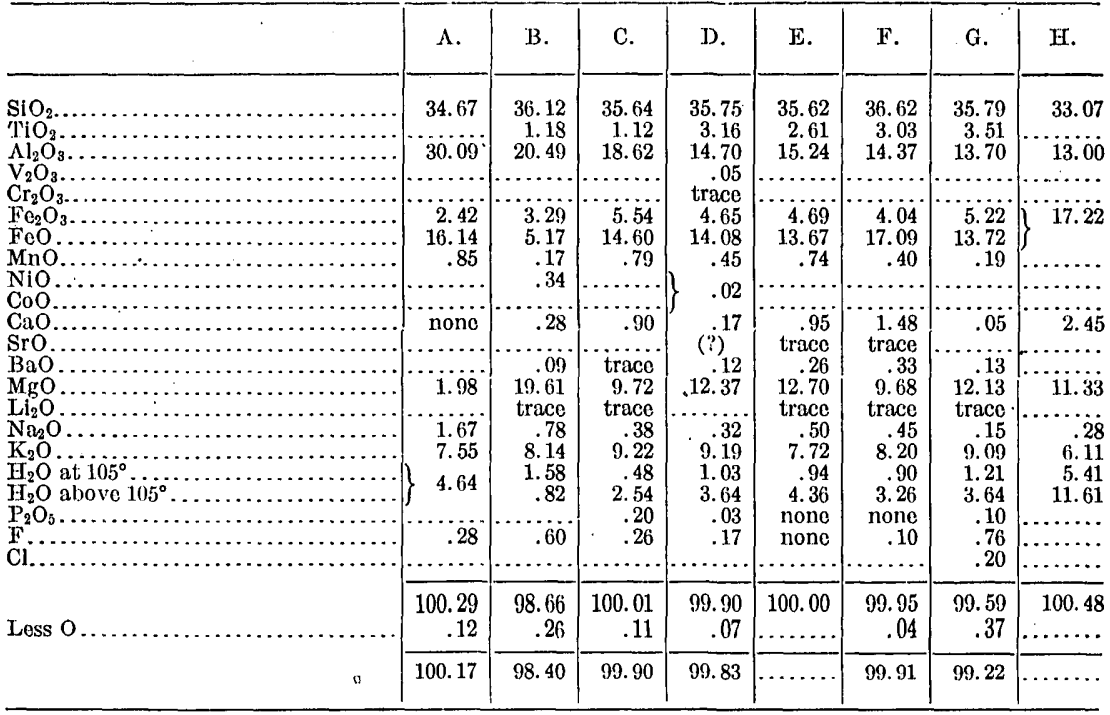

PHLOGOPITE.

A. From Edwards, St. Lawrence County, New York. Outwardly resembles talc. Analysis by E. A. Schneider. Discussed by Clarke and Schneider in Bull. 78.

B. From Burgess, Canada. Brown. Analysis by E. A. Schneider. See Clarke and Schneider, Bull. 78.

C. Phlogopite separated from the wyomingite of the Leucite Hills, Wyoming. Described by Cross in Am. Jour. Sei., 4th ser., vol. 4, p. 1.15. Analysis by W. F. Hillebrand.

The following micas of doubtful character occur in serpentine. Analyses by Charles Catlett. See Bull. 64 .

D. Brown mica, from the serpentine of Montville, New Jersey.

E. Yellowish mica, same locality as D.

F. White mica, same locality.

G. White mica from near Easton, Pennsylvania.

$\mathrm{E}, \mathrm{F}$, and $\mathrm{G}$ are perhaps to be called vermiculites rather than micas.

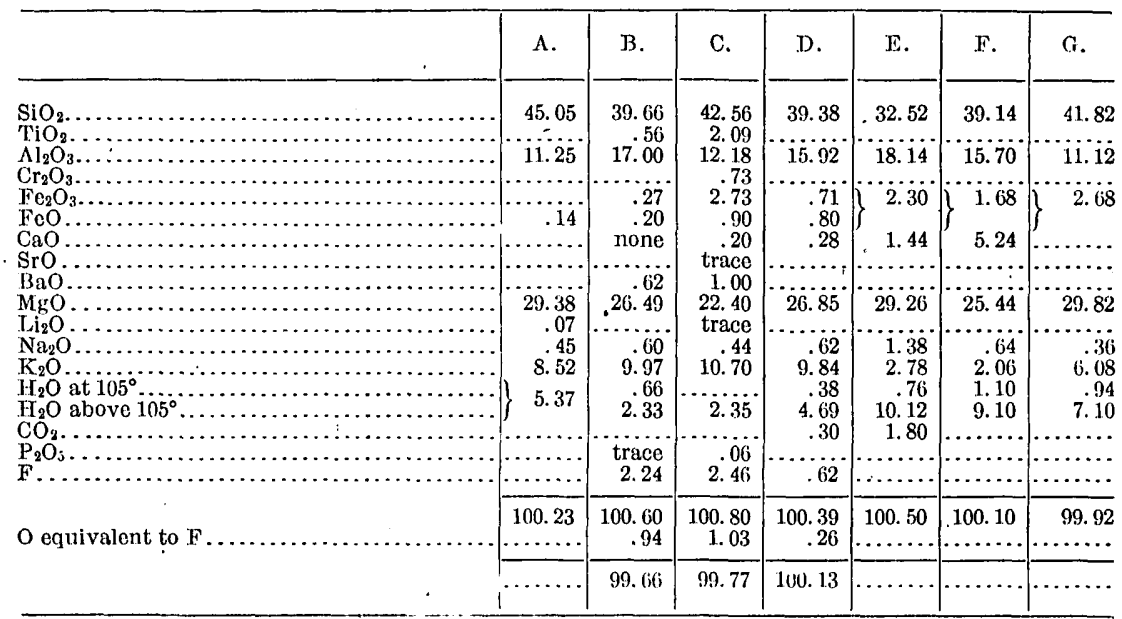




\section{LEPIDOMELANE.}

A. From Litchfield, Maine. Analyzed by F. W. Clarke, and described in Bull. 42.

B. Same as A. Later analysis by Clarke. See Bull. 55.

C. Annite. From Rockport, Massachusetts. Analysis by R. B. Riggs. Discussion by Clarke in Bull. 42.

D. From Port Henry, New York. Analysis by E. A. Schneider. See paper by Clarke and Schneider in Bull. 78. 55.

E. From Baltimore, Maryland. Analysis by F. W. Clarke, and discussed in Bull.

F. From near Pikes Peak, Colorado. Siderophyllite? The slightly altered margin of a large crystal.

G. Much-altered central portion of specimen F. Analyses F and G by F. W. Clarke and R. B. Riggs. Described by Clarke in Bull. 55. Classed by Dana under biotite.

\begin{tabular}{|c|c|c|c|c|c|c|c|}
\hline & A. & B. & $\mathrm{C}$. & D. & E. & F. & G. \\
\hline $\begin{array}{l}\mathrm{SiO}_{2} \ldots . . \\
\mathrm{TiO}_{2} \ldots\end{array}$ & 32.09 & $\begin{array}{l}32.35 \\
\text { trace }\end{array}$ & $\begin{array}{r}32.03 \\
3.42\end{array}$ & $\begin{array}{r}34.52 \\
2.70\end{array}$ & 35.78 & 34.21 & 34.63 \\
\hline $\mathrm{Al}_{2} \mathrm{O}_{3} \ldots$ & 18.52 & 17. 47 & 11. 92 & 13. 22 & 16.39 & 16.53 & 17.95 \\
\hline $\mathrm{Fe}_{2} \mathrm{O}_{3}$ & 19.49 & 24.22 & 8.00 & 7.80 & 14.55 & 20.15 & 31.25 \\
\hline $\mathrm{FeO}$ & 14.10 & 13.11 & 30.41 & 22.27 & 11.02 & 14.17 & 3.01 \\
\hline $\mathrm{MnO}$. & 1. 42 & 1.02 & .21 & .41 & 1.08 & .91 & .34 \\
\hline $\mathrm{CaO} \ldots \ldots \ldots \ldots$ & & none & .23 & none & none & .48 & .81 \\
\hline 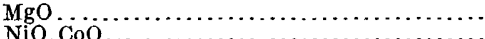 & 1.01 & .89 & .06 & 5.82 & 8.67 & 1.34 & 1.08 \\
\hline $\begin{array}{l}\mathrm{NiO}, \mathrm{CoO} \ldots \ldots \ldots \\
\mathrm{Li}_{2} \mathrm{O} \ldots \ldots \ldots \ldots\end{array}$ & & & trace & $\begin{array}{l}.30 \\
.04\end{array}$ & & & \\
\hline $\begin{array}{l}\mathrm{Na}_{2} \mathrm{O} \ldots \ldots \ldots \ldots \\
\mathrm{K}_{2} \mathrm{O} \ldots \ldots \ldots \ldots\end{array}$ & $\begin{array}{l}1.55 \\
8.12\end{array}$ & $\begin{array}{r}.70 \\
6.40\end{array}$ & $\begin{array}{l}1.54 \\
8.46\end{array}$ & $\begin{array}{r}.16 \\
8.59\end{array}$ & $\begin{array}{l}.56 \\
7.76\end{array}$ & $\begin{array}{l}1.33 \\
6.50\end{array}$ & $\begin{array}{r}.89 \\
1.96\end{array}$ \\
\hline $\begin{array}{l}\mathrm{H}_{2} \mathrm{O} \text { at } 105^{\circ} \ldots \ldots \\
\mathrm{H}_{2} \mathrm{O} \text { above } 105^{\circ} \ldots \\
\mathrm{P}_{2} \mathrm{O}_{5} \ldots \ldots \ldots \ldots \ldots\end{array}$ & 4. 62 & 4. 67 & 4.19 & $\begin{array}{r}.57 \\
3.82 \\
\text { trace }\end{array}$ & 4.48 & 4.54 & 7.82 \\
\hline & none & none & trace & .34 & none & .08 & .54 \\
\hline \multirow[t]{2}{*}{ O equivalent'to $\mathrm{F}$.. } & $\begin{array}{c}100.92 \\
\cdots \cdots \cdots\end{array}$ & 100.83 & $\begin{array}{l}100.47 \\
\ldots \ldots \ldots .\end{array}$ & $\begin{array}{r}100.56 \\
.14\end{array}$ & $\begin{array}{l}100.29 \\
\ldots \ldots \ldots\end{array}$ & $\begin{array}{r}100.34 \\
.03\end{array}$ & $\begin{array}{r}100.28 \\
.22\end{array}$ \\
\hline & & & & 100.42 & & 100.31 & 100.06 \\
\hline
\end{tabular}

\section{ROSCOELITE.}

Analyses by W. F. Hillebrand.

A. From the Stockslager mine, near Lotus, Eldorado County, California. Described by Hillebrand in Bull. 167.

B. Soluble portion of a vanadiferous sandstone from Placerville, Colorado. Described by Hillebrand in Am. Jour. Sci., 4th ser., vol. 10, p. 130.

\begin{tabular}{|c|c|c|}
\hline & A. & B. \\
\hline 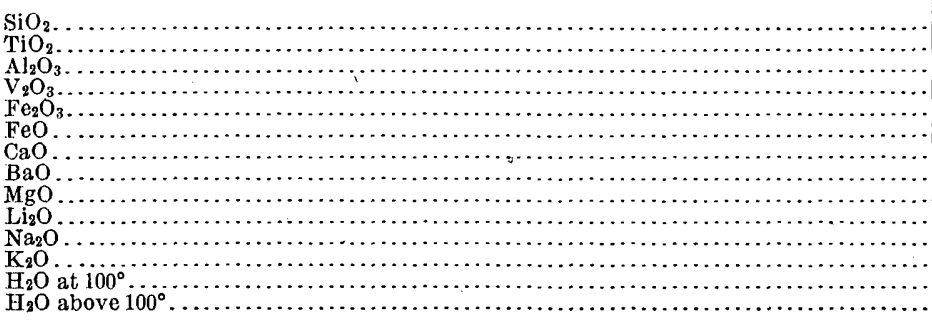 & $\begin{array}{r}45.17 \\
11.78 \\
24.01 \\
1.60 \\
\ldots \\
1.64 \\
\text { trace } \\
\text { trace } \\
10.37 \\
4.40 \\
4.29\end{array}$ & $\begin{array}{r}46.06 \\
22.55 \\
12.84 \\
.73 \\
\ldots . .8 \\
.44 \\
1.35 \\
.92 \\
\ldots \ldots .22 \\
8.84 \\
1.98 \\
4.07\end{array}$ \\
\hline & 99.80 & 100.00 \\
\hline
\end{tabular}




\section{MARGARITE.}

A. Brownish yellow, from Iredell County, North Carolina. Analysis by T. M. Chatard. See Bull. 9.

B. Bright green, associated with pink corundum. From Gainesville, Georgia. Specific gravity, 3.00. Analysis by T. M. Chatard. See Bull. 9.

C. Inclusion in diorite, Crugers station, near Peekskill, New York. Analysis by T. M. Chatard.

\begin{tabular}{|c|c|c|c|}
\hline & A. & B. & C. \\
\hline 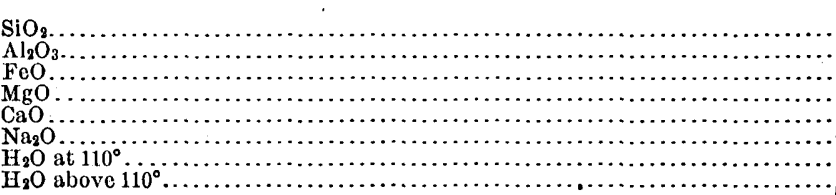 & $\begin{array}{r}31.15 \\
49.51 \\
\text { trace } \\
.45 \\
11.13 \\
2.74 \\
5.68\end{array}$ & $\begin{array}{r}31.72 \\
50.03 \\
\text { trace } \\
.12 \\
11.57 \\
2.26 \\
4.88\end{array}$ & $\begin{array}{r}32.73 \\
46.58 \\
5.12 \\
1.00 \\
11.04 \\
\ldots . .12 \\
.12 \\
4.49\end{array}$ \\
\hline & 100.66 & 100.58 & 101.08 \\
\hline
\end{tabular}

CHLORITOID.

From a phyllite rock near Liberty, Maryland. Analysis by L. G. Eakins. See Bull. 168, p. 50.

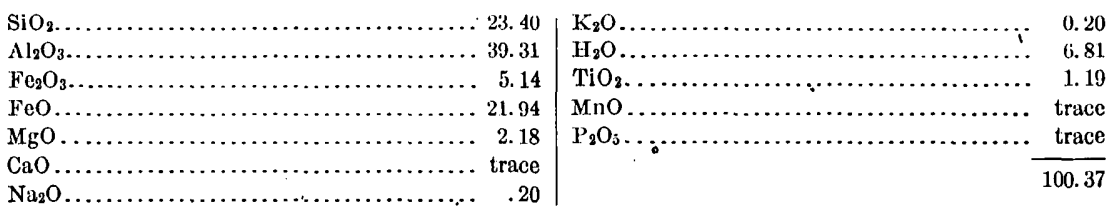

XANTHOPHYLLITE.

Variety waluewite, from the Nikolai-Maximilian mine, district of Slatoust, Urals, Siberia. Analysis by E. A. Schneider. Discussion by Clarke and Schneider in Bull. 113.

\begin{tabular}{|c|c|c|c|}
\hline $\mathrm{SiO}_{2} \ldots \ldots \ldots \ldots \ldots \ldots \ldots \ldots \ldots$ & 16.85 & MgO ................... & 20.77 \\
\hline $\mathrm{TiO}_{2}, \ldots \ldots \ldots \ldots \ldots \ldots \ldots \ldots \ldots \ldots \ldots \ldots \ldots \ldots$ & trace & $\mathrm{H}_{2} \mathrm{O}$ at $105^{\circ} \ldots \ldots \ldots \ldots \ldots \ldots \ldots \ldots \ldots \ldots \ldots \ldots$ & .04 \\
\hline 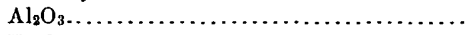 & 42.33 & $\mathrm{H}_{2} \mathrm{O}$ above $105^{\circ} \ldots \ldots \ldots \ldots \ldots \ldots \ldots \ldots \ldots \ldots \ldots$ & 4.56 \\
\hline $\mathrm{Fe}_{2} \mathrm{O}_{3} \ldots \ldots \ldots \ldots \ldots \ldots \ldots \ldots \ldots \ldots \ldots \ldots \ldots \ldots \ldots$ & 2.35 & & 100.40 \\
\hline $\begin{array}{c}\mathrm{Fe} \\
\mathrm{Ca}\end{array}$ & $\begin{array}{r}.20 \\
13.30\end{array}$ & & \\
\hline
\end{tabular}

\section{THE VERMICULITES.}

A. Jefferisite from Westchester, Pennsylvania. Analysis by E. A. Schneider. Discussed by Clarke and Schneider in Bull. 78.

B. Vermiculite, near jefferisite or culsageeite, from Corundum Hill, North Carolina. Analyzed by T. M. Chatard and described by him in Bull. 42 .

C. Altered biotite from the zircon mine, Green River, Henderson County, North Carolina. Analysis by E. A. Schneider. Discussed by Clarke and Schneider in Bull. 90.

D. Kerrite, from near Frauklin, Macon County, North Carolina. Analysis by E. A. Schneider. Discussed by Clarke and Schneider in Bull. 78. 
E. Lucasite. Described as a new species by Chatard in Bull. 42. Analysis by T. M. Chatard. From Corundum Hill, North Carolina. Sp. gr., 2.613 at $25.5^{\circ}$.

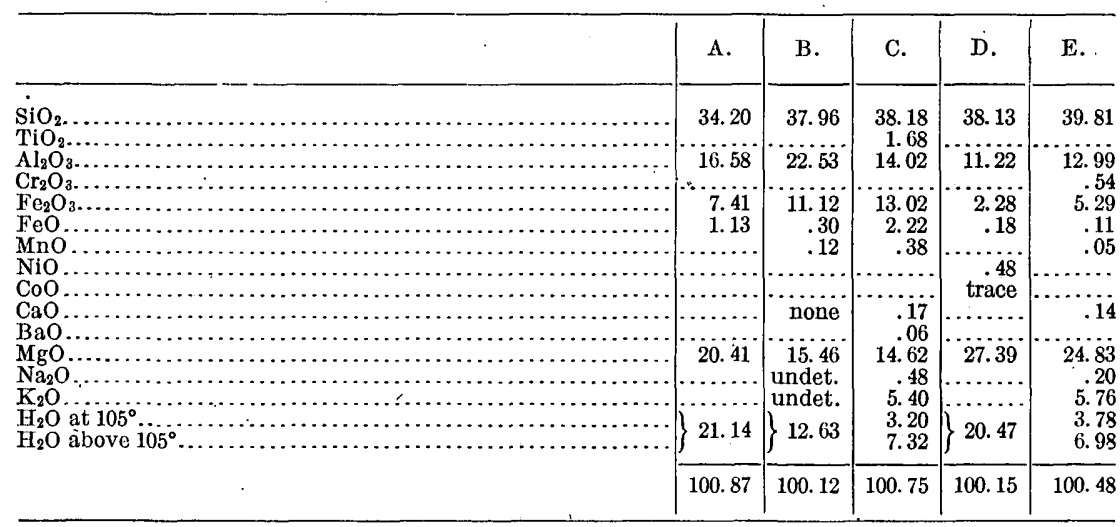

F. Hallite from Nottingham, Chester County, Pennsylvania.

G. White lennilite from Lenni, Delware County, Pennsylvania.

H. Brown lennilite, same locality.

I. Green lennilite, same locality.

Analyses F to I by E. A. Schneider. Discussion by Clarke and Schneider in Bull. 90 .

\begin{tabular}{|c|c|c|c|c|}
\hline . & F. & G. & H. & I. \\
\hline 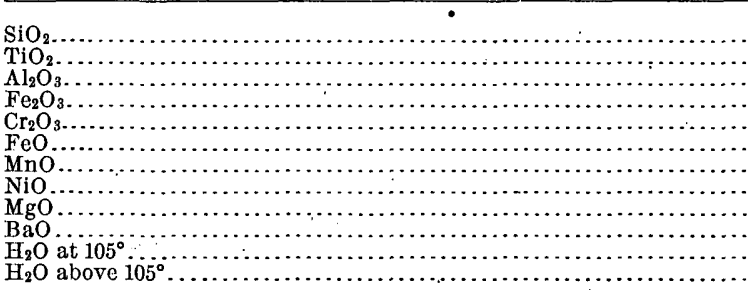 & $\begin{array}{r}35.54 \\
\text { undet. } \\
9.74 \\
9.07 \\
.28 \\
.25 \\
.16 \\
30.05 \\
\cdots \\
2.64 \\
12.14\end{array}$ & $\begin{array}{r}36.72 \\
.18 \\
10.06 \\
5.37 \\
.26 \\
.12 \\
.31 \\
.20 \\
29.40 \\
6.40 \\
11.37\end{array}$ & $\begin{array}{r}35.09 \\
.58 \\
12.05 \\
6.67 \\
.46 \\
.11 \\
.27 \\
.20 \\
27.62 \\
\text { trace } \\
5.70 \\
11.20\end{array}$ & $\begin{array}{r}34.90 \\
.10 \\
10.60 \\
8.57 \\
.23 \\
.22 \\
.17 \\
.19 \\
28.21 \\
\ldots . .99 \\
11.48\end{array}$ \\
\hline & 99.87 & 100.39 & 99.95 & 99.66 \\
\hline
\end{tabular}

J. A vermiculite from Newlin, Chester County, Pennsylvania.

K. Painterite from Middletown, Delaware County, Pennsylvania.

L. Another sample of painterite.

Analyses J, K, L, by E. A. Schneider. Discussion by Clarke and Schneider in Bull. 90.

M. Hydromica from Rocky Hill, New Jersey. Analysis by George Steiger. Described by Clarke and Darton in Bull. 167.

$\mathrm{N}$. Analysis M corrected by deduction of calcite, union of like radicles, and recalculation to 100 per cent.

O. Protovermiculite from Magnet Cove, Arkansas. Analysis by E. A. Schneider. Discussed by Clarke and Schneider in Bull. 90. 


\begin{tabular}{|c|c|c|c|c|c|c|}
\hline - & J. & K. & L. & M. & N. & O. \\
\hline 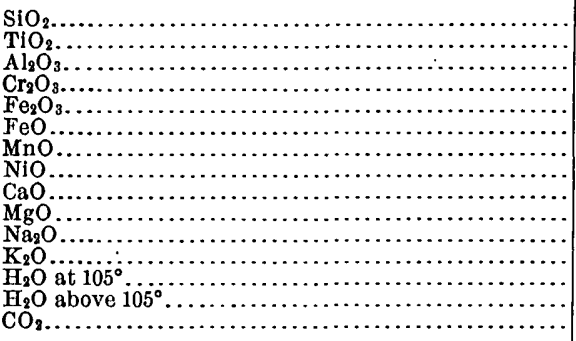 & $\begin{array}{r}31.23 \\
17.52 \\
.14 \\
4.70 \\
1.20 \\
.20 \\
.33 \\
\ldots \ldots .36 \\
\ldots \ldots \ldots \\
\ldots \ldots .08 \\
12.55 \\
\ldots \ldots . .\end{array}$ & $\begin{array}{r}34.86 \\
\text { trace } \\
11.64 \\
\ldots .78 \\
3.78 \\
.20 \\
\ldots .1 .14 \\
.07 \\
31.32 \\
\ldots . . . \\
1.64 \\
16.78 \\
\ldots \ldots . .\end{array}$ & $\begin{array}{r}33.95 \\
\text { trace } \\
12.52 \\
\ldots . \ldots . \\
4.40 \\
.20 \\
\ldots \ldots . . \\
.23 \\
\text { none } \\
30.56 \\
\ldots \ldots \ldots \\
17.56 \\
\ldots \ldots \ldots\end{array}$ & \begin{tabular}{r}
32.72 \\
.24 \\
8.41 \\
$\ldots \ldots . .9$ \\
19.99 \\
4.24 \\
$\ldots . . .$. \\
\hdashline 10.30 \\
5.51 \\
.63 \\
.85 \\
2.47 \\
6.22 \\
8.21
\end{tabular} & $\begin{array}{r}40.24 \\
\cdots \\
10.34 \\
\cdots 24.57 \\
5.21 \\
\ldots \ldots \ldots \ldots \\
\cdots \ldots \ldots . \\
\cdots 6.78 \\
\ldots 2.20 \\
3.03 \\
7.63 \\
\ldots \ldots \ldots\end{array}$ & $\begin{array}{r}34.03 \\
\text { undet. } \\
14.49 \\
\ldots .7 . \\
7.71 \\
.14 \\
.09 \\
\ldots \ldots .88 \\
20.89 \\
11.23 \\
9.96 \\
\ldots \ldots . . .\end{array}$ \\
\hline . & 100.31 & 100.43 & 100.47 & 99.79 & 100.00 & 100.42 \\
\hline
\end{tabular}

P. Chloritic vermiculite from Corundum Hill, North Carolina.

Q. Altered chlorite from Corundum Hill. Analyses P, Q by T. M. Chatard. Description in Bull. 42 .

\begin{tabular}{|c|c|c|}
\hline . & P. & Q. \\
\hline 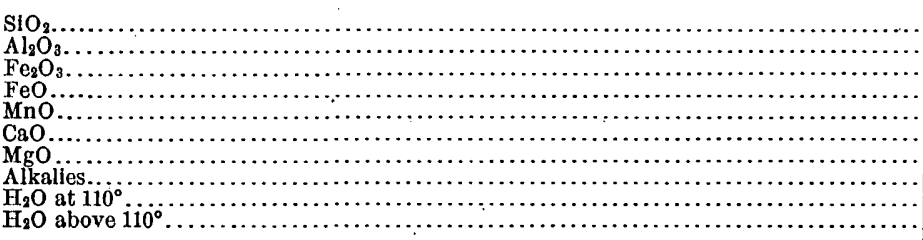 & $\begin{array}{r}35.88 \\
20.90 \\
6.55 \\
3.68 \\
\ldots . .14 \\
19.90 \\
\text { traces } \\
12.71\end{array}$ & $\begin{array}{r}32.97 \\
17.88 \\
4.76 \\
.57 \\
\text { trace } \\
\text { none } \\
22.36 \\
\text { undet. } \\
11.42 \\
10.05\end{array}$ \\
\hline$\cdot$ & 99.76 & 100.01 \\
\hline
\end{tabular}

\section{CLINOCHLORE.}

A. From Westchester, Pennsylvania. Broadly foliated. Analysis by E. A. Schneider. Discussed by Clarke and Schneider in Bull. 78.

B. From the Nikolai-Maximilian mine, district of Slatoust, Urals, Siberia. Analysis by E. A. Schneider. Discussed by Clarke and Schneider in Bull. 113.

C. Leuchtenbergite from Slatoust. Analyst and reference as under B.

D. Kotschubeite from Green Valley, American River canyon, California. Sp. gr., 2.69. Analysis by W. H. Melville. Described by Melville and Lindgren in Bull. 61.

\begin{tabular}{|c|c|c|c|c|}
\hline & A. & $\mathrm{B}$. & C. & D. \\
\hline 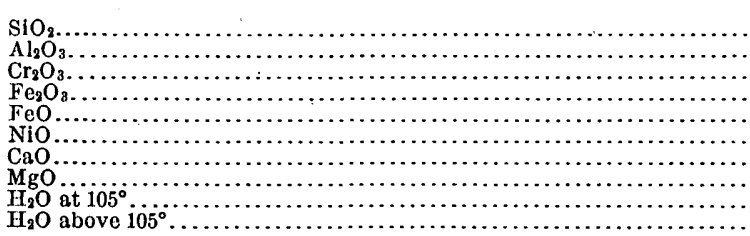 & $\begin{array}{r}29.87 \\
14.48 \\
1.56 \\
5.52 \\
1.93 \\
.17 \\
33.06 \\
13.60\end{array}$ & \begin{tabular}{r}
30.84 \\
18.31 \\
$\cdots \ldots . .$. \\
1.94 \\
1.08 \\
$\cdots \ldots .$. \\
\hdashline 34.38 \\
13.55 \\
13.33
\end{tabular} & $\begin{array}{r}30.00 \\
20.43 \\
\cdots . . . \\
1.68 \\
.14 \\
\cdots .21 \\
34.20 \\
13.25\end{array}$ & \begin{tabular}{r}
31.74 \\
6.74 \\
11.39 \\
\hdashline 1.23 \\
.49 \\
.18 \\
35.18 \\
.37 \\
12.68
\end{tabular} \\
\hline 、 & 100.19 & 100.43 & 100.47 & 100.00 \\
\hline
\end{tabular}

15619-Bull. 419-10--20 
PROCHLORITE.

From the Aqueduct tunnel, Washington, District of Columbia. Analysis by E. A. Schneider. Described by Clarke and Schneider in Bull. 78.

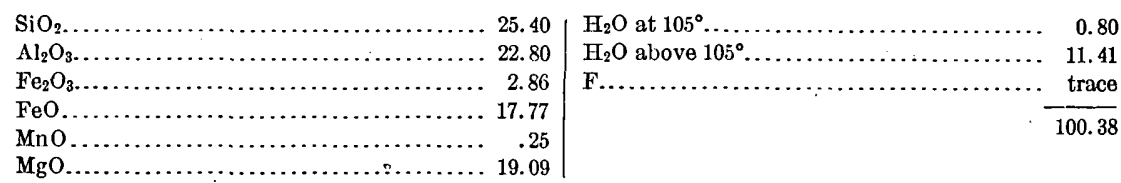

\section{SERPENTINE. $a$}

A. From Newburyport, Massachusetts. Analysis by F. W. Clarke.

B. Same locality as $\Lambda$. Analysis by E. A. Schneider. Discussion by Clarke and Schneider in Bull. 78.

C. From Moriah, New York. Analysis by Charles Catlett. Described by Merrill, Proc. U. S. Nat. Mus., vol. 12, p. 596.

D. From the aqueduct shaft, New York City. Analysis by Charles Catlett. See Merrill, loc. cit., p. 598.

E. From Montville, New Jersey, light yellowish green.

F. From Montville, darker green. Analyses E, F by Charles Catlett. See Merrill, Proc. U. S. Nat. Mus., vol. 11, p. 105.

G. From Montville, massive.

H. From Montville, chrysotile. Aualyses G, H by E: A. Schneider. Discussion by Clarke and Schneider in Bull. 78.

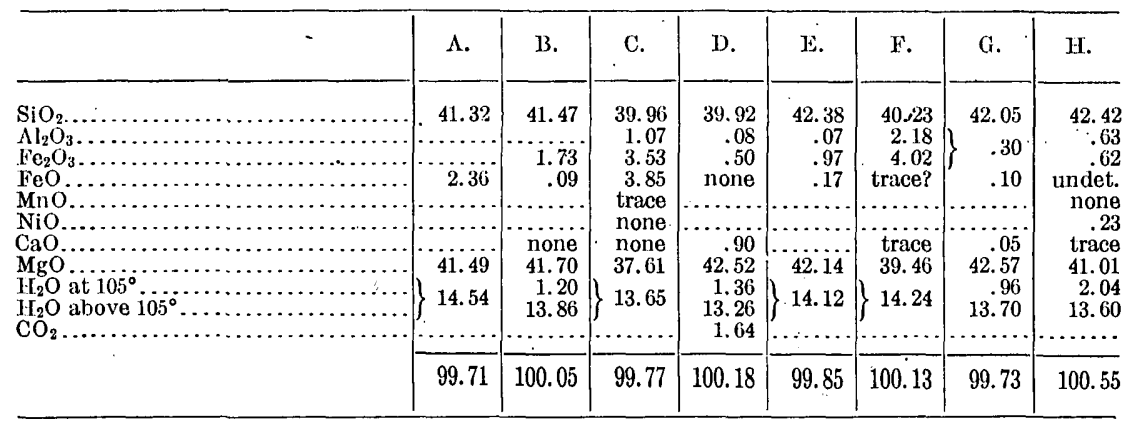

I. From Easton, Pennsylvania. Resembles a vermiculite. Analysis by E. A. Schneider. See Clarke and Schneider, Bull. 90.

J. Grayish-green serpentine from Corundum Hill, North Carolina.

K. Picrolite from Buck Creek, Clay County, North Carolina. Analyses R and S by E. A. Schneider. See Clarke and Schneider, Bull. 78 .

L. From the river Poldnewaja, district of Syssert, Urals, Siberia. Analysis by E. A. Schneider. See Clarke and Schneider, Bull. 113.

M. From Greenville, Plumas County, California. Analysis by W. H. Melville: Described by Diller in Bull. 150.

$a$ Other analyses of serpentine are given in the section on igneous and crystalline rocks. See pp. 18, 19, $146,161,162,169,172$, 


\begin{tabular}{|c|c|c|c|c|c|}
\hline . & I: & J. & K. & L: & M. \\
\hline 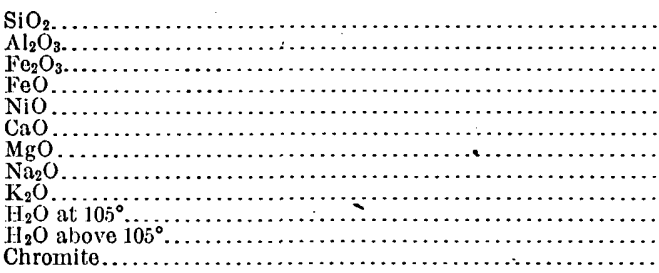 & $\begin{array}{r}43.71 \\
3.59 \\
.90 \\
\ldots . . \\
\ldots . . . \\
\ldots . . . \\
38.58 \\
.13 \\
2.22 \\
.46 \\
10.79\end{array}$ & \begin{tabular}{r}
41.90 \\
.71 \\
.91 \\
undet. \\
.10 \\
$\ldots . .16$ \\
40.16 \\
\hdashline$\ldots$. \\
2.26 \\
13.90
\end{tabular} & \begin{tabular}{r}
42.94 \\
5.05 \\
1.88 \\
.61 \\
$\ldots 36.53$ \\
$\ldots \ldots \ldots$ \\
\hdashline 1.53 \\
11.68
\end{tabular} & $\begin{array}{r}42.55 \\
1.25 \\
1.56 \\
1.52 \\
\text { none } \\
40.05 \\
\ldots \ldots \\
.21 \\
12.26 \\
.37\end{array}$ & $\begin{array}{r}39.14 \\
2.08 \\
4.27 \\
2.04 \\
\text { trace } \\
39.84 \\
\ldots \\
12.70 \\
.11\end{array}$ \\
\hline & 100.38 & 99.94 & 100.22 & 99.77 & 100.18 \\
\hline
\end{tabular}

GENTHITE.

From Riddles, Douglas County, Oregon. Analyzed by I. W. Clarke, and described by Diller and Clarke in Bull. 60.

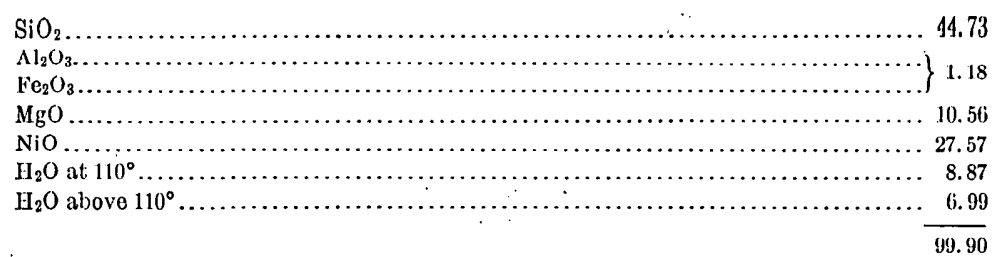

TALC.

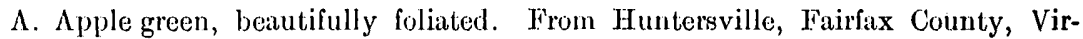
ginia. Analysis by E. A. Schreider.

B. From Deep River, North Carolina. Analysis by H. C. McNeil.

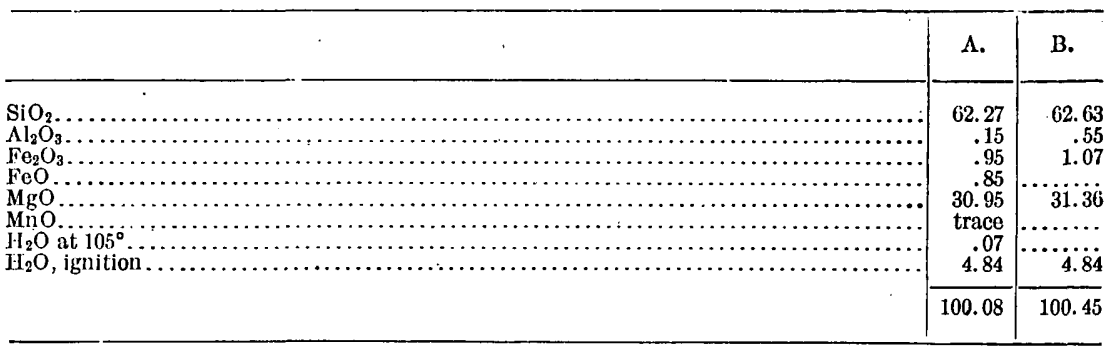

\section{MEERSCHAUM.}

Three magnesian silicates of uncertain character.

A. From Arroyo de San Jose de Gracia, Lower California. Received from W. F. Ferrier. Analysis by W. T. Schaller.

B. From Alunogen district, Grant County, New Mexico. Received from A. R. Ledoux. Analysis by Schaller.

C. From the Dorsey mine, 12 miles northwest of Silver City, New Mexico. Analysis by $\mathrm{G}$. Sțeiger.

$A$ is near saponite. C may be sepiolite. 


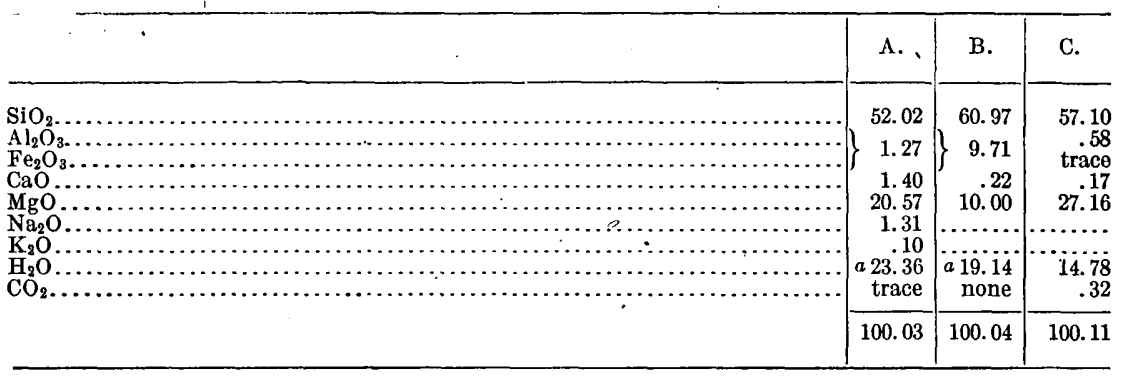

a Loss on ignition.

\section{GLAUCONITE.}

From Big Goose Canyon, 15 miles southwest of Sheridan, Bighorn Mountans, Wyoming. Analysis by George Steiger. Sp. gr., 2.73.

\begin{tabular}{|c|c|}
\hline $\mathrm{SiO}_{2} \ldots \ldots \ldots \ldots \ldots \ldots \ldots \ldots \ldots \ldots \ldots \ldots \ldots \ldots \ldots \ldots \ldots$ & $\mathrm{Na}_{2} \mathrm{O} \ldots \ldots \ldots \ldots \ldots$ \\
\hline 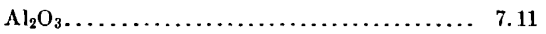 & $\mathrm{K}_{2} \mathrm{O} \ldots \ldots \ldots$ \\
\hline 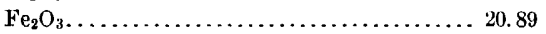 & $\mathrm{H}_{2} \mathrm{O}$ at $100^{\circ} \ldots \ldots$ \\
\hline 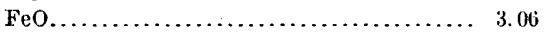 & $\mathrm{H}_{2} \mathrm{O}$ above $100^{\circ}$. \\
\hline 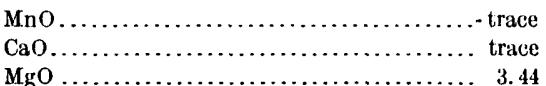 & 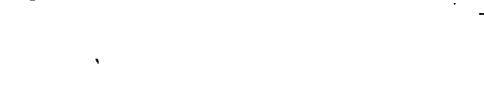 \\
\hline
\end{tabular}

KAOLINITE.

A. From Hockessen, Delaware. Analysis by George Steiger.

B. From Aiken, South Carolina. Analysis by F. W. Clarke.

C. From Aiken. Analysis by Steiger.

D. From Talladega, Alabama. Analysis by Charles Catlett.

E. From the Eureka vein, St. Peters Dome, near Pikes Peak, Colorado. Analysis by W. F. Hillebrand. Described by Cross and Hillebrand in Bull. 20.

F. From New Discovery mine, Lead ville, Colorado. Analysis by W. F. Hillebrand.

G. From National Belle mine, Ouray County, Colorado. Analysis by W. F. Hillebrand. Sp. gr., 2.611 at 18.5 . Described in Bull. 20.

\begin{tabular}{|c|c|c|c|c|c|c|c|}
\hline & A. & B. & c. & D. & E. & F. & G. \\
\hline 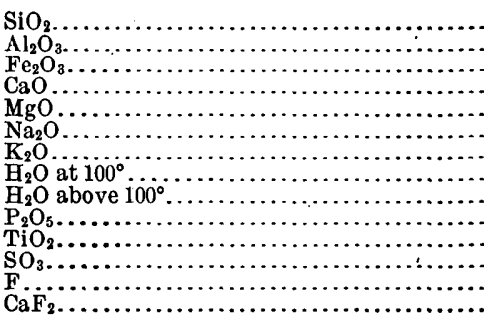 & $\begin{array}{r}48.73 \\
37.02 \\
.79 \\
.10 \\
.11 \\
.04 \\
.41 \\
.52 \\
12.83 \\
.03 \\
.17 \\
\ldots \ldots . .\end{array}$ & $\begin{array}{r}45.56 \\
40.25 \\
\cdots \cdots \cdots \\
\cdots \cdots \cdots \\
\cdots \cdots \cdots \\
\cdots \cdots \cdots \\
14.10 \\
\cdots \cdots \cdots \\
\cdots \cdots \cdots \\
\cdots\end{array}$ & 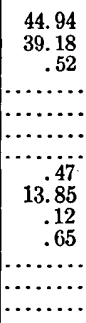 & 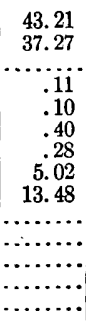 & 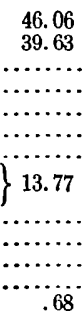 & 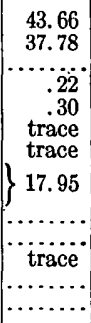 & 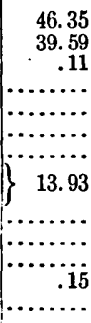 \\
\hline \multirow[t]{2}{*}{$\mathrm{O}$ equivalent to $\mathrm{F} \ldots \ldots \ldots \ldots \ldots \ldots \ldots \ldots \ldots \ldots \ldots \ldots \ldots$} & $\begin{array}{c}100.81 \\
\cdots \cdots \cdots \cdots\end{array}$ & $\begin{array}{r}99.91 \\
\cdots \cdots \cdots \\
\end{array}$ & $\begin{array}{r}99.73 \\
\cdots \cdots \cdots\end{array}$ & $\begin{array}{r}99.87 \\
\cdots \cdots \cdots \\
\end{array}$ & $\begin{array}{c}100.14 \\
\cdots \cdots \\
\ldots \ldots\end{array}$ & $\begin{array}{r}99.91 \\
\cdots \cdots \cdots \\
\end{array}$ & $\begin{array}{r}100.13 \\
.06 \\
\end{array}$ \\
\hline & & & & & & & 100.07 \\
\hline
\end{tabular}

HALLOYSITE.

A. From Horse Cave, Kentucky. Analysis by H. C. McNeil.

B. From Edwards County, Texas. Analysis by McNeil.

C. From the Logan mine, Rico, Colorado. Analysis by W. F. Hillebrand. 
D. From the Lucia mining district, Elko County, Nevada. Pale greenish. Analysis by G. Steiger.

E. From the Detroit copper mine, near Mono Lake, California. Analyzed by F. W. Clarke, and described in Bull. 9.

F. From Pala, San Diego County, California. Pink. Analysis by W. T. Schaller.

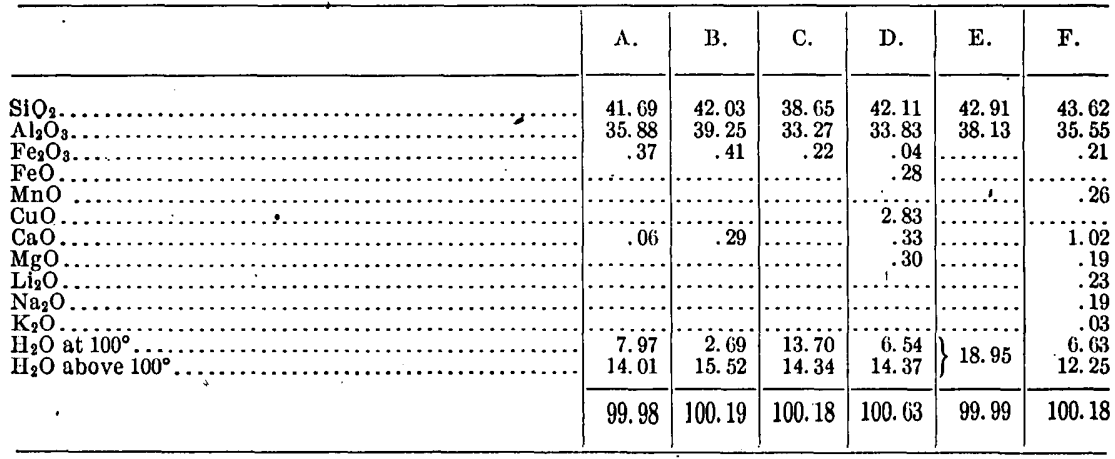

\section{CIMOLITE.}

Rose-red, earthy variety, from Norway, Maine. Analysis by R. B. Riggs. Described by Clarke in Bull. 42.

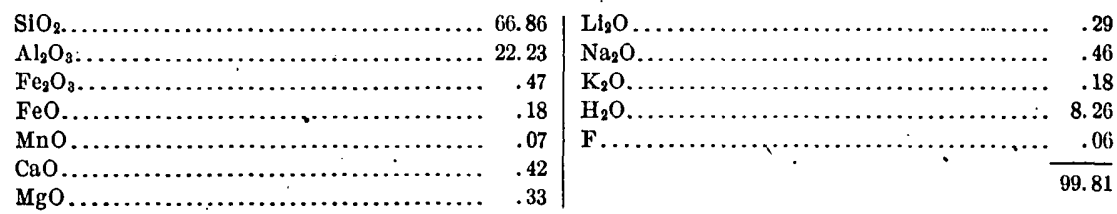

\section{PYROPHYLLITE.}

A. Compact, white. From Deep River, North Carolina. Analysis by George Steiger.

B. Radiated. From East Tres Cerritos, California. Analysis by H. C. McNeil.

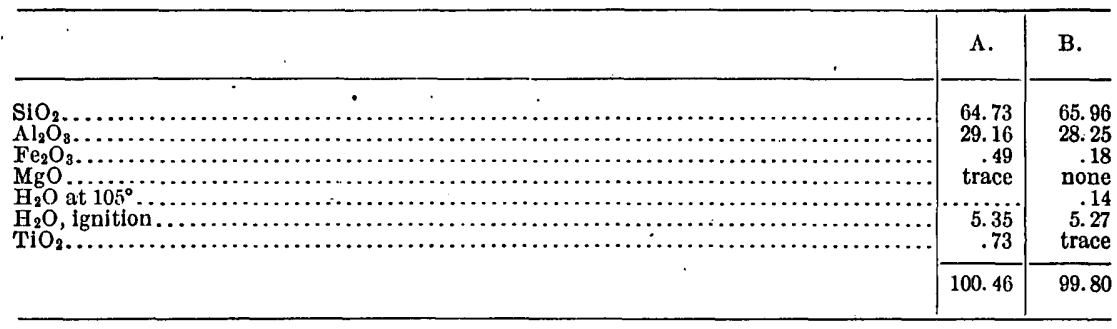

\section{BEMENTITE.}

From the Trotter mine, Franklin Furnace, New Jersey, collected by C. Palache. Analysis by G. Steiger.

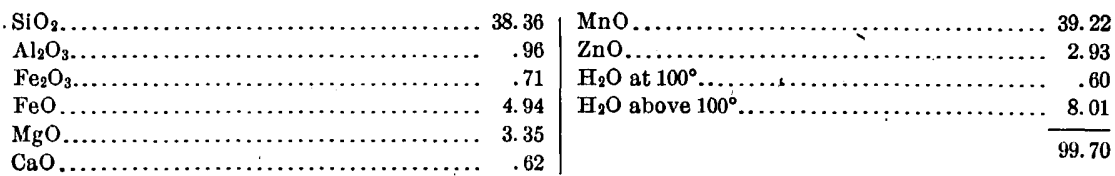




\section{MORENCITE.}

Described as a new mineral from the Arizona Central mine, Morenci, Arizona, by Lindgren and Hillebrand, in Bull. 262, p. 49. Analysis by W. F. Hillebrand.

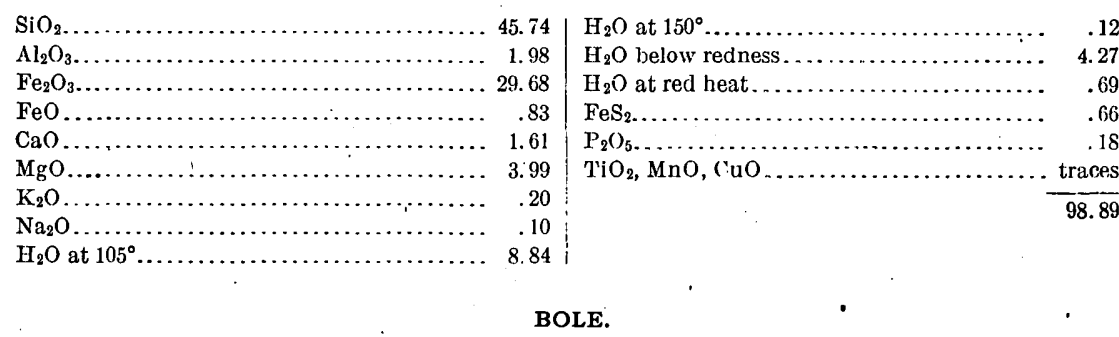

From South Table Mountain, Golden, Colorado. Described by Cross and Hillebrand.in Bull. 20. Analyses by W. F. Hillebrand.

A. Dark brown.

B. Light brown.

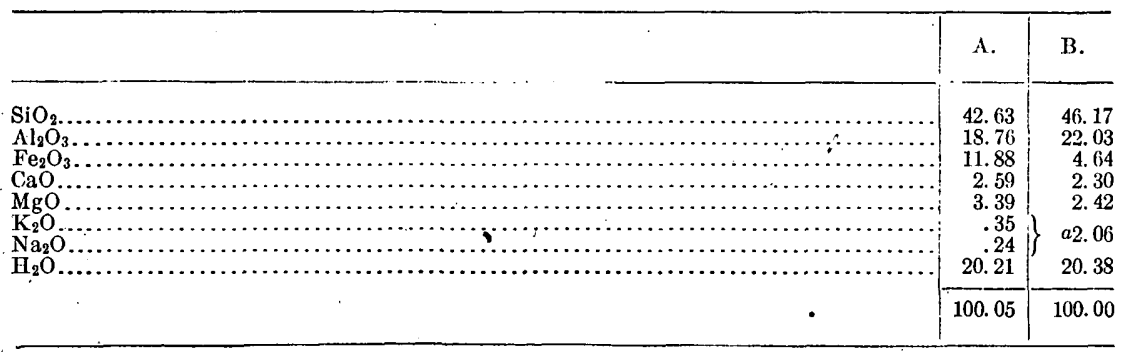

a By difference.

VIII. TITANATES AND TITANO-SILICATES.

\section{ILMENITE.}

From the peridotite dike of Elliott County, Kentucky. Described by Diller in Bull. 38. Analysis by T. M. Chatard.

\begin{tabular}{|c|c|c|}
\hline $\mathrm{TiO}_{2}+\ldots \ldots \ldots$ & 49. 32 & $\mathrm{CaO}$. \\
\hline $\mathrm{SiO}_{2} \ldots \ldots \ldots$ & .76 & 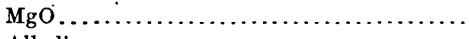 \\
\hline 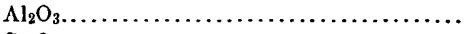 & 2.84 & Alkalies................................ \\
\hline 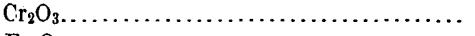 & .74 & 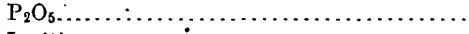 \\
\hline 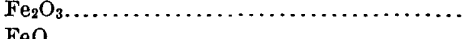 & 9.13 & Ignition.......... \\
\hline $\begin{array}{l}\mathrm{FeO} \\
\mathrm{MnO}\end{array}$ & $\begin{array}{r}27.81 \\
.20\end{array}$ & \\
\hline
\end{tabular}

\section{ARIZONITE.}

New species from '25 miles southeast of Hackberry, Arizona. Analyzed by Chase Palmer, and described by him in Am. Jour. Sci., October, 1909. The following analysis shows the mineral to be a ferric metatitanate.

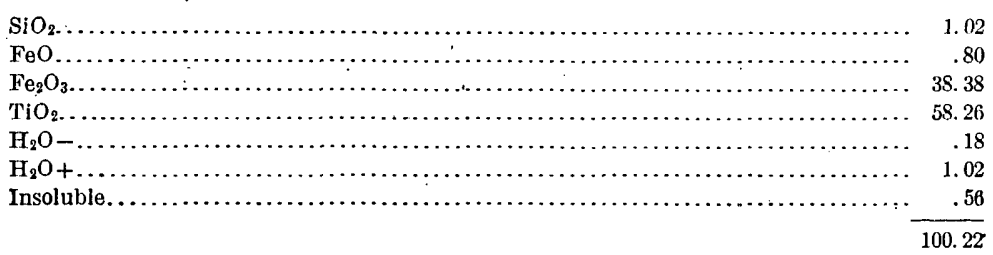




\section{XANTHITANE.}

From 'Green River, Henderson County, North Carolina: Alteration product of titanite. Analyzed by L. G. Eakins, and described in Bull. 60. Sp. gr., 2.941 at $24^{\circ}$. Material dried at $100^{\circ}$.

\begin{tabular}{|c|c|c|c|}
\hline $\mathrm{TiO}_{2}$. & 61.54 & 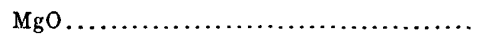 & trace \\
\hline $\mathrm{SiO}_{2}$. & 1.76 & 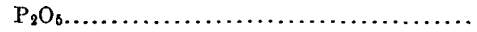 & 4.17 \\
\hline $\mathrm{Al}_{2} \mathrm{O}_{3}$ & 17.59 &,$\Pi_{2} \mathrm{O} \ldots \ldots \ldots \ldots \ldots \ldots \ldots \ldots \ldots \ldots \ldots \ldots \ldots \ldots \ldots \ldots \ldots \ldots \ldots \ldots$ & 9.92 \\
\hline $\begin{array}{l}\mathrm{Fe}_{2} \mathrm{O}_{3} \\
\mathrm{CaO} .\end{array}$ & $\begin{array}{r}4.46 \\
.90\end{array}$ & & 100.34 \\
\hline
\end{tabular}

\section{TITANITE.}

Pale-yellow, semitranslucent. From the waterworks tunnel, Washington, D. C. Occurs embedded in prochlorite. Analysis by F. W. Clarke. Sp.. gr., 3.452.

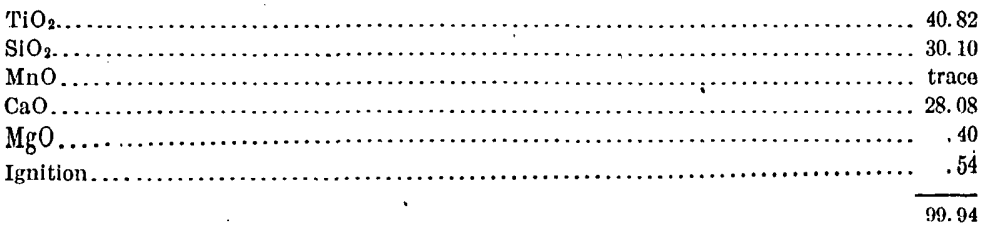

\section{TSCHEFFKINITE.}

From Bedford County, Virginia. Analyzed by L. G. Eakins and described by him in Bull. 90.

A. Lustrous portion. Sp. gr., 4.33 at $27^{\circ}$.

B. Dull portion. Sp. gr., 4.38 at $22^{\circ} .2$.

\begin{tabular}{|c|c|c|}
\hline & A. & B. \\
\hline 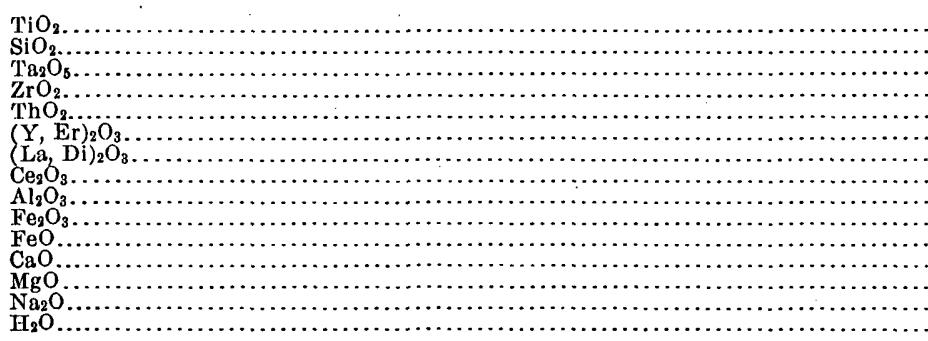 & $\begin{array}{r}18.78 \\
20.21 \\
.08 \\
\text { trace? } \\
.85 \\
1.82 \\
19.72 \\
20.05 \\
3.60 \\
1.88 \\
6.91 \\
4.05 \\
.55 \\
.06 \\
.94\end{array}$ & $\begin{array}{r}18.99 \\
21.49 \\
.08 \\
\text { trace? } \\
.75 \\
1.64 \\
17.16 \\
19.08 \\
3.65 \\
2.89 \\
5.92 \\
5.24 \\
.48 \\
.04 \\
2.06\end{array}$ \\
\hline & 99.50 & 99.47 \\
\hline
\end{tabular}

ASTROPHYLLITE.

From St. Peters Dome, near Pikes Peak, Colorado. Analyzed by I. G: Eakins and described by him in Bull. 90 .

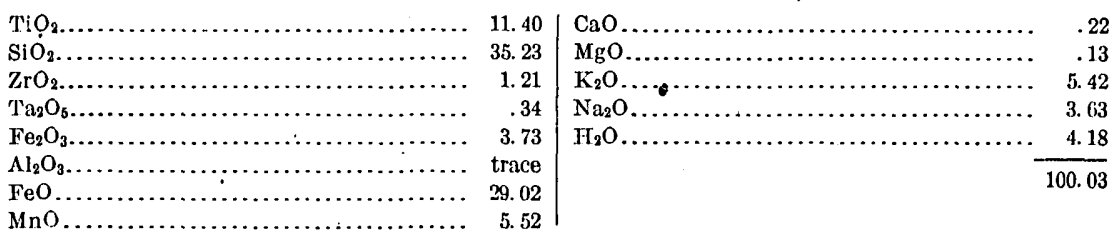


IX. COLUMBO-TANTALATES.

\section{SAMARSKITE.}

Mineral near samarskite, from Devils Head Mountain, near Pikes Peak, Colorado. Analyzed by W. F. Hillebrand and described in Bulletin 55 .

A. Pitch-black variety, streak dirty brown. Sp. gr., 6.18 at $22^{\circ}$.

B. Black, streak salmon colored. Sp. gr., 6.12.at $25^{\circ}$.

C. Altered variety. Sp. gr., 5.45 at $16^{\circ}$.

\begin{tabular}{|c|c|c|c|}
\hline & A. & B. & C. \\
\hline 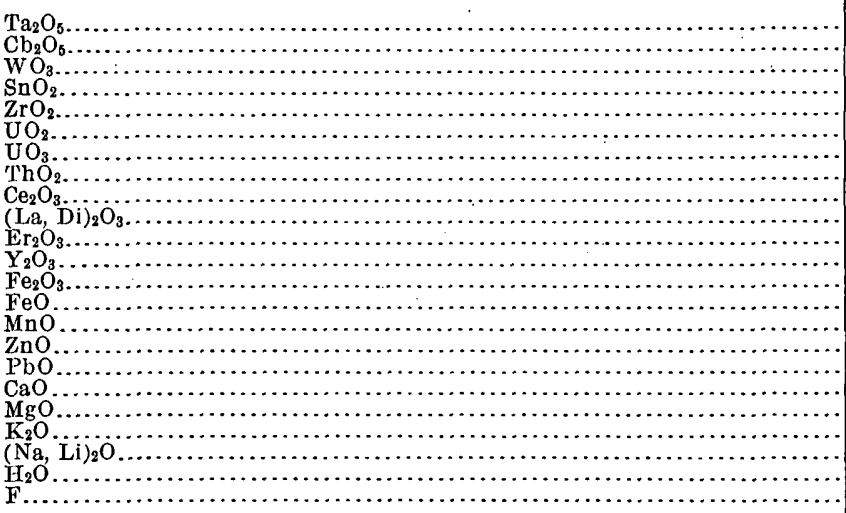 & $\begin{array}{r}27.03 \\
27.77 \\
2.25 \\
.95 \\
2.29 \\
4.02 \\
\ldots . . . \\
3.64 \\
.54 \\
1.80 \\
10.71 \\
6.41 \\
8.77 \\
.32 \\
.78 \\
.05 \\
.72 \\
.27 \\
\ldots . .17 \\
.17 \\
.24 \\
1.58 \\
(?)\end{array}$ & $\begin{array}{r}28.11 \\
26.16 \\
2.08 \\
1.09 \\
2.60 \\
4.22 \\
\ldots . . . \\
3.60 \\
.49 \\
2.12 \\
10.70 \\
5.96 \\
8.72 \\
.35 \\
.75 \\
.07 \\
.80 \\
.33 \\
\ldots . .13 \\
.13 \\
.17 \\
1.30 \\
(?)\end{array}$ & $\begin{array}{r}19.34 \\
27.56 \\
5.51 \\
. .82 \\
a 3.10 \\
\ldots 6.20 \\
3.19 \\
.41 \\
1.44 \\
9.82 \\
5.64 \\
8.90 \\
b .39 \\
.77 \\
1.07 \\
1.61 \\
.11 \\
.36 \\
3.94 \\
(?)\end{array}$ \\
\hline & 100.31 & 99.75 & 100.18 \\
\hline
\end{tabular}

a With $\mathrm{TiO}_{2}$.

$b$ Or $0.74 \mathrm{UO}_{2}$.

X. BORATES.

COLEMANITE.

A. Transparent crystal, ordinary type, Death Valley, California.

B, C. Bladelike crystals, Death Valley.

D. Priceite, Curry County, Oregon.

E. Pandermite, Island of Panderma, Black Sea.

Analyses by J. E. Whitfield, with details in Bull. 55 .

\begin{tabular}{|c|c|c|c|c|c|}
\hline . & A. & B. & C. & D. & E. \\
\hline \multirow{3}{*}{ 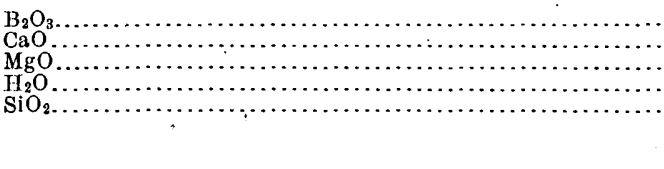 } & $\begin{array}{l}50.70 \\
27.31\end{array}$ & $\begin{array}{l}\text { 49. } 56 \\
\text { 27. } 36\end{array}$ & $\begin{array}{l}49.62 \\
27.40\end{array}$ & $\begin{array}{l}48.44 \\
32.15\end{array}$ & $\begin{array}{l}48.63 \\
32.16\end{array}$ \\
\hline & $\begin{array}{r}21.87 \\
\cdots \cdots\end{array}$ & $\begin{array}{r}22.66 \\
.44\end{array}$ & $\begin{array}{r}22.70 \\
.47\end{array}$ & $\begin{array}{c}19.42 \\
\ldots \ldots\end{array}$ & 19.40 \\
\hline & 99.98 & 100.27 & 100.45 & 100.01 & 100. 19 \\
\hline
\end{tabular}


ULEXITE.

From Rhodes Marsh, Esmeralda County, Nevada. Analysis by.J. E. Whitfield, with description in Bull. 55 .

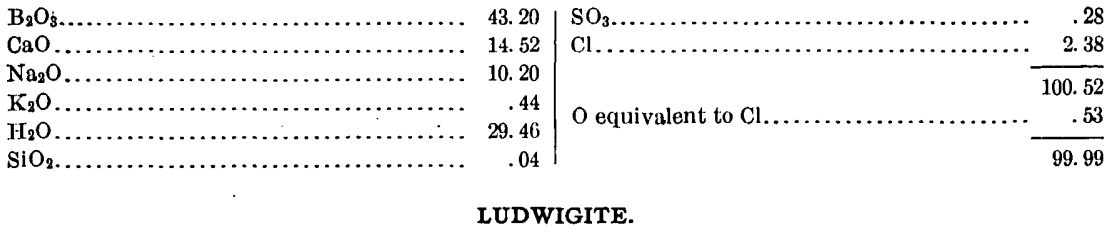

A. From Morawitza, Banat, Hungary. Analysis by J. E. Whitfield, with description in Bull. 55.

B. Same locality as A. Analysis by W. T. Schaller.

C. From Phillipsburg, Montana. Contents some admixed olivine. Analysis by Schaller.

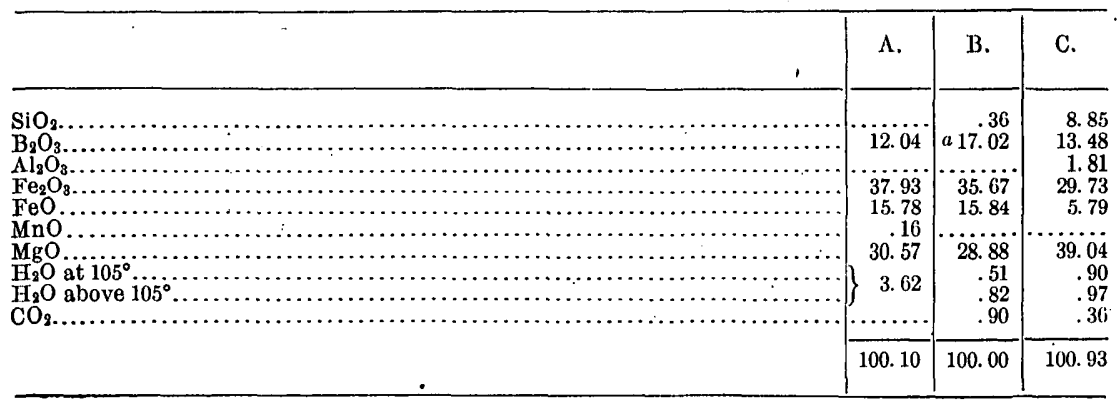

$a$ By difference.

WARWICKITE.

From Edenville, Orange County, New York. Somewhat contaminated by spinel, from which the warwickite could not be entirely freed. Analysis by J. E. Whitfield. See Bull. 64.

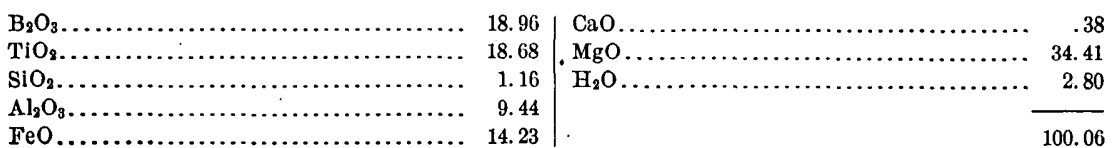

\section{NITRATES.}

\section{SODA NITER.}

From the Leucite Hills, Wyoming. 'Analysis by L. G Eakins. The $\mathrm{N}_{2} \mathrm{O}_{5}$ was not determined directly, but calculated to satisfy $\mathrm{Na}_{2} \mathrm{O}+\mathrm{K}_{2} \mathrm{O}$.

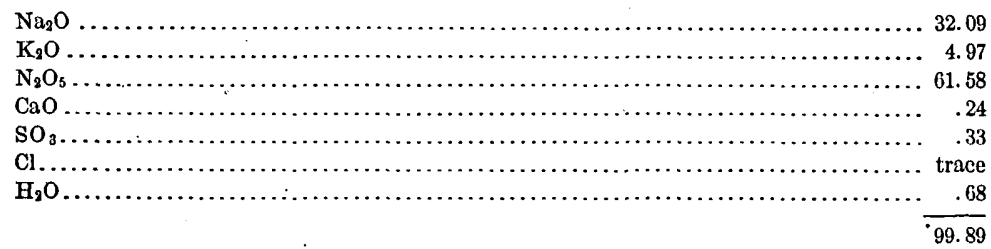




\section{NITER.}

A. From Utah, exact locality unknown. Analysis by T. M. Chatard.

B. From the Leucite Hills, Wyoming. Analysis by L. G. Eakins, with the $\mathrm{N}_{2} \mathrm{O}_{5}$ calculated to satisfy the alkalies.

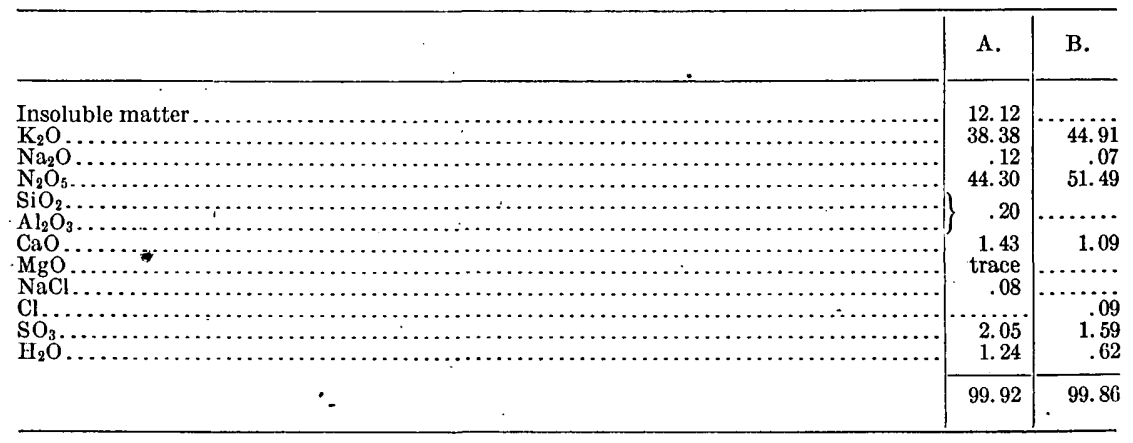

\section{PHOSPHATES.}

\section{XENOTIME.}

From the gold washings at Brindletown, North Carolina. Analyses by I. G. Eakins.

A. Green; sp. gr., 4.68 at $24.2^{\circ}$.

B. Brown; sp. gr!, 4.46 at $24.4^{\circ}$.

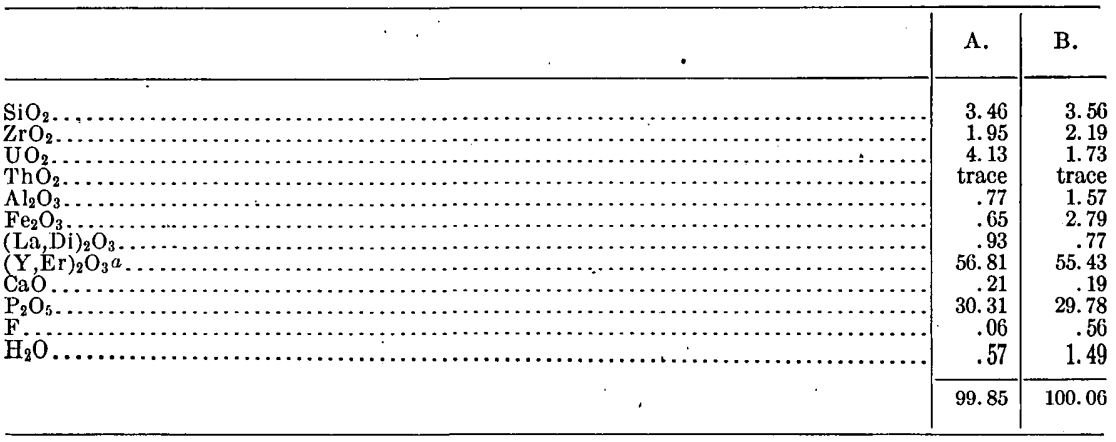

a Molecular weight, 260.

APATITE.

Dark-green, massive apatite from the topaz locality at Stoneham, Maine. Analysis by J. E. Whitfield. See Bull. 27. Sp. gr., 3.27.

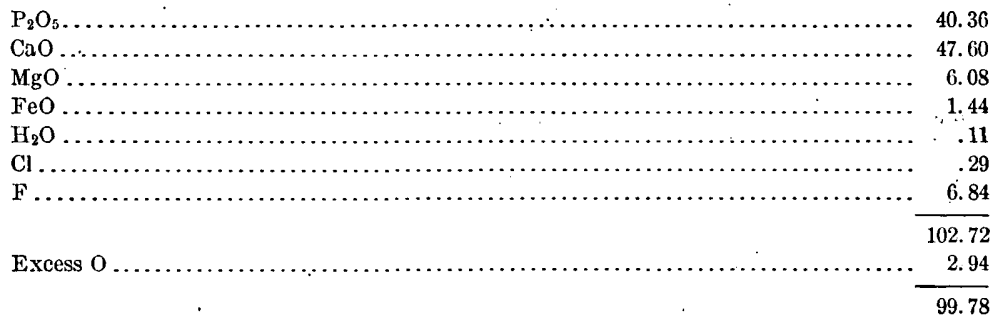


TRIPLITE.

From a tin mine near Rapid City, South Dakota. Probably identical with Headden's "griphite." Analysis by L. G. Eakins, with description in Bull. 60.

\begin{tabular}{|c|c|c|c|}
\hline${ }_{2} \mathrm{O}_{5}$ & 39.68 & $\mathrm{~K}_{2} \mathrm{O}$. & trace \\
\hline $\mathrm{SiO}_{2} \ldots \ldots \ldots$ & .43 & $\mathrm{H}_{2} \mathrm{O}$. & .3 .67 \\
\hline 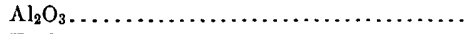 & 8.74 & 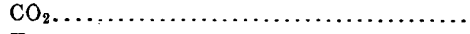 & .20 \\
\hline 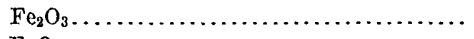 & 2.36 & 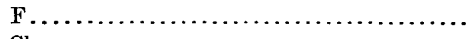 & 2.35 \\
\hline 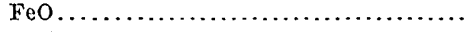 & 1.97 & 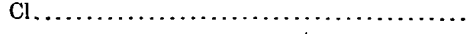 & .25 \\
\hline MnO.. & 29.13 & & \\
\hline 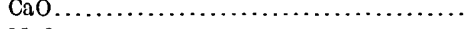 & 6.72 & & 100.94 \\
\hline 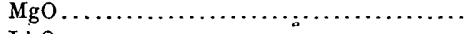 & trace & Excess $0 \ldots$ & 1.05 \\
\hline 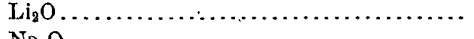 & .13 & & \\
\hline 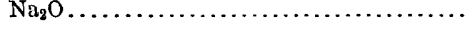 & 5.25 & & \\
\hline
\end{tabular}

\section{AMBLYGONITE.}

From Pala, San Diego County, California. Analysis by W. T. Schaller

\begin{tabular}{|c|c|c|c|}
\hline $\mathrm{P}_{2} \mathrm{O}_{5}$. & 48.83 & $\mathrm{H}_{2} \mathrm{O} \ldots \ldots \ldots \ldots \ldots \ldots \ldots \ldots \ldots \ldots$ & 5.95 \\
\hline $\mathrm{Al}_{2} \mathrm{O}_{3}$ & 33.70 & F................ & 2.29 \\
\hline $\mathrm{Fe}_{2} \mathrm{O}_{3}$ & .12 & - & \\
\hline $\mathrm{MnO}$. & .09 & & 101.31 \\
\hline $\mathrm{MgO}$. & .31 & Less $0 \ldots \ldots$ & .96 \\
\hline $\mathrm{Li}_{2} \mathrm{O}$. & 9.88 & & \\
\hline $\mathrm{Na}_{2} \mathrm{O}$ & .14 & & 100.45 \\
\hline
\end{tabular}

\section{DUFRENITE (?).}

Two samples of a fibrous phosphate from Grafton, New Hampshire. Near dufrenite, but uncertain. Analyses by W. T. Schaller.

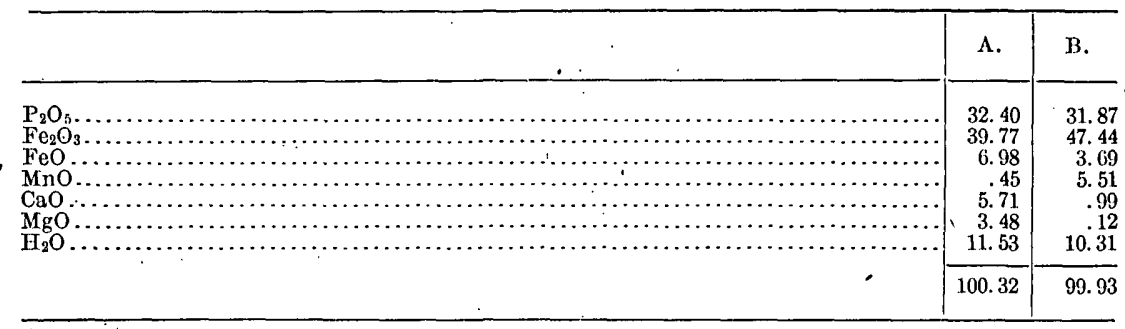

PURPURITE.

Described as a new species by Graton and Schaller. Analyses by W. T. Schaller.

A. From Faries mine, Kings Mountain, North Carolina.

B. From Hill City, South Dakota.

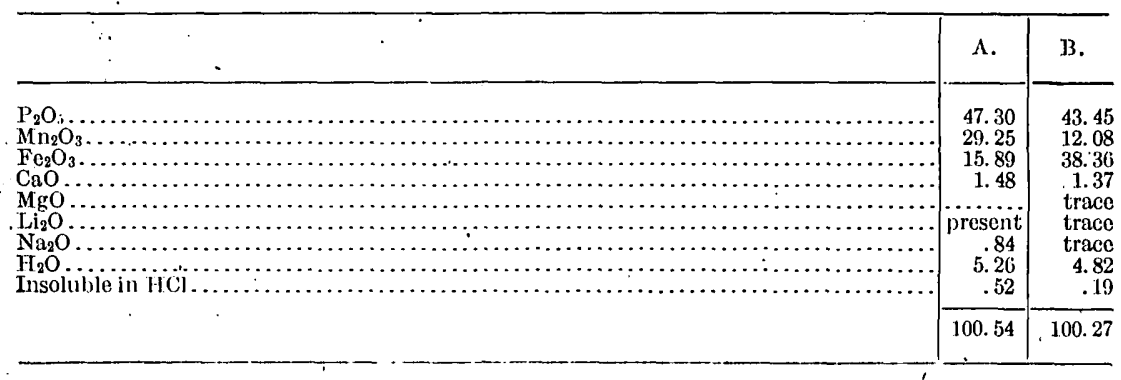


EVANSITE.

A. From near Goldburg, Idaho.

B. From Columbiana, Alabama. Analyses by W. T. Schaller.

\begin{tabular}{|c|c|c|}
\hline$\cdot$ & A. & B. \\
\hline $\begin{array}{r}\mathrm{P}_{3} \mathrm{O}_{5} \\
\mathrm{Al}_{2} \mathrm{O}_{3} \\
\mathrm{Fe}_{2} \mathrm{O}_{3} \\
\mathrm{Ca} \mathrm{O}_{2}\end{array}$ & $\begin{array}{r}19.14 \\
34.48 \\
5.49 \\
4.32 \\
\text { trace } \\
36.96\end{array}$ & $\begin{array}{r}a 21.70 \\
38.33 \\
1.03 \\
.75 \\
38.19\end{array}$ \\
\hline & 100.39 & 100.00 \\
\hline
\end{tabular}

$a$ By difference. Determination lost.

\section{TURQUOISE.}

From Los Cerrillos, New Mexico. Described by Clarke and Diller in Bull. 42. Analyses by F. W. Clarke.

A. Bright blue, faintly translucent in thin splinters.

B. Pale blue, with a slight greenish cast. Opaque and earthy in texture. Sp. gr.; 2.805 .

C. Dark green, opaque.

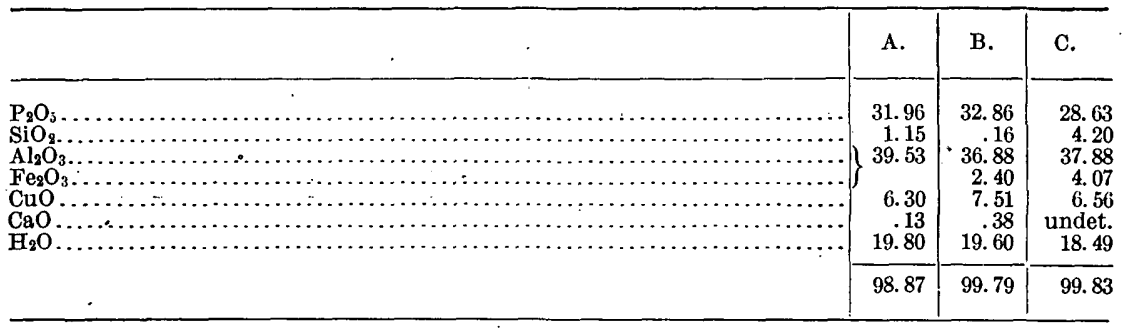

\section{VANADATES.}

\section{DESCLOIZITE.}

A. From the Mayflower mine, Bald Mountain mining district, Beaverhead County, Montana. Yellow, friable, not crystallized.

B. From the Commercial mine, Georgetown, New Mexico. Brilliantly crystallized.

C. Cuprodescloizite from the Lucky Cuss mine, Tombstone, Arizona. Sp. gr., 5.88 at $19^{\circ}$.

Analyses by W. F. Hillebrand, with description in Bull. 64 .

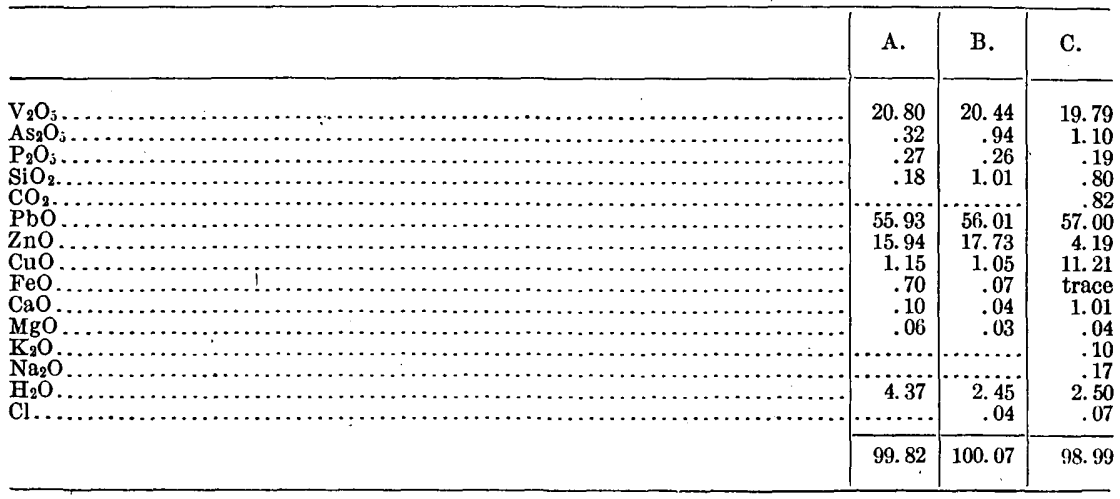




\section{CARNOTITE.}

From Montrose County, Colorado. See memoir by Hillebrand and Ransome. Am. Jour. Sci., 4th ser., vol. 10, p. 120.

A, B, C. From Copper Prince claim, Roc Creek.

D, E. From Yellow Boy claim, La Sal Creek.

Analyses by W. F. Hillebrand.

\begin{tabular}{|c|c|c|c|c|c|}
\hline & A. & B. & C. & D. & E. \\
\hline 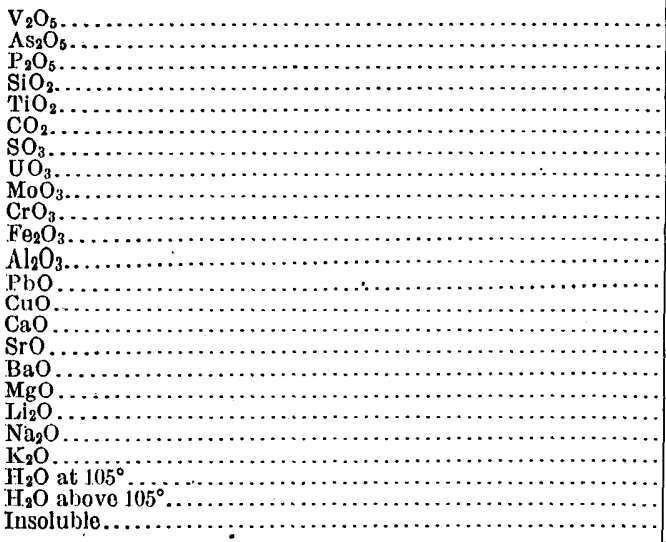 & $\begin{array}{r}18.35 \\
.25 \\
.33 \\
\ldots .10 \\
.33 \\
.12 \\
52.25 \\
.23 \\
\text { trace } \\
1.77 \\
1.08 \\
.25 \\
.20 \\
2.85 \\
\ldots .72 \\
.20 \\
\text { none } \\
.09 \\
6.73 \\
2.59 \\
3.06 \\
8.34\end{array}$ & $\begin{array}{r}18.49 \\
\text { trace } \\
.80 \\
.15 \\
.03 \\
.56 \\
\ldots 7 . \\
54.89 \\
.18 \\
\ldots .21 \\
.21 \\
.09 \\
.13 \\
.15 \\
3.34 \\
.02 \\
.90 \\
.22 \\
\text { trace } \\
.14 \\
6.52 \\
2.43 \\
2.11 \\
7.10\end{array}$ & $\begin{array}{r}15.76 \\
\text { none } \\
.40 \\
.13 \\
(?) \\
\text { none } \\
.18 \\
47.42 \\
.18 \\
\ldots .72 \\
.72 \\
.08 \\
.18 \\
.22 \\
2.57 \\
(?) \\
.65 \\
.24 \\
(?) \\
.07 \\
6.57 \\
1.85 \\
2.79 \\
19.00\end{array}$ & $\begin{array}{r}3.36 \\
\text { undet. } \\
\text { undet. } \\
\ldots \ldots \ldots . \\
1.85 \\
\ldots 3.21 \\
.17 \\
\text { trace } \\
.07 \\
5.32 \\
4.52 \\
3.87 \\
\ldots \ldots \ldots .\end{array}$ & $\begin{array}{r}18.05 \\
\text { none } \\
.05 \\
.20 \\
(?)^{2} \\
\text { none } \\
\text { none } \\
54.00 \\
.05 \\
\ldots . . \\
.42 \\
.29 \\
.07 \\
\text { trace } \\
1.86 \\
\text { trace } \\
2.83 \\
.14 \\
\text { trace } \\
.13 \\
5.46 \\
3.16 \\
2.21 \\
10.33\end{array}$ \\
\hline - & 99.84 & 98.46 & 99.01 & 97.50 & 99.25 \\
\hline
\end{tabular}

XIV. ARSENATES AND ANTIMONATES.

OLIVENITE.

From the American Eagle mine, Tintic mining district, Utah. Described by Hillebrand in Bull. 20. Analysis by W. F. Hillebrand.

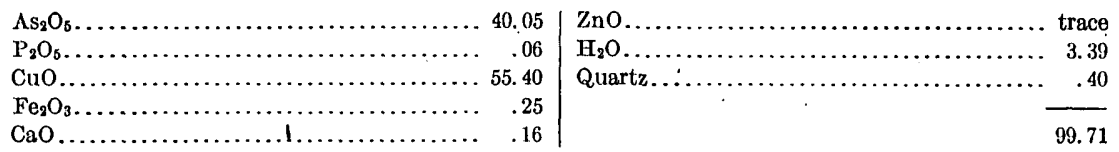

ERINITE.

From the Mammoth mine, Tintic district, Utah. Described by Hillebrand and Washington in Bull. 55. Analyses by W. F. Hillebrand.

\begin{tabular}{|c|c|c|}
\hline & A. & B. \\
\hline 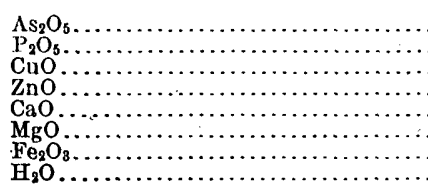 & $\begin{array}{r}33.53 \\
.10 \\
57.67 \\
1.06 \\
.32 \\
\text { trace } \\
.14 \\
7.22\end{array}$ & $\begin{array}{r}31.91 \\
57.51 \\
.59 \\
.51 \\
\text { trace } \\
.20 \\
9.15\end{array}$ \\
\hline . & 100.04 & 99.87 \\
\hline
\end{tabular}




\section{CLINOCLASITE.}

From the Mammoth mine, Tintic district, Utah. Described by Hillebrand and Washington in Bull. 55. Analyses by W. F. Hillebrand. Sp. gr., 4.38 at $19^{\circ}$.

\begin{tabular}{|c|c|c|}
\hline & A. & $B$. \\
\hline 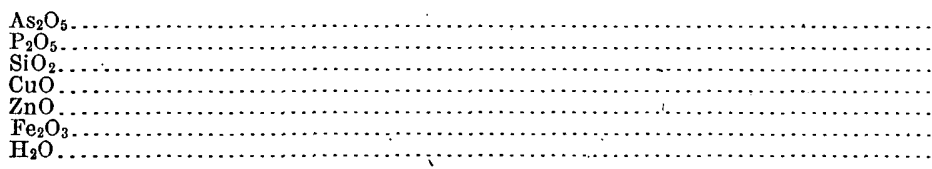 & $\begin{array}{r}29.59 \\
.05 \\
.06 \\
62.34 \\
.06 \\
.12 \\
7.73\end{array}$ & $\begin{array}{r}29.60 \\
\text { a. } 05 \\
6.06 \\
62.54 \\
.04 \\
.12 \\
7.72\end{array}$ \\
\hline - & 99.95 & 100.13 \\
\hline
\end{tabular}

$a_{\Lambda}$ ssumed the same as in $A$.

\section{CONICHALCITE.}

From the American Eagle mine, Tintic district, Utah. Analysis by W. F. Hillebrand, with description in Bull. 20.

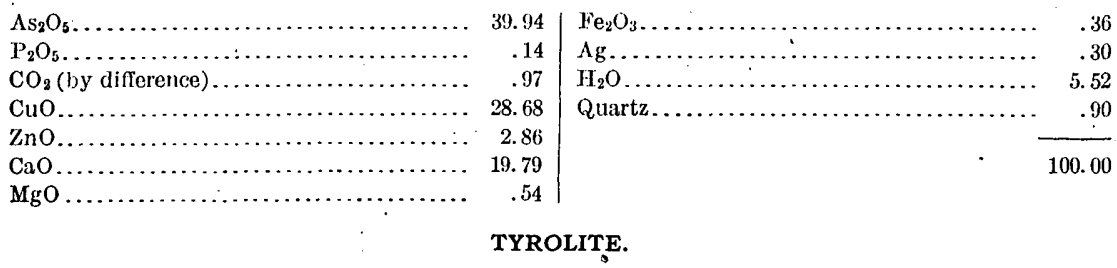

From the Mammoth mine, Tintic district, Utah. Described by Hillebrand and Washington in Bull. 55. Analyses by W. F. Hillebrand. Sp. gr., 3.27 at $20^{\circ} .5$.

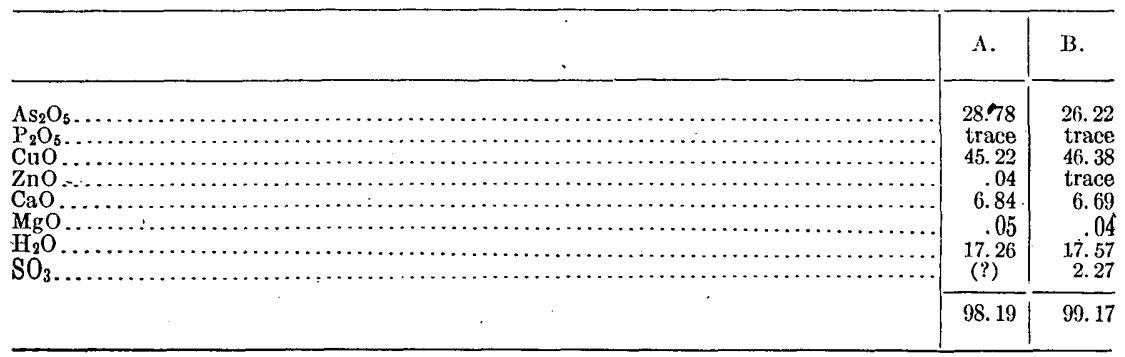

CHENEVIXITE.

From the American Eagle mine, Tintic district, Utah. Analyzed by W. F. Hillebrand and described in Bull. 20.

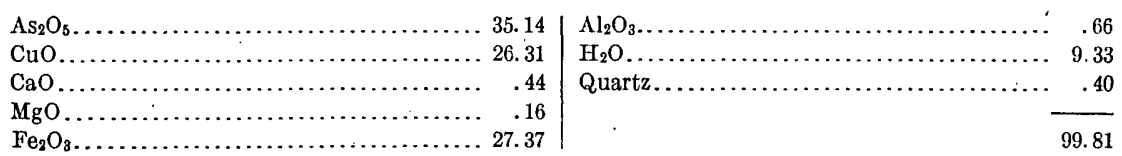

\section{MIXITE.}

From the Mammoth mine, Tintic district, Utah. Described by Hillebrand and Washington in Bull. 55. Analysis by W. F. Hillebrand.

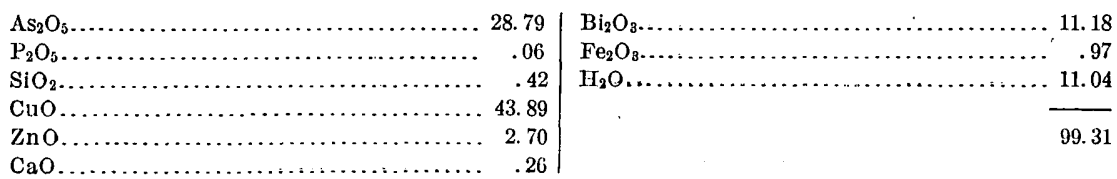


SCORODITE.

An incrustation on hot-spring deposits, from Joseph's Coat Spring, Broad Creek, Yellowstone Park. Analyzed by J. E. Whitfield and described in Bull. 55.

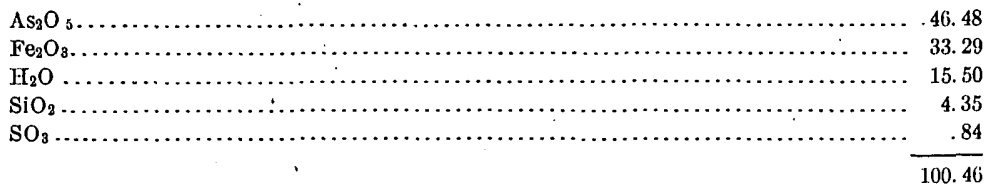

\section{BINDHEIMITE.}

From a claim near the Bertrand mine, Secret Canyon, Nevada. Analyzed by W. F. Hillebrand and described in Bull. 20. Sp. gr., 5.01 at $1.9^{\circ}$, after correction for admixed quartz and cerussite.

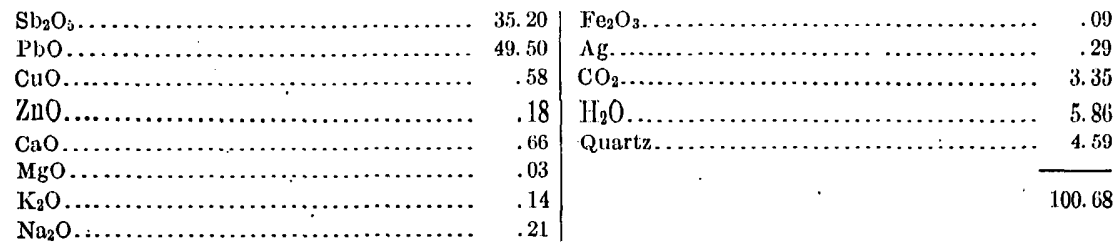

XV. SULPHATES AND TELLURITES.

ANHYDRITE.

From Newhouse, Utah. Approximate analysis by W. T. Schaller.

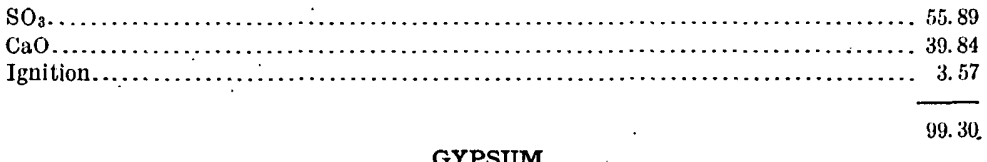

\section{GYPSUM.}

A. From Hillsboro, New Brunswick. Analysis by George Steiger.

B. From the Western Plaster Works, Alabaster, Michigan. Mnalysis by George Steiger.

C. From east of Cascade, Black Hills, South Dakota. Analysis by Steiger.

D. From Rico-Aspen mine, Rico district, Colorado. Analysis by W. F. Fillebrand.

E, F. From Nephi, Utah. Analyses by E. T. Allen. Some anhydrite must be present.

\begin{tabular}{|c|c|c|c|c|c|c|}
\hline & A. & B. & C. & D. & E. & F. \\
\hline 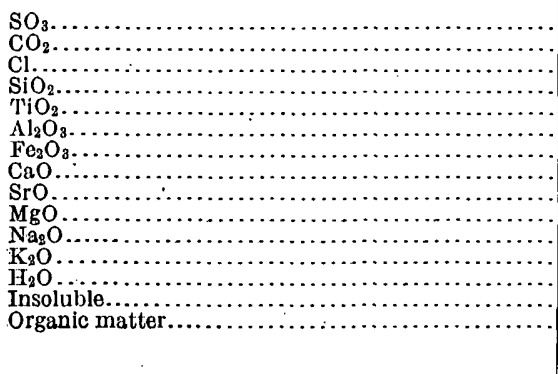 & $\begin{array}{r}46.18 \\
\text { trace } \\
\ldots \ldots \ldots \\
\ldots \ldots \\
.10 \\
32.37 \\
\ldots \ldots \\
\text { trace } \\
.10 \\
20.94 \\
.10 \\
\ldots \ldots \ldots \\
99.79\end{array}$ & $\begin{array}{r}46.18 \\
.03 \\
\ldots \ldots \\
\cdots \cdots \\
.08 \\
32.33 \\
\cdots \\
.05 \\
.14 \\
20.96 \\
.05 \\
\cdots \cdots\end{array}$ & 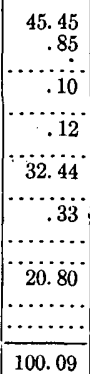 & $\begin{array}{r}45.07 \\
1.54 \\
.51 \\
\text { trace } \\
.03 \\
.09 \\
32.49 \\
.10 \\
.92 \\
\text { trace } \\
19.67 \\
\text { present } \\
100.42\end{array}$ & 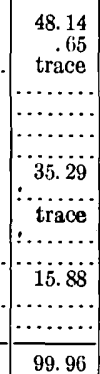 & $\begin{array}{r}39.53 \\
7.73 \\
.04 \\
\ldots \ldots . . \\
\ldots \ldots . . \\
.14 \\
38.46 \\
\ldots . .24 \\
.07 \\
.19 \\
12.69 \\
.45 \\
\ldots \ldots . . \\
99.54\end{array}$ \\
\hline
\end{tabular}


G. Gypsite, Watonga, Oklahoma. Analysis by Chase Palmer.

H. From west point of Sierra Nacimiento, New Mexico. Analysis by W. T. Schaller. I, J. From Lost Hills, San Joaquin Valley, California. Analyses by R. C. Wells.

\begin{tabular}{|c|c|c|c|c|}
\hline • & G. & H. & I. & J. \\
\hline 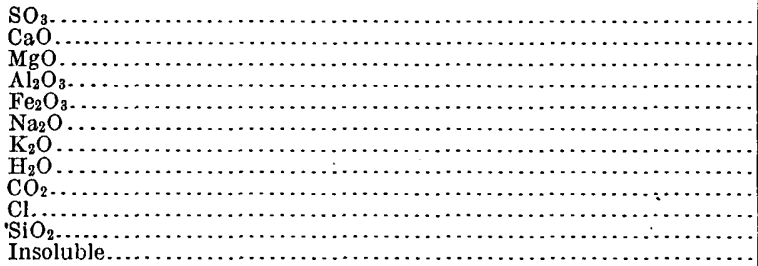 & $\begin{array}{r}40.51 \\
29.54 \\
.23 \\
2.11 \\
.49 \\
\cdots 18.58 \\
.29 \\
\text { present } \\
7.91 \\
\ldots . . .\end{array}$ & 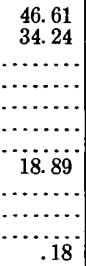 & $\begin{array}{r}40.7 \\
29.5 \\
\text { none } \\
1.7 \\
.4 \\
1.2 \\
.6 \\
19.1 \\
.7 \\
\text { none } \\
5.3\end{array}$ & $\begin{array}{r}40.8 \\
29.9 \\
\text { none } \\
1.4 \\
.3 \\
2.0 \\
.5 \\
19.4 \\
\text { none } \\
\text { none } \\
6.1\end{array}$ \\
\hline & 99.66 & 99.92 & 99.2 & 100.4 \\
\hline
\end{tabular}

BOOTHITE.

From Campo Seco, California. Analysis by W. T. Schaller.

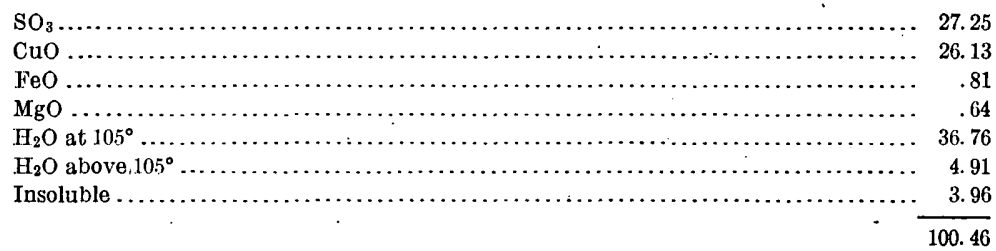

PISANITE.

From Bingham, Utah. Analysis by W. F. Hillebrand.

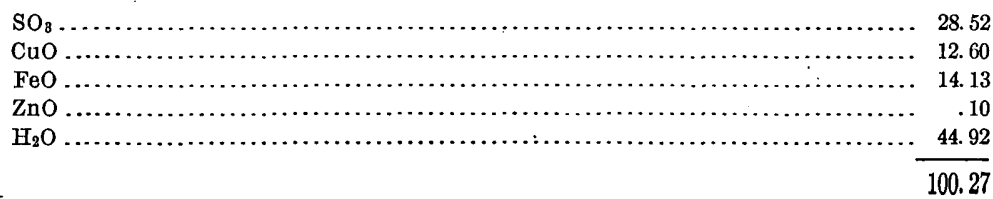

BROCHANTITE.

From the United Verde mine, Jerome, Arizona. Analysis by W. F. Hillebrand.

\begin{tabular}{|c|c|c|c|}
\hline 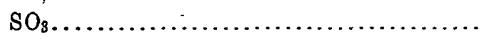 & 16.75 & 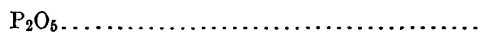 & trace \\
\hline $\mathrm{CuO} \ldots$ & 69.45 & $\mathrm{CO}_{2} \ldots$ & .71 \\
\hline 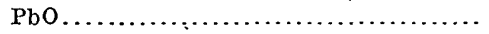 & .04 & Insoluble................................ & .70 \\
\hline $\mathrm{Fe}_{2} \mathrm{O}_{3}$ (from gangue) $\ldots \ldots \ldots \ldots \ldots \ldots \ldots \ldots$ & .28 & . & \\
\hline 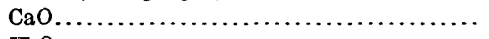 & trace & & 99.98 \\
\hline $\mathrm{H}_{2} \mathrm{O} \ldots \ldots \ldots \ldots \ldots \ldots \ldots \ldots \ldots \ldots \ldots \ldots \ldots \ldots \ldots \ldots \ldots \ldots \ldots \ldots$ & 12.05 & & \\
\hline
\end{tabular}

\section{ANTLERITE.}

From the Antler mine, Yucca Station, Mohave County, Arizona. Described by Hillebrand as a new species in Bull. 55. Sp. gr., 3.93 at $16^{\circ}$, corrected for gangue. Analyses by W. F. Hillebrand.

A, B. First lot received.

C. Later sample. 


\begin{tabular}{|c|c|c|c|}
\hline & A. & B. & C. \\
\hline \multirow[t]{2}{*}{ 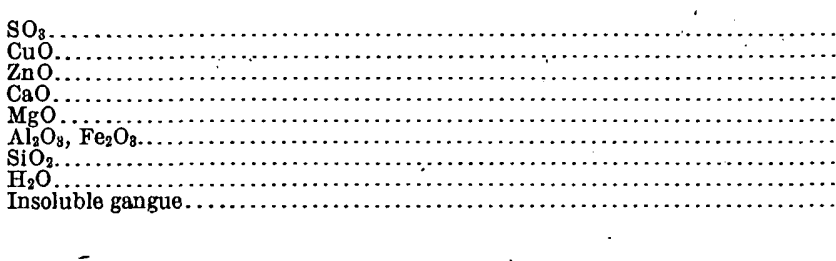 } & $\begin{array}{r}18.78 \\
62.48 \\
.27 \\
.06 \\
.03 \\
.14 \\
.06 \\
10.21 \\
8.01\end{array}$ & $\begin{array}{r}18.48 \\
62.69 \\
.27 \\
.05 \\
.03 \\
.12 \\
\text { undet. } \\
10.18 \\
8.02\end{array}$ & $\begin{array}{r}20.11 \\
63.26 \\
.04 \\
.04 \\
\ldots .07 \\
.15 \\
.05 \\
10.05 \\
6.27\end{array}$ \\
\hline & 100.04 & 99.84 & 99.97 \\
\hline
\end{tabular}

\section{PICRALLUMOGENE.}

From near Las Vegas, New Mexico. Analysis by W: F. Hillebrand.

\begin{tabular}{|c|c|c|}
\hline 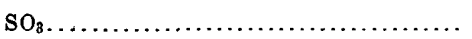 & 37.51 & $\mathrm{Na}_{2} \mathrm{O} \ldots \ldots \ldots \ldots \ldots \ldots \ldots \ldots \ldots \ldots \ldots \ldots \ldots \ldots \ldots \ldots \ldots$ \\
\hline 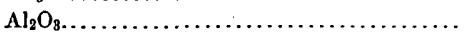 & 9:71 & 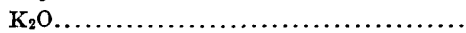 \\
\hline 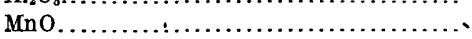 & .06 & 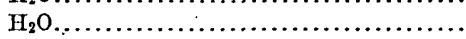 \\
\hline 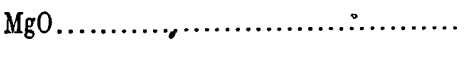 & 6.75 & 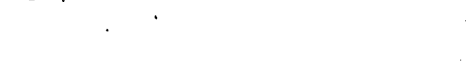 \\
\hline
\end{tabular}

\section{HALOTRICHITE.}

A. From the headwaters of Gila River, Grant County, New Mexico, about 40 miles north of Silver City. Analyzed by F. W. Clarke, and described in Bull. 9.

B. From Alum Creek, New Mexico. Possibly the same locality as A. Analysis by W. T. Schaller.

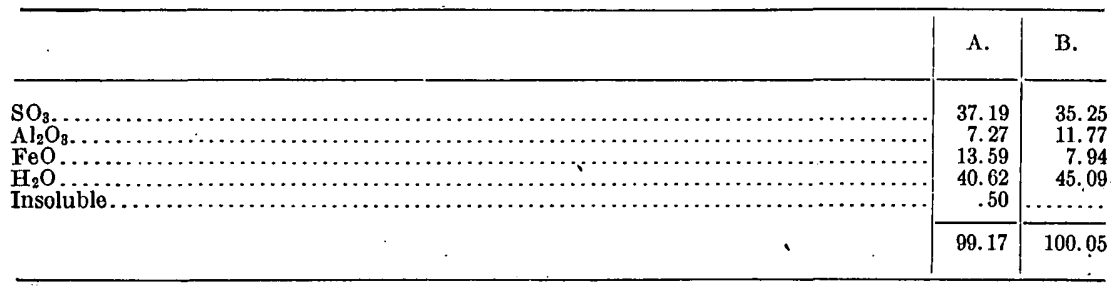

ALUNOGEN.

A. From headwaters of Gila River, Grant County, New Mexico, about 40 miles north of Silver City. Analyzed by F. W. Clarke, and described in Bull. 9. Color, pinkish.

B. From the calcite spring, Yellowstone Park. Fine, white, silky fibers. Analysis by J. E. Whitfield.

C. From the Grand Canyon, Yellowstone Park. Analysis by J. E. Whitfield.

\begin{tabular}{|c|c|c|c|}
\hline & A. & B. & C. \\
\hline 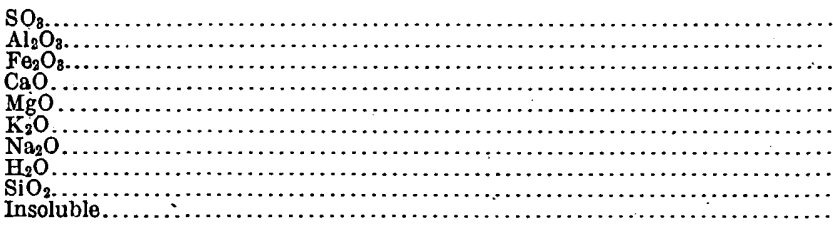 & 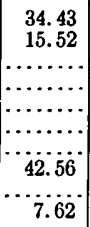 & $\begin{array}{r}40.65 \\
15.72 \\
\text { trace } \\
\text { trace } \\
1.53 \\
\quad .1 \% \\
.09\end{array}$ & $\begin{array}{r}38.22 \\
16.80 \\
\text { trace } \\
\text { none } \\
.18 \\
.01 \\
.63 \\
43.64 \\
1.38\end{array}$ \\
\hline & 100.13 & 99.80 & 100.86 \\
\hline
\end{tabular}

15619-Bull. 419-10-21 


\section{COPIAPITE.}

A. From the Redington mine, Knoxville, California.

B. From Sulphur Bank, Lake County, California.

Analyses by W. H. Melville. Description by Melville and Lindgren in Bull. 61 .

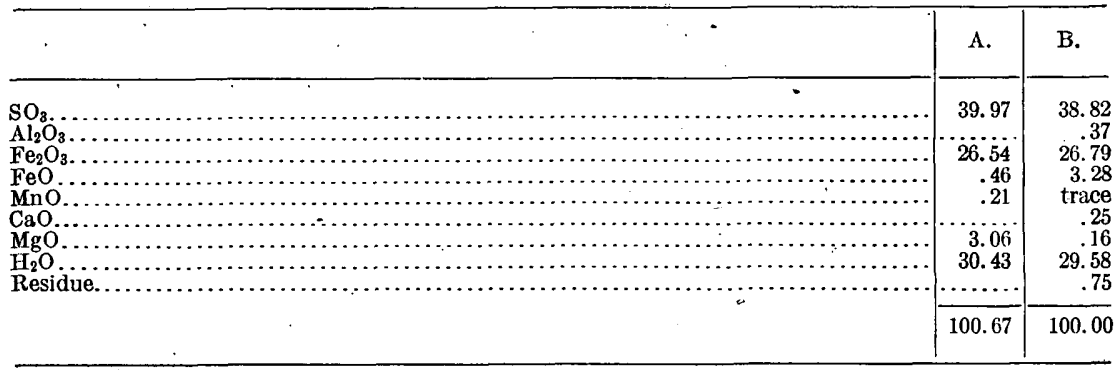

KNOXVILLITE.

From the Redington mine, Knoxville, California. Described as a new species by Melville and Lindgren in Bull. 61. Analysis by W. H. Melville.

\begin{tabular}{|c|c|c|c|}
\hline $\mathrm{SO}_{3}$ & 35.91 & $\mathrm{MgO} . . . \ldots \ldots . .$. & 3.22 \\
\hline $\mathrm{Cr}_{2} \mathrm{O}_{3}$ & 7.41 & $\mathrm{H}_{2} \mathrm{O}$ at $100^{\circ} \ldots \ldots \ldots \ldots \ldots \ldots \ldots \ldots \ldots \ldots \ldots \ldots \ldots$ & 9.30 \\
\hline $\mathrm{Al}_{2} \mathrm{O}_{3}$ & 4. 83 & $\mathrm{H}_{2} \mathrm{O}$ above $100^{\circ} \ldots \ldots \ldots \ldots \ldots \ldots \ldots \ldots \ldots \ldots$ & 17.60 \\
\hline $\mathrm{Fe}_{2} \mathrm{O}_{3}$ & 15.36 & Residue..................... & 1.73 \\
\hline $\begin{array}{l}\mathrm{FeO} . \\
\mathrm{NiO}\end{array}$ & $\begin{array}{r}3.81 \\
83\end{array}$ & & 00.00 \\
\hline
\end{tabular}

\section{REDINGTONITE.}

From the Redington mine, Knoxville, California. Described as a new species by Melville and Lindgren in Bull. 61. Analysis by W. H. Melville. Sp. gr., 1.761.

\begin{tabular}{|c|c|c|c|}
\hline $\mathrm{sO}_{\mathrm{s}} \ldots \ldots \ldots \ldots \ldots \ldots \ldots \ldots \ldots \ldots \ldots \ldots \ldots \ldots \ldots \ldots \ldots$ & 35.35 & 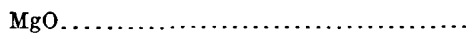 & 1.85 \\
\hline $\mathrm{Cr}_{2} \mathrm{O}_{3} \ldots \ldots \ldots \ldots \ldots$ & 7.51 & $\mathrm{H}_{2} \mathrm{O}$ at $100^{\circ} \ldots .$. & 27.09 \\
\hline $\mathrm{Al}_{2} \mathrm{O}_{3} \ldots \ldots \ldots \ldots$ & 5.14 & $\mathrm{H}_{2} \mathrm{O}$ above $100^{\circ} \ldots \ldots .$. & 14.34 \\
\hline 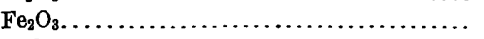 & .19 & Residue.............. & 3.46 \\
\hline $\begin{array}{l}\text { FeO } \\
\text { NiO } \ldots \ldots \ldots, \ldots\end{array}$ & $\begin{array}{l}4.58 \\
1.00\end{array}$ & & 100.51 \\
\hline
\end{tabular}

ALUNITE.

A. Form Knickerbocker Hill, Custer County, Colorado. Analysis by L. G. Eakins. B. From Tres Cerritos Buttes, southwest of Indian Gulch, Mariposa County, California. Analysis by William Valentine.

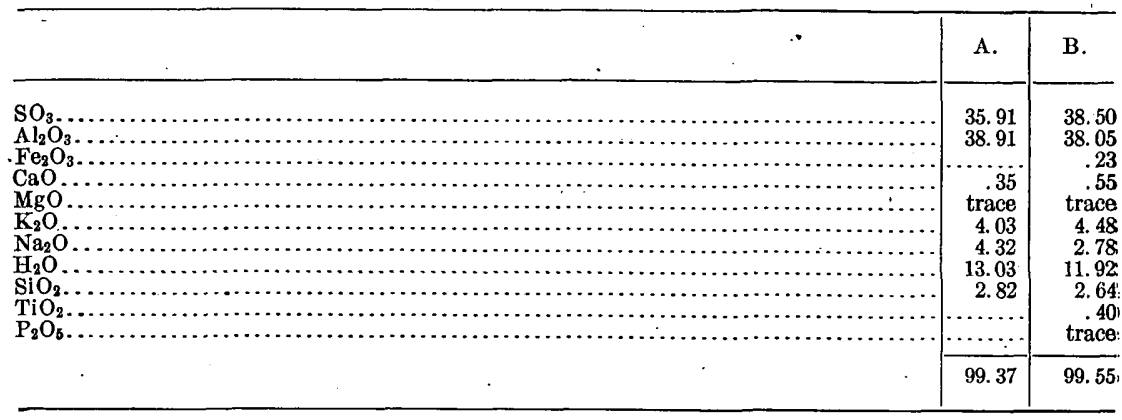


JAROSITE.

A. From Black Iron mine, Eagile County, Colorado.

B. From Pigeon mine, Rico district, Colorado.

Analyses by W. F. Hillebrand.

\begin{tabular}{|c|c|c|c|}
\hline . & $0^{0}$ & A. & B. \\
\hline \multirow{2}{*}{\multicolumn{2}{|c|}{ 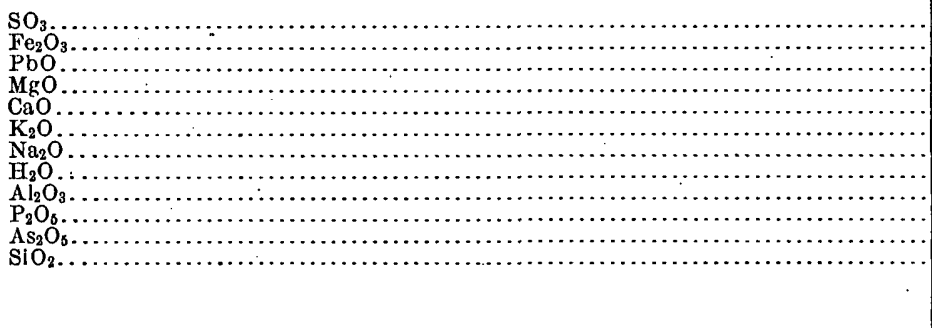 }} & $\begin{array}{r}30.72 \\
46.37 \\
1.80 \\
\text { trace } \\
\times 8.14 \\
.27 \\
11.40 \\
\cdots \\
\cdots \\
. \\
.71\end{array}$ & $\begin{array}{r}28.20 \\
43.81 \\
\ldots . . . \\
\text { trace } \\
.06 \\
7.44 \\
.08 \\
11.64 \\
1.00 \\
.33 \\
\ldots .35\end{array}$ \\
\hline & & 99.93 & 99.91 \\
\hline
\end{tabular}

\section{NATROJAROSITE.}

From Soda Springs Valley, on road from Sodaville to Vulcan copper mine, Nevada. Described by Hillebrand and Penfield in Am. Jour. Sci., 4th ser., vol. 14, p. 211. Analysis by W. F. Hillebrand. Sp. gr., 3.18 at $30.5^{\circ}$.

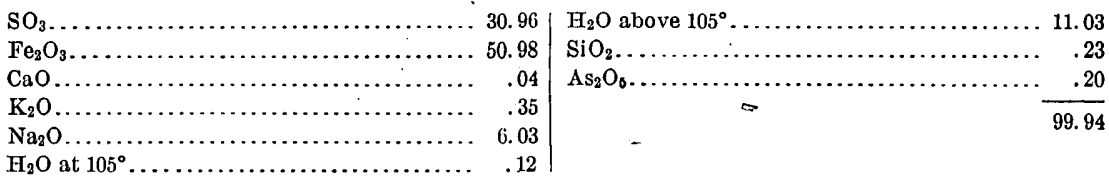

\section{PLUMBOJAROSITE.}

From Cooks Peak, New Mexico. Described as a new species by Hillebrand and Penfield in Am. Jour. Sci., 4th ser., vol. 14, p. 211. Analysis by W. F. Hillebrand. Sp. gr., 3.668 at $30.5^{\circ}$.

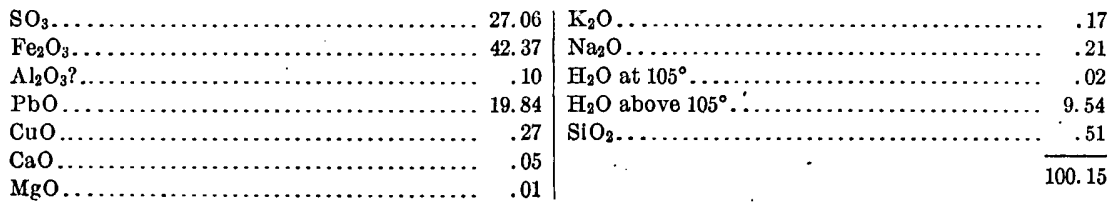

\section{EMMONSITE.}

A hydrous ferric tellurite from near Tombstone, Arizona. Analyzed partially by W. F. Hillebrand, and described by him as a new species in Proc. Colorado Sci. Soc., vol. 2, pt. 1, p. 21. The data relative to the composition of emmonsite are as follows:

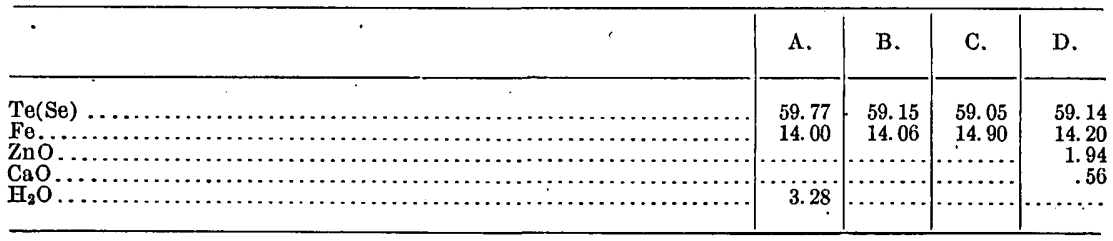


Another mineral, apparently emmonsite, from the W. P. H. mine, Cripple Creek, Colorado, has also been analyzed by Hillebrand. A, B, C are partial analyses; D is the mean of all.

\begin{tabular}{|c|c|c|c|c|}
\hline & A. & B. & C. & D. \\
\hline \multirow[t]{2}{*}{ 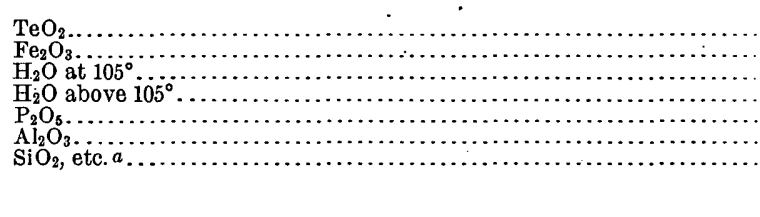 } & $\left\{\begin{array}{r}70.83 \\
22.67 \\
4.68 \\
\cdots \cdots \\
\cdots \cdots\end{array}\right.$ & $\begin{array}{r}71.80 \\
22.81 \\
4.82 \\
.34 \\
.58 \\
\end{array}$ & $\begin{array}{r}70.20 \\
22.79 \\
.21\end{array}$ & $\begin{array}{r}70.71 \\
22.76 \\
.21 \\
4.54 \\
.34 \\
.56 \\
.88\end{array}$ \\
\hline & & & & 100.00 \\
\hline
\end{tabular}

a By difference. Includes alkalies, and traces of magnesia, gold, and metals precipitable by $\mathrm{H}_{2} \mathrm{~S}$.

\section{MOLYBDATES, TUNGSTATES, AND URANATES.}

\section{POWELLITE.}

A. From the Seven Devils mining district, Idaho. Analyzed by W. H. Melville, and described by him as a new mineral species in Bull, 90. Sp. gr., 4.526.

B. From Baringer Hill, Llano County, Texas. Ochreous, coating molybdenite. Sp. gr., 4.15 .

C. From 2 miles south of Oak Springs, Nye County, Nevada. Analyses B, C by W. T. Schaller.

\begin{tabular}{|c|c|c|c|}
\hline & A. & B. & C. \\
\hline 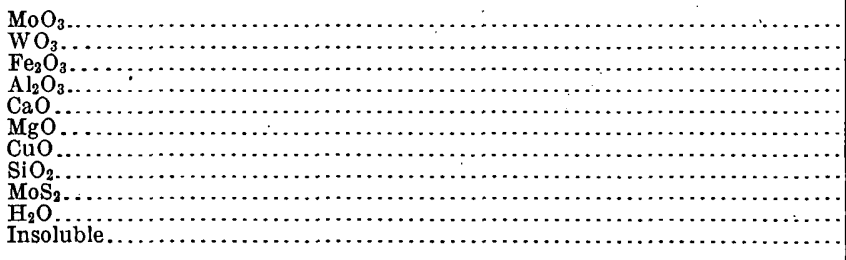 & $\begin{array}{r}58.58 \\
10.28 \\
1.65 \\
\text { trace } \\
25.55 \\
.16 \\
\text { trace } \\
3.25 \\
\cdots \\
\cdots\end{array}$ & \begin{tabular}{|r}
67.90 \\
$\cdots \cdots$ \\
$\cdots \cdots$ \\
$\cdots \cdots$ \\
27.46 \\
$\cdots \cdots$ \\
$\cdots$ \\
$\cdots$ \\
1.50 \\
2.33 \\
.88
\end{tabular} & $\begin{array}{r}62.43 \\
\ldots .17 \\
1.17 \\
26.44 \\
\ldots \ldots . \\
\ldots .80 \\
6.69 \\
\ldots \ldots\end{array}$ \\
\hline & 99.47 & 100.07 & 99.53 \\
\hline
\end{tabular}

$a$ By ignition.

HÜBNERITE.

From the Royal Albert vein, Uncompahgre district, Ouray County, Colorado. Analyzed by W. F. Hillebrand, and described by him in Bull. 20. Sp. gr., 7.177 at $24^{\circ}$.

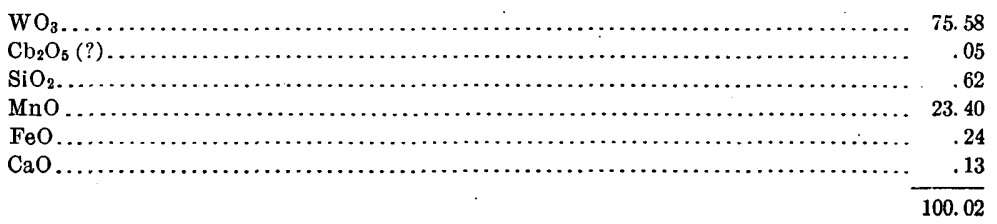




\section{MOLYBDIC OCHER,}

A. From Renfrew, Ontario. I

B. From Westmoreland, New Hampshire.

C. From Telluride, Colorado.

D. From Hortense, Colorado.

E. From California, exact locality unknown. Analyses by W. T. Schaller; A, C, and $\mathrm{E}$ on very little material.

\begin{tabular}{|c|c|c|c|c|c|}
\hline & A. & B. & C. & D. & E. \\
\hline 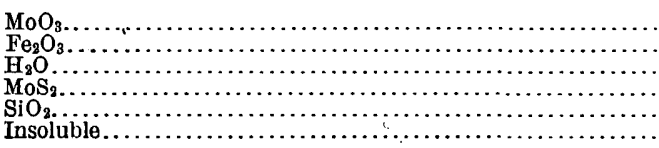 & $\begin{array}{r}55.7 \\
17.3 \\
15.5 \\
\cdots \\
\cdots \\
\cdots .9\end{array}$ & $\begin{array}{r}57.69 \\
21.08 \\
17.62 \\
\cdots \cdots \\
4.66\end{array}$ & $\begin{array}{r}59.3 \\
19.0 \\
15.8 \\
\cdots \\
\cdots \\
\end{array}$ & $\begin{array}{r}46.77 \\
15.95 \\
15.87 \\
5.50 \\
16.51 \\
\cdots\end{array}$ & $\begin{array}{r}47.7 \\
15.8 \\
15.4 \\
\cdots \\
\hdashline .0 .0\end{array}$ \\
\hline & 97.9 & 101.05 & 100.0 & 100.60 & 102.9 \\
\hline
\end{tabular}

$a$ By difference.

\section{URANINITE.}

Analyses by W. F. Hillebrand. Discussed in Bulls. 78 and 90 . In the original publications the gaseous constituent of uraninite was supposed to be nitrogen, as indeed it was in part. The discovery of helium, however, has shed new light upon the subject, and the analyses have been corrected accordingly.

A, B, C, D, E. Crystallized uraninite from Hale's quarry, Glastonbury, Connecticut. Nitrogen was certainly present in these specimens.

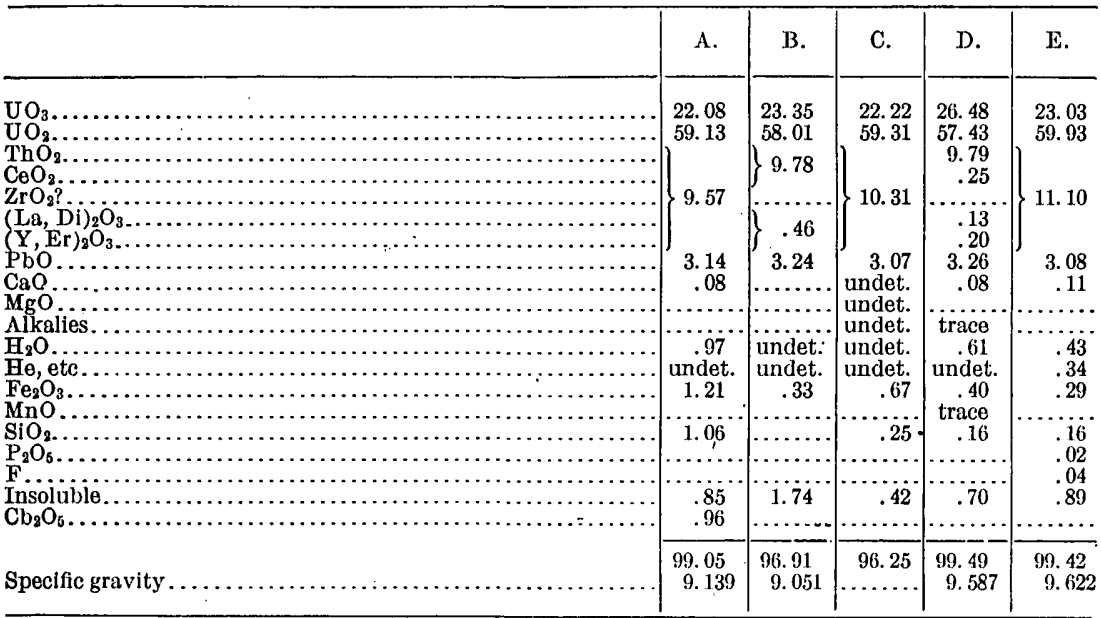


F, G, H. Brilliantly crystallized, from Branchville, Connecticut.

I, J. Altered uraninite, from the Flat Rock mine, Mitchell County, North Carolina.

K. Massive, from near Blackhawk, Colorado.

L. From Marietta, South Carolina:

\begin{tabular}{|c|c|c|c|c|c|c|c|}
\hline & F. & G. & H. & I. & J. & K. & L. \\
\hline $\begin{array}{l}\mathrm{UO}_{3} \ldots \\
\mathrm{UO}_{2} . .\end{array}$ & $\begin{array}{l}13.27 \\
72.25\end{array}$ & $\begin{array}{l}21.54 \\
64.72\end{array}$ & $\begin{array}{l}14.00 \\
70.99\end{array}$ & $\begin{array}{l}50.83 \\
39.31\end{array}$ & $\begin{array}{l}44.11 \\
46.56\end{array}$ & \multirow{2}{*}{$\begin{array}{r}25.26 \\
58.51 \\
\text { trace } \\
7.59\end{array}$} & 83.95 \\
\hline ive & \multirow{2}{*}{7.20} & & & & ……. & & \\
\hline $\mathrm{ThO}_{2} \ldots \ldots$ & & 6.93 & 6.52 & 2.78 & & \multirow[b]{2}{*}{$\begin{array}{c}.22 \\
\ldots .2 \\
\ldots .2\end{array}$} & 1. 65 \\
\hline $\left.\mathrm{CeO}_{2}, \mathrm{Di}_{2}\right)_{2} \mathrm{O}_{3}$ & & & & $\begin{array}{l}.26 \\
.50\end{array}$ & 3.04 & & $\begin{array}{r}.19 \\
2.05\end{array}$ \\
\hline$(\mathrm{Y}, \mathrm{Er})_{2} \mathrm{O}_{3}$. & 435 & & & .20 & & & 6.16 \\
\hline $\mathrm{ZnO}$ & 4.35 & 4.34 & 4.35 & 4.20 & 4.53 & \multirow{2}{*}{$\begin{array}{l}.70 \\
.44 \\
.32\end{array}$} & 3.58 \\
\hline & $\cdots$ & & & trace & 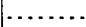 & & \\
\hline & .11 & .28 & .27 & $\therefore \ldots$. & & \multirow{10}{*}{$\begin{array}{r}.16 \\
.84 \\
\text { trace? } \\
\text { trace? } \\
1.96 \\
.02 \\
2.79 \\
.22 \\
.43 \\
.12 \\
.24\end{array}$} & trace \\
\hline $\mathrm{Mn}$ & .10 & .07 & (?) & $\cdots \cdots$ & 33 & & ii \\
\hline & .18 & .22 & .30 & .85 & $\begin{array}{r}.23 \\
25\end{array}$ & & trace \\
\hline & ........ & & .15 & .30 & \} .25. & & trace \\
\hline $\mathrm{H}_{2} \mathrm{O}$ & .68 & .67 & .68 & 1.21 & undet. & & undet. \\
\hline etc. & undet. & undet. & .38 & .05 & undet. & & undet. \\
\hline & .03 & .13 & .20 & $\begin{array}{l}.08 \\
(?)\end{array}$ & $\begin{array}{c}.13 \\
\ldots . .\end{array}$ & & …...... \\
\hline & ......... & & ...... & & ........ & & ......... \\
\hline & & & & & & & 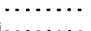 \\
\hline Insoluble.. & .04 & .14 & 1.40 & .10 & .06 & & .20 \\
\hline 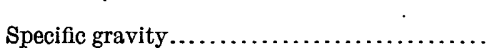 & $\begin{array}{c}98.21 \\
9.733\end{array}$ & $\begin{array}{c}99.37 \\
9.560\end{array}$ & $\begin{array}{c}99.24 \\
9.348\end{array}$ & $\begin{array}{c}100.67 \\
8.086\end{array}$ & $\begin{array}{c}98.91 \\
9.492\end{array}$ & $\begin{array}{c}99.82 \\
8.068\end{array}$ & 98.39 \\
\hline & & & & & & & \\
\hline
\end{tabular}

M. Nivenite from Llano County, Texas.

N. Somewhat altered uraninite from Villeneuve, Province of Quebec; Canada.

O. Massive uraninite from Johanngeorgenstadt, Saxony.

\begin{tabular}{|c|c|c|c|}
\hline - & M. & N. & 0. \\
\hline 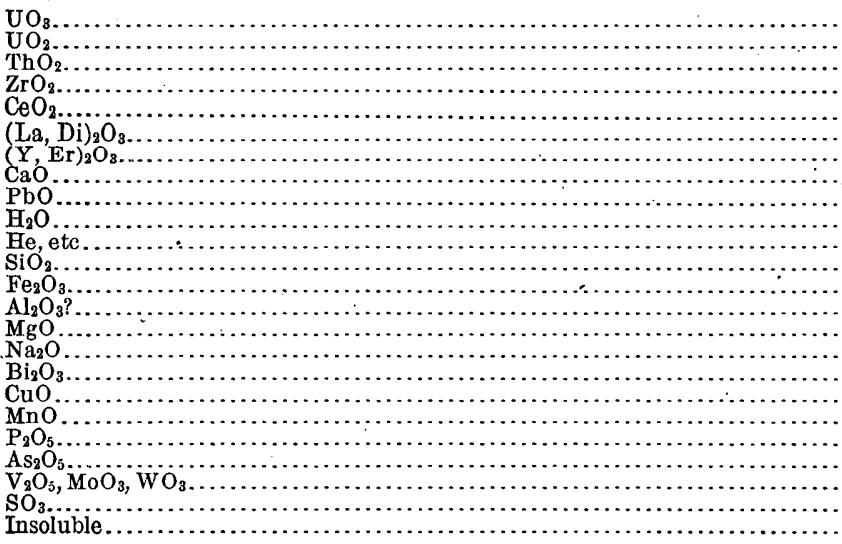 & $\begin{array}{r}20.89 \\
44.17 \\
6.69 \\
.34 \\
.34 \\
2.36 \\
9.46 \\
.32 \\
10.08 \\
1.48 \\
.08 \\
.46 \\
.14 \\
\ldots . . \\
\ldots . .\end{array}$ & 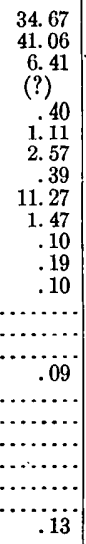 & $\begin{array}{r} \\
1.00 \\
6.39 \\
3.17 \\
\text { trace } \\
.50 \\
.21 \\
.20 \\
.17 \\
.31 \\
.75 \\
.17 \\
.09 \\
.06 \\
2.34 \\
.75 \\
.19 \\
\ldots \ldots . .\end{array}$ \\
\hline Specific gravity & $\begin{array}{r}98.28 \\
8.29\end{array}$ & $\begin{array}{c}99.96 \\
\cdots \ldots . .\end{array}$ & $\begin{array}{r}97.93 \\
6.89\end{array}$ \\
\hline
\end{tabular}


The following uraninites are all from Norway, and were analyzed for comparison with the American material.

P. Bröggerite from Anneröd, near Moss. Sp. gr., 8.893.

Q. From Elvestad. Sp. gr., 9.145.

R. From Elvestad. Sp. gr., 8.320.

S. From Skraatorp. Sp. gr., 8.966 .

T. From Huggenäskilen. Sp. gr., 8.930 .

U. Cleveite from Arendal. Sp. gr., 7.500.

V. From Arendal.

\begin{tabular}{|c|c|c|c|c|c|c|c|}
\hline • & P. & Q. & R. & S. & $\mathrm{T}$. & U. & V. \\
\hline 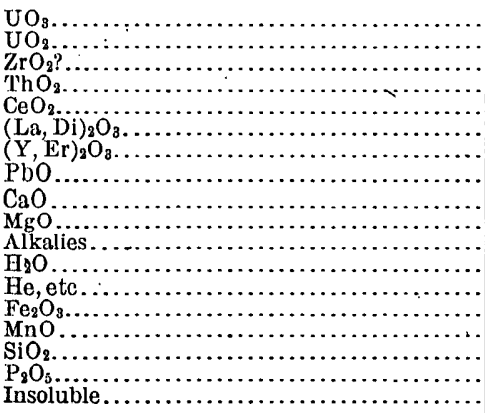 & $\begin{array}{r}30.63 \\
46.13 \\
.06 \\
6.00 \\
.18 \\
.27 \\
1.11 \\
9.04 \\
.37 \\
\operatorname{traces} \\
.74 \\
.17 \\
.25 \\
. .22 \\
.22 \\
.02 \\
4.42\end{array}$ & $\begin{array}{r}25.36 \\
50.74 \\
.08 \\
8.48 \\
.21 \\
.26 \\
1.10 \\
10.06 \\
.77 \\
\text { traces } \\
.73 \\
.18 \\
.21 \\
.06 \\
.38 \\
.04 \\
.45\end{array}$ & $\begin{array}{r}8.43 \\
\\
8.58 \\
.37 \\
.13 \\
.74 \\
.15 \\
.30 \\
\ldots . . . \\
.29 \\
\text { trace } \\
15.45\end{array}$ & $\begin{array}{r}32.00 \\
43.88 \\
\ldots \ldots \ldots \\
8.98 \\
.17 \\
.36 \\
.97 \\
9.46 \\
.36 \\
\text { traces } \\
.77 \\
.15 \\
.09 \\
\ldots . .7 \\
.53 \\
(?) \\
1.54\end{array}$ & $\begin{array}{r}35.54 \\
43.38 \\
\ldots \ldots . . \\
6.63 \\
.20 \\
.23 \\
1.03 \\
9.44 \\
.41 \\
.13 \\
.79 \\
.15 \\
.32 \\
\ldots . . . \\
.49 \\
\text { trace } \\
.42\end{array}$ & $\left\{\begin{array}{r}41.71 \\
24.18 \\
3.66 \\
\\
9.76 \\
10.54 \\
1.06 \\
.10 \\
.23 \\
1.23 \\
\text { undet. } \\
.03 \\
\ldots \ldots .90 \\
\ldots .90 \\
1.10\end{array}\right.$ & $\begin{array}{r}26.80 \\
44.18 \\
\ldots . .15 \\
4.15 \\
\text { none } \\
.67 \\
9.05 \\
10.95 \\
.61 \\
.04 \\
.15 \\
\text { undet. } \\
.18 \\
.24 \\
\ldots . .50 \\
.50 \\
\text { trace } \\
1.19\end{array}$ \\
\hline & 99.61 & 99. 11 & $99.5 \mathrm{i}$ & 99.26 & 99.16 & 94.50 & 98. 7.1 \\
\hline
\end{tabular}

XVII. CARBONACEOUS MINERALS.

\section{QUISQUEITE.}

A remarkable Bubstance associated with the patronite of Minasragra, Peru. Analyzed and described by W. F. Hillebrand.

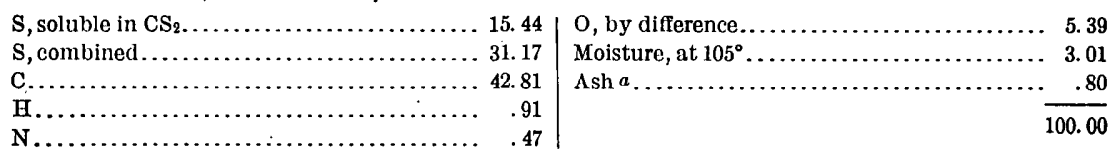





\section{INDEX.}

A.

Absarokite

Absarokose:

Acmite trachyte.

Actinolite.

Actinolite-magnetite schist.

Adamellite.

Adamellose...

$28,36,51,64,84,87,94,111,113$

Adinole.

Adirondackiase........................ $\quad 27$

Adobe soil............................ 229, 230

Egirite............................ 263

Isirite granite porphyry................ 120

Ægirite tinguaite....................... 21

A kerose............................ 13,

$15,16,17,59,67,80,81,86,101,102,105,120,126$

Alaskase............................ 128, 134

Alaskite............................. 164, 177

Alaskite porphyry....................... 177

Alaskose.......................... 30, 37, $43,70,71,78,99,106,112,116,134,144,152,177$

Albanose............................. 88 Albite............................... 23, 258

Albite diorite......................... 174

Albite schist............................ 23

Aleutite............................. 164,177

Allanite.............................. 273

Alluvium.............................. 228

Almandite........................... $\quad 269$

Almerose. . ............................. 181

Alsbachite............................ 22

Alsbachose................. 34,71,111, 142,171

Alunite.............................. 310

Alunogen........................... 309

Amadorose .......................... 22,153

A mblygonite......................... 303

Amiatose.....................

$80,111,113,117,130,131,132,133,134,152$

Amphibole ........ 77, 146, 156, 158, 264, 265, 266, 267

Amphibole-biotite granite............... 157

Amphibole gabbro...................... 156

Amphibole picrite........................ 158

Amphibole pyroxene rock .............. 158 Amphibolite............. 17, 19, 20, 25, 46, 150, 174 Amphibolitic schist.................... 163 Amygdaloid ............................... 37

Analcite.......................... 90, 104, 283

Analcite basalt...................... 88, 104

Andalusite hornfels ..................... 159

Andalusite schist....................... 159

Andesite......................... $35,62,64,66,67 ; 72,73,76,80,92,98,105,108$, $113,117,119,121,123,124,128,129,130,133,135$, $140,141,142,145,148,160,167,168,173,175,176$ Andesite-basalt:
Pảge.

Andesite porphyry..................... 80

Andesitic perlite........................ 132

Andesitic tuff......................... 142

Andose .......................... 16,21, $60,62,63,64,65,73,79,81,84,86,93,95,103,108$, $109,112,115,122,124,133,141,142,143,144,146$, $151,152,153,160,163,164,168,169,172,176,177$ Andradite........................... $\quad 269$ Anhydrite........................... 307

Annite............................ 290

Anorthite............................ 259

Anorthoclase. ..................... 52,82, 258

Anorthosite.......................... 26

Antlerite............................ 308

A patite.............................. 302

A plite.............. 22, 23, 64, 78, 92, 95, 148, 157

A plitic granite....................... 86, 100

A pophyllite........................... 281

A porhyolite.......................... $\quad 29$

Argillite.............................. 149

Arizonite .......................... 298

Arkose gneiss...........................

Arsenopyrite......................... 244

Asperite ............................ 160

Astrophyllite.......................... 299

A ugite.................... 43,98, 104, 123, 262, 263

A ugite aleutite........................ 177

Augite andesite...............6 61,67,98, 167, 175

$\Lambda$ ugite andesite porphyry $\ldots \ldots \ldots \ldots \ldots \ldots 661,64$

A ugite belugite....................... 177

A ugite-biotite andesite................. 130

Augite-bronzite andesite................... 176

Augite diorite...................... 105,116

A ugite granite.......................... 29

A ugite latite.......................... 155

A ugite-mica syenite ...................... 98

Augite-microcline granite ................ ' 38

Augite porphyrite...................... $\quad 119$

Augite porphyry $\ldots \ldots \ldots \ldots \ldots \ldots \ldots \ldots \ldots \ldots \ldots \ldots, 75$

Augite syenite.................. 65, 112, 155

Augite syenite porphyry ................. 65

Augite vogesite...................... 59,87

Auvergnase......................... 24

Auvergnose ............. 17, 24, 25, 28, 30, 34, $36,46,47,48,49,50,55,59,73,75,83,123$ $126,127,143,144,159,161,163,170,173,178$

Axinite........................... 277

B.

Baltimoriase....................... 30

Baltimorose......................... $\quad 30$

Banakite.......................... 68, 69

Banatite......................... 77

Bandose......................... 25, 32, $42,56,80,98,141,143,149,153,176,179,180$

Barkevikite..................... 90,266 
Page.

Basalt .... 24, 28,41, 43, 63, 73, 74, 75, 86, 88, 96, 97, 98 , $103,104,118,122,123,124,126,127,132$, $133,135,142,143,144,146,147,160,163$, $164,166,168,169,170,171,172,173,178$

Basaltic glass.

Basanite............................. 41,124

Bastite............................... 162

Bastnüsite........................... 256

Bauxite............................. 254

Beemerose............................ 28

Beerbachose............. 13, 47, 135, 146, 169, 170

Belcherose............................ 18

Belugite............................. 177

Bementite........................... $\quad 297$

Beryl................................ 267

Bindheimite......................... 307

Biotite............................. 77,288

Biotite andesite....................... 130

Biotite-augite latite...................... 155

Biotite dacite...................... 126,130,131

Biotite diorite......................... $\quad 32$

Biotite gneiss.......................... 49

Biotite granite............. 15,22,33,34,134,157

Biotite-hornblende-quartz latite ........... 134

Biotite latite.......................... 115

Biotite-orthoclase gabbro.................. 94

Biotite porphyrite...................... 109

Biotite-pyroxene andesite ................ 130

Biotite-quartz monzonite................ 133,148

Biotite rhyolite......................... 134

Biotite trachyte......................6 67,102

Bismuthinite......................... $\quad 242$

Bole.................................. .298

Boltonite............................ . 18

Boothite............................. 308

Borolanose......................... 87,88,89

Bostonite............................... 14

Bournonite............................ 246

Bravoite............................. $\quad 243$

Brochantite............................ 308

Bröggerite............................ 315

Bronzite............................ 31,260

Bronzite norite $. . \ldots \ldots \ldots \ldots \ldots \ldots \ldots \ldots . .46$

Bronzite-olivine aleutite................... 164

Brucite............................. 254

Brucite serpentine....................... 174

Calamine............................. 274

cCalaverite............................ 247

Calcareous sinter...................... . 201,202

Calcareous tufa......................... 201

Calcite.................................. 255

Californite............................ 271

Camptonite .................. 15, 17,73, 126, 150

Camptonose ...................... 17, 26,

$27,40,45,48,63,66,67,73,96,97$, $112,124,128,143,146,147,148,155$

Cancrinite........................... 268

Carnotite.............................. 305

Cascadose............................. 88

Casseliase............................... $\quad 39$

Casselose............................... 43

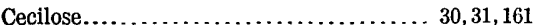

Cement rock...................... 190, 194, 195 Chabazite............................. 283

Champlainiase .......................... $\quad 27$

Chenevixite.
Chert........................ $\begin{gathered}\text { Page. } \\ \text { Ch, 55, } 186\end{gathered}$

Chiastolite schist...................... 159

Chlorite.............................. 293

Chloritic granite........................ 17

Chloritoid ........................... 34, 291

Chotose.............................. 91

Chromite .......................... 233,253

Chrysolite............................. 270

Chrysotile........................... 294

Cimolite............................... 297

Clay ............................ 219,220 , $221,222,223,224,225,226,227,228,229,230,231$

Cleveite.............................. 315

Clinoclasite .......................... 306

Clinochlore............................. 293

Colemanite.......................... $\quad 300$

Conichalcite............................ 306

Cookeite............................. 288

Cookose........................... 18, 55,75

Copiapite............................ $\quad 310$

Coquina............................... 192

Coral ............................... 193

Coral rock...................... 193, 209, 210

Cordierite hornfels....................... 17

Coronadite........................... 255

Corsose............................... 156

Cortlandtite $\ldots \ldots \ldots \ldots \ldots \ldots \ldots \ldots \ldots \ldots, 18,25,31$

Cortlandtose.......................... 31

Cosalite............................... 246

Cotton rock.......................... 186

Covellite...............................

Cryolite.............................. $\quad 250$

Cryophyllite........................... 288

Culsageeite........................... 291

Cuprobismutite........................ $\quad 245$

Cuprodescloizite........................... 304

Custerose............................... 104

Cyrtolite.............................. 276

D.

Dacase.................. 120

Dacite.............................. 58

$62,106,123,126,130,131,132,139,140,144$

Dacitè porphyry ............... 71, 136, 167, 171

Dacite tuff.......................... $\quad 140$

Dacose.............. 72, 80, 84, 124, 127, 152,176

Danburite............................. $\quad 277$

Dargase............................. 134

Datolite.............................. $\quad 277$

Descloizite............................ 304

Devonite............................... 40

Diabase........................... 14, 17,

$21,23,34,36,48,50,51,65,115$,

$126,138,149,159,161,164,172$

Diabase-diorite........................ 161

Diabase-porphyrite..................... 81, 163

Diabase porphyry ..................... $\quad 148$

Diabasic basalt......................... $\quad 96$

Diabasic norite.......................... $\quad 26$

Diallage............................ 51, 262

Diallage gabbro........................ 55

Diaspore............................... ${ }_{253}$

Diopside.......................... 31, 58, 261

Diorite............................. 16, 23,

$25,32,36,38,46,59,60,61 ; 64,65,79,81,84,86$, $92,93,94,95,105,111,112,116,121,126,133,136$, $138,146,147,148,151,153,155,159,164,174,176$

Diorite-monzonite ..................... 115 
Diorite-porphyrite

Diorite porphyry.

Page.

Dolerite.

Dolomite

$197,108,200,202,203,204,205,206,209,255$

Dufrenite............................ $\quad 303$

Dumortierite..................... 165, 174, 277

Dunite ............................... 37

Dunose............................. 37, 178

Dysluite............................. 252

Elæolite.............................. 15,267

Elæolite syenite................... 14, 15, 28, 82

Elpasolite............................. $\quad 250$

Embolite.............................. 249

Emmonsite.......................... 311, 312

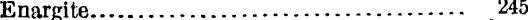

Enstatite......................... 18, 237, 260

Enstatite diabase porphyry............... . 98

Epidote.............................. 272

Epidote-chlorite schist................... $\quad 37$

Erinite.............................. 305

Essexite............................... 44

Essexose................. 41, 44, 59, 83, 102, 178

Etindase............................. 41

Evansite........................... 304

Feldspar.....................

$20,21,23,50,51,52,55,56,82$, $109,123,166,257,258,259,260$

Feldspar-epidote rock................... 138

Feldspar porphyry ..................... 86

Feldspathic conglomerate................. 21

Felsite................................ 35

Felsophyre.......................... 35

Fergusite.............................. 89

Fergusose............................. 89

Ferrodolomite....................... $\quad 199$

Fibrolite............................. 274

Fluorite............................ 250

Franklinite............................. 253

Freieslebenite........................ 246

Friedelite............................. 271

Fuchsite............................. 286

Fulgurite........................... 166

G.

Cabbro......................... $25,26,27,30$,
$31,32,38,40,46,48,55,56,63,64,65,66$, $82,92,94,115,124,151,156,162,170,172$

Gabbro-diorite .................. 32,49,56,64

Gabbro porphyry $\ldots \ldots \ldots \ldots \ldots \ldots \ldots, 63,66,115,124$

Gadolinitė............................ 275

Gahnite............................... 252

Garnet............................ 38, 269

Gauteite............................ $\quad 87$

Gearksutite......................... 251

Gedrite.............................. 266

Genthite............................. '295

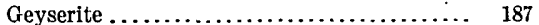

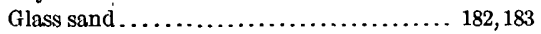

Glaucodot............................... 244

Glauconite........................... 296

Glaucophane schist ................. 160, 1.61

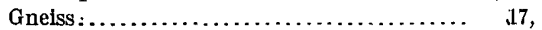

$23,28,29,30,33,34,37,45,46,49,56,103,153$
Gneissold granite.............

Gold ............................... 241

Goldfieldite............................ 248

Gordonose.......................... 38

Granite................. 14, 15, 17, 22, 25, 29, 33, $34,38,40,41,46,52,53,77,78,79,86,99$, $100,102,116,121,128,134,144,151,157$

Granite gneiss....................... 23,99,103

Granite porphyry . $65,80,83,84,110,125,128,137,157$

Granite-syenite porphyry ................ 90

Granitite...................... 80,99,125, 152

Granitoid gneiss....................... 17

Granodiorite ........................ $96,144,146,149,150,151,152,167,171,172,173$

Granodiorite porphyry................. 94,172

Granulite............................. 148

Graywacke............................ 51

Greenalite............................... 54

Greenstone..................... 45, 49, 167

Griphite........................... 303

Grorudite........................... 44,120

Grorudose ........................... 44

Grossularite........................ 269

Grossularite hornfels................... $\quad 92$

Grossularite hornstone................... 92

Grünerite-magnetite schist............... . $\quad 45$

Guitermannite...................... 247

Gypsite............................. 308

Gypsum........................... 307

Gyrolite.............................. 281

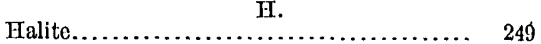

Fallite................................. 292

Halloysite.......................... 296,297

Halotrichite.........................., 309

Harzose...................... $38,77,79$, $92,94,113,119,120,130,131,133,148,149,155$

Hessite............................., 247

Hessose..... 17, 25, 26, 27, 30, 32, 40, 46, 51, 55, 56, 92, $94,115,131,132,133,135,139,140,142$, $144,145,146,153,156,164,170,172,177$

Hetærolite.......................... 254

Heulandite.......................... 282

Highwoodose........................ 87,155

Holyokeite.......................... 22

Hornblende...................... 20,90,266

Hornblende andesite. ... 62, 72, 129, 135, 140, 145, 176

Hornblende andesite porphyry ............ 92

Hornblende-augite andesite .............. 66

Hornblende basalt.................... 144

Hornblende-biotite andesite.............. 130

Hornblende-biotite-quartz diorite........... 133

Hornblende diorite ..................... 25, 32

Hornblende gabbro..................... 46

Hornblende gneiss...................... 30

Hornblende granite...................... 17

Hornblende granitite.................... 80

Hornblende-mica andesite...............66, 62,72

Hornblende-mica andesite porphyry....... 64,72

Hornblende-mica porphyrite............. 111

Hornblende-mica porphyry................ 61

Hornblende-mica syenite................. 86

Hornblende microdiorite.................., 93

Hornblende norite...................... 25

Hornblende paisanite..................... 15,16

Hornblende picrite.................... 75,83 
Page.
Hornblende porphyrite......... 93,116,119,127, 149 Hornblende porphyry .

Hornblende-pyroxene andesite . ..... $62,130,145,148$ Hornblende-pyroxene-biotite-quartz latite... 133

Hornblende-quartz andesite............... 133

Hornblende-quartz diorite................ 164

Hornblendite......................... 25

Hornfels.......................... 17,92,159

Hornstone........................... 82,92

Hübnerite........................... $\quad 312$

Hydronephelite....................... 285

Hypersthene.................. 55, 108, 166, 261

Hypersthene andesite................. 76, $108,128,135,141,142,145,148,173,175$

Hypersthene-augite andesite.............. 168 Hypersthene basalt.................... 166,169

Hypersthene gabbro................. 30,32, 55

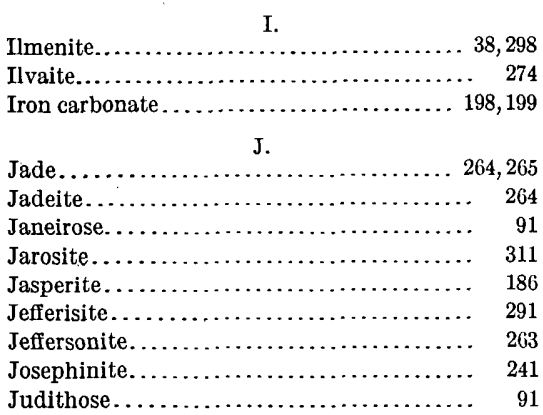
$99,123,129,171,175$

Kamerunose 83

Kaolin ............................ 223, 296

Kaolinite........................... 296

Kedabekase........................... 126

Kedabekose......................... 32, 162

Kentallenose..................... 59,61,67, $69,73,75,79,87,112$

Keratophyre......................... 21 Kerrite............................. $\quad 291$

Kersantite........................... 73

Ki ${ }_{1}$ auase............................ $\quad \mathbf{1 7 0}$

Kilauose........................... $\quad 86$

Kimberlite......................... $\quad 38$

Kleinite............................. $\quad 249$

Knoxvillite......................... 310

Kotschubeite........................ $\quad 293$

Kyanite............................ 274

Kyanitic schist..................... 180

L.

Lamarose. $66,67,75$

Lamprophyre.......... 74, 75, 100, 112, 115, 117 Lassenose. ................... $22,61,62,65$, $70,71,72,78,80,84,86,96,106,108,109,110,111$, $115,116,123,125,127,128,132,134,136,139,140$, $146,148,151,154,157,168,169,171,175,177$ Latite................ 103, 113, 114, 115, 123,155 Latite-phonolite..................... 101, 102

Laumontite........................ 283

Laurdalose........................... 42,117

Laurvikose...................... 59, 65, 105, 119

Lava soil......................... 229, 230
- $\quad$ Page

Lennilite................................. ${ }_{292}^{274}$

Lepidolite............................. 287

Lepidomelane.......................... $\quad 290$

Lermondose ............................. 14

Leuchtenbergite........................ 293

Leucite............................. $\quad 260$

Leucite absarokite...................... 66,67

Leucite banakite....................... 69

Leucite basalt..................... $88,91,132$

Leucite shoshonite...................... 68, 88

Leucitite............................ 91

Levynite.................................. 283

Lherzolite............................. 30

Liebenerite........................... 286

Limburgite............................ $\quad 27$

Limburgose...................... 41, 124

Limestone..... 12, 189, 190, 191, 193, 194, 195, 196, 197, $198,200,201,202,203,204,205,206,207,208,209$ Liparose.......................... 13, 15, $21,33,40,41,43,52,53,70,80,86,90,99,105,106$, $109,116,120,122,124,128,134,160,165,173,177$

Litchfieldite............................. 14

Lithographic stone.................... $\quad 194$ Lithoidite........................... 70 Loess....................... 223, 224, 225, 227 Löllingite............................ $\quad 244$ Lucasite................................ 292

Ludwigite........................... 301

Lujavrose........................... 41

M.

Mackintoshite........................ 276

Madupite............................. $\quad 57$

Madupose........................... 57

Magdeburgose............... 36, 46,99,106, 152

Magnetite........................ 27, 51, 56,253

Malignose.......................... 83

Manganosite......................... 252

Marble........... 189, 190,191, 192, 194, 202, 206, 255 Margarite .............................. 291

Maricose............................ 30

Mariposite........................... 286

Mariposose............................ 157

Marl......................... 192, 198, 200

Marquettose........................... 45

Meerschaum........................... 295

Melaphyre tuff ....................... 153

Melilite-nephelite basalt.................. 179

Melonite............................. 248

Mesolite................................... 285

Meta-andesite......................... 154

Meta-andesite tuff ....................... 147

Metabasalt............................ 47,136

Metacinnabarite........................ $\quad 242$

Metadacite............................. 154

Metadolerite.......................... 47

Metagabbro........................... $\quad 170$

Metaquartz diorite..................... $\quad 38$

Metarhyolite...................... 35, 136, 144

Meteoric iron ........................ 239,240

Meteoric stone....................... 232-238

Miaskose............... 21, 57, 100,101,120,123

Mica............... 34, 94, 153, 157, 158, 164, 285-290

Mica basalt........................... 88, 127

Mica dacite............................. 106

Mica dacite porphyry .................. 71,72 
Yage.

Mica diorite..................... 46,92

Mica diorite porphyry .................. 49

Mica dolerite.......................... 131

Mica gabbro........................ 63

Mica gabbro porphyry.$\ldots \ldots \ldots \ldots \ldots \ldots \ldots .63$

Mica gneiss....................... 30

Mica-hornblende gabbro. . . . . . . . . ${ }_{25}$

Mica peridotite ................... $\quad 39$

Mica schist. . . . . . . . . . . . . . 46, 56, 103, 159

Microcline ...................... 257, 258

Micropegmatite....................... 157

Mihalose........................... 132

Minette. ................... 28, 85, 128, 174

Minnesotase........................... 158

Missourite.......................... 88

Mixite......................... 300

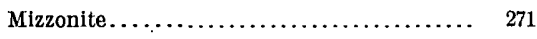

Molybdic ocher..................... 313

Monchiquite.................. 85, 87, 102

Monchiquose ................. 82,85,88, 104

Montanose.......................... 89

Montroydite...................... 252

Monzonase.................... 47, 170

Monzonite.......6 64, 85, 89,91, 112,119, 133, 137, 148

Monzonitic porphyry................ 113, 120

Monzonose............... 38, 61, 68, 69, 74, 84, $85,87,91,94,97,102,103,106,112,120,127,155$

Morencite......................... 298

Muscovite......................... 285, 286

N.

Natrojarosite $\ldots \ldots \ldots \ldots \ldots \ldots \ldots \ldots \ldots \ldots \ldots, 311$

Natrolite. ............................. 284

Nepheline basalt. ................ 42,43,101

Nepheline basanite.................... 124

Nepheline-melilite basalt.

43

Nephelino syenite ................. 28, 101

Nepheline tephrite................... 118

Nephelite (elæolite) .................. 15, 267

Nephrite....................... 265

Nevadite.......................... 109

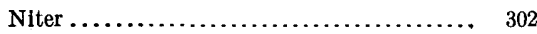

Nivenite.......................... 314

Nordmarkite . . . . . . . . . . . . . . . . . . 15,16

Nordmarkose....... 14, 44, 67, 82, 90, 120, 123, 178

Norite.................... 25, 26, 32,46, 59

Noselite syenite porphyry ............. 120

Novaculite.

Obsidian

O.

$70,78,124,160,163,165$

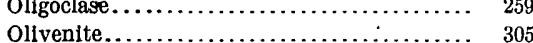

Olivine.................. 38,55, 167, 235, 270

Olivine basalt. ............. 24,118, 133, 146, 164

Olivine diabase................48,51,126

Olivine dolerite. .................... 131

Olivine gabbro............. $31,55,82,94,156$

Omeose..................... 99,106,120,128

Oolitic sand ......................... 206

Opal.............................. 187

Orendase............................. 35

Orendite.......................... 58

Orendose..................... 57,58

Ornose................. 13, 21, 147, 149, 160, 161

Orthoclase. ....................... 109, 257

Orthoclase gabbro.

Orthoclase gabbro-diorite $\ldots \ldots \ldots \ldots \ldots \ldots \ldots \quad 64$

Ottrelite phyllite rock .................. 34

Ouachitite........................ 41

Ourose....................... 87, 102

Pachnolite.$\ldots \ldots \ldots \ldots \ldots \ldots \ldots \ldots \ldots \ldots \ldots .250$

Painterite........................ 292

Paisanite. .......................... 15, 16

Palagonite $. \ldots \ldots \ldots \ldots \ldots \ldots \ldots \ldots \ldots \ldots \ldots, 23$

Pandermite...................... 300

Pantellerite. . . . . . . . . . . . . 41

Pantellerose........................ 44, 129

Patronite............................ 245

Pectolite........................... 264

Pegmatite........................ 14

Peridotite. . . . . . 14, 27, 38, 39, 45, 76, 104, 147, 166, 178

Perknite ......................... 158

Perlite. . . ...................... 72, 132

Petalite........................ 257

Petzite........................... 247

Phlegrose.............. 15, 16,44, 102, 105, 120

Phlogopite....................... 57, 289

Phonolite............. 42,57,59,100,101,123

Phthanite........................ 218

Phyllite....................... 17

Picrallumogene..................... 309

Picrite. .................... 75, 83, 158

Picrite porphyry $\ldots \ldots \ldots \ldots \ldots \ldots \ldots \ldots \ldots . \quad 47$

Picrolite.......................... 294

Piedmontite....................... 272

Pisanite........................... 308

Pitchstone................. 24, 78, 86, 106, 122

Placerose................. 150, 151, 154, 176, 180

Plagioclase basalt.......... 41, 103, 124, 135, 178

Plagioclase gneiss..................... 153

Plagioclase-scapolite-biotite rock.......... 178

Pleonaste........................ 252

Plumbojarosite...................... 311

Polydymite.......................... 242

Porphyrite.......... 74, 80,81, 109, 111, 119, 152

Porphyrite-diorite.................... 111

Porphyry............ 14, 40, 108, 115, 120,127

Powellite............................ 312

Prehnite......................... 273

Priceite........................ 300

Prochlorite. . . . . . . . . . . . . . . . . . . . . . 294

Prosopite.......................... 251

Protovermiculite....................... 292

Prowersose..................... 14,117

Pseudodiabase ....................... 160

Pseudodiorite...................... 160

Pseudoleucite-sodalite tinguaite .......... 91

Psilomelane........................... 254

Ptilolite......................... 282

Pulaskite.......................... 44

Pulaskose..... 59,67,69,87,90,97, 103, 105, 115, 120

Pumice..................... 165, 179, 180

Purpurite....................... 303

Pyrope........................ 263

Pyrophyllite....................... 297

Pyroxene........ 74, 94, 115, 117, 166, 261, 262, 263

Pyroxene andesite...... 62, 72,73, 113, 124, 135, 140

Pyroxene aplite.................... 95

Pyroxene-biotite andesite.............. 130

Pyroxene-hornblende andesite......... 117, 130 


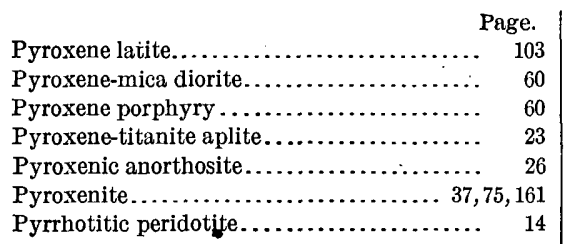

\section{Q.}

Quartz-alunite rock ..................... 107

Quartz andesite......................... 148

Quartz-augite rock..................... 165

Quartz banakite........................ 69

Quartz basalt..................... 132,142, 143

Quartz-biotite-garnet gneiss............... 28

Quartz diabase........................ $\quad 23$

Quartz-diaspore rock.................. 107

Quartz diorite........... 25, 81, 83,94, 121, 146, 151

Quartz diorite gneiss................... 152

Quartz diorite porphyry............. 64, 121, 148

Quartz gabbro......................... 38

Quartz-hornblende-mica porphyrite....... 109

Quartzite............................. 52,53

Quartzite gneiss........................ 23

Quartz keratophyre.................... 52

Quartz latite................... 103,113,114,123

Quartz-mica diorite...........61,64,126,148,159

Quartz-mica diorite porphyrý............61,64

Quartz-mica-hornblende diorite................. 32

Quartz monzonite.................. 77,79,84 $92,93,95,96,97,114,115,117,125,126,152$

Quartz-muscovite rock................. 133,134

Quartz norite gneiss..................... 56

Quartz-orthoclase-augite rock............... $\quad 29$

Quartz pantellerite....................... 41

Quartz porphyrite............. 109,111,149,152

Quartz porphyry........ 15,30,36, 50,78, 86,118, 176

Quartz-pyroxene diorite................. 155

Quartz-pyroxene latite................... 114

Quartz-pyroxene-mica diorite............. 60

Quartz schist.......................... 34

Quartz-sericite schist..................... 17

Quartz syenite................... 65,90,124

Quartz syenite porphyry................. 90

Quartz-tourmaline porphyry.............. 86

Quartz trachyte....................... 13

Quisqueite............................ 315

Redingtonite.

R.

Rhyolite............. 13,36,37,43,70,71,78, 80, 86, $96,103,106,109,111,116,117,118,122,130$, $132,134,138,139,145,152,163,165,168,173$

Rhyolite-dacite obsidian

Rhyolite pitchstone.

78

Rhyolite porphyry........

Rhyolitic glass......................... 107,122,132

Rhyolitic obsidian...................... 163

Rhyolitic perlite........................ 72

Rhyolitic tuff...................... 86, 106,139

Rhyolitic vitrophyre.................... 117

Riebeckite granite...................... 100

Riesenase............................... 134

Riesenose.......................... 106,108

Roscoelite............................. 290

Rossweinose...................... 46,159,160
Page.

Rowlandite........................... 276

Rutile............................. 253

S.

Salemose............................ 105

Samarskite.......................... 300

Sandstone....... 12, 13, 39, 182, 183, 184, 185, 186, 227

Sanidine.............................. 109

Sanidine-quartz rhyolite................. 71

Saponite.............................. 295

Saussurite ............................. 272

Saussurite gabbro..................... $\quad 48$

Saxonite............................... 166

Scapolite-pyroxene aplite................ 95

Schist.... 17, 23, 30, 34, $35,37,45,46,49,50,56,57,180$

Scolecite.............................. 284

Scorodite.............................. 307

Sepiolite............................... 295

Sericite schist.................... 30,35, 45

Serpentine........ 18, 19, 146, 161, 162, 169, 172, 294

Shale.................. 12,39, 214, 216,217, 218

Shonkinite..................... 85, 88, 89, 91

Shonkinose............................ 85, 88

Shoshonite.......................... 68, 69

Shoshonose........................... 46,

$63,66,67,68,69,75,81,89,93$,

$94,98,124,126,128,155,176$

Siderophyllite.......................... 290

Siliceous sinter...................... 187, 188

Sillimanite............................. 274

Sitkose.............................. $\quad 176$

Slate............ 53,211,212, 213, 214, 215, 216, 218

Smaragdite........................... 30

Smithsonite.......................... $\quad 256$

Soda granite......................... 52, 157

Soda granulite........................... $\quad 157$

Sodalite................................ 90,268

Sodalite syenite........................ 90

Soda niter............................ 301

Soda syenite........................... 165

Soda syenite porphyry................... 155

Sölvsbergite......................... 82, 120

Sölvsbergite porphyry................. 87

Spessartite............................. $\quad 269$

Spherulite. ............................... 107

Spilosite............................ 47, 48

Spinel............................... 252

Staurolite ............................. $\quad 275$

Stilbite............................... 282

Stromeyerite......................... 243

Syenite.................... 28,65,81,84,86,

$88,89,91,98,102,106,112,124,155,165$

Syenite-aplite porphyry ................ 120

Syenite porphyry... 14, 44, 65, 84, 87, $97,115,120,124$

Sylvanite........................... 247

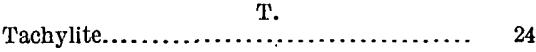

Talc.................................. 295

Tallow clay.......................... 225, 226 Tehamose..... 22, 34, 70, 78, 92, 138, 139, 152, 163, 164

Tengerite............................ 256

Tephrite............................. 118

Terlinguaite............................ $\quad 249$

Teschenite............................... 13

Tetradymite............................ 248 


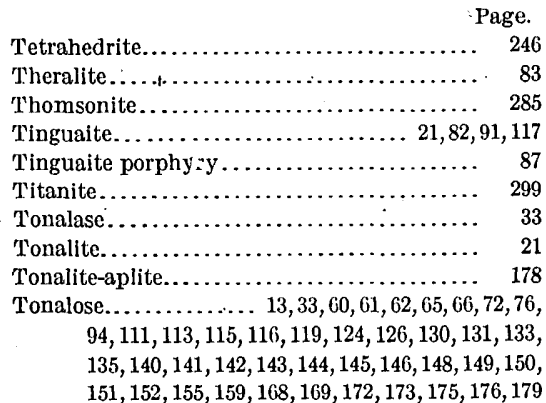

Topaz............................ 273

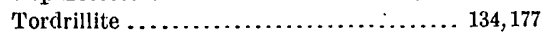

Toscanose.......................... 15,

$16,22,23,33,35,38,40,50,52,64,70,72,78,79$, $80,83,84,92,93,95,103,105,106,108,109,110$, $111,113,115,117,118,122,125,126,130,132,134$, $138,144,145,148,152,155,157,163,164,165,177$

Tourmaline...................... 278, 279,280

Tourmaline-biotite schist. ............ 57

Trachyandesite ..................... 87

Trachydolerite .................... 102, 126

Trachyte...... 13, 67, 102, 103, 105, 117, 124, 128, 178

Trachyte-andesite.................. 67

Trachytic phonolite.................... 101

Trachytic rhyolite. . . . . . . . . . . . . . . 71

Travertine......................... 201,202

Tremolite...................... 264,265

Triplite............................ 303

Troilite........................ 239, 240

Tscheffkinite......................... 299

Tuff.............. 13,71, 86, 106, 139, 163,171

Tuolumnose.................. 22, 155, 174

Turquois............................. 304

Tyrolite........................... 306

Tysonite.......................... 251

U.

Ulexite......................... 301

Umptekose.................. 15, 82,178

Uralite diorite. . . . . . . . . . . . . . . . 147

Uraninite..................... 313,314,315

Urao............................ 256

Uvaldose................. 42,43,158, 179

$\mathrm{V}$.

Vaalase.......................... 113, 126

Vaalose....................... 23, 151, 172

Varingose........... $\begin{aligned} & \text { Page } \\ & 23,160\end{aligned}$

Vermiculite ...................... 291, 292

Vesuvianite........................ 271

Viezzenose.......................... 28

Vitrophyre................... 115,131

Vogesite...................... 59,87,102

Volcanic ash . . . . . . . . . . . . . . . 57,165

Volcanic bombs...................... 142,181

Volcanic dust. . . . . . . . . . . . . . . . 179, 180

Volcanic glass. ...................... 76

Volcanic sand . . . . . . . . . . . $76,97,142,179$

Vulcanose........................... 35,154

W.

Waluewite ........................... 291

Warrenite....................... 246

Warwickite........................ 301

Websterite.................... 30,31,37

Websterose...................... 37

Wehrlite................. 18,46,76

Wehrlose..................... 75,76

Willemite......................... 270

Windsorite........................ 16

Wollarstonite...................... 264

Wollastonite gneiss.................. 153

Wyomingase..................... 91,174

Wyomingite...................... 57

Wyomingose.................... 57,85

$\mathbf{X}$.

Xanthitane......................... 299

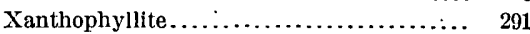

Xenotime......................... 302

$\mathrm{Y}$.

Yellowstonose............. 17,30,33,61, 62, 64, $65,72,93,96,98,108,109,110,111,113,115,130$ $135,136,140,142,144,148,149,160,170,171,179$

Yogoite........................... 91

Yttrialite........................ 275

Yukonite........................... 178

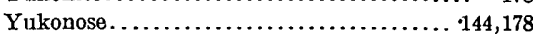

Z.

Zinkenite......................... 245

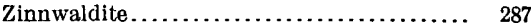

Zoisite........................ 272

Zoisite-hornblende diorite............... 126

Zunyite.......................... $\quad 269$ 